\title{
Numerical simulation of low salinity waterflood on fractured chalk outcrop-based models
}

Andrianov, Nikolai; Nick, H. M.

Published in:

InterPore2019 Valencia - Book of Abstracts

Publication date:

2019

Document Version

Publisher's PDF, also known as Version of record

Link back to DTU Orbit

Citation (APA):

Andrianov, N., \& Nick, H. M. (2019). Numerical simulation of low salinity waterflood on fractured chalk outcropbased models. In InterPore2019 Valencia - Book of Abstracts (pp. 34-35). [416] Universitat Politecnica De Valencia.

\section{General rights}

Copyright and moral rights for the publications made accessible in the public portal are retained by the authors and/or other copyright owners and it is a condition of accessing publications that users recognise and abide by the legal requirements associated with these rights.

- Users may download and print one copy of any publication from the public portal for the purpose of private study or research.

- You may not further distribute the material or use it for any profit-making activity or commercial gain

- You may freely distribute the URL identifying the publication in the public portal

If you believe that this document breaches copyright please contact us providing details, and we will remove access to the work immediately and investigate your claim. 


\section{InterPore2019 Valencia}

Monday 06 May 2019 - Friday 10 May 2019

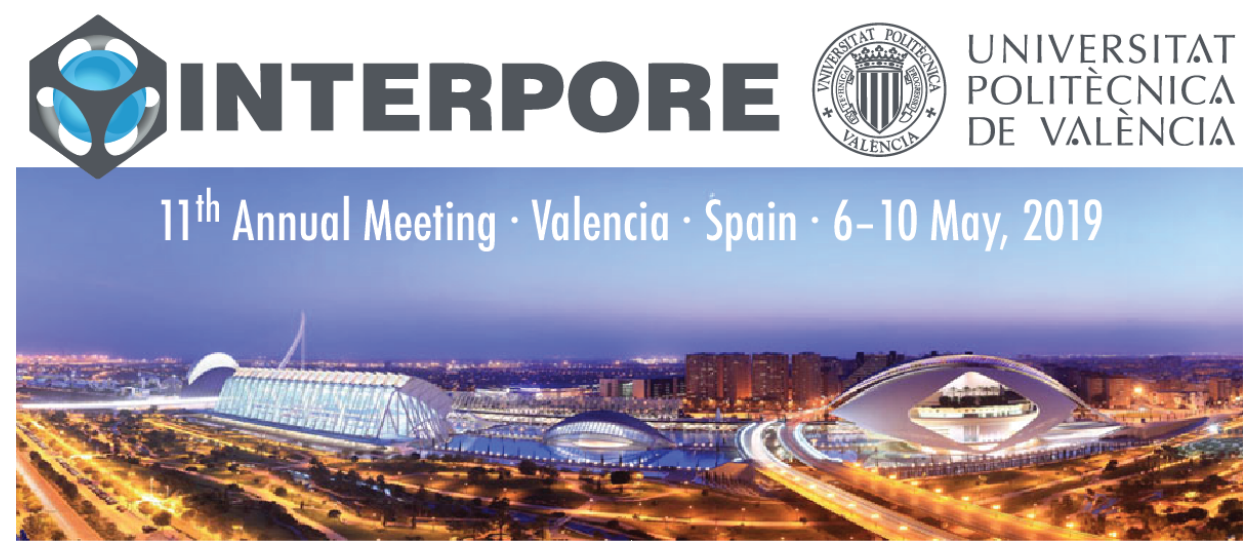

\section{Book of Abstracts}



This is an auto-updated version of the submitted contributions for InterPore2019 Valencia 


\section{Contents}

Dynamics of foam flow in a rock fracture: Effects of aperture variation on apparent shear viscosity and bubble morphology $169 \ldots \ldots \ldots \ldots \ldots$

Stochastic upscaling for hydraulic conductivity based on laboratory experiments 969 . . 1

Modeling and simulation of transport and reaction processes through digitally generated

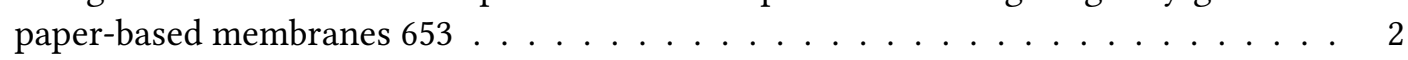

A Multiscale Study on the Effects of Dynamic Capillary Pressure in Two-Phase Flow in

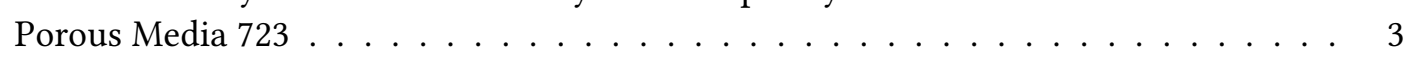

The Behavior of Liquid Percolation and Water Uptake in Catalytic Porous Materials used in Proton Exchange Membrane Fuel Cells (PEMFC) $171 \ldots \ldots \ldots$. . . . . . . 4

new correlation to predict effective thermal conductivity of open cell metal foams 736 . 5

A high-order conservative finite element method for Darcy flow problem with transport

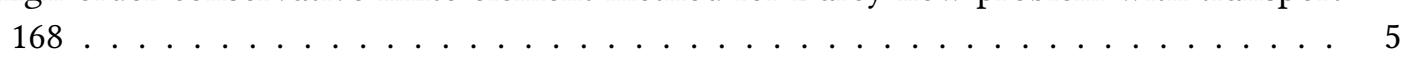

Dynamic Pore-Scale Study of Water-Salinity Effect on a Moving Oil Droplet 426 . . . . . 6

Upscaling of coupled free-flow and porous-media-flow processes $16 \quad \ldots \ldots \ldots$. . . . . 7

Application of Laplace-Differential Transform method to transport problems in porous me-

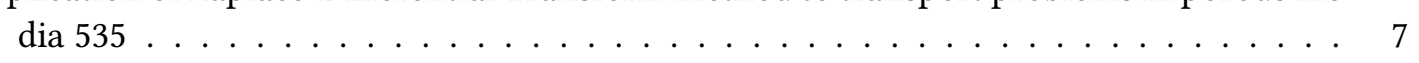

Modeling of transport phenomena and pollutant degradation during DBD discharge used for soil remediation $774 \ldots \ldots \ldots \ldots \ldots$

Modeling Water Treatment by Capacitive Deionization Using Multiphysics Pore Network

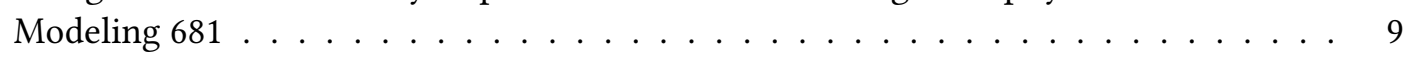

Pore-scale investigation of the surface roughness impacts on reaction rate $801 \ldots$. . . . 10

Fracture-matrix flow interaction characterizations using a temporo-ensemble PIV method

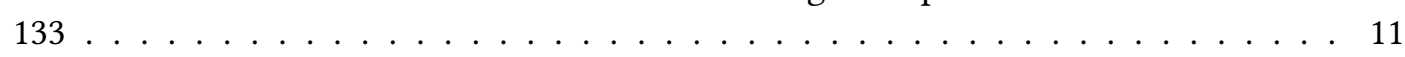

Two-equation continuum model of drying: a limiting case of immobile phase 60 . . . . . 12

Adaptive fixed-stress iterative coupling schemes based on a posteriori error estimates for Biot's consolidation model $613 \ldots \ldots \ldots \ldots \ldots$

Porosity of Pervious Concretes Based on 2D scan images 607 . . . . . . . . . . . . . 13 
MULTIFUNCTIONALITY OF LIQUID-FILLED POROUS MATERIALS: FROM ENCRYPTION AND ANTI-FOULING TO EFFICIENT CATALYSIS $948 \ldots \ldots \ldots$

Direct Numerical Simulations on Mixed-Wet Carbonates: A Comprehensive Workflow from Experiments to Simulations $222 \ldots \ldots \ldots \ldots$. . . . . . . . . . . 14

Experimental Analysis and Mathematical Modelling of Inorganic Scaling in Seawater Reverse Osmosis Membranes $928 \ldots \ldots \ldots \ldots \ldots$

Thermal stability of urease produced by Sporosarcina pasteurii 845 . . . . . . . . . . . . 16

Insight into Influence of Crossflow in layered Sandstone porous media during Miscible and Immiscible CO2 WAG Flooding $7 \ldots \ldots \ldots \ldots$

Characterisation of the Effect of Microstructure on the Liquid Transport in Porous Media Using Terahertz Spectroscopy and Imaging $834 \ldots \ldots . \ldots 20$

Thermally Induced Calcium Carbonate Precipitation in the Bakken Oilfields 793 . . . . . 20

non-Gaussian multi-scale heterogeneity patterns of equivalent conductivities of a fractured

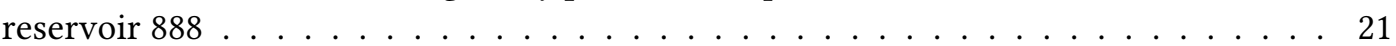

Investigation of the Erodibility of Fungal Treated Soils Using a Laboratory JET Apparatus

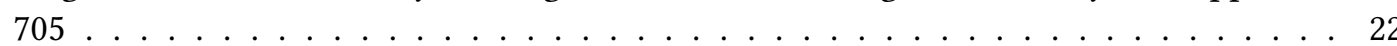

Pore-scale imaging and measurement of relative permeability in a mixed-wet carbonate reservoir rock at reservoir conditions $839 \ldots \ldots \ldots \ldots$

Simulation of Asphaltene Precipitation and Deposition in Porous Media: Thermodynamics and LBM Approaches $878 \ldots \ldots \ldots$. . . . . . . . . . . . . . . . 24

Pore-Scale Study of Multi-Species Ion Transports through Inhomogeneously Charged Nanoporous Media $460 \ldots \ldots \ldots \ldots \ldots \ldots \ldots \ldots$. . . . . . . . . . . . . . . . . . . . . . .

Convergence Analysis Of The Fixed Stress Split Iterative Coupling Scheme In Fractured Heterogeneous Poro-Elastic Media 680 . . . . . . . . . . . . . . . . 27

Assessment of the Low-Re RANS based turbulence models in two simplified pore geome-

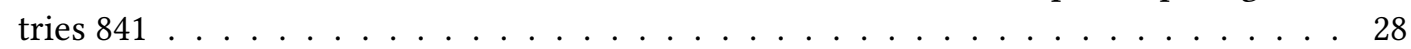

Thermally Induced Nanoparticle Delivery to Porous Media via Emulsions 604 . . . . . . 29

A posteriori error estimates for flow modeling using an Enhanced Velocity scheme 178 . 29

Multiscale modelling of NAPL dissolution in porous media in the presence of pore and continuum scale heterogeneity $297 \ldots \ldots \ldots \ldots$

A universal law for slip flow in tubes and porous media $771 \ldots$. . . . . . . . . . . . . 31

GPU-based pore-scale transport modelling in heterogeneous rocks 246 . . . . . . . . . . 31

Permeability prediction in porous media using multivariant structural regression 953 . . 32

Wettability in porous rocks, from macroscopical measurements to pore scale characteriza-

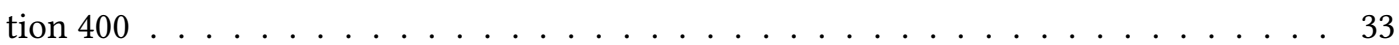


Numerical simulation of low salinity waterflood on fractured chalk outcrop-based models

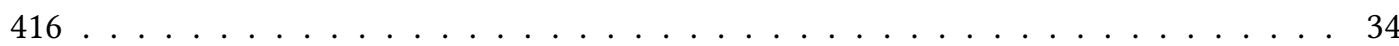

Poroelastic coupling and rupture directivity in injection-induced earthquakes 461 . . . . 35

Relating mechanical properties of biofilm-mineral composites to bulk porous media prop-

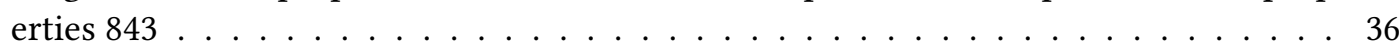

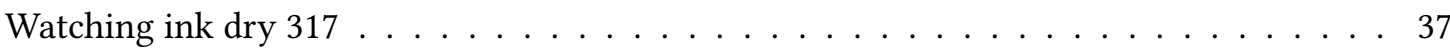

Anomalous Reaction-Diffusion under Quenched Heterogeneity 129 . . . . . . . . . . . . 37

Experimental Investigations of Calcite Crystals Nucleation and Growth in 3D Printed Mi-

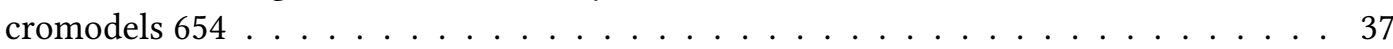

Computational Tool Developed for Simulation of Nano-fluids Behaviors 391 . . . . . . . 38

Foam flow and its blocking effect in high permeable saturated porous media 898 . . . . . 39

Surface influence on the dynamics of molecules confined inside mesoporous xerogels: low field NMR investigations $844 \ldots \ldots \ldots$. . . . . . . . . . . . . . . . 40

On the wettability of porous systems $76 \ldots \ldots \ldots \ldots \ldots$. . . . . . . . . 41

Probing small-scale connectivity for a high-permeability carbonate rock via two-phase

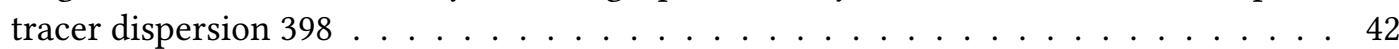

A Multiphase Extension of Darcy's Law with Non-Local Support 983 . . . . . . . . . . . 43

Evaluation of Interaction Between Corrosive Fluid and Crude Oil in Porous Media and its Side-Product Effect during Matrix Acidizing 193 . . . . . . . . . . . . . . . . 43

Utilization of Lattice Boltzmann Method to Study Disjoining Pressure in Porous Media $73 \quad 44$

Shear and Tensile Failure Modeling in the Context of Embedded Discrete Fractures 689 . 45

Extensions of poromechanical dual-continuum models for anisotropic elastic properties

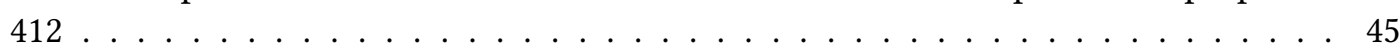

Droplet movement into thin porous layer: an inkjet printing case study 758 . . . . . . . 46

Density Functional Theory for Fluid Adsorption on Rough Surfaces 538 . . . . . . . . . . 46

Natural convection in coarse-grained porous media: pore-scale velocity and temperature

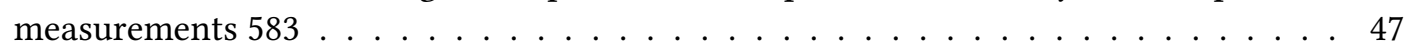

Reactive transport simulations throughout the continuum "Unsaturated - Saturated Zones" of a complex multiphase system using the O-ZNS platform $600 \ldots$. . . . . . . . . 48

Preparation of porous and magnetic carbon from waste cellulosic cloth by microwave irradiation, and its application as an adsorbent for dye removal from water 139 . . . . . 49

Solute mixing in the hyporheic region $135 \ldots \ldots \ldots$. . . . . . . . . . . . 50

From 3D Images to Adsorption and Deformation in Porous Media 475 . . . . . . . . . . . 51 
Pore-Scale Simulation of Wettability Effects on CO2 Storage Efficiency in Deep Saline

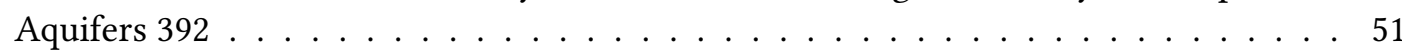

CFD modelling of Wettability and Temperature effect on Oil Recovery at Pore-Scale level.

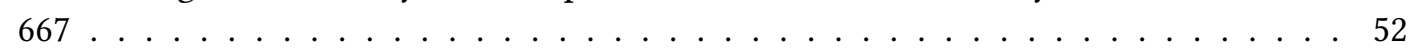

Modelling Liquid Transport on Gas Diffusion Layers with Heterogenous Surfaces in PEM

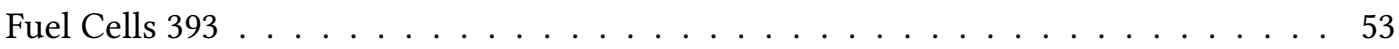

Gas Permeability in Micro/Nano-Porous Media: An extended Kozeny-Carman-Klinkenberg Model 341 . . . . . . . . . . . . . . . . . . . 53

Surface electric charge inside mesoporous silica at different pore sizes and porosities 347

An electrochemical impedance spectrocopy technique for determining ionic resistance of ion-exchange membranes $626 \ldots \ldots \ldots \ldots \ldots \ldots$

A method for determining through-plane thermal conductivity of polymeric membranes

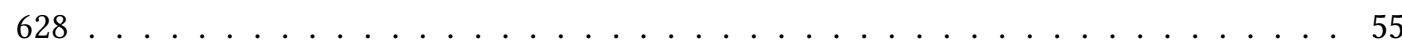

Pore Network Modeling for Topological Characterization of Porous Media 937 . . . . . . 56

Pore-scale simulation of interphase mass transfer across fluids interfaces using the Phase-

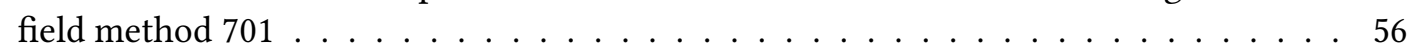

Adaptive numerical homogenization: linearization procedure to solve non-linear multiscale problems. 143 . . . . . . . . . . . . . . . . 57

Precipitation characteristics of enzyme-induced calcite - Experimental study using mi-

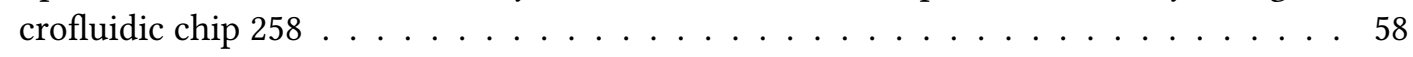

Macro-scale simulation of fibrous liquid aerosol filters $432 \ldots \ldots$. . . . . . . . . . . 58

Exploring the Robustness of Terahertz-Based Porosity Measurements using Embossed and Unembossed Pharmaceutical Tablets 773 . . . . . . . . . . . . . . . . . . . 59

Adaptive pores for oil permeation from oil-in-water emulsions $704 \ldots \ldots$. . . . . . . 60

Scour shape dependency on hydro-mechanical state of compacted clay 668 . . . . . . . 61

Multicomponent transport in hydrated polymer membranes: Alcohols, Formate and Acetate in Nation $11731 \ldots \ldots$. . . . . . . . . . . . . . . . . . . 62

Impact of image resolution on quantification of mineral properties and simulated mineral reactions and reaction rates $50 \ldots \ldots \ldots \ldots \ldots$

Non-wetting liquid flow in nanoporous media under the impulse pressure change 738 . . 63

Homogeneous wetting by controlled imbibition 268 . . . . . . . . . . . . . . . . 64

On the separate treatment of mixing and spreading by the reactive-particle-tracking algorithm: An example of accurate upscaling of reactive Poiseuille flow 671 . . . . . . . 65

Lagrangian Mass Transfer/Reaction Models: Is Probabilistic Mass Transfer Numerically Equivalent to SPH? $669 \ldots \ldots \ldots \ldots \ldots \ldots$. . . . . . . . . . . . . 66

A mixed MoL-TMoL approach for solving 2D Richards' equation in layered soils 717 . . 66 


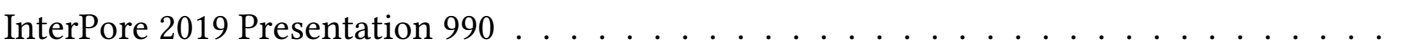

Molecular Dynamics Simulations of Polymeric Fibre Bundles under Tensile Load 362 . .

Effects of Inertia, Péclet Number, and Heterogeneity on Flow and (Anomalous) Transport in Heterogeneous Porous Media $180 \ldots \ldots$. . . . . . . . . . . 68

Adaptive mesh refinement for large scale Discrete Fracture Network flow simulations 380

Industrial gas storage monitoring based on measurement of soil respiration model 552 .

A posteriori error estimation by stress and flux reconstruction for Biot's consolidation

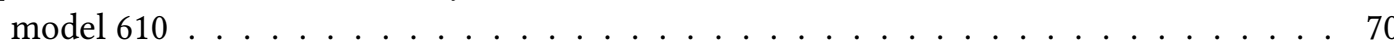

Swelling of gel rods $740 \ldots \ldots \ldots \ldots \ldots \ldots \ldots \ldots$

Coarse-grained simulations to guide an understanding of capillary pressure scanning curves in fibrous porous media $913 \ldots \ldots \ldots \ldots \ldots \ldots$. . . . . . . . . . . 72

Coupled Impact of Physical and Chemical Heterogeneity on Reaction Rates and Dissolution Patterns in Mixed-Mineralogy Porous Rock 794 . . . . . . . . . . . . . . . . . 72

Experimental investigation of oil recovery and recovery mechanisms of silica nanoparticles in water-wet reservoirs $642 \ldots \ldots \ldots \ldots \ldots \ldots$. . . . . . . . . . . . . . . .

INNOVATIVE TOOLS FOR FIELD SCALE INTERPRETATION OF ATTENUATION PROCESSES AND QUANTIFICATION OF CONTAMINANT MASS DISCHARGE AT THE GROUNDWATER-SURFACE WATER INTERFACE 123 . . . . . . . . . . . . . 74

Determination of thermodynamic contact angle based on interfacial energy balance in multiphase flow displacement in porous media $209 \ldots \ldots$. . . . . . . . . . . 76

Population balance modelling of particle deposition and aggregation in porous media 337

A robust upscaling of the effective particle deposition rate in porous media 338 . . . . . 77

"Hot Spots" observed in pore scale simulation of flow in carbon fibre felt electrodes limits the efficiency of Redox Flow Battery operation $965 \ldots \ldots$. . . . . . . . . 78

Wet granular materials: from building sandcastles to the construction of the Egyptian pyra-

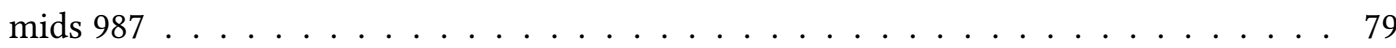

Reactive transport modeling in carbonate reservoirs and assessment of the adsorption of bil polar components on the rock surface $547 \ldots \ldots \ldots$. . . . . . . . . 80

The impact of small-scale rock heterogeneity on $\mathrm{CO} 2$ plume migration and residual trap-

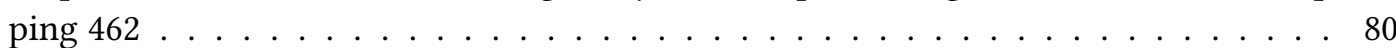

Auxiliary space preconditioners for mixed-dimensional H(div) problems 427 . . . . . . . 81

ADAPTIVE MCMC METHODS APPLIED TO THE CHARACTERIZATION OF ROCK PROPERTIES IN FLOW IN POROUS MEDIA $571 \ldots \ldots$. . . . . . . . . . . . . 82

Impact of mixing dynamics on fluid-solid reactions in porous media: pore-scale imaging and modelling $511 \ldots \ldots \ldots \ldots \ldots \ldots \ldots$ 
The Virtual Element method for simulations of physical phenomena on Discrete Fracture Matrix models $323 \ldots \ldots \ldots \ldots \ldots$. . . . . . . . . . . . . . . 84

Gradient flow perspective on poroelasticity and energy minimizing splitting schemes 92

Visualization of dynamic wormhole development in polymer gel using Positron Emission

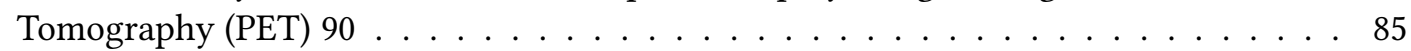

Effective behavior of an upscaled phase field model for reactive transport 492 . . . . . . 86

The effect of saturation dependent capillary diffusion on radial Buckley-Leverett flow $470 \quad 87$

Iterative algorithms for coupled thermo-poroelasticity $442 \ldots \ldots$. . . . . . . . . . . 87

Multiphase Flow Simulations in Large Domains of Upper Maastrichtian Chalk 935 . . . . 88

Fourier-Transform Laser Speckle Imaging for fast, quantitative and real-time flow imaging

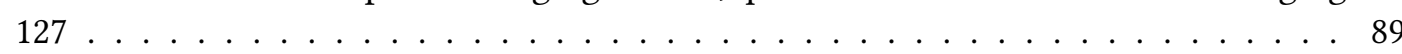

Improving the description of two-phase flow in rocks by integrating pore scale models and

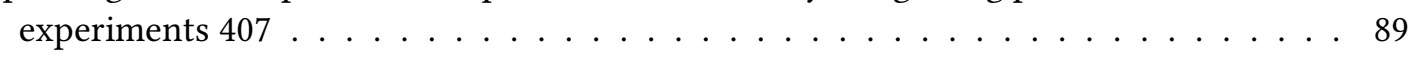

A Finite-Volume Approach for Two-Phase Flow in Fracturing Porous Media 643 . . . . . 90

Understanding the increased microbial activity of soils after rainfall events: a modeling

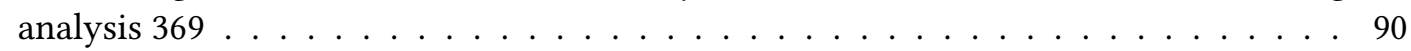

A new gas transport model of shale stratum based on fractal porous media 214 . . . . . . 91

Phase-field modeling of shrinkage-induced cracking in cement mortar $28 \ldots \ldots$. . . . 92

Research on productivity evaluation and development optimization of tight oil-Taking the Chang 7 Hua H6 Platform as an Example 964 . . . . . . . . . . . . . . . . . . . 93

Unraveling the interplay of different sorption-induced deformation mechanisms in a slit pore: an atomistic simulation approach $675 \ldots \ldots$. . . . . . . . . . . 93

The various scenarios of liquid water formation in the cathode gas diffusion layer of PEM

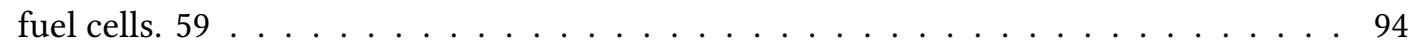

A Multiphase Darcy-Brinkman-Biot Approach to Modeling the Hydrology and Mechanics

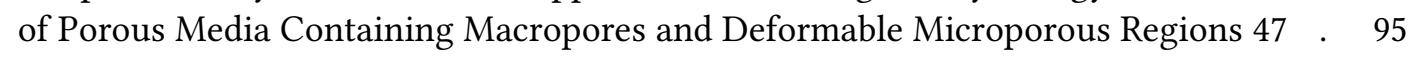

Removal of organic pollutants from water by non-thermal plasma techniques 800 . . . . 98

CO2 injection impairment: simple model for fracturing risk associated with mineral dissolution and precipitation $772 \ldots \ldots \ldots \ldots$. . . . . . . . . . . . . . . . 99

Modeling solute transport in segregated porous media: sensitivity-driven calibration of a new double continuum model $213 \ldots \ldots \ldots$. . . . . . . . . . . . . . . . . . . .

Force chain analysis during particle migration using community detection 443 . . . . . . 100

A Dynamical System for Gas Phase Cycling in Porous Media 785 . . . . . . . . . . . . . 101

Dynamics of water imbibition through paper 918 . . . . . . . . . . . . . . . . 102 
Multiscale Porosity Measurements in Shale Rocks using Gas Adsorption and Mercury In-

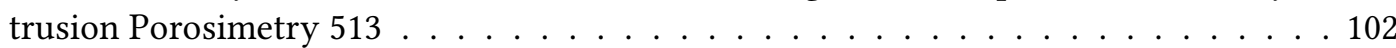

Characterization of sands and sandstones using electrical impedance spectroscopy 636 . 103

Identification of fractures in porous medium 795 . . . . . . . . . . . . . . . . . . 104

Quantifying Uncertainty Reduction in Geological CO2 Sequestration 602 . . . . . . . . . 104

Dynamic Formation Damage in Porous Media induced by Particle Transport 115 . . . . . 105

Application of online nuclear magnetic resonance technology to analysis of displacement

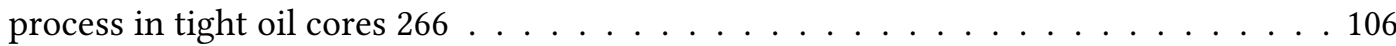

Pore-scale simulation of fluid displacement in micromodels and real rock samples using the $\begin{array}{lll}\text { CSF-based lattice Boltzmann multiphase model and geometrical wetting model } 303 & 107\end{array}$

An improved LBM-DEM model for hydro-mechanical coupled process 25 . . . . . . . . . 107

Joint identification of contaminant source and non-Guassian conductivities via the restart normal-score Ensemble Kalman filter 968 . . . . . . . . . . . . . . . . . . . 108

A unified model for liquid rise in a single capillary tube 23 . . . . . . . . . . . . . . 109

Nonlinear upscaling for flows in porous and fracture media 30 . . . . . . . . . . . . . . . 109

Voxels agglomeration for a fast permeability estimation on micro-CT images 962 . . . . 110

Relative permeability in discrete fracture network model of coal micro-CT images 457 . 111

Fundamentals of Geometrical and Physical Concept of Pore Space Tortuosity 780 . . . . 111

Limit Models of Pore Space Structure of Porous Materials for Determination of Limit Pore Size Distributions Based on Mercury Intrusion Data 782 . . . . . . . . . . . . . . 112

Correlation of magnetic resonance imaging and High-Resolution X-ray Tomography to characterise pore size distributions in polymeric open - cell sponges 543 . . . . . . . 113

Crystalline calcium silicate hydrates could enable tailored permeability control in the deep

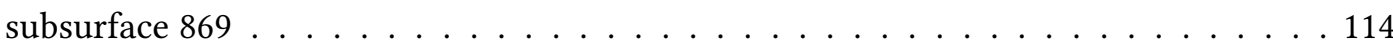

Imaging pore scale processes in geomaterials $950 \ldots \ldots$. . . . . . . . . . . . . . 115

Investigation of fluid/fluid couplings in two-phase flows: application to soil remediation

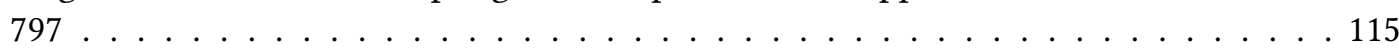

Multiscale characterization of effective conductivity of random heterogeneous porous media: multiple versus single realization approaches. $296 \ldots \ldots$. . . . . . . . . 116

The Soil-Atmosphere Interface: Numerical Analysis of Coupled Free-flow Porous Media Flow Systems 908 . . . . . . . . . . . . . . . . . . . . . 117

A predictive spatial Markov model for transport in heterogeneous Darcy flows 292 . . . 118

Properties of A $+\mathrm{B}->\mathrm{C}$ reaction fronts under radial advection in three-dimensional systems

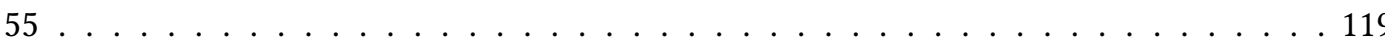


Multiphase Flow Modeling for the Estimation of the Interphase Mass Transfer of Enhanced DNAPL Solubilization $540 \ldots \ldots$. . . . . . . . . . . . . . . 120

A Study of the Total Compressibility in Three-Phase Immiscible Flow in Poroelastic Media

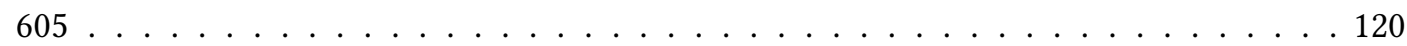

Drying thanks to nano-films $?^{98} \ldots \ldots \ldots \ldots$. . . . . . . . . . . . . . 121

Direct simulation of the effect of over-burden pressure on porosity and permeability of unconsolidated sands $377 \ldots \ldots \ldots \ldots . \ldots \ldots \ldots$. . . . . . . . . . . . . . . . . . . . .

A comparison between 2D, quasi-3D and fully-3D phase-field simulations of two-phase displacements at the pore scale $750 \ldots \ldots$. . . . . . . . . . . . . 123

IFBattery Max Power, Internal Resistance and Specific Energy for an n-Cell System in Series

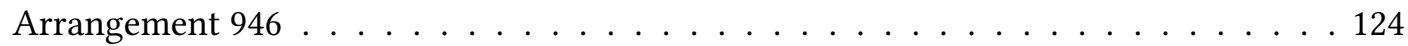

Simulations of coupled flow and geomechanics in porous media with embedded discrete fractures $660 \ldots \ldots \ldots \ldots \ldots \ldots$

A local time-stepping (LTS) strategy for fully-implicit simulation of multiphase flow in

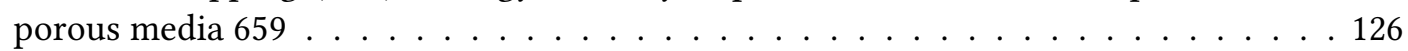

History Matching of Hydraulic Fracture Network in Shale-Gas Reservoir囚Application of Ensemble Kalman Filter Coupled with Embedded Discrete Fracture Modeling 36 . . . 126

mpact of the initial condition on concrete behavior during severe loading conditions 751

The importance of passive materials in Li-Ion battery electrodes 559 . . . . . . . . . . . 128

Development of a Novel Method based on MLP-ANN to Investigate Carbon dioxide capture

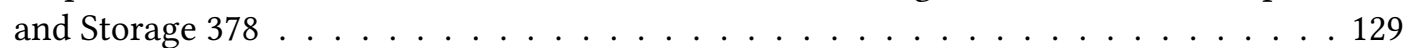

Determination of effect of impurities on carbon dioxide capture and storage by a novel LSSVM-GA method 376 . . . . . . . . . . . . . . . . . . . . . 129

Impact of disorder on flows and transport in porous media 329 . . . . . . . . . . . . 130

\begin{tabular}{lll}
\hline Impact of particle size correlation on immiscible fluid displacement in porous media 687 & 131
\end{tabular}

An adaptive upscaling method for unstable multiphase flows in heterogeneous porous media using advanced numerical methods $227 \ldots \ldots$. . . . . . . . . . . . 132

Pore Network Modeling of Reactive Transport in CO2 Storage Reservoirs 479 . . . . . . 132

\begin{tabular}{lll}
\hline Geometrical 2D to 3D Transform: Estimation of Material Properties from 2D Images 984 & 133
\end{tabular}

Changes in Porous Media Properties by Modeling Diagenetic Processes in Micro-CT Images

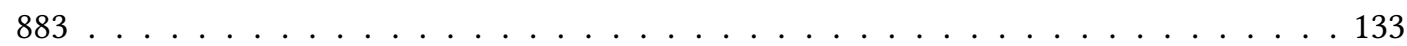

Microbial growth in confined flows: the role of physical heterogeneity 621 . . . . . . . . 134

Prediction of low velocity distribution from pore structure in simple porous media 733 . 135

The influence of nominal resolution and focal spot size on analysis results in porous media.

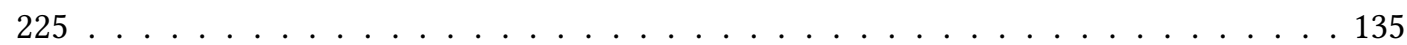


Cross pumping test to characterize the heterogeneity of hydraulic properties of a contami-

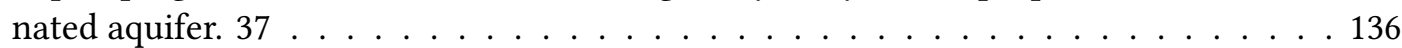

On the applicability of superposition principle to multiphase flow for the assessment of

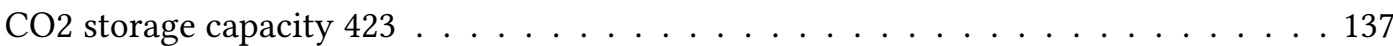

Experimental observations of coupling of free flow and non-uniform porous structures in

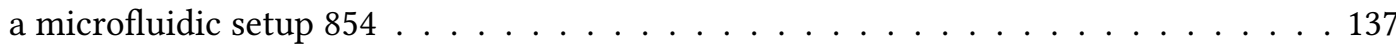

Effect of precipitation mineralization reactions on convective dissolution of $\mathrm{CO}_{2}$ : an ex-

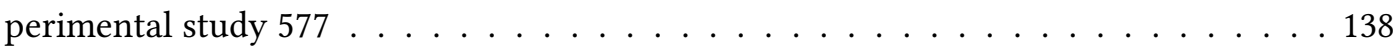

Moment-based Metrics for Global Sensitivity for multiple Models with Uncertain Parame-

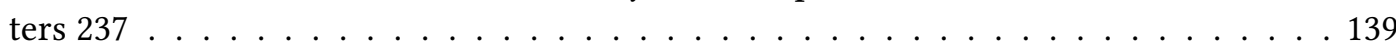

Feed-Forward Neural Networks for Flux Regression in Discrete Fracture Networks 290 . 139

Numerical simulations of underground carbon dioxide storage with hydrogen impurities

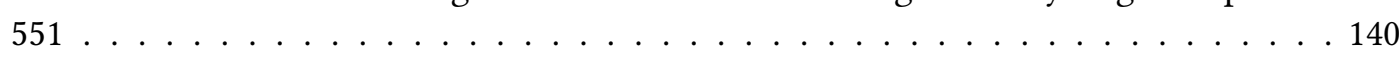

Conservative multirate multiscale method for multiphase flow in heterogeneous porous

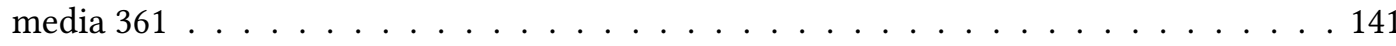

Stochastic mechanisms of dispersion in a porous medium: Upscaling flow and transport from the pore to the Darcy scale 188 . . . . . . . . . . . . . . . . 142

Hysteresis, sorption, swelling and mechanical behavior of wood: a multiscale study 787 . 143

Dynamic X-ray Tomography in the Laboratory: Exploring foam stability in 4D 512 . . . 144

Structural adaptation of biological tissues 167 . . . . . . . . . . . . . . . . . . . . . 144

Probabilistic Design Optimization for $\mathrm{CO}_{2}$ Sequestration With Leakage Risk Control 484 . . . . . . . . . . . . . . . . 145

Water retention behaviour of smectite-rich materials 851 . . . . . . . . . . . . . . . . . 146

Effective steady-state flow regimes during the stranding and mobilization of NAPL ganglia within stochastically reconstructed porous domains $304 \ldots$. . . . . . . . . . . . 147

Investigation of N2 and CO2 Huff-Puff Characteristics in Tight Sand Based on Dual T2C Model $522 \ldots \ldots \ldots \ldots \ldots \ldots \ldots \ldots \ldots$. . . . . . . . . . . . . . . . . . . .

Applying low-field nuclear magnetic resonance for characterization of nanoparticle transport through low permeability heterogeneous geomaterials 187 . . . . . . . . . . . 149

Coupled CFD-DEM modelling of granular porous media under shear: implications for dynamics of faults with granular gouge $810 \ldots \ldots$. . . . . . . . . . . 150

Numerical challenges in modelling spontaneous imbibition flows in throat-pore body systems: an assessment of the volume of fluid formulations $558 \ldots \ldots$. . . . . . . . 150

Prediction of the Effective Thermal Conductivity of the Porous Media based on Digitally Reconstructed Matrix Structures $219 \ldots \ldots$. . . . . . . . . . . . . 151

Numerical Simulation of Foam Three Phase Displacement Characteristics in porous media Using Stochastic Bubble Population Model 262 . . . . . . . . . . . . . . . . . . . 152 
Characteristics of Aggregate Size Distribution of Nanoscale Zero-valent Iron in Aqueous Suspensions and Its Effect on Transport Process in Porous Media 186 . . . . . . . . 152

Modelling of nanochannels imbibition with Smoothed Particles Hydrodynamics method

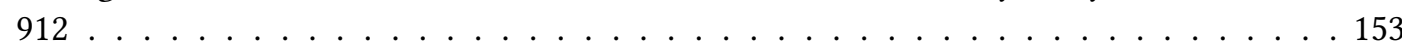

Estimation of permeability reduction during drilling operations in naturally fractured reser-

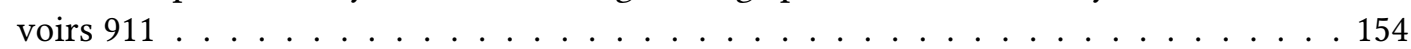

Fracture width prediction and particle size distribution design to wellbore strengthening: A comprehensive workflow for effective operations. 906 . . . . . . . . . . . . . 154

Predicting fluid flow via convolutional neural networks 769 . . . . . . . . . . . . . . . 155

Experimental Study of Induced Seismicity by Designed Porous Media 519 . . . . . . . . 156

The impact of the calcite surface charge on multicomponent transport in chalk aquifers 567

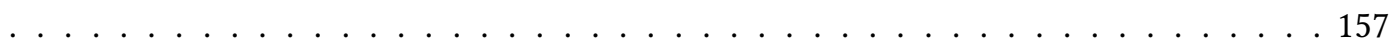

Interface Conditions for Infiltration Problems 39 . . . . . . . . . . . . . . . . . . . . 157

Shear thickening of flexible coiled polymer solutions in non-inertial shear and extensional

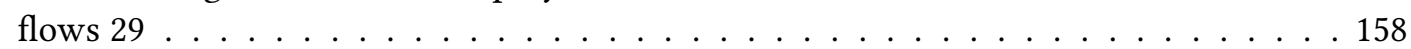

Micromorphic contributions to granular and porous materials 808 . . . . . . . . . . . . . 159

Viscosity Membrane - A Novel Paradigm for Polymer Flow in Sand 892 . . . . . . . . . 160

Viscosity Membrane - A Novel Paradigm for Polymer Flow in Sand 893 . . . . . . . . . 160

Solute transport for non-Darcy flow in porous media using pore network modelling 504 161

Behavior of turbulence at Fluid/Porous interfaces of different Permeabilities 686 . . . . 162

$10 \mathrm{~Hz}$ Operando X-ray Tomographic Imaging of Liquid Water in Gas Diffusion Layers of Polymer Electrolyte Fuel Cells 864 . . . . . . . . . . . . . . . . . . 163

An upscaled model for the transport of inertial particles in a water-saturated porous medium

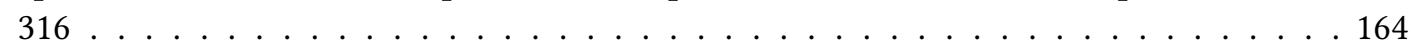

Balancing complexity and parsimony to accurately model, and upscale, the transport of large, fibrous colloids through porous media 17 . . . . . . . . . . . . . . 164

Predicting diameter changes of the vasculature to regulate blood flow during activation 763

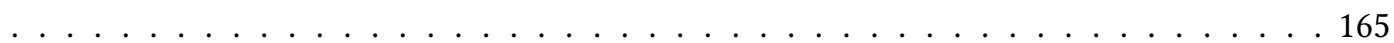

Pore Scale Modelling of Reactive Transport in Porous Media: Implications to Geological Energy Applications 243 . . . . . . . . . . . . . . . . . . . . . 166

Reactive Density-Driven CO2 Mixing in Sandstone Aquifers: Role of Geochemistry 245 . 166

Pilot scale "in pile" thermal desorption remediation of mercury and mixed pesticides con-

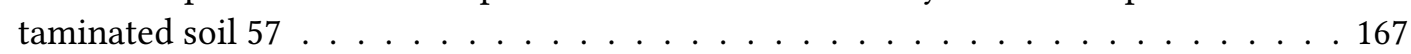

Pore-Scale Visualization of Hydrate Formation and Dissociation 465 . . . . . . . . . . . 168 
Grid convergence studies for lattice Boltzmann method based pore-scale reactive transport

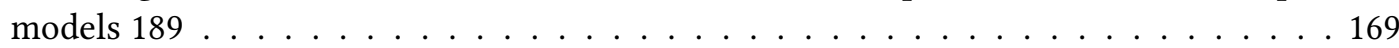

Characterization of Foam Flow in Porous Media: Effect of Different Chemical Concentrations, Flow Rates and GLR Ratio 731 . . . . . . . . . . . . . . . . . . . . . 169

Uncertainity analysis for Seawater Intrusion in Fractured Coastal Aquifers: Effect of Fractures Apparture, Location, Density and Hydraulic Properites 518 . . . . . . . . . . 173

Fluid flow in a channel partially filled with thin ordered porous media: pore scale simulations vs volume averaging continuum modeling $429 \ldots$. . . . . . . . . . . . 174

An upscaled transport model of shale gas considering multiple mechanisms and heterogeneity based on homogenization theory $75 \ldots \ldots$. . . . . . . . . . . 174

Size effects of molecular sub-region in a multiscale, multiphase process 24 . . . . . . . 175

Modified salinity water flooding: pore-scale simulations and microfluidic experiments 818

Towards Bayesian quantification of permeability in micro-scale porous structures - Sandstones and Carbonates $805 \ldots \ldots \ldots$. . . . . . . . . . . . . . 176

Upscaling of reactive transport from pore-scale to core plug-scale in shale samples: the pore-scale part $959 \ldots \ldots \ldots \ldots$. . . . . . . . . . . . . . . . . . . . . . . . .

Accelerated Rate Calorimeter Application for Heavy Oil Characterization 383 . . . . . . 177

Effect of the rigidity of a granular material on heat transfer 53 . . . . . . . . . . . 178

a New Method to Predict the Performance in Gas Assisted Gravity Drainage Using Dimensionless Groups 900 . . . . . . . . . . . . . . . . . . . 178

Role of Surface Area Density of Porous Paper-like Materials on the Detection Performance of Lateral Flow Assay $807 \ldots$. . . . . . . . . . . . . . . . . 179

Impact of creep on the permeability evolution of coal by the thermo-mechanical coupling

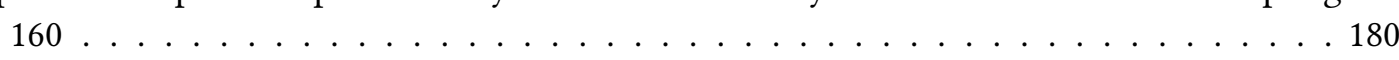

Three-Dimensional Finite Deformation Measurements of Soft Porous Media using Lens Free

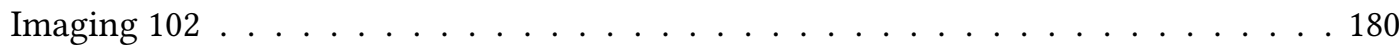

The Porous Microstructure Analysis (PuMA) software for high-temperature microscale modeling $708 \ldots \ldots \ldots \ldots \ldots \ldots 1 . \ldots \ldots \ldots \ldots \ldots$

Design of multilayer porous pavements for optimizing their evaporative cooling potential as a strategy for urban heat island mitigation $646 \ldots \ldots \ldots$. . . . . . . . . 182

A Recursive Mixed Multiscale Method for Darcy problem 299 . . . . . . . . . . . . . . . 183

ADVANCES IN LINEAR SOLVERS FOR COUPLED MULTI-PHYSICS PROBLEMS 493 . . 184

Influence of yarn configuration on wicking processes studied by fast X-ray micro-tomography

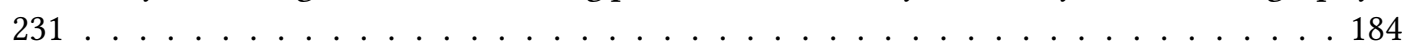

Integrating publication, software and data in simulation sciences 986 . . . . . . . . . 185 
Flow in porous media of foams reinforced with polymers or particles: application to sub-

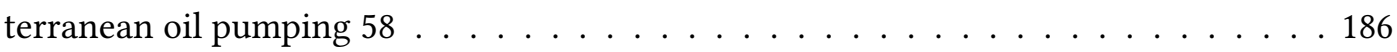

Advanced Water Management in Fuel Cells Using Engineered Diffusion Layers with Patterned Wettability $720 \ldots \ldots \ldots$. . . . . . . . . . . . 187

Numerical modeling of thin porous media with LBM 454 . . . . . . . . . . . . . . . . 188

Cut, overlap and locate: 3D localization of highly overlapping tracer particles in defocal microscope images 934 . . . . . . . . . . . . . . . . . . . . . . 189

Bringing techniques from soil science to contaminated sites 307 . . . . . . . . . . . . . . 190

Urease Immobilization for Advancing Enzyme-Induced Calcium Carbonate Precipitation Applications 756 . . . . . . . . . . . . . . . . . . 191

Dynamics of paper fiber during liquid imbibition; capillary pull and swelling 874 . . . . 191

Impact of PTFE distribution on water transport in a gas diffusion layer of polymer elec-

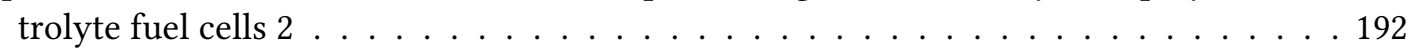

Gas transport in PEFC gas diffusion layers and an analysis of surface characteristics 421

Reverse Imbibitions Damages during Gas-Water Two-Phase Flow in Tight Sand Gas Reservoir: An Experimental Based Case Study 580 . . . . . . . . . . . . . . . . . . . 194

An improved model to predict irreducible water saturation in tight gas reservoir 579 . . 194

Modeling of pore throat distribution and capillary effect in synthesized artificial core 241 195

Quantifying Transport Uncertainty in Two-Phase Flow Using Probability Distribution Method

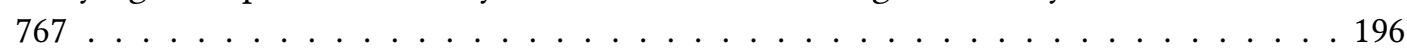

Homogenization of reactive transport processes through thin heterogeneous layers 548 . 197

Thermodynamic Evidence of Structural Transition of ZIF-8 upon Methane Adsorption 386197

Nanoimbibition in Artificial Opals -a Photonic Approach for Nanoporous Media 306 . . 198

(Nano)pore scale modeling: can we learn from molecular simulations ? 321 . . . . . . . 199

Pressure inside a nano-porous medium. The case of a single phase fluid. 54 . . . . . . . . 199

Modelling immiscible fingering in hierarchically structured porous media 781 . . . . . . 200

Study of Evaporation from Wavy Surfaces by Coupled Turbulent Air Flow and Porous Me-

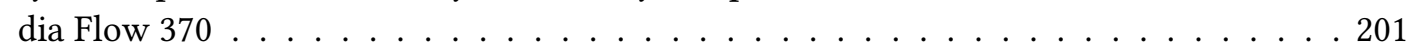

Characteristics of Micropore-Nano Structure in Shale Oil Reservoir 291 . . . . . . . . . . 202

Dynamic pore-scale flow regimes study during steady-state multiphase flow using synchrotron X-ray micro-tomography $348 \ldots \ldots \ldots 202$

Porous building materials and their degradation process due to the absorption of solution salts: a Markov approach to modeling the evolution of the degradation process. 477 . 203 
Pore-scale Water Draining in Gas Diffusion Layers of Polymer Electrolyte Membrane Fuel Cells Using X-ray Tomography and Lattice Boltzmann Method 564 . . . . . . . . . . 204

The role of chemical heterogeneities in controlling the wetting state of porous media 503 204

The role of convection for $\mathrm{CO} 2$ migration and trapping in CO2-EOR reservoirs 855 . . . 205

Variable Permeability and Reactions in a Hele-Shaw Cell model system of Carbon Dioxide Convective Dissolution for CCS 494 . . . . . . . . . . . . . . . 206

On the use of fixed-stress split scheme for the solution of flow problems in deformable

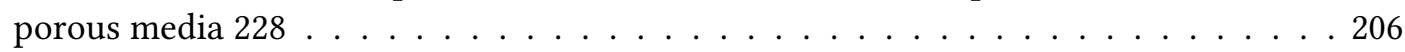

\begin{tabular}{|l|l|}
\hline A stabilized mixed formulation for phase-field modeling of fracture in porous media 544 & 207
\end{tabular}

Modelling chemical deterioration processes in building materials - Poromechanical approach

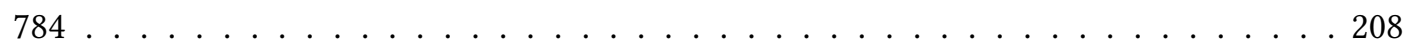

From streamlines and transfer functions to flow diagnostics for naturally fractured reser-

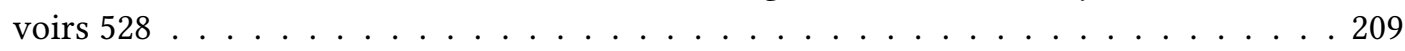

Lattice Boltzmann Method for effective diffusion coefficient computation at the pore scale

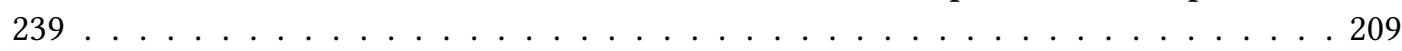

Measuring swelling and shrinkage of porous materials with a digital camera 650 . . . . 210

Colloidal deposition in 3D porous media, a multi-scale approach 527 . . . . . . . . . . . 211

Permeability upscaling from pore to core scale 279 . . . . . . . . . . . . . . . . . . . . . 212

Combusting in the Deep 989 . . . . . . . . . . . . . . . . . . . . . . . . . . . . 213

Mesh-free simulation for precipitation and dissolution in a 2D discrete fracture network

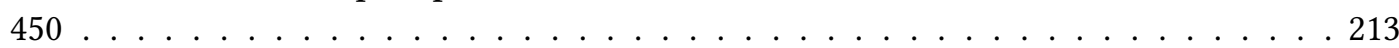

Increasing capacity of $\mathrm{CO} 2$ sequestration by brine production 848 . . . . . . . . . . . . 214

Coupled Electrohydrodynamic transport through fractures 779 . . . . . . . . . . . . . . 215

GEOMATICS FOR THE PREDICTION OF SAND BOIL PRONE AREAS 282 . . . . . . . . 216

Ballisticules, mobile-mobile mass exchange, and mixing-limited reactions 114 . . . . . . 217

Modeling a large scale experiment in MICP using independently determined parameters

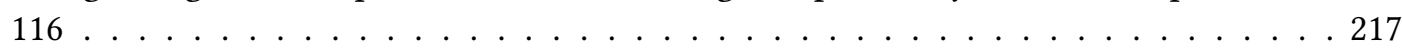

Determination of the solute diffusivity, dispersion, skewness , kurtosis and higher-order moments in heterogeneous porous flow via Extended Method of Moments. 46 . . . 218

Network model studies of relations between flow velocities in two-phase flow in porous

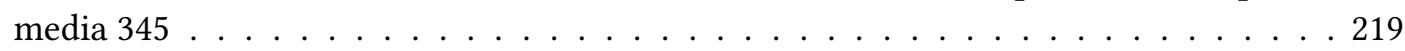

Coupled 1D-3D Flow Models 136 . . . . . . . . . . . . . . . . . . . . . . . . . . . 220

Optimizing the performance of batteries using digital material engineering 313 . . . . . 221 
Pore scale effects of electrical charge in porous media under electrical and mechanically

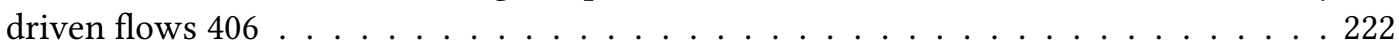

Salt polygons and porous media convection 359 . . . . . . . . . . . . . . . . . . 222

Drying and percolation in correlated porous media 349 . . . . . . . . . . . . . . . . . . 223

Modelling blood flow in mouse brain microvascular networks 641 . . . . . . . . . . . . . 223

Lattice-Boltzmann modeling of osmosis during low-salinity flooding 696 . . . . . . . . . 224

Evolution of Pore Structure during Organic Acid Injection: A Pore Network Modeling Ap-

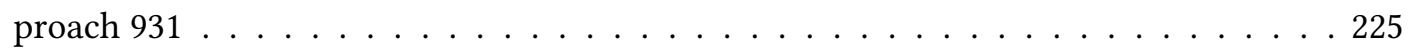

Pore scale Numerical Modeling of Fluid Flow through Porous Media( Direct Method Ap-

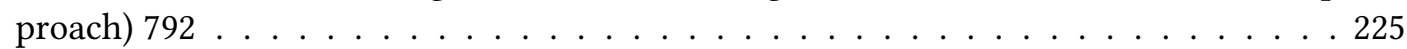

A computational pore-scale model of NAPL remediation -Investigating the impact of biofilm growth on bio-enhanced dissolution and biodegradation $761 \ldots \ldots 226$

Structural changes in bulk materials for thermochemical energy storage 277 . . . . . . . 227

Liquid Injectivity in Surfactant-Alternating-Gas Foam Enhanced Oil Recovery: Effect of

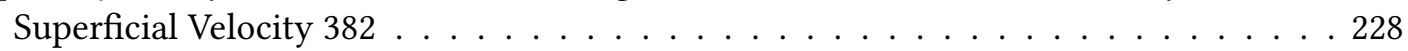

Modeling of coupled conductive-radiative heat transfer in a 3D numerical material by a

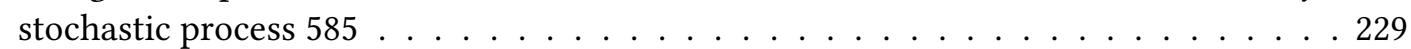

Experimental and numerical study of gas migration through compacted MX- 80 bentonite

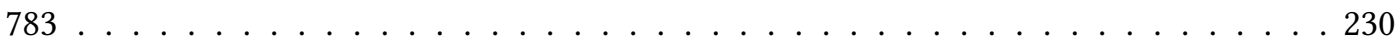

The effect of water adsorption on deformation and elastic properties of Bentheim sandstone

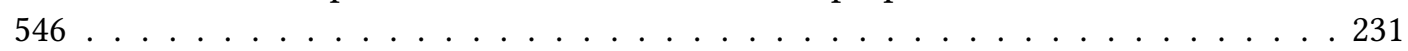

Modeling Dispersion in Pore Networks: Comparison of Pore-Scale Model Accuracy 684 . 232

Influence of Charge-Coupled Diffusion and Non-Linear Reaction Kinetics on the Dissolution of Carbonate Rocks: Pore-scale Modelling and Experiments 716 . . . . . . . . . 233

Thermal conductivity of partially sintered oxide ceramics predicted via cross-property relations and measured via laser flash $661 \ldots \ldots$. . . . . . . . . . . . . 234

Analysis on the existing state of pore water in Gas Hydrate Reservoir 919 . . . . . . . . 235

Pore-scale characterization of velocity probability distributions in partially saturated porous

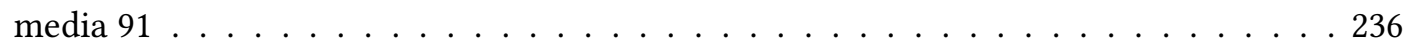

Impacts of brine and reservoir properties on the transport of HPAM solutions in low per-

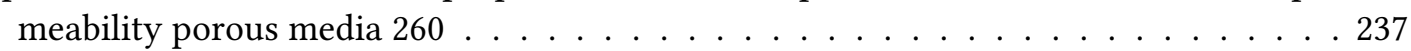

Permeability in different scales comparing Darcy and Darcy-Brinkman-Stokes (DBS) 938238

Pore Network Modeling of Mixed-Wet Carbonates Based on Pore Classification and Fluid

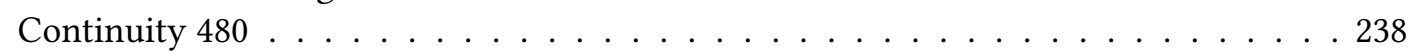

Peltier Heat of porous LiFePO4 electrodes in a thermoelectric cell 44 . . . . . . . . . . . 239 
Thermal analysis and design optimization of multi-layer insulation with fiber-reinforced aerogel and reflective screen $895 \ldots \ldots$. . . . . . . . . . . . . . . 240

Permeation and separation of $\mathrm{CH} 4 / \mathrm{CO} 2$ mixtures through single-layer nanoporous graphene membranes : theory and molecular simulations. 971 . . . . . . . . . . . . . 241

Permeation and separation of $\mathrm{CH} 4 / \mathrm{CO} 2$ mixtures through single-layer nanoporous graphene membranes : theory and molecular simulations. $309 \ldots \ldots . . \ldots 242$

Pore-scale investigation of residual oil distribution and mechanisms of enhanced oil recovery methods 69 . . . . . . . . . . . . . . . . . . . . . . 243

Age of Mixing Ratios. 106 . . . . . . . . . . . . . . . . . . . . . . 245

Pore-filling mechanics in capillaries with variable-wettability patterns 638 . . . . . . . 245

Effects of gas adsorption-induced deformation on carbon dioxide storage in coal 860 . . 246

Hydrodynamic instabilities of immiscible fluids flow in oil reservoir rocks during enhanced

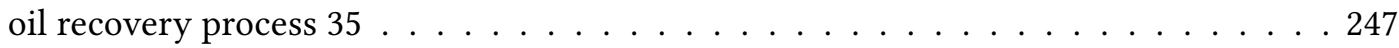

Thermal diffusion in porous media 399 . . . . . . . . . . . . . . . . . . . . 247

Soil respiration under climate extremes: On the role of root hydraulic redistribution delin-

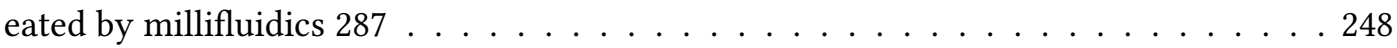

What key physical factors yield a good horizontal hydrofractured gas well in a mudrock?

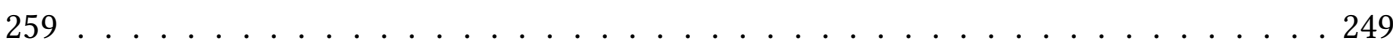

Hydro-mechanical effects on the stability of RJD wells and production rate 824 . . . . . 250

Multiscale finite volume method for finite-volume-based simulation of poroelasticity 858251

Stochastic velocity transitions and continuous time random walks to model solute transport in heterogeneous porous media $536 \ldots \ldots$. . . . . . . . . . . . . . . . . . . . . .

Solute mixing in porous media: the role of confinement 233 . . . . . . . . . . . . 252

Experimental study of biogenic gas bubble formation - Microfluidic chip and core-scale

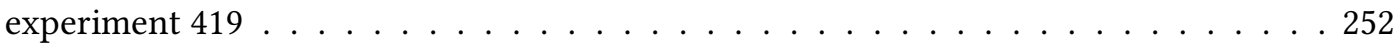

Understanding the Co-Moving Velocity 288 . . . . . . . . . . . . . . . . . . 253

Displacement of methane by injecting water into shale nanopores using molecular dynam-

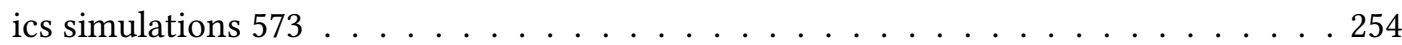

Saturation-Dependence of Non-Fickian Transport in Porous Media 242 . . . . . . . . . . 255

Fast 4D Micro-Tomography Imaging of Anomalous Transport in Partially-Saturated Porous

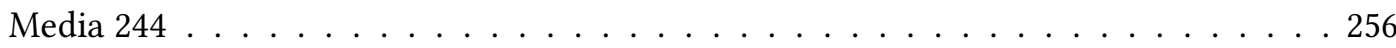

Wettability Alteration Modeling by Pore Network Approach for Low-salinity Water Injection Process 354 . . . . . . . . . . . . . . . . . . . . . . . 257

Probing Chemical Causes of Asphaltene Deposition onto Silica and Calcium Carbonate

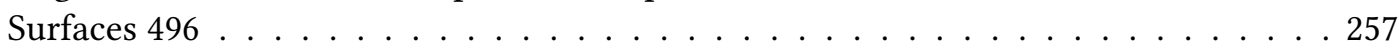


Two-phase flow in industrial porous media; Experiments, theory, and modelling 314 .

Microbial Growth and its Influence on Hydraulic Properties in Saturated Porous Media - a Microfluidic Study $510 \ldots \ldots$. . . . . . . . . . . . . . . . 260

Study on salt precipitation behavior during carbon dioxide injected into underground porous

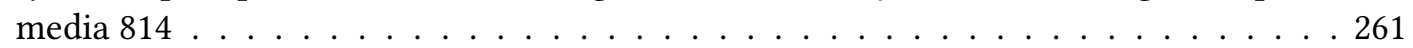

Temperature effects on swelling behaviour of smectite: insight into coupled chemo-mechanical phenomena in charged porous systems. 198 . . . . . . . . . . . . . . 262

Analytical and Numerical Assessment of Hydraulic Properties of 3D Partially Open Fractures with Variable Roughness 677 . . . . . . . . . . . . . . . . . . 263

Evaporation in multi-component systems 413 . . . . . . . . . . . . . . . . . . . . . 263

Bimodal imaging of water flow in samples of green infrastructure soils 562 . . . . . . . . 264

Microfluidic Investigation of Brine-hydrocarbon Interactions during Low-salinity Water-

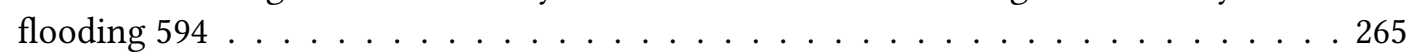

Simulation of capillary-dominated three-phase flow experiments on 3-D bead packs 637 266

A Numerical Simulation of Fluid Flow through a Thin Porous Media Confined within a Narrow Duct 802 . . . . . . . . . . . . . . . . . . . . . . 267

Asymptotic analysis for the coupling between subdomains in Discrete Fracture Matrix mod-

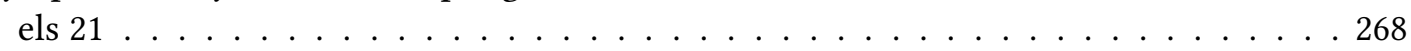

Estimating Permeability Decrease in Deformation Bands in Unconsolidated Sandstones by Digital Rock Simulation 914 . . . . . . . . . . . . . . . . . . . . 268

Mass dispersion between a fluid region and a porous medium 726 . . . . . . . . . . . 269

4D Structural and Chemical Characterization of Reactive Magnesium Cement-Based Con-

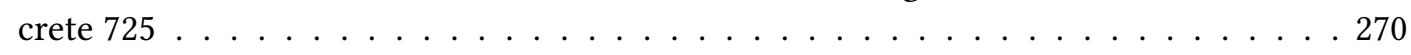

How does chaos rule concentration gradients in porous flow? 555 . . . . . . . . . . . . 272

Pore scale mechanisms of chaotic advection 957 . . . . . . . . . . . . . . . . . . . 272

Convective mixing in heterogenous porous media 289 . . . . . . . . . . . . . . . . . . 273

SYNTHESIS, STRUCTURAL EVOLUTION AND APPLICATIONS OF METAL ORGANIC FRAMEWORK AND ZEOLITIC IMIDAZOLATE FRAMEWORKS 122 . . . . . . . . . 273

Gas flow in shale nanopores by solving Boltzmann model equation 709 . . . . . . . . . . 274

Micro-scale simulation of fibrous liquid aerosol filters 436 . . . . . . . . . . . . . . . . . 275

Hysteresis of Multiphase Flow in Porous and Fractured Media 759 . . . . . . . . . . . . 275

Impact of spatial correlations in particle sizes on fluid displacement in porous media 762 276 
Investigating induced calcium carbonate precipitation with a focus on changing hydraulic

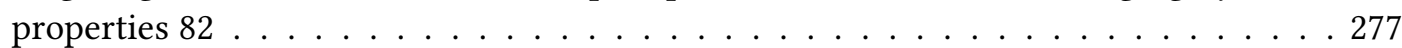

Dynamic Multiscale Simulation of Fractured Geothermal Reservoirs 364 . . . . . . . . . 278

Free Energy of Immiscible Two-Fluid Flow in Porous Media by Integral Geometry and Ther-

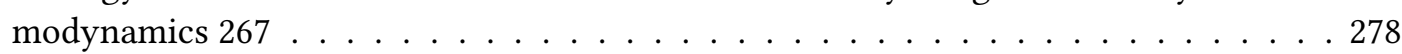

Combination of the in-situ loading scanning experiment and digital core deformation simulation for investigating stress sensitivity mechanism 12 . . . . . . . . . . . . 279

Experimental Investigations of Flow Boiling inside Porous Media at Pore-scale with Micro-

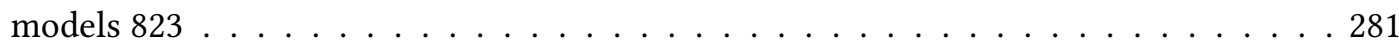

Numerical Simulation of Multiphase Flow inside Porous Media at Low Pressure 820 . . 282

Energy conversion reveals regime transition of imbibition in a rough fracture 777 . . . . 282

Robust Preconditioners for Mixed-dimemsional Models of Flow in Fractured Porous Media

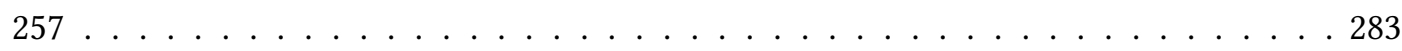

Experimental study on mechanical properties of granite subjuected to temperature and

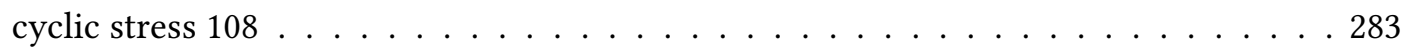

Thermal conductivity measurement of porous materials: challenges, examples and prospects

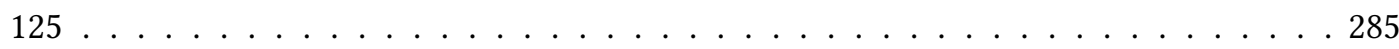

Pore-scale experiments on the behavior of $\mathrm{CO} 2$ exsolution in thermal recovery and $\mathrm{CO} 2$

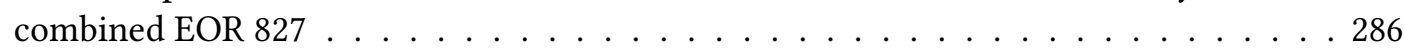

An efficient numerical simulator based on embedded discrete fracture model for fractured

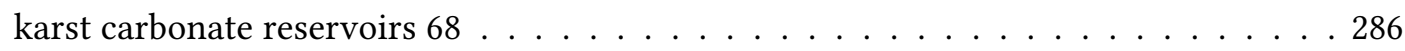

Liquid-Infused Nanoporous Solids as Photonic Metamaterials 121 . . . . . . . . . . . . . 287

Electrosorption in Polypyrrole-Silicon Hybrid Nanopores: Insights from Cyclic Voltamme-

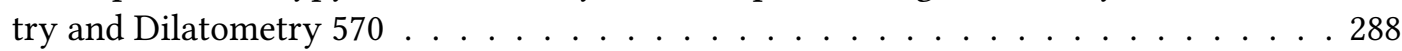

Mesoscopic modelling of ink imbibition in paper $263 \ldots \ldots$. . . . . . . . . . 288

Applications of Graph Theory and Machine Learning in Discrete Fracture Network Simu-

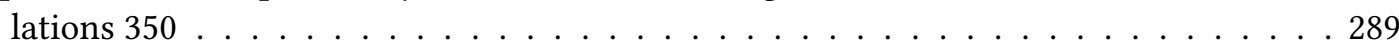

Initiation and propagation of strain localization in cohesive soil using a novel miniature

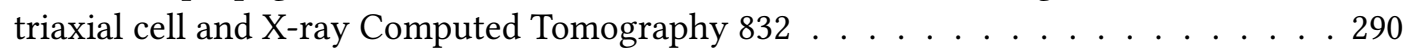

Novel approaches for upscaling transport beyond homogenisation 357 . . . . . . . . . . 290

On modeling and simulation of multiscale processes in catalytic filters 857 . . . . . . . . 291

Domain decomposition techniques for fully coupled reactive transport and multiphase flow

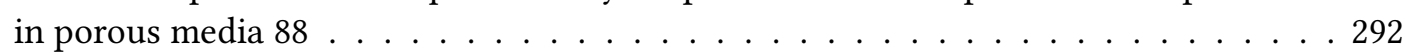

In-pore solidification: from nanoscale origin to mesoscale damage 218 . . . . . . . . . . 293

CapillaryStress and Structural Relaxation in Disordered Porous Materials 105 . . . . . . 293 
Dependence of shallow landslide stability onset on terrain porosity - an experimental study

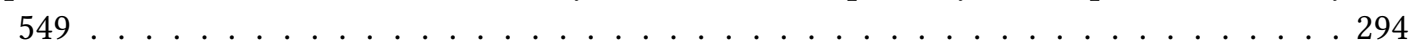

Reliable Multiscale Models for Manufacturing of Lightweight Composite Structures 152 . 295

Capillary pressure heterogeneity across scales: Insights from micro-CT imaging and con-

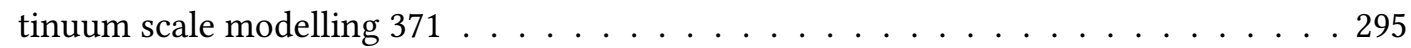

Fostering microbial enhanced oil recovery? About general mechanisms and technical tools to explore new avenues to prevent or stimulate bacterial adhesion 635 . . . . . . . 297

Application of numerical modelling and flow experiments to optimize the design of microfluidic devices representing porous media 374 . . . . . . . . . . . . . . 297

Non-isothermal Reservoir Souring Simulations 873 . . . . . . . . . . . . . . . . . . . . . 299

Modeling of transport, accumulation and replication of bacteria in porous media 933 . . 299

Fluid flow and heat transfer modeling through metal foams or lamella structures 765 . . 300

Gas bubble migration and trapping in porous media - Pore-scale simulation 640 . . . . . 301

The significance of porous cell walls for water transport in plants 459 . . . . . . . . . . 302

Molecular dynamics simulation of ink imbibition in porous media 346 . . . . . . . . 303

Effect of flow-path tortuosity and contact area fraction on permeability of a rough rock

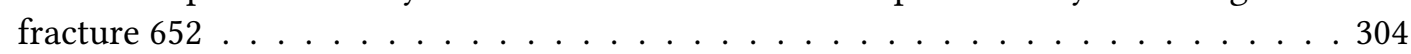

Multiscale numerical simulation and analysis on reactive acid flow in carbonate rock. 182305

Gas-Water Flow of Tight Core Using Pore Scale Observation and Characterization 8 . . . 305

Carbon aerogel composites with SiCO aerogels as inner coatings for ultra-high temperature

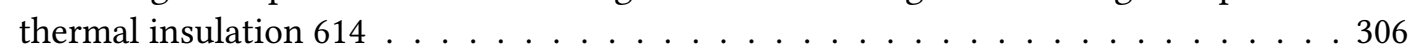

Rock-typing of heterogeneous rock samples via Minkowski functionals and hydraulic at-

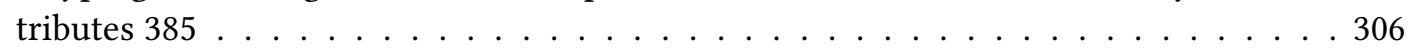

Carbonate pore space segmentation and classification by a combination of multiscale imaging and deep learning $651 \ldots \ldots$. . . . . . . . . . . . . . . . . . . . . . . . . . .

The influence of fracture on the gas reservoir development 545 . . . . . . . . . . . . 308

Graph theory to infer mixing capacity in complex systems 325 . . . . . . . . . . . . . . 308

Fracture Modelling of Unconventional Reservoirs with X-ray micro-computed tomography

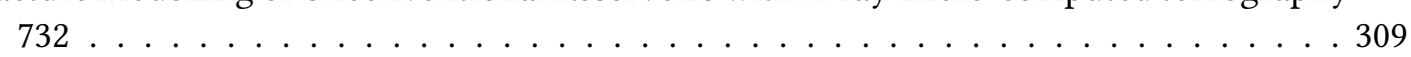

Porous Media Characterisation relavent to Enhanced Gas Recovery 51 . . . . . . . . . 310

Simulation and Experimental Measurements of Internal Magnetic Field Gradients and NMR Transverse Relaxation Times (T2) in Sandstone Rocks 119 . . . . . . . . . . . . . . . 311

Enhanced convective dissolution flux due to an $\mathrm{A}+\mathrm{B}->\mathrm{C}$ reaction 124 . . . . . . . . . 312

Imaging and modeling Martin Blunt at the pore scale 74 . . . . . . . . . . . . . . . . 312 
Study of contact angle variation considering geological CO2 sequestartion 608 . . . . . 313

Monitoring Pressure Fluctuations in Artificial Porous Media. 644 . . . . . . . . . . . . . 313

Carbon aerogels with high microporous specific surface area and low thermal conductivity prepared through self-sacrificial salt templating route with ambient drying 612 . . . 314

Multi-scale modeling in poroelasticity: Computational homogenization and Numerical Model

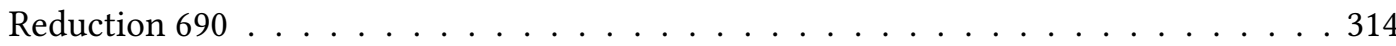

Impact of Heterogeneous Fracture Aperture on the Well Productivity of Deformable Fractured Porous Media 826 . . . . . . . . . . . . . . . . . . 315

A Mixed-dimensional Discontinuous Galerkin Method for Coupled Flow and Transport in Fractured Porous Media 185 . . . . . . . . . . . . . . . . . . 316

Atomistic Simulation of the Adsorption and Transport of Naturally Occurring Radioactive Materials in Clay Nanopores in the Context of Shale Gas Exploration 588 . . . . . . 317

Atomistic Computational Modeling of Fluid Transport in Cement Nanopores in the Context of Geological CO2 Sequestration 589 . . . . . . . . . . . . . . . . . . 318

Adaptive Discontinuous Galerkin Methods for complex flow in porous media 488 . . . . 319

Stress-induced Anomalous Transport in Natural Fracture Networks 842 . . . . . . . . . . 320

Understanding Mixing and Reaction at Rough Fracture Intersections 791 . . . . . . . . . 321

The effect of ionic strength on the development and evolution of two phase flow in an artificial porous medium $615 \ldots \ldots . \ldots . \ldots \ldots 21$

Impact of Time-dependent wettability alteration on Capillary pressure 753 . . . . . . . . 322

Robust Superhyrophobic/Superoleophilic Nanomaterial Coated Porous Medium for Selective Separation and Expulsion of Oil Pollutants from Water 358 . . . . . . . . . . . 323

Pore-Scale Flow of Water and Supercritical CO2 in 2D Porous Micromodels: High-Speed Quantifications of Velocity Fields and Interface Dynamics at Reservoir Conditions 867324

Interfacial curvature, contact angle and capillary pressure measurements during drainage process in micromodels $394 \ldots \ldots \ldots$. . . . . . . . . . . . . . . . . . . . . . . . . . .

Numerical simulation of residual oil distribution in middle porosity \& low permeability carbonate reservoir developed by WAG-Take RS oil field in Abu Dhabi for example 972

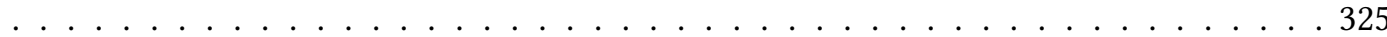

One-particle layer drying kinetics to assess recycled concrete aggregates absorption 275

Influence of non-adsorbing polymers on drying of fresh mortars: measurement and physi-

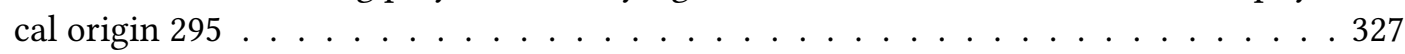

Understanding the Critical Role of Capillary Suction on Rock Mechanical Responses during Post-fracturing Spontaneous Imbibition in Tight Formations 159 . . . . . . . . . . . . 327

Geostatistical multi-scale estimating the effective elastic parameters of sandstone from dig-

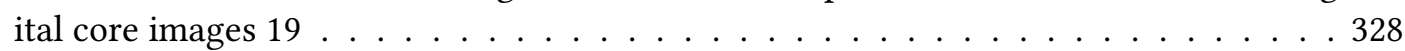


Performance modeling of Li-ion batteries using multiphysics-based pore network modeling

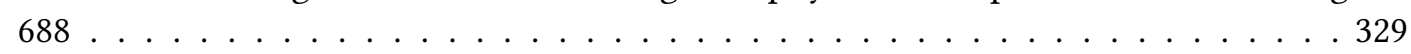

Fluid-solid interaction during particle aggregates drying $760 \ldots \ldots 330$

3D simulation of flow and active-passive tracer propagation in Voronoi tessellation-based

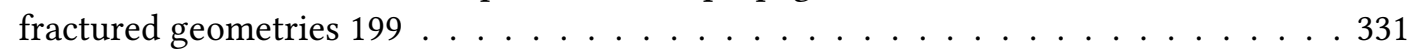

Analysis of numerical error in lattice-Boltzmann simulations of Stokes flow in porous media

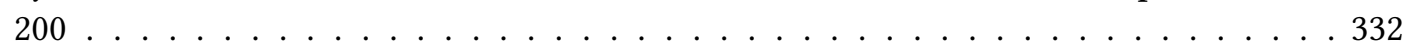

The effect of kinetics on the efficiency of biologically induced carbonate precipitation via urea hydrolysis for soil improvement applications $566 \ldots 332$

Pulsed waterflood performance in oil reservoirs: a pore-scale visualization experiment 395

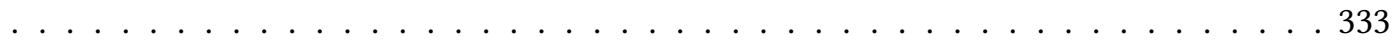

Experimental and theoretical study of formation of filtration structures in swelling clays

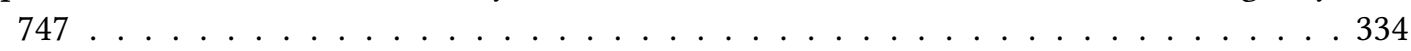

Stability and transport behavior of DNA-tagged silica particle tracers in undisturbed labo-

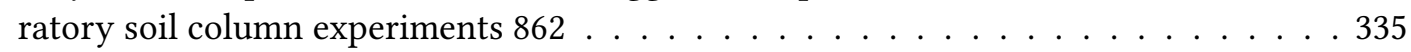

Pore-scale Characterization of Biogenic Gas Formation in Porous Media: The Effect of Gas Production Rate 739 . . . . . . . . . . . . . . . . . . . . 336

Deep learning-based fracture extraction from X-ray computed tomographic images of frac-

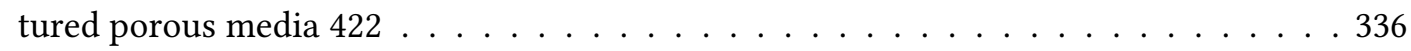

MICP in the Field: continuous injection to reduce permeability and enhance wellbore in-

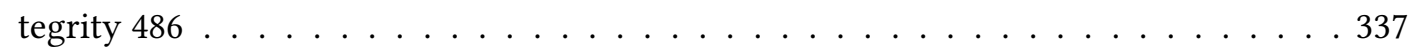

Imaging of porosity of concrete hydrates by (Cryo/LMI-) BIB-SEM 846 . . . . . . . . . 338

On the scalability of MGSR- and CWY-based GMRES variants for the solver of two-phase flow in porous media using MHFEM discretization $812 \ldots 339$

Beyond Kozeny Carman 89 . . . . . . . . . . . . . . . . . . . . . . . 340

Hepatitis C virus replication within the porous medium of the Endoplasmatic Reticulum

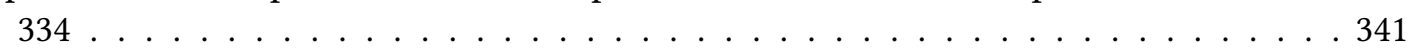

Multiphase multicomponent flow in porous media with general reactions 328 . . . . . . 342

Pore-scale modeling of catalytic filters for automotive exhaust gas aftertreatment 11 . . . 344

Smart \& cost efficient solvers for poroelastic wave propagation 452 . . . . . . . . . . . 345

Hybrid models for simulating blood flow in microvascular networks 132 . . . . . . . . 346

SoilJ - A software for the semi-automatic processing and analyses of X-ray images of soil

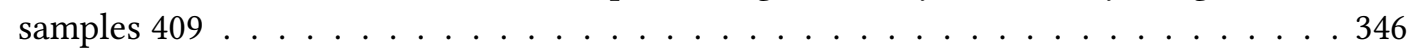

Quantifying and Analysing Transport Phenomena in Electrospun Electrodes by X-ray Com-

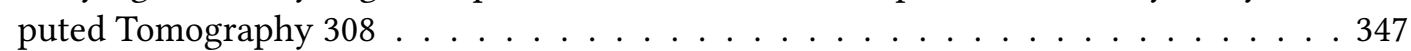


Snap-Off Phenomenon in a Pore Network Model For Retrograde Gas Flow in Porous Media

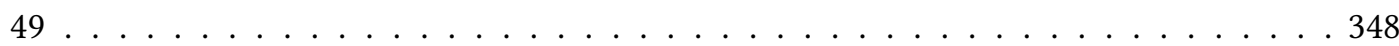

Investigation of time dependent liquid property influence on paper wetting and penetration using pico-liter contact angle measurement 26 . . . . . . . . . . . . . . . . 349

Iterative coupling methods for multi-permeability poroelasticity models 196 . . . . . . . 349

Influence of Small Scale Geological Heterogeneity on Capillary Trapping of CO2 606 . 350

Low-Temperature Waste Heat Recovery by Reverse Electrodialysis Cells 84 . . . . . . . . 351

Assessing pore network model performance for predicting flow and transport in three dimensional porous media $691 \ldots \ldots \ldots 352$

Robust simulation of mineral precipitation-dissolution problems with non-Lipschitz min-

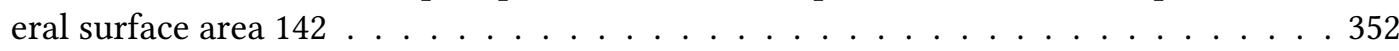

A numerical tool for design and explicit chemical interpretation of low salinity water flood-

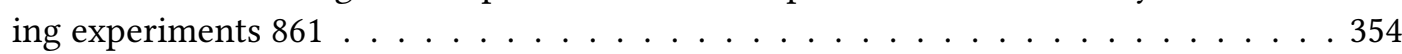

Experimental observations of the spatial structure of the concentration field during solute transport in reservoir rocks by Positron Emission Tomography 440 . . . . . . . . . 355

A microfluidic study on biofilm and preferential flow path formation in porous media 226355

Influence of Porous Structure on Electrokinetic Properties of Polymer Membranes 871 . 356

A Domain Decomposition Projection Method for the Navier-Stokes Equations Based on the Multiscale Robin Coupled Method 203 . . . . . . . . . . . . . . . 357

A relaxation projection analytical-numerical approach in hysteretic two-phase flows in

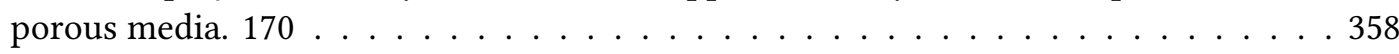

Phase diagram of quasi-static immiscible displacement in disordered porous media 786 . 359

Biofilms in porous media: laboratory experiments, mathematical modelling, and upscaling.

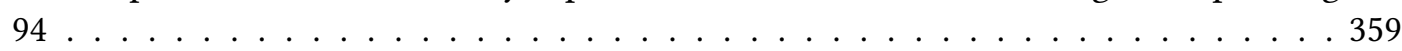

Tomographic PIV of low to high Re flow through quadratic and hexagonal arrangements

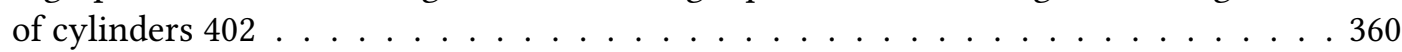

Upscaling unsteady and inertial single-phase flow in homogeneous porous media 674 . . 361

Study of coupled finite volume schemes minimizing the grid orientation effect in reservoir

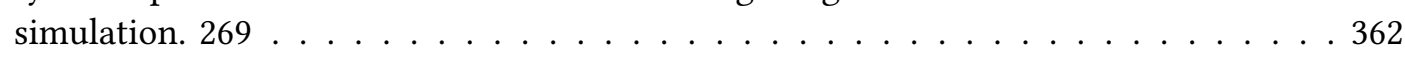

Non-linear biogeochemical reactions in chemical gradients 629 . . . . . . . . . . . . 362

Viscoelastic multiphase flow simulations for tumor growth 417 . . . . . . . . . . . . 363

Optimal thickness of porous micro-electrodes operating a single reduction reaction 778 . 364

Dynamic effect in capillary pressure for fractal porous media: a theoretical model 891 . . 365

Transport mechanism of emulsion micro-gel particles: Synergistic effect of emulsion and

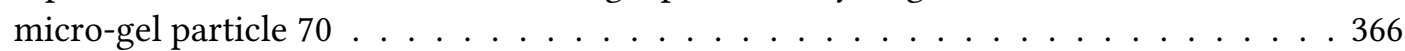


Hygromorphic response dynamics of oak: an NMR study $202 \ldots$. . . . . . . . . . 367

Multifunctional doubly-porous materials with controlled hydrophilicity 803 . . . . . . . 367

High Pressure Small Angle Scattering Investigation of Methane and CO2 Sorption in Shales and Nano-porous Shale Analogues $332 \ldots \ldots 368$

The influence of interfacial tension on spontaneous imbibition of low-permeability waterwet reservoir $261 \ldots \ldots 369$

Investigation on the multi-parameter of hydrate-bearing media using nano-focus X-ray computed tomography $221 \ldots \ldots$. . . . . . . . . . . . . . . . . . . . . . . . . . .

Genetic Mechanism of Diverse Pores in Bioclastic Limestone and Impacts on Reservoir

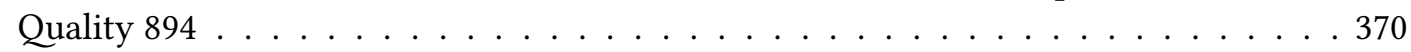

Micro Gas Displacing potential of horizontal well in tight reservoirs 232 . . . . . . . . . 370

The analysis of the effect of ethane fraction on the carbon dioxide displacement in coal using molecular simulation $217 \ldots \ldots$. . . . . . . . . . . . . . . . . . . . . .

Investigation of CO2 Huff-n-Puff EOR Mechanisms in Liquid-Rich Shale Reservoirs based on Microscopic Visualization Experiments 533 . . . . . . . . . . . . . . . . . 372

Advanced CO2 Injection Enhanced Oil Recovery in Shale Oil Reservoirs: Experiments, Molecular Dynamic Simulations and Theoretical Analysis 532 . . . . . . . . . . . . 373

Simulation of Subsurface CH4 Storage if Using CO2 as Cushion Gas 195 . . . . . . . . . 374

Studying the generation of nanoparticle-stabilized foam at pore scale 315 . . . . . . . . 375

Modeling nanoparticle transport in porous media in the presence of a foam 319 . . . . . 375

Role of the interaction of the calcite-water interface in the wettability alteration during low salinity waterflooding $449 \ldots \ldots \ldots$. . . . . . . . . . . . . . . . . . . . . . . .

Object-based modeling of braided river reservoir interbed constrained by genetic density

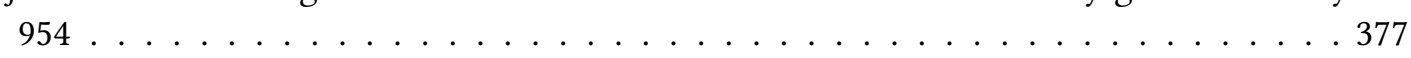

Calculating the exact volume of intersections between spheres and arbitrary polyhedra 859

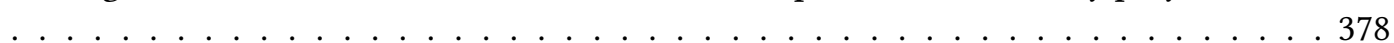

Research on water and rock interaction of groundwater contamination in abandoned coal

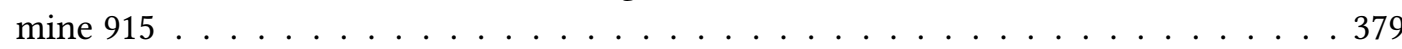

Heavy oil recovery and asphaltenes deposition mechanism in solvent based recovery tech-

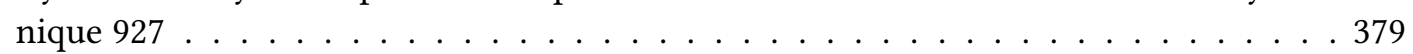

Moisture and Heat Transfer in Layered soil: Implications for Inversely Estimating Layered Soil Hydraulic Property 516 . . . . . . . . . . . . . . . . . . . 380

Slip length of oil-water mixtures in organic nanopores 177 . . . . . . . . . . . . . . . . 381

Flow regulation analysis in rough fractures considering time variable conductivity 581 . 381

Data Assimilation Based on Ensemble Kalman Filters Using KL-Expansion and Stochastic

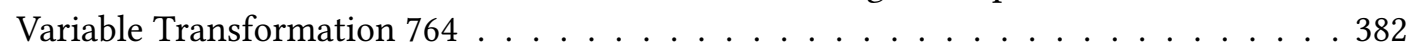


Pore scale observations of the impact of wettability alteration on fluid interfacial curvature

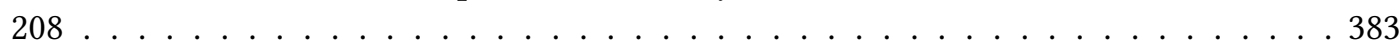

\begin{tabular}{lll}
\hline Two-phase electrohydrodynamics in complex geometries - modelling and simulation 48 & 384
\end{tabular}

Coupling free flow and porous-medium flow: Comparison of solution strategies for solving

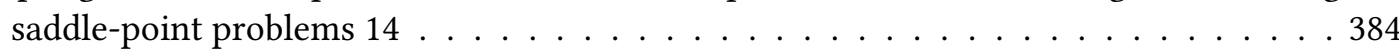

Evaluation of pore space structure due to chemical dissolution of rock matrix 15 . . . . . 385

Coupling Continuum and Pore-Network Models to Study the Dispersed Nanostructured Thin Film Electrodes of Polymer Electrolyte Fuel Cells 336 . . . . . . . . . . . . . . 386

A New Method for Analysis of Nano-Scale Dual-Pore-Shape Pore Size Distributions in shale and its application $576 \ldots \ldots \ldots$. . . . . . . . . . . . . . . . . . . . . . . . . . .

Shale Oil Transport through Nano Slit of Kerogen by Molecular Dynamic Simulation 517387

Hydrate growth in porous media - implications to the water permeability of hydrate-bearing

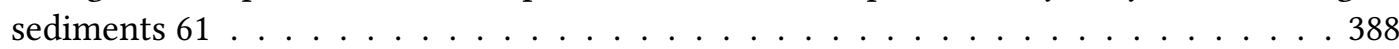

Grain Boundary Effects on Mineral Dissolution in Porous Media 887 . . . . . . . . . . . 389

Pore-scale investigation of Bingham fluids flow through tight sandstone on representative element volume 627 . . . . . . . . . . . . . . . . . . . . . . . 389

Poro-Elasto-Plastic Modeling of Hydraulic Fracture Propagation in Naturally Fractured Deep Shale Reservoir 438 . . . . . . . . . . . . . . . . . . . . . . . . . 390

Study on modification experiment of pore structure of coal by response surface optimiza-

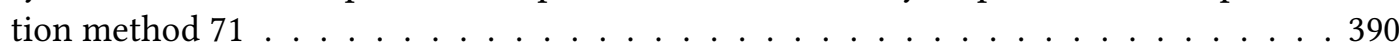

Characterization of pore interconnectivity and adsorption-induced deformation in mesoporous thin films by ellipsometry porosimetry : an experimental study 700 . . . . . 391

Adsorption-induced deformation in mesoporous materials: comparison between pore-load modulus and Young's modulus $703 \ldots \ldots$. . . . . . . . . . . . . 392

Pore-filling model for micro-structure of cement hydration using level-set 439 . . . . . . 392

Experimental Investigations of Nanopore Adsorption Measurement by Low-field Nuclear Magnetic Resonance 815 . . . . . . . . . . . . . . . . . . . . . 393

Insights into evaporation from the surface of capillary porous media gained by discrete

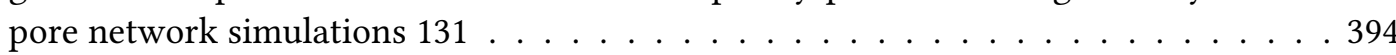

Linearization and Domain Decomposition Methods for Two-Phase Flow in Porous Media

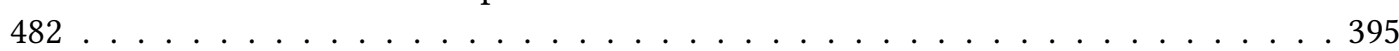

An Ensemble-based Learning Framework For History Matching With Imperfect Forward

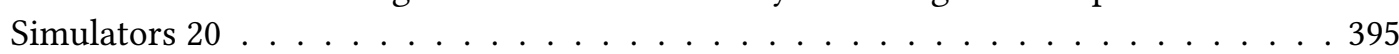

Precisely Studies on Petrophysical Parameters and Interface Properties of Cores from Tight Oil Reservoirs 45 . . . . . . . . . . . . . . . . . . . . . . . 396

One-domain approach for immiscible two-phase dispersed flow in stratified porous media

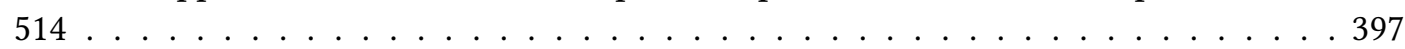


Operator-based linearization method for modelling of multiphase flow with buoyancy and

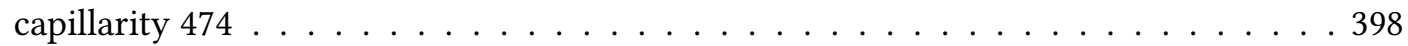

Numerical calculation of effective thermal conductivity for aerogel porous materials at high

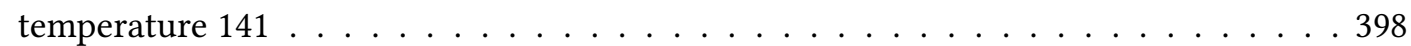

Research on influential characters of low- permeability coal rock under microwave radia-

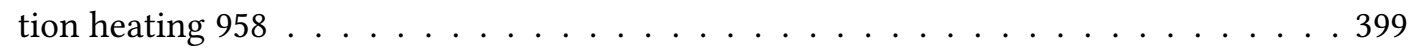

New Yield Stress fluid porosimetry Method (YSM) 137 . . . . . . . . . . . . . . . . . . . 400

Viscous fingering in a soft porous medium $742 \ldots \ldots$. . . . . . . . . . 401

Direct Pore-scale modelling of CO2 solubility and mineral trapping 41 . . . . . . . . . . 401

Pore Network Simulation of Biogenic Gas Nucleation and Migration in Porous Media 768 402

An Integrated History-Matching of Enhanced Oil Recovery and Geochemical Processes for Carbonated Water Injection in Carbonate Rocks 1 . . . . . . . . . . . . . . . . 403

Two-Phase Flow Upscaling For Fractured Reservoirs 557 . . . . . . . . . . . . . . . . . 403

Simulation of Flow and Transport in Microfractures using Discrete Fracture Network Ap-

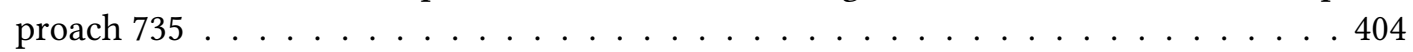

Particle tracking velocimetry for the multiphase fluid flow through a three-dimensional

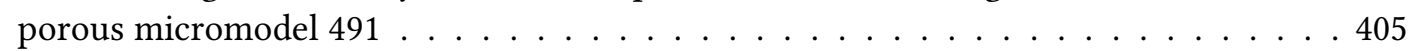

The impact of fracture slip on the fracture contact pressure 476 . . . . . . . . . . . . 405

The Multiscale Restriction Smoothed Basis Method for Thermal Water-flooding in Porous Media 850 . . . . . . . . . . . . . . . . . . . . 406

Analyzing tissue remodeling in avascular tumors 62 . . . . . . . . . . . . . . . . 407

Pore-by-pore investigation of multiphase flow characteristics using dynamic micro-computed tomography $471 \ldots \ldots \ldots \ldots \ldots$. . . . . . . . . . . . . . . . . . . . . .

In-situ investigation of aging protocol effects on relative permeability measurements using high throughput experimentation methods $561 \ldots \ldots$. . . . . . . . . 408

Pushing of a liquid drop through a non-wettable porous media 273 . . . . . . . . . . . . 409

Irreversible salinization and sodification $884 \ldots \ldots \ldots \ldots$. . . . . . . . . 410

Efficient DG-based simulation of coupled surface subsurface flow 942 . . . . . . . . . . 411

Lattice Boltzmann simulation of drop impact on textile materials placed on a substrate 495

Deep learning from multiphase digital rock simulations 706 . . . . . . . . . . . . . 412

The fluorescence microscopy toolkit in investigations of paper: High-speed, super-resolution

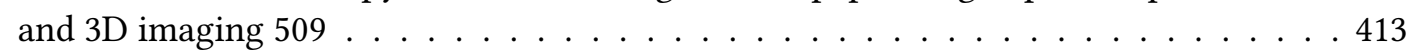

Coreflood on a Chip: Scaling up of a Micromodel for EOR Experiments 766 . . . . . . . . 413 
DEPENDENCY OF STRESS REGIME AND ORIENTATION ON FRACTURE PERMEABILITY IN NATURALLY FRACTURED RESERVOIRS- A DISCRETE FRACTURE APPROACH

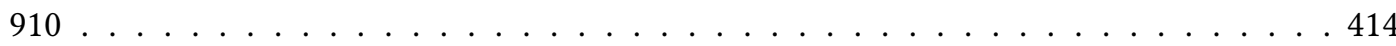

Numerical study of dispersed foamer injection in a gas stream for Enhanced Oil Recovery in Naturally Fractured Reservoirs 909 . . . . . . . . . . . . . . . . . . . 415

Reactive silicate mineral carbonation under diffusive transport and confining stress 875 . 416

Using nanoCT and high contrast imaging to inform microporosity permeability during Stokes-Brinkman single and two-phase flow simulations on microCT images 333 . . 416

Regeneration of capillary state for water occluded in dual-porosity systems 620 . . . . . 417

Generalizing the Non-Local Darcy Formulation for Settings with Boundaries 424 . . . . 418

Development of a 3D dual pore-system leaching model with application on metal extraction from an oxide copper ore 238 . . . . . . . . . . . . . . . . . . . . . . 4419

Modelling of the liquid water transport in the porous layers of a PEM fuel cell using porenetwork modelling techniques $727 \ldots \ldots \ldots$. . . . . . . . . . . 421

Comparative study of voids in minerals aggregates of virtual aggregate assembly using a

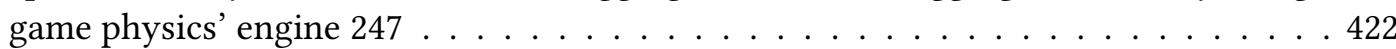

Filtration by porous media: the role of flow disorder 183 . . . . . . . . . . . . . . 423

Modeling of asphaltene precipitation and deposition in porous medium under a natural gas injection process. 616 . . . . . . . . . . . . . . . . . . . . . . . . . . . . . . . . . .

Experimental observations of Sporosarcina Pasteurii attachment in sands and soils for a range of MICP treatment strategies $437 \ldots \ldots$. . . . . . . . . . . . . . 424

Pore network modeling in unsaturated soil mechanics: current state, challenges, and future

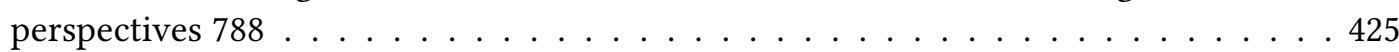

The impact of intraformational baffles on carbon mineralization in $\mathrm{CO} 2$ storage reservoirs

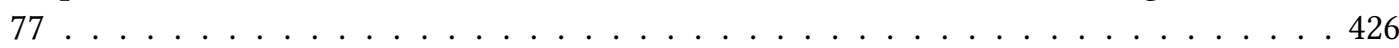

Laboratory Investigation of Infiltration of Non-Newtonian Fluids through Cold Porous Media for Effective Remediation of Adsorbed Contaminants 721 . . . . . . . . . . . . 428

Fronts in two-phase flow in porous media: hysteresis and dynamic capillarity 271 . . . . 428

Microbial ecology of seawater flooded petroleum reservoirs: a modelling and field study

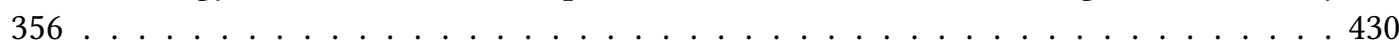

Modified Low Salinity Water Efficiency by Advanced Core-Flooding under X-Ray Tomo-

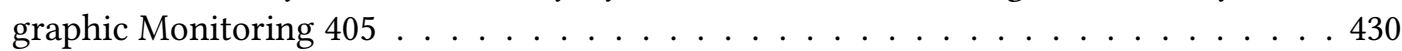

Bayesian Validation of a Coupled Porous Medium/Free-flow Model using Adaptive arbitrary Polynomial Chaos Expansion $184 \ldots \ldots$. . . . . . . . . . . . . . . 431

A General, Data-Driven Dispersion Model for Stochastic Transport in Heterogeneous Me-

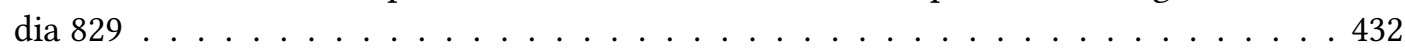


Coupled Thermally-Enhanced Bioremediation and Renewable Energy Storage System: Conceptual Framework and Modeling Investigation 901 . . . . . . . . . . . . . . . 433

Correlations between cross-over and critical flow conditions for steady-state two-phase flows in porous media $978 \ldots \ldots \ldots$. . . . . . . . . . . . . . . . . . . . . . .

Binary solvents in nanoporous confinement: how different are they? 138 . . . . . . . . 435

Multiphysics gas transport in coal seams $162 \ldots \ldots$. . . . . . . . . . . 436

3D model-based interpretation of tracer tests in large undisturbed columns from fractured

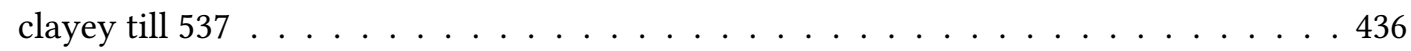

A Novel Method to Evaluate Reservoir Net pay by Using Cut-offs and Hydro Flow Units

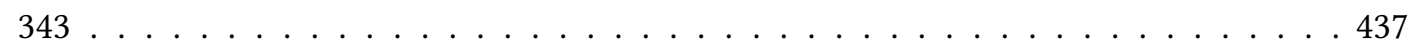

Unconventional transport mechanisms in porous media: connectivity enhancement due to thin film flow $524 \ldots \ldots \ldots$. . . . . . . . . . . . . . . . . . . . . . . . . . . . . . . . .

Examining the limitations and advantages of multiphase flow meters 197 . . . . . . . . 438

Mathematical model of microbiological oil recovery with wetting inversion by bio-surfactants

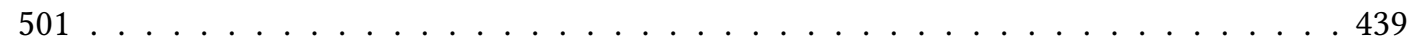

Convective Flow in Fuel Cell Gas Diffusion Layers and its Impact on Evaporation 806 . . 439

Multiphase multi-rate mass transfer model for anomalous transport in fractured and heterogeneous media 65 . . . . . . . . . . . . . . . . . . . . 440

Water sorption into and evaporation from thin porous media- a thermographic study 253441

Analysis of species mass transport in fibrous electrodes of redox flow batteries 101 . . . 442

Effect of inhomogeneous compression on pressure drop and species transport in electrodes of redox flow batteries $161 \ldots \ldots \ldots$. . . . . . . . . . . . . . . . . . . . . . . . . . .

Pattern formation of frictional fingers in a gravitational potential 831 . . . . . . . . . 444

Environmental Constraints of Non-Mechanical Excavation of Water from Gypsum on the Martian Surface 158 . . . . . . . . . . . . . . . . . . . . . . . . 444

Water Vapor Diffusive Transport in a Smectite Clay: Cationic Control of Normal vs. Anomalous Diffusion $718 \ldots \ldots \ldots 4 . \ldots \ldots \ldots$

Specific Surface Area Determination on Chalk Drill Cuttings 924 . . . . . . . . . . . . . 446

Upscaling transport of nanoparticles in porous media $956 \ldots$. . . . . . . . . . . . 446

Mathematical Models for Skin Penetration 352 . . . . . . . . . . . . . . . . . . . . . . 447

Uncertainty Quantification in Multiphysical Process Simulation of Porous and Granular Media for Geotechnical Applications 448 . . . . . . . . . . . . . . . . . . . . 448

Modelling deformation and flow during fault-reactivation in argillacieous rocks following

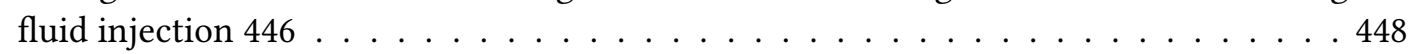

Flow through a regular arrays of complex shapes 886 . . . . . . . . . . . . . . . . 449 
Is it possible to introduce 3D complex heterogeneities in a real transient groundwater model used to manage a sedimentary aquifer? $639 \ldots \ldots$. . . . . . . . . . . . . 450

Pore size distribution effects on the mechanical behaviour of nanoporous silicon structures

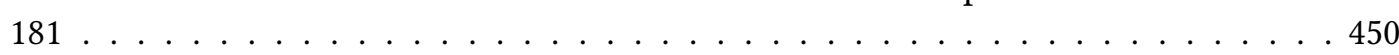

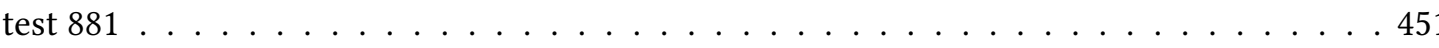

Phase-field method vs. volume of fluid (VOF) for simulating immiscible two-phase flow in

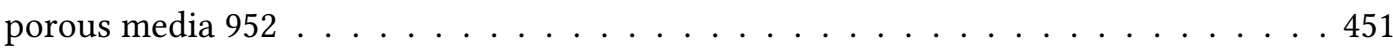

Finite element analysis of LTE and LTNE processes during steam injection process in porous

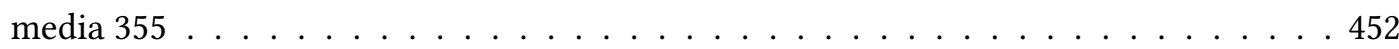

A versatile model for mass transport abnormalities applicable to molecular displacements complemented by tracer test $649 \ldots \ldots$. . . . . . . . . . . . . . . 4 452

Accuracy of Hydraulic Rock Properties derived from X-ray-Tomographic Images - Insights from Laboratory Experiments on Porous and Fractured Rocks 311 . . . . . . . . . . . 453

Quantifying the influence of microstructure on effective conductivity and permeability:

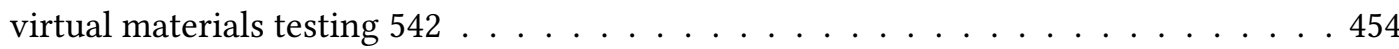

Permanent Deformation of Permeable Base Based on Repeated Load Triaxial Test 609 .

The Effect of Injection Rate on Fluid Invasion Patterns: Immiscible Two-Phase Fluid Displacement in 2D Laser-Manufactured Micromodels 925 . . . . . . . . . . . . . . 456

Novel insights into two-phase flow dynamics due to wettability alteration during low salin-

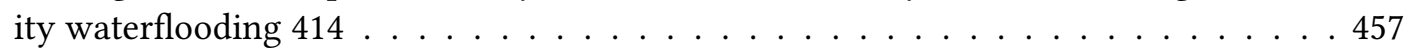

Using direct numerical simulations to uncover the mechanisms of two-phase flow between GDL interfaces in fuel cells $435 \ldots \ldots$. . . . . . . . . . . . . . 458

How seismic faults and well test data analysis may help to better model the sub-seismic faults: a study of impact of a fractal dimension choice on flow. 798 . . . . . . . . . 458

Experimental gas permeability studies on synthetic microporous materials 330

Apparent Permeability of Gas Shales - Caution when comparing simulation results with

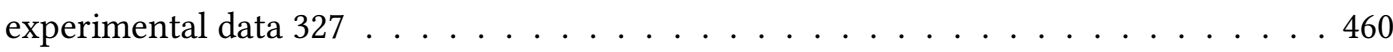

Formation dry-out and salt precipitation in porous and fractured media: Laboratory insights on physics and dynamics of CO2-induced halite accumulations 38 . . . . . . 461

Numerical methods for detection of kidney disease using tissue deformation fields 81 . . 462

Numerical modeling of wave propagation in fractured porous fluid-saturated media - fracture connectivity effect on seismic wave attenuation $18 \ldots \ldots$. . . . . . . 463

Numerical assessment of organic source rocks' flexibility at the atomistic scale an its implications on transport at the nanoscale $154 \ldots \ldots$. . . . . . . . . . . 464

Impact of Physical Heterogeneity on Multispecies Reactive Transport in a Microporous Carbonate 847 . . . . . . . . . . . . . . . . . . . 464

Non-Newtonian behaviour of foam flow in porous media: experiments and upscaling 420466 
Observation and modelling of capillary instabilities, front pinning and drainage/imbibition hysteresis in two-phase displacements across a single constriction 565 . . . . . . . 467

Controlling the drying Induced Peeling of Colloidal Films 93 . . . . . . . . . . . . 468

Experimental study of the influence of flow rate, temperature and pressure on the carbonation of natural serpentinites induced by CO2 geological storage 737 . . . . . . . . . 469

Screening of EOR Potential on the Pore Scale - Application of Microfluidics to Alkaline

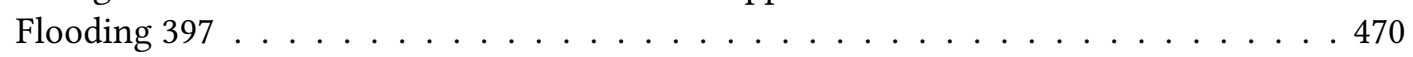

Cross-property relations between elastic properties and thermal conductivity of partially sintered ceramics and fractured rocks $662 \ldots \ldots$. . . . . . . . . . . . 4 . . . . . .

Pore shape influence on the thermal conductivity of porous ceramics and sedimentary rocks with empty, water-saturated or high-conductivity pore space 665 . . . . . . . . . 472

Groundwater Remediation using Nanotechnology 151 . . . . . . . . . . . . . . . . . . . 473

Mobility of zero-valent iron nanoparticles in porous media - A study using X-ray computed micro-tomography $698 \ldots \ldots \ldots$. . . . . . . . . . . . . . . . . . . . . . .

Experimental and Numerical Studies of Chemical Flooding Under Impacts of Solutal Marangoni

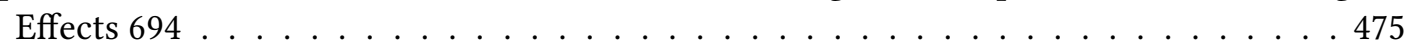

On the impact of asphaltene and resin on stability of water in oil emulsions 840 . . . . . 476

Supershear rupture promoted by poroelastic coupling in induced earthquakes 487 . . . . 476

A novel nano-based filter to combat oil spills 699 . . . . . . . . . . . . . . . . . . 477

Evaluating Storage Capacity of a Depleted Oil Reservoir during CO2 sequestration process using advanced numerical simulation technique $240 \ldots \ldots$. . . . . . . . . 478

Beyond failure of geomaterials $879 \ldots \ldots$. . . . . . . . . . . . . . 479

About the Use of 3D Printed Micromodels to Investigate single- and two-phase Flow Pro-

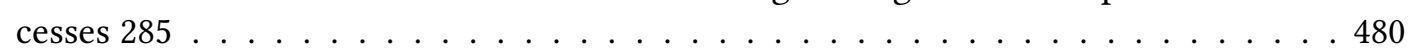

A study on the microstructure of colloidal silica hydrogel when used as soil grouting material for ground barriers 666 . . . . . . . . . . . . . . . . . 481

Fluid-Particle Interactions: Image-based Discrete Element Method (iDEM) 529 . . . . . . 481

Direct observation of the moisture distribution in castables during first dry out at high temperatures: an NMR study $204 \ldots \ldots$. . . . . . . . . . . . . . 482

Coupled evolution of ecohydrologic processes and pore-size distributions 724 . . . . . . 482

Analyzing ultrasonic data of nanoporous materials without using effective medium theories: pore sizes and pressure-dependence of elastic moduli. 310 . . . . . . . . . . 483

Influence of sub-micrometer porosity on permeabilty of Rotliegend reservoir sandstones

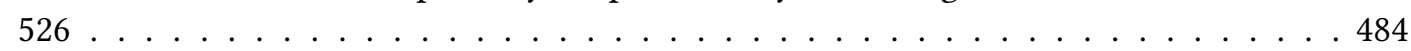


Homogenized modeling for vascularized poroelastic materials 351 . . . . . . . . . . . . . 484

A comparative study of elastic, single- and two-phase REV of porous rocks 64 . . . . . . 485

Effective elastic parameters of porous heterogeneous materials computed from tomographid image large datasets using personal computers $707 \ldots$. . . . . . . . . . . . . 486

A Two-Stage Markov Chain Monte Carlo Method for Full Waveform Inversion 155 . . . 487

Mixing-limited bimolecular chemical reactions at pore-scale 428 . . . . . . . . . . . . 487

Network models for physical and biological processes in porous media 945 . . . . . . . . 488

Adaptive Stratified Sampling in Geomechanics and Fractured Porous Media 415 . . . . . 489

In Situ Bioremediation of Selenium and Nitrate for Mine Waste Management in the Elk Valley, British Columbia 885 . . . . . . . . . . . . . . . . . . . . . 490

Two-fluid model for supercritically adsorbed films of hydrogen and methane in nanoporous

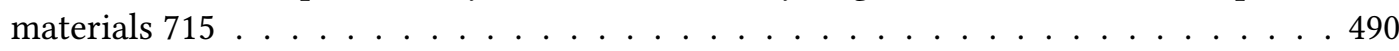

Numerical and experimental immiscible two-phase flow under non-isothermal conditions: application to thermally enhanced DNAPLs recovery $563 \ldots \ldots 492$

Uncertainty Quantification in Discrete Fracture Networks flow simulations 375 . . . . . 493

Phase Separation of Hydrogen 941 . . . . . . . . . . . . . . . . . . . . . . . 493

Dynamic measurements of drainage capillary pressure curves in carbonate rocks 302 . . 495

Dynamic synchrotron microtomography for direct in-situ capillary flow visualization in functionalized porous material for passive microfluidics $444 \ldots$. . . . . . . . . 495

Experimental modelling of the oscillating multiphase flow in porous medium with chemi-

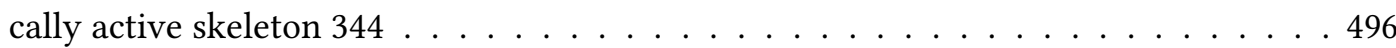

Magnetic resonance imaging to assess changes in transport properties of porous media due to dissolution and precipitation processes $248 \ldots \ldots$. . . . . . . . . 498

Ecohydrology: interactions of life and water around a porous medium 981 . . . . . . . . 499

Impact of wind action and medium physical properties on horizontal pore gas flow in nearsurface porous media. 5 . . . . . . . . . . . . . . . . . . . 499

Swelling behavior of shale/clay: Discrete element modeling, based on Monte-Carlo tech-

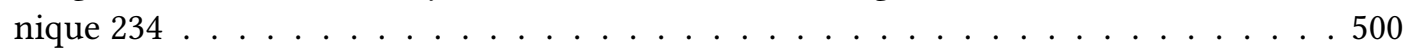

Dissolution - precipitation driven migration of a salt crust $207 \ldots \ldots$. . . . . . . . 501

Modelling cell migration in confined environemnts 877 . . . . . . . . . . . . . . . . . . 501

On the Upscaling of Reactive Flow in Plug-Flow Experiments in the Project ResKin 682 . 502

Experimental Study of Gas Mass Transfer between Gas Bubbles and Water in Otherwise

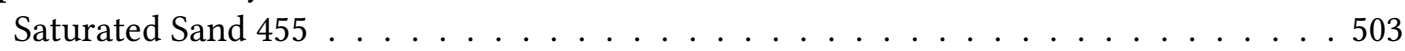


Pathways of heat and mass transfer in adsorption of methane in nanoporous monolithic

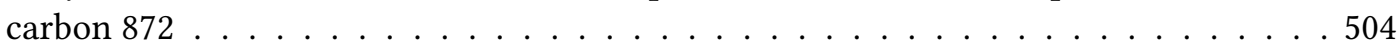

Experimental determination of fluid and solute transport parameters in textiles 498 . . . 505

Pore-network - lattice Boltzmann method hybrid model for multiphase flow 936 . . . . . 506

Pore-network - lattice Boltzmann method hybrid model for multiphase flow 678 . . . . . 507

Comparative Analysis of Image Segmentation Techniques Applied for Measuring PoreScale Parameters in Saturated Media $902 \ldots . . \ldots 508$

Upscaling hydrodynamic transport in heterogeneous porous media from fully advective to

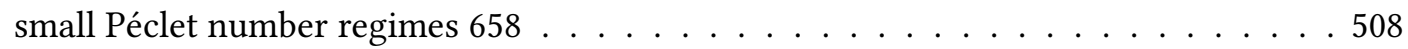

Generalized network modelling: validation using micro-CT images of two-phase flow 838509

Quantitative Predictions of Two-Phase Flow Dynamics in Porous Media by the Pore-Network Modeling 730 . . . . . . . . . . . . . . . . . . . . . 510

Hybrid LBM simulation of non-isothermal drying of colloid suspension in a micro-pore

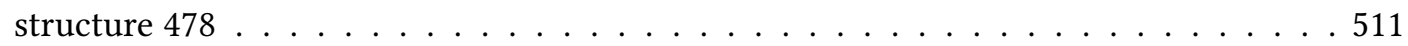

Impact of subcore-scale permeability heterogeneity on solute mixing in a microporous limestone: experiments and numerical study $430 \ldots \ldots$. . . . . . . . . . . 512

The effect of carbonate cementation on the pore space structure 456 . . . . . . . . . . 513

A Convergence Analysis of MCMC Methods for Porous Media Flows 388 . . . . . . . . . 514

Injection Strategies and Benefits of Using Flue Gas as Working fluid for Enhanced Geothermal Systems 485 . . . . . . . . . . . . . . . . . . . . . . . 5 514

Effect of fractures geometrical and hydrodynamic characteristics on natural convection in fractured porous enclosure $235 \ldots \ldots \ldots 515$

A multi-scale asymptotic homogenization technique to the material remodeling of hetero-

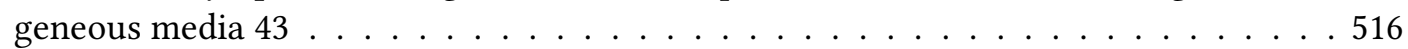

Viscous fingering instability in oscillating systems $468 \ldots \ldots$. . . . . . . . . . . 516

Some improvements to projection-based embedded discrete fracture model (pEDFM) 890517

Numerical simulation of coupling flow and geomechanics for fractured reservoirs 117 . . 517

Thermal Driving Forces for Transport in Porous Media 975 . . . . . . . . . . . . . . . 519

Upscaling reactive transport in an evolving porous medium 27 . . . . . . . . . . . . . 520

Discrete-continuum multiscale model for evolving porous media 80 . . . . . . . . . . . 520

A pore-scale model of microbially induced calcium carbonate precipitation 502 . . . . . 521

Flexible Framework for Two-Phase-Flow in Extremely Heterogeneous Media 256 . . . . 522

Modeling of wavefields in saturated elastic porous medium based on thermodynamically compatible system theory for multiphase mixture $757 \ldots$. . . . . . . . . . . . . 523 
Machine Learning for Data Assimilation, Uncertainty Quantification of System Performance and Optimization of Controls $366 \ldots \ldots \ldots$. . . . . . . . . . . . . . . 524

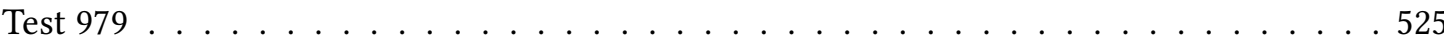

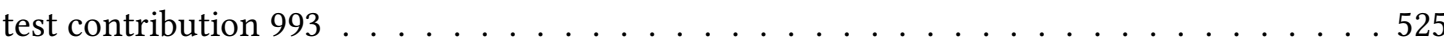

Experimental and numerical study of the compression dependency of the permeability of

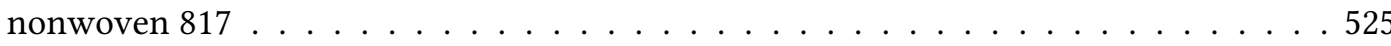

The Multiscale Robin Coupled Method for two-phase flows in porous media 201 . . . . . 526

Robust discretizations for fluid-flow problems in deformable porous media 980 . . . . . . 527

Understanding the behavior of BTEX on the vadose zone using simple 1-D finite differences

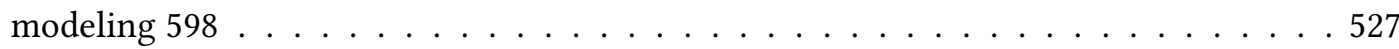

Application of Yield Stress fluids porosimetry Method and pore-network modelling to characterize the pore size distribution of packs of spherical beads 657 . . . . . . . . . 528

Dynamics and reversibility of tracer dispersion in time dependent flows inside rough frac-

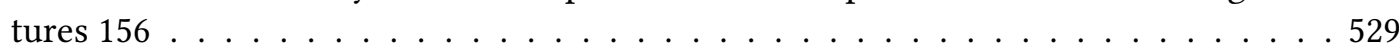

Emptying of mesoporous materials: direct evidence for cavitation 926 . . . . . . . . . . 530

Dissipative processes during two-phase flows: microfluidic experiments 284 . . . . . . . 531

Do deformation bands influence CO2 storage volume? 331 . . . . . . . . . . . . . . . . 532

Chemically-driven convective dissolution in a porous medium 592 . . . . . . . . . . . 533

Multiscale Image Based Modelling of Plant-Soil Interaction 939 . . . . . . . . . . . . . . 533

Flow velocity distribution of a shear-thinning fluid in porous media 360 . . . . . . . . 534

A numerical tool for computing the thermal radiative properties of digitally generated cel-

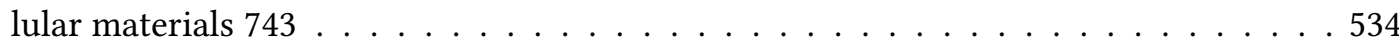

Study of the coupled liquid and oxygen transfer in wood: Application to oak for cooperage

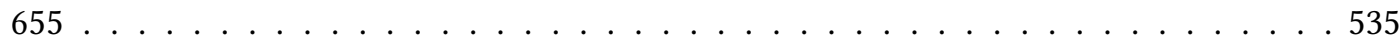

Modeling moisture sorption and desorption in porous materials: A finite volume approach

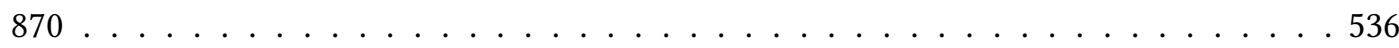

A Study in Earthquake and Porous Medium: A Numerical Approach in Disordered Systems

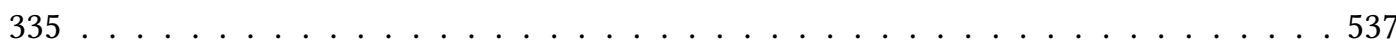

The effect of pore-scale heterogeneities on capillary trapping for geological storage of $\mathrm{CO} 2$

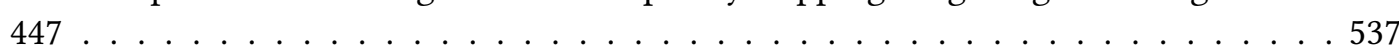

Reduced Darcy-Stokes model for flow in fractured porous media 318 . . . . . . . . . 538

Wettability - Capillary Pressure Relationships in Ketton Rock: From Nano-Scale Fluid Films to Core-Scale 372 . . . . . . . . . . . . . . . . . . . . . . . . 5 539

Dynamics of Capillarity-Driven Water Invasion into Mixed-Wet Angular Pores 463 . . . 540 
Mathematical Modeling and Simulation of Asphaltene Precipitation and Deposition during Miscible Displacement of Fluids in Porous Media 367 . . . . . . . . . . . . . . . . . 541

Mixing by dispersion in heterogeneous porous medium 940 . . . . . . . . . . . . . . . . 542

Scale-up of Laboratory Data for Surfactant-Alternating-Gas Foam EOR 353 . . . . . . . 543

Potential application of fungal biogeotechnology as irrigation canal lining material for agri-

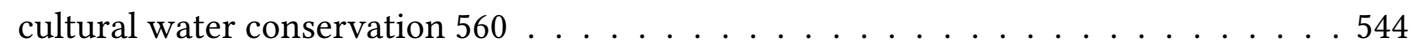

Constitutive relations for primary, secondary drainage and imbibition from percolation

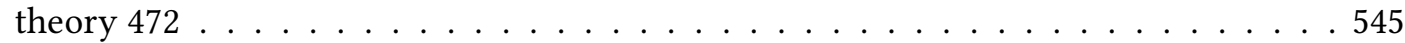

Hydraulic Fracturing In Layered Media: Comparison of Different Scenarios 211 . . . . . 546

Numerical Modelling of Coupled Flow and Fluid-Driven Fracturing in Fractured Porous Media using the Immersed Body Method 145 . . . . . . . . . . . . . . . . . . . 547

An efficient control volume finite element method for multi-phase flow in fractured porous

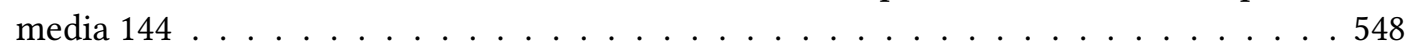

The Effects of Coating Morphology on Complex Liquid - Porous Substrates Interaction 140

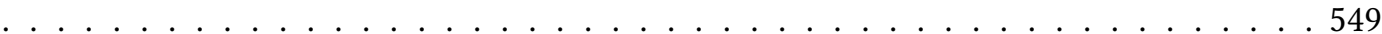

A Conservative Streamline Method for In-Situ Combustion Processes 693 . . . . . . . . . 550

Phase-field modelling of desiccation cracks in multiphase porous media 863 . . . . . . . 551

Multiscale, pore-scale modeling of fluid mechanics using digital images and direct numerical solutions on unstructured meshes $702 \ldots \ldots$. . . . . . . . . . . . 552

Structural characterization of nanoporous materials by small-angle X-ray scattering 951

Finite element/finite volume framework for phase-field fracture in saturated porous media

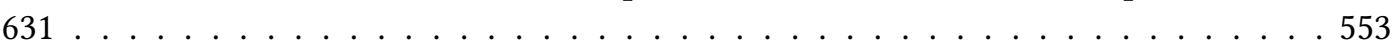

Seeking minimum entropy production for flow-field patterns and their geometries in fuel

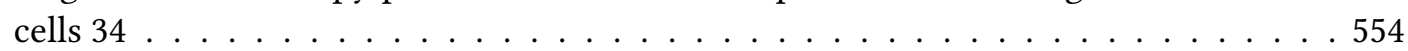

Three-Phase Flow Visualization and Characterization for Water- and Mixed-Wet Carbonate

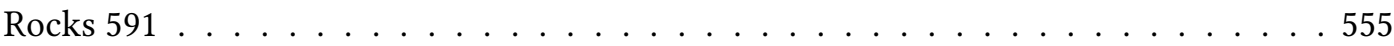

Biofilm architectural differentiation in porous systems $363 \ldots \ldots 556$

Coupling of Adsorption and Transport in Hierachical Porous Materials 103 . . . . . . . . 556

The role of porous media in PEM fuel cells 929 . . . . . . . . . . . . . . . . . . 557

Imaging and quantifying the microstructure of polymers and composites using Cryo-BIB-

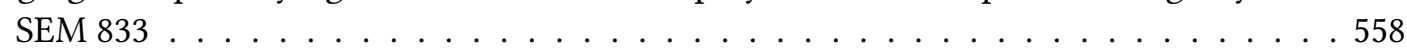

Depth-dependent Flow and Pressure Characteristics in Brain Microvascular Networks 744 558 Modeling Mass Transport in Porous Transport Layers of PEM Water Electrolysis Cells 976559 Non-linear hydro-mechanics of fluid-filled fractures $618 \ldots \ldots$. . . . . . . . . . . 560 
Modelling microbially enhanced coal-bed methane production: From a validated batch model to column scale $434 \ldots \ldots \ldots$. . . . . . . . . . . . . . . . . . . . . . . . .

Microscale Analysis of deforming saturated porous media: Terzaghi stress principle and the Significance of Shear and Plasticity in Hydrostatic Compression 172 . . . . . . . 562

Comparing Upscaled Governing Equations for Transport and Flow Derived via Hybrid Mixture Theory and Homogenization $722 \ldots \ldots$. . . . . . . . . . . . 563

Comparing simple and complex continuum-mechanical models for lung-cancer prolifera-

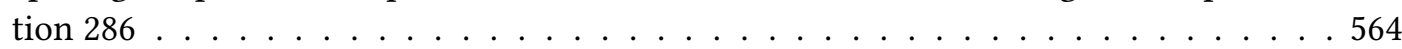

Effective Model for Crystal Precipitation and Dissolution in a Porous Medium with Perforated Solid Matrix 96 . . . . . . . . . . . . . . . . . . . . 564

\begin{tabular}{|l|l}
\hline Digital Rock Physics for Carbonate Reservoirs - Simulation of Acidizing Treatments 473 & 565
\end{tabular}

A Lagrange multiplier method for single-phase flow in 3D discrete fractured porous media

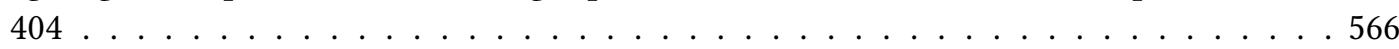

Simulation of fluid flow in porous materials with embedded fractures via an optimization

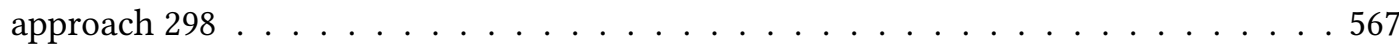

A bi-compartment porous medium approach for modeling of vascularized solid tumors 550

The influence of flow on bacterial transport and surface colonisation in a model porous medium 134 . . . . . . . . . . . . . . . . . . . . . 569

Effects of adsorption-induced deformation of microstructure on fluid flow in smectite 569569

Model coupling in thermochemical heat storage 469 . . . . . . . . . . . . . . . . . . . . 570

Microscale modeling of high-temperature heat transfer in anisotropic porous materials 713

Opposite effects of water repellency phenomenon on transport and release of Escherichia coli and PTCC1767 Rhodococcus erythropolis through dry natural soils 712 . . . . . 574

Numerical simulation of Santa Olalla lagoon through MODFLOW and Local Grid Refinement package. $453 \ldots \ldots \ldots \ldots \ldots$. . . . . . . . . . . . . . . . . . . . . . . .

An LDD method for coupled two-phase flow in porous media 230 . . . . . . . . . . . . . 575

Creating synthetic porous media for multiphase flow experiments under controlled condi-

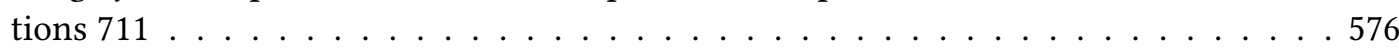

Biological activity affects rheological properties in surface water sediments 664 . . . . 577

Microscopic investigation of polymer flow in porous media 645 . . . . . . . . . . 578

Direct numerical simulation of two-phase flow at the micro-scale using a volume-of-fluid

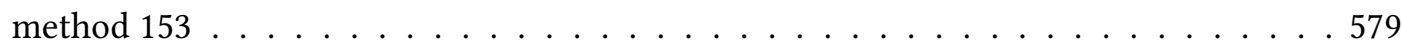

Reservoir Physical Analysis of Ellj Gas Field in West Amu Darya 970 . . . . . . . . . . . 579 
Upscaling of two-phase flow models in porous media: from the pore scale to the Darcy

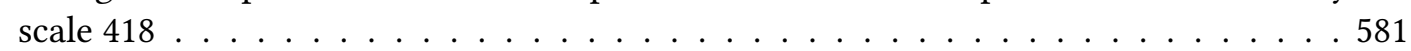

In-Situ High Resolution Dynamic X-ray Microtomographic Imaging of Olive Oil Removal in Kitchen Sponges by Squeezing and Rinsing 625 . . . . . . . . . . . . . . . 581

Cellular 3D printed concrete with disconnected pores 729 . . . . . . . . . . . . . . 582

Gas flows in nano channels: coupling molecular dynamics to a kinetic method 943 . . . 583

A Dual Domain Stochastic Lagrangian Model for Predicting Transport in Open Channels with Hyporheic Exchange 695 . . . . . . . . . . . . . . . . . . . . 584

Production Forecasting of Horizontal Wells with Acid Etching Hole Completion in Unconventional Reservoirs $252 \ldots \ldots \ldots$. . . . . . . . . . . . . . . . . 584

A New Relative Permeability Correlation for Gas Condensate Reservoirs Based on Microscale Force Analysis 250 . . . . . . . . . . . . . . . . . . 585

Porous materials for nutrient recirculation from industry to agriculture 305 . . . . . . . 586

Foam: A potential vehicle for fertilizer delivery in soil 464 . . . . . . . . . . . . . . . 587

Water table depth and soil salinity: Pore-scale physics defining large-scale responses 586588

Quasi-2D Phase Transition of Methane Adsorbed in Cylindrical Silica Mesopores 917 . . 589

Natural designs of efficient heat and mass exchangers 87 . . . . . . . . . . . . . . . 589

Representative Elementary Volume using Second-order Grayscale Entropy: A Sandstone and Carbonate case study 966 . . . . . . . . . . . . . . . . . . 590

Dynamics of multiphase flow in porous media: pore-filling I-n mechanisms, snap-off and

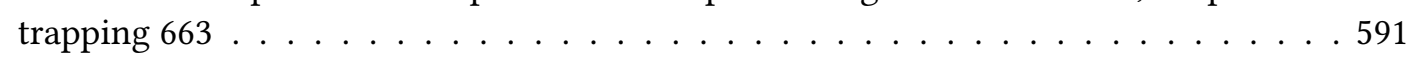

Nonisothermal modeling of $\mathrm{CO} 2$ dissolution trapping in deep saline aquifers 466 . . . . 592

Effective Viscosity of Immiscible Two-phase Flow in Porous Media at High Capillary Num-

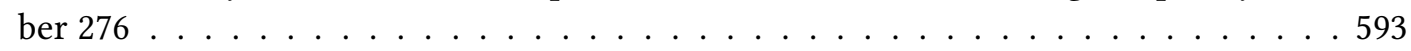

An alternative approach for solving the VTN-stability testing problem of a multicomponent

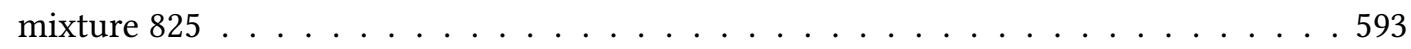

Dissolution Dynamics of Air Bubbles Entrained in Porous Media 368 . . . . . . . . . . 594

Effects of Land-Atmosphere Interactions on Methane Emissions from Natural Gas Pipelines

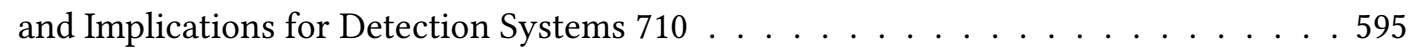

Magnetic resonance imaging of freezing and thawing of water in two contrasting porous

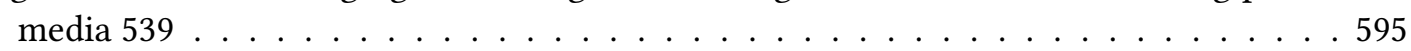

Direct numerical simulation and characterization of flame propagation regimes in porous

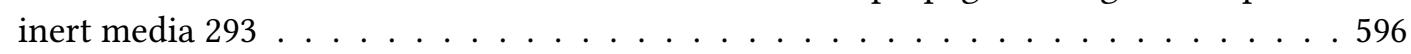

Freezing and thawing of water in fully saturated packed sand sample 595 . . . . . . . . . 597 
A Lagrangian Model of Mixing-Limited Reactive Transport 340 . . . . . . . . . . . . . 597

Lagrangian Modeling of Solute Transport with Equilibrium Reactions 342 . . . . . . . . 598

MHFEM with BDDC for two-phase flow in porous media in 2D and 3D 811 . . . . . . . . 599

Concentration fluctuations and dilution of non-reactive solutes in hierarchical and multiscale geological media 111 . . . . . . . . . . . . . . . . . . 599

STUDY ON THE FLOW CHARACTERISTICS OF OIL IN NANO-CHANNELS 109 . . . . 600

Understanding fluid transport through hardened cement paste from 3D image observation

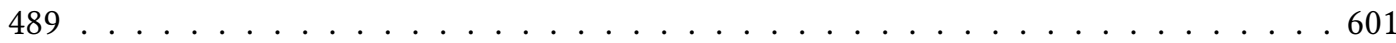

Towards a continuum-mechanical model of fluid-driven fracture for fully and partially saturated porous materials $322 \ldots \ldots \ldots 01$

Pore-scale modeling of coupled hydro-geochemical processes 505 . . . . . . . . . . 602

The multiscale perturbation method for the solution of time-dependent porous media flows

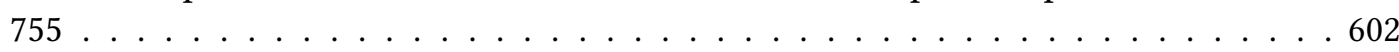

Dynamic interface rearrangement during steady-state multi-phase flow in porous media

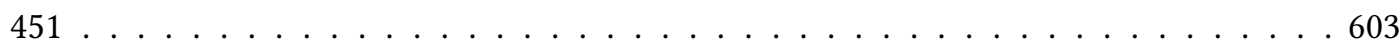

INVESTIGATING THE ENHANCED MASS FLOW RATES IN PRESSURE-DRIVEN WATER FLOW THROUGH NANOPORES $967 \ldots \ldots$. . . . . . . . . . . . . . . 605

CONSISTENT DISCRETIZATION OF FLOW FOR INHOMOGENEOUS GRAVITATIONAL

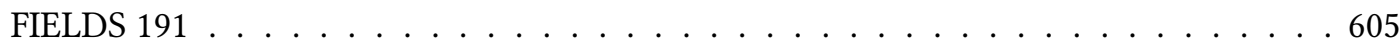

Two-scale approach for the simulation of fluid-structure interaction in thin fibrous filters

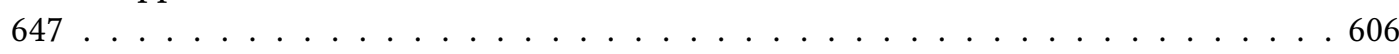

Calcium carbonate crystal/biofilm attachment in MICP grouted sands under different wet-

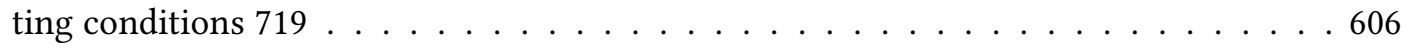

Multiphysics modeling of porous media acid dissolution: the effects of acid fluid properties and geometry on wormhole formation and directionality $852 \ldots \ldots 67$

Relative permeability model of two-phase flow in nanopores considering liquid-solid molecular interactions $531 \ldots \ldots$. . . . . . . . . . . . . . . . . . . . . . . . . . . . . . . . .

NMR Experimental Study of CO2 Huff-Puff Characteristics on Matrix and Fractured Tight Sand Based on Four-Pore Model 525 . . . . . . . . . . . . . . . . . . . . 609

A hybrid numerical model (CFD-DEM) for proppant transport in dynamically propagating hydraulic fractures in shale gas reservoirs 648 . . . . . . . . . . . . . . . . 6610

Topological characterization of 3D printing fracture networks $458 \ldots \ldots \ldots 11$

Karst-on-a-chip: microfluidic studies of dissolution of an analogue fracture 176 . . . . . 611

Natural Time Scales of the Monod type kinetics: experimental support by photocatalysis and oxygen consumption in saturated porous media $714 \ldots$. . . . . . . . . . 612

Mathematical modelling of modified salinity water flooding in carbonate rocks 520 . . . 613 
Modeling the effect of time-dependent wettability modification in modified salinity water

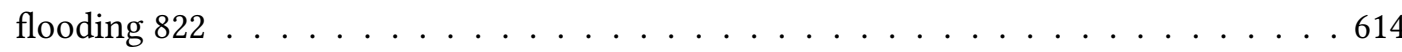

Effective Rheology of Bi-Viscous Non-Newtonian Fluids in Porous Media 272

New Capillary Number Definition for Micromodels: the Impact of Pore Microstructure 312

Three-Phase Fractional-Flow Theory of Oil Displacement by Foam with Multiple Steady

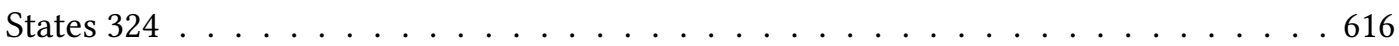

Experimental study on effect of effective stress on coal and gas outburst in crosscutting 281

Accurate thermodynamic analysis of supercritical fluids adsorption on shale and coal 32

Learning Parameters and Constitutive Relationships with Physics-Informed Deep Neural Networks $988 \ldots \ldots \ldots$. . . . . . . . . . . . . . . . . . 618

Introducing a New Conceptual-Physical Model for Simulation of Groundwater Flow in a Porous Medium Using a Network of Pipes 205 . . . . . . . . . . . . . . . . . . . 619

Permeability change during the growth of dense 3D geomechanical fracture networks 752620

Modeling dynamic particle filtration processes using coupled pore-to-continuum multi-

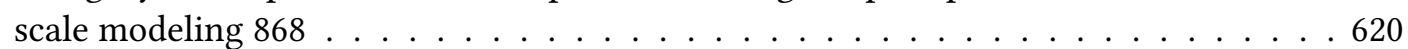

Advances in conformal mixed finite element methods for generalized poroelasticity 254 . 621

Plug formation of a ferromagnetic and glass beads mix $955 \ldots \ldots$. . . . . . . . . . . 622

Numerical estimation of compacted illite multiphase transport properties from pore-scale simulations using FIB-SEM images 403 . . . . . . . . . . . . . . . . . . 6623

Insights into the physical interactions of water based ink with porous paper 165 . . . . . 624

Microemulsion-Based Conformance Treatment: Fluid Optimization and Microfluidics Im-

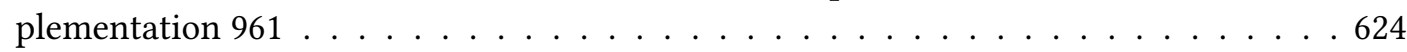

Direct Visualization of the Inaccessible Pore Volume Effect of Polymer Chains in a Dual Porosity Medium Using a Microfluidic Technique 960 . . . . . . . . . . . . . . . 625

Experimental investigation of the impacts of microtopographic and vegetative roughness elements on near-surface airflow and soil moisture dynamics using a coupled wind tunnel-porous media test-system $856 \ldots \ldots \ldots 626$

Downscaling Strategies for Multiscale Mixed Methods 754 . . . . . . . . . . . . . . . 627

Modelling of bone-cement-injection processes in cancellous vertebral bones 923 . . . . 628

Numerical investigation of fracture propagation in porous medium under shear stimulation

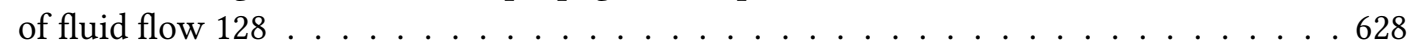

Green synthesis of nZVI suspensions from plant extracts for in situ remediation of polluted groundwater $578 \ldots \ldots \ldots$. . . . . . . . . . . . . . . . . . . 629

Two-dimensional flows in fluid coupled with porous medium 896 . . . . . . . . . . . . 630 
Boundary layers at "fluid - porous medium" interface 897 . . . . . . . . . . . . . . 631

Bone Dry: Hydroxyapatite as a Water-Resistant Cement Coating Material 33 . . . . . . . 631

Numerical characterization of intrinsic permeability based on 2D and 3D data $623 \ldots 632$

Recent advances in the DeProF theoretical framework for two-phase flows in porous media - Where we stand and where we could go. $728 \ldots \ldots$. . . . . . . . . . . 633

Energy efficiency optimization in n-spot recovery processes based on critical flow conditions and integration of flow-dependent relative permeability scaling functions. 809

Pore-scale visualization and quantification of saturated solute transport using fast micro-

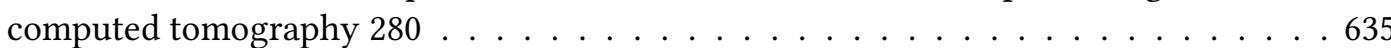

Liquefaction mitigation of Fraser River sand with Microbial Induced Desaturation and Precipitation (MIDP) $597 \ldots \ldots \ldots$. . . . . . . . . . . . . . . . . . . . . . . . . . .

Long-term deformation rate of sealed concrete samples as a viscous response of the material

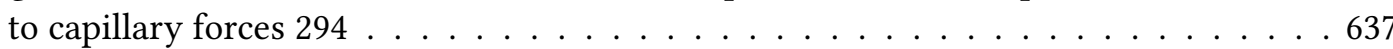

Validation of functional-structural root system models using MRI-monitored tracer exper-

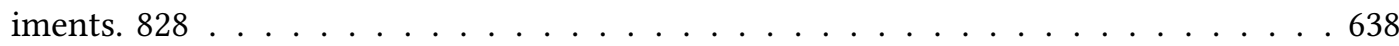

Pore-scale modelling of carbonation reaction in fractured hardened cement paste 775

A Multi-instrument Approach to the Characterisation of Irradiated and Virgin Nuclear Graphite 977 . . . . . . . . . . . . . . . . . . . . . 6639

Effects of spatial heterogeneity on flow and transport in variably saturated porous media

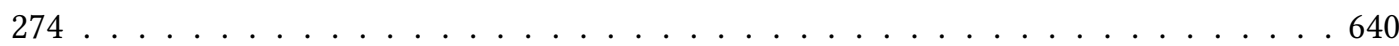

\begin{tabular}{|l|l|l|l|}
\hline Effect of surfactants on film formation in inkjet printing on dense and porous media 148 & 641
\end{tabular}

Multiscale dentinal porous medium modeling using image restoration techniques 22 . . 642

Application of Micellar Flooding for Soil Remediation at a DNAPL contaminated site 746 643

Elucidating the effects of tapered flow channels on the performance of vanadium redox

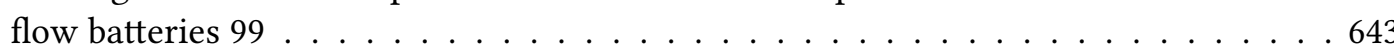

Permeation of gas through single-layer nanoporous graphene membranes: theory and

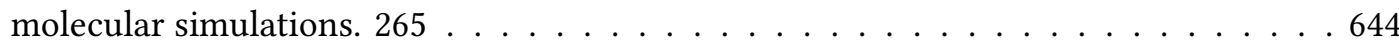

Novel Unsupervised Machine Learning Methods for Characterization Information Content of Data and Model Results 790 . . . . . . . . . . . . . . . . . . . 645

Imbibition, pervaporation and drying in $3 \mathrm{~nm}$ diameter pores $431 \ldots$. . . . . . . . . 646

Microfluidics Experiments and Lattice Boltzmann Simulations to Characterize Multi-phase Flow and Particle Transport in Fracture Networks 146 . . . . . . . . . . . . . . . . 647

Computing Fluid Flow through Discrete Fracture Networks by Using Combinatorial Optimization on Graph Networks 249 . . . . . . . . . . . . . . . . . . . . . . 648

Immersed boundary methods for HM-simulations of fractures 408 . . . . . . . . . . . . . 649 
Pore-Scale Phase Field Models for Multi-Phase Flow with Precipitation 553 . . . . . . . . 649

Lyphilization front structures in thin layers of frozen solutions 56 . . . . . . . . . . 650

Experiment based pore network study on secondary capillary transport through liquid films of different morphology $40 \ldots \ldots$. . . . . . . . . . . . . . . . . . . . . . . . . .

Pore network analysis of the steady state water saturation of hydrophilic GDLs at high current densities $42 \ldots \ldots \ldots \ldots \ldots \ldots$. . . . . . . . . . . . . . . . . . . . .

Improved performance of geothermal systems through modelling with uncertainties 837653

Exploring the Impact of Electrode Microstructure on the Performance of Non-Aqueous Redox Flow Batteries 673 . . . . . . . . . . . . . . . . . . 654

A new method of quantifying pore structure and extracting pore networks from 2D images

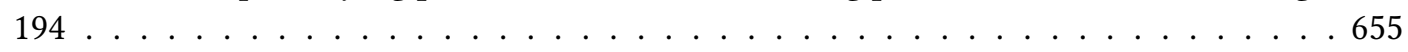

Effect of gas hydrate formation and decomposition on flow properties of fine-grained sediments using X-CT based pore network modeling $390 \ldots 656$

A New Approach to Enhance Oil Recovery in the Tight Oil Reservoir: Advance Gas Injec-

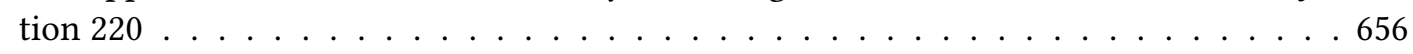

Analysis of Dynamic Structure Characteristics of Foam Fluid Transport in Porous Media Based on Fractal Theory 216 . . . . . . . . . . . . . . . . . . 657

Experimental Study on the Variation of Micro structure of Coal under surfactant 72 . . . 657

Research on Non-linear Porous Seepage Model of Multi-Fractured Horizontal Well in Shale Gas Reservoir 603 . . . . . . . . . . . . . . . . . . . . . . 668

Hysteresis in Adsorption and Desorption Isotherm for Shale Gas in Realistic Kerogen mod-

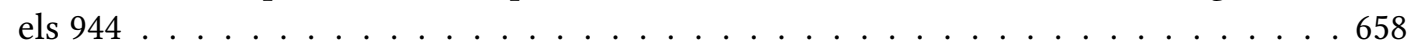

Hydraulic fracture optimization in tight oil reservoirs using artificial intelligence 816 . . 659

Pressure-driven two-phase flow of Gas and water in shale nanopores 697 . . . . . . . 660

Data Driven Models Using Machine Learning Methods to Predict Well Productivity in Montney Formations $789 \ldots \ldots \ldots 61$

Effect of cyclic injection on migration and trapping mechanisms of immiscible fluids in

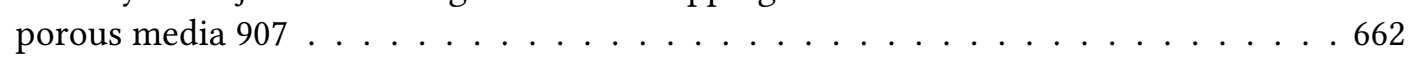

Application of Machine Learning Ideas to Shale Oil Reservoir Permeability Estimation 575

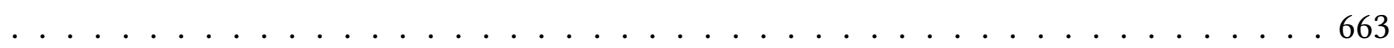

An research and application of yield variation law of fractured wells in tight oil straight well囚A case of the Daqing Oilfield, China 174 . . . . . . . . . . . . . . 663

Pore Scale Investigating of Multi-phase Flow in Tight Reservoirs 13 . . . . . . . . . . . 664

CO2-Induced Structure Alteration of Oil Well Cement: a Micro CT Study 130 . . . . . . 665

High-enthalpy Geothermal Simulation with Multi-level Physics Parameterization 490 . . 665 
Dynamic Effects of Capillary Number on Relative Permeability of Porous Sandstone and Carbonate Rocks 899 . . . . . . . . . . . . . . . . . . . 666

Derivation of new water drive characteristic curve formula for ultra high water-cut oilfield

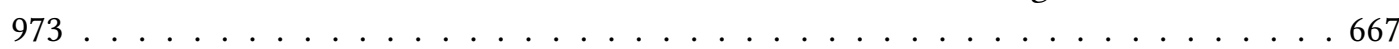

A semi-analytical interpretation model of pulse decay measurements on ultra-tight rocks

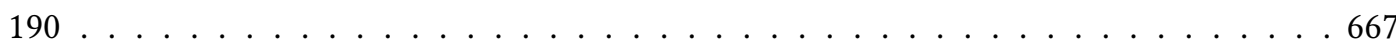

Disorder characterization of porous media and its effect on fluid displacement 387 . . . . 668

Reduced Complexity Modelling of $\mathrm{CO}_{2}$ Leakage Through Faults 126 . . . . . . . . . . . 668

\begin{tabular}{|l|l|l}
\hline Fast artificial $\mu \mathrm{CT}$-scans from 3D-structure models using Gaussian Random Fields 224 & 669
\end{tabular}

The critical factor for relationship between permeability and formation factor of porous media: Body-to-throat ratio, tortuosity and connectivity 179 . . . . . . . . . . 670

Numerical simulations of turbulent flows and heat transfer in regular porous structures 920

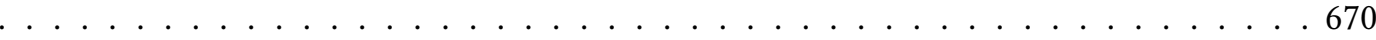

A pore-scale approach to couple mass, momentum and energy at the interface between free flow and porous-medium flow $433 \ldots \ldots \ldots 71$

Controlling the mechanics of nanoporous metals by adsorption and superficial electric

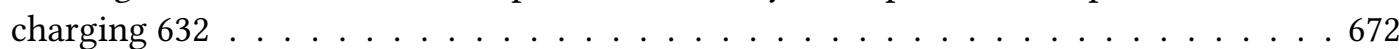

Microbial processes that allow pollutant biodegradation in toxic and inaccessible microenvironments within porous media $932 \ldots$. . . . . . . . . . . . 672

Scalable Solvers for Multiphase Poromechanics 982 . . . . . . . . . . . . . . . . . . . . . 673

AI-based identification of binder and fibers in 3D images of nonwoven 584 . . . . . . . . 673

Time-Correlation Functions of Immiscible Two-Phase Flow in Porous Media 734 . . . . . 674

Taylor Dispersion: Evolution from the Initial Condition 10 . . . . . . . . . . . . . . . . . 675

Hydrogel-Enhanced EICP Application to Fugitive Dust Mitigation: Impacts on Surface Permeability and Erosion Resistance 866 . . . . . . . . . . . . . . . . . . 676

Pore network modeling of cathode catalyst layer of proton exchange membrane fuel cell

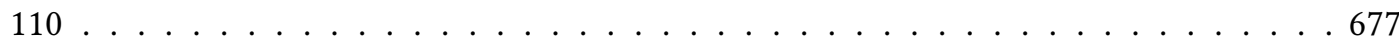

Evaporation from thin porous media with mixed intermediately-wet and hydrophobic net-

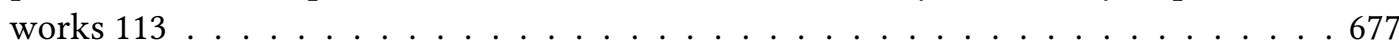

Numerical Simulation of fluid flow in unsaturated porous media using the SPH method 497

Simultaneous determination of pore space and tortuosity fractal dimensions based on 3D CT images 215 . . . . . . . . . . . . . . . . . . . . 6679

A Machine-learning Based Subdomain POD-TPWL for Reservoir History Matching 500 . 680

Particle Transport and Deposition Characteristics in 3D Horizontal Fracture with Rough-

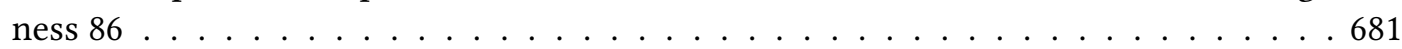


Flow and heat transfer in a microchannel partially filled with a microporous foam involving effects of flow inertia, flow/thermal slips, thermal non-equilibrium and thermal asym-

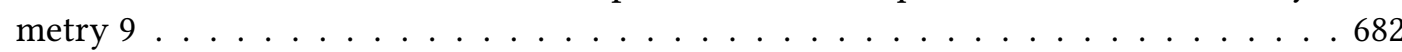

The laplace transform embedded discrete fracture model method for the simulation of com-

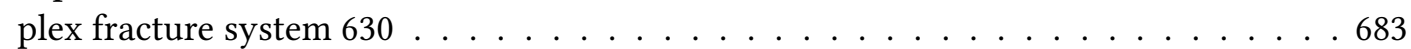

A machine learning method for joint optimization of well location and hydraulic fracture

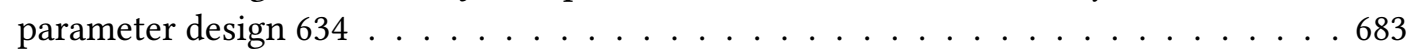

Experimental Study on Gas Accumulation Mechanism of Sandstone Gas Reservoirs in Sulige Gas Field, Ordos Basin, China 770 . . . . . . . . . . . . . . . . . . . . 684

Enhanced Thermal Recovery Mechanism of CO2 - Assisted Steam 83 . . . . . . . . . . . 685

The Effect of Rock Deformation during CO2 Flooding and Storage Using Pore Scale Char-

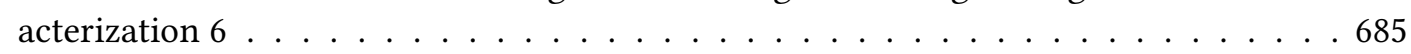

Experimental investigation on the stress sensitivity of permeability in naturally fractured

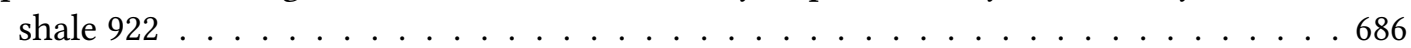

Experimental investigation on anisotropic permeability and its relationship with anisotropic thermal cracking of oil shale under high temperature and triaxial stress 107 . . . . . 687

Integrated simulation of Darcy and Stokes flow for porous rock by lattice Boltzmann method

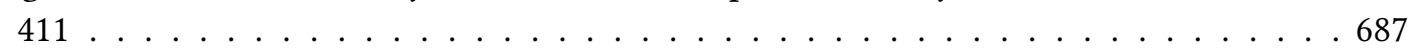

A new method for simultaneous determination of in situ porosity and permeability under \begin{tabular}{lll}
\hline reservoir conditions: insights from gas diffusion on gas shales with nanopores 776 & 688
\end{tabular}

A Digital Image Correlation Analysis on the Rapid Reduction Mechanism of Permeability in Low-Permeability Sandstone Reservoirs 930 . . . . . . . . . . . . . . . . . . 689

Multiphase SPH simulation based on an improved boundary model 79 . . . . . . . . . . 690

The Research of Microscopic Remaining Oil on the Basis of Displacement Experiment and Digital Core 401 . . . . . . . . . . . . . . . . . . . . 6 690

Fluid displacement and trapping during two-phase steady-state flow in complex carbonate imaged by synchrotron x-ray microtomography $3 \ldots$. . . . . . . . . . . 6691

Pore-scale simulation of shale oil flow based on pore network model 523 . . . . . . . . . 692

Impact of Temperature on Sandstone based on CT images 521 . . . . . . . . . . . . . 693

Characterizing the Plugging Capability of New Bilayer-Coating Microspheres for In-Depth Conformance Control: Mathematical Modeling and Laboratory Study 889 . . . . . . . 693

Research and Application of Numerical Method of Evaluation of Fracturing Effects in Large Scale Volume Reform of Vertical Wells 212 . . . . . . . . . . . . . . . . . . . 694

Unsaturated flow through fracture intersections: competition between gravity and capillary force controls fluid splitting of droplets $283 \ldots$. . . . . . . . . . . . . 695 
Atomic Force Microscopy (AFM) Study of Wettability Modification and Mineralogy of Bandera Brown. 85 . . . . . . . . . . . . . . . . . 665

Asymptotic mathematical model of gas filtration through thin porous wall $821 \ldots$. . . . 696

On volume averaging in porous electrode theory $611 \ldots$. . . . . . . . . . . . . 697

A numerical and experimental study of drainage processes in predominantly 2D microflu-

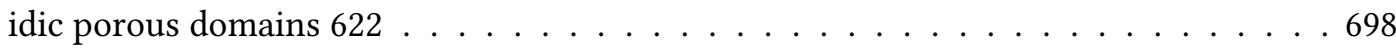

Study on occurrence state of fluid in nanopores by combining nanofluidic and simplified local density model $904 \ldots \ldots$. . . . . . . . . . . . . . . . 699

3D printing application for flow and mechanical deformation in a single fracture network

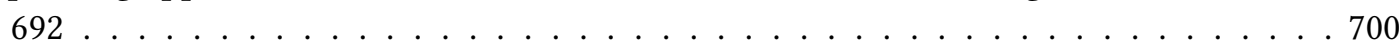

Interplay among Roughness, Flow, and Transport in Rough Single Fractures: The Critical Role of Velocity Correlation on Anomalous Transport 534 . . . . . . . . . . . . . 700

Flow and transport in fractured poroelastic media 97 . . . . . . . . . . . . . . . . 701

Foam Propagation at Low Superficial Velocity: Implications for Long-Distance Foam Prop-

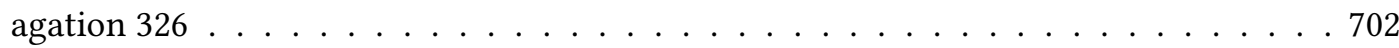

Reactive Flow in Unconsolidated Sandstone: Implications for Carbon Geosequestration 903

A dynamic simulator for gas diffusion and surface adsorption-desorption in fractured coal

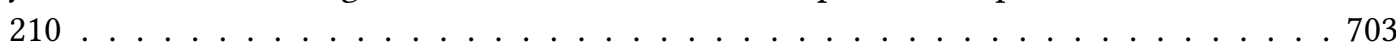

High resolution sequence stratigraphic characteristics of ahe formation,Tarim basin $921 \quad 704$

High resolution sequence stratigraphic characteristics of ahe formation, Tarim basin $947 \quad 705$

An innovative workflow for designing ion-tuned water flooding of carbonate reservoirs

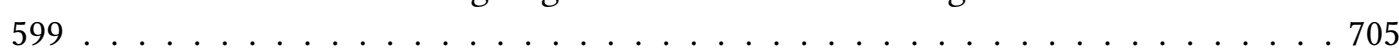

Dust suppression by enzyme induced carbonate precipitation (EICP) in soils 410 . . . . . 706

Equilibrium Constraints in Low Salinity Waterflooding of Carbonate Reservoirs 506 . . . 707

Thermodynamic modeling of adsorption kinetics 300 . . . . . . . . . . . . . . . . . 709

Lattice Boltzmann Modelling of surfactant adsorption and transport in porous media 301710

Advanced X-ray Computed Tomography in Additive Manufacturing 617 . . . . . . . . . 711

Positron Emission Tomography in Water Resources and Subsurface Energy Resources Engineering Research $104 \ldots \ldots$. . . . . . . . . . . . . . . 712

Lithium Ion Battery Separators: understanding the importance of surface interactions and pore space topology for cell operation and manufacturing $741 \ldots \ldots 712$

Micro-Scale Mathematical Model of Freezing in a Porous Medium 853 . . . . . . . . . . 714

Improved understanding of microbe-mineral interactions using droplet-based microfluidics

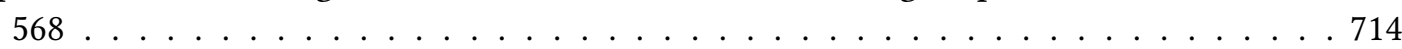


Multipoint flux mixed finite element methods for a double-porosity model of single-phase flow in naturally fractured reservoirs $804 \ldots \ldots$. . . . . . . . . . . . . . . . . . . . . .

Modeling of lithium ion batteries with emphasis on spatial fluctuations and mechanical effects on the electrode pore scale 633 . . . . . . . . . . . . . . . . . 716

Experimental investigation of microbial induced calcium carbonate precipitation induced by Sporosarcina pasteurii on the microscale 656 . . . . . . . . . . . . . . . 717

Numerical modelling of microbially induced calcite precipitation in field trials 530 . . . . 718

Apparent Permeability Model of Complex Fracture Network in Shale Reservoir 384 . . . 718

Fractal Forced Spontaneous Imbibition Model of Water Phase in Shale 481 . . . . . . . . 719

Molecular Simulation of Water Adsorption in Shale Organic Nano-Pores 813 . . . . . . . 720

Coupling virus and salinity transport through porous media. 365 . . . . . . . . . . . 721

Numerical study on the mechanism of fluid flow through thin rough fractures $880 \ldots 722$

A discrete Boltzmann method investigation for the micro-compressible-thermal flow in porous media with nonequilibrium effect 255 . . . . . . . . . . . . . . 723

A coupled flow-DEM approach to investigate the effect of embedded gravel on the propagation of hydraulic fractures in glutenite reservoirs $574 \ldots$. . . . . . . . . . . 723

Dynamic simulation of water invasion in multi-well collaborative mining of gas reservoirs

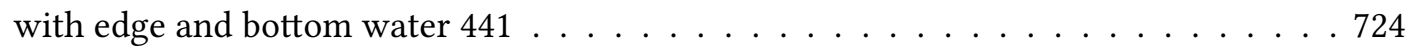

Study on the Factors Affecting the Productivity of Coal-bed Gas Well -A Case Study of Block S of D Gas Field in Australia 974 . . . . . . . . . . . . . . . . . . . 725

Pore Scale Simulation of Coupled multiple physicochemical processes for Methane Hydrate Dissociation using Lattice Boltzmann Method 118 . . . . . . . . . . . . . . . . 725

The Adaptability of Foam Profile Control in Low Permeable Heterogeneous Reservoir 112726

Uranium release and migration in porous rock under geologic $\mathrm{CO} 2$ storage conditions: a

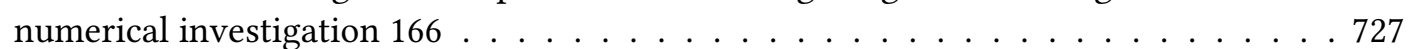

Development and Calibration of an Analytical Model for Unconventional Wells Using Machine Learning Techniques 4 . . . . . . . . . . . . . . . . . . . 727

Oil adsorption and diffusion in shale nanopores: Insight of molecular dynamics simulation

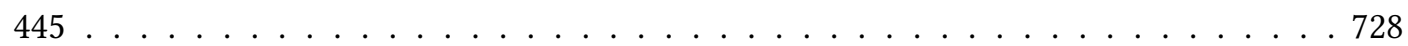

Numerical simulation of fluid flow and heat extraction in Enhanced Geothermal System based on discrete fractures model 95 . . . . . . . . . . . . . . . . . . 729

Why permeability reduction during carbon geosequestration in coal seam: an experimental study via direct in-situ microCT scanning 541 . . . . . . . . . . . . . . . . . . 729

Porous beds to mitigate fluctuated fluid across channel flow 799 . . . . . . . . . . . . . . 730

Computational exploration of the performances of mechanically constrained MOFs for gas

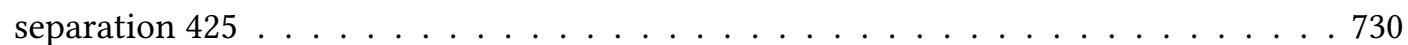


Pore-scale Two-phase Flow Simulation in Porous Media Coupling Pore Network Model and Lattice Boltzmann Method $508 \ldots \ldots$. . . . . . . . . . . . . . . 731

Study on the Change of Seepage Field in the Condition of Extreme Water Consumption 175

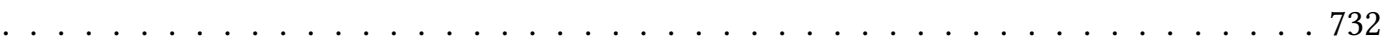

Seepage characteristics and fracture numerical experiments on the action of stress-seepage coupling of the rock with micro random fractures $173 \ldots \ldots$. . . . . . . . . 732

Fractal analysis of gas relative diffusion coefficient in porous nanofibers with rough sur-

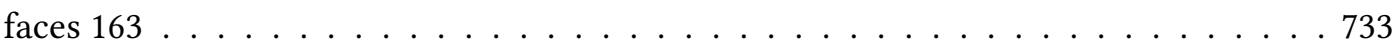

Microscopic Residual Oil Recovery Effect by Different Displacement Methods 270 . . . . 734

Direct Visualization of Fluid in Nanopores - Fundamental and Application in Shale with Nanofluidics 849 . . . . . . . . . . . . . . . . . . . . . 734

Capillary imbibition governed by water adsorption in hygroscopic plant-like structure 100

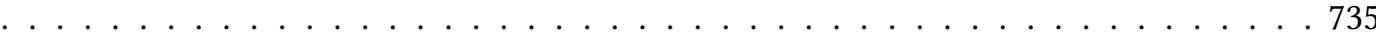

Similarity between Modeling of Solute and Heat Transport in Fractured Reservoirs with a Generalized Multirate Memory Function 515 . . . . . . . . . . . . . . . . 736

A phase-field moving contact line model with soluble surfactants 67 . . . . . . . . . . . 736

Pore-scale simulation of imbibition process in a 3D digital rock $264 \ldots$. . . . . . . . . 737

Pore-scale direct numerical simulations of non-continuum flows by solving kinetic model equations in OpenFOAM $150 \ldots \ldots \ldots$. . . . . . . . . . . . . . . . . . . . . . .

Experimental Studies of the Hydraulic Properties of Thin Porous Media 916 . . . . . . . 738

An Auxiliary Space Method for Mixed Finite Element Discretizations 320 . . . . . . . . . 739

Wicking simulations of cryogenic liquid into superheated porous structures 147 . . . . . 739

Three-Dimensional Simulation of Density-Driven Natural Convection in Porous media for CO2 Geological Storage 865 . . . . . . . . . . . . . . . . . . . . . . . 740

Capillary-driven transport of dissolved salt to the drying zone during $\mathrm{CO} 2$ injection in homogeneous and layered porous media. $949 \ldots \ldots$. . . . . . . . . 740

Multiscale and multiphysics models for geomechanics 278 . . . . . . . . . . . . . . 741 


\title{
Dynamics of foam flow in a rock fracture: Effects of aperture variation on apparent shear viscosity and bubble morphology
}

\author{
Mohammad Javad Shojaei ${ }^{1}$; Antonio Rodríguez de Castro ${ }^{2}$; Yves Méheust ${ }^{3}$; Nima Shokri ${ }^{1}$ \\ 1 The University of Manchester \\ 2 Arts et Métiers ParisTech \\ ${ }^{3}$ Géosciences Rennes
}

Corresponding Author(s): mohammadjavad.shojaei@postgrad.manchester.ac.uk

Foams are widely used in hydrogeological applications and Enhanced Oil Recovery (EOR) as mobility control agents for flow in porous media [1-3]. In the particular case of flow through fractured media, the local aperture distribution strongly influences the effective viscosity of the injected foam and the distribution of fluxes along preferential flow paths [4]. Although several experimental studies have been previously performed to study foam flow in fractured porous media, none of them has specifically addressed the effects of aperture distribution on the rheological behaviour of the injected foams. The specific objective of this study was to elucidate the effects of aperture variation on the "in situ" shear viscosity of the foam and the size of the bubbles. To do so, a compressive series of single-phase experiments have been performed by injecting pre-generated foam at six different foam qualities and constant flow rate through two types of media: 1) a Hele-Shaw cell with constant aperture [5] and 2) a replica of a Vosges sandstone fracture of well-characterized aperture map. The effect of variable flow rate on the "in situ" shear viscosity of a $85 \%$ quality foam was also analysed. To go further, the velocity vector map of the foam flow was computed by capturing the movement of the bubbles with a digital camera. The effective shear rate map was then calculated from local velocities and apertures. The results show the significant impact of aperture variation on the the spatial distribution of the foam flow and bubble dimensions of the foam, echoing the necessity to take these parameters into account when predicting the pressure drop through the fracture and the effective viscosity of the foam.

References

1. Osei-Bonsu, K., P. Grassia, and N. Shokri, Investigation of foam flow in a 3D printed porous medium in the presence of oil. Journal of colloid and interface science, 2017. 490: p. 850-858.

2. Osei-Bonsu, K., N. Shokri, and P. Grassia, Foam stability in the presence and absence of hydrocarbons: from bubble-to bulk-scale. Colloids and Surfaces A: Physicochemical and Engineering Aspects, 2015. 481: p. 514-526.

3. Osei-Bonsu, K., P. Grassia, and N. Shokri, Relationship between bulk foam stability, surfactant formulation and oil displacement efficiency in porous media. Fuel, 2017. 203: p. 403-410.

4. Buchgraber, M., L.M. Castanier, and A.R. Kovscek. Microvisual investigation of foam flow in ideal fractures: role of fracture aperture and surface roughness. in SPE Annual Technical Conference and Exhibition. 2012. Society of Petroleum Engineers.

5. Osei-Bonsu, K., N. Shokri, and P. Grassia, Fundamental investigation of foam flow in a liquid-filled Hele-Shaw cell. Journal of colloid and interface science, 2016. 462: p. 288-296.

Procter and Gamble Student poster award:

I would like to compete in the Procter and Gamble Student award References:

Acceptance of Terms and Conditions:

Click here to agree 


\title{
Stochastic upscaling for hydraulic conductivity based on labora- tory experiments
}

\author{
VANESSA A. GODOY ${ }^{1}$; LAZARO V. ZUQUETTE ${ }^{2}$; JAIME GOMEZ-HERNANDEZ ${ }^{1}$ \\ ${ }^{1}$ Institute for Water and Environmental Engineering, Universitat Politècnica de València \\ ${ }^{2}$ Geotechnical Engineering Department, São Carlos School of Engineering, University of São Paulo
}

Corresponding Author(s): godoyalmeida@gmail.com

\begin{abstract}
The use of deterministic approaches that consider hydraulic conductivity $(\mathrm{K})$ as a constant value for an entire soil layer are usually employed in numerical modeling in geotechnical engineering. However, $\mathrm{K}$ can present high spatial variability and its heterogeneity is important even in apparently homogeneous soils. Due to this heterogeneity K presents scale effects, that is, the change of support (from the measurement scale or fine scale to the numerical scale or coarse scale) implies a change in the parameter. The scale effect is especially important if we consider that those $\mathrm{K}$ values are used to feed the $\mathrm{K}$ values of a numerical model, where the discretization support is generally orders of magnitude larger than the measurement support. The objective of this research is to perform stochastic upscaling for $\mathrm{K}$ based on laboratory experiments. We use the theory of stochastic random fields to deal with the uncertainty associated with having limited information about the spatial variability of $\mathrm{K}$. The scale effect problem was addressed using upscaling, more precisely, the method known as Laplacian-with-skin, which transfers the information obtained at the fine scale into the coarse scale to be used by the numerical code accounting for the heterogeneity within the block being upscaled plus a 'skin' area surrounding it. The hydraulic conductivity of 55 undisturbed cylindrical samples of radius $0.10 \mathrm{~m}$ and height $0.15 \mathrm{~m}$ was measured in the laboratory using a rigid-wall permeameter. The natural logarithm of $\mathrm{K}(\mathrm{lnK})$ was modeled by a Gaussian distribution with mean of $-0.38(\ln (\mathrm{m} / \mathrm{d}))$ and standard deviation of $1.25(\ln (\mathrm{m} / \mathrm{d}))$. The best fit to the experimental variogram of $\operatorname{lnK}$ was an isotropic spherical variogram model with a range of $4 \mathrm{~m}$ and no nugget effect. The Sequential Gaussian Simulation (SGS) algorithm implemented in the code GCOSIM3D was used to generate 70 equally likely realizations of the $\mathrm{K}$ field at the fine scale (cubic grid of $0.1 \mathrm{~m}$ ), that were used as input to 70 numerical models of the groundwater flow. Each of the 70 realizations of the $\mathrm{K}$ fields generated at the fine-scale was upscaled. We performed upscaling with cubic block sizes 2, 4, $5,8,10$, and 40 times the side size of the block at the fine scale $(0.1 \mathrm{~m})$. For each realization at the fine and at the coarse scales, three-dimensional flow was solved by the finite element method (FEM) using FEFLOW 7.1. Our results show that the upscaling method is very effective for the purpose of reproducing the average flux crossing the model despite causing a loss of information because the small-scale heterogeneity is not preserved. Our results also show that upscaling not only produces models with a small RBq but also preserves the uncertainty of the specific discharge at the fine scale, even for a block size equal to the entire domain $(12 \mathrm{~m} \times 8 \mathrm{~m} \times 4 \mathrm{~m})$.
\end{abstract}

Procter and Gamble Student poster award:

I don't want to compete References:

Godoy, V. A., Zuquette, L. V., \& Gómez-Hernández, J. J. (2018). Stochastic analysis of three-dimensional hydraulic conductivity upscaling in a heterogeneous tropical soil. Computers and Geotechnics, 100, 174-187.

Zhou, H., Li, L., \& Gómez-Hernández, J. J. (2010). Three-dimensional hydraulic conductivity upscaling in groundwater modeling. Computers \& Geosciences, 36(10), 1224-1235. Acceptance of Terms and Conditions:

Click here to agree

\section{Modeling and simulation of transport and reaction processes through digitally generated paper-based membranes}

Andres Abad Gonzalez ${ }^{1}$; Patrick Altschuh ${ }^{2}$; Jamshidi Farshid ${ }^{1}$; Willfried Kunz ${ }^{1}$; Andreas Reiter ${ }^{1}$; Marcel Bremerich $^{3}$
; Annette Reiche ${ }^{3}$; Michael Selzer ${ }^{2}$; Britta Nestler 
${ }^{1}$ Karlsruhe University of Applied Sciences

${ }^{2}$ Karlsruhe Institute of Technology

${ }^{3}$ Sartorius Stedim Biotech $\mathrm{GmbH}$

Corresponding Author(s): andres.abad_gonzalez@hs-karlsruhe.de

One of the most widespread uses for paper-based membranes is in the field of analytical testing and Lateral Flow Assays (LFA) are probably the most common and relevant exponents. The most important component of LFA is a thin porous membrane used to transport a fluid, which may contain an analyte, through the porous body and to give a clear optical signal by a coloured line (test line) if the analyte is detected. Thus indicating, the presence of a molecule or substance responsible for a certain condition or sickness.

Nevertheless, much effort is still being invested regarding the understanding of the transport and reaction processes taking place in the LFA membrane aiming to improve the design for better assay results. The enhancement of the test line analyte sensitivity, the reduction of the test time and the minimization of the sample volume used for the analyte detection are a few of the desired optimization outcomes.

It is necessary to emphasize that the development of LFA has been until now, strongly dependent on experimental results. The use of numerical digital models and simulations is an important asset for the design and optimization of LFA, which allows more flexibility and the access to design information and operational predictions that otherwise could not be possible, or would be very expensive to get experimentally.

Fluid propagation, analyte diffusion through the porous membrane and analyte-sample surface reactions, i.e. adsorption and desorption in the test line volume, depend not just on the fluid but on the membrane properties as well. The advantage of using a digital model is the possibility to perform controlled structural/morphological membrane variations in addition to the variation of the involved substances parameters and quantities.

Pore-scale and macro-scale numerical models are employed to algorithmically evaluate generated three-dimensional LFA membranes. Important processes involved in the preparation and utilization of LFA membranes such as the hydrophobic surfactant coating, the analyte transport and diffusion through the porous structure, the analyte adsorption at available adsorption sites and the surface analyte unbinding are taken into account for the simulations.

All these processes together are able to describe the operation and signal quality of a complete Lateral Flow Assay.

Various approaches providing insight into the propagation, dispersion and reaction processes in thin porous media are presented in this work.

Procter and Gamble Student poster award:

I would like to compete in the Procter and Gamble Student award References:

Acceptance of Terms and Conditions:

Click here to agree

\section{A Multiscale Study on the Effects of Dynamic Capillary Pressure in Two-Phase Flow in Porous Media}

Jassem Abbasi ${ }^{1}$; Mojtaba Ghaedi ${ }^{2}$; Masoud Riazi ${ }^{3}$

\footnotetext{
${ }^{1}$ Research Assistant

${ }^{2}$ Assistant Professor

${ }^{3}$ Associate Professor
} 
Corresponding Author(s): jassem.abbasi@gmail.com

The fluid flow in porous media can be highly influenced by capillary forces. It is convenient that the capillary pressure is considered as a function of the rock and fluid properties and also relative saturation of wetting phase. However, recent studies have shown that the capillary forces also are a function of the rate of change of saturation in porous media. This functionality has been investigated in numerous core scale studies and it was proved that the flow in porous media, especially in low capillary number fluxes, can be heavily affected by these dynamic capillary forces. Moreover, although it was observed that the dynamic forces are highly scale dependent, the role of these effects in large scale practices is still unclear. In this study, using a novel simulation approach, the impacts of the mentioned parameters were studied in a large scale and highly heterogeneous oil reservoir that is under waterflooding process. At first, the primary investigations showed that the role of capillary pressure in the considered geometry was significant. Then, it was observed that the role of dynamic capillary effects in low dynamic capillary coefficient values is not important in large scale problems. However, it would be important in the high capillary coefficient values that are not reported in previous experimental studies. Moreover, some noises may be observed in production curves as it was reported by previous studies. The findings of this work shows that a large unknown area still exists in this field of study that needs the attention of the researchers in future.

Procter and Gamble Student poster award:

I would like to compete in the Procter and Gamble Student award References:

Acceptance of Terms and Conditions:

Click here to agree

171

\title{
The Behavior of Liquid Percolation and Water Uptake in Catalytic Porous Materials used in Proton Exchange Membrane Fuel Cells (PEMFC)
}

\author{
Author(s): Karrar Alofari ${ }^{1}$; Sofyane Abbou ${ }^{1}$ \\ Co-author(s): Ezequiel Médici ${ }^{1}$; Kazuya Tajiri ${ }^{1}$; Jeffrey Allen ${ }^{1}$ \\ ${ }^{1}$ Michigan Technological University \\ Corresponding Author(s): sabbou@mtu.edu, efmedici@mtu.edu
}

Proton exchange membrane fuel cells (PEMFC) are low temperature $\left(<90^{\circ} \mathrm{C}\right)$ electrochemical devices which combine hydrogen and oxygen to produce electricity (and heat). The only product of the reaction is water. An excessive presence of water in the thin (<10 um) porous catalyst layers (CLs), where the electrochemical reactions take place, may significantly lower the PEMFC performance and lifetime. Therefore, a proper water removal from the CLs is crucial. The behavior of liquid water percolating through the porous CLs is still not well understood. Up to date, no experimental characterization have been made due to the CLs complex structure: mix of a thermally and electrically conductive support (mostly carbon); dispersed catalyst nanoparticles (typically Pt or Pt-alloys); a few nanometer thick ionomer film which assures protonic conductivity and a pore phase for reactant and water transport. An additional complexity is that the hydrophilic ionomer absorbs water and swells affecting the CLs morphology and therefore transport properties. In this work, we investigate the relationship between catalyst layer morphology and water transport. A pseudo-Hele-Shaw test has been developed in which a liquid is injected from the bottom into a catalyst layer under controlled conditions. The injected liquid displace air to generate an imbibition flow pattern. We showed that the ionomer swelling caused a shift in the pore size distribution with a subsequent increase in injection pressure during percolation testing. In addition, the Washburn method was used to assess ionomer water uptake and estimate CLs contact angles to water. Using a Kruss K100 tensiometer, the samples were attached from the top to a sensitive microbalance and immersed into water to extract: ionomer liquid uptake and (external) contact angles to water. The results showed that the ionomer to carbon ratio and the ionomer chemistry have a significant impact on the CLs wettability.

Procter and Gamble Student poster award: 
I would like to compete in the Procter and Gamble Student award References:

Acceptance of Terms and Conditions:

Click here to agree

736

\title{
new correlation to predict effective thermal conductivity of open cell metal foams
}

\author{
Author(s): Arash Abdoli ${ }^{1}$ \\ Co-author(s): milad saljooghi ${ }^{2}$ \\ ${ }^{1}$ University of Hormozgan \\ ${ }^{2}$ university of hormozgan
}

Corresponding Author(s): arashabdoli.sci@gmail.com

\begin{abstract}
Since long time Conception of thermophysical properties of porous materials has been a challenging task for scientists to conquer. Knowledge of heat transfer mechanism in porous media due to developing of various application in industries causes efficiency upgrades. Miniaturization in technology, energy optimization, multifunctional equipment manufacturing requires engineers to develop new material that satisfy different functional, thermal, mechanical and physical features. As a revolutionary idea, open cell metal foams has been great improvement in heat transfer process. These materials increase heat transfer while energy dissipation, dimension and density of them which are constraints for modern technologies significantly reduce. So various numerical, analytical and experimental efforts have been made to obtain better understanding of their thermo-hydraulic behavior. In present study a brief history of proposed methods and correlations is presented and finally a correlation based on experimental data is derived to predict effective thermal conductivity of open cell metal foams. Presented correlation demonstrates dependency of conduction heat transfer on pore size, porosity, fluid and solid material properties.
\end{abstract}

Keyword. Open cell, metal foams, heat transfer, porous media, effective thermal conductivity, porosity.

Procter and Gamble Student poster award:

I would like to compete in the Procter and Gamble Student award References:

Acceptance of Terms and Conditions:

Click here to agree

168

\section{A high-order conservative finite element method for Darcy flow problem with transport}

\author{
Eduardo Abreu ${ }^{1}$; Ciro Díaz ${ }^{2}$; Juan Galvis ${ }^{3}$; John Perez ${ }^{4}$ \\ ${ }^{1}$ University of Campinas, Sao Paulo, Brazil \\ ${ }^{2}$ University of Campinas \\ ${ }^{3}$ Universidad Nacional de Colombia \\ ${ }^{4}$ ITM - University Institution
}

Corresponding Author(s): eabreu@ime.unicamp.br 
A mathematical and numerical model describing a two-phase flow system in porous medium is treated. For the Darcy flow problem we describe and analyze a volumetric and residual-based Lagrange multipliers saddle point reformulation of the standard high-order finite method, to impose conservation of mass constraints for simulating the pressure equation on two dimensional convex polygons, with sufficiently smooth solution and mobility phase. We establish high-order a priori error estimates with locally conservative fluxes. For the transport problem we employ a new locally conservative Lagrangian-Eulerian finite volume method. This approach was applied to several nontrivial examples to show that we are calculating the correct qualitatively good (entropic) solutions with accurate resolution. We combine these procedures for solving the fundamental two-phase flow problem with high-contrast discontinuous porous medium. We provide numerical examples for verifying the theory and illustrating the capabilities of the approach being presented.

\section{Procter and Gamble Student poster award:}

\section{References:}

[ 1 ] E. Abreu, C. Diaz, J. Galvis, M. Sarkis, On high-order conservative finite element methods, Computers and Mathematics with Applications 75 (2018) 1852-1867.

[ 2 ] E. Abreu, C. Diaz, J. Galvis, M. Sarkis, Domain Decomposition Methods in Science and Engineering XXIV https://www.springer.com/us/book/9783319938721 (2018).

[ 3 ] E. Abreu, W. Lambert, J. Perez and A. Santo, A new finite volume approach for transport models and related applications with balancing source terms, Mathematics and Computers in Simulation, 137 (2017) 2-28. Acceptance of Terms and Conditions:

Click here to agree

\section{Dynamic Pore-Scale Study of Water-Salinity Effect on a Moving Oil Droplet}

Moataz Abu-Al-Saud ${ }^{1}$; Hamdi Tchelepi ${ }^{2}$

\footnotetext{
${ }^{1}$ Saudi Aramco

${ }^{2}$ Stanford University
}

Corresponding Author(s): moataz.abualsaud@aramco.com

The salinity of the injected water has been shown to have a substantial impact on oil recovery. Various experimental studies have concluded that the system wettability is altered when the water ioniccomposition is changed. In this work, a numerical investigation of an oil droplet mobilized by water is conducted inside a single pore. The presented model studies the synergy effect of multiphase flow and water salinity at the pore level.

The flow dynamics is modeled by solving the full hydrodynamic Navier-Stokes equations using direct numerical simulation (DNS), and the fluid-fluid interface is captured by the level-set method. The effect of brine ionic-composition is examined through the DLVO (Derjaguin, Landau, Verwey, and Overbeek) theory of intermolecular forces, where experimental values of the zeta potential have been used in the model. The system (oil/water/solid) wettability is determined by the intermolecular forces.

The simulation results show that the water wetting film surrounding the oil-droplet collapses to an adsorbed nanometer water layer when high salinity water is used. As a result, a large pressure gradient is required to mobilize the oil inside the pore and overcome the attractive surface forces between the water-oil and water-solid interfaces. In the case of tailored water salinity, the oil/water/solid system becomes more water-wet. The wetting film adhered to the solid is stable due to the repulsive electric double layer force. Therefore, it takes a smaller pressure gradient to mobilize the oil droplet inside the pore compared to high salinity water, which indicates that waterflooding efficiency increases with tailored salinity water injection. The effect of solid roughness is studied. 
The novelty of the numerical method lies in efficiently capturing the nanoscale effect of the electric double layer in DNS pore-scale multiphase flow. The new results shed light on the dynamics of smart waterflooding at the pore level.

Procter and Gamble Student poster award:

References:

Acceptance of Terms and Conditions:

Click here to agree

16

\title{
Upscaling of coupled free-flow and porous-media-flow processes
}

\author{
Author(s): Sina Ackermann ${ }^{1}$ \\ Co-author(s): Rainer Helmig ${ }^{1}$ \\ ${ }^{1}$ University of Stuttgart
}

Corresponding Author(s): sina.ackermann@iws.uni-stuttgart.de

Drops at the interface between a coupled free flow and a flow in a porous medium can significantly influence the exchange of mass, momentum and energy between the two flow compartments. A physically correct description of the transfer processes in and around the drops would require a model on the pore-scale, while averaged macro-scale equations can be used to describe the free flow and the flow in the porous medium.

Therefore, we develop a coupling concept which takes the drop-related pore-scale processes into account by upscaling them to the macro-scale. The drops are modeled in a lower-dimensional interface domain. This concept has already been applied to fractures in porous media [2] and allows the storage of mass, momentum and energy within the interface.

References:

1. Baber, K., Flemisch, B. and R. Helmig: Modelling drop dynamics at the interface between free and porous-medium flow using the mortar method. International Journal of Heat and Mass Transfer. (2016),99, 660-671

2. Gläser,D., Helmig, R., Flemisch, B. and Class, H.: A discrete fracture model for two-phase flow in fractured porous media. Advances in Water Resources (2017), 110, 335-348

Acceptance of Terms and Conditions:

Click here to agree

535

\section{Application of Laplace-Differential Transform method to trans- port problems in porous media}

Temitayo Adeyemi ${ }^{1}$

\footnotetext{
${ }^{1}$ University of Ibadan
}

Corresponding Author(s): spegenius@gmail.com 
In the field of reservoir engineering, several solutions to equations that govern transport of fluids in porous media have been developed. A solution to the diffusivity equation with infinite boundary condition was first introduced into the industry by Everdingen and Hurst in 1949. Furthermore, Carslaw and Jeagar in their book (conduction of heat in solids) also solved the diffusivity equation for different boundary conditions. Unfortunately, all these solution are too complex and require a lot of approximations and assumptions for most practical applications like well testing because they involve infinite summation of Bessel and Exponential functions and this in turn reduces the accuracy of their real life applications.

Therefore, this paper presents a novel method called Laplace-Differential Transform Method (LDTM), which is suitable for obtaining very simple and excellently accurate solutions to equations that governs fluid transport in porous media. The results show that this technique is very effective, efficient and convenient.

Procter and Gamble Student poster award:

I would like to compete in the Procter and Gamble Student award References:

Acceptance of Terms and Conditions:

Click here to agree

\title{
Modeling of transport phenomena and pollutant degradation dur- ing DBD discharge used for soil remediation
}

\author{
Author(s): Nadia Bali ${ }^{1}$ \\ Co-author(s): Christos Aggelopoulos ${ }^{1}$; Skouras Eugene ${ }^{1}$; Christos Tsakiroglou $^{1}$; Vasilis Burganos ${ }^{1}$ \\ ${ }^{1}$ Foundation for Research and Technology, Institute of Chemical Engineering Sciences
}

Corresponding Author(s): caggelop@iceht.forth.gr

A numerical simulator has been developed to model DBD plasma discharges that generate suitable oxidation processes in the interior of a porous soil structure, as presented in Figure 1. This simulator assesses the favorable pollutant (atrazine) degradation inside the soil structure, and examines the transport phenomena inside this specific pore network. The complex phenomena that take place in the reactor are spanned over two characteristic time scales. The first scale refers to plasma discharge phenomena extended from a few nanoseconds up to the order of milliseconds, while the second one refers to the momentum, mass, and thermal transport phenomena that take place at characteristic timescales on the order of minutes. In the former scale, a 100-reaction model is formulated and the resulting equations are solved numerically (using commercial CFD software) to assess the oxidized species generation, as well as the electric power production, under specific DBD operating conditions. For the transition from the micro-timescale (plasma phenomena) description to the macrotimescale (transport phenomena), the species calculated at the micro-timescale are introduced as source terms for the description of the degradation of the pollutant inside the soil porous structure. In this study, the effect of the pore structure on the concentration of the oxidized species at the micro-timescale is assessed by varying the pore volume. Furthermore, the effect of the heterogeneity of the pore structure is estimated by changing the characteristic dimensions of the soil grains. In addition, the dielectric permittivity and thickness are investigated in order to optimize the reactor characteristics under specific operation conditions. At the macro-timescale level, the transport phenomena are monitored inside the pore geometry, initially assessing the degradation rate inside a single pore, and then extending over a pore network. This pore-scale analysis offers the advantage of understanding the degradation process from the level of a single pore up to a complex pore network, avoiding the use of oversimplifying approximations, such as the Effective Medium Theory (EMT). Experimental data of a similar DBD reactor setup for the atrazine degradation are used for comparison with numerical results. 
Indico rendering error

Could not include image: Cannot read image data. Maybe not an image file?

Acknowledgements

This project has received funding from the Hellenic Foundation for Research and Innovation (HFRI) and the General Secretariat for Research and Technology (GSRT), under grant agreement No [1754].

Procter and Gamble Student poster award:

I don't want to compete References:

Acceptance of Terms and Conditions:

Click here to agree

681

\section{Modeling Water Treatment by Capacitive Deionization Using Mul- tiphysics Pore Network Modeling}

Mehrez Agnaou ${ }^{1} ;$ Jeff Gostick $^{1}$

${ }^{1}$ University of Waterloo

Corresponding Author(s): magnaou@uwaterloo.ca

Water desalination and wastewater purification by capacitive deionization is a fast-growing water treatment technology. It is based on ion removal from feed-water through electrosorption in carbon porous electrodes electrically insulated by a spacer and subject to a low voltage.

The process consists of two half-cycles. The first one, the desalination or ion removal stage, occurs when the charged ions in the feedwater migrate to oppositely charged electrodes, where they are electrostatically contained in electric double layers within carbon electrodes micropores. The second half-cycle, the discharge stage, occurs when the ions are released from the fully charged electrodes by short-circuiting them.

Whereas many capacitive deionization cell architectures exist in the literature, we focus in this work on the two most common ones; the flow-by and the flow-through. In both cases, the cell is made of two porous carbon electrodes separated by a permeable spacer. For the flow-by cell architecture, the feedwater flows through the spacer. Hence, the macroscopic flow field and the electric field are perpendicular. On the other hand, in the flow-through architecture, the feedwater flows through the carbon electrodes macropores, and is thus parallel to the electric field. Each of the two architectures presents advantages and drawbacks. Chiefly, while the flow-through cell enables the use of spacer of reduced thickness resulting in more compact cells, it requires a higher pressure difference to induce water flow compared to the flow-by architecture.

Several studies have focused on modeling and simulation of capacitive deionization processes; however, due to the high numerical costs of pore-scale simulations, the are typically restricted to the macroscopic scale and were carried out using averaged models. In this work, a pore network modeling approach was adopted to perform pore-scale simulations at lower computational costs compared to other continuum methods, while still focusing on the key impact of electrode structure. The developed multiphysics solver consists of three coupled algorithms solved iteratively. In the first and second steps the flow problem and the Poisson equation for the electrostatic potential are solve, respectively. The third step solves the Nernst-Planck equation for each of the ionic species using the velocity and the potential fields produced by the two first steps. The sorption and desorption occurring in the electrodes is modeled by means of a nonlinear source/sink term.

The developed solver scheme was used to study the adsorption and desorption of ions in the electrodes micropores and a parametric study was performed to analyze the quantity of adsorbed ions 
as a function of the pore-scale parameters, which are easily adjusted in the pore network modeling approach.

Procter and Gamble Student poster award:

I don't want to compete References:

Acceptance of Terms and Conditions:

Click here to agree

801

\title{
Pore-scale investigation of the surface roughness impacts on re- action rate
}

\author{
Priyanka Agrawal ${ }^{1}$; Till Bollermann ${ }^{2} ;$ Amir Raoof $^{1} ;$ Oleg Iliev $^{3}$; Cornelius Fischer $^{2}$; Mariëtte Wolthers ${ }^{1}$ \\ ${ }^{1}$ Department of Earth Sciences, Utrecht University, Utrecht, Netherlands \\ ${ }^{2}$ Helmholtz-Zentrum Dresden-Rossendorf, Inst. f. Ressourcenökologie, Abt. Reaktiver Transport, Leipzig, Germany \\ ${ }^{3}$ Fraunhofer Institute for Industrial Mathematics ITWM, Kaiserslautern, Germany
}

Corresponding Author(s): p.agrawal@uu.nl

The dissolution process of calcite mineral is vital in context of several fields i.e. drinking water, $\mathrm{CO} 2$ storage in carbonate reservoirs, enhanced oil recovery from carbonate reservoirs etc. Confident assessment of these processes requires an understanding of the relationship between dissolution rate and surface area. Most often, these two are considered to be linearly related. However, it has been found that dissolution rates calculated using specific surface area (SSA) based on BET gas-adsorption methods are four orders of magnitude lower than the laboratory-measured batchreaction rate (Menke et al., 2014). BET-based rates account for the impact of surface roughness, but with the assumption that each point at the surface is equally probable to react. It has been shown at both molecular and pore scale that surface roughness not only increases the surface area but also alters the overall reactivity of the surface (Fischer et al., 2014 and Jeschke et al., 2002). Consequently, the concept of rate maps and rate spectra has been introduced in order to quantify the heterogeneity of crystal surface reactivity (Fischer et al., 2012). Nevertheless, most of such work has been limited to the respective scale, and not investigated the interplay between roughness, reactivity and hydrodynamics. In this study we aim to answer two questions:

1. What is the magnitude of the control of pore scale roughness on overall reactions rate?

2. How do molecular and pore scale roughness, reactivity and hydrodynamics inter-relate?

For the first question, we utilize a multi-component single pore model, in which pore-scale roughness is defined with sinusoidal curves corresponding to a roughness factor $\beta$ i.e. ratio of amplitude and wavelength of the curve. We observe that in such converging-diverging pore geometries, recirculation zones develop in the trough part of the pore. In these zones, reaction products such as $\mathrm{Ca}$ and $\mathrm{CO} 3$ ions get trapped. Their increasing concentrations negatively impact on dissolution reaction rate. Consequently, the contribution of reaction rate of this point into overall average reaction rate is lower compare to a point located at the crest part of the pore. This surface reaction rate heterogeneity along the pore wall eventually reflects into the relationship between surface area and the average reaction rate. This impact is, however, a function of the flow rate and the roughness magnitude. Deeper the crest or higher the fluid velocity, the heterogeneity in the reaction rate increases and the RSA based reaction rate has more deviation compare to SSA based reaction rate.

While investigating the impact of molecular scale roughness, which is defined by the distribution of different structural sites, the hydrodynamic impacts have thus far been neglected. The derived reaction rate variability across surface is solely a function of density of these different surface sites and molecular dynamics' processes such as diffusion and water exchange kinetics. The assumption of a uniform diffusion boundary layer can be valid at the start of dissolution of calcite surface. We aim to investigate the validity of this assumption through the long-duration of the dissolution course.

Procter and Gamble Student poster award:

I would like to compete in the Procter and Gamble Student award References: 
(1) Menke, H.; Bijeljic, B.; Andrew, M.; Blunt, M. J. Dynamic Pore-Scale Imaging of Reactive Transport in Heterogeneous Carbonates at Reservoir Conditions. Energy Procedia 2014, 63 (June), 5503-5511.

(2) Fischer, C.; Kurganskaya, I.; Schäfer, T.; Lüttge, A. Variability of Crystal Surface Reactivity: What Do We Know? Appl. Geochemistry 2014, 43, 132-157.

(3) Jeschke, A. A.; Dreybrodt, W. Dissolution Rates of Minerals and Their Relation to Surface Morphology. Geochim. Cosmochim. Acta 2002, 66 (17), 3055-3062.

(4) Fischer, C.; Arvidson, R. S.; Lüttge, A., How predictable are dissolution rates of crystalline material? Geochimica et Cosmochimica Acta 2012, 98, 177-185. Acceptance of Terms and Conditions:

Click here to agree

\title{
Fracture-matrix flow interaction characterizations using a temporo- ensemble PIV method
}

\author{
Author(s): Mehrdad Ahkami ${ }^{1}$ \\ Co-author(s): Martin O. Saar ${ }^{1}$; Xiang-Zhao Kong ${ }^{1}$ \\ ${ }^{1}$ Geothermal Energy and Geofluids Group, Institute of Geophysics, ETH Zurich, Zurich, Switzerland
}

Corresponding Author(s): mahkami@ethz.ch

\begin{abstract}
In reservoir applications, such as groundwater management, waste geological disposal, and geothermal energy utilization in particular Engineered Geothermal Systems, a fractured reservoir is often considered to be an interacting double-continuum medium, where open fractures contribute high permeabilities and rock matrix bears low permeability. In such a medium, mass and energy are considered to be transported mainly through the fracture networks. In contrast, the heat is retained in the rock matrices. Therefore, the efficiency of heat extraction during geothermal applications largely depends on the fracture-matrix flow interactions. Several physical models (e.g., the flow transfer function (Kazemi et al., 1976; Lu et al., 2008; Abushaikha and Gosselin, 2008)) have been proposed in order to describe the fluid flow interactions between fractures and rock matrices. However, validation of these models requires laboratory experimental observations.

Here we present an experimental study on analysis of fracture-matrix flow interactions in a 3Dprinted fractured porous medium. The 3D-printed medium consists of well-defined heterogeneities, i.e. two porous matrices with two different pore sizes and one dead-end and one flow-through fracture in each matrix. The interaction analysis takes the two-dimensional velocity field, which is delineated using a temporo-ensemble Particle Image Velocimetry (PIV) method (Ahkami et al., 2018). This temporo-ensemble PIV method is capable of reducing the interrogation window down to a single pixel. Such small interrogation windows enable resolving velocity fields in micro structures, while still capturing the flow in larger features, such as fractures with a much smaller overall pixel number

The fracture-matrix flow interaction of this study focuses on the fluid flow field of each matrix and embedded fractures. The velocity analyses suggest that the effect of fracture geometry and background matrices on fracture-matrix interactions can be quantified by the longitudinal and lateral components of the velocities in fractures. Comparisons of lateral and longitudinal velocities between different regions indicate that the background matrices have a significant effect on fracture-matrix flow interactions, which can dominate over the effect of fracture geometry. These results should facilitate the calibration of numerical simulations on fracture-matrix flow interactions in fractured porous media.
\end{abstract}

Procter and Gamble Student poster award:

I would like to compete in the Procter and Gamble Student award References:

Ahkami, M., Roesgen, T., Saar, M.O. and Kong, X.Z., 2018. High-Resolution Temporo-Ensemble PIV to Resolve Pore-Scale Flow in 3D-Printed Fractured Porous Media. Transport in Porous Media, pp.117.

Abushaikha, A.S. and Gosselin, O.R., 2008. Matrix-fracture transfer function in dual-media flow simulation: Review, comparison and validation. EAGE Conference and Exhibition. Society of Petroleum Engineers, SPE Europe, pp.1-25. 
Kazemi, H., Merrill Jr, L.S., Porterfield, K.L. and Zeman, P.R., 1976. Numerical simulation of water-oil flow in naturally fractured reservoirs. Society of Petroleum Engineers Journal, 16(06), pp.317-326.

Lu, H., Di Donato, G. and Blunt, M.J., 2008. General transfer functions for multiphase flow in fractured reservoirs. SPE Journal, 13(03), pp.289-297. Acceptance of Terms and Conditions:

Click here to agree

\title{
Two-equation continuum model of drying: a limiting case of im- mobile phase
}

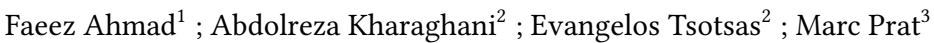

\author{
${ }^{1}$ Thermal Process Engineering, Otto von Guericke University \\ ${ }^{2}$ Thermal Process Engineering, Otto von Guericke University \\ ${ }^{3}$ Institut de Mécanique des Fluides de Toulouse (IMFT), Université de Toulouse, CNRS, Toulouse, France
}

Corresponding Author(s): faeez.ahmad@ovgu.de

The modeling of the drying process in porous media has been a subject of research for decades. For this purpose, the classical continuum-scale model 1 is widely developed and used in many environmental and engineering applications. This model requires (effective) parameters which depend on the local saturation. These parameters are often obtained from tedious experiments. Very recently, a new method was proposed to extract these parameters from numerical pore network simulations [2]. In this method the entire computational domain is artificially cut into local slices (volumes) such that each slice may contain hundreds of pores. In fact, each slice represents the local element of the one-dimensional continuum model and the averaged values of the parameters are obtained at the scale of these local averaging volumes. The values of parameters are thus calculated in all slices during the drying process. The parameters, which depend only on the local saturation, are fed into the one-equation continuum model [2]. The most interesting outcome in [2] was the observation of a non-local equilibrium (NLE) effect, meaning that the equilibrium condition of the vapor is established at the meniscus scale, not at the scale of the local averaging volume. In this work, we push our previous work forward and thus develop a two-equation NLE continuum model that accounts for the liquid and vapor transport coupled by a source/sink term. As a first step, we chose a limiting case of immobile liquid phase, where liquid is distributed as isolated clusters in the entire porous medium, neglecting the capillary pumping in the liquid phase. This regime corresponds to the last drying period, where the mass transport is dominated by vapor diffusion. In order to solve the two-equation NLE model, the effective vapor diffusivity and the specific interfacial area (both are functions of the local saturation), as well as the mass exchange coefficient are required. These parameters are determined from the pore network simulations. The solution of the two-equation model reveals that the non-local equilibrium effect can be simulated with a reasonable degree of accuracy. The source/sink term successfully captures the non-equilibrium mass transfer at the local scale. The model can independently reproduce drying kinetics, which was not possible with the previous one-equation continuum model. The effective vapor diffusivity in the unsaturated region of the pore network is formulated with an expression which depends on the dimensions of pores (radius and length), the pore-scale binary diffusion coefficient and the network porosity. Our next step is to move towards further extension of the NLE continuum model in order to simulate the drying process in a more resolute and comprehensive manner, i.e. not only when the liquid is present under the form of small disconnected clusters (last period of drying) but also when liquid clusters spans the entire network (first and second drying periods).

\section{Procter and Gamble Student poster award:}

I would like to compete in the Procter and Gamble Student award References:

1. Whitaker, S.: Simultaneous heat, mass, and momentum transfer in porous media: A theory of drying. Advances in Heat Transfer, 13 (1977) 119-203.

2. Attari Moghaddam, A., Prat, M., Tsotsas, E., Kharaghani, A.: Evaporation in capillary porous media at the perfect piston-like invasion limit: Evidence of nonlocal equilibrium effects. Water Resources Research, 53 (2017) 10433-10449. 


\title{
Adaptive fixed-stress iterative coupling schemes based on a pos- teriori error estimates for Biot's consolidation model
}

\author{
Elyes Ahmed ${ }^{1}$; Jan Martin Nordbotten ${ }^{1}$; Florin Adrian Radu ${ }^{1}$ \\ ${ }^{1}$ Department of Mathematics, University of Bergen, Norway
}

Corresponding Author(s): elyes.ahmed@uib.no

\begin{abstract}
We develop in this paper adaptive iterative coupling schemes for the Biot system modeling coupled flow and geomechanics in a poro-elastic medium. We particularly consider the space-time formulation of the fixed-stress iterative scheme, in which we first solve the problem of flow over the whole space-time interval, then exchanging the space-time information for solving the problem of mechanics. Two common discretizations of this algorithm is then introduced based on two coupled mixed finite elements methods in-space and the backward Euler scheme in-time. Therefrom, adaptive fixed-stress algorithms are build on conforming reconstructions of the pressure and displacement together with equilibrated flux and stresses reconstructions delivering a posteriori error estimate distinguishing the different error components, namely the spatial discretization, the temporal discretization, and the fixed-stress iteration components. Precisely, at the iteration $k \geq 1$ of the adaptive algorithm, we prove that our estimate gives a guaranteed and fully computable upper bound on the energy-type error measuring the differences between the exact and the approximate pressure and displacement. These error components are efficiently used to design adaptive and multiscale time-stepping strategy and adaptive stopping criteria for the aforementioned fixed-stress algorithms. Numerical experiments illustrate the efficiency of our estimates and the performance of the adaptive iterative coupling algorithms.
\end{abstract}

Procter and Gamble Student poster award:

References:

1 E. Ahmed, F. A. Radu, and J. M. Nordbotten, Adaptive poromechanics computations based on a posteriori error estimates for fully mixed formulations of Biot's consolidation model, research report, Department of Mathematics, University of Bergen, Nov. 2018, https://hal.inria.fr/hal-01687026.

[2] A. Ern and M. Vohralík, Adaptive Inexact Newton Methods with A Posteriori Stopping Criteria for Nonlinear Diffusion PDEs, SIAM Journal on Scientific Computing, 35 (2013), pp. A1761-A1791, doi:10.1137/120896918, https://doi.org/10.1137/120896918.

[3] M. Bause, F. Radu, and U. Köcher, Space-time finite element approximation of the Biot poroelasticity system with iterative coupling, Computer Methods in Applied Mechanics and Engineering, 320 (2017), pp. 745-768. Acceptance of Terms and Conditions:

Click here to agree

607

\section{Porosity of Pervious Concretes Based on 2D scan images}

Author(s): Aryssa Kathreen Marcaida ${ }^{1}$

Co-author(s): Jaehun Ahn $^{1}$; Seongwoo Lee ${ }^{2}$; Jongwon Jung ${ }^{3}$

${ }^{1}$ Department of Civil and Environmental Engineering, Pusan National University

${ }^{2}$ The GL Company Ltd. 
${ }^{3}$ School of Civil Engineering, Chungbuk National University

Corresponding Author(s): jahn@pusan.ac.kr

Pervious concrete is a sustainable pavement material that helps revive the natural hydrologic functions in urban watershed. One of its main characteristics is its pore structure, which influences its capacity to infiltrate stormwater through its layers. The objective of the present study is to utilize an image processing technique in order to determine the porosity of pervious concrete. $25 \mathrm{~mm}$ slices from 20 different pervious concrete mixtures were obtained and scanned at 1200 dpi resolution. Using ImageJ software, the 2D scanned images were scaled, cropped, thresholded, and analyzed. The estimated porosity is the area fraction extracted from the pore structure analysis. From the results of image analysis, it was found out that the addition of fine aggregates result to a lower porosity for most test cases. Similar trend was observed when the cement-aggregate ratio was increased.

Procter and Gamble Student poster award:

References:

Acceptance of Terms and Conditions:

Click here to agree

\section{MULTIFUNCTIONALITY OF LIQUID-FILLED POROUS MATERI- ALS: FROM ENCRYPTION AND ANTI-FOULING TO EFFICIENT CATALYSIS}

Joanna Aizenberg ${ }^{1}$

${ }^{1}$ Harvard University

Corresponding Author(s): jaiz@seas.harvard.edu

Liquids entrapped within a structured solid begin to exhibit unique behaviors often providing the surrounding material with unprecedented properties. Recently we have developed two award-winning materials platforms (R\&D 100 awards in 2012 and 2013), both based on the infusion of a liquid into a porous substrate. First, we introduced a new strategy to create self-healing, anti-fouling materials (so-called Slippery, Lubricant-Infused Porous Surfaces, or SLIPS) that outperform state-of-the-art materials in their ability to resist ice and microbial adhesion and repel various simple and complex liquids. Second, we pioneered a technique for patterning 3D photonic crystals, generating complex wettability patterns, and illustrated multilevel encryption, with selective decoding by specific liquids, so-called Watermark Ink, or W-INK. Generalized, low-cost, and scalable methods to manufacture SLIPS and W-Ink on glass, ceramics, polymers, fabrics and metals will be presented. We anticipate that slippery surfaces can find important applications as antifouling materials in medicine, construction, fluid handling and transportation, while chemically patterned photonic structures can serve as colorimetric indicators for liquids, used in encryption and anti-tampering applications, and provide a novel platform for efficient catalytic materials.

Procter and Gamble Student poster award:

References:

Acceptance of Terms and Conditions:

\section{Direct Numerical Simulations on Mixed-Wet Carbonates: A Com- prehensive Workflow from Experiments to Simulations}

Author(s): Takashi Akai ${ }^{1}$ 
Co-author(s): Amer Alhammadi ${ }^{1}$; Martin Blunt $^{1}$; Branko Bijeljic $^{2}$

${ }^{1}$ Imperial College London

${ }^{2}$ Imperial College

Corresponding Author(s): t.akai17@imperial.ac.uk

We present direct multiphase numerical simulations on micro-CT images of a mixed-wet reservoir carbonate. Our objectives are 1) to accurately model multiphase flow in a mixed-wet rock incorporating experimentally measured contact angles and 2) to study the impact of enhanced oil recovery (EOR) applications on the microscopic sweep efficiency using direct numerical simulations.

We used a water-flooding experiment of Alhammadi et al. (2017) in which the fluid configuration after water-flooding was imaged using a micro-CT. Direct numerical simulations were performed on a pore structure obtained from segmented micro-CT images using the two-phase colour gradient lattice Boltzmann method. In the experiment, the wettability of the sample was altered to a mixedwet state by aging the sample at subsurface conditions using crude oil from the same reservoir. The mixed-wettability was confirmed by a range of in situ contact angles measured by an automated algorithm from AlRatrout et al. (2017). Using a wetting boundary condition presented in Akai et al. (2018), the measured contact angles were employed in our simulations. We demonstrate how to use measured contact angle data to improve the predictability of direct numerical simulations, highlighting the difference between the contact angle required for the simulation of dynamic displacement process and the contact angle measured at equilibrium after water-flooding through the comparison between the simulations and experiment.

Furthermore, a well-calibrated simulation model to the water-flooding experiment was then used to study the impact of three EOR schemes on the microscopic displacement efficiency: low salinity water flooding, surfactant flooding and polymer flooding. Taking the well-calibrated simulation model as a base case, rock and/or fluid properties were changed to mimic these EOR schemes. Additional oil recovery by these EORs were quantitatively evaluated by comparing the remaining oil saturation of EOR cases to that of the base case. As a result, surfactant flooding showed the highest impact on microscopic displacement efficiency, while the impact of polymer flooding was not significant in our case. In addition, we investigated the mechanisms of the additional oil recovery at the pore-scale, through the analysis of location of remaining oil as a function of pore sizes and as a function of distance from pore walls.

Overall, we demonstrate a comprehensive workflow for pore-scale modelling from experiments to simulations to better understand water-flooding and EOR processes at the pore-scale.

Procter and Gamble Student poster award:

I would like to compete in the Procter and Gamble Student award References:

Acceptance of Terms and Conditions:

Click here to agree

\title{
Experimental Analysis and Mathematical Modelling of Inorganic Scaling in Seawater Reverse Osmosis Membranes
}

\author{
Mayo Akele ${ }^{1}$ \\ ${ }^{1}$ University of Nottingham \\ Corresponding Author(s): ooakele@gmail.com
}

A key challenge associated with desalination is inorganic scaling/clogging. These scales deposit on the thin membrane surface, decreasing the performance of Reverse Osmosis (RO) desalination plants. It is common practice to use the fouling rates, indices and prediction models that have been developed in this field. However, inconsistency arise when field measurements are compared with model predictions making these techniques ineffective in mitigating and controlling industrial scale clogging. 
Research on the subject has been mostly restricted to individual or ideal solutions with well-controlled operating conditions. However, this fails to mimic the complex water chemistries and operating conditions found in RO plants. This project seeks to understand scaling of different water chemistries and the subsequent precipitation on membrane surfaces. The goal is to model inorganic scaling of RO membranes under industrial scale operating conditions and validate the model developed by history matching real field data from a RO desalination plant.

A combination of experimental analysis and mathematical modelling is being used in the analysis. As a first step towards quantifying the effects of complex water chemistries, an experimental study was conducted with mixed salt solution simulating the molar ratio found in natural waters. The experiments demonstrated co-precipitation of more than one type of inorganic scale, which is consistent with what is typically observed in RO operations. A mathematical model is currently being developed to describe the formation of the scales and subsequent co-precipitation observed experimentally.

Procter and Gamble Student poster award:

I would like to compete in the Procter and Gamble Student award References:

Acceptance of Terms and Conditions:

Click here to agree

845

\section{Thermal stability of urease produced by Sporosarcina pasteurii}

Arda Akyel ${ }^{1}$; Adrienne Phillips ${ }^{2}$; Robin Gerlach ${ }^{2}$

${ }^{1}$ Ph.D. Student
${ }^{2}$ Montana State University

Corresponding Author(s): arda.akyel@montana.edu

Microbially induced calcium carbonate precipitation (MICP) is a technology touted for the stabilization of porous media such as soils, sealing of leaky wells and the remediation of contaminants in water and soil. The application in the deeper subsurface might occur at elevated temperatures and the most commonly used organism Sporosarcina pasteurii does not appear to grow reliably at temperatures above approximately $40^{\circ} \mathrm{C}$. However, it has been demonstrated that urease can remain active at elevated temperatures and hence, we assessed the kinetics and thermal stability of urease produced by $S$. pasteurii over a temperature range from 20 to $80^{\circ} \mathrm{C}$. It was observed by us that the enzyme half-life decreases with increasing temperature while enzyme activity increases; when both, enzyme inactivation and activity, are considered, $60^{\circ} \mathrm{C}$ was determined to be the optimal temperature for the ureolysis reaction over a 2-hour reference timeframe, which is deemed relevant for field deployment. We have developed a method to produce urease using $S$. pasteurii, thermally inactivate it and make it available for enzyme-induced calcium carbonate mineral precipitation (EICP) technology implementation in the field. Column studies demonstrating the successful use of $S$. pasteurii-produced enzyme for the consolidation of porous media will be presented.

Procter and Gamble Student poster award:

I would like to compete in the Procter and Gamble Student award References:

Acceptance of Terms and Conditions:

Click here to agree 


\title{
Insight into Influence of Crossflow in layered Sandstone porous media during Miscible and Immiscible CO2 WAG Flooding
}

\author{
Duraid Al-Bayati ${ }^{1}$; Ali Saeedi ${ }^{1}$; Ipek $\mathrm{Ktao}^{2}{ }^{\text {; Matthew }}$ Myers $^{3}$; Cameron White ${ }^{3}$; Quan Xie $^{1}$ \\ ${ }^{1}$ Faculty of Science and Engineering, School of WASM-MECE, Curtin University \\ ${ }^{2}$ Private researcher \\ ${ }^{3}$ Commonwealth Scientific and Industrial Research Organisation (CSIRO-Energy)
}

Corresponding Author(s): d.al-bayati@postgrad.curtin.edu.au

\begin{abstract}
For the layered system, cross-flow is one of the mechanisms for recovery enhancement during an IOR/EOR process. Thus, the results from this paper are very important to overcome the current challenges in capturing the importance of cross-flow influence as well as mitigating the effect of geological uncertainties on current and future IOR/EOR projects.

This manuscript presents the results of an experimental investigation into the effect of cross-flow on ultimate oil recovery during miscible and immiscible $\mathrm{CO} 2$ WAG flooding in layered sandstone porous media. A manufactured core sample constructed by attaching two half-cylindrical homogeneous natural sandstone plugs of different permeabilities. The core flooding tests using n-Decane Synthetic brine $\mathrm{CO} 2$ were conducted at a constant temperature of $343 \mathrm{~K}$ and under two different pressure conditions, namely, 9.6 MPa and 17.23 MPa to attain both immiscible and miscible conditions, respectively.

The results indicated that cross-flow in the layered sample has a negative impact on the ultimate oil recovery (i.e. decreasing oil recovery factor). The degree of heterogeneity seems to strongly influence the effectiveness of cross-flow during $\mathrm{CO} 2 \mathrm{EOR}$ with the oil recovery decreases as the permeability ratio (PR) between the two half plugs included in every samples increase. For instance, during miscible $\mathrm{CO} 2 \mathrm{WAG}$ flooding, a decrease in incremental oil recoveries from $3.3 \%$ to $11.3 \%$ and eventually to only $4.8 \%$ occurred when the permeability ratios were increased from 2.5 to 5 and 12.5 , respectively. Similarly, during immiscible displacement, the recorded oil recoveries were $6.1 \%$, $6.9 \%$ and $4.7 \%$ reflecting the same increases in permeability ratios as above. These results revealed that cross-flow works against the influence of the dominant active forces. For instance, in noncommunicating layers, the dominance of viscous forces prevailed while there is a preferential flow path exists in flow in communicating layers. However, with increasing permeability ratio a considerable channelling of $\mathrm{CO} 2$ into the high permeability layer leaving the low permeability layer touched partially, implying that heterogeneity in vertical direction indeed significantly affects remaining oil saturations, thus oil recovery.
\end{abstract}

Procter and Gamble Student poster award:

\section{References:}

AL-BAYATI, D., SAEEDI, A., MYERS, M., WHITE, C. \& XIE, Q. 2018a. An Experimental Investigation of Immiscible CO2 Flooding Efficiency in Sandstone Reservoirs: Influence of Permeability Heterogeneity. SPE Europec featured at 80th EAGE Conference and Exhibition. Copenhagen, Denmark: Society of Petroleum Engineers.

AL-BAYATI, D., SAEEDI, A., MYERS, M., WHITE, C., XIE, Q. \& CLENNELL, B. 2018b. Insight investigation of miscible SCCO2 Water Alternating Gas (WAG) injection performance in heterogeneous sandstone reservoirs. Journal of CO2 Utilization, 28, 255-263.

AL-BAYATI, D., SAEEDI, A., XIE, Q., MYERS, M. B. \& WHITE, C. 2018c. Influence of Permeability Heterogeneity on Miscible CO2 Flooding Efficiency in Sandstone Reservoirs: An Experimental Investigation. Transport in Porous Media, 125, PP 341-356.

AL EIDAN, A. A., MAMORA, D. D. \& SCHECHTER, D. S. 2011. Experimental and Numerical Simulation Studies of Different Modes of CO2 Injection in Fractured Carbonate Cores. Society of Petroleum Engineers.

AL HAMDAN, M. R. S. 2013. An experimental and numerical investigation of compositional displacements in heterogeneous reservoirs. The University of New South Wales.

AL WAHAIBI, Y. M. \& AL HADHRAMI, A. K. 2011. First-Contact-Miscible, Vaporizing- and CondensingGas Drive Processes in a Channeling Heterogeneity System. Society of Petroleum Engineers.

ALAJMI, A. F. \& GRADER, A. Analysis of fracture-matrix fluid flow interactions using X-ray CT. SPE Eastern Regional Meeting, 2000. Society of Petroleum Engineers.

ALHAMDAN, M., CINAR, Y., SUICMEZ, V. S. \& DINDORUK, B. 2012. Experimental and numerical study of compositional two-phase displacements in layered porous media. Journal of Petroleum Science and Engineering, 98, 107-121.

ALVARADO, V. \& MANRIQUE, E. 2010. Enhanced oil recovery: an update review. Energies, 3, 15291575 . 
ASGHARI, K. \& TORABI, F. 2008. Effect of Miscible and Immiscible CO2 Injection on Gravity Drainage: Experimental and Simulation Results. Society of Petroleum Engineers.

BAHRALOLOM, I. M., BRETZ, R. E. \& ORR, F. M., JR. 1988. Experimental Investigation of the Interaction of Phase Behavior With Microscopic Heterogeneity in a CO2 Flood. Society of Petroleum Engineers Journal, 3, 662-672.

BIKKINA, P., WAN, J., KIM, Y., KNEAFSEY, T. J. \& TOKUNAGA, T. K. 2016. Influence of wettability and permeability heterogeneity on miscible CO2 flooding efficiency. Fuel, 166, 219-226.

BLUNT, M., FAYERS, F. J. \& ORR JR, F. M. 1993. Carbon dioxide in enhanced oil recovery. Energy Conversion and Management, 34, 1197-1204.

BROCK, D. \& ORR JR, F. Flow visualization of viscous fingering in heterogeneous porous media. SPE Annual Technical Conference and Exhibition, 1991. Society of Petroleum Engineers.

BURGER, J., SPRINGATE, G. \& MOHANTY, K. 1996. Experiments on bypassing during gasfloods in heterogeneous porous media. SPE Reservoir Engineering, 11, 109-115.

CAO, M. \& GU, Y. 2012. Physicochemical characterization of produced oils and gases in immiscible and miscible CO2 flooding processes. Energy \& Fuels, 27, 440-453.

CHAOUCHE, M., RAKOTOMALALA, N., SALIN, D. \& YORTSOS, Y. 1993. Capillary effects in immiscible flows in heterogeneous porous media. EPL (Europhysics Letters), 21, 19.

DEBBABI, Y., JACKSON, M. D., HAMPSON, G. J., FITCH, P. J. R. \& SALINAS, P. 2017a. Viscous Crossflow in Layered Porous Media. Transport in Porous Media, 117, 281-309.

DEBBABI, Y., JACKSON, M. D., HAMPSON, G. J. \& SALINAS, P. 2017b. Capillary Heterogeneity Trapping and Crossflow in Layered Porous Media. Transport in Porous Media, 120, 183-206.

DINDORUK, B. \& FIROOZABADI, A. 1997. Crossflow in Fractured/Layered Media Incorporating Gravity, Viscous, and Phase Behavior Effects: Part I-Formulation and Features in Layered Media. SPE Journal, 2, 120-135.

DING, M., YUAN, F., WANG, Y., XIA, X., CHEN, W. \& LIU, D. 2017. Oil recovery from a CO2 injection in heterogeneous reservoirs: The influence of permeability heterogeneity, CO2-oil miscibility and injection pattern. Journal of Natural Gas Science and Engineering, 44, 140-149.

DING, M., YUE, X. A., ZHAO, H. \& ZHANG, W. 2013. Extraction and Its Effects on Crude Oil Properties During CO2 Flooding. Energy Sources, Part A: Recovery, Utilization, and Environmental Effects, 35, 2233-2241.

DONALDSON, ERLE, CHILINGARIAN, G. V. \& YEN, T. F. 1985. Enhanced Oil Recovery. I. Fundamentals and Analysis, The Netherlands, Elsevier Science.

FAYERS, F. J. \& LEE, S.-T. 1992. Crossflow Mechanisms by Gas Drive in Heterogeneous Reservoirs. Society of Petroleum Engineers.

GEORGIADIS, A., LLOVELL, F., BISMARCK, A., BLAS, F. J., GALINDO, A., MAITLAND, G. C., TRUSLER, J. P. M. \& JACKSON, G. 2010. Interfacial tension measurements and modelling of (carbon dioxide $+n-$ alkane) and (carbon dioxide+water) binary mixtures at elevated pressures and temperatures. The Journal of Supercritical Fluids, 55, 743-754.

GREENKORN, R. A. \& KESSLER, D. 1969. Dispersion in heterogeneous nonuniform anisotropic porous media. Industrial \& Engineering Chemistry, 61, 14-32.

GRIGG, R. B. \& SCHECHTER, D. S. 1997. State of the Industry in CO2 Floods. Society of Petroleum Engineers.

HAQ, S. \& REIS, J. C. 1993. Predicting Capillary Crossflow in Layered Reservoirs. SPE Annual Technical Conference and Exhibition. Houston, Texas: Society of Petroleum Engineers.

HICKS JR, P. J., NARAYANAN, R. \& DEANS, H. A. 1994. An experimental study of miscible displacements in heterogeneous carbonate cores using X-ray CT. SPE Formation Evaluation, 9, 55-60.

HOLM, L. \& JOSENDAL, V. 1974. Mechanisms of oil displacement by carbon dioxide. Journal of petroleum Technology, 26, 1,427-1,438.

KAMALI, F., HUSSAIN, F. \& CINAR, Y. 2016. An Experimental and Numerical Analysis of WaterAlternating-Gas and Simultaneous-Water-and-Gas Displacements for Carbon Dioxide Enhanced Oil Recovery and Storage. SPE Journal, 22, $521-538$.

KATZ, M. L. \& TEK, M. R. 1962. A Theoretical Study of Pressure Distribution and Fluid Flux in Bounded Stratified Porous Systems with Crossflow.

KRAUSE, M. H., PERRIN, J.-C. \& BENSON, S. M. 2011. Modeling permeability distributions in a sandstone core for history matching coreflood experiments. SPE Journal, 16, 768-777.

LAKE, L. \& JENSEN, J. 1989. A review of heterogeneity measures used in reservoir characterization. SPE. LAKE, L. W. \& HIRASAKI, G. J. 1981. Taylor's dispersion in stratified porous media. Society of Petroleum Engineers Journal, 21, 459-468.

LAKE, W. 1989. Enhanced oil recovery.

LEI, H., YANG, S., ZU, L., WANG, Z. \& LI, Y. 2016. Oil Recovery Performance and CO2 Storage Potential of $\mathrm{CO} 2$ Water-Alternating-Gas Injection after Continuous CO2 Injection in a Multilayer Formation. Energy \& Fuels, 30, 8922-8931.

MACALLISTER, D., MILLER, K., GRAHAM, S. \& YANG, C. 1990. Application of X-ray CT scanning to the determination of gas-water relative permeabilities. SPE, 20494, 23-26.

MORANVILLE, M., KESSLER, D. \& GREENKORN, R. 1977. Dispersion in layered porous media. AIChE 
Journal, 23, 786-794.

NAGARAJAN, N. \& ROBINSON JR, R. 1986. Equilibrium phase compositions, phase densities, and interfacial tensions for carbon dioxide+ hydrocarbon systems. 2. Carbon dioxide+ n-decane. Journal of Chemical and Engineering Data, 31, 168-171.

NOBAKHT, M., MOGHADAM, S. \& GU, Y. 2008. Mutual interactions between crude oil and CO 2 under different pressures. Fluid phase equilibria, 265, 94-103.

PANDE, K. \& ORR, F. M. 1994. Effect of Viscous Crossflow on Miscibility Development in a Two-Layer Flow System: Part I - Ternary Vaporizing Gas Drives.

PERKINS, T. K., JOHNSTON, O. C. \& HOFFMAN, R. N. 1965. Mechanics of viscous fingering in miscible systems. Society of Petroleum Engineers Journal, 5, 301-317.

PERRIN, J.-C. \& BENSON, S. 2010. An Experimental Study on the Influence of Sub-Core Scale Heterogeneities on CO2 Distribution in Reservoir Rocks. Transport in Porous Media, 82, 93-109.

PETERS, B. M., ZHOU, D. \& BLUNT, M. J. Experimental Investigation of Scaling Factors that Describe Miscible Floods in Layered. SPE/DOE Improved Oil Recovery Symposium, 1998. Society of Petroleum Engineers.

RAO, D. N. \& LEE, J. I. 2003. Determination of gas-oil miscibility conditions by interfacial tension measurements. Journal of colloid and interface science, 262, 474-482.

RAPOPORT, L. \& LEAS, W. 1953. Properties of linear waterfloods. Journal of Petroleum Technology, 5, 139-148.

SAEEDI, A. 2012. Experimental study of multiphase flow in porous media during CO2 Geo-Sequestration processes, Springer Science \& Business Media.

SCHEIDEGGER, A. E. 1961. General theory of dispersion in porous media. Journal of Geophysical Research, 66, 3273-3278.

SHAVER, R., ROBINSON, R. \& GASEM, K. 2001. An automated apparatus for equilibrium phase compositions, densities, and interfacial tensions: data for carbon dioxide+ decane. Fluid phase equilibria, 179, 43-66.

SHEDID, S. 2009. Influences of different modes of reservoir heterogeneity on performance and oil recovery of carbon dioxide miscible flooding. Journal of Canadian Petroleum Technology, 48, 29-36.

SONG, Y., ZHU, N., ZHAO, Y., LIU, Y., JIANG, L. \& WANG, T. 2013. Magnetic resonance imaging study on near miscible supercritical CO2 flooding in porous media. Physics of Fluids, 25, 053301.

SORBIE, K., SHEB, M., HOSSEINI, A. \& WAT, R. Scaled miscible floods in layered beadpacks investigating viscous crossflow, the effects of gravity, and the dynamics of viscous slug breakdown. SPE Annual Technical Conference and Exhibition, 1990. Society of Petroleum Engineers.

SORBIE, K. S. \& SERIGHT, R. S. 1992. Gel Placement in Heterogeneous Systems With Crossflow. SPE/DOE Enhanced Oil Recovery Symposium. Tulsa, Oklahoma: Society of Petroleum Engineers.

TRIVEDI, J. \& BABADALI, T. 2006. Optimal Injection Strategies for CO2 and Flue Gas Sequestration During Tertiary Oil Recovery. OIL GAS European Magazine

TUNIO, S. Q., TUNIO, A. H., GHIRANO, N. A. \& EL ADAWY, Z. M. 2011. Comparison of different enhanced oil recovery techniques for better oil productivity. International Journal of Applied Science and Technology, 1.

VERMA, M. K. 2015. Fundamentals of carbon dioxide-enhanced oil recovery (CO2-EOR): A supporting document of the assessment methodology for hydrocarbon recovery using CO2-EOR associated with carbon sequestration, US Department of the Interior, US Geological Survey.

VINEGAR, H. J. \& WELLINGTON, S. L. 1987. Tomographic imaging of three-phase flow experiments. Review of Scientific Instruments, 58, 96-107.

WANG, S. Y., HUANG, Y., PEREIRA, V. \& GRYTE, C. C. 1985. Application of computed tomography to oil recovery from porous media. Applied optics, 24, 4021-4027.

WELLINGTON, S. \& VINEGAR, H. CT studies of surfactant-induced CO2 mobility control. SPE Annual Technical Conference and Exhibition, 1985. Society of Petroleum Engineers.

WITHJACK, E. 1988. Computed tomography for rock-property determination and fluid-flow visualization. SPE formation evaluation, 3, 696-704.

YAMAMOTO, J., SATOH, T., ISHII, H. \& OKATSU, K. An Analysis of CO2 WAG Coreflood by Use of Xray CT. SPE Asia Pacific Oil and Gas Conference and Exhibition, 1997. Society of Petroleum Engineers. YOKOYAMA, Y. \& LAKE, L. W. The effects of capillary pressure on immiscible displacements in stratified porous media. SPE Annual Technical Conference and Exhibition, 1981. Society of Petroleum Engineers. YONGMAO, H., ZENGGUI, W., BINSHAN, J., YUEMING, C. \& XIANGJIE, L. 2004. Laboratory Investigation of $\mathrm{CO} 2$ Flooding. Society of Petroleum Engineers.

ZAPATA, V. J. \& LAKE, L. W. 1981. A Theoretical Analysis of Viscous Crossflow. Society of Petroleum Engineers.

ZHAO, F., HAO, H., HOU, J., HOU, L. \& SONG, Z. 2015. CO2 mobility control and sweep efficiency improvement using starch gel or ethylenediamine in ultra-low permeability oil layers with different types of heterogeneity. Journal of Petroleum Science and Engineering, 133, 52-65.

ZHOU, D., FAYERS, F. J. \& ORR, F. M., JR. 1994. Scaling of Multiphase Flow in Simple Heterogeneous Porous Media.

ZHOU, X., AL-OTAIBI, F. M., KOKAL, S. L., AL-HASHBOUL, A. A., BALASUBRAMANIAN, S. \& AL- 
GHAMD, F. A. 2015. Novel Insights into IOR/EOR by Seawater and Supercritical CO2 Miscible Flooding Using Dual Carbonate Cores at Reservoir Conditions. Saudi Aramco Journal of Technology.

ZHOU, X., ALOTAIBI, F., KOKAL, S., ALHASHBOUL, A. \& AL-QAHTANI, J. 2017. A New Approach of Pressure Profile and Oil Recovery during Dual and Single Carbonate Core Flooding by Seawater and CO2 Injection Process at Reservoir Conditions. SPE/IATMI Asia Pacific Oil \& Gas Conference and Exhibition. Jakarta, Indonesia: Society of Petroleum Engineers. Acceptance of Terms and Conditions:

Click here to agree

\section{Characterisation of the Effect of Microstructure on the Liquid Transport in Porous Media Using Terahertz Spectroscopy and Imag- ing}

Author(s): Mohammed Al-Sharabi ${ }^{1}$

Co-author(s): Theona Mudley ${ }^{2}$; Daniel Markl ; Prince Bawuah ; Anssi-Pekka Karttunen ; Cathy Ridgway ; Patrick Gane ; Jarkko Ketolainen ; Kai-Erik Peiponen ; Thomas Rades ; J. Axel Zeitler

\footnotetext{
${ }^{1}$ University of Cambridge

${ }^{2}$ University of Cambridge
}

Corresponding Author(s): mdfa2@cam.ac.uk

Immediate release tablets are one of the most common and convenient ways of drug delivery to the patient. The overall microstructure of the tablet influences the hydration of such formulations and hence affects the disintegration time and the release of the active pharmaceutical ingredients (APIs) in the body. The disintegration mechanisms of these drug products are complex and poorly understood due to the limitation of the appropriate characterisation techniques 1 . Terahertz timedomain spectroscopy (THz-TDS) and terahertz pulsed imaging (TPI) techniques were used as a new method to measure fast disintegrating tablets and provide a quantitative analysis of the results to understand better the disintegration process. The terahertz techniques are concerned with characterising the properties of materials using electromagnetic radiation in the frequency range of 0.1$6 \mathrm{THz}$ [2]. Functionalised calcium carbonate (FCC) based tablets with porosities of 45\% and $60 \%$ and microcrystalline cellulose (MCC) based tablets with porosities of $25 \%$ and $10 \%$ with two different APIs, theophylline and paracetamol, were used for the terahertz measurements. TPI was able to study the liquid penetration and swelling of the tablets with different porosities upon contact with the dissolution medium. The total duration for the water to fully hydrate the tablet is less for the FCC and MCC tablets with the higher porosity compared to the tablets with the lower porosity. THz-TDS and TPI are useful tools for studying the immediate release tablets and fast disintegrating tablets in particular. Investigating the effect of the microstructure characteristics on the liquid penetration and swelling kinetics is important for a better understanding of the disintegration process and in turn controlling the quality and improving the development and performance of the drug in the body.

\section{Procter and Gamble Student poster award:}

I would like to compete in the Procter and Gamble Student award References:

1 Markl, D., Wang, P., Ridgway, C., Karttunen, A-P., Bawuah, P., Ketolainen, J., Gane, P., Peiponen, K-E., Zeitler, J. A. Resolving the rapid water absorption of porous functionalised calcium carbonate powder compacts by terahertz pulsed imaging. Chemical Engineering Research and Design. 132, 1082-1090 (2018).

[2] Zeitler, J. A. Pharmaceutical terahertz spectroscopy and imaging. Muellertz, A., Rades, T., Perrie, Y. editors, Analytical Techniques in the Pharmaceutical Sciences. New York, NY: Springer. 171- 222(2016). Acceptance of Terms and Conditions:

Click here to agree 


\title{
Thermally Induced Calcium Carbonate Precipitation in the Bakken Oilfields
}

\author{
Author(s): Dalton Albers ${ }^{1}$ \\ Co-author(s): Adrienne Phillips ${ }^{1}$; Bjorn Worum ${ }^{1}$; Catherine Kirkland ${ }^{1} ;$ Kyle Deverna ${ }^{1} ;$ Logan Schultz ${ }^{1}$; Robin \\ Gerlach $^{1}$ \\ ${ }^{1}$ Montana State University \\ Corresponding Author(s): albersdalton365@gmail.com
}

Fractures and high permeability zones in oil bearing formations can greatly complicate oil recovery by creating preferential flow paths which can lead to reduced recovery and the contamination of water supplies. Microbially induced calcium carbonate precipitation (MICP) is a technology capable of modifying the permeability of such zones, wherein ureolytic microbes catalyze the precipitation of calcium carbonate 1 . In deep wells, however, the high temperatures cause rapid inactivation of the necessary urease enzyme. Fortunately, urea hydrolysis also occurs spontaneously at temperatures above approximately $100 \mathrm{oC}$. Thus, thermally induced calcium carbonate precipitation (TICP) is possible for deep wells, such as those in the Bakken fields of North Dakota. While previous researchers have studied TICP as a sealing method, their experiments focused on the reaction in deionized (DI) water. Therefore, the existing models might not be adequate for environmental conditions. Bakken water in particular can have very high concentrations of various ions, including $\mathrm{Ca} 2+$ and $\mathrm{Na}+$. (21 $\mathrm{g} / \mathrm{L}$ and $81 \mathrm{~g} / \mathrm{L}$, respectively).

In this study, TICP kinetic studies were performed in Bakken and DI water at $130 \mathrm{oC}$ to assess the influence of dissolved salts on the ureolysis rate and subsequent precipitation of calcium carbonate. Our data show that ureolysis (and therefore TICP) proceeds at a much slower rate in Bakken water than in DI water. Approximating the reaction as first-order, the ureolysis rate constants were calculated to be $0.028 \mathrm{~h}-1$ in the Bakken water versus $0.14 \mathrm{~h}-1$ in DI water (when urea was the only added solute). High concentrations of calcium had been previously observed to slow the rate of ureolysis, therefore the rate difference was postulated to be due to the native calcium in the Bakken water In follow-up experiments which controlled for calcium concentration, Bakken water samples still showed a significantly slower reaction rate. Under equimolar conditions (1 M urea and $1 \mathrm{M} \mathrm{Ca} 2+$ ) the first order rate constants were estimated at 0.028 h- 1 in Bakken water versus $0.043 \mathrm{~h}-1$ in DI water. This discrepancy may be due to the high ionic strength of the Bakken water, for which the effect on activity is not accounted for in concentration-based kinetics models.

The results of these experiments suggest that while the Bakken oilfields will be more time-consuming to seal than earlier laboratory models would predict, TICP might be a viable option for deep zones where microbially or enzymatically induced calcium carbonate precipitation is impossible due to the elevated temperatures.

Procter and Gamble Student poster award:

References:

1 Adrienne J. Phillips, Robin Gerlach, Ellen Lauchnor, Andrew C. Mitchell, Alfred B. Cunningham \& Lee Spangler (2013) Engineered applications of ureolytic biomineralization: a review, Biofouling, 29:6, 715-733, DOI: 10.1080/08927014.2013.796550 Acceptance of Terms and Conditions:

Click here to agree

\section{non-Gaussian multi-scale heterogeneity patterns of equivalent conductivities of a fractured reservoir}

\author{
Author(s): Guadagnini Alberto ${ }^{1}$ \\ Co-author(s): Riva Monica ${ }^{1}$; Siena Martina ${ }^{1}$; Papini Monica ${ }^{1}$; Longoni Laura ${ }^{1}$; Scesi Laura ${ }^{1}$ \\ ${ }^{1}$ Department of Civil and Environmental Engineering, Politecnico di Milano (Italy)
}


Corresponding Author(s): alberto.guadagnini@polimi.it

We assess scaling of the probability density of equivalent hydraulic conductivity of a fractured rock system. We do so upon considering jointly data from an outcrop of a fractured system and their increments evaluated between two points separated by a given distance (or lag). The study is set in the context of a geostatistical framework which has enabled us to quantify seemingly random spatial variations of environmentally relevant quantities such as rock mineral content, permeability, or porosity. While traditional approaches rely on a view of such quantities as multivariate Gaussian random functions characterized by well-defined spatial correlation scales, there are evidences documenting that many spatially varying hydrologic (and other) quantities exhibit non-Gaussian behavior over a variety of (spatial and/or temporal) scales. In this context, quantities such as log hydraulic conductivity and their spatial increments are known to be generally non-Gaussian. Documented evidences of such behavior include symmetry of increment distributions at all separation scales (or lags) between incremental values of the given (hydrological) quantity of interest, with sharp peaks and heavy tails that appear to decay as lag increases. These and other aspects of statistical scaling are manifest in porous as well as fractured media characterized by either one or a hierarchy of spatial correlation scales. We illustrate typical aspects of such scalable non-Gaussian behavior upon relying on data collected across a natural outcrop of a fractured system. Our data-set comprises aperture, spacing, orientation, and persistency of fractures which are categorized into a collection of families. Spatial distributions of directional equivalent conductivities are evaluated at two differing observation scales/windows across the investigated domain. These data and their increments evaluated at a set of lags are then subject to statistical analysis. They are interpreted through a recent generalized sub-Gaussian theoretical model that captures scaling behavioral aspects in a comprehensive manner. Major results of the study include: (i) an accessible high-quality dataset; and (ii) the assessment of the ability of the considered generalized sub-Gaussian model to characterize the observed statistical scaling features displayed by sample statistics.

Keywords: equivalent hydraulic conductivity, fractured reservoir, geostatistics, scaling, Generalized Sub-Gaussian model.

\title{
Procter and Gamble Student poster award:
}

I don't want to compete References:

Acceptance of Terms and Conditions:

Click here to agree

\section{Investigation of the Erodibility of Fungal Treated Soils Using a Laboratory JET Apparatus}

\author{
Author(s): Gráinne El Mountassir ${ }^{1}$ \\ Co-author(s): Emmanuel Salifu ${ }^{2}$; Schellenger Alexandra ${ }^{3}$; Livia Adinolfi ${ }^{3}$; Raniero Beber ${ }^{3}$; Alessandro \\ Tarantino $^{3}$ \\ ${ }^{1}$ University of Strathclyde, Glasgow UK \\ ${ }^{2}$ University of Strathclyde Glasgow and Università di Napoli Federico II, Napoli \\ ${ }^{3}$ University of Strathclyde, Department of Civil and Environmental Engineering, fames Weir Building, 75 Montrose \\ Street, Glasgow, Scotland, United Kingdom.
}

Corresponding Author(s): alexandra.schellenger@strath.ac.uk

Conventional ground improvement techniques are energy intensive, highly invasive and require the introduction of environmentally damaging chemicals or carbon intensive materials. There is a clear need for the development of sustainable, low-carbon technologies for ground improvement. Over the past decade, geotechnical engineers have started to consider biological-based solutions including engineered vegetation/plant root systems, the use of biopolymers, and more recently, biomineralisation (bio-clogging and bio-cementation) for ground improvement. This has resulted in the 
creation of the sub-discipline of biogeotechnics, with the overarching aim of 'engineering nature' to create sustainable geo-structures.

We present here results from investigations of a novel bio-geotechnical technique involving the use of fungal hyphae to mitigate surface erosion. Fungal hyphae (long filamentous branches) are known to contribute to soil aggregation and soil hydrophobicity. This study evaluates the influence of different fungal species and treatment strategies on soil erodibility. Sterile sandy soils amended with known quantities of organic matter were placed in a mould, inoculated with fungal spores of different species (including Pleurotus ostreatus and Trichoderma reesei) and placed in incubators to grow at $25^{\circ} \mathrm{C}$. JET tests were performed on treated and untreated specimens with periods of incubation up to 9 weeks to determine erosion parameters: erodibility coefficient, Kd and critical stress, $\tau c$ as well as volume of soil eroded. Results show that fungal hyphae significantly reduced the erodibility of the soil. Erodibility is shown to be dependent on the method of inoculation, growth period and nature of hyphal growth obtained. Erosion resistance of fungal treated soils could be further improved by optimising and enhancing growth techniques.

Procter and Gamble Student poster award:

I don't want to compete References:

Acceptance of Terms and Conditions:

Click here to agree

839

\title{
Pore-scale imaging and measurement of relative permeability in a mixed-wet carbonate reservoir rock at reservoir conditions
}

\author{
Author(s): Amer Alhammadi ${ }^{1}$ \\ Co-author(s): Ying Gao ${ }^{1}$; Martin Blunt ${ }^{1}$; Branko Bijeljic ${ }^{2}$ \\ ${ }^{1}$ Imperial College London \\ ${ }^{2}$ Imperial College
}

Corresponding Author(s): a.alhammadi15@imperial.ac.uk

The key parameters describing multiphase flow in porous media are relative permeability and capillary pressure. In this study we use X-ray microtomography combined with high-pressure hightemperature flow apparatus to conduct co-injection of crude oil and formation brine simultaneously in a mixed-wet reservoir carbonate while measuring the pressure drop. We are therefore able to measure relative permeability and the pore-scale arrangement of fluids simultaneously.

We use a carbonate rock sample extracted from a very large producing oil field from the Middle East saturated with crude oil and formation brine from the same reservoir. The sample was dynamically aged at $10 \mathrm{MPa}$ and $80{ }^{\circ} \mathrm{C}$ to establish the mixed-wettability conditions known to exist in hydrocarbon reservoirs (Alhammadi et al., 2017). After completing the aging process, eight water fractional flows were injected $(\mathrm{fw}=0,0.15,0.3,0.5,0.7,0.85,0.95,1)$. The relative permeability was measured from the water saturation obtained on the segmented X-ray images (each image is 10 billion voxels) and pressure drop from pressure transducer readings. A high concentration of potassium iodide (30 weight \%) was used as a dopant in the formation brine to resolve the brine in the sub-resolution pores. To this end, differential imaging was applied to accurately quantify brine saturation in macro-pores and micro-pores.

The mixed-wettability conditions of the rock were quantified using an automated contact angle method (AlRatrout et al., 2017). The carbonate rock found to be mostly weakly oil-wet with a contact angle distribution of $119^{\circ} \pm 18^{\circ}$ from 194,000 points. In oil-wet pores the brine was found in the centre of the pores and oil was present in layers. In contrast, in water-wet pores, the brine was in small crevices and pore corners. Oil layers helped maintain oil connectivity, leading to favourable waterflood recovery. This has important implications on improved oil recovery and, potentially, on carbon storage. In future, the measured relative permeability and pore-scale fluid distribution could be used to benchmark and validate pore-scale models.

Procter and Gamble Student poster award: 
References:

1. Alhammadi A.M., AlRatrout, A., Singh, K., Bijeljic, B., Blunt, M.J. In situ characterization of mixedwettability in a reservoir rock at subsurface conditions. Scientific Reports. 7 (1), 10753 (2017).

2. AlRatrout, A., Raeini, A.Q., Bijeljic, B., Blunt, M.J. Automatic measurement of contact angle in pore-space images. Advances in Water Resources. 109, 158-169 (2017).

Acceptance of Terms and Conditions:

Click here to agree

\title{
Simulation of Asphaltene Precipitation and Deposition in Porous Media: Thermodynamics and LBM Approaches
}

\author{
Sepideh Alimohammadi ${ }^{1}$; Sohrab Zendehboudi ${ }^{1}$; Lesley James ${ }^{1}$ \\ ${ }^{1}$ Faculty of Engineering and Applied Science, Memorial University, St. John's, NL, Canada \\ Corresponding Author(s): salimohammad@mun.ca
}

\begin{abstract}
Asphaltene -the cholesterol of crude oil- may negatively affect the oil and gas industry in terms of production, processing, transportation, economic, and environmental aspects. To obtain a comprehensive understanding of asphaltene and its behaviours, a variety of thermodynamic equations of state (EOSs), particularly cubic-plus-association (CPA) and perturbed-chain statistical associating fluid theory (PC-SAFT) are used in this research work to investigate the effect of pressure and solvent ratio on the precipitation phenomena. Association property of asphaltene leads to precipitation and deposition of asphaltene. These two EOSs are able to accurately determine the amount of asphaltene precipitation on the basis of physical properties and association term, where saturate, aromatic, resin, and asphaltene (SARA) analysis in the form of solubility fractions, are the input data to the model. Based on the research output, a good agreement between the modelling results and real data is noticed. Moreover, a single phase two dimensional lattice Boltzmann method (LBM) in conjunction with multi-relaxation time collision operator is implemented to simulate permeability impairment due to asphaltene deposition. A pressure drop and different solvent flow rates were imposed to obtain asphaltene deposition. The permeability impairment was modelled in different rates of solvent injection. According to the output of this study, the introduced model (the thermodynamic approach in conjunction with LBM) offers a good strategy to assess the permeability impairment due to asphaltene deposition. It was shown that a decrease in permeability at higher flow rates is expected due to higher deposition of asphaltene.
\end{abstract}

Procter and Gamble Student poster award:

I would like to compete in the Procter and Gamble Student award References:

Acceptance of Terms and Conditions:

Click here to agree

460

\section{Pore-Scale Study of Multi-Species Ion Transports through Inho- mogeneously Charged Nanoporous Media}

\author{
Amer Alizadeh $^{1} ;$ Moran Wang $^{2}$ \\ ${ }^{1}$ Department of Engineering Mechanics and CNMM, Tsinghua University, Beijing 100084, China \\ ${ }^{2}$ Department of Engineering Mechanics and CNMM, Tsinghua University, Beijing 100084, China,
}


Corresponding Author(s): aalizadeh@outlook.com, mrwang@tsinghua.edu.cn

The electrokinetic transport mechanisms of multi-species ions through 3D nanoporous membranes with chemical reaction at the solid-aqueous solution interfaces are investigated. We systematically study the multi-physics transport phenomena by considering either inhomogeneous (locally charged based on local solution $\mathrm{pH}$ and ion concentrations) or prescribed homogeneous surface charge at solid-aqueous solution interface while the pores are screened via electric double layers. To this aim, a lattice Boltzmann numerical framework is developed to solve the set of coupled multi-physicochemical governing equations (PNP+NS). Our modeling results reveal that averaged electric potential of the membrane is greatly underestimated (about 83\%) when the locally acquired surface charge in pores of the membrane is ignored. It is shown that increasing the porosity of the membrane considerably increases the absolute values and inhomogeneity of the surface charge. The averaging of the ion concentrations over membrane cross-sections uncovers an internal ion depletion by approaching toward the outlet which resembles the reverse osmosis phenomenon. The streaming and electrical conductivity of nanoporous membrane exhibit a nonlinear behavior with porosity. Moreover, the electrical conductivity demonstrates limiting and over limiting-like regimes with the variation of the porosity.

Charged porous membranes and ion-selective nanochannels have attracted numerous interests in recent decades owing to their ever-increasing applications for water desalination [1-5], pre-concentration of species in micro- and nanochannels [6, 7], power generation via reverse electrodialysis [8], molecular sorting, separations, and sensing [9-12]. A crucial common factor among these applications is the presence of surface charge at the membrane-aqueous solution interface (Fig. 2.1). For instance, this charge plays a substantial role in the ion selectivity feature of the ion exchange membranes (IEM) [13-16]. Understanding the physics underlying of the charge acquiring and ion transport through mechanisms these membranes paves the way for improvements in water treatment and biologicalrelated technologies.

It is well-understood that a membrane could be locally charged by the chemical reactions of the solid surface (i.e. silica) with the aqueous solution. The local solution $\mathrm{pH}$, temperature, and ion concentration (thermos-chemical properties) determine the final acquired surface charge [17]. Modeling the electrokinetic transport phenomena through electrically charged nanoporous membranes is challenging due to three main difficulties. Firstly, the complex geometry of the membrane which brings such a great complexity to the governing equations. Essentially, the manufacturing process of the nanoporous membranes makes randomly oriented pores with various pore size distribution which cannot be easily manipulated [11]. Secondly, obtaining the local surface charge based on the local solution thermo-chemical properties which postures as a main bottleneck. Finally, the thickness of the electric double layer (EDL) within the pores of the membrane. The thickness of the EDL in comparison with the representative pore size of the nanoporous membrane has a key role in electrokinetic transport phenomena. For instance, when the thickness of the EDL is comparative with the pore size (or even larger), the whole pore space would be electrically charged. This means that the ion selectivity of the nanoporous membrane is significantly enhanced. However, the conventional theories which lied on the Helmholtz-Smoluchowski equation are failed to predict the behavior of the nanoporous membrane for when the pores are screened via the EDLs. For sake of simplicity, the previous works tried to model ion transport through homogeneously charged nanotubes and nanochannels [18-21] or structured straight nanochannels [22]. Moreover, except a few studies which relaxed the assumption related to the thickness of the EDL $[18,19,21,23,24]$, the majority of the literatures investigated the ion transport under the assumption of thin double layer thickness; where and denote the reverse of Debye length and the average radius or height of the nanopores, respectively [25-28]. In this relation, denotes the vacuum electrical permittivity, the relative permittivity of the solution to the vacuum, the Boltzmann constant, the elementary charge of the electron, the number density of the bulk ions, and the absolute temperature of the solution. The assumption of the thin double layer lets us use the electroosmotic velocity at the edge of the diffuse layer and as a result, one can ignore the presence of the EDL. This assumption justifies the employing of the Poisson-Boltzmann equation instead of the complex advection-diffusion (NernstPlanck) equation. Only a few studies in history tried to tackle the electrokinetic transport through the porous membranes under the thick double layer conditions (e.g. [29, 30]).

Evidently, the surface charge within a complex geometry like nanoporous membrane could not be considered as homogeneous, namely, constant surface charge medium. Essentially, the surface charge heterogeneity within the nanoporous membrane arises from the local solution properties ( $\mathrm{pH}$, ion concentration) or even chemically or structurally inhomogeneity in solid surfaces which are in contact with the solution.

Despite the aforementioned attempts, one can deduce that two main bottlenecks have not been tackled yet: taking into account the effects of the inhomogeneous structure of the real nanoporous membrane on the electrokinetic transport mechanisms and the local surface charge in the pores due to the local $\mathrm{pH}$ and ion concentration. For understanding the physics underlies ion transport mechanism through the charged nanoporous membranes, one has to solve the coupled Poisson, Nernst-Planck, 
and Navier-Stokes equations within a randomly-generated nanoporous membrane. By utilizing a randomly-generated porous medium, Wang and Chen [31] investigated the electro-osmosis in a homogeneously charged porous medium. Recently Zhang and Wang [25] studied the ion transport through the inhomogeneously charged randomly-generated porous medium by the aid of a simple 1-pK model [32] for very thin electric double layers ( ). In their work, the effect of the thin electric double layer has been introduced to the hydrodynamic equations by considering a slip velocity based on the Helmholtz-Smoluchowski classical equation [33].

In this paper, for the first time, we conduct a pore-scale study of the nonlinear electrokinetic transport phenomena for a 3D randomly-generated nanoporous membrane which acquires local surface charge due to chemical adsorption of the ions. Regarding the local surface charge, a representative bulk layer (RBL) model [17] has been recently introduced to take into account not only the effects of the electric double layers (EDLs) interaction (overlapped EDLs regime) but also the complex geometry of the randomly-generated nanoporous membrane. Introducing the obtained local zeta potential to the Poisson's equation coupled with the Nernst-Planck and Navier-Stokes equations enables us to study the electroosmotic permeability and tortuosity for inhomogeneously charged nanoporous membrane with overlapped EDLs. It is noteworthy that, although in the present contribution we do not consider the inhomogeneity of surface chemical properties (i.e. pure silica), the proposed numerical framework can be simply extended to consider the chemical inhomogeneity within the synthesized nanoporous membrane. In terms of the electrokinetic conductivity of the nanoporous membrane, we obtained the total streaming and electrical conductance of the nanoporous membrane as a function of the porosity and compared with when the membrane is assumed to be homogeneously charged.

\section{Procter and Gamble Student poster award:}

I would like to compete in the Procter and Gamble Student award References:

1 K. Sung Jae, K. Sung Hee, K. Kwan Hyoung, H. Jongyoon, Direct seawater desalination by ion concentration polarization, Nature Nanotechnology, 5 (2010) 297-301.

[2] S.V. Pham, H. Kwon, B. Kim, J.K. White, G. Lim, J. Han, Helical vortex formation in three-dimensional electrochemical systems with ion-selective membranes, Physical Review E, 93 (2016).

[3] V.V. Nikonenko, A.V. Kovalenko, M.K. Urtenov, N.D. Pismenskaya, J. Han, P. Sistat, G. Pourcelly, Desalination at overlimiting currents: State-of-the-art and perspectives, Desalination, 342 (2014) 85-106.

[4] P.M. Biesheuvel, M.Z. Bazant, Nonlinear dynamics of capacitive charging and desalination by porous electrodes, Physical Review E, 81 (2010).

[5] M.E. Suss, T.F. Baumann, W.L. Bourcier, C.M. Spadaccini, K.A. Rose, J.G. Santiago, M. Stadermann, Capacitive desalination with flow-through electrodes, Energy \& Environmental Science, 5 (2012) 95119519.

[6] S.H. Ko, Y.-A. Song, S.J. Kim, M. Kim, J. Han, K.H. Kang, Nanofluidic preconcentration device in a straight microchannel using ion concentration polarization, Lab on a Chip, 12 (2012) 4472-4482.

[7] D. Hlushkou, R. Dhopeshwarkar, R.M. Crooks, U. Tallarek, The influence of membrane ion-permselectivity on electrokinetic concentration enrichment in membrane-based preconcentration units, Lab on a Chip, 8 (2008) 1153-1162.

[8] D.K. Kim, C.H. Duan, Y.F. Chen, A. Majumdar, Power generation from concentration gradient by reverse electrodialysis in ion-selective nanochannels, Microfluid. Nanofluid., 9 (2010) 1215-1224.

[9] D.L. Gin, R.D. Noble, Designing the Next Generation of Chemical Separation Membranes, Science, 332 (2011) 674-676.

[10] K. Hotta, A. Yamaguchi, N. Teramae, Deposition of Polyelectrolyte Multilayer Film on a Nanoporous Alumina Membrane for Stable Label-Free Optical Biosensing, The Journal of Physical Chemistry C, 116 (2012) 23533-23539.

[11] P. Bhadra, S. Sengupta, N.P. Ratchagar, B. Achar, A. Chadha, E. Bhattacharya, Selective transportation of charged $\mathrm{ZnO}$ nanoparticles and microorganism dialysis through silicon nanoporous membranes, Journal of Membrane Science, 503 (2016) 16-24.

[12] J.L. Snyder, A. Clark, D.Z. Fang, T.R. Gaborski, C.C. Striemer, P.M. Fauchet, J.L. McGrath, An experimental and theoretical analysis of molecular separations by diffusion through ultrathin nanoporous membranes, Journal of Membrane Science, 369 (2011) 119-129.

[13] V.A. Shaposhnik, O.A. Kozaderova, Transport of hydrogen and hydroxyl ions through ion-exchange membranes under overlimiting current densities, Russian Journal of Electrochemistry, 48 (2012) 791-796. [14] M. Tedesco, H.V.M. Hamelers, P.M. Biesheuvel, Nernst-Planck transport theory for (reverse) electrodialysis: III. Optimal membrane thickness for enhanced process performance, Journal of Membrane Science, 565 (2018) 480-487.

[15] M. Tedesco, H.V.M. Hamelers, P.M. Biesheuvel, Nernst-Planck transport theory for (reverse) electrodialysis: I. Effect of co-ion transport through the membranes, Journal of Membrane Science, 510 (2016) 370-381.

[16] P.M. Biesheuvel, A. van der Wal, Membrane capacitive deionization, Journal of Membrane Science, 346 (2010) 256-262. 
[17] A. Alizadeh, M. Wang, Reverse electrodialysis through nanochannels with inhomogeneously charged surfaces and overlapped electric double layers, Journal of Colloid and Interface Science, 529 (2018) 214223.

[18] J.C. Fair, J.F. Osterle, reverse electrodialysis in charged capillary membranes, J. Chem. Phys., 54 (1971) 3307-3316.

[19] P.B. Peters, R. van Roij, M.Z. Bazant, P.M. Biesheuvel, Analysis of electrolyte transport through charged nanopores, Physical Review E, 93 (2016) 14.

[20] J. Catalano, R.G.H. Lammertink, P.M. Biesheuvel, Theory of fluid slip in charged capillary nanopores, arXiv, (2016) 8 pp.-8 pp.

[21] R.J. Gross, J.F. Osterle, Membrane transport characteristic of ultrafine capillaries, J. Chem. Phys., 49 (1968) 228-234.

[22] M. Schmuck, M.Z. Bazant, Homogenization of the poisson-nernst-planck equations for ion transport in charged porous media, SIAM J. Appl. Math., 75 (2015) 1369-1401.

[23] A. Alizadeh, M.E. Warkiani, M. Wang, Manipulating electrokinetic conductance of nanofluidic channel by varying inlet $\mathrm{pH}$ of solution, Microfluid. Nanofluid., 21 (2017) 52.

[24] M. Wang, Q.J. Kang, E. Ben-Naim, Modeling of electrokinetic transport in silica nanofluidic channels, Anal. Chim. Acta, 664 (2010) 158-164.

[25] L. Zhang, M. Wang, Electro-osmosis in inhomogeneously charged microporous media by pore-scale modeling, Journal of Colloid and Interface Science, 486 (2017) 219-231.

[26] J.T.G. Overbeek, P.W.O. Wijga, On electro-osmosis and streaming-potentials in diaphragms, Recueil des Travaux Chimiques des Pays-Bas, 65 (1946) 556-563.

[27] D. Burgreen, F.R. Nakache, Electrokinetic Flow in Ultrafine Capillary Slits1, The Journal of Physical Chemistry, 68 (1964) 1084-1091.

[28] C.L. Rice, R. Whitehead, Electrokinetic Flow in a Narrow Cylindrical Capillary, The Journal of Physical Chemistry, 69 (1965) 4017-4024.

[29] A. Revil, P. Leroy, Constitutive equations for ionic transport in porous shales, J. Geophys. Res., Solid Earth, 109 (2004).

[30] A.H. Galama, J.W. Post, M.A. Cohen Stuart, P.M. Biesheuvel, Validity of the Boltzmann equation to describe Donnan equilibrium at the membrane-solution interface, Journal of Membrane Science, 442 (2013) 131-139.

[31] M. Wang, S. Chen, Electroosmosis in homogeneously charged micro- and nanoscale random porous media, Journal of Colloid and Interface Science, 314 (2007) 264-273.

[32] S.A. de Lima, M.A. Murad, C. Moyne, D. Stemmelen, A three-scale model for pH-dependent steady flows in 1:1 clays, Acta Geotechnica, 3 (2008) 153.

[33] R.J. Hunter, Foundations of colloid science, 2nd ed., Oxford University Press, (2001). Acceptance of Terms and Conditions:

Click here to agree

\title{
Convergence Analysis Of The Fixed Stress Split Iterative Coupling Scheme In Fractured Heterogeneous Poro-Elastic Media
}

\author{
Tameem Almani ${ }^{1}$; Abdulrahman Manea ${ }^{1}$; Kundan Kumar ${ }^{2}$ \\ ${ }^{1}$ Saudi Aramco \\ ${ }^{2}$ University of Bergen, Norway
}

Corresponding Author(s): tameem.almani@aramco.com

The accurate coupling of geomechanics and flow in poroelastic media plays an important role in modeling surface subsidence, reservoir production, ground water remediation, hydraulic fracturing, and $\mathrm{CO} 2$ sequestration. In addition, such an accurate and precise coupling is needed for uncertainty quantification in applications incorporating geomechanical parameters. Since fractures have significant effects on reservoir flow profiles, incorporating a realistic model for fractures when studying the coupled geomechanics and flow problem is a must. The main objective of this work is to establish the convergence of an adaptation of the fixed-stress split coupling scheme (for coupling flow with geomechanics) in fractured heterogeneous poro-elastic media. In our approach, fractures are modeled as possibly non-planar interfaces, and the flow in the fracture is described by a lubrication type system 1. The flow in the reservoir matrix and in the fracture are coupled to the geomechanics model through a fixed-stress split coupling scheme, in which mass balance equations (for both 
flow in the matrix, and in the fracture) are augmented with fixed-stress split regularization terms. The convergence proof, based on a Banach contraction argument, shall determine the appropriate values of these regularization terms, which ensure the convergence of the coupling scheme in heterogeneous media. Geometric convergence to the unique solution of the system follows immediately as the sequence of iterates represents a convergent Cauchy sequence. To the best of our knowledge, this is the first time in literature a rigorous convergence result is established for coupling flow with geomechanics in fractured heterogeneous poroelastic media.

\title{
Procter and Gamble Student poster award:
}

I don't want to compete References:

1 Vivette Girault, Kundan Kumar, Mary F Wheeler, "Convergence of iterative coupling of geomechanics with flow in a fractured poroelastic medium”, Computational Geosciences 20 (5), 997-1011, 2016. Acceptance of Terms and Conditions:

Click here to agree

\section{Assessment of the Low-Re RANS based turbulence models in two simplified pore geometries}

\author{
Alejandro Alonzo-García ${ }^{1}$; Ana Mendoza-Rosas ${ }^{1}$; Martín Díaz-Viera $^{2}$; Edgar Martínez-Mendoza $^{3}$ \\ ${ }^{1}$ CONACYT-Centro de Ingeniería y Desarrollo Industrial \\ ${ }^{2}$ Instituto Mexicano del Petróleo (IMP) \\ ${ }^{3}$ Universidad Nacional Autónoma de México (UNAM)
}

Corresponding Author(s): alejandro_1980@hotmail.com

Understanding the mean and turbulent flow structures existent within pore spaces is important in a wide variety of engineering fields as mechanical, chemical, oilfield, environmental, geological among others. For example, network modeling is making progress towards predictive capabilities and absolute and relative permeability, among other flow-based characteristics, are required for static and dynamic oil-field modeling. In this sense, when high inertial effects drive the flow displacement in porous media, the values of permeability should be corrected by introducing non-linear terms in the pressure gradient (Forchheimer relation). Thus adequate predictions of flow turbulence are important. For its capabilities and reasonable computational requirements, the Reynolds Average Navier Stokes (RANS) based turbulence models are extensively used in the engineering fields to account for turbulence at macro-scale. However, at micro-scale, a complete assessment of the particular strengths and weaknesses of the RANS models has not been performed under the same framework, and it is important, as the theoretical basis of such models obeys to deep physical assumptions. Accordingly, this work presents an evaluation the Low-Re (Yangh-Shi, Lam-Bremhorst and LaunderSharma) and the classic (Standard k- $\varepsilon$, RNG, Realizable and k- $\omega$ ) RANS based turbulence models in pore domains defined by periodic arrangements of squares and circles. The Reynolds number $(\mathrm{Re})$ was varied from $100-40000$ and the porosity $\varphi$ was fixed to 0.3 . The grids were built in $2 \mathrm{~d}$ domains, using triangular cells, as this kind of elements are especially suited to map complex geometries, as those required to represent realistic scenarios. Grid independence analysis was completed, and the validation was made by comparing turbulence kinetic energy (TKE), dissipation rate ( $\varepsilon$ ) and pressure gradients with experimental data and other numerical studies reported in the literature. Results show the existence of a critical Re where all models predictions are independent of Re. Strong differences in the TKE and $\varepsilon$ predictions were found for the Low-Re models below such critical Re, suggesting the existence of a strong flow transition, and peculiar flow patterns.

Procter and Gamble Student poster award:

I don't want to compete References:

Acceptance of Terms and Conditions:

Click here to agree 


\title{
Thermally Induced Nanoparticle Delivery to Porous Media via Emulsions
}

\author{
Usua U. Amanam ${ }^{1}$; Anthony Kovscek ${ }^{1}$; Huang Zeng ${ }^{2}$ \\ ${ }^{1}$ Stanford University \\ ${ }^{2} B P$
}

Corresponding Author(s): uamanam@stanford.edu

\begin{abstract}
We have developed a novel payload delivery technique to transport nanoparticles deep into geological formations. Such nanoparticles are useful for facilitating oxidation of organic phases, such as crude-oil, and enabling gas-phase reactions. Flow rate, solution ionic strength, particle surface charge, $\mathrm{pH}$, and the interaction with inorganic colloids, among other factors, affect the mobility of nanoparticles (Petosa et al., 2012; Heidmann, 2013). The number of variables makes it difficult to predict how particles will respond to a complex hydrocarbon reservoir environment, and for most applications, this understanding is critical for maintaining the nanoparticle size distribution necessary for success. While the size of primary nanoparticles improves their transportability, it also makes them susceptible to agglomeration as a result of increased attractive van der Waals (vdW) forces. This can lead to premature settling, pore plugging, and a subsequent critical loss in the available active surface area. Our technique decreases particle interactions with other elements in the reservoir by providing a protective microcapsule that releases nanocatalysts at specified conditions (e.g., temperature). The capsules are the dispersed phase of an oil-in-water emulsion and move through water pathways in the porous medium. We use an established empirical formulation to manage and plan for different reservoir conditions that affect capsule stability and visualize pore-scale nanoparticle delivery using microfluidic devices with representative sandstone pore network patterns.

Here, we report fundamental colloidal calculations, corrected for size, quantify particle-particle interactions experimentally, and observe payload delivery using experimental microfluidics. We show that Lifshitz and Derjaguin-Landau-Verwey-Overbeek (DLVO) theory are useful to screen potential encapsulating mediums to limit interparticle interaction within the capsules. These calculations provide insight into the relative strength of net attractive/repulsive forces of particles in different solvents. Zeta potential and dynamic light scattering measurements presented quantify these interactions in the colloidal system by computing the effective surface charge of particles and the associated mean size, respectively. Our microfluidics results show that delivery occurs by destabilizing the emulsion at temperatures near or slightly greater than formation temperature. Once the emulsion breaks, particles move freely in a continuous oil phase and mix with the in-situ crude. Moreover, we show that an essential benefit is that the temperature that triggers the release is tunable to reservoir conditions with the appropriate choice of solvent, surfactant, and fluid ratio. Our work also leads us to believe that in the absence of an easily accessible crude-oil phase, particles will deposit on grains or clay minerals at or above the saturation temperature of the encapsulating medium.
\end{abstract}

Procter and Gamble Student poster award:

I would like to compete in the Procter and Gamble Student award References:

A. R. Petosa, S. J. Brennan, F. Rajput, and N. Tufenkji. Transport of two metal oxide nanoparticles in saturated granular porous media: role of water chemistry and particle coating. water research, 46(4):1273 1285, 2012.

I. Heidmann. Metal oxide nanoparticle transport in porous media-an analysis about (un) certainties in environmental research. In Journal of Physics: Conference Series, volume 429, page 012042. IOP Publishing, 2013. Acceptance of Terms and Conditions:

Click here to agree

\section{A posteriori error estimates for flow modeling using an Enhanced Velocity scheme}


Yerlan Amanbek ${ }^{1}$; Gurpreet Singh ${ }^{2}$; Gergina Pencheva ${ }^{2}$; Mary Wheeler ${ }^{\text {None }}$

\footnotetext{
${ }^{1}$ Nazarbayev University

${ }^{2}$ The University of Texas at Austin
}

Corresponding Author(s): yerlan.amanbek@nu.edu.kz

\begin{abstract}
A novel and an efficient method, adaptive numerical homogenization, is used for modeling of flow and transport in the heterogeneous porous media 1 . The key component of method is a selection of appropriate adaptivity criteria to achieve better accuracy of simulation. In the flow, the traditional error indicator relies on pressure error indicator, however, most of the previous studies do not take into account velocity error in the adaptivity strategy. The velocity plays important role in the coupling flow and transport problems.

We investigate a posteriori error estimator for the Darcy flow using Enhanced Velocity Mixed FEM [2]. We show the error indicators for pressure error and velocity error. Residual-based error indicator and implicit error indicator will be derived theoretically and will be demonstrated numerically. In the implicit error analysis, we used the post-processing of pressure from [3] to achieve better indicator of flux. The residual estimators are better indicators for pressure error in the incompressible flow. Moreover, we show the advantages of the implicit error estimators with postprocessing in the detection of velocity error numerically. Numerical experiments are also presented. The proposed indicators can be successfully used for a number of adaptive methods with Enhanced Velocity scheme in subsurface simulations.
\end{abstract}

\title{
Procter and Gamble Student poster award:
}

I would like to compete in the Procter and Gamble Student award References:

1) Amanbek, Y., Singh, G., Wheeler, M. F., \& van Duijn, H. (2017). Adaptive numerical homogenization for upscaling single phase flow and transport. ICES Report, 12, 17.

2) Wheeler, J. A., Wheeler, M. F., \& Yotov, I. (2002). Enhanced velocity mixed finite element methods for flow in multiblock domains. Computational Geosciences, 6(3-4), 315-332.

3) Arbogast, T., \& Chen, Z. (1995). On the implementation of mixed methods as nonconforming methods for second-order elliptic problems. Mathematics of Computation, 64(211), 943-972. Acceptance of Terms and Conditions:

Click here to agree

\section{Multiscale modelling of NAPL dissolution in porous media in the presence of pore and continuum scale heterogeneity}

Morteza Aminnaji ${ }^{1}$; Masoud Babaei ${ }^{2}$; Arash Rabbani $^{1}$

\footnotetext{
${ }^{1}$ University of Manchester

${ }^{2}$ The University of Manchester
}

Corresponding Author(s): morteza.aminnaji@manchester.ac.uk

Dissolution coefficient of non-aqueous phase liquids (NAPL) is one of the most important parameters in predicting the transport in porous media for contaminant removal from the aquifers. A multiscale (pore-to-continuum) numerical method is developed in which the dissolution coefficient has been derived from pore scale physics for heterogeneous pore network models. The method replaces the need to use commonly applied empirical correlations for dissolution coefficient. To this end, a threedimensional pore-scale model is developed to simulate interphase mass transfer over different synthetic and extracted pore network structures. Using pore network modelling, both NAPL dissolution rate coefficient (i.e., interfacial area is explicitly taken into account) and lumped dissolution rate coefficient (i.e., interfacial area is incorporated in dissolution coefficient) are calculated for the various pore network structures. Sherwood and modified Sherwood numbers are formulated for different rock types. Additionally several unstructured pore networks are extracted from micro-tomography 
images of rock samples using watershed segmentation algorithm. In parallel, continuum scale modelling is conducted for NAPL removal in porous media. Dissolution rate coefficients calculated using pore network are used as an up-scaled mass transfer coefficient for simulation of NAPL removal at continuum scale. Therefore, the impacts of pore network heterogeneity, pore scale parameters (e.g. throat diameter, pore diameter, and coordination number), NAPL saturation/distribution and aqueous phase velocity on NAPL dissolution coefficient are underpinned.

Procter and Gamble Student poster award:

References:

Acceptance of Terms and Conditions:

Click here to agree

771

\title{
A universal law for slip flow in tubes and porous media
}

\author{
Mohammad Aminpour ${ }^{1}$; Sergio Galindo Torres ${ }^{2}$; Alexander Scheuermann ${ }^{3}$; Ling LI $^{3}$ \\ ${ }^{1}$ Geotechnical Engineering Centre. School of Civil Engineering, The University of Queensland, Brisbane QLD 4072, \\ Australia \\ ${ }^{2}$ University of Liverpool \\ ${ }^{3}$ University of Queensland
}

Corresponding Author(s): m.aminpour@uq.edu.au

Slip flow due to wettability effects has significant potential applications in industry, with flow enhancement ratios observed in nanotubes up to several orders of magnitudes. Our simulation results using lattice-Boltzmann method in porous media and tubes considering different wettability conditions are collapsed as a universal law for slip flow. With a nondimentioanl number defined, we show that the flow enhancement is governed by physical parameters attributed to the hydrodynamics of small-scale flow systems. We show the agreement of the model with the experimental and numerical data from literature.

\section{Procter and Gamble Student poster award:}

I would like to compete in the Procter and Gamble Student award References:

1 Jason K Holt, Hyung Gyu Park, Yinmin Wang, Michael Stadermann,Alexander B Artyukhin, Costas P Grigoropoulos, Aleksandr Noy, and Ol-gica Bakajin. Fast mass transport through sub-2-nanometer carbon nan-otubes.Science, 312(5776):1034-1037, 2006.

[2] Mainak Majumder, Nitin Chopra, Rodney Andrews, and Bruce J Hinds.Nanoscale hydrodynamics: enhanced flow in carbon nanotubes.Nature,438(7064):44, 2005.

[3] Keliu Wu, Zhangxin Chen, Jing Li, Xiangfang Li, Jinze Xu, and XiaohuDong. Wettability effect on nanoconfined water flow.Proceedings of theNational Academy of Sciences, page 201612608, 2017. Acceptance of Terms and Conditions:

Click here to agree

\section{GPU-based pore-scale transport modelling in heterogeneous rocks}

\author{
Author(s): Senyou $\mathrm{An}^{1}$ \\ Co-author(s): Vahid Joekar-Niasar ${ }^{1}$ \\ ${ }^{1}$ University of Manchester
}


Corresponding Author(s): senyou.an@postgrad.manchester.ac.uk

The structure with all length scales existing in geological media is an inherent natural, and the scale of a few pores is no exception. Herein, we incorporated heterogeneity into the microscale advectiondispersion simulation using correlated random pore network. Anisotropic exponential variogram is used to generate random auto-correlated fields with different correlation length, considering the mean and variance value of Berea sandstone. These fields are superimposed on one same unstructured irregular topology network, which only has pore locations and throat connection relationship. Lognormal distributed pore body radii can be mapped form the radius fields. Setting pressure boundaries, we designed the GPU accelerated preconditioned conjugate gradient algorithm with data optimization to solve the diagonal dominant symmetric matrix Hagen-Poiseuille flow equation. The transport equation containing advection and dispersion is solved explicitly after solving the pressure distribution. As high-density iteration calculation, the transport evolution is ideally suitable for GPU parallelization by allocating different pore into different calculation kernels. With one-time input and output data transformation, the transport modeling for about 1.2 million pore bodies can be finished in 2 minutes. Our simulation results show an increase in the magnitudes of the estimated dispersion coefficients in correlated networks compared to uncorrelated ones in the advection-controlled regime. The range of the Péclet numbers which dictate mixed advectiondiffusion regime considerably reduces in the correlated networks. The findings emphasize the critical role of correlation length illustrated by pore-scale models.

\title{
Procter and Gamble Student poster award:
}

I would like to compete in the Procter and Gamble Student award References:

Acceptance of Terms and Conditions:

Click here to agree

\section{Permeability prediction in porous media using multivariant struc- tural regression}

\author{
Matthew Andrew ${ }^{1}$ \\ ${ }^{1}$ Carl Zeiss X-ray Microscopy \\ Corresponding Author(s): matthew.andrew@zeiss.com
}

Traditional approaches to the prediction of effective properties from porous media focus on coupling the 3D structural imaging of porous media with numerical simulation of the partial differential equations governing the property of interest $[1,2]$. Such an approach has at its heart the idea that a single emergent "steady state" will arise, given the defined domain and conditions - there is a single map which deterministically predicts effective property from structure. The past 10 years have seen an explosion in the availability of open-source machine learning tools, allowing for an both in-depth multivariant analysis of porous media structure[3] and powerful new regression techniques, allowing for the usage of such multivariant statistics for effective prediction. In this study a new technique for prediction of permeability from pore scale images of rocks using multivariant statistical regression is presented.

A series of over 2,000 synthetic pore networks were generated using object-based techniques. Flow was simulated on these synthetic volumes using traditional digital rock techniques, giving the permeability of each network. The networks were then analyzed for the statistics of their structural properties and these statistics were used to construct a multivariant feature vector for each network. This feature vector set was then extended using the Hagen-Poiseuille equation, creating an ultimate training set of over 10,000 permeabilities and rock structures. The multivariant structural statistics were then regressed against permeability using open-source machine learning tools to create a predictive model, with predicted values varying over 10 orders of magnitude with a Root-MeanSquare-Fractional-Error (RMSFE) of $<4 \%$ in the test set. This predictive model was then validated using a composite set of real digital rock images (using both open source datasets from the Digital Rock Portal (www.digitalrocksportal.org), previously published data, and new nano-CT datasets of micritic carbonate microporosity) ranging in voxel size from $10 \mu \mathrm{m}$, to $32 \mathrm{~nm}$. Permeability was predicted on these data using the trained model, with predicted values ranging from $10 \mu \mathrm{D}$ to $10 \mathrm{D}$. These 
predictions were then compared with full-physics simulations with a RMSFE of $<25 \%$.

Such a technique offers significant advantages of traditional (full physics) simulations. It is significantly faster than traditional techniques, with a large volume prediction taking less than a minute on standard computational resources, where a full physics simulation might take many hours. Perhaps the most important potential this technique is that the features used for both regression and prediction can be extracted in $2 \mathrm{D}$ as well as 3D. This allows for effective property prediction from structural analysis of 2D data (e.g. light or electron microscopy), which can be acquired over much larger areas at high resolution (allowing a better characterization of heterogeneity), can be acquired faster, and offers qualitatively richer data (potentially including mineralogical or textural information not present when in 3D). This approach was validated using a correlated light, electron and (3D) nano-CT data. Future work will focus on the examination of the usage of such large area predictive statistical techniques to validate and inform upscaling techniques, allowing for integration of multiple scales of data in single predictive models.

Procter and Gamble Student poster award:

References:

1 Andrä H, Combaret N, Dvorkin J, Glatt E, Han J, Kabel M, et al. Digital rock physics benchmarks-Part I: Imaging and segmentation. Comput Geosci 2013;50:25-32. doi:10.1016/j.cageo.2012.09.005.

[2] Andrä H, Combaret N, Dvorkin J, Glatt E, Han J, Kabel M, et al. Digital rock physics benchmarkspart II: Computing effective properties. Comput Geosci 2013;50:33-43. doi:10.1016/j.cageo.2012.09.008. [3] Andrew M. Correction to: A quantified study of segmentation techniques on synthetic geological XRM and FIB-SEM images. Comput Geosci 2018;22:1513. Acceptance of Terms and Conditions:

Click here to agree

\section{Wettability in porous rocks, from macroscopical measurements to pore scale characterization}

Author(s): Prisca Andriamananjaona ${ }^{1}$

Co-author(s): Manuel Chamerois ${ }^{2}$; Peter Moonen ${ }^{3}$; Richard Rivenq ${ }^{2}$

${ }^{1}$ Université Pau \& Pays Adour, CNRS, E2S UPPA, Développement des Méthodologies Expérimentales - IPRA ; Université Pau I\& Pays Adour, CNRS, E2S UPPA, Laboratoire des Fluides Complexes et leurs Réservoirs - IPRA, Total E\&P

${ }^{2}$ Total E\&P, Pau, France

${ }^{3}$ Université Pau \& Pays Adour, CNRS, E2S UPPA, Développement des Méthodologies Expérimentales - IPRA ; Université Pau I\& Pays Adour, CNRS, E2S UPPA, Laboratoire des Fluides Complexes et leurs Réservoirs- IPRA, Pau, France

Corresponding Author(s): prisca.andriamananjaona@total.com

Wettability refers to the relative preference of a solid material for one fluid in a diphasic or multiphasic system. As this property greatly affects the distribution of fluids in the pore space, it has a critical influence on the oil recovery. Despite the great importance of wettability for the oil industry, its experimental characterization and its exact impact at the pore scale remains poorly known. The wettability of a reservoir rock is generally determined on centimeter-sized samples through recovered fluids volumetric measurements, and expressed by a parameter such as the Amott index. The current study aims at establishing a correlation between such macroscopic measurements and physical characteristics at the pore scale. We focus on a Bentheimer outcrop sandstone, saturated with brine and oil. Two twin samples with equal length but different diameter are subject to the classical Amott procedure: a sample of $36 \mathrm{~mm}$ in diameter enables to obtain the Amott index by volumetric measurement of the produced fluids, while a sample of around $5 \mathrm{~mm}$ in diameter enables direct observation of the fluids distribution inside the sample's pore space. This observation is done by acquiring tomographic scans with a typical resolution around $2 \mu \mathrm{m}$ to $3 \mu \mathrm{m}$. As both samples are subject to the same constraints, we impose the same capillary pressure, and should obtain the same saturation state at each phase of the test. For time considerations, and as we are mostly interested in Amott Indexes, we only record saturation states at the endpoints, i.e. after primary drainage, 
spontaneous imbibition, forced imbibition, spontaneous drainage, and forced drainage. When equilibrium is reached, we perform X-ray computed tomography on the small sample. The bulk part of this work focuses on the adaptation and validation of the Amott procedure on the small sample. We first applied this methodology to extremely water wet cases, i.e. Bentheimer "as received". The indexes calculated by means of recovered volume on the centimeter-scale and through saturation profile in the millimeter-scale sample proved to be consistent. Moreover, a good agreement was found between the observed behavior of the fluids in the rocks, the expectations from the literature, and the macroscopically measured Amott Index. Future work will focus on moderately water wet to oil wet cases.

Keywords: Wettability, X-Ray microcomputed tomography, Pore scale

\title{
Procter and Gamble Student poster award:
}

\section{References:}

E. Amott - Observations relating to the wettability of porous rock. Trans., AIME, Vol. 216, pp. 156-162, 1959.

L. Cuiec - Evaluation of reservoir wettability and its effects on oil recovery. Interfacial Phenomena in Oil Recovery, 319-375, 1991.

E. C. Donaldson, R. D. Thomas - Microscopic Observations of Oil Displacement in Water-Wet and OilWet Systems. SPE, New Orleans, 1971.

N. R. Morrow - Wettability and its effect on Oil recovery.

SPE, New Mexico 1990. Acceptance of Terms and Conditions:

Click here to agree

\section{Numerical simulation of low salinity waterflood on fractured chalk outcrop-based models}

\author{
Nikolai Andrianov ${ }^{1}$; H. M. Nick ${ }^{2}$ \\ ${ }^{1}$ Technical University of Denmark \\ ${ }^{2}$ Danish Hydrocarbon Research and Technology Centre, Denmark
}

Corresponding Author(s): nandria@dtu.dk

Waterflood optimization for mature North Sea fractured reservoirs requires more accurate fracture representations and improved abilities to model rock-fluid interaction in dynamic reservoir models, as compared to industry-standard approaches. We gain insights into waterflood performance by considering a 2D model of an outcrop at Lägerdorf quarry in northwest Germany, which reveals an extensive fracture network together with several major faults, see [Koestler and Rekstein 1995]. The model is populated with rock and fluid properties, representative for North Sea chalk reservoirs (see [Graue et al, 1999]).

We assess the waterflood efficiency using low-salinity water (LSW), which is an emerging IOR technique that aims at improving microscopic sweep efficiency by changing the reservoir wettability towards more water-wet state. We study the dependency of oil recovery factor with respect to water injection rate under uncertainty in fracture apertures and orientations, and compare the results to the case of conventional sea water (SW) flooding.

The domain is discretized using a Discrete Fracture Matrix (DFM) approach so that the fractures are represented as low-dimensional finite volumes, see [Gläser et al 2017]. Low salinity waterflood is modelled as a two-phase immiscible displacement, whereas the water phase is represented with two components - brine and low-salinity injection water. The phase relative permeabilities are then modified as a function of the salinity. This model was implemented in DuMuX, a free and opensource simulator for flow and transport processes in porous media, see [Flemisch et al 2011]. 
Key observations are the following:

- Oil recovery is slower if fractures are mostly aligned with waterflood direction as compared to the case of water injection in the perpendicular direction to major fractures orientation;

- Depending on the shape of relative permeabilities curves, SW may initially yield faster recovery than LSW; as oil saturation approaches the residual value, the trend reverses and LSW leads to improved recovery;

- For high permeability fractures, high injection rates lead to lower oil recovery with respect to water pore volumes injected; for low permeability fractures the flow tends to be matrix dominated and high injection rates lead to higher oil recovery;

- Even relatively poor connected fracture network can yield early water breakthrough;

- Large fractures apertures lead to better sweep efficiency due to more pronounced water imbibition effects as compared to the case of small fractures.

Procter and Gamble Student poster award:

\section{References:}

1. A. G. Koestler and K. Reksten, SPE 28728, 1995.

2. B. Flemisch et al. - Advances in Water Resources 2011; 34: 1102-1112.

3. D. Gläser et al. - Advances in Water Resources 2017; 110: 335-348.

\section{Acceptance of Terms and Conditions:}

Click here to agree

461

\section{Poroelastic coupling and rupture directivity in injection-induced earthquakes}

Sandro Andrés ${ }^{1}$; David Santillán ${ }^{1}$; Juan Carlos Mosquera ${ }^{1}$; Luis Cueto-Felgueroso ${ }^{1}$

${ }^{1}$ Universidad Politécnica de Madrid

Corresponding Author(s): sandro.andres.martinez@alumnos.upm.es

Anthropogenic earthquakes induced by pore pressure changes associated with the injection or extraction of fluids have recently become a major concern in subsurface energy technologies. When hydromechanical and frictional conditions lead to fault reactivation and unstable slip, rupture propagates across the fault with a pattern analogous to two crack tips spreading from the hypocenter. The final magnitude of the earthquake is in part determined by the symmetry of the rupture process: whether rupture fronts propagate with equal speeds in all directions or, in contrast, a preferred directionality emerges during rupture.

Here we study how poroelastic coupling controls rupture directivity in injection-induced earthquakes in rate-and-state faults embedded in poroelastic saturated media. Previous studies have shown that a contrast in material properties across the fault may explain some of the directivity biases observed in earthquakes ruptures. We show that pore pressure and stress changes induced by fluid injection prior to rupture, together with the undrained pressure response during coseismic slip, may lead to rupture asymmetries. Using fully coupled hydromechanical simulations, we observe that, depending on the fault stress state and flow conditions, rupture patterns range from almost-symmetric to almost-unilateral.

We characterize the rupture directivity bias in terms of the conditions of fault confinement, pressure evolution and fluid flow. We identify two mechanisms that govern the symmetry of the rupture. 
Firstly, there is an undrained effect due to coseismic fault slip that leads to an increase or decrease of pore pressure on either side of the fault, which is directly related to the initial confinement. Secondly, the pore pressure distribution prior to earthquake rupture, which depends on the injection protocol and on the distance between the injection well and the fault, controls the heterogeneity of fault strength along and across the fault.

Our results help to understand the poroelastic impact on rupture directivity in injection-induced seismicity, offering a feasible explanation of the predominance of unilateral rupture patterns in global catalogs of large earthquakes.

Procter and Gamble Student poster award:

References:

Acceptance of Terms and Conditions:

Click here to agree

\title{
Relating mechanical properties of biofilm-mineral composites to bulk porous media properties
}

\author{
Sobia Anjum ${ }^{1}$; Betsey Pitts ${ }^{\text {None }}$; Adrienne Phillips ${ }^{\text {None }}$; Robin Gerlach ${ }^{\text {None }}$ \\ ${ }^{1}$ Center for Biofilm Engineering, Montana State University
}

Corresponding Author(s): sobia.anjum@montana.edu

Biomineralization carried out by bacterial biofilms can lead to formation of conglomerates consisting of biofilm-produced minerals and the surrounding biological materials. Such biomineral conglomerates can be used for the consolidation of soils, improvement of wellbore cement integrity and to seal subsurface fractures. The composition, spatiotemporal distribution, and interaction of the biofilm and mineral components will affect the material and mechanical properties of biofilm-mineral composites, which will likely determine the mechanical properties of e.g. consolidated porous media. The study of structural and mechanical features of biofilm-mineral composites has been limited, and we are striving to relate the microscale properties of biofilm composites to macroscale properties such as bulk strength and stiffness of consolidated porous media. In this study, spatiotemporal distribution as well as cohesive and adhesive strength of biofilm-mineral composites produced by Sporosarcina pasteurii strain ATCC11859 and Escherichia coli strain MJK2 biofilms are being assessed. The structural features of and the mineral distribution in biofilm-mineral composites grown in Drip Flow Reactors (DFRs) are observed using Confocal Laser Scanning Microscopy (CLSM) and Field Emission Electron Microscopy (FE-SEM). The composition of the biofilm-mineral composites is analyzed as a ratio of organic to inorganic content using Thermogravimetric Analysis (TGA). The analyses show that the structure, composition and distribution of the minerals varies with the bacterial strain and the availability of calcium chloride and urea. Dynamic Mechanical Analysis (DMA) in compression and tension modes are being optimized to measure elasticity and ductility of samples while a Double Cantilever Beam (DCB) method is being optimized to measure the fracture strength of biofilm composites grown between two flat plates. We have demonstrated that the mechanical and structural properties of biofilm-mineral composites can be varied with operational parameters such as availability of calcium and urea, and the bacterial strain used. We are striving to develop relationships between biofilm structure, composition and mechanical properties (such as elasticity, ductility and toughness) to aid in the design of engineering applications in porous media and elsewhere.

\section{Procter and Gamble Student poster award:}

I don't want to compete References:

Acceptance of Terms and Conditions:

Click here to agree 


\section{Watching ink dry}

Author(s): riccardo antonelli ${ }^{\text {None }}$

Co-author(s): Joris Sprakel ; Jasper van der Gucht

Corresponding Author(s): riccardo.antonelli@wur.nl

Exploration of phenomenon as evaporation and imbibition for small droplets have great importance in many application, especially in the printing industry. Employing the techinque of Laser Speckle Imaging (LSI) it is possible to acheive the dynamic of te porous media beneath and in proximity of the drop. Pico-liter drops are ink-jetted on a pourus substrate: in this study we used different types of common printing paper, both coated and uncoated. After the deposition of the droplet a fast camera (20.000 fps) records the speckle pattern, which is analyzed by means of home-made MATLAB script. Experimental results will be compared with numerical simulation.

Procter and Gamble Student poster award:

References:

Acceptance of Terms and Conditions:

Click here to agree

\section{Anomalous Reaction-Diffusion under Quenched Heterogeneity}

Tomas Aquino $^{1}$; John Lapeyre ${ }^{1}$; Marco Dentz ${ }^{1}$

${ }^{1}$ IDAEA - CSIC

Corresponding Author(s): tomas.aquino@idaea.csic.es

It is well-known that reaction rates measured in the lab under well-mixed conditions significantly overpredict reaction when applied directly at the field scale. Effective reaction dynamics are a result of the interplay between fluctuating physical and chemical properties and their impact on transport and local reaction. They are impacted by mass transfer limitations, especially when transport is slow compared to the chemical kinetics at the scales of interest, and the resulting chemical rate laws may be fundamentally different from the classical mass-action laws. We consider diffusive solute transport under broadly distributed retention times, which are often at the root of anomalous transport and typically result from stagnation regions and low-permeability inclusions at the Darcy scale. We then study different chemical heterogeneity scenarios corresponding to the spatial reaction rate distribution for first order, irreversible decay kinetics. This type of kinetics may describe, for example, reaction in dilute solutions that do not lead to appreciable depletion of a mineral phase. In contrast to most available stochastic models, the disorder is taken to be quenched, i.e., the reaction rates and retention times result from local physical or chemical properties and are fixed at each spatial location. We derive the late-time behavior of the evolution of the total solute mass in transport-limited conditions. We find asymptotic power-law decay as a result of the heterogeneity, in sharp contrast to the classical exponential decay.

Procter and Gamble Student poster award:

References:

Acceptance of Terms and Conditions:

Click here to agree 


\title{
Experimental Investigations of Calcite Crystals Nucleation and Growth in 3D Printed Micromodels
}

\author{
Author(s): Mohamed Arab ${ }^{1}$ \\ Co-author(s): Julien Maes ${ }^{1}$; Shuo Zhang ${ }^{2}$ \\ ${ }^{1}$ Heriot-Watt University \\ ${ }^{2}$ Aramco Services Company \\ Corresponding Author(s): mohamed.m.arab@gmail.com
}

Understanding the diagenetic evolution of carbonate sediments and their pore system is essential to evaluate the potential of reservoirs for oil and gas recovery, thermal energy storage or CO2 trapping. Crystal nucleation and ion transport in the pore volume are competitive processes that occur during chemical diagenesis and greatly influence the pore sizes and shapes. Greater knowledge of the factors that influence the growth and dissolution rates as well as the diffusion and dispersion rates in the porous domain will lead to more accurate prediction of porosity and permeability in the forming rocks.

In this work, microfluidic experiments were conducted either at constant flowrate or pressures to observe diagenetic cementation in homogeneous and heterogeneous micromodels. The micromodels were printed using Formlabs Form 2 3D printer, which employs stereolithography (SLA) printing techniques. Clear resin was used in the printing to enable visualisation. The pore networks for the homogeneous and heterogeneous micromodels were obtained from the Digital Rocks Portal. Two incompatible brines were injected into the micromodels to precipitate calcium carbonate. Acid Red 18 and Acid Blue 9 dyes were used to: 1) track the mixing zones in the micromodel, and 2) control the rate of crystal growth due to their chemistry. The results of the experiment were modelled using OpenFOAM.

The results highlight how cementation occurs in an oil-free environment in a way that has not been illustrated before, and promise to pave the way to forming a more concrete understanding of the fundamental processes governing diagenetic cementation and the role of the oil once it has been introduced to the mix in future experiments. The experimental techniques are also unique in providing additional parameters for controlling and fine tuning the carbonate system by exploiting the chemistry of the dyes, thereby enabling more accurate experiments to be conducted.

Procter and Gamble Student poster award:

\section{References:}

Acceptance of Terms and Conditions:

Click here to agree

\section{Computational Tool Developed for Simulation of Nano-fluids Be- haviors}

\author{
Author(s): Rasoul Arabjamaloei ${ }^{1}$ \\ Co-author(s): Steven Bryant ${ }^{2}$; Milana Trifkovic ${ }^{3}$ \\ ${ }^{1}$ Post-Doctoral Associate, Department of Chemical and Petroleum Engineering, University of Calgary \\ ${ }^{2}$ Canada Excellence Research Chair in Materials Engineering for Unconventional Oil Reservoirs, University of Calgary \\ ${ }^{3}$ Associate Professor, Department of Chemical and Petroleum Engineering, University of Calgary
}


Corresponding Author(s): rasoul.arabjamaloei@ucalgary.ca

Solid nanoparticles (NPs) has been extensively used to produce novel soft materials such as Pickering emulsions and bicontinuous interfacially jammed emulsion gels (bijels). In this study, the contribution of NPs in hydrodynamics of multi-phase systems and formation of bicontinuous multiphase systems was studied. The free energy Lattice Boltzmann Method was used to solve the Cahn-Hilliard convection-diffusion and the Navier-Stokes equations in a two dimensional Cartesian domain. The NPs were added to the system as hard spheres and their spatial location was traced individually by Newton's laws of motion. A potential function was assumed to represent the chemical potential alteration of the multi-phase system due to the presence of NPs. Attractive and repulsive particleparticle interaction was entered into the model by a Morse potential function.

Two particle collision models were tested in this research. The first model was the classic elastic hard sphere collision model. In this method the particles move freely, collide with each other elastically and exchange momentum. Using this collision model, the particles could be packed to occupy the minimum space. A second collision model was developed where the particles collide with each other and form permanent bonds. This model represents the particles that form permanent bonds by van der Waals or electrostatic forces. Such bonds approximate interparticle stickiness that arises for some types of nanoparticles but does not lead to large-scale aggregation of the particles.

The two collision models were coupled to the multiphase simulator and were used to study the formation of the newly discovered bicontinuous intraphase jammed emulsion gels (bipjels). Bipjels are cocontinuous multiphase systems that are formed when spinodal decomposition is arrested by the solid nanoparticles that stay in one of the phases. The simulations showed that simultaneous spinodal decomposition and particle aggregation can arrest the phase separation and preserve cocontinuous gels when the new bonding collision model is used. In contrast, simulations with the elastic particle collision model always leads to formation of emulsions with only one continuous phase. The results also showed that spinodal decomposition and interface movement has a prominent effect on the collision process: particles are forced to form stretched structures instead of packed structures. The effect of particle size distribution (normal, bimodal, linear and constant) on the structure of the cocontinuous gel was observed to strongly affect bipjel characteristics such as cocontinuity extent, domain size and curvature. The fractal dimension of the particle aggregates was calculated and it was shown that it can be used as the main indicator for bipjel formation criteria. The particle structures formed in bipjels have lower fractal dimensions comparing to the ones formed in single-phase systems.

The developed simulation model can be used for optimal design of porous materials, such as aerogels, manufactured by aggregation of nanoparticles in fluid systems.

Procter and Gamble Student poster award:

References:

Acceptance of Terms and Conditions:

Click here to agree

\section{Foam flow and its blocking effect in high permeable saturated porous media}

Author(s): Romain Aranda ${ }^{1}$

Co-author(s): Hossein Davarzani ${ }^{2}$; Fabien Laurent ${ }^{3}$; Henri Bertin ${ }^{4}$

${ }^{1}$ Université de Bordeaux

${ }^{2}$ BRGM (French geological survey)

${ }^{3}$ Solvay

${ }^{4} \mathrm{I} 2 \mathrm{M}$

Corresponding Author(s): r.aranda@brgm.fr

Liquid foam has been studied as a blocking agent in porous media, especially for Enhanced Oil Recovery applications 1. Initially, the goal of foam injection consists to block high-permeability 
layers in the media in order to reach the oil in low-permeability layers. This approach can also be applied in the remediation field to allow the accessibility of low permeability layers that are usually more contaminated and difficult to treat [2] as reaching pollutants in low-permeability layers can also be difficult during remediation processes. However, contrary to the deep stratums in oil industry, upper soils are non-consolidated porous media with high permeability as well as low pressure and temperature operational conditions. These differences present specific challenges regarding stability and flow of the foam, as there is still little literature on these issues.

The main goal of this study is to isolate the pollution treatment zone downstream of the groundwater flow by diverting it upstream. This study aims to give a better understanding of foam flow in heterogeneous and high permeability (100-10 000 darcys) porous media, with competition of high velocity groundwater flow (10 m/day).

Two different experimental setups were used to obtain the results. The first one is composed of a column of sand or glass beads, instrumented with pressure sensors to monitor the pressure gradient during foam flow. Mass balance was also conducted to measure the water saturation in the column. The second one is composed of a thin $1 \mathrm{~m}$ large and $50 \mathrm{~cm}$ high tank to model a 2D flow. The tank is instrumented with pressure and water saturation sensors. In addition, an imaging technique is used to measure the evolution of foam volumes, streamlines and water saturations. During the experiments, foam is injected from the bottom of the tank and water is injected from left to right to model a groundwater flow. Finally, the experimental results are compared with numerical models in order to validate them. The validated model can be then used to simulate foam injection for field scale studies.

The surfactant has been chosen based on foamability and foam stability tests. Then, thanks to the column experiments, specific behaviors of foam in high permeable porous media was identified with two successive different regimes of foam flow ("weak" and "strong" foam) as well as necessary injection conditions for its generation. Finally, the influence of injection parameters on foam blocking properties, such as the "Resistance Factor", the residual water saturation, the radius of influence or the stability under a groundwater flow, has been investigated. The parameters studied are the injection method (gas and surfactant co-injection, Surfactant-Alternating-Foam (SAF), foam pre-generation), the flow rates, and the foam quality. The macroscopic experimental observations have been correlated to pore-scale phenomenon such as bubble generation and destruction, and viscous interactions between bubbles and pores or between bubbles themselves (several bubbles in one pore).

Procter and Gamble Student poster award:

I don't want to compete References:

1 R. Farajzadeh, A. Andrianov, and P. L. J. Zitha, Investigation of immiscible and miscible foam for enhancing oil recovery, Industrial \& Engineering chemistry research, 49.4, 1910-1919 (2009).

[2] G. J. Hirasaki et al., Surfactant/foam process for aquifer remediation, International symposium on oilfield chemistry, Society of Petroleum Engineers (1997). Acceptance of Terms and Conditions:

Click here to agree

\title{
Surface influence on the dynamics of molecules confined inside mesoporous xerogels: low field NMR investigations
}

\author{
Ioan Ardelean ${ }^{1}$; Calin Cadar $^{2}$ \\ ${ }^{1}$ Technical University of Cluj-Napoca, Romania \\ ${ }^{2}$ Technical University of Cluj-Napoca
}

Corresponding Author(s): ioan.ardelean@phys.utcluj.ro

Low field nuclear magnetic resonance (NMR) relaxometry and diffusometry techniques are widely used for the characterization of porous materials. NMR relaxometry techniques exploit the proportionality between the relaxation rate of the confined molecules and the surface to volume ratio of 
the investigated pores. The proportionality constant, also called relaxivity, is determined by the adsorption properties of molecules on the pore surface 1, the magnetic impurity content of the solid matrix [2] and the magnitude of the external magnetic field [1-3]. Consequently, the relaxation experiments provide access to the pore size distribution and the wettability of the confined molecules [1-3]. NMR diffusometry techniques [4] use pulse field gradients to encode and decode position of the investigated molecules and thus allow determination of the effective diffusion coefficients as a function of the diffusion time. This in turn provides information about the transport properties of the pore system.

In our contribution, low-field NMR relaxometry and diffusometry techniques are employed to extract information about the effects introduced by the interaction with the surface on the rotational and translational dynamics of molecules confined inside mesoporous carbon xerogels. The molecules under study were water, cyclohexane and hexane. They were chosen due to their different interaction strength with the carbonaceous matrix [3]. Frequency dependent longitudinal relaxation measurements, using the fast field cycling technique [5], allowed extraction of the fractal dimension for the carbon xerogel surface. It was observed that the measured value is influenced by the molecule affinity to the surface. Diffusion measurements, using the pulse field gradient technique, have revealed that the stronger interaction with the surface of cyclohexane and hexane molecules leads to an increased diffusive tortuosity, as compared with water.

\section{Procter and Gamble Student poster award:}

I don't want to compete References:

1 S. Stapf and R. Kimmich, J. Chem.Phys. 103, 2247 (1995).

[2] J.-P. Korb, Curr. Opin. Colloid Interface Sci. 14, 192(2009)

[3] C. Cadar, C. Cotet, L. Baia, L. Barbu-Tudoran, I. Ardelean, Microporous Mesoporous Mater. 251, 19(2017).

[4] I. Ardelean, R. Kimmich, Annu. Reports NMR Spectrosc. 49, 43(2003)

[5] R. Kimmich, E. Anoardo, Prog. Nucl. Magn. Reson. Spectrosc. 44, 257(2004) Acceptance of Terms and Conditions:

Click here to agree

\section{On the wettability of porous systems}

Ryan Armstrong $^{\text {None }}$; Chenhao Sun ${ }^{1}$; James McClure ${ }^{2}$; Anna Herring ${ }^{3}$; Peyman Mostaghimi ${ }^{\text {None }}$; Steffen Berg ${ }^{4}$

${ }^{1}$ University of New South Wales

${ }^{2}$ Virginia Tech

${ }^{3}$ Australian National University

${ }^{4}$ Shell Global Solutions International B.V.

Corresponding Author(s): ryan.armstrong@unsw.edu.au

The wetting condition of surfaces is one of the key parameters controlling their behaviour towards fluids. That holds in particular for porous systems subjected to immiscible fluids such as membranes, filters and natural porous media. So far there is no satisfactory definition of wettability for porous media. At the pore scale the concept of contact angle is used providing a consistent link with surface science and thermodynamics, but it fails to capture the chemical heterogeneity, e.g. for rock consisting of complex mineralogy with spatially varying wetting properties. At the Darcy scale wettability indices defined in an arbitrary way via capillary pressure-saturation relationships are used. They do capture the effective behaviour of spatially varying systems but are not unique and do not provide a consistent link to the pore-scale contact angles and their spatial distribution. In this work, we close this gap by describing the wetting state of porous systems in terms of the geometrical state of immiscible fluids that co-exist within the pore space. The concept is based on integral geometry principles where contact angle is related to deficit (integral) curvature caused by the intersect of fluids and solid. For geometry consisting of many pores it provides geometrical and topological constraints for the movement of the fluids. It describes the range of possible contact angles and interfacial curvatures that can exist even in the case of a chemically homogeneous systems. For homogeneous surface 
energies, the range of possible contact angles are narrow. However, for more heterogeneous distributions of surface energies, the range of contact angles are broader, which resulting in more degrees of freedom for possible dynamics. This demonstrates that geometric/topological description unifies the thermodynamic picture of the intrinsic contact angle and spatial variations in heterogeneous systems. The concept is universal and applicable beyond porous media.

Procter and Gamble Student poster award:

References:

Acceptance of Terms and Conditions:

Click here to agree

398

\title{
Probing small-scale connectivity for a high-permeability carbon- ate rock via two-phase tracer dispersion
}

\author{
Pierre M Adler ${ }^{1}$; Igor Shikhov ${ }^{2}$; Christoph Arns ${ }^{2}$ \\ ${ }^{1}$ Metis, Sorbonne Universite \\ ${ }^{2}$ The University of New South Wales \\ Corresponding Author(s): c.arns@unsw.edu.au
}

The contribution of micro-porosity in carbonates to hydrocarbon recovery is not easily quantified, yet is of considerable impact in the context of enhanced oil recovery. Of particular importance is the level of connectivity of fluids under mixed-wet conditions. While resistivity measurements can be utilized to characterize the connectivity of the water phase, the oil-phase is non-conducting. In this work we introduce a framework to test fluid-connectivity for single- and two-phase fluid distributions under water-wet conditions on a heterogeneous limestone sample, utilizing the much faster diffusion and higher solubility of oxygen in oil. The framework consists of a combination of micro-CT imaging, image-based computation, and NMR relaxometry to measure tortuosity in the long-time diffusion limit.

We consider a $25.4 \mathrm{~mm}$ diameter plug ( $50 \mathrm{~mm}$ length) of Mt Gambier limestone. The sample exhibits a measured permeability of 4.5 Darcy. Oxygen dissolved in water or oil affects NMR relaxation due to the effect of oxygen paramagnetic enhancement as compared to oxygen-free fluids. We initiate an oxygen-free initial fluid state by purging the fluids with nitrogen, then allowing oxygen to enter from one end of the cylindrical plug (inlet). The propagation of oxygen is then dynamically resolved using NMR 1D low field T2-weighted imaging at 2MHz.

The full plug is imaged via micro-CT at 11 micron resolution. We solve the concentration-diffusion equation with constant concentration boundary conditions of full oxygen saturated oil at the inlet of the cylindrical core and closed boundary conditions otherwise. Different discretisation levels (original 11um, 22um, 44um) and both single-phase and two-phase fluid distributions are considered. When the rock is only saturated with oil, we observe a strong dependence of the computed effective diffusion coefficient on discretisation. Counter-intuitively, resolution reduction increases the measured diffusion coefficient by a factor 2 for a $4 \mathrm{x}$ coarsening. For two-phase calculations we consider the solubility and exchange of oxygen between fluid phases by including a partition coefficient and notice strong fingering of oxygen through the well-connected oil phase, before water residing in small-scale porosity is also saturated.

\section{Procter and Gamble Student poster award:}

\section{References:}

1 I. Shikhov and C.H. Arns, Tortuosity prediction from oxygen diffusion via T2(z,t) relaxation NMR, Int. Symp. Soc. Core Analysts, SCA2016-063, Snowmass, USA, 21-26 August 2016, pp. 1-6.

[2] I. Shikhov and C.H. Arns, Application of tracers and low-field NMR relaxometry to characterisation of two-phase transport in sedimentary rocks, Advances in Water Resources, pre-print. 
[3] P.M. Adler, I. Shikhov, C.H. Arns, Tracer-dispersion account for coupling across fluid boundaries in carbonate rock at intermediate saturations, Transport in Porous Media, pre-print. Acceptance of Terms and Conditions:

Click here to agree

\section{A Multiphase Extension of Darcy's Law with Non-Local Support}

Saman Aryana ${ }^{1}$; Yuhang Wang ${ }^{1}$

${ }^{1}$ University of Wyoming

Corresponding Author(s): saryana@uwyo.edu

An accurate mathematical description of multiphase flow in porous media is essential in many practical applications, such as environmental remediation of the vadose zone and mitigation of greenhouse effects by geological storage of $\mathrm{CO} 2$ in saline aquifers and depleted petroleum reservoirs. Macroscale models, which are indispensable for simulating flow through porous media in large-scale systems, often suffer from lack of predictive capabilities due to flow instabilities. This work proposes a physicsbased, macroscale formulation of multiphase porous-media flows that honors the validity of Darcy's law in steady or near-steady flows. The new formulation recognizes the multiscale nature of various parameters that characterize the system and the nonlinear nature of these flows. Constitutive relations operate under length scales that are dynamic, may be extracted directly from experimental observations, and are different from the length scale associated with the static Representative Elementary Volume used to define variables such as porosity and saturation. Weighted local upscaling of instantaneous local saturation data over the appropriate dynamic length scale, informed by the direction of the propagation of information, will honor the fundamental premise underlying the formulation. The proposed formulation is validated by comparing highly accurate, numerical solutions against core-scale displacement experiments. The classical multiphase Darcy formulation represents a limiting case of the proposed formulation with the size of the dynamic length scale approaching the static ones used to define macroscopic properties such as porosity.

Procter and Gamble Student poster award:

References:

Acceptance of Terms and Conditions:

Click here to agree

193

\section{Evaluation of Interaction Between Corrosive Fluid and Crude Oil in Porous Media and its Side-Product Effect during Matrix Acidiz- ing}

Author(s): Hamidreza Asaadian ${ }^{1}$

Co-author(s): Bahram Soltani Soulgani ${ }^{1}$; Mohammad Zare ${ }^{1}$

${ }^{1}$ Petroleum University of Technology

Corresponding Author(s): hamidreza.asaadian@hotmail.com

Matrix acidizing is defined as injection of corrosive fluid into near wellbore porous area that its permeability is declined by induced damage, in order to dissolve the material presence and pore walls to create more efficient path for oil production 1 . Presence of immiscible crude oil with injected acid in pore space and interaction between them may result destructive side products which leads 
the operation to failure. Asphaltene molecules as one of the main oil sub-fractions are susceptible to interact with acid and create a third phase named as asphaltic sludge [2]. The formed phase alters the rock properties like permeability and wettability by settling on pore throats and rock surface. Volume of formed sludge and quantity of wettability alteration are directly depend on acid and asphaltene content percentages in medium [3]. In this work the influence of oil and acid properties on three types of rock (sandstone, fractured and vuggy carbonate rocks) is investigated. Then a model is suggested to determine the rock permeability and induced skin factor in case of sludge formation. Also a novel bio-surfactant is extracted from Zizyphus spina-christi shrub leaves, is used to control and inhibit changing in rock wettability and pore plugging.

Measured volume results indicate that sludge and emulsion volume is increased $113 \%$ by $28 \mathrm{wt} \% \mathrm{HCl}$ acid while this value was $7 \%$ for $15 \mathrm{wt} \% \mathrm{HCl}$ acid one average for all asphaltene concentration. It is noticeable that used bio-surfactant avoids any sludge formation on the interface between oil and acid. The increase in sludge concentration in media from $1 \mathrm{wt} \%$ to $2 \mathrm{wt} \%$ leads to an increase in contact angle and alter the surface wettability oil-wetted for every aged time and rock type indicating that more sludge structures are adsorbed on rock surface. The highest contact angle was observed for vuggy carbonate samples. Existence of large pore space in this rock type encourages sludge phase to settle in and alter the wettability. In case of bio-surfactant ZDCE utilization, no significant sludge phase is created on oil and acid interface. Also it was able to keep the rock wettability water-wetted and contact angle lower than 60 degree. Since ZSCE molecules compete with sludge structure for adsorption onto rock surface by their hydrophilic part.

\title{
Procter and Gamble Student poster award:
}

I would like to compete in the Procter and Gamble Student award References:

1 M. Karimi, M. M. Shirazi, and S. Ayatollahi, "Investigating the effects of rock and fluid properties in Iranian carbonate matrix acidizing during pre-flush stage,” J. Pet. Sci. Eng., vol. 166, pp. 121-130, 2018. [2] H. Asaadian, B. Soltani Soulgani, and A. Karimi, "An experimental study on electrical effect on asphaltene deposition,” Pet. Sci. Technol., vol. 35, no. 24, pp. 2255-2261, 2017.

[3] R. Morales and C. Diaz, "Microemulsion Additive to Mitigate Impacts of Acid Flow Back," in SPE Abu Dhabi International Petroleum Exhibition \& Conference, 2017. Acceptance of Terms and Conditions:

Click here to agree

\section{Utilization of Lattice Boltzmann Method to Study Disjoining Pres- sure in Porous Media}

\author{
Author(s): Mohammad bagher asadi ${ }^{\text {None }}$ \\ Co-author(s): Sohrab Zendehboudi ${ }^{1}$ \\ ${ }^{1}$ Faculty of Engineering and Applied Science, Memorial University
}

Corresponding Author(s): mbasadi@mun.ca

Wetting phenomena play a crucial role in applied surface science and molecular thermodynamics. In this study, the Lattice Boltzmann Method (LBM) is used to investigate the effect of disjoining pressure on the structure and stability of nano-droplets formed on a solid substrate. The pseudo-potential method is implemented to incorporate the interparticle potentials in the interface modeling. MultiRelaxation Time (MRT) collision operator is utilized to decrease the spurious current and to enhance the numerical stability at low values of viscosity. A new stability criterion is thus introduced and examined in terms of applicability and reliability. The interfacial region is then determined, and the smoothing splines method is applied to attain a continuous interfacial profile. Based on the profile, the adsorbed layer is determined, and the magnitude of the macroscopic contact angle is obtained. Through calculating the curvature of the droplet, the disjoining pressure is evaluated. Finally, the role of thin liquid film in the multiphase flow is modeled at the pore scale. According to the output of this study, the introduced model offers a better strategy to assess the microscale wettability at the multiphase pore scale, compared to conventional methods. 
References:

Acceptance of Terms and Conditions:

Click here to agree

689

\title{
Shear and Tensile Failure Modeling in the Context of Embedded Discrete Fractures
}

Amir Ashrafi Habibabadi ${ }^{1}$; Rajdeep Deb ${ }^{1}$; Patrick Jenny ${ }^{1}$

${ }^{1}$ Institute of Fluid Dynamics, ETH Zurich

Corresponding Author(s): ashrafi@mavt.ethz.ch

\begin{abstract}
Hydraulic fracturing is a widely applied strategy to enhance permeability in geological reservoirs, e.g. to create enhanced geothermal systems (EGS) or to increase the production rates of oil and gas reservoirs. Here we focus on EGS, where shear slip in preexisting natural fractures is triggered by high-pressure fluid. As a consequence of these shear failure events, new fractures are created adjacent to the tips of the preexisting ones due to tensile failure.

Thus, numerical studies of EGS require efficient modeling of the coupled solid and fluid mechanics including shear and tensile failure. We employ an embedded discrete fracture representation, where large fractures are described by discrete manifolds embedded in an elastic damaged matrix domain. To properly account for the displacement discontinuities due to irreversible failure of fractures, we employ a previously developed extended finite volume method (XFVM) 1. For each fracture segment, a discontinuity function is introduced; similar as in the extended finite element method (XFEM), but with less additional degrees of freedom.

Fluid flow is computed within both the damaged matrix and the fractures, while mass exchange is accounted for by modeled transfer rates. Failure and fracture propagation is based on the local mechanical stress situation and on the fracture fluid pressure. To reduce computational cost, resolving the timescale of individual slip events is avoided by directly solving for the final slip displacement This is achieved based on the requirement that the local shear force matches the local shear strength limit. New fracture segments are initiated once the principle stress at the crack tip exceeds the strength of the material, and the orientation of a new fracture segment is based on the principle stress directions.

The accuracy of the new model is demonstrated for various test cases involving tensile failure and fracture propagation. Especially if considering its low computational cost (compared to alternative models), the results are very promising.
\end{abstract}

Procter and Gamble Student poster award:

\section{References:}

1 Deb, Rajdeep, and Patrick Jenny. "Finite volume-based modeling of flow-induced shear failure along fracture manifolds." International Journal for Numerical and Analytical Methods in Geomechanics 41.18 (2017): 1922-1942. Acceptance of Terms and Conditions:

Click here to agree

\section{Extensions of poromechanical dual-continuum models for anisotropic elastic properties}

\author{
Author(s): Mark Ashworth ${ }^{\text {None }}$ \\ Co-author(s): Florian Doster
}

Corresponding Author(s): ma174@hw.ac.uk 
Fractured porous media can exhibit complicated multiscale, multiphysics behaviours due to their inherent heterogeneous nature. The focus of this work is on dual-continuum modelling of poromechanically coupled fracture systems at the field scale. Continuum modelling approaches are attractive for developing insight into first order behaviours, as well as having lower requirements on computational power and data compared to explicit modelling methods. The primary goal of this work is to introduce a poromechanical dual-continuum model that can account for anisotropic behaviours. Such a model is beneficial, as it is well known that fractures can be preferentially aligned within the subsurface due to mineralogy and stress history. The poromechanical dual-continuum models introduced in the literature to date have seldom incorporated anisotropy within their formulation. To derive this model we make use of approaches developed within the field of microporomechanics. Within this theoretical framework we are also able to derive explicit equations for intrinsic matrix and fracture strains. Having access to such quantities is desirable for developing our understanding of the processes occurring within these complex systems. Further, isolation of fracture strain could be useful when trying to incorporate non-linear stress dependent fracture permeabilities within the model. Finally, we compare both isotropic and anisotropic dual-continuum models to fine-scale numerical equivalents.

Procter and Gamble Student poster award:

I would like to compete in the Procter and Gamble Student award References:

Acceptance of Terms and Conditions:

Click here to agree

\section{Droplet movement into thin porous layer: an inkjet printing case study}

Hamed Aslannejad $^{1}$; S. Majid Hassanizadeh ${ }^{1}$; Sergey Loginov ${ }^{\text {None }}$

${ }^{1}$ Utrecht University

Corresponding Author(s): h.aslannejad@gmail.com

Continuum-scale modeling of fluid flow in Thin Porous Layers (TPL) are usually doubtful since they do not fulfill the enough REVs (Representative Elementary Volume) requirement along the cross section of layer. Therefore, pore-scale modeling approaches are needed in case of flow modeling into thin layers. In this work, pore-scale modeling of ink droplet movement into a thin $\mathrm{CaCO} 3$ layer was done using OpenFOAM CFD toolbox. The effect of contact angle, droplet impingement velocity, and droplet fluid properties impact on final distribution were studied. Later on, the results of the modelling work was validated comparing with imaging results. For the imaging, a novel combination of FIB-SEM and confocal laser microscopy were used. The results comparison showed a good match between modeling and imaging works. Therefore, the developed modeling tool can be used for further studies of droplet movement in thin porous industrial layers such as paper, diaper, and fuel cell GDL layers.

\section{Procter and Gamble Student poster award:}

I would like to compete in the Procter and Gamble Student award References:

Acceptance of Terms and Conditions:

Click here to agree

\section{Density Functional Theory for Fluid Adsorption on Rough Sur- faces}


Timur Aslyamov ${ }^{1}$; Aleksey Khluypin ${ }^{2}$; Vera Pletneva ${ }^{1}$

${ }^{1}$ Schlumberger Moscow Research Center

${ }^{2}$ Moscow Institute of Physics and Technology

Corresponding Author(s): t.aslyamov@gmail.com

Random Surface Density Functional Theory (RSDFT) allows to describe the fluid distribution near rough surfaces. The major difference of our theoretical approach from existing ones is stochastic model of solid surface which takes into account the correlation properties of geometry. Efficiency of RSDFT is demonstrated in calculation of argon and nitrogen low temperature adsorption on real heterogeneous surfaces (BP280 carbon black). These results are in good agreement with experimental data published in the literature. Also this approach is used as the part of the workflow allowing to analyse surface geometry from experiments with simple gases $\left(N_{2}\right.$, Ar) and then to predict adsorption properties of more complex fluids such as n-alkanes [2].

1 Aslyamov, T. and Khlyupin, A., 2017. Density functional theory formulation for fluid adsorption on correlated random surfaces. The Journal of chemical physics, 147(15), p.154703.

[2] Aslyamov, T., Pletneva, V. and Khlyupin, A., 2018. Random Surface Statistical Associating Fluid Theory: Adsorption of n-Alkanes on Rough Surface. arXiv preprint arXiv:1808.05876.

Procter and Gamble Student poster award:

\title{
References:
}

1 Aslyamov, T. and Khlyupin, A., 2017. Density functional theory formulation for fluid adsorption on correlated random surfaces. The Journal of chemical physics, 147(15), p.154703.

[2] Aslyamov, T., Pletneva, V. and Khlyupin, A., 2018. Random Surface Statistical Associating Fluid Theory: Adsorption of n-Alkanes on Rough Surface. arXiv preprint arXiv:1808.05876. Acceptance of Terms and Conditions:

Click here to agree

\section{Natural convection in coarse-grained porous media: pore-scale velocity and temperature measurements}

\author{
Iman Ataei Dadavi ${ }^{1}$; Chris R. Kleijn ${ }^{1}$; Mark J. Tummers ${ }^{2}$ \\ ${ }^{1}$ Transport Phenomena Section, Department of Chemical Engineering, Delft University of Technology \\ ${ }^{2}$ Fluid Mechanics Section, Department of Process and Energy, Delft University of Technology
}

Corresponding Author(s): i.ataeidadavi@tudelft.nl

Natural convection in porous media has been widely studied due to its many applications in steel and iron production, geothermal energy, chemical industries, oil production, and hydrogeology. However, these studies have been based almost exclusively on Darcy's model for porous media, assuming that the porous length scales are small compared to the flow and thermal length scales. This paper reports on an experimental study of natural convection in an enclosure filled with a packed bed of relatively large solid spheres, heated at the bottom and cooled at the top for Rayleigh numbers varying between 107 and 109. Global heat transfer measurements showed that at lower Rayleigh numbers, the heat transfer is lower than that for pure Rayleigh-Bénard convection, with the difference depending on packing type and size of the spheres. However, at high Rayleigh numbers, there exists an asymptotic regime where the heat transfer for all sphere sizes and packing types collapse on a single curve which is very close to the curve for pure Rayleigh-Bénard convection.

To better understand the physics of natural convection flow in coarse-grained porous media and enhance knowledge of the mechanisms influencing the heat transfer in the two regimes, velocity and temperature fields need to be visualized. Optical flow measurement techniques such as Particle Image Velocimetry (PIV) and Liquid Crystal Thermography (LCT) can be used in porous media, only if the refractive index of a (transparent) porous medium matches that of the liquid. Refractive index matching has been used in several studies to visualize fluid flow in porous media. However, the refractive indices of all frequently-used refractive index matched liquids are strongly temperature 
dependent. This adversely affects the refractive index matching and causes optical distortions in non-isothermal flows. In this study, water was chosen as the liquid since its refractive index has the lowest sensitivity to temperature variations among all other refractive index matched liquids. Polyacrylamide hydrogel spheres were used to match the refractive index of water.

Refractive index-matching of the fluid and the solid spheres enabled the use of PIV and LCT to obtain highly resolved velocity and temperature fields. Visualization of the velocity and temperature fields of the two heat transfer regimes showed that in the reduced heat transfer regime, the flow hardly penetrates into the pores, and it is mostly confined to a (thin) region along the periphery of the cell. However, in the asymptotic regime, since the thermal boundary layers become sufficiently thin, the flow penetrates considerably into the porous layer leading to much higher velocity magnitudes in the core region, a deeper penetration of hot and cold fluid up to the opposite solid boundaries, and consequently higher heat transfer across the porous medium. The obtained results confirm that the comparison between thermal length scales and porous length scales determine the flow structure and the corresponding heat transfer in natural convection in porous media.

Procter and Gamble Student poster award:

I would like to compete in the Procter and Gamble Student award References:

Acceptance of Terms and Conditions:

Click here to agree

\title{
Reactive transport simulations throughout the continuum "Un- saturated - Saturated Zones" of a complex multiphase system us- ing the O-ZNS platform
}

\author{
Mohamed Azaroual $^{1}$; Arnaud Isch ${ }^{2}$; Clara Jodry $^{2} ;$ Marion Klintzing $^{2}$; Carlos Aldana ${ }^{2}$
}

\author{
${ }^{1}$ ISTO - BRGM \\ ${ }^{2}$ ISTO
}

Corresponding Author(s): m.azaroual@brgm.fr

The PIVOTS (Environmental Technology Innovation, Development and Optimization Platforms) program is a coordinated set of experimental and analytical platforms focused on environmental quality monitoring and sustainable management of natural resources (soil, subsurface, surface water, groundwater, sediment and air) within a context of global change. The observatory (O-ZNS platform), a part of this family, aimed to understand the water-rock-biosphere interactions and the mass (water and contaminants) and heat transfers in the unsaturated zone (UZ). This observatory, developed around an exceptional well dimensions (depth - $20 \mathrm{~m} \&$ dimeter - 4m), offers a unique support for deciphering, at the relevant scales, coupled phenomena in the environmental geoscience. This project goal is the understanding of the vadose dynamics, from the ground to the groundwater table, throughout the entire vadose zone. The long-term objective is to identify the key processes promoted by the anthropogenic pressure along the soil-aquifer continuum, founding stone of a new generation of predictive modeling tools efficient for policy makers and industrials decision.

The design of the well associated with laboratory experiments and numerical models allows the development/adaptation of innovative geophysical and physico-chemical sensors for quantitative water transfers in UZ, redox barriers, capillary fringe, in a porous/fissured carbonated structure. The research field aimed identifying the level of coupling between the interfacial properties of phases, and the electrical/electrokinetic/electrochemical properties associated with moving fluids and biogeochemical reactions. Interestingly, an important amount of knowledge's have been shared over the last 15 years bringing together electrokinetic characterizations (redox potential, electron activity, chargeability, etc.) and geophysical techniques including mainly Spontaneous Potential (SP), Spectral Induced Polarization (SIP), etc. A field observatory, associating geochemical and geophysical processes, thanks to the O_ZNS observatory, gathering geophysicists, geochemists, microbiologists, and numerical modelers is the action asset of the project.

The preliminary studies carried out with the aim of characterizing the initial state of the O-ZNS site in Villamblain (35 km north-west of Orléans, Centre Val de Loire, France) through three core drill holes allowed the collection of soil, sediment and rock samples every meter over the entire $20 \mathrm{~m}$ 
depth of the vadose zone. The geophysical measurements, made from the surface as well as in boreholes, consisted in particular of the realization of wall imaging, logging (gamma-ray, gamma-gamma, neutron) and tomography (electrical, seismic, radar). All of these prospections made it possible to obtain information on the lithology as well as on the presence of clay levels, the density, the porosity and the resistivity of the vadose zone. The laboratory tests carried out on selected samples, made it possible to obtain information on the physical, petrophysical and mechanical properties of the samples. The hydric properties (water retention and saturated or unsaturated hydraulic conductivity) of the vadose zone materials were also determined.

During its exploitation (planned for several decades), the project will allows acquiring original and unique data on the mass (water, solutes, organic and inorganic contaminants, gases, etc.) and heat (induced by gradients temperature, microbiological reactions, etc.) transfers across the continuum "Soil-UZ-Capillary Fringe - Aquifer" for environmental issues and georesources needs (water, energy, etc.).

Procter and Gamble Student poster award:

References:

Acceptance of Terms and Conditions:

Click here to agree

\title{
Preparation of porous and magnetic carbon from waste cellulosic cloth by microwave irradiation, and its application as an adsor- bent for dye removal from water
}

\author{
Saeid Azizian ${ }^{1}$; Mohamad Reza Khodadadian ${ }^{2}$ \\ ${ }^{1}$ Department of Chemistry, Bu-Ali Sina University \\ ${ }^{2}$ Department of Physical Chemistry, Bu-Ali Sina University
}

Corresponding Author(s): sdazizian@yahoo.com

Preparation of porous and magnetic carbon from waste cellulosic cloth by microwave irradiation, and its application as an adsorbent for dye removal from water

Saeid Azizian*, Mohamad Reza Khodadadian

Department of Physical Chemistry, Faculty of Chemistry, Bu-Ali Sina University, Hamedan, Iran ${ }^{*}$ E-mail address: sazizian@basu.ac.ir

Magnetic, porous and low cost carbon is a promising adsorbent for pollutant removal from water $[1,2]$. In the present work, waste cellulosic cloth was used as the raw material to prepare porous and magnetic carbon by microwave irradiation, as a source of energy. To optimize the synthesis of nanostructured graphitic carbon, the effects of different parameters on the removal efficiency, such as the power of microwave radiation, the duration of microwave irradiation and the exposure time of cellulosic cloth to the pyrrole vapor were investigated. The prepared sample was characterized by different techniques such as scanning electron microscopy (SEM), energy dispersive X-ray spectroscopy (EDX), elemental analysis map, transmission electron microscopy (TEM), X-ray diffraction (XRD), thermo gravimetric analysis (TGA) and N2 adsorption/desorption methods.

Also adsorption of methyl violet was performed from both equilibrium and kinetics point of views. The effect of various parameters (such as adsorbent mass, temperature and $\mathrm{pH}$ ) on the removal efficiency of the prepared graphitic carbon was studied for removal of methyl violet from water.

TEM and SEM images showed that the prepared carbon has graphitic structure with iron oxide particles, deposited on its surface. Based on BET analysis, the synthesized carbon has a high surface area around $452 \mathrm{~m} 2 / \mathrm{g}$. Total pore volume of the prepared sample is $0.27 \mathrm{~cm} 3 / \mathrm{g}$ with pore size distribution within the range of 1 to $100 \mathrm{~nm}$ and average value of $2.4 \mathrm{~nm}$.

The obtained results from adsorption experiments were fitted with appropriate isotherm equations and also kinetics models. By regarding the obtained results, surprisingly, the prepared porous and 
magnetic carbon adsorbs most of the dye molecules from water within few minutes.

The most important advantages of the prepared magnetic carbon are easy separation from the solution (just by using a magnet), low cost, noticeable adsorption capacity and high rate of adsorption.

References

1 C. Wang., D. Ma, X. Bao, Journal of Physical Chemistry C 112 (2008) 17596.

[2] M. Khoshnood, S. Azizian, Journal of Industrial and Engineering Chemistry 18 (2012) 1796.

\title{
Procter and Gamble Student poster award:
}

\section{References:}

1 C. Wang., D. Ma, X. Bao, Journal of Physical Chemistry C 112 (2008) 17596.

[2] M. Khoshnood, S. Azizian, Journal of Industrial and Engineering Chemistry 18 (2012) 1796. Acceptance of Terms and Conditions:

Click here to agree

\section{Solute mixing in the hyporheic region}

\author{
Elisa Baioni ${ }^{1}$; Giovanni Porta ${ }^{2}$; Alberto Guadagnini ${ }^{2}$; Mohaddeseh Mousavi Nezhad $^{\text {None }}$ \\ ${ }^{1}$ University of Warwick \\ ${ }^{2}$ Politecnico di Milano
}

Corresponding Author(s): e.baioni@warwick.ac.uk

We investigate solute mixing in a porous medium in the presence of turbulent flow conditions. Our setup is representative of mixing processes taking place within the hyporheic zone and considers transport of dissolved chemicals close to the interface between a free fluid system and a porous medium. Turbulence is assumed at the boundary between the free fluid and the porous column in order to simulate the effect of the river flow. Interaction and mixing between surface water and groundwater takes place in such a critical area, where key processes can contribute to decreasing contamination in surface waters. In general, mixing smooths out non-uniformities in fluid systems, such as gradients of concentration. Quantification of mixing is particularly critical for the assessment of reactive processes taking place in the porous domain.

Our study is grounded on the use of an appropriate fit-to-purpose Lagrangian mixing model. Resorting to the latter enables us to predict the temporal evolution chemical concentration in the hyporheic zone, as driven by mixing. We benchmark our theoretical and numerical results against recently published experimental results (Chandler et al., 2016), quantifying the vertical variation of the effective dispersion coefficient with depth below the sediment-water interface. The available data display an exponential decrease of the dispersion coefficient with depth. Our study is keyed at providing a proof-of-concept by assessing the ability of our modeling strategy to reproduce solute breakthrough curves observed at various depths as well as the documented variation of the diffusion coefficient. Our results will provide additional information on solute mixing and its temporal evolution and will be relevant to the parameterization of reactive transport processes in the hyporheic zone.

Keywords: solute transport, mixing model, hyporheic zone, porous media

\section{Procter and Gamble Student poster award:}

I would like to compete in the Procter and Gamble Student award References:

Chandler, I. D., I. Guymer, J. M. Pearson,and R. van Egmond. (2016). Vertical variation of the mixing within porous sediment beds below turbulent flows. Water Resour. Res., 52, 3493-3509,doi:10.1002/2015WR018274

Hester, E. T., M. B. Cardenas, R. Haggerty, and S. V. Apte. (2017). The importance and challenge of hyporheic mixing. Water Resour. Res, 53, 3565-3575, doi:10.1002/2016WR020005. Acceptance of Terms and Conditions: 
Click here to agree

\title{
From 3D Images to Adsorption and Deformation in Porous Me- dia
}

Sahar Bakhshian ${ }^{1}$; Seyyed Abolfazl Hosseini ${ }^{1}$

${ }^{1}$ Bureau of Economic Geology, University of Texas at Austin

Corresponding Author(s): sahar.bakhshian@beg.utexas.edu, seyyed.hosseini@beg.utexas.edu

\begin{abstract}
We have established a framework for fluid adsorption and the deformation that it induces in a porous medium. The model establishes a fundamental link between the thermodynamics of adsorption and the mechanical response of the porous media. This is based on the total energy (Hamiltonian) of the system that takes into account the interaction between the solid matrix of a porous medium and the adsorbing fluid, as well as the elastic energy stored in the material, and the interaction energy between the adsorptive fluids themselves. Finite-element computations are applied in order to compute fluid adsorption isotherms along with the induced strain in the medium. The simulations are performed directly on realistic 3D image of the porous samples, therefore, no assumption is made regarding the shapes and sizes of the pore bodies and pore throats of the pore space.

To show the versatility and capability of the proposed framework in predicting the adsorptioninduced deformation in porous materials, we applied the model for gas adsorption in metal-organic frameworks (MOFs), geological formations such as clay materials and sandstone reservoirs.
\end{abstract}

\section{Procter and Gamble Student poster award:}

I would like to compete in the Procter and Gamble Student award References:

Acceptance of Terms and Conditions:

Click here to agree

\section{Pore-Scale Simulation of Wettability Effects on CO2 Storage Effi- ciency in Deep Saline Aquifers}

\author{
Sahar Bakhshian ${ }^{1}$; Seyyed Abolfazl Hosseini ${ }^{1}$; Nima Shokri ${ }^{2}$ \\ ${ }^{1}$ Bureau of Economic Geology, University of Texas at Austin \\ ${ }^{2}$ University of Manchester
}

Corresponding Author(s): sahar.bakhshian@beg.utexas.edu, seyyed.hosseini@beg.utexas.edu, nima.shokri@manchester.ac.uk

Wetting properties of reservoir rocks and caprocks can vary remarkably, and these variations have a significant effect on the performance of $\mathrm{CO} 2$ sequestration in deep saline aquifers. Understanding the effect of wettability on pore-scale fluid displacement is crucial for prediction of large-scale flow models and improving storage efficiency and security. The objective of this study is to investigate the effect of wettability on pore-scale characteristics of $\mathrm{CO} 2$ displacement dynamics and its capillary trapping mechanism during $\mathrm{CO} 2$ /brine drainage and imbibition cycles. A multiphase lattice Boltzmann (LB) model is adopted to simulate $\mathrm{CO}$ /brine two-phase flow in rock samples, by employing three-dimensional micro-CT images of Tuscaloosa sandstone taken from the Cranfield $\mathrm{CO} 2$ injection site. The LB model that has been applied in this study is an extended Colour-Gradient approach with improved numerical stability and can handle multiphase flow simulations with low capillary number and high mobility ratio. To improve the computational efficiency of the LB simulations, the 
model has been applied to a parallel scheme written in $\mathrm{C}++$ using the Message Passing Interface (MPI).

Various sets of $\mathrm{CO} 2 /$ brine drainage and imbibition simulations have been performed in rock samples with both uniform and non-uniform (fractional) wettability conditions and the effect of wettability on $\mathrm{CO} 2$ migration patterns, its capillary trapping and storage efficiency has been investigated. To gain a better insight into the effect of wettability on the $\mathrm{CO} 2$ displacement patterns during drainage, the evolution of $\mathrm{CO} 2$ interface with brine and rock surface is quantified by calculating $\mathrm{CO} 2$ interfacial area for rock samples with various wettability preferences. The results show that the wettability controls $\mathrm{CO} 2$ entrapment pattern and spatial distribution of residual $\mathrm{CO} 2$ clusters during brine flooding. For fractional-wet conditions, the fraction of $\mathrm{CO} 2$-wet regions plays an important role in $\mathrm{CO} 2$ sweep efficiency and its capillary trapping.

Procter and Gamble Student poster award:

I would like to compete in the Procter and Gamble Student award References:

Acceptance of Terms and Conditions:

Click here to agree

\title{
CFD modelling of Wettability and Temperature effect on Oil Re- covery at Pore-Scale level.
}

Yakubu Balogun $^{1}$; ${\text { Draco } \text { Iyi }^{1} \text {; Faisal Nadimul }}^{1}$; Babs Oyeneyin ${ }^{2}$

\author{
${ }^{1}$ Robert Gordon University, Aberdeen
}

${ }^{2}$ Robert Gordon University

Corresponding Author(s): y.balogun@rgu.ac.uk

The effect of wettability on waterflood oil recovery in a reservoir of varying temperature using porescale structure was investigated numerically. Three different cases of the two-dimensional geometry comprising varying sizes of pore spaces and interconnected pore-throats was used for the study. The Volume of Fluid approach in ANSYS-FLUENT ${ }^{\circledR}$ was used to simulate the two-phase flow and heat transfer physics. The configuration involves the injection of relatively cold water into a reservoir of varying temperature under different wettability condition in order to study the flow behaviour, the wettability effect and temperature on the oil recovery processes. Also, the wettability contact angle for each of the three different cases of the two-dimensional geometry was simulated for water-wet, oil-wet and intermediate-wet.

The result showed that in the oil-wet system the displacement behaviour was affected by the contact angle and then a significant effect on the oil recovery factor. In the water-wet system with the water wetting the matrix wall and the oil phase surrounded by water, more oil was relatively displaced from the domain thereby improving the oil recovery factor. The water-wet system resulted in about 25$35 \%$ increase in oil recovery than with the oil-wet system, with the unrecovered oil mainly adhering to the wall region of the pore bodies for the oil-wet system. For the intermediate wet system, initial fluid distribution is seen to have a more significant effect on the displacement behaviour than the contact angle in the study.

The general observation is that by altering the wettability from oil-wet to water-wet condition, the oil recovery rate can be improved. The results from this study are consistent with reported experimental and numerical studies in literature and offer a better understanding of the phenomenon which is critical for other recovery mechanisms such as surfactant and polymer flooding process.

\section{Procter and Gamble Student poster award:}

I would like to compete in the Procter and Gamble Student award References:

Acceptance of Terms and Conditions:

Click here to agree 


\title{
Modelling Liquid Transport on Gas Diffusion Layers with Het- erogenous Surfaces in PEM Fuel Cells
}

\author{
Author(s): Yanyao Bao ${ }^{1}$ \\ Co-author(s): Yixiang Gan ${ }^{1}$ \\ ${ }^{1}$ The University of Sydney \\ Corresponding Author(s): yanyao.bao@sydney.edu.au
}

\begin{abstract}
Gas diffusion layer (GDL) is a critical component in proton exchange membrane (PEM) fuel cells for diffusion of reactants and removal of by-product water in the pore space. Numerical simulations with volume of fluid (VOF) method are applied to investigate the dynamic liquid transport process in a micro gas channel in fuel cells. The liquid is injected from a single inlet in GDLs with different surface roughness, generated by gaussian height distribution function and correlation function, comparable to commercial carbon paper GDLs. The surfaces are characterised by root mean square height of surfaces and roughness wavelengths. The simulation results show that surface roughness, liquid injection rate, and air flow rate can influence the transport of water droplets. The increase of surface roughness generally assists the transport of the droplet inside the gas channel. The droplet radius at the time of detachment is dominated by air flow rate, and two types of movement mechanism, i.e., rolling and sliding, are identified for droplets with different volume. In addition, the volume of remaining liquid water on GDL surface and pressure drop in the gas channel are investigated under different conditions. The amount of remaining liquid water decreases as GDL surface roughness increases, and the pressure drop increases when the GDL has a rougher surface. Furthermore, an analytical model is proposed to predict the detachment and movement of the droplet by considering the detachment and retentive force of the droplet in the channel, and the solutions are compared with simulation results.
\end{abstract}

Procter and Gamble Student poster award:

I would like to compete in the Procter and Gamble Student award References:

Acceptance of Terms and Conditions:

Click here to agree

\section{Gas Permeability in Micro/Nano-Porous Media: An extended Kozeny- Carman-Klinkenberg Model}

\author{
Author(s): Murat Barisik ${ }^{1}$ \\ Co-author(s): Safa Sabet ${ }^{2}$; Ali Beskok ${ }^{3}$ \\ ${ }^{1}$ Assistant Professor \\ ${ }^{2}$ Izmir Institute of Technology, Department of Mechanical Engineering, \\ ${ }^{3}$ Southern Methodist University, Lyle School of Engineering,
}

Corresponding Author(s): muratbarisik@iyte.edu.tr

Gas transport in micro/nano-pores deviates from classical continuum calculations due to non-equilibrium in gas dynamics. In such a case, transport can be classified by Knudsen number (Kn) as the ratio of gas mean free path and characteristic flow diameter. The well-known Klinkenberg correction and its successors estimate deviation from existing permeability values as a function of Kn through a vast number of modeling attempts. However, the non-equilibrium in a porous system cannot be simply modeled using the classical definition of Kn number calculated from the Darcy's definition of pore size or hydraulic dimeter. Instead, a proper flow dimension should consider pore connectivity in order to characterize the rarefaction level. This study performs a wide range of pore-level 
analysis for gas dynamics with different porosities, pore sizes, and pore throat sizes at different Kn values in slip flow regime. First, intrinsic permeability values were calculated without any rarefaction effect and an extended Kozeny-Carman model was developed by formulating Kozeny-Carman constant by porosity and pore to throat size ratio. Permeability increased by increasing porosity and decreasing pore to throat size ratio. Next, velocity slip was applied on pore surfaces to calculate apparent permeability values. Permeability increased by increasing $\mathrm{Kn}$ at different rates depending on the pore parameters. While the characterization by Kn value calculated with pore height or hydraulic diameter did not display unified behavior, relating permeability values with the Kn number calculated from the equivalent height definition created a general characterization based on porosity independent from pore to throat size ratio. Next, we extended the Klinkenberg equation by calculating unknown Klinkenberg coefficients which were found as a simple first order function of porosity regardless of the corresponding pore connectivity. The extended model as a combination of Kozeny-Carman for intrinsic permeability and Klinkenberg for apparent permeability correction yield successful results.

Procter and Gamble Student poster award:

References:

Acceptance of Terms and Conditions:

Click here to agree

\title{
Surface electric charge inside mesoporous silica at different pore sizes and porosities
}

\author{
Author(s): Murat Barisik ${ }^{1}$ \\ Co-author(s): Tumcan Sen ${ }^{2}$ \\ ${ }^{1}$ Assistant Professor \\ ${ }^{2}$ Izmir Institute of Technology, Department of Mechanical Engineering,
}

Corresponding Author(s): muratbarisik@iyte.edu.tr

Mesoporous silica is an emerging technology to solve existing problems and support projected revolutionary applications ranging from targeted drug delivery to artificial kidney. However, one of the major driving mechanisms, electric charging of internal mesoporous surfaces, has not been characterized yet. In the nanoscale confinements of mesoporous structures made of pore throats and pore voids, surface charges diverge from existing theoretical calculations and show local variation due to two occurrences. First, when the size of pore throat becomes comparable with the thickness of ionic layering forming on throats' surfaces, ionic layers from opposite surfaces overlap so that ionic concentration on the surface becomes different than Boltzmann distribution predicts, and there will no longer be an equilibrium of zero electric potential at pore throat centers. Second, when this non zero potential inside throats becomes different than the potential of pore voids, ionic diffusion from void to throat creates axial ionic variation on surfaces. For such a case, we performed a pore level analysis on mesoporous internal surface charge at various porosities and ionic conditions. Pore parameters strongly affected the average internal charge which we characterized as a function of overlap ratio and porosity, first time in literature. Using this, a phenomenological model was developed as an extension of the existing theory to include nano-effects, to predict the average mesoporous internal surface charge as a function of EDL thickness, pore size and porosity.

Procter and Gamble Student poster award:

References:

Acceptance of Terms and Conditions:

Click here to agree 


\title{
An electrochemical impedance spectrocopy technique for deter- mining ionic resistance of ion-exchange membranes
}

\author{
V. María Barragán ${ }^{1}$; Pedro Antoranz ${ }^{1}$; María Amparo Izquierdo-Gil ${ }^{1}$; Sagrario Muñoz ${ }^{1}$ \\ ${ }^{1}$ Complutense University of Madrid
}

Corresponding Author(s): vmabarra@ucm.es

\begin{abstract}
Ion-exchange membranes (IEMs) are used in many applications in different fields such as sustainable energy generation or separation techniques. The resistance to ion transport in the membrane is a key parameter that contributes with a major portion of the overall internal resistance of the system, strongly affecting the performance of the IEM-based process. The membrane resistance is affected by the concentration and composition of solutions near the solution membrane interface and the development of techniques for separating the different contributions is necessary to improve the understanding of the relationship between electrolyte and IEM resistance. We present an electrochemical impedance spectroscopy technique applied to a modified coaxial line enclosing, the membrane under investigation, to be characterized in the frequency range from $100 \mathrm{kHz}$ to $100 \mathrm{MHz}$ The text fixture consists of a brass coaxial air-filled transmission line of length $67 \mathrm{~mm}$ and radii for inner and outer conductors of $1.75 \mathrm{~mm}$ and $4 \mathrm{~mm}$ respectively. The cell consisted of two separate compartments filled with the solution and in direct contact with the membrane holder. The inner conductor of the coaxial line is fixed at one end whereas it can be dismantled at the opposite end so that the system can be easily filled with the different solutions. The main advantage of this proposed setup is the relatively small membrane area required. To validate the performance of the system, we have used deionized water as a test standard of well-known conductivity value. The final value of the resistance of the different ion-exchange membranes was determined by means of an iterative process so that the simulated impedance spectra (real and imaginary parts) of the equivalent circuit matched the experimental one. The analysis of the experimental data allows the determination of the different contributions to the overall electrical resistance, membrane and solution contributions. Financial support from Banco de Santander and Universidad Complutense de Madrid under Projects PR26/16-20296 and PR75/18-21589 are gratefully acknowledged.
\end{abstract}

Procter and Gamble Student poster award:

References:

Acceptance of Terms and Conditions:

Click here to agree

\section{A method for determining through-plane thermal conductivity of polymeric membranes}

Sagrario Muñoz ${ }^{1}$; María Amparo Izquierdo-Gil ${ }^{1}$; Pedro Antoranz ${ }^{1}$; V. María Barragán ${ }^{1}$

${ }^{1}$ Complutense University of Madrid

Corresponding Author(s): vmabarra@ucm.es

Thermal conductivity of polymeric membranes is a critical property for thermal management in membrane-based applications. However, scarce information is available in the literature about this property in ion-exchange membranes. An experimental investigation has been performed to measure the through-plane thermal conductivity of polymeric membranes. A simple experimental apparatus based on the steady state method was designed consisting on two cylindrical copper setups with the test sample placed between them, with or without membrane holder. A Pt-100 sensor array inserted in the cylinders permitted to measure the temperature profile long them at both sides of the test sample. This experimental temperature profile together a numerical simulation permit to determine the through-plane thermal conductivity of the test sample. The numerical simulation was 
performed using Comsol (Comsol Multiphysics ${ }^{\circledR}$, Los Angeles, CA). A simple cylindrical setup with no isolation, was designed to validate the experimental setup. Thus, the thermal properties of the solid materials used in the experimental model were characterized. This process leads to have, as the only unknown variable for the simulation, the membrane thermal conductivity. Different tests were carried out to verify the known thermal properties of each one of the different materials: Copper, cork and the pla filament used in the $3 \mathrm{D}$ printer to design the membrane holder. We used an iterative process to derive the thermal conductivity of the membrane sample fitting the numerical temperature profile to the measured one. To test the applicability of the method in the determination of the thermal conductivity of polymer membranes, it was applied to determine the thermal conductivity of a Nafion membrane. Values according to the values found in the literature for this same membrane were found.

Financial support from Banco de Santander and Universidad Complutense de Madrid under Projects PR26/16-20296 and PR75/18-21589 are gratefully acknowledged.

Procter and Gamble Student poster award:

References:

Acceptance of Terms and Conditions:

Click here to agree

\title{
Pore Network Modeling for Topological Characterization of Porous Media
}

\author{
Author(s): Mariane Barsi-Andreeta ${ }^{1}$ \\ Co-author(s): Everton Lucas-Oliveira ${ }^{1}$; Arthur Gustavo de Araujo Ferreira ${ }^{1}$; Tito José Bonagamba ${ }^{1}$ \\ ${ }^{1}$ Instituto de Física de São Carlos, Universidade de São Paulo
}

Corresponding Author(s): mariane.andreeta@usp.br

The advances in micro-CT imaging and other techniques have improved our knowledge of the internal structure of porous materials. Currently, most of the efforts in digital porous media and pore network extraction are focused in using the digital data as a tool for estimation of parameters such as permeability and capillary pressure curves of the rock cores. We show in this work that porenetwork extraction can be used for analyzing the morphology and topology of the internal pore structure as well. The main impediment to the application of the pore-network parameters on the topology characterization of different samples is that different algorithms of pore-network extraction can lead to different networks describing the same sample. The purpose of this work is to present a method that can overcome this issue through a simple algorithm that unifies the Max Ball and medial axis approaches. The network extraction method maps the pore space into a network of spheres of different sizes and interconnected based on the Maximum Spheres Algorithm (MS). The final mapping procedure is an accurate method to separate the medium into pores and throats, and an efficient procedure to extract a medial axis network that can map structures such as fractures and channels. The recovering of the pore's local geometry is done by applying graph theory analysis of the centrality of the nodes and efficiency of its connections, instead of a geometrical definition. This way the pore-space separation is governed by the sample's intrinsic morphology. These networks can provide important data to better understand the morphology and topology of the samples. This method was successfully applied to samples of sandstones, carbonates, and to characterize the morphology of processes of acidification.

Procter and Gamble Student poster award:

I don't want to compete References:

Acceptance of Terms and Conditions:

Click here to agree 
701

\title{
Pore-scale simulation of interphase mass transfer across fluids interfaces using the Phase-field method
}

\author{
Farzad Basirat $^{1}$; Auli Niemi ${ }^{2}$ \\ ${ }^{1}$ Department of Earth Sciences, Uppsala University \\ ${ }^{2}$ Uppsala University \\ Corresponding Author(s): farzad.basirat@geo.uu.se
}

We present a micro-continuum approach to simulate interfacial mass transfer across two fluids interface. We used the Continuous Species Transfer method (Haroun et al., 2010) for interfacial mass transfer along with the Phase-Field method (Yue et al., 2006) as the interface-capturing technique for the two-phase flow system. The main objective is to investigate the multicomponent multiphase flow at the pore level during geological storage of $\mathrm{CO} 2$. The model has been applied to simulate the interfacial mass transfer in a single pore and 2D porous media under various flow conditions. The results show that the model can capture the mass transfer between the two phases and at the same time the interface adjustment.

Procter and Gamble Student poster award:

\section{References:}

Haroun, Y., Legendre, D., Raynal, L., 2010. Direct numerical simulation of reactive absorption in gasliquid flow on structured packing using interface capturing method. Chem. Eng. Sci. 65 (1), 351-356. Yue, P., Zhou, C., Feng, J.J., Ollivier-Gooch, C.F. and Hu, H.H., 2006. Phase-field Simulations of Interfacial Dynamics in Viscoelastic Fluids Using Finite Elements with Adaptive Meshing. J. Comp. Phys. 219, 4767. Acceptance of Terms and Conditions:

Click here to agree

\section{Adaptive numerical homogenization: linearization procedure to solve non-linear multi-scale problems.}

\author{
Iuliu Sorin Pop $^{1}$; Carina Bringedal ${ }^{\text {None }}$; Florin Adrian Radu $^{2}$; Manuela Bastidas ${ }^{1}$ \\ ${ }^{1}$ Hasselt University \\ ${ }^{2}$ University of Bergen, Norway
}

Corresponding Author(s): manuela.bastidas@uhasselt.be

Non-linear and multi-scale models are encountered in many real-life applications, ranging from oil recovery and environmental pollution to biosystems and fuel cells. Direct simulation of models involving behavior over multiple scales are challenging due to the need of a very fine grid.

In this context, multi-scale techniques are a good strategy for the numerical simulations of nonlinear, multi-scale models. Here we present a numerical strategy based on the classical homogenization theory and the mixed finite element formulation combined with a-posteriori indicators for mesh refinement. Next to deriving the effective (homogenized) models at the macro-scale, we develop an efficient numerical method employing adaptive grid refinement base on a-posteriori error estimates.

Subsequently, this scheme is combined with a linearization technique for solving the non-linear, homogenized models. The main idea is to apply a non-linear solver that takes the advantages of the standard Newton's method combined with the unconditional convergence of the modified Lscheme. 
Finally, we present some numerical examples to show the efficiency of the adaptive strategies. In particular, for some of these cases the classical schemes and non-linear solvers either fail to converge or become inefficient.

\section{Procter and Gamble Student poster award:}

I would like to compete in the Procter and Gamble Student award References:

I.S. Pop, F.A. Radu and P. Knabner, Mixed finite elements for the Richards' equation: linearization procedure. J. Comput. and Appl. Math. 168, 2004, pp. 365-373

F. List and F.A. Radu, A study on iterative methods for Richards' equation. Computational Geosciences 20 (2), 2016, pp. 341-353.

Bringedal, C., Berre, I., Pop, I. S., \& Radu, F. A. (2016). Upscaling of non-isothermal reactive porous media flow with changing porosity. Transport in Porous Media, 114(2), 371-393.

Mitra, K., \& Pop, I. S. (2018). A modified L-Scheme to solve nonlinear diffusion problems. Computers \& Mathematics with Applications. Acceptance of Terms and Conditions:

Click here to agree

\section{Precipitation characteristics of enzyme-induced calcite - Experi- mental study using microfluidic chip}

Raul Batista Araujo de Sousa ${ }^{\text {None }}$; Jaewon Jang ${ }^{\text {None }}$

Corresponding Author(s): raulbatista01@gmail.com, jwj@hanyang.ac.kr

Enzyme-induced calcite precipitation (EICP) is a promising soil improvement technique. There are a couple of advantages in using EICP: (1) there is no need to grow bacteria in soils, (2) the process of mineral precipitation is quick compared to microbial-induced calcite precipitation, and (3) the treatment can be repeated until a target mineral concentration is reached. The shear strength improved by EICP is dependent on the concentration of calcite precipitated in soils and the precipitation pattern in pore space. The shear strength of sediment increases with increasing concentration of calcite. And at a given calcite concentration, if the precipitation is concentrated on the contact between soil particles, the increase in the shear strength is greater than the case of the precipitation by coating on soil particles. In this study, the pattern of mineral precipitation is explored by using transparent microfluidic chips. The precipitation characteristics is investigated under different conditions including the concentration of EICP solution, wettability of pore surface, and the use of nucleating minerals. The nucleation pattern and growth pattern is monitored as a function of those variables.

Procter and Gamble Student poster award:

References:

Acceptance of Terms and Conditions:

Click here to agree

432

\section{Macro-scale simulation of fibrous liquid aerosol filters}

Author(s): André Baumann ${ }^{1}$

Co-author(s): Dennis Hoch ${ }^{1}$; Jennifer Niessner ${ }^{1}$

${ }^{1}$ Heilbronn University of Applied Sciences 
Corresponding Author(s): andre.baumann@hs-heilbronn.de

Today, liquid aerosols are produced in many industrial processes like machining, manufacturing of catalysts or in pneumatic compressors. While the harmfulness of fine dust aerosols is well known the equally dangerous liquid aerosols (mist) receive less attention. In order to be prepared for future legal regulations we develop efficient mist filters with less energy consumption.

In our research, we couple micro-scale simulations of two-phase flow in a fibrous porous medium with macro-scale simulations to develop efficient filters for a stationary (oil mist separator) and a mobile (pneumatic truck compressor system) application. Because the computational effort to simulate mist deposition in a whole filter on the micro-scale would be much too high, on the macro-scale, the information of the micro-scale is adopted and the whole filter is considered as porous continuum. In other words, the output of the micro-scale becomes the input of the macro-scale. Specifically, parameters like porosity, intrinsic permeability as well as relative permeability - and capillary pressure - saturation relationship determined from micro-scale simulations are used as input to the macroscale to simulate the filtration process on the domain of the whole filter. Furthermore, a source term of the liquid (oil) phase is derived from micro-scale simulations which may depend on parameters such as velocity and initial saturation. Thanks to this multi-scale coupling, it is possible to predict the pressure drop and the separation efficiency of a filter and figure out the factors to optimize these two properties.

A sensitivity analysis is made to find out which parameters have the strongest influence on pressure drop and separation efficiency. Furthermore, different parameterizations of the capillary pressure - and the relative permeability - saturationships (e.g. Brooks \& Corey and Van Genuchten) are compared. We also present the validation of our simulations by comparing both pressure drop and separation efficiency to measurement results. Using this validated model it is possible to predict both the pressure drop and the separation efficiency for the unsteady as well as for the stationary phase. In the first step, deposition on existing filters is simulated. In the second step, the filter setup is optimized with respect to both reduced pressure drop and enhanced separation efficiency.

Procter and Gamble Student poster award:

I would like to compete in the Procter and Gamble Student award References:

Acceptance of Terms and Conditions:

Click here to agree

773

\title{
Exploring the Robustness of Terahertz-Based Porosity Measure- ments using Embossed and Unembossed Pharmaceutical Tablets
}

\author{
Author(s): Prince Bawuah ${ }^{1}$ \\ Co-author(s): Alessia Portieri ${ }^{2}$; Daniel Markl ${ }^{3}$; Daniel Farrell ${ }^{2}$; J. Axel Zeitler ${ }^{1}$; Mike Evans ${ }^{2}$; Ralph Lucas \\ ${ }^{1}$ University of Cambridge \\ ${ }^{2}$ TeraView Limited \\ ${ }^{3}$ University of Strathclyde \\ ${ }^{4}$ Huxley Bertram Engineering Ltd
}

Corresponding Author(s): pb612@cam.ac.uk

\section{INTRODUCTION}

To achieve a continuous downstream manufacturing process with in-process quality monitoring and real-time release testing capabilities, different process analytical technologies (PATs) such as laser scattering methods, near-infrared (NIR) spectroscopy, and Raman spectroscopy can be deployed to, for example, measure the particle size of raw materials and powder blends as well as detect the content uniformity of powder blends and tablets. However, the use of the above-mentioned PAT methods to directly measure and quantify the bulk properties of tablets, e.g. porosity, that govern the mass transport processes and mechanical changes in tablets during disintegration and dissolution 
is limited. For example, NIR typically probes surface properties only and these measurements are not necessarily representative of the bulk tablet property, hence the need for suitable analytical technologies, such as terahertz time-domain spectroscopy (THz-TDS), that can reliably measure the porosity (volumetric property) of tablets [1,2].

This study showcases the robustness of the use of a terahertz-based approach [1-4] in the extraction of the porosity of several batches of tablets with and without embossment. To achieve a minimum measuring time for possible in-line application of the terahertz porosity method, the study further investigates the minimum average waveforms required to extract porosity values with maximum acceptable error.

\title{
METHOD AND MATERIALS
}

Terahertz time-domain measurements of the batches of tablets were acquired using a TeraPulse 4000 (TeraView Ltd., Cambridge, UK). The porosity was extracted from neff based on the Bruggeman's effective medium theory 1. Several batches of biconvex tablets with different porosities within the range of $10-26 \%$ that are either embossed or unembossed were used for the study.

\section{RESULTS AND CONCLUSIONS}

A comparison made between the terahertz porosity values of the embossed and unembossed tablets with respect to their respective nominal porosity has proven that the presence of the embossment has a negligible effect on the porosity measurement. Despite the use of biconvex tablets, the excellent linear correlation observed between the nominal and the terahertz porosity manifests the robustness of the terahertz approach for tablet porosity measurement. It is also observed that terahertz porosity with a root mean square error (RMSE) value of less than 0.006 with respect the nominal porosity can be achieved under one second of signal measurement.

Acknowledgments: The authors would like to thank Innovate UK for funding and value contributions of our collaborators from the GlaxoSmithKline (GSK).

Procter and Gamble Student poster award:

\section{References:}

1. Bawuah, P.; Pierotic Mendia A.; Silfsten, P.; Pääkkönen P.; Ervasti, T.; Ketolainen, J.; Zeitler, J.A. and Peiponen, K.E. Detection of porosity of pharmaceutical compacts by terahertz radiation transmission and light reflection measurement techniques. Int. J. Pharm. 465, 70-76 (2014).

[2]. Bawuah, P.; Silfsten, P.; Ervasti, T.; Ketolainen, J.; Zeitler, J.A. and Peiponen, K.E. Non-contact weight measurement of flat-faced pharmaceutical tablets using terahertz transmission pulse delay measurements. Int. J. Pharm. 476, 16-22 (2014).

[3]. Markl, D.; Strobel, A.; Schlossnikl, R.; Bøtker, J.; Bawuah, P.; Ridgway, C.; Rantanen, J.; Rades, T.; Gane, P.; Peiponen, K.-E. and Zeitler, J. A. Characterisation of pore structures of pharmaceutical tablets: A review. Int. J. Pharm. 538, 188-214 (2018).

[4]. Markl, D.; Sauerwein, J.; Goodwin, D.J.; van den Ban, S. and Zeitler, J. A. Non-destructive determination of disintegration time and dissolution in immediate release tablets by terahertz transmission measurements. Pharm. Res. 34, 1012-1022 (2017). Acceptance of Terms and Conditions:

Click here to agree

\section{Adaptive pores for oil permeation from oil-in-water emulsions}

\author{
Author(s): Hanieh Bazyar ${ }^{1}$ \\ Co-author(s): Thejas Hulikal Chakrapani ${ }^{2}$; Wouter K. den Otter ${ }^{2}$; Stefan Luding ${ }^{2}$; Rob G. H. Lammertink \\ ${ }^{1}$ University of Twente, Wetsus \\ ${ }^{2}$ University of Twente
}


Corresponding Author(s): h.bazyar@utwente.nl

Slippery liquid-infused membranes (SLIMs) have been introduced to membrane technology recently $[1,2]$. The liquid-filled pores can be opened and closed in response to system pressure giving rise to the formation of pore-sized gates (gating mechanism). In the open state, the pore wall is covered with the infusion liquid forming the so-called liquid-lined pores. Liquid-lining is expected to give anti-fouling properties to SLIMs. The pressure responsive pores can be used for efficient sorting of fluids from a mixture. For example in a two-phase mixture of immiscible liquids, the required system pressure, i.e. breakthrough pressure, is different for the constituent liquids. As a consequence, by setting the pressure properly, liquid-gated pores can let one phase through while retaining the other phase. Here, we investigate the capability of SLIMs for gravity-assisted permeation of the dispersed phase, i.e. oil from the oil-in-water $(\mathrm{O} / \mathrm{W})$ emulsion. The separation experiments are conducted in a dead-end pressure controlled filtration cell using liquid-infused and non-infused (dry) membranes. In order to permeate oil, oil droplets should migrate through the bulk of the $\mathrm{O} / \mathrm{W}$ emulsion and come in to contact with the membrane surface. Our results reveal that by setting the system pressure between the breakthrough pressure of oil and that of the surfactant solution, oil can be successfully permeated. The experimental findings are complimented with multi-body dissipative particle dynamics (MDPD) simulations [3]. In the simulations, piston induced penetration of immiscible multi-component mixtures (surfactant-solution and oil) into non-infused (dry) and liquidinfused cylindrical pores is investigated. Subsequently, the amount of water permeating the pore in the non-infused (dry) and liquid-infused case is measured, and, compared to experiments.

\section{Procter and Gamble Student poster award:}

\section{References:}

1 Bazyar H., Javadpour S., Lammertink R. G. H., On the gating mechanism of slippery liquid infused porous membranes, Advanced materials interfaces (2016), 3, 1600025 (1-6), DOI: https://doi.org/10.1002/admi.201600025.

[2] Bazyar H., Lv P., Wood J. A., Porada S., Lohse D., Lammertink R. G. H., Liquid-liquid displacement in slippery liquid-infused membranes (SLIMs), Soft Matter (2018), 14, 1780-1788, DOI: 10.1039/c7sm02337e. [3] P. B. Warren, Vapor-liquid coexistence in many-body dissipative particle dynamics, Phys. Rev. E. (2003), 68, 066702, .DOI:10.1103/PhysRevE.68.066702. Acceptance of Terms and Conditions:

Click here to agree

\section{Scour shape dependency on hydro-mechanical state of compacted clay}

Author(s): Raniero Beber ${ }^{1}$

Co-author(s): Alessandro Tarantino ${ }^{1}$; Matteo Pedrotti ${ }^{1}$; Rebecca Lunn ${ }^{1}$

${ }^{1}$ University of Strathclyde

Corresponding Author(s): raniero.beber@strath.ac.uk

The increase of extreme weather events, both in frequency and magnitude, is a well-established trend observed as a consequence of climate change. In the management and mitigation of flood risk earthen structures, such as dikes and levees, are the essential barriers to prevent inundation. Government policies on flood defense now tend toward the design of flood-able areas, where embankments can be overflown and the safe discharge of excess water managed, instead of continually raising the crest.

The understanding of the onset of breaching induced by surface erosion is thus fundamental to defining the levels of protection afforded by embankments and to provide standards for the design of new structures and strategies for upgrading existing ones.

Almost all earthen structures are built by soil compaction to improve their mechanical properties; therefore, it is important to understand how compacted soil responds to the load posed by flood water. At the onset of the overflow process, compacted embankment materials are generally partially saturated. The degree of saturation at overflow start, and change in saturation of the outer portion of the embankment slope during overflow, controls its resistance to erosion.

The focus of this research is to understand the interplay between hydraulic conditions and surface 
loading, in the prediction of clay erosion with the analysis of the scour acquired via laser scanner. Erosion behavior is known to be a counterbalance between gravity forces and shear erosion forces. As the particle size decreases, electrochemical interaction between soil particles become more and more relevant, while gravitational forces become negligible. Particle configuration and inter-particle forces in clays are known to change with hydro-mechanical stresses history (i.e. compaction and water content). We investigate the erosion behavior of Speswithe kaolin clay by means of the Jet Erosion Test (JET). Experiments are designed to mimic erosion of soil on the landward slope of an embankment during overflow; scour profiles are acquired via laser scanner.

The role of clay microstructure on erosion mechanisms and scour evolution in kaolin have been investigated in terms of at formation conditions (i.e. dry and wet side of compaction optimum), hydraulic conditions (i.e. wetting and drying cycles) and surface loading imparted by the jet (i.e. the fluid induced tangential and normal stresses). Results of these JET tests show that the different microstructures (i.e. aggregated structure or matrix-like structure) control the erodibility of the sample. We propose a conceptual model to explain counterintuitive findings. For example, samples with the same density but compacted on the dry side of optimum are more erodible than samples compacted on the wet side; samples with the same formation conditions, but with a different hydro-mechanic history (i.e. swelling upon wetting) produce different erosion behavior. Our results contribute to the fundamental understanding of the time-dependent mechanisms that influence erosion of clay embankments (i.e. the progression of the saturation front) during overtopping and, hence, to embankment failure. In addition, these tests show how basic concepts of unsaturated soil mechanics can serve as a guidance to 'design' the compaction of embankment material.

Procter and Gamble Student poster award:

I don't want to compete References:

Acceptance of Terms and Conditions:

Click here to agree

\title{
Multicomponent transport in hydrated polymer membranes: Al- cohols, Formate and Acetate in Nation 117
}

\author{
Breanna Dobyns $^{1}$; Daniel Miller ${ }^{2}$; Bryan Beckingham ${ }^{1}$ \\ ${ }^{1}$ Auburn University \\ ${ }^{2}$ Lawrence Berkeley National Laboratory
}

Corresponding Author(s): bsb0025@auburn.edu

Ion conducting membranes are of interest for various energy applications including fuel cells and artificial photosynthesis systems. Membranes in such systems are required to facilitate the ion transport necessary to feed the electrochemical reactions while meeting various additional selectivity and permeability demands depending on the application. Accurate descriptions of transport of species, and in many cases simultaneous transport of multiple species, in these hydrated polymer membranes is thereby critically important for a range of membrane separations and fuel cell operation. The transport of solutes through these dense, hydrated polymer membranes is described by the well-known solution diffusion model, $P i=(K i) x(D i)$, where $P i$ is the apparent permeability of the membrane to component $i, K i$ is the solubility of component $i$ in the membrane, and $D i$ is the apparent diffusivity of component $i$ in the membrane. Transport behavior of small-molecule solutes in these systems is typically characterized in single-component permeation experiments where permeation of the solute through the membrane is often monitored using chromatographic techniques that require periodic aliquot sampling. Here, we utilize in-situ ATR FTIR spectroscopy to quantify solution concentrations, preempting the need for aliquotic sampling and ex situ analysis. We apply this technique towards understanding how the presence of co-permeants impact solute uptake and permeability in Nafion, a commonly used ion exchange membrane. In particular, we examine the permeation of a series of single solute and multicomponent solute mixtures of both charged and uncharged species through Nafion. Membrane permeabilities extracted from in situ monitored diffusion cell experiments are coupled with solute solubility measurements to fully describe the permeability, solubility and diffusivity in these systems. Critically, we find substantial deviations in transport behavior between 
ideal permselectivities calculated from single solute diffusion cell experiments and real permselectivities extracted from multicomponent diffusion cell experiments. For instance, in the case of acetate and methanol co-diffusion this deviation is found to be driven by changes in solubility selectivity attributed to the screening of electrostatic interactions between acetate and Nafion's pendant anions by methanol.

Procter and Gamble Student poster award:

References:

Acceptance of Terms and Conditions:

Click here to agree

\title{
Impact of image resolution on quantification of mineral proper- ties and simulated mineral reactions and reaction rates
}

\author{
Lauren Beckingham ${ }^{1}$; Fanqi Qin ${ }^{1}$ \\ ${ }^{1}$ Auburn University \\ Corresponding Author(s): leb0071@auburn.edu
}

\begin{abstract}
Reactive transport modeling is a powerful means of enhancing understanding of mineral reactions and reaction rates in porous media. Imaging has emerged as a valuable tool to characterize geological samples and parameterize reactive transport simulations. Analysis of scanning electron microscopy (SEM) and X-ray computed tomography (CT) images of rock samples can provide information on porosity, mineral composition and mineral accessible surface area at the pore scale. These data can be upscaled and applied in continuum scale modeling of mineral reactions. Images can be collected using a variety of techniques and at a range of resolutions, yet the impact of image resolution on measured mineral properties, and simulated mineral reactions and reaction rates, is largely unknown. In this work, the impact of 2D image resolution on the calculated porosity, mineral volume fractions, accessibilities and effective surface areas were evaluated for a sample from the Paluxy formation, Kemper County, Mississippi. SEM backscatter electron (BSE) images of thin sections were captured under resolutions ranging from $0.3 \mu \mathrm{m}$ to $6 \mu \mathrm{m}$ and used to calculate mineral volume fractions and mineral accessibilities. This was combined with 3D X-ray CT imaging to calculate mineral accessible surface areas. Minimum variations in mineral volume fractions occurred with changing image resolution. For high resolution images, $0.3 \mu \mathrm{m}$ to $1 \mu \mathrm{m}$, mineral accessibilities agreed relatively well. For images with resolutions from $1 \mu \mathrm{m}$ to $6 \mu \mathrm{m}$, the calculated accessibility of smectite/illite and muscovite decreased with decreasing resolution while the accessibility of quartz increased. This in turn resulted in higher estimated effective surface areas for quartz with decreasing resolution. No significant variations were observed for calcite, siderite and K-feldspar. The impact of observed variations in mineral properties on simulated mineral reactions and reaction rates will be assessed through continuum scale reactive transport modeling in the context of geologic CO2 sequestration. Continuum scale models will be developed using data from each image resolution and results compared to assess variations in simulated dissolution rates and the overall evolution of mineralogy and porosity. The "Establishing an Early CO2 Storage Complex in Kemper, MS" project is funded by the U.S. Department of Energy's National Energy Technology Laboratory and cost-sharing partners.
\end{abstract}

Procter and Gamble Student poster award:

References:

Acceptance of Terms and Conditions:

Click here to agree 


\title{
Non-wetting liquid flow in nanoporous media under the impulse pressure change
}

\author{
Anton Belogorlov ${ }^{1}$; Nikolai Demin ${ }^{1}$ \\ ${ }^{1}$ National Research Nuclear University MEPhI \\ Corresponding Author(s): aabelogorlov@mephi.ru
}

\begin{abstract}
A lot of works are dedicated to study a rate of liquid flow in porous media 1 . And now, actively developed a new field of mechanics of liquid as nanofluidics, especially in case of damping of mechanical energy by intrusion of non-wetting liquid in nanoporous media process [2-5]. Some of authors are modeling fluid flow in a one channel by a molecular dynamics method [3], another authors are calculating the rate of fluid flow from experimental data by the recalculating the time dependence of force (acceleration) [4]. But the direct volume measurements in experiments with impulse (impact) pressure change are not presented, except of work [5].

In this work we present results of experimental study of non-wetting liquid intrusion in nanoporous media under the impulse pressure change obtained by an experimental method presented in work [5]. This experimental method and experimental setup allow to obtain measurements both of pressure and volume changes in experimental cell in time during external impact. Pressure and volume changes dependence on time analyzing allow calculating the flux of non-wetting liquid in nanoporous media under the impulse pressure change.
\end{abstract}

\section{Procter and Gamble Student poster award:}

I don't want to compete References:

1 Vafai, K. (2015). Handbook of porous media. Crc Press.

[2] Sun, Y., Guo, Z., Xu, J., Xu, X., Liu, C., \& Li, Y. (2015). A candidate of mechanical energy mitigation system: Dynamic and quasi-static behaviors and mechanisms of zeolite $\beta /$ water system. Materials \& Design, 66, 545-551. doi:10.1016/j.matdes.2014.04.028

[3] Cao, G. (2012). Working mechanism of nanoporous energy absorption system under high speed loading. The Journal of Physical Chemistry C, 116(14), 8278-8286.

[4] Li, M., Lu, W. Adaptive liquid flow behavior in 3D nanopores (2017) Physical Chemistry Chemical Physics, 19 (26), pp. 17167-17172. DOI: 10.1039/c7cp02981k

[5] Borman, V.D., Belogorlov, A.A., Lisichkin, G.V., Tronin, V.N., Troyan, V.I. Investigation of the dynamics of a percolation transition under rapid compression of a nanoporous body-nonwetting liquid system (2009) Journal of Experimental and Theoretical Physics, 108 (3), pp. 389-410. DOI: 10.1134/S1063776109030042 Acceptance of Terms and Conditions:

Click here to agree

\section{Homogeneous wetting by controlled imbibition}

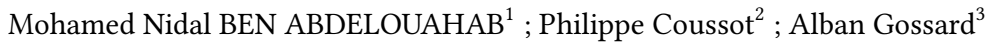 \\ ${ }^{1}$ CEA Marcoule - Laboratoire Navier \\ ${ }^{2}$ Univ. Paris-Est \\ ${ }^{3}$ CEA
}

Corresponding Author(s): mohamednidal.benabdelouahab@cea.fr

Keywords : Porous materials, Spontaneous imbibition, precursor films, kaolin paste, shrinkage, MRI

Poultice technology is currently mainly used for the desalination of masonry structures in the field of architectural heritage conservation 1 . Wet poultices are spread on the porous material surface to be treated, and kept in place before being removed when dry. The efficiency of the process depends on the capability of the paste to saturate the substrate before the system dries. However, little is 
known concerning porous substrate imbibition when put in contact with a soft porous material (a kaolin paste for example).

We followed the imbibition of an initially dry substrate (glass microbeads packing) whose top surface is coated by a wet kaolin paste. Nuclear Magnetic Resonance (NMR) was used to follow liquid distribution in time inside the system. This provided us the evolution of key internal characteristics in time: 1) the water saturation distribution along the sample axis and 2) the shape of the shrinking paste.

In contrast with classical imbibition from a free water reservoir where a saturated front progresses inside the substrate and which may be described by the Washburn model [2], here the liquid is observed to invade the porous medium while maintaining a partial saturation. In a first period, a liquid film progresses inside the substrate until reaching substrate bottom. Then, during a second period, the saturation in the substrate increases homogenously and stops before full saturation. We explain this remarkable behavior by the development of a precursor film during the first regime that would progress faster than the extraction of the main liquid amount from the paste. In the second regime the slow liquid extraction from the paste then goes on filling the medium and capillary effects can now ensure a homogeneous distribution of the concentration. The process stops when the stress associated with capillary effects is insufficient to further compress the paste. 
with fully described velocity and diffusion. Both models use the same thermodynamic reaction rate, because the rate is not forced to absorb the loss of information upon upscaling. Analytic and semianalytic upscaling is also performed using volume averaging and ensemble streamtube techniques. Volume averaging does not perform as well as the RPT, while the streamtube approach (using an effective dispersion coefficient along with macro-dispersion) performs almost exactly the same as RPT.

Procter and Gamble Student poster award:

I don't want to compete References:

Acceptance of Terms and Conditions:

Click here to agree

\title{
Lagrangian Mass Transfer/Reaction Models: Is Probabilistic Mass Transfer Numerically Equivalent to SPH?
}

\author{
Guillem Sole-Mari ${ }^{1}$; Michael Schmidt ${ }^{2}$; David Benson ${ }^{2}$ \\ ${ }^{1}$ Universitat Politècnica de Catalunya \\ ${ }^{2}$ Colorado School of Mines
}

Corresponding Author(s): dbenson@mines.edu

Some of the existing Lagrangian techniques for simulating solute transport in fluids represent diffusive processes as proximity-based mass exchanges between numerical particles. Two main approaches of this kind can be distinguished in the hydrology literature: (a) Smoothed Particle Hydrodynamics (SPH), which can be derived from the assumption that each particle's mass can be projected on the continuum according to some smoothing kernel (e.g., Herrera et al., 2009), and (b) probabilistic motion-based formulations, often referred to as Mass Transfer Particle Tracking (MTPT), which can be derived heuristically by considering the probability of collocation of any pair of particles according to the Green's function of diffusion in a time step (e.g. Benson and Bolster, 2016). Here, we show that MTPT can be numerically equivalent to (i.e., a specific case of) SPH for specific kernel choices. Following this key finding, we perform a numerical study to shed some light on the influence of the kernel bandwidth (related to the mass and time discretization) on the results of SPH transport simulations (MTPT here being a specific choice of bandwidth), for different spatial distributions of particles. We also consider a hybrid Random Walk / SPH approach. We discuss the trends observed in the deviation from the analytical solution for the different scenarios. This deviation from the analytical solution can be interpreted simply as an error or as a representation of incomplete mixing, as has been traditionally done by SPH and by MTPT modelers, respectively.

Procter and Gamble Student poster award:

I don't want to compete References:

Herrera, P. A., Massabl'o, M., \& Beckie, R. D. (2009). A meshless method to simulate solute transport in heterogeneous porous media. Advances in Water Resources, 32(3), 413-429. https://doi.org/10.1016/j.advwatres.2008.12.0

Benson, D. A., and D. Bolster (2016), Arbitrarily complex chemical reactions on particles, Water Resour. Res., 52, 9190-9200, doi: 10.1002/2016WR019368. Acceptance of Terms and Conditions:

Click here to agree

\section{A mixed MoL-TMoL approach for solving 2D Richards' equation in layered soils}


Marco Berardi $^{1}$; Fabio Difonzo ${ }^{2}$; Michele Vurro ${ }^{1}$; Luciano Lopez ${ }^{3}$

${ }^{1}$ CNR - Istituto di Ricerca sulle Acque

${ }^{2}$ Code Architects Automation

${ }^{3}$ Università di Bari

Corresponding Author(s): marco.berardi@ba.irsa.cnr.it

Water infiltration into layered soils is studied, considering a two dimensional spatial domain. The physics of the problem suggests a subtle analysis of discontinuity at the interface between different soils, which is carried on by means of Filippov theory, a sophisticated tool developed in the contest of control theory that is now spreading out also in the PDEs framework.

The novelty of our approach is based on a mixed MoL-TMoL approach, which merges desirable features of both the classical Method of Lines (MoL) and Transversal Method of Lines (TMoL), a numerical scheme originally proposed for the 1D Richards' equation modeling the case of two layered soils. The numerical method numerical, together with corresponding numerical simulations are provided.

Procter and Gamble Student poster award:

I don't want to compete References:

Acceptance of Terms and Conditions:

Click here to agree

990

\section{InterPore 2019 Presentation}

Steffen Berg ${ }^{1}$; Steffen Berg ${ }^{2}$

${ }^{1}$ Shell Global Solutions International B.V,

${ }^{2}$ Shell Global Solutions International B.V.

Corresponding Author(s): s.berg@shell.com, steffen.berg@shell.com

Procter and Gamble Student poster award:

References:

Acceptance of Terms and Conditions:

362

\section{Molecular Dynamics Simulations of Polymeric Fibre Bundles un- der Tensile Load}

Author(s): Eivind Bering ${ }^{1}$

Co-author(s): Signe Kjelstrup ${ }^{2}$; Dick Bedeaux ${ }^{3}$; Astrid S. de Wijn ${ }^{4}$

\footnotetext{
${ }^{1}$ PoreLab, NTNU

${ }^{2}$ Norwegian University of Science and Technology, NTNU, Trondheim

${ }^{3}$ Norwegian University of Science and Technology - NTNU

${ }^{4} N T N U$
} 
Corresponding Author(s): eivind.bering@ntnu.no

Bundles of polymeric materials play essential and ubiquitous roles in biological systems, and often display remarkable mechanical properties 1 . By utilizing non-equilibrium molecular dynamics simulations to preform force controlled and length controlled virtual stretching experiments of polymeric fibre bundles one may get valuable insight into the mechanical properties of nanofibrous materials. A minimalistic reactive force field is constructed from existing force fields to investigate the yield behavior of the samples [2]. The supra-molecular structure of fibrils in nanometric bundles is studied, which have been shown experimentally to have great impact on e.g. the Young's modulus of the bundles [3]. The chains in the bundle typically self organize to a semi-crystaline hexagonal pattern with entropically induced defects due to non-zero temperatures, with higher degree of amorphicity for low external forces.

The main material investigated here is polyethylene oxide (PEO), a polyether compound with applications ranging from industrial manufacturing to medicine. The simple chemical composition makes it well suited as a starting point for the study of the general behavior of polymeric fibre bundles, and this procedure of virtual stretching is directly applicable to more complex structures such as cellulose or collagen with and without the presence of third species, such as room temperature ionic liquids and aqueous environments. The simulations are carried out for a range of sizes to study finite size effects.

The thermodynamic potentials of the bundles are studied in more detail. The smallest systems under investigation consists of single molecules of PEO with 32 units, and on this scale it is still debated whether a non-equilibrium thermodynamic description even exists. According to the theory of Hill (small system thermodynamics), the thermodynamic functions cease to be extensive at small length scales. They obtain additional terms, from surface or line energy contributions. Strøm, Schnell and Kjelstrup have previously found a scaling law between the thermodynamic and the molecular limit [4]. This indicates that the thermodynamic laws will indeed apply to the molecular level, provided that we use the additional terms arising from the system smallness. It is an aim of the project to verify the theoretical formulation of Rubi et al. 5. This may have a large fundamental impact on how one can describe biomolecular and other events related to energy conversion on the small scale.

\title{
Procter and Gamble Student poster award:
}

I would like to compete in the Procter and Gamble Student award References:

1 N. G. McCrum, C. P. Buckley, C. B. Bucknall, Principles of Polymer Engineering, Oxford University Press, Oxford, New York (1997).

[2] C. Chen, P. Depa, V. G. Sakai, J. K. Maranas, J. W. Lynn, I. Peral \& J. R. D. Copley. J. Phys. Chem. 124 (2006).

[3] A. Arinstein, M. Burman, O. Gendelman, E. Zussman, Nat. Nanotechnol. 2, 59 (2007).

[4] B. A. Strøm, J.-M. Simon, S. K. Schnell, S. Kjelstrup, J. He \& D. Bedeaux. J. Phys. Chem. 19 (2017).

5 J. M. Rubi, D. Bedeaux \& S. Kjelstrup, J. Phys. Chem. 110 (2006). Acceptance of Terms and Conditions:

Click here to agree

180

\section{Effects of Inertia, Péclet Number, and Heterogeneity on Flow and (Anomalous) Transport in Heterogeneous Porous Media}

\author{
Brian Berkowitz ${ }^{1}$; Alon Nissan ${ }^{1}$ \\ ${ }^{1}$ Weizmann Institute of Science
}

Corresponding Author(s): brian.berkowitz@weizmann.ac.il

We investigate the effects of high velocities (inertial effects) in various heterogeneous porous media on fluid flow and tracer transport. We generate three pore-scale configurations that differ in terms of their structural complexity, and investigate 10 realizations of each type. For each realization, we solve the Navier-Stokes equations to obtain the spatial flow field; tracer transport is then 
modeled by a particle tracking algorithm that uses a semi-analytic, streamline-based method to simulate advective motion. At a sufficiently high Reynolds number (within the laminar regime), inertial effects become relevant, and we observe a transition from linear to nonlinear flow that deviates from Darcy's law. The sensitivity to this transition and the strength of the nonlinear behavior are controlled by the structure of the medium: higher complexity tends to amplify effects of inertia, which lead to spatial homogenization of the flow field. In the context of transport, at increasingly high Reynolds numbers, the portion of the velocity field that actively contributes to tracer transport exhibits increasingly narrower distributions of velocity values. This result leads to more uniform tracer transport, reducing the degree of anomalous behavior. We interpret the effects of high velocities in porous media on transport within the Continuous Time Random Walk (CTRW) framework, using the spatial distribution of the kinetic energy within the fluid - which is itself a function of the Reynolds number - as a characteristic measure. Finally, we investigate the effects of the Péclet number $(\mathrm{Pe})$ on tracer transport in heterogeneous porous media. With increasing $\mathrm{Pe}$, anomalous transport becomes enhanced as the host domain becomes more heterogeneous, due to the increasingly dominant effects of the complex velocity field. We quantify the effects of Pe by interpreting the numerical simulations within the CTRW framework, and incorporate Pe within the underlying tracer transition time distribution.

Procter and Gamble Student poster award:

References:

Acceptance of Terms and Conditions:

Click here to agree

\section{Adaptive mesh refinement for large scale Discrete Fracture Net- work flow simulations}

Stefano Berrone ${ }^{1}$; Andrea Borio ${ }^{2}$; Sandra Pieraccini ${ }^{\text {None }}$; Stefano Scialò ${ }^{2}$; Fabio Vicini ${ }^{2}$

${ }^{1}$ Politecnico di Torino, Italy

${ }^{2}$ Politecnico di Torino

Corresponding Author(s): stefano.berrone@polito.it

In the talk the issue of reliable and efficient flow simulations in large scale Discrete Fracture Networks is tackled. The stochastic generation of DFNs introduces fractures sampled from given distributions for position, orientation, dimension, and hydro-geological properties. From a computational point of view, among the several numerical difficulties of these simulations, reliability and efficiency of numerical solutions, as well as mesh generation, take a relevant rule. Recently, a new approach has been proposed, based on a reformulation of the flow problem as a PDE-constrained optimization problem and capable to circumvent mesh generation problems avoiding any mesh conformity requirement among the fractures.

Thanks to its robustness with respect to mesh generation problems, the method has proven to allow for very general Uncertainty Quantification analyses, also in the case of geometrical stochasticity, resorting to suitable Multi-Level Monte Carlo methods. Moreover, a tailored parallel approach has been derived and very high parallel efficiency has been obtained allowing very large scale simulations.

For this recasting of the flow problem, reliable a posteriori error estimates have been derived. Their application to flow simulation in realistic DFNs yield a large improvement of the quality and reliability of the solution.

The automatic choice of a suitable computing mesh can be useful to ensure, for a fixed level of accuracy, the same precision in the large number of simulations needed in Uncertainty quantification analyses, reducing the effect on UQ analysis of numerical noise in the solution. Moreover, adaptive mesh refinement can take advantage from the mesh independence between intersecting fractures, making the mesh adaptivity easier and computationally faster, providing a suitable mesh with an 
equidistributed and controlled error. The great advantage of the adaptive mesh refinement with respect to uniform refinement is proven on realistic networks with tens of thousand fractures.

\title{
Procter and Gamble Student poster award:
}

\section{References:}

S. Berrone, S. Pieraccini, S. Scialò, A PDE-constrained optimization formulation for discrete fracture network flows, SIAM J. Sci. Comput. 35 (2) (2013) B487-B510.

S. Berrone, A. Borio, S. Scialò, A posteriori error estimate for a PDE-constrained optimization formulation for the flow in DFNs, SIAM J. Numer. Anal. 54 (1) (2016) 242-261.

S. Berrone, S. Pieraccini, S. Scialò, Towards effective flow simulations in realistic discrete fracture networks, J. Comput. Phys. 310 (2016) 181-201.

S. Berrone, C. Canuto, S. Pieraccini, S. Scialò, Uncertainty quantification in discrete fracture network models: Stochastic geometry, Water Resources Research 54 (2) (2018) 1338-1352. Acceptance of Terms and Conditions:

Click here to agree

\section{Industrial gas storage monitoring based on measurement of soil respiration model}

\author{
Author(s): Guillaume Berthe $\mathrm{None}$ \\ Co-author(s): Ricardo Huerta ; Abdelaziz Faraj ; Virgile Rouchon ; Bruno Garcia
}

Corresponding Author(s): guillaume.berthe@ifpen.fr

Soil monitoring is necessary in the surrounding of the industrial sites impacted by geological gas storage. Measuring the physical and biochemical reactions is a typical tool to ensure storage integrity from industrial sites. Measurements of natural gas flows between the soil and the atmosphere can be used to detect eventual abnormal emissions. Indeed, it is possible to distinguish natural flows (soil respiration considered as soil natural emissions from additional gas flows that have migrated from deep reservoirs, allowing thus to detect leaks).

A prototype station (ESCORT ${ }^{\circledR}$ ) for monitoring the temporal evolution of gas emissions (qualitatively and quantitatively) has been developed at IFPEN (Rueil-Malmaison, France). This station records gas concentrations $(\mathrm{CO} 2, \mathrm{CH} 4, \mathrm{O} 2)$ in soil at four different depths (in this study : on the ground's surface, at $60 \mathrm{~cm}, 120 \mathrm{~cm}$ and $180 \mathrm{~cm}$ of depth). ESCORT ${ }^{\circledR}$ also integrates meteorology data as temperature, atmospheric pressure and rainfall to correlate the meteorological conditions with gas variations in the soil. During 2 years, from 2016 to 2017, measurements have been acquired providing thus a rich database of $\mathrm{CO} 2$ and $\mathrm{O} 2$ measurements that allow to model the soil respiration of the site.

Several soil respiration models were tested to predict natural $\mathrm{CO} 2$ emissions. This paper presents the approach proposed to validate the soil respiration model, using available weather data from 2017 year to estimate the $\mathrm{CO} 2$ fluxes. In addition $\mathrm{CO} 2$ injections experiments in soil were also carried out to mimic leaks and test the model in such a context. Results show that the $\mathrm{CO} 2$ flow during injection period is higher than the flux calculated from the natural flow of soil respiration. The distinction between natural emission and leak was observed. Furthermore a mobile station is now developed and provides on map a real-time measurements of abnormal $\mathrm{CO} 2 / \mathrm{CH} 4$ concentrations on the ground's surface.

Procter and Gamble Student poster award:

\section{References:}

Acceptance of Terms and Conditions:

Click here to agree 


\title{
A posteriori error estimation by stress and flux reconstruction for Biot's consolidation model
}

\author{
Fleurianne Bertrand ${ }^{1}$ \\ ${ }^{1}$ HU Berlin \\ Corresponding Author(s): fleurianne@gmail.com
}

\begin{abstract}
Flux and stress equilibration procedure for the Biot's consolidation (see 1) problem using RaviartThomas elements with weakly symmetry as in 2 are proposed and analyzed. The stress tensor is reconstructed from a displacement-pressure approximation computed with a stable finite element pair. The Darcy velocity is reconstructed in the Raviart-Thomas finite element space, such that both reconstructions are $\mathrm{H}$ (div)-conforming, and the equilibration procedure offering serveral advantages (see 3) can be used. In particular, these reconstructions are build on vertex patches (see also 4) such that they lead to a local efficient a posteriori error estimator for the Biot's consolidation problem, involving constants that depends only on the shape regularity of the triangulation.
\end{abstract}

Procter and Gamble Student poster award:

\section{References:}

1 M. A. Biot. General theory of three-dimensional consolidation. J. Appl. Phys., 12:155-169, 1941.

[2] A posteriori error estimation for planar linear elasticity by stress reconstruction, https://arxiv.org/pdf/1703.00436.pdf

[3] M. Ainsworth and R. Rankin, Guaranteed computable error bounds for conforming and nonconforming finite element analyses in planar elasticity, Elsevier, 2005.

[4] D. Braess, V. Pillwein and J. Schöberl Equilibrated residual error estimates are p-robust, Comput. Methods Appl. Mech. Engrg 198, 1189-1197. Acceptance of Terms and Conditions:

Click here to agree

\section{Swelling of gel rods}

\author{
Author(s): Thibault Bertrand ${ }^{1}$ \\ ${ }^{1}$ Imperial College London \\ ${ }^{2}$ Princeton University \\ ${ }^{3}$ University of Oxford
}

Co-author(s): H. Jeremy Cho ${ }^{2}$; Sujit S. Datta ${ }^{2}$; Christopher W. MacMinn ${ }^{3}$

Corresponding Author(s): t.bertrand@imperial.ac.uk

Swelling is a process in which a porous material grows spontaneously by absorbing additional pore fluid. Polymeric hydrogels are highly deformable materials that can experience extreme volume changes during swelling, allowing a small amount of dry hydrogel to absorb a very large amount of fluid. This feature makes hydrogels extremely useful in applications ranging from moisture control to drug delivery. The effective use of hydrogels in these applications requires a detailed characterisation of their constitutive properties, including osmotic pressure, stiffness, and permeability as functions of water content. Permeability is particularly important for applications that are sensitive to the rate of swelling and drying. The constitutive properties of hydrogels are typically measured at equilibrium, but equilibrium states provide no information about permeability. Measurement of the permeability of hydrogels is further complicated by their extreme softness, and by the transient nature of swelling. Here, we address this difficulty by studying the swelling of a hydrogel rod that is suspended in dry air and has one end in contact with a water bath. The rod evolves from a uniform initial state to a non-uniform (non-equilibrium) steady state in which the rate of water imbibition from the bath and conduction through the rod is exactly balanced by the rate of water loss through the sides via evaporation. We study this steady shape with laboratory experiments and a dynamic 
model based on large-deformation poromechanics. This shape is easy to measure and also very sensitive to the permeability function, making it a novel and convenient method of characterising the permeability of hydrogels.

Procter and Gamble Student poster award:

I don't want to compete References:

Acceptance of Terms and Conditions:

Click here to agree

913

\section{Coarse-grained simulations to guide an understanding of capil- lary pressure scanning curves in fibrous porous media}

Divesh Bhatt ${ }^{1}$

${ }^{1}$ Kimberly-Clark Corporation

Corresponding Author(s): divesh.bhatt@kcc.com

Quantification of capillary pressure scanning curves is important in estimating the efficacy of porous absorbent structures when operated under reversals of scanning direction in partially saturated conditions. Experiments for thin fibrous deformable porous media are difficult. This work details a coarse-grained approach to study the scanning behavior in such porous media and identify its qualitative features.

Procter and Gamble Student poster award:

References:

Acceptance of Terms and Conditions:

Click here to agree

794

\section{Coupled Impact of Physical and Chemical Heterogeneity on Re- action Rates and Dissolution Patterns in Mixed-Mineralogy Porous Rock}

Yousef Al-khulaifi ${ }^{1}$; Qingyang Lin $^{1}$; Martin Blunt ${ }^{1}$; Branko Bijeljic $^{2}$

${ }^{1}$ Imperial College London

${ }^{2}$ Imperial College

Corresponding Author(s): b.bijeljic@imperial.ac.uk

Reactive transport plays an important role in changing flow behaviour during carbon dioxide (CO2) injection into carbonate aquifers for sequestration or oil recovery. In-situ brine is acidified by the injected $\mathrm{CO} 2$, which may lead to a more rapid mineral dissolution. However, the manner in which dissolution occurs could be strongly influenced by the physical and chemical heterogeneities inherent in the host rock (Al-khulaifi et al.,2017).

We apply a combined experimental and modelling approach (Al-khulaifi et al., 2018). to study the coupled impact of physical and chemical heterogeneity on reaction rates and dissolution patterns. We use micro-CT to image in situ dissolution of carbonate rocks flooded with supercritical CO2 
saturated brine at reservoir conditions. We select two pairs of mixed mineralogy rock samples with similar physical heterogeneity characterised by velocity distribution obtained by a Navier-Stokes flow solver (Raeini et al.,2012; Bijeljic et al., 2013). All four samples have approximately 8:1 ratio of dolomite to calcite, which are non-uniformly distributed, having different chemical heterogeneity. We run experiments at two flowrates associated with different transport conditions.

Distinct physical heterogeneity (initial pore structure and associated velocity field) and chemical heterogeneity (intrinsic reaction rates and mineral distribution) exhibit a markedly different impact on the effective reaction rates and dissolution patterns. Average reaction rates for calcite and dolomite are an order of magnitude lower than the corresponding batch rates due to mass transfer limitations. The calcite effective reaction rates in proximity to fast flow channels are initially higher than those of dolomite. However, after the formation of a single dominant channel, the dolomite rates are higher, since calcite becomes shielded by dolomite. The latter is also true for the dolomite effective reaction rates in proximity to slow regions.

We introduce a new metrics quantifying coupled reactive transport behaviour, which describes proximity of reacted minerals to the fast channels and slow regions. Overall, a higher degree of physical and/or chemical heterogeneity promotes the preferential channelling effect, as opposed to uniform dissolution.

Procter and Gamble Student poster award:

I don't want to compete References:

Al-Khulaifi, Y., Lin, Q., Blunt M.J., Bijeljic B. (2018). Reservoir-Condition Pore-Scale Imaging of Dolomite Reaction with supercritical CO2 Acidified Brine: Effect of Pore-Structure on Reaction Rate using Velocity Distribution Analysis, International Journal of Greenhouse Gas Control, 68, 99-111, doi.org/10.1016/j.ijggc.2017.11.01.

Al-Khulaifi, Y., Lin, Q., Blunt M.J., Bijeljic B. (2017). Effective Reaction Rates in Chemically Heterogeneous Rock - The Coupled Impact of Structure and Flow Properties Studied by X-ray Microtomography, Environmental Science and Technology, 51 (7), 4108-4116, doi: 10.1021/acs.est.6b06224.

Raeini Q. A., Blunt M.J., Bijeljic B. (2012). Modelling Two-Phase Flow in Porous Media at the Pore Scale Using the Volume-of-Fluid Method, Journal of Computational Physics, 231, 5653-5668.

Bijeljic B., Raeini A., Mostaghimi P., Blunt, M.J. (2013). Predictions of Non-Fickian Solute Transport in Different Classes of Porous Media using Direct Simulation on Pore-scale Images, Physical Review E. 87, 013011, doi: 10.1103/PhysRevE.87.013011. Acceptance of Terms and Conditions:

Click here to agree

642

\section{Experimental investigation of oil recovery and recovery mecha- nisms of silica nanoparticles in water-wet reservoirs}

Author(s): Alberto Bila ${ }^{1}$

Co-author(s): Torsæter Ole

${ }^{1}$ Luis

Corresponding Author(s): alberto.bila@ntnu.no

The era of "easy oil" production is ending, and it is necessary to develop new enhanced oil recovery (EOR) technologies to run the production economically. Previous studies have shown that hydrophilic silica nanoparticles can improve water-flooding performance significantly due to their small size $(1-100 \mathrm{~nm})$ and high surface area-to-volume ratio. However, the major challenge is the development of the nanoparticle types capable of meeting the oil field screening conditions while providing the highest oil recovery. In addition, a proper understanding of the underlying EOR mechanisms of the nanoparticles is needed.

In this work, we experimentally investigated four different types of silica nanoparticles as additives to injection water for oil recovery applications. The nanoparticles were developed to remain stable 
in the injection water and the hypothesis is that the particles improve the performance of water injection in a specific oil reservoir.

The nanoparticles were prepared to $0.1 \mathrm{wt} . \%$ concentration in synthetic North Sea water. Crude oil was obtained from a field in the North Sea. Two-phase flow experiments were conducted, at surface conditions, to verify the oil recovery potential of the nanoparticles at the breakthrough point and at the end of flooding in water-wet Berea sandstone rocks. The best injection scheme were also evaluated. 1) The prepared nanoparticles were injected from start (at Swir) and the EOR performance evaluated with respect to reference water flood; 2) the nanoparticles were injected after reference water flood; and 3) the displacement mechanisms of the nanoparticles were investigated by interfacial tension measurements between the nanofluids and crude oil, Amott-wettability test and by analyzing the differential pressure across the core and the nanoparticle size distribution.

Our experimental results from core floods show that the silica nanoparticles can boost oil recovery. The secondary recoveries ranged from $44.4 \%$ to $55.7 \%$ of original oil in place (OOIP) compared to $39.7 \%$ of reference water flood. Oil recoveries of nanoparticles applied after water flood varied from $4.6 \%$ to $9.8 \%$ of OOIP. The presence of nanoparticles in the injection water delayed the breakthrough point and resulted in addtional oil recovery over water flood. The results suggest that it is preferable to inject the nanoparticles from start, rather than the injection of nanoparticles after the water flood. Oil displacement studies showed that oil recovery is affected by a contribution of interfacial tension reduction and wettability. However, the increased differential pressure observed throughout the nanoparticle injection and the particle filtration may support the log jamming effect in the recovery of oil. High flowrate applied at the end of low rate generated nanoparticle-stabilized emulsions, which is also thought to contribute in the reduction of residual oil saturation.

Procter and Gamble Student poster award:

I would like to compete in the Procter and Gamble Student award References:

Acceptance of Terms and Conditions:

Click here to agree

\title{
INNOVATIVE TOOLS FOR FIELD SCALE INTERPRETATION OF ATTENUATION PROCESSES AND QUANTIFICATION OF CON- TAMINANT MASS DISCHARGE AT THE GROUNDWATER-SURFACE WATER INTERFACE
}

\author{
Poul L. Bjerg ${ }^{1}$; Vinni Rønde ${ }^{1}$; Nicola Balbarini ${ }^{1}$; Majken Frederiksen ${ }^{1}$; Anne Sonne ${ }^{1}$; Frederick Devlin ${ }^{2}$; MacKen- \\ zie Cremeans ${ }^{3}$; Mike Annable ${ }^{4}$; Philip Binning ${ }^{5}$; Ursula McKnight ${ }^{1}$ \\ ${ }^{1}$ Technical University of Denmark \\ ${ }^{2}$ Kansas University \\ ${ }^{3}$ Kansa University \\ ${ }^{4}$ University of Florida \\ ${ }^{5}$ Technical University of Denmark
}

Corresponding Author(s): plbj@env.dtu.dk

Contaminants such as chlorinated solvents, pharmaceuticals and pesticides, as well as emerging micropollutants are released to streams from multiple point and diffuse sources. Sustainable management of water resources requires assessment of multiple contamination sources within a watershed in order to assess their direct impact on water quality. Determination of flow paths and groundwater fluxes are essential for evaluating the transport, fate and potential impact of contaminant plumes discharging to streams. This implies that investigators have the tools to evaluate the governing parameters, including an appreciation of the scale of variability, as well as conceptual and numerical models that incorporate the various mechanisms affecting flow, transport and fate.

A major multidisciplinary field-scale investigation of the Grindsted stream area was carried out in 2012-2018 to develop the scientific basis for conducting risk assessments for contaminated sites 
impacting groundwater and stream water. The contamination originating from a former pharmaceutical industry discharges into a multilayered aquifer system and a downgradient stream (1). The groundwater plume contains high concentrations of pharmaceutical compounds, chlorinated ethenes and benzene. The contamination in the source area has been depleted and today the main challenge is related with the complex plume discharging into the stream. The main impact zone is located within a mixed land-use stream system, comprising urban areas and agricultural production, with multiple chemical stressors impacting the stream corridor $(8,9)$.

Our overall aim of the field investigations was to (i) advance our understanding of transport and fate of chlorinated ethenes and pharmaceutical compounds in a multilayered aquifer-stream system; (ii) test the applicability of different methods for mapping groundwater flow and pollution as it enters streams at a complex site, (iii) perform a risk assessment of the stream using the contaminant mass discharge approach; and (IV) assess the stream's chemical and ecological status in a multiple stressor context.

The study included development of geological and hydrogeological models, geophysical measurements, numerical modeling of the flow and transport, mapping of the contaminant plume at various scales, and detailed field investigations at the main entry point of the plume into the stream. We quantified the flow, contaminant mass discharge (CMD) and attenuation of the plume at the groundwater-surface interface using different approaches and innovative tools. This included for example:

- high resolution groundwater sampling in a control plane at the stream bank in conjuction with application of Point Velocity Probes for determination of flow velocities and directions at the stream bank $(2,7)$

-identification of inflow zones using traditional and innovative tools e.g sediment bed Passive Flux Meters and streambed Point Velocity Probes $(3,4,5,7,9)$

-Comparison of CMD estimates at control planes in stream bank, stream bed and in- stream $(5,7,9)$. -geophysics based quantification of CMD by use of geostatistical methods $(2,6)$

Our current understanding of the attenuation processes for key contaminants at the groundwatersurface water interface and the value of innovative field methods for quantification of CMD will be discussed in the presentation.

\section{Procter and Gamble Student poster award:}

\section{References:}

1) Balbarini, N., Frederiksen, M., Rønde, V., Møller, I.B., Sonne, A.T., McKnight, U.S., Pedersen, J.K., Binning, P.J., Bjerg, P.L. (2018). Assessment of the fate of pharmaceutical compounds in a layered aquifer discharging to a stream. Submitted.

2) Balbarini, N.; Rønde, V.; Maurya, P.; Fiandaca, G.; Ingelise Møller, I.; Klint, K.E.; Christiansen, A.V.; Philip J. Binning, P.J.; Bjerg, P.L. (2018). Geophysics based contaminant mass discharge quantification downgradient of a landfill and a former pharmaceutical factory. Water Resources Research. 54, 8, 54365456.

3) Balbarini, N.; Boon, W.M.; Nicolajsen, E.; Nordbotten, J.M.; Bjerg, P.L.; Binning, P.J. (2017). A 3-D numerical model of the influence of meanders on groundwater discharge to a gaining stream in an unconfined sandy aquifer. Journal of Hydrology. 552, 168-181.

4) Cremeans, Mackenzie; Devlin, J.; McKnight, U. S.; Bjerg, P. L. (2018). A Comparison of Tools and Methods for Estimating Groundwater-Surface Water Exchange. Submitted.

5) Cremeans, Mackenzie; Devlin, J.; McKnight, U. S.; Bjerg, P. L. (2018). Application of New Point Measurement Device to Quantify Groundwater-Surface Water Interactions. Journal of Contaminant Hydrology. 211, 85-93.

6) Maurya, P.K.; Balbarini, N.; Balling, I.; Rønde, V.; Christiansen, A.V.; Bjerg, P.L.; Auken, E.; Fiandaca, G. (2018) Subsurface imaging of water electrical conductivity, hydraulic permeability and lithology at contaminated sites by induced polarization, Geophysical Journal International. 213, 2, 770-785.

7) Rønde, V.; McKnight, U.S.; Sonne, A.Th.; Balbarini, N.; Devlin, J.F.; Bjerg, P.L. (2017). Contaminant mass discharge to streams: comparing direct groundwater velocity measurements and multilevel groundwater sampling with an in-stream approach. Journal of Contaminant Hydrology. 206, 43-54.

8) Sonne; A.T.; Rasmussen, J.J. Höss, S.; Traunspurger, W.; Bjerg, P.L.; McKnight, U.S. (2018). Linking 
ecological health to co-occurring organic and inorganic chemical stressors in a groundwater-fed stream system. Science of the Total Environment. 642, 1153-1162.

9) Sonne, A.T.; McKnight, U.S.; Rønde, V.; Bjerg, P.L. (2017). Assessing the chemical contamination dynamics in a mixed land use stream system. Water Research, 125, 141-151. Acceptance of Terms and Conditions:

Click here to agree

\title{
Determination of thermodynamic contact angle based on inter- facial energy balance in multiphase flow displacement in porous media
}

\author{
Qingyang $\operatorname{Lin}^{1}$; Takashi Akai $^{1}$; Branko Bijeljic ${ }^{2}$; Martin Blunt ${ }^{1}$ \\ ${ }^{1}$ Imperial College London \\ ${ }^{2}$ Imperial College \\ Corresponding Author(s): m.blunt@imperial.ac.uk
}

Recently, the in situ contact angle estimated from the geometry of the fluid/fluid interface where it meets the solid has been used to characterize wettability. This geometric contact angle is normally measured from segmented pore-scale X-ray tomography images with interface identification, meshing and smoothing. However, the quality of the contact angle directly measured from voxelised images is highly dependent on image quality and resolution, which is related to the pore/throat size, and segmentation method. Furthermore, the contact angle may be an equilibrium value, or represent a pinned interface, and so is not necessarily representative of a displacement process. We have developed an alternative approach to obtain the thermodynamic contact angle based on the interfacial energy balance during fluid displacement (brine displacing oil). This angle can be determined from the local capillary pressure through measuring the interfacial curvature, the changes of fluid saturation, and the change of interfacial areas between different phases. We validate this approach by simulating immiscible two-phase flow at the pore-scale in a water wet Bentheimer sandstone using the colour-gradient lattice Boltzmann method. Simulations with both geometric and thermodynamic contact angles were performed and compared with a steady-state two-phase flow experiment on the same sample. The results show that the simulation using the thermodynamic contact angle consistently gave better agreement for the fluid distribution and capillary pressure . This approach has also been further applied to porous media with more complex wettability (a Bentheimer sandstone with altered wettability) to provide a more representative wettability input for pore-scale modelling

Procter and Gamble Student poster award:

I would like to compete in the Procter and Gamble Student award References:

Acceptance of Terms and Conditions:

Click here to agree

\section{Population balance modelling of particle deposition and aggrega- tion in porous media}

\author{
Gianluca Boccardo $^{1}$; Rajandrea Sethi ${ }^{2}$; Daniele Marchisio ${ }^{2}$ \\ ${ }^{1}$ Politecnico di Torino, Italy \\ ${ }^{2}$ Politecnico di Torino
}


Corresponding Author(s): boccardo.gianluca@gmail.com

Our work deals with the investigation of particle transport and deposition in porous media, a problem with practical implications in many processes, concerning both natural and engineered systems: aquifer remediation for freshwater extraction, packed bed chemical reactors, enhanced oil recovery are some examples. In all of these cases, it is of the utmost importance to analyse the transport of particulate matter in the porous medium and investigate their evolution due to deposition and aggregation phenomena, in order to eventually obtain an upscaled transport model employable at the macroscopic scale.

The deposition of particles through the system is quantitatively expressed by the deposition efficiency in the porous bed, whose theoretical reference lies in the classical colloid filtration theory (CFT), and for which the the most important parameter is particle size.

Theoretical works based on the CFT studying particle deposition at the pore-scale suffer a big disadvantage: mainly, they ignore the effect of packing randomness on macroscale fluid flow behaviour, or are limited to very simple particle shapes, thus neglecting the effects of the grains roughness and shape on mass transport. Moreover, no comprehensive pore-scale study of the influence of the geometrical features of the porous medium on particle removal mechanisms is still available, whereas both the packed bed porosity and tortuosity have been shown to have a marked influence on particle deposition efficiency. Last but not least deposition involving a population of particles characterized by a particle size distribution has not been studied before.

Thus, our novel approach relies on the construction of an array of 3D realistic models using the open-source code Blender, which allows for a fast and robust modelling of packings of arbitrary grain size distribution and grain shape. Having obtained the geometrical models of the porous media considered, flow field and particle transport were then investigated using a finite-volume CFD code (OpenFoam). This methodology allowed us to perform a comprehensive study of all the relevant geometric and fluid dynamic variabilities at the pore-scale and obtain the particle deposition efficiency for each case.

Another important innovation in our work is the introduction of the population balance equation, whose solution is dealt with using the Quadrature Method of Moments (QMOM). This approach allowed us to consider the evolution of a non-uniformly distributed population of particles which remains unexplored in the current state of the art regarding particle deposition and will allow for a description of aggregation phenomena at the macroscopic scale.

The effects of these innovations are made apparent by the results of this work, which clearly show how the standard CFT theory fails to correctly describe particle deposition in realistic, random, packed beds.

Other important differences are highlighted between studying an uniform particle distribution and non-uniform ones. For instance, the deposition process will remove preferentially the smaller particles, increasing the average particle diameter during the evolution of the dispersion process. This has an effect on reaction rates, dispersion coefficient, et c. that would be impossible to estimate with the classical methodology.

Procter and Gamble Student poster award:

\section{References:}

Boccardo, G., Sethi, R. and Marchisio, D.L., 2018. Fine and ultrafine particle deposition in packed-bed catalytic reactors. Chemical Engineering Science. Acceptance of Terms and Conditions:

Click here to agree

\section{A robust upscaling of the effective particle deposition rate in porous media}

Gianluca Boccardo ${ }^{1}$; Rajandrea Sethi ${ }^{2}$; Matteo Icardi ${ }^{3}$ 


\footnotetext{
${ }^{1}$ Politecnico di Torino, Italy

${ }^{2}$ Politecnico di Torino

${ }^{3}$ University of Nottingham
}

Corresponding Author(s): boccardo.gianluca@gmail.com

In the upscaling from pore to continuum (Darcy) scale, reaction and deposition phenomena at the solid-liquid interface of a porous medium have to be represented by macroscopic reaction source terms. The effective rates can be computed, in the case of periodic media, from three-dimensional microscopic simulations of the periodic cell. Several computational and semi-analytical models have been studied in the field of colloid filtration to describe this problem. They typically rely on effective deposition rates defined by complex fitting procedures, neglecting the advection-diffusion interplay, the pore-scale flow complexity, and assuming slow reactions (or large Péclet numbers). Therefore, when these rates are inserted into general macroscopic transport equations, they can lead to several conceptual inconsistencies and significant errors. To study more accurately the dependence of deposition on the flow parameters, in this work we advocate a clear distinction between the surface processes (that altogether defines the so-called attachment efficiency), and the pore-scale processes. With this approach, valid when colloidal particles are small enough, we study Brownian and gravitydriven deposition on a face-centered cubic (FCC) arrangement of spherical grains, and define a robust upscaling based on a linear effective reaction rate. The case of partial deposition, defined by an attachment probability, is studied and the limit of perfect sink is retrieved as a particular case. We introduce a novel upscaling approach and a particularly convenient computational setup that allows the direct computation of the asymptotic stationary value of effective rates. This allows to drastically reduce the computational domain down to the scale of the single repeating periodic unit. The savings are ever more noticeable in the case of higher Péclet numbers, when larger physical times are needed to reach the asymptotic regime and thus, equivalently, much larger computational domain and simulation time would be needed in a traditional setup. We show how this new definition of deposition rate is more robust and extendable to the whole range of Péclet numbers; it also is consistent with the classical heat and mass transfer literature.

Procter and Gamble Student poster award:

References:

Boccardo, G., Crevacore, E., Sethi, R. and Icardi, M., 2018. A robust upscaling of the effective particle deposition rate in porous media. Journal of contaminant hydrology, 212, pp.3-13. Acceptance of Terms and Conditions:

Click here to agree

\title{
"Hot Spots" observed in pore scale simulation of flow in carbon fibre felt electrodes limits the efficiency of Redox Flow Battery operation
}

\author{
Edo Boek $^{1}$; Jervis Rhodri ${ }^{2}$; Farrel Gray ${ }^{3}$ \\ ${ }^{1}$ Queen Mary University of London \\ ${ }^{2}$ University College London \\ ${ }^{3}$ Imperial College London
}

Corresponding Author(s): e.boek@qmul.ac.uk

The Vanadium Redox Flow Battery (VRFB) is one of the most promising Electro-Chemical Device (ECD) technologies for large scale local storage of renewable energy, such as wind and solar. Commercial exploitation of this technology has emerged, but a lack of fundamental understanding of VRFB operation is limiting the development of this new technology. In particular, battery longevity is a problem due to degradation of the porous graphite felt carbon fibre electrode material under electrochemical cycling. In addition, the energy density must be improved, as this is low compared to Li-ion battery performance. For these reasons, we investigated here the performance of 
micro-porous carbon fibre electrode materials using a combination of computational modelling and experimental characterisation. In particular, the surface area of the porous electrode is critical to the device performance. To optimize electrochemical reactions in the electrode, the reactive surface area has to be as large as possible. In terms of micro-structure, this means that the felt fibres must be distributed homogeneously. In current VRFB technology, however, the felts are woven bundles of fibres yielding a large local variation in voids and bundles. Recently, synchrotron micro-CT scanning was used 1 to image the 3D pore structure of a graphite felt in-operando. However, the resulting alteration of the actual flow field could not be quantified, as it was very difficult to measure in-situ due to limited spatio-temporal resolution. Also, it is difficult to obtain the altered flow field from average tortuosity and porosity calculations, as the relation between permeability and tortuosity / porosity is only known empirically. Here we calculate, for the first time, the relation between flow and altered micro-structural properties using direct flow calculations in pore space images of a representative volume of a carbon fibre material obtained from micro-CT imaging. We present detailed spatio-temporal pore space images of a representative 3D fibre felt geometry, and the corresponding flow field. The flow field was calculated using our home-grown Lattice-Boltz-mann (LB) code (see [2], [3] and [4]) on a big data set of 15 billion voxels using HPC facilities.

Our simulations show that the electrolyte is concentrated in local areas ("hot spots"), thus limiting electro-chemical reactions. The heterogeneity of the commercial carbon fibre material therefore reduces the efficiency of the electrode, as it may cause high voltage spots and therefore damage in the electrode. To mitigate problems associated with the heterogeneous nature of carbon fibre graphite felts, we propose a rational design approach to develop new fibrous carbon materials with superior properties.

Procter and Gamble Student poster award:

I don't want to compete References:

1 Jervis, R., Brown, L.D., Neville, T.P., Millichamp, J., Finegan, D.P., Heenan, T.M., Brett, D.J. and Shearing, P.R., (2016) Journal of Physics D: Applied Physics, 49(43), p.434002

[2] Zacharoudiou, I., Boek, E.S., \& Crawshaw, J. (2018), Nature Scientific Reports, 8:15561

[3] Zacharoudiou, I., Chapman, E., Boek, E.S. \& Crawshaw, J. (2017), Journal of Fluid Mechanics, 824, 550-573. doi:10.1017/jfm.2017.363

[4] Gray, F., Shah, S., Crawshaw, J., Anabaraonye, B. and Boek, E.S. (2018), Advances in Water Resources 121, 369-387 Acceptance of Terms and Conditions:

Click here to agree

\section{Wet granular materials: from building sandcastles to the con- struction of the Egyptian pyramids}

Daniel Bonn ${ }^{1}$

${ }^{1}$ Van der Waals-Zeeman Institute, Institute of Physics, University of Amsterdam, Science Park 904, 1098 XH Amsterdam, Netherlands

Corresponding Author(s): d.bonn@uva.nl

I will discuss the rheology and mechanical properties of wet granular materials, and show why the behavior can be very subtle. Once one understands the mechanical properties, I will show that one can use this knowledge to construct the perfect sandcastle, or to understand why the ancient Egyptians wetted the desert sand with water before sliding heavy stones over it (Figure).

I will then go on to show some new results on friction at the microscopic scale, between 2 grains. Amonton's famous friction law states that the friction force is proportional to the normal force since both are proportional to the area of contact. However for spherical grains, the contact area is not proportional to the normal force, as shown by Hertz long ago. We use a new fluorescence technique that allows us to probe the real area of contact between 2 rough surfaces. In our case, we conclude that important deviations from Amonton's law are observed.

Procter and Gamble Student poster award:

References: 


\title{
Reactive transport modeling in carbonate reservoirs and assess- ment of the adsorption of oil polar components on the rock sur- face
}

\author{
Maria Bonto $^{1}$; Ali Akbar Eftekhari ${ }^{1}$; Hamid Nick ${ }^{1}$ \\ ${ }^{1}$ Technical University of Denmark-DHRTC
}

Corresponding Author(s): mabon@dtu.dk

The calcite surface plays an important role in the development of hydrocarbon reserves, since this mineral is the main constituent of the carbonate reservoirs, which are estimated to hold more than $60 \%$ of the oil reserves. Although initially water-wet, most of these reservoirs become neutral to preferential oil-wet during the oil migration due to the adsorption of acidic groups from the oil on the calcite surface. It is observed that by altering the brine composition, the wettability of the rock shifts towards more water wet for certain brine composition. The phenomenon is called modified salinity water flooding. Despite many efforts, the modeling of this phenomenon is still based on simple correlations (i.e., interpolation of relative permeability curves as a function of brine salinity) that poorly reflect the physicochemical interaction in the chalk/brine/oil system. Moreover, the role of the crude oil composition has been traditionally completely disregarded. However, new experimental evidence suggests that the fluid-fluid interactions can even outweigh the role of the rock-fluid interactions on the wettability alteration. Therefore, in this work we first review the few available thermodynamic (surface complexation) models (SCM) for the brine-oil interface. We found that these models have important limitations: the chemistry of each crude oil is not considered, they cannot capture the water/non-polar hydrocarbons surface charge, the interactions between $\mathrm{Na}+$ and the acid sites are not included, and the equilibrium constants for the adsorption reactions are not validated against experimental data. We address the mentioned shortcomings by proposing an improved diffuse-layer SCM for the oil-brine interface. The new model accounts for the chemistry of crude oils by considering surface sites that are linearly dependent on the total acid number (TAN) and total base number (TBN). We define weak sites to account for the negative surface charge observed for non-polar hydrocarbons in water. We optimize the parameters of our model by fitting the model to reported zeta potential measurements of oil in aqueous solutions. We validate the optimized model against different experimental data sets, which generally shows a good performance in predicting the surface charge of oil in different brines with different $\mathrm{pHs}$. We show that the acid and base numbers are only useful as a qualitative estimation of the distribution of polar groups at the oil surface, and more sophisticated analysis is necessary to quantify the chemistry of the oil-brine interface. Later, we couple the optimized oil-brine diffuse layer model to a CD-MUSIC (Charge Distribution MultiSite Complexation) model for the calcite-brine interface, to investigate the interactions between the negatively charged carboxylic acids (dependent on the TAN) and the positively charged carbonate. These thermodynamic models are coupled to a finite volume solver, providing a numerical approximation of the transport of species and fluid and energy flow. The model is then validated against core flooding experiments that monitor the acid number at the outlet, which gives an indication of the extent of polar components adsorption/desorption on/from the surface of calcite.

Procter and Gamble Student poster award:

References:

Acceptance of Terms and Conditions:

Click here to agree

\section{The impact of small-scale rock heterogeneity on $\mathrm{CO} 2$ plume mi- gration and residual trapping}


Maartje Boon ${ }^{1}$; Hailun $\mathrm{Ni}^{1}$; Sally M. Benson ${ }^{1}$

${ }^{1}$ Stanford University

Corresponding Author(s): mmboon@stanford.edu

The lateral migration of the $\mathrm{CO} 2$ plume is often not well predicted by reservoir simulators where small-scale rock heterogeneity is not taken into account. Moreover, small-scale rock heterogeneity has a significant impact on the residual trapping potential of reservoir rock. In this research, we have measured and characterized the impact of small-scale rock heterogeneity on multiphase flow parameters and residual trapping of $\mathrm{CO} 2$. Furthermore, we have developed methods to find upscaled effective parameters that can be used to incorporate the effects of small-scale heterogeneities into larger scale models. This could significantly improve the prediction of plume migration which will increase our confidence in assessing storage capacity and leakage risks.

For this purpose, we performed experimental and numerical $\mathrm{CO} 2 /$ water core-flood tests at reservoir conditions for a range of sandstone rock cores (including the Fontainebleau, Bentheimer, Massillon and several cores of the Paraatte formation) containing different degrees and types of heterogeneity. The experimentally obtained $\mathrm{CO} 2$ saturation distributions, visualized with the use of a medical X-ray CT-scanner, were used to construct models of the sub-core scale permeability fields of the rock cores studied.

For each rock sample, accurate petrophysical properties such as porosity, permeability, degree of sub-core scale heterogeneity were extracted. The degree of heterogeneity, measured by the variance of the saturation distribution during drainage, proved to be the best predictor of residual gas trapping. By extrapolating the correlation relationship between the degree of heterogeneity and the linear trapping coefficient, we were able to show that pore-scale trapping mechanisms account for $47-99 \%$ of the residually trapped CO2 and capillary heterogeneity trapping mechanisms accounts for $1-53 \%$ of the residually trapped CO2 for the samples we tested. This suggests that capillary heterogeneity trapping has great potential in contributing to $\mathrm{CO} 2$ residual trapping.

The interplay of gravitational, viscous and capillary forces determines the $\mathrm{CO} 2$ saturation distribution and the amount of residual trapping that takes place. The relative permeablity function is flow rate dependent as a result of this. We used the models of the rock cores to simulate the core-flood tests for a range of flow rates and quantified the relative importance of each of the forces at the scale of the heterogeneity. The results show that the direction, magnitude and spatial extent of the heterogeneity impact the local capillary forces and, therefore, the capillary pressure and saturation distribution. Furthermore, our experimental and numerical results indicate that for layered rock with small variations in permeability, lamination direction has minimal impact on the local capillary forces and does not affect the residual trapping potential.

From the numerical simulations, upscaled flow-rate dependent effective relative permeabilties were obtained which can be used to incorporate flow rate dependency into larger scale models. In addition for the special cases of horizontally and vertically layered systems, analytical solutions were derived to obtain relative permeability as a function of flow rate and fractional flow. These solutions provide an easy way to investigate the impact of using multiple characteristic relative permeability curves, and different permeability ratio between layers, on the upscaled effective relative permeability.

Procter and Gamble Student poster award:

References:

Acceptance of Terms and Conditions:

Click here to agree

\section{Auxiliary space preconditioners for mixed-dimensional H(div) prob- lems}

Author(s): Wietse Boon ${ }^{1}$

Co-author(s): Ana Budiša ${ }^{2}$; Xiaozhe $\mathrm{Hu}^{3}$; Jan Martin Nordbotten ${ }^{2}$

${ }^{1}$ University of Stuttgart 


\footnotetext{
${ }^{2}$ University of Bergen

${ }^{3}$ Tufts University
}

Corresponding Author(s): wietse.boon@iws.uni-stuttgart.de

Mixed-dimensional modeling has become an active field of research, especially in the context of porous media applications. These models arise whenever thin structures such as fractures or faults, as well as subsurface layers such as aquifers, are represented as lower-dimensional manifolds embedded within the computational domain. In turn, the resulting model typically consists of coupled equations defined on manifolds of different dimensions, referred to as mixed-dimensional partial differential equations.

We focus on a subclass of such problems, namely those concerning mixed-dimensional $\mathrm{H}(\mathrm{div})$ spaces. These problems are common due to their direct connection to conservation laws, with examples including mass conservation in fracture flow and momentum balance in linear elasticity. One of the key challenges in this field is that the inherent scaling with small length parameters often leads to an ill-conditioned system of equations.

To tackle this problem, we introduce function spaces and differential operators on the mixed-dimensional geometry which ultimately leads to the formation of a mixed-dimensional de Rham complex. With the use of this complex, we prove that well-known decompositions of functions including the Hodge decomposition exist in the mixed-dimensional setting. Such a decomposition, which uses an auxiliary space corresponding to $\mathrm{H}(\mathrm{curl})$, then serves as the main ingredient for the proposed preconditioner.

By employing conforming, mixed finite elements of lowest order, we show both theoretically and numerically that these decompositions translate directly to discretized systems. To conclude, we present numerical experiments showing the performance of the mixed-dimensional auxiliary space preconditioner.

Procter and Gamble Student poster award:

References:

Acceptance of Terms and Conditions:

Click here to agree

571

\title{
ADAPTIVE MCMC METHODS APPLIED TO THE CHARACTER- IZATION OF ROCK PROPERTIES IN FLOW IN POROUS MEDIA
}

\author{
Marcio Borges $^{1}$; Maicon Correa ${ }^{2}$ \\ ${ }^{1}$ National Laboratory for Scientific Computing - LNCC \\ ${ }^{2}$ Unuversity of Campinas - UNICAMP
}

Corresponding Author(s): mrborges@lncc.br

The Metropolis algorithm (Metropolis et al., 1953) and its variants have been used extensively in many areas to generate target distributions. However, the convergence of this kind of method can be extremely slow due an inappropriate selection of shape and size of the proposal distribution used to generate trial moves in the Markov chain Monte Carlo methods, becoming the bottleneck of this methodology, mainly in high-dimensional problems. To overcome such problem several adaptive methodologies have been proposed in order to choose (or tuning) the proposal distribution (Haario et al., 1999, 2001; Mira, 2001; Haario et al., 2006). In this work we compare the classical random walk Metropolis algorithm, Differential Evolution -DE- (Ter Braak, 2006) and Differential Evolution Adaptive Metropolis -DREAM- (Vrugt et al., 2008) to draw samples from high-dimensional permeability fields in a two-phase flow problem. 
Procter and Gamble Student poster award:

\title{
References:
}

H. Haario, E. Saksman, and J. Tamminen. Adaptive proposal distribution for random walk Metropolis algorithm. Computational Statistics, 14(3):375-395, 1999.

Heikki Haario, Marko Laine, Antonietta Mira, and Eero Saksman. Dram: Efficient adaptive MCMC. Statistics and Computing, 16(4):339-354, 2006.

Heikki Haario, Eero Saksman, and Johanna Tamminen. An adaptive Metropolis algorithm. Bernoulli, 7(2):223-242, 042001.

Nicholas Metropolis, Arianna W. Rosenbluth, Marshall N. Rosenbluth, Augusta H. Teller, and Edward Teller. Equation of state calculations by fast computing machines. The Journal of

Chemical Physics, 21(6):1087-1092, 1953.

Antonietta Mira. On Metropolis-Hastings algorithms with delayed rejection. Metron, 59:3-4, 2001.

Cajo J. F. Ter Braak. A Markov chain Monte Carlo version of the genetic algorithm differential evolution: easy bayesian computing for real parameter spaces. Statistics and Computing, 16(3):239-249, Sep 2006. Jasper A. Vrugt, Cajo J. F. Ter Braak, Martyn P. Clark, James M. Hyman, and Bruce A. Robinson. Treatment of input uncertainty in hydrologic modeling: Doing hydrology backward with Markov chain Monte Carlo simulation. Water Resources Research, 44(12):n/a-n/a, 2008. Acceptance of Terms and Conditions:

Click here to agree

511

\section{Impact of mixing dynamics on fluid-solid reactions in porous me- dia: pore-scale imaging and modelling}

\author{
Hojjat Borhany $^{1}$; Joris Heyman ${ }^{2}$; Khalil Hanna ${ }^{3}$; Tanguy Le Borgne ${ }^{2}$ \\ ${ }^{1}$ Geosciences Rennes, École nationale supérieure de chimie de Rennes \\ ${ }^{2}$ Géosciences Rennes \\ ${ }^{3}$ Ecole Nationale Supérieure de Chimie de Rennes
}

Corresponding Author(s): hojjat.borhany@univ-rennes1.fr

\begin{abstract}
A key challenge for modelling reactive transport in porous media is to upscale the effect of solute concentration fluctuations at the pore scale on effective macroscale reaction kinetics (Battiato et al., 2009, Dentz et al. 2011). Experimental observations have shown the effect of solute mixing on reactions in mixing limited reactive fronts where reactions occur in the fluid phase (e.g. Gramling et al., 2002, Knutson et al. 2007, de Anna et al., 2013). However, investigating the control of solute mixing on fluid-solid reaction, which concerns a large spectrum of natural dissolution, adsorption or complexation reactions, requires new experimental approaches allowing the joint characterization of the pore scale distribution of solute concentrations and reaction rates at solid-fluid interfaces. Here we develop a three-dimensional experimental set up to image the pore scale distribution of solid-fluid reaction rate in order to explore the link between concentration fluctuations induced by mixing and effective reaction rates.

To quantify reaction rates at the solid-fluid interface, we graft fluorescence probe molecules on the functionalized surface of glass beads. Pore scale imaging is then obtained using laser induced fluorescence in optical-index matched porous media. Image processing provides quantitative pore scale measurements of key properties including the PDFs of concentration and reaction rates in threedimensions. Results are interpreted by developing recent mixing theories (Lester et al., 2016, Le Borgne et al., 2017, Souzy et al. 2018) to link the dynamics of fluid mixing in porous media to effective solid-fluid reaction kinetics.
\end{abstract}

\section{Procter and Gamble Student poster award:}

I would like to compete in the Procter and Gamble Student award References:

Battiato, I., Tartakovsky, D., Tartakovsky, A., \& Scheibe, T. (2009). On breakdown of macroscopic models of mixing-controlled heterogeneous reactions in porous media. Advances in Water Resources, 16641673.

de Anna, P., Jimenez-Martinez, J., Tabuteau, H., Turuban, R., Le Borgne, T., Derrien, M., \& Méheust, 
Y. (2013). Mixing and Reaction Kinetics in Porous Media: An Experimental Pore Scale Quantification. Environmental Science \& Technology, 508-516.

Dentz, M., Le Borgne, T., Englert, A., \& Bijeljic, B. (2011). Mixing, spreading and reaction in heterogeneous media: A brief review. Contaminant Hydrology, 1-17.

Gramling, C., H arvey, C. F., \& Meigs, L. C. (2002). Reactive Transport in Porous Media: A Comparison of Model Prediction with Laboratory Visualization. Environ. Sci. Technol, 2508-2514.

Knutson, C., Valocchi, A., \& Werth, C. (2007). Comparison of continuum and pore-scale models of nutrient biodegradation under transverse mixing conditions. Advances in Water Resources, 1421-1431.

Lester, D., Dentz, M., \& Borgne, T. L. (2016). Chaotic mixing in three-dimensional porous media. Journal of Fluid Mechanics, 803.

Souzy, M. Z., Lhuissier, H., Borgne, T. L., \& Metzger, B. (2018). Mixing lamellae in a shear flow. Journal of Fluid Mechanics, 838. Acceptance of Terms and Conditions:

Click here to agree

\title{
The Virtual Element method for simulations of physical phenom- ena on Discrete Fracture Matrix models
}

\author{
Andrea Borio $^{1}$; Stefano Berrone ${ }^{2}$; Stefano Scialò $^{1}$; Fabio Vicini ${ }^{1}$; Matias Fernando Benedetto ${ }^{3}$ \\ 1 Politecnico di Torino \\ ${ }^{2}$ Politecnico di Torino, Italy \\ ${ }^{3}$ University of Buenos Aires
}

Corresponding Author(s): andrea.borio@polito.it

The simulation of flow and transport phenomena in poro-fractured media is a very challenging task, mainly because of the high geometrical complexity of the domains, that introduces great difficulties to the efficient generation of good quality computational grids. The reliability and computational efficiency of these kind of simulations is of crucial importance for uncertainty quantification techniques, were many computations have to be performed on networks of fractures that are randomly generated from soil property distributions. To circumvent these issues, we propose a series of approaches [1-5] that exploit the flexibility of the Virtual Element method in dealing with polygonal and polyhedral meshes. We consider a fractured medium represented as a 3D Discrete Fracture network immersed in a rock matrix and discretize the domain by a minimal mesh where polyhedra are delimited by the polygons representing the fractures. Such approach guarantees a conforming mesh where the continuity of the solution and the balance of fluxes can be imposed strongly, thus obtaining good quality solutions.

Procter and Gamble Student poster award:

\section{References:}

1 M. F. Benedetto, S. Berrone, and A. Borio. "The Virtual Element Method for underground flow simulations in fractured media”. In: Advances in Discretization Methods. Vol. 12. SEMA SIMAI Springer Series. Switzerland: Springer International Publishing, 2016, pp. 167-186. doi: 10.1007/9783-319-41246-7_8.

[2] M.F. Benedetto, S. Berrone, and S. Scialò. "A Globally Conforming Method For Solving Flow in Discrete Fracture Networks Using the Virtual Element Method”. In: Finite Elem. Anal. Des. 109 (2016), pp. 23-36. doi: 10.1016/j.finel.2015.10.003.

[3] M.F. Benedetto et al. "A Hybrid Mortar Virtual Element Method For Discrete Fracture Network Simulations”. In: J. Comput. Phys. 306 (2016), pp. 148-166. doi: 10.1016/j.jcp.2015.11.034.

[4] Matías Fernando Benedetto, Andrea Borio, and Stefano Scialò. "Mixed Virtual Elements for discrete fracture network simulations". In: Finite Elements in Analysis \& Design 134 (2017), pp. 55-67. doi: 10.1016/j.finel.2017.05.011.

5 S. Berrone et al. "Advanced computation of steady-state fluid flow in Discrete Fracture-Matrix models: FEM-BEM and VEM-VEM fracture-block coupling”. In: GEM - International Journal on Geomathematics 9.2 (July 2018), pp. 377-399. issn: 1869-2680. doi: 10.1007/s13137-018-0105-3. Acceptance of Terms and Conditions:

Click here to agree 


\section{Gradient flow perspective on poroelasticity and energy minimiz- ing splitting schemes}

Jakub Both $^{1}$; Kundan Kumar $^{1}$; Jan Martin Nordbotten ${ }^{1}$; Florin Adrian Radu ${ }^{2}$

${ }^{1}$ University of Bergen

${ }^{2}$ University of Bergen, Norway

Corresponding Author(s): jakub.both@uib.no

Irreversible, dissipative processes can be naturally modeled as gradient flows; flow in deformable porous media modelled by the Biot equations is such a process. In this talk, we exploit the gradient flow structure of poroelasticity in order to analyze well-posedness of the problem and to accelerate classical splitting schemes - undrained split and fixed-stress split - using abstract gradient flow theory and convex optimization, respectively.

After time discretization, a gradient flow structure translates to an optimization problem, allowing the use of mature optimization theory to construct numerical solvers. For the particular case of the linear Biot equations, its time-discrete version translates into a quadratic optimization problem subject to linear constraints. Furthermore, the standard classical splitting schemes can be identified as alternating and hence successive minimization. Based on these observations, we propose two novel techniques to improve the performance of classical splitting schemes while reducing userinput:

- The stabilization parameter included in the splitting schemes is chosen such that energy is reduced maximally. This thereby resolves the question of optimal splitting parameters in the splitting scheme

- By exploiting the quadratic structure of the optimization problem, we note that the optimal damping factor can be calculated explicitly, thus accelerating convergence.

Both techniques are cheap and can be build in on top of existing implementations of splitting schemes. We note, acceleration via line search increases robustness and can be also applied to other iterative solvers than the classical splitting schemes, ensuring energy minimization. We also discuss the applicability of the approach for nonlinear processes in deformable porous media.

Procter and Gamble Student poster award:

I would like to compete in the Procter and Gamble Student award References:

Acceptance of Terms and Conditions:

Click here to agree

90

\section{Visualization of dynamic wormhole development in polymer gel using Positron Emission Tomography (PET)}

Author(s): Bergit Brattekås ${ }^{1}$

Co-author(s): Martin Ferno ${ }^{2}$

${ }^{1}$ Dept. of Physics and Technology, University of Bergen, Norway

${ }^{2}$ University of Bergen

Corresponding Author(s): bergit.brattekas@uib.no 
Highly conductive fractures through porous media may dominate fluid flow, hence injected fluids mainly channel through the fracture network without contacting large portions of the matrix volume. Establishing fluid flow within the porous matrix is important in many applications, e.g. during oil or natural gas recovery from, and $\mathrm{CO} 2$ storage in, geological structures. Several options exist to minimize fracture flow, therein the placement of polymer gel in the fracture network. With polymer gel in place, subsequently injected fluids may be diverted into the porous matrix. Polymer gel may be perceived as a flexible porous medium in itself, where the polymer structure of the gel constitutes a matrix of constant solid volume and the gel porosity is defined by the volume fraction of solvent. The behavior of polymer gel after placement in a fracture is complex and largely controlled by external conditions. We used core scale experiments and high-resolution Positron Emission Tomography (PET) to investigate the behavior of gel during water injection into gel-filled fractures through porous rock. The properties of water injection were varied, applying either: 1) a stepwise variation in the imposed pressure gradient (i.e. external mechanical stimuli), or 2) injection of low-salinity water, where the salinity of the injected water phase was decreased compared to the gel solvent (i.e. a change in external chemical composition).

The structural integrity of gel placed in a fracture may often be maintained at low differential pressure gradients. An increase in the pressure gradient may cause the gel to rupture, creating a pattern of void space, often termed wormholes. Injected fluids may resume flow through the partially gelfilled fracture, preferentially flowing through wormholes. A significant reduction in fracture conductivity may be maintained over an extended period of time due to the elasticity of gel, where the wormholes collapse or expand depending on the imposed pressure gradient. PET enabled visualization of dynamic wormhole development as a function of imposed pressure and water throughput. The blocking capacity of a ruptured polymer gel is expected to decrease with water throughput, i.e. the wormholes are expected to increase in size during water injection. However; gel also has the ability to shrink and swell. Volumetric changes in gel may occur due to changes in a number of external conditions. Recent work shows that a salinity contrast between the gel solvent and surrounding brine implemented volumetric changes; gels generally swell in lower salinity water and shrink in higher salinity water. We show that injection of low-salinity water may reverse the damages of gel rupture, thus increasing the blocking capacity of gel with water throughput, contradictory to previous findings. Visualization by PET was used to search for the mechanism behind improved gel blocking.

Procter and Gamble Student poster award:

\title{
References:
}

Acceptance of Terms and Conditions:

Click here to agree

\section{Effective behavior of an upscaled phase field model for reactive transport}

\author{
Carina Bringedal $^{\text {None }}$; Iuliu Sorin Pop ${ }^{1}$; Manuela Bastidas ${ }^{1}$ \\ ${ }^{1}$ Hasselt University
}

Corresponding Author(s): carina.bringedal@uhasselt.be

Reactive flow with mineral precipitation and dissolution includes an evolving interface at the pore scale as ions precipitate and minerals dissolve, affecting the pore scale structure. Large-scale properties such as the effective diffusivity and the permeability of the porous medium is affected. Such a problem is difficult to handle numerically, both due to the evolving interface but also due to the difference in scales and the highly oscillatory behavior at the pore scale. A direct simulation would require a very fine grid to resolve the oscillatory behavior.

We here consider a phase field formulation for the coupled flow and reactive transport resolving the evolving interface at the pore scale, which, using periodic homogenization, is upscaled to Darcy 
scale. The upscaled model provides the effective behavior and introduces Darcy velocities where the permeability is found through a cell problem incorporating the phase field at the pore scale.

In a numerical simulation the Darcy-scale equations are time-stepped, where they in each time step receive information from the updated cell problems as the phase field evolves. However, the cell problems are decoupled from each other through the periodicity assumption and can be solved in parallel. Hence, the upscaled model is much faster compared to a direct simulation.

Procter and Gamble Student poster award:

References:

Acceptance of Terms and Conditions:

Click here to agree

\title{
The effect of saturation dependent capillary diffusion on radial Buckley-Leverett flow
}

Bernard Meulenbroek $^{1}$; Negar Khoshnevis Gargar ${ }^{2}$; Hans Bruining ${ }^{1}$

${ }^{1}$ TU-Delft

${ }^{2}$ Deltares

Corresponding Author(s): j.bruining@tudelft.nl

One-D Water oil displacement in porous media is usually described by the Buckley-Leverett equation or the Rapoport-Lease equation when capillary diffusion is included. The 1-D geometry is not representative of a general oil displacement problem. It is therefore of interest to describe the Buckley-Leverett or Rapoport-Lease equation in radial geometry. We can show that under appropriate conditions one apply a similarity transformation $\eta=r^{\wedge} 2 / 2 t$ that reduces the PDE in radial geometry to an ODE, even (as opposed to the situation in the linear geometry) when capillary diffusion is included. We consider two cases (1) where the capillary diffusion is constant independent of the saturation and (2) when we include saturation dependent capillary diffusion. It turns out that the solution with a constant capillary diffusion coefficient is fundamentally different from the solution, with a saturation dependent capillary diffusion. The most conspicuous difference is the behavior around the dispersed shock, where we obtain a smoothly dispersed "shock" in the constant diffusion case and a power-law behavior around the shock for a saturation dependent capillary diffusion. We compare the numerical solution of the initial value problem obtained with a finite element software package to a partially analytical solution of the problem in terms of the similarity variable $\eta$.

Procter and Gamble Student poster award:

References:

Acceptance of Terms and Conditions:

Click here to agree

\section{Iterative algorithms for coupled thermo-poroelasticity}

\author{
Elyes Ahmed ${ }^{\text {None }}$; Florin Adrian Radu ${ }^{1}$; Inga Berre ${ }^{2}$; Jan Martin Nordbotten ${ }^{\text {None }}$; Mats Brun ${ }^{2}$ \\ ${ }^{1}$ University of Bergen, Norway \\ ${ }^{2}$ University of Bergen
}


Corresponding Author(s): mats.brun@uib.no

This work is concerned with iterative solution procedures for coupled thermo-poroelasticity. The thermo-poroelastic model problem we consider is formulated as a three-field system of PDE's, consisting of an energy balance equation, a mass balance equation and a momentum balance equation, where the primary variables are temperature, fluid pressure, and elastic displacement. Due to the presence of a nonlinear convective transport term in the energy balance equation, it is convenient to have access to both the pressure and temperature gradients. Hence, we introduce these as two additional variables and extend the original three-field model to a five-field model. Based on this formulation, we propose six different iterative algorithms, where we at each iteration either split the problem into several subproblems, or solve monolithically a linearized system. These methods are based on the well-known 'fixed stress splitting algorithm' from poroelasticity, extended to also include thermal effects, and also capable of resolving the nonlinearity in the model. We also provide a convergence proof for the algorithms, and validate our results through numerical examples.

Procter and Gamble Student poster award:

References:

Acceptance of Terms and Conditions:

Click here to agree

\title{
Multiphase Flow Simulations in Large Domains of Upper Maas- trichtian Chalk
}

\author{
Author(s): Stefan Bruns ${ }^{1}$ \\ Co-author(s): Henning Osholm Sørensen ${ }^{2}$ \\ ${ }^{1}$ Technical University of Denmark \\ ${ }^{2}$ Teechnical University of Denmark
}

Corresponding Author(s): bruns@fysik.dtu.dk

Simulation of multiphase flow parameters needs to be carried out in domains that are large enough for capturing the representative pore connectivity. For pore scale imaging of chalk nanometer resolution is required. Reconstructed domains of chalk from nanotomography barely meet the connectivity requirement. Such simulations are performed on limited size volumes and therefore only provide snapshots of the larger scale properties, which implies that statistical significance can only be achieved by repeated sampling.

Until recently the computational burden associated with multiphase simulations prohibited comprehensive studies on the pore scale without the use of supercomputing facilities. With up to 32 GB of memory modern graphic card technology is now capable of handling large scale domains in multiphase Lattice Boltzmann simulations enabling the computation of results within a reasonable timeframe of days or even hours.

We developed a three-dimensional phase-field Lattice Boltzmann solver for GPUs, which is fast and memory efficient. Here we present benchmarks for the solver and initial results obtained from simulating drainage and imbibition processes in samples of Upper Maastrichtian chalk. The samples were extracted core plugs and imaged by X-ray nanotomography. For each core plug several samples were evaluated with respect to relative permeability and capillary pressure. Finally, we discuss different drainage protocols and the influence of finite size effects on the estimated water saturation.

\section{Procter and Gamble Student poster award:}

I don't want to compete References:

Acceptance of Terms and Conditions:

Click here to agree 


\section{Fourier-Transform Laser Speckle Imaging for fast, quantitative and real-time flow imaging}

Author(s): Jesse Buijs ${ }^{1}$

Co-author(s): Jasper van der Gucht ; Joris Sprakel

${ }^{1}$ Wageningen University

Corresponding Author(s): jesse.buijs@gmail.com

Laser Speckle imaging (LSI) is a very powerful imaging technique that visualizes flow. Compared to other optical imaging techniques it has several advantages: it works well within turbid materials, has very high spatial and temporal resolution, is sensitive to extremely small displacements and set-ups are generally very low-cost. However the two existing data analysis algorithms each have a serious drawback. One algorithm gives quantitative results but takes a huge amount of calculation time, while the other algorithm is fast but not quantitative.

We propose a new algorithm that is both fast and quantitative. This new algorithm uses the fast Fourier transform to quantify dynamics and visualize flow. The existing quantitative method is based on the auto-correlation function which mathematically holds the same information as the Fourier spectrum. However the Fourier spectrum is up to 500 times faster to calculate! We have implemented this new algorithm on a portable LSI set-up where the real-time analysis is performed on a tablet. This set-up increases the accessebility and applicability of LSI for imaging flow.

Procter and Gamble Student poster award:

I would like to compete in the Procter and Gamble Student award References:

Acceptance of Terms and Conditions:

Click here to agree

407

\section{Improving the description of two-phase flow in rocks by integrat- ing pore scale models and experiments}

Tom Bultreys ${ }^{1}$; Kamaljit Singh ${ }^{2}$; Ali Q. Raeini ${ }^{2}$; Leonardo C. Ruspini ${ }^{3}$; Pål-Eric Øren ${ }^{3}$; Steffen Berg ${ }^{4}$; Branko Bijeljic $^{2}$; Martin J. Blunt ${ }^{2}$

${ }^{1}$ Department of Earth Science and Engineering, Imperial College London, London, UK

${ }^{2}$ Department of Earth Science and Engineering, Imperial College London, London, UK

${ }^{3}$ Petricore Norway AS

${ }^{4}$ Shell International Exploration and Production BV

Corresponding Author(s): tom.bultreys@ugent.be

Two-phase fluid flow in rocks depends crucially on the underlying pore-scale physics. While numerical modelling at this scale has received great attention in recent years, it has remained unclear whether simulation discrepancies derive from a lacking description of the physics or from other sources. In this work, we investigate this by directly comparing the sequence in which pores are invaded during imbibition in numerical models to experimental observations based on fast synchrotron micro-computed tomography. Our methodology allows to compare models to experiments on a pore-to-pore basis in sandstone and carbonate samples, and captures the importance of filling discrepancies on the averaged flow properties. The analysis proves that quasi-static physics which take capillary dominated pore filling and throat snap-off into account can provide a good first-order approximation of imbibition in strongly-wetted rocks at low capillary numbers. Furthermore, we investigate how this experimental analysis can be extended into data-assisted pore-scale models, 
aiming to make pore-scale models with low computational cost more reliable and more broadly applicable.

Procter and Gamble Student poster award:

References:

Acceptance of Terms and Conditions:

Click here to agree

\title{
A Finite-Volume Approach for Two-Phase Flow in Fracturing Porous Media
}

Samuel Burbulla ${ }^{1}$; Christian Rohde ${ }^{1}$

${ }^{1}$ University of Stuttgart

Corresponding Author(s): samuel.burbulla@mathematik.uni-stuttgart.de

The mutual interaction between the fluid flow in fractured porous media and propagating, possibly bifurcating fractures is important for the overall systems' behavior in many natural and technical applications.

We consider porous media where dominant fractures are kept in the mathematical model as geometric structures up to the Darcy scale. Those will be understood as sharp interfaces and will be represented by dimension reduced manifolds. Incompressible two-phase flow formulated in the fractional flow formulation will be considered in the bulk as well as in the fracturing domain.

We propose a fully conforming Finite-Volume approach where all fractures coincide with volume edges. For the fracture network we rely on a Finite-Volume scheme formulated on manifolds. Appropriate coupling conditions are used to fix numerical fluxes orthogonal to the lower-dimensional fracture network. To track fracture propagation we will employ a moving-mesh concept that restricts the topological changes to locally marked regions.

Procter and Gamble Student poster award:

References:

Acceptance of Terms and Conditions:

Click here to agree

\section{Understanding the increased microbial activity of soils after rain- fall events: a modeling analysis}

\author{
Author(s): Albert C. Brangarí ${ }^{1}$ \\ Co-author(s): Stefano Manzoni ${ }^{2}$; Johannes Rousk ${ }^{3}$ \\ ${ }^{1}$ Centre for Environmental and Climate Research, Lund University \\ ${ }^{2}$ Department of Physical Geography, Stockholm University \\ ${ }^{3}$ Microbial Ecology, Department of Biology, Lund University
}


Corresponding Author(s): albert.brangari@cec.lu.se

The terrestrial ecosystems in arid, semi-arid and Mediterranean areas are characterized by extended periods of drought followed by rainfall events, creating pronounced cycles of drying-rewetting (DRW). As a consequence, the resident microorganisms need to respond to these extreme dynamics in environmental conditions, which is known to induce some of the most dynamic patterns of microbial growth and respiration documented. Thus, DRW events can account for the dominant fraction of carbon dioxide emissions to the atmosphere in ecosystems affected by drought, with strong implications for the global carbon cycle and climate change.

Despite their great significance, the mechanisms underlying microbial metabolic dynamics induced by DRW in soils are still debated and several questions remain open - What is the carbon source sustaining the increased proliferation of microbes? Which mechanisms control the intensity and duration of the respiration pulses? How the growth and respiration rates are connected after rewetting? Specifically, two categorically different microbial responses to rewetting have been identified; a more efficient way of growing where microorganisms start growing immediately upon rewetting, coinciding with respiration rates that peak immediately and then decrease exponentially ("Type 1"), or more 'wasteful' response in which bacteria grow exponentially after an extensive lag period (up to $20 \mathrm{~h}$ ), with a sustained period of elevated respiration, sometimes followed by a further increase coinciding with the emergence of bacterial growth ("Type 2"). Previous studies have suggested that the response upon rewetting could be related to the harshness of the disturbance as experienced by the microbes, with more "harsh" (i.e. longer or more severe) drying resulting in a Type 2 response [Meisner et al., 2017]. However, the specific underlying mechanisms remain unknown.

Empirical evidence and theoretical work point to several potential processes and mechanisms that strongly influence the nature of microbial dynamics. On the one hand, the amount of labile carbon is increased after rewetting because the organic matter accumulated during the preceding dry period becomes available, sourced from e.g. dead microbial biomass and extracellular microbial products, and previously physically protected soil aggregates are disrupted during intense dry periods. On the other hand, microbial physiological processes such as osmoregulation, dormancy/reactivation of cells, and synthesis/reuse of extracellular polymeric substances are activated as a direct response to the extreme environmental changes from wet to dry, or dry to wet, conditions.

In this work, we address the hypotheses proposed and formalize them via a modeling approach built on the process-based soil biofilm model by Brangarí et al. [2018]. Specifically, we evaluate the simplest scheme that allows capturing the general microbial patterns observed after rewetting of dry soils. We also identify the environmental and microbial factors that can trigger a switch from the type 1 to the type 2 response to rewetting, and vice versa. These scenarios allow disentangling the putative drivers of microbial growth and respiration pulses in a set of virtual experiments that subsequently can be interrogated by empirical experiments.

Procter and Gamble Student poster award:

References:

Acceptance of Terms and Conditions:

Click here to agree

\title{
A new gas transport model of shale stratum based on fractal porous media
}

\author{
Author(s): Jianchao Cai ${ }^{1}$ \\ Co-author(s): Wei Wei ; Duanlin Lin \\ ${ }^{1}$ China University of Geosciences (Wuhan)
}

Corresponding Author(s): caijc@cug.edu.cn 
Shale gas, an unconventional energy resource, has proven to be a boon in many ways. Its reservoirs have extremely low permeability, low porosity, are rich in organic matter, and have complex pore structures. Many models have been proposed to describe gas flow in shale reservoirs where all the models account for some additional flow mechanism besides the viscous flow (Darcy's law). In this work, a model for gas transport in shale is proposed by accounting for three major fluid flow mechanisms in shale stratum, which is modeled as a 3D fractal media. The proposed apparent permeability of shale is an analytical expression that also accounts for heterogeneous pore sizes in shale stratum, and is verified using experimental datasets for methane and helium flow in shale. Results of sensitivity analysis indicate that surface diffusion of adsorbed gas plays an important role, specifically in smaller pores, while surface diffusion would be negligible in larger pores. Further, the proposed model shows that flow due to surface diffusion decreases moderately with the increase of isosteric adsorption heat, while it increases significantly with the increase of the maximum adsorption capacity. One of the key novelties of the proposed permeability model is that it accounts for pore size distribution to reveal novel insights on gas transport in shale that can be used to optimize gas production by operational controls (e.g. controlling reservoir pressure) as flow regimes change with time.

Procter and Gamble Student poster award:

\title{
References:
}

1. Javadpour, F., Fisher, D., Unsworth, M., 2007. Nanoscale gas flow in shale gas sediments. J. Can. Pet. Technol. 46, 55-61.

2. Jarrahian, A., Heidaryan, E., 2014. A simple correlation to estimate natural gas viscosity. J. Nat. Gas Sci. Eng. 20, 50-57.

3. Yu, B., Li, J., 2001. Some fractal characters of porous media. Fractals 9, 365-372.

\section{Acceptance of Terms and Conditions:}

Click here to agree

28

\section{Phase-field modeling of shrinkage-induced cracking in cement mortar}

\author{
Tuanny Cajuhi ${ }^{1}$; Pietro Lura ${ }^{2}$; Laura De Lorenzis ${ }^{1}$ \\ ${ }^{1}$ Institute of Applied Mechanics, Technische Universität Braunschweig, Germany \\ ${ }^{2}$ Department of Civil, Environmental and Geomatic Engineering, ETH Zürich, Zürich, Switzerland
}

Corresponding Author(s): t.cajuhi@tu-braunschweig.de

Due to the multiphase nature of porous media, pore fluid redistribution induces a variation of strains in the solid matrix according to the theory of effective stress $[1,2]$. In cement-based materials, this phenomenon is related to the change of volume at early states because of thermal shrinkage, autogenous and drying shrinkage. In the presence of restraints (self-, internal or external), cracks may develop [3, 4]. Such early-age cracks can affect the durability of the material as they facilitate the transport of harmful chemical substances into the medium. Objective of this work is to describe drying shrinkage and cracking in cementitious materials with the theory of porous media and the phase-field approach to fracture. The phenomenon of drying is modeled within the poromechanical theory of 5, where the average properties of the solid and the fluid water phases are taken into account. Crack initiation,propagation and branching can be numerically modeled by the phasefield approach, which describes cracks as diffusive interfaces and offers an unprecedented flexibility in describing crack patterns with arbitrarily complex topology. The poromechanical-phase-field framework in the context of variable saturation [8] is calibrated with original experimental data. We present the calibration procedure and validation of the framework with some examples of shrinkageinduced cracks observed experimentally.

Keywords: phase-field modeling of fracture, shrinkage, drying, cement, mortar. 
1 K. Terzaghi. Erdbaumechanik auf Bodenphysikalischer Grundlage. Franz Deuticke, Liepzig-Vienna, 1925.

[2] A.W. Bishop and G.E. Blight. Some aspects of effective stress in saturated and partly saturated soils. Geotechnique, 13(3):177\{197, 1963.

[3] J. Bisschop. Drying shrinkage microcracking in cement-based materials. TU Delft, Delft University of Technology, 2002.

[4] P. Lura. Autogenous deformation and internal curing of concrete. PhD thesis, TU Delft, Delft University of Technology, 2003.

5 O.C. Zienkiewicz, A.H.C. Chan, M. Pastor, B.A. Schrefler, and T. Shiomi. Computational geomechanics - with special

reference to earthquake engineering. John Wiley \& Sons, 1999.

[6] T. Cajuhi, L. Sanavia, and L. De Lorenzis. Phase-field modeling of fracture in variably saturated porous media. Computational Mechanics, 61(3):299-318, 2017.

Procter and Gamble Student poster award:

References:

Acceptance of Terms and Conditions:

Click here to agree

\title{
Research on productivity evaluation and development optimiza- tion of tight oil-Taking the Chang 7 Hua H6 Platform as an Ex- ample
}

Jinjian Cao None

Corresponding Author(s): 1577362614@qq.com

\begin{abstract}
With the rapid increasing demand of energy in the world, unconventional oil and gas resources have received more and more attention, especially the tight oil -it has become a hot spot in global oil and gas exploration, and an important replacement resource for the future oil and gas production. In recent years, tight oil exploration and development has been extremely frequent and active. As an important form of energy supply, most countries and regions in the world have discovered this resource.

Tight oil reservoirs of Chang 7 Formation in the Ordos Basin are abundant, but the reservoir physical property is poor and the formation pressure is insufficient, so there are many complicated problems for its exploration and development. In order to achieve the goal of profitable production, we selected the Hua H6 platform as a case study, planned to forecast and evaluate its productivity, and further to optimize its productivity- based on the condition that we have determined the main control factors. Eventually, suitable tight oil exploration and development program for Chang 7 Formation was established.
\end{abstract}

Key word: tight oil; productivity evaluation; development optimization; Hua H6 platform

Procter and Gamble Student poster award:

References:

Acceptance of Terms and Conditions:

Click here to agree

675

Unraveling the interplay of different sorption-induced deformation mechanisms in a slit pore: an atomistic simulation approach 
Author(s): Mingyang Chen ${ }^{1}$

Co-author(s): Benoit Coasne ${ }^{2}$; Robert Guyer ; Dominique Derome ${ }^{3}$; Jan Carmeliet ${ }^{4}$

\author{
${ }^{1}$ ETH Zurich \\ ${ }^{2}$ CNRS/University Grenoble Alpes \\ ${ }^{3}$ Empa \\ ${ }^{4}$ ETHZ
}

Corresponding Author(s): cajan@ethz.ch

Common phenomena in porous materials ranging from nano- to meso- and macro-pore scale are sorption hysteresis and sorption-induced deformations. In this paper, we focus on meso-porous materials and analyze sorption hysteresis and sorption-induced deformations in a slit pore. Sorptioninduced deformations are generally attributed to the interaction of three mechanisms: a disjoining pressure, a surface stress in the film forming region, as described by Bangham or Shuttleworth, and a Laplace pressure when capillary condensation occurs and menisci between liquid and vapor phase appear.

Several studies have been conducted on the different mechanisms at play of sorption-induced deformations, as well as their interplay both experimentally and theoretically, but an integrated approach is still lacking. Since the origin of these phenomena arises from the atomistic/molecular level, where intermolecular forces are induced due the fluid-solid and fluid-fluid interactions, we perform hybrid atomistic simulations including both Grand Canonical Monte Carlo (GCMC) and Molecular Dynamics (MD). In this study, we aim to characterize the contributions of different mechanisms at play that explain the sorption-induced deformation when argon atoms ad- and desorb in an academic, but realistic slit pore model.

The simulated sorption isotherms, characterized by first a film thickening and then a capillary filled regime, are consistent with experimental and theoretical studies. They show hysteresis, attributed to the capillary condensation and evaporation process at different relative pressures. With respect to deformation in the film thickening region, the slit pore shows negligible deformation in normal direction, while a significant shrinkage occurs in longitudinal direction at very low absorbed amount. This longitudinal shrinkage is generally explained by the surface stress, but we show that this deformation already occurs much before a single film layer is formed. The shrinkage is found to result from isolated adsorbed fluid atoms trying to attract the surrounding solid atoms, making the solid substrate to shrink. This shrinkage can turn over to swelling when the first adsorbed layer is formed and becoming repulsive, turning to the classical Bangham effect. When capillary condensation occurs, a sudden shrinkage occurs in normal and longitudinal directions as explained and properly modelled by the Laplace pressure. During further adsorption, the menisci at the ends of the slit pore flatten, decreasing the shrinkage as the relative pressure increases. The small mismatch between deformations predicted by Laplace law and atomistic simulations is attributed to the disjoining pressure. Analysis of isosteric heat of adsorption shows that the deformation-related isosteric heat is relative small compared to sorption-related isosteric heat, which indicates that the influence of deformation on the sorption process is limited. Finally, the effect of temperature on the different mechanisms is analyzed.

Procter and Gamble Student poster award:

I don't want to compete References:

Acceptance of Terms and Conditions:

Click here to agree

\title{
The various scenarios of liquid water formation in the cathode gas diffusion layer of PEM fuel cells.
}

Pierre Carrere $^{1}$; Marc Prat $^{1}$ 
${ }^{1}$ Institut de Mécanique des Fluides de Toulouse (IMFT), Université de Toulouse, CNRS, Toulouse, FRANCE

Corresponding Author(s): pierre.carrere@imft.fr

The cathode Gas Diffusion Layer (GDL) is considered as a critical component as regards the water management in Proton Exchange Membrane Fuel Cells (PEMFC), a key issue in order to improve the PEMFC efficiency and durability. In this context, the identification and the understanding of the exact mechanisms responsible for the liquid water formation in cathode GDLs are therefore of the uttermost importance for improving the water management.

This topic has been widely studied through experimental works and pore network model (PNM) simulations. So far, two liquid water formation scenarios have been considered in the PNM models. In the first scenario, referred to as the liquid invasion scenario, the produced water enters the GDL in liquid phase and crosses it finding paths of lower capillary resistance. In the second scenario, namely the condensation scenario, the produced water enters the GDL in vapour phase, diffuses through it and condenses in colder areas, e.g. 1. However, those PNM models are only valid for very specific conditions and are therefore not able to simulate the whole range of fuel cell operating conditions. In particular, none of them are in agreement with the observations in fuel cells operating with a reactant gas high relative humidity $(\sim 100 \%)$ at standard temperatures $\left(\sim 80^{\circ} \mathrm{C}\right)$, which are conditions very frequently encountered in PEMFC.

To overcome these limitations, we present a new pore network model [2] aiming at simulating the full range of operating conditions. This model combines the main features of previous models: evaporation condensation phenomena are taken into account and water can enter the GDL both in liquid and vapour phases.

Good agreements with experimental observations are obtained over an extended range of PEM fuel cell operating conditions. As a result, the new model can simulate not only the operating conditions for which the previous models were satisfactory but also the conditions that could not be adequately simulated with previous models. This leads to identify three main liquid formation scenarios depending on the operating conditions: the liquid invasion scenario where liquid - vapour phase change phenomena are negligible, the condensation scenario where phase change phenomena control the liquid water formation and the mixed scenario where the invasion in liquid phase from the catalyst layer and the condensation - evaporation phenomena are both important.

The model can also take into account wettability heterogeneities in relation with the study of degradation mechanisms in GDLs (loss of hydrophobicity) or for optimising the GDLs (in terms of microstructure and/or heterogeneous wettability properties).

1 B. Straubhaar, J. Pauchet, M. Prat, Pore network modelling of condensation in gas diffusion layers of proton exchange membrane fuel cells, Int. J. Heat and Mass Transf. 102, 891-901 (2016)

[2] P. Carrere, M. Prat, Liquid water in cathode gas diffusion layers of PEM fuel cells: Identification of various pore filling regimes from pore network simulations, Int. J. Heat Mass Transf. 129, 1043-1056 (2019)

Procter and Gamble Student poster award:

\section{References:}

B. Straubhaar, J. Pauchet, M. Prat, Pore network modelling of condensation in gas diffusion layers of proton exchange membrane fuel cells, Int. J. Heat and Mass Transf. 102, 891-901 (2016)

P. Carrere, M. Prat, Liquid water in cathode gas diffusion layers of PEM fuel cells: Identification of various pore filling regimes from pore network simulations, Int. J. Heat Mass Transf. 129, 1043-1056 (2019) Acceptance of Terms and Conditions:

Click here to agree

\section{A Multiphase Darcy-Brinkman-Biot Approach to Modeling the Hydrology and Mechanics of Porous Media Containing Macrop- ores and Deformable Microporous Regions}

Author(s): Francisco Carrillo ${ }^{1}$ 
Co-author(s): Ian Bourg ${ }^{2}$

${ }^{1}$ Princeton University

${ }^{2}$ Princeton Univeristy

Corresponding Author(s): franjcf@outlook.com

The hydrology of soft porous materials such as clays, hydrogels, membranes, or biofilms is an active research area with important implications in water and energy technology as well as biomedical engineering. A key challenge in these areas is that many soft porous materials (including clay-rich sediments and sedimentary rocks) contain both macropores and micropores, i.e., they are inherently multi-scale structures. Well-established models based on poromechanics theory exist for describing the hydrology and mechanics of soft porous materials, but these models are not adapted to describe systems with more than one characteristic length scale. In this paper, we expand upon the wellknown Darcy-Brinkman formulation to develop a "Darcy-Brinkman-Biot" formulation, i.e., a general coupled system of equations that resemble the equations for poroelasticity in the microporous regions and approximate the Navier-Stokes equations in fluid-filled macropores. Previously we have shown that this model can be used to model single-phase flow within both plastic solids (swelling clays) and elastic solids (membranes) by comparison to experimental and analytical data. More recently, we have expanded this modeling framework to capture porous media dynamics driven by multi-phase couplings through the inclusion of capillary forces, wettability effects, and relative permeabilities. We will show that the resulting model can be used as a tool to simulate and characterize complex dynamic systems and phenomena: from reservoir fluid injection to clay-soil drying, and from fracture propagation to viscous fingering. This is an exciting development, since, to the best of our knowledge, this is the first CFD model to capture the dynamic couplings between a deformable porous medium and multiple fluid phases in a manner compatible with Digital Rock Physics. 


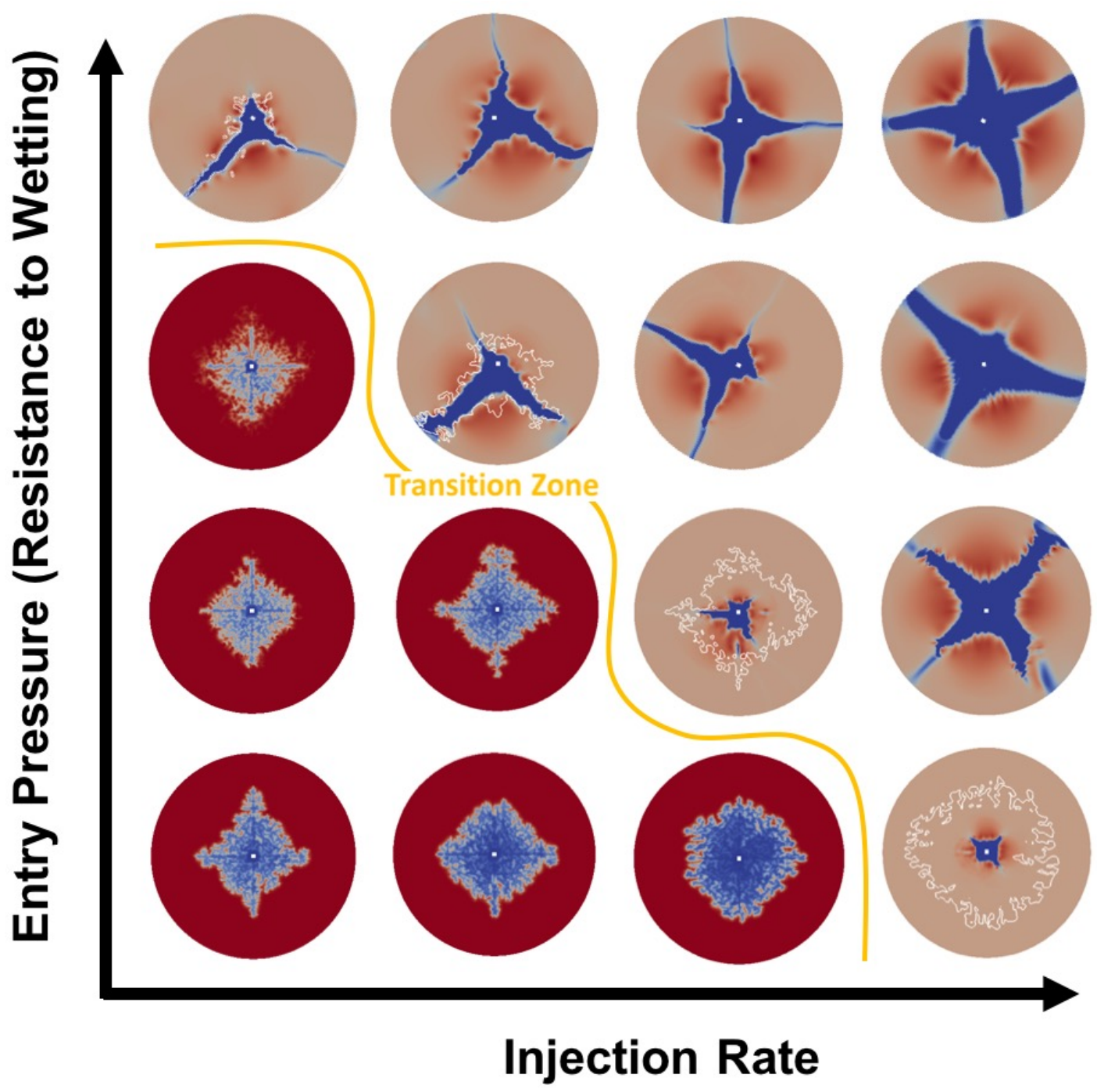

Figure 2: Wettability vs Fluid Injection Rate Phase Diagram 


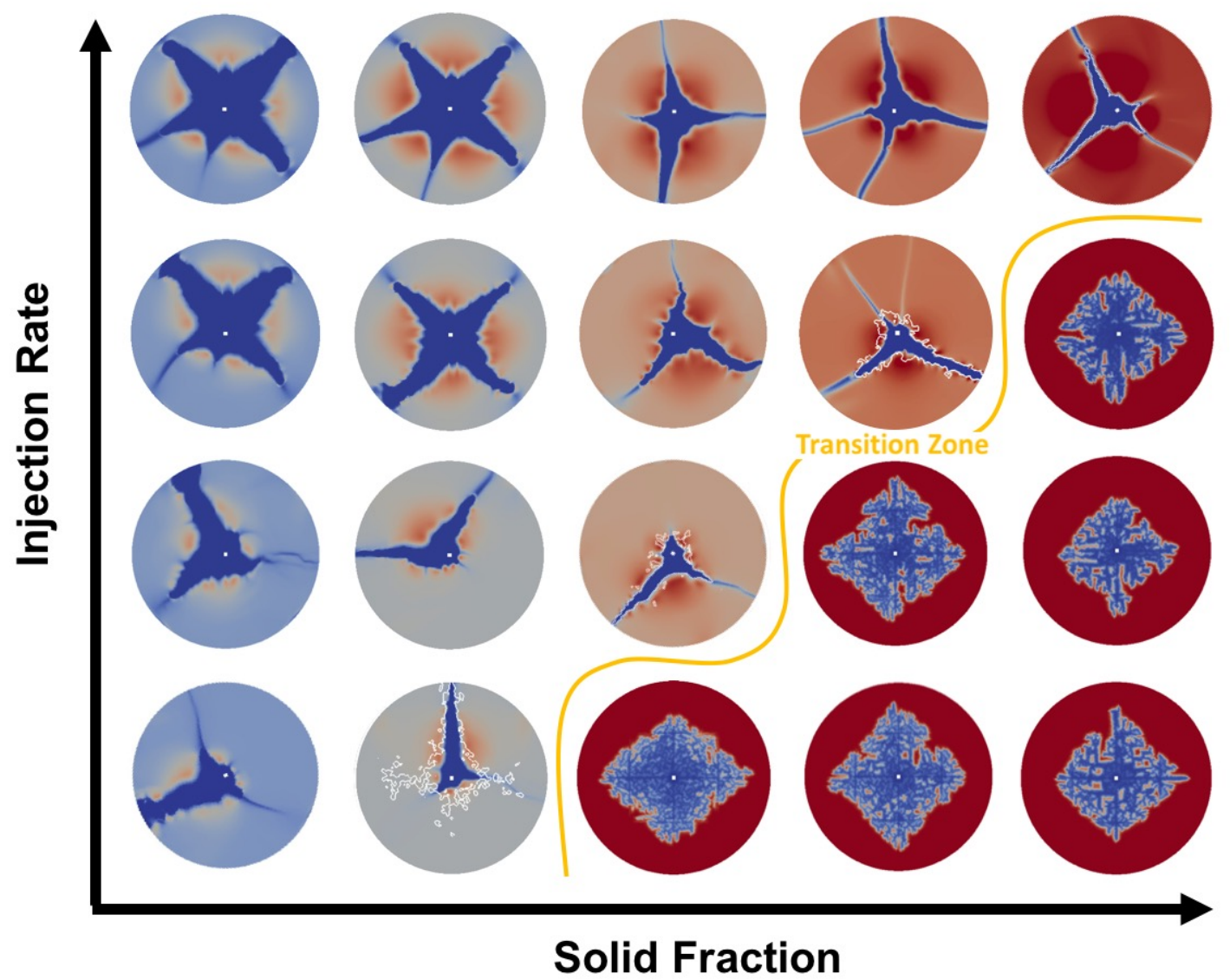

Figure 3: Fluid Injection Rate vs Solid Fraction Phase Diagram

Procter and Gamble Student poster award:

I would like to compete in the Procter and Gamble Student award References:

Acceptance of Terms and Conditions:

Click here to agree

800

\title{
Removal of organic pollutants from water by non-thermal plasma techniques
}

\author{
Simeon Cavadias ${ }^{1}$; Stephanie Ognier ${ }^{1}$ \\ ${ }^{1}$ Chimie-Paristech / PSL University
}

Corresponding Author(s): simeon.cavadias@chimieparistech.psl.eu

The uncontrolled management of water resources for agricultural and industrial uses has caused significant problems of water quality (e.g. pollution of groundwater, rivers and lakes by organic compounds) thatbmay cause diseases either directly through human consumption, or indirectly through the food chain.

The removal of contaminants by modern wastewater treatment plants is often incomplete. With stricter current and anticipated regulations, more efficient and cheaper removal technologies need to be developed and optimized.

Low temperature plasma technologies have been proved to be sustainable and efficient alternatives amongst the different proposed water treatment methods. During non-thermal plasma treatment, 
highly reactive oxidative species $(\mathrm{OH}, \mathrm{H} 2 \mathrm{O} 2, \mathrm{O} 3$, atomic $\mathrm{O}$, singlet $\mathrm{O} 2$ and UV/VUV photons) of short life-time (order of milliseconds) are generated in the neighborhood of the water-gas interface. These species are capable of oxidizing a wide variety of organic molecules ranging from viruses and bacteria to poly-aromatic hydrocarbons, insecticides, and endocrine disruptors .

Nevertheless the energy cost for the direct treatment by non-thermal plasma of organic pollutants is of the order of $30 \mathrm{Wh}$ per liter of treated water. Also because of the dielectric constant of the water which is 80 , while that of air and other gases is 1 and of solids like, Alumina, glass, PTFE is between 4 and 5 , the voltage to maitain the discharge is above $15 \mathrm{kV}$, which can lead to strong instabilities.

A viable option for water and wastewater remediation is based on the development of adsorbents to trap the pollutants. The adsorbents can be treated by non-thermal plasma that can oxidize the pollutants, converting them into less complex and less hazardous intermediates or even fully mineralizing them to $\mathrm{CO} 2$ and $\mathrm{H} 2 \mathrm{O}$. As the dielectric constante of the adsorbants, in this case the view is slightly greater than 5 , the power to maintain the discharge and the energy consumption are much lower..

In this paper we review the possibilities of treatment of pollutants by non-thermal plasma and we demonstrate that the treatment of pollutants adsorbed on porous materials is much more economical than the direct treatment in the liquid.

Procter and Gamble Student poster award:

References:

Acceptance of Terms and Conditions:

Click here to agree

\title{
CO2 injection impairment: simple model for fracturing risk as- sociated with mineral dissolution and precipitation
}

\author{
Pierre Cerasi ${ }^{1}$ \\ ${ }^{1}$ SINTEF \\ Corresponding Author(s): pierre.cerasi@sintef.no
}

Several CO2 sequestration pilots and demonstration projects have been compromised by injection impairment, decreasing injectivity, and influencing the shape of the $\mathrm{CO} 2$ plume in the reservoir The latter effect also potentially reduces the total storage capacity of the reservoir by leaving large swathes unexplored by the injected fluid. Loss of injectivity is usually associated with super-critical injected $\mathrm{CO} 2$ drying the reservoir formation inducing salt precipitation from resident brine. On the other hand, mixing of the $\mathrm{CO} 2$ with resident brine results in a lower $\mathrm{pH}$, low enough to dissolve certain minerals acting as cement at formation grain contacts. These dissolved minerals will be transported outwards from the well until they are deposited due to lowered superficial flow velocity or aggregation and trapping at pore throats. A simplified model is constructed simulating dissolution and precipitation without going into detailed geochemical reaction descriptions; instead, adjustable coefficients specify increase or decrease in pore radius, influencing local velocity in the different pore volumes. The evolving pore throat size distribution in turn influences the stability of the injected fluid front, potentially leaving areas behind with no or less $\mathrm{CO} 2$ saturation. A similar simplified representation of the local effective stresses is established in terms of the pore pressure, whereby criteria are established based on pressure difference between neighbor pores for fracture opening and propagation. The fluid pressure in each pore being a function of flowing velocity, in turn itself a function of pore throat radius and thus of the competition between dissolution and precipitation. The mineral transport along the flow direction also implies that the competing chemical effects are not occurring at the same distance from the injection point, instead separated by a distance depending on the local velocity field. Variation in dissolution and precipitation propensity thus also leads to different fracture patterns.

Procter and Gamble Student poster award:

I don't want to compete References: 


\title{
Modeling solute transport in segregated porous media: sensitivity- driven calibration of a new double continuum model
}

\author{
Giulia Ceriotti $^{1}$; Anna Russian ${ }^{1}$; Diogo Bolster ${ }^{2}$; Giovanni Porta ${ }^{1}$ \\ ${ }^{1}$ Politecnico di Milano \\ ${ }^{2}$ Notre Dame
}

Corresponding Author(s): giulia.ceriotti@polimi.it

Some real porous media, such as carbonates, show very complex structures characterized by the presence of poorly connected cavities which impacts on flow and transport leading to stagnant zones formation and solute segregation. The double or multiple continua approach is appealing for pore to continuum upscaling of transport in these scenarios.

We present the derivation and the calibration of a novel double-continuum model, which explicitly accounts for the exchange process between high and low-velocity regions but avoids cumbersome non-local formulations. Our modeling approach relies on the simplification of the porous medium system into a unit cell made up of an immobile continuum and mobile one, where a uniform shear flow is present. The numerically resolved pore-scale velocity distribution in the porous medium is employed to characterize the volume fraction assigned to each continuum and the velocity profile in the unit cell. Using the simplified unit cell, we derive a closed-form model, which includes two effective parameters that need to be estimated: a characteristic length scale (L) and a ratio of waiting times RD which lumps the solute segregation effect defined as the time required by the solute to escape/explore the stagnant regions of the porous medium to the characteristic diffusive time scale. We explore the impact of the two effective parameters on the solute concentration profile shape. Our results showcase the flexibility of the model using global sensitivity analysis (GSA) considering three different initial conditions for solute transport. These results are then used to define a tailor-made objective function for optimizing the efficacy of model calibration compared to classical calibration approaches. To calibrate and validate our model, we rely on a set of pore-scale numerical simulations performed on a disordered segregated periodic porous medium and the same initial solute distributions investigated in the sensitivity analysis.

Our results show that our model can predict both symmetric and highly skewed solute concentration profiles. Using a sensitivity analysis driven calibration, our model provides a good interpretation of the solute concentration profile evolution independent of the given initial condition relying on a unique set of effective parameter values. These results support the robustness of the approach proposed and allow better understanding the solute transport phenomena itself, particularly on the influence of the mass exchange process between mobile and immobile regions and how it impacts on solute profile shape, depending on the initial condition.

Procter and Gamble Student poster award:

References:

Acceptance of Terms and Conditions:

Click here to agree

\section{Force chain analysis during particle migration using community detection}


${ }^{1}$ China University of Petroleum

\title{
Corresponding Author(s): coc1993@163.com
}

In the last decade, the study of the behavior of granular materials, such as dense packing of spherical particles and particle migration process, etc., has great important significance for chemical engineering and petroleum engineering. However, it mainly focuses on the macroscopic performance of granular materials, but there are few reports on the mechanism of microscopic mechanism such as the mechanism of rupture and evolution. To address this challenge, we treat granular materials as spatially-embedded networks in which the particles (nodes) are connected by weighted edges. We use community detection network analysis method to find sets of closely connected particle clusters and extract chain-like structure that are reminiscent of force chains. We investigate the packing granular material in three dimensional which is compressed by fluid with different pressure. The results show that Granular contact network exhibit a very clear community structure and network analysis method provide new viewpoints to underlying and generate new perspective of describing granular material problem compared with traditional methods. The distribution of force chain community size is consistent with an exponential distribution and the high-pressure force chain network exhibit compact instead of branching communities. And granular matter displays traits of self-organization forming complex force network which arrange in response to applied load or compression and more specifically force network rearranges prominently when applied pressure is disturbed.

Procter and Gamble Student poster award:

References:

Acceptance of Terms and Conditions:

Click here to agree

\section{A Dynamical System for Gas Phase Cycling in Porous Media}

\author{
Alex Chang $^{1}$; Brent Lindquist ${ }^{2}$ \\ ${ }^{1}$ National Pingtung University \\ ${ }^{2}$ Texas Tech University
}

Corresponding Author(s): chang@mail.nptu.edu.tw

Compositional flow involving a gas phase is of importance in many areas, including oil reservoir production, pipeline transport, CO2 sequestration, and the disposal of radioactive waste. Compositional flow involves the inherent possibility of phase creation and its subsequent transport. Keeping any potential gas components dissolved in the brine phase is important for efficient extraction in reservoirs; the presence of gas bubbles and the resultant fluid-gas menisci complicates flow and can compete with brine movement.

A challenge to the numerical simulation of compositional flow in porous media is the change in the system of equations that accompanies the appearance and disappearance of a gas phase. We developed a network flow model that checked on the flow condition (two-phase, single-phase undersaturated, single-phase over-saturated) in each pore to determine the appropriate equations to apply. In our computations of two-phase, two-component $(\mathrm{H} 2 \mathrm{O}, \mathrm{CO} 2)$ flow in a 3D pore network1. We noted the periodic appearance and dissolution of the gas phase in certain pores. Intensive evaluation of our algorithms led us to conclude that the phenomenon was not numerical in origin. We develop and analyze a $2 \times 2$ dynamical system describing flow through a single pore to study the dynamics of the appearance and dissolution of gas bubbles during two-component (CO_2, H_2 O), two-phase (gas, liquid) flow.

Procter and Gamble Student poster award:

References: 
1. Chang, K. A., Lindquist, W. B., 2013, A network flow model for the genesis and migration of gas phase. Computational Geosciences 17, 67-81.

Acceptance of Terms and Conditions:

Click here to agree

918

\title{
Dynamics of water imbibition through paper
}

\author{
Sooyoung Chang ${ }^{1}$; Wonjung Kim ${ }^{1}$ \\ ${ }^{1}$ Sogang University \\ Corresponding Author(s): chswim@sogang.ac.kr
}

Paper is one of the most widely used typical porous media for absorbing liquid, and accurate control of water imbibition in paper is crucial in developing paper-based microfluidic devices. Washburn equation is usually used to describe the dynamics of the liquid flow through the cellulose matrix of paper. However, it is well known that this equation has limitations in predicting water flow in paper. We report that swelling and intra-fiber pores that have not been considered in developing Washburn equation are mainly responsible for the limited accuracy when predicting imbibition length of water. We demonstrate that cellulose fibers have significant internal voids that absorb water. In addition, we quantify water induced swelling that leads to the expansion of inter-fiber space. We develop a hydrodynamic model of water imbibition with the consideration of intra-fiber voids and cellulose fiber swelling that well explain experimental observations. Our study provides a new insight into not only porous media flow with intra-void structure and swelling effects, but also a theoretical background to design $\mu$ PADs.

\section{Procter and Gamble Student poster award:}

I would like to compete in the Procter and Gamble Student award References:

Acceptance of Terms and Conditions:

Click here to agree

\section{Multiscale Porosity Measurements in Shale Rocks using Gas Ad- sorption and Mercury Intrusion Porosimetry}

\author{
Nicolas Chanut ${ }^{1}$; Thibaut Divoux ${ }^{1}$; Rénal Backov ${ }^{1}$; Jeffrey Kenvin ${ }^{2}$; Franz Ulm ${ }^{3}$; Roland Pellenq ${ }^{4}$ \\ ${ }^{1}$ Massachusetts Institute of Technology \\ ${ }^{2}$ Micromeritics Instruments Corporation \\ ${ }^{3}$ MIT \\ ${ }^{4}$ MIT / CNRS
}

Corresponding Author(s): nchanut@mit.edu

Unconventional oil and gas production from shale has revolutionized the world energy landscape, and particularly within the united states where unconventional gas represents today more than $50 \%$ of U.S total production, as compared to $1 \%$ in 2000. Shale is a fine-grained sedimentary rock, composed of solid organic matter (OM) scattered in a mineral framework. The decomposition of this $\mathrm{OM}$ at high temperature leads to the generation of hydrocarbons during a process known as maturation. The resulting organic matter develops a network of nanoscale pores that governs the ability of a shale petroleum reservoir to store and ultimately yield oil and gas. The mineral matrix 
may also contribute, mainly through inter-particle voids, to the overall porosity leading to a pore size distribution spanning from a few $\AA$ to the $\mu \mathrm{m}$ range. It is therefore crucial to develop accurate tools to characterize the multiscale pore network of shale rocks in order to better understand the link between their texture and their adsorption and diffusion properties controlling the efficiency of a production well. In this work, we have combined adsorption of multiple gases (N2, Ar, CO2, H2 and O2) with Mercury Intrusion Porosimetry (MIP) to study the pore system of 5 samples from the Vaca Muerta Shale formation in Argentina. While the combination of low-pressure adsorption enables probing the micro and meso-porosity (from a few $\AA$ to $\approx 50 \mathrm{~nm}$ ), MIP allows for characterization of the meso and macro-porosity (from $3 \mathrm{~nm}$ to $\approx 500 \mu \mathrm{m}$ ). Rather than using a fragmented approach of simple overlays from individual techniques, a unified approach utilizing a kernel constructed from model isotherms and model intrusion curves is used to calculate the complete pore size distribution and the total pore volume of the material. This technique has then been used to follow the evolution of the porous network over the course of the demineralization of the original shale rocks by various acid treatments, ultimately leading to isolated OM. This sequential approach gives insights of the contribution of the different mineral phases to the overall porosity and makes the link between the textural properties of these samples and their high-pressure adsorption properties.

Procter and Gamble Student poster award:

References:

Acceptance of Terms and Conditions:

Click here to agree

\title{
Characterization of sands and sandstones using electrical impedance spectroscopy
}

\author{
Alex Kirichek $^{\text {None }}$; Claire Chassagne ${ }^{1}$ \\ ${ }^{1}$ TU Delft
}

Corresponding Author(s): c.chassagne@tudelft.nl

The low-frequency dielectric spectroscopy of granular material, where the porosity is representative for sands and sandstones, is until now always modelled using theories based on the work of Schwartz (Schwartz, 1962). The theory for the low-frequency dielectric spectroscopy of suspensions, on the other hand, has been developed much further over the last decades both numerically and analytically.

We present new analytical expressions for the complex conductivity of granular material, such as sands and sandstones in an electrolyte solution, adapting the theories developed for suspensions (Kirichek, 2017). We show that the new expressions enable to predict the measured complex conductivity of various granular material, such as packed glass beads, sands and sandstones. Because of the typical grain size of sand and sandstone particles, for any ionic strength the double layer is much thinner that the particle size. Contrary to existing theories for granular materials, the expressions we derived are valid for any ionic strength and no adjustable parameters are required.

Figure 1: Equivalent circuit representation of the measured electrical impedance for a sandstone. Left: the sandstone (white) saturated with electrolyte (red and blue) in between two electrodes (grey). Right: the equivalent circuit where $\mathrm{Rb}, \mathrm{Cep}$ and $\mathrm{Cb}$ are resistance and capacitances of the EP and bulk. L is a fitting parameter.

We furthermore demonstrate that the measured impedance of an electrolyte bearing sandstone can correctly be modelled using an equivalent circuit approach (Kirichek, 2018), see Fig.1. The following impedances of the circuit can be mathematically correlated to a polarisation phenomenon: electrode polarization (EP) at low frequencies and bulk polarization at middle frequencies $(\mathrm{kHz})$. EP is correctly predicted in our case using the model presented in (Chassagne, 2016) for all investigated concentrations. The bulk contribution is derived from the model we present here and requires no 
adjustable parameter in the case of fully electrolyte-saturated pores when all material properties (porosity, salinity, dielectric constants) are known.

In the measurements, an additional polarization effect appeared at higher frequencies. We defined it as a "pseudo-inductance" in the model and it is symbolized by L in Fig.1. We show that even though this pseudo-inductance might be of parasitic origin and originate from an unwanted interaction between the electric circuit and the sample it can be used to our advantage in the case of sandstones saturated with a complex fluid. In the case of a sandstone saturated with a mixture of electrolyte and supercritical $\mathrm{CO} 2$ we indeed demonstrate that two of the system parameters (the electrolyte concentration and the $\mathrm{CO} 2$ saturation) can be uniquely determined by simultaneously fitting the bulk polarization and the pseudo-inductance polarization. The fits are given in Fig.2.

Figure 2: Magnitude $/ \sigma /$ and phase conductivity spectra in a sandstone saturated with $10 \mathrm{mmol} / \mathrm{L} \mathrm{NaCl}$ for different $\mathrm{CO} 2$ saturation. The CO2 saturation is given by (1-Swn) where Swn is given in the figure. Using the electrolyte concentration and Swn as adjustable parameters, the data could be uniquely fitted and the found parameters corresponded to the expected ones.

Procter and Gamble Student poster award:

\section{References:}

G. Schwartz, A theory of the low-frequency dielectric dispersion of colloidal particles in electrolyte solution, J. Phys. Chem. 66 (12) (1962) 2636-2642.

Chassagne, Claire, et al. "Compensating for electrode polarization in dielectric spectroscopy studies of colloidal suspensions: theoretical assessment of existing methods.” Frontiers in chemistry 4 (2016): 30. Kirichek, A., C. Chassagne, and R. Ghose. "Dielectric spectroscopy of granular material in an electrolyte solution of any ionic strength." Colloids and Surfaces A: Physicochemical and Engineering Aspects 533 (2017): 356-370.

Kirichek, A., C. Chassagne, and R. Ghose. "Predicting the dielectric response of saturated sandstones using a 2-electrode measuring system" accepted in Frontiers in physics (2018). Acceptance of Terms and Conditions:

Click here to agree

\section{Identification of fractures in porous medium}

Fatma Cheikh ${ }^{\text {None }}$

Corresponding Author(s): fatma.cheikh@utc.fr

Fractures can drastically affect the flow patterns in porous media.

We propose a new iterative algorithm for the identification of a small number of large fractures in a porous medium from given distributed measurements.

This algorithm is based on the use of fracture indicators that give a first order information concerning the effect of the addition of a new fracture. As these indicators are cheap to compute, a large number of configurations of new fractures is tested at each iteration.

This algorithm gives very satisfactory results for a series of numerical examples representative of typical situations, although this problem is considered difficult.

Procter and Gamble Student poster award:

I don't want to compete References:

Acceptance of Terms and Conditions:

Click here to agree 


\section{Quantifying Uncertainty Reduction in Geological CO2 Sequestra- tion}

Bailian Chen ${ }^{1}$; Dylan Harp ${ }^{1}$; Shaoping $\mathrm{Chu}^{1}$; Nataliia Makedonska ${ }^{1}$; Rajesh Pawar $^{1}$

${ }^{1}$ Los Alamos National Laboratory, US

Corresponding Author(s): chenbailian719@gmail.com

Geologic CO2 sequestration sites usually have large uncertainty in geological properties, such as uncertainty in permeability fields. Geological uncertainty leads to significant uncertainty in the risk metrics such as $\mathrm{CO} 2$ plume stability in storage reservoir, $\mathrm{CO} 2$ /brine leakage rates through wellbores, and impact to drinking water quality (such as total dissolved solid (TDS) and $\mathrm{pH}$ ) in groundwater aquifer due to $\mathrm{CO} 2 /$ brine leakage, all of which would have large impact on the post injection site care (PISC) and the determination of the storage site closure time. Recently, a CO2 stability evaluation approach and Integrated Assessment Model (IAM) have been developed by National Risk Assessment Partnership (NRAP) to quantify the uncertainty of risk metrics. However, these models do not account for the potential value of monitoring data (e.g., CO2 saturation and pressure measurements) acquired during the operation of $\mathrm{CO} 2$ storage.

In this study, we integrate monitoring data into a reservoir model(s) using a novel data assimilation approach called Ensemble Smother with Multiple Data Assimilation (ES-MDA). With the integration of monitoring data, reservoir model(s) are calibrated. Based on the calibrated reservoirs, three sets of uncertainties, i.e., uncertainties in the risk metrics for $\mathrm{CO} 2$ plume stability, uncertainties in $\mathrm{CO} 2$ and brine leakages from potentially leaky wellbores, and uncertainties in groundwater aquifer impact (TDS/pH plume size), are quantified using the $\mathrm{CO} 2$ stability evaluation approach and IAM. We demonstrate how assimilating monitoring data into reservoir models can reduce the uncertainties in different risk metrics of interest using the Rock Spring Uplift site at southwestern Wyoming in US as a field case. The value of information (VOI) for monitoring data over time is also quantified in this study. To the best of our knowledge, this is the first study in geologic $\mathrm{CO} 2$ sequestration to comprehensively quantify the uncertainty reduction in all risk metrics of interest from storage reservoir to wellbore to groundwater aquifer.

Procter and Gamble Student poster award:

References:

Acceptance of Terms and Conditions:

Click here to agree

115

\section{Dynamic Formation Damage in Porous Media induced by Particle Transport}

Jessie Chen $^{1}$

${ }^{1}$ University of New South Wales

Corresponding Author(s): jlchen2594@gmail.com

Reservoir management is critical for the petroleum industry, $\mathrm{CO} 2$ sequestration, and groundwater quality evaluation. By understanding the effect that particulates have on the permeability of reservoirs, we can better evaluate field performance, as well as the concentration of contaminants in aquifers.

The aim of this research is to investigate the effect of rock structure and particle properties on formation damage to sandstone reservoirs. It has been shown both experimentally and numerically that permeability reduction can occur when particles such as sand grains or fines travel through the reservoir and clog the pore spaces. Pore clogging is predominantly initiated due to size exclusion for sand 
grains, which comprise of larger quartz particles. However, with shaly sandstone, pore clogging can also be a result of electrostatic forces between the clay fines and the porous medium.

In this research, we study the phenomenon numerically in three dimensions and validate the results with experiments. In order to obtain the porous media structure, X-ray microtomography (microCT) scans are taken of sandstone samples to obtain realistic pore geometry. The micro-CT images are then meshed in preparation for numerical simulation. In order to solve both the fluid and particle flow, the mesh is smoothed for increased accuracy before being imported into the numerical simulator. For the numerical simulations, we use an opensource numerical package to simulate the particle transport and retention through porous media. The software couples the Lattice Boltzmann method for fluid flow with the discrete element method for particle-particle interactions. For the particle-fluid interactions, the immersed boundary method is used for both dilute and dense particle flow scenarios.

The experimental work verifies the physics of the phenomenon and validates the numerical results. Firstly, a micro-CT image of the rock is taken as a reference geometry. Particles are then injected at selected fluid rates and in specified numbers. For each case, steady state is reached before obtaining a second micro-CT image. The fate of the particles within the samples are observed and compared against the reference image. From this, the physics of the phenomenon is verified through observing size exclusion, amongst other secondary trapping mechanisms, and the permeability reduction obtained experimentally is used to validate the numerical model.

The pore-scale numerical model can provide the basis for more realistic reservoir models through upscaling to field scale. The outcome of this work is a dynamic skin factor, which is a function of rock geometry and particle properties. This information can be fed into reservoir models to account for particle retention during production or injection. Furthermore, the resultant reservoir model can be coupled with a geomechanical model to obtain the concentration of sand particles travelling through the porous media under different operating conditions. This coupling would also enable a more accurate sand production rate prediction due to the inclusion of particle retention. The significance in this work lies in the improvement of production forecasting, injectivity estimation, sand production prediction. Our results show that the rock structure and particle properties play an important role for determination of formation damage.

\title{
Procter and Gamble Student poster award:
}

I would like to compete in the Procter and Gamble Student award References:

Acceptance of Terms and Conditions:

Click here to agree

\section{Application of online nuclear magnetic resonance technology to analysis of displacement process in tight oil cores}

\author{
Author(s): Ting Chen ${ }^{1}$; zhengming yang ${ }^{2}$ \\ Co-author(s): Yutian Luo ; SHENGCHUN XIONG ; JIaxiang Xu ${ }^{2}$ \\ ${ }^{1}$ University of Chinese Academy of Sciences \\ ${ }^{2}$ PetroChina Research Institute of Petroleum Exploration \& Development
}

Corresponding Author(s): chenting15@mails.ucas.edu.cn

The analysis of oil and water content in the cores is of great significance for the exploration and development of tight oil reservoirs. Online nuclear magnetic resonance (NMR) technology combines low field NMR spectrometer with high temperature and high pressure displacement technology, and NMR testing can be carried out in core displacement experiments. In this study, online NMR technology was used to test T2 spectra, stratified T2 spectra and Magnetic resonance imaging (MRI) of tight oil sandstone cores during deuterium water flooding. Through the analysis of the measured T2 spectra data, it can be concluded that the injected deuterium water preferentially entered the macropores. When the proportion of pore with inner diameter greater than $1 \mu \mathrm{m}$ in tight core 
exceeded $30 \%$, it was difficult to displace oil in small pores for waterflooding development. It can be seen from stratified T2 spectra that with the injection of deuterium water, the right peak of T2 spectrum at the injection end decreased significantly, which indicated that the injection medium preferentially enters the large pores. By adding fake colors to MRI images, the displacement process can be seen intuitively. With the amount of deuterium water injected from 0.2 pore volume (PV) to $5 \mathrm{PV}$, the core color begins to darken from the injection end, and the whole core color darkens later, indicating that the oil in the cores was continuously expelled.

Procter and Gamble Student poster award:

References:

Acceptance of Terms and Conditions:

Click here to agree

303

\title{
Pore-scale simulation of fluid displacement in micromodels and real rock samples using the CSF-based lattice Boltzmann multi- phase model and geometrical wetting model
}

\author{
Yu Chen ${ }^{1}$; Albert Valocchi ${ }^{2}$; Qinjun Kang ${ }^{3}$; Hari Viswanathan ${ }^{3}$ \\ ${ }^{1}$ Los Alamos National Lab \\ ${ }^{2}$ Univ Illinois \\ ${ }^{3}$ Los Alamos National Laboratory
}

Corresponding Author(s): yu_chen@lanl.gov

The lattice Boltzmann method has become very popular for pore-scale flow simulations in micromodels and real rock samples, thanks to its high fidelity and high efficiency on Manycore processors such as graphics processing unit (GPU). However, the current color-gradient multiphase model suffers from large spurious currents and low numerical stability, leading to a very limited range of surface tension to viscosity ratio. These have hindered the predictive capability of the color-gradient model. For example, recent studies have confirmed that inertial effects impact the invasion patterns during the process of scCO2 displacing brine in a heterogeneous micromodel 1 . However, the existing color-gradient model failed to match the capillary number and Reynolds number simultaneously due to the limited range of surface tension to viscosity ratio, thus cannot fully reproduce the invasion patterns observed in the experiment.

In this work, we employ the CSF (continuum-surface-force) based color-gradient model and geometrical wetting model to reduce spurious currents and eliminate artificial films, thus we are able to match both the Reynolds number and capillary number of the scCO2 and brine displacing process. The simulations show good agreement with micromodel experiments and fully reproduce the invasion patterns. With efficient implementation of the code on Manycore processors, we are also able to study the inertial effects on a real rock sample during fluid displacing process.

Procter and Gamble Student poster award:

References:

1. Chen, Y., et al., Lattice Boltzmann simulations of liquid CO2 displacing water in a 2D heterogeneous micromodel at reservoir pressure conditions. Journal of Contaminant Hydrology, 2018. 212: p. $14-27$.

Acceptance of Terms and Conditions:

Click here to agree 


\title{
An improved LBM-DEM model for hydro-mechanical coupled pro- cess
}

Zhiqiang Chen ${ }^{1}$; Moran Wang ${ }^{1}$

${ }^{1}$ Tsinghua University

Corresponding Author(s): 1452087789@qq.com

Hydro-mechanical coupled processes are considerably prevalent in petroleum industry such as hydraulic fracture in unconventional reservoirs and sand production in sandstone reservoirs. Owing to the high cost of experiments, numerical study has been an efficient way to explore the underlying physics of hydro-mechanical coupled process. Generally, there are two kinds of methods to simulate this process, continuum-based models and discontinuum-based models. In continuum-based models, different nonlinear equations based on continuum theory should be solved iteratively, so fully hydro-mechanical coupled schemes are difficult to be achieved. In addition, little information can be provided by continuum-based model to understand the mechanism at particle scale. Thus, discontinuum-based models are developed, and among them LBM-DEM is a popular one. Compared with continuum-based models, DEM can capture the discrete nature of rock by treating it as an assembly of discrete particles. Secondly, the influence of micro parameters on the macroscopic behaviors can be considered, which is important for understanding the mechanism of hydro-mechanical coupled process.

In LBM-DEM model, rock deformation and fluid flow are simulated by discrete element method (DEM) and lattice Boltzmann method (LBM), respectively, and then DEM and LBM are coupled to capture the fluid solid interaction. There are some schemes to couple these two methods, such as bounce-back scheme (BB), immersed boundary method (IBM) and immersed moving boundary (IMB). Among them immersed moving boundary is commonly used to explore hydro-mechanical coupled processes involved in geophysical system such as hydraulic fracture and sand production, because this scheme is computationally efficient especially in dense particles cases. However, a limitation of original immersed moving boundary exists that is the hydraulic force calculation is not accurate enough. Thus, the purpose of this work is to overcome this limitation and simulate hydro-mechanical coupled processes with improved LBM-DEM model.

In this work we develop an improved LBM-DEM coupled model, which can give more accurate hydraulic force calculation than original IMB method but with little additional computational cost. To validate current model, sphere particle sedimentation in Newtonian fluid is simulated, and better agreement with FEM results can be obtained. Finally, hydro-mechanical coupled process is simulated, which gives an understanding of this process at particle scale.

Procter and Gamble Student poster award:

I would like to compete in the Procter and Gamble Student award References:

Acceptance of Terms and Conditions:

Click here to agree

\section{Joint identification of contaminant source and non-Guassian con- ductivities via the restart normal-score Ensemble Kalman filter}

\author{
Zi Chen ${ }^{1}$; Jaime Gomez ${ }^{\text {None }}$; Teng $\mathrm{Xu}^{1}$; Andrea Zanini ${ }^{2}$ \\ ${ }^{1} U P V$ \\ ${ }^{2}$ Department of Engineering and Architecture, University of Parma
}

Corresponding Author(s): dtpenguincz@gmail.com 
The normal-score ensemble Kalman filter (NS-EnKF) has been proven to work well for the identification of a contaminant source in an aquifer together with the estimation of the spatial variability of hydraulic conductivity, even for highly heterogeneous, non-Gaussian conductivity distributions, in synthetic aquifers. In this work, the NS-EnKF is tested with real data coming from a sandbox experiment with a binary spatial distribution of conductivities. The sandbox contains patches of high conductivity mixed with patches of low conductivity. The main difference with the synthetic cases in which the technique had been tested previously is that no piezometric head data are available, and therefore, an important amount of information regarding the spatial distribution of conductivities will be missing. After some preliminary tests, it was found that for the filter to meet the goal of jointly identifying the contaminant source parameters and the non-Gaussian conductivities, it was needed to use a large number of members in the ensemble or with inflation techniques to prevent filter inbreeding. The best results were obtained with a large ensemble size or with the Bauser's inflation method. The results show the ability of the NS-EnKF method to jointly identify contaminant source and conductivities in a sandbox experiment.

Procter and Gamble Student poster award:

I don't want to compete References:

Acceptance of Terms and Conditions:

Click here to agree

\title{
A unified model for liquid rise in a single capillary tube
}

\author{
Author(s): Zhilin Cheng ${ }^{\text {None }}$ \\ Co-author(s): Weibo Sui ; Yanan Hou \\ Corresponding Author(s): , ,
}

The study of the dynamic capillary rise is crucial for many industries and applications, such as flipchip encapsulation, design of micro-/nanoelectrical mechanical systems, fluid flow in porous media, as well as enhanced oil recovery.

A comprehensive study of liquid penetrating into capillary tubes is of great significance and necessary. Based on the energy balance theory, a normalized governing equation for the capillary rise was derived in this paper, which accounts for the kinetic, gravity, viscous force, and capillary tube effects. Among them, the friction dissipations of gas resulting from long capillary tubes are usually ignored by previous research.

This model was validated the by comparing the results with some published experimental data. Then, the origin of capillary oscillation phenomena and some relevant effects on it were investigated in detail. The results show that this model can accurately capture characteristics of rebounds of liquids in a cylindrical tube. As the capillary tube's length increases, the magnitude of capillary oscillation is weakened owing to the resistance of air. Theoretically, the capillary oscillation would vanish if the length of the tube is infinite. In addition, the liquid viscosities also pose a remarkable effect on the oscillating behavior, increasing the viscosity of liquid leads to a greater viscous resistance of the wetting liquid, thus the oscillation behavior is inhibited and even disappears. Moreover, as there is a positive relationship between the liquid density and the inertial force. Therefore, a more drastic oscillation behavior will emerge when using a higher density of liquid for simulation. Overall, the capillary oscillation is controlled by a variety of factors. The inertial force of the liquid is the primary reason that gives rise to the oscillation of liquid column. We suggest that the unified mathematical model could provide us new insights into the capillary rise of liquid, and it can be readily upscaled into porous media using homogenization techniques, fractal theory, statistical models, etc.

\section{References:}

Acceptance of Terms and Conditions:

Click here to agree 


\title{
Nonlinear upscaling for flows in porous and fracture media
}

\author{
Eric Chung ${ }^{1}$; Maria Vasilyeva ${ }^{\text {None }}$ \\ ${ }^{1}$ The Chinese University of Hong Kong \\ Corresponding Author(s): tschung@math.cuhk.edu.hk
}

In this talk, we present upscaling methods for nonlinear multiscale problems. We use recently developed multiscale concepts for linear problems and extend them to nonlinear problems. The main idea of these approaches is to use local constraints and solve problems in oversampled regions for constructing macroscopic equations. These techniques are intended for problems without scale separation and high contrast, which often occur in applications. In our method, the local solutions are used as a nonlinear forward map from local averages (constraints) of the solution in oversampling region. This local fine-grid solution is further used to formulate the coarse-grid problem. Our approach is discussed on several examples and applied to single-phase and two-phase flow problems, which are challenging because of convection-dominated nature of the concentration equation. The numerical results show that we can achieve good accuracy using our new concepts for these complex problems. This research is partially supported by Hong Kong RGC General Research Fund (Project 14304217) and CUHK Direct Grant for Research 2017-18.

Procter and Gamble Student poster award:

References:

Acceptance of Terms and Conditions:

Click here to agree

962

\section{Voxels agglomeration for a fast permeability estimation on micro- CT images}

Traiwit Chung $^{\text {None }}$; Ying Da Wang ${ }^{1}$; Peyman Mostaghimi ${ }^{\text {None }}$

${ }^{1}$ UNSW

Corresponding Author(s): traiwit.chung@student.unsw.edu.au

Traditionally, direct numerical methods are widely used to solve for flow on micro-computed tomography (micro-CT) images of rock samples. However, it is computationally demanding, especially on large micro-CT images (10003 voxels and more). We introduce a fast and robust approach in order to solve the elliptic flow equation and estimate the absolute permeability of micro-CT imaged rocks. The approach involves a common technique in computational fluid dynamics, an agglomeration. By allowing specific locations of pore voxels to be agglomerated, number of pore voxels or active cells in a sparse matrix can be reduced by approximately $60 \%$ to $80 \%$ depending on the type of rock and its pore size distribution. Meanwhile, the fine details obtained from micro-CT scan are maintained. The results compared with Pore-scale finite volume solver (PFVS) are within 1.6\% difference (3.7\% difference for carbonate sample) on level 1 agglomerated grids and 1.9\% for level 2 agglomerated grids while fluxes are being conserved. Moreover, the computation time reductions are at least 59\% from all the micro-CT image tested in this study. This approach is suitable for a rapid estimation of an absolute permeability from micro-CT images of rocks, especially on the system with more pore voxels.

\section{Procter and Gamble Student poster award:}

I would like to compete in the Procter and Gamble Student award References:

Acceptance of Terms and Conditions: 


\title{
Relative permeability in discrete fracture network model of coal micro-CT images
}

\author{
Traiwit Chung $^{\text {None }}$; Ying Da Wang ${ }^{\text {None }}$; Peyman Mostaghimi ${ }^{\text {None }}$; Ryan Armstrong ${ }^{\text {None }}$; Jing Yu $^{\text {None }}$
}

Corresponding Author(s): traiwit.chung@student.unsw.edu.au

Recently, Coal seam gas (CSG) has recently been globally gaining interests, because its environmental benefits compared to traditional hydrocarbon sources, as well as its abundance. Nowadays, direct flow simulations are widely used for simulating flow on microcomputed-tomography (micro-CT) images of conventional reservoir rocks. However, not many have been done on micro-CT images of coal, due to the complexity that makes coal images difficult to characterise.

In this work, we use Lattice Boltzmann Methods for Porous Media (LBPM) in order to study for fluid flow (both single-phase and multiphase) on coal images. The coal images we use in this study are reconstructed using a discrete fracture network (DFN) model from the actual micro-CT images of coal. The results are compared with the laboratory experiment results. Understanding of absolute and relative permeabilities and other petrophysical properties from coal images in pore-scale will lead to better development of larger scale simulation for CSG

Procter and Gamble Student poster award:

I would like to compete in the Procter and Gamble Student award References:

Acceptance of Terms and Conditions:

Click here to agree

\section{Fundamentals of Geometrical and Physical Concept of Pore Space Tortuosity}

Mieczysław Cieszko ${ }^{1}$

${ }^{1}$ Kazimierz Wielki University

Corresponding Author(s): cieszko@ukw.edu.pl

Parameter of the pore tortuosity is a basic parameter characterizing macroscopic pore space structure of permeable porous materials. This parameter plays important role in all transport processes taking place in porous materials (Carman 1937, Clennel 1997). In spite of its fundamental character and great number of publications devoted to its definition and analysis of the physical and geometrical meaning and the methods of determination, there is still no commonly accepted general definition of this macroscopic notion and its microscopic representation. This problem becomes even more complicated in materials with anisotropic pore space structure.

The aim of the paper is to present the general solution of the problem of macroscopic description of the anisotropic pore space structure, which allows precise and consistent formulation of definitions of macroscopic parameters of pore space structure: pore tortuosity and surface porosity, and also their natural introduction into macroscopic description of processes occurring in porous materials. Considerations have been based on the model assumptions presented in the papers Cieszko 2005 and Cieszko 2009. It was assumed that at the macroscopic point of view interconnected pores in permeable porous materials form anisotropic space, the structure of which is determined by its metric and this space is modelled as Minkowski metric space. Such approach to this problem results in a number of consequences: a) modelling of the pore space structure is a primary problem in comparison to the modelling of processes occurring in the pore space; $b$ ) parameters of the pore space structure are defined by the metric of the space; c) pore structure parameters co-determine the 
course of each process occurring in the pore space and are independent of them. It was shown that pore structure parameters and their tensor characteristics are directly defined by the metric tensor of the pore space. This means that character of these parameters is purely geometrical.

The proposed definition of the pore tortuosity parameter based on the concept of Minkowski metric space is also the basis for precise determination of their relation with quantities characterizing microscopic pore structure. General form of such relation for pore tortuosity have been obtained requiring the full representation of macroscopic density of fluid kinetic energy in the potential flow, by microscopic velocity field. It was shown that such approach is directly related with the variational problem of minimization of scalar field inhomogeneity defined in the pore region the measure of which is the integral of square of gradient of this scalar field, called Dirichlet integral or Dirichlet energy. Euler equation for this problem takes form of the Laplace equation that is the basic equation describing various types of potential transport. This equation does not contain any material characteristics, and due to the pure geometrical character of the variational problem, its solutions are contingent also upon geometry of the region on which it is defined.

\title{
Procter and Gamble Student poster award:
}

I don't want to compete References:

Carman P., Fluid Flow through Granular Beds, Trans. Inst. Chem. Engng, 15, 150-166, 1937.

Clennel M., Tortuosity: A Guide through the Maze, in Developments in Petrophysics, eds Lovell M.A. \& Harvey P.K., Geological Society London Special Publication No 122: 299-344, 1997.

Cieszko M., Extended description of pore space structure and fluid flow through anisotropic porous materials. Application of Minkowski space. Eds: Wang Y., Hutter K., Trends in Application of Mathematics to Mechanics, Shaker Verlag 2005.

Cieszko M., Description of Anisotropic Pore Space Structure of Permeable Materials Based on Minkowski Metric Space, Archives of Mechanics, 61, 6 (425-444), 2009. Acceptance of Terms and Conditions:

Click here to agree

\section{Limit Models of Pore Space Structure of Porous Materials for De- termination of Limit Pore Size Distributions Based on Mercury Intrusion Data}

\author{
Author(s): Mieczysław Cieszko ${ }^{1}$ \\ Co-author(s): Marcin Kempiński ${ }^{1}$; Tomasz Czerwiński ${ }^{2}$ \\ ${ }^{1}$ Kazimierz Wielki University \\ ${ }^{2}$ Kazimierz Wielki Uniwersity
}

Corresponding Author(s): cieszko@ukw.edu.pl

This paper proposes application of capillary and chain random models of pore space structure for determination of limit pore diameter distributions of porous materials (Cieszko et all 2018), based on the mercury intrusion curves. Both distributions determine the range in which the pore diameter distribution of the investigated material occurs and defines the degree of inaccuracy of the method based on the mercury intrusion data caused by the indeterminacy of the sample shape and its pore space architecture.

We derived equations describing the quasi-static process of mercury intrusion into the porous layer and porous ball with a random chain pore space structure and analysed the influence of the model parameters on the mercury intrusion curves. It was shown that distribution of link length in the chain model of the pore space, random location of chain capillaries in the sample and the length distribution of the capillaries do not influence significantly the intrusion process. Therefore, a simple model of the mercury intrusion into the layer is proposed in which chain links of the pore space have random diameters and constant length. This model is used as a basic model of the intrusion process into a sample of any shape and size and with homogeneous and isotropic chain pore space architecture. The thickness of the layer then represents the mean length of chain capillaries in the sample. It was also proved that the capillary and chain models of pore space architecture are limit models of the network model identified in this paper with the pore architecture of the investigated sample. This justifies application of both models for determination of limit cumulative distributions 
of pore diameters in porous materials based on the mercury intrusion data.

The limit distributions are compared with distributions determined from microscopic image analysis of samples obtained by the micro computed tomography $(\bigotimes \mathrm{CT})$ method. These distributions are considered as actual distributions of the pore space and are determined by prescription to each point (voxel) of the pore space of porous material, the diameter of the maximal sphere that contains this point and is completely inside the pore space (Hildebrand and Ruegsegger 1997).

Procter and Gamble Student poster award:

I don't want to compete References:

M. Cieszko, M. Kempiński, T. Czerwiński, Limit Models of Pore Space Structure of Porous Materials for Determination of Limit Pore Size DistributionsBased on Mercury Intrusion Data, Transport in Porous Media, DOI: 10.1007/s11242-018-1200-5, 2018.

Hildebrand T., Ruegsegger P., A new Method for the Model-Independent Assessment of Thickness in Three-Dimensional Images, Journal of Microscopy, 185, 1, pp. 67-75, 1997. Acceptance of Terms and Conditions:

Click here to agree

\title{
Correlation of magnetic resonance imaging and High-Resolution $\mathrm{X}$-ray Tomography to characterise pore size distributions in poly- meric open - cell sponges
}

\author{
Author(s): Gabriele M. Cimmarusti ${ }^{1}$ \\ Co-author(s): Abhishek Shastry ${ }^{2}$; Matthieu N. Boone ${ }^{2}$; Kostas Gkatzionis ${ }^{3}$; Melanie M. Britton ${ }^{1}$ \\ ${ }^{1}$ School of Chemistry, University of Birmingham \\ ${ }^{2}$ Department of Physics and Astronomy, Ghent University \\ ${ }^{3}$ School of Chemical Engineering, University of Birmingham
}

Corresponding Author(s): g.m.cimmarusti@bham.ac.uk

Nowadays, the applications of polymeric sponges are extensive, ranging from classical uses in cleaning 1 and filtration tasks 2, to more advanced uses in sampling devices [3] or eye surgery [4]. Irrespective of its actual application, characterisation of a sponge's properties is fundamental to its application. These properties, such as pore size and connectivity, which influence liquid uptake, retention and transport, are dependent on production methods and can change over time, depending on the way they are used 5. However, in order to gain a better understanding of sponge properties and behaviour, new methodologies are needed that allow the characterisation of these systems, preferably in situ, enabling a more complete characterisation. Magnetic resonance imaging (MRI) and High resolution X-ray Tomography $(\mu \mathrm{CT})$ offer complementary information about the structure and properties of porous media [6,7]. $\mu \mathrm{CT}$ provides precise information about the 3D structure of porous media, due to the typically high resolution of the technique, which can go down to the $\mu \mathrm{m}$ scale [6]. While MRI is unable to achieve the same spatial resolution, it can probe the 3D structure of porous media at a resolution of $10-100 \mu \mathrm{m}$ and is also able to probe the diffusion and flow of fluid within the system, providing an understanding of transport within the pore network [8]. Thus, by combining $\mu \mathrm{CT}$ and MRI, a more-complete understanding of the relationship between structure and transport becomes possible. While $\mu \mathrm{CT}$ has been used to study sponges [9], MRI has mostly studied porous media composed of inorganic materials, such as rocks or glass [10] and limited studies have been conducted on polymeric porous system. In this study, we have employed MRI, in combination with $\mu \mathrm{Ct}$, to study the structure and properties of a polymeric open-cell foam. T2 nuclear magnetic resonance (NMR) relaxation times of water have been measured for a range of open-cell sponges and correlated with the average pore size distribution determined by $\mu \mathrm{Ct}$. This methodology is found to demonstrate the complementarity of $\mathrm{MRI}$ and $\mu \mathrm{CT}$, leading to new insight in the characterisation of polymerics open-cell sponges.

Procter and Gamble Student poster award:

I would like to compete in the Procter and Gamble Student award References: 
1. G.L. Starry, Google Patents (1967).

2. Q. Ma et al., Small 12(2016) 2186.

3. W.S. Lambert, Google Patents (1993).

4. S. Itoh, Google Patents (1994).

5. B. Obi, Elsevier (2017).

6. V. Cnudde and M.N. Boone, Earth Sci. Rev.123 (2013)1.

7. M.M Britton, eMagRes (2011).

8. A. Vallatos et al., Ind. Eng. Chem. Res., 54(2015) 6825.

9. W. Y. Jang et al., Int. J. Solids Struct. 45(2008) 1845.

10. F. Marica et al., J. Magn. Reson. 178(2006)136.

Acceptance of Terms and Conditions:

Click here to agree

\title{
Crystalline calcium silicate hydrates could enable tailored per- meability control in the deep subsurface
}

\author{
Dan Plattenberger ${ }^{1}$; Catherine Peters $^{\text {None }}$; Florence Ling $^{\text {None }}$; Andres Clarens ${ }^{1}$ \\ ${ }^{1}$ University of Virginia
}

Corresponding Author(s): andres@virginia.edu

Efforts to develop next-generation energy technologies in the subsurface hinge on the ability to selectively control fluid migration in heterogeneous formations. In this work we report on the use of pseudowollastonite carbonation as a means to dramatically reduce the permeability of porous media in situ. Pseudowollastonite is a polymorph of calcium silicate ( $\mathrm{CaSiO} 3)$ with a strained ring crystal structure. It has been found to generate layered crystalline calcium silicates in addition to calcium carbonate when reacted at elevated pressures, temperatures, and $\mathrm{pH}$ conditions with $\mathrm{CO} 2$. The reaction products are noteworthy because they are extremely hard and resistant to acid attack, unlike carbonates and the calcium silicate gels that form cement, but also because they could precipitate selectively within pore throats, which would maximize their efficiency in controlling permeability in porous media. A series of sand column experiments were carried out in which pseudowollastonite was injected and then reacted with $\mathrm{CO} 2$. The reacted columns were then characterized using a suite of micro- and macroscale techniques including air permeability, scanning electron microscopy and energy dispersive X-ray spectroscopy (SEM-EDS), synchrotron $\mu \mathrm{X}$-ray diffraction ( $\mu \mathrm{XRD})$, and synchrotron $\mu \mathrm{X}$-ray fluorescence (XRF) mapping. To fully describe the reactive transport occurring in the column experiments, small powder batch experiments were conducted with pseudowollastonite and were analyzed via SEM-EDS and transmission electron microscopy (TEM). The results suggest that in subsurface applications the chemistry reported here may react in ways that are highly desirable for controlling the fate of fluid. The gas permeability of experimental columns was found to drop by three orders of magnitude before and after reaction. MicroXRF analysis of the columns reveals the ways in which the precipitated phases seem to crystalize in pore walls as opposed to pore bodies. The samples were doped with strontium to differentiate the carbonate and hydrated calcium silicate precipitates and bromide was added to the water to evaluate its penetration into the column. When these columns were exposed to acidic solutions the carbonate-based cements dissolved and the reaction front of precipitation followed be redissolution and precipitation further downstream. In columns containing pseudowollastonite on the other hand, very little change to the column was observed before and after exposure to the acidic solution. Additional experiments were carried out to evaluate the temperature sensitivity of these reactions, which are slower but still observed at temperature below $120^{\circ} \mathrm{C}$. Silicon to calcium ratios of the $\mathrm{CCSH}$ phases suggest that the reaction products are likely a combination of products including phyllosilicates, inosilicates, nesosilicates, and sorosilicates. These results could enable new techniques to deploy targeted deployment 
of permeability control in the deep subsurface for geologic carbon storage, enhanced geothermal energy, compressed air storage and other similar technologies.

Procter and Gamble Student poster award:

References:

Acceptance of Terms and Conditions:

Click here to agree

950

\section{Imaging pore scale processes in geomaterials}

Veerle Cnudde ${ }^{1}$

${ }^{1}$ Ghent University- UGCT

Corresponding Author(s): veerle.cnudde@ugent.be

Mass transport, fluid flow, evolving pores, mineral crystallization and dissolution, pore clogging and micro-fractures propagation of are all important phenomena closely related to durability studies of geomaterials. The study of these phenomena bears importance to a variety of real-world problems. To fully comprehend the impact of these processes on geomaterials in general, a good understanding of the underlying pore scale dynamics is vital. In this context, $4 \mathrm{D}$ pore-scale X-ray imaging offers new and exciting insights and the resulting $4 \mathrm{D}$ datasets are valuable to complement modelling studies. Besides providing input, 4D X-ray CT is also a valuable tool for validation of theories and models. We will discuss some of the current possibilities and challenges related to 4D imaging. Examples will be given of different experiments related to the imaging of dynamic pore scale processes in geomaterials.

Procter and Gamble Student poster award:

I don't want to compete References:

Acceptance of Terms and Conditions:

Click here to agree

797

\section{Investigation of fluid/fluid couplings in two-phase flows: appli- cation to soil remediation}

Author(s): Maxime Cochennec ${ }^{1}$

Co-author(s): Hossein Davarzani ${ }^{2}$; Yohan Davit ${ }^{3}$; Stéfan Colombano ${ }^{2}$; Ioannis Ignatiadis ${ }^{4}$; Michel Quintard

${ }^{1} B R G M$

${ }^{2}$ BRGM (French geological survey)

${ }^{3}$ Institut de Mécanique des Fluides de Toulouse

${ }^{4}$ BRGM

Corresponding Author(s): m.cochennec@brgm.fr

The main objective of this work is to derive effective parameters (e. g. permeabilities and additional coupling terms) for two-phase flows in highly permeable porous structures. We are interested here 
in the transport of Non-Aqueous Phase Liquid (NAPL) in groundwater and soils that have large permeabilities, such as sands with a permeability of $10^{-11}$ to $10^{-10} \mathrm{~m}^{2}$.

The derivation of effective parameters is well known for creeping single-phase subsurface flows in low permeability media. Phenomenological relationships combined with extension of the law obtained for single-phase flows are mainly used to model two-phase flows in low permeability system. These classes of models are generally termed generalized Darcy's law. Inherent assumption of such models is that, locally over a Representative Elementary Volume, phase configuration can be considered as quasi-static and therefore, the capillary pressure defined as the pressure difference between the averaged pressures is mainly determined by the averaging of Young-Laplace equation assuming a constant curvature.

Multiphase flows in highly permeable structures, such as coarse sands or distillation columns, requires a specific treatment due to the fact that, when the pore size becomes very large, Reynolds number, capillary number and Bond number attached to the flow can no longer be considered small, as it is assumed for the quasi-static assumption, i.e., the system can no longer be described as mainly controlled by capillarity. As a consequence, the classical generalized Darcy's law model must be adapted. While this is largely an open question, experimental and theoretical evidence suggest that, at least, terms representing the drag force between the two phases must be added to the generalized Darcy's law. This has been emphasized in recent studies showing, for instance, a deviation from the hydrostatic pressure drop in water observed in a vertical column subject to an upward gas flow 1.

Models exist to take into account the coupling between phases and are written, for a creeping flow

$\mathbf{V}_{\alpha}=-\frac{1}{\mu_{\alpha}} \mathbf{K}_{\alpha \alpha} \cdot\left(\nabla P_{\alpha}-\rho_{\alpha} \mathbf{g}\right)$

$-\frac{1}{\mu_{\kappa}} \mathbf{K}_{\alpha \kappa} \cdot\left(\nabla P_{\kappa}-\rho_{\kappa} \mathbf{g}\right) \quad \alpha, \kappa=\beta, \gamma \quad \alpha \neq \kappa$

where $\beta$ and $\gamma$ label fluid phases and $\mathbf{K}_{\beta \gamma}$ and $\mathbf{K}_{\gamma \beta}$ are permeability cross terms .

For flows with strong inertia effect, one can derive the following generalized form accounting for inertia effects

$\mathbf{V}_{\alpha}=-\frac{1}{\mu_{\alpha}} \mathbf{K}_{\alpha \alpha} \cdot\left(\nabla P_{\alpha}-\rho_{\alpha} \mathbf{g}\right)-\mathbf{F}_{\alpha \alpha} \cdot \mathbf{V}_{\alpha}$

$-\frac{1}{\mu_{\kappa}} \mathbf{K}_{\alpha \kappa} \cdot\left(\nabla P_{\kappa}-\rho_{\kappa} \mathbf{g}\right)-\mathbf{F}_{\alpha \kappa} \cdot \mathbf{V}_{\kappa} \quad \alpha, \kappa=\beta, \gamma \quad \alpha \neq \kappa$

where $\mathbf{F}_{\beta \gamma}$ and $\mathbf{F}_{\gamma \beta}$ are cross terms associated with the inertial correction and $\mathbf{F}_{\beta \beta}$ and $\mathbf{F}_{\gamma \gamma}$ refers to terms similar to those appearing in the generalized Ergun's law. Such models can be derived, for instance, from upscaling procedures such as volume averaging applied to systems respecting the quasi-static hypothesis 2 .

By conducting direct numerical simulations in cylinder bundles, we investigate the sensibility of the effective parameters on capillary length, capillary number and permeability within the limit of creeping flows. Numerical simulations are based on the Boundary Element Method, which is particularly suitable for modeling creeping flow with free boundaries. A Brinkman formulation is used to include some of the $3 \mathrm{D}$ effects on the flow. This work provides information about the dependency of effective terms for flows that challenge the assumptions underlying the construction of conventional macroscopic models.

\section{Procter and Gamble Student poster award:}

I would like to compete in the Procter and Gamble Student award References:

1 Chikhi, N., Clavier, R., Laurent, J. P., Fichot, F., \& Quintard, M. (2016). Pressure drop and average void fraction measurements for two-phase flow through highly permeable porous media. Annals of Nuclear Energy, 94, 422-432.

2 Davit, Y., \& Quintard, M. (2018). One-Phase and Two-Phase Flow in Highly Permeable Porous Media. Heat Transfer Engineering, 1-19. Acceptance of Terms and Conditions:

Click here to agree 


\title{
Multiscale characterization of effective conductivity of random heterogeneous porous media: multiple versus single realization approaches.
}

\author{
${\text { Ivan } \text { Colecchio }^{1} \text {; Benoit Noetinger }}^{2}$; Alejandro Boschan ${ }^{3}$; Alejandro Otero ${ }^{4}$ \\ ${ }^{1} 5$ Facultad de Ingeniería, Universidad de Buenos Aires \\ ${ }^{2}$ IFPEN \\ ${ }^{3}$ Facultad de Ingeniería, Universidad de Buenos Aires \\ ${ }^{4}$ Centro de Simulación Computacional, CSC - CONICET
}

Corresponding Author(s): colecchio.ivan@gmail.com

\begin{abstract}
Most porous natural or synthetic materials possess a multiscale structure that have a major impact on large scale transport properties. In particular, the large scale effective conductivity associated to thermal, electrical or hydraulic fluxes depends on the media microstructure as well as on the spatial scale considered.

In this talk, the potential field that solves the up scaling closure problem for a single large realization $\left(4096^{\wedge}\right.$ 2grid blocks) of a 2D media sample (a Log normal with Gaussian or exponential covariance, or binary media of high conductivity contrast of $10^{\wedge} 4$ ) is first determined.
\end{abstract}

Then, using two different post treatment of that potential, we calculate two descriptors of the equivalent conductivity for any subsample. These descriptors correspond to the evaluation of the viscous dissipation and of the average flow rate in that subsample, both estimators requiring negligible extra cost once the full potential has been determined.

The probability density function (pdf) of these descriptors are studied as a function of the subsample size and are compared with reference pdf determined by directly solving the subsample up-scaling closure problem by a complete and far more expansive Monte Carlo study. Each descriptor converges towards the full sample value if the REV size is smaller that the full sample size. Numerical results are compared in the asymptotic case to analytical estimations. For log normal media, these estimations are found to be accurate, even for large input Log $\mathrm{k}$ variance. This also happens for binary media, except near percolation, where slow convergence to the asymptotic regime is observed. These post-treatment strategies are discussed for the REV determination, as well as for a deeper understanding of the convergence of the effective properties distribution to a limiting one.

Future work using $3 \mathrm{D}$ cases, power law conductivity distributions and long ranged correlations will be discussed, as well as applications of such finding to uncertainty quantification.

Procter and Gamble Student poster award:

References:

Boschan, A., and B. Noetinger (2012), Scale dependence of effective hydraulic conductivity distributions in 3d heterogeneous media: a numerical study, Transport in porous media, 94(1), 101-121.

Bauer, D., L. Talon, and A. Ehrlacher (2008), Computation of the equivalent macroscopic permeability tensor of discrete networks with heterogeneous segment length, Journal of Hydraulic Engineering, 134(6), 784-793

Rubin, Y., and J. J. Gómez-Hernández (1990), A stochastic approach to the problem of up scaling of conductivity in disordered media: Theory and unconditional numerical simulations, Water Resources Research, 26(4), 691-701.

Sánchez-Vila, X., J. P. Girardi, and J. Carrera (1995), A synthesis of approaches to upscaling of hydraulic conductivities, Water Resources Research, 31(4), 867-882. Acceptance of Terms and Conditions:

Click here to agree 


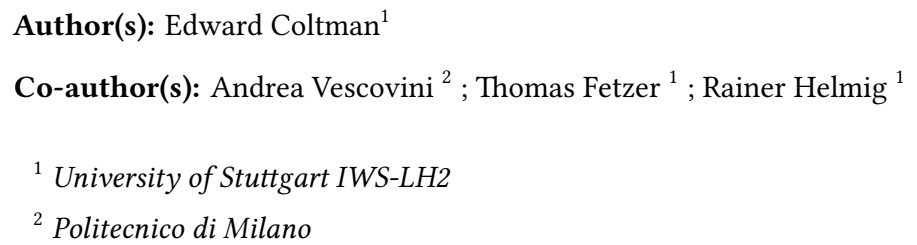

Corresponding Author(s): edward.coltman@iws.uni-stuttgart.de

Mass, momentum, and energy exchange processes at the soil-atmosphere interface depend on the dynamics of flow both above, below, and at the surface interface. This leads to a strongly coupled behavior across flow domains.

In the free-flow domain, the development of boundary layers in free-flows depends largely on the momentum, energy, and compositional content of the atmosphere. In the subsurface, diffusive evaporation and capillary effects will dominate the thermo- and multi-phase flow dynamics in the subsurface. The size of the boundary layers developed in the free flow will define the primary evaporation rate at the surface, exchanging mass and energy and altering the rates beneath the surface. Changes to the subsurface saturation and the undulating shape of the interface will influence momentum exchange from one domain to the other, further modifying the exchange rates between the domains. In this work, a coupled turbulent free-flow and multi-phase, compositional, non-isothermal porous media flow model has been developed, where soil-water evaporation across undulating surfaces is simulated on the REV scale. This simulation data has been compared to experimental work, and the validity of the model concept evaluated.

Procter and Gamble Student poster award:

I would like to compete in the Procter and Gamble Student award References:

Acceptance of Terms and Conditions:

Click here to agree

\title{
A predictive spatial Markov model for transport in heterogeneous Darcy flows
}

\author{
Alessandro Comolli ${ }^{1}$; Vivien Hakoun ${ }^{2}$; Marco Dentz ${ }^{2}$ \\ ${ }^{1}$ Université libre de Bruxelles - NLPC \\ ${ }^{2}$ IDAEA - CSIC
}

Corresponding Author(s): alessandro.comolli@ulb.ac.be

Solute transport in porous media generally exhibits a non-Fickian character that can be traced back to multi-scale heterogeneity. This anomalous character manifests itself in heavy-tailed breakthrough curves and non-Gaussian solute concentration plumes.

Understanding and predicting these transport behaviours is crucial for several environmental issues, including aquifer contamination remediation and risk assessment in nuclear waste repositories.

In order to develop a predictive large-scale transport formulation, it is essential to understand the relationships between transport features, and medium and flow attributes, as well as the impact of the injection conditions on early- and late-time dynamics. We investigate these relationships by considering different scenarios of Darcy flows in porous media characterised by spatially-varying hydraulic properties. In particular, we consider broad distributions of hydraulic conductivity with point values spanning over several orders of magnitude.

We propose a correlated continuous time random walk model (cCTRW) to describe and predict transport in several scenarios of increasing heterogeneity. In the spirit of the recent works by Dentz et al. (2016) and Hakoun et al. (under review), this cCTRW is based on a Markov velocity process which accounts for injection conditions that lead to non-stationary Lagrangian velocity statistics. 
The effective transport model that we propose is parameterised in terms of the velocity correlation length, which relates to the correlation length of the hydraulic properties, and of Eulerian statistics, i.e. in terms of transport-independent quantities, which provides the model with a predictive character.

The presented approach allows us to quantify the impact of advective heterogeneity and of different injection conditions on average transport behaviour. We show that both processes are crucial for the fate of effective transport characteristics such as the moments of particle displacements and the breakthrough curves.

Within our modelling framework, we derive analytical predictions that show good agreement with cCTRW and direct numerical simulations.

Procter and Gamble Student poster award:

References:

- Dentz, M., P. K. Kang, A. Comolli, T. Le Borgne, and D. R. Lester (2016), Continuous time random walks for the evolution of lagrangian velocities, Physical Review Fluids, 1 (7), 074,004.

- Hakoun, V., A. Comolli, and M. Dentz, under review.

Acceptance of Terms and Conditions:

Click here to agree

\title{
Properties of $A+B->C$ reaction fronts under radial advection in three-dimensional systems
}

\author{
Alessandro Comolli ${ }^{1}$; Anne De Wit ${ }^{2}$; Fabian Brau ${ }^{2}$ \\ ${ }^{1}$ Université libre de Bruxelles, NLPC \\ ${ }^{2}$ Université libre de Bruxelles - NLPC
}

Corresponding Author(s): alessandro.comolli@ulb.ac.be

Understanding the properties of reactive transport in porous media is crucial for several environmental issues, including carbon dioxide sequestration and aquifer contamination remediation, among others. In particular, the bimolecular irreversible $\mathrm{A}+\mathrm{B} \rightarrow \mathrm{C}$ chemical reaction is ubiquitously observed in nature and the properties of the related reaction-diffusion fronts have been the subject of intense investigation from both theoretical and experimental points of view since the founding paper of Gálfi \& Rácz (1988), describing their scalings in a rectilinear geometry.

Most of the studies since then have focused on the case of a reactant A being injected into a region filled with a reactant B along a rectilinear front. In contrast, only few recent works (Brau et al. 2017, Trevelyan and Walker 2018) have investigated the properties of A + B -> C fronts in radial symmetries. In $2 \mathrm{D}$ systems where the reaction front is subjected to passive radial injection, the front position, its width and the reaction rate keep the same asymptotic temporal scaling as in the rectilinear case. However, the proportionality constants depend on the injection flow rate, which means that the front position and the reaction production rate can be tuned by the injection flow velocity.

Here, we extend the results from Brau et al. to $d=3$ dimensions. Analytical results for the asymptotic regimes are provided and compared to the results of numerical simulations. We show that the properties of the radial front in 3D in presence of advection are quite different from those in 2D. We discuss the consequences of these properties on the production of $\mathrm{C}$ and their implications on remediation strategies.

Procter and Gamble Student poster award:

References: 
- Gálfi, L., \& Rácz, Z. (1988). Properties of the reaction front in an A + B -> C type reaction-diffusion process. Physical Review A, 38(6), 3151.

- Brau, F., Schuszter, G., \& De Wit, A. (2017). Flow control of A + B -> C fronts by radial injection. Physical Review Letters, 118(13), 134101.

- Trevelyan, P. M. J., \& Walker, A. J. (2018). Asymptotic properties of radial A + B -> C reaction fronts. Physical Review E, 98(3), 032118.

Acceptance of Terms and Conditions:

Click here to agree

540

\title{
Multiphase Flow Modeling for the Estimation of the Interphase Mass Transfer of Enhanced DNAPL Solubilization
}

\author{
Aybike Gul Karaoglu ${ }^{\text {None }}$; Nadim Copty ${ }^{1}$; Nihat Hakan Akyol $^{2}$; Seda Aslan Kilavuz ${ }^{2}$; Masoud Babaei ${ }^{3}$ \\ ${ }^{1}$ Bogazici University \\ ${ }^{2}$ Kocaeli University \\ 3 The University of Manchester
}

Corresponding Author(s): ncopty@boun.edu.tr

Enhanced solubilization of entrapped DNAPLs has emerged as one of the most effective techniques for the remediation of subsurface DNAPLs zones. A key process governing the effectiveness of these remediation techniques is the interphase mass transfer. Multiphase flow models can be used to estimate the interphase mass transfer coefficient from effluent concentration data. The calibration of such models is a challenging task due to the lack of detailed data describing the DNAPL spatial distribution and the governing the fate and transport processes. In this study laboratory scale experiments were conducted to evaluate reagent-enhanced DNAPL solubilization in saturated heterogeneous media. The DNAPL consisted of both pooled and residual forms. To gain insight into the influence of various input parameters on effluent concentrations, sensitivity coefficients of key parameters were numerically computed. The sensitivity coefficients were, in turn, used to underpin the difficulties associated with the calibration of multiphase flow models, most notably the non-uniqueness of calibration process when partial input data are available. To alleviate this uncertainty and provide additional constraints, the conducted flushing experiments were jointly used in the calibration process. The calibrated model was then used to estimate the interphase mass transfer coefficient. The results suggest that the interphase mass transfer coefficient is also dependent on the flushing solution viscosity and the interfacial tension between the DNAPL and aqueous phase.

Procter and Gamble Student poster award:

References:

Acceptance of Terms and Conditions:

Click here to agree

\section{A Study of the Total Compressibility in Three-Phase Immiscible Flow in Poroelastic Media}

Author(s): Maicon Correa ${ }^{1}$

Co-author(s): Marcio Borges ${ }^{2}$; Marcio Murad $^{2}$ 
${ }^{1}$ University of Campinas - Brazil

${ }^{2}$ LNCC - Brazil

Corresponding Author(s): maicon.correa@gmail.com

In 1 a new computational model is developed to solve three-phase immiscible compressible flow in strongly heterogeneous poroelastic media where, within the context of an iterative coupled formulation based on the fixed stress split algorithm, the governing equations are decomposed into three subsystems associated with the geomechanics, hydrodynamics and transport problems [2,3]. The proposed methodology gives rise to a new pressure equation supplemented by a source term involving the time derivative of the total mean stress with more complex coefficients, dependent on pressure and saturations that extends the two-phase incompressible model discussed in [4]. The hydrodynamics subsystem involves an overall compressibility which plays an essential role in the magnitude of the additional source in the pressure equation associated the time derivative of the total mean stress. The additional coefficients in the source can be decomposed into a rock/grain and fluid components involving the formation volume factors of each phase. In this work, by using the one-way and the two-way numerical methodologies proposed in 1 , we study the influence of the fluid and rock properties on the total compressibility and study the numerical dependence of the sequential iterative scheme on the saturations of the phases. Numerical results illustrate the role of the transient nature of the total compressibility upon subsidence, rock compaction, oil and gas production and finger grow in primary and secondary recovery regimes.

Procter and Gamble Student poster award:

References:

1 M. R. Correa, M. A. Murad, A new sequential method for the three-phase immiscible flow in poroelastic media. Journal of Computational Physics, 373, 493-532, 2018.

2 A. Mikelic and M.F. Wheeler. Convergence of iterative coupling for coupled flow and geomechanics. Computational Geosciences, 17(3):455--461, 2013.

[3] J. Kim, H.A. Tchelepi and R. Juanes. Stability and convergence of sequential methods for coupled flow and geomechanics: Fixed-stress and fixed-strain splits. Computer Methods in Applied Mechanics and Engineering, 200(13-16):1591-1606, 2011.

[4] M. A. Murad, M. R. Borges, J. A. Obregon, and M. R. Correa. A new locally conservative numerical method for two-phase flow in heterogeneous poroelastic media. Computer and Geotechnics, 42:192-207. 2013.

5 M. R. Correa and M. R. Borges. A semi-discrete central scheme for scalar hyperbolic conservation laws with heterogeneous storage coefficient and its application to porous media flow. International Journal for Numerical Methods in Fluids. 73:205-224. 2013 Acceptance of Terms and Conditions:

Click here to agree

\title{
Drying thanks to nano-films?
}

\author{
Alban Gossard $^{1}$; Benoit Coasne ${ }^{2}$; Mohamed Nidal BEN ABDELOUAHAB ${ }^{3}$; Philippe Coussot $^{4}$ \\ ${ }^{1} C E A$ \\ ${ }^{2}$ CNRS/University Grenoble Alpes \\ ${ }^{3}$ CEA Marcoule - Laboratoire Navier \\ ${ }^{4}$ Univ. Paris-Est
}

Corresponding Author(s): philippe.coussot@ifsttar.fr

Basic phenomenological mechanisms of drying in simple solid porous systems initially filled with pure liquid and in the absence of gravity effects have been identified 1. First, a constant (drying) rate period $(\mathrm{CRP})$, with a homogeneous desaturation of the medium, occurs. In this regime capillary 
equilibration processes allow for water redistribution throughout the medium so that the saturation decreases uniformly. This is followed by a falling-rate period (FRP) associated with the development of a heterogeneous profile of saturation. It is generally considered that the onset of this results from a demand of liquid, through the imposed evaporation rate at the free surface, larger than the liquid transport within the porous system induced by a gradient of capillary pressure 2. The mechanism assumed to govern this second regime is the inward growth of a dry region from the sample free surface, through which vapor diffuses before reaching the free surface. However, existing internal observations do not provide unambiguous evidence that such a dry region effectively develops [3-4]. Moreover, it was shown that, surprisingly, as it dries, a model porous medium with pore size of the order of a few nanometers, desaturates almost homogeneously [3,5]. This suggests that some kind of continuous molecular liquid film remains present along the (hydrophilic) solid surface in that case, to allow the liquid transport towards the sample exit.

To clarify this problem, we look at the mechanisms of liquid extraction under different boundary conditions. The fundamental observation is obtained from the "drying" of a model (hydrophilic) porous medium covered with a wet paste. NMR measurements show that the resulting extraction rate and the liquid distribution in the porous medium in time, are similar to those for drying of the same porous medium directly submitted to a dry air flux, whereas in the former case air is saturated with vapour so that vapor diffusion is a priori absent. This suggests that a continuous, molecular liquid film exists throughout a hydrophilic porous medium and this at any time during its convective drying at any stage. This contrasts with the usual assumption of dry front formation in the last stages of the process. This leads to a change of paradigm: drying simply results from the suction of liquid imposed by the evaporation of the molecular film along the available liquid-air interface at the sample free surface. As a consequence, the drying rate of a material with a given structure type can be described by a unique master curve for the saturation vs time scaled by initial drying rate, whatever the pore size (or, equivalently, the permeability), the sample height and the air flux velocity, and whatever the resulting liquid distribution in the porous medium (more or less heterogeneous).

References

1 J. Van Brakel, Mass transfer in convective drying, Adv. Drying, 1, 217-267 (1980)

2 P. Coussot, European Physical Journal B 15, 557-566 (2000)

[3] J. Thiery, S. Rodts, D.A. Weitz, P. Coussot, Phys. Rev. Fluids, 2, 074201 (2017)

[4] K.U. Goss, M. Madliger, Water Resources Research, 43, W05433 (2007)

5 J. Thiery, S. Rodts, E. Keita, X. Chateau, P. Faure, D. Courtier-Murias, T.E. Kodger, P. Coussot, Phys. Rev.E, 91, 042407 (2015)

Procter and Gamble Student poster award:

References:

Acceptance of Terms and Conditions:

Click here to agree

\title{
Direct simulation of the effect of over-burden pressure on poros- ity and permeability of unconsolidated sands
}

\author{
Bernd Crouse $^{1}$; Juan Bautista ${ }^{1}$; Shaun Gill ${ }^{1}$; David Freed ${ }^{1}$ \\ ${ }^{1}$ Dassault Systemes
}

Corresponding Author(s): bernd.crouse@3ds.com

The mechanical stresses on a rock in the subsurface depend on reservoir depth and pore pressure. The resulting over-burden pressure (often referred to as Net Confining stress NCS) can affect rock properties, such as porosity and permeability. Reservoir conditions, and in particular variations of reservoir conditions are often not matched in laboratory testing.

Most commercial micro-CT scanners operate at ambient conditions when taking a digital 3D image 
of a pore space. This may lead to some differences in pore space geometry when the effect of compression is not modeled.

We present a digital workflow to simulate both, the compaction of an unconsolidated sand using a finite element (FE) method as well as the permeability for various NCS levels using a lattice-Boltzmann (LB) solver. An in-depth analysis of the porosity and permeability variation is provided to provide better understanding.

We create a finite element mesh for each individual grain of a sand pack, and initialize all grains randomly within a test container, modeled as a FE mesh, in such a way that they do not touch each other. Gravity is applied to simulate the movement of all grains falling into the container using a finite element solver. The process of obtaining the initial location and orientation of all grains is finalized when all grains are not moving anymore. A rigid plate FE model is put on top of the sand pack within the container. We then perform a second FE calculation to simulate the effect of overburden pressure, by defining a step-wise translation of the rigid plate and measuring the resulting force on the rigid plate. During the compaction we observe relocation of sand grains and some elastic deformations.

For every NCS we derive a pore space from the compressed grain space and perform pore scale single-phase flow and multi-phase flow simulations 1 to study the impact of NCS on absolute and relative permeability.

We observe that the stress dependent porosity is consistent with laboratory data of an unconsolidated sand reported in 2. The slight under-prediction could be related to the lack of modeling of plastic deformations such as grain crushing and grain-grain penetration.

The reduction of absolute permeability for various NCS levels follows the trend of weakly consolidated sandstones up to 3000 PSI. For stress levels above 3000 PSI, we observe larger deviations which we associate with not including plastic deformation in the compaction process, which would likely lead to a stronger pore space alteration.

In summary, the presented compaction process models grain relocation and elastic deformations. For moderate NCS the simulated porosity and permeability trends compare well with published data. Large NCS levels require to include plastic deformation in the FE calculation.

Procter and Gamble Student poster award:

References:

1 Gary Jerauld et al, "Validation of a Workflow for Digitally Measuring Relative Permeability", SPE188688-MS, ADIPEC, 2017

2 Matthew J. Saul, "Pressure-dependent elastic properties of sandstones, with applications to seismic reservoir characterization and monitoring", PhD thesis, July 2014, University of Western Australia Acceptance of Terms and Conditions:

Click here to agree

\section{A comparison between 2D, quasi-3D and fully-3D phase-field sim- ulations of two-phase displacements at the pore scale}

Luis Cueto-Felgueroso ${ }^{1}$; Amir Pahlavan ${ }^{2}$; Robin Zhao $^{3}$; Ruben Juanes ${ }^{2}$

\footnotetext{
${ }^{1}$ Universidad Politécnica de Madrid

${ }^{2}$ MIT

${ }^{3}$ Massachusetts Institute of Technology
}

Corresponding Author(s): luis.cueto@upm.es

The combination of high-resolution visualization techniques and pore-scale flow modeling is a powerful tool to understand multiphase flow mechanisms in porous media and their impact on reservoirscale processes. Phase-field, or diffuse-interface, modeling is an effective framework to construct 
multiphase theories that capture interfacial and wetting phenomena in flow through porous media.

In the context of a recent benchmark study on pore-scale models for multiphase flow in porous media, we compare the flow processes and displacement patterns predicted by three different modeling approaches of increasing complexity. The problem under study is the viscously unfavorable fluidfluid displacement in a microfluidic flow cell patterned with vertical posts. By varying flow rates and wetting conditions, the experimental results explore a rich phase diagram of displacement patterns and processes.

We consider three approaches to simulate this flow problem: a purely 2D phase-field formulation, where flow is assumed to be uniform in the gap; a quasi-3D formulation that partially captures the $3 \mathrm{D}$ viscous and capillary coupling between fluids in the cell gap, in the context of a 2D, gap-averaged Darcy formulation; and, finally a fully-3D Cahn-Hilliard-Stokes formulation that is conceived as a high-fidelity flow model for this problem. We discuss the range of applicability of each modeling approach, depending on the capillary number and wetting conditions, and discuss the open challenges in modeling unstable imbibition processes in porous media.

Procter and Gamble Student poster award:

I don't want to compete References:

Acceptance of Terms and Conditions:

Click here to agree

\title{
IFBattery Max Power, Internal Resistance and Specific Energy for an n-Cell System in Series Arrangement
}

\author{
John Cushman ${ }^{1}$; Eric Nauman ${ }^{2}$; Mika Dziekan ${ }^{2}$ \\ ${ }^{1}$ IFBattery and Purdue University \\ ${ }^{2}$ IFBattery and Purdue
}

Corresponding Author(s): jcushman@purdue.edu

The IFBattery flow-battery system is very different from a classical system-power, internal resistance and energy density are nonlinear in the number of cells in series. In a classical battery system, each cell is isolated, possessing its own anode, cathode and electrolyte, and the only motion interior to the cell is diffusion of ions on an electrochemical gradient. In the IFBattery system all cells are immersed in a common electrolyte that is in rapid convective motion relative to the anodes and cathodes. As we next illustrate, this makes the internal resistance a nonlinear function of the number of cells in series, which in turn makes specific energy and power a nonlinear function of the number of cells in series-and fortunately for the IFBattery battery, the nonlinearity works very positively in favor of the IFBattery battery relative to classical systems.

The battery produces electricity directly, and by judicious choice of the chemistry, can simultaneously produce vast quantities of hydrogen gas (no Oxygen) that can be directed at low pressure to a fuel cell. Here we focus on only the direct electricity generation.

Increasing the convective flow velocity from zero to some critical value increases power and decreases internal resistance. Beyond this critical value, further increases in convective velocity decreases the power and increases internal resistance. For this presentation, two types of flow cells have been employed to create convective flow: one uses a rectangular acrylic flow cell, open at the top to allow Hydrogen to escape, and mounted on an orbital shaker; the other uses stationary, sealed, clear, PVC tubes with the flow directed vertically upwards and driven by a pump, Hydrogen is removed at the pump instead of directly from the cell. The later design produces higher power with a 3-cell system reaching 30 Watts.

Typical statistics are presented below for the orbital shaker system:

Specific energy $(\mathrm{Wh} / \mathrm{kg})$, direct electric only, cells arranged in series in common electrolyte:

1-cell 273

2-cell 544

4-cell 1140 
9-cell 1365

Max power and internal resistance with cells in series arrangement:

Cells, max power, internal resistance

$1,3.16 \mathrm{~W}, 0.435 \mathrm{Ohms}$

2, 9.01W, 0.517 Ohms

$3,19.0 \mathrm{~W}, 0.515 \mathrm{Ohms}$

$5,32.5 \mathrm{~W}, 0.532 \mathrm{Ohms}$

Using the above data for max power, $\mathrm{Wn}$,for an n-node series arrangement of the cells on the 3- and 5 -cell systems we find $\mathrm{Wn} \sim \mathrm{n}^{\wedge} 1.84$ and $\mathrm{n}^{\wedge} 1.86$, respectively, which is quite close to $\mathrm{n}^{\wedge} 2$. The punch line is that classical systems have power scaling with $\mathrm{n}$ and for the IFBattery system in this example power scales with $\mathrm{n}^{\wedge} 1.85$, a very favorable result.

Procter and Gamble Student poster award:

I don't want to compete References:

Acceptance of Terms and Conditions:

Click here to agree

660

\section{Simulations of coupled flow and geomechanics in porous media with embedded discrete fractures}

Matteo Cusini ${ }^{1}$; Nicola Castelletto ${ }^{\text {None }}$; Joshua A. White ${ }^{2}$

${ }^{1}$ Delft University of Technology

${ }^{2} L L N L$

Corresponding Author(s): m.cusini@tudelft.nl

The decision making process in many geoengineering applications has to be supported by accurate numerical models that help to predict how fluids displace in the underground and the geomechanical response of the surrounding rock. For some systems the geomechanical and the flow and transport problems are only loosely coupled and can be solved separately. However, in fractured natural formations the coupling is much stronger since the hydrodynamical properties (i.e., aperture, conductivity) of the fractures are highly dependent on the geomechanical configuration. Thus, accurate simulation requires to solve the coupled system of equations describing flow and geomechanics. In this work, we employ a finite-volume discretization for the flow equation coupled with a finiteelement scheme for the geomechanics. A friction model is also considered to describe the contact behaviour at the location of closed fractures. Fractures are embedded in the rock matrix grid and their effect is captured by employing the embedded discrete fracture model (EDFM) $[1,2]$ and the Assumed Enhanced Strain (AES) method [3,4], to represent their contribution to the flow and the mechanics, respectively. This embedded discretization reduces considerably the complexity of the grid and allows for different grids to be used for the fractures and the matrix. EDFM considers fractures as lower dimensional entities which exchange a mass flux with the rock matrix. The discrete representation of the flux between the two media is directly related to how the fractures cells intersect the matrix ones. The AES method, on the other hand, assumes a local enrichment of the FEM solution space inside each matrix cell cut by a fracture element.

Numerical experiments are presented to study the convergence and the accuracy of the proposed method.

\section{Procter and Gamble Student poster award:}

\section{References:}

1 S. Lee, M. Lough, C. Jensen, Hierarchical modeling of flow in naturally fractured formations with multiple length scales, Water Resour. Res. 37(3): 443-455,2001.

2 H. Hajibeygi, D. Karvounis, P. Jenny, A hierarchical fracture model for the iterative multiscale finite volume method, J. Comput. Phys. 230(24): 8729-8743, 2011. 
[3] J. C. Simo, M. S. Rifai. A class of mixed assumed strain methods and the method of incompatible modes, International journal for numerical methods in engineering, 29(8):1595-1638, 1990.

[4] R. I. Borja. Assumed enhanced strain and the extended finite element methods: A unification of concepts, Computer Methods in Applied Mechanics and Engineering, 197(33):2789-2803, 2008. Acceptance of Terms and Conditions:

Click here to agree

\title{
A local time-stepping (LTS) strategy for fully-implicit simulation of multiphase flow in porous media
}

\author{
Matteo Cusini ${ }^{1}$; Fanxiang $\mathrm{Xu}^{1}$; Hadi Hajibeygi ${ }^{1}$ \\ ${ }^{1}$ TU Delft \\ Corresponding Author(s): m.cusini@tudelft.nl
}

\begin{abstract}
Most challenges of simulating multiphase flow in natural porous media derive from the variety of length and time-scales involved. While a considerable effort has been done by the scientific community to deal with the disparity of length-scales by introducing, for example, multiscale methods and adaptive mesh refinement techniques, less attention has been given to how to tackle the different time scales.

In this work, we propose a local time-stepping (LTS) method for fully-implicit (FIM) simulation of multiphase flow in natural porous media. The application of local time-stepping techniques to hyperbolic problems has been thoroughly studied. However, the fully-coupled system of equations describing flow and transport in porous media has a mixed elliptic (or parabolic) - hyperbolic nature. As such, it is more challenging to devise a LTS method for such a system. Here, we propose to employ a predictor-corrector strategy. Starting from simulation time $t$, first, the full system is solved, implicitly, with the use of a coarse time-step $d t_{c}$. Then, based on an error criterion, the regions of the domain where a smaller time-step should have been employed are identified (e.g., around the advancing saturation front). Finally, the non-linear coupled system of equations is solved with a much finer time-step, $d t_{f}$ in these regions until the time $t+d t_{c}$ is reached. A flux boundary condition that ensures mass conservation is applied at the interface between the fine and the coarse time-step regions.

Numerical test cases are presented to demonstrate the accuracy and the efficiency of the method. The LTS solution is compared against a reference one obtained by employing a uniform high resolution time-step. It is shown that results are in good agreement even though LTS only employs the smaller time-step in a small fraction of the domain.
\end{abstract}

Procter and Gamble Student poster award:

References:

Acceptance of Terms and Conditions:

Click here to agree

36

History Matching of Hydraulic Fracture Network in Shale-Gas Reservoir囚Application of Ensemble Kalman Filter Coupled with Embedded Discrete Fracture Modeling

\author{
Author(s): Cheng Dai ${ }^{1}$ \\ Co-author(s): Hua Liu ${ }^{1}$; Haibin Chang ${ }^{2}$
}


${ }^{1}$ State Key Laboratory of Shale Oil and Gas Enrichment Mechanisms and Effective Development, SINOPEC Group, Beijing, China

${ }^{2}$ Peking university

Corresponding Author(s): daicheng.syky@sinopec.com

The most distinctive characteristic of a shale gas reservoir is its extremely low permeability and porosity. Therefore, multistage hydraulic fracturing and horizontal wells (MHFHWs) are widely used to enhance the production. Many previous study have revealed that the spatial extent and properties of hydraulic fracture was critical to MHFHWs' production. However, limited information of activated natural fracture in the formation leads to high uncertainty of stimulated area as well as the well performance. History matching is always used to determine the uncertain data in reservoir simulation and it could be a feasible way to determine the extent of hydraulic fracture network.

In this study, we proposed a new method for history-matching of the Hydraulic fracture by use of Ensemble Kalman Filter(EnKF) coupled with embedded discrete fracture modeling冈EDFM】. For each hydraulic fracturing stage, the fracture network is parameterized by a set of uncertain parameters including main fracture length, width of stimulated area, fracture density et al. With these parameters, the fracture network in which each fracture is treaded as a discrete fracture embedded into the background can be generated stage by stage. After parameterization, EnKF is applied to perform the history-matching. As each fracture is treaded as embedded fracture, ones do not need to adjust the background grid system during the history-matching which make the approach much more convenient in some case with the complex geologic structures. As Microseismic surveillance is used quite often in field nowadays, we also consider the prior constraint in our proposed approach. We first define the effective microseismic events area for each stage. Then, the events within the effective area are used to generate discrete fracture while events without the area are abandoned. The length, width and height of the effective microseismic events area are adjusted gradually by historymatching and the reasonable effective area is provided with the assimilation of all production history. In this study, both synthetic and real case are proposed to validate the approach. The numerical results show that by use of the proposed approach, the extent of hydraulic fracture network can be well recognized and the production history can be well matched.

Procter and Gamble Student poster award:

I would like to compete in the Procter and Gamble Student award References:

Acceptance of Terms and Conditions:

Click here to agree

\title{
mpact of the initial condition on concrete behavior during severe loading conditions
}

\author{
Stefano Dal Pont ${ }^{1}$; Giuseppe Sciumè ${ }^{2}$ \\ ${ }^{1}$ University Grenoble-Alpes \\ ${ }^{2}$ University of Bordeaux
}

Corresponding Author(s): stefano.dalpont@3sr-grenoble.fr

This work focuses on the impact of concerte early age behavior and structure service-life conditions on an accidental condition such as a fire or a Loss-of-Coolant Accident. Concrete structure exposed to fire or, more generally, to high temperatures/high pressure, are sensitive to spalling, i.e. the violent detachment of flakes that exposes steel reinforcement and may lead to structure failure. Numerical experiments are performed using a fully coupled multi-phase model based of porous media mechanics taking into account dependencies of material parameters on hydration advancement degree. Concrete hydration at early age and dehydration/degradation due to high temperature exposure are here treated by means of an original unified approach. The mathematical model is based on general conservation equations of mass, energy and linear momentum: the explicit introduction of the stoichiometric model of Powers allows deriving some of the needed closure relationship; 
a new constitutive model is also proposed for the desorption isotherm. The paper underlines the importance of describing a more realistic condition to properly gather local phenomena (moisture accumulation, gas pressure increase) that might trigger spalling.

Procter and Gamble Student poster award:

I don't want to compete References:

Acceptance of Terms and Conditions:

Click here to agree

\title{
The importance of passive materials in Li-Ion battery electrodes
}

\author{
Timo Danner ${ }^{1}$; Simon Hein $^{2}$; Daniel Westhoff ${ }^{3}$; Benedikt Prifling ${ }^{3}$; Volker Schmidt ${ }^{3}$; Arnulf Latz $^{1}$ \\ ${ }^{1}$ German Aerospace Center (DLR) \\ ${ }^{2}$ Helmholtz-Institute Ulm (HIU) \\ ${ }^{3}$ Ulm University, Institute of Stochastics
}

Corresponding Author(s): timo.danner@dlr.de

Li-Ion batteries are commonly used in portable electronic devices and state-of-the-art electric vehicles due to their outstanding energy and power density. A typical Li-Ion battery electrode is a porous composite consisting of active material (particle diameter $\sim 10-20 \mu \mathrm{m}$ ), conductive carbon (particle diameter $\sim 100 \mathrm{~nm}$ ), and polymeric binder. The carbon black and binder form a microporous phase ( $\mathrm{CB}$ domain) which is distributed in the macro-pores of the active material network and ensures mechanical stability and electrical contact. However, at high current densities, e.g. during fast charging, the transport of Li-ions in the electrolyte is decisive for the performance of the battery cell and the CB domain increases mass transport limitations. Furthermore, it was shown that the production process, e.g. harsh drying conditions cause binder migration to the electrode surface which enhances performance losses 1 .

In our presentation we will show results of pore-scale simulations of Li-Ion batteries 2 which additionally take into account the morphology and distribution of the CB domain. In order to assess the effect of the $\mathrm{CB}$ domain we performed intensive simulation studies on reconstructions of NMC electrodes with different thickness and density which were created with the help of synchrotron tomography and a 3D stochastic microstructure generator[3]. The simulations are in quantitative agreement with galvanostatic cycling data and impedance measurements on symmetrical cells [4] which are especially advantageous for the characterization of electrode tortuosity. Based on these results different electrode configurations were evaluated regarding their performance improvements at high C-rates. This virtual screening provides material-structure-function relationships which are a helpful tool for the development of improved functional materials and electrochemical devices.

Acknowledgement

This work has been funded by the 'Bundesministerium für Bildung und Forschung' within the project HighEnergy under the reference numbers 03XP0073D, and 03XP0073E.

References:

1. Jaiser, S. et al. Investigation of film solidification and binder migration during drying of Li-Ion battery anodes. J. Power Sources 318, 210-219 (2016).

2. Danner, T. et al. Thick electrodes for Li-ion batteries: A model based analysis. J. Power Sources 334, 191-201 (2016).

3. Westhoff, D., Manke, I. \& Schmidt, V. Generation of virtual lithium-ion battery electrode microstructures based on spatial stochastic modeling. Comput. Mater. Sci. 151, 53-64 (2018).

4. Landesfeind, J., Hattendorff, J., Ehrl, A., Wall, W. A. \& Gasteiger, H. A. Tortuosity Determination of Battery Electrodes and Separators by Impedance Spectroscopy. J. Electrochem. Soc. 163, A1373-A1387 (2016). 
Procter and Gamble Student poster award:

References:

Acceptance of Terms and Conditions:

Click here to agree

378

\title{
Development of a Novel Method based on MLP-ANN to Investi- gate Carbon dioxide capture and Storage
}

\author{
Houman Darvish $^{\text {None }}$; Karim Rouhibakhsh ${ }^{1}$; Mohsen Zare ${ }^{2}$; Abdolreza Kazemi Abadshapoori ${ }^{3}$ \\ ${ }^{1}$ Department of Petroleum Engineering, School of Chemical, Petroleum and Gas Eng, Shiraz University, Shiraz, Iran \\ ${ }^{2}$ Department of Petroleum Engineering, Marvdasht Branch, Islamic Azad University, Marvdasht, Iran \\ ${ }^{3}$ Department of Petroleum Engineering, School of Chemical and Petroleum Engineering, Shiraz University, Shiraz, \\ Iran
}

Corresponding Author(s): houman_d70@yahoo.com, reza.pixy@gmail.com

\begin{abstract}
Nowadays global warming is known as one of challenging problems which threat human future on the earth. The main reason of global warming refers to carbon dioxide emission so the researchers attract to the study of carbon dioxide capture and storage (CSS) process. Accurate investigation of vapour liquid equilibrium (VLE) of impure carbon dioxide system has dominant impact on designing CCS process such as injection and storage in porous media. In the present study, multi-layer perceptron artificial neural network (MLP-ANN) algorithm was developed to forecast VLE data of binary system of carbon dioxide with oxygen, Nitrogen and Argon. ANN algorithm is proposed based on statistical learning methodologies, which has wide applications in different sciences $[1,2]$. According to this end, a reliable source of experimental data was gathered to train and test the proposed algorithm. a comprehensive experimental data which contains 191 experimental vapor liquid equilibrium data for $\mathrm{CO} 2+\mathrm{N} 2, \mathrm{CO} 2+\mathrm{O} 2$ and $\mathrm{CO} 2+\mathrm{Ar}$ were assembled from a reliable paper [3]. The actual dataset contains 57 data points of $\mathrm{CO} 2+\mathrm{N} 2,68$ of data points $\mathrm{CO} 2+\mathrm{O} 2$ and 66 of data points $\mathrm{CO} 2+$ Ar. The utilized MLP-ANN algorithms were developed for determination of vapor and liquid mole fraction of carbon dioxide separately. The accuracy of predicting models were investigated by graphical and statistical manners. The determined error indexes lie near the zero and predicted VLE data has great agreement with experimental data. The comparison of MLP-ANN outputs and experimental VLE data showed that predicting algorithm has great potential to predict behaviour of binary system of impure carbon dioxide so MLP-ANN can be used as applicable method for prediction of carbon dioxide phase behaviour in porous media.
\end{abstract}

Procter and Gamble Student poster award:

I would like to compete in the Procter and Gamble Student award References:

Development of a Novel Method based on MLP-ANN to Investigate Carbon dioxide capture and Storage1. Abdi-Khanghah, M., et al., Prediction of solubility of N-alkanes in supercritical CO 2 using RBF-ANN and MLP-ANN. Journal of CO2 Utilization, 2018. 25: p. 108-119.

2. Keybondorian, E., et al., Application of MLP-ANN strategy to predict higher heating value of biomass in terms of proximate analysis. Energy Sources, Part A: Recovery, Utilization, and Environmental Effects, 2017. 39(22): p. 2105-2111.

3. Lasala, S., et al., VLE properties of CO 2-based binary systems containing N 2, O 2 and Ar: experimental measurements and modelling results with advanced cubic equations of state. Fluid Phase Equilibria, 2016. 428: p. 18-31. Acceptance of Terms and Conditions:

Click here to agree 


\title{
Determination of effect of impurities on carbon dioxide capture and storage by a novel LSSVM-GA method
}

\author{
Houman Darvish $^{\text {None }}$; Karim Rouhibakhsh ${ }^{1}$; Mohsen Zare $^{2}$ \\ ${ }^{1}$ Department of Petroleum Engineering, School of Chemical, Petroleum and Gas Eng, Shiraz University, Shiraz, Iran \\ 2 Department of Petroleum Engineering, Marvdasht Branch, Islamic Azad University, Marvdasht, Iran
}

Corresponding Author(s): houman_d70@yahoo.com

In the recent years, earth surface temperature have elevated significantly. One of the dominant reasons of this elevation is emission of carbon dioxide so the importance of carbon dioxide capture and storage (CCS) technology has been growing recently. As the storage of carbon dioxide is a complex process especially in porous media and accurate knowledge about vapor liquid equilibrium (VLE) and phase behavior of impure carbon dioxide has noticeable effect on designing CCS processes. The impurities of carbon dioxide has vital effect on CCS technology however as the impurities of carbon dioxide streams increase the cost of capturing process decreases but it causes further problems in other processes of CCS such as storage and transportation phases1. In the current work, a novel computational method namely least square support vector machine (LSSVM) optimized by genetic algorithm (GA) was used to predict VLE data of binary system of carbon dioxide with Nitrogen, Oxygen and Argon. The LSSVM approach was prorogated by Suykens as a form of SVM method [2, 3]. The LSSVM method can be considered as a simple computational method because it has two optimization parameters. To this end, two models based on LSSVM-GA were developed for vapor and liquid phases. The accuracy of predicting models were investigated by different graphical demonstrations and statistical indexes such as determination of coefficients of determination near one for training and testing phases. The results express that the proposed algorithms are dependable for estimation of phase behavior of impure carbon dioxide. Due to obtained results and analysis, LSSVM-GA can be considered as an accurate tool for prediction of phase behavior and thermodynamics of carbon dioxide.

\section{Procter and Gamble Student poster award:}

I would like to compete in the Procter and Gamble Student award References:

1. Blanco, S.T., et al., Influence of methane in CO2 transport and storage for CCS technology. Environmental science \& technology, 2012. 46(23): p. 13016-13023.

2. Keybondorian, E., et al., Estimation of the higher heating value of biomass using proximate analysis. Energy Sources, Part A: Recovery, Utilization, and Environmental Effects, 2017. 39(20): p. 2025-2030.

3. Suykens, J.A. and J. Vandewalle, Least squares support vector machine classifiers. Neural processing letters, 1999. 9(3): p. 293-300.

Acceptance of Terms and Conditions:

Click here to agree

329

\section{Impact of disorder on flows and transport in porous media}

\author{
Author(s): Thomas Darwent ${ }^{1}$ \\ Co-author(s): Felix Meigel ${ }^{2}$; Karen Alim $^{2}$; Lucas Goehring ${ }^{1}$ \\ ${ }^{1}$ Nottingham Trent University \\ ${ }^{2}$ Max Planck Institute for Dynamics and Self-Organization \\ Corresponding Author(s): thomasmdarwent@gmail.com
}


Fluid flows in a porous media have been widely studied due to their importance in a variety of fields including oil recovery, solute transport (e.g. groundwater contamination) and biological applications (e.g. flow of nutrients in capillaries). Flow in porous media can be influenced by variations in the microscopic pore throats, which lead to a range of invasion patterns and flow distributions. Here we use numerical and experimental methods to investigate flow distributions for a range of Péclet numbers with increasingly disordered porous media at a micro-scale. We examine the resulting effect that disorder has on the dispersion and diffusion of solutes at the macro-scale. For this, soft lithography is used to create PDMS chips (micro-models) that contain an array of microscopic pillars and channels for fluid injection. We use particle tracking to determine the distributions of flow speeds through the disordered pores throughout a sample of our system, which are then compared to numerical and theoretical models of the flows. These measurements are complemented by fluorescence imaging to track dye concentration across the system as a function of time, and hence to evaluate the effect of pore-scale disorder on how a contaminant or nutrient is transported through a porous body.

Procter and Gamble Student poster award:

\title{
References:
}

Acceptance of Terms and Conditions:

Click here to agree

\section{Impact of particle size correlation on immiscible fluid displace- ment in porous media}

\author{
Author(s): Thomas Darwent ${ }^{1}$ \\ Co-author(s): Oshri Borgman ${ }^{2}$; Enrico Segre ${ }^{3}$; Ran Holtzman ${ }^{4}$; Lucas Goehring ${ }^{1}$ \\ ${ }^{1}$ Nottingham Trent University \\ ${ }^{2}$ The Hebrew University of ferusalem, \\ ${ }^{3}$ Weizmann Institute of Science, \\ ${ }^{4}$ Institute of Environmental Assessment and Water Research (IDAEA) Spanish National Research Council (CSIC)
}

Corresponding Author(s): thomasmdarwent@gmail.com

The displacement of immiscible fluids in porous media affects processes in both industry and natural environment, such as geological $\mathrm{CO} 2$ sequestration, hydrocarbon recovery and water infiltration in soil. As these processes are determined by pore-scale physics, the distribution of pore and grain sizes have a significant impact on the observable behaviour of fluid displacement. Here we investigate how a correlation length, i.e. the length over which nearby particle sizes are similar, impacts drainage patterns at the pore-scale using experimental methods. We saturate PDMS micromodels, containing thousands of microscopic pillars, with different concentrations of a water and glycerol mixture at various viscosities. This mixture is then withdrawn from an outlet to either side of the chip so that air enters through a central inlet and invades radially outwards at different capillary numbers. Images are studied at the moment when the air exits the porous medium (breakthrough). These results coincide with work done numerically, where simulations of the same correlation lengths are undertaken. We find that as the correlation length is increased, the invaded volume and trapping fraction at breakthrough decreases, whereas the asymmetry of the patterns increases. Our results show that the structure of the porous medium is an important factor controlling fluid displacement, and should be considered when modelling and predicting flow and transport behaviour.

\section{Procter and Gamble Student poster award:}

I don't want to compete References:

Acceptance of Terms and Conditions:

Click here to agree 


\title{
An adaptive upscaling method for unstable multiphase flows in heterogeneous porous media using advanced numerical methods
}

\author{
Author(s): Narges Dashtbesh ${ }^{1}$ \\ Co-author(s): Guillaume Enchéry ${ }^{1}$; Benoit Noetinger ${ }^{1}$; Beatrice Riviere \\ ${ }^{1}$ IFPEN
}

Corresponding Author(s): narges.dashtbesh@ifpen.fr

The main goal in the development of any upscaling method is to capture the flow physics as closely as possible while reducing the computational cost. Upscaling of immiscible multiphase flow is still challenging, mainly due to instabilities occurring especially at the fluid front. We propose an adaptive upscaling approach, using robust mathematical homogenization theory and high order discontinuous Galerkin numerical modeling while minimizing the computational time. This approach involves modeling the position of the fluid interface in a multiphase flow problem, at the coarse scale, without running the time-consuming full-field simulations. The proposed method for modeling the interface of flowing fluids don't have any restriction regarding the complexities of multiphase flows at the interface. The computational efficiency is achieved by solving a coarse scale problem away from the front, in the single-phase regions, where homogenization is used appropriately to obtain the macroscopic equations and effective parameters at the coarse scale, with minimum loss in accuracy. Local flow details close to the fluid interface which gives rise to the complexities of the multiphase flow problem is taken into account using more advanced higher order discontinuous Galerkin methods. We present some numerical results to show the accuracy and computational efficiency of the approach in comparison to the fine-scale flow simulations.

Procter and Gamble Student poster award:

I would like to compete in the Procter and Gamble Student award References:

Acceptance of Terms and Conditions:

Click here to agree

\section{Pore Network Modeling of Reactive Transport in CO2 Storage Reservoirs}

Hassan Dashtian $^{1}$; Sahar Bakhshian ${ }^{1}$; Seyyed Abolfazl Hosseini ${ }^{1}$

\footnotetext{
${ }^{1}$ Bureau of Economic Geology, University of Texas at Austin
}

Corresponding Author(s): dashtian@utexas.edu, sahar.bakhshian@beg.utexas.edu, seyyed.hosseini@beg.utexas.edu

$\mathrm{CO} 2$ enriched brine interaction with reservoir minerals and the subsequent mineral trapping of $\mathrm{CO} 2$ alters the morphology of pore structure. Such changes in the pore space modify the storage capacity of reservoirs and increases the long-term security of CO2 storage. The extent of pore space modification depends on the relative weight of advection, diffusion and reaction mechanisms. We present a general framework for direct simulation of reactive transport in digitized reservoir rock samples based on pore-scale modeling in 2D and 3D pore throat networks. We examine the impacts of transport properties such as Peclet $(\mathrm{Pe})$ and Damkohler $(\mathrm{Da})$ numbers, on the petrophysical properties and dissolution of mineral in pore space. We implement a modified version of the marker-based watershed segmentation to extract pore-network of 3D micro-CT images. The extracted network is used to simulate the reactive solute transport and dissolution in real rock samples. We implement our approach to study the $\mathrm{CO} 2$ injection in Cranfield site, Mississippi, U.S.A. We show the effect of PeDa number on the spreading of solute and found that high diffusion rates enhance the dispersion of concentration in porous media and results in higher porosity alteration rates. 
Procter and Gamble Student poster award:

I would like to compete in the Procter and Gamble Student award References:

Acceptance of Terms and Conditions:

Click here to agree

984

\title{
Geometrical 2D to 3D Transform: Estimation of Material Proper- ties from 2D Images
}

\author{
Juan Pablo Daza ${ }^{1}$ \\ ${ }^{1}$ Stanford University \\ Corresponding Author(s): jpdaza@stanford.edu
}

Recent advances in 3D imaging allow us to get high resolution geometry of different porous media. However, 3D imaging is expensive and time consuming compared with 2D imaging. 2D imaging gives us high resolution and quality, quickly and with little economical investment.

In this work we propose a geometrical transformation of thin slices to a 3D volume. This transformation is unique, isotropic, analytical, and enables us to compute physical properties such as permeability, bulk modulus, and conductivity. We rely completely in the geometrical information available in $2 \mathrm{D}$ images, and use this information to construct a $3 \mathrm{D}$ volume that preserves the geometrical measurements in $2 \mathrm{D}$. The result is a $3 \mathrm{D}$ volume that has the same porosity as the $2 \mathrm{D}$ image, as well as a well connected pore-space, geometrically isomorphic to the pore space of the original sample.

We test the success of this 2D to 3D transform in its ability to predict the physical properties of the sample, in our case the bulk and shear modulus, conductivity and permeability. Obtaining and excellent match of properties computed in volumes obtained from micro-CT, and those properties obtained just from 2D thin slices.

Procter and Gamble Student poster award:

I would like to compete in the Procter and Gamble Student award References:

Acceptance of Terms and Conditions:

Click here to agree

883

\section{Changes in Porous Media Properties by Modeling Diagenetic Pro- cesses in Micro-CT Images}

Juan Pablo Daza ${ }^{1}$; Gerald Mavko ${ }^{1}$; Amos Nur ${ }^{1}$

${ }^{1}$ Stanford University

Corresponding Author(s): jpdaza@stanford.edu

Understanding thermo-chemo-mechanical processes with fluids in porous media and rocks is important. Because of the likelihood of fluid-rock chemical interactions, and our limited ability to decipher the mechanical, and fluid flow effects of these coupled processes. One of the missing links is understanding the evolution of elastic and transport properties together with reactive transport. Because the properties of porous media evolve as a result of chemical reactions and vice versa. Capturing 
this coupling experimentally and theoretically is one of the missing elements in the existing literature. We describe here recent advances in theoretical modeling and simulation of reactive fluids processes in rocks with complex pore structure, in order to understand the effects of dissolutioninduced changes on acoustic velocity, porosity, permeability and electrical properties.

To deal with the problem of modeling the effects of reactive fluids in porous media properties, we adopt the approach by Osher and Sethian (1988) - a conceptual framework for using level sets as a tool for numerical analysis of surfaces and shapes - together with 2D and 3D scanned and segmented pore structure images. The advantage of the level-set approach is that one can perform numerical computations involving complex curves and irregular surfaces on a fixed Cartesian grid without having to parameterize these objects. With this we have already shown how material evolution problems can be mathematically/computationally solved accurately, efficiently (fast), while honoring the coupling between physics and chemistry.

We can get different versions of the pore geometry by using the Osher's approach with the simplest velocity field to change the pore-space interface. Properties such as permeability are then calculated using the Lattice-Boltzmann method (Keehm, Mukerji, and Nur 2001), for each change in the geometry. The results of the calculated permeability are compared with the experimental observations (Bourbié 1985) for Fountainebleau sandstone. Thus the method used in our work is the enabling technology to model and simulate the structural changes of the different components of the porous media, under different physical and chemical processes.

\title{
Procter and Gamble Student poster award:
}

I would like to compete in the Procter and Gamble Student award References:

Osher, S., \& Sethian, J. A. (1988). Fronts propagating with curvature-dependent speed: Algorithms based on Hamilton-Jacobi formulations. Journal of Computational Physics, 79(1), 12-49

Keehm, Y., Mukerji, T., \& Nur, A. (2001). Computational rock physics at the pore scale: Transport properties and diagenesis in realistic pore geometries. The Leading Edge, 20(2), 180-183.

Bourbié, T., \& Zinszner, B. (1985). Hydraulic and acoustic properties as a function of porosity in Fontainebleau Sandstone. Journal of Geophysical Research, 90(B13), 11524. Acceptance of Terms and Conditions:

Click here to agree

\section{Microbial growth in confined flows: the role of physical hetero- geneity}

\author{
Author(s): Camille Kerboas ${ }^{1}$ \\ Co-author(s): Pietro De Anna ; Stephane Mahe ${ }^{1}$; Mayumi Hamada ${ }^{1}$ \\ ${ }^{1}$ University of Lausanne
}

Corresponding Author(s): pietro.deanna@unil.ch

Within the shallow subsurface, soil, rock, fluids, gases and living organisms are in close interaction: the macroscopic resulting phenomena derive from the coupling between fluid flow, solutes mixing and microorganisms displacement, growth and adaptation. The common challenge in all these processes is their spatial variability (heterogeneity). The consequent complexity that rises from the coupling of these processes makes predictions, based on rates measured under homogenized, wellmixed, conditions, different by orders of magnitudes from laboratory and field observations. Using microfluidics and time-lapse video-microscopy we investigate the coupling between non uniform nutrient transport through confined micro-structures and the microbial growth of an isolate population.

Procter and Gamble Student poster award:

References:

Acceptance of Terms and Conditions: 


\title{
Prediction of low velocity distribution from pore structure in sim- ple porous media
}

\author{
Author(s): Pietro De Anna ${ }^{1}$ \\ Co-author(s): Bryan Quaife ${ }^{2}$; George Biros ${ }^{3}$ \\ ${ }^{1}$ Universite de Lausanne \\ ${ }^{2}$ Florida State University \\ ${ }^{3}$ The University of Texas
}

Corresponding Author(s): pietro.deanna@unil.ch

The macroscopic properties of fluid flow and transport through porous media are a direct consequence of the underlying pore structure. However, precise relations that characterize flow and transport from the statistics of pore-scale disorder have remained elusive. Here, we investigate the relationship between pore structure and the resulting fluid flow and asymptotic transport behavior in 2D geometries of non overlapping circular posts. We derive an analytical relationship between the pore throat size distribution $f^{-}$and the distribution of the low fluid velocities $f_{u} u^{-/ 2}$, based on a conceptual model of porelets (the flow established within each pore throat, here a HagenPoiseuille). Our model allows us to make predictions-within a Continuous Time Random Walk (CTRW) framework-for the asymptotic statistics of spreading of fluid particles along their own trajectories. These predictions are confirmed by high fidelity simulations of Stokes flow and advective transport. The proposed framework can be extended to other configurations which can be represented as a collection of known flow distributions.

Procter and Gamble Student poster award:

References:

Acceptance of Terms and Conditions:

\section{The influence of nominal resolution and focal spot size on analy- sis results in porous media.}

\author{
Wesley De Boever ${ }^{1}$; Andreas Grießer ${ }^{2}$ \\ ${ }^{1}$ Bruker microCT \\ ${ }^{2}$ Math2Market
}

Corresponding Author(s): wesley.deboever@bruker.com

Over the past years, 3D image analysis based on computed tomography data has become a standard method in many fields of porous media research. The technology is used to calculate volume fractions of ingredients, pore and particle size distribution, connectivity and many more parameters.

One important field in particular, is core analysis based on 3D models, or digital rock physics. Assessment of transport phenomena through digital rock physics has the advantage of being faster than traditional laboratory measurements. Furthermore, it is not needed to take expensive cores from the well, but small drill cuttings can be enough to obtain petrophysical properties of reservoir rocks.

As in most cases models derived from micro-CT scans are used as a basis for transport modelling, 
the rapidly increasing availability of micro-CT scanners means that more and more researchers can benefit from this type of data.

These micro-CT scanners come in many different makes, shapes and sizes, but the overall technology is in general the same. Although the maximum X-ray energies, scan times or key components can differ from model to model, the one key parameter for adequate digital rock measurements is resolution. Although this resolution is one of the most important scanner specifications, it is also one of the hardest to quantify. In micro-CT terminology, resolution is often put identical to 'voxel size'. This voxel size is the real physical size of one voxel, but can in practice be much smaller than the resolution. It is therefore more correct to refer to the smallest achievable voxel size, limited by the system's geometry, as the 'nominal resolution' of a micro-CT system. True spatial resolution on the other hand is limited by the focal spot size of the X-ray source on one side, and the physical size of the pixels in the X-ray detector on the other side.

In this experimental study, we assessed what is the real difference between high-resolution microCT systems with a true sub-micron spot size and a 2-3 $\mu \mathrm{m}$ spot size. The experiment was performed using an open-type X-ray source, where different focal spot modes allow scanning using the same nominal resolution, but different spatial resolution. All other system components (X-ray detector, rotation stage, etc.) are kept the same. This experiment allows to quantify in which cases it is needed to take the extra hours of scanning, as small, submicron spot sizes are typically only reached at low $\mathrm{X}$-ray power and flux, therefore drastically increasing scan time. In the study, several well-known reservoir analogues are used, with different porosity and composition characteristics. The GeoDict software package is then used to calculate relevant flow parameters on these samples. The first results indicate that - especially for sandstones - similar results are reached with a lower spatial resolution but the same voxel size, in roughly 4 times less scan time.

Procter and Gamble Student poster award:

References:

Acceptance of Terms and Conditions:

Click here to agree

\title{
Cross pumping test to characterize the heterogeneity of hydraulic properties of a contaminated aquifer.
}

\author{
Author(s): Théo De Clercq ${ }^{1}$ \\ Co-author(s): Abderrahim Jardani ${ }^{2}$; Laurent Thannberger ${ }^{1}$ \\ ${ }^{1}$ VALGO, France \\ ${ }^{2}$ M2C Rouen, France
}

Corresponding Author(s): theo.de-clercq@etu.univ-rouen.fr, theo.de-clercq@univ-rouen.fr

The assessment of hydrodynamic properties of an aquifer is usually carried out by pumping test in which the hydraulic responses to a constant or an harmonic pumping flow are analyzed by the analytical equations to only get the average values of the transmissivity hydraulic and storativity coefficient. In this work we have developed the harmonic pumping test as an interesting and innovative approach to characterize spatially the heterogeneities of a contaminated aquifer by identifying the preferential paths of the groundwater flow and contaminants. The approach relies on a harmonic extraction of the water at the pumping well and recording the piezometric fluctuations in many neighboring wells. The use of such a tool permits: i) to limit the quantity of pumped water in particular if it is contaminated; ii) to analyze and exploit even the hydraulic signals with the small amplitudes; iii) to facilitate the extraction of hydraulic oscillations due to harmonic pumping test from other hydraulic fluctuations such as tide; iv) to reduce the computing time needed to interpret the hydraulic signal in term of hydraulic properties by solving the hydraulic diffusion equation in frequency mode instead to the transitient mode; v) to image the spatial heterogeinity of the hydraulic properities.

This approach was successfully applied in reconstruction of hydraulic parameters of an experimental site located in Rouen (France), in which 30 wells were placed on a hydrocarbon-contaminated 
alluvial aquifer in order to improve and study the effectiveness of hydrogeophysical tools to monitor the remediation activities.

Procter and Gamble Student poster award:

I would like to compete in the Procter and Gamble Student award References:

Acceptance of Terms and Conditions:

Click here to agree

\title{
On the applicability of superposition principle to multiphase flow for the assessment of $\mathrm{CO} 2$ storage capacity
}

\author{
Silvia De Simone ${ }^{1}$; Samuel Jackson ${ }^{1}$; Robert Zimmerman ${ }^{2}$; Sam Krevor ${ }^{\text {None }}$ \\ ${ }^{1}$ Imperial College London \\ ${ }^{2}$ Imperial College
}

Corresponding Author(s): s.de-simone@imperial.ac.uk

The results of energy systems modelling used in the IPCC synthesis reports on mitigating climate change identify carbon capture and storage (CCS) as essential in technology pathways limiting global warming to less than $1.5^{\circ} \mathrm{C}$ and $2^{\circ} \mathrm{C}$. Energy system models including CCS usually assume unlimited storage capacity or very few constraints on rates of injection. However, pressure limitations arising from the risk of induced seismicity or fracturing of sealing caprocks may significantly limit rates at which CO2 may be injected over regional and global scales. The adoption of simplified analytical solutions to pressurisation with subsurface injection can provide dynamic estimates of the rates at which regional storage resources may approach these limits. The analytical models are computationally inexpensive and may be included in energy system models to identify potential bottlenecks to CCS deployment. Analytical solutions for the pressure response to the injection of $\mathrm{CO} 2$ into a single well exhibit accurate estimation of pressure build-up. However, large scale carbon storage involves more complex features, like injection into multiple wells, variable injection rate and presence of structural barriers to flow. In this work, we investigate if the traditional procedure of superposing effects, valid for linear single phase flow, can be extended to the case of multiphase flow. We evaluate the error associated with the application of superposition to the case of $\mathrm{CO} 2$ injection. For the multiple well scenario, the application of superposition overestimates the pressure build-up because it neglects the presence of multiple $\mathrm{CO} 2$ plumes that increases the total fluid mobility in the reservoir. This error increases with time and with the number of wells, as the dimension of the $\mathrm{CO} 2$ saturated area increases. The adoption of a novel non-dimensionalization allows us to define a general model for the error which can be used to correct the pressure estimated by superposition. By means of this corrective factor we define a simplified procedure for the analytical assessment of reservoir pressurization during multiwell $\mathrm{CO} 2$ injection that can be included in regional energy system models.

Procter and Gamble Student poster award:

References:

Acceptance of Terms and Conditions:

Click here to agree

\section{Experimental observations of coupling of free flow and non-uniform porous structures in a microfluidic setup}

Matthijs de Winter ${ }^{1}$; Kilian Weishaupt ${ }^{2}$; Majid Hassanizadeh ${ }^{1}$; Rainer Helmig ${ }^{2}$ 


\footnotetext{
${ }^{1}$ Environmental Hydrogeology, Faculty of Geosciences, Utrecht University

${ }^{2}$ Institute for Modelling Hydraulic and Environmental Systems University of Stuttgart
}

Corresponding Author(s): d.a.m.dewinter@uu.nl

The exchange of momentum of a free flow fluid and a fluid in a porous structure is a common phenomenon in e.g. river beds, air-soil interactions, cooling applications, and petrochemical riser reactors. We present our experimental observations of a PDMS (poly-dimethyl-siloxane) micro-model, where single-phase flow in a channel and single- or two-phase flow in a porous structure is considered.

The semi-3D PDMS models consist of a 10-cm long free-flow channel (about $2 \mathrm{~mm}$ wide and 0.15 mm deep) and a network of pores with overall dimensions of $0.25 \mathrm{~mm}$ by $0.25 \mathrm{~mm}$, representing a porous medium. The micro models have a depth of $0.01-0.25 \mathrm{~mm}$ and pore sizes vary between 0.005 $\mathrm{mm}$ and $0.5 \mathrm{~mm}$. The pores are shaped and positioned in a regular or random pattern. The porous structure is in contact with the free channel over a length of about $20 \mathrm{~mm}$.

The PDMS model is fully or partially saturated prior to the experiment. Saturation levels less than $100 \%$ add to the heterogeneity of the pore structure, due to the randomness of the locations and shapes of the blobs, droplets and ganglia of, for instance, air. Then, once (partially) saturated, a fluid (water or oil) is injected at a constant flowrate into the free flow channel using a syringe pump. The fluid is laden with fluorescent nanoparticles with average diameter of $0.75 \mu \mathrm{m}$. In case of two immiscible fluid phases (water-oil), both phases can be loaded with fluorescent particles with a different color.

The fluorescent particles can be observed in a confocal scanning laser microscope (CSLM). Time series of images are recorded with a sufficient number of frames per second to observe the movement of individual particles. Particle Image Velocimetry (PIV) is applied to the image series to extract the spatial distribution of flow velocities and directions.

The field of view of CSLM is not large enough to view the entire PDMS model. Therefore, mapping of the entire PDMS model is achieved sequentially with an array of observations (each observation represents a time series of images). A full PIV map is stitched together after post-processing the individual observations.

The number of data points per PDMS model and the required number of post-processing steps per data point are considerably large. Hence, a reliable automation of the acquisition and postprocessing steps is crucial. In particular, when investigating a non-uniform network of pores, as the effect of non-uniformity can only be characterized when performing a sufficient number of experiments.

Procter and Gamble Student poster award:

I don't want to compete References:

Acceptance of Terms and Conditions:

Click here to agree

\title{
Effect of precipitation mineralization reactions on convective dis- solution of $\mathrm{CO}_{2}$ : an experimental study
}

\author{
Author(s): Anne De $\mathrm{Wit}^{1}$ \\ Co-author(s): Carelle Thomas ${ }^{1}$; Sam Dehaeck ${ }^{1}$ \\ ${ }^{1} U L B$ \\ Corresponding Author(s): adewit@ulb.ac.be
}


In order to decrease the amount of greenhouse gases in the atmosphere and reduce global warming, carbon capture and sequestration (CCS), whereby $\mathrm{CO}_{2}$ is injected into geological formations, is receiving increased attention. To explore the influence in CCS of a mineralization reaction between dissolved $\mathrm{CO}_{2}$ and calcium ions on the convective transfer of $\mathrm{CO}_{2}$ towards a saline aquifer, the convective dissolution of $\mathrm{CO}_{2}$ into aqueous solutions of $\mathrm{Ca}(\mathrm{OH})_{2}$ and $\mathrm{CaCl}_{2}$ is analyzed experimentally at the laboratory scale.

We show that different precipitation patterns develop in the aqueous solution depending on the nature and concentration of the reactant in the host phase. In the case of $\mathrm{Ca}(\mathrm{OH})_{2}$, precipitation coupled to convection leads to vigorous convective mixing in the host phase and sedimentation of solid particles of $\mathrm{CaCO}_{3}$ down to the bulk of the reservoir. Conversely, dissolution of $\mathrm{CO}_{2}$ in buffered $\mathrm{CaCl}_{2}$ solutions leads to a stabilisation of the buoyancy-driven convection due to a decrease in density and the adherence of the precipitate to the cell walls.

Procter and Gamble Student poster award:

\title{
References:
}

C. Thomas, S. Dehaeck, A. De Wit, Convective dissolution of $\mathrm{CO}_{2}$ in water and salt solutions, Int. J. of Greenhouse Gas Control 72, 105 (2018). Acceptance of Terms and Conditions:

Click here to agree

\section{Moment-based Metrics for Global Sensitivity for multiple Models with Uncertain Parameters}

Aronne Dell'Oca $^{1}$; Alberto Guadagnini ${ }^{1}$; Monica Riva ${ }^{1}$

\footnotetext{
${ }^{1}$ Politecnico di Milano
}

Corresponding Author(s): aronne.delloca@polimi.it

\begin{abstract}
We propose a set of new metrics to assist global sensitivity analysis (GSA) in the presence of data allowing for interpretations based on a collection of diverse models whose parameters could be affected by uncertainty. Our GSA indices enable us to assess the sensitivity of diverse features of the probability density function (pdf) of a quantity of interest with respect to imperfect knowledge of (i) the interpretive model to be employed to characterize the system and (ii) the ensuing model parameters. We exemplify our methodology for the scenario of heavy metal sorption onto soil, for which we consider three interpretative models (i.e., isotherm models) widely used in the literature. We perform our analysis considering (a) an uninformed scenario, i.e., when no data are available to constrain parameters uncertainty and to evaluate the (relative) plausibility of each considered model, and (b) an informed scenario, i.e., when the analysis is constrained against observed experimental data. Our Moment-based indices are structured according to two major components: (a) a betweenmodel contribution, which takes into account the possibility of analyzing the system of interest by taking advantage of diverse competing model conceptualizations (or mathematical rendering); and (b) a within-model contribution, due to the uncertainty in the parameters of a selected model. Our results indicate that a given parameter can be associated with diverse degrees of importance, depending on the statistical moment of considered for the target model output (y). The influence on y of parameter uncertainty evolves according with the available level of information about the modeled system behavior.
\end{abstract}

Procter and Gamble Student poster award:

References:

Acceptance of Terms and Conditions:

Click here to agree 


\title{
Feed-Forward Neural Networks for Flux Regression in Discrete Fracture Networks
}

\author{
Stefano Berrone $^{1}$; Francesco Della Santa ${ }^{2}$; Sandra Pieraccini $^{\text {None }}$; Francesco Vaccarino $^{2}$ \\ ${ }^{1}$ Politecnico di Torino, Italy \\ ${ }^{2}$ Politecnico di Torino
}

Corresponding Author(s): francesco.dellasanta@polito.it

Characterization of flow and transport through fractured media in the subsurface is a crucial issue in many applications concerning civil, environmental and industrial engineering, e.g. in oil and gas extraction or in avoidance of drinking water pollution due to industrial waste. All these applications require models that can accurately simulate flow through networks of subsurface fractures.

Unfortunately, there is a lack of full knowledge of the topology of these sparse systems of fractures, since usually exact position, size, orientation and hydrogeological properties of all fractures (located hundreds of meters below the ground) can't be determined. Statistical representations of fracture networks (the so-called Discrete Fracture Networks - DFNs) are then introduced and used to simulate flow and transport through fractured media.

Due to the probabilistic nature of DFNs, flow and transport characterization in a real fractured medium usually requires a statistical analysis of thousands of DFNs simulations; therefore, in order to speed up simulation processes and to build alternative model reduction methods, it is worth considering the application of machine learning (ML) techniques and, more specifically, Neural Networks (NNs).

NNs are a particular kind of ML algorithms that were born more than fifty years ago but only in the last decade they started to be used in practice, due to computer hardware improvements and increasing amount of data of these years. The most interesting property of such a kind of algorithms is their ability to approximate functions through a training (or learning) phase.

In this work, the application of Neural Networks to flux regression problems in a DFN will be described. We will discuss the behavior of the regression quality with respect to the number of stochastic fractures and to NNs regularization and architecture; after this discussion we will also study how regression outputs and errors could help in the understanding of the given DFN hidden properties.

Procter and Gamble Student poster award:

\section{References:}

Acceptance of Terms and Conditions:

Click here to agree

551

\section{Numerical simulations of underground carbon dioxide storage with hydrogen impurities}

\author{
Anwar Al Assadi ${ }^{1}$; Joan Delort Ylla ${ }^{2}$; Beatrix Becker ${ }^{3}$; Allan Leal ${ }^{\text {None }}$; Holger Class ${ }^{\text {None }}$; Martin Saar ${ }^{2}$; Anozie \\ Ebigbo $^{4}$

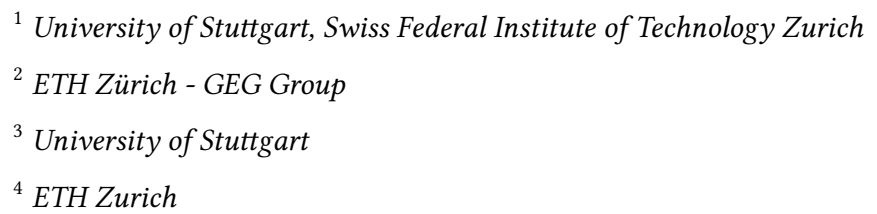


Corresponding Author(s): joan.delort@gmail.com

Natural gas reforming combined with carbon capture and storage (CCS) or carbon dioxide $\left(\mathrm{CO}_{2}\right)$ plume geothermal (CPG) can be a means of accelerating the use of hydrogen $\left(\mathrm{H}_{2}\right)$ as a low-carbon fuel 1. $\mathrm{CO}_{2}$ emissions can be captured at power plants, while clean energy is distributed, e.g., for transportation, heating/cooling etc. For process-related reasons, the $\mathrm{CO}_{2}$ that is to be stored will contain impurities, such as $\mathrm{H}_{2}$, methane, and nitrogen. $\mathrm{H}_{2}$, in particular, may change the behavior of the system in the subsurface. $\mathrm{A} \mathrm{H}_{2}$ concentration of $2 \%$ lowers the density of a $\mathrm{CO}_{2}-\mathrm{H}_{2}$ mixture at depth by as much as $25 \%$ compared to pure $\mathrm{CO}_{2} 2$. High concentrations of dissolved $\mathrm{H}_{2}$ are a driving factor of hydrogenotrophic microbial activity in the subsurface [3], which can lead to a reduced reservoir permeability. An accumulation of $\mathrm{H}_{2}$ in the $\mathrm{CO}_{2}-\mathrm{H}_{2}$ mixture at the CPG production borehole can enhance the corrosion of steel borehole casings. Numerical simulations can help to understand and quantify the effect these factors have on CCS and CPG operations.

This work is divided into two parts. In the first part, we use a 3D numerical model of a saline aquifer at a depth of $2 \backslash, \mathrm{km}$. The simulations were conducted using the open-source multiphase-flow simulator $\mathrm{DuMu}^{\mathrm{X}}$ [4].

We observe that the injection of supercritical $\mathrm{CO}_{2}$ containing $1 \% \mathrm{~mol}$ of $\mathrm{H}_{2}$ can lead to an accumulation of $\mathrm{H}_{2}$ in the $\mathrm{CO}_{2}$-rich phase, locally up to $5.5 \%$ mol. However, this accumulation tends to be at the fringe of the $\mathrm{CO}_{2}$ plume, in areas where the $\mathrm{CO}_{2}$-rich phase is immobile.

Nonetheless, this accumulation of $\mathrm{H}_{2}$ in the gas phase leads to an increase in concentration of dissolved $\mathrm{H}_{2}$ in the liquid phase of an order of magnitude, up to values of $0.023 \% \mathrm{~mol}$.

In the second part, we assess the consequences of the increased dissolved concentrations of $\mathrm{H}_{2}$ on the activity of methanogens and acetogens/acetotrophs using the numerical framework for modelling reactive systems, Reaktoro 5 .

Procter and Gamble Student poster award:

I would like to compete in the Procter and Gamble Student award References:

[1 ] ELEGANCY. (2018). ELEGANCY. [online] Available at: https://www.sintef.no/projectweb/elegancy/ [Accessed 26 Nov. 2018].

2 Sanchez-Vicente, Y., Drage, T.C., Poliakoff, M., Ke, J. and George, M.W., 2013. Densities of the carbon dioxide+ hydrogen, a system of relevance to carbon capture and storage. International Journal of Greenhouse Gas Control, 13, pp.78-86.

[3] Schwartz, E., Fritsch, J. and Friedrich, B., 2013. H2-metabolizing prokaryotes. In The Prokaryotes (pp. 119-199). Springer, Berlin, Heidelberg.

[4] Fetzer, Thomas, Becker, Beatrix, Flemisch, Bernd, Gläser, Dennis, Heck, Katharina, Koch, Timo, Schneider, Martin, Scholz, Simon, Weishaupt, Kilian, 2017. DuMuX 2.12.0.

5 Leal, A.M., Kulik, D.A., Smith, W.R. and Saar, M.O., 2017. An overview of computational methods for chemical equilibrium and kinetic calculations for geochemical and reactive transport modeling. Pure and Applied Chemistry, 89(5), pp.597-643. Acceptance of Terms and Conditions:

Click here to agree

\section{Conservative multirate multiscale method for multiphase flow in heterogeneous porous media}

Ludovica Delpopolo Carciopolo $^{1}$; Luca Formaggia $^{2}$; Hadi Hajibeygi ${ }^{3}$; Anna Scotti $^{2}$

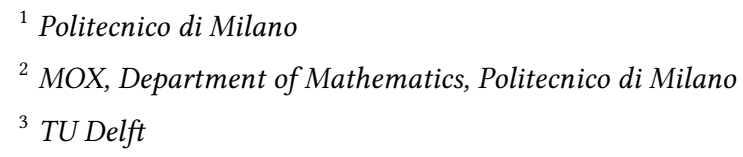

Corresponding Author(s): ludovica.delpopolo@polimi.it

We propose a conservative multirate multiscale method, which allows for simulation of multiphase flow in heterogeneous media adaptively in time and space. Motivated by the co-existence of fast 
and slow processes, the multirate method constructs an adaptive conservative time discretization scheme which imposes different time steps in different sub-domains. Yet, these sub-domains are consistently integrated with a conservative flux partitioning strategy. On the other hand, to resolve the challenge of imposing fine-scale spatial grids, to heterogeneous fine-scale properties, we integrated our multirate approach with the multiscale finite volume method. The multiscale method allows for construction of coarse-scale systems while the fine-scale heterogeneity is being captured by using local basis functions. To this end, we develop a sequential simulation approach where we solve the pressure equation with the multiscale technique and then advance the phase saturation adaptively by solving the transport equation using multirate approach. Preliminary numerical results show that the proposed adaptive strategy yields efficient and accurate solutions. In the talk, we will illustrate the numerical procedure and the latest developments.

Procter and Gamble Student poster award:

I would like to compete in the Procter and Gamble Student award References:

Acceptance of Terms and Conditions:

Click here to agree

188

\title{
Stochastic mechanisms of dispersion in a porous medium: Up- scaling flow and transport from the pore to the Darcy scale
}

\author{
Author(s): Marco Dentz ${ }^{1}$ \\ Co-author(s): Matteo Icardi ${ }^{2}$; Juan J. Hidalgo ${ }^{1}$ \\ ${ }^{1}$ IDAEA-CSIC \\ ${ }^{2}$ University of Nottingham
}

Corresponding Author(s): marco.dentz@csic.es

We present an approach to upscale solute dispersion in porous media from the pore to the Darcy scale. We use a particle based point of view to model the impact of medium geometry, flow heterogeneity and diffusion on average solute transport. In this framework, the solute is represented by an ensemble of solute particles, whose distribution is equivalent to the solute distribution. The fluctuating microscopic particle motion is formulated within a stochastic modeling framework, which allows to systematically derive the average macroscopic transport dynamics. Particle motion is conditioned by the pore geometry in two ways. Firstly, it provides the characteristic length scale for the evolution of particle velocities, and secondly, it determines the distribution of flow velocities in the medium. Also diffusion has a dual role. It allows individual particles to sample flow velocities across streamlines, and causes the trapping of particles in low velocity zones in the wake of solid grains. These mechanisms are studied through high performance numerical flow and transport simulations in 3-dimensional porous media for different Peclet numbers. We observe deviations from the hydrodynamic dispersion paradigm, which predicts linear growth of the particle displacement variance and inverse Gaussian breakthrough curves. For a sand-like porous medium we identify two distinct transport regimes. The first regime is characterized by advective heterogeneity, the second by retention through diffusion into low velocity regions. Particle motion is modeled as a stochastic process that moves particles over the characteristic pore length at transition times, which are determined by advection and diffusion. A purely advective transport models fails and significantly overpredicts the breakthrough curve tailing. At the Peclet numbers under consideration, particles can sample the intrapore velocity by diffusion and the interpore (mean) velocities due to advection. In order to quantify this mechanism, we develop a model for the statistics of the Eulerian velocity magnitude based on Poiseuille's law for individual pores, and for statistics of the mean pore velocity, both of which are linked to the distribution of pore diameters. Diffusion across streamlines into low velocity zones is characterized by an exponential distribution of residence times, which can be determined by solving a local diffusion problem. The trapping frequency is related to the low velocity end of the Eulerian velocity distribution. The resulting stochastic transport model is parameterized by the medium and flow properties and captures non-Fickain transport in both pre-asymptotic regimes, and the transition to the Fickian regime. It quantifies the physical non-equilibrium that 
is caused by a broad distribution of advective and diffusive mass transfer time scales on the representative elementary volume. Based on this stochastic model for particle motion, we derive the non-local advection-dispersion equation governing the evolution of the average concentration distribution.

Procter and Gamble Student poster award:

\title{
References:
}

Dentz, M., M. Icardi, J. J. Hidalgo, Mechanisms of dispersion in a porous medium, J. Fluid Mech. 841, 851-882, 2018. Acceptance of Terms and Conditions:

Click here to agree

\section{Hysteresis, sorption, swelling and mechanical behavior of wood: a multiscale study}

\author{
Author(s): Dominique Derome ${ }^{1}$

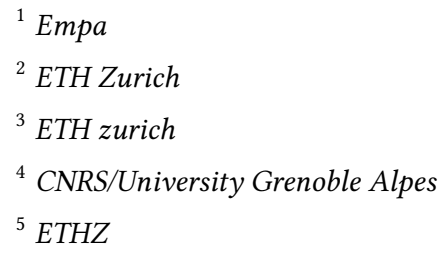

Co-author(s): Mingyang Chen ${ }^{2}$; chi zhang ${ }^{3}$; Benoit Coasne ${ }^{4}$; Sinan Keten ; Robert Guyer ; Jan Carmeliet

Corresponding Author(s): dominique.derome@empa.ch

Wood is a well-used material, in buildings, furniture, art artefacts, etc., where its capacity to absorb water is not only an issue of dimensional stability and modified material properties, but also of durability. Wood is a hierarchical material, where the configuration at different scales (lumber, growth ring, cellular and cell wall material) plays different roles in adsorption/desorption and also in the resulting swelling/shrinkage and mechanical softening. Different modeling approaches are required at different scales. As well, upscaling between models is required. This approach allows us to study holistically this material hygromechanical hysteretical behavior.

Clearly, the origin of swelling lies at the nanoporous material scale. As water molecules are adsorbed into the hydrophilic matrix in the cell walls, the induced fluid-solid interaction forces result in swelling of these cell walls. The interaction of the composite polymeric material, that is the layer S2 of wood cell wall, with water is known to rearrange its internal structure, make it moisture sensitive and influence its physical properties. The in-depth study of the coupled effects of water sorption on hygric and mechanical properties of different polymeric components is performed with atomistic modeling. Our aim is to understand all the ramifications of this intricate nanocomposite, with the specific aim of upscaling the results to cellular and macroscopic scales. In order to study the behavior of S2 layer, we analyse the different configurations of cellulose microfibril aggregates and S2 matrix using Molecular Dynamics (MD) and Grand Canonical Monte Carlo (GCMC) simulations. Atomistic simulations are used to mimic water adsorption and desorption in amorphous cellulose, make observations on hysteresis and relate this hygromechanical behavior as observed from the breaking and reforming of hydrogen bonds to the hysteretical behavior of more complex polymeric composites.

We upscale the hygromechanical observations using a poromechanical constitutive model. Further, upscaling to cellular scale is informed through accurate geometrical description using X-ray CT at different relative humidity, at different scales, namely sub-cellular, cellular and growth ring scales. The ensemble of results documents the full co-occurrence of sorption and swelling. Swelling is shown to undergo constraints by the multilayer wall composition and the cellular structure of wood. This modeling methodology provides new insights in understanding wood behavior and its material 
properties. Such insights cannot be directly determined from experiments. This multiscale methodology allows to explore new pathways for material development and durability of wooden components.

\title{
Procter and Gamble Student poster award:
}

I don't want to compete References:

Chen M, Coasne B, Guyer R, Derome D, Carmeliet J. 2018. Role of hydrogen bonding in hysteresis observed in sorption-induced swelling of soft nanoporous polymers, Nature Comm., 9:3507. Acceptance of Terms and Conditions:

Click here to agree

\section{Dynamic X-ray Tomography in the Laboratory: Exploring foam stability in $4 \mathrm{D}$}

\author{
Jan Dewanckele ${ }^{1}$; Marijn Boone ${ }^{1}$; Arno Merkle ${ }^{1}$ \\ ${ }^{1}$ TESCAN XRE
}

Corresponding Author(s): jan.dewanckele@tescan.com, marijn.boone@xre.be

Time-resolved 3D imaging with X-rays has rapidly emerged as an essential technique to understand materials evolution, facilitating in situ investigations ranging from mechanical deformation to fluid flow in porous materials and beyond. This push toward time-resolved dynamic studies has been spearheaded by synchrotron radiation facilities, with temporal resolutions going below 1 second for a full 3D acquisition. Meanwhile, in the laboratory X-ray imaging spatial resolutions and image quality have continued to improve significantly 1 , often at a significant cost of temporal resolution however.

Recent advances in laboratory-based approaches have pushed achievable temporal resolutions from hours down to seconds, enabling the visualization of dynamic processes and real-time imaging 2 . Dynamic in situ studies represent some of the most important scientific drivers of this technology, however, their widespread adoption has been limited to proof-of-concept excursions due to significant technical barriers preventing routine use.

Here we present progress in our approach to dynamic tomography workflows, intended to facilitate more routine application of such $4 \mathrm{D}$ experiments. Dynamic acquisitions do generate vast sums of raw projection data, which need to be reconstructed and further post-processed and quantified. It is therefore essential to devise workflow strategies to quickly identify the interesting moments prior to reconstruction to optimize the amount of data that is generated, but also incorporate the added time dimension in the $3 \mathrm{D}$ analysis workflow to improve image quality. Here we present challenges and possibilities for dynamic micro-CT imaging related to acquisition, reconstruction and analysis. The methodology and dedicated workflow from acquisition through analysis is illustrated by way of example through a foam collapse study. Formation and movement of individual bubbles are monitored and analyzed over time, providing valuable insights on the stability of the overall foam structure.

\section{Procter and Gamble Student poster award:}

\section{References:}

1 E Maire and PJ Withers, Int. Materials Review 59 (2014) p. 1-43.

2 T Bultreys et al, Advances in Water Resources 95 (2016) p. 341-351. Acceptance of Terms and Conditions:

Click here to agree 


\section{Structural adaptation of biological tissues}

Salvatore Di Stefano ${ }^{1}$; Kotaybah Haslamoun ${ }^{2}$; Salvatore Federico ${ }^{3}$; Alfio Grillo ${ }^{4}$

${ }^{1}$ Politecnico di Torino

${ }^{2}$ Graduate Programme in Biomedical Engineering, The University of Calgary

${ }^{3}$ Department of Mechanical and Manufacturing Engineering, The University of Calgary

${ }^{4}$ Department of Mathematical Sciences “'G. L. Lagrange”, Politecnico di Torino, Torino, 10129, Italy

Corresponding Author(s): salvatore.distefano@polito.it

In this work, biological tissues are studied as fibre-reinforced biphasic media, filled with an interstitial fluid and exhibiting remodelling [3,2].

The latter, understood as the structural reorganisation of the tissue, is characterised by the onset and evolution of irreversible strains, which accompany the tissue's overall change of shape as well as the flow of the intertistial fluid 2 .

Furthermore, remodelling is the result of the adaptation of the tissue to external stimuli, and our aim is to try to understand in which way the mechanical properties of the tissue are influenced when structural rearrangement occurs [2, 4].

To this purpose, by taking inspiration from the Theory of Elastoplasticity, remodelling is regarded as the production of anelastic distortions. To keep track of these, a new kinematic variable is added to the standard ones and a suitable evolution law for plastic-like distortions is derived $[1,4]$.

\section{References}

1 Epstein, M., Maugin, G., A.: On the geometrical material structure of anelasticity. Acta Mechanica 115.1-4, 119-131 (1996).

2 Grillo, A., Prohl, R., Wittum, G.: A poroplastic model of structural reorganisation in porous media of biomechanical interest, Continuum Mechanics and Thermodynamics, 28, 579-601 (2016).

[3] Federico, S., Grillo, A.: Elasticity and permeability of porous fibrereinforced materials under large deformations. Mechanics of Materials, 44, 58-71 (2012).

[4] Di Stefano, S., Carfagna, M., Knodel, M., Hashlamoun, K., Federico, S.,

Grillo, A.: Anelastic reorganisation in fibre-reinforced biological tissues. Submitted.

Procter and Gamble Student poster award:

\section{References:}

Acceptance of Terms and Conditions:

Click here to agree

\section{Probabilistic Design Optimization for $\mathrm{CO}_{2}$ Sequestration With Leakage Risk Control}

Author(s): Ben Mansour Dia ${ }^{1}$

Co-author(s): Bilal Saad ${ }^{2}$

${ }^{1}$ CPG, King Fahd University of Petroleum and Minerals

${ }^{2}$ Baker Hughes Research Center, Dhahran

Corresponding Author(s): diabenmansour@gmail.com

The physical process of

$\mathrm{CO}_{2}$ injection in geologic reservoirs, including

solubility trapping, is a non-isothermal two-phase two-component flow in porous 
media, which is governed by a system of coupled nonlinear partial differential equations. In this model, the water-rich phase (brine) and the carbon dioxide-rich phase (

$\mathrm{CO}_{2}$ ) consist of two components (water and

$\mathrm{CO}_{2}$ component), as the solubility of the components in the phases has to be taken into account. Local equilibrium phase exchange of the components in the phases is assumed to hold.

To close the system, the fluid properties of

$\mathrm{CO}_{2}$ are calculated as functions of pressure and temperature. The properties of brine additionally depend on the salinity and on the mole fraction of

$\mathrm{CO}_{2}$ in brine.

For simulations purpose, a reservoir composed of two aquifers separated by an aquitard is considered. The leaky well is modelled as a porous medium with higher permeability compared to the formation. We count the effect of uncertainties in reservoir porosity, reservoir absolute permeability, and permeability of the leakage well, on the model response. Also, we consider spatial heterogeneity only through the different layers according to different geological media, and we count the changes in fluid properties of $\mathrm{CO}_{2}$ and that the $\mathrm{CO}_{2}$ and brine fluid properties (e.g., density and viscosity) depend on the aquifer conditions, the temperature, the

$\mathrm{CO}_{2}$ pressure, the brine salinity, and the mass fraction of

$\mathrm{CO}_{2}$ in brine.

In the considered Benchmark,

$\mathrm{CO}_{2}$ is injected to be stored in the lower aquifer and the injected

$\mathrm{CO}_{2}$ spreads within the aquifer and once it reaches the leaky well, it connects the two aquifers and rises to a shallower aquifer.

The following sketch summarizes the model geometry and illustrates a $2 \mathrm{D}$ section of the $3 \mathrm{D}$ domain.

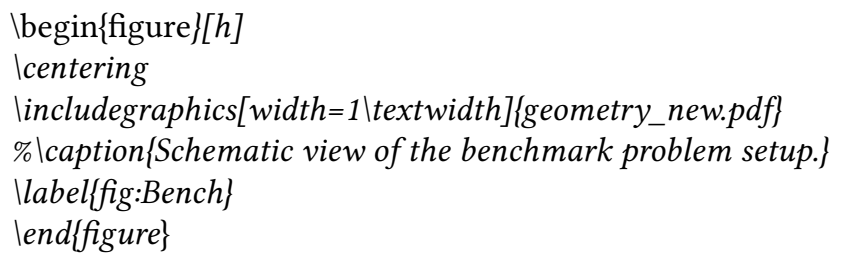

We address an optimal experimental design problem under uncertainties with the main objective of searching the optimal injection rate of $\mathrm{CO}_{2}$ for the learning of the leakage rate in order to consistently maintain the harmful leakage risk.

\title{
Procter and Gamble Student poster award:
}

\section{References:}

Acceptance of Terms and Conditions:

Click here to agree

\section{Water retention behaviour of smectite-rich materials}

\author{
Anne-Catherine Dieudonné ${ }^{1}$; Claude Gatabin ${ }^{2}$; Jean Talandier ${ }^{3}$; Frédéric Collin ${ }^{4}$; Robert Charlier ${ }^{4}$

\footnotetext{
${ }^{1}$ Delft University of Tehchnology

${ }^{2}$ Commissariat à l'énergie atomique et aux énergies nouvelles (CEA)
} \\ ${ }^{3}$ Agence nationale pour la gestion des déchets radioactifs (ANDRA)
}


${ }^{4}$ University of Liege

Corresponding Author(s): a.a.m.dieudonne@tudelft.nl

Water retention models have traditionally been formulated as a unique relationship between suction and the degree of saturation or water content. Accordingly, materials compacted to different dry densities and which exhibit different water retention properties have been described using different sets of model parameters. If this approach has been successfully applied for granular soils and low activity clay materials, it cannot describe accurately the water retention behaviour of smectite-rich materials. Indeed, these expansive materials swell significantly upon wetting, resulting in important changes in dry density. Consequently, the dependency of the water retention curve on the dry density of the material is a major issue and explains why classical water retention models have failed in describing the water retention properties of expansive materials.

This paper presents an experimental study conducted in order to characterize the water retention behaviour of compacted bentonite. Water retention curves under constant volume and free-swelling conditions were determined. The results show that, for high suction values, the wetting branch in terms of water ratio appeared to be density-independent. On the contrary, the boundary conditions significantly influenced the water uptake capacity of the bentonite in the lower suction domain. The quantity of water stored under free-swelling conditions is much higher than under constant volume.

In terms of degree of saturation, the samples at high initial density wetted under free volume conditions do no exhibit significant changes of degree of saturation. This is explained by the important competing effects of bentonite swelling and water uptake along wetting paths, as a consequence of strong hydro-mechanical coupling in compacted bentonites. Indeed, the high-density samples exhibit important swelling, i.e. mechanical drying when suction decreases.

In order to complement the interpretation and model the experimental results, a water retention model was developed. This model explicitly takes into account the aggregated and evolving double structure of compacted smectite-rich materials. The model is calibrated and validated against the experimental data. The model succeeds in reproducing the stability of the degree of saturation upon wetting under unconfined conditions. The model provides a better understanding of the influence of the complex hydromechanical processes on the water retention curve. The influence of the microstructure evolution is highlighted.

While classical water retention models relating suction to the degree of saturation are generally used in numerical modelling (their parameters being determined from constant volume water retention curves), they are not able to represent the observed behaviour. In this case, the predicted degree of saturation of a bentonite plug under in situ conditions can be considerably overestimated if technological voids are important and not taken into account in modelling. A good representation of the water retention properties of compacted smectite-rich materials is thus a fundamental issue.

Procter and Gamble Student poster award:

I don't want to compete References:

Acceptance of Terms and Conditions:

Click here to agree

\title{
Effective steady-state flow regimes during the stranding and mo- bilization of NAPL ganglia within stochastically reconstructed porous domains
}

\author{
Andreas Yiotis ${ }^{1}$; Anastasia Dollari ${ }^{1}$; Laurent Talon ${ }^{2}$; Dominique Salin ${ }^{2}$ \\ ${ }^{1}$ Environmental Research Laboratory, NCSR "Demokritos" \\ ${ }^{2}$ Laboratoire FAST, Centre National de la Recherche Scientifique
}


Corresponding Author(s): natasa@ipta.demokritos.gr

We study the flow dynamics of Non Aqueous Phase Liquid residuals within stochastically reconstructed porous domains over a typical range of Capillary, $C a$, and saturation, $S_{n w}$, values where both mobile and stranded ganglia coexist. Using an elaborate Lattice-Boltzmann immiscible twophase flow simulator, we monitor the dynamics of mass re-partitioning between the mobile and stranded ganglia populations at the pore scale and at steady-state flow conditions, i.e. when the volume-averaged phase velocities and ganglia size distributions reach an 'apparent' dynamic equilibrium state (starting from a sufficiently disordered initial size distribution). Our analysis reveals the existence of a Non-Darcy flow regime, spanning over a non-trivial range of $C a$ values, where the non-wetting phase flux, $u_{n w}$, is a linear function of the non-wetting phase saturation (for $S_{n w}<0.4$ ) and exhibits a power-law dependence on the applied body force difference $\left(B o-B o_{c}\right)$. We show that this regime is related to the gradual mobilization of stranded ganglia and the formation of new two-phase flow paths that are increasing in number as the body force, $B o$, increases above a clearly identified threshold value, $B o_{c}$ (which is independent of $S_{n w}$ ). Our study also focuses on the size and velocity distributions of mobile and stranded populations at steady-state. Both populations exhibit distinct statistical characteristics which evolve with $B o$ in the capillarity-dominated regime.

Procter and Gamble Student poster award:

References:

1 T. Chevalier, D. Salin, L. Talon, and A. Yiotis, Physical Review E 91, 043015 (2015).

2 A. Yiotis, L. Talon and D. Salin, Physical Review E 87, 033001 (2013).

[3] K. T. Tallakstad, H. A. Knudsen, T. Ramstad, G. Løvoll, K. J.

Maløy, R. Toussaint, and E. G. Flekkøy, Physical Review. Letters 102, 074502 (2009). Acceptance of Terms and Conditions:

Click here to agree

\title{
Investigation of N2 and CO2 Huff-Puff Characteristics in Tight Sand Based on Dual T2C Model
}

\author{
XU DONG ${ }^{1}$; YULI SUN ${ }^{1}$; LICHEN ZHENG $^{1}$; DONGXING DU ${ }^{1}$
}

${ }^{1}$ Qingdao University of Science \& Technology

Corresponding Author(s): 382234706@qq.com

Huff-puff is a good solution to improve the oil recovery of tight reservoirs, which are typically characterized by low permeability $(<1 \mathrm{mD})$. In this study, the $\mathrm{N} 2$ and $\mathrm{CO} 2$ huff-puff characteristics in simulation oil $(10.5 \mathrm{cp}, 0.821 \mathrm{~g} / \mathrm{cm} 3)$ saturated tight sand plugs are experimentally investigated using NMR ( $\mathrm{Te}=60 \mathrm{us}, \mathrm{Tw}=10 \mathrm{~s}$ ). A dual T2 cutoff model is constructed to illustrate the dynamic distribution of oil in a full pore scale of cores. For a typical T2 spectrum of $100 \%$ saturated tight sand, the movable fluid T2 cutoff (T2C1) and unrecoverable fluid cutoff (T2C2) are derived from centrifuge and heat-treated experiments to distinguish the unrecoverable fluid (T2 < T2C2), capillary bound fluid (T2C2 $<\mathrm{T} 2<\mathrm{T} 2 \mathrm{C} 1)$ and movable fluid $(\mathrm{T} 2>\mathrm{T} 2 \mathrm{C} 1)$. The moveable fluid corresponding to seepage pores can be drained, whereas the bound fluid cannot be easily drained due to capillary forces in small pores and clay particle pores.

Our results on the investigated aliquots show that the $\mathrm{T} 2 \mathrm{C} 1$ locates around 10-30 ms, and the T2C2 range at about $1 \mathrm{~ms}$.

About 3 rounds of individual N2 and $\mathrm{CO} 2$ huff-puff can basically stable the residual oil saturations in tight sand samples. The oil displacement laws in the full-scale pores are similar in N2 and CO2 huff-puff processes. The moveable oil is recovered first and then followed by the capillary bound oil. Note that not all moveable oil can be recovered. After rounds of individual or alternative N2 and $\mathrm{CO} 2$ huff-puff, the right edge of all $\mathrm{T} 2$ spectrums do not move close to the $\mathrm{T} 2 \mathrm{C} 1$ simply, but shrink gradually and remain stable at about 110-140 ms. The right boundaries of residual oil in moveable pores will not be obviously changed by the gas or injection mode used in huff-puff. But the amplitude of the residual peaks can be decreased obviously when the $\mathrm{CO} 2$ is injected. The peak represents the residual oil adhered to the surface of the particles in the form of film, which is resulted by the wettability differences between the gas and oil to the matrix. The stability of T2 spectrum 
on the left of T2C2 (T2 < T2C2) in the whole process means the $\mathrm{N} 2$ or CO2 huff-n-puff is unable to recover the oil in clay particle pores (namely the unrecoverable pores). The enhanced recoveries in the last rounds of $\mathrm{N} 2$ and $\mathrm{CO} 2$ huff-puff are both mainly contributed by the oil recovered from the capillary bound pores. CO2 huff-puff can furtherly enhance the oil recovery rate about $12 \%$. Most of it is consisted by the capillary bound oil, and also includes a small amount of oil films that can be weakly recovered by the N2 huff-puff. The dual T2C mode based on NMR described in this study provides new insights into the understanding of the dynamic distribution of oil on the N2 and CO2 huff-puff processes in the full pore scale of tight oil reservoirs.

Procter and Gamble Student poster award:

I would like to compete in the Procter and Gamble Student award References:

Acceptance of Terms and Conditions:

Click here to agree

\title{
Applying low-field nuclear magnetic resonance for characteriza- tion of nanoparticle transport through low permeability hetero- geneous geomaterials
}

\author{
Author(s): Yanhui Dong ${ }^{\text {None }}$ \\ Co-author(s): Qian Zhang \\ Corresponding Author(s): dongyh@mail.iggcas.ac.cn
}

\begin{abstract}
Understanding the transport of nanoparticles (NPs), such as nanoscale zero-valent iron (nZVI) particles, through water-saturated porous media has important implications for many natural and engineered systems. Compared to traditionally used high-field Nuclear Magnetic Resonance (HF-NMR), low-field NMR is considered to be more suitable for characterization of transport processes in rocks, because it is less subject to the impacts of internal gradients. In this study, we used the spin-echo single point imaging (SE-SPI) sequence of LF-NMR to monitor nanoparticle transport processes through a heterogeneous porous medium for the first time. This method has the advantages of fast data acquisition and being able to measure paramagnetic NP concentrations in low permeability porous media. In the experiments, a solution of modified nZVI particles was injected into an artificial sandstone core initially, and NMR data were acquired at regular time intervals to quantify concentration profiles. The concentration data were used to constrain the modeling in HYDRUS-1D software to evaluate transport parameters at multiple slices along the sandstone core. Two sets of simulations were performed. One assumed spatial homogeneity and a single set of the transport parameters was fitted using the concentration data at the outlet, similar to standard breakthrough curve (BTC) analysis. The other simulation considered spatial heterogeneity by dividing the core into four regions, each with a distinctive set of transport parameters. This was made possible because the NMR data provided the evolution of NP concentration at multiple points along the core. A significant role of spatial heterogeneity in NP transport was proved that the simulation with a homogenous assumption failed to predict BTCs of interior slices due to variations in pore structures. In addition, the impacts of pore structure properties, obtained by means of micro X-ray Computed Tomography $(\mu \mathrm{CT}) \mathrm{im}$ ages, on transport behaviors were discussed in terms of transport parameters. Simulation results showed that dispersion coefficient $D$ decreased, attachment rate coefficient Kac increased, while detachment rate coefficient $K d c$ varied in a narrow range with the decreasing porosity. Consequently, it may lead to a misunderstanding of transport behaviors on the basis of fitted transport parameters when neglecting the influences of spatial heterogeneity. With the successful application of SE-SPI sequences in detecting transport processes affected by spatial heterogeneity, it becomes possible to explore more transport mechanism of NPs in a multi-dimensional and complex system.
\end{abstract}

Procter and Gamble Student poster award:

References:

Acceptance of Terms and Conditions:

Click here to agree 


\title{
Coupled CFD-DEM modelling of granular porous media under shear: implications for dynamics of faults with granular gouge
}

Omid Dorostkar $^{1}$; Jan Carmeliet ${ }^{2}$

\author{
${ }^{1}$ ETH Zurich \\ ${ }^{2}$ ETHZ
}

Corresponding Author(s): domid@ethz.ch

Granular porous materials subjected to shear stress are involved in many geological catastrophes including earthquakes, landslides and avalanches. The dynamics of a sheared granular layer is controlled by its particle properties, loading configuration, temperature and pore fluids. While field and laboratory experiments have studied the role of pore fluid in granular interactions within geological systems, they lack detailed information about pore- and grain-scale mechanisms that dictate mechanics of deformation and evolution of macroscopic characteristics. Here a 3D coupled Computational Fluid Dynamics-Discrete Element Method (CFD-DEM) is used to model stick-slip dynamics in a granular porous system, saturated with fluids, aiming to better understand the hydro-mechanical influence of fluid flow. Our focus here is dedicated to the effect of fluid flow on the characteristics of slip events i.e. friction coefficient drop, potential energy drop, thickness change etc. that are measured and analysed statistically using information of hundreds of slip events in a drained system. Our results show that, slip events are characterized by a higher drop in friction coefficient, in potential energy and thickness of the granular porous layer compared to the dry conditions. Our results show a fluid-assisted type of particle mobilization in the fluid saturated system, where the high dynamic fluid pressure is found to stem from the fast fluid flow during slip caused by particles rearrangements. The spatial correlation of regions with high fluid velocity, particle-fluid interaction and particle kinetic energy during slip demonstrates the strongly coupled mechanisms of particle rearrangement, increase of fluid pressure and particle-fluid interaction forces. This study emphasizes the important role of fluid-particle interactions at play in sheared granular porous media present at tectonic fault damage zones showing how numerical models, and in particular the coupled CFDDEM approach, can help understand the hydro-mechanical processes that dictate fault slip from a grain-scale point of view.

Procter and Gamble Student poster award:

References:

Acceptance of Terms and Conditions:

Click here to agree

\section{Numerical challenges in modelling spontaneous imbibition flows in throat-pore body systems: an assessment of the volume of fluid formulations}

Author(s): Saideep Pavuluri ${ }^{1}$

Co-author(s): Julien Maes ${ }^{1}$; Florian Doster ${ }^{1}$

${ }^{1}$ Heriot-Watt University

Corresponding Author(s): f.doster@hw.ac.uk

Flows driven by capillary forces are observed in a broad range of porous media applications. While modelling capillary flows, parasitic currents are generated around the interface due to the difficulty in accurately approximating the capillary forces. To alleviate parasitic currents, several volume of fluid (VOF) formulations have been proposed on top of the standard Continuum Surface Force (CSF) 
model, namely the Sharp Surface Force (SSF) and the Filtered Surface Force (FSF). In a previous related study, we have demonstrated analytically the occurrence of a dynamic capillary barrier zone during spontaneous imbibition in throat-pore body systems when the contact angle is larger than a critical value $\theta$ c, which depends on the sharpness of the transition zone. In the present study, we investigate the capability of the different VOF formulations to model accurately the imbibition in such systems. We observe that all formulations struggle to capture the process while using the conventional curvature computation. To solve this issue, we propose to apply a curvature correction which insures that the norm of the interface normal vector remains equal to one during the computation. Using this correction, the numerical estimation of the critical contact angles using the CSF formulation for various shape of the transition zone is in close proximity to the analytic expression. However, sharpening and filtering may result in wrong prediction of $\theta$ _c. Our results suggest that it is more important to accurately compute the interface curvature than to eliminate parasitic velocities. Finally, we investigate spontaneous imbibition in a pore network comprising of ten pores having different aspect ratios and shapes of the transition zones. For the pore network, using CSF, we notice a capillary barrier zone that is overcome by the meniscus after stopping at the expected position temporarily. This could potentially occur due to numerical diffusion. Applying a minimal amount of sharpening helps to prevent this numerical artefact and capture the accurate flow dynamics.

Procter and Gamble Student poster award:

References:

Acceptance of Terms and Conditions:

Click here to agree

\title{
Prediction of the Effective Thermal Conductivity of the Porous Media based on Digitally Reconstructed Matrix Structures
}

\author{
Dongxing $\mathrm{DU}^{1}$; Xu Zhang ${ }^{1}$; Fei Wang ${ }^{\text {None }}$; Xu Dong ${ }^{1}$; Zhifeng Sun ${ }^{1}$; Yingge Li $^{1}$ \\ ${ }^{1}$ Qingdao University of Science and Technology
}

Corresponding Author(s): du-dongxing@163.com

The effective thermal conductivity (ETC) of a porous media reflects the interaction of the matrix thermal conductivity and the pore fluid thermal conductivity, therefore its successful prediction relies heavily on the exact description of the microscale structure of the porous medium. In recent years, Digital rock physics (DRP) technology for simulative analysis of rock physical properties based on extracted CT scanning images plays more and more important roles in geosciences, soil science, petroleum engineering and many other fields.

In this paper, a novel procedure is proposed for accurate reconstruction of the 3-D internal skeletal structures, based on which the solid part of the porous medium is successfully meshed for detailed numerical studies on the thermal conductivity properties. The first step is to determine the REV of the studied rock after processing all the CT images with the software of ImageJ ${ }^{\circledR}$, with which the image noise reduction, threshold segmentation and binary processes are performed. The second step is to transform the binary black and white series of rock images into a digital 3-D matrix consists of 0 and 1 with Matlab ${ }^{\circledR}$ software. Then reconstruction of the rock frame is performed through the software of ProE ${ }^{\circledR}$ to supply the compatible format of the Fluent ${ }^{\circledR}$ software for heat conduction analysis.

Simulations are carried out to anticipate the ETC of a porous medium along different directions with fixed heat flux and temperature at the upper and lower boundaries respectively. Numerical results reveal obvious anisotropy characteristics with distinctively different ETC values along different heat flux directions. It is concluded the exact description of the skeletal structure is essential on successful ETC predictions and the novel methods proposed in this paper have practical significance for the accurate characterization of thermal properties of porous media materials. 
Procter and Gamble Student poster award:

References:

Acceptance of Terms and Conditions:

Click here to agree

262

\title{
Numerical Simulation of Foam Three Phase Displacement Char- acteristics in porous media Using Stochastic Bubble Population Model
}

Dongxing $\mathrm{DU}^{1}$; Yingge $\mathrm{Li}^{1}$

${ }^{1}$ Qingdao University of Science and Technology

Corresponding Author(s): du-dongxing@163.com

Foam technology has found wide applications in enhanced oil recovery (EOR) and greenhouse geological storage practices. In this paper, a numerical simulation is carried out with the stochastic bubble population balance model on the foam three phase displacement process in a homogeneous oil/water/gas coexistence porous media. The effects of the maximum equilibrium bubble density nmax and the foam generation rate $\mathrm{Kg}$ on foam displacement process is discussed and the simulation results are validated with corresponding experiments. Following conclusions are obtained:

(1)Foam could produce extra oil component from oil/gas/water pre-saturated porous media due to its high apparent viscosity;

(2) Elevated maximum foam density value leads to higher pressure drop along the sample and therefore results in higher liquid phase recovery rate;

(3)Higher bubble generate rate $\mathrm{Kg}$ values could generate fully developed foam in a shorter distance after gas injection, however shows little effect on total liquid phase displacement efficiency of the foam flooding process.

(4) Numerical results could qualitatively reproduce the experimental observations.

It is expected the reported work could improve the understanding of the EOR mechanism of the foam flooding processes in porous media.

Procter and Gamble Student poster award:

References:

Acceptance of Terms and Conditions:

Click here to agree

186

\section{Characteristics of Aggregate Size Distribution of Nanoscale Zero- valent Iron in Aqueous Suspensions and Its Effect on Transport Process in Porous Media}

\author{
Ruiqi Duan ${ }^{1}$; Liheng Wang ${ }^{1}$ \\ ${ }^{1}$ Institute of Geology and Geophysics, Chinese Academy of Sciences \\ Corresponding Author(s): duanruiqi1993@163.com
}

Bare nanoscale zero-valent iron (NZVI) particles in aqueous suspensions aggregate into micron to submicron sizes. The transport process of enlarged aggregates or multi-sized aggregates is different from that of nanoparticles. In this work, we performed aggregate size distribution analysis of NZVI 
suspension using a laser grain size analyzer and conducted a series of continuous injection column experiments with different injected NZVI concentrations. The results show that aggregates in NZVI suspensions range from submicron to submillimeter size and are mainly distributed around 5-9 $\mu \mathrm{m}$ and $50-100 \mu \mathrm{m}$. Quantitative calculation of iron transport and retention showed that the retained iron linearly correlates with injected concentration. The cross-section images revealed that clogging weakened from inlet to outlet. Further, larger aggregates $(>40 \mu \mathrm{m})$ appeared more often in the risingdeclining stages of breakthrough curves, whereas small aggregates $(<30 \mu \mathrm{m})$ dominated the steady stage. Indeed, relatively preferential flow facilitated the transport and discharge of both large and small iron aggregates. Straining of grains especially for the large iron aggregates resulted in a decline in breakthrough. Moreover, the blocking of attached and plugged iron prevented later retention of iron, resulting in certain concentration of iron in the effluents. Our study provides greater insight into the transport of NZVI.

Procter and Gamble Student poster award:

References:

Acceptance of Terms and Conditions:

Click here to agree

912

\section{Modelling of nanochannels imbibition with Smoothed Particles Hydrodynamics method}

Magdalena Dymitrowska ${ }^{1}$; Aliaksei Pazdniakou ${ }^{1}$

${ }^{1}$ IRSN

Corresponding Author(s): magdalena.dymitrowska@irsn.fr

A large number of natural and engineered porous materials present a part of their pore space with nanometric pore sizes. This is the case for example for natural indurated clays, which are studied in several countries as potential hosts for deep geological repositories of radioactive wastes. In such clay rocks the connectivity of micrometric pores is ensured though very small throats, with diameters as thin as $2-5 \mathrm{~nm}$. To reliably describe macroscopic flow properties at different time and space scales of such materials it becomes necessary to relate them with microscopic pore structure. It becomes increasingly popular to exploit to this aim 3D pore networks obtained from different tomographic methods like X-ray microtomography (resolution from 0.2 m to 40 冈m) or destructive FIBSEM (resolution down to 5-10 $\mathrm{nm}$ ). These real porous structures are used to conduct pore-scale flow simulations. However caution must be payed to correctly take into account the confinement effects that may both change flow parameters, or even require adding new terms to governing equations. This is particularly the case for two phase flow within porous media where capillary effects dominate over viscous forces. In this work we focus on studying the imbibition of dead-end nano-channels, initially filled with air $[1,2]$. It has been observed experimentally, that the imbibition process can be divided into two parts: initial capillary rise following Washburn equation with a compression of the trapped gas, followed by a dissolution-diffusion stage during which the entrapped gas dissolves in the surrounding liquid. However in 2, authors reported a particular blocking-moving regime where the liquid fills dead-ends behind gas bubbles, while its front meniscus stays immobile. During this regime, the gas bubbles diminish asymmetrically, with only the rear meniscus moving. This stage ends with a symmetrical regime, where the mass center of the gas bubble is immobile and both menisci are receding towards it. The first, asymmetrical, stage is unusual and the driving phenomena involved are not clear. Using the home-made Smoothed Particle Hydrodynamics code [3], we explore possible origins of this behavior while keeping the physics as simple as possible (isothermal, non-compressible, Navier-Stokes based). We show that geometrical factors are sufficient to induce liquid flow through wetting films and filling of dead-ends behind gas bubbles. It is also possible to generate the blocking-moving regime, especially when taking into account the roughness of the solid walls. This observation implies that the two-phase flow depends on the nanometric details of the pore structure, which are difficult to resolve by imaging technics. Thus the pore-scale simulations may be more appropriate and reliable in bigger, micrometric, pores only.

Procter and Gamble Student poster award: 
I don't want to compete References:

1 Vinh Nguyen Phan, et al. (2010), Capillary Filling in Closed End Nanochannels, Langmuir, 26(16), 13251-13255

2 A.Naillon (2016) Ecoulements liquide-gaz, évaporation, cristallisation dans les milieux poreux et nanoporeux. Etudes à partir de systèmes micro et nanofuidique. PhD Thesis of University of Toulouse

[3] A.Pazdniakou, M.Dymitrowska (2018), Migration of Gas in Water Saturated Clays by Coupled Hydraulic-Mechanical Model, Geofluids, vol. 2018, article ID 6873298, https://doi.org/10.1155/2018/6873298 Acceptance of Terms and Conditions:

Click here to agree

\section{Estimation of permeability reduction during drilling operations in naturally fractured reservoirs}

Kelly Lorena Díez Hernández ${ }^{1}$; Nicolás Bueno Zapata ${ }^{2}$; Juan Mejía ${ }^{2}$; Juan David Vallejo Restrepo ${ }^{3}$

\footnotetext{
${ }^{1}$ Facultad de Minas, Universidad Nacional del Colombia

${ }^{2}$ Facultad de Minas, Universidad Nacional de Colombia

${ }^{3}$ Exergy - Modeling and Analytics
}

Corresponding Author(s): kldiezh@unal.edu.co

The productivity of many tight hydrocarbon reservoirs depends on natural fractures networks. Keeping the high fracture permeability remains a challenge during drilling operations. The finer particles invade fractures and pore throats, restricting the flow of fluids from the reservoir to the well. The industry lacks of models that integrates these phenomena, involving dynamic operating conditions during drilling. This work presents a new model to evaluate filtrate and particle invasion processes into naturally fractured reservoirs. This model is based on a three-phase simulator (oil, gas and water) coupled to a set of mass transport equations of solids and chemical species. In addition, mudcake formation in the wellbore face is also modeled under dynamic conditions. Phenomenological equations are used to model the invasion of solids and their entrapment in the formation. These equations correlate multiple particle diameters with pore diameters and the fracture widths. Therefore, it is possible to estimate the contribution of suspended solids to the reduction of permeability, i.e., the formation damage. The model is validated using results obtained from core-flooding experiments. The results are also supported by a large amount of technical literature. The simulation model allows varying the operative conditions, such as overpressure, circulation and drilling rate in order to evaluate their impact on permeability impairment. The model allows for estimating the permeability return after the displacement of the invasive fluids by the in-situ fluids. As a valuable conclusion of this work, optimizing the suspended particles size distribution leads to a less severe permeability reduction. Hence, this work contributes to increasing the productivity of naturally fractured reservoirs.

\section{Procter and Gamble Student poster award:}

\section{References:}

1. D. Jiao, M, Sharma. "Mud-Induced Formation Damage in Fractured Reservoirs". SPE Drilling \& Completion, 1996. 2. T. Bratton, D.V. Canh. "The Nature of Naturally Fractured Reservoirs". Oilfield Review, 2006. [3]. M. Taha, H.D. "Drilling and Completions Strategies in Naturally Fractured Rservoirs". SPE 59057, 2000. [4]. Dewan, J. \& Chenevert, M. A Model for Filtration of Water-base Mud During Drilling: Determination of Mudcake Parameters. Petrophysics, pp. 237-250, 2001. 5. Lohne, A. Formation Damage and Well Productivity Simulation. SPE Journal, pp. 751-769, 2010. Acceptance of Terms and Conditions:

Click here to agree 


\title{
Fracture width prediction and particle size distribution design to wellbore strengthening: A comprehensive workflow for effective operations.
}

\author{
Kelly Lorena Díez Hernández ${ }^{1}$; Carlos Nuñez Perez ${ }^{2}$; Juan Mejía ${ }^{1}$; Juan David Valencia Londoño ${ }^{1}$ \\ ${ }^{1}$ Universidad Nacional de Colombia \\ 2 Total S.A
}

Corresponding Author(s): kldiezh@unal.edu.co

\begin{abstract}
The operating drilling window between the pore pressure and the fracture gradient is very narrow in many geologically complex reservoirs, depleted formations, and deepwater reservoirs. As a consequence, lost circulation remains a recurring problem. Lost circulation increases significantly well costs during the non-productive time as well as in high expensive remediation treatments. To mitigate this, wellbore strengthening technique emerged some years ago. These techniques consist in inducing shallow fractures in the formation and propping them with an impermeable bridge of lost control material. In this way, the tangential stress in the near-wellbore region is increased and the drilling window is improved.

Despite the uninterrupted use of this technology, there is a lack of understanding of mechanisms by which wellbore strengthening works properly. The success of a wellbore strengthening depends on the coupling between the fracture induced and lost control material. Influential geomechanical aspects on fracture width prediction, often are not taken account on mathematical models yet. In this work, we evidence the effect of anisotropic stress state, rock elastic modules and wellbore deviation in fracture width prediction. Sensitivity analysis are carried out over the analytical model proposed by (Zhang 2016) to determinate fracture width. Finally, we show a comprehensive workflow for effective wellbore strengthening operations from the fracture width prediction, through particle size distribution to the application on site.
\end{abstract}

Procter and Gamble Student poster award:

References:

Zhang, J., Alberty, M., \& Blangy, J. P. (2016). A semi-analytical solution for estimating the fracture width in wellbore strengthening applications. Society of Petroleum Engineers - SPE Deepwater Drilling and Completions Conference. Acceptance of Terms and Conditions:

Click here to agree

\section{Predicting fluid flow via convolutional neural networks}

\author{
Author(s): Javier E. Santos ${ }^{1}$ \\ Co-author(s): Masa Prodanovic ${ }^{2}$; Duo Xu ${ }^{3}$; Honggeun Jo ${ }^{1}$; Michael Pyrcz ${ }^{4}$ \\ ${ }^{1}$ University of Texas at Austin \\ ${ }^{2}$ The University of Texas at Austin \\ ${ }^{3}$ Department of Astronomy \\ ${ }^{4}$ Hildebrand Department of Petroleum and Geosystems Engineering
}

Corresponding Author(s): javierestradasantos@gmail.com

Recent advances in imaging techniques have made it possible to study micron and sub-micron scales with more detail. These results have enhanced our understanding of pore-scale structures and how they impact flow via direct pore-scale simulations based on images. However, performing direct simulations at a representative scale has proven computationally prohibitive. Furthermore, linking results from multiple length-scales is still an open question, and usually simplifications are require 
to solve for the flow field. In this work, we aim to speed up pore-scale lithology-independent predictions by learning from an ensemble of existing simulations, supporting efficient upscaling.

Via an artificial neural network architecture, we trained an algorithm to understand the relationship between pore-scale morphology and flow properties under different flow conditions. We first created an extensive training set using a multi-relaxation-time lattice-Boltzmann algorithm that runs in our supercomputer cluster. Afterwards, different transformations were tested to select the most suitable mapping technique that is able to capture the main morphological relationships that affect flow at the desired scale. Finally, we validate our model with 3D x-ray images from cores with different lithology.

Simulating flow at the pore-scale is a powerful tool to understand the processes underlying fieldscale operations. Nevertheless, long simulation times have limited the size of the predictions that are feasible to carry out by personal computers. Through appending a statistical learning toolbox to the digital-rock petrophysics workflow, we add value by supporting fast predictions from large datasets that support physics-informed decisions by the end-user.

\title{
Procter and Gamble Student poster award:
}

I would like to compete in the Procter and Gamble Student award References:

Acceptance of Terms and Conditions:

Click here to agree

\section{Experimental Study of Induced Seismicity by Designed Porous Media}

\author{
yaniv edery $^{1}$; Tajudeen M. Iwalewa ${ }^{2} ;$ R. Rice James ${ }^{2}$ \\ ${ }^{1}$ Technion \\ ${ }^{2}$ Harvard
}

Corresponding Author(s): yanivedery@gmail.com

Induced seismicity, where earthquakes are initiated anthropogenically, is omnipresent in many anthropogenic processes such as $\mathrm{CO} 2$ sequestration, wastewater injection and hydraulic fracturing, to name a few. The frequency of these induced earthquakes has increased significantly in the past decade as a result of the increase in wastewater injection, leading to considerable public concern. As such, understanding the basic parameters that control the initiating mechanism for the earthquake is essential. Fundamentally, the controlling parameters for the initiation, namely, pore pressure and shear strength, are known. Naively, the injection of wastewater leads directly to an increase in pore pressure, which reduces the effective strength of a nearby fault. However, the way in which the pore pressure diffuses to the fault through the permeability heterogeneity of the sub-surface porous media is largely unknown. Since the permeability generally has significant variation in natural rock, and more so around the fault where the earthquake begins, it is imperative to test the effect of permeability on the pore pressure increase. Understanding the impact of permeability on the pore pressure increase can improve the risk assessment for an induced earthquake.

In this study we use layers of polymethylmethacrylate (PMMA) beads, in the 45 to $200 \mu \mathrm{m}$-size range, to simulate the variable fault permeability architecture. The beads were chemically sintered to form a rock-like structure, and their refractive index is matched with a wetting and a non-wetting fluid. Thus, we can visualize the replacement of one fluid with another in 3D using a confocal microscope. We can control the infiltration rate by imposing the pressure of the invading phase while following the plume invasion within the porous medium at the micron scale. An important observation showed that the invading phase deforms the domain, and locally the domain permeability, leading to a focusing of the plume invasion towards the outlet.

Procter and Gamble Student poster award:

References: 
Click here to agree

\title{
The impact of the calcite surface charge on multicomponent trans- port in chalk aquifers
}

\author{
María Bonto $^{1}$; Ali Akbar Eftekhari ${ }^{1}$; Hamid M. Nick ${ }^{2}$ \\ ${ }^{1}$ Technical University of Denmark \\ ${ }^{2} D T U$
}

Corresponding Author(s): aliak@dtu.dk

\begin{abstract}
Critical aspects of the reactivity of the natural and artificial calcite (e.g., carbonates) in aqueous solutions are still not entirely understood, although crucial in many applications (i.e. CO2 sequestration, contaminant transport, development of hydrocarbon reservoirs, etc.). The high sensitivity of the calcite dissolution and precipitation to changes in the solution chemistry makes it challenging to elucidate experimentally the ion adsorption equilibria. This becomes even more difficult when the experimental system can exchange $\mathrm{CO} 2$ with a gas phase. In order to understand and describe the reactivity of the calcite mineral surface, several surface complexation models have been developed over the last few decades. These models could be the key to predicting the behavior of the calcitebrine complicated systems, e.g., the wettability of natural calcite in contact with different brine and hydrocarbons, where probably different mechanisms occur concurrently. The prerequisite for the development of a fully mechanistic model for, e.g., the adsorption of water pollutants or polar organic groups on the carbonate surfaces in groundwater-remediation and improved oil recovery, is a thermodynamic model that can describe the physicochemical interactions between the minerals on the surface of carbonates (mainly calcite) and the ionic species in the aqueous phase. The adsorption, dissolution, ion exchange, and precipitation not only affect the surface charge of the carbonate, but also its interaction with other existing phases (i.e., DNAPL, LNAPL). The calcite surface potential is traditionally quantified experimentally through zeta potential measurements. We revise some of the existing surface complexation models for the calcite-brine interface and implement them. We assess their performance in the prediction of available zeta potential measurements reported in the literature. We optimize one of the models and we couple it to a finite volume solver (Eftekhari et al., 2017) to model the nonisothermal reactive flow of brine in porous media. In our model, the apparent equilibrium constants of the adsorption reactions are dependent on the calcite surface charge, the adsorption is a function of not only the $\mathrm{pH}$ and temperature, but is also affected by other physicochemical interactions that occur at the calcite/brine interface. Therefore, this model accounts not only for the species adsorption and transport, but also the effect of these latter on the properties of the porous media, which enable us to evaluate more realistically the fate and transport of contaminants.
\end{abstract}

Procter and Gamble Student poster award:

\section{References:}

References

Eftekhari, A. A., Thomsen, K., Stenby, E. H., \& Nick, H. M. (2017). Thermodynamic Analysis of ChalkBrine-Oil Interactions. Energy and Fuels, 31(11), 11773-11782. Acceptance of Terms and Conditions:

Click here to agree

\section{Interface Conditions for Infiltration Problems}

Elissa Eggenweiler $^{1} ; \operatorname{Jim}_{\text {Magiera }^{1}} ;$ Iryna Rybak $^{1}$ 
${ }^{1}$ University of Stuttgart

Corresponding Author(s): elissa.eggenweiler@mathematik.uni-stuttgart.de

Coupled flow systems containing a porous medium domain and a free flow region appear in many environmental and technical applications, e.g. surface water/groundwater flow, industrial filtration and water management in fuel cells. The interaction of the porous medium and the free flow is dominated by the processes which take place at the interface between the two flow regions. Modelling such coupled flow systems is a challenging task because of the complexity of the interface driven processes.

Although mathematical modelling and numerical simulation of coupled free flow and porous medium systems has been an active research area, there is a lack of interface concepts for flows non-parallel to the porous layer. Coupling conditions existing in the literature for flows perpendicular to the interface are physically inconsistent. Moreover, there aren't any interface concepts found yet for arbitrary flow directions.

In this talk, we will show by means of microscale numerical simulations that the available interface conditions for infiltration problems (flow normal to the interface) are physically questionable. We will present the newly developed conditions valid at the interface for non-parallel single-fluid-phase flow. These conditions are derived using homogenisation with two-scale asymptotic expansions and validated via microscale numerical simulations.

Procter and Gamble Student poster award:

I would like to compete in the Procter and Gamble Student award References:

Acceptance of Terms and Conditions:

Click here to agree

29

\title{
Shear thickening of flexible coiled polymer solutions in non-inertial shear and extensional flows
}

\author{
Author(s): Eseosa Eguagie ${ }^{1}$ \\ Co-author(s): Steffen Berg ${ }^{2}$; John Crawshaw ${ }^{1}$; Shauvik De ${ }^{3}$; Paul Luckham ${ }^{1}$ \\ ${ }^{1}$ Imperial College London \\ ${ }^{2}$ Shell Global Solutions International B.V, \\ ${ }^{3}$ Shell India Markets Private Limited
}

Corresponding Author(s): e.eguagie16@imperial.ac.uk

We investigate the challenges involved in the use of polymer flooding as a chemical enhanced oil recovery (cEOR) technique for improving mobility ratio and enhancing macroscopic sweep efficiency. Flexible coiled polymers in porous media undergo stretching in a spatially heterogeneous structure. Due to the viscoelasticity of these polymers, they stretch continuously depending on their previous deformation until their elastic limit is reached and relaxation occurs. Previous research has proposed that at a certain critical flow rate, the relaxation of polymers cause an increase in viscosity and in turn a better mobility for enhancing microscopic sweep in porous media. However, others have reported that the increased viscosity in porous media is not so much related to the elasticity but more on the normal stresses that occur when polymers are sheared in porous media flow. One similar fact is that as increased viscosity is observed an enhanced pressured drop occurs and the 
flow becomes highly unstable even at laminar flow regime. This unstable flow is termed the elastic instability or turbulence but the details of this kind of turbulence, its consequences and applicability on the impact of oil recovery is not understood.

In this work, we experimentally investigate the flow behaviours of flexible coiled polymers of hydrolysed polyacrylamide (HPAM) based on a single pore throat geometry using a microfluidic device. The aim is to adequately parameterise the effects of the normal stress difference in shear and extension as a function of the geometry and intrinsic characteristics of the polymer solutions at different Deborah (De) numbers. Hence, we compare pressure drop and particle image velocimetry experimental data for solutions of varying salinity. Results showed a critical flow rate and De at which polymer viscosity increases as well as the normal stress difference and different salinities were observed to affect the scale of instabilities upstream of the single contraction geometry. It was also observed that the flow resistance might be a function of both the elasticity and the normal stresses occurring in shear flow, however, extensional stresses cannot be neglected.

Procter and Gamble Student poster award:

References:

Acceptance of Terms and Conditions:

Click here to agree

\section{Micromorphic contributions to granular and porous materials}

Wolfgang Ehlers ${ }^{1}$; Sami Bidier ${ }^{1}$

${ }^{1}$ University of Stuttgart

Corresponding Author(s): wolfgang.ehlers@mechbau.uni-stuttgart.de

From a microscopic point of view, a large number of natural and engineering materials exhibit a highly heterogeneous structure. Natural granular materials, such as sand, for example, can consist of millions of grains of different materials that form a loose porous solid skeleton. However, in engineering applications, sand is also frequently used as a basis material to generate highly stiff solids, compare, for example, sand-cores in metal casting applications, where quartz sand is combined with a polyurethane-based binder material. Thereby, the overall macroscopic response of such media stems from the composition and the interaction of the materials on various length and time scales. Understanding the different processes on and the interactions between the scales has been one of the main driving forces in research over the last decades.

The group of kinematically extended continuum theories, especially including micropolar and micromorphic continua, aims at taking specific microstructural mechanisms into account without direct modelling or simulation of the underlying microstructure. In general, this is done by enriching the local material point of the continuum description with an independent microrotation, associated to an attached rigid (micropolar) or deformable (micromorphic) microcontinuum.

In this context, the focus of the present contribution lies in the identification, the detection and the evaluation of extended micromorphic and micropolar contributions on the macroscopic scale of granular materials. This aim is achieved by taking a particle-based microstructural point of view and deriving a consistent homogenisation strategy that links particle interactions, displacement and local deformation with extended stress and deformation states of micromorphic continua. The homogenisation procedure is based on the introduction of Representative Elementary Volumes (REV) that collect a number of particles on the mesocopic scale and the subsequent derivation of balance relations for embedded REV. Ultimately, the exploitation of the derived relations on the mesoscopic REV level yields the identification of averaging formalisms for all relevant stress and deformation quantities.

In order to demonstrate the transfer of particle-based information from the micro- to the macroscale, Discrete-Element (DE) computations are carried out as simulations of small-scale experiments with emphasis on the initiation and the full evolution of shear-bands, as it is well known that extended micropolar quantities are active in localising granular materials that consist of rigid unbonded grains (micropolar setting). In order to detect micromorphic characteristics after homogenisation, the possibility of local deformation is included in the DE model by introducing deformable beam elements as surrogates for binder material (micromorphic setting). 
The evaluation of the homogenised results shows the activation of micromorphic and micropolar contributions in granular materials, but also concentrates on their dependency on the chosen REV size.

Procter and Gamble Student poster award:

References:

Acceptance of Terms and Conditions:

Click here to agree

\title{
Viscosity Membrane - A Novel Paradigm for Polymer Flow in Sand
}

John Ejezie $^{1}$; Stephan Jefferis ${ }^{2}$

\author{
${ }^{1}$ Stantec \\ ${ }^{2}$ University of Oxford
}

Corresponding Author(s): johnejezie@gmail.com

Partially hydrolysed polyacrylamide (PHPA) based polymers are used in a variety of civil engineering applications including as excavation support fluids in construction work such as piling and borehole drilling. For these applications, the role of the fluid is to keep the excavation open during digging. PHPA based polymers offer promising solutions to challenging problems such as stabilising coarse grained soils. They also require a smaller site plant set-up than conventional bentonite clay based support fluids and can show reduced environmental impacts. However, there are problems, for example, excessive loss of polymer from the excavation by permeation of the fluid into the adjacent ground. This is not only detrimental to excavation stability but also wastes polymer.

To tailor polymers to meet the demands of specific engineering projects it is necessary to develop a fuller understanding of their flow and sorption behaviours. The research presented sets out the results of some tests to develop the understanding of the underpinning science as related to the rheological and sorption behaviour of polymers fluids in coarse grained soils.

The results of a series of laboratory studies of the flow of PHPA polymer fluids in sands over a range of grain sizes and pressure heads will be presented. The data were obtained using a constant head permeability set-up modified to obtain data over a range of depths into the sand bed. In parallel, theoretical aspects of polymer fluid flow in porous media have been revisited and applied to obtain a better insight into the underpinning microscopic and macroscopic phenomena involved.

Measurements made under transient flow conditions show an initial pressure drop and a subsequent rise in pressure as the fluid front passes a point in the soil. This phenomenon is described as a viscosity membrane and is the stabilising mechanism of polymer support fluids in porous media. A novel theoretical framework to explain the complete time dependent behaviour was tested against the experimental data. The results indicate that such a model is adequate to address the transient penetration behaviour of the PHPA polymer solutions in soil pores and thus can predict the length of penetration at any time. Uncertainties regarding the theoretical description of polymer fluid flow in coarse grained soils using capillary model will be further discussed. The microscopic interactions of polymers and solid particles showing a profound effect on the macroscopic flow process in sand also will be presented.

Procter and Gamble Student poster award:

I would like to compete in the Procter and Gamble Student award References:

\section{Acceptance of Terms and Conditions:}

Click here to agree 


\title{
Viscosity Membrane - A Novel Paradigm for Polymer Flow in Sand
}

\author{
John Ejezie $^{1}$; Stephan Jefferis ${ }^{2}$ \\ ${ }^{1}$ Stantec \\ ${ }^{2}$ University of Oxford
}

Corresponding Author(s): johnejezie@gmail.com

Partially hydrolysed polyacrylamide (PHPA) based polymers are used in a variety of civil engineering applications including as excavation support fluids in construction work such as piling and borehole drilling. For these applications, the role of the fluid is to keep the excavation open during digging. PHPA based polymers offer promising solutions to challenging problems such as stabilising coarse grained soils. They also require a smaller site plant set-up than conventional bentonite clay based support fluids and can show reduced environmental impacts. However, there are problems, for example, excessive loss of polymer from the excavation by permeation of the fluid into the adjacent ground. This is not only detrimental to excavation stability but also wastes polymer.

To tailor polymers to meet the demands of specific engineering projects it is necessary to develop a fuller understanding of their flow and sorption behaviours. The research presented sets out the results of some tests to develop the understanding of the underpinning science as related to the rheological and sorption behaviour of polymers fluids in coarse grained soils.

The results of a series of laboratory studies of the flow of PHPA polymer fluids in sands over a range of grain sizes and pressure heads will be presented. The data were obtained using a constant head permeability set-up modified to obtain data over a range of depths into the sand bed. In parallel, theoretical aspects of polymer fluid flow in porous media have been revisited and applied to obtain a better insight into the underpinning microscopic and macroscopic phenomena involved.

Measurements made under transient flow conditions show an initial pressure drop and a subsequent rise in pressure as the fluid front passes a point in the soil. This phenomenon is described as a viscosity membrane and is the stabilising mechanism of polymer support fluids in porous media. A novel theoretical framework to explain the complete time dependent behaviour was tested against the experimental data. The results indicate that such a model is adequate to address the transient penetration behaviour of the PHPA polymer solutions in soil pores and thus can predict the length of penetration at any time. Uncertainties regarding the theoretical description of polymer fluid flow in coarse grained soils using capillary model will be further discussed. The microscopic interactions of polymers and solid particles showing a profound effect on the macroscopic flow process in sand also will be presented.

Procter and Gamble Student poster award:

I would like to compete in the Procter and Gamble Student award References:

Acceptance of Terms and Conditions:

Click here to agree

\section{Solute transport for non-Darcy flow in porous media using pore network modelling}

Author(s): Amr Abbas El-Zehairy ${ }^{1}$

Co-author(s): Mohaddeseh Mousavi Nezhad ; Vahid.J Niasar ; Ian Guymer ${ }^{2}$

${ }^{1}$ PhD researcher, School of Engineering, University of Warwick, Uk.

${ }^{2}$ Department of Civil and Structural Engineering, University of Sheffield, Sheffield, UK

Corresponding Author(s): a.el-zehairy@warwick.ac.uk

Darcy's law, which describes a linear relationship between pressure gradient and fluid velocity, is normally applied for modelling fluid flow with low velocities through porous media. However, due 
to relatively higher velocities in some applications such as new wellbore, the non-Darcy flow can be created and Darcy's law loses its validity. In such circumstances, the relationship between pressure gradient and fluid velocity becomes nonlinear as a result of flow inertial effects. The inertial effects can be observed as a result of convergence, divergence and tortuosity in the flow path geometry $[1,2]$. To our knowledge, all previous studies that modelled solute transport in porous media using Pore Network Modelling (PNM) have been carried out within the Darcy regime. Solute dispersion in porous media has many applications in hydrology, geology and oil recovery [3-5]. In this study, a computational framework, based on pore network theory, has been developed to investigate the effects of the pore-scale structure of porous media on the macro-scale transport process within the non-Darcy flow regime. For non-Darcy flow, due to high flow velocity in each pore throat, the asymptotic Fickian regime (i.e., the regime in which the longitudinal dispersion coefficient reaches a constant value over time) is not likely to be reached. For that purpose, three different transport regimes are defined for each pore throat based on the solute residence time and fluid velocity; asymptotic-Fickian regime, pre-asymptotic time dependent regime and pure advection regime. For each regime a suitable equation has been used to calculate the effective longitudinal dispersion coefficient for each pore throat, then the mass balance equation is invoked at each node. The longitudinal dispersion coefficient has been estimated by fitting the resulting break through curves from PNM to the one-dimensional (1-D) Advection-Dispersion Equation (ADE). The results show a very good match between the break through curves obtained from the proposed model and the analytical solution of the 1-D ADE. In contrast to the Darcy flow regime, the transport process within the non-Dacry flow regime is advection dominated. At high flow velocities, the longitudinal dispersion coefficient is directly proportional to the fluid velocity. If the inertial forces are not accounted in the flow simulation, this causes overestimation of the Péclet number $(\mathrm{Pe})$ and longitudinal dispersion coefficient (DL) through the medium which can be up to three times.

\section{Procter and Gamble Student poster award:}

I would like to compete in the Procter and Gamble Student award References:

1 F. Thauvin and K. K. Mohanty, "Network Modeling of Non-Darcy Flow Through Porous Media," Transport in Porous Media, vol. 31, pp. 19-37, 1998.

2 M. T. Balhoff and M. F. Wheeler, "A Predictive Pore-Scale Model for Non-Darcy Flow in Porous Media," SPE Journal, vol. 14, pp. 579-87, 2009/12/1/ 2009.

[3] B. Bijeljic and M. J. Blunt, "Pore-scale modeling and continuous time random walk analysis of dispersion in porous media," Water Resources Research, vol. 42, p. W01202, 2006.

[4] M. Sahimi, Flow and Transport in Porous Media and Fractured Rock: From Classical Methods to Modern Approaches, Second Edition: John Wiley and Sons, 2011.

5 R. Aziz, V. Joekar-Niasar, and P. Martinez-Ferrer, "Pore-scale insights into transport and mixing in steady-state two-phase flow in porous media," International Journal of Multiphase Flow, vol. 109, pp. 51-62, 2018/12/01/2018. Acceptance of Terms and Conditions:

Click here to agree

686

\section{Behavior of turbulence at Fluid/Porous interfaces of different Per- meabilities}

Mahmoud Elhalwagy $^{1}$; Anthony Gerald Straatman ${ }^{2}$

\footnotetext{
${ }^{1}$ Postdoctoral associate, Western University, Canada

${ }^{2}$ Professor, Western University, Canada
}

Corresponding Author(s): melhalwa@uwo.ca, melhalwa@gmail.com

This work describes a comparison between the turbulence characteristics at Fluid/Porous interfaces of different permeability values between pore-level simulations and Conjugate volume-averaged Fluid/Porous simulations, introducing a novel interface treatment for conjugate simulations that is capable of controlling numerically the penetration/dissipation of turbulence at the interface according to different levels of permeability. The interface treatment is based on an enhanced turbulent wall-like treatment that is free from refinement restrictions. Computational domains are those of channel flow partially blocked by a porous layer, similar to the classical Beavers and Joseph problem 1 as described by Betchen et al. 2. The development follows an analytical framework that was 
established by De Lemos [3] for turbulence characteristics. A recently-published pore-level digital technique [4] is utilized to produce geometric models with the use of a stack up of representative elementary volumes (REVs), a fluid layer on top of Porous layer, manifesting the fluid/porous interface. Porosities of as low as $5 \%$ were produced and simulated successfully. Distinct flow behaviors are observed in Conjugate simulations (and confirmed by pore-level simulations) for cases of relatively high permeability values (i.e. for 0.3 porosities and higher), and low permeability values (i.e. as low as 0.05 of and lower). Applications with such low porosities are related to produce drying processes in which treating the produce as porous media is important to represent the mechanisms of both vapor and liquid heat and moisture diffusion. $k-\varepsilon$ turbulence models have been used for both simulations. Profiles at the fluid transition layer for turbulent kinetic energy and dissipation rate are investigated and compared between the two types of simulations as well as the jump coefficient interface model by De Lemos [3]. The profiles for turbulence kinetic energy and its dissipation rate show a peak near the interface that decays in both directions towards the porous and the fluid regions for low permeability interfaces while a different behavior is observed for moderate-to-high permeabilities. In this case, a considerable turbulence penetration is observed at the porous region with a much slower turbulence decay towards the fluid region. The approach is comparable in its results to the work of De Lemos [3] without introduction of empirical ad-hoc jump coefficients.

\title{
Procter and Gamble Student poster award:
}

\section{I don't want to compete References:}

1 G. Beavers and D. D. Joseph, Boundary Conditions at a Naturally Permeable Wall, J. Fluid Mech., vol. 30, pp. 197-207, 1967.

2 Betchen, L., Straatman, A. G., \& Thompson, B. E. (2006). A nonequilibrium finite-volume model for conjugate fluid/porous/solid domains. Numerical Heat Transfer, Part A: Applications, 49(6), 543-565.

[3] De Lemos, M. J. (2009). Turbulent flow around fluid-porous interfaces computed with a diffusionjump model for $\mathrm{k}$ and $\varepsilon$ transport equations. Transport in porous media, 78(3), 331-346.

[4] Dyck, N., Straatman, A. G., 2015 "A new approach to digital generation of spherical void phase porous media microstructures,” Int. J. Heat Mass Transfer 81, 470-477. Acceptance of Terms and Conditions:

Click here to agree

\section{$10 \mathrm{~Hz}$ Operando X-ray Tomographic Imaging of Liquid Water in Gas Diffusion Layers of Polymer Electrolyte Fuel Cells}

\author{
Hong $\mathrm{Xu}^{1}$; Minna Büher ${ }^{1}$; Federica Marone ${ }^{1}$; Thomas J. Schmidt ${ }^{1}$; Felix N. Büchi ${ }^{2}$; Jens Eller ${ }^{1}$ \\ ${ }^{1}$ Paul Scherrer Institut \\ ${ }^{2}$ Electrochemistry Laboratory, Paul Scherrer Institut, Villigen, Switzerland
}

Corresponding Author(s): jens.eller@psi.ch

In the last decade X-ray tomographic microscopy (XTM) has become a powerful 3D characterization technique for the fundamental understanding of the behavior of liquid water in the gas diffusion layers (GDLs) of polymer electrolyte fuel cells (PEFCs), which is vitally important for improving their performance, cost and durability.

In early 2018 a new high numerical aperture 4x microscope from Optique Peter has been commissioned at the TOMCAT beamline of the Swiss Light Source at Paul Scherrer Institut. It provides a higher light collection efficiency and a higher spatial resolution compared to the previous $2 \mathrm{x}-4 \mathrm{x}$ white beam microscope from Elya Solutions. With the new microscope PEFC-XTM scan times have been improved by about one order of magnitude down to $0.1 \mathrm{~s}$ with sufficient image quality for post processing. For such short scan times the supply of the feed gases for operando XTM at elevated cell temperatures of up to $80{ }^{\circ} \mathrm{C}$ became a bottleneck of the setup. As the acceleration of the sample to scan speed requires about two full rotations of the sample stage it prevents the use of flexible heated tubes which where previously used to provide feed gases with dew points of up to $85{ }^{\circ} \mathrm{C}$ to the fuel cell on the rotation stage. As solution a heated and motorized rotary union setup that revolves synchronized with the rotation stage at TOMCAT was developed.

This contribution will discuss a) the image quality improvements by the hardware upgrade and improved imaging settings, b) the development of the motorized rotary union setup and it's coupling 
to the beamline as well as c) present some results of recent subsecond operando PEFC-XTM imaging experiments exploiting the new setup

Procter and Gamble Student poster award:

I don't want to compete References:

Acceptance of Terms and Conditions:

Click here to agree

316

\title{
An upscaled model for the transport of inertial particles in a water- saturated porous medium
}

\author{
Max Endo Kokubun ${ }^{1}$; Florin Adrian Radu ${ }^{2}$; Eirik Keilegavlen ${ }^{1}$; Kundan Kumar $^{3}$; Kristine Spildo ${ }^{1}$ \\ ${ }^{1}$ University of Bergen \\ ${ }^{2}$ University of Bergen, Norway \\ ${ }^{3}$ University of Karlstad
}

Corresponding Author(s): max.akira@gmail.com

We derive an upscaled model for the problem of transport of inertial particles by water phase in a porous medium. At the pore scale, we consider an Euler-Euler flow model, in which the particles are accounted as a dispersed phase embebbed in the fluid phase. Stokes drag is the only interphase interaction, which renders different velocities for the phases. The non-homogeneities in the flow field, created by the tortuous paths of the porous medium, create regions of preferential accumulation or dispersion of particles, which can affect the overal properties of the multiphase flow. We upscale the pore-scale model by means of formal homogenization, obtaining a set of governing equations valid in the Darcy scale. We consider both homogeneous and heterogeneous random media.

Procter and Gamble Student poster award:

References:

Acceptance of Terms and Conditions:

Click here to agree

17

\section{Balancing complexity and parsimony to accurately model, and upscale, the transport of large, fibrous colloids through porous media}

\author{
Nick Engdahl ${ }^{1}$ \\ ${ }^{1}$ Washington State University \\ Corresponding Author(s): nick.engdahl@wsu.edu
}

Large fibrous, colloids such as microplastic synthetic fibers, organic matter fragments, and even bacteria can strongly affect flow and transport in porous media, with impacts that include pore-clogging and hyper-accumulation of contaminants, among many others. The mobility of these fiber-like objects in porous media is particularly challenging to model because of the wide range of physical and chemical processes in porous media that affect their mobility. Pore-scale flow and transport of fibrous objects can now be modeled using physically based simulation tools like constrained random 
walks, which have already shown that fibers have complicated transport tendencies that involve tradeoffs between fiber length and Peclet number. However, the computational effort required for these explicit simulations is immense and not practical at field scales. Upscaling methods are needed to bridge the pore- and field scales but the question of how to scale the transport of these fibers is unresolved. This presentation considers whether or not classical upscaling tools from the decades of research on solute transport can be applied to describe fiber mobility. The methods include simple advection-dispersion based homogenization, dual-domain transport models, exposure time dependent transport, and several random walk-based approaches. We consider transport of an ensemble of fibers through a simple, periodic model of porous media and compare the parameters of the trial, effective models, to the observed transport at different Peclet numbers and over different length scales. Preliminary results suggest that a scale-dependent advection-dispersion model can approximate fiber transport, and that a random-walk based model provides a less parameterized upscaling approach, but the results also suggest that simple upscaling techniques break down when the fibers are allowed to interact with each other and/or the porous substrate. Our preliminary results suggest that exposure time-based models are good candidate for modeling the mobility of interacting fibers and that these relatively simple models may afford robust modeling of the complex processes that affect fiber transport.

Procter and Gamble Student poster award:

References:

Acceptance of Terms and Conditions:

Click here to agree

\title{
Predicting diameter changes of the vasculature to regulate blood flow during activation
}

\author{
Author(s): Robert Epp \\ Co-author(s): Franca Schmid ${ }^{2}$; Bruno Weber $^{2}$; Patrick Jenny ${ }^{1}$ \\ ${ }^{1}$ Institute of Fluid Dynamics, ETH Zurich \\ ${ }^{2}$ University of Zurich
}

Corresponding Author(s): eppr@student.ethz.ch

The brain is capable of up-regulating cerebral blood flow in response to local changes in neural activity. However, the precise pattern of the underlying vasodynamics is still poorly understood. Our goal is to improve our understanding of the impact of diameter changes on blood flow regulation in the vasculature.

We present a numerical framework, which is based on a previously developed blood flow model 1 and on well-established optimization algorithms. The strength of our model is that we can compute diameter changes necessary to achieve desired flow distributions. This is done by adjusting the diameters of the network, in order to minimize a predefined cost function J. The required diameter changes are computed iteratively by using a gradient based optimization algorithm, where the sensitivity of J with regard to the diameters is calculated with the adjoint method.

In our blood flow model, red blood cells (RBCs) are tracked individually as they move through the vascular network and their dynamic impact on the flow resistance is considered. If a RBC reaches a divergent bifurcation, it is assigned to a daughter branch based on a stochastic bifurcation rule. Due to this stochastic nature of the blood flow, flow rates and pressures can vary over time. This has to be taken into account while calculating the partial derivatives for the adjoint method and therefore, our optimization algorithm is based on time averaged flow rates and pressures.

Another challenge is that the solution for this inverse problem can be ambiguous, since multiple possible diameter distributions can minimize J. Therefore, the cost function is augmented with a regularization term that aims to find the most likely solution, e.g. the solution that minimizes the total change of all diameters.

This method allows us to investigate different scenarios related to blood flow regulation. Furthermore, we can use the same method to tune model parameters such as boundary conditions or to reduce uncertainties of diameters and lengths of blood vessels in realistic microvascular networks 
based on sparse experimental data.

We performed simulations in artificial and realistic microvascular networks to compute the vessel diameters required to increase the mean flow rate at different locations in the network. In our studies, we investigated how the dilation and constriction of different vessel types affect the flow distribution. First results reveal that a localized blood flow increase can only be obtained, if arterioles and capillaries can change their diameters. These findings suggest that not only arteriole-, but also capillary dilation may play an important role in the local regulation of cerebral blood flow.

Procter and Gamble Student poster award:

I would like to compete in the Procter and Gamble Student award References:

1 Schmid et al., 2017, PloS Comput Biol Acceptance of Terms and Conditions:

Click here to agree

\title{
Pore Scale Modelling of Reactive Transport in Porous Media: Im- plications to Geological Energy Applications
}

\author{
Author(s): Hamidreza Erfani Gahrooei ${ }^{1}$ \\ Co-author(s): Vahid Joekar-Niasar ${ }^{1}$ \\ ${ }^{1}$ The University of Manchester \\ Corresponding Author(s): hamidreza.erfanigahrooei@postgrad.manchester.ac.uk
}

\begin{abstract}
Simulation of reactive transport of subsurface flow helps us to improve our understanding of complex processes of rock-fluid and fluid-fluid interactions. Such phenomena are important to be considered in different processes ranging from Enhanced Oil Recovery (EOR) and geological energy storage/recovery. The aim of this research is to develop a pore-network modelling of reactive flow in sandstone rock samples. For this purpose, a geochemical module, PhreeqcRM is coupled with a geostatistical-realistic pore network model, where advection-diffusion transport along with equilibrium/kinetic geochemical reactions are solved using sequential non-iterative approach (SNIA). Reactive transport models, which consider mineral dissolution/precipitation, mostly use reaction rates, measured in the laboratory, to calculate reaction rates in natural porous media. As laboratory systems are designed in a way to eliminate the effect of mass transport limitations, they can be very different from natural systems. Also, natural sedimentary rocks (carbonate and sandstone) are usually composed of multiple minerals, with considerably different reaction rates. The developed coupled pore-network model is able to simulate the interaction of water with different $\mathrm{pH}$ and compositions with a wide range of minerals. In this study pore scale chemical heterogeneity and the effect of different averaging methods on reactive model parameters are studied.
\end{abstract}

\section{Procter and Gamble Student poster award:}

I would like to compete in the Procter and Gamble Student award References:

Acceptance of Terms and Conditions:

Click here to agree

\section{Reactive Density-Driven CO2 Mixing in Sandstone Aquifers: Role of Geochemistry}

\author{
Author(s): Hamidreza Erfani Gahrooei ${ }^{1}$ \\ Co-author(s): Vahid.J Niasar ; Masoud Babaei ${ }^{2}$
}




\footnotetext{
${ }^{1}$ University of Manchester

${ }^{2}$ The University of Manchester
}

Corresponding Author(s): hamidreza.erfanigahrooei@postgrad.manchester.ac.uk

\begin{abstract}
During geological carbon sequestration, $\mathrm{CO} 2$ overrides to the top of the aquifer is inevitable. Consequently, far from injection wells, $\mathrm{CO} 2$ phase dissolves into the aqueous phase by molecular diffusion and density-driven mixing takes place. The phenomenon has been extensively studied in the literature, however, crucially the coupled flow-geochemistry and effects of geochemistry on the process in sandstone rock has not been adequately addressed. This research presents a $2 \mathrm{D}$ multicomponent reactive convective-diffusive formulation to simulate density-driven mixing in sandstone aquifers A realistic field scale model is designed based on the literature to investigate the effect of rock-fluid interactions on density-driven mixing and the role of carbon mineralization in carbon capture and storage process over the project life time. A typical sandstone mineralogical assemblage is considered and solid-phase reactions are assumed to be kinetic to present a realistic geochemistry. Also, the impact of mineral dissolution and precipitation on porosity are calculated. The results show that in moderate Rayleigh numbers, rock-fluid interactions adversely affect dissolution trapping, while stimulating the total carbon capture through mineral trapping process. In this situation dawsonite along with magnesite minerals are the most important precipitating carbonates and mineral trapping contributes to $28 \%$ of total sequestered carbon after almost 10 years. The largest porosity reduction is $1.3 \%$ and happens near the upper boundary of the domain. The results of this study shed light on the effect of rock-fluid interaction on density driven mixing and solubility trapping in sandstone aquifers and help to better estimate the capacity of saline aquifers for carbon storage.
\end{abstract}

\title{
Procter and Gamble Student poster award:
}

I would like to compete in the Procter and Gamble Student award References:

Acceptance of Terms and Conditions:

Click here to agree

57

\section{Pilot scale "in pile" thermal desorption remediation of mercury and mixed pesticides contaminated soil}

Author(s): Søren Eriksen ${ }^{1}$

Co-author(s): Jocob Brix ${ }^{1}$; Jesper Holm ${ }^{1}$

${ }^{1}$ Krüger $A / S$

Corresponding Author(s): soe@kruger.dk

Thermal desorption remediation of sandy soil from a former chemical waste land fill site (Groyne 42) has been tested in laboratory and pilot scale.

Contaminants are mercury, thiophosphate ester pesticides - parathion, methyl parathion, malathion and sulfotep - smaller amounts of chloro phenols and chloro cresols as well as waste from pesticide production - mixed (thio) phosphate esters, elemental sulphur and solvents.

The contaminated soil volume of the site is $27000 \mathrm{~m} 3$ containing 100 tons of contamination including 67 tons of parathion and 7 tons of mercury. /1/

High remediation efficiencies of well over $99 \%$ are required due to high concentrations of contaminants.

Complete removal of pesticides and an $85 \%$ reduction of mercury to a level of $6 \mathrm{mg} / \mathrm{kg}$ was reported in previous thermal desorption laboratory testing at $300^{\circ} \mathrm{C} . / 2 /$

Based on results from lab scale testing removing all contaminants to $<1 \mathrm{mg} / \mathrm{kg}$ by maintaining $350^{\circ} \mathrm{C}$ for 2-4 days a pilot scale test was designed. The pilot set up for thermal treatment of 40 tons soil is a $2.4 \times 2.4 \times 6.0 \mathrm{~m}$ steel box fitted with $6 \times 5 \mathrm{~kW}$ electrical insertions heaters and off gas treatment plant consisting of water injection, condenser, mist separator, activated carbon filters and blower. 
The soil for the pilot test contained $440 \mathrm{mg} / \mathrm{kg}$ mercury and $1400 \mathrm{mg} / \mathrm{kg}$ combined pesticides, primarily parathion.

Five step sequential selective extraction analysis revealed that $71 \%$ of the mercury is only soluble in the last step, aqua regia, and hence most probably is mercury sulfide. $24 \%$ was soluble in concentrated nitric acid probably elemental mercury.

During the pilot test, the soil volume was slowly heated while aerated at a moderate flow of $10 \mathrm{~m} 3 / \mathrm{h}$. The air flow serves two purposes: carrying evaporated contaminants out of the soil and oxidizing mercury sulphide to volatize the mercury in line with processes for winning mercury from roasting sulphide ore.

Safety considerations for operating the pilot scale test includes: Exothermal reactions, production of flammable gasses from pesticide decomposition, concentration of flammable vapors of solvents, sulphur, phenols.

Simulation of chemical reactions and evaporation rates has been used to find safe heating rates keeping concentrations of flammable gasses well below lower explosion limit.

Preliminary results of the pilot testing as of Nov. 2018 are elimination of pesticides and a 75\% reduction of mercury at $200^{\circ} \mathrm{C}$. The operational experience has shown that the closed system approach is ideal when negotiating complex contaminant mixtures.

References

/1/ Høfde 42, Afgrænsning af nedsivningsområdet, rapport udarbejdet af Rambøll/Cowi/Kogsgaard, 2013, p.18

http://www.northpestclean.dk/siteassets/northpestclean/publikationer/npc/2013/2013-12-06-hofde-42afgransning-af-nedsivningsomrade-1.pdf

/2/ Steffen Griepke Nielsen et al.: Termisk assisteret oprensning af høfdedepotet, Høfde 42, Harboøre Tange, Miljøprojekt Nr. 1193, Miljøstyrrelsen, 2007, p. 70

https://www2.mst.dk/Udgiv/publikationer/2007/978-87-7052-604-3/pdf/978-87-7052-605-0.pdf

/3/ N. S. Bloom et al., Analytica Chimica Acta 479 (2003) 233-248

Procter and Gamble Student poster award:

References:

Acceptance of Terms and Conditions:

Click here to agree

465

\title{
Pore-Scale Visualization of Hydrate Formation and Dissociation
}

\author{
Geir Ersland $^{1}$; Martin Ferno ${ }^{2}$; Arne Graue ${ }^{2}$; Stian Almenningen ${ }^{2}$; Maksim Lysyy ${ }^{2}$ \\ ${ }^{1}$ University of Bergen (UiB) \\ ${ }^{2}$ University of Bergen
}

Corresponding Author(s): geir.ersland@uib.no

We report a large data-set on visualization of $\mathrm{CH} 4$ gas hydrate formation and dissociation patterns using micromodels with sandstone pore shapes and sizes. The effect of salinity and initial fluid saturation on hydrate growth and dissociation within porous media is identified. Direct observations of hydrate phase transition patterns in pores using micromodels at reservoir conditions provides an improved understanding of sedimentary gas hydrate and how such system respond to pressure depletion and thermal stimulation. The occurrence and dynamics of the three-phase system of saline water, methane gas and solid gas hydrate is characterized. The hydrate growth rates were studied with varying salinity, initial water saturation, pore sizes and degree of sub-cooling. Hydrate dissociation was studied with respect to two hydrate configurations: 1) non-porous and 2) porous 
hydrate films/shell. A strong correlation between initial methane gas hydrate distribution and dissociation characteristic, and subsequent mobilization of methane gas, was observed. The dissociation of non-porous hydrate was slower with a gas front that propagated through the pore space. The direct contact with mobile gas rather than water favored the hydrate dissociation and induced a dissociation front. Local water salinities affected the methane gas hydrate structure leading to distinct dissociation patterns of self-preservation due to water freshening.

Procter and Gamble Student poster award:

References:

Acceptance of Terms and Conditions:

Click here to agree

\title{
Grid convergence studies for lattice Boltzmann method based pore- scale reactive transport models
}

\author{
Author(s): Omid EsmaeelipoorJahromi ${ }^{1}$ \\ Co-author(s): Ravi Patel ${ }^{2}$; Mohammad J Amani ${ }^{1}$; Mojtaba Ghaedi ${ }^{1}$; Mohammad H Golestan ${ }^{3}$ \\ ${ }^{1}$ Department of Petroleum Engineering, School of Chemical and Petroleum Eng., Shiraz University \\ ${ }^{2}$ Laboratory for Waste Management (LES), Paul Scherrer Institute \\ ${ }^{3}$ Department of Petroleum Engineering and Applied Geophysics, Norwegian University of Science and Technology
}

Corresponding Author(s): o.esmaeelipoor@shirazu.ac.ir, mohammad.h.golestan@ntnu.no

\begin{abstract}
The reactive transport processes such as dissolution/precipitation are of interest for varieties of engineering fields. Such processes exhibit highly non-linear behavior and many time difficult to upscale at continuum-scale. As a result in recent years, increasing focus has been laid on developing pore-scale reactive transport models. Due to the high computing cost involved in such pore-scale studies often detail validation and grid convergence studies are not carried out. It has been observed in case on continuum scale reactive transport modeling that in some conditions grid convergent solutions are not attainable. This study aims to provide discussion on this aspect using lattice Boltzmann method-based pore-scale reactive transport model. The lattice Boltzmann method (LBM) has proved to be efficient numerical tool for simulating such pore-scale processes involving evolution of solid phases due to dissolution/precipitation. In particular this study will focus on effect of grid discretization on dissolution, precipitation and dissolution-precipitation simultaneously. A detail set of simulations are carried out to establish grid convergence of lattice Boltzmann method based pore-scale reactive transport model by comparing with existing analytical models for different geometrical configuration, transport parameters and reaction rates. It is finally aimed that such study will provide a set of benchmarks to which pore-scale models can be validated.
\end{abstract}

Procter and Gamble Student poster award:

References:

Acceptance of Terms and Conditions:

Click here to agree

\section{Characterization of Foam Flow in Porous Media: Effect of Differ- ent Chemical Concentrations, Flow Rates and GLR Ratio}

Author(s): Sahand Etemad ${ }^{1}$ 
Co-author(s): Apostolos Kantzas ${ }^{1}$; Steven Bryant

${ }^{1}$ University of Calgary

Corresponding Author(s): sahand.etemad@ucalgary.ca

Foam can be used as a subsurface mobility control agent to improve oil recovery. High quality and long-lasting foam inside reservoir guarantees higher mobility reduction and improved recovery factor. In this study, two different quality regimes were investigated; low-quality coarse-textured foam versus high quality strong foam with fine texture. Flooding experiments were conducted in sand packs contained in a HP/HT mini-rig apparatus, and pressure buildup across the sand pack was measured. The foam apparent viscosity was calculated, and the effluent of the sand pack was collected in a visual cell at test conditions. The foam structure was qualitatively monitored. Conventional surfactant stabilized foam was compared against fortified foam with nanoparticles. Changing surfactant and nanoparticle concentrations, as well as the effect of quantity of chemicals in the foaming solution on foam stability were tested. Finally, the effect of injection velocity on foam performance in porous media was also investigated.

Several core flood experiments were conducted in sand packs at elevated temperature and pressure conditions $\left(2.7 \mathrm{MPa}\right.$ and $\left.60^{\circ} \mathrm{C}\right)$. CO2 gas and [AOS 14-16 surfactant + Cellulose nanocrystal] solution was co-injected into a foam generator. Later, the pre-generated foam was injected into the water saturated sand pack at the same conditions.

It was observed that foam properties, especially pressure behavior of the foam during flow experiments, are highly dependent on the chemical concentrations and gas to liquid ratio (GLR). By increasing the injection velocity from $8.8 \times 10-5 \mathrm{~m} / \mathrm{s}$ to $7 \times 10-4 \mathrm{~m} / \mathrm{s}$, it was observed that above a critical shear rate, foam flow performance inside the porous media does not change significantly. Very strong foam with apparent viscosity around $650 \mathrm{mPas}$ was generated at $80 \%$ quality and [ $1 \mathrm{wt} \%$ surfactant $+1 \mathrm{wt} \% \mathrm{NP}$ ] concentration. Nanoparticle addition increased the stability during core flood. CNC Nanotubes prevent the bubble collapse at elevated temperature and pressure by making a barrier in between the lamella. The apparent viscosity is also measured to be twice that of the conventional surfactant stabilized foam.

\section{Procter and Gamble Student poster award:}

I don't want to compete References:

Adebanjo, F.O. and Olusegun, O., 2015. Evaluating the application of foam injection as an enhanced oil recovery in unconsolidated sand. Journal of Petroleum and Gas Engineering, 6(2): 22-37.

Alargova, R. G., D. S. Warhadpande, V. N. Paunov, and O. D. Velev, 2004, Foam superstabilization by polymer microrods: Langmuir, v. 20, no. 24, p. 10371-10374, doi:10.1021/la048647a.

Aroonsri, A., A. Worthen, T. Hariz, K. Johnston, C. Huh, and S. Bryant, 2013, Conditions for Generating Nanoparticle-Stabilized CO 2 Foams in Fracture and Matrix Flow, in SPE Annual Technical Conference and Exhibition: p. 1-19, doi:10.2118/166319-MS.

Bernard, G. G., and L. Holm (1964), Effect of foam on permeability of porous media to gas, Society of Petroleum Engineers Journal, 4(03), 267-274.

Bernard, G.G. and Holm, L., 1964. Effect of foam on permeability of porous media to gas. Society of Petroleum Engineers Journal, 4(03): 267-274.

Binks, B. P., 2002, Particles as surfactants-similarities and differences: Current opinion in colloid \& interface science, v. 7, p. 21-41.

Bondor, P. L. (1992), Applications of carbon dioxide in enhanced oil recovery, Energy Conversion and Management, 33(5), 579-586, doi: https://doi.org/10.1016/0196-8904(92)90059-6.

Bondor, P.L., 1992. Applications of carbon dioxide in enhanced oil recovery. Energy Conversion and Management, 33(5): 579-586.

Denkov, Nikolai D., Krastanka G. Marinova, and Slavka S. Tcholakova. "Mechanistic understanding of the modes of action of foam control agents." Advances in colloid and interface science 206 (2014): 57-67. Espinoza, D. A., F. M. Caldelas, K. P. Johnston, S. L. Bryant, and C. Huh, 2010, Nanoparticle-stabilized supercritical CO2 foams for potential mobility control applications: SPE Improved Oil Recovery Symposium.

Espinoza, D. A., F. M. Caldelas, K. P. Johnston, S. L. Bryant, and C. Huh, 2010, Nanoparticle-Stabilized Supercritical CO2 Foams for Potential Mobility Control Applications, in SPE Improved Oil Recovery Symposium: doi:10.2118/129925-MS.

Etemad, S., A. Behrang, P. Mohammadmoradi, and A. Kantzas, 2017, Pore level investigation of steam injection processes; visualization of oil entrapment and steam propagation: Journal of Petroleum Science and Engineering, v. 158, doi:10.1016/j.petrol.2017.08.057. 
Etemad, S., A. Behrang, P. Mohammadmoradi, S. H. Hejazi, and A. Kantzas, 2017, Effects of surface roughness and mineral heterogeneity on pore-scale steam condensation: Journal of Petroleum Science and Engineering, v. 159, p. 624-634, doi:10.1016/j.petrol.2017.09.055.

Ettinger, R., and C. Radke (1992), Influence of texture on steady foam flow in Berea sandstone, SPE reservoir engineering, 7(01), 83-90.

Farajzadeh, R., A. Andrianov, R. Krastev, G. J. Hirasaki, and W. R. Rossen, 2012, Foam-oil interaction in porous media: Implications for foam assisted enhanced oil recovery: Advances in Colloid and Interface Science, v. 183-184, p. 1-13, doi:10.1016/j.cis.2012.07.002.

Farajzadeh, R., Andrianov, A., Krastev, R., Hirasaki, G.J. and Rossen, W.R., 2012. Foam-oil interaction in porous media: Implications for foam assisted enhanced oil recovery. Advances in Colloid and Interface Science, 183-184(Supplement C): 1-13.

Fjelde, I., J. Zuta, and O. V. Duyilemi Oil Recovery from Matrix during CO2-Foam Flooding of Fractured Carbonate Oil Reservoirs, edited, Society of Petroleum Engineers.

Fjelde, I., Zuta, J. and Duyilemi, O.V., Oil Recovery from Matrix during CO2-Foam Flooding of Fractured Carbonate Oil Reservoirs. Society of Petroleum Engineers.

Friedmann, F., and J. Jensen (1986), Some parameters influencing the formation and propagation of foams in porous media, paper presented at SPE California Regional Meeting, Society of Petroleum Engineers.

Friedmann, F., W. H. Chen, and P. A. Gauglitz, 1991, Experimental and simulation study of high-temperature foam displacement in porous media: SPE Reservoir Engineering (Society of Petroleum Engineers), v. 6, no. 1, p. 37-45, doi:10.2118/17357-PA.

Georges Lwisa, E., and A. R. Abdulkhalek, 2018, Enhanced oil recovery by nitrogen and carbon dioxide injection followed by low salinity water flooding for tight carbonate reservoir: Experimental approach, in IOP Conference Series: Materials Science and Engineering: doi:10.1088/1757-899X/323/1/012009.

Garrett PR, editor. Defoaming: theory and industrial applications. CRC Press; 1992 Nov 12.

Gonzenbach, U. T., A. R. Studart, E. Tervoort, and L. J. Gauckler, 2006, Ultrastable Particle-Stabilized Foams: Angewandte Chemie International Edition, v. 45, p. 3526-3530.

Hamdi, Z., M. Awang, A. Basyouni, H. Hematpour, and M. Bataee (2016), Comparison of CO 2 and Nitrogen Injection at Low Temperatures in High Temperature Reservoirs for Improving Oil Recovery, paper presented at Abu Dhabi International Petroleum Exhibition \& Conference, Society of Petroleum Engineers.

Haugen, A., Fernø, M.A., Graue, A. and Bertin, H.J., Experimental Study of Foam Flow in Fractured Oil-Wet Limestone for Enhanced Oil Recovery.

Haugen, Å., M. A. Fernø, A. Graue, and H. J. Bertin Experimental Study of Foam Flow in Fractured OilWet Limestone for Enhanced Oil Recovery, doi: 10.2118/129763-PA.

Hematpour, H., Mahmood, S.M., Akbari, S. and Asl, A.S., 2016. Foam modeling approaches in enhanced oil recovery: A review. Indian Journal of Science and Technology, 9(22).

Hematpour, H., S. M. Mahmood, S. Akbari, and A. S. Asl (2016), Foam modeling approaches in enhanced oil recovery: A review, Indian Journal of Science and Technology, 9(22).

Hirasaki, G. (1989), Supplement to SPE 19505, The Steam-Foam Process-Review of Steam-Foam Process Mechanisms.

Hirasaki, G., 1989. Supplement to SPE 19505, The Steam-Foam Process-Review of Steam-Foam Process Mechanisms.

Horozov, T. S., and B. P. Binks, 2004, Stability of suspensions, emulsions, and foams studied by a novel automated analyzer: Langmuir, v. 20, no. 21, p. 9007-9013, doi:10.1021/la0489155.

Kovscek, A., and C. Radke (1993), Fundamentals of foam transport in porous mediaRep., Lawrence Berkeley Lab., CA (United States).

Lawson, J. B., and J. Reisberg (1980), Alternate slugs of gas and dilute surfactant for mobility control during chemical flooding, paper presented at SPE/DOE enhanced oil recovery symposium, Society of Petroleum Engineers.

Lawson, J.B. and Reisberg, J., 1980. Alternate slugs of gas and dilute surfactant for mobility control during chemical flooding, SPE/DOE enhanced oil recovery symposium. Society of Petroleum Engineers.

Li, R. F., W. Yan, S. Liu, G. Hirasaki, and C. A. Miller Foam Mobility Control for Surfactant Enhanced Oil Recovery, doi: 10.2118/113910-PA.

Li, R.F., Yan, W., Liu, S., Hirasaki, G. and Miller, C.A., Foam Mobility Control for Surfactant Enhanced Oil Recovery.

Liu, N. et al., 2015. Nanoparticle-stabilized $\mathrm{CO}_{2}$ foam for $\mathrm{CO}_{2} \mathrm{EOR}$ application, United States. Liu, N., et al. (2015), Nanoparticle-stabilized $\mathrm{CO}_{2}$ foam for $\mathrm{CO}_{2}$ EOR applicationRep., United States. Liu, Z., B. Sunden, and J. Yuan (2012), VOF modeling and analysis of filmwise condensation between vertical parallel plates, Heat Transfer Research, 43(1). 
Liu, Z., Sunden, B. and Yuan, J., 2012. VOF modeling and analysis of filmwise condensation between vertical parallel plates. Heat Transfer Research, 43(1).

Lohne, A., and I. Fjelde, 2012, Surfactant Flooding in Heterogeneous Formations: The SPE Imroved Oil Recovery Symposium-USA, no. April, p. 14-18, doi:10.2118/154178-MS.

Lv, Q., Z. Li, B. Li, S. Li, and Q. Sun, 2015, Study of nanoparticle-surfactant-stabilized foam as a fracturing fluid: Industrial \& Engineering Chemistry Research, v. 54, p. 9468-9477.

Manrique, E. J., V. E. Muci, and M. E. Gurfinkel (2007), EOR field experiences in carbonate reservoirs in the United States, SPE Reservoir Evaluation \& Engineering, 10(06), 667-686.

Manrique, E.J., Muci, V.E. and Gurfinkel, M.E., 2007. EOR field experiences in carbonate reservoirs in the United States. SPE Reservoir Evaluation \& Engineering, 10(06): 667-686.

Mo, D., J. Yu, N. Liu, and R. L. Lee, 2012, Study of the Effect of Different Factors on Nanoparticle-Stablized CO2 Foam for Mobility Control, in SPE Annual Technical Conference and Exhibition: doi:10.2118/159282MS.

Nguyen, P., H. Fadaei, and D. Sinton, 2014, Pore-scale assessment of nanoparticle-stabilized CO2 foam for enhanced oil recovery: Energy \& Fuels, v. 28, p. 6221-6227.

Nguyen, P., H. Fadaei, and D. Sinton, 2014, Pore-scale assessment of nanoparticle-stabilized CO2foam for enhanced oil recovery: Energy and Fuels, v. 28, no. 10, doi:10.1021/ef5011995.

Robinson JV, Woods WW. A method of selecting foam inhibitors. Journal of the Society of Chemical Industry. 1948 Sep;67(9):361-5.

Rafati, R., H. Hamidi, A. K. Idris, and M. A. Manan (2012), Application of sustainable foaming agents to control the mobility of carbon dioxide in enhanced oil recovery, Egyptian Journal of Petroleum, 21(2), 155-163, doi: https://doi.org/10.1016/j.ejpe.2012.11.010.

Rafati, R., Hamidi, H., Idris, A.K. and Manan, M.A., 2012. Application of sustainable foaming agents to control the mobility of carbon dioxide in enhanced oil recovery. Egyptian Journal of Petroleum, 21(2): 155-163.

S. Owete, O., and W. E. Brigham (1987), Flow Behavior of Foam: A Porous Micromodel Study, 315-323 pp.

Saha, M. C., M. E. Kabir, and S. Jeelani, 2008, Enhancement in thermal and mechanical properties of polyurethane foam infused with nanoparticles: Materials Science and Engineering: A, v. 479, p. 213222.

San, J., S. Wang, J. Yu, N. Liu, and R. Lee (2017), Nanoparticle-Stabilized Carbon Dioxide Foam Used In Enhanced Oil Recovery: Effect of Different Ions and Temperatures, SPE Journal.

San, J., S. Wang, J. Yu, N. Liu, and R. Lee Nanoparticle-Stabilized Carbon Dioxide Foam Used In Enhanced Oil Recovery: Effect of Different Ions and Temperatures, doi: 10.2118/179628-PA.

San, J., S. Wang, J. Yu, N. Liu, and R. Lee, 2017, Nanoparticle-Stabilized Carbon Dioxide Foam Used In Enhanced Oil Recovery: Effect of Different Ions and Temperatures: SPE Journal.

San, J., S. Wang, J. Yu, N. Liu, and R. Lee, 2017, Nanoparticle-Stabilized Carbon Dioxide Foam Used In Enhanced Oil Recovery: Effect of Different Ions and Temperatures: SPE Journal, v. 22, no. 5, p. 14161423, doi:10.2118/179628-PA.

San, J., Wang, S., Yu, J., Liu, N. and Lee, R., Nanoparticle-Stabilized Carbon Dioxide Foam Used In Enhanced Oil Recovery: Effect of Different Ions and Temperatures.

Singh, R., \& Mohanty, K. K. (2017, October 9). Nanoparticle-Stabilized Foams for High-Temperature, High-Salinity Oil Reservoirs. Society of Petroleum Engineers. doi:10.2118/187165-MS

Singh, R., and K. K. Mohanty, 2014, Synergistic Stabilization of Foams by a Mixture of Nanoparticles and Surfactants, in SPE Improved Oil Recovery Symposium: doi:10.2118/169126-MS.

Sun, Q., N. Zhang, Z. Li, and Y. Wang, 2016a, Nanoparticle-Stabilized Foam for Effective Displacement in Porous Media and Enhanced Oil Recovery: Energy Technology, v. 4, p. 1053-1063.

Sun, Q., N. Zhang, Z. Li, and Y. Wang, 2016b, Nanoparticle-Stabilized Foam for Mobility Control in Enhanced Oil Recovery: Energy Technology, v. 4, p. 1084-1096.

Sunmonu, R. M., and M. Onyekonwu, 2013, Enhanced oil recovery using foam injection; a mechanistic approach, in Society of Petroleum Engineers - 37th Nigeria Annual Int. Conf. and Exhibition, NAICE 2013 - To Grow Africa's Oil and Gas Production: Required Policy, Funding, Technol., Techniques and Capabilities: p. 30 July-1 August. Lagos, Nigeria, 20131061-1073.

Sunmonu, R.M. and Onyekonwu, M., Enhanced Oil Recovery using Foam Injection; a Mechanistic Approach. Society of Petroleum Engineers.

Turta, A. T., and A. K. Singhal Field Foam Applications in Enhanced Oil Recovery Projects: Screening and Design Aspects, doi: 10.2118/02-10-14.

Turta, A.T. and Singhal, A.K., Field Foam Applications in Enhanced Oil Recovery Projects: Screening and Design Aspects.

Tyrode, E., A. Pizzino, and O. J. Rojas, 2003, Foamability and foam stability at high pressures and temperatures. I. Instrument validation: Review of Scientific Instruments, v. 74, no. 5, p. 2925-2932, doi:10.1063/1.1569403.

Wo, A. J., H. G. Bagaria, C. Yunshen, S. L. Bryant, and K. P. Jo, 2012, Nanoparticle Stabilized Carbon 
Dioxide in Water Foams for Enhanced Oil Recovery: Eighteenth SPE Improved Oil Recovery Symposium, p. 1-7, doi:10.2118/154285-ms.

Worthen, A. J., S. L. Bryant, C. Huh, and K. P. Johnston, 2013, Carbon dioxide-in-water foams stabilized with nanoparticles and surfactant acting in synergy: AIChE Journal, v. 59, no. 9, p. 3490-3501, doi:10.1002/aic.14124.

Worthen, A., H. Bagaria, Y. Chen, S. L. Bryant, C. Huh, and K. P. Johnston, 2012, Nanoparticle stabilized carbon dioxide in water foams for enhanced oil recovery: SPE Improved Oil Recovery Symposium.

Zhang, S., D. Sun, X. Dong, C. Li, and J. Xu, 2008, Aqueous foams stabilized with particles and nonionic surfactants: Colloids and Surfaces A: Physicochemical and Engineering Aspects, v. 324, no. 1-3, p. 1-8, doi:10.1016/j.colsurfa.2008.03.020. Acceptance of Terms and Conditions:

Click here to agree

\title{
Uncertainity analysis for Seawater Intrusion in Fractured Coastal Aquifers: Effect of Fractures Apparture, Location, Density and Hydraulic Properites
}

Behshad Koohbor ${ }^{1}$; Marwan Fahs $^{2}$; Benjamin Belfort ${ }^{1}$; Behzad Ataie-Ashtiani ${ }^{3}$; Craig T. Simmons ${ }^{4}$; Anis Younes $^{1}$

\author{
${ }^{1}$ LHYGES \\ ${ }^{2}$ ENGEES-LHYGES \\ ${ }^{3}$ Flinders University/ Sharif University \\ ${ }^{4}$ Flinders University
}

\section{Corresponding Author(s): fahs@unistra.fr}

\begin{abstract}
Seawater Intrusion (SWI) is a global hazard for coastal aquifers which can result in the salinization of freshwater resources and adversly influencing the lives of millions of people in coastal zones. Fractured coastal aquifers (FCAs) are widespread worldwide and especially in Mediterranean regions. In these aquifers, fracture networks represent the preferential pathways for fluid flow and transport in the subsurface that can intensify SWI. Therefore, understanding the hydrogeological processes associated with SWI in fractured coastal aquifers are significantly important for prevention of such threats. Numerical modelling can act as an initial step towards this understanding and it is widely employed for practical purposes as site investigation, safety report assessment and performing predictive computations. Modeling SWI in FCAs can be perofmred by coupling the discrete fracture network (DFN) and variable-density flow (VDF) models. The DFN-VDF model requires detailed discontinuous analysis of the fractures. In real field applications, these characteristics are usually uncertain which may have a major effect on the predictive capability of the model. Thus main goal of this work is to provide a preliminary assessment on how these uncertainties can affect the model outputs.

As our conceptual model, we consider fractured configurations of the Henry Problem which is widely used to understand SWI processes. A finite element DFN-VDF model is developed in the framework of COMSOL Multiphysics ${ }^{\circledR}$. We examine the uncertainty of several SWI metrics and salinity distribution due to the incomplete knowledge of fracture characteristics. Polynomial chaos expansion (PCE) is used as a surrogate model to reduce the computational burden. A new sparse PCE technique is used to allow for high polynomials orders at low computational cost. The Sobol' indices (SIs) are used as sensitivity measures to identify the key variables driving the model outputs uncertainties. The proposed methodology based on PCE and SIs is useful for identifying the source of uncertainties on the model outputs with an affordable computational cost and an acceptable accuracy. It shows that fracture hydraulic conductivity is the first source of uncertainty on the salinity distribution. The imperfect knowledge of fracture location and density affects mainly the toe position and the total flux of saltwater entering the aquifer. Marginal effects based on the PCE are used to understand the effects of fracture characteristics on SWI. The findings provide a technical support for monitoring, controlling and preventing SWI in FCAs.
\end{abstract}

Procter and Gamble Student poster award:

References: 


\title{
Fluid flow in a channel partially filled with thin ordered porous media: pore scale simulations vs volume averaging continuum modeling
}

\author{
Nima Fallahjoybari ${ }^{1}$; Staffan Lundström ${ }^{2}$; Majid Eshagh Nimvari ${ }^{3}$ \\ ${ }^{1}$ Lulea University of Technology \\ ${ }^{2}$ Fluid Mechanics, Luleå University of Technology, Sweden \\ ${ }^{3}$ Amol University of Special Modern Technologies \\ Corresponding Author(s): nima.jouybari@gmail.com
}

The present study deals with the investigation of the widely used volume averaged method accuracy in the case of fluid flow within a thin porous layer. For the direct simulations, a staggered arrangement of square cylinders with a thickness of one unit cell is considered as porous medium covering the area close to the walls of a channel. For the comparison, the cylinders are replaced with a thin continuum layer of a porous medium with prescribed permeability and porosity using the volume averaging approach. While a two-equation turbulence model is used to solve turbulent flow in direct simulation, the averaged method uses the modifies k-epsilon model to capture the effects of solid matrix on the production of turbulent kinetic energy and dissipation rate within porous matrix. The simulations are carried out for different Darcy numbers (Da), Reynolds numbers (Re) and channel widths. The results obtained from direct simulation are then averaged over the corresponding unit cell and compared with those calculated by the volume averaged method.

Procter and Gamble Student poster award:

References:

Acceptance of Terms and Conditions:

Click here to agree

75

\section{An upscaled transport model of shale gas considering multiple mechanisms and heterogeneity based on homogenization theory}

\author{
Weipeng Fan ${ }^{\text {None }} ;$ Hai Sun $^{\text {None }} ;$ Jun Yao $^{\text {None }}$ \\ Corresponding Author(s): fanweipeng2017@126.com, sunhaiupc@sina.com
}

Due to complex pore structure and gas storage, traditional Darcy permeability model is not valid to describe shale gas flow. In this paper, an upscaled transport model is established based on homogenization theory to consider multiple transport mechanisms and heterogeneity of shale sample. This new transport model is employed to explain the pressure decay experiment data successfully. Finally, the sensibility of transport parameters is analyzed. The results show as follows. Absorbed gas cannot be ignored in the pressure decay experiment and the pressure difference will drop rapidly without considering the absorbed gas. Then shale gas transport is dominated by Knudsen diffusion when the pore pressure is smaller than $2 \mathrm{MPa}$ and the contribution of surface diffusion increases with pore pressure increasing. The macroscopic effective permeability of whole domain is dominated by the permeability of connected phase meanwhile it can be influenced by the permeability of disconnected phase. When the pore pressure is smaller than $2 \mathrm{MPa}$ the effective permeability increases with the organic matter content (TOC) decreasing. However, the effective permeability increases 
with the TOC increasing when the pore pressure is greater than $2 \mathrm{MPa}$, which is different from the traditional discussions without considering the relationship between TOC and gas absorption capacity of shale matrix. The effective increases especially for high pore pressure with Langmuir volume increasing. In addition, the effective increases with Langmuir pressure decreasing, however, which becomes apparent when the pore pressure is smaller.

Procter and Gamble Student poster award:

I would like to compete in the Procter and Gamble Student award References:

Acceptance of Terms and Conditions:

Click here to agree

24

\title{
Size effects of molecular sub-region in a multiscale, multiphase process
}

\author{
Liu Fanli ${ }^{1}$; Wang Moran ${ }^{2}$ \\ ${ }^{1}$ Tsinghua University \\ ${ }^{2}$ Tsinghua Univerisity
}

Corresponding Author(s): liu-fl17@mails.tsinghua.edu.cn

The process of displacing tiny droplet in a microchannel by another fluid is ubiquitous in unconventional oil recovery, and is also closely related to the formation of thrombus in blood vessel. It is generally accepted that purely macroscopic approach is not suitable for such multiscale problem where the whole system is big with a tiny sub-region at molecular scale, because it is unable to resolve extremely small scales where continuum assumption may not hold. However, no clear evaluation has been made on the size effects of the sub-region, which determines when macroscopic approaches become invalid. This work investigates the effects of varying drop size on the droplet displacement process in microchannel in detail by comparing results of multiscale hybrid modeling and continuum method. The results indicate that the change of physical properties of droplet due to huge Laplace pressure has considerable effect once the drop diameter approaches 30 molecular diameters, corresponding to 10 nanometers in our study, but continuum method is applicable with macroscopic correction done beforehand. When the drop diameter decreases below 20 molecular diameters, the macroscopic correction gradually becomes invalid as well but continuum method is still usable with parameters obtained by upscaling from molecular dynamics. The continuum method totally breaks down for drop diameter in the order of several molecular diameters, where macroscopic descriptions may no longer apply.

Procter and Gamble Student poster award:

\section{References:}

Acceptance of Terms and Conditions:

Click here to agree

818

\section{Modified salinity water flooding: pore-scale simulations and mi- crofluidic experiments}

Author(s): Mohsen Farhadzadeh ${ }^{1}$

Co-author(s): Maria Bonto ${ }^{1}$; Ali A. Eftekhari ${ }^{1}$; Murat Nulati Yesibolati ${ }^{2}$; Kristian Mølhave ${ }^{2}$; Hamidreza M. Nick ${ }^{1}$ 


\footnotetext{
${ }^{1}$ The Danish Hydrocarbon Research and Technology Centre, Technical University of Denmark, Lyngby, Denmark

${ }^{2}$ Department of Micro- and Nanotechnology, Technical University of Denmark, Lyngby, Denmark
}

Corresponding Author(s): mohsen@dtu.dk

Modified salinity water flooding is an efficient oil recovery technique to unlock residual oil. While wettability alternation is considered as one of the main mechanisms behind the modified salinity effect, the factors that control the wettability variation have not been completely elucidated. This is due to the complex coupling between two-phase flow, species transport, and chemical reactions. Detailed understanding of oil, brine, and rock interaction in pore-scale is an essential step to pinpoint the mechanism of wettability alternation. Experimental investigations as well as numerical simulations will lead to a better understanding of these systems. Due to the spatial and temporal scales of EOR processes, we frequently encounter technical barriers to obtaining accurate measurements. Unlike conventional core flooding tests, microfluidic devices offer a means of observing interface displacement in immiscible two-phase flow. Hence, we designed and performed a set of two-phase flow simulations and experiments to provide better insights into microscopic sweep efficiency of two-phase reactive systems. To this end, a numerical model based on Direct Numerical Simulation of the Navier-Stokes equations 1 coupled with a calibrated surface complexation model 2 is implemented to describe interaction in the oil-brine-rock system. Besides, we used microfluidic chips integrated with a high-resolution microscope to perform multiphase interfacial tests. Thus, fluid configurations inside the microfluidic chip with length scales comparable to the pore sizes of oil reservoir rock were imaged and compared with numerical model results for different brines. The development of numerical models validated and compared by experimental microfluidic results are shown here to serve as a powerful complementary approach to gain in-depth insight into the fundamental of these complicated physico-chemical systems, hence better control over the processes of oil recovery.

\title{
Procter and Gamble Student poster award:
}

I would like to compete in the Procter and Gamble Student award References:

1 J. Maes, C. Soulaine, A new compressive scheme to simulate species transfer across fluid interfaces using the volume-of-fluid method. Chem, Engng Sci., 190 (2018), pp. 405-418.

2 A.A. Eftekhari, K. Thomsen, E.H. Stenby, H.M. Nick, Thermodynamic analysis of chalk-brine-oil interactions, Energy Fuels, 31 (2017), pp. 11773-11782. Acceptance of Terms and Conditions:

Click here to agree

\section{Towards Bayesian quantification of permeability in micro-scale porous structures - Sandstones and Carbonates}

\author{
Babak Fazel $^{1}$; Mohammad Hossein Golestan ${ }^{2}$ \\ ${ }^{1}$ Center for Exploration and Production Studies and Research, Research Institute of Petroleum Industry (RIPI) \\ ${ }^{2}$ Department of Petroleum Engineering and Applied Geophysics, Norwegian University of Science and Technology
}

\section{Corresponding Author(s): fazelb@ripi.ir}

This article develops a Bayesian framework to quantify the absolute permeability of water in a porous structure from the geometry and clustering parameters of its underlying pore-throat network. These parameters include the network's diameter, transivity, degree, centrality, assortativity, edge density, K-core decomposition, Kleinberg's hub centrality scores, Kleinberg's authority centrality scores, length, porosity, as well as the Nitrogen-accessible surface area of its porous structure. In addition, the incorporated clustering aspects of the networks have been determined with respect to several clustering criteria - edge betweenness, greedy optimization of modularity, multi-level optimization of modularity, and short random walks. As such, the article takes the first footsteps of creating a Database of Micro Networks for sandstones and carbonates, to be used as main input stream for the proposed Bayesian scheme. The Bayesian Network prediction of the absolute permeability exhibits a mean error value of less than $5 \%$, which is favorable given the associated subsurface uncertainties. 
Procter and Gamble Student poster award:

I would like to compete in the Procter and Gamble Student award References:

Acceptance of Terms and Conditions:

Click here to agree

\title{
Upscaling of reactive transport from pore-scale to core plug-scale in shale samples: the pore-scale part
}

\author{
hossein fazeli ${ }^{1}$; Bagus Muljadi ${ }^{2}$; Veerle Vandeginste ${ }^{3}$ \\ ${ }^{1}$ University of Oslo \\ ${ }^{2}$ Department of Chemical and Environmental Engineering, University of Nottingham, University Park, NG9 2RD, \\ Nottingham, UK \\ ${ }^{3}$ School of Chemistry, University of Nottingham, University Park, NG9 2RD, Nottingham, UK
}

Corresponding Author(s): hossein.fazeli@geo.uio.no

Once oxygenated, slightly acidic fracking fluid is injected into targeted shale units, fluid-rock interactions cause a change in porosity and permeability characteristics of shale rocks which consequently impact the flow and reactive transport processes. Large scale reactive transport models can help simulate these interactions and predict these behaviors. However, presence of micro-scale featuressuch as mineral heterogeneity-which strongly affect the reactive transport processes, cannot be implemented in large scale models and hence pore scale models are needed to take these micro-scale features into account. In other words, there is a need for a sophisticated upscaling approach that can capture micro-scale heterogeneity from pore scale. Our final aim is to have such an upscaling approach such as the one presented by Rhodes et al. (2008) where a Continuous Time Random Walk (CTRW)-based method was used for the upscaling. The upscaling method needs some input parameters from pore scale that can either be obtained from experiments, such as the nuclear magnetic resonance (NMR) experiments presented by Muljadi et al. (2018) to probe molecular displacements (propagators), or from pore scale simulations. In this work, we only focus on the pore scale part and use a 3D pore-scale reactive transport Lattice Boltzmann (LB) based model to simulate the interactions between a fracking fluid and a shale sample. The pore-scale LB model is able to track the geometry evolution of the shale rock triggered by dissolution (or precipitation) of minerals. Moreover, the pore-scale LB simulator is coupled with an external geochemical solver enabling us to include different mineral reactions. In the future work, we will use this pore scale model to provide inputs for the upscaling algorithm.

Procter and Gamble Student poster award:

I don't want to compete References:

Acceptance of Terms and Conditions:

Click here to agree

\section{Accelerated Rate Calorimeter Application for Heavy Oil Charac- terization}

Rita Fazlyeva ${ }^{\text {None }}$

Corresponding Author(s): rita.fazlyeva1@ucalgary.ca

Air injection has proven to be a high recovery potential method for both heavy and light types of oil. One of the screening techniques that allow to examine an oil-candidate for air injection 
process is Accelerated Rate Calorimeter (ARC). The unique feature of the instrument is to mimic a reservoir conditions in terms of pressure and temperature supporting an adiabacity. This paper reviews four tests performed in the closed and flowing ARCs to fingerprint and observe the thermal behavior of an oil. Investigated hydrocarbons characterized as heavy with the specific gravity of $13^{\circ} \mathrm{API}$ was undergone to the combustion reaction in two scenarios of oil-only and oil in native carbonate core. Initial running conditions of test are characterized by the temperature of $23^{\circ} \mathrm{C}$ and reservoir pressure of 2000 psig. Experiments showed that an ignition occurs at temperature about $350^{\circ} \mathrm{C}$ when High-Temperature Oxidation region is dominant. Calculation of kinetic parameters are also discussed in this work. Observation of the temperature change per gram of consumed oxygen that is a representation of the apparent heats of combustion versus inverse temperature is proposed as a novel approach of ARC. This technique also can be useful for understanding of occurred reactions during the combustion process.

Procter and Gamble Student poster award:

\title{
References:
}

Acceptance of Terms and Conditions:

Click here to agree

\section{Effect of the rigidity of a granular material on heat transfer}

\author{
Author(s): Wenbin $\mathrm{Fei}^{1}$ \\ Co-author(s): Guillermo Narsilio ${ }^{1}$; Joost van der Linden ${ }^{2}$; Mahdi Disfani ${ }^{1}$ \\ ${ }^{1}$ The University of Melbourne \\ ${ }^{2}$ The Unviersity of Melbourne
}

Corresponding Author(s): wenbin.fei@unimelb.edu.au

Coordination number is widely used to quantify the deformability of a granular material. However, it is a local feature and the use of average coordination number is not able to fully characterise the variation of the microstructure in the granular material. Instead, contact networks can be used to investigate the rich physical behaviour of granular assemblies, allowing for many other multi-scale structural features beyond coordination number to be accounted for, not only on the mechanical behaviour but also on a variety of other physics. In the contact network, particles are considered as nodes and edges are assigned to neighbouring nodes when the represented particles contact. At the particle scale, when considering 'granular rigidity', triangular-like structures tend to be rigid while square-like structures tend to be deformable. 'Cycles' (i.e., triangular or square like structures) in the contact network can indicate rigid or deformable structures. However, the effect of contact network features on heat transfer has not been studied yet. This work uses code developed in-house to extract contact network features such as 'cycles', 'clustering coefficients' and 'centralities'. We use a thermal network model to calculate effective thermal conductivity of a deforming granular material. In the thermal network model, each node represents a particle and each edge represents a thermal conductor (i.e., interparticle contact and/or small void gap through which heat can be also transferred between particles). Here we show that a change of structural features from the contact network can capture the variation of effective thermal conductivity of granular materials.

\section{Procter and Gamble Student poster award:}

I would like to compete in the Procter and Gamble Student award References:

\section{Acceptance of Terms and Conditions:}

Click here to agree 


\title{
a New Method to Predict the Performance in Gas Assisted Gravity Drainage Using Dimensionless Groups
}

\author{
Kewen FENG $^{1}$
}

${ }^{1}$ petrochina research institute of petroleum exploration \& development

Corresponding Author(s): fkw2580969576@outlook.com

\begin{abstract}
Many of core-flood experiments and field-scale applications have demonstrated that the Gas-Assisted Gravity Drainage (GAGD) process has gravity-stable oil displacement and thus enhances the oil recovery. One of the effective reservoir development plans based on this process is injecting gas into the gas cap through vertical wells and displace water and oil downward to the horizontal producers. The corresponding methods to predict the oil recovery of the GAGD process in the previous literature is limited in terms of universality and accuracy, because they attempt to consider either the capillary number or the Bond number independently. In addition, only one formula is attempted for all cases. In this paper, we propose a novel empirical dimensionless formula $(\mathrm{Nd})$ which takes all capillary number, Bond number, and viscosity ratio into consideration. Instead of previous dimensionless number given directly, we use mathematical approach and experimental data to regress three exponents so that the predictive method is improved in terms of both accuracy and universality. Last but not least, we coded this approach into an in-house software, of which the input is the oil viscosity, Darcy velocity, permeability, porosity etc. The software then calculates the expression of $\mathrm{Nd}$ and regression straight line of as well as oil recovery. This method has been compared with core-flood experiment and oil field data in immiscible GAGD process, and we observe a good alignment in this validation.
\end{abstract}

Keywords: Gas-Assisted Gravity Drainage; oil recovery

Procter and Gamble Student poster award:

I would like to compete in the Procter and Gamble Student award References:

Acceptance of Terms and Conditions:

Click here to agree

\section{Role of Surface Area Density of Porous Paper-like Materials on the Detection Performance of Lateral Flow Assay}

\author{
Shangsheng Feng ${ }^{1}$ \\ ${ }^{1} X i$ 'an fiaotong University, Xi'an, China \\ Corresponding Author(s): shangshengfeng@xjtu.edu.cn
}

As a simple, portable, cost-effective, and user-friendly diagnostic tool, paper-based detection platforms (e.g., paper diagnostics) have found widespread applications in diagnosis and monitoring of diseases, environment pollution, food safety, etc. A variety of paper materials have been used to fabricate the paper-based devices, among which two main kinds are widely used. One is cellulose fiber based materials, such as filter paper and chromatography paper, which constitute the major substrates of dipsticks and microfluidic paper-based analytical devices ( $\mu$ PADs). The other is nitrocellulose membranes (NC membranes), which are the most important materials for LFAs (lateral flow assays) as the decisive reactions take place in the NC membranes. The detection performance of LFAs is notably affected by the reactive fluid flow in the porous NC membranes.

The microstructures of NC membranes affect the capillary flow properties. The capillary flow properties in turn affect reagent deposition, assay sensitivity, assay specificity, and test line consistency. Surface area density is one of the most important geometrical parameters of porous materials which determines the chemical reaction or heat transfer surface area that a porous material can provide. In this study, relation between the detection performance of LFAs and the surface area density of NC membranes is revealed using combined experimental and theoretical approaches. First, experiments 
using NC membranes with different micro-structures commercially provided by MilliporeTM are conducted to compare their detection performance when used as the substrate materials in LFAs. Then a theoretical model coupling surface $\mathrm{Ag}-\mathrm{Ab}$ reaction with fluid flow is developed which allows one predict the surface area density effects on the detection performance. By comparing the experimental and theoretical results, the importance of surface area density on the detection performance is demonstrated. Based on the validated model, the trade-off effects between the surface area density and flow speed is analyzed.

Procter and Gamble Student poster award:

I would like to compete in the Procter and Gamble Student award References:

Acceptance of Terms and Conditions:

Click here to agree

160

\title{
Impact of creep on the permeability evolution of coal by the thermo- mechanical coupling
}

Zengchao Feng ${ }^{\text {None }}$

Corresponding Author(s): zc-feng@163.com

Coal permeability is the key factor determining the effectiveness of coal-bed methane (CBM) exploitation. In the CBM production cycle, the long-term high temperature and stress make the seepage paths in coal evolve with time. In this paper, the creep-seepage law of coal by temperature-stress coupling effect is studied, and the results show that the creep strain of coal undergoes three phases: the hardening phase before the creep start stress threshold, the creep phase with compressive volume deformation and the creep phase with expanding volume deformation. In the whole process, the permeability of coal gradually decreases and then increases with the creep strains and there is a good consistency between the permeability evolution and time-dependent volumetric strain. The creep of coal shows different effects on the coal permeability at different temperatures. Temperature increase is helpful for the occurrence of creep strain of coal. When temperature is increased, the creep start stress threshold and the ultimate failure strength of coal samples decrease. Meanwhile, the stress of coal at the permeability turning point decreases. At high temperature, the creep of coal shows a greater influence on the decrease of permeability, and the maximum loss of coal permeability can reach $35.45 \%$. The results can provide theoretical support for the effective exploitation of CBM.

Procter and Gamble Student poster award:

References:

Acceptance of Terms and Conditions:

Click here to agree

102

\section{Three-Dimensional Finite Deformation Measurements of Soft Porous Media using Lens Free Imaging}

\author{
Author(s): Eanna Fennell ${ }^{1}$ \\ Co-author(s): Tairan Liu ${ }^{2}$; Yibo Zhang ${ }^{3}$; Aydogan Ozcan ${ }^{3}$; Jacques Huyghe ${ }^{4}$ \\ ${ }^{1}$ Bio-materials Research, Bernal Institute, University of Limerick. \\ ${ }^{2}$ Bioengineering Department, University of California, Los Angeles, USA \\ ${ }^{3}$ Bioengineering Department, University of California, Los Angeles, USA.
}


${ }^{4}$ Biomaterials Research, Bernal Institute, University of Limerick, Ireland

Corresponding Author(s): eanna.fennell@ul.ie

A hydrogel is a network of cross-linked polymer chains. Industrially used hydrogels, known as superabsorbent polymers, have the ability to osmotically swell up to 100 times their original volume. The volume of solution absorbed by a gel is a function of several external stimuli, predominately the $\mathrm{pH}$, temperature and ionic strength of the solution. Specialist mixed hybrid finite element software has been developed to mimic the swelling of these gels under several external and internal parameters. However, experimental verification of this software is essential in demonstrating its efficacy. Standard imaging techniques such as confocal microscopy or particle tracking velocimetry have limitations. They lack the requirements of a system needed to image such a highly dynamic three dimensional process. Therefore, a novel lens free technique is utilized. This technique incorporates a CMOS image sensor and partially coherent illumination to create digital holograms of the specimen under investigation. Transparent micro-particles embedded within swelling polyacrylate gels are illuminated by two separate wavelengths at 45 degrees to each other. This multi-angle and multicolor illumination permits three-dimensional tracking of particles at 10 frames per second over an imaging volume of roughly $80 \mathrm{~mm} 3$. Digital post-processing algorithms are then implemented to dramatically increase the spatial resolution of the system achieving sub-micron accuracy. The spatial coordinates of the transparent micro-particles will enable verification of the numerical model with a particular emphasis on the constitutive and permeability laws.

Procter and Gamble Student poster award:

References:

Acceptance of Terms and Conditions:

Click here to agree

\title{
The Porous Microstructure Analysis (PuMA) software for high- temperature microscale modeling
}

Joseph C. Ferguson $^{1}$; Francesco Panerai ${ }^{2}$; John Thornton ${ }^{1}$; Federico Semeraro ${ }^{3}$; Arnaud Borner ${ }^{1}$; Nagi N. Mansour $^{4}$

\author{
${ }^{1}$ STC at NASA Ames Research Center \\ ${ }^{2}$ University of Illinois, Urbana-Champaign \\ ${ }^{3}$ UIUC at NASA Ames Research Center \\ ${ }^{4}$ NASA Ames Research Center
}

Corresponding Author(s): joseph.c.ferguson@nasa.gov

The Porous Microstructure Analysis (PuMA) software 1 is a collection of tools for the analysis of porous materials and generation of material microstructures. From 3D microstructural images, often obtained from X-ray microtomography 2, PuMA can compute a number of effective material properties and perform material response simulations, with an emphasis on high temperature applications. PuMA was designed to be a modular framework, such that users can implement custom solvers and tools, utilizing the existing capabilities in the software. Version 3.0 includes capabilities for computing volume fractions, porosity, specific surface area, effective thermal and electrical conductivities, and continuum and rarefied diffusive tortuosity factors. PuMA can also simulate competitive diffusion/reaction processes at the micro-scale, such as surface oxidation.

The effective thermal and electrical conductivity for isotropic materials, as well as continuum tortuosity, can be computed using a finite-volume solver with a variety of boundary conditions. For computing effective anisotropic thermal and electrical conduction, a numerical scheme

based on the Multipoint Flux-Approximation (MPFA) method [3] was implemented. The MPFA method enables the solution of solid conduction in materials with anisotropic local thermal conductivity, such as in woven materials. 
For material properties and response in non-continuum conditions, particle-based random walk solvers have been implemented into the PuMA software. The particle solver allows for the computation of mean intercept length (MIL), as well as tortuosity factors of a porous material in transitional and rarefied regimes, such as those found in a porous heat shield during atmospheric entry. A particle method was also implemented to solve the competing diffusion/reaction problem in porous media, which has been applied to simulate the oxidation of fibrous and woven materials at high temperatures. Finally, a particle-based solver for simulating molecular beam-surface scattering experiments [4] was implemented and parallelized within PuMA. The solver can simulate high speed gas transport and complex surface reaction mechanisms and is being used to develop finite-rate surface chemistry models.

Recently, advanced material generation capabilities have also been implemented, including the creation of complex 2D and 3D woven structures, curved fibers, multiple cross-sectional designs, and fiber clustering. PuMA is freely available to the scientific community as a NASA software under a US \& Foreign license.

\title{
Procter and Gamble Student poster award:
}

I don't want to compete References:

1 J. C. Ferguson, F. Panerai, A. Borner, N. N. Mansour, PuMA: the Porous Microstructure Analysis software, SoftwareX 7 (2018) 81-87.

2 F. Panerai, J. C. Ferguson, J. Lachaud, A. Martin, M. J. Gasch, N. N. Mansour, Micro-tomography based analysis of thermal conductivity, diffusivity and oxidation behavior of rigid and flexible fibrous insulators, International Journal of Heat and Mass Transfer 108 (2017) 801-811.

[3] I. Aavatsmark, An introduction to multipoint flux approximations for quadrilateral grids, Computational Geosciences 6 (3-4) (2002) 405-432.

[4] V. J. Murray, B. C. Marshall, P. J. Woodburn, T. K. Minton, Inelastic and reactive scattering dynamics of hyperthermal O and O2 on hot vitreous carbon surfaces, The Journal of Physical Chemistry C 119 (26) (2015) 14780-14796. Acceptance of Terms and Conditions:

Click here to agree

\section{Design of multilayer porous pavements for optimizing their evap- orative cooling potential as a strategy for urban heat island miti- gation}

\author{
Author(s): Andrea Ferrari ${ }^{1}$ \\ Co-author(s): Aytaç Kubilay ${ }^{1}$; Dominique Derome ${ }^{1}$; Jan Carmeliet ${ }^{2}$ \\ ${ }^{1}$ Swiss Federal Laboratories for Materials Science and Technology (EMPA) \\ ${ }^{2}$ Swiss Federal Institute of Technology Zurich (ETHZ)
}

Corresponding Author(s): andrea.ferrari@empa.ch

The urban heat island effect rises the temperature of cities, increases the building energy consumption and affects the thermal comfort adversely in urban environments. Pavements cover a very high fraction of the urban areas and highly con-tribute to the urban heat island development. A promising mitigation technique to counteract this phenomenon consists of replacing conventional pavements, typically made by impervious materials, with porous and water-retentive pavements. Due to evaporative cooling, a part of the net downward radiation can be convert-ed into latent heat, keeping the surface temperature lower compared to conventional pavements. As a result, the underground heat storage is reduced, as well as the sensible and thermal heat exchange between the ground surface and the atmosphere. These new pavements can provide effective mitigation solutions for urban heat island. The present study investigates the impact of porous pavements on the urban microclimate by means of a fully-integrated urban climate model, which couples turbulent wind flow in the air with heat and moisture transport in the porous building materials. The proposed approach yields detailed analysis of the drying behavior of different types of pavements under different meteorological conditions (wind, sun, rain). For the case study, three permeable pavements presenting different 
porosities and hydraulic properties are designed and combined in single- and multi-layer configurations. Their thermal performances and evaporative cooling potentials are investigated in comparison to standard pavement systems. Porous pavements exhibit a longer first drying phase compared to impervious surfaces, thus benefiting from the cooling due to evaporation for a longer duration and contributing to reduce air temperature and improve thermal comfort over urban areas.

Procter and Gamble Student poster award:

References:

Acceptance of Terms and Conditions:

Click here to agree

299

\title{
A Recursive Mixed Multiscale Method for Darcy problem
}

\author{
Paola Ferraz ${ }^{1}$; Eduardo Abreu ${ }^{2}$; Felipe Pereira ${ }^{3}$; Fabricio Sousa ${ }^{4}$; Het Mankad ${ }^{5}$ \\ ${ }^{1}$ University of Campinas (UNICAMP) \\ ${ }^{2}$ University of Campinas, Sao Paulo, Brazil \\ ${ }^{3}$ Mathematical Sciences Department, The University of Texas at Dallas, Richardson, TX, USA \\ ${ }^{4}$ University of Sao Paulo \\ ${ }^{5}$ University of Texas at Dallas
}

Corresponding Author(s): paola.ferraz@gmail.com

In this work, we develop a new efficient parallel solver for Darcy problem in heterogeneous porous media with high-contrast. This is a fundamental problem appearing in many relevant applications where uncertainty quantification (UQ) of complex modeling of processes and data in geophysical flows demand fast algorithms. The new recursive method is an improvement of the iterative multiscale mixed method MuMM introduced in 1 (see also 2 for its variational formulation), based on a non-overlapping domain decomposition iterative procedure with Robin interface conditions. The new method uses a clustering of the multiscale basis functions on each pair of subdomains leading to a local, and small, algebraic linear system for the interface between the subdomains. Cheap local multiscale basis functions are calculated in each subdomain to deal with the discrete local solutions and can be efficiently computed in CPU-GPU clusters. The global solution is obtained by recursively applying the MuMM over of all pairs of subdomains until the union of subdomains reaches the whole domain. The interface problem are solved by an efficient implementation of a Schur decomposition with a LU factorization. The new recursive algorithm bypass the need of iterations. Numerical experiments for Darcy problem with permeability field of the SPE10 benchmark geometry is presented to confirm efficiency of the procedure.

1 A. Francisco, V. Ginting, F. Pereira and J. Rigelo, (2014). "Design and implementation of a multiscale mixed method based on a nonoverlapping domain decomposition procedure", Math. Comput. Simul., 99, 125-138.

2 R.T. Guiraldello, R.F. Ausas, F.S. Sousa, F. Pereira and G.C. Buscaglia, (2018). "The Multiscale Robin Coupled Method for flows in porous media”, Journal of Computational Physics, 355, 1-21.

Procter and Gamble Student poster award:

References: 
1 A. Francisco, V. Ginting, F. Pereira and J. Rigelo, (2014). "Design and implementation of a multiscale mixed method based on a nonoverlapping domain decomposition procedure", Math. Comput. Simul., 99, 125-138.

2 R.T. Guiraldello, R.F. Ausas, F.S. Sousa, F. Pereira and G.C. Buscaglia, (2018). "The Multiscale Robin Coupled Method for flows in porous media”, Journal of Computational Physics, 355, 1-21. Acceptance of Terms and Conditions:

Click here to agree

\title{
ADVANCES IN LINEAR SOLVERS FOR COUPLED MULTI-PHYSICS PROBLEMS
}

Massimiliano Ferronato $^{1}$; Matteo Frigo $^{1}$; Nicola Castelletto ${ }^{\text {None }}$; Andrea Franceschini ${ }^{2}$; Hamdi Tchelepi $^{\text {None }}$

\author{
${ }^{1}$ University of Padova \\ ${ }^{2}$ Stanford University \\ Corresponding Author(s): ferronat@dmsa.unipd.it
}

The fully coupled numerical simulation of different physical processes in porous media, which can typically occur at variable time and space scales, is often a very challenging task. A common feature of such models is that their discretization gives rise to systems of linearized equations with an inherent block structure reflecting the properties of the set of governing PDEs. The efficient solution of the sequence of block linear systems is usually the most time- and memory-demanding issue in a coupled simulation. This effort can be carried out by using either staggered schemes, where easier sub-problems are solved separately iterating until coupled convergence is achieved, or monolithic approaches, which tackle the problem of the system solution as a whole.

This communication aims to discuss recent advances in the monolithic solution of coupled simulations in a specific class of multi-physics problems, namely poromechanics of fractured media. The problem is addressed either by proper sparse approximations of the Schur complements or special splittings that can partially uncouple the variables related to different physical processes. The selected approaches can be included in a more general preconditioning framework that can help accelerate the convergence of Krylov subspace solvers. The generalized preconditioner relies on approximately decoupling the different processes (e.g., momentum balance, contact mechanics, fluid flow dynamics, ...), so as to address each single-physics problem independently of the others. The objective is to provide an algebraic framework that can be employed as a general "black-box" tool and can be regarded as a common starting point to be later specialized for the particular multi-physics problem at hand. The use of this preconditioning framework for simulating real-world examples of poromechanical problems and fractured media will be demonstrated.

Acknowledgements. Portions of this work were performed under the auspices of the U.S. Department of Energy by Lawrence Livermore National Laboratory under Contract DE-AC52-07NA27344.

Procter and Gamble Student poster award

References:

Acceptance of Terms and Conditions:

Click here to agree

\section{Influence of yarn configuration on wicking processes studied by fast X-ray micro-tomography}




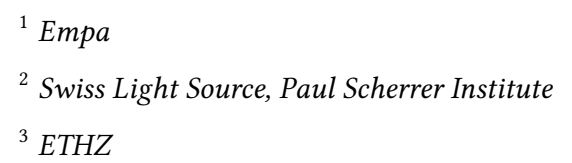

Corresponding Author(s): robert.fischer@empa.ch

Textiles show a large variability in materials, yarns and patterns and thus respond very differently to moisture uptake. A yarn is a twisted bundle of filaments used to produce textile by knitting or weaving. Liquid uptake into textiles happens mainly by capillary uptake in complex voids between yarn filaments. The role of yarn configuration, and particularly of yarn twisting on transport, is far from being documented. Yet, understanding liquid transport in yarns is key to optimize moisture management in garments. The goal of this study was to document in situ capillary water uptake in yarns of different pore configurations.

The documented yarns are composed of 10 or 32 circular continuous filaments made of polyethylene terephthalate (polyester), of $55 \mu \mathrm{m}$ diameter (3.3tex). The filaments were plasma coated for uniform contact angle $\left(50^{\circ}\right)$. Filaments were spun directly inside the sample holders at tension levels of 2.5 , 10 and $30 \mathrm{mN} /$ tex to twists of 50, 200 and 500 tpm using a twist-tester and fixed at this state. A reservoir is attached to the holder base.

Fast synchrotron X-ray micro-tomography is applied to document the influence of twist and tension on pore configuration of dry and wetting yarns. Remote-controlled injection of water on the holder base allows documenting water uptake driven by capillary forces over $5 \mathrm{~mm}$ of height of the yarn. A voxel size of $2.75 \mu \mathrm{m}$ with one full 3D-scan per second at high image quality allows the extraction of detailed pore geometry data and water detection almost per voxel. The Paganin phase filtered and reconstructed images undergo a multistep procedure to segment the different phases, i.e. air, polymer and water. We employ the Weka-plugin, ImageJ, a machine-learning-based algorithm. Voxels which are not belonging to fibers are examined separately in a following step as a time series of their intensity value and we track when the transition from air to water occurs (Parada, Derome et al. 2018) . Imaging is acquired at the Tomcat beamline of Swiss Light Source of Paul Scherrer Institute, Villigen, Switzerland.

Our experiments show that single uptake paths can conduct liquid undisturbedly and rapidly millimeters away from the inlet. We observe faster unsaturated flow in fillets at fiber contacts that can bridge sections between saturated pores. These stable water bodies in angular pores partially pre-wet uptake paths from multiple entry points. Once a vertically advancing piston-like flow front reaches such an unsaturated pore, the water bodies coalesce with formerly stable menisci leading to further transport in single pores. The abundance of the three filling processes depends on the yarn configuration, e.g. low-twist yarns contain more undisturbed large pores while high-twist yarns show shorter free paths and more angular pores.

Contrary to typical hydrophilic porous media, the sequential filling of pores does not correspond to an advancing moisture front. Due to the small number of pores per yarn cross section, single filling events have a large impact on the overall uptake process and we conclude that averaged uptake models, such as Washburn's or Darcy's, cannot describe moisture transport in polyester yarns.

Procter and Gamble Student poster award:

\section{References:}

Parada, M., et al. (2018). "Two stage wicking of yarns at fiber scale investigated by synchrotron X-ray phase contrast fast tomography." submitted to Textile Research Journal. Acceptance of Terms and Conditions:

Click here to agree 


\title{
Integrating publication, software and data in simulation sciences
}

Bernd Flemisch ${ }^{1}$

${ }^{1}$ University of Stuttgart

Corresponding Author(s): bernd.flemisch@iws.uni-stuttgart.de

While the process of publishing scientific findings in form of journal articles can be considered to be mature, this doesn't hold in general for the supplementary material associated with such an article. In porous-media modeling, this material may consist of the software that has been used to produce the presented results as well as data such as simulation results or experimental measurements used for model validation. Although methods and tools for developing and publishing research software as well as for managing research data already exist and are under continuous development, standardized workflows for their employment in porous-media modeling are still in their infancy. Especially the integration of the three components publication, software and data poses special challenges.

We present our efforts for making our publications transparent and reproducible by accompanying them with the associated software, data and metadata. Concerning the software, our aim is to provide for each publication a containerized environment storing the complete software stack that is necessary to run the corresponding simulations. Concerning the research data, we present the workflow that is being developed currently within the SFB 1313 and the SimTech Cluster of Excellence, together with the university library and the computing centers in Stuttgart.

Procter and Gamble Student poster award:

References:

Acceptance of Terms and Conditions:

\section{Flow in porous media of foams reinforced with polymers or par- ticles: application to subterranean oil pumping}

\author{
Author(s): Natacha Forey ${ }^{1}$ \\ Co-author(s): Olivier Atteia ${ }^{2}$; Abdelaziz Omari ${ }^{1}$; Henri Bertin ${ }^{1}$ \\ ${ }^{1} \mathrm{I} 2 \mathrm{M}$ \\ ${ }^{2}$ ENSEGID
}

Corresponding Author(s): natacha.forey@u-bordeaux.fr

\section{Introduction}

To prevent aquifer pollution due to industrial leaks, and remediate soil pollution, foams can be used as by blocking water beneath and by improving hydrocarbon oil pumping. However, when it comes in contact with oil, foam may collapse and thus has a very limited life time, well below the characteristic time of the process. Therefore, a suitable foam formulation that can resist oil contact is needed for application purpose.

In that respect, two main ways were investigated: use of polymer or colloidal solid particles as stabilizing agents. Polymer addition should, at least, increase foam stability by decreasing the film drainage velocity and colloidal particles may adsorb at the air-water interface and create a mechanical barrier towards oil induced foam destruction. Experiments are first performed in 1D sand column to study foams propagation in porous media, before injecting foam in a vertical metric $2 \mathrm{D}$-tank to observe gravity impact and foam behavior at the oil-foam contact. 


\section{Experiments:}

Beforehand, several surfactants and additives were screened by investigating foamability and stability in bulk in order to choose the best candidates. Experiments were then performed in columns of unconsolidated porous media, through a gas-water co-injection process. Final saturation and resistance factor were recorded and operating parameters were adjusted to obtain strong foams and the influence of surfactants and additives concentrations were of concern in absence as in presence of oil.

In a second part, a vertical 2D-tank of unconsolidated porous media was used to observe gravity influence on foam displacement. The front shape, total saturation and local resistance factors were continuously determined thanks to 12 pressure measurement taps. Experiments were then realized with an oil layer disposed at the top to observe foam resistance to the oil.

\section{Results:}

The results from sand columns bring to light the importance of foam quality, total flow rate and surfactant concentration on foam strength. Concerning foam strengthening with additives, polymer addition shows an increase in the resistance factor but such increase is only due to the induced increase of solution viscosity bringing out a loss of foam strength.

On the contrary, particle addition leads to foam strengthening both in absence and in presence of oil with an increase of the resistance factor (compared to the surfactant alone).

In 2D-tank experiments, stronger foams are seen to lead to a shift from a net $45^{\circ}$-sloping sweeping front to a vertical front. Pressure measurements show, as usual, a weak foam behavior at the entrance, followed by progressive foam strengthening along the porous media. Following experiments performed with setting an oil layer at the top obviously show foam degradation resulting in a modification of the front slope. This will be explained by proposing a phenomenological description of the sweeping process in that case.

Procter and Gamble Student poster award:

References:

Acceptance of Terms and Conditions:

Click here to agree

720

\section{Advanced Water Management in Fuel Cells Using Engineered Dif- fusion Layers with Patterned Wettability}

Antoni Forner-Cuenca $^{1}$; Victoria Manzi-Orezzoli ${ }^{2}$; Lorenz Gubler ${ }^{2}$; Thomas J. Schmidt ${ }^{1}$; Pierre Boillat $^{2}$

${ }^{1}$ Paul Scherrer Institute and ETH Zurich

${ }^{2}$ Paul Scherrer Institute

Corresponding Author(s): aforner@mit.edu

The water management in polymer electrolyte fuel cells is a rather complex issue 1. On one hand side, some water is needed in order to hydrate a proton conductive membrane and ionomer in the catalyst layers. Therefore, humidified gases are fed into the cell. On the other hand, excessive amounts of water in the porous layers lead to blockage of available pores for gas transport and consequently increased mass transfer limitations, especially at high current densities when large amounts of water are produced. The gas diffusion layers (GDLs) are highly porous carbon fiber materials which are sandwiched between electrodes and flow fields and provide several functionalities: conduction of electricity and heat, mechanical integrity of the membrane, distribution of reactant gases and 
removal of the liquid water produced. These functions imply a triple set of contradictory requirements: high diffusivity of the gas phase, high permeability of the water phase, and high thermal and electrical conductivity of the solid phase.

The creation of artificial pathways for water removal within the porous material enables the development of advanced water management strategies; however, none of the existing approaches proposed a method to produce such materials in a way compatible with mass productions. To tackle this issue, we invented a method to produce GDLs with patterned wettability based on electron radiation grafting 2. The method permits creating hydrophilic patterns throughout the complete material thickness while the remaining areas remain hydrophobic 2. In this talk, the synthetic method will be discussed fist [3]. Later, I will discuss the results of capillary pressure characterization obtained using microfluidic cells combined with neutron radiography and X-ray tomographic microscopy [4].

1 T. V. Nguyen et al., 1993 J. Electrochem. Soc. 140(8), 2178-2186.

2 A. Forner-Cuenca et al., Adv. Mater. 2015, 27, 6317-6322.

[3] A. Forner-Cuenca et al., J. Electrochem. Soc. 2016, 163 (8), F788-F801.

[4] A. Forner-Cuenca et al., J. Electrochem. Soc. 2016, 163 (9), F1038-F1048.

Procter and Gamble Student poster award:

References:

Acceptance of Terms and Conditions:

Click here to agree

454

\title{
Numerical modeling of thin porous media with LBM
}

\author{
Author(s): Tobias Forslund ${ }^{\text {None }}$ \\ Co-author(s): Sofia Larsson ; Staffan Lundström ${ }^{1}$ \\ ${ }^{1}$ Fluid Mechanics, Luleå University of Technology, Sweden
}

Corresponding Author(s): tobfor@ltu.se

The study of Thin Porous Media (TPM) have been gathering substantial interest lately 1. As noted in the article understanding flow in thin porous materials are of interest to many industrial processes including but not limited to fuel-cells, textiles and composite manufacturing. 2 defines three classes of TPM namely:

- VTPM - Very Thin Porous Media $\rightarrow(\delta » h)$

- PTPM - Proportionally Thin Porous Media $\rightarrow(\delta \approx \mathrm{h})$

- HTPM - Homogeneously Thin Porous Media $\rightarrow(\delta \ll h)$

Here $\mathrm{h}$ is the height of the cell and $\delta$ is the side length. The flow domains of interest include the stokes region, the laminar region, the transition region and the turbulent region. A numerical model that can take these domains and TPM classes into account should ideally be able to handle widely varying geometries and be stable over a wide range of Reynolds numbers. Lattice Boltzmann Methods (LBM) has proven to be effective for numerical modeling of porous media. Reasons for choosing LBM over traditional Finite Volume Methods (FVM) includes faster parallel computation allowing highly efficient implementation on massively parallel architectures (GPUs) and ease of varying the geometry.

In this study a model problem consisting of staggered and simple cubic arrays of cylinders are studied both numerically and experimentally. For the numerical modeling a Multiple Relaxation Time (MRT-LBM) D3Q27-Method is chosen so that high Reynolds-number flows are stable; for the turbulence modeling a subgrid-scale model is also utilized. The simulation results are validated against 
tomographic PIV measurement of a physical model. Results indicate satisfactory agreement between model and experiment.

References:

1 - Lilit Yeghiazarian, Krishna Pillai, and Rodrigo Rosati. "Thin Porous Media”. In: Transport in Porous Media 15.3 (2016), pp. 407-410. ISSN : 15731634. DOI : 10.1007/s11242-016-0793-9.

2 - John Fabricius et al. "Darcy's Law for Flow in a Periodic Thin Porous Medium Confined Between Two Parallel Plates”. In: Transport in Porous Media 115.3 (2016), pp. 473-493. ISSN : 15731634. DOI : 10.1007/s11242-016-0702-2.

\title{
Procter and Gamble Student poster award:
}

\section{References:}

1 - Lilit Yeghiazarian, Krishna Pillai, and Rodrigo Rosati. “Thin Porous Media”. In: Transport in Porous Media 15.3 (2016), pp. 407-410. ISSN : 15731634. DOI : 10.1007/s11242-016-0793-9.

2 - John Fabricius et al. "Darcy's Law for Flow in a Periodic Thin Porous Medium Confined Between Two Parallel Plates". In: Transport in Porous Media 115.3 (2016), pp. 473-493. ISSN : 15731634. DOI : 10.1007/s11242-016-0702-2. Acceptance of Terms and Conditions:

Click here to agree

\section{Cut, overlap and locate: 3D localization of highly overlapping tracer particles in defocal microscope images}

\author{
Simon Franchini ${ }^{1}$; Sam Krevor ${ }^{\text {None }}$ \\ ${ }^{1}$ Imperial College London \\ Corresponding Author(s): s.franchini15@imperial.ac.uk
}

Tracer particles are a powerful tool to reveal velocities of fluids in experiments. When the appropriate size and density of the tracer particles is chosen, the tracer particles follow directly the motion of the fluid. The carrying fluid can therefore be observed by tracking the tracer particles. High particle concentrations are desirable to provide a high measurement density in such experiments. In quasi $2 \mathrm{D}$ experiments, this can easily be achieved as all particle images come from one plane. In 3D experiments, the particle density is much lower to avoid overlapping of particle images when particles are in front of each other along the out-of-plane axis. Current techniques cannot reliably analyze these overlapping particle images. Instead, overlapping particle images are simply filtered out and ignored by conventional 3D particle tracking techniques.

We propose a new workflow which overcomes this limitation and allows for the localization of overlapping particle images. We first generate a semi-synthetic dataset of highly overlapping particle images and use this dataset to train a deep neural network in the detection and simultaneous 3D localization of highly overlapping tracer particles.

Generation of synthetic dataset

A large set of cropped particle images is generated and $\mathrm{x}, \mathrm{y}$ and $\mathrm{z}$ locations of particles are determined. A set number of these images are chosen and placed in random positions of the training images. It is ensured that a set fraction of these object images overlap with neighboring object images. The background of the training images is stochastically generated and simulates the noise as well as strongly defocused object images. The resulting training images have a defined number and degree of particle image overlapping, a stochastic image background and all particle images in the training images are $\mathrm{x}, \mathrm{y}$ and $\mathrm{z}$-annotated.

Training of Deep Neural Network for 3D object localization

For the localization of the particles in images, we adapted the model Tensorbox for our purpose. The original version of the model is able to locate highly overlapping objects within the image plane. We adjusted the network architecture to be able to locate objects not only in 2D, but also in 3D. The 
semi-synthetic dataset with its 3D annotated and overlapping particle images is used to train the model end-to-end. The accuracy of the system is then evaluated on the semi-synthetic dataset as well as on real videos of moving tracer particles.

\title{
Procter and Gamble Student poster award:
}

I would like to compete in the Procter and Gamble Student award References:

Acceptance of Terms and Conditions:

Click here to agree

\section{Bringing techniques from soil science to contaminated sites}

\author{
Juliana Freitas $^{1}$; Sheila Furquim ${ }^{1}$; Mirian Shinzato ${ }^{2}$; Miguel Cooper $^{3}$; Everton Oliveira $^{4}$; Mariana Favero ${ }^{1}$; Ellen \\ Leite $^{1}$; Fábio Minzon ${ }^{1}$; Thomás Bodelão ${ }^{5}$ \\ ${ }^{1}$ Universidade Federal de São Paulo \\ ${ }^{2}$ Federal University of São Paulo \\ ${ }^{3}$ Universidade de São Paulo \\ ${ }^{4}$ Hidroplan \\ ${ }^{5}$ Unesp
}

Corresponding Author(s): jugardenalli@gmail.com

Contaminants transport in soils is strongly affected by the soil physical properties. Similarly, the efficacy of in-situ remediation techniques is highly dependent on these properties, and, at the same time, can interfere and alter the soil physical properties. However, the techniques more frequently applied in soil science to study soil formation, its properties, classification and mapping, are not commonly used in studies of soil contamination and remediation. For example, the description and analysis of soil micromorphology is considered in less than $0.7 \%$ of the papers published about soil remediation. Techniques used for soil characterization at the pore scale are also not frequently used. For example, scanning electron microscopy (SEM) has been mentioned in less than $10 \%$ of papers that refer to soil remediation. In this study, we evaluated how techniques that are used in soil science can be applied for the study of contaminants transport and to assess the impact of in-situ remediation techniques.

One of the typical methods applied in soil sciences is the procedure for morphological soil description in the field, which includes the observation of several properties and the horizons identification. This type of description is rarely conducted in in-situ remediation studies. To evaluate if this characterization could be used to address the contaminants fate in the unsaturated zone, one controlled release of $27 \mathrm{~L}$ of gasoline with $20 \%$ ethanol was performed at field scale. The soil was characterized according to the guidelines for soil description established in soil sciences, and classified as an Oxisol, a typical tropical soil. Seven days after the release, the contaminants distribution was investigated. It was verified that the contaminants transport was strongly controlled by the horizons as characterized by the morphological description, accumulating in the interfaces between horizons.

The impact of remediation techniques in the soil physical properties was also investigated using techniques typical to soil science. To assess the impact of in-situ chemical oxidation using persulfate, column tests were conducted simulating a persulfate injection in a tropical soil. Micromorphological analysis of porosity were conducted before and after the oxidation using impregnated soil thin sections and blocks. Changes in poroids types were observed, mainly an increase of complex packing voids. The impacts of thermal remediation were also evaluated, using morphological soil description and SEM to observe the changes in the soil physical properties at the pore scale. A physical model was constructed using undisturbed soil samples and heated to temperatures up to $450^{\circ} \mathrm{C}$. The soil morphological description indicated significant changes in color and texture. The SEM analysis also indicated changes in texture, with a decrease in roughness and more clear lamellar structures.

These examples illustrate how methods from soil science can help construct a more complete understanding of the processes involved in contaminants transport and in-situ remediation. The number of papers about in-situ remediation that mention this type of techniques has increased significantly in the past years, indicating that more researchers are becoming aware of the need to include other disciplines techniques in the studies of contaminated soils. 
Procter and Gamble Student poster award:

References:

Acceptance of Terms and Conditions:

Click here to agree

\title{
Urease Immobilization for Advancing Enzyme-Induced Calcium Carbonate Precipitation Applications
}

\author{
Zachary Frieling $^{1}$; Robin Gerlach ${ }^{1}$; Adrienne Phillips ${ }^{1}$ \\ ${ }^{1}$ Montana State University \\ Corresponding Author(s): zachary.frieling@gmail.com
}

\begin{abstract}
Microbially induced calcium carbonate precipitation (MICP) is widely studied and exploits bacterial activity to form a calcium carbonate precipitate that has been used to modify porous media. MICP is an enzymatically driven process that uses the enzyme urease to change solution chemistry to favor calcium carbonate precipitation. However, in some application environments, temperature can stunt microbial growth and enzyme induced calcium carbonate precipitation (EICP) might be the preferred method to promote precipitation. Urease from plant-based sources can be used in lieu of microbial growth or microbially produced enzyme. The plant sourced urease can be applied in a similar fashion and this process is referred to as EICP. However, as the temperature increases the urease enzyme is thermally inactivated as it becomes denatured inhibiting calcium carbonate precipitation. This factor limits the potential use of EICP in higher (approximately $>50^{\circ} \mathrm{C}$ ) temperature environments. To combat this thermal inactivation and to extend the practical temperature range of EICP, immobilization of the urease enzyme through entrapment in silica gel and adsorption on an internally porous ceramic proppant was performed. The first order inactivation coefficient, kd, was determined for temperatures from $60^{\circ} \mathrm{C}-90^{\circ} \mathrm{C}$. It was found that immobilization of the urease enzyme drastically reduced the apparent $\mathrm{kd}$ when compared to the free, non-immobilized form indicating that the enzyme was protected by immobilization. Column experiments were performed using the urease immobilized on the ceramic proppant at room temperature $\left(\sim 23^{\circ} \mathrm{C}\right)$ and at $60^{\circ} \mathrm{C}$. It was found that the immobilized urease retained ureolytic activity for the duration of the experiments even when subjected to the elevated temperature condition. This indicated that the immobilized form of the urease enzyme was indeed protected from thermal degradation. It also appeared that the immobilized form of the urease enzyme was shielded from inactivation from the calcium carbonate precipitation process. In previous EICP and MICP experiments ureolytic activity decreased rapidly as calcium carbonate precipitated. This immobilized form of the urease enzyme shows promise for more economical EICP applications due to its prolonged activity at higher temperature and during calcium carbonate precipitation.
\end{abstract}

Procter and Gamble Student poster award:

I don't want to compete References:

Acceptance of Terms and Conditions:

Click here to agree

\section{Dynamics of paper fiber during liquid imbibition; capillary pull and swelling}

Author(s): Raoul Frijns ${ }^{1}$

Co-author(s): Joris Sprakel ; Jasper van der Gucht 
${ }^{1}$ Wageningen University

\section{Corresponding Author(s): raoul.frijns@wur.nl}

When studying the dynamics of porous imbibition processes, the focus is often on the dynamics of the fluid and the imbibition imbibition speed. However, in paper materials the solid is also dynamically active as the paper fibers swell with the imbibing liquid. This swelling can change imbibition parameters like the pore size and surface roughness. The dynamics of this swelling process is important to understand in order to understand its effects on industrial processes that use paper imbibition like inkjet printing.

Using the light scattering technique LSI (Laser Speckle Imaging), we measure the dynamical behaviour of the paper fibers throughout the imbibition process. A dynamical front, a short burst in fiber dynamics, is observed slightly preceding the actual water front. The intensity of this dynamical front is dependent on the local imbibition speed and the type of liquid. This dynamical front is caused by the reactionary capillary force of the liquid on the solid, locally pulling on the fibers that make up the capillaries that the liquid travels through.

After the bulk water front has passed, we observe a slow increase in dynamics. This is caused by the swelling of the paper fibers by the liquid. The LSI technique allows us to follow the whole swelling process and how it is influenced by different solvent properties.

Procter and Gamble Student poster award:

I don't want to compete References:

Acceptance of Terms and Conditions:

Click here to agree

\section{Impact of PTFE distribution on water transport in a gas diffusion layer of polymer electrolyte fuel cells}

Dieter Froning ${ }^{1}$; Junliang $\mathrm{Yu}^{1}$; Uwe Reimer $^{1}$; Werner Lehnert ${ }^{1}$

${ }^{1}$ Forschungszentrum fülich GmbH, Germany

Corresponding Author(s): d.froning@fz-juelich.de

In polymer electrolyte fuel cells (PEFC) the transport of liquid water is highly relevant for efficient operation of the stack. For this reason the liquid water transport in gas diffusion layers (GDL) and in channels is addressed by many researchers. The GDL/channel interface is characterized by $\mathrm{Yu}$ et al. [1,2], its coupling to channel simulations is shown by Andersson et al. [3]. In that work the GDL fibers were assumed being hydrophobic by covering them with polytetrafluorethylene (PTFE) completely. On the other hand it is known that the PTFE distribution on the outer surfaces and inside the GDL is not homogeneous [4].

In this work the impact of PTFE distribution inside the GDL on transport properties will be discussed.

The water transport simulations in the GDLs are performed in micro structures created by a stochastic geometry model 5. These geometries are transferred to the lattice Boltzmann (LB) simulations via a series of binary images. Since the local hydrophilic / hydrophobic properties of the micro structure have a strong impact on two-phase flow behaviour inside the GDL, a distribution of the hydrophobing agent PTFE on the fiber surface is taken into account. A certain range around random positions within the GDL can be hydrophobized until a total amount of the GDL surface is covered. This algorithm can be applied to the whole GDL or to particular sub-regions of it resulting in local different contact angles of water on the solid fibers.

The water transport in a hydrophobized GDL is simulated for several total amounts of PTFE. The studies are applied for homogeneous and also for inhomogeneous PTFE distribution. The amount of PTFE and its homogeneity of distribution can cause systematic impact on the liquid water transport. 
However; the distribution of a given total amount of PTFE lower than $100 \%$ leads to statistical spread when several realizations of PTFE distribution are applied to a fixed geometry.

The impact of the pore-scale distribution of PTFE on macrospcopic flow behaviour is discussed.

Part of this work was funded by the Chinese Scholarship Council (CSC), grant 201408080011. Transport simulations are running on hardware of the Jülich Supercomputing Centre, grant JIEK30.

Procter and Gamble Student poster award:

References:

Acceptance of Terms and Conditions:

Click here to agree

421

\section{Gas transport in PEFC gas diffusion layers and an analysis of sur- face characteristics}

Dieter Froning ${ }^{1}$; Junliang $\mathrm{Yu}^{1}$; Uwe Reimer ${ }^{1}$; Werner Lehnert ${ }^{1}$

${ }^{1}$ Forschungszentrum fülich GmbH, Germany

Corresponding Author(s): d.froning@fz-juelich.de

In polymer electrolyte fuel cells (PEFCs), including the direct methanol (DMFC) and high temperature (HT-PEFC) variants, the gas diffusion layer (GDL), with its geometric structure on the mesoscale, connects the electrodes with the feeding channels of the bipolar plate on the cell and stack scales. Efficient fuel cell operation requires that the electrodes be sufficiently supplied by fluid fuels from the channels. Furthermore, reaction products must be transported away from the electrodes. The GDL must also provide electric contact to the bipolar plates, but its major task is the mass transport of these fluids. The GDL is typically composed of materials based on carbon fibers, e.g., paper, woven and non-woven textiles.

The structure of non-woven and paper-type GDLs in real fuel cells was analyzed by x-ray synchrotron under different local compressions 1. The gas transport is simulated in compressed and uncompressed microstructures in through-plane and in-plane directions of the GDL, both in real structures and stochastic equivalent geometries. In order to support multi-scale simulation, effective properties can be calculated from meso-scale simulation results to provide model parameters for homogenized approaches in cell-scale simulations. Furthermore, the resulting gas flow is analyzed with statistical methods that were introduced in a previous study 2 . This approach provides the opportunity to detect quantitative relationships between functionality and microstructure and to design virtual GDL materials with improved transport properties [3]. The evaluation with stochastic methods provides substantiated properties that are suitable for connecting the meso-scale to the other spatial scales.

The analysis of simulated through-plane gas flow at the exit layer of the GDL can identify the regions where the gas is entering the adjacent one. For instance, $70 \%$ of the volume flow is leaving the GDL at less than $30 \%$ of the surface area. For transport simulations in smaller micro structures, e.g., catalyst layers, the results from the above may affect a significant part of the simulation domain [4].

Part of this work was funded by the Chinese Scholarship Council (CSC), grant 201408080011. Transport simulations were run on the hardware of the Jülich Supercomputing Centre, grant JIEK30.

References:

1 C. Tötzke et al., Journal of Power Sources 253 (2014) 123-131.

2 D. Froning et al., Transport in Porous Media 103 (2014) 469-495.

[3] D. Froning et al., Journal of Power Sources 318 (2016) 26-34.

[4] D. Froning et al., Transport in Porous Media 123 (2018) 403-420.

Procter and Gamble Student poster award: 
References:

Acceptance of Terms and Conditions:

Click here to agree

580

\title{
Reverse Imbibitions Damages during Gas-Water Two-Phase Flow in Tight Sand Gas Reservoir: An Experimental Based Case Study
}

\author{
Jingang $\mathrm{Fu}^{1}$; Yuliang $\mathrm{Su}^{1}$ \\ ${ }^{1}$ China University of Petroleum (East China)
}

Corresponding Author(s): fujingang86@sina.com

Reverse imbibitions damages are occurred due to fluid accumulation in horizontal well during the advance stage of depletion of gas reservoir and also during the practicing of hydraulic fracturing. This affects gas well productivity and economics of development of low-permeability gas field. In this research, degree of reverse imbibitions damage for parameters calculation, dynamic analysis and numerical simulation in tight sand gas reservoir have been studied thoroughly on the basis of laboratory experiments and as well as numerical simulation.

In this vast research, several full diameter cores are selected from Daniudi block of Ordos Basin, China. Reverse imbibitions damage experiment is carried out by pressure imbibitions method with the sets of different pressure and time under high pressure and temperature. Imbibitions rate can be obtained in matrix and fracture system with injection pump and computer system. Gas-water relative permeability was also tested through unsteady experiment in matrix and fracture cores to analyze features of reverse imbibitions damage in different reverse imbibitions conditions. The MRI techniques allowed us to observe and measure process of the fluid into the reservoir formation and the gas-water distribution in pores/fractures in sandstone core.

The results showed that imbibitions rate slowed down with time and pressure has positive effect on imbibitions rate. The high pressure difference between reservoir and wellbore is the key factor of reverse imbibitions damage. MRI shows that the fluid fills fractures first and then enters the matrix system and the scope of reverse imbibitions becomes larger as time passes. In addition, the injecting side tends to absorb more fluid and hence increases the apparent water saturation. The gas permeability test results of the core after reverse imbibitions shows that there is a relation between reverse imbibitions volume, time and gas permeability. The reduced mobility of gas is a major consequence of the water saturation increased during reverse imbibitions process. The process of water saturation changing and water migration can also be observed by MRI techniques.

Keywords: Gas-Water relative permeability, MRI techniques, Reverse imbibitions, Tight gas reservoir, Water saturation

Procter and Gamble Student poster award:

I would like to compete in the Procter and Gamble Student award References:

Acceptance of Terms and Conditions:

Click here to agree

579

\section{An improved model to predict irreducible water saturation in tight gas reservoir}

Jingang $\mathrm{Fu}^{\text {None }}$; Yuliang $\mathrm{Su}^{1}$ 
${ }^{1}$ China University of Petroleum (East China)

Corresponding Author(s): fujingang86@sina.com

The irreducible water saturation (Swir) is a vital parameter for permeability prediction and initial hydrocarbon reserves estimation. However, the complex pore structure of the rocks and multiple factors of formation condition makes the parameter difficult to be accurately calculated from both laboratory and conventional well logging methods in tight gas reservoir.

In this study, an improved model to predict Swir is derived based on the capillary tubes model with the fractal theory, which considers different combined forms of immobile water and stress dependence effect of the capillary tube. Some special immobile water called the lost kinematic water (LKW) is considered in this model and it is remaining in small pores due to flow around effect and complex pore structure. The embedded cone on capillary tubes wall is used to simulate volume of LKW. The model also was considered that during the depressurization development of actual tight sandstone gas reservoir, the change of stress field can induce the radius of capillary tubes, thickness of immobile water film and volume of LKW, which resulted in change irreducible water saturation in the reservoirs.

The validity of the new model is tested and an ideal consistency between predicted results and experimental data is found. It is proved that the new model is applicable for accurate calculation of the irreducible water saturation in tight sandstone gas reservoir. It reveals that Swir is controlled by the critical capillary radius (maximum displacement pressure), LKW coefficient, effective stress and formation temperature. The results show that at a certain temperature, the smaller the critical capillary radius is, the lower the irreducible water saturation is. When the critical capillary radius is constant, the higher the formation temperature is, the lower the irreducible water saturation is When the porosity is constant, the higher LKW coefficient is, the higher the irreducible water saturation is. The irreducible water saturation would increase with effective stress if other parameters are constant.

Keywords: Fractal theory, Stress dependence effect, Capillary tubes model, Irreducible water saturation, Tight sandstone

Procter and Gamble Student poster award:

I would like to compete in the Procter and Gamble Student award References:

Acceptance of Terms and Conditions:

Click here to agree

\title{
Modeling of pore throat distribution and capillary effect in syn- thesized artificial core
}

\author{
Joseph $\mathrm{Fu}^{1}$; Xiang'an Yue ${ }^{1}$; Bo Zhang ${ }^{1}$ \\ ${ }^{1}$ State Key Laboratory of Petroleum Resources and Prospecting, Key Laboratory of Petroleum Engineering Ministry \\ of Education, China University of Petroleum, Beijing 102249, China
}

Corresponding Author(s): josephfu100@yahoo.com

In petroleum engineering, application of enhanced oil recovery (EOR) often involves coreflood testing using reservoir core with live fluids to get a more realistic picture of possible recovery enhancement using a certain EOR techniques. These types of testing often requires the use of natural reservoir core for which there are issues such as poor repeatability of petrophysical properties, fixed petrophysical properties that can't be tailored to suit the research need, complex mineralogy, small size and quantity, and localized nature of the core with its regional characteristics making it hard to model the macroscopic heterogeneity of the true reservoir. Artificial core is an invaluable tool for investigation into the physics of multiphase flow in porous media for the enabling of contrast and repeat experiments to examine the influence of individual petrophysical factor under specified physical conditions as well as tailoring of macroscopic material properties according to the research 
needs. Following our previous work in which we demonstrated how to model the primary petrophysical property of particle size distribution and degree of cementation to thus model the secondarily derived properties of porosity and permeability in an artificial core, in this work, we continued in our modeling effort to mimic the pore throat distribution in order to ultimately model the capillary pressure effects of the target core in the synthesized artificial core. To start, we recognized the pore scale capillary effect is a amalgamated summation of key petrophysical factors such as particle size distribution, cementation, pore throat distribution, wettability, and mineralization. Due to the realistic constraints of difficulty in obtaining sources of various sands of sufficiently pure mineralogy, we limited the scope of this effort to of a medium wet, pure quartz artificial core model to model that of a target natural core of medium wettability. In so doing, the modeling of the capillary effect in artificial core shown in this work is simplified to just the result of two primary factors: the particle size distribution and cementation. With the role of the wettability being fixed to medium wet, the pore throat distribution of the artificial core is then controlled through use of different sieving fractions of finer sands that are small relative to the pore scale as well as compression pressure. By manipulating these two parameters, the capillary effect and the pore throat distribution of the target core as is represented by the mercury injection capillary pressure test with its two main parameters of entry pressure and irreducible water saturation is shown to be matched to by the synthesized artificial core.

Procter and Gamble Student poster award:

\title{
References:
}

Acceptance of Terms and Conditions:

Click here to agree

\section{Quantifying Transport Uncertainty in Two-Phase Flow Using Prob- ability Distribution Method}

\author{
Olga Fuks $^{1}$; Daniel W. Meyer ${ }^{2}$; Hamdi Tchelepi ${ }^{1}$ \\ 1 Stanford University \\ ${ }^{2}$ ETH Zurich
}

Corresponding Author(s): ofuks@stanford.edu

In the applications dealing with the two-phase flow in the heterogeneous subsurface, uncertainties of the phase saturations arise due to the substantial geological uncertainties and need to be quantified in order to enable the management decisions. This task is complicated by the high computational costs typically associated with the accurate numerical solution of the underlying hyperbolic transport problem. In this work, we apply for the quantification of transport uncertainties a probability distribution method that is well suited for the highly heterogeneous porous media. This method relies on a streamline-based description and thus, does not suffer from the numerical diffusion. First, we show an excellent performance of the method under the fixed streamline assumption in the challenging channelized porous medium. Next, we propose a method to account for the mobility changes in the more complex setting where the streamline fluxes change with time. This aspect is particularly important in the porous systems with high phase viscosity differences. We demonstrate the capabilities of the method by accurately quantifying saturation uncertainties in the channelized systems and log-Gaussian permeability fields for different phase viscosity ratios.

\section{Procter and Gamble Student poster award:}

I don't want to compete References:

O. Fuks, F. Ibrahima, P. Tomin, H. A. Tchelepi., Analysis of Travel Time Distributions for Uncertainty Propagation in Channelized Porous Systems, Transport in Porous Media, 2018.

Ibrahima, F., Tchelepi, H. A. and Meyer, D. W. (2018), An efficient distribution method for nonlinear two-phase flow in highly heterogeneous multidimensional stochastic porous media, Computational Geosciences 22(1), 389-412. Acceptance of Terms and Conditions: 


\title{
Homogenization of reactive transport processes through thin het- erogeneous layers
}

\author{
Author(s): Markus Gahn ${ }^{1}$ \\ Co-author(s): Maria Neuss-Radu ${ }^{2}$; Peter Knabner ${ }^{2}$ \\ ${ }^{1}$ University of Heidelberg, Center for Modelling and Simulation in the Biosciences - Interdisciplinary Center for Sci- \\ entific Computing \\ ${ }^{2}$ Friedrich-Alexander-Universität Erlangen-Nürnberg, Chair of Applied Mathematics I
}

Corresponding Author(s): markus.gahn@iwr.uni-heidelberg.de

We deal with the mathematical modeling and analysis of nonlinear reactive transport processes through thin heterogeneous layers. The aim is the rigorous derivation of homogenized models, where the thin layer is replaced by a lower dimensional interface, and the transport processes through the membrane are described by effective interface transmission conditions. Our models are particularly motivated by biological applications, as transport processes through biological membranes. A crucial example are epithelial layers occurring for example in the wall of blood vessels or the gastrointestinal wall.

We start from a microscopic model depending on a small scaling parameter $\epsilon$. This model describes all the chemical and physical processes on the microscopic scale. We consider a system of nonlinear partial differential equations for reactive transport in a domain consisting of two bulk regions separated by a thin layer $\Omega_{\epsilon}^{M}$ with a periodic heterogeneous structure. The thickness of the layer and the period within the layer are of order $\epsilon$. Additionally, the equations within the layer depend on the scaling parameter $\epsilon$. Across the interface between the bulk-domains and the thin layer, we consider two types of transmission conditions: Continuous transmission conditions and nonlinear Neumann-transmission conditions.

The aim is the derivation of a homogenized model for $\epsilon \rightarrow 0$, the solution of which approximates the solution of the microscopic model. In the singular limit for $\epsilon \rightarrow 0$, the thin layer reduces to an interface $\Sigma$ separating the two bulk-regions. The limit equations in the bulk-regions carry the same structure as in the microscopic model. However, the crucial point is the derivation of the effective transmission conditions across the interface $\Sigma$. In fact, one has to deal with the coupling between the bulk-domains and the thin layer, the heterogeneous structure within the thin layer and the periodic coefficients in the microscopic equations, the singular limit, when the thin layer reduces to an interface, and the nonlinear reaction-kinetics. To overcome these difficulties, we use the method of two-scale convergence and the unfolding operator for thin domains.

Procter and Gamble Student poster award:

References:

Acceptance of Terms and Conditions:

Click here to agree

\section{Thermodynamic Evidence of Structural Transition of ZIF-8 upon Methane Adsorption}

Dinuka H. Gallaba ${ }^{1}$; Aldo Migone ${ }^{1}$ 
${ }^{1}$ Department of Physics, Southern Illinois University Carbondale USA

Corresponding Author(s): dinuka4@siu.edu

In this study we report methane adsorption on Zeolitic Imidazolate framework-8 (ZIF-8) which is one of the porous MOF that has zeolite-like structure with higher thermal and chemical stability. Structural studies at room temperature and high pressures, found that at $1.47 \mathrm{GPa}$ ZIF- 8 undergoes a structural phase transition. What occurs is that the pressure-transmitting fluid is forced into the ZIF8 resulting in a reorientation (gate-opening) of the imidazolate windows in the ZIF- 8 that increases their size. The larger windows allow more fluid to get into the ZIF-8; this additional fluid produces an expansion of the ZIF-8. This is an unusual behavior for a sorbent. This structural transition was also observed at cryogenic temperatures with different adsorbates. We have previously observed this phenomena with $\mathrm{N} 2, \mathrm{CO}, \mathrm{O} 2$ and Xe adsorption. We will present the results of low temperature adsorption isotherms of methane on ZIF-8 ranging from $85 \mathrm{~K}$ to $107 \mathrm{~K}$. We have observed some influences of the structural transition in adsorption isotherms. In addition to presenting results for the isotherm characteristics as a function of temperature we will present the loading dependence of the isosteric heat of adsorption for $\mathrm{CH} 4$ in ZIF-8. We have also explored the kinetics of adsorption for methane in ZIF-8 as a function of loading. Results on the loading dependence of the equilibration time for a fixed temperature will be presented.We will compare our results for methane with those that others and us have obtained for the sorption of other gases in ZIF-8.

Procter and Gamble Student poster award:

I would like to compete in the Procter and Gamble Student award References:

W. Zhou, H. Wui, M.R. Hartman and T. Yildirim "Hydrogen and Methane Adsorption in Metal-Organic Frameworks: A High-Pressure Volumetric Study”, J. Phys. Chem. C 111, 16131-16137 (2007)

D. Fairen-Jimenez, R. Gavelis, A. Torrisi, A.D. Gellan, M.T. Wharmby, P.A. Wright, C. Mellot-Draznieks, T. Duren, "Flexibility and swing effect on the adsorption of energy-related gases on ZIF-8: combined experimental and simulation study” Dalton Trans. 41, 10752-10762 (2012)

Gallaba, D.H., Villarroel-Rocha, J., Sapag, K. and Migone, A.D, Microporous and Mesoporous Materials 265 (2018): 227-233. Acceptance of Terms and Conditions:

Click here to agree

\title{
Nanoimbibition in Artificial Opals -a Photonic Approach for Nanoporous Media
}

\author{
Author(s): Francisco Gallego-Gómez ${ }^{1}$ \\ Co-author(s): Cefe López ${ }^{1}$ \\ ${ }^{1}$ CSIC, Spain
}

Corresponding Author(s): francisco.gallego@icmm.csic.es

The so-called artificial opals are colloidal crystal systems made of submicron spheres, exhibiting a photonic bandgap with application in fields such as photonics, light harvesting or sensing. As porous materials, opals are prone to adsorb and confine fluids, affecting correspondingly the photonic response. Latest advances have allowed accurate transduction of the photonic performance in order to investigate the liquid-solid interplay in the nanoporous opal network [1-3]. More generally, our approach enables to use the opal as a photonic lab-on-a-chip to study and model fluid phenomena in porous microsystems [4-5]. Thereby, in situ monitoring of the photonic bandgap by simple spectroscopy suffices to gain insight about vapor adsorption and condensation [6, 7], dew formation [8], pore characterization $[9,10]$, wetting or confined flow. Beside practical benefits such as the versatile and easy manufacturing and post-fabrication treatments, artificial opals offer a fundamental, intrinsic advantage: the photonic nature of the output (the photonic bandgap) ensures high sensitivity and immediate response. 
In this talk I will show the most recent results exploiting the potential of opals for fundamental investigation in nanoporous media. In particular, imbibition of the nanoporous void network of opals (referred to as nanoimbibition) is studied in detail. A number of experimental configurations (immersion, lateral liquid injection), geometries (direct and inverse opals), porous materials (silica and polymer spheres) and imbibing liquids (water, nonpolar solvents) are considered. The spectral analysis of the photonic response of the opals revealed the main features of the nanoimbibition process and their correlation with factors such as the wetting properties, pore size and homogeneity, etc. As an additional advantage, the vanishing of the photonic bandgap in the imbibed parts of the opal (due to refractive index matching) allowed direct imaging of the imbibition front across the sample at the micrometer scale. In view of our results, macroscopic theories are tested and the appearance of new aspects regarding the filling and drainage of 10-100 nm pores is discussed.

\section{Procter and Gamble Student poster award:}

\section{References:}

1 Gallego-Gómez, F.; Blanco, A.; Canalejas-Tejero, V.; López, C. Small 2011, 7, 1838-1845.

2 Gallego-Gómez, F.; Blanco, A.; Golmayo, D; López, C. Adv. Funct. Mater. 2011, 21, 4109-4119.

[3] Gallego-Gómez, F.; Blanco, A.; López, C. J. Phys. Chem. C 2012, 116, 18222-18229.

[4] Gallego-Gómez, F.; Blanco, A.; López, C. Adv. Mater. 2015, 27, 2686-2714.

5 Gallego-Gómez, F.; Morales-Flórez, V.; Morales, M.; Blanco, A.; López, C. Adv. Colloid Interface Sci. 2016, 234, 142-160.

[6] Gallego-Gómez, F.; Blanco, A.; López, C. Adv. Mater. 2012, 24, 6204-6209.

[7] Gallego-Gómez, F.; Morales, M.; Blanco, A.; López, C. Adv. Mater. Technol. 2018, 1800493, 1-10.

[8] Gallego-Gómez, F.; Morales, M.; Blanco, A.; López, C. Adv. Funct. Mater. 2018, 28, 1800591, 1-9.

[9] Gil-Herrera L.K., Pariente J.A., Gallego-Gómez F., Gándara, F. Juárez B.H., Blanco A., López C. Adv. Funct. Mater. 2017, 1703885,1-9.

[10] J. Farrando-Pérez, C. López, J. Silvestre-Albero, F. Gallego-Gómez, J. Phys. Chem. C 2018, 122, 22008-22017. Acceptance of Terms and Conditions:

Click here to agree

\section{(Nano)pore scale modeling: can we learn from molecular simula- tions?}

Guillaume Galliero $^{1}$; Hai Hoang ${ }^{2}$; Dominique Legendre ${ }^{3}$

${ }^{1}$ LFCR, Université de Pau et des Pays de l'Adour, France

${ }^{2}$ Duy Tan University, Vietnam

${ }^{3}$ IMFT, Université de Toulouse, France

Corresponding Author(s): guillaume.galliero@univ-pau.fr

Molecular simulations have proven to be useful in the context of (nano)porous media, both to generate quasi-experimental data and to improve the physical modeling. In this presentation, we will focus on some fundamental and applied examples on what has been done in our group with these numerical tools to better understand and quantify what is occurring at interfaces and in low permeability media. These examples will concern slip at fluid-solid and fluid-fluid interfaces, moving contact line, modification of effective transport properties under confinement, multicomponent transport ... and will illustrate the possibilities, and limitations, of the use of molecular simulations to deal with pore scale modeling and how this information could be introduced into macroscopic simulations.

\section{Procter and Gamble Student poster award:}

\section{References:}

Acceptance of Terms and Conditions:

Click here to agree 


\title{
Pressure inside a nano-porous medium. The case of a single phase fluid.
}

Olav Galteland ${ }^{\text {None }}$; Dick Bedeaux ${ }^{1}$; Signe Kjelstrup ${ }^{2}$; Bjørn Hafskjold ${ }^{3}$

\author{
${ }^{1}$ Norwegian University of Science and Technology - NTNU \\ ${ }^{2}$ Norwegian University of Science and Technology, NTNU, Trondheim \\ ${ }^{3}$ Norwegian University of Science and Technology
}

Corresponding Author(s): olav.galteland@ntnu.no

Pressure is an essential property for transport processes in porous media, however there is no consensus on how to describe the pressure of a fluid in a nano-porous medium. We have developed a description of the pressure via the additivity of the grand potential using thermodynamics of small systems, described by Hill 1. We find that the pressure is a weighted sum of the pressures in each phase minus the surface tension of each surface. Using non-equilibrium thermodynamics we use this expression to describe the coupled transport processes in porous media [2,3]. We test the viability and validity of the pressure expression using molecular dynamics simulations by studying two idealized models for nano-porous media, a single spherical grain and a lattice of spherical grains.

We find that the application of Hill's thermodynamics of small systems is essential to describe pressure in a nano-porous medium, as it is needed to reproduce Young-Laplace's law of pressure differences across curved interfaces. We present data for how the phase pressures and surface tensions depend on each other, and we show how this can be used in the understanding of transport processes in nano-porous medium. We also show how the pressure in nano-porous media is connected to the pressure on macro scale.

Acknowledgement

The calculation power was granted by The Norwegian Metacenter of Computational Science (NOTUR). Thanks to the Research Council of Norway through its Centres of Excellence funding scheme, project number 262644, PoreLab.

\section{Procter and Gamble Student poster award:}

I would like to compete in the Procter and Gamble Student award References:

1 Terrell L. Hill, “Thermodynamics of Small Systems," New York: Dover, 1964.

2 S. Kjelstrup, D. Bedeaux, A. Hansen, B. Hafskjold, and O. Galteland, "Nonisothermal transport of immiscible fluids in porous media. I. The entropy production," Fontiers in Physics, accepted, 2018.

[3] S. Kjelstrup, D. Bedeaux, A. Hansen, B. Hafskjold, and O. Galteland, "Nonisothermal transport of immiscible fluids in porous media. II. Constitutive equations," Fontiers in Physics, submitted, 2018. Acceptance of Terms and Conditions:

Click here to agree

\section{Modelling immiscible fingering in hierarchically structured porous media}


${ }^{1}$ The University of Sydney

Corresponding Author(s): yixiang.gan@sydney.edu.au

Porous media with hierarchical structures are commonly encountered in nature and synthetic materials, e.g., fractured rocks, electrode materials, and fibrous materials, which are generally composed of two components with distinct characteristic length scales. In this paper, we utilise the volume of fluid (VOF) to capture the interface dynamics and to model the imbibition processes in porous media with variable secondary pore sizes and wettability. We emphasise the following aspects: (1) how such hierarchical structures influence immiscible fingering during fluid-fluid displacement, and most importantly, (2) to what extent the immiscible fingering can be suppressed due to the existence of secondary porous structures compared with single-pore-size media. To characterise fingering dynamics in hierarchical porous media, we provide a phase diagram and propose a scaling law based on a non-dimensional number, which takes multiple length scales and wettability conditions into account. The present study provides an alternative mechanism for controlling the temporal and spatial distribution of interface within porous media for various applications, e.g., increased drainage efficiency, or enhanced chemical reaction.

Procter and Gamble Student poster award:

I don't want to compete References:

Acceptance of Terms and Conditions:

Click here to agree

\title{
Study of Evaporation from Wavy Surfaces by Coupled Turbulent Air Flow and Porous Media Flow
}

\author{
Author(s): Bo Gao ${ }^{1}$ \\ Co-author(s): Kathleen Smits ${ }^{2}$ \\ ${ }^{1}$ Colorado School of Mines \\ ${ }^{2}$ University of Texas Arlington
}

Corresponding Author(s): gaobo@mymail.mines.edu

The undulation of the soil surface (e.g. tilled soil) has significant influences on the mass/energy interactions between the soil and the atmosphere during evaporation. The naturally occurring turbulent air flow above undulated surfaces may enhance the influences. However, most previous works only consider evaporation behavior from flat surfaces, or do not incorporate both porous media flow and atmospheric flow for studying turbulence over undulated soil surfaces. The goal of this work is to provide an initial understanding of evaporation from undulated soil surfaces at the REV scale by experimental and numerical approaches. For simplicity, the surface undulation is represented by sinusoidal-type wavy surfaces in this study. A coupled free flow and porous media flow model was developed to describe evaporation under non-isothermal conditions. Turbulent air flow was taken into consideration. Experiments using an open-ended wind tunnel and soil tank were conducted and two-period wavy surfaces were built. The experimental system was instrumented with various environmental sensors to continuously collect atmospheric and subsurface data. Both particle imaging velocimetry (PIV) and hotwire anemometry were applied to capture the velocity profile above the soil surface. Experimental results show that slower speeds on upstream portions of troughs as predicted and indicated by lower levels of evaporation in these regions. Evaporation first happened at the uphill area of the upward wavy surface. Initial modeling results considering turbulence show that the existence of a recirculation area leads to a local thicker boundary layer and a local minimum value of the diffusive flux. In addition, this preliminary study demonstrated the application of velocimetry technology in evaporation from wavy soil surfaces under turbulent air flow. Future work will keep focusing on the influence of wavy surfaces and turbulence on evaporation by experiments with velocimetry technology and numerical modeling approaches.

Procter and Gamble Student poster award: 


\title{
References:
}

Acceptance of Terms and Conditions:

Click here to agree

\section{Characteristics of Micropore-Nano Structure in Shale Oil Reser- voir}

\author{
Author(s): TieNing Gao ${ }^{\text {None }}$ \\ Co-author(s): zhengming yang ${ }^{1}$; Haibo Li \\ ${ }^{1}$ PetroChina Research Institute of Petroleum Exploration \& Development \\ Corresponding Author(s): 1579195331@qq.com
}

This paper analyzes the characteristics of micropore-Nano structure and calculates the fractal dimension. The main research object is shale oil reservoir, and the high-pressure pressure mercury experiment is used as the method. Studies have shown that the micropore-Nanos of shale oil reservoirs are very concentrated and continuous and the main distribution range of pore radius is $30-500$ $\mathrm{nm}$ by comparing the shale reservoir with two typical dense reservoirs in Songliao Basin and Ordos Basin. Nano porosity is an important part of reservoir porosity. The fractal dimension of micronano pore in shale oil reservoir is significantly larger than that of typical dense oil reservoir, which indicates that the heterogeneity is stronger. The research results are of great theoretical and practical significance for inter-salt shale oil reservoir extraction.

\section{Procter and Gamble Student poster award:}

I would like to compete in the Procter and Gamble Student award References:

Acceptance of Terms and Conditions:

Click here to agree

\section{Dynamic pore-scale flow regimes study during steady-state mul- tiphase flow using synchrotron X-ray micro-tomography}

\author{
Ying Gao ${ }^{1}$; Qingyang Lin $^{1}$; Branko Bijeljic ${ }^{1}$; Martin Blunt ${ }^{1}$ \\ ${ }^{1}$ Imperial College London
}

Corresponding Author(s): y.gao15@imperial.ac.uk

Fast synchrotron-based X-ray micro-tomography was used to image the steady-state flow of brine and decane at equal fractional flows during co-injection through a sandstone core at the pore scale as a function of capillary number from $C a=10^{\wedge}(-7)$ to $10^{\wedge}(-5)$. Three-dimensional images with a voxel size of $5.2 \mu \mathrm{m}$ were acquired every minute during the experiment. An accurate differential pressure transducer was used to measure the pressure gradient along the sample at all the flow rates.

When $C a$ was lower than $10^{\wedge}(-6)$, which represents the capillary dominated regime, both oil and brine were observed to flow through their own connected pathways with a linear dependence of pressure gradient vs $\mathrm{Ca}$ 1. This confirmed to the traditional conceptual picture of steady-state multiphase Darcy flow in porous media. When $\mathrm{Ca}$ was larger than $10^{\wedge}(-6)$, local fluid rearrangements were observed [2-7], which caused pressure fluctuations, but there was still a linear dependence of pressure gradient on $\mathrm{Ca}$. However, when $\mathrm{Ca}$ was larger than $10^{\wedge}(-5)$, we observed a power-law dependence between pressure gradient and $C a$ with $\nabla P \sim \mathrm{q}^{\wedge}$ a where a $\approx 0.6$ [8]. In addition, we observed that the oil saturation decreased when $\mathrm{Ca}$ was increased at fixed fractional flow. 
The ganglia populations were subdivided in accordance with their size to assess the connectivity for the range of $\mathrm{Ca}$ studied. The brine/oil saturation profile along the core remained approximately constant throughout each experimental stage at each flow rate. However, the amount contributed by the different ganglia populations changed with both time and flow rate. As the flow rate increased, the proportion of small and medium ganglia increased, while the proportion of large ganglia decreased. The large connected pathway was not stable and varied in both volume and spatial position over time. For intermittent flow, we observed that oil had sufficient energy to make short-cuts through the pore space, occasionally opening up a more conductive flow path through a narrow throat, as opposed to oil flow through tortuous and long pathways through larger pore spaces [3,9] emphasized text. In conclusion, we present a new phase diagram describing the arrangement of non-wetting phase (as transient connected pathways or as disconnected ganglia) as a function of wetting and non-wetting phase capillary numbers for oil and brine flow in Bentheimer sandstone.

\section{Procter and Gamble Student poster award:}

I would like to compete in the Procter and Gamble Student award References:

1 Blunt MJ. Multiphase flow in permeable media: a pore-scale perspective. Cambridge University Press; 2017.

2 Berg S, Ott H, Klapp SA, Schwing A, Neiteler R, Brussee N, et al. Real-time 3D imaging of Haines jumps in porous media flow. Proc Natl Acad Sci 2013;110:3755-9. doi:10.1073/pnas.1221373110.

[3] Reynolds CA, Menke H, Andrew M, Blunt MJ, Krevor S. Dynamic fluid connectivity during steadystate multiphase flow in a sandstone. Proc Natl Acad Sci 2017:201702834.

[4] Rücker M, Berg S, Armstrong RT, Georgiadis A, Ott H, Schwing A, et al. From connected pathway flow to ganglion dynamics. Geophys Res Lett 2015;42:3888-94. doi:10.1002/2015GL064007.

5 Datta SS, Dupin J-B, Weitz DA. Fluid breakup during simultaneous two-phase flow through a threedimensional porous medium. Phys Fluids 2014;26:62004. doi:10.1063/1.4884955.

[6] Datta SS, Ramakrishnan TS, Weitz DA. Mobilization of a trapped non-wetting fluid from a threedimensional porous medium. Phys Fluids 2014;26:22002.

[7] Gao Y, Lin Q, Bijeljic B, Blunt MJ. X-ray Microtomography of Intermittency in Multiphase Flow at Steady State Using a Differential Imaging Method. Water Resour Res 2017;53:10274-92. doi:10.1002/2017WR021736.

[8] Sinha S, Hansen A. Effective rheology of immiscible two-phase flow in porous media. EPL (Europhysics Lett 2012;99:44004.

[9] Cueto-Felgueroso L, Juanes R. A discrete-domain description of multiphase flow in porous media: Rugged energy landscapes and the origin of hysteresis. Geophys Res Lett 2016;43:1615-22. doi:10.1002/2015GL067015.

Acceptance of Terms and Conditions:

Click here to agree

\section{Porous building materials and their degradation process due to the absorption of solution salts: a Markov approach to modeling the evolution of the degradation process.}

Elsa Garavaglia ${ }^{1}$; Cristina Tedeschi ${ }^{1}$

\footnotetext{
${ }^{1}$ Politecnico di Milano
}

Corresponding Author(s): elsa.garavaglia@polimi.it

A building material is often subject to aggressive attacks for the absorption of salts in solution. The porosity of materials such as mortar or concrete significantly influences the degradation process due to such attacks and the rapidity of the degradation process is affected by the aggressiveness of the attack, the size and the distribution of the pores, the degree of diffusivity of the material, and so on. The damage caused by such attacks is the loss of surface material by exfoliation or pulverization, whereas the underlying material keeps the initial properties as long as it is not exposed to the air itself.

Each of these parameters suffers of uncertainty that can not be easily determined.

The proposed paper intends to face the degradation process as a stochastic renewal process: after the achievement of a certain level of damage with consequent loss of the first layer of material, the process seems to renew itself equal to itself. Considered the uncertainty involved in the process its 
modelling requires a stochastic approach. A particularly suitable approach seems to be the Markovian approach-

On the basis of experimental surveys and analysis of porosity on different types of mortars and construction concretes, the authors propose a first deterministic reading of the damage as a function of the porosity and the its evolution time. Afterwards, starting from the results obtained, a probabilistic evaluation of the damage process is proposed through a Markov approach that will lead to the construction of fragility curves of the probability of exceed each of the established damage thresholds, as well as the evaluation of the probability of transition from the current state in a future one characterized by a lower level of performance. Considerations on the choice of the damage thresholds and on the choice of the distributions to be adopted will be discussed.

Procter and Gamble Student poster award:

References:

Acceptance of Terms and Conditions:

Click here to agree

564

\section{Pore-scale Water Draining in Gas Diffusion Layers of Polymer Electrolyte Membrane Fuel Cells Using X-ray Tomography and Lattice Boltzmann Method}

Pablo A. García-Salaberri ${ }^{1}$; Gisuk Hwang ${ }^{2}$; Mohammad Borumand ${ }^{2}$; Jeff T. Gostick ${ }^{3}$; Adam Weber ${ }^{4}$

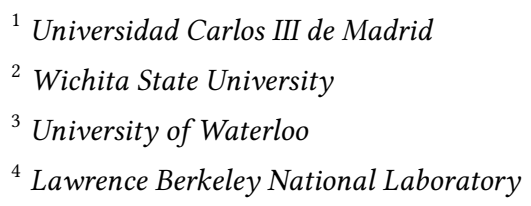

Corresponding Author(s):

Understanding of water draining is crucial for optimal water management in gas diffusion layers (GDLs) of polymer electrolyte membrane fuel cells (PEMFCs). In this study, pore-scale water draining processes in GDLs are visualized using micro X-ray tomography at the spatial resolution of 1.3 microns. The pore-scale water saturation with and without hydrophobic coating agent, i.e., PTFE, is observed at controlled capillary pressure. The PTFE-coated GDL shows water drainage with isolated water saturation at higher capillary pressure compared to GDL without PTFE coating. To further understand the dynamic draining process, the pore-scale saturation distribution is simulated using single-component, multiphase lattice Boltzmann method, and the capillary meniscus front movement is compared to experimental data. Preliminary results show that the PTFE-coated small pores initiate the water draining process by increasing the local capillary pressure, whereas the uncoated larger pores drain the water at low capillary pressure.

Procter and Gamble Student poster award:

References:

Acceptance of Terms and Conditions:

Click here to agree

\section{The role of chemical heterogeneities in controlling the wetting state of porous media}

Author(s): Gaetano Garfi ${ }^{1}$ 
Co-author(s): Cedric M. John ${ }^{1}$; Sam Krevor

${ }^{1}$ Imperial College London

Corresponding Author(s): g.garfi17@imperial.ac.uk

Multiphase flow phenomena are at the core of several research fields, such as fuel cell design, carbon sequestration and oil and gas recovery. With reference to the latter, a better understanding of complex subsurface flow phenomena would allow us to design more efficient and environmentally friendly recovery processes. In industrial practice, two-phase flow phenomena in porous media are typically described as a function of fluid saturation by means of relative permeability and capillary pressure. The behaviour of these functions is known to depend on the wetting state of the system considered. The wetting state of a system is controlled by several factors such as mineralogy, rock texture and fluid saturation history.

In this work we focused on the investigation of the role that chemical heterogeneities play in controlling the wetting state of rocks in oil-brine systems. We performed an unsteady-state experiment drainage-waterflooding cycle - in a water-wet Berea sandstone sample, imaging it with X-ray micro$\mathrm{CT}$. The images were processed to segment the rock matrix in four mineral groups and the pore space in decane and brine phases. Involving the identification of six phases, the segmentation task played a crucial role. For this reason, we also included a sensitivity analysis of some properties of interest - contact angle measurement, fluid-fluid interfacial curvature, mineral volume fraction and specific surface area - to the choice of the image processing pipeline. We compared two pipelines: non-local means filtering followed by watershed segmentation; machine learning based segmentation consisting of a manually trainable fast random forest classifier. This allowed us to analyse with confidence fluid arrangement in relation to mineral spatial distribution. The same experiment and analysis were repeated on another Berea sandstone sample after having exposed it to crude oil for the aging process. The results of the two experiments were compared to highlight different minerals response to the aging procedure.

Procter and Gamble Student poster award:

I would like to compete in the Procter and Gamble Student award References:

Acceptance of Terms and Conditions:

Click here to agree

\section{The role of convection for $\mathrm{CO} 2$ migration and trapping in $\mathrm{CO} 2-$ EOR reservoirs}

Sarah Gasda ${ }^{1}$; Tor Harald Sandve ${ }^{1}$; Roland Kaufmann ${ }^{1}$; Robert Klöfkorn ${ }^{1}$; Ivar Aavatsmark ${ }^{1}$

${ }^{1}$ NORCE Energy

Corresponding Author(s): sgas@norceresearch.no

Sustainable offshore oil production can be achieved through capture of platform-based emissions followed by storage or EOR in a nearby reservoir. Platform-based CCS could contribute to substantial reduction of offshore emissions (currently accounting for $25 \%$ of Norway's domestic emissions), while also providing carbon-free oil that will likely be a valuable commodity in future green-energy markets. $\mathrm{CO}_{2}$-EOR provides the most financial incentive for disposal of $\mathrm{CO}_{2}$, however the economic constraints for offshore $\mathrm{CO}_{2}$-EOR may restrict the number and placement of wells thus impacting flow regimes in the reservoir. In addition, the lower volume of $\mathrm{CO}_{2}$ available offshore is a key factor. New approaches to EOR can be designed to take advantage of emergent phenomena, such as convective mixing of $\mathrm{CO}_{2}$ in oil, to increase trapping of $\mathrm{CO}_{2}$ and improve the efficiency of oil production. This implies that interaction between $\mathrm{CO}_{2}$ and hydrocarbons at the fine scale will play an increasingly important role. Therefore, detailed understanding is required for effectively managing $\mathrm{CO}_{2}$ migration and storage efficiency in $\mathrm{CO}_{2}$-EOR reservoirs, or any storage reservoirs with existing hydrocarbons. 
Fine-scale interactions between $\mathrm{CO}_{2}$ and hydrocarbons occur in zones where fluids mix in a fully miscible setting. The mixing process occurs at the sub-centimeter-scale and involves complex convectivediffusive processes. Most oils exhibit non-monotonic change in density when mixed with $\mathrm{CO}_{2}$, which leads to density instabilities in the mixing zone. These density differences can play a significant role in parts of the reservoir that are gravity dominated, either by design or constraint. One key factor is that density instabilities may occur that drive convective mixing and impact the flow behavior of mixed fluids in these regimes. In this paper, we employ the use of high-resolution simulation technology to understand he interaction between gravity convection within an EOR reservoir that involves a mix of viscous- and gravity-dominated regions. We explore the impact of $\mathrm{CO}_{2}$ gravity fingers in oil at the field-scale and its impact on $\mathrm{CO}_{2}$ storage and oil recovery. We propose a hybrid modeling approach that is better suited to field-scale simulation using smart model reduction combined with subscale solutions.

Procter and Gamble Student poster award:

I don't want to compete References:

Acceptance of Terms and Conditions:

Click here to agree

494

\section{Variable Permeability and Reactions in a Hele-Shaw Cell model system of Carbon Dioxide Convective Dissolution for CCS}

Delora Gaskins $^{1}$; Sam Dehaeck ${ }^{1}$; Anne De Wit ${ }^{1}$

${ }^{1} U L B$

Corresponding Author(s): delora.gaskins@ulb.ac.be

The convective dissolution of carbon dioxide and subsequent mineralization in the form of carbonate containing minerals in saline aquifers for CCS are functions of coupled geochemistry and hydrodynamics. Basalt aquifers like CarbFix and the Wallula Basalt Pilot Project contain minerals like olivine which can liberate ions to mineralize and deposit the carbonates as they themselves dissolve. Flow through experiments in olivine show evidence for a dual control of carbonation by reactive and transport processes (1) and the modification of the permeability of the host rock (2). To discuss the effect of such variable permeability and reactions on the efficiency of $\mathrm{CO}_{2}$ storage, we report our experimental study of convective dissolution of carbon dioxide and mineral deposition in a vertical Hele-Shaw cell modified to account for permeability variations and reactive compositional changes.

Procter and Gamble Student poster award:

References:

1. Peuble, S. et al. $\mathrm{CO} 2$ geological storage in olivine rich basaltic aquifers: New insights from reactive-percolation experiments. Appl. Geochemistry 52, 174-190 (2015).

2. Luhmann, A. J. et al. Permeability, porosity, and mineral surface area changes in basalt cores induced by reactive transport of CO 2 -rich brine. Water Resour. Res. 53, 1908-1927 (2017).

Acceptance of Terms and Conditions:

Click here to agree

On the use of fixed-stress split scheme for the solution of flow problems in deformable porous media 
Francisco Gaspar ${ }^{1}$; Kundan Kumar ${ }^{2}$; Carmen Rodrigo ${ }^{1}$; Florin Adrian Radu ${ }^{3}$; Manuel Borregales ${ }^{\text {None }}$

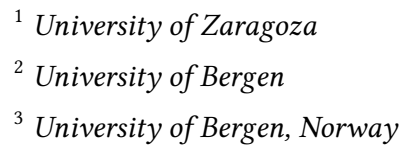

Corresponding Author(s): fjgaspar@unizar.es

The time dependent interaction between the deformation of an elastic porous material and the fluid flow inside of its pore network plays an important role in a vast number of real application problems in fields like geomechanics, hydrogeology and biomechanics, for example. Biot's equations are used to model this type of problems and therefore the numerical simulation of this model is an important issue. There are mainly two approaches to deal with this problem, the monolithic or fully coupled methods, which solve the linear system simultaneously for all the unknowns, and the iterative coupling schemes, which solve sequentially the equations for fluid flow and geomechanics, at each time step, until a converged solution within a prescribed tolerance is achieved.

The most used iterative coupling method is the fixed stress split scheme. Different approaches to this algorithm, however, have been used for both the coupled and decoupled solution of the Biot's problem. In this talk, we will focus on some of these solution strategies.

Procter and Gamble Student poster award:

References:

Acceptance of Terms and Conditions:

Click here to agree

\section{A stabilized mixed formulation for phase-field modeling of frac- ture in porous media}

Claudio Gavagnin ${ }^{1}$; Lorenzo Sanavia ${ }^{2}$; Laura De Lorenzis ${ }^{1}$

${ }^{1}$ TU Braunschweig

${ }^{2}$ Università degli Studi di Padova

Corresponding Author(s): gavagninclaudio@gmail.com

In the numerical approximation of a phase-field model of fracture in porous media with the Finite Element Method (FEM), the problem of the numerical locking due to high volumetric stiffness of the medium can occur. The causes of this state of incompressibility can be traced both to the hydraulic and the mechanical properties of the material. Regarding the hydraulic properties, it is well known that soils characterized by a low value of permeability show a local incompressible behaviour immediately after the application of a load. This causes oscillations of the numerical solution for the water pressure field when the inf-sup condition for the finite element interpolation functions is not fulfilled. Regarding the mechanical properties, when cracks are modeled with a phase-field approach based on a decomposition of the elastic energy into volumetric and deviatoric parts, the deviatoric stiffness becomes several order of magnitudes smaller than the volumetric one, causing an enlargement of the localization band of the phase-field variable and a dissipative residual force in the crack To solve these problems, a mixed formulation for the mechanical part fulfilling the inf-sup condition for the choice of the interpolation functions has been developed.

In this work, we first present a mixed $u-p-d$ (displacement-pressure-phase field) finite element formulation for the phase-field modeling of fracture in single phase elastic materials. Then, this approach is extended to variably saturated porous media, developing a $u-p-p^{w}-d$ formulation (with $p^{w}$ the pore liquid water pressure). Both models are shown to be stable. However, the higher degree of interpolation for the displacements field due to the inf-sup condition and the fine discretization required by the solution of the phase-field evolution equation lead to a very large number of degrees of freedom.

To reduce its computational cost a lower order of interpolation is proposed and a stabilization technique based on polynomial pressure projections is applied. This stabilization stems directly from the 
analogy between the incompressible Stokes equations 1 and the mixed formulation for incompressible elasticity, and has two interesting properties: the stabilization term is local, requiring only a few additional lines in the finite element code, and its influence is controlled by a material parameter 2. Four numerical examples obtained using several unstable, stable and stabilized finite elements are provided. They are: a one-dimensional tension test of an elastic bar; a two-dimensional shear test in a single phase material and in a water saturated material, and the study of the development of cracks in a clay domain during a desiccation process [3].

\title{
Procter and Gamble Student poster award:
}

\section{References:}

1 Dohrmann, Clark R., and Pavel B. Bochev. "A stabilized finite element method for the Stokes problem based on polynomial pressure projections.” International Journal for Numerical Methods in Fluids 46.2 (2004): 183-201.

2 White, Joshua A., and Ronaldo I. Borja. "Stabilized low-order finite elements for coupled solid-deformation/fluiddiffusion and their application to fault zone transients." Computer Methods in Applied Mechanics and Engineering 197.49-50 (2008): 4353-4366.

[3] Cajuhi, Tuanny, L. Sanavia, and L. De Lorenzis. "Phase-field modeling of fracture in variably saturated porous media." Computational Mechanics 61.3 (2018): 299-318. Acceptance of Terms and Conditions:

Click here to agree

\section{Modelling chemical deterioration processes in building materials - Poromechanical approach}

\author{
Dariusz Gawin $^{1}$; Marcin Koniorczyk ${ }^{1}$; Francesco Pesavento ${ }^{2}$ \\ ${ }^{1}$ Lodz University of Technology \\ ${ }^{2}$ University of Padova
}

Corresponding Author(s): dariusz.gawin@p.lodz.pl

A general approach 1 to modelling various deterioration processes in porous building materials, due to combined action of variable chemical, hygro-thermal and mechanical loads, is presented. Mechanics of multiphase porous media and damage mechanics are applied for this purpose. Kinetics of physico-chemical processes, like: salt crystallization/dissolution 2, calcium leaching [3], Alkali Silica Reaction (ASR) [4], is described with evolution equations based on thermo-dynamics of chemical reactions. The mass-, energy- and momentum balances, the evolution equations describing progress of chemical reactions, as well as the constitutive and physical relations are briefly summarized. The mutual couplings between the chemical, hygral, thermal and mechanical processes are presented and discussed, both from the viewpoint of physicochemical mechanisms and mathematical modelling. Numerical methods used for solution of the model governing equations are presented. For this purpose the finite element method is applied for space discretization and the finite difference method for integration in the time domain.

Three examples of the model application for analysis of transient chemo-hygro-thermo-mechanical processes in porous building materials are presented and discussed. The first example concerns the salt crystallization during drying of a wall made of concrete or ceramic brick, causing degradation of surface layer due to development of crystallization pressure. The second one deals with calcium leaching from a concrete wall due to chemical attack of pure water, exposed to gradients of temperature and pressure. The third one describes cracking of concrete element, caused by development of expanding products of ASR.

Procter and Gamble Student poster award:

I don't want to compete References: 
1 D. Gawin, M. Koniorczyk and F. Pesavento, "Modelling of hydro-thermo-chemo-mechanical phenomena in building materials", Bulletin of The Polish Academy of Sciences: Technical Sciences, Vol. 61(1), pp. 51-63, (2013).

2 M. Koniorczyk and D. Gawin, "Modelling of salt crystallization in building materials with microstructure - poromechanical approach", Construction and Building Materials, Vol. 36, pp. 860-873, (2012).

[3] D. Gawin, F. Pesavento and B.A. Schrefler, "Modeling deterioration of cementitious materials exposed to calcium leaching in non-isothermal conditions", Computer Methods in Applied Mechanics and Engineering, Vol. 198(37-40), pp. 3051-3083, (2009).

[4] F. Pesavento, D. Gawin, M. Wyrzykowski, B.A. Schrefler and L. Simoni, "Modeling alkali-silica reaction in non-isothermal, partially saturated cement based materials", Computer Methods in Applied Mechanics and Engineering, Vol. 225-228, pp. 95-115, (2012). Acceptance of Terms and Conditions:

Click here to agree

\section{From streamlines and transfer functions to flow diagnostics for naturally fractured reservoirs}

Sebastian Geiger ${ }^{\text {None }}$

Corresponding Author(s): s.geiger@hw.ac.uk

Streamline-based reservoir simulation, which was pioneered by Martin Blunt and his students Marco Thiele and Rod Batycky at Stanford University in the 1990ties, provides a computationally fast way to approximate flow and transport processes in geological reservoirs. Streamline-based reservoir simulations are hence an effective means to quantify the impact of geological uncertainties on future reservoir management decisions and forecast reservoir performance more accurately. In the 2000s, now at Imperial College London, Martin demonstrated with his $\mathrm{PhD}$ students and postdocs how streamline-based reservoir simulation can be extended to naturally fractured reservoirs through the use of new transfer functions.

This presentation will revisit some of Martin's pioneering research in this field and discuss how it can be extended to perform flow diagnostics for naturally fractured reservoirs directly on the grid of a geological model without the need of running streamline simulations. The fundamental idea of flow diagnostics, originally pioneered by Moyner et al. (2014), is to provide a very fast way to approximate the dynamic behaviour of a reservoir. By incorporating new physics-based transfer functions which account for a variety of physical processes (e.g. gravity drainage, imbibition, and deformation) into flow diagnostics, we can now efficiently screen large numbers of geological models and consider a much broader range of dynamic uncertainties when we forecast flow and transport in naturally fractured reservoirs. Considering that the performance of naturally fractured reservoirs is notoriously difficult to forecast, flow diagnostics offer a step change in how we account for geological uncertainties in modern reservoir simulation workflows.

Procter and Gamble Student poster award:

References:

Acceptance of Terms and Conditions:

Click here to agree

\section{Lattice Boltzmann Method for effective diffusion coefficient com- putation at the pore scale}

Alain Genty ${ }^{1}$ 
Corresponding Author(s): alain.genty@cea.fr

In the context of radioactive waste management, deep geological repository in indurated argillaceous media appears as a potential solution. Radionuclides transport through argillaceous media is then of concern and in particular diffusion processes in pore water. The Excavated Damaged Zone (EDZ) surrounding the storage cells and the galleries of the repository presents a complex network of micro- and macro-fractures where radionuclides transport may be enhanced with respect to the diffusion in intact host rock. Especially, the micro-fractures are supposed to form a connected pathway and thus the diffusion of solutes in this network is of high interest in the context of the storage safety assessment. During the transitory period after the excavation of the repository, the fractures undergo several desaturation-resaturation cycles resulting from desaturation process during the excavation phase or from hydrogen flow where hydrogen is produced from corrosion of the waste metallic canisters after repository closure. Their state can vary from fully saturated with water to almost complete desaturation depending on their location, aperture and on the instant in the history of the repository. The saturation state of porous media has an important impact on the diffusion processes as it modifies the connectivity and the tortuosity of residual pore water.

In order to simulate diffusion process inside porous geometry, we chose to use a Lattice Boltzmann Model (LBM) that allows to i) to easily take into account the geometry as available from X-ray tomographic images or reconstructed through mathematical models, ii) simulate water-gas distribution inside the porous media for different saturation level, and iii) simulate diffusion inside the resulting connected water pathway.

If the presented work was initiated focusing on radionuclide diffusion through unsaturated microfractures, diffusion process in unsaturated porous media is of concern in many other fields like pesticides or carbonated nutriments behaviour in soils or solute migration in reservoirs. We present here the application of our technique in several kind of porous media, namely on an argillite microfracture, a soil macro-pore and a generic reservoir representative elementary volume.

The LBM we used in this work is based on a Two-Relaxation-Time (TRT) collision operator. Watergas distribution was simulated using the LBM described in 1 which follow the Shen-Chen approach through a particular source term. At initial time, a mean density was imposed inside the void space of the porous media. The presence of the wetting walls induces spontaneous phase separation leading to a low-density phase (gas) and a higher density phase (water). On the basis of the calculated liquid-gas distributions inside the pore space, a basic thresholding algorithm allowed to extract the connected liquid phase. For the diffusion computations, we considered a non-volatile tracer and only took into account diffusion in the liquid phase. We conducted diffusion simulations for different saturation in the connected liquid phase. Computations were performed starting from an initial Dirac imposed concentration on the symmetry line of the media. The mean concentration was calculated as a function of distance to the central line and then fitted with an analytical diffusion equation candidate curve. Effective diffusion coefficient was deduced as a function of saturation.

\title{
Procter and Gamble Student poster award:
}

\section{References:}

1 A. Genty and V. Pot. Numerical Simulation of 3D Liquid Gas Distribution in Porous Media by a TwoPhase TRT

Lattice Boltzmann Method. Transport Porous Med., 96(2): 271-294, (2013).

2 A. Genty and V. Pot. Numerical Calculation of Effective Diffusion in Unsaturated Porous Media by the TRT

Lattice Boltzmann Method. Transport Porous Med., 105(2): 391-410, (2014).

[3] A. Genty, S. Gueddani and M. Dymitrowska. Computation of Saturation Dependence of Effective Diffusion

Coefficient in Unsaturated Argillite Micro-fracture by Lattice Boltzmann Method. Transport Porous Med., 117(1): 149-168, (2017). Acceptance of Terms and Conditions:

Click here to agree

\section{Measuring swelling and shrinkage of porous materials with a dig- ital camera}

\author{
Author(s): Nikolaj Georgi ${ }^{1}$ \\ Co-author(s): Andrei Kolesnikov ${ }^{2}$
}


${ }^{1} G M B U$

${ }^{2} I N C$

Corresponding Author(s): nikolaj.georgi@gmx.net

On adsorption gases and vapors exert a force on the pore walls resulting in an excess pressure compared to the bulk. The disjoining pressure1 can result in swelling as well as shrinkage of the material. Depending on the rigidity of the porous material the relative length changes can be quite small, as for cement 2 or large - 10\%, as for wood and cellulose fibers[3]. Despite the small length changes the effects of the large forces exerted on the porous material can lead to a reversible as well as to an irreversible modification of the pore size distribution[6]. Given that many porous materials of practical importance like concrete are exposed to humidity changes the mechanical response and the long term stability of such structural materials may be quite relevant [4].

In the present contribution we present an optical technique to measure small length changes of porous materials that are exposed to humidity changes. The technique involves a digital camera recording images of the sample and an algorithm calculating the mechanical response of the material. The algorithm is based on the so called Digital Image Correlation5, that has already been used to study the stress of rigid materials. We present measurements of deformation isotherms of concrete and wood obtained by the optical technique.

1 B. Derjaguin, N. Churaev, V. Muller, Surface Forces, Consultants Bureau,New York, 1987.

2 M. J. Setzer,F. H. Wittmann, Appl. Phys. 3, 403-409, 1974.

[3] F. T. Wallenberger et al.,Natural Fibers, Plastics and Composites, (c) Kluwer Academic Publishers 2004.

[4] A.V. Tvardovskiy, Sorption Deformation, Elsevier Ltd., Amsterdam, 2006.

5 M.A. Sutton et al., Image Correlation for Shape, Motion and Deformation Measurements: Basic Concepts, Theory and Applications, Springer, 2009.

[6] A.L. Kolesnikov, N. Georgi, et al. Langmuir, 34, 25,7575, 2018.

Procter and Gamble Student poster award:

References:

Acceptance of Terms and Conditions:

Click here to agree

\section{Colloidal deposition in 3D porous media, a multi-scale approach}

Gaétan Gerber $^{1}$; Philippe Coussot ${ }^{2}$

${ }^{1}$ ENPC - Harvard SEAS

${ }^{2}$ Univ. Paris-Est

Corresponding Author(s): gaetan.gerber@ifsttar.fr

The flow of suspended solid elements through saturated porous structures is of great importance in a multitude of industrial, geochemical and hydrologic processes like filtration, contamination and remediation of groundwater aquifers, or enhanced oil recovery. Depending on their size, concentration and charge properties, the suspended elements will be subject to various events, including size-exclusion clogging in narrow pore throats 1 , surface adsorption, or interactions with previously attached bodies. In all cases, these deposition events take place at the colloid scale but can affect areas at the kilometre scale.

For a long time, the mechanisms have been studied through break-through analysis at the sample's scale in column experiments [2,3]. More recently, 2D pore-scale studies provided direct observations and quantification of the statistics of deposition [4]. Here, we propose to link the two approaches 
through a direct multiscale observation of the adsorption of colloidal particles on the internal surface of a 3D porous structure.

We consider the transport, deposition and accumulation of charged colloids $(1 \mu \mathrm{m})$ in a model column of negatively charged borosilicate packed grains $(63 \mu \mathrm{m})$. We vary the inter-colloidal interactions via the ionic strength of the suspensions to modulate the dynamics of aggregation of the colloids. The deposition and accumulation events are followed in-situ by confocal microscopy; simultaneously over multiple length scales: observation of individual colloidal deposition at the grain's scale $(100 \mu \mathrm{m})$ allows us to identify the precise deposition mechanisms. Then, up to the sample's scale $(1 \mathrm{~cm})$, evolution of the colloid distribution over space and time can be described by models deduced from the local mechanisms.

First, we verify that in the case of repulsive inter-colloidal interactions, deposition is limited to a Langmuirian single-layer of particles at the surface of the porous medium. We highlight the influence of the flow direction and pore throat sizes on the distribution of particles, in comparison with a model pore (single spherical collector approach). Secondly, screening of the inter-colloidal repulsive interactions leads to additional deposition mechanisms in the form of multi-layer deposits. We show that these deposits grow to a critical size defined by an equilibrium between electrostatic cohesive forces and drag forces, following the coupling between the probability of particle deposition and the evolution of the local porosity and velocity. Lastly, extreme screening of inter-particle repulsion leads to deposits entirely filling the pore space, until clogging of the system.

We believe these results provide a new understanding of deposition mechanisms through direct observations at the pore scale, as well as a set of references to better predict the transport and deposition of suspensions over large length scales.

Procter and Gamble Student poster award:

I would like to compete in the Procter and Gamble Student award References:

1 G. Gerber et al., PRL, 2018

2 M. Elimelech, Journal of Colloid and Interface Science, 1991

[3] S. A. Bradford et al., Env. Sci. Tech., 2003

[4] B. Dersoir et al., Microfluid Nanofluid, 2015 Acceptance of Terms and Conditions:

Click here to agree

\title{
Permeability upscaling from pore to core scale
}

\author{
Author(s): Lefki Germanou ${ }^{1}$ \\ Co-author(s): Lei $\mathrm{Wu}^{1}$; Yonghao Zhang ${ }^{1}$ \\ ${ }^{1}$ University of Strathclyde
}

Corresponding Author(s): lefki.germanou@strath.ac.uk

As the global interest in unconventional reservoirs has increased, accurate prediction of shale gas production is indispensable. Direct calculation of intrinsic or apparent gas permeability is only feasible in high-resolution imaging samples where connectivity allows the solution of the Stokes or Boltzmann equation respectively. The effective permeability of the largest low-resolution samples can be provided through various upscaling techniques, which use as input the permeability tensor derived from these direct simulations. The main objective of this research is to identify the challenges and enhance the accuracy of permeability upscaling from the nano to the micro-scale where fractures appear. To this end, intrinsic and apparent permeability of numerous two-dimensional random porous media structures is obtained solving the Stokes equations and the linearized BGK equation (using the Discrete Velocity Method) respectively. This permeability is used as input for the upscaling process, herein performed by two types of solvers. The first one solves Darcy's law in the porous medium while the second solves the Brinkman equation for a domain consisting of both porous and free regions. In this way, computational domains containing either matrix only or both matrix and fractures can be simulated accordingly. In this study, numerical upscaling using 
the aforementioned solvers is compared with the direct simulation results and methods to improve its accuracy are proposed. Special focus is given on the region near the porous and the free medium interface where the effect of the drastic variations of velocity is taken into consideration and incorporated into the model. Additionally, several flow configurations and different type of porous media are studied in detail and their impact on upscaling is investigated. Overall, this analysis provides an insight into permeability upscaling of ultra-tight porous media.

Procter and Gamble Student poster award:

I would like to compete in the Procter and Gamble Student award References:

Acceptance of Terms and Conditions:

Click here to agree

989

\section{Combusting in the Deep}

Margot Gerritsen ${ }^{1}$

${ }^{1}$ ERE-Stanford University

Corresponding Author(s): margot.gerritsen@stanford.edu

There are few systems of partial differential equations as interesting, and challenging, as those governing

in-situ combustion (ISC). ISC is an environmentally friendlier alternative to steam injection for enhanced production of heavy oils. A part of the oil is burned in-situ to generate heat that reduces oil viscosity and often also leads to in-situ upgrading of the hydrocarbons. The ISC process involves

flow and transport of multiple strongly interacting phases. The chemical reactions and intrinsic reservoir properties render the systems fiercely multi-scale also.

Our poster will give you an introduction to this fascinating research topic.

We conduct research experimentally, to learn more about the intricate physics, as well as computationally,

to predict process performance and develop new numerical tools (which can often be applied to similar systems of PDEs arising in other applications).

The poster will contain a wee challenge to you also, with a prize, which we will explain in the poster pitch on Monday.

Procter and Gamble Student poster award:

References:

Acceptance of Terms and Conditions:

450

\section{Mesh-free simulation for precipitation and dissolution in a 2D discrete fracture network}

Masoud Ghaderi Zefreh ${ }^{1}$; Florian Doster ${ }^{1}$

${ }^{1}$ Heriot Watt University

Corresponding Author(s): mg65@hw.ac.uk

We propose a simulation method for reactive flow with dissolution and precipitation in discrete fracture networks. We consider the corresponding reactions for two salts that share an ion. The intersections of fractures are locations where fluids with potentially different composition mix. Therefore, 
precipitation of minerals may occur at the intersection points. Accumulation of precipitates at the intersection affects the flow and potentially changes the topology of the network. Therefore, it is important that mixing in intersections is accurately calculated. This usually requires an extremely fine mesh to capture the mixing around the intersections as the volume of the mixing is very small.

A fracture network in 2D can be effectively modelled as a set of 1D domains. Hence, the analytical 1D solution can be used for each subdomain. We use the graph representation of fractures for the flow part and a front-tracking algorithm based on the analytical solution for advection-dominated transport. The intersections of fractures require special attention. We represent them by semi-continuous reactors. Each reactor has a characteristic radius that determines its rate of clogging and dissolution. The simulator is mesh-free as none of the components of the simulator requires a mesh.

\title{
Procter and Gamble Student poster award:
}

I would like to compete in the Procter and Gamble Student award References:

Karra, S., O’Malley, D., Hyman, J. D., Viswanathan, H. S., \& Srinivasan, G. (2018). Modeling flow and transport in fracture networks using graphs. Physical Review E, 97(3), 033304. https://doi.org/10.1103/PhysRevE.97.03330

Ghaderi Zefreh, M., Nilsen, H. M., Lie, K. A., Raynaud, X., \& Doster, F. (2018). Streamline simulation of a reactive advective flow with discontinuous flux function. Computational Geosciences. https://doi.org/10.1007/s10596018-9771-3 Acceptance of Terms and Conditions:

Click here to agree

\section{Increasing capacity of $\mathrm{CO} 2$ sequestration by brine production}

\author{
Author(s): Seyyed Ghaderi ${ }^{1}$ \\ Co-author(s): Yuri Leonenko ${ }^{2}$ \\ ${ }^{1}$ University of Calgary \\ ${ }^{2}$ University of Waterloo, Canada
}

Corresponding Author(s): s.amir.ghaderi@gmail.com, leonenko@uwaterloo.ca

In this study we present how the storage capacity can be increased by adding brine production to reduce rapid pressure build up during large volume of $\mathrm{CO} 2$ injection.

It is well recognized that deep underground aquifers offer a very large potential storage capacity for $\mathrm{CO} 2$ sequestration 1 . But it is not clear how to fill the storage with a large volumes of $\mathrm{CO} 2$ when it is taken place within a limited injection area and in a relatively short period of time. Wabamun Lake Sequestration Project or WASP was one of the first comprehensive studies conducted to evaluate storage of large $\mathrm{CO} 2$ volumes into a saline formation. Nisku aquifer located in Alberta, Canada 2 was considered in this study. To put it into perspective, the project aimed at storing 20 megatons (Mt) of $\mathrm{CO} 2$ per year over 50 years of solely injection operation. However, previous studies have indicated that, pure injection operation would require a massive landscape usage of the reservoir and large space between the injectors to manage the rapid pressure increase in the bulk of the reservoir and especially at the vicinity of the injectors. With all favourable formation properties, such as high permeability, high fracture pressure, and adequate thickness, maximization of distances between the injectors which translates to minimum number of injectors, was not a successful option and the storage target is far from fulfilment. For example, our previous study [3] showed that the maximum number of vertical injectors cannot exceed 10 (no significant capacity increase was observed above this number of wells) and as such, the maximum injected volume barely exceeds $40 \%$ of target value of 1 Gigatons (Gt). Adding wells above Application of horizontal injectors can only slightly improve the storage volume capacity [4]. From economic point of view, although drilling less injectors will be more favourable, for injection pipeline to cover such a huge area, capital cost requirement would be significant. Therefore, such operation is not the most practical option due to limited achievable volumes and viability constraints. 
Based on the above observations, it seems production of the formation water is an inevitable option to manage the rapid pressure evolution. In this study, a commercial black oil simulator (IMEX, Computer Modelling Group) is used to determine the number of required injectors/production wells, their orientation (vertical, horizontal, or combination), and their operation constraint (rate vs. bottomhole pressure) to achieve the cumulative volume target of $1 \mathrm{Gt}$ in 50 years.

\title{
Procter and Gamble Student poster award:
}

\section{References:}

1 Metz, B.; Davidson, R.; De Coninck, H.; Loos, M.; Meyer, A. Carbon Dioxide Capture and Storage: Special Report of the Intergovernmental Panel on Climate Change. Cambridge University Press: New York 2005.

2 B. Hitchon, Aquifer Disposal of Carbon Dioxide, Hydrodynamic and Mineral Trapping - Proof of Concept, 1996, Geoscience Publishing Ltd., Sherwood Park, Alberta, Canada.

[3] Ghaderi, S., Keith, D., and Leonenko, Y., Feasibility of Injecting Large Volumes of CO2 into Aquifers, Energy Procedia, 1, (1), 3113-3120. (2009)

[4] Ghaderi, S.M. and Leonenko, Y.: Reservoir modeling for Wabamun lake sequestration project. Energy Science \& Engineering 3(2), 98-114 (2015) Acceptance of Terms and Conditions:

Click here to agree

\section{Coupled Electrohydrodynamic transport through fractures}

\author{
Uddipta Ghosh $^{1}$; Tanguy Le Borgne ${ }^{2}$; Yves Méheust ${ }^{2}$ \\ ${ }^{1}$ Indian Institute of Technology Gandhinagar \\ ${ }^{2}$ Géosciences Rennes
}

Corresponding Author(s): uddipta.ghosh@iitgn.ac.in

Geological fractures constitute the basic structural units controlling the flow of fluids and the transport of solutes in subsurface crystalline rocks. Fracture wall roughness is responsible for flow channeling within the fracture plane, which impacts the fracture's transmissivitty 1 , and can also impact the distribution of fluxes in-between fractures of the fracture networks 2. The most prevalent way of computing the distribution of local fluxes in (and transmissivitty of) a rough fracture without resorting to a full 3D flow simulation, is to use the lubrication approximation, which leads to a simple linear equation for pressure: the Reynolds equation. However, the effect of the electrical properties of the solid walls on the transport properties of a fracture still remains an open question. Since dissolved minerals and salts are present in the fluids, Electrical Double Layers (EDLs) form at the fluid-solid interface [3]. Hence, the occurrence of externally-imposed or naturally-occurring gradients in electrical potential and/or ionic concentration leads to significant changes in the fluid flow and solute transport as compared to flows driven primarily by hydraulic head differences. We consider geological fractures with a realistic aperture field and explore the flow dynamics resulting from such coupled electro-hydrodynamic forces. To this end we generalize the standard lubrication theory for flow to include a description of the coupled transport of fluid mass, solutes, and electrical current under the application of fixed differences in hydraulic head (or pressure), electrical potential and concentration across the fracture. The generalized lubrication theory finally leads to a set of coupled global conservation equations, which are solved using an iterative Finite Volume Method.

Procter and Gamble Student poster award:

I don't want to compete References:

1. S. R. Brown, J. Geophys. Res. Solid Earth, 92(B2):1337, 1987.

2. J.-R. de Dreuzy, Y. M\'eheust, and G. Pichot. J. Geophys. Res.: Solid Earth, 117(B11), 2012. 
3. U. Ghosh, S. Mandal, and S. Chakraborty. Phys. Rev. Fluids, 2(6):064203, 2017.

\title{
Acceptance of Terms and Conditions:
}

Click here to agree

\section{GEOMATICS FOR THE PREDICTION OF SAND BOIL PRONE AR- EAS}

\author{
Author(s): maria daniela giliberti ${ }^{1}$; massimiliano cirincione ${ }^{2}$ \\ Co-author(s): alessandro cavagni ${ }^{3}$ \\ ${ }^{1}$ Interregional Agency for the Po river-AIPo \\ ${ }^{2}$ IHO - Cat. A Hydrographer \\ ${ }^{3}$ Officine Maccaferri Italia s.r.l.
}

Corresponding Author(s): mariadaniela.giliberti@agenziapo.it, massimiliano.cirincione@gmail.com, a.cavagni@maccaferri.com

3-D Numerical models can be useful in in the design of efficient hydraulic barriers intended to prevent flood induced sand boil formation. Seepage under levees presents serious hazard due to the difficulty in forecasting where erosion, that can lead to levee breaches, initiates.

Results show that the proposed procedure allows identification of sand boils -prone areas pointing out preferential, and sometimes unexpected, flows suggesting a new perspective for remediation measures.

The procedure was tested on three piping - sensitive subsoil areas in Italy, along the Po river: Sacca di Colorno (Cavagni, 2017), Caselle Landi (Cremonesi, 2017) and Boretto (Virderi, 2018) using documentation of sand boil occurrence produced by the Interregional Agency for the Po river.

The analysis was carried out using FEMWATER (Hsin-Chi, 1997) a 3-D Finite Element model, able to solve the saturated-unsaturated flow field both in steady and unsteady conditions. The embankments and surrounding land have been described by means of a detailed Digital Terrain Model with $1 \mathrm{~m}$ cell grid. The foundation soil and hydraulic parameters have been recovered from existing investigations: boreholes, Le Franc tests and ERT. These data was processed by the graphical interface of the Groundwater Modeling System (GMS, 2017) software. The soil properties of the unsaturated zones have been represented with the Van Genuchten curves (1980). All analyzed in unsteady conditions using historical flood waves.

3D simulations proved to be more able to distinguish where sand boils occurred and where not, delivering results more adherent to reality.

The method is sensitive to DTM resolution and a cell size of $1 \mathrm{~m}$ was sufficient for good performance. This study highlights the role and weight of the DTM for the delineation of sand boil-prone areas.

\section{Procter and Gamble Student poster award:}

\section{References:}

Carsel R., Parrish R. (1988). Developing joint probability distributions of soil water retention characteristics, Water Resour Res.

Cavagni A. (2017). 3D modeling of the seepage and piping phenomena in a Po River embankment in the Parma province, MSc Thesis in Civil Engineering, Parma University, Italy (in Italian).

Cremonesi S. (2017). 3D modeling of the seepage and piping phenomena in a Po River embankment in the Lodi province, MSc Thesis in Environmental and Land Engineering, Parma University, Italy (in Italian).

De Marsily (1986). Quantitative Hydrogeology, Academic Press Inc., London.

GMS (2017). http://www.aquaveo.com/software/gms-groundwater-modeling-system-introduction

Hsin-Chi J.Lin, Richards D. R., Talbot C. A., Yeh G. T., Cheng J. R., Cheng H. P., Jones N. L. (1997). FEMWATER A Three-Dimensional Finite Element Computer Model for Simulating Density-Dependent Flow and Transport in Variably Saturated Media, U.S. Army Engineer Waterways Experiment Station, Vicksburg.

VICENZETTO S.r.l. (2017) Technical Report on "Geognostic investigations to design works for functional adjustment of Po river main levee in the municipality of Boretto (RE) to control seepage". Villa 
Estense (PD) Italy.

L. Virderi (2018). Internship report 2017/2018 - Civil Engineer, Parma University, Italy.

Zhang G., Zhang J. (2004). A fem seepage analysis for upstream cofferdam of Xiluodu hydraulic power station. Conference proceedings International Conference on Case Histories in Geotechnical Engineering.10. New York, USA Acceptance of Terms and Conditions:

Click here to agree

114

\title{
Ballisticules, mobile-mobile mass exchange, and mixing-limited reactions
}

Author(s): Timothy Ginn ${ }^{1}$

Co-author(s): Deviyani Gurung ${ }^{1}$

${ }^{1}$ Washington State University

Corresponding Author(s): tim.ginn@wsu.edu

Reaction extent between displacing and displaced solutes depends on mixing at the pore scale that is overestimated by continuum scale quantification by dispersive fluxes. Experiments suggest that this is due to formation of segregation zones, termed here ballisticules because they arise from locally correlated pore-scale velocities giving rise to short-range ballistic transports. Diffusive mixing between ballisticules is addressed via a first-order mobile-mobile mass exchange based on the phase exposure-dependent exchange (PhEDEx) concept. This results in time-delayed diffusive mixing of reactants, and in models honoring both independently measured reaction rates and dispersivities, for both kinetically-controlled and equilibrium cases. The solution for the equilibrium case is a remarkably simple closed-form expression involving a hydrodynamic activity coefficient and one fitting variable. The approach is applied to published experimental data on both fast and slow bimolecular reactions for homogeneous porous media under postasymptotic dispersive conditions with good results.

Procter and Gamble Student poster award:

References:

Acceptance of Terms and Conditions:

Click here to agree

116

\section{Modeling a large scale experiment in MICP using independently determined parameters}

\author{
Mohamed Nassar ${ }^{1}$; Deviyani Gurung ${ }^{2}$; Timothy Ginn ${ }^{2}$; Michael Gomez ${ }^{3}$; Charles Graddy ${ }^{3}$; Doug Nelson ${ }^{3}$; Jason \\ DeJong $^{3}$

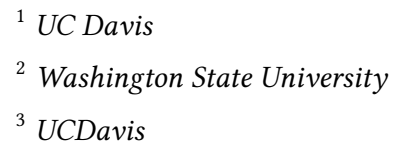

Corresponding Author(s): tim.ginn@wsu.edu

We develop a reactive transport model of microbial-induced calcite precipitation (MICP) in a meterscale tank experiment with transient nonuniform transport in a natural soil, using independently determined parameters. Flow in the tank was controlled via cyclic injection/withdrawals followed by no-flow intervals among three wells. Different injection solution recipes were used in sequence 
for transport characterization, biostimulation, cementation, and groundwater rinse phases of the 17 day experiment. Reaction kinetics were calibrated using separate column experiments designed with a similar flow sequence. This allowed for a parsimonious approach to modeling the tank experiment with zero fitting parameters. The tank data were simulated using PHT3-D, involving transient nonuniform flow and a multicomponent reaction network combining equilibrium and kinetically controlled biogeochemical reactions. The assumption that microbes mediating the reaction were exclusively sessile with constant activity provided for efficient and accurate modeling of the resulting nonuniform calcite precipitate. This analysis suggests that under the biostimulation conditions applied here the assumption of steady state sessile biocatalyst suffices to describe the microbially mediated calcite precipitation.

Procter and Gamble Student poster award:

References:

Acceptance of Terms and Conditions:

Click here to agree

46

\title{
Determination of the solute diffusivity, dispersion, skewness, kurtosis and higher-order moments in heterogeneous porous flow via Extended Method of Moments.
}

\author{
Irina Ginzburg ${ }^{1}$; Alexander Vikhansky² \\ ${ }^{1}$ Irstea, Antony Regional Centre, HYCAR \\ ${ }^{2}$ CD-adapco
}

Corresponding Author(s): irina.ginzburg@irstea.fr

Prediction of the first four moments of the solute distribution is decisive in the optimization of the dispersion,asymmetry, peakedness and heavy-tails of the solute distributions, through an adequate design of the composite materials, chemical devices or in oil recovery. Although the relative importance of the higher moments decays downstream, they manifest the non-Gaussian behavior of the breakthrough curves approaching the Taylor-Aris regime [1-2], especially if the solute can diffuse into less porous phase. The extended method

of moments (EMM) is a new mathematical algorithm [7,10] for the recursive

prediction of the two systems of Taylor moments: the spatial (mean-concentration) and the temporal (residence time distribution, RTD). The method applies in any streamwise-periodic stationary d-dimensional, Newtonian or non-Newtonian, velocity field resolved in the piecewise continuous heterogeneous porosity field. The EMM looks for the solution of the transport equation in a single periodic cell, as a product of a long harmonic wave and a spatially periodic oscillating component, based on the ideas [5-6] for RTD.

The method extends the Brenner's generalized dispersion microscopic approach [3] and volume averaging boundary-value formulation [4], from the second spatial moment to any-order, spatial and temporal, moment in multi-scale flow. The EMM is supported with the entropy-maximization procedure for reconstruction of distribution from its moments, allowing to visualize the deviation from the classical ADE solution in heterogeneous structure without resorting to solving of the highorder upscaled PDE [4] or modeling of the direct microscopic transport. The method provides the non-trivial benchmarks $[7,10]$ for direct numerical schemes; it also allows to analytically and numerically examine an interference of their fourth-order truncation corrections with the effective diffusivity, dispersion coefficient, skewness and kurtosis: this is demonstrated for the direct Lattice Boltzmann LBM-ADE transport solver [9]. The simple exact algebraic transforms [7,10] constructed between the temporal and spatial systems of moments are also applicable to any direct ADE computations.

In porous rock, the EMM quantifies the Taylor dispersion and two principal bi-modality effects: sharp peaks and elongated tails, versus the porosity contrast, aspect ratio, Péclet and Darcy numbers, 
as illustrated [10] by the symbolic solutions constructed in a stratified system composed from a fracture surrounded by penetrable diffusive matrix or embedded in porous flow. In multi-dimensional flow, the EMM can be handled by any linear advection-diffusion method, supported with the precomputed space-variable mass-source and diffusive-flux jump on the abrupt-porosity streamwisenormal interface. A sequentially solved chain of the steady-state boundary-value ADE problems [10], independent for each mean-velocity direction, provides three longitudinal Taylor coefficients: dispersion, skewness and kurtosis; the full dispersion tensor can be restored from $\mathrm{d}$-independent runs and a recursive extension to the next-order longitudinal moments is straightforward.

The simple local multi-dimensional LBM-EMM numerical formulation with an implicit interface tracking is examined [11]. Operating in a single cell, the LBM-EMM

is hundreds time faster already in the small Péclet range than the direct LBM-ADE solver, and it copes very well with the grid refining for high Péclet range. The method proves its efficiency in prediction of the dispersion reduction through porous obstruction geometry and allows to establish the structure-depending Péclet scaling in dispersion coefficients and the next-order moments.

Procter and Gamble Student poster award:

\section{References:}

1 G. I. Taylor. Dispersion of soluble matter in solvent flowing slowly through a tube. Proc. R. Soc. Lond, Ser. A. 219, 186-2003, 1953.

2 R. Aris. On the dispersion of a solute in a fluid flowing through a tube.

Proc. R. Soc. Lond 235: 67-77, 1956.

[3] H. Brenner. A general theory of dispersion phenomena. Phys.Chem. Hydrodyn. 1: 91-123, 1980.

[4] G. N.Mercer and A. J.Roberts.

A Centre Manifold Description of Contaminant Dispersion in Channels with Varying Flow Properties. SIAM, J. Appl. Math, 50, 1547, 1990.

5 A. Vikhansky. Effect of diffusion on residence time distribution in chaotic channel flow. Chem.Eng. Sci. 63: 1866-1870, 2008.

[6] A. Vikhansky and W. Wang. Taylor dispersion in finite-length capillaries. Chem. Eng. Sci. 66, 642-649, 2011.

[7] A.Vikhansky and I.Ginzburg.

Taylor dispersion in heterogeneous porous media:

Extended method of moments, theory, and modelling with

two-relaxation-times Lattice Boltzmann scheme.

Physics of Fluids 26: 022104-52, 2014.

[8] F. J. Valdès-Parada, D. Lasseux, F. Bellet. A new formulation of the dispersion in homogeneous porous media. Adv. Water Res, 90, 70-82, 2016.

[9] I. Ginzburg. Prediction of the moments in advection-diffusion lattice Boltzmann method. I. Truncation dispersion, skewness, and kurtosis. Physical Review E 95, 013304-34, 2017.

[10] I. Ginzburg and A. Vikhansky. Determination of the diffusivity, dispersion, skewness and kurtosis in heterogeneous porous flow. Part I: Analytical solutions with the Extended Method of Moments. Adw. Wat. Res., 115:60-87, 2018.

[11]. I. Ginzburg. Determination of the diffusivity, dispersion, skewness and kurtosis in heterogeneous porous flow. Part II: Lattice Boltzmann schemes with implicit interface. Adw. Wat. Res., 118:49-82, 2018. Acceptance of Terms and Conditions:

Click here to agree 
Magnus Aa. Gjennestad ${ }^{1}$; Mathias Winkler ${ }^{\text {None }}$; Alex Hansen ${ }^{2}$

${ }^{1}$ Norwegian University of Science and Technology

${ }^{2}$ NTNU

Corresponding Author(s): magnus.aa.gjennestad@ntnu.no

It was recently found that applying Euler's theorem of homogeneous functions to steady-state twophase flow in porous media leads to certain very specific relations between the flow velocities of each phase 1. These relations are

lbegin $\{$ equation $\} \mathrm{v} \_w=\mathrm{v}+\mathrm{S} \_\mathrm{n} \backslash \operatorname{left}\left(\backslash \operatorname{frac}\{\mathrm{dv}\}\left\{\mathrm{dS} \_\mathrm{w}\right\}-\mathrm{v} \_\mathrm{m} \backslash\right.$ right$)$, end $\{$ equation $\}$

$\backslash$ begin $\left\{\right.$ equation\} $\mathrm{v} \_\mathrm{n}=\mathrm{v}-\mathrm{S} \_\mathrm{w} \backslash \operatorname{left}\left(\backslash \operatorname{frac}\{\mathrm{dv}\}\left\{\mathrm{dS} \_\mathrm{w}\right\}-\mathrm{v} \_\mathrm{m} \backslash\right.$ right$)$, ,end\{equation $\}$

where $S_{n}$ and $S_{w}$ are the saturations of the non-wetting and wetting fluids, respectively, and $v_{n}$ and $v_{w}$ are the flow velocities of the non-wetting fluid and the wetting fluid, respectively. The total velocity is

|begin $\{$ equation $\} \mathrm{v}=\mathrm{S} \_\mathrm{w}$ v_w + S_n v_n, lend\{equation $\}$

and

|begin $\{$ equation $\}$ v_m $=$ S_w $\backslash$ frac $\left\{\mathrm{dv} \_w\right\}\left\{\mathrm{dS} \_w\right\}+\mathrm{S} \_\mathrm{n} \backslash$ frac $\left\{\mathrm{dv} \_\mathrm{n}\right\}\left\{\mathrm{dS} \_\mathrm{w}\right\}$, end $\{$ equation $\}$

is called the co-moving velocity. Remarkably, these relations arise from no other assumptions than steady-state and Euler homogeneity, and are therefore expected to be general.

Here, we explore $v$ and $v_{m}$ further using a dynamic pore network model. In particular, we shed light on their functional forms and their importance to the flow under different conditions. The model is documented in 2, along with novel solution procedures for low capillary numbers.

\section{Procter and Gamble Student poster award:}

I would like to compete in the Procter and Gamble Student award References:

1 A. Hansen, S. Sinha, D. Bedeaux, S. Kjelstrup, M. Aa. Gjennestad, M. Vassvik. Relations between seepage velocities in immiscible, incompressible two-phase flow in porous media. Transport in Porous Media 125 (2018), 565-587.

2 M. Aa. Gjennestad, M. Vassvik, S. Kjelstrup, A. Hansen. Stable and efficient time integration of a dynamic pore network model for two-phase flow in porous media. Frontiers in Physics 6 (2018). doi:10.3389/fphy.2018.00056. Acceptance of Terms and Conditions:

Click here to agree

136

\section{Coupled 1D-3D Flow Models}

Jan Martin Nordbotten ${ }^{1} ;{\text { Kundan } \text { Kumar }^{2} \text {; Ingeborg Gåseby Gjerde }}^{1}$

${ }^{1}$ University of Bergen

${ }^{2}$ University of Karlstad

Corresponding Author(s): ingeborg.gjerde@uib.no

Coupled 1D-3D flow models are used for a variety of applications, such as modeling fluid flow through vascularized tissue, modeling the flow of water and nutrients through soil embedded with a root system, or modeling flow in a reservoir to or from a well. Veins, arteries, roots and wells all have in common that their radius is negligible compared to the domain as a whole; for this reason, they are idealized as being 1D geometries. The 1D structures can then be endowed with their own $1 \mathrm{D}$ flow equation, and coupled to the $3 \mathrm{D}$ flow equation by the use of a line source.

The main challenge of coupled 1D-3D models is that the line source makes the solution singular. This complicates both the analysis and approximation of the problem. In this talk, we show that the solution admits a splitting into two parts: (i) a term that explicitly captures the singularity and (ii) some smooth remainder. Via this splitting, we can then reformulate the model so that all variables 
are smooth. The solution can then be approximated using any standard numerical method. We conclude by showing numerical experiments relevant for biomedical applications.

Procter and Gamble Student poster award:

References:

Acceptance of Terms and Conditions:

Click here to agree

313

\section{Optimizing the performance of batteries using digital material engineering}

Erik Glatt $^{1}$; Fabian Biebl ${ }^{1}$; Ilona Glatt ${ }^{1}$; Christian Wagner ${ }^{1}$; Wiegmann Andreas ${ }^{1}$

${ }^{1}$ Math2Market $\mathrm{GmbH}$

Corresponding Author(s): erik.glatt@math2market.de

Renewable energy plays an important role in reducing greenhouse gas emissions from the use of fossil fuels. Key challenges to transform renewable energy sources into a reliable energy supply lie in optimizing its storage through increased performance of batteries and in improving efficiency and durability of portable devices, such as those in hybrid, electric or fuel cell vehicles. The battery performance is strongly influenced by the micro-structure of the battery materials and, thus, engineering better batteries and optimizing their behavior strongly depends on understanding the micro-structure and its impact on material properties.

Here, we use the simulation software GeoDict ${ }^{\circledR}$ to show such an optimization on a state-of-the-art cathode, which contains a blend of the two active materials Lithium Nickel Cobalt Aluminum Oxide (NCA) and Lithium Cobalt Oxide (LCO).

The starting point for the optimization is a focused ion beam scanning electron microscopy (FIBSEM) image of an NCA-LCO cathode. FIB-SEM is a technique to obtain high resolution 3D images, although it presents several challenges for the subsequent image analysis and segmentation process. Image processing and segmentation are done using the import module of GeoDict巴. The result is a structure segmented into three materials: electrolyte, binder and active material. The two active materials cannot be discerned based on gray values alone, because they have very similar gray-values. In a next step, the two active materials (NCA and LCO) of the cathode are separated. The GrainFind module is used to identify the single grains in the image and to characterize each grain by size, shape, location, and orientation. The two active materials have different grain shapes and so, based on the grain shape analysis, the single grains are assigned as being NCA or LCO. The result is a fully segmented cathode material containing electrolyte, binder, NCA, and LCO.

Next, we use the fully segmented cathode structure in a battery charging simulation with the BatteryDict module of GeoDict ${ }^{\circledR}$. BatteryDict computes the cell potential over the state of charge and the evolution of the 3D Lithium concentration, including the two active materials and the binder (see figure). In addition, other modules of GeoDict ${ }^{\circledR}$ can be used to compute other important material properties such as pore-sizes, diffusivity, conductivity, tortuosity, and mechanical properties.

Finally, we focus on finding the optimal amount of binder in the cathode for improved battery charging performance. The binder's conductive additives enhance the electronic conductivity in the (otherwise poorly) electronically conducting cathode. Unfortunately, because the binder also acts as a good insulator to ionic diffusion, the lithium ions cannot enter the electrolyte if the cathode contains too much binder and lithium transport becomes impossible. To find the ideal amount of binder and study its influence on the charging performance, variations of the original material differing only in the amount of binder are modelled with GeoDict ${ }^{\circledR}$ and the results are shown.

Procter and Gamble Student poster award:

References:

Acceptance of Terms and Conditions:

Click here to agree 


\title{
Pore scale effects of electrical charge in porous media under elec- trical and mechanically driven flows
}

Omar Emmanuel Godinez Brizuela ${ }^{1}$; Vahid.J Niasar ${ }^{\text {None }}$

${ }^{1}$ The University of Manchester

Corresponding Author(s): omar.godinezbrizuela@manchester.ac.uk

Electrically charged components are common in natural porous materials such as rocks and soils. Under certain conditions, charged material in the solid matrix, such as clays or other polar species may affect the fluid dynamics in the pore space through electrostatic interaction with the charged components of the fluid. These microscopic electrokinetic effects are the source of important phenomena with macroscopic repercussions, such as electro-osmosis, and streaming potentials in porous materials and other microfluidic systems, with applications in areas such as contaminant transport and enhanced oil recovery.

In this work, we studied the pore-scale hydrodynamic effect of the presence of negatively charged grains randomly allocated in a porous medium, while varying the distribution and amount of charged surface area present in the system. With this, we aim to emulate the effect of clays randomly scattered in a porous medium. We studied this under flow conditions driven by a pressure gradient, an electric field or both simultaneously and observed the effect these phenomena have on the permeability of the system.

Procter and Gamble Student poster award:

I would like to compete in the Procter and Gamble Student award References:

Acceptance of Terms and Conditions:

Click here to agree

\section{Salt polygons and porous media convection}

\author{
Lucas Goehring $^{1}$; Jana Lasser ${ }^{2}$; Joanna Nield ${ }^{3}$ \\ ${ }^{1}$ Nottingham Trent University \\ ${ }^{2}$ Max Planck Institute for Dynamics and Self-Organisation \\ ${ }^{3}$ University of Southampton
}

Corresponding Author(s):

The polygonal patterns of ridges in salt flats, playa, and pans are shockingly regular features of arid landscapes. They are most well-known from places like Death Valley, or Salar de Ununi. They also form in the salt crusts covering large pans like the Sua or Etosha pans, which are some of the primary sources of airborne dust in sub-saharan Africa. Such crusts typically display a pattern of polygonal cells, a few meters across, bounded by ridges a few centimetres in size. No conclusive explanation for these features has ever been given, but their resemblance to polygonal terrain, or columnar joints, has led to speculation that they are formed by fracture patterns of the salt crust. Here, we instead argue that the pattern cannot be properly understood unless it is considered in light of porous media convection of salt-rich ground-water beneath the crust. In particular, we propose that surface evaporation creates a salt-rich surface layer of water that is unstable to Rayleigh-Bénard convection within the pore space of the playa soil. This drying-induced convection creates variations in salt concentration, which allow for the selective deposition of salt around the perimeters of the convection cells, hence appearing as a regular surface pattern. We support this argument with a linear stability analysis of the geophysical situation, numerical simulations of the stratified fluids, analogue experiments, and field observations. 
Procter and Gamble Student poster award:

References:

Acceptance of Terms and Conditions:

Click here to agree

\title{
Drying and percolation in correlated porous media
}

\author{
Soumyajyoti Biswas ${ }^{1}$; Paolo Fantinel ${ }^{1}$; Oshri Borgman ${ }^{2}$; Ran Holtzman ${ }^{2}$; Lucas Goehring ${ }^{3}$ \\ ${ }^{1}$ Max Planck Institute for Dynamics and Self-Organisation \\ ${ }^{2}$ Hebrew University of Jerusalem \\ ${ }^{3}$ Nottingham Trent University \\ Corresponding Author(s):
}

We study how the dynamics of a drying front propagating through a porous medium are affected by small-scale correlations in material properties. For this, we first present drying experiments in micro-fluidic micro-models of porous media. Here, the fluid pressures develop more intermittent dynamics as local correlations are added to the structure of the pore spaces. We also consider this problem numerically, using a model of invasion percolation with trapping, and find that there is a crossover in invasion behaviour associated with the length-scale of the disorder in the system. The critical exponents that describe large enough events are similar to the classic invasion percolation problem, while the addition of a finite correlation length significantly affects the exponent values of avalanches and bursts, up to some characteristic size. We thus find that even a weak local structure can interfere with the universality of invasion percolation phenomena. This has implications for a variety of multi-phase flow problems, such as drying, drainage, and fluid invasion.

Procter and Gamble Student poster award:

References:

arxiv:1809.03564 (accepted to Physical Review Fluids) Acceptance of Terms and Conditions:

Click here to agree

641

\section{Modelling blood flow in mouse brain microvascular networks}

Author(s): Florian Goirand ${ }^{1}$

Co-author(s): Maxime Berg ${ }^{1}$; Tanguy Le-Borgne ${ }^{2}$; Sylvie Lorthois ${ }^{1}$

${ }^{1}$ Institut de Mécanique des Fluides de Toulouse (IMFT)

${ }^{2}$ Observatoire des Sciences de l'Univers de Rennes (OSUR)

Corresponding Author(s): florian.goirand@imft.fr

Key words : Brain microcirculation, Pore-Network Modeling, Blood Rheology, Advection-Diffusion(Reaction) Equation, Lagrangian Analysis, Travel-Time Distributions

Introduction

The vascular system of the mouse brain cortex is composed of a space filling mesh-like capillary network connected upstream and downstream to branched quasi-fractal arterioles and venules. This network allows for delivery of oxygen into brain cells and removal of metabolism products. The 
distribution of blood flow rates in these networks affects the efficiency of oxygen transfer processes and its resiliency to local obstructions. However, the statistics of blood flow in the microcirculation and their consequences on transport properties are unknown. Here, we investigate the distribution of blood flow from numerical resolutions in a large 3D mouse intra-cortical vascular network (anatomical data kindly provided by P. Blinder and P. Tsai).

\title{
Methods
}

In each segment, the flow is solved from a stationnary 1D non-linear model taking account of the complex rheological properties of blood flow in microcirculation to deduce the blood pressure, blood flow and red blood cell volume fraction distributions throughout the network. Conservative transport is then solved using particle tracking through the network to quantify Lagrangian transport properties.

\section{Results}

The distribution of flow rates is found to follow a broad PDF with power law decays characterizing the flow resulting from a two-well system. We relate the corresponding exponents and transition flow rate to the structural properties of the network. We then quantify the effect of the distribution of flow and path lengths on the travel time distribution through the networks to develop a stochastic transport model.

Using the model, we investigate the effect of random obstructions in the network on flow and transport properties and discuss the consequences of these findings for oxygen transport in brain microcirculatory networks.

Funding sources

ERC BrainMicroFlow GA615102, ERC ReactiveFronts GA648377

\section{Procter and Gamble Student poster award:}

I would like to compete in the Procter and Gamble Student award References:

\section{Acceptance of Terms and Conditions:}

Click here to agree

696

\section{Lattice-Boltzmann modeling of osmosis during low-salinity flood- ing}

\author{
Mohammad Hossein Golestan ${ }^{1}$; Olav Aursjø ${ }^{2}$; Carl Fredrik Berg ${ }^{1}$; eirik flekkøy ${ }^{3}$; Espen Jettestuen ${ }^{4}$ \\ ${ }^{1}$ PoreLab, Dept. Geoscience \& Petroleum, Norwegian University of Science and Technology (NTNU) \\ ${ }^{2}$ International Research Institute of Stavanger (IRIS) \\ ${ }^{3}$ PoreLab, Dep. Physic University of Oslo, Norway \\ ${ }^{4}$ research scientist at International research institute of Stavanger
}

Corresponding Author(s): mohammad.h.golestan@ntnu.no

Flooding of oil-reservoirs by low-salinity water has been proposed as a technique to enhance oil production. Such low-salinity flooding has been tested both experimentally in laboratory experiments and in field cases. Positive experimental results of low-salinity flooding have been reported for both sandstone and carbonate samples. Different mechanisms for oil mobilization by low-salinity waterflooding have been proposed, including wettability alteration, reduced interfacial tension, increased $\mathrm{PH}$ and dissolution. There has been a significant amount of research on low-salinity flooding during the last two decades, however, this has not resulted in consensus on the dominant low-salinity mechanisms.

A recently proposed low salinity mechanism is osmosis: This require a situation where oil separate the higher-salinity reservoir water from the lower-salinity injected water. Due to the difference in 
chemical potential between high- and low-salinity water, the water will be transported as a solvent in the oil phase from the low-salinity water to the high-salinity water. This leads to swelling of the high-salinity water, which can induce enhanced oil production.

We use a lattice-Boltzmann model to simulate osmosis during low-salinity flooding. The underlying two-phase lattice-Boltzmann model has a single relaxation time collision operator, while fluid phase separation is obtained by the color gradient method. Further, we allow for water to be partially solvable in the oil phase. The employed fluid separation yields a diffuse interface where the fluids partially mix. The dissolved water and the brine equilibrate at this diffusive interface, and the equilibrium is related to the salt concentration. At equilibrium, there is a higher concentration of water dissolved in the oil phase at the low-salinity interface than at the high-salinity interface. The osmotic transport is then obtained by modeling an advection-diffusion process for the water solvent in the oil phase.

We will present results where we have employed this lattice-Boltzmann simulation code to model experiments where osmosis is assumed to be the main mechanism. This include an oil-wet tube with a high-salinity and low-salinity droplet separated by oil, and micro-model experiments of lowsalinity flooding. We will compare the lattice-Boltzmann model results to the experimental results, and discuss the predictability of our model.

\title{
Procter and Gamble Student poster award:
}

I would like to compete in the Procter and Gamble Student award References:

Acceptance of Terms and Conditions:

Click here to agree

\section{Evolution of Pore Structure during Organic Acid Injection: A Pore Network Modeling Approach}

\author{
Hossein Younesian-Farid ${ }^{\text {None }}$; Mohammad.h Golestan ${ }^{1}$ \\ ${ }^{1}$ Department of Petroleum Engineering and Applied Geophysics, Norwegian University of Science and Technology
}

Corresponding Author(s): mohammad.h.golestan@ntnu.no

A multicomponent reactive transport model is developed in order to investigate dissolution mechanisms in high-pressure condition during acidizing processes. A synthetic pore network model is built and an operator splitting approach is implemented for solving advection-diffusion and reaction equations. In this study, the dominant equilibrium and kinetic reactions are considered in the model. A general reaction rate equation is used for calculating dissolution/precipitation rate near to or far from equilibrium conditions. Organic acids are injected into the system, under different flooding conditions, and the evolution of porosity is monitored. Due to the uncertainty in the way that permeability evolves by porosity evolution, permeability changes are prognosticated using several well-known equations. Because of high acid residence time in low injection rates, pores that are near to the inlet experience more dissolution in comparison to the downstream pores, which results in having not so much permeability increment. In contrast, during higher injection rates, porosity changes uniformly across the system. In this condition, permeability increases more rapidly.

Procter and Gamble Student poster award:

\section{References:}

Acceptance of Terms and Conditions:

Click here to agree 


\title{
Pore scale Numerical Modeling of Fluid Flow through Porous Me- dia( Direct Method Approach)
}

\author{
Author(s): Mohammad.h Golestan ${ }^{1}$; Soroor Pashang ${ }^{\text {None }}$ \\ Co-author(s): Eghbal Sahraei \\ ${ }^{1}$ Department of Petroleum Engineering and Applied Geophysics, Norwegian University of Science and Technology
}

Corresponding Author(s): mohammad.h.golestan@ntnu.no, soroor.pashang@gmail.com

In this study, Digital Rock Physics (DRP) is used to increase the accuracy of the reservoir rock properties calculation. To specify the size of the Representative Elementary Volume (REV), the values of porosity and permeability are obtained from two separate methods, Direct Numerical Simulation (DNS) and Pore Network Modeling (PNM). Three types of digital images studied in this project are including Bentheimer sandstone, Grosmont carbonate and the Spherepack; five distinct subvolumes in 50, 100, 150, 200 and 250 voxel sizes were extracted from each rock sample. In order to run direct numerical simulation, the Avizo 9.0.1 commercial software was used. The steps of image pre-processing for each of the separated volumes, including the filtering, segmentation or thresholding (binarization), and connectivity have been done to prepare the rock images for fluid flow simulations. Avizo (XLab Hydro Extension) solves the navier stokes equation through the generated volume mesh in the void space of the rock by finite volume method (FVM). Porosity values are just the voxels belonging to the connected void space divided by the total number of the voxels. In order to obtain porosity and permeability values based on the PNM method, an equivalent network is extracted using the images that pre-processed by avizo software, and then the fluid flow was modeled inside the 3D network extracted by Maximal Balls (MB) Algorithm. Finally, the results shown that DNS method generate better prediction of porous media characteristics and also can decrease the uncertainty in oil field developments, although it is undeniable to improve the $\mathrm{MB}$ algorithm for extraction of pore network and increasing the accuracy of the PNM method. It was observed that the size of REV was estimated in two samples of Bentheimer and the Spherepack for the permeability, respectively, is a cube with side lengths equal to 150 and 100 voxel size. Also, for porosity of Bentheimer and Spherepack, the $\mathrm{REV}$ value is respectively a cube with lengths equal to 100 and 50 voxel size. In the Grosmont carbonate rock due to its high heterogeneity, there was no characteristic trend for permeability data and thus estimating the value of its REV was not possible; and based on the results of the DNS method, it is predicted that REV size for the Grosmont porosity is a cube with side lengths equal to 150 voxels, Which is expected to increase the accuracy of the REV parameter by increasing the dimensions of the individual volumes that will be considered.

Key Words:

Digital Rock Physics (DRP), Direct Numerical Simulation (DNS), Pore Network Modeling (PNM), Maximal Ball Algorithm (MB), Representative Elementary Volume (REV).

Procter and Gamble Student poster award:

I would like to compete in the Procter and Gamble Student award References:

Dong, H., Micro-CT imaging and pore network extraction. 2008, Department of Earth Science and Engineering, Imperial College London.

Valvatne, P.H. and M.J. Blunt. Predictive pore-scale network modeling. in SPE Annual Technical Conference and Exhibition. 2003. Society of Petroleum Engineers.

Blunt, M.J., et al., Pore-scale imaging and modelling. Advances in Water Resources, 2013. 51: p. 197216.

Moukalled, F., L. Mangani, and M. Darwish, The finite volume method in computational fluid dynamics. 2016. Acceptance of Terms and Conditions:

Click here to agree

\section{A computational pore-scale model of NAPL remediation -Investigating the impact of biofilm growth on bio-enhanced dissolution and}




\title{
biodegradation
}

\author{
Author(s): Fabrice GOLFIER ${ }^{1}$ \\ Co-author(s): Marbe Benioug ${ }^{2}$; Xiaofan Yang ${ }^{3}$; Constantin Oltéan ${ }^{4}$ \\ ${ }^{1}$ Université de Lorraine - GeoRessources Laboratory \\ ${ }^{2}$ Al-Aasriya Nouakchott University \\ ${ }^{3}$ Faculty of Geographical Science, Beijing Normal University \\ ${ }^{4}$ Université de Lorraine - GeoRessources
}

Corresponding Author(s): fabrice.golfier@univ-lorraine.fr

\begin{abstract}
Non-aqueous phase liquids (NAPLs) are important but challenging problems for traditional groundwater remediation. They usually contaminate the subsoil following an accidental spill or due to a defect in the structures storage. These pollutants remain trapped in the form of droplets and/or immiscible clusters within the aquifers, becoming a persistent source of pollution that is difficult to decontaminate. Predicting the fate of this pollutant requires characterizing all the mechanisms involved and in particular the biodegradation, which can occur in the vicinity of the pollutant source or further, within the dissolved plume.

In the current work, we introduce a pore-scale model to study the interaction between biofilm growth and non-aqueous phase-liquid (NAPL) dissolution. Fluid flow and dissolved NAPL transport are coupled with a biofilm growth model to correctly describe the complex dynamics of the processes including fluid flow, NAPL dissolution/biodegradation and biofilm growth. Fluid flow is simulated using an immersed boundary-lattice Boltzmann model; while solute transport is described by a cutcell finite volume method. A uniform dissolution approach is also adopted to capture the temporal evolution of trapped blobs. Spatio-temporal distribution of the biomass is investigated using a cellular automaton algorithm combined with the immersed boundary method. Simulations focused on the dissolution of NAPL in both abiotic and biotic conditions are conducted to assess the capability of the model. In abiotic conditions, we analyze the effects of the hydrodynamic regimes and the spatial distribution of NAPL blobs on the dissolution rate under different assumptions (blob size and Péclet number). In biotic conditions, a series of scenarios are also investigated (spatial distribution, reaction kinetics and NAPL-induced toxicity). Finally, this model is used to evaluate the pore scale relevance of a local equilibrium assumption between fluid phase and biofilm phase in the vicinity of the NAPL source.
\end{abstract}

\section{Procter and Gamble Student poster award:}

I don't want to compete References:

Benioug, M., Golfier, F., Fischer, P., Oltéan, C., Buès, M.A. and Yang, X. 2018, Interaction between biofilm growth and NAPL remediation: a pore-scale study, Advances in Water Resources (under review).

Benioug, M., Golfier, F., Oltéan, C., Buès, M.A., Bahar T. and Cuny, J. 2017, An immersed-boundary lattice Boltzmann model of biofilm growth in porous media, Advances in Water Resources, 107:65-82. doi:10.1016/j.advwatres.2017.06.009

Benioug, M., Golfier, F., Tinet, AJ., Buès, M.A. and Oltéan, C. 2015, Numerical efficiency assessment of IB-LB method for 3D pore-scale modeling of flow and transport, Transport in Porous Media 109(1), 1-23, doi: 10.1007/s11242-015-0497-6. Acceptance of Terms and Conditions:

Click here to agree

\section{Structural changes in bulk materials for thermochemical energy storage}

Marie Gollsch ${ }^{1}$; Matthias Bisdorf ${ }^{1} ;$ Marc Linder $^{2}$

\footnotetext{
${ }^{1}$ German Aerospace Center (DLR)

${ }^{2}$ German Aerospace Center
} 
Corresponding Author(s): marie.gollsch@dlr.de

One major challenge regarding the intended shift to renewable energy sources lies within their fluctuating nature. In answer to this challenge and to ensure a consistently sustainable energy supply, the development of advanced energy storage technologies is required. In many cases - such as in concentrating solar power plants - storage of thermal energy is reasonable not only technically but also in view of economic efficiency 1 .

Reversible chemical reactions, wherein thermal energy can be stored as reaction enthalpy $\triangle H R$, represent a highly promising approach. Compared to sensible and latent thermal energy storage, advantages of thermochemical storage (TCS) include higher potential energy storage densities and long-term storage with minimal losses. Reaction systems of the generic equation

$\mathrm{AB}(\mathrm{s})+\Delta H \mathrm{R} \rightleftharpoons \mathrm{A}(\mathrm{s})+\mathrm{B}(\mathrm{g})$

consisting of a solid and a gaseous component, present additional benefits. Not only are the components easily separated, due to the dependency of the reaction's equilibrium temperature on the reaction gas partial pressure, gas-solid reactions also offer adjustable charging and discharging temperatures. For a number of applications, various gas-solid reaction systems are currently investigated as thermochemical storage materials $[2,3]$. However, particularly with regard to reactor design, a unique challenge appears: with each reaction cycle, the structure of the solid bulk changes significantly. Dependent on the investigated sample mass (analysis, lab-scale, pilot scale), design of the reaction chamber and handling of the solid material, these structural changes seem to differ. Reactor development is thus complex as material behaviour is so far unpredictable and vital properties such as gas permeability and thermal conductivity of the solid bulk cannot be estimated within a satisfactory range. Upscaling of reaction systems showing promise at lab-scale [4] is consequently costly as prediction of material properties and therefore simulation of reactor and system behaviour is inaccurate. Consequently, heat and mass transfer within the solid are also difficult to predict as they change due to structural changes of the solid. Caused by these changes, prediction of the reaction behaviour is suffering accordingly as the thermodynamic equilibrium depends on solid temperature and gas pressure. Reactor studies are therefore often conducted by trial-and-error.

Motivated by this predicament, our contribution features ongoing experimental studies regarding the structural changes of the solid components of gas-solid reaction systems for TCS. We aim to gain a better understanding of the crucial mechanisms. Furthermore, we investigate the impact of possible modifications of the materials, such as particle size stabilization, on the structural changes.

\title{
Procter and Gamble Student poster award:
}

\section{References:}

1 S. Kuravi, J. Trahan, D. Y. Goswami, M. M. Rahman and E. K. Stefanakos, Thermal energy storage technologies and systems for concentrating solar power plants, Progress in Energy and Combustion Science 39 (4), 285-319, 2013.

2 A. Gutierrez, S. Ushak and M. Linder, High Carnallite-Bearing Material for Thermochemical Storage: Thermophysical Characterization, ACS Sustainable Chemistry and Engineering 6 (5), 6135-6145, 2018.

[3] S. Afflerbach, T. Kowald and R. Trettin, Phase transformations during de- and rehydration of scholzite CaZn2(PO4)2 2 H2O, Journal of Solid State Chemistry 254, 184-194, 2017.

[4] M. Schmidt, M. Gollsch, F. Giger, M. Grün and M. Linder, Development of a moving bed pilot plant for thermochemical energy storage with $\mathrm{CaO} / \mathrm{Ca}(\mathrm{OH}) 2$, AIP Conference Proceedings 1734 (1), 0500411-050041-8, 2016. Acceptance of Terms and Conditions:

Click here to agree

\section{Liquid Injectivity in Surfactant-Alternating-Gas Foam Enhanced Oil Recovery: Effect of Superficial Velocity}

\author{
Author(s): JIAKUN GONG ${ }^{1}$ \\ R. Rossen ${ }^{6}$ \\ ${ }^{1}$ Delft University of Technology
}

Co-author(s): Wendy Flores Martinez ${ }^{1}$; Sebastien Vincent-Bonnieu ${ }^{2}$; Ridhwan Zhafri Kamarul Bahrim ${ }^{3}$; Nasser Bakri Che Mamat ${ }^{3}$; Raj Deo Tewari ${ }^{3}$; Iqbal Mahamad Amir ${ }^{3}$; Jeroen Groenenboom ${ }^{4}$; Rouhi Farajzadeh ${ }^{5}$; Willaim 


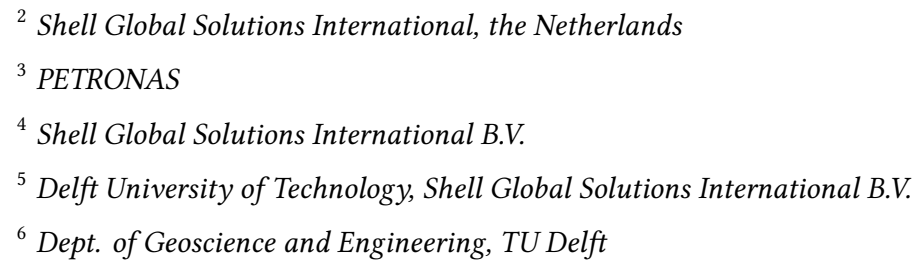

Corresponding Author(s): j.gong@tudelft.nl

Surfactant-alternating-gas (SAG) is a favored method of foam injection for EOR, but liquid injectivity in SAG is problematic. Our previous studies suggest that the injectivity in a SAG process is determined by propagation of several banks (Gong et al., 2018). During gas injection, a collapsed-foam region slowly propagates from the inlet which greatly improves subsequent liquid injectivity. This region represents a complex interplay of evaporation, gas dissolution, capillary and viscous effects. During the subsequent liquid-injection period, liquid first quickly fills the collapsed-foam region and then fingers through the weakened-foam region beyond of it. Subsequently, a second bank develops within the liquid-fingering bank, in which gas within the fingers dissolves into unsaturated liquid or is displaced, and mobility rises substantially. However, it is not clear how gas and liquid injectivities would be affected by the superficial velocities and volumes of gas and liquid.

In this study, we first examine gas injection following foam at different superficial velocities. We then study the effect of liquid superficial velocity on the liquid injectivity following a similar amount of gas injection. We also investigate the effect of the propagation of the collapsed-foam bank on liquid penetration of trapped foam downstream of the collapsed-foam bank. Finally, we scale-up the experimental results using a radial bank-propagation model and, for comparison the Peaceman equation which is widely used in conventional foam simulators.

During prolonged gas injection following foam, the propagation velocity and the total mobility of the collapsed-foam bank are not significantly affected by the gas superficial velocity. During liquid injection after gas, the liquid mobility holds constant until gas within the fingers dissolves into liquid. The constant liquid mobility is hardly affected by the duration of the period of gas injection. However, it takes longer for liquid to dissolve gas within the fingers (the plateau in mobility lasts longer) if more gas was injected previously. This suggests that the fingers are wider if more gas was injected. The total mobilities of the liquid-fingering bank and the gas-dissolution bank follow a power-law against the liquid superficial velocity. Liquid fingering through the weakened-foam region shows shear-thinning behavior with the superficial velocity. It is also observed that the liquid fingers are wider if the liquid superficial velocity is greater.

In radial flow, velocity varies with distance from the well. Knowing the effect of superficial velocity is crucial for scaling-up laboratory results to injectivity in the field. However, these effects are not described by current foam models. We scale-up the model for radial flow around an injection well. The results show the impact of non-uniform velocity in radial flow and overall injection rate on injectivity of gas and liquid in SAG.

\title{
Procter and Gamble Student poster award:
}

\section{References:}

Gong, J., S. Vincent Bonnieu, R. Z. Kamarul Bahrim et al.: Modelling of Liquid Injectivity in SurfactantAlternating-Gas Foam Enhanced Oil Recovery. Accepted for publication by SPE Journal. SPE-190435-PA (2018). Acceptance of Terms and Conditions:

Click here to agree

\section{Modeling of coupled conductive-radiative heat transfer in a 3D numerical material by a stochastic process}

Vincent Gonneau $^{1}$; Denis Rochais ${ }^{1}$; Franck Enguehard ${ }^{2}$; Gérard Louis Vignoles ${ }^{3}$

\author{
${ }^{1}$ CEA DAM Le Ripault \\ ${ }^{2}$ Institut Pprime, CNRS, Université de Poitiers, ISAE-ENSMA \\ ${ }^{3}$ LCTS, UMR 5801 CNRS
}


Corresponding Author(s): vincent.gonneau@cea.fr

In many high temperature applications, porous refractory materials (for example: alumina $\mathrm{Al} 2 \mathrm{O} 3$ or zirconia $\mathrm{ZrO} 2$ felts) have become serious candidates as insulating materials. However, the modeling of heat transfer in these porous materials requires to solve several difficulties. First of all, the complex morphology of the medium makes it very difficult to realize a mesh for finite elements or finite differences methods. Moreover, these methods require a very high computing memory and/or are time consuming. Thus, a 3D numerical voxel structure, issued from X-ray tomography for example, has been chosen to represent the material, allowing to avoid the meshing process. A second difficulty is to take into account the semi-transparent behavior of the solid phase (i.e. the $\mathrm{Al} 2 \mathrm{O} 3$ or $\mathrm{ZrO} 2$ fibers) in the modeling and to manage the conductive-radiative coupling at the voxel scale.

The aim of this study is to present a new modeling procedure of the transient conductive and radiative transfer in a numerical felt of overlapping semi-transparent (absorbing) fibers. The transient thermal conduction is described by a stochastic process based on the motion of brownian walkers. Every walker carries an elementary enthalpy and the temperature of a volume $\mathrm{V}$ is proportional to the number of walkers within it. Different boundary conditions can be prescribed like adiabatic wall, imposed thermal flux or imposed temperature. In a former work 1, the statistical distribution functions which describe the extinction, absorption and scattering phenomena within the two phases of the material were quantified. The knowledge of these functions allows to evaluate the radiative power deposited within each voxel of the spatial discretization. This modeling strategy ensures the continuity of heat flux at interfaces, and its implementation, based on an iterative algorithm updating successively temperatures and radiative powers, allows to take into account finely the presence of the internal sources due to the radiative contribution and to solve the transient coupled heat transfer equation.

Our first results show the transient temperature evolution in a felt of zirconia fibers in the case of the simulation of the flash method 2 .

\title{
Procter and Gamble Student poster award:
}

I would like to compete in the Procter and Gamble Student award References:

1 Y. Dauvois, D. Rochais, F. Enguehard, J. Taine, Statistical radiative modeling of a porous medium with semi-transparent and transparent phases : Application to a felt of overlapping fibres,International Journal of Heat and Mass Transfer 106 (2017), 601-618.

2 M. Niezgoda, D. Rochais, F. Enguehard, P. Echegut, B. Rousseau, Modeling of time-resolved coupled radiative and conductive heat transfer in multilayer semitransparent materials up to very high temperatures, Applied Physics Letters 99 (2011), 224101. Acceptance of Terms and Conditions:

Click here to agree

\section{Experimental and numerical study of gas migration through com- pacted MX-80 bentonite}

\author{
Laura Gonzalez-Blanco $^{1}$; Enrique Romero ${ }^{2}$; Paul Marschall ${ }^{3}$ \\ ${ }^{1}$ International Centre of Numerical Methods in Engineering (CIMNE) \\ ${ }^{2}$ Universitat Politècnica de Catalunya (UPC) \\ ${ }^{3}$ Nationale Genossenschaft für die Lagerung radioaktiver Abfälle (NAGRA)
}

Corresponding Author(s): laura.gonzalez.blanco@upc.edu

Understanding gas transport in saturated compacted bentonite is of great importance for the assessment of the long-term safety of engineered barriers for disposal of heat-emitting radioactive wastes. Gas migration in bentonite materials is strongly affected by the initial microstructure and state (dry density and degree of saturation) set on compaction, as well as by the stress state/history and the deformation undergone on gas injection. Particularly, the present study focuses on studying, both 
experimentally and numerically, how the heterogeneity of the sample that is created during compaction, may influence the initiation and propagation of preferential pathways for gas flow after complete saturation. These pathways are expected to develop through low-density (clay gel) zones between coarser clay aggregates.

An experimental program was launched to perform tests on statically compacted MX- 80 bentonite samples (dry density of $1.55 \mathrm{Mg} / \mathrm{m} 3$ and degree of saturation of 0.70 ) with large-size aggregates. The sample microstructure was studied using micro-computed tomography $(\mu-\mathrm{CT})$ at different states: compacted, saturated and after a gas injection and dissipation test. A high-pressure oedometer cell was used to perform the saturation and the gas tests at constant volume injection rate.

The evolution of axial strain during gas injection/dissipation tests revealed different deformation stages. The sample underwent some small expansion at the early injection stage. Once the injection system was stopped, expansion continued as a consequence of the gas pressure front propagation into the sample, which induced the pore fluid pressure to increase and the effective stress to decrease. Maximum expansion of the sample was associated with the breakthrough process, in which increasing outflow volumes were recorded. During the dissipation stage, the pore fluid pressure decreased, inducing sample compression.

$\mu$-CT images at the as-compacted state clearly indicated a grain structure made of aggregates. This initial structure did not completely extinguish during the saturation process. It appeared that the gas injection process followed the low-density pathways (clay gel) between aggregates by inducing their opening and desaturation. These results were fundamental for a correct understanding and simulation of the coupled hydro-mechanical processes occurring during gas migration through a medium with local heterogeneity (random variation of porosity affecting intrinsic permeability and gas-entry value).

The calibration between CT gray values and densities with reference materials (aluminum, distilled water and air) allowed reconstructing the local distribution of the initial dry density of the saturated sample (representative of the state before gas injection). This initial heterogeneity was implemented in the modelling approach by a random distribution of different porosities. The two-phase flow finite element Code_Bright was used for the coupled hydro-mechanical simulations. Exponential laws as a function of porosity for intrinsic permeability and parameters of the water retention curve were used. The results clearly showed that gas migration initiated and propagated due to the desaturation of paths in areas with low-density material. Good agreements between measured and simulated results were obtained in terms of the evolution of deformation, the pressures at the inlet and outlet chambers, as well as the evolution of the outflow volume.

Procter and Gamble Student poster award:

I would like to compete in the Procter and Gamble Student award References:

Acceptance of Terms and Conditions:

Click here to agree

546

\title{
The effect of water adsorption on deformation and elastic prop- erties of Bentheim sandstone
}

\author{
Alexey Yurikov ${ }^{1}$; Maxim Lebedev ${ }^{1}$; Gennady Gor ${ }^{2}$; Boris Gurevich ${ }^{1}$ \\ ${ }^{1}$ Curtin University \\ ${ }^{2}$ New fersey Institute of Technology
}

Corresponding Author(s): gor@njit.edu

Adsorption of water in nano-porous materials and rocks (concrete, shales, coal, etc.) causes deformation of such media (e.g., Gor et al., 2017; Yurikov et al., 2018). Additionally, adsorption of water leads to variations in elastic properties of natural rocks (e.g., Pimienta et al., 2014; Mikhaltsevich et al., 2017; Yurikov et al., 2018). For example, Yurikov et al. (2018) showed that adsorption of water in Opalinus shale results in drastic decrease of the shear moduli and swelling of the sample. Similar effects of reduction of elastic moduli with adsorption of water are also observed in sandstones (e.g., Pimienta et al., 2014), rocks that conventionally are not considered as nano-porous. However, sandstones contain compliant pores at grain contacts, which may be of nanometer scale. Therefore, 
water adsorbed in these pores can produce effects, which are similar to those observed in nanoporous shales and clays. In order to validate this, we conduct simultaneous measurements of the elastic moduli and deformation of the Bentheim sandstone with adsorption of water.

The Bentheim sandstone used in the experiment is a nearly isotropic and homogeneous rock composed by $95 \%$ quartz, $3 \%$ kaolinite, and $2 \%$ orthoclase. We regulate adsorption processes by maintaining the sample in the atmosphere with controlled relative humidity $(\mathrm{RH})$. Elastic properties of the sample are measured using ultrasonic pulse transmission technique. Deformation of the sample caused by changes in $\mathrm{RH}$ is measured with a semiconductor strain gauge. Transition of the sample from the driest state $(\mathrm{RH}=13 \%)$ to the wettest state $(\mathrm{RH}=97 \%)$ leads to increase of saturation from 0.1 to $1-2 \%$. The measured bulk and shear moduli of the sample decrease with increasing saturation exhibiting the difference between the driest and the wettest states of $\sim 20 \%$. The strain caused by adsorption of water in the sample is of order of $10^{-4}$.

The effects observed in the Bentheim sandstone can be of similar nature as the effects observed in nano-porous shales. Deformation and reduction of the elastic moduli of the sample can be caused by changes in pressure in the fluid adsorbed in compliant nano-pores at grain contacts. We estimate that such change in the fluid pressure should be of several megapascals to cause the observed deformation. If the change in fluid pressure is a reason of observed effects, the measured variation in elastic moduli should be consistent with stress-dependency of the elastic moduli of the sandstone measured, for example, in a triaxial cell. We compare the measured variations in the bulk and shear moduli related to the estimated change in the fluid pressure against dependencies of the moduli of the Bentheim sandstone on the confining pressure applied in a triaxial cell. The two sets of measurements show broadly similar trends at low pressures. This confirms that adsorption-induced deformation and variations in the moduli of the sandstone can be explained by changes in the pressure of fluid confined in compliant pores at grain contacts.

\section{Procter and Gamble Student poster award:}

\section{References:}

Gor, G. Y., Huber, P., \& Bernstein, N. (2017), Adsorption-induced deformation of nanoporous materials - A review:Applied Physics Reviews, 4, 011303.

Mikhaltsevich, V., Lebedev, M., \& Gurevich, B. (2017), Effect of water saturation on P-wave anisotropy in the Mancos Shale at seismic frequencies: 79th EAGE Conference and Exhibition, France, Extended Abstracts, Tu P3 11, https://doi.org/10.3997/2214-4609.201701042.

Pimienta, L., Fortin, J., \& Guéguen, Y. (2014), Investigation of elastic weakening in limestone and sandstone samples from moisture adsorption: Geophysical Journal International, 199(1), 335-347.

Yurikov A., Lebedev, M., Pervukhina, M., \& Gurevich, B. (2018), Water retention effects on elastic properties of Opalinus shale: Geophysical Prospecting, Special Issue, doi: 10.1111/1365-2478.12673. Acceptance of Terms and Conditions:

Click here to agree

\section{Modeling Dispersion in Pore Networks: Comparison of Pore-Scale Model Accuracy}

Jeff Gostick $^{1}$; Mehrez Agnaou ${ }^{1}$; Jake Baralett ${ }^{2}$; Amin Sadeghi ${ }^{2}$

\footnotetext{
${ }^{1}$ University of Waterloo

${ }^{2}$ McGill University
}

Corresponding Author(s): jgostick@uwaterloo.ca

Mass transfer in porous media resulting from dispersion occurs in a wide variety of applications such as water treatment, flow batteries, flow in aquifers, enhanced oil recovery, and packed-bed reactors. The underlying mechanisms of dispersion are the molecular diffusion superimposed on the advective transport induced by the fluid flow. Many efforts have been made to model dispersion in porous media. Macroscopic continuum models require the knowledge of effective properties (ex. the dispersion tensor) whose evaluation may be a numerically intensive task. On the other hand, modeling dispersion in pore networks can be performed at a relatively lower computational cost. At 
the pore-scale, based on the assumption that dispersion between neighbor pores can be described by the convection-diffusion equation, several discrete pore-scale models have been employed.

In this work, three novel models were derived and their accuracy was compared to existing approaches. The derivations are based on a spatial discretization of the convection-diffusion equation using the hybrid and power-law finite difference schemes and the exact solution of the onedimensional convection-diffusion equation. Perfect mixing at the pore space is assumed and the Hagen-Poiseuille model is used to describe the incompressible flow of a Newtonian fluid in the creeping regime, where the local Reynolds number is well below unity.

Finally, considering dispersion problems over arbitrary porous structures, the developed pore-scale discrete models were validated through comparisons with direct numerical simulations using a finite element solver. We show that under a wide range of dispersion regimes, the relative error (with respect to finite element results) introduced by the power-law and exact solution-based models is consistently below $1 \%$, whereas the existing models show up to $10 \%$ of relative error, depending on the dispersion regime. Furthermore, the impact of including the Taylor-Aris effect in modeling dispersion in pore networks is discussed for the different pore-scale models considered in this study.

Procter and Gamble Student poster award:

I don't want to compete References:

Acceptance of Terms and Conditions:

Click here to agree

\title{
Influence of Charge-Coupled Diffusion and Non-Linear Reaction Kinetics on the Dissolution of Carbonate Rocks: Pore-scale Mod- elling and Experiments
}

\author{
Author(s): Farrel Gray ${ }^{1}$ \\ Co-author(s): Benaiah Anabaraonye ${ }^{1}$; John Crawshaw ${ }^{1}$ \\ ${ }^{1}$ Imperial College London
}

Corresponding Author(s): farrel.gray@imperial.ac.uk

Reactive transport phenomena play an important role in the transport of sequestered $\mathrm{CO} 2$, since flow and storage properties of rocks can be dramatically altered by dissolution and precipitation during the injection of acidic $\mathrm{CO} 2$-rich brine. The resulting effect on the rock structure is determined by the interplay of a number of physical and chemical processes occurring at the pore-scale which require sophisticated numerical models to be able to predict.

In this contribution, we present a comprehensive pore-scale reactive transport model which couples flow using the lattice Boltzmann method, chemical transport of multiple species using a finitevolume method, reactions between aqueous species, and mineral surface reactions. Time-dependent changes in the mineral geometry due to dissolution are captured 1 . We also include two further physical and chemical models: a charge-coupled diffusion model using the Nernst-Plank equation, and non-linear saturation kinetics. The effects of these models are considered by comparison with experiment in both simple calcite channel systems, and in large 3D images of rock pore-spaces.

In many pore-scale reactive flow modelling studies, the diffusion coefficients of all chemical species are set to the same value, which ensures an electrically neutral solution, however fails to capture charge-migration effects. In our pore-scale model, we include the effects of ion charges on diffusion, which allows chemical components to diffuse at different rates, whilst maintaining electroneutrality. We then examine how this affects overall dissolution rates in cases of two acids: $\mathrm{HCl}$ and carbonic acid as $\mathrm{CO} 2$-saturated solution. For the case of $\mathrm{HCl}$ acid injected into calcite channels, we show this leads to large differences in reactant transport rates compared to the use of a single constant diffusion 
coefficient for all species. Use of the charge-coupled model strongly affects the resulting dissolution rate, and leads to better agreement with corresponding experiments. For the case of CO2-saturated brine, use of the charge-coupled diffusion model leads to only slight changes in overall dissolution rate compared to the use of a constant diffusion coefficient. This is understood by considering the charge interaction of the reactant and product ions with the brine solution.

Then we consider how non-linear reaction kinetics affect overall reaction rates. In many cases, a linear dependence on saturation state is used. However, according to recent batch reactor experiments, the carbonate system reaches saturation in a highly non-linear way 2 . Here we use the saturation model of Anabaraonye et al. 2. We find that using the non-linear model leads to considerable differences to the overall dissolution rate of $\mathrm{CO} 2$-saturated brine systems, both in simple channel flow experiments and during injection into a real calcite rock, when compared to linear saturation models. Comparisons with the corresponding experiments are again shown and discussed.

Our results point to the need to include the effects charge coupled diffusion and non-linear reaction kinetics to more accurately model experiments.
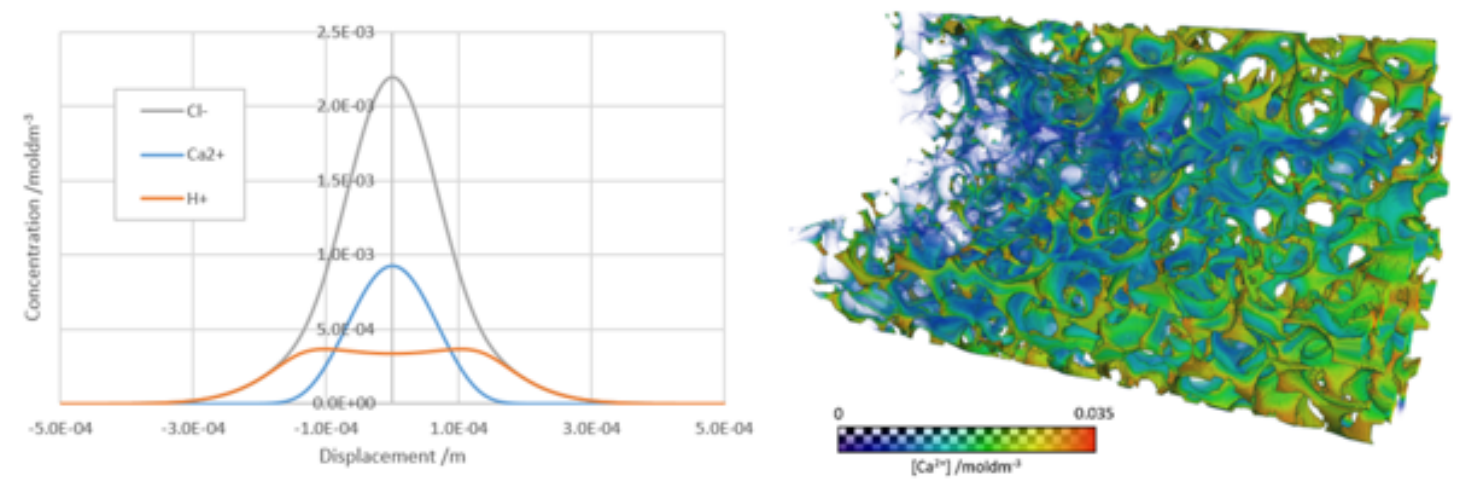

Figure 4: Figure 1

Figure 1 - Left: charge-coupled diffusion processes lead to electrochemical migration effects in systems with multiple ions; right: the calcium ion distribution inside a carbonate rock sample undergoing dissolution during the injection of $\mathrm{CO} 2$-saturated brine solution.

Procter and Gamble Student poster award:

I don't want to compete References:

1. Gray, F., et al., Chemical mechanisms of dissolution of calcite by $\mathrm{HCl}$ in porous media: Simulations and experiment. Advances in Water Resources, 2018. 121: p. 369-387.

2. Anabaraonye, B.U., Experimental and Modelling Studies of Reservoir Mineral Dissolution Following Carbon Dioxide Injection, in Department of Chemical Engineering. 2017, Imperial College London.

Acceptance of Terms and Conditions:

Click here to agree

661

\section{Thermal conductivity of partially sintered oxide ceramics predicted via cross-property relations and measured via laser flash}

Eva Gregorová ${ }^{1}$; Willi Pabst ${ }^{1}$; Vojtěch Nečina ${ }^{2}$; Tereza Uhlířová ${ }^{2}$

\footnotetext{
${ }^{1}$ University of Chemistry and Technology, Prague

${ }^{2}$ UCT Prague
} 
Corresponding Author(s): eva.gregorova@vscht.cz

It is well known that the thermal conductivity of partially sintered ceramics, which may be viewed as model systems for porous sedimentary rocks, is much lower than predicted by analytical models based on the single-inclusion solutions for spherical pores [1-3]. Although so-called "minimum solid area models" have been proposed in order to account for these low thermal conductivity values [4], it has been shown recently that these models are not useful and should be avoided 5. However, an alternative model is not available, and only rough estimates can be given when the bulk density of the ceramic powder compact before firing is known [6]. In this contribution we present experimental data obtained for partially sintered oxide ceramics via the laser-flash method and confront them with predictions via so-called cross-property relations, in which a prior knowledge of the relative elastic properties (Young's modulus) is used to predict the relative thermal conductivity. We show that our cross-property relation between Young's modulus and thermal conductivity [7] can indeed predict the thermal conductivity reasonably well, without the need for any additional information on the microstructure.

\section{Procter and Gamble Student poster award:}

I don't want to compete References:

1. Pabst W., Gregorová E.: Elastic and thermal properties of porous materials - rigorous bounds and cross-property relations (Critical assessment 18), Mater. Sci. Technol. 31 (15), 1801-1808 (2015).

2. Pabst W., Uhlírová T., Gregorová E., Wiegmann A.: Young's modulus and thermal conductivity of model materials with convex or concave pores - from analytical predictions to numerical results, J. Eur. Ceram. Soc. 38 (7), 2694-2707 (2018).

3. Uhlířová T., Nečina V., Pabst W.: Modeling of Young's modulus and thermal conductivity evolution of partially sintered alumina ceramics with pore changes from concave to convex, J. Eur. Ceram. Soc. 38 (8), 3004-3011 (2018).

4. Rice R. W.: Porosity of Ceramics. Marcel Dekker, New York (1998).

5. Pabst W. Gregorová E.: Minimum solid area models for the effective properties of porous materials - a refutation, Ceram. Silik. 59 (3), 244-249 (2015).

6. Gregorová E., Pabst W., Sofer Z., Jankovský O., Matějíček J.: Porous alumina and zirconia ceramics with tailored thermal conductivity, J. Phys. Conf. Series 395, 012022, 8 pp. (2012).

7. Pabst W., Gregorová E.: A cross-property relation between the tensile modulus and the thermal conductivity of porous materials, Ceram. Int. 33 (1), 9-12 (2007).

Acceptance of Terms and Conditions:

Click here to agree

\section{Analysis on the existing state of pore water in Gas Hydrate Reser- voir}

Wen Guan ${ }^{\text {None }}$

Corresponding Author(s): 1701210249@pku.edu.cn

Temperature is a factor affecting nuclear magnetic resonance (NMR) relaxation. However, most of the current NMR measurements are limited to room temperature, so the study about of how temperature affected the NMR relaxation of saturated water deposit and the existance of pore water is meaningful. In this talk, we first saturated the sediment of the hydrate reservoir with deionized water, and placed the sample in an NMR device for temperature-changing measurement. During the whole process, the experimental temperature increased gradually from $-10^{\circ} \mathrm{C}$ to $25^{\circ} \mathrm{C}$.

In the experiment, T2 spectrum showed two distinct peaks and the amplitude of left peak signal was stronger than the right peak. This phenomena indicated that the pores in the sediment were mainly micropores and mesopores, with a few fractures. 
When temperature increased geadually from $-10^{\circ} \mathrm{C}$ to $-1{ }^{\circ} \mathrm{C}$, the increase in fluidity of water in micropores and mesopores was larger than that in macropores, and the peak intensity of water in micropores and mesopores was increasing all the time. These phenomena all indicated that the phase transition of water was severe, and the ice first melt in the micropores and mesopores.

When the temperature increased gradually from $-1^{\circ} \mathrm{C}$ to $25^{\circ} \mathrm{C}$, the $\mathrm{T} 2$ spectrum was stable throughout the process. The left peaks had little change, while the right peak changed slightly, which indicated that water in mesopores and macropores had not reached the stable state, so the volume of fluid increased irregularly.

Except the NMR, we also used X-ray computed tomography (X-ray CT) and nitrogen adsorption method to characterize the pore structures. we got the 3D models of porosity distribution by X-ray $\mathrm{CT}$, which can reflect the pore morphology and space configuration. However it was difficult for us to choose the parameters of threshold segmentation in CT image processing, and the result of the reconstruction will be affected by the large selection of parameters. Finally, we combined with nitrogen adsorption method to quantitatively characterize the pore size. The combination of the three methods can characterize the pore structure more accurately and comprehensively, which can help to get better pore structure parameters of the samples.

Procter and Gamble Student poster award:

I would like to compete in the Procter and Gamble Student award References:

Acceptance of Terms and Conditions:

Click here to agree

91

\title{
Pore-scale characterization of velocity probability distributions in partially saturated porous media
}

\author{
Author(s): Gael Raymond Guedon ${ }^{1}$ \\ Co-author(s): Fabio Inzoli ${ }^{1}$; Monica Riva ${ }^{1}$; Alberto Guadagnini ${ }^{1}$ \\ ${ }^{1}$ Politecnico di Milano
}

Corresponding Author(s): gaelraymond.guedon@polimi.it

We investigate the key characteristics of steady-state pore-scale velocity probability distributions in the presence of multiple immiscible fluid phases flowing in heterogeneous porous spaces. We focus on the identification of the scaling behavior of low and high velocities in natural rocks, which is critical to the characterization of anomalous transport phenomena occurring in a variety of natural and engineered processes. For instance, strong tailing affecting high velocities leads to non-Fickian contaminant transport and early breakthrough, while the scaling behavior of low velocities influences late time spreading of fluid particles and mixing processes. It is well established that fluid flow and transport in porous media are direct consequences of the underlying pore structure. However, relating the statistics of the complex pore geometry with major features of fluid flow and transport is still an open challenge. Here, we consider unsaturated media (i.e., systems within which two or more fluid phases coexist) and focus on numerical analyses of immiscible two-phase flow processes leading to a stationary spatial distribution of the fluids. We consider three-dimensional pore spaces and use a finite volume-based solver to determine the pore-scale velocity probability distributions of the flowing fluid phases. We compare the results achieved for diverse wettability conditions in such settings and use data from fully saturated (single-phase) simulations as a reference for the analysis. Our results suggest the existence of power-law scaling behavior in the low velocity range, heavy tails following stretched exponential laws being observed in the high velocity range. These heavy tails appear to depend on the spatial distribution of the phases. The latter, in turn, depends on phase wettability and on the flow history. A non-wetting fluid phase tends to remain trapped in the form of ganglia, obstructing pores and throats otherwise contributing to preferential paths. This contributes to modifying the spatial distribution of high velocity channels which can be observed under singlephase flow. Otherwise, a wetting fluid phase is likely to fill the minute crevices and corners of the porous medium, yielding a less pronounced modification of the topology of preferential paths. Flow history also influences the spatial patterns observed during fluid-fluid displacements. For instance, dead end pores will likely remain filled with the phase initially present in the pore space. Otherwise, 
pore spaces associated with high entry pressure will be invaded by the displacing fluid under favorable local pressure gradient and depending on the relative strength between capillary and viscous forces (as driven by boundary conditions). For a given degree of saturation of a fluid phase, differing flow features can then be observed depending on whether the trapped phases are distributed in isolated pores or obstruct channels otherwise participating to preferential pathways.

Procter and Gamble Student poster award:

References:

Acceptance of Terms and Conditions:

Click here to agree

260

\title{
Impacts of brine and reservoir properties on the transport of HPAM solutions in low permeability porous media
}

\author{
Imane Guetni $^{1}$; Claire Marlière ${ }^{2}$; David Rousseau ${ }^{2}$; Isabelle Bihannic ${ }^{3}$; Manuel Pelletier $^{3}$; Frédéric Villiéras ${ }^{3}$ \\ ${ }^{1}$ IFP Energies nouvelles, Rueil-malmaison and Université de Lorraine, CNRS, Laboratoire Interdisciplinaire des Envi- \\ ronnements Continentaux, Nancy \\ ${ }^{2}$ IFP Energies nouvelles \\ ${ }^{3}$ Université de Lorraine, CNRS, Laboratoire Interdisciplinaire des Environnements Continentaux, Nancy
}

Corresponding Author(s): imane.guetni@ifpen.fr

Chemical EOR is now considered as an attractive option for low permeability reservoirs, in particular where lack of gas supply does not allow gas injection processes. However, its application can be challenging for permeabilities below $100 \mathrm{mD}$ as poor injectivity and high chemical retention are frequently observed. This work aimed at investigating the impact of both chemical and mineralogical parameters on the transport of polymer solutions in well-controlled low permeability porous media. Solutions of partially hydrolyzed polyacrylamide (HPAM) in waters of variable ionic strengths and hardnesses were injected in granular sand and clays packs having similar petrophysical characteristics (permeability range $6080 \mathrm{mD}$ ) but variable and well controlled mineralogical compositions of quartz and various clays (kaolinite, illite and smectite investigated separately). Specific preparation methodologies were designed to make these porous media homogenous and reproducible. Their textural properties were characterized in terms of structure (SEM), and specific surface area and porosity (BET). The main observables of the tests were the mobility and permeability reductions generated by the polymer and its irreversible retention.

Viscometric analysis showed that the HPAM solutions intrinsic viscosity decreases with increasing total salinity, as expected from charge screening, with a sharp decrease in presence of divalent cations, even at low ionic strength, which was less expected. Injection experiments revealed that polymer retention expressed by unit surface area, mobility and permeability reductions increase significantly: (a) with increasing ionic strength and hardness for porous media of a given mineralogical composition; (b) in presence of clays, even at low ionic strength and hardness.

Assuming that polymer retention originated in polymer adsorption, irreversible permeability reductions were translated into adsorbed layer thicknesses according to a straightforward capillary bundle model. This allowed discussing the results in terms of adsorbed layer density, which was showed to increase with increasing brine hardness and to be lower in presence of illite and smectite than kaolinite and pure quartz.

The results regarding the impact of hardness are consistent with the outcomes of the viscometric study and confirms the major role played by hardness, not only on the polymer conformation but also on the polymer-minerals interactions.

The results obtained with the various clays were more intriguing. For the porous media containing illite and smectite, we observed in SEM images the formation of clay aggregates. In these cases, the layer densities as well as the polymer irreversible retention were lower than with kaolinite. The interpretation proposed is that polymer molecules cannot enter the inside of the clays aggregates and only interact with their outer surface and that the concept of layer density was not relevant to describe these situations. 
This systematic study allowed dissociating the impacts of salinity, hardness and clay contents/types on the transport properties of polymer solutions in low permeability porous media. These results present new insights on the versatile polymer - clays interactions during flow in confined geometries. They should help building accurate predictive models for the transport of polymer solutions in porous media with challenging properties for chemical EOR.

Procter and Gamble Student poster award:

References:

Acceptance of Terms and Conditions:

Click here to agree

\title{
Permeability in different scales comparing Darcy and Darcy-Brinkman- Stokes (DBS)
}

Sonia Gumbau Rodriguez ${ }^{1}$; Daniel Fernàndez-Garcia ${ }^{2}$; Xavier Sanchez-Vila ${ }^{3}$

\footnotetext{
${ }^{1}$ University Politecnic Catalonia

${ }^{2}$ Universitat Politècnica de Catalunya

${ }^{3}$ University Politecnica de Catalunya
}

Corresponding Author(s): soniagumbau@gmail.com

The calculation of the permeability is difficult of getting in small scale, for this reason there is a discussion about which method is better and until which dimension is useful. The target is to obtain values of the permeability from different methods and apply them in upscaling scenarios moving from micron to millimeter scale. The investigation is based on samples of Imperial Colleague of London. They apply a technique called X-ray micro tomography (XMT), which consist of taking 2D images of the sample from different angles and processing them to reconstruct a detailed 3D image. The advantages of the XMT are that technique is not-invasive and non-destructible and you can obtain a resolution from 0.5 micron to 5 microns, which allows to obtain $3 \mathrm{D}$ images of objects that measure from $1 \mathrm{~mm}$ to $10 \mathrm{~mm}$. The study is based on a sandpack with a $37 \%$ of porosity, where Imperial Colleague have apply Navier Stokes equations to calculate velocities and pressure fields for single-phase incompressible flow. Our particular sample is called LVC60 is a block of 300 voxels, each voxel has a dimension of 10 micron, and the flow is only in the direction $\mathrm{X}$, the other sides are impermeable and the boundary condition is a gradient of pressure of value 1 . In global, it has a permeability in the direction X of $3.41806 \mathrm{e}-11 \mathrm{~m} 2$, the aim of our investigation is compare the results of the methods: Darcy and Darcy-Brinkman-Stokes (DBS) to examine in which scale method is better than Darcy or in which scale we can use each method. It is expected obtain a comparative of the upscaled permeability using their probability density functions (pdf).

Procter and Gamble Student poster award:

I would like to compete in the Procter and Gamble Student award References:

Acceptance of Terms and Conditions:

Click here to agree

\section{Pore Network Modeling of Mixed-Wet Carbonates Based on Pore Classification and Fluid Continuity}

\author{
Author(s): Asli S. Gundogar ${ }^{1}$ \\ Co-author(s): Serhat Akin ${ }^{2}$; Cynthia M. Ross ${ }^{3}$; Anthony R. Kovscek ${ }^{3}$
}




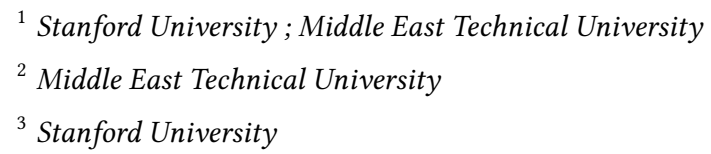

Corresponding Author(s): asligun@stanford.edu

A pore network model approach is used to investigate the interplay between structural and wettability heterogeneities on macroscopic capillary pressure $(\mathrm{Pc})$ and relative permeability (kr) functions. The scope of this study is to construct structurally representative pore networks of Middle East carbonates and to develop a capillary-dominated two-phase flow simulator for a complete primary drainage-secondary imbibition-secondary drainage flooding cycle under arbitrary wettability conditions. In comparison with conventional pore networks, multiscale pore structure characterization and pore-throat classification approaches are utilized. This study is carried out using carbonate samples from a giant Middle East carbonate oil field. Pore system properties determined from scanning electron microscopy (SEM) at multiple-magnifications (75X and 750X) and high-pressure mercury intrusion porosimetry (MIP) are used as network inputs. Mosaics constructed from overlapping 75X views reveal abundant evidence of unresolved microporosity within the carbonate samples. After renormalizing $750 \mathrm{X}$ porosities to compensate for the missing large pore areas in the whole carbonate thin sections, the low and high magnification data are integrated into a multiscale pore size distribution. Six distinct pore-throat classes, identified by relating mercury-volume intruded through throats to the corresponding SEM pore-area, are implemented in the proposed model. Model-derived throatsize distributions are calibrated by experimental MIP data. In flow simulations, all possible generic fluid configurations in irregular triangle-shaped pore cross-sections are evaluated with appropriate pore-scale physics and wettability description. Model results display a significant dependency on pore structure, wettability and saturation history. In primary drainage, the smallest-sized throats $(\leq 0.711 \mu \mathrm{m})$ covering microporous regions of bimodal MIP-curves make a major contribution to initial water saturation. Carbonate networks with larger microporosity are found to have lower absolute permeability. Incorporating wettability-alteration and contact-angle hysteresis in the model, different mixed-wettability levels varying from strongly-water-wet to strongly-oil-wet cases are realized in the consecutive floods. Wetting films and intermediate layers on the corners of mixed-wet pores allow better fluid connectivity and accordingly less trapping. Corner water saturation accounts for no more than $3 \%$ of total pore volume despite its significant role in water continuity. Oil recovery increases with oil-wetness through inhibited snap-off and further oil layer formation. There is an apparent coincidence between saturation trends of intermediate oil and water layers during secondary drainage process. The continuity of a phase is deduced to be critical for its relative permeability rather than its abundance in the porous system. The hysteresis between primary drainage-imbibition-secondary drainage Pc and $\mathrm{kr}$ curves grows with the increase in oil-wetness of the medium. Simulator predictions are consistent with complex experimental hysteresis trends and micromodel observations on carbonates. Integrating with realistic pore-networks, our newlydeveloped model succeeds to represent experimentally difficult-to-measure Pc and kr curves with their hysteresis loops and residual saturation trends in mixed-wet carbonates.

Procter and Gamble Student poster award:

References:

Acceptance of Terms and Conditions:

Click here to agree

\title{
Peltier Heat of porous LiFePO4 electrodes in a thermoelectric cell
}

\author{
Author(s): Astrid F. Gunnarshaug ${ }^{1}$ \\ Co-author(s): Frank Richter ${ }^{2}$; Odne S. Burheim ${ }^{3}$; Preben J. S. Vie ${ }^{4}$; Signe Kjelstrup ${ }^{1}$ \\ ${ }^{1}$ PoreLab, Department of Chemistry, Norwegian University of Science and Technology \\ ${ }^{2}$ Department of Chemistry, Norwegian University of Science and Technology \\ ${ }^{3}$ Department of Process and Energy, Norwegian University of Science and Technology
}


${ }^{4}$ Institute for Energy Technology

Corresponding Author(s): astrid.f.gunnarshaug@ntnu.no

Temperature is known to affect ageing and performance of lithium ion batteries 1 . We need to know the heat sources in the electrode compartments in order to accurately model internal temperature profiles. Reports of reversible heat effects local to electrode surfaces in lithium-ion batteries are therefore important, but are scarce in literature 2. The reversible heat production at a battery electrode interface is investigated in this experimental work, measuring the Peltier heat of the electrode LiFePO4 electrode. The Peltier heat at an electrode can be found by measuring the change in electric potential across the cell with two identical electrodes when a temperature difference is applied 3. We present the Seebeck coefficient and Peltier heat of a symmetric pouch cell consisting of two commercial porous LiFePO4 electrodes and a 1M LiPF6 and Ethylene Carbonate (EC) /Diethyl Carbonate (DEC) (50:50 w\%) electrolyte-soaked separator. The electrodes have been thermostatted to the wanted temperatures $\mathrm{T}$ and $\mathrm{T}+\Delta \mathrm{T}$.

$\mathrm{Al}(\mathrm{s}, \mathrm{T})|\mathrm{LiFePO} 4(\mathrm{~s}, \mathrm{~T}) \mathrm{EC}, \mathrm{DEC}, \mathrm{LiPF} 6| \mathrm{LiFePO} 4(\mathrm{~s}, \mathrm{~T}+\Delta \mathrm{T}) \mid \mathrm{Al}(\mathrm{s}, \mathrm{T}+\Delta \mathrm{T})$

We show that the Peltier heat effect is time dependent due to a build up of concentration gradients within the cell, and the establishment of a Soret equilibrium. The steady state value is obtained after 2-3 days. The Peltier heat of the LiFePO4 electrode will be compared to that reported for $\mathrm{LiCoO} 2$, see ref. 2, and its consequences for the battery will be discussed.

Acknowledgment

The authors are grateful to the Research Council of Norway through its Centres of Excellence funding scheme, project number 262644, PoreLab, and project no. 228739 SafeLiLife as well as to ENERSENSE, NTNU.

\title{
Procter and Gamble Student poster award:
}

I would like to compete in the Procter and Gamble Student award References:

1 J. Vetter, P. Novak, M. Wagner, C. Veit, K.-C. Möller, J. Besenhard, M. Winter, M. Wohlfahrt-Mehrens, C. Vogler,' A. Hammouche, Ageing mechanisms in lithium-ion batteries, J. Power Sources 147 (12) (2005) $269-281$.

2 Frank Richter, Astrid Gunnarshaug, Odne Stokke Burheim, Preben J. S. Vie, and Signe Kjelstrup. Single Electrode Entropy Change for LiCoO2 Electrodes. ECS Trans., 80(10):219-238, 2017.

3 K. S. Førland, T. Førland, and S. Kjelstrup. Irreversible Thermodynamics: Theory and Applications. Tapir, Trondheim, 2001. Acceptance of Terms and Conditions:

Click here to agree

\section{Thermal analysis and design optimization of multi-layer insula- tion with fiber-reinforced aerogel and reflective screen}

\author{
Jiangfeng Guo ${ }^{1}$; Guihua Tang ${ }^{2}$; Jian Feng $^{3}$; Bo Fu $^{1}$; Yuan $\mathrm{Ma}^{1}$ \\ ${ }^{1}$ Xi'an fiaotong University, PR China \\ ${ }^{2} X i$ 'an fiaotong University \\ ${ }^{3}$ National University of Defense Technology, PR China
}

Corresponding Author(s): 89184606@qq.com

Thermal analysis and design optimization of multi-layer insulation with fiber-reinforced aerogel and reflective screen

Jiang-Feng Guo1, Gui-Hua Tang1, Jian Feng2, Bo Fu1, Yuan Ma1

1. Key Laboratory of Thermo-Fluid and Science and Engineering, Xi'an Jiaotong University, Xi'an 710049, PR China

2. Science and Technology on Advanced Ceramic Fibers and Composites Laboratory, National University of Defense Technology, Changsha 410073, PR China 


\begin{abstract}
ABSRACT: Fiber-reinforced silica aerogel has been widely used in high temperature insulation field due to its higher mechanical property and thermal insulation capability in high temperature compared to pure silica aerogel. The heat transfer character of fiber-reinforced silica aerogel is numerically investigated in this work. We determine the optimal temperature-dependent size for silica fiber in composite aerogel by combining the spectral extinction coefficient with blackbody radiation. The optimal temperature-dependent doping amount is obtained by minimizing the effective thermal conductivity. Moreover, numerical model of steady heat transfer is established to obtain the effective thermal conductivity of multi-layer thermal insulation (MTIM) comprised of fiber-reinforced aerogel and reflective screen. The Finite Volume method and Discrete Ordinate method are employed to consider the combined conduction and radiation heat transfer in this model, respectively. Based on the obtained temperature-dependent optimal parameters of fiber-reinforced silica aerogel, the optimal number of reflecting screens and thickness of each insulation layer could be obtained in different temperatures when the screen distribution character in multilayer thermal insulation is determined. The screen distribution with unvaried thickness and unvaried temperature drop are also numerically investigated with present model. The results indicate that: (1) The optimal fiber particle diameter decreases with the increase in temperature and lager fiber doping volume fraction is more appropriate for higher temperature in fiber-reinforced aerogel; (2) optimal number of reflecting screens increases with the increase in temperature, and optimal thickness of each insulation layer increases with the increase in temperature; (3) Screen distribution with unvaried temperature drop has higher thermal insulation property than the unvaried thickness, and putting the screens at the middle of the MTIM can enhance the thermal insulation performance of the MTIM successfully compared to putting the screens at the top and bottom of the MTIM. The measured back temperature curves of MTIM in the experiment qualitatively verify the optimization predictions. The conclusions can provide useful guidance for improving heat-insulating property of fiber-reinforced aerogel and MTIM in engineering.
\end{abstract}

Procter and Gamble Student poster award:

I would like to compete in the Procter and Gamble Student award References:

Acceptance of Terms and Conditions:

Click here to agree

971

\title{
Permeation and separation of $\mathrm{CH} 4 / \mathrm{CO} 2$ mixtures through single- layer nanoporous graphene membranes : theory and molecular simulations.
}

\author{
Juncheng GUO $^{\text {None }}$; Romain Vermorel ${ }^{1}$; Guillaume Galliero $^{2}$ \\ ${ }^{1}$ LFCR, E2S-UPPA \\ ${ }^{2}$ Université de Pau et des Pays de l'Adour, France
}

Corresponding Author(s): juncheng.guo@etud.univ-pau.fr

Nanoporous graphene membranes are gaining attention in the field of water desalination 1 and gas separation 2. In the field of gas separation, perm-selective membranes technology consumes less energy than other conventional technologies. Due to nanoporous graphene's atomic thickness and controllable pore size in molecular diameter, it is considered as the most favorable membrane material showing a high selectivity. For instance, in the context of natural gas production, the separation of $\mathrm{CH} 4 / \mathrm{CO} 2$ mixtures would greatly benefit from these new materials. With the rapid development in graphene fabrication technology, breakthroughs in nanoporous graphene membranes are expected in the next few years and quite sufficient data can be found in publications. However, there is no accurate theory with which we can predict gas permeation and separation factor quantitively.

In most researches, analytical frameworks are expressed using Arrhenius-type equations which assume activated diffusion is the permeation mechanism. Nevertheless, recent work from our group 3 shows that Arrhenius-type equations are too simple to predict the diffusive transport coefficient correctly. The authors performed molecular dynamics simulations on a simplified model of single-layer 
porous membranes. They found that the transport coefficient is related to a well-defined accessible porosity and a thermodynamic factor, which can be computed from the potential of mean force (PMF) between the membrane atoms and the permeating gas molecules. Simulated transport coefficients are in good agreement with the predictions of this theory.

In this work, we show how to extend and apply this theoretical model to more realistic molecular models of nanoporous graphene membranes. By means of Equilibrium (EMD) and Non Equilibrium (NEMD) molecular dynamics simulations, we explore the permeation and separation of $\mathrm{CH} 4 / \mathrm{CO} 2$ mixtures through porous graphenes exhibiting different pore sizes and geometry. Furthermore, we investigate the effect of thermodynamic conditions (pressure, temperature, mixture composition) on the diffusive transport coefficients. We will report our latest results and compare simulation data to the extended theoretical model.

\title{
Procter and Gamble Student poster award:
}

I would like to compete in the Procter and Gamble Student award References:

1. Cohen-Tanugi, D.; Grossman, J. C. Water Desalination across Nanoporous Graphene. Nano Lett. 2012, $12,3602-3608$.

2 Yuan, Z. et al. Mechanism and prediction of gas permeation through sub-nanometer graphene pores: comparison of theory and simulation. ACS Nano 2017, 11, 7974-7987.『3 Oulebsir, F.; Vermorel, R.; Galliero, G. Diffusion of Supercritical Fluids through Single-Layer Nanoporous Solids: Theory and Molecular Simulations. Langmuir 2018, 34, 2, 561-571. Acceptance of Terms and Conditions:

Click here to agree

\section{Permeation and separation of $\mathrm{CH} 4 / \mathrm{CO} 2$ mixtures through single- layer nanoporous graphene membranes : theory and molecular simulations.}

\author{
Juncheng Guo $^{1}$; Romain Vermorel $^{1}$; Guillaume Galliero $^{1}$ \\ ${ }^{1}$ LFCR, E2S-UPPA, University of Pau and Pays de l'Adour
}

Corresponding Author(s): juncheng.guo@etud.univ-pau.fr

Nanoporous graphene membranes are gaining attention in the field of water desalination 1 and gas separation 2. In the field of gas separation, perm-selective membranes technology consumes less energy than other conventional technologies. Due to nanoporous graphene's atomic thickness and controllable pore size in molecular diameter, it is considered as the most favorable membrane material showing a high selectivity. For instance, in the context of natural gas production, the separation of $\mathrm{CH} 4 / \mathrm{CO} 2$ mixtures would greatly benefit from these new materials. With the rapid development in graphene fabrication technology, breakthroughs in nanoporous graphene membranes are expected in the next few years and quite sufficient data can be found in publications. However, there is no accurate theory with which we can predict gas permeation and separation factor quantitively.

In most researches, analytical frameworks are expressed using Arrhenius-type equations which assume activated diffusion is the permeation mechanism. Nevertheless, recent work from our group 3 shows that Arrhenius-type equations are too simple to predict the diffusive transport coefficient correctly. The authors performed molecular dynamics simulations on a simplified model of single-layer porous membranes. They found that the transport coefficient is related to a well-defined accessible porosity and a thermodynamic factor, which can be computed from the potential of mean force (PMF) between the membrane atoms and the permeating gas molecules. Simulated transport coefficients are in good agreement with the predictions of this theory.

In this work, we show how to extend and apply this theoretical model to more realistic molecular models of nanoporous graphene membranes. By means of Equilibrium (EMD) and Non Equilibrium (NEMD) molecular dynamics simulations, we explore the permeation and separation of $\mathrm{CH} 4 / \mathrm{CO} 2$ mixtures through porous graphenes exhibiting different pore sizes and geometry. Furthermore, we 
investigate the effect of thermodynamic conditions (pressure, temperature, mixture composition) on the diffusive transport coefficients. We will report our latest results and compare simulation data to the extended theoretical model.

\title{
Procter and Gamble Student poster award:
}

I would like to compete in the Procter and Gamble Student award References:

1 Cohen-Tanugi, D.; Grossman, J. C. Water Desalination across Nanoporous Graphene. Nano Lett. 2012, $12,3602-3608$.

2 Yuan, Z. et al. Mechanism and prediction of gas permeation through sub-nanometer graphene pores: comparison of theory and simulation. ACS Nano 2017, 11, 7974-7987.『3 Oulebsir, F.; Vermorel, R.; Galliero, G. Diffusion of Supercritical Fluids through Single-Layer Nanoporous Solids: Theory and Molecular Simulations. Langmuir 2018, 34, 2, 561-571. Acceptance of Terms and Conditions:

Click here to agree

69

\section{Pore-scale investigation of residual oil distribution and mecha- nisms of enhanced oil recovery methods}

\author{
Author(s): Yaohao Guo ${ }^{1}$ \\ Co-author(s): Jun Yao ${ }^{2}$; Lei Zhang ${ }^{2}$; Guangpu Zhu ${ }^{3}$; Hai Sun ${ }^{3}$ \\ ${ }^{1}$ China university of Petroleum(East China) \\ ${ }^{2}$ China University of Petroleum \\ ${ }^{3}$ China University of Petroleum (East China)
}

Corresponding Author(s): guoyh9409@gmail.com

\begin{abstract}
Water flooding is an economic method commonly used in secondary recovery, but the poor sweep efficiency of water flooding results in a large amount of crude oil trapped in reservoirs. Accurate understanding the distribution of residual oil is essential for the subsequent development of water flooding. In this study, a pore-scale model is developed to study the distribution characteristics and formation process of residual oil. The Navier-Stokes equation coupled the phase field method is employed to describe the fluid flow and track two phase interface. The results show a significant difference in residual oil distribution under different wettability conditions. The difference is also reflected in oil recovery and the water cut curves. Much more remaining oil is displaced out in water-wet porous media than oil-wet porous media after water breakthrough. Furthermore, mechanisms of enhanced oil recovery (EOR) methods are investigated at the pore scale including surfactant flooding and polymer flooding. Appropriate time of implementing EOR methods is also studied. Surfactant solution reduces the entry pressure of micropores due to a low interface tension and increases oil displacement efficiency. Polymer flooding weakens the effect of capillary force by increasing the viscous force, which leads to the improvement in sweep efficiency. Injection time of surfactant has an important impact on the field development, but polymer flooding does not have this feature. Injecting at the low water-cut stage is an economical and appropriate solution for surfactant flooding.
\end{abstract}

Procter and Gamble Student poster award:

References:

1 J. Melrose, Role of capillary forces in detennining microscopic displacement efficiency for oil recovery by waterflooding, J. Can. Pet. Technol. , 13(04) (1974).

2 S.A. Shedid, Influences of fracture orientation on oil recovery by water and polymer flooding processes: An experimental approach, J. Pet. Sci. Eng., 50(3) (2006) 285-292.

3 P. Daripa, G. Paşa, An optimal viscosity profile in enhanced oil recovery by polymer flooding, Int. J. Eng Sci 42(19) (2004) 2029-2039.

[4] P. Janssen, W. Van Den Broek, C. Harris, Laboratory study investigating emulsion formation in the near-wellbore region of a high water-cut oil well, SPE Journal, 6(01) (2001) 71-79.

5 J. Waddell, R.A. Berthelet, Novel approach to high water cut measurement in a mature oil field, in: 
Abu Dhabi International Petroleum Exhibition and Conference, Society of Petroleum Engineers, 2008. [6] O.J. Jaramillo, R. Romero, G. Lucuara, A. Ortega, A.W. Milne, E. Rodrigues, Combining stimulation and water control in high-water-cut wells, in: SPE International Symposium and Exhibiton on Formation Damage Control, Society of Petroleum Engineers, 2010.

[7] S. Zhaojie, L. Zhiping, L. Fengpeng, L. Gang, G. Huohua, Derivation of water flooding characteristic curve for high water-cut oilfields, Petroleum Exploration and Development, 40(2) (2013) 216-223.

[8] W. Hongyan, C. Xulong, Z. Jichao, Z. Aimei, Development and application of dilute surfactantpolymer flooding system for Shengli oilfield, J. Pet. Sci. Eng., 65(1) (2009) 45-50.

[9] J. Shuhong, T. Changbing, S. Chengfang, Y. Jigen, Z. ZHANG, F. Xiujuan, New understanding on water-oil displacement efficiency in a high water-cut stage, Petroleum Exploration and Development, 39(3) (2012) 362-370.

[10] A.Z. Abidin, T. Puspasari, W.A. Nugroho, Polymers for Enhanced Oil Recovery Technology, Procedia Chemistry, 4(12) (2012) 11-16.

[11] V. Alvarado, E. Manrique, Enhanced Oil Recovery: An Update Review, Energies, 3(9) (2010) 15291575.

[12] G. Lideng, D. Xiaofeng, X. Zhang, L. Linggao, D. Wenhui, L. Xiaohong, G. Yinbo, L. Minghui, M. Shufang, Z. HUANG, Key technologies for seismic reservoir characterization of high water-cut oilfields, Petroleum exploration and development, 39(3) (2012) 391-404.

[13] X. Yan, Z. Huang, J. Yao, Y. Li, D. Fan, An efficient embedded discrete fracture model based on mimetic finite difference method, Journal of Petroleum Science \& Engineering, 145 (2016) 11-21.

[14] K. Falk, B. Coasne, R. Pellenq, F.J. Ulm, L. Bocquet, Subcontinuum mass transport of condensed hydrocarbons in nanoporous media, Nature Communications, 6 (2014) 6949.

[15] C. Zhang, M. Oostrom, T.W. Wietsma, J.W. Grate, M.G. Warner, Influence of viscous and capillary forces on immiscible fluid displacement: Pore-scale experimental study in a water-wet micromodel demonstrating viscous and capillary fingering, Energy Fuels, 25(8) (2011) 3493-3505.

[16] H. Liu, A.J. Valocchi, C. Werth, Q. Kang, M. Oostrom, Pore-scale simulation of liquid CO 2 displacement of water using a two-phase lattice Boltzmann model, Adv. Water Res. , 73 (2014) 144-158.

[17] K. Chaudhary, M. Bayani Cardenas, W.W. Wolfe, J.A. Maisano, R.A. Ketcham, P.C. Bennett, Pore-scale trapping of supercritical CO2 and the role of grain wettability and shape, Geophys. Res. Lett. , 40(15) (2013) 3878-3882.

[18] R. Lenormand, E. Touboul, C. Zarcone, Numerical models and experiments on immiscible displacements in porous media, J. Fluid Mech. , 189 (1988) 165-187.

[19] C. Zhang, M. Oostrom, J.W. Grate, T.W. Wietsma, M.G. Warner, Liquid CO2 displacement of water in a dual-permeability pore network micromodel, Environmental science \& technology, 45(17) (2011) 7581-7588.

[20] H. Liu, Y. Zhang, A.J. Valocchi, Lattice Boltzmann simulation of immiscible fluid displacement in porous media: Homogeneous versus heterogeneous pore network, Physics of Fluids (1994-present), 27(5) (2015) 052103.

[21] A.M. Tartakovsky, P. Meakin, Pore scale modeling of immiscible and miscible fluid flows using smoothed particle hydrodynamics, Adv. Water Res. , 29(10) (2006) 1464-1478.

[22] A. Raoof, H. Nick, S. Hassanizadeh, C. Spiers, PoreFlow: A complex pore-network model for simulation of reactive transport in variably saturated porous media, Comput. Geosci. , 61 (2013) 160-174.

[23] L. Zhang, Q. Kang, J. Yao, Y. Gao, Z. Sun, H. Liu, A.J. Valocchi, Pore scale simulation of liquid and gas two-phase flow based on digital core technology, Sci. China: Technol. Sci., 58 (2015) 1375-1384.

[24] L. Chen, H.-B. Luan, Y.-L. He, W.-Q. Tao, Pore-scale flow and mass transport in gas diffusion layer of proton exchange membrane fuel cell with interdigitated flow fields, Int. J. Therm. Sci. , 51 (2012) 132-144.

[25] G. Zhu, J. Yao, L. Zhang, H. Sun, A. Li, B. Shams, Investigation of the Dynamic Contact Angle Using a Direct Numerical Simulation Method, Langmuir, 32(45) (2016) 11736-11744.

[26] Z. Qiao, S. Sun, Two-Phase Fluid Simulation Using a Diffuse Interface Model with Peng-Robinson Equation of State, SIAM Journal on Scientific Computing, 36(4) (2014) B708-B728.

[27] A.Q. Raeini, M.J. Blunt, B. Bijeljic, Direct simulations of two-phase flow on micro-CT images of porous media and upscaling of pore-scale forces, Adv. Water Res. , 74 (2014) 116-126.

[28] M. Peszynska, A. Trykozko, Pore-to-core simulations of flow with large velocities using continuum models and imaging data, Comput. Geosci., 17 (2013) 623-645.

[29] G. Tryggvason, B. Bunner, A. Esmaeeli, D. Juric, N. Al-Rawahi, W. Tauber, J. Han, S. Nas, Y.-J. Jan, A front-tracking method for the computations of multiphase flow, J. Comput. Phys. , 169(2) (2001) 708759.

[30] F. Boyer, C. Lapuerta, S. Minjeaud, B. Piar, M. Quintard, Cahn-Hilliard/Navier-Stokes model for the simulation of three-phase flows, Transp. Porous Media 82 (2010) 463-483.

[31] P. Yue, C. Zhou, J.J. Feng, C.F. Ollivier-Gooch, H.H. Hu, Phase-field simulations of interfacial dynamics in viscoelastic fluids using finite elements with adaptive meshing, J. Comput. Phys. , 219 (2006) 47-67.

[32] A.C. Gunde, B. Bera, S.K. Mitra, Investigation of water and CO 2 (carbon dioxide) flooding using micro-CT (micro-computed tomography) images of Berea sandstone core using finite element simula- 
tions, Energy, 35 (2010) 5209-5216.

[33] G. Zhu, J. Yao, A. Li, H. Sun, L. Zhang, Pore-scale investigation of carbon dioxide enhanced oil recovery, Energy \& Fuels, 31(5) (2017).

[34] W. Villanueva, G. Amberg, Some generic capillary-driven flows, Int. J. Multiphase Flow 32 (2006) $1072-1086$

[35] G. Zhu, J. Kou, S. Sun, J. Yao, A. Li, Decoupled, energy stable schemes for a phase-field surfactant model, Computer Physics Communications, (2018).

[36] G.P. Zhu, J. Yao, H. Sun, M. Zhang, M.J. Xie, Z.X. Sun, T. Lu, The numerical simulation of thermal recovery based on hydraulic fracture heating technology in shale gas reservoir, Journal of Natural Gas Science \& Engineering, 28 (2016) 305-316.

[37] H. Huang, X.-y. Lu, Relative permeabilities and coupling effects in steady-state gas-liquid flow in porous media: A lattice Boltzmann study, Phys. Fluids, 21(9) (2009) 092104.

[38] Y.J. Zhu G P, Zhang L, el at., Pore-scale investigation of residual oil distributions and formation mechanisms at the extra-high water-cut stage, Chin Sci Bull, 62 (2017) 2553-2563.

[39] B. Hou, Y. Wang, X. Cao, J. Zhang, X. Song, M. Ding, W. Chen, Mechanisms of Enhanced Oil Recovery by Surfactant-Induced Wettability Alteration, J. Dispersion Sci. Technol. , 37(9) (2016) 1259-1267.

[40] B.Y. Jamaloei, R. Babolmorad, R. Kharrat, Visualization and analysis of viscous fingering in alcoholassisted surfactant waterflooding of heavy oil in a two-dimensional sandstone micromodel, Fuel, 184 (2016) 169-179.

[41] A. Seethepalli, B. Adibhatla, K. Mohanty, Physicochemical Interactions During Surfactant Flooding of Fractured Carbonate Reservoirs, Spe Journal, 9(9) (2004) 411-418. Acceptance of Terms and Conditions:

Click here to agree

106

\section{Age of Mixing Ratios.}

Deviyani Gurung ${ }^{1}$; Timothy Ginn ${ }^{1}$

${ }^{1}$ Washington State University

Corresponding Author(s): deviyani.gurung@wsu.edu, tim.ginn@wsu.edu

The concept of mixing ratios has proven useful in analysis of reactive transport in natural and engineered porous media, and in particular as a basis for mathematical separation of transport and reactions processes. Also the use of age of solutes, that is exposure time to the flow field, has been recently explored as a useful proxy for reaction extent. Here we develop mixing ratio models that are structured on age, for one-dimensional cases. We show how to develop age-structured mixing ratios in general and compare with conventional formulations of mixing ratio models, demonstrating that age is often a more natural independent variable than absolute time. We then apply age-structured mixing ratios to the problem of mixing-limited reactive transport in one-dimension, and explore its application to data with comparison to prior models of mixing-limited reactions.

Procter and Gamble Student poster award:

I would like to compete in the Procter and Gamble Student award References:

Acceptance of Terms and Conditions:

Click here to agree

638

\section{Pore-filling mechanics in capillaries with variable-wettability pat- terns}

Victor Raul Gutierrez Cortes ${ }^{1}$; Jingsheng $\mathrm{Ma}^{1}$; Steven R. McDougall ${ }^{1}$

${ }^{1}$ Heriot-Watt University 


\title{
Corresponding Author(s): vrg1@hw.ac.uk
}

The modelling and simulation of multiphase flow in shale gas is a considerable challenge due to its pore structure and surface phenomena, which leads to complex underlying physics. Because of the depositional environment and organic maturity, clay minerals produce pores of multiple sizes, which shrink by close compaction during diagenesis and eventually, change the morphology of the porous medium. Consequently, some organic nano-pores may come into contact with other minerals, leading to a dual-wettability condition on the pore surface. This affects the multiphase flow dynamics, making it difficult for network modelling techniques to predict macroscopic flow properties. Although Washburn's equation adequately describes pore-filling processes under homogeneous mineralogical conditions, it does not accurately reproduce displacement phenomena associated with variable wettability patterns in capillary tubes. This work studies the quantitative dependency of pore-filling rate on mineral pattern distributions and initial momentum for fluids of different density and viscosity ratios. Results show the applicability of the developed analytical relationships for a 2D channel with given wettability patterns. These analytical models allow the determination of average filling rates from mineral characterisation of real porous media samples and can subsequently be used in multi-phase pore-network models. In the future, this study can be extended to a $3 \mathrm{D}$ fracture to define lower and upper limits of pore-filling rates.

\section{Procter and Gamble Student poster award:}

I would like to compete in the Procter and Gamble Student award References:

Acceptance of Terms and Conditions:

Click here to agree

\section{Effects of gas adsorption-induced deformation on carbon dioxide storage in coal}

\author{
Mojgan Hadi Mosleh ${ }^{1}$ \\ ${ }^{1}$ Lecturer \\ Corresponding Author(s): mojgan.hadimosleh@manchester.ac.uk
}

Coal is considered to be a dual porosity rock containing micropores $(<2 \mathrm{~nm})$ and macropores $(>50 \mathrm{~nm})$. Micropores in the coal matrix constitute approximately $70 \%$ of its total porosity and account for approximately $95 \%$ of the total internal surface area. Macropores, on the other hand, consist of naturally occurring networks of fractures or cleat system. It is well established that gas transport in coal includes the flow through naturally fractured porous network (cleats), diffusion into the organic coal matrix, and storage within the micropores in an adsorbed state. It is also known that the microstructure of coal can exhibit a strong deformation response when it interacts with gas species. The gas adsorption-induced deformation in coal has created uncertainties on the efficiency of $\mathrm{CO} 2$ injection and storage in deep unminable coal seems due to the potential reduction in the permeability and gas injectivity. Based on a series of experimental investigations, this work will provide new insight into the effects of carbon dioxide adsorption in coal on flow properties and gas exchanges.

The interactions between carbon dioxide and methane in a high rank coal will be discussed based on the results of a series of core flooding experiments. The permeability evolution in response to CO2 injection will be presented and the reversibility of the adsorption-induced deformation processes during the carbon dioxide storage and methane recovery (i.e. CO2-ECBM) will be reviewed. In comparison with a series of flooding experiments of N2 into the same coal, new insights into the permeability evolution under the effects of adsorption induced matrix deformation will be provided. In addition, the results of a series of core flooding experiments in which $\mathrm{N} 2$ and $\mathrm{CO} 2$ were injected into the $\mathrm{CH} 4$-saturated coal sample will be presented. Finally, the competitive displacement of $\mathrm{CH} 4$ with $\mathrm{N} 2$ and $\mathrm{CO} 2$ under simulated underground conditions will be presented and the displacement process, gas breakthrough, and recovery ratios will be discussed. 
References:

Acceptance of Terms and Conditions:

Click here to agree

\title{
Hydrodynamic instabilities of immiscible fluids flow in oil reser- voir rocks during enhanced oil recovery process
}

\author{
Zahreddine Hafsi ${ }^{1}$ \\ ${ }^{1}$ Laboratory of Applied Fluids Mechanics Process and Environment Engineering, National Engineering School of Sfax, \\ Sfax, 3038, Tunisia
}

Corresponding Author(s): hafsi.zahreddine@gmail.com

\begin{abstract}
Through this paper, equations governing oil-water flow in oil reservoir rocks are developed and numerically solved following a finite element scheme. Numerical results are obtained using Comsol Multiphysics software. We discussed different retention models and the effect of the model parameters on the hydrodynamic instabilities that occur during an Enhanced Oil Recovery (EOR) process was investigated. An oil reservoir is a porous medium consisted of many fractures of tiny dimensions. In this work and for modeling purposes, the oil reservoir rock is considered as a collection of capillary tubes which provides useful insights into how fluids behave in the reservoir pore spaces. In the presence of two immiscible fluids in capillarities, a pressure difference known as capillary pressure arises across the interface separating the fluids. The capillary pressure plays a significant role in determining the fluid flow configuration. After conventional oil recovery processes and in order to retrieve oil left behind, water flooding, as one of several techniques used for enhanced oil recovery, is numerically modeled. Considering the imbibition case where wetting phase (water) is pushing non wetting phase (oil), a fingering phenomenon is observed. Such hydrodynamic instability is followed up versus time in order to give a deep insight into how to control the oil-water interface for a complete oil recovery.
\end{abstract}

Key words: immiscible flow, capillary pressure, EOR process, Retention models, numerical modeling.

\section{Procter and Gamble Student poster award:}

I would like to compete in the Procter and Gamble Student award References:

1 Prodanovic, M. and S.L. Bryant: Physics-driven interface modeling for drainage and imbibition in fractures SPE 11044. 2007

2 Brooks, R. H., and Corey, A. T. Hydraulic properties of porous media. Hydrology paper No. 3, Colorado State Univ., Fort Collins, 1964

3 Van Genuchten, M. T. (1980). A closed-form equation for predicting the hydraulic conductivity of unsaturated soils. Soil Sci. Soc. Am. J., 44, 892-898.

[4] Mualem Y. A new model for predicting the hydraulic conductivity of unsaturated porous media. Water Resources Research 12(3): 513-522, 1976.

5 Lenhard R, Parker J, Mishra S. On the correspondence between Brooks-Corey and van Genuchten models. Journal of Irrigation and Drainage Engineering ASCE 115:744-751, 1989. Acceptance of Terms and Conditions:

Click here to agree 
Author(s): Bjørn Hafskjold ${ }^{1}$

Co-author(s): Signe Kjelstrup ${ }^{1}$; Trygve Røsæg ${ }^{2}$; Olav Galteland ${ }^{1}$; Astrid F. Gunnarshaug ${ }^{1}$; Dick Bedeaux ${ }^{1}$; Anders Lervik $^{2}$; Mathias Winkler ${ }^{3}$

\footnotetext{
${ }^{1}$ PoreLab, Department of Chemistry, Norwegian University of Science and Technology - NTNU, Trondheim, Norway

${ }^{2}$ Department of Chemistry, Norwegian University of Science and Technology - NTNU, Trondheim, Norway

${ }^{3}$ PoreLab, Department of Physics, Norwegian University of Science and Technology - NTNU, Trondheim, Norway
}

Corresponding Author(s): bjorn.hafskjold@ntnu.no

Thermal diffusion, the Ludwig - Soret effect, plays an important role in transport of heat and mass in fluid mixtures. The coupling between heat- and mass transport extends Fourier's law for heat conduction and Fick's law for mass diffusion and is quantified by the Soret coefficient. The effect has applications in industrial processes, such as utilization of waste heat 1 and analysis of composition gradients in oil reservoirs 2, as well as novel use in nanomachines 3 and microswimmers [4]. Many experimental techniques have been used to measure Soret coefficients in bulk fluids 5. It is known that a porous medium may have an impact on the Soret effect, but experimental data are not conclusive on its origin. For instance, porosity, permeability, wettability, and tortuosity will all change diffusion relative to bulk fluid, but the magnitude and mechanism of the coupling of mass diffusion and thermal diffusion is still unknown.

We will present results from non-equilibrium molecular dynamics simulations of the Soret effect for a Lennard-Jones model with two miscible fluid components in a porous medium. The medium has different porosity and wettability preferences for the two fluid components. We show that the wettability preferences change the Soret coefficient and discuss the mechanisms that lead to such change. The effect of gravity on component separation is examined by combining gravity and a temperature gradient acting in the same direction, opposite directions, and orthogonal directions.

Procter and Gamble Student poster award

\title{
References:
}

1 L. Keulen, L.V. van der Ham, J. Haanemaijer, N.J.M. Kuipers, Thijs Vlugt, S. Kjelstrup, «Membrane distillation against a pressure difference», J. Membr. Science, 524 (2017) 151-162.

2 F. Montel, J. Bickert, A. Lagisquet, and G. Galliero, «Initial state of petroleum reservoirs: A comprehensive approach», J. Pet. Sci. Eng., 58, (2007) 391-402 2007.

3 M. Yang and M. Ripoll, «Thermoosmotic microfluidics», Soft matter, 12 (2016) $8564-8573$.

[4] H.-R. Jiang, N. Yoshinaga, and M. Sano, «Active motion of a Janus particle by self-thermophoresis in a defocused laser beam», Phys. Rev. Lett. 105 (2010) 268302.

5 W. Köhler and K. I. Morozov, «The Soret Effect in Liquid Mixtures - A Review», J. Non-Equilib. Thermodyn. 41 (2016) 151-197. Acceptance of Terms and Conditions:

Click here to agree

\section{Soil respiration under climate extremes: On the role of root hy- draulic redistribution delineated by millifluidics}

\author{
Erfan Haghighi ${ }^{1}$; Joaquin Jimenez-Martinez ${ }^{2}$ \\ ${ }^{1}$ Swiss Federal Institute of Aquatic Science and Technology \\ ${ }^{2}$ EAWAG-ETHZ
}

Corresponding Author(s): ehaghighi.mech@gmail.com

The respiratory release of carbon dioxide (CO2) from the Earth's soil into the atmosphere is a major, yet poorly understood, flux regulating land's carbon sink potential. Understanding soil respiration sensitivity to climate extremes (i.e., global warming and drought) remains one of the key sources of uncertainty in quantifying/projecting atmospheric $\mathrm{CO} 2$ growth rate. In particular, compensatory mechanisms accounting for global observations of soil $\mathrm{CO} 2$ efflux insensitivity to combined heat and water stress, leading to strongest reduction in land's carbon sink potential, are insufficiently 
studied. We seek to explore the passive movement of water between different soil parts via plant roots (i.e., root hydraulic redistribution), triggered by plant response to climate extremes, mitigating local water stress in the rhizosphere and thus facilitating rhizosphere respiration under favorable hot extreme. To this end, we design a millifluidic setup enabling direct $2 \mathrm{D}$ visual observations of spatio-temporal variations in hydration conditions and oxygen content (i.e., the two factors primarily controlling microbial activities) in the root zone, as the model plant responds to controlled climate conditions. This offers an opportunity to quantify subsurface fluid dynamics modulating soil respiration hotspots (with higher rates of oxygen consumption) that account for the fluctuations in land's carbon sink potential under extreme events. These readily observable root-mediated controls on soil biogeochemical fluxes will provide new insights into hidden mechanisms, neither accounted for by large-scale Earth system models (ESMs) nor readily quantified by field observations; thereby facilitating improved (multiscale) representation of this important boundary condition in current ESMs.

Procter and Gamble Student poster award:

\section{References:}

Acceptance of Terms and Conditions:

Click here to agree

\section{What key physical factors yield a good horizontal hydrofractured gas well in a mudrock?}

Author(s): Syed Haider ${ }^{1}$

Co-author(s): Tadeusz Patzek ${ }^{1}$

${ }^{1}$ King Abdullah University of Science and Technology

Corresponding Author(s): syed.haider@kaust.edu.sa

Gas flow in mudrocks (shales) depends on the complex, multiscale connectivity among nanopores, microfractures and macrofractures. Hydraulic fractures stimulate the reservoir volume near a horizontal well and create fractures at all scales. We describe the Stimulated Reservoir Volume (SRV) as a fractal with its own fracture network and that accesses the organic-rich matrix. The physical structure of the SRV, i.e., the number of macrofractures and surface area created after fracking is constrained by the conservation of mass of frac water and proppant. Nanopores in the organic matrix acts as the source of almost all gas. Poor connectivity of organic matter at the nanoscale is compensated by the percolating backbone of the multiscale fractures created during hydrofracking. These multiscale fractures bridge the otherwise disconnected specs of organic matter and increase overall permeability of the reservoir. With most of the organic pore radii in the range of 2-50 $\mathrm{nm}$, the smallest pores $(\mathrm{R}<2 \mathrm{~nm})$ are responsible for the low-rate prolonged production over most of well life. Dense and highly ordered gas molecules in the nanopores (5-15\% of PV) can maintain the latestage well production for 3-5 years. Initial production decline that defines well quality depends on the coupling between the fracture network and the organic matrix. The physical parameters that establish this coupling are the fracture network permeability, $k_{f}$, and the fracture shape factor, $s$, that in turn controls fluid transfer from the organic matrix to the fractures. Values of $k_{f}$ and $s$ are determined by numerical fitting of production data with an optimization function. A large value of the ratio $k_{f} / s$ causes gradual production decline because of high fracture network permeability at macro scale. The effective kerogen surface area exposed to the fractures is smaller, but gas flow in the fractures is more efficient, thus nullifying the negative effect of the smaller surface area. For low values of $k_{f} / s$, the decline is faster. In summary, the fracture network permeability at the macro scale and the shape factor at the microscale control the production rate of shale wells. The best quality wells have better macroscale connectivity. 
Could not include image: Cannot read image data. Maybe not an image file?

\section{Procter and Gamble Student poster award:}

I would like to compete in the Procter and Gamble Student award References:

Marder M, Chen CH, Patzek T. Simple models of the hydrofracture process. Physical Review E. 2015 Dec 29;92(6):062408.

Patzek T. Knudsen-Like Scaling May Be Inappropriate for Gas Shales. InSPE Annual Technical Conference and Exhibition 2017 Oct 2. Society of Petroleum Engineers.

Fan D, Ettehadtavakkol A. Semi-analytical modeling of shale gas flow through fractal induced fracture networks with microseismic data. Fuel. 2017 Apr 1;193:444-59.

Miao T, Yu B, Duan Y, Fang Q. A fractal analysis of permeability for fractured rocks. International Journal of Heat and Mass Transfer. 2015 Feb 1;81:75-80.

Thomas LK, Dixon TN, Pierson RG. Fractured reservoir simulation. Society of Petroleum Engineers Journal. 1983 Feb 1;23(01):42-54.

Chang J, Yortsos YC. Pressure transient analysis of fractal reservoirs. SPE Formation Evaluation. 1990 Mar 1;5(01):31-8.

Yuan Y, Doonechaly NG, Rahman S. An analytical model of apparent gas permeability for tight porous media. Transport in porous media. 2016 Jan 1;111(1):193-214.

Patzek TW, Male F, Marder M. Gas production in the Barnett Shale obeys a simple scaling theory. Proceedings of the National Academy of Sciences. 2013 Dec 3;110(49):19731-6. Acceptance of Terms and Conditions:

Click here to agree

\section{Hydro-mechanical effects on the stability of RJD wells and pro- duction rate}

Mohammad Reza Hajiabadi ${ }^{1}$; Hamid M. Nick ${ }^{1}$

${ }^{1}$ Danish Hydrocarbon Research and Technology Centre, DTU

Corresponding Author(s): mreza@dtu.dk

Radial Jet Drilling (RJD) is an emerging technology in oil recovery enhancement and geothermal energy production that uses high pressurized fluid often composing of acid or just water to drill radial patterns of lateral paths into a producing formation. The laterals cut through the formation in different directions from the main well and extend up to more than $100 \mathrm{~m}$. Depending on the type of the nozzle used, jetting fluid chemistry and confining stress, the cross-section shape and size of the laterals varies between a round circular hole to a star shape.

Bypassing the damage formation near the main well, RJD increases the production shortly after the jetting, but in long time it may gradually loses the efficiency. Since the RJD laterals are not cased, the instability issue in laterals can be more crucial. During the several production / shut-in sequences, the material around the hole has the potential for breaking into the laterals and plugging the hole. Moreover, pore-collapse induced compaction can be responsible for failure and reduction in permeability near the laterals. The coupled hydro-mechanical simulation results show that large decrease in permeability due to pore collapse can lead to significant reduction in hydrocarbon recovery. This study considers the hydro-mechanical effects on the stability of RJD wells and production rate.

Procter and Gamble Student poster award: 
References:

Acceptance of Terms and Conditions:

Click here to agree

858

\title{
Multiscale finite volume method for finite-volume-based simula- tion of poroelasticity
}

Hadi Hajibeygi ${ }^{1}$; Irina Sokolova $^{1}$

${ }^{1}$ TU Delft

Corresponding Author(s): h.hajibeygi@tudelft.nl

We propose a multiscale finite volume method (MSFV) for simulation of fully-coupled flow-deformation in heterogeneous porous media under elastic deformation. The fine-scale fully implicit system is obtained based on a conservative finite-volume method with staggered pressure-displacement nodes. We propose independent coarse-scale grids for flow and deformation, over which local multiscale basis functions for scalar pore pressure and vectorial displacement unknowns are introduced. The basis functions are solved only at the beginning of the simulation, and reused for the rest of the timedependent simulations. As for the finite-volume formulation for all unknowns, the fine-scale and coarse-scale stress fields are locally conservative, as well as the fluid mass flux. Several numerical test cases are provided first to validate the fine-scale finite-volume discrete fully-implicit simulation, and then to investigate the accuracy of the proposed multiscale formulation for synthetic and real-field applications. Specially, we show that our multiscale method provides a good estimate of surface subsidence as a result of gas production, with only a few degrees of freedom (compared with the fine-scale resolved solver). As such, our multiscale method casts a promising approach for field-scale simulation of coupled flow and geo-mechanics.

Procter and Gamble Student poster award:

I don't want to compete References:

I. Sokolova, M. Bastisyaa, H. Hajibeygi, Journal Of Computational Physics, (in press) 2019. Acceptance of Terms and Conditions:

Click here to agree

536

\section{Stochastic velocity transitions and continuous time random walks to model solute transport in heterogeneous porous media}

\author{
Vivien Hakoun ${ }^{1}$; Arash Massoudieh ${ }^{2}$; Marco Dentz ${ }^{1}$ \\ ${ }^{1}$ IDAEA-CSIC \\ ${ }^{2}$ The Catholic University of America, Washington, D.C., USA.
}

Corresponding Author(s): vivien.hakoun@gmail.com

We study anomalous transport in heterogeneous porous media using a continuous time random walk (CTRW) approach based on two Markov models for the stochastic evolution of particle velocities. These approaches allow for the systematic quantification of the impact of flow heterogeneity and injection conditions on average solute transport. Specifically, we consider a Bernoulli and an Ornstein-Uhlenbeck model for velocity transitions combined with Lognormal and Gamma distributions for the steady state velocity. We analyze the properties of these Markov models and their impact on solute transport based on the copula function underlying each model. This analysis gives insight in the model specific correlation length and convergence rates towards steady state, which 
are key features when applying these models to experimental data. Using two different velocity distributions, we investigate how the choice of the velocity transition model impacts on the transport behavior in terms of solute dispersion and breakthrough curves. This study sheds new light on the representation of the interplay between velocity transition and velocity distribution in upscaled models for the prediction of transport in heterogeneous porous media.

Procter and Gamble Student poster award:

References:

Acceptance of Terms and Conditions:

Click here to agree

\title{
Solute mixing in porous media: the role of confinement
}

\author{
Author(s): Mayumi Hamada ${ }^{1}$ \\ Co-author(s): Pietro De Anna \\ ${ }^{1}$ University of Lausanne
}

Corresponding Author(s): mayumi.hamada@protonmail.com

\begin{abstract}
A pore-scale description of the transport and mixing processes is particularly relevant when looking at biological and chemical reactions. For instance, a microbial population growth is controlled by local concentrations of nutrients and oxygen, and chemical reaction are driven by molecular-scale concentration gradients. The heterogeneous flow field typically found in porous media results from the contrast of velocities that deforms and elongates the mixing fronts between solutes that often evolves through a lamella-like topology. For continuous Darcy type flow field a novel framework that describes the statistical distribution of concentration being transported was recently developed (Le Borgne et al., JFM 2015). In this model, concentrations in each lamella are distributed as a Gaussianlike profile which experiences diffusion in the transverse direction while the lamella is elongated by advection along the local flow direction. The evolving concentration field is described as the superposition of each lamella. We hypothesize that this novel view, while perfectly predicting the distribution of concentration for Darcy scale mixing processes, will breakdown when the processes description is at the pore scale. Indeed the presence of solid and impermeable boundaries prevents lamella concentration to diffuse freely according to the a Gaussian shape, and therefore changes the mixing front profile, the lamella superposition and elongation rules. We developed an experimental set-up to visualize and quantify, at the pore scale, the dynamics of solutes diffusion, transport and mixing in a confined space, where we use microfluidics chips to built synthetic fracture and porous material. First, we measured, with microfluidics and video-microscopy, the distribution of a displacing solute concentration, the results are not well reproduced either qualitatively or quantitatively by the Darcy scale model. Secondly, laboratory experiment, theoretical and numerical models of diffusion in a confined medium show deviation of the concentration profile from the Gaussian distribution, characteristic and building block of Darcy scale model. Our ongoing work is to take into consideration this pore-scale effect on the global mixing dynamics.
\end{abstract}

Procter and Gamble Student poster award:

References:

Acceptance of Terms and Conditions:

Click here to agree 
JUHEE HAN ${ }^{\text {None }}$; Jaewon Jang ${ }^{1}$

${ }^{1}$ Hanyang University

Corresponding Author(s): hanjhhy@naver.com

It has been shown that a little reduction in water saturation of in-situ sediments can dramatically increase the resistance to liquefaction induced by earthquake loading. Biogenic gas bubble formation via denitrification is a promising method to reduce water saturation of saturated sediment. Gas bubble formation characteristics should be investigated further to apply the denitrification process for the reduction of saturation. In this study, the characteristics of biogenic gas bubble formation via denitrification process is investigated through microfluidic chip experiment and core-scale experiment. The microfluidic chip experiment provides visual observation on the size and spatial distribution of nucleated gas bubbles and the behavior of gas bubble migration by buoyancy force and trapping in pore space. The core-scale experimental results provide the final water saturation reduced by biogas formation and long-term stability of gas bubbles trapped in the sediment. In addition, the packing of particles with different size shows the characteristic behavior of gas bubbles as a function of pore size of the sediment. The results of this study will help to predict the saturation reduction and stability of bubbles for the in-situ application.

Procter and Gamble Student poster award:

References:

Acceptance of Terms and Conditions:

Click here to agree

\section{Understanding the Co-Moving Velocity}

Author(s): Alex Hansen ${ }^{1}$

Co-author(s): Dick Bedeaux ${ }^{2}$; Signe Kjelstrup ${ }^{3}$

${ }^{1} N T N U$

${ }^{2}$ Norwegian University of Science and Technology - NTNU

${ }^{3}$ Norwegian University of Science and Technology, NTNU, Trondheim

Corresponding Author(s): alex.hansen@ntnu.no

In a recent paper 1 , we demonstrated that the seepage velocities of each fluid species in immiscible two-phase flow in porous media can be expressed in terms of the volume averaged seepage velocity and a co-moving velocity. The mathematical structure presented in that paper is based on the Euler theorem for homogeneous functions. Even though the mathematical manipulations never went beyond elementary theory for functions of several variables, it was still hard to build an intuition as to what precisely is the co-moving velocity.

By considering the geometrical structure of the space spanned by the relevant flow parameters, the role of the co-moving velocity may be clarified. From these considerations, we also demonstrate how it may be measured experimentally.

\section{Procter and Gamble Student poster award:}

\section{References:}

1 A. Hansen, S. Sinha, D. Bedeaux, S. Kjelstrup, M. Aa. Gjennestad and M. Vassvik, Relations between seepage velocities in immiscible, incompressible two-phase flow in porous media, Transp. Porous Media, 125, 565-587 (2018). Acceptance of Terms and Conditions:

Click here to agree 


\title{
Displacement of methane by injecting water into shale nanopores using molecular dynamics simulations
}

\author{
Author(s): Youzhi Hao ${ }^{1}$ \\ Co-author(s): Detang Lu ${ }^{1}$ \\ ${ }^{1}$ University of Science and Technology of China
}

Corresponding Author(s): hyzlvxg@mail.ustc.edu.cn

The coexistence of water and methane is important during hydraulic fracturing process in shale gas development. In this work, the coexistence of water and methane in shale nanopores under shale gas reservoir conditions is investigated using molecular dynamics simulations. The shale nanopores are modelled by kaolinite and illite cuboid pores with basal and edge surfaces. At initial state, the slit pores are filled with methane molecules at equilibrium state. Then water molecules are added outside the pore. We observed that as time went on, the water molecules entered into the cuboid pores, driving much methane molecules outside the pore, which leaves only a small portion of methane inside the pore. Water films are formed on the surface of the circular or square nanopores of the clay mineral, serving as a water channel and the methane molecules can flow in the channel. The nanoscale confinement makes the water channel in the cuboid pore exhibit quasi-round shape. Compared with a non-polar, methane molecule with a zero dipole moment, water molecules with polar and strong dipole moments have more affinity with clay minerals. Additionally, the smaller the pore is, the greater the proportion of methane molecules being displaced. This work reveals the microscopic dynamic process of methane molecules displaced outside the pores by water molecules. Water molecule is absorbed by shale fractures or pores, and methane is displaced out of the pores to the fractures by the water, resulting in lower water flowback ratio. This phenomenon explains that the low rate of fracturing water flowback return can achieve high gas production in shale gas development. 

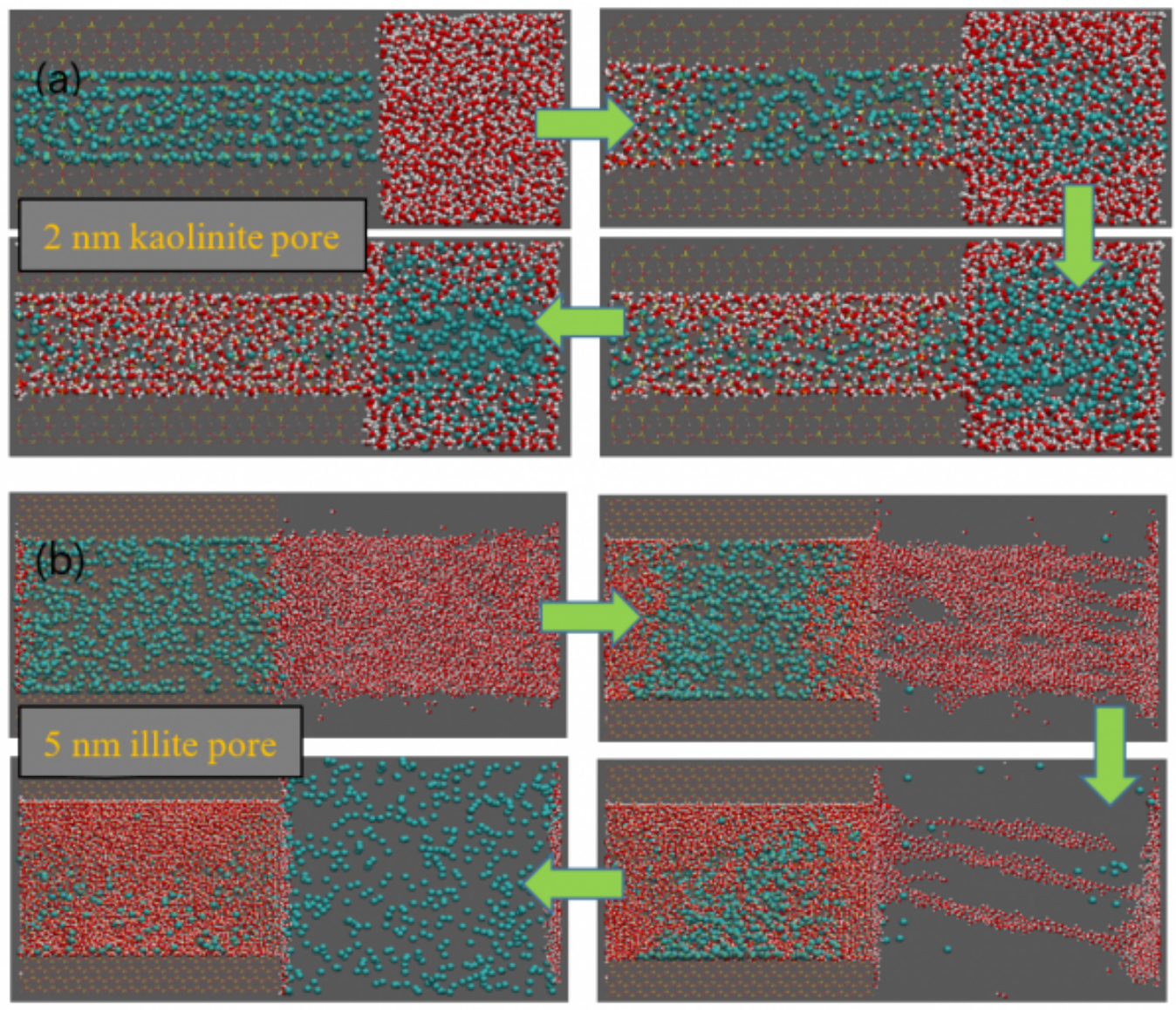

Figure 1: Dynamic processes of water molecules moving into (a) kaolinite and (b) illite cuboid nanopores, displacing the methane molecules outside the pores.

Figure 5: Figure 1: Dynamic processes of water molecules moving into (a) kaolinite and (b) illite cuboid nanopores, displacing the methane molecules outside the pores

Procter and Gamble Student poster award:

I would like to compete in the Procter and Gamble Student award References:

Acceptance of Terms and Conditions:

Click here to agree

\section{Saturation-Dependence of Non-Fickian Transport in Porous Me- dia}

Author(s): Sharul Hasan ${ }^{1}$; Vahid.J Niasar ${ }^{\text {None }}$

Co-author(s): Nikolaos Karadimitriou ${ }^{2}$; Muhammad Sahimi ${ }^{3}$

\footnotetext{
${ }^{1}$ University of Manchester

${ }^{2}$ Stuttgart University

${ }^{3}$ University of Southern California
}

Corresponding Author(s): sharulnizam.hasan@manchester.ac.uk 
In two-phase flow through porous media the percolating pathways can be hydrodynamically split into the flowing and stagnant regions. Although the stagnant regions are connected to the flow pathways, mass transfer into and out of them is diffusion-, and not advection-controlled. The highlyvariable velocity field in the flowing region leads to significant differences in the transport time scales in the two regions that cannot be explained by the Fickian (Gaussian) advection-dispersion equation. Up to now very limited efforts have been devoted to characterizing the transport properties in twophase flow, using pore-scale information. In this paper, we simulated advection-diffusion transport in steady-state two-phase flow in porous media using a pore-network model as an upscaling tool. The simulation results were upscaled to analyse three main aspects of transport in two-phase flow, namely, stagnant saturation, mass-transfer coefficient and rate between the flowing and stagnant saturation, and longitudinal dispersion in the flowing regions. The results were compared with the mobile-immobile (MIM) model, which is a popular model for simulating non-Fickian transport in porous media. The disagreement between the directly-estimated parameters from the pore-network simulations and those obtained by using the MIM model implies fundamental shortcomings of the latter for describing transport in two-phase flow. The simulation results indicate that the relative permeabilities may be used to obtain accurate estimates of the stagnant saturation, which links between two-phase fluid flow and transport that. To our knowledge, this is currently absent in the literature.

\title{
Procter and Gamble Student poster award:
}

I would like to compete in the Procter and Gamble Student award References:

\section{Acceptance of Terms and Conditions:}

Click here to agree

\section{Fast 4D Micro-Tomography Imaging of Anomalous Transport in Partially-Saturated Porous Media}

\author{
Author(s): Sharul Nizam Hasan ${ }^{1}$; Vahid.J Niasar ${ }^{\text {None }}$ \\ Co-author(s): Jose Godinho ${ }^{2}$; Holger Steeb ${ }^{3}$; Nghia Vo $^{4}$; Nikolaos Karadimitriou ${ }^{5}$; David Uribe ; Maria Osorno \\ 6 \\ ${ }^{1}$ university of manchester \\ ${ }^{2}$ Helmholtz-Zentrum Dresden-Rossendorf \\ ${ }^{3}$ University of Stuttgart \\ ${ }^{4}$ Diamond Light Source \\ ${ }^{5}$ Stuttgart University \\ ${ }^{6}$ University Stuttgart
}

Corresponding Author(s): sharulnizam.hasan@manchester.ac.uk

Solute transport in partially-saturated porous media is the key process in many engineering and natural systems. Current major understanding is based on inverse modelling using different theories which have never been directly evaluated against the experiment. For the first time, we directly visualise transport of a non-reactive tracer in a partially-saturated porous medium using the very fast high-resolution synchrotron-based X-ray computed microtomography. After establishing a steady-state two-phase flow (water-Fluorinert) in a glass-bead packing, we imaged the transport of a water-based tracer (KI solution) under the same steady-state conditions to directly visualise and characterise the advection-diffusion transport at 5 second time resolution and $3.25 \mu \mathrm{m}$ spatial resolution. The temporal evolution of the concentration field clearly showed that transport time scale within the water-filled area was spatially heterogeneous which led to non-Gaussian transport within the partially-saturated porous medium. The heterogeneity of the saturation topology influences the relationship between the total permeability of the advancing and receding phase and stagnant saturation of the advancing phase.

Keywords: two-phase flow, stagnant saturation, 4D x-ray imaging

Procter and Gamble Student poster award: 
I would like to compete in the Procter and Gamble Student award References:

Acceptance of Terms and Conditions:

Click here to agree

\title{
Wettability Alteration Modeling by Pore Network Approach for Low-salinity Water Injection Process
}

\author{
Author(s): Leila Hashemi ${ }^{1}$ \\ Co-author(s): Mohammad.h Golestan ${ }^{2}$; Riyaz Kharrat ${ }^{3}$; Mehdi Zallaghi ${ }^{4}$ \\ ${ }^{1}$ Petroleum University of Technology, Department of Tehran \\ ${ }^{2}$ Department of Petroleum Engineering and Applied Geophysics, Norwegian University of Science and Technology \\ ${ }^{3}$ Professor at Montan University Austria \\ ${ }^{4}$ Tehran Petroleum Research Center - Petroleum University of Technology
}

Corresponding Author(s): leila_hashemi94@yahoo.com

\begin{abstract}
Increasing oil production from reservoirs by changing composition (e.g. by reducing salinity) of the injected water during water-flooding has been subject of many research and shown in laboratory and field studies. In the literature, several mechanisms have been presented for low-salinity effects on enhanced oil recovery, such as $\mathrm{pH}$ variation, multi-component ion exchange and double layer expansion which are leading to wettability alteration, interfacial tension reduction and emulsification, fine migration, microscopic diversion and selective plugging via clay swelling. Notwithstanding, there are many challenges of determining dominant mechanism during low-salinity water flooding. Moreover contradictory results demonstrate more than one mechanism can affect on increasing oil recovery. Thus, it seems likely several parameters control the process. Clearly experimental study of this process to assess one parameter separately is impossible. Therefore, other techniques such as pore network modeling can be chosen as an appropriate remedy. In addition, some macroscopic reactions are governed by implementation reactive transport at the pore-scale. So capability of pore network modeling to simulate various scenarios of multiphase flow transportation makes it as an alternative method to calculate network and pertinent fluid properties which has been widely considered.

Wettability alteration is the most accepted mechanisms for the increasing oil recovery by low-salinity water injection which is the consequence of adsorption, dissolution and desorption process. It is assumed that wettability alteration described by changing contact angle as a function of water salinity. The purpose of this study is to evaluate the oil recovery by wettability alteration in low-salinity water injection via pore network approach. The displacement process including drainage and imbibition are modeled and the effect of contact angle changes investigated. Various contact angles at pore network model can make by several factors, in this work we focus on the salinity of injected water and geochemical reactions at rock surface and phases. In this regard, the effects of salinity on changing surface potential investigate by using pore network modeling which is coupled with geochemical reaction models. Then, the model used to determine critical salinity of water injection for oil recovery process.
\end{abstract}

Procter and Gamble Student poster award:

References:

Acceptance of Terms and Conditions:

Click here to agree 


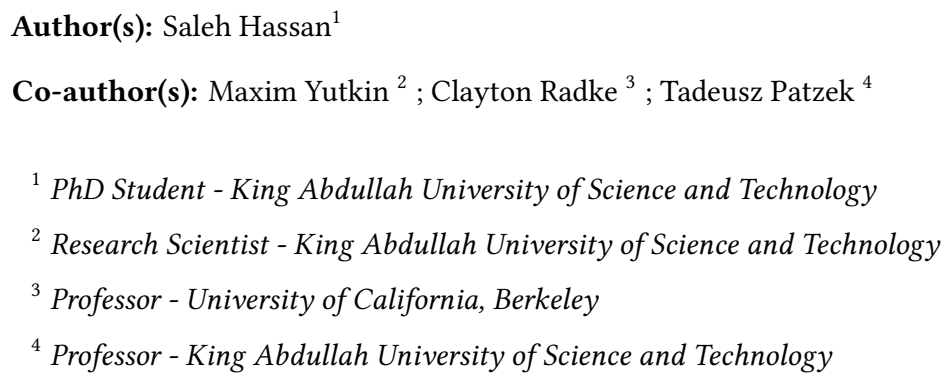

Corresponding Author(s): saleh.hassan@kaust.edu.sa

Recovery of subsurface hydrocarbons is related to crude oil interaction with mineral surfaces. Crude oil interactions with minerals are believed to be strong, almost irreversible, and caused by complex asphaltenes with several active functional groups. A few likely candidates have been highlighted in the literature. The surface-active carboxyl and amine (quaternary ammonia) groups seem to be the main causes of asphaltene adsorption. However, no direct experimental confirmation of their roles in asphaltene adsorption has been published.

In this work, we study adsorption of different functional groups onto silica and calcium carbonate surfaces. Sodium hexanoate ( $\mathrm{NaHex}$ ), sodium dodecyl sulfate ( $\mathrm{NaDS}$ ), and cetyl trimethylammonium chloride (CTAC) were served as sources of these functional groups. Their interactions with silica and calcium carbonate surfaces in different brines were probed via the QCM-D technique.

Silica surface is negatively charged in almost any brine with weak $\mathrm{pH}$ dependence. CTAC is a positively charged surfactant, and is expected to adsorb easily on silica. Indeed, we observe that CTAC adsorbs and almost completely desorbs upon flush from silica regardless of brine composition. In contrast, the negatively charged surfactants like NaHex or NaDS hardly adsorb onto silica surface even in the presence of calcium.

In contrast to silica, calcium carbonate surfaces can be positively or negatively charged. From literature, we know that calcium carbonate has a positively charged surface in calcium chloride brines and a negatively charged surface in sodium carbonate or sodium chloride brines [Hirasaki 2017]. We found that CTAC adsorbs on calcium carbonate in comparable amount for both types of brine. $\mathrm{NaDS}$ also adsorbs onto calcium carbonate surface especially in the presence of calcium ions. In general, calcium carbonate seems to adsorb surfactants regardless of its own surface charge and the surfactant charge.

Adsorption of all the above surfactants, CTAC, NaHex, and $\mathrm{NaDS}$ is reversible and not representative of asphaltenes. To better mimic asphaltene behavior, we studied adsorption of polymers with carboxylic (sodium carboxymethyl cellulose, Na-CMC) and quaternary ammonium (Polydiallyldimethylammonium chloride, PolyDADMAC) functionalities.

The positively charged PolyDADMAC readily adsorbs onto a negatively charged silica surface, and its adsorption is irreversible. We have failed to wash it away with highly concentrated brines of calcium chloride. Interestingly enough, $\mathrm{Na}-\mathrm{CMC}$ also adsorbs to silica, but its adsorption is rather slow. The effect seems to be stronger in the presence of calcium ions. In this case, adsorption is also irreversible.

Therefore, polymers with carboxylic and quaternary ammonia groups do mimic irreversibility behavior of asphaltenes contrary to mono-functional surfactants. We made a more thorough comparison of asphaltene adsorption features with those of polymers. For example, from the literature we know that asphaltenes can exhibit fast and slow adsorption depending on surface and asphaltene type. They also can form both rigid and elastic films upon adsorption [Kornfeldt 2002]. This behavior is controlled by concentration and reaction time of asphaltenes with surface [Xu 2014]. We found that polymers share some similarities here as well. For example, Na-CMC can produce elastic films if high molecular weight is used. PolyDADMAC shows fast adsorption and forms elastic films depending on brine composition.

Could not include image: Cannot read image data. Maybe not an image file? 
Procter and Gamble Student poster award:

References:

Acceptance of Terms and Conditions:

Click here to agree

587

\title{
Lake Urmia basin: soil, water, and climate nexus
}

\author{
Amirhossein Hassani $^{1}$; Adisa Azapagic ${ }^{2}$; Amir Keshmiri $^{3}$; Nima Shokri ${ }^{2}$ \\ ${ }^{1}$ The University of Manchester \\ ${ }^{2}$ School of Chemical Engineering and Analytical Science, The University of Manchester, Manchester, UK \\ ${ }^{3}$ School of Mechanical, Aerospace \& Civil Engineering, The University of Manchester, Manchester, UK
}

Corresponding Author(s): amirh.hassani@yahoo.com

Lake Urmia, second largest hypersaline lake in the world and the largest internal lake of Iran has confronted a sever water shrinkage (nearly $90 \%$ of its initial volume) in less than 25 years. The salt and dust storms originated from the lake dried bed have the potential to endanger the sustainability of local ecosystems, agriculture and livelihoods, regional health, and tourism due to soil salinization. Here, using dispersion modelling, we estimate what would be the mass flux and spatial scale of likely salt/sand storms, created from the dried lake bed and how this can affect the soils around Lake. Also through a combination of climatic, agronomic and hydrologic assessments, a comprehensive scheme reliant on land management is proposed for rehabilitation of the lake water body as well as assuring the agriculture and food production sustainability in Lake Basin. The proposed study and its findings have important implications beyond Lake Urmia and therefore will be attractive for policy makers and those who are interested to quantify the effectiveness of several strategies applied for the lake restoration and will also contribute to the efforts toward food and water supply sustainability.

Procter and Gamble Student poster award:

References:

Acceptance of Terms and Conditions:

Click here to agree

314

\section{Two-phase flow in industrial porous media; Experiments, theory, and modelling}

S. Majid Hassanizadeh ${ }^{1}$; Hamed Aslannejad ${ }^{\text {None }} ;$ Amir Hossein Tavangarrad $^{1}$

${ }^{1}$ Utrecht University

Corresponding Author(s): smhfba@yahoo.com

Various industrial processes involve two-phase flow in porous media. Examples are found in fuel cells, filtration, paper, food, concrete, ceramics, moisture absorbents, and membranes, to name a few. The common practice in modelling flow and transport in such porous media is to employ the concepts,

models, and algorithms developed in geosciences. However, many industrial porous media are significantly different from soil, and flow and transport processes occur in different regimes. Some major examples are: 
- Constitutive relations (e.g., capillary pressure curve) are obtained under equilibrium conditions whereas many industrial flows are very fast.

- Porous media in geosciences are usually hydrophilic; in industrial processes they can be completely or partially hydrophobic.

- Electrochemical reactions usually do not influence flow in geosciences. Often not so in certain industrial processes (e.g. fuel cells, filtration, food industry)

- Industrial porous media often have the form of a (stack) of very think porous layers.

- Industrial porous media often have a very wide range of porosity values (1 to $90 \%$ ).

- Soils/rocks usually have granular packings; industrial porous media have much more complex topologies (e.g., granular, fibrous, planar, and combinations)

- Deformations of soils/rocks are negligible or slow; so, the solid matrix doesn't change much. Often large deformations are encountered in industrial porous media, and the solid matrix may change drastically.

There is a clear need for developing theories, models, and measurement techniques specifically applicable to industrial porous media. In this presentation, we highlight the special features of industrial porous media through the discussion of results or studies of two industrial applications: penetration of ink into paper and the absorption of liquids in diapers. We also discuss how formulation of two-phase flow in such systems should be modified.

\section{Procter and Gamble Student poster award:}

I would like to compete in the Procter and Gamble Student award References:

\section{Acceptance of Terms and Conditions:}

Click here to agree

\section{Microbial Growth and its Influence on Hydraulic Properties in Saturated Porous Media - a Microfluidic Study}

Neda Hassannayebi ${ }^{1}$; Schritter Johanna ${ }^{2}$; Martin Ferno ${ }^{3}$; Frieder Enzmann ${ }^{\text {None }}$; Andreas Loibner ${ }^{2}$; Holger $\mathrm{Ott}^{1}$

\footnotetext{
${ }^{1}$ Montanuniversität Leoben

${ }^{2}$ Universität für Bodenkultur Wien

${ }^{3}$ University of Bergen
}

Corresponding Author(s): neda.nayebi@gmail.com

Understanding growth and transport of microorganisms in porous media is essential for understanding natural subsurface systems and the application of subsurface engineering measures. Storage of renewable energy as hydrogen gas in exploited reservoirs aims at the use of porous space in the subsurface. A first attempt to store hydrogen in an underground gas storage was recently carried out in Austria (www.underground-sun-storage.at). In the frame of this flagship project, a methane/hydrogen mixture was injected and stored in a depleted gas field to estimate the risk of hydrogen loss due to physical, chemical and biological processes potentially occurring in the reservoir. In particular microbial processes resulted in hydrogen loss and consequently in a partial loss of energy. The prevailing microbial process for hydrogen reduction was its conversion to methane in the presence of carbon dioxide.

In a follow-up project, "Underground Sun Conversion", the possibility of using microorganisms to convert hydrogen to methane in-situ and on purpose is investigated. However, in-situ gas conversion may result in excessive formation of biomass in the pore space of the reservoir rock. As a consequence, biomass will reduce the pore space for gas storage and likely the permeability of the reservoir rock as well. As a result, biomass may substantially compromise storage capacity and injectivity.

In the presented work, we systematically investigate accumulation of microbes and microbial growth in the pore scale and its influence on hydraulic properties of porous media. Even though hydrogen storage comprises in principle a two-phase-flow system, the present study focuses on saturated flow, which provides sufficient complexity. 
The study takes advantage of the high spatial and temporal resolution of optical microscopy in order to investigate transport and accumulation of microorganisms in microfluidics, i.e. 2D porous media etched in glass. The experiments were performed in two experimental phases by using Lactobacillus casei as model organisms. In phase 1, a bacterial suspension solution in a stationary growth phase was flooded through microfluidic chips leading to initial accumulation of microbes in the pore space. In phase 2, a nutrient (substrate) solution was injected; the supply of nutrients changes bacterial growth to an exponential mode. Biomass accumulates with a high rate in the pore space.

The change of porosity over time has been determined by segmentation of the pore space and the accumulation of biomass in the image sequences. Furthermore, the segmented images allow for direct numerical simulations on the "digital twin" - the digital representation of the experimental data. By solving the Stokes-flow and the Navier-Stokes equations in the pore space, changes in flow field and permeability with increasing biomass could be determined. The study indicates (a) the formation of preferred flow pathways with increasing biomass, and (b) an associated porosity-permeability relationship, with a qualitatively different behaviour in suspension flooding (phase 1) and nutrient flooding (phase 2). Furthermore, an intermittent release of biomass and subsequent filtration have been observed, leading to a stepwise local increase of biomass by filtration. A first attempt to simulate transport and filtration of microbes has been started by particle-flow simulations in the Digital Twin.

Procter and Gamble Student poster award:

References:

Acceptance of Terms and Conditions:

Click here to agree

814

\section{Study on salt precipitation behavior during carbon dioxide in- jected into underground porous media}

Di $\mathrm{He}^{1}$; Peixue Jiang ${ }^{1}$; Ruina $\mathrm{Xu}^{1}$

${ }^{1}$ Tsinghua University

Corresponding Author(s): hed15@mails.tsinghua.edu.cn

With the rapid development of the global economy and industry, the consumption of fossil energy has also increased sharply, and the global climate change triggered by it has attracted more and more attention. Geological carbon sequestration (GCS) is a promising way to reduce carbon dioxide emissions. The saline aquifers is an ideal reservoir for geological carbon sequestration. However, due to the high concentration of salts and minerals in the saline aquifers, when a large amount of dry carbon dioxide is injected from the injection well, the water in solution evaporates continuously. Therefore, the concentration of the salt in the solution is continuously increased, eventually leading to reach the solubility limit then precipitation happening, which will reduce the porosity and permeability of the reservoir, resulting in poor or even complete clogging of the carbon dioxide. Therefore, this paper will carry out pore scale experimental research to study the effects of carbon dioxide injection rate on salt precipitation behavior by designing porous media chip with cracks, revealing the influence of salt distribution on permeability.

In this paper, the effects of different carbon dioxide injection rates on the precipitation and distribution of salt in different cracks were studied by designing two kinds of crack microfluidic chips. Furthermore, the influence of distributions of salt on the permeability of cracks was also studied. The results show that in the straight crack, when the carbon dioxide injection rate is small, the capillary reflow is more serious, so that the salt solution is continuously replenished to the injection end, and a two-phase flow is formed in the microporous medium of the salt to increase the capillary pressure. Thereby, the salt is locally aggregated at the injection end, resulting in a greatly reduced permeability of the chip to lower than 0.0023 times of the permeability before salting out. When the flow rate of carbon dioxide is increased, the capillary reflow is suppressed, so that the solution cannot be replenished to the injection end. Two-phase flow has not been observed in the porous medium of the salt. The salt is more uniformly distributed in space, so it has less influence on the permeability, which only reduced to about half of the original. In the curved crack, under different 
flow injections, it is found that the salting out near the injection end is less, which has small influence on the permeability of the chip. When the flow rate is small, the precipitated salt accumulates at the front end of the crack and blocks the entire crack in the form of large crystals区the structure of the precipitated salt is mostly in the shape of a crystal. Since most of the solute is locally aggregated in the porous medium in the form of crystals, there is a relatively clean and complete carbon dioxide channel in other areas, so the final impact on the chip permeability is small, which only decreased to 0.68 times of original.

Procter and Gamble Student poster award:

I don't want to compete References:

Acceptance of Terms and Conditions:

Click here to agree

\section{Temperature effects on swelling behaviour of smectite: insight into coupled chemo-mechanical phenomena in charged porous systems.}

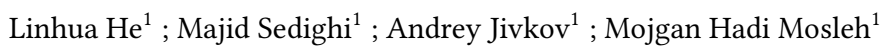

${ }^{1}$ The University of Manchester

Corresponding Author(s): linhua.he@manchester.ac.uk

Smectite is a clay mineral which exhibits a high degree of swelling in presence of water. Smectite is a negatively charged porous system and its pore water contains multiple ionic species and minerals. It is well established that the swelling behaviour of the soils containing high amount of smectite (e.g. commercial bentonite), is primarily governed by the physico-chemical interactions in the clay-waterchemical system. The high swelling capacity of bentonite is among critical characteristics which promote this material as a key component of several barrier technologies in geo-environmental applications. An example is the concept of geological disposal of high level nuclear waste in which the use of compacted bentonite as a buffer material is envisaged. Temperature of the clay buffer can be elevated to a range of 80 to $100 \mathrm{oC}$ whilst scenarios beyond this range are also studied. It is critical in such environmental applications that the clay barrier sustains its swelling capacity under the effects of elevated temperature to a large extent and for a long period of time.

We will present the results of an investigation on the effects of temperature on swelling pressure evolution of compacted bentonite. This research provides new insights into the thermo-chemically coupled processes which govern the mechanics of swelling of compacted bentonite. Based on the results of a series of experimental investigations on compacted MX-80 bentonite, the development and evolution of swelling pressure under the effects of temperature for up to $80 \mathrm{oC}$ will be discussed. The results of a series of re-saturation of compacted MX-80 under constant volume at different temperature and for a range of dry densities from low $(1200 \mathrm{~kg} / \mathrm{m} 3)$ to high $(1800 \mathrm{~kg} / \mathrm{m} 3)$ will be presented. Two main aspects of swelling behaviour will be discussed: i) the equilibrium state of swelling pressure as the results of temperature change and ii) the kinetics of swelling pressure development for a range of temperature. We will present theoretical aspects of hydro-mechanical behaviour by re-visiting coupled processes. In comparisons with the experimental results we will present new findings on the chemically driven processes which control the mechanical response of clay system to the elevated temperature effects.

Procter and Gamble Student poster award:

\section{References:}

Acceptance of Terms and Conditions:

Click here to agree 


\title{
Analytical and Numerical Assessment of Hydraulic Properties of 3D Partially Open Fractures with Variable Roughness
}

\author{
Xupeng $\mathrm{He}^{1}$; Hussein Hoteit ${ }^{2}$ \\ ${ }^{1}$ King Abdullah University of Science and Technology \\ ${ }^{2}$ KAUST
}

Corresponding Author(s): xupeng.he@kaust.edu.sa

\begin{abstract}
Assessment of the effective hydraulic properties of rock fractures is of considerable interest in numerous applications in the context of subsurface fluid dynamics. Natural fractures typically exhibit irregular fracture aperture and roughness resulting from shearing, compaction, deposition, and other mechanical and chemical processes. This work introduces a new analytical model to estimate the effective hydraulic properties of partially open fractures with rough walls and irregular contactarea distributions. We first briefly review the applicability of the relevant models in the literature. Most of the existing models exhibit major limitations related to prediction accuracy and practicality For instance, most models rely on static indicators to quantify the fracture contact-area proportion but do not account for the contact-area distribution. As a result, they fail to estimate properly the anisotropic flow behavior caused by fracture filling and heterogeneous aperture distribution. Alternatively, we propose a dynamic indicator using a particle-percolation approach to quantify the effect of the contact-area distribution. We then introduce a modified cubic low for 3D fractures which accounts for different factors including irregular fracture aperture, nonparallel walls, wall roughness, and fracture filling distribution. We assess the predictability of our model and benchmark it with eight other models from the literature for a large number of cases. The model predictions are compared with the solutions of 3D Navier-Stokes equation using high-precision simulations computed in parallel. We show significant superiority of our model especially for cases with pronounced fracture anisotropy where the impact of the fracture contact-area and filling is dominant. We then demonstrate the model for real rock fractures digitized using a profilometer and present a workflow to extend the model applicability for complex fracture networks.
\end{abstract}

Procter and Gamble Student poster award:

I would like to compete in the Procter and Gamble Student award References:

Acceptance of Terms and Conditions:

Click here to agree

\section{Evaporation in multi-component systems}

\author{
Author(s): Katharina Heck ${ }^{1}$ \\ Co-author(s): Lisa Bahlmann ${ }^{2}$; Kilian Weishaupt ${ }^{3}$; Rainer Helmig ${ }^{3}$; Bernd Flemisch ${ }^{3}$ \\ ${ }^{1}$ University Stuttgart \\ ${ }^{2}$ Leibniz Universität Hannover \\ ${ }^{3}$ University of Stuttgart
}

Corresponding Author(s): katharina.heck@iws.uni-stuttgart.de

A standard approach to model diffusion in porous media is the assumption of the validity of Fick's Law. Although widely used, that description can only be employed for binary mixtures or low concentrations of the components as it neglects molecular interactions of the different species.

When looking e.g. at gas migration of an organic component in soil where higher concentrations of 
components can occur, more complex laws need to be employed. In this work we present a multiphase, multi-component model incorporating the Maxwell-Stefan's approach to diffusion which takes into account all interactions between the molecules of different species.

We present a coupled free-flow porous-medium model, where the Maxwell-Stefan diffusion approach is employed in both domains including consistent coupling conditions.

The model is implemented in the numerical software framework DuMuX and can be used for various applications. The example application presented is a study on evaporation together with gas migration of $\mathrm{CO} 2$ in the porous medium and across the porous-medium free-flow interface. The numerical results are compared to experimental results conducted in a climate controlled wind tunnel.

Procter and Gamble Student poster award:

References:

Acceptance of Terms and Conditions:

Click here to agree

\title{
Bimodal imaging of water flow in samples of green infrastructure soils
}

\author{
Petra Heckova $^{1}$; Jitka Hanzlikova ${ }^{1}$; Michal Snehota $^{2}$; Anders Kaestner $^{3}$; Johannes Koestel ${ }^{4}$ \\ ${ }^{1}$ University Centre for Energy Efficient Buildings, Czech Technical University in Prague, Czech Republic \\ ${ }^{2}$ CTU in Prague, Prague, Czech Republic \\ ${ }^{3}$ Paul Scherrer Institut, Laboratory for Neutron Scattering and Imaging, Villigen PSI, Switzerland \\ ${ }^{4}$ Swedish University of Agricultural Sciences, Uppsala, Sweden
}

Corresponding Author(s): petra.heckova@cvut.cz

Green infrastructure, such as bioretention cells and green roofs, help adaptation to climate change and sustainable storm water management in cities. Bioretention cells are artificial surface depressions which uses a natural process of stormwater filtration, cleaning and retention. A green roof is a vegetated layer on building rooftops.

The long term aim of our research is to experimentally enlighten the initial formation of soil structure of the green infrastructure soils and its effect on its performance (Jelinkova et al. 2016). The neutron imaging proofed as an effective method to study the flow processes in soil (Snehota et al. 2015). In the current study, we used a bi-modal tomographic imaging method to visualize and quantify the soil structure and internal pore geometry as well as the water flow (Kaestner et al. 2017). The fast bi-modal neutron and X-ray tomographic imaging was conducted at ICON beamline of the Paul Scherrer Institut, (Switzerland).

Two stages of initial soil formation were compared. The first batch of 22 samples was collected from a freshly established green roof and bioretention cells, while the second batch of 48 was collected two months later. The aluminum sampling cylinders had an internal diameter of $29 \mathrm{~mm}$ and height of $80 \mathrm{~mm}$. The height of the samples was in average $50 \mathrm{~mm}$. We selected 8 samples from the first batch and 8 samples from the second batch for the experiment.

Two rainfall episodes were simulated in the beamline on each sample, respectively. Heavy water was used as a flowing liquid. The duration of each episode was 15 minutes with a maximal rainfall intensity of $25.73 \mathrm{~mm} / \mathrm{min}$ for the soil from the bioretention cell and $2.57 \mathrm{~mm} / \mathrm{min}$ for the green roof samples. The water was gravitationally drained from the samples for 30 minutes in between the rainfall episodes. In case of the samples from the bioretention cell, the water flow was reduced whenever ponding of the surface exceeded approximately $15 \mathrm{~mm}$. Changes in outflow and inflow were recorded gravimetrically.

Time lapse series of 11 to 13 tomographic images were recorded for each sample. Each tomogram was reconstructed using 300 projection, acquired during a $360^{\circ}$ sample rotation. The time needed to collect one tomogram was $60 \mathrm{~s}$. The voxel size of each neutron image reached $68 \mu \mathrm{m}$ the image dimensions were 900x900x900. The X-ray image resolution was $41 \mu \mathrm{m}$, the image dimensions were $2170 \mathrm{x} 2170 \mathrm{x} 1784$. Both neutron and X-ray tomograms were reconstructed using the software MuhRec (Kaestner 2011). 
The time lapse series of neutron tomograms showed that samples taken after two months of initial soil formation already contained larger macropores in comparison to the samples taken shortly after setting up the experiments. The presence of macropores resulted in faster wetting front advancement in the second batch of samples. The samples taken in the second batch retained less water in contrast to samples collected in first batch. The time lapse of X-ray tomographic images were used to assess changes in pore geometry occurring during the infiltration experiments.

Procter and Gamble Student poster award:

\title{
References:
}

KAESTNER, A.P., J. HOVIND, P. BOILlAT, C. MUEHLEBACH, C. CARMINATI, M. ZAREBANADKOUKI, a E.H. LEHMANN (2017). Bimodal imaging at ICON using neutrons and X-rays. Physics Procedia, 88, 314-321.

KAESTNER, A. P. (2011). MuhRec - A new tomography reconstructor, Nuclear Instruments and Method in Physics Research Section A - Accelerator Spectrometers Detectors and Associated Equipment, 651(1), 156-160.

JELINKOVA, V., M. DOHNAL, J. SACHA (2016). Thermal and water regime studied in a thin soil layer of green roof systems at early stage of pedogenesis, Journal of Soils and Sediments, 16(11), 2568-2579.

SNEHOTA, M., V. JELINKOVA, M. SOBOTKOVA, J. SACHA, P. VONTOBEL, a J. HOVIND (2015). Water and entrapped air redistribution in heterogeneous sand sample: Quantitative neutron imaging of the process. Water Resources Research, 51(2), 1359-1371. Acceptance of Terms and Conditions:

Click here to agree

\section{Microfluidic Investigation of Brine-hydrocarbon Interactions dur- ing Low-salinity Waterflooding}

\author{
Yaqi Zhang $^{1}$; Amir Sanati-Nezhad ${ }^{1}$; S. H. Hejazi ${ }^{1}$ \\ ${ }^{1}$ University of Calgary
}

Corresponding Author(s): shhejazi@ucalgary.ca

In the present study, an important but less-characterized phenomenon, i.e., the osmosis, during the oil production by water flooding is systematically investigated. Chemical osmosis describes a physical process where water molecules spontaneously move in the direction of lower-water potential, i.e., higher solute concentration, through a semipermeable membrane while the solutes are retained. The non-equilibrium chemical potentials in the two fluid phases and a semipermeable membrane in between are the essential elements for the occurrence of osmosis.

In the underground reservoirs, the osmotic phenomenon is widely recognized in analyzing the wellbore stability during drilling operations [1, 2], the formation damage during fracturing treatment in shale or tight sandstone reservoirs [3-5], the high-pressure anomalies in geologic environment $[6,7]$ and the contaminant preservation by compacted clay liners in landfill sites [8]. Tight porous media are deemed to be the membrane in these cases. The role of crude oil acting as semipermeable membranes has recently been recognized in the studies of enhanced oil recovery (EOR) mechanisms of low-salinity waterflooding (LSWF) $[9,10]$. When brines with tuned ionic compositions are injected into the formations, an osmotic pressure is generated by the incompatibility or the chemical potential difference between the formation water and the injected water solution. However, the semipermeable nature of crude oils and the contribution of chemical osmosis-driven mass transfer to oil recovery remain largely uncharacterized.

We use a microfluidic device with custom-designed post structures where samples of synthetic formation water, crude oil, and low-salinity water are placed in contact. The water transition through the oil phase under the chemical potential gradient is recorded. We use fluorescent microscopy to quantify the rate of water molecule transport under different osmotic pressures. Crude oils of various compositions and viscosities are subjected to act as the membrane. We measure the membrane efficiency $(\sigma)$ of crude oils, a parameter describes the ability of a material to act as the membrane, with different viscosity and compositional properties. Furthermore, a microchip with complex porenetwork is used to quantify the contribution of osmotic pressure to oil recovery processes. The study of osmosis in crude oil, brine, and rock (COBR) systems is not only beneficial to exploring the 
mechanisms of LSWF but also applicable to the geological processes related to various brines, such as chemical enhanced oil recovery and fracturing fluid infiltration.

\title{
Procter and Gamble Student poster award:
}

I would like to compete in the Procter and Gamble Student award References:

(1) Schlemmer, R.; Friedheim, J. E.; Growcock, F. B.; Bloys, J. B.; Headley, J. A.; Polnaszek, S. C. Chemical osmosis, shale, and drilling fluids. Spe Drilling \& Completion 2003, 18 (4), 318.

(2) Nguyen, V. X.; Abousleiman, Y. N.; Hoang, S. K. Analyses of Wellbore Instability in Drilling Through Chemically Active Fractured-Rock Formations. Spe Journal 2009, 14 (2), 283.

(3) Zhou, Z.; Abass, H.; Li, X.; Bearinger, D.; Frank, W. Mechanisms of imbibition during hydraulic fracturing in shale formations. Journal of Petroleum Science and Engineering 2016, 141, 125.

(4) Teklu, T. W.; Li, X.; Zhou, Z.; Alharthy, N.; Wang, L.; Abass, H. Low-salinity water and surfactants for hydraulic fracturing and EOR of shales. Journal of Petroleum Science and Engineering 2018, 162, 367.

(5) Wang, F.; Pan, Z. Q.; Zhang, S. C. Impact of chemical osmosis on water leakoff and flowback behavior from hydraulically fractured gas shale. Journal of Petroleum Science and Engineering 2017, 151, 264.

(6) Neuzil, C. E. Osmotic generation of 'anomalous' fluid pressures in geological environments. Nature 2000, 403, 182.

(7) Neuzil, C. E.; Provost, A. M. Recent experimental data may point to a greater role for osmotic pressures in the subsurface. Water Resour. Res. 2009, 45 (3).

(8) Keijzer, T. J. S.; Loch, J. P. G. Chemical osmosis in compacted dredging sludge. Soil Science Society of America Journal 2001, 65 (4), 1045.

(9) Sandengen, K.; Kristoffersen, A.; Melhuus, K.; Josang, L. O. Osmosis as Mechanism for Low-Salinity Enhanced Oil Recovery. Spe Journal 2016, 21 (4), 1227.

(10) Fredriksen, S. B.; Rognmo, A. U.; Ferno, M. A. Pore-scale mechanisms during low salinity waterflooding: Oil mobilization by diffusion and osmosis. Journal of Petroleum Science and Engineering 2018, 163, 650. Acceptance of Terms and Conditions:

Click here to agree

\section{Simulation of capillary-dominated three-phase flow experiments on 3-D bead packs}

Johan Olav Helland ${ }^{1}$; Helmer André Friis ${ }^{1}$; Espen Jettestuen ${ }^{1}$; Rebecca Lynn Paustian ${ }^{2}$; Dorthe Wildenschild ${ }^{2}$

\author{
${ }^{1}$ NORCE Norwegian Research Centre \\ ${ }^{2}$ Oregon State University
}

Corresponding Author(s): jhel@norceresearch.no

Three-phase flow in porous media occurs in many engineering science applications, including oil recovery and $\mathrm{CO} 2$ storage in mature hydrocarbon reservoirs where water-alternate-gas injections and cyclic injections are relevant processes. In this context, it is important to understand three-phase displacement mechanisms at the pore scale for the different invasion processes to develop reliable constitutive relationships for capillary pressure and relative permeability, as well as their hysteresis behavior, for use in three-phase flow simulation on larger porous media scales. To this end, we have developed a multiphase level set (MLS) approach for investigating three-phase, capillary-controlled displacement at pore scale. The model is implemented in a software framework for massive parallelization and adaptive mesh refinement (AMR).

Here, we compare MLS simulations directly with segmented, high-resolution microtomography images of three-phase fluid distributions obtained during capillary-dominated flow experiments of gas and water invasion cycles on 3-D bead packs, for both spreading and non-spreading oils. Primary drainage and imbibition constitute the oil/water saturation history prior to gas invasion. Analyses of the segmented three-phase images provide fluid/fluid interfacial areas and capillary pressures (from interfacial curvature) as functions of saturations. We validate MLS simulations based on these 
criteria, as well as the correspondence between simulated and experimental three-phase fluid configurations.

The experiments use a quasi-static displacement protocol where invasion with low, constant flow rate are shut off regularly for interface equilibration and imaging. MLS simulations use a corresponding saturation-controlled displacement mode that can track the experimental saturation paths exactly and predict three-phase capillary pressures along these paths. A consequence of controlling fluid saturations (or flow rates) during drainage is that pore invasion occurs with temporary imbibition events in other pore regions (i.e., cooperative behavior). However, three-phase simulations with contact angle hysteresis shows that pinning of contact lines arrest most of these temporary imbibition events, whereas three-phase capillary pressure jumps associated with the cooperative behavior persist irrespective of the difference between receding and advancing contact angles. Overall, our results show that MLS method describes the experimental three-phase capillary pressure curves and bulk pore occupancies very well for the gas and water invasion cycles. For extracted image subsets, we show that using AMR in the simulations significantly improves representation of oil layers and calculation of interfacial area. We also perform two-phase gas/oil and oil/water simulations from which we construct three-phase configurations for comparison with experimental images and investigate the conjecture that two two-phase systems can represent capillary pressures in three-phase systems.

Finally, we demonstrate that, by using the MLS method directly on an imaged three-phase configuration with preserved saturations and a realistic range of contact angle hysteresis, we can predict capillary pressure and a spatial contact angle distribution simultaneously when the equilibrium configuration remains unchanged during the simulation. This is an attractive supplement to other manual and automatic measurement techniques for contact angles on pore-scale images with two or three fluids present.

Procter and Gamble Student poster award:

References:

Acceptance of Terms and Conditions:

Click here to agree

\title{
A Numerical Simulation of Fluid Flow through a Thin Porous Me- dia Confined within a Narrow Duct
}

\author{
Ammar Hazim Saber ${ }^{1}$; Gunnar Hellström ${ }^{2}$; Staffan Lundström ${ }^{3}$ \\ ${ }^{1}$ Luleå University of Technology, Div. Fluid and Experimental Mechanics \\ ${ }^{2}$ Luleå University of Technology \\ ${ }^{3}$ Fluid Mechanics, Luleå University of Technology, Sweden
}

Corresponding Author(s): gunnar.hellstrom@ltu.se, staffan.lundstrom@ltu.se

Fluid flow through a mould containing reinforcing material during manufacturing processes such as RTM or vacuum infusion experiences a pressure drop owing to the influence of the walls and the porous material. The contribution of the confining walls and the porous bed, to the pressure drop, depends upon the thickness of the bed and the permeability of it. The commercial software Ansys CFX 18, was here used in order to solve the governing equations and to disclose characteristics of the flow field in such a set-up. Pressure and shear forces exerted by the fluid on the confined and porous bed are disclosed. The geometric model, chosen to mimic the reinforced composite material, consists of a square array of uniform cylinders, with three different heights, mounted normal to the flow direction and confined within a rectangular narrow duct. The comparison of forces gives an indication of the importance of each of them. When the flow regime changes from laminar to turbulent through transition, due to increase flow velocity, the ratio of the forces will change. The results show that inertia effect increases with increasing velocity for all bed thickness. However, the inertial to the viscous forces ratio increases more for thick beds than for a thin bed with increasing Reynolds number. 
Procter and Gamble Student poster award:

References:

Acceptance of Terms and Conditions:

Click here to agree

21

\section{Asymptotic analysis for the coupling between subdomains in Dis- crete Fracture Matrix models}

Julian Hennicker ${ }^{1}$; Martin Jakob Gander ${ }^{1}$; Roland Masson ${ }^{2}$

\footnotetext{
${ }^{1}$ University of Geneva

${ }^{2} L F A D$
}

Corresponding Author(s): julian.hennicker@unige.ch

Current Discrete Fracture Matrix (DFM) models, such as 2, 3, 1, rely on ad hoc approximations in order to derive the matrix fracture coupling conditions. In our work, we present a derivation of the exact coupling conditions for DFM models based on Fourier analysis, and further obtain approximations of these coupling conditions, up to a certain order of a given quantity (fracture width, conductivity, resistivity), by truncating the corresponding asymptotic expansions. In a next step, we give estimates for the error of the so derived approximate models and compare them to existing models in the literature. This way, we find that the error of the coupling conditions proposed by 3 is of third order in the fracture aperture. Finally, we present some numerical tests, in order to illustrate the theoretical results.

\footnotetext{
1 Angot, P., Boyer, F., Hubert, F., 2009 Asymptotic and numerical modelling of flows in fractured porous media, ESAIM Mathematical Modelling and Numerical Analysis 23, 239-275.

2 Flauraud, E., Nataf, F., Faille, I., Masson, R., 2003, Domain Decomposition for an asymptotic geological fault modeling, Comptes Rendus à l'académie des Sciences, Mécanique, 331, 849-855.

3 Martin, V., Jaffré, J., Roberts, J. E., 2005, Modeling fractures and barriers as interfaces for flow in porous media, SIAM J. Sci. Comput. 26 (5), 1667-1691.
}

Procter and Gamble Student poster award:

\section{References:}

Acceptance of Terms and Conditions:

Click here to agree

\section{Estimating Permeability Decrease in Deformation Bands in Un- consolidated Sandstones by Digital Rock Simulation}

\footnotetext{
Ronaldo Herlinger $\mathrm{Jr}^{1}$; João Paulo Pereira Nunes ${ }^{1}$; Rodrigo Surmas ${ }^{1}$; Andreidy Andry Andrade ${ }^{1}$; Abraham Grader $^{2}$

${ }^{1}$ Petrobras S.A.

${ }^{2}$ Halliburton
} 
Corresponding Author(s): ronaldo.herlinger@petrobras.com.br

Deformation bands in unconsolidated sandstones are a common feature found in fault damage zones, which can cause compartmentalization within hydrocarbon reservoirs. They are low-displacement deformation zones ( $\mathrm{mm}$ to $\mathrm{cm}$ thick) and are formed by continuous processes of grain displacement, including rotation and translation, as well as fracturing and deformation at grain scale. Those bands are frequently seen in outcrops, but only scarcely observed in well cores. Due to its morphology, permeability reduction within the band is hard to be measured in routine core analysis. Hence, we estimate the reduction of the permeability generated by deformation processes performing simulations directly on the milimetric band. The study was conducted in representative plugs cut from well cores. The digital model was obtained via CT (core $-0.5 \mathrm{~mm}$ voxel size) and micro-CT (plug - $3.84 \mu \mathrm{m}$ voxel size) imaging under three confining pressures $(500,1300$, and $2500 \mathrm{psi})$. The flow simulations were performed using Lattice Boltzmann method in subsamples of the plugs in order to characterize petrophysical properties within and outside the deformation band. Moreover, porosity was obtained by segmentation, and elastic parameters (Vp, Vs, K, G, E, and Poisson's ratio) by finite element method. Results show significant decrease from thousands (average $3612 \mathrm{mD}$ ) to hundreds (average $371 \mathrm{mD}$ ) of milidarcies on the permeability within the band. The porosity decreased about $40 \%$, from an average of $25.8 \%$ to $17.4 \%$. Additionally, the elastic parameters were equally affected by the textural changes in deformation bands. This work provided useful information to understand and quantify the flow impact of the deformation bands in friable sandstones. Although the bands tend to be very thin, they occur periodically spaced, restricting the flow across the reservoir. Prior to this study, the reduction of permeability by fault zones was successfully incorporated to adjust well match history, corroborating the results of the flow simulation. Moreover, the results are important in reservoir modeling to estimate the permeability reduction along fault damage zones, which could improve production forecast.

Procter and Gamble Student poster award:

References:

Acceptance of Terms and Conditions:

Click here to agree

726

\title{
Mass dispersion between a fluid region and a porous medium
}

\author{
Roel Hernandez-Rodriguez ${ }^{1}$; O. A. Luévano-Rivas ${ }^{1}$; J. Alberto Ochoa-Tapia ${ }^{1}$ \\ ${ }^{1}$ Universidad Autónoma Metropolitana
}

Corresponding Author(s): iqrhr@hotmail.com

The transfer of mass by convection in porous media has been extensively studied in the literature due to the large number of systems in which it can be found. However, in many practical systems with mass transfer by convection the porous medium is adjacent to a region of free fluid. A challenging problem in the modeling of mass transfer by convection in such a configuration consists in describing the phenomena in the transition zone between the region and the medium, which is known as inter-region. From a macroscopic perspective, an alternative to model these systems with this configuration is known as the one domain approach (ODA), which considers the entire system as a continuum because it considers the spatial variations of the effective coefficients in the interregion. However, heuristic interpolations between the properties of the fluid region and the porous medium have been frequently used. Therefore, there is no guarantee that such models can provide an accurate description of physical phenomena. In this work we derive the closed macroscopic equations of convective mass transfer using the volumetric average method, which is expressed in terms of the position-dependent total dispersion tensor. The total dispersion tensor is calculated by two alternatives, (i) through direct numerical simulations (DNS) and (ii) by solving a previously reported local closure problem 1. Our results show the strong dependence of the spatial variations of the total dispersion tensor with respect to the Péclet number $(\mathrm{Pe})$. In addition, we have also analyzed the effects of the microstructure of the inter-region in calculating the total mass dispersion tensor. 
It should be noted that, the relevance of the prediction of the spatial variations of the total mass dispersion tensor are: first, these coefficients will allow the determination of the concentration profiles by means of the ODA solution and second, they are necessary to calculate the coefficients involved in the jump conditions that apply in the dividing surface between homogeneous regions, which will be developed in a later work.

Procter and Gamble Student poster award:

I would like to compete in the Procter and Gamble Student award References:

Valdés-Parada, F. J., Alvarez-Ramirez, J., Goyeau, B., \& Ochoa-Tapia, J. A. (2009). Jump condition for diffusive and convective mass transfer between a porous medium and a fluid involving adsorption and chemical reaction. Transport in porous media, 78(3), 459-476. Acceptance of Terms and Conditions:

Click here to agree

\title{
4D Structural and Chemical Characterization of Reactive Magne- sium Cement-Based Concrete
}

\author{
Anna Herring ${ }^{1}$; Mohammad Saadatfar ${ }^{1}$; Fatin Mahdini ${ }^{1}$; Penelope King ${ }^{1}$; Ulrike Troitzsch $^{1}$ \\ ${ }^{1}$ Australian National University
}

Corresponding Author(s): annalisaherring@gmail.com,mohammad.saadatfar@anu.edu.au

Cements made of reactive magnesium minerals ("reactive magnesium cements", or RMCs) have been proposed as an alternative to commonly used Portland cement (PC). PC production is a significant carbon emissions source, producing on the order of $5 \%$ of the world's carbon emissions annually [Worrell et al., 2001]. RMC production requires a lower calcination temperature compared to PC (800 vs. $1450 \mathrm{oC}$ ), enabling the usage of renewable energy for calcination process; and RMCs necessarily consume significant amounts of CO2 during cementation [Ruan and Unluer, 2016]. These factors combined indicate that replacement of PC with RMCs in construction and industrial applications could largely reduce, or even eliminate, the carbon emissions derived from cement production and concrete usage.

Upon initial mixing, RMC-aggregate mixtures are porous and relatively weak, but over time, and with exposure to water and gaseous $\mathrm{CO} 2$, RMCs react to form stable magnesium-carbonate solids within the pore structure of the original aggregate matrix. The formation of carbonate cements strengthens the concrete, but can also clog the pore space and limit complete reaction.

This study reports on a suite of experiments focused on RMC curing in different aggregate compositions, under accelerated and ambient $\mathrm{CO}$ concentration conditions. The evolution of the 3D concrete microstructure was characterized using high resolution 3D X-ray computed tomography at the ANU CTLab (Figure 1); accompanying temporally-resolved mineral characterization was accomplished using diffraction infrared spectroscopy. Endpoint chemical composition and CO2 uptake of samples was determined used X-ray diffraction and thermogravitational analysis. The results provide insight into the bulk curing mechanism, enabling us to optimise initial aggregate composition such that the RMCs enhance $\mathrm{CO} 2$ uptake and cement strength. Curing under accelerated $\mathrm{CO} 2$ conditions is shown to enhance reaction rates, minimize clogging, and enhance strength. 


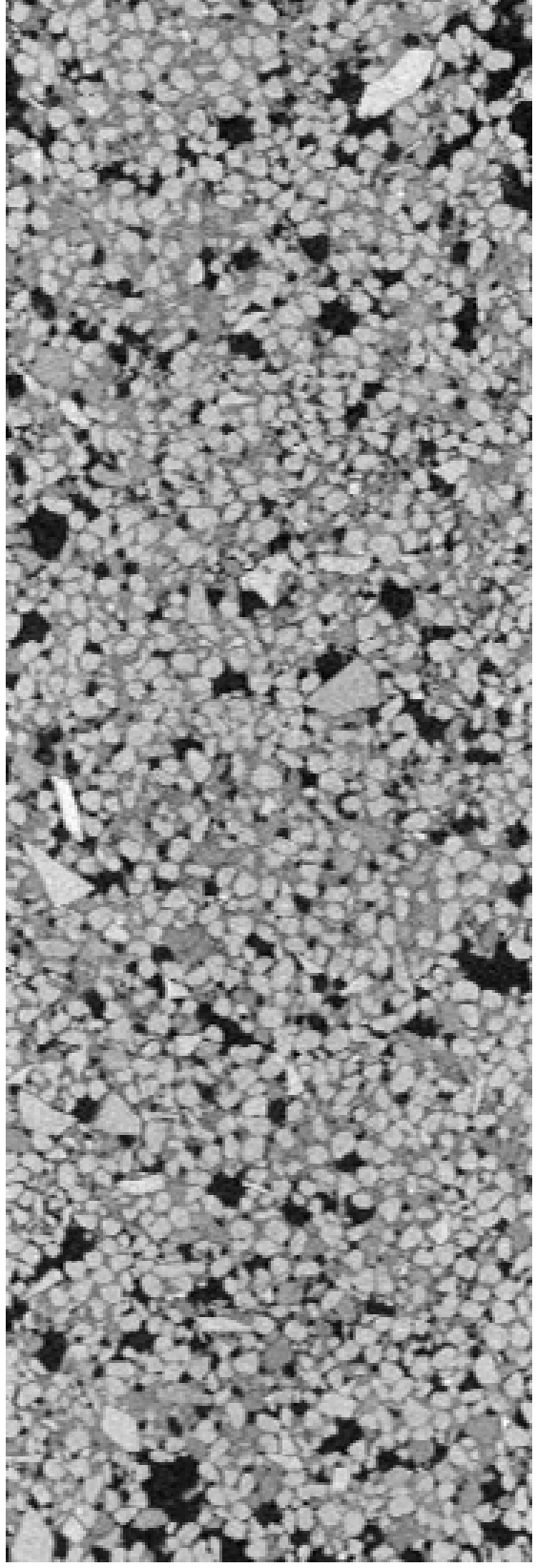

(a)

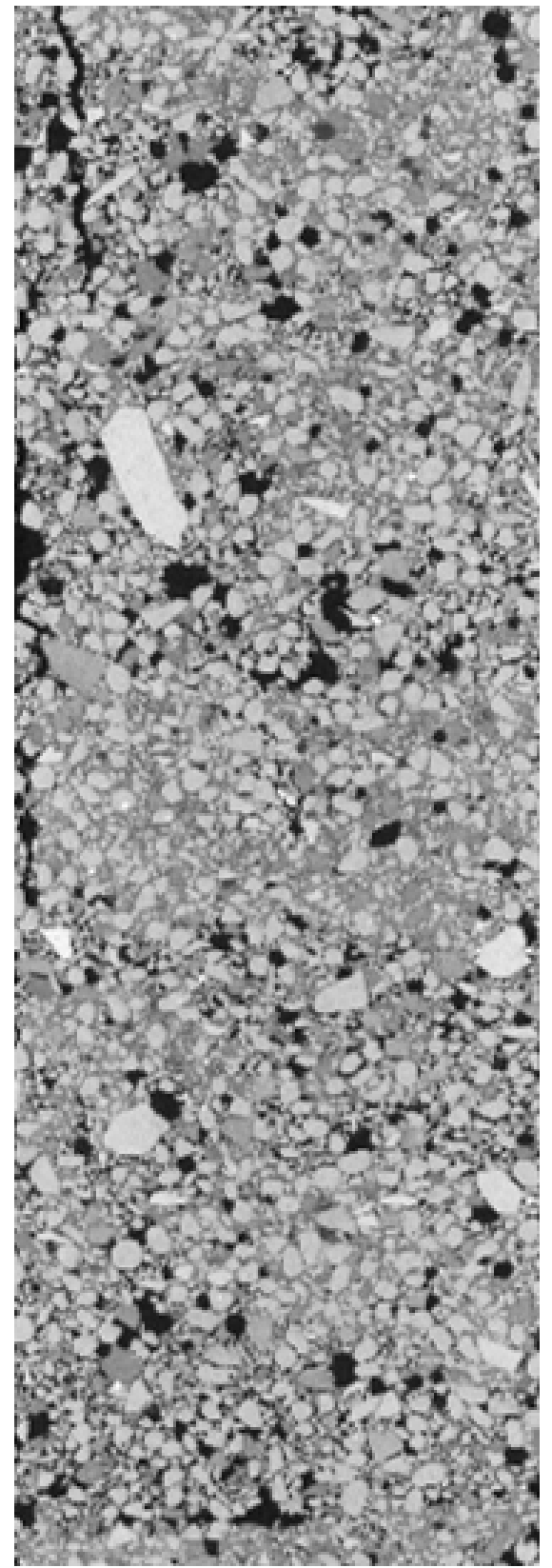

(b)

Figure 1. 2D slices from a 3D data volume of concrete micro-cores after 3 days of accelerated $\mathrm{CO}_{2}$ curing. Both samples had the same RMC:aggregate:water ratios, but were mixed with two different aggregate recipes: (a) 700 micron quartz grains and (b) a 50:50 mix of 350:700 micron quartz grains. 
Procter and Gamble Student poster award:

I don't want to compete References:

Ruan S., and C. Unluer (2016), Comparative life cycle assessment of reactive $\mathrm{MgO}$ and Portland cement production, J. Clean. Prod., 137, 258-273, doi:10.1016/J.JCLEPRO.2016.07.071.

Worrell, E., L. Price, N. Martin, C. Hendriks, and L. O. Meida (2001), CARBON DIOXIDE EMISSIONS FROM THE GLOBAL CEMENT INDUSTRY, Annu. Rev. Energy Environ., 26(1), 303-329, doi:10.1146/annurev.energy.26.1 Acceptance of Terms and Conditions:

Click here to agree

\title{
How does chaos rule concentration gradients in porous flow?
}

\author{
Joris Heyman $^{1}$; Daniel Lester ${ }^{2}$; Yves Méheust ${ }^{3}$; Tanguy Le Borgne ${ }^{4}$ \\ ${ }^{1}$ CNRS \\ ${ }^{2} \mathrm{RMIT}$ \\ ${ }^{3}$ Géosciences Rennes \\ ${ }^{4}$ Geosciences Rennes
}

Corresponding Author(s): joris.heyman@univ-rennes1.fr

In this talk, we present recent experimental and theoretical results about the mixing potential of flows in granular porous media. While these flows are observed in many natural contexts ( $96 \%$ of the fresh water resource is underground), their dispersive and reactive dynamics are poorly constrained. As an example, no universal relationship has yet been able to relate the effective macroscopic dispersion coefficients to the porous media structure and flow properties. Based on recent experiments, we show how the lamellar description of mixing together with the chaotic properties of porous flow may partly explain the evolution of solute concentrations and gradients, preparing the grounds for reactive model formulations based on the physics of pore-scale mixing.

Procter and Gamble Student poster award:

References:

Acceptance of Terms and Conditions:

Click here to agree

957

\section{Pore scale mechanisms of chaotic advection}

\author{
Joris Heyman $^{1}$; Daniel Lester ${ }^{2}$; Yves Méheust ${ }^{3}$; Tanguy Le Borgne ${ }^{3}$ \\ ${ }^{1}$ CNRS \\ ${ }^{2}$ RMIT \\ ${ }^{3}$ Géosciences Rennes
}

Corresponding Author(s): joris.heyman@univ-rennes1.fr

In this poster, we complement the talk by Heyman et al. (555) "How does chaos rule concentration gradients in porous flow?" explaining the mechanisms generating chaotic streamlines at pore scale. We propose a general model capable of predicting Lyapunov exponents for a large range of porous media. We show that granular porous media generally leads to stronger chaos than continuous 
porous media and that it is possible to tune the media properties (in terms of volume fraction and coordination number) to achieve a target mixing rate.

Procter and Gamble Student poster award:

I don't want to compete References:

Acceptance of Terms and Conditions:

Click here to agree

289

\section{Convective mixing in heterogenous porous media}

Juan J. Hidalgo ${ }^{1}$; Marco Dentz ${ }^{1}$

${ }^{1}$ IDAEA-CSIC

Corresponding Author(s): juanj.hidalgo@idaea.csic.es

We study the relation between convective instabilities and the heterogeneity of the porous medium. We consider a Rayleigh-Bénard instability driven by the density changes created by a sustained temperature difference in a porous medium characterized by a multi-Gaussian permeability field. Heterogeneity alters the shape and persistence of the scalar patterns as it interacts with the velocity field structure. The mixing efficiency of the system is also analyzed. We observe that the boundary fluxes are proportional to the variance of the permeability field. However, the system tends to be less well-mixed as the heterogeneity creates preferential flow paths that segregate the system.

Procter and Gamble Student poster award:

\section{References:}

Acceptance of Terms and Conditions:

Click here to agree

122

\section{SYNTHESIS, STRUCTURAL EVOLUTION AND APPLICATIONS OF METAL ORGANIC FRAMEWORK AND ZEOLITIC IMIDAZO- LATE FRAMEWORKS}

Mohamad Hmadeh ${ }^{1}$; Mohamad Hmadeh ${ }^{\text {None }}$

${ }^{1}$ AUB, Department of Chemistry, Beirut, Lebanon

Corresponding Author(s): mohamad.hmadeh@aub.edu.lb

Metal-Organic Frameworks (MOFs) and Zeolitic Imidazolate Frameworks( ZIFs) are an incipient class of highly crystalline porous extended frameworks 1 . These materials are characterized by their hybrid identity as they are formed by anchoring organic linkers mainly containing carboxylate (for MOFs) and Imidazolates (for ZIFs) moieties with metal clusters via strong coordination bonds2. These materials are renowned by their ultrahigh porosity, high surface area, flexibility and good thermal stability overcoming zeolites, activated carbons and other ordinary porous materials. Traditionally, MOFs and ZIFs are prepared through solvothermal methods, but alternative synthetic strategies have been developed based on exploiting conventional electric or microwave heating, electrochemistry, mechanochemistry, and ultrasonication. Herein, we exploit a new method of synthesizing MOFs and ZIFs via a reaction diffusion process at room temperature 3. Our novel method of synthesis is advantageous in that it can be easily carried out under facile conditions and provides control 
of the morphology and the size of the crystals by adjusting the thickness of the gel, the concentrations of the electrolytes and temperature. Moreover, more than one kind of metals and/or ligands could be incorporated within the same topology. Furthermore, application of these new materials on adsorption and catalysis are investigated and discussed.

Procter and Gamble Student poster award:

\section{References:}

References

1. H.-C. Zhou, J. R. Long and O. M. Yaghi, Chem. Reviews, 112, 673, (2012).

2. H. Furukawa, et al. Science, 341, 1230444, (2013).

3. Daniel Saliba et al., J. Am. Chem. Soc., 140, 1812, (2018). Acceptance of Terms and Conditions:

Click here to agree

\section{Gas flow in shale nanopores by solving Boltzmann model equa- tion}

Author(s): Minh Tuan $\mathrm{Ho}^{1}$

Co-author(s): Lei $\mathrm{Wu}^{1}$; Yonghao Zhang ${ }^{1}$

${ }^{1}$ University of Strathclyde, UK

Corresponding Author(s): minh-tuan.ho@strath.ac.uk

An in-depth understanding of gas transport in nanopores is the key to quantify flow properties of shale gas reservoirs. In shale nanopores, the gas flow deviates from the description of continuum fluid mechanics because of the rarefaction effects, which can be characterized by the Knudsen numbers 1. It has been shown in experimental study that significant amount of natural gas is adsorbed on organic-rich shale 2. Recently, a few transport models considering rarefaction and adsorption effects have been proposed for nanopores [3,4]. However, these models include empirical parameters determined by fitting with numerical or experimental data.

In modelling nanopore flows, molecular dynamics (MD) is widely used; however, its extremely high computational cost restricts size of the simulation domain. The gas kinetic theory approach, such as the lattice Boltzmann method (LBM), has been employed recently to simulate gas flow in nanopores [5,6]. Although the conventional LBM can be derived from the Bhatnagar-Gross-Krook (BGK) kinetic equation [7], which are simplified model of the Boltzmann equation, it fails to capture rarefaction effects in simple flows due to limited number of discrete velocities.

In this work, we employ the discrete velocity method (DVM) to solve the Boltzmann model equation, which can accurately capture rarefaction effects. Appropriate boundary condition is implemented in DVM to take into account the adsorption effects. We illustrate the influence of pore size and pressure on flow rate.

\section{Procter and Gamble Student poster award:}

\section{References:}

1 H. Darabi, A. Ettehad, F. Javadpour, K. Sepehrnoori. Gas flow in ultra-tight shale strata. Journal of Fluid Mechanics, 710:641-658, 2012.

2 S. M. Kang, E. Fathi, R. J. Ambrose, I. Y. Akkutlu, R. F. Sigal. Carbon dioxide storage capacity of organic-rich shales. SPE Journal, 16(04):842-855, 2011.

3 A. Sakhaee-Pour and S. Bryant. Gas permeability of shale. SPE Reservoir Evalu-

ation \& Engineering, 15(4):401-409, 2012.

[4] X.Xiong,D. Devegowda, G. G. Michel, R. F. Sigal, F. Civan. A fully-coupled free and adsorptive phase transport model for shale gas reservoirs including non-Darcy flow effects. In Proceedings of SPE Annual 
Technical Conference and Exhibition,

SPE 159758, 2012.

5 E. Fathi and I. Y. Akkutlu. Lattice Boltzmann method for simulation of shale gas transport in kerogen. SPE Journal, 18(01):27-37, 2013.

[6] J. Ren, P. Guo, Z. Guo, Z. Wang. A lattice Boltzmann model for simulating gas flow in kerogen pores. Transport in Porous Media, 106(2):285-301, 2015.

[7] P. Bhatnagar, E. Gross, and M. Krook. A model for collision processes in gases. I. Small amplitude processes in charged and neutral one-component systems. Physical Review, 94(3):511-525, 1954. Acceptance of Terms and Conditions:

Click here to agree

\section{Micro-scale simulation of fibrous liquid aerosol filters}

Author(s): Dennis Hoch ${ }^{1}$

Co-author(s): Andre' Baumann ${ }^{1}$; Jennifer Niessner ${ }^{1}$

${ }^{1}$ Heilbronn University of Applied Sciences, Germany

Corresponding Author(s): dennis.hoch@hs-heilbronn.de

Today aerosols are produced in many industrial processes like machining, manufacturing of catalysts or in pneumatic compressors. While the harmfulness of fine dust aerosols is well known the equally dangerous liquid aerosols (mist) receive less attention. In order to be prepared for future legal regulations we develop efficient mist filters with less energy consumption.

We present results of micro-scale simulations of mist deposition on fibrous structures. In our framework, the fibrous structure is obtained from $\mu \mathrm{CT}$ scans. Micro-scale simulations provide information on fractional efficiency as well as parameters and constitutive relationships for macro-scale simulation used to investigate pressure drop. Therefore, the micro-scale simulation is closely linked to the macro-scale simulation.

The aim is to develop fibrous mist filters with less energy consumption (reduced pressure drop) and increased fractional efficiency compared to existing filters. Both energy consumption and the fractional efficiency depend on the microscopic structure of the liquid aerosol filter. To improve both properties, it is necessary to analyze the microscopic structure of currently used mist filters first. Therefore, in the first step $\mu \mathrm{CT}$ scans are generated and analyzed. In order to obtain statistically representative microscopic and macroscopic properties, it is necessary to evaluate a sufficient number of $\mu \mathrm{CT}$ scans. The required amount of $\mu \mathrm{CT}$ scans has to be defined with a statistic method and depends of the material fluctuation.

The second step is to generate $\mathrm{CAD}$ data out of the $\mu \mathrm{CT}$ scans to run multiphase flow simulations on the CAD data. The multiphase flow simulation considers all processes and interactions between phases, such as droplet deposition, fluid film formation, droplet to droplet and droplet to wall as well as droplet to film interactions. Also the microscopic parameters like fiber diameter distribution, and fiber orientation are calculated. Subsequently, we determine macro scale parameters like porosity und permeability as well as capillary pressure- and relative permeability-saturation relationships. The micro-macro multi-scale coupling is put into practice by determining a liquid-phase source term as a function of parameters like initial saturation and velocity by micro-scale simulations. This source term is used along with the above mentioned parameters and constitutive relationships as an input to the macro-scale simulations.

The third step is to generate virtual fibrous structures with improved properties to optimize pressure drop (as an output of macro-scale simulations) and fractionally efficiency (output of micro-scale simulation).

Procter and Gamble Student poster award:

I would like to compete in the Procter and Gamble Student award References:

Acceptance of Terms and Conditions:

Click here to agree 


\title{
Hysteresis of Multiphase Flow in Porous and Fractured Media
}

\author{
Ran Holtzman ${ }^{1}$; Marco Dentz ${ }^{2}$; Ramon Planet ${ }^{3}$; Jordi Ortin ${ }^{3}$ \\ ${ }^{1}$ Institute of Environmental Assessment and Water Research (IDAEA) Spanish National Research Council (CSIC) \\ ${ }^{2}$ IDAEA-CSIC \\ ${ }^{3}$ Universitat de Barcelona
}

Corresponding Author(s): rholtzman4@gmail.com

Multiphase flow in porous and fractured media often exhibits hysteresis or path-dependency. In particular, at a given state in terms of capillary pressure, the saturation and fluid configuration can vary significantly between wetting (imbibition) and dewetting (drainage), resulting in a hysteretic capillary pressure-saturation (retention) function. This behaviour has important consequences in a variety of applications, including soil moisture, subsurface remediation, carbon sequestration and hydrocarbon recovery. The retention function is a key component in the classical continuum model for multiphase flow, the Richards equation.

Despite the importance of drainage-imbibition hysteresis, existing models rely on phenomenological or semi-empirical formulations. Here instead we propose a novel model based on physical considerations of the local pressure balance at the fluid-fluid interface. The model resolves a sequence of equilibrium configurations corresponding to local energy minima; numerically this is implemented through simple synchronous rules of high computational efficiency. In contrast to existing models, all the parameters involved have physical meaning and can be measured experimentally. The model and the results of our numerical simulations are validated against laboratory experiments of quasistatic displacements in a slightly inclined Hele-Shaw cell of variable depth -a laboratory analogue of a rough fracture, in which viscous fingering is suppressed by the stabilizing effect of gravity.

Our results establish a direct link between local, microscopic capillary instabilities (Haines jumps) and hysteresis in the macroscopic pressure-saturation curve. Our approach provides a physicallygrounded description of the nonequilibrium (metastable) nature of multiphase flow in porous and fractured media, and of the memory properties of partial wetting-dewetting cycles associated with it.

Procter and Gamble Student poster award:

References:

Acceptance of Terms and Conditions:

Click here to agree

\section{Impact of spatial correlations in particle sizes on fluid displace- ment in porous media}

Oshri Borgman $^{1}$; Thomas Darwent ${ }^{2}$; Enrico Segre ${ }^{3}$; Lucas Goehring ${ }^{2}$; Ran Holtzman ${ }^{4}$

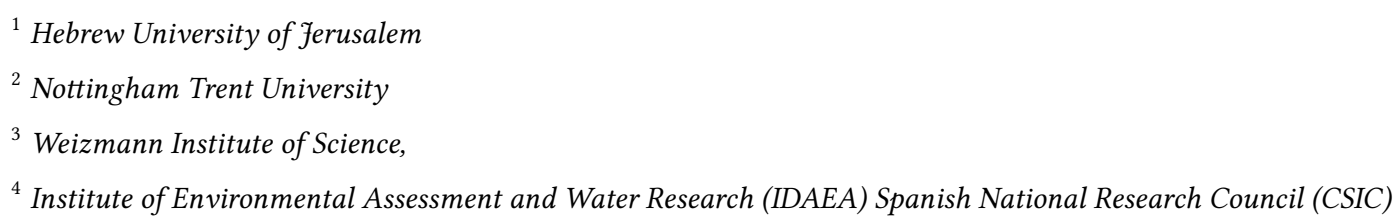

Corresponding Author(s): rholtzman4@gmail.com 
Immiscible fluid displacement in porous media is key in many environmental processes, including infiltration of water in soils, groundwater remediation, enhanced recovery of hydrocarbons and carbon geosequestration. While it is known that microstructural heterogeneity can significantly impact fluid displacement, quantification of its effect is lacking. We present a systematic pore-scale study that quantifies the impact of spatial correlations in pore sizes on the drainage of a partiallywetting fluid. We perform pore-network simulations at varying flow rates and different degrees of spatial correlation, complemented with microfluidic experiments at similar pore geometry. We find that spatial correlation leads to more preferential invasion, with reduced trapping of the defending fluid, especially at low flow rates. Increasing the correlation length reduces the fluid-fluid interfacial area and the trapping of the defending fluid, and increases the invasion pattern asymmetry and selectivity.

Procter and Gamble Student poster award:

References:

Acceptance of Terms and Conditions:

Click here to agree

\title{
Investigating induced calcium carbonate precipitation with a fo- cus on changing hydraulic properties
}

\author{
Johannes Hommel $^{1}$; Felix Weinhardt ${ }^{1}$; Robin Gerlach ${ }^{2}$; Holger Steeb ${ }^{1} ;$ Holger Class $^{1}$
}

${ }^{1}$ University of Stuttgart

${ }^{2}$ Montana State University

Corresponding Author(s): johannes.hommel@iws.uni-stuttgart.de, felix.weinhardt@iws.uni-stuttgart.de

Fluid storage in the subsurface is important to reduce climate change (sequestration of CO2) or for energy storage $(\mathrm{CH} 4, \mathrm{H} 2)$ to cope with the intermittent, unpredictable production of renewable sources like wind and solar. However, the fluids have the potential to leak through damaged cap rocks or wellbores. One method to remediate these problems is inducing calcium carbonate precipitation (ICP). Currently, most applications of ICP rely on urea hydrolysis by microbes (MICP) to promote precipitation within the porous media. However, precipitation may also be induced by the enzyme urease (EICP) extracted from microbes or plants. The applicability of a certain method is largely determined by the depth below ground surface and the local geothermal gradient. MICP has been demonstrated to have immense potential to seal leakage pathways, even at field scale 1 but is only effective within a limited temperature range, as it relies on the activity of living bacterial cells. For such field applications, numerical models are important tools to aid in the design, monitoring, and the evaluation of the application 2.

In such models, e.g. 3 developed for MICP, the sealing is predicted based on the volume of precipitated minerals using porosity-permeability relations [4]. The choice of a representative porositypermeability relation is necessary to model the large spatial scales of field-scale applications, as no pore-scale information on the location of the precipitates is available in REV-scale models. However, this is no straightforward choice to make, as the effect of a porosity reduction on permeability will depend on the location of that porosity reduction at the pore-scale and is likely to be specific for the porosity-reducing process. But ultimately, the predictions of the sealing efficiency will strongly depend on the used porosity-permeability relation. Additionally, when considering fluid storage in the subsurface, multi-phase systems may develop in the case that the present and introduced fluids are not fully miscible. In this case, also REV-scale properties such as the capillary pressure-saturation relation or the relative permeability-saturation relation will be affected by the precipitation of carbonates.

To improve REV-scale models, we investigate the effect of both MICP and EICP on hydraulic properties in microfluidic experiments, measuring the pressure drop to calculate the permeability while quantifying the change in porosity by microscopy (2D experiments) and $\mu \mathrm{CT}$ imaging $(2 \mathrm{D}$ and $3 \mathrm{D}$ experiments). Samples mineralized to various degrees will then also be subjected to capillary-pressure measurements during primary drainage to estimate the effect of ICP on the capillary pressuresaturation relation. Additionally to the experimental investigations, pore-scale simulations using the Stokes Equations on the actual geometries obtained by imaging and pore-network simulations 
will be used to confirm the measured impact of the porosity change on the permeability and to derive a process-and porous-medium-specific porosity-permeability relation.

\title{
Procter and Gamble Student poster award:
}

\section{References:}

1 Phillips, A. J., Cunningham, A. B., Gerlach, R., Hiebert, R., Hwang, C., Lomans, B. P., Westrich, J., Mantilla, C., Kirksey, J., Esposito, R., Spangler, L.; Fracture Sealing with Microbially-Induced Calcium Carbonate Precipitation: A Field Study. Environ. Sci. Technol. 2016, 50 (7), 4111-4117.

2 Cunningham, A. B., Class, H., Ebigbo, A., Gerlach, R., Phillips, A., Hommel, J.: Field-scale modeling of microbially induced calcite precipitation. Computational Geosciences (2018) accepted.

3 Hommel, J., Lauchnor, E., Phillips, A., Gerlach, R., Cunningham, A.B., Helmig, R., Ebigbo, A., Class, H.; A revised model for microbially induced calcite precipitation: Improvements and new insights based on recent experiments. Water Resour. Res. 2015; 51 (5), 3695-3715.

[4] Hommel, J., Coltman, E., Class, H.: Porosity-Permeability Relations for Evolving Pore Space: A Review with a Focus on (Bio-)geochemically Altered Porous Media. Transport in Porous Media (2018), 124 (2), 589-629. Acceptance of Terms and Conditions:

Click here to agree

\section{Dynamic Multiscale Simulation of Fractured Geothermal Reser- voirs}

\author{
Author(s): Mousa HosseiniMehr ${ }^{1}$ \\ Co-author(s): Cornelis Vuik ${ }^{1}$; Hadi Hajibeygi ${ }^{1}$ \\ ${ }^{1}$ TU Delft \\ Corresponding Author(s): s.hosseinimehr@tudelft.nl
}

A new dynamic multiscale method for fully-coupled simulation of flow and heat transfer in fractured geothermal reservoirs is presented (FG-ADM). The FG-ADM develops an advanced simulation method which maintains its efficiency when scaled up to field-scale applications. At the same time, it remains accurate in the presence of complex fluid physics and heterogeneous rock properties. The embedded discrete fracture model is employed to accurately represent explicit fractures while maintaining computational grid flexibility. On this given fine-scale system, FG-ADM introduces a multi-resolution nested dynamic grid based on the time-dependent solution of the heat and mass transport equations. The fully-coupled implicit simulation strategy, as well as the multiscale framework, makes the FG-ADM be stable and efficient in presence of strong flow-heat coupling terms. Furthermore, its finite-volume formulation preserves local conservation for both mass and heat fluxes. The FG-ADM employs multiscale multi-level local basis functions for pressure and temperature, in order to accurately represent the heterogeneous fractured rocks at coarse scales. These basis functions are constructed at the beginning of the simulation and are reused during the entire time-dependent simulation. For several heterogeneous test cases with complex fracture networks (including outcrop-based geo-models), we show that, by employing only a fraction of the fine grid cells, FG-ADM can accurately represent the flow-heat solutions in the fractured subsurface formations. The sensitivity of the method to the changes in the reservoir properties is studied for different coarsening criteria. The proposed method represents the first dynamic multilevel method for simulation of fractured geothermal reservoirs and it casts a promising approach for field-scale geothermal applications.

\section{Procter and Gamble Student poster award:}

I would like to compete in the Procter and Gamble Student award References:

Acceptance of Terms and Conditions:

Click here to agree 


\title{
Free Energy of Immiscible Two-Fluid Flow in Porous Media by Integral Geometry and Thermodynamics
}

\author{
Hamid Hosseinzade Khanamiri ${ }^{1}$; Carl Fredrik Berg ${ }^{1}$; Per Arne Slotte ${ }^{1}$; Steffen Schlüter ${ }^{2}$; Ole Torsæter ${ }^{1}$ \\ ${ }^{1} N T N U$ \\ ${ }^{2}$ Helmholtz-Centre for Environmental Research - UFZ
}

Corresponding Author(s): hamid.hosseinzade@ntnu.no

\begin{abstract}
In integral geometry, intrinsic volumes are a set of geometrical variables to characterize spatial structures, for example, distribution of fluids in two-fluid flow in porous media. McClure et al. (2018) utilized this principle and proposed a geometric state function based on the intrinsic volumes. In a similar approach, we find a geometrical description for free energy of a porous system with two fluids. This is also an extension of the work by Mecke (2000) for energy of a single fluid. Several geometrical sets of spatial objects were defined, including bulk of the two fluids, interfaces, and three-phase contact lines. We have simplified the description of free energy by showing how the intrinsic volumes of these sets are geometrically related. We obtain a description for energy as a function of seven microscopic geometrically independent variables. In addition, using a thermodynamic approach, we find an approximation for the free energy as a function of macroscopic parameters of saturation and pressure under quasi-static conditions. The combination of the two energy descriptions, by integral geometry and thermodynamics, completes the relation between the associated variables and enables us to find the unknown coefficients of the intrinsic volumes and to calculate the amount of dissipated energy in drainage and imbibition processes. We show that the theory is consistent with a set of experiments performed by Schlüter et al. $(2016,2017)$.
\end{abstract}

Procter and Gamble Student poster award:

References:

McClure, J. E., Armstrong, R. T., Berrill, M. A., Schlüter, S., Berg, S., Gray, W. G., \& Miller, C. T. (2018). Geometric state function for two-fluid flow in porous media. Physical Review Fluids, 3, 084306. https://doi.org/10.1103/PhysRevFluids.3.084306

Mecke K.R. (2000), Additivity, Convexity, and Beyond: Applications of Minkowski Functionals in Statistical Physics (Springer Lecture Notes in Physics vol 554) (Berlin: Springer) pp 111-84.

Schlüter, S., Berg S., Li T., Vogel H.-J., Wildenschild, D. (2017), Time scales of relaxation dynamics during transient conditions in two-phase flow, Water Resour. Res., 53, doi:10.1002/2016WR019815.

Schlüter, S., Berg, S., Rücker, M., Armstrong, R.T., Vogel H.-J., Hilfer, R., \& Wildenschild, D. (2016), Porescale displacement mechanisms as a source of hysteresis for two-phase flow in porous media, Water Resour. Res., 52 (3), 2194-2205, doi: 10.1002/2015WR018254. Acceptance of Terms and Conditions:

Click here to agree

\section{Combination of the in-situ loading scanning experiment and dig- ital core deformation simulation for investigating stress sensitiv- ity mechanism}

\author{
Author(s): Yanan $\mathrm{Hou}^{1}$ \\ Co-author(s): Weibo Sui ${ }^{1}$; Zhilin Cheng ${ }^{1}$ \\ ${ }^{1}$ China University of Petroleum Beijing
}

Corresponding Author(s): hyn930802@163.com, suiweibo@cup.edu.cn, zhilin_cheng1992@163.com

Rock stress sensitivity was mostly investigated through macro-scale experiments. A new method combining a high-resolution two-dimensional (2D) in-situ loading scanning experiment and digital core deformation simulation was developed in this paper, which provides an effective way to 
investigate the relationship between micro-structural deformation and permeability decrease. The simulation method may also be a possible replacement of in-situ loading scanning experiments under certain scenarios.

Firstly, the 2D in-situ loading scanning experiment combining Field Emission Scanning Electron Microscope (FE-SEM) and in-situ loading equipment were carried out. The micro structure deformation of the rock sample during uniaxial loading had been observed and recorded, and the FE-SEM images at different stress states were analyzed by Digital Image Correlation Method (DIC) for investigating the principles of rock micro structure deformation. The deformation simulation method was proposed based on the investigation. The simulation was demonstrated to be effective by comparing the results of simulation and 2D in-situ experiments. Secondly, to validate the simulation method for three-dimensional (3D) digital core, a 3D digital core of tight sandstone was reconstructed by MicroCT imaging experiment. With the same rock sample, porosity-permeability integrated measurements under triaxial stresses were conducted for obtaining the macro-scale porosity-permeability data under different stress states. With the constraints of measured porosity changes, a 3D digital core deformation simulation was realized by applying the simulation method. Then the series of simulated 3D digital cores at different stress states were used for pore network model extraction, a group of permeabilities were calculated respectively. Comparing the permeability changes of simulation and porosity-permeability integrated measurements, the consistent results showed that the simulation method can be used for 3D digital core micro stress sensitivity investigation.

Besides, the SEM images analysis in 2D in-situ loading scanning experiments showed that the throats generally lead to more severe deformation than the pores did. Pore and throat diameter frequency distributions were calculated from pore network models. By analyzing the frequency distributions, we can see that the amounts of connected throats and pores decreased with increasing stress, and the amount of throats changed more intensely than that of pores. The changing percentage of the diameter of throats was larger than that of pores. Therefore, we concluded that the deformation of throats is more critical than pores for permeability reduction.

\section{References:}

An S, Yao J, Yang Y, et al. Influence of pore structure parameters on flow characteristics based on a digital rock and the pore network model[J]. Journal of Natural Gas Science \& Engineering, 2016, 31:156-163. Ataman E, Aatre V K, Wong K M. Some statistical properties of median filters[J]. IEEE Transactions on Acoustics Speech \& Signal Processing, 1981, 29(5):1073-1075.

Biot M A. General Theory of Three-Dimensional Consolidation[J]. Journal of Applied Physics, 1941, 12(2):155-164.

Blaber J, Adair B, Antoniou A. Ncorr: Open-Source 2D Digital Image Correlation Matlab Software[J]. Experimental Mechanics, 2015, 55(6):1105-1122.

Curtis M, Ambrose R, Sondergeld C. Structural Characterization of Gas Shales on the Micro and NanoScales[C]// Society of Petroleum Engineers, 2010.

Dimanov A, Bornert M, Dautriat J, et al. Micromechanical Investigation of the Hydromechanical Behaviors of Carbonates Contribution of In-Situ Strain Field Measurement by Means of SEM and Optic Digital Image Correlation[J]. Petrophysics, 2010, 51(6):1477-1507.

Dong H, Blunt M J. Pore-network extraction from micro-computerized-tomography images.[J]. Phys Rev E Stat Nonlin Soft Matter Phys, 2009, 80(2):036307.

He W, Hayatdavoudi A. A comprehensive analysis of fracture initiation and propagation in sandstones based on micro-level observation and digital imaging correlation[J]. Journal of Petroleum Science \& Engineering, 2018, 164:75-86.

Lai J, Wang G, Wang Z, et al. A review on pore structure characterization in tight sandstones[J]. EarthScience Reviews, 2018, 177:436-457.

Loucks R G, Reed R M, Ruppel S C, et al. Morphology, Genesis, and Distribution of Nanometer-Scale Pores in Siliceous Mudstones of the Mississippian Barnett Shale[J]. Journal of Sedimentary Research, 2015, 79(12): 848-861.

Jie Liu, Joel Sarout, Minchao Zhang, Jeremie Dautriat, Emmanouil Veveakis, Klaus Regenauer-Lieb; Computational upscaling of Drucker-Prager plasticity from micro-CT images of synthetic porous rock, Geophysical Journal International, Volume 212, Issue 1, 1 January 2018, Pages 151-163, https://doi.org/10.1093/gji/ggx409 Martin J. Blunt, Branko Bijeljic, Hu Dong, et al. Pore-scale imaging and modelling[J]. Advances in Water Resources, 2013, 51(1):197-216.

Otsu, N. 1975. A threshold selection method from gray-level histograms. Automatica 11(285-296): 2327.

Sun H, Tao G, Vega S, et al. Simulation of gas flow in organic-rich mudrocks using digital rock physics[J]. Journal of Natural Gas Science \& Engineering, 2017, 41:17-29.

Wang, F. P., \& Reed, R. M. (2009, January 1). Pore Networks and Fluid Flow in Gas Shales. Society of Petroleum Engineers. doi:10.2118/124253-MS

Wang L L, Bornert M, Héripré E, et al. Full-Field Measurements on Low-Strained Geomaterials Using Environmental Scanning Electron Microscopy and Digital Image Correlation: Improved Imaging Con- 
ditions[J]. Strain, 2015, 50(5):370-380.

Wang L L, Bornert M, Héripré E, et al. The Mechanisms of Deformation and Damage of Mudstones: A Micro-scale Study Combining ESEM and DIC[J]. Rock Mechanics \& Rock Engineering, 2015, 48(5):19131926.

Wang L L, Yang D S, Yang R W, et al. Investigating the mechanical behavior of shale: A micro-scale approach[J]. Journal of Natural Gas Science \& Engineering, 2016.

Warpinski N R, Teufel L W. Determination of the Effective-Stress Law for Permeability and Deformation in Low-Permeability Rocks[J]. Spe Formation Evaluation, 1992, 7(2):123-131.

Yang F, Ning Z, Wang Q, et al. Pore structure characteristics of lower Silurian shales in the southern Sichuan Basin, China: Insights to pore development and gas storage mechanism[J].International Journal of Coal Geology, 2016, 156: 12-24.

Zhang Y, Xu X, Lebedev M, et al. Multi-scale x-ray computed tomography analysis of coal microstructure and permeability changes as a function of effective stress[J]. International Journal of Coal Geology, 2016, 165:149-156. Acceptance of Terms and Conditions:

Click here to agree

\title{
Experimental Investigations of Flow Boiling inside Porous Media at Pore-scale with Micromodels
}

\author{
Haowei $\mathrm{Hu}^{1}$; Ruina $\mathrm{Xu}^{1}$; Peixue Jiang ${ }^{1}$ \\ ${ }^{1}$ Tsinghua University \\ Corresponding Author(s): hwhu1994@163.com
}

Flow boiling inside porous media is widely used in many applications, such as thermal-removal system for electronic devices, heat pipes, and high-efficiency heat exchangers. During phase transition inside the pore, there are too many uncertainties to accurately measure temperature distribution and clearly know what's happening inside the pore. With these considerations, in this paper, we conduct micro-scale experiment with Particle Image Velocimetry (PIV) to take deeper insight into flow boiling inside porous media, and provide a fundamental knowledge to optimize the phase-changed applications.

The pore-scale test is carried out to investigate the non-continuous phenomenon inside microfluidics device, including the process of bubble nucleation, coalescence, flow-pattern transition, and dry-out inside the pore. We designed different micromodels to figure out the effect of wettability, porosity and geometry, all of which are in micro-size. In this experiment, as an application for electronic devices cooling, the critical heat flux was measured with different mass flow rate and heat flux, and the mechanism of local heat transfer deterioration is explained. To deeply grasp the random flow inside the pore and the effect of porosity, Particle Image Velocimetry (PIV) technology is applied to quantify the velocity distribution at pore-scale, which is different from large-scale. Backflow can be investigated with the help of capillary force, which is vital to enhance replenishment of coolant to the hot wall and delay the critical heat flux. What's more, different pore-structure can lead to different heat transfer performance, which is favorable for thermal-removal system for electronic devices as well.

In all, in this paper, we take new insight into two-phase flow inside porous media at micro-scale, which can enhance the understanding of small scale processes taking place within tiny fluid volumes with non-continuous effect, and give further knowledge of the effect of phase change, capillary forces, trapping process, and so on. Micromodels with different wettability, porosity and geometry are designed and investigated at the scale of individual pores. The results are applicable to learn microfluidics in porous systems, which provides a fundamental knowledge to optimize the structure and control strategy of phase-changed applications.

Procter and Gamble Student poster award:

I don't want to compete References:

Acceptance of Terms and Conditions:

Click here to agree 


\title{
Numerical Simulation of Multiphase Flow inside Porous Media at Low Pressure
}

Haowei $\mathrm{Hu}^{1}$; Ruina $\mathrm{Xu}^{1}$; Peixue Jiang ${ }^{1}$

${ }^{1}$ Tsinghua University

Corresponding Author(s): hwhu1994@163.com

Flow boiling inside porous media has many applications, such as bio-systems coordination, heat pipes, high-efficiency heat exchangers, and aerospace thermal protection. As the structure of porous media is complex, it's hard to accurately measure the parameters distribution and the mechanism of local heat transfer deterioration inside the pore, especially with dynamic phase transition. Therefore, numerical simulation provides an alternative way for the research during the past 60 years. An extensive work has been conducted at REV (Representative Elementary Volume) scale, and two of the typical models are multiphase flow model (MPM) and multiphase mixture model (MPMM). Recently, pore-scale model becomes a new trend to take deeper insight into this problem and consider the interaction between the pore and throat, such as pore network model. However, till now the existing study on the mechanism of deterioration is insufficient, thus, it's difficult to give reasonable support for the applications. Besides, flow and heat transfer inside porous media is a quite non-linear problem, which calls for endeavor to enhance the convergence of the algorithm.

Based on these problems, this paper puts forward a modified model to link the phase-change phenomenon during flow boiling at low pressure. First, we modified multiphase mixture model at REV scale. To enhance model convergence and accuracy, the energy equation is rearranged with kinetic enthalpy, which has a great linearity and a clear physical meaning, and can catch some slight variation without missing some important data. Besides, to figure out the mechanism of local heat transfer deterioration, with the help of micromodel experiment, we take pore-scale effect into consideration, such as capillary pressure, saturation temperature change with slight pressure drop at low pressure, and the geometry effect into the model. We found that at low pressure, slight pressure drop may cause large temperature drop at two-phase zone, which is usually neglected at high pressure, and concluded that capillary force plays an important role during flow boiling inside porous media, and the vapor formation affects flow and heat transfer well, which is coupled with the interaction of mass flow rate and pore-structure. We then added these effects into our model to study the relation between the pore geometry and the flow and heat input. The results show that with proper geometry design, local heat transfer deterioration can be delayed, which is important in many thermalprotection applications.

In all, in this paper, numerical simulation of multiphase flow is conducted to take deeper insight into the internal mechanism during phase change inside porous media. This paper reveals the mechanism of local deterioration during flow boiling in porous media inside the pore, and summarizes the response mechanism during dynamic process, which provides a fundamental knowledge to optimize the structure and control strategy of phase-changed applications.

Procter and Gamble Student poster award:

I don't want to compete References:

Acceptance of Terms and Conditions:

Click here to agree

\section{Energy conversion reveals regime transition of imbibition in a rough fracture}

\author{
Ran $\mathrm{Hu}^{1}$; Dong-Sheng $\mathrm{Wu}^{1}$; Yi-Feng $\mathrm{Chen}^{1}$ \\ ${ }^{1}$ Wuhan University
}


Corresponding Author(s): whuran@whu.edu.cn

The displacement of non-wetting phase by wetting phase in permeable media, known as imbibition, is central to diverse processes including enhanced oil recovery and geological carbon sequestration. As externally imposed flow rate increases during imbibition, viscous force increasingly dominates the imbibition over the capillary force, leading to the transition of imbibition from capillary to capillary-viscous regimes. Since imbibition involves energy conversion among surface energy, dissipated energy, external work and kinetic energy, is there a fundamental link between energy conversion and regime transition? To explore this issue, we perform imbibition experiments in a rough fracture and analyze energy balance enabled by real-time imaging. We find that regime transition is intrinsically linked with energy conversion. In capillary regime, surface energy is partially transformed into external work, and $51 \sim 58 \%$ of the total surface energy is dissipated. In capillaryviscous regime, surface energy together with external work is transformed into kinetic and dissipated energies. Such transition is evidenced by quantitative analysis of invasion morphologies. Our findings bridge the gap between energy conversion/dissipation and multiphase flow, and have important implications for identifying imbibition regimes in enhanced oil recovery and geological CO2 sequestration.

Procter and Gamble Student poster award:

References:

Acceptance of Terms and Conditions:

Click here to agree

\title{
Robust Preconditioners for Mixed-dimemsional Models of Flow in Fractured Porous Media
}

\author{
Author(s): Xiaozhe $\mathrm{Hu}^{1}$ \\ Co-author(s): Wietse Boon ${ }^{2}$; Ana Budiša ${ }^{3}$ \\ ${ }^{1}$ Tufts University \\ ${ }^{2}$ University of Stuttgart \\ ${ }^{3}$ University of Bergen
}

Corresponding Author(s): xiaozhe.hu@tufts.edu

Mixed-dimensional partial differential equations arise in many physical applications including flow in fractured porous media, where the fractures and their intersections form a hierarchy of lowerdimensional submanifolds. An essential component, and usually the most time-consuming part of simulating PDEs, is solving the large-scale and ill-conditioned linear systems of equations arising from discretizations. In this work, we generalize the traditional framework of designing preconditioners for the saddle point systems and develop effective preconditioners that are robust with respect to the physical and discretization parameters for mixed-dimensional models for flow in fractured porous media. Preliminary numerical experiments are presented to support the theory and demonstrate the robustness of our preconditioners.

Procter and Gamble Student poster award:

References:

Acceptance of Terms and Conditions:

Click here to agree 


\title{
Experimental study on mechanical properties of granite subjuected to temperature and cyclic stress
}

\author{
Yaoqing $\mathrm{Hu}^{\text {None }}$
}

Corresponding Author(s): huyaoqing@tyut.edu.cn

\begin{abstract}
1 INTRODUCTION
Temperature and stress are important factors affecting the mechanical properties of rocks, such as the treatment of high-temperature nuclear waste, geothermal development and utilization, in-situ pyrolysis of oil shale, underground coal gasification and other fields are involved in high temperature and alternating load. So the high temperature and alternating load seriously affect all aspects of the project. The study of physical and mechanical properties of rock has become a very active research direction in rock mechanics. Relevant research results are mainly concentrated in two aspects, one is the study of physical and mechanical properties of granite after high temperature action, the other is the study of physical and mechanical properties under real-time temperature conditions.

Most of the research results about the effect of cyclic loading on the mechanical properties of granite do not consider the effect of temperature. Through uniaxial compression and fatigue deformation tests of granite and white sandstone, a new method for determining the critical stress of rock under cyclic loading is proposed (Feng,2010). The evolution law of plastic hysteresis loops under cyclic loading is studied (Wang and Li 2006, 1017). It is concluded that the plastic hysteresis loops will tend to be stable from the second cycle and the relationship between the plastic hysteresis loops and the number of cycles is obtained. Taking into account the influence of temperature, Xia (2015) carried out an experimental study on the mechanical properties of basalt under cyclic uniaxial stresstemperature. The results show that cyclic stress and cyclic temperature have a "superposition" effect, and the failure mode is related to the upper limit of cyclic stress and temperature. The highest temperature of this experiment is $90 \%$.

In order to further reveal the mechanical properties of granite under high temperature, the mechanical properties of granite under real-time high temperature and the influence of cyclic loading on its mechanical properties at different temperatures are studied in this paper, which provides a reference for the study of related engineering fields.
\end{abstract}

2 METHODOLOGY

The experiment is carried out by a multi-functional rock triaxial testing machine developed by Taiyuan University of Technology. The maximum axial pressure of the testing machine is $1000 \mathrm{KN}$, and the maximum temperature is $650 \mathrm{C}$. Granite samples were processed into $30 \rrbracket 60 \mathrm{~mm}$ specimens. The first group measured the uniaxial stress-strain characteristics under real-time temperature conditions. The temperature ranged from $25^{\circ} \mathrm{C} \sim 600^{\circ} \mathrm{C}$, and there were 3 pieces in each temperature state, totaling 21 pieces. The second group measured the stress-strain characteristics of granite under cyclic loading at different temperatures. The temperature level and the number of specimens were the same as the first group. The upper and lower limit distributions of stress cycles were taken as $20 \%$ and $80 \%$ of uniaxial compressive strength at corresponding temperatures. The stress is loaded to the preset stress values at a rate of $0.1 \mathrm{KN} / \mathrm{s}$ and then unloaded at a rate of $0.1 \mathrm{KN} / \mathrm{s}$. It is repeated 50 times. If the failure occurs within 50 times, the test is completed. If no failure occurs, the stress is reduced to 0 , and then loaded at a rate of $0.1 \mathrm{KN} / \mathrm{s}$ until the specimen fails.

3 RESULTS AND CONCLUSION

1)the uniaxial compressive strength and elastic modulus of granite are drops gradually with the increase of temperature, while the elastic modulus reaches its maximum in $100^{\circ} \mathrm{C} ; 2$ ) The influence law of temperature on the ultimate strain generally exhibits an $\mathrm{W}$ shape.In other words, $25^{\circ} \mathrm{C}$ to $200^{\circ} \mathrm{C}$, the ultimate strain of granite is drops with the increase of temperature; $200^{\circ} \mathrm{C}$ to $300^{\circ} \mathrm{C}$,increases with temperature; $300^{\circ} \mathrm{C}$ to $500^{\circ} \mathrm{C}$ decreases with temperature rise; and after $500^{\circ} \mathrm{C}$ increased with temperature increase;3) The cyclic stress has little effect on the change of elastic modulus from cycles 2 to 50, but it is greater than the elastic modulus during the first loading.4)The elastic modulus of granite subjected to cyclic stress under high temperature is generally increased, $100^{\circ} \mathrm{C}$ and $400^{\circ} \mathrm{C}$ are its two extremities;5) $100^{\circ} \mathrm{C}$ not only is the highest temperature threshold value in the elastic modulus of granite, is also the temperature of elastic best threshold value;6) The effect of cyclic stress on uniaxial compressive strength of granite is related to its temperature;7) Sample subjected to 50 times cyclic stress at $100{ }^{\circ} \mathrm{C}$ and $400{ }^{\circ} \mathrm{C}$ temperature, the ultimate strain value is greater than the ultimate strain without stress cycle, and its changes is slightly on another temperature.Such results has important theoretical significance and application value for the study of engineering stability under the simultaneous action of temperature and cyclic stress. 
KEYWORDS: Granite, Cyclic stress, Temperature, Mechanical properties

Procter and Gamble Student poster award:

I would like to compete in the Procter and Gamble Student award References:

Acceptance of Terms and Conditions:

Click here to agree

\title{
Thermal conductivity measurement of porous materials: chal- lenges, examples and prospects
}

\author{
Zhang $\mathrm{Hu}^{1}$; Shang Chen-Yang ${ }^{1}$; Tang Gui-Hua ${ }^{1}$ \\ ${ }^{1}$ Xi'an fiaotong University \\ Corresponding Author(s): huzhang@xjtu.edu.cn
}

Materials with pore size ranging from nanoscale to macroscale are widely used as thermal insulation materials for they possess very low thermal conductivity. The thermal conductivity is an effective and key feature of porous thermal insulation materials and its test accuracy is of great significance. Many steady state and unsteady state methods have been used to measure the thermal conductivity of porous materials. However, there have some challenges on measuring it accurately. Porous materials have some specific features. For example, the thermal conductivity of solid structure in porous materials can be 10 to 10,000 times higher than that of gaseous molecules. Thermal radiation happens in porous materials and heat convection also exists in large size pores. For moisture materials, liquid molecules also contributes to the effective thermal conductivity which is affected by the varied moisture distribution under non-uniform temperature field.

Traditional thermal conductivity methods have some challenges when measuring porous materials. The laser flash method, hot wire method, hot strip method and transient plane source method are all based on the unsteady energy equation via heat conduction for uniform and isotropic medium. When measuring porous materials with high porosity, large pore size or high transparency, the accuracy will be questionable due to the participating of thermal radiation. The effective thermal conductivity of porous materials is related to the sample size when the pore size is close to the heat penetration depth of dynamic test process or close to the sample thickness for steady state method. For the thermal conductivity measurement of moisture materials, the existence of temperature gradient during the test will affect the moisture distribution which will lead to some uncertainty. When measuring porous materials, the influence of nature convection within the pores is regarded as negligible for pore size smaller than $1 \mathrm{~mm}$. However, the assumption is lacking of verification and the critical value varies with temperature and the magnitude of effective thermal conductivity.

Due to the characteristics of porous materials and the different heat transfer processes among steady state method and unsteady method, the thermal conductivity measured by different methods can be different for the same porous materials. In this study, the thermal conductivity of porous materials with pore size from nanoscale to microscale and macroscale will be measured by steady state method and unsteady state method. The comparison among these examples will be made to illustrate the difference. The difference is not only caused by the theoretical basis of different test methods, but related to the uncertainties of input and output parameters as well. Thus, it is difficult to evaluate the uncertainties introduced by the test theory and test parameters separately, quantitatively and exactly. Numerical experiment is a novel and prospective method to evaluate the influences of different factors and to reveal the responding uncertainty quantitatively.

Procter and Gamble Student poster award:

I would like to compete in the Procter and Gamble Student award References:

Acceptance of Terms and Conditions: 
Click here to agree

\title{
Pore-scale experiments on the behavior of $\mathrm{CO} 2$ exsolution in ther- mal recovery and $\mathrm{CO} 2$ combined EOR
}

\author{
Feng Huang $^{\text {None }}$; Ruina $\mathrm{Xu}^{1}$; Peixue Jiang ${ }^{\text {None }}$ \\ ${ }^{1}$ Tsinghua University \\ Corresponding Author(s): thuhuangfeng@163.com
}

With the decline in recoverable conventional oil reserves and rising costs caused by the continuous exploitation of conventional reservoirs, more and more attention has been paid to unconventional reservoirs with huge reserves. However, unconventional reservoirs are often more difficult to mine due to the smaller pore size, larger viscosity, deeper reservoir depth and the like. For tertiary oil recovery, both the thermal recovery and $\mathrm{CO} 2$ have been successfully applied to enhance oil recovery. In the process of jointly utilizing the thermal recovery and $\mathrm{CO} 2$, the heating effect induced by the high temperature steam will cause the solubility of $\mathrm{CO} 2$ in oil to decrease. Subsequently, the $\mathrm{CO} 2$ which dissolves in the oil phase before becomes supersaturated, nucleate on the pores wall and grow up with supply of $\mathrm{CO} 2$ component from the surrounding oil phase. The process of $\mathrm{CO} 2 \mathrm{bubble}$ nucleation, growth, migration, and coalesce affects the $\mathrm{CO} 2$-oil two-phase fluids morphology, distribution and flow characteristics in the underground porous medium, thereby having a nonnegligible impact on the EOR efficiency. Therefore, revealing the law of heating induced CO2 exsolution in oil phase from the pore scale plays a significant role in understanding formation multi-phase seepage and EOR.In this study, a pore-scale high pressure visualization experimental system which can provide a variable temperature (in range of room temperature to $120^{\circ} \mathrm{C}$ ) was applied. And the $\mathrm{CO} 2$ exsolution behavior from the supersaturation oil in microporous due to the heating induced solubility reduction had been directly observed and analyzed. Using a micro-heating system, we explored the influence of heating rate and the degree of superheat on the nucleation, growth and migration of $\mathrm{CO} 2$ during the exsolution process. With a relatively higher heating rate and degree of superheat, the $\mathrm{CO} 2$ bubbles could grow faster and have a larger volume. However, once the heating rate and degree of superheat were too large, dense $\mathrm{CO} 2$ bubbles emerged in the oil phase and grew up slowly. The quantitative temperature dependency of the exsolved $\mathrm{CO} 2$ volume and mass for the depressurization and heating process are obtained based on the pore-scale images. We also compared the observed phenomenon to a pore-scale gas exsolution model, and modify the model to fit the case of $\mathrm{CO} 2$ exsolution from oil in microporus better.

Procter and Gamble Student poster award:

I would like to compete in the Procter and Gamble Student award References:

Acceptance of Terms and Conditions:

Click here to agree

68

\section{An efficient numerical simulator based on embedded discrete frac- ture model for fractured karst carbonate reservoirs}

\author{
Author(s): Zhaoqin Huang ${ }^{1}$ \\ Co-author(s): Jun Yao ${ }^{2}$; Lijun Liu $^{1}$; Liang Gong ${ }^{1}$; Huang Tao ${ }^{1}$; Xiaoguang Wang ${ }^{3}$; Hervé Jourde ${ }^{3}$ \\ ${ }^{1}$ China University of Petroleum (East China) \\ ${ }^{2}$ China University of Petroleum \\ ${ }^{3}$ Laboratoire HydroSciences Montpellier (HSM)
}


Corresponding Author(s): huangzhqin@upc.edu.cn

Fractured karst reservoir is a typical kind of carbonate reservoir, which have significant contributions for the world oil \& gas reserves and productions. The key issue of numerical simulation for such reservoirs is how to model the 3D complex fracture and cavities. However, this is still a challenging for real fractured karst carbonate reservoirs. In this work, a hybrid model for simulating multiphase fluid flow in 3D complicated fractured karst reservoir is presented. In the hybrid model, an improved embedded discrete fracture model (EDFM) and a cavity model are proposed. The improved EDFM improves the discretization of fractures by using two sets of independent grids for the matrix and fracture systems, which promotes the modeling of 3D complex fracture in real geologic models (corner-point grids). The proposed cavity model simplifies the coupled porous and free flow by the assumption of multi-phase instantaneous gravity differentiation. We demonstrate the accuracy of the improved EDFM and the cavity model by comparing the results with the conventional EDFM and volume of fluid (VOF) method. Then based on the proposed hybrid model, an efficient numerical simulator is developed based on the integrated finite difference method. And then based on the typical outcrops of TAHE oilfields, three typical fracture-cavity unit models are designed to analyze their flow characteristics, which indicate the effect of fracture and cavity on the production performance. Finally, a real fractured karst carbonate reservoir model with 3D complicated fractures and cavities is simulated to demonstrate the applicability of the proposed model.

Procter and Gamble Student poster award:

References:

Acceptance of Terms and Conditions:

Click here to agree

121

\title{
Liquid-Infused Nanoporous Solids as Photonic Metamaterials
}

\author{
Kathrin Sentker ${ }^{1}$; Mark Busch ${ }^{1}$; Andriy Kityk ${ }^{2}$; Patrick Huber $^{1}$ \\ ${ }^{1}$ Hamburg University of Technology \\ ${ }^{2}$ Czestochowa University of Technology
}

Corresponding Author(s): patrick.huber@tuhh.de

\begin{abstract}
Metamaterials derive their functional properties not from the properties of the base materials, but from their newly designed, often multiscale structures, in terms of precise shape, geometry, size, orientation, and elastic properties. Such hybrid material systems can be designed by infusing liquids in porous solids. In particular nanoporous media offer the possibility to establish structures significantly smaller than optical wavelengths and thus to act as photonic metamaterials, where the optical properties can be fine-tuned by the filling of the pore space.

Here we present Laser-optical, $x$-ray and neutron diffraction experiments on monolithic nanoporous silicon, silica and alumina filled with simple (n-alkanes, water) and complex liquids (polymers, liquid crystals). These experiments allow us to gain detailed insights on the liquid distribution in the nanoporous media and the phase behaviour of the confined fluids with regard to the unconfined state $[1,2,3,4]$. By the same token the resulting soft-hard hybrid materials exhibit novel properties typical of photonics metamaterials, encompassing optical birefringence step-wise changing with temperature 1, switchable optical transmission 2 and fast electro-optical activity 3.
\end{abstract}

Procter and Gamble Student poster award:

\section{References:}

1 Quantized self-assembly of discotic rings in a liquid crystal confined in nanopores. K. Sentker, A.W. Zantop, M. Lippmann, T. Hofmann, O.H. Seeck, A.V. Kityk, A. Yildirim, A. Schoenhals, M.G. Mazza, and P. Huber; Physical Review Letters 120067801 (2018).

2 Liquid n-hexane condensed in silica nanochannels: A combined optical birefringence and vapor sorption isotherm study. A.V. Kityk, K. Knorr, and P. Huber; Physical Review B 80, 035421 (2009). 
3 A ferroelectric liquid crystal confined in cylindrical nanopores: Reversible smectic layer buckling, enhanced light rotation and extremely fast electro-optically active Goldstone excitations M. Busch, A.V. Kityk, W. Piecek, T. Hofmann, D. Wallacher, S. Calus, P. Kula, M. Steinhart, M. Eich, and P. Huber; Nanoscale 9, 19086 (2017).

[4] Chiral phases of a confined cholesteric liquid crystal: Anchoring-dependent helical and smectic selfassembly in nanochannels; S. Calus, M. Busch, A.V. Kityk, W. Piecek, and P. Huber Journal of Physical Chemistry C 120, 11727 (2016). Acceptance of Terms and Conditions:

Click here to agree

570

\title{
Electrosorption in Polypyrrole-Silicon Hybrid Nanopores: Insights from Cyclic Voltammetry and Dilatometry
}

\author{
Manuel Brinker ${ }^{1}$; Guido Dittrich ${ }^{1}$; Pirmin Lakner ${ }^{2}$; Thomas Keller $^{2}$; Patrick Huber $^{1}$ \\ ${ }^{1}$ Hamburg University of Technology \\ ${ }^{2}$ Deutsches Elektronen-Synchrotron (DESY)
}

Corresponding Author(s): patrick.huber@tuhh.de

Porous silicon provides a versatile matrix to study confinement effects in soft matter systems, since its pore space can be tailored over a wide range of pore diameters, pore morphologies, and pore wall chemistries $[1,2]$. We present a study on the electrosorption behaviour of acidic electrolytes in polypyrrole/nanoporous silicon (PPy/pSi) hybrids. The PPy/pSi hybrids are prepared by electropolymerization of PPy in an array of tubular nanopores with $10 \mathrm{~nm}$ diameter and 80 micrometer length in a silicon membrane. The monolithic character of this nanoporous medium allows us to study the macroscopic deformation behaviour of the systems by dilatometry during the electrosorption experiments 3 . The findings are discussed with regard to the electrolyte/pore surface interaction, electrolyte transport in extreme spatial confinement and sorption stresses in nanoporous media under external electrical control.

Procter and Gamble Student poster award:

References:

1. Soft matter in hard confinement: phase transition thermodynamics, structure, texture, diffusion and flow in nanoporous media. Patrick Huber; Journal of Physics: Condensed Matter 27, 103102 (2015).

2. Tuning the pore wall morphology of mesoporous silicon. Pushpendra Kumar, Tommy Hofmann, Klaus Knorr, Patrick Huber, Patric Scheib and Peter Lemmens; Journal of Applied Physics 103, 024303 (2008).

3. Elastic response of mesoporous silicon to capillary pressures in the pores. Gennady Gor, Luca Bertinetti, Noam Bernstein, Peter Fratzl, and Patrick Huber; Applied Physics Letters 106, 261901 (2015).

Acceptance of Terms and Conditions

Click here to agree

\section{Mesoscopic modelling of ink imbibition in paper}

Thejas Hulikal Chakrapani ${ }^{1}$; Stefan Luding $^{1}$; Wouter K. den Otter ${ }^{1}$ 
${ }^{1}$ University of Twente

Corresponding Author(s): t.hulikalchakrapani@utwente.nl

Jettable ink is a mixture of water, co-solvent like glycerol, surfactants and pigment particles for color. The typical size of these pigment particles is between $20-200 \mathrm{~nm}$. On the other hand, office paper consists of dried hollow natural fibres of $\sim 2 \mathrm{~mm}$ length, and pores which are tens of nanometer in size. Further, these fibres are compressed into a 100 micrometer layer, which generates a complex network of pores 1. In addition, paper coatings are granular in nature and contain pores of width between 10-400 nm. Hence, ink-imbibition into paper is a highly complex process which encompasses the complexity of the fluid, complex geometry of paper, and the multi-scale nature of the problem.

We have started on this topic, using the particle-based mesoscopic simulation technique called MDPD 2. The first topic to be presented is the surface-tension-driven flow of various fluids into cylindrical pores, to assess the impact of the fluid on the expected Lucas-Washburn imbibition rate. Next, results will be presented on the surface-tension-driven flow of a simple fluid into a variety of idealized arrays of (hollow) cylinders, representing our first step to a model for paper.

References:

1 Mikko Alava and Kaarlo Niskanen. "The physics of paper”. In: Reports on Progress in Physics 69.3 (2006), p. 669. url: http://stacks.iop.org/0034-4885/69/i=3/a=R03.

2 P. B. Warren. "Vapor-liquid coexistence in many-body dissipative particle dynamics". In: Phys.

Rev. E 68 (6 Dec. 2003), p. 066702. doi: 10.1103/PhysRevE.68.066702. url: https://link.aps.org/doi/10.1103/PhysRevE.68.066702.

\section{Procter and Gamble Student poster award:}

I would like to compete in the Procter and Gamble Student award References:

Acceptance of Terms and Conditions:

Click here to agree

\section{Applications of Graph Theory and Machine Learning in Discrete Fracture Network Simulations}

Jeffrey Hyman ${ }^{1}$

${ }^{1}$ Los Alamos National Laboratory

Corresponding Author(s): jhyman@lanl.gov

Flow channeling is commonly observed in field and laboratory experiments of flow and transport in fractured media. This phenomenon indicates the existence of primary sub-networks, also referred to as the network backbones, where the majority of flow and transport occurs. We use graph representations of discrete fracture networks in conjunction with machine learning classification methods, such as support vector machines and random forest, to identify these backbones. We focus on two basic units of classification, individual fractures and paths. Features used in the classification are based on topological, geometric, and physical properties of the fractures. Flow and transport simulations in the backbones provides insights into the physical properties that lead to their formation and the impact of their existence.

Procter and Gamble Student poster award:

References:

Acceptance of Terms and Conditions:

Click here to agree 


\title{
Initiation and propagation of strain localization in cohesive soil using a novel miniature triaxial cell and $\mathrm{X}$-ray Computed Tomog- raphy
}

Christopher Ibeh ${ }^{1}$; Matteo Pedrotti ${ }^{1}$; Alessandro Tarantino ${ }^{1}$; Rebecca J. Lunn ${ }^{1}$

${ }^{1}$ University of Strathclyde

Corresponding Author(s): christopher.ibeh@strath.ac.uk

Particle scale coupled hydro-mechanical interactions play a major role in clay macroscopic behavior and Clays, like other geomaterials, deform in a localized manner (failure and strain localization), but conventional laboratory test measurements are made at the sample scale rather than at the particle scale. Understanding the micro-mechanisms underlying the initiation and propagation of strain localization in cohesive soil, can form the foundation for quantitative prediction of strain localization in practical fine cohesive soil. Furthermore, clay microstructure is highly dependent on the presence of water and on the hydraulic history, but conventional microscopy methods such as SEM require sample dehydration which generates a high suction that deforms the microstructure and prevents a clear understanding.

In the last decade, soil microstructure has been intensively investigated thanks to in-situ microscopy technologies such as X-ray computed tomography (X-CT), which allows 3D imaging of the sample microstructure without the need for dehydration. However, this step foward in the general understanding of soil microstructure is restricted to sandy soils, as the resolution of the X-CT (in the order of few microns) hinders investigation of the microstructure of clayey soils, which generally; present a particle size lower than 2 microns and a pore space in the sub-micron range.

In this study, $2 \%$ of fine silt to sand sized mica (average particle size of 90 micron) has been; mixed with kaolinite clay (average particle size of 0.4 micron) as a strain marker. Mica is mineralogically a clay, and as a clay it is platy in shape and electrically charged, yet big enough to be clearly imaged with an $\mathrm{X}-\mathrm{CT}$ at high resolution.

A novel miniature triaxial compression cell $(7 \mathrm{~mm}$ in diameter) has been manufactured such that the X-CT can be used to image the in-situ soil microstructural behavior upon undrained triaxial compression at different strain levels. The cell has been additionally instrumented with a high capacity tensiometer sensor to measure negative pore-water pressure developed upon shear, therefore allowing mechanical comparison with macroscopic behavior. A particle matching code has been developed to match mica particles in consecutive scans at the different strain levels and their kinematics computed. A conceptual model of the particle configuration, based on the strain localization evolution observed, will be presented.

Procter and Gamble Student poster award:

I would like to compete in the Procter and Gamble Student award References:

Acceptance of Terms and Conditions:

Click here to agree

\section{Novel approaches for upscaling transport beyond homogenisa- tion}

\author{
Author(s): Matteo Icardi ${ }^{1}$ \\ Co-author(s): Thomas Hudon ${ }^{2}$; Simona Perotto ${ }^{3}$; Giovanni Porta ${ }^{3}$ \\ ${ }^{1}$ University of Nottingham
}




\footnotetext{
${ }^{2}$ University of Warwick
}

${ }^{3}$ Politecnico di Milano

Corresponding Author(s): matteo.icardi@nottingham.ac.uk

\begin{abstract}
Athough the macroscopic limit of linear advection-diffusion equations is well understood since the early developments of porous media transport theory (through, for example, homogenisation, and volume averaging), its extension to complex flow regimes is still an open question, even in presence of well-separated spatial scales. This is due to the presence of non-trivial microscopic equilibrium configurations (compared to the trivial constant solution obtained by standard periodic homogenisation), or of dynamic equilibrium configurations. For example, when dealing with fast surface reactions, high microscopic gradients can develop locally. Similarly, a conservative solute in the neighborhood of a concentration source (injection) undergoes a dynamic evolution of the local microscopic configuration in time and space before reaching the asymptotic self-similar profile. These are only two examples when the classical upscaling approaches fail, and effective macroscopic equations are often found either empirically or by resorting to generic random walk models.

In this talk, we present some (old and) new theoretical frameworks to overcome these limitations without the need of ad-hoc calibration or stochastic particle models. These generally involve solving more auxiliary local problems, and often computing local spectral properties (eigenvalues and eigenfunctions) of the underlying transport operators. In particular we will focus on i) an extension of the approach presented in 1 and 3 , considering a large-deviation (exponential) form for the concentration field, and on ii) model-order reduction based on projections from the full microscale formulation onto low-dimensional spaces, borrowing concepts from the finite element community (such as Hi-Mod ([4]), MsFEM, and VMM), and from statistical mechanics. New macroscopic equations are obtained for one or more macroscopic quantities, that can be seen as extended homogenised formulations. Applications of these techniques to the classical 2D Taylor dispersion in a channel and other heterogeneous periodic flows will be discussed.
\end{abstract}

Procter and Gamble Student poster award:

References:

1) Allaire, G., \& Raphael, A. L. (2007). Homogenization of a convection-diffusion model with reaction in a porous medium. Comptes Rendus Mathematique, 344(8), 523-528.

2) Battiato, I., \& Tartakovsky, D. M. (2011). Applicability regimes for macroscopic models of reactive transport in porous media. Journal of contaminant hydrology, 120, 18-26.

3) Haynes, P. H., \& Vanneste, J. (2014). Dispersion in the large-deviation regime. Part 1: shear flows and periodic flows. Journal of Fluid Mechanics, 745, 321-350.

4) Perotto, S. (2014). A survey of hierarchical model (Hi-Mod) reduction methods for elliptic problems. In Numerical simulations of coupled problems in engineering (pp. 217-241). Springer, Cham.

5) Parish, E. J., \& Duraisamy, K. (2018). A Unified Framework for Multiscale Modeling Using MoriZwanzig and the Variational Multiscale Method. ArXiV preprint. Acceptance of Terms and Conditions:

Click here to agree

\title{
On modeling and simulation of multiscale processes in catalytic filters
}

\author{
Oleg Iliev $^{\text {None }}$; Torben Prill ${ }^{1}$; Pavel Gavrilenko ${ }^{1}$; Andro Mikelic ${ }^{2}$ \\ ${ }^{1}$ Fraunhofer ITWM \\ ${ }^{2}$ Univ. Lyon 1
}

Corresponding Author(s): oleg.iliev@itwm.fraunhofer.de

In catalytic filters the transport and reaction processes occur at three scales. The washcoat (active) particles are nanoporous, and the adsorption processes occur at the surface of the nanopores. The washcoat is usually placed within a microporous filter, often by a kind of coating procedure for the inert ceramic matrix. The complete filter element or its parts are usually modeled and simulated at 
Darcy scale. Due to the manufacturing procedures, inert matrices with different level of heterogeneity need to be considered, and different level of heterogeneity of the washcoat particles also has to be considered. The nanoporosity is usually considered to be homogene-ous.

The contaminants are transported through micropores by means of convection and diffusion. The transport from the micropores to the washcoat particles is usually dif-fusion dominated, but in some case convection is also possible. The adsorption oc-curs at the surface of the nanopores.

At filter element scale (Darcy scale) usually the reactive transport is modeled with Darcy equation for the flow and convection-diffusion-reaction, CDR, equation for the contaminant. A postulated macroscopic CDR equation is usually used in the engineering practice. This modeling approach, however, is valid only for totally ho-mogeneous media and diffusion dominated processes.

In the current talk, we start with homogeneous media and diffusion dominated pro-cesses, for which asymptotic homogenization results can be applied, and investi-gate how the increased heterogeneity influences the validity of the macroscopic model. Pore scale simulations on synthetic domains and on 3D images are used to calculate effective coefficients at macro scale, as well as to compare the averaged results from the pore scale simulations to the solution of the macroscopic equations.

Procter and Gamble Student poster award:

References:

Acceptance of Terms and Conditions:

Click here to agree

88

\title{
Domain decomposition techniques for fully coupled reactive trans- port and multiphase flow in porous media
}

\author{
Author(s): Davide Illiano ${ }^{1}$ \\ Co-author(s): Florin Adrian Radu ${ }^{2}$; Olav Møyner ${ }^{3}$ \\ ${ }^{1}$ University of Bergen \\ ${ }^{2}$ University of Bergen, Norway \\ ${ }^{3}$ SINTEF, NTNU
}

Corresponding Author(s): davide.illiano@uib.no

Fully coupled flow and reactive transport are a classical example of non-linear coupled equations on a porous medium. Whenever studying soil remediation, $\mathrm{CO}_{2}$ storage or enhance oil recovery these equations must be modeled.

We investigate a domain $\Omega$ partitioned into two disjoint sub-domains, $\Omega_{1}$ and $\Omega_{2}$, separated by a Lipschitz continuous interface $\Gamma$ [Seus, Mitra, Pop, Radu, Rohde, 2018]. Such domains will be characterized by highly different soil properties, such as porosity and permeability, and different initial conditions. In this paper, only two domains are investigated for the ease of the presentation, anyhow the scheme can be easily extended to regions with a higher numbers of sub-domains.

After a Euler implicit discretisation is implemented, we propose three different linearisation schemes to threat the non-linearity of the expressions involved. We investigate the Newton method, the modified Picard [Celia, Bouloutas, 1990] and the L-scheme [Radu, Nordbotten, Pop, Kumar, 2015]. The Newton method is the only method which is quadratic convergent. We will anyhow observe that this method, being only locally convergent can be inefficient in case of complex configurations. The L-scheme appears to be a valid alternative to the other linearization schemes because, although only linear, it is globally convergent.

We implement fully implicit solvers, applied to the entire domain, and domain decomposition (DD) schemes. Such DD algorithms allow us to solve the different sub-regions in parallel, reducing the complexity of the problem and the computational times.

All the models have been implemented and compared in MRST a reservoir simulation toolbox based on Matlab [Lie, 2016]. 
Procter and Gamble Student poster award:

I would like to compete in the Procter and Gamble Student award References:

1) D, Seus, K. Mitra, I. S. Pop, F. A. Radu, A linear domain decomposition method for partially saturated flow in porous media, Computational methods in applied mechanics and engineering, 2018

2) M. Celia, E. Bouloutas, A General Mass-Conservative Numerical Solution for the Unsaturated Flow Equation, Water Resources Research 26(7):1483-1496, 1990

3) K.-A. Lie, An Introduction to Reservoir Simulation Using MATLAB/GNU Octave, Cambridge University Press, 2016 Acceptance of Terms and Conditions:

Click here to agree

\section{In-pore solidification: from nanoscale origin to mesoscale dam- age}

Katerina Ioannidou ${ }^{1}$; Edmond Tingtao Zhou ${ }^{2}$; Martin Bazant ${ }^{3}$; Roland Pellenq ${ }^{4}$

${ }^{1}$ Massachusetts Institute of Technology - CNRS

${ }^{2}$ MIT Dpt of Physics

${ }^{3}$ MIT Dpt Chemical Engineering

${ }^{4}$ MIT / CNRS

Corresponding Author(s): hekate@mit.edu, pellenq@mit.edu

Mechanical and viscoelastic behavior of concrete crucially depends on cement hydrates, the glue of cement. Even more than the atomistic structure, the mesoscale amorphous texture of cement hydrates over hundreds of nanometers plays a crucial role for material properties. Common degradation mechanisms of concrete are Freeze-Thaw, Alkali-silica-Reaction and Delayed Ettringite Formation. These damage mechanisms occur under different thermodynamic conditions, nevertheless with necessary condition the suffiient saturation of cement's pore network with water. The damage occurs when a new phase solidifies inside the pore network of cement paste. In this talk, I present a unified computational framework that links the nanoscale origin of in pore crystallization to mesoscale damage in cement. Grand Canonical Monte Carlo and Molecular Dynamics simulations of in-pore crystallization were performed in the realistic structure and pore network of cement hydrates 1 . The results provide a new insight on how in-pore solidification creates cracks in cement paste and suggest solutions to mitigate the problem. 1. Ioannidou K. et al, The mesoscale texture of cement hydrates, Proceedings of National Academy of Science USA, 113 (8), 2029-2034 (2016)

Procter and Gamble Student poster award:

References:

Acceptance of Terms and Conditions:

Click here to agree

105

\section{CapillaryStress and Structural Relaxation in Disordered Porous Materials}

Edmond Tingtao Zhou $^{1}$; Katerina Ioannidou ${ }^{2}$; Martin Bazant ${ }^{3}$; Roland Pellenq ${ }^{4}$

\footnotetext{
${ }^{1}$ MIT Dpt of Physics

${ }^{2}$ LMGC, CNRS and University of Montpellier

${ }^{3}$ MIT Dpt Chemical Engineering
} 
${ }^{4}$ CNRS / MIT-CEE

Corresponding Author(s): hekate@mit.edu, pellenq@mit.edu

Capillary condensation is a ubiquitous process of vapor-liquid phase transition in porous media, such

as sand piles, plaster, silica gels, cementitious materials, which has an important yet poorly understood effect on mechanical behavior. The confined fluid can generate enormous local stresses, as observed in granular material aging, wet floor friction, nano-tribology, cement drying shrinkage and in everyday life experiences such as hardening of a drying sponge or building a sand castle on the beach. Capillary condensation and evaporation potentially bring undesirable fracture processes,as in drying cracking of colloidal films and paints, but capillary stress can also can be exploited innanomaterials fabrication by capillary force lithography, capillary rise infiltration, evaporation-driven assembly and self-organization and composite imbibition and even used to evaluate the atmosphere of planets.

Despite the broad importance of capillary forces, they remain challenging to predict in complex porous materials over the full range of liquid saturation, either in equilibrium or during a dynamical process of drainage/imbibition. For granular or colloidal materials, existing models addressing partial saturations are oversimplified and only apply either to low humidity (so called pendular, funicular regimes) or to idealized geometries (slit/cylindrical independent pores or single sphere against a wall). At higher saturation, models based on geometrical analysis of Young-Laplace equation for smaller clusters are proposed but restricted to only equilibrium liquid distributions inside mono disperse packings. Molecular simulations methods are also difficult to use, since the porous structure considered at the mesoscale (micron-scale).

In this work, we propose a numerical and theoretical framework to quantitatively predict capillary condensation/evaporation, compute associated capillary forces and structural relaxation in a 3D realistic nano-granular cement paste model using lattice-gas simulations of adsorbed water parametrized from atomistic simulations. In particular, this allowed us to access the adsorption-desorption mechanism and assess the role of (metastable) cavitation. In addition, we present for the first time to our knowledge a phase field model of the liquid-vapor mixture spatial distribution, whose inhomogeneous stress tensor is integrated over Voronoi polyhedra in order to calculate forces on each grains. Minimizing system free energy upon the conjugate action of capillary forces applied to the cement hydrate nano-grains, together with the cohesive interactions between these nano-grains predict the overall stress relaxation. We show that capillary stress is an effective mechanism for eigenstress relaxation in granular heterogeneous porous media, which contributes to the durability of cement.

Procter and Gamble Student poster award:

References:

Acceptance of Terms and Conditions:

Click here to agree

\title{
Dependence of shallow landslide stability onset on terrain poros- ity - an experimental study
}

\author{
Author(s): Vladislav Ivanov ${ }^{1}$ \\ Co-author(s): Laura Longoni ${ }^{1}$; Monica Papini ${ }^{1}$ \\ ${ }^{1}$ Politecnico di Milano
}

Corresponding Author(s): vladislavivov.ivanov@polimi.it

Rainfall triggered shallow landslides are notoriously known to be a severe threat even though mobilization of shallow layers rarely exceeds several meters of depth. On the other hand, such soil slips 
could certainly involve large amounts of highly energetic soil prone to evolve into debris flows of destructive nature. Affected areas could encompass great extents, even far from the origin of the slip. Identification of unstable soil layers could be a challenging task as signs of instability are rarely present. Mathematical models are thus used for the evaluation of areas with an increased risk of shallow movement development. Such models rely on a number of physical parameters and yield threshold values for precipitation or soil moisture content so as to distinguish instability conditions. However, soil heterogeneity could have a drastic impact on the redistribution of precipitation within the terrain. Variations in porosity of the material are therefore to be considered crucial for the development of soil slips. In this study we design and carry out experimental works to investigate the parameters governing stability onset. A number of tests involving layers of variable porosity, moisture content and precipitation have been carried out. Results indicate the strong effect of porosity on the infiltration process and thus the decrease of effective stresses and consequent development of a slip surface. Physical models could therefore be effectively used to calibrate mathematical solutions for pore processes in soil stability problems. An interesting future development of this work will involve the transition from reduced- to a real-scale problem by exploring the scale effects relevant to the various processes. Field investigations aimed at the investigation of the aforementioned transition is currently being programmed.

Procter and Gamble Student poster award:

I would like to compete in the Procter and Gamble Student award References:

Acceptance of Terms and Conditions:

Click here to agree

152

\section{Reliable Multiscale Models for Manufacturing of Lightweight Com- posite Structures}

Masoud Jabbari ${ }^{1}$; Mohammad Nasr Esfahani ${ }^{2}$

${ }^{1}$ School of Mechanical, Aerospace and Civil Engineering, University of Manchester

${ }^{2}$ WMG, University of Warwick

Corresponding Author(s): m.jabbari@manchester.ac.uk

The need for weight reduction is driven by the demand for better fuel efficiency and reduced $\mathrm{CO}_{2}$ emissions in transportation industry. Fibre-reinforced composites (FRCs) are attracting more interest in the market as their excellent stiffness and strength are combined with a low density. However, there are several surmountable industrialisation hurdles due to complex interplay between manufacturing process parameters, multiscale nature of the fibre preform, and different chemistry of resin, which are mostly related to the liquid composite moulding (LCM) stage of composite manufacturing. Previous works have reported on different methods to simulate saturated/unsaturated flow during LCM using coupled local and global (dual-scale) solver for system of equations. This study will introduce a rigorous numerical implementation to simulate multiscale/multiphase flow of resin in porous composite preforms with strong meso/macro coupling. Results show that the developed model can be used for increased reliable manufacturing of FRCs that are capable of predicting most process induced damages, e.g. voids, dry spots and resin rich areas.

Procter and Gamble Student poster award:

References:

Acceptance of Terms and Conditions:

Click here to agree 


\section{Capillary pressure heterogeneity across scales: Insights from micro- CT imaging and continuum scale modelling}

Samuel Jackson ${ }^{1}$; Qingyang $\operatorname{Lin}^{1}$; Sam Krevor ${ }^{\text {None }}$

${ }^{1}$ Imperial College London

Corresponding Author(s): samuel.jackson@imperial.ac.uk

In order to tractably model the large scale displacement of fluids in the subsurface, effective, macroscopic parameters that describe continuum multiphase flow are required. These parameters should ideally be upscaled rigorously from the pore scale, ensuring that any heterogeneity below the averaging scale is accounted for and that unique, well defined functions are obtained.

In current Darcy formulations of multiphase flow, the capillary pressure - saturation - relative permeability functions are the primary controls on fluid displacement at the continuum scale. When deriving these functions at relatively large scale (i.e. on cylindrical rock samples with $\Phi \sim 4 \mathrm{~cm}, \mathrm{~L} \sim 15$ $\mathrm{cm}$ ), generally little consideration is given to the pore scale displacement mechanisms, the impacts of rock heterogeneity or the REV. The resulting functions are therefore largely sample dependent and non-unique; only suitable for very specific modeling scenarios in a limited range of flow regimes 1 .

Recently it has been shown that spatial heterogeneities in the capillary pressure - saturation function and permeability at the REV scale $\left(>10 \mathrm{~mm}^{3}\right.$ in Berea sandstones 2$)$, caused by variations in the rock micro-structure can lead to large variations in upscaled effective flow properties, which become dependent on the capillary number and the specific structure of the heterogeneity 1 . These heterogeneities are generally inferred from core flooding experiments and are ultimately derived from observations based on fluid saturations linked with phenomenological concepts [1-3]. Understanding the nature of these heterogeneities, and their emergence from the pore scale is therefore of vital importance in both the practical derivation of macroscopic parameters and in the rigorous validation of upscaling methodologies, whether phenomenological or thermodynamically based.

In this work we image meso scale (i.e. $\Phi \sim 1 \mathrm{~cm}, \mathrm{~L} \sim 5 \mathrm{~cm}$ ) Bentheimer rock cores during the steadystate multiphase flow of Decane and Brine using high resolution X-Ray micro-CT scanning. We image the entire core at a resolution of $6 \mu \mathrm{m}$ allowing accurate reconstruction of fluid interfaces and saturations from the pore to continuum scale. Using the methods of $[4,5]$, we evaluate the interfacial curvature and resulting capillary pressure in the core, analysing the spatial variability in the capillary pressure and associated REV. At the relatively coarse resolution, we are only able to accurately reconstruct capillary pressures during imbibition, due to partial volume effects. To analyse drainage displacements with much higher capillary pressures, we perform zoom in scans on regions of interest, allowing accurate determination of interfacial curvature and capillary pressures on several $O\left(10 \mathrm{~mm}^{3}\right)$ size sub volumes.

Using the continuum scale characterization methods of [1-3] the capillary pressure heterogeneity is inferred from the saturation distributions in the core, assuming capillary equilibrium and scaling from the intrinsic, average capillary pressure curve - obtained using both mercury intrusion and porous plate methods. We compare this to the directly measured capillary pressures at the pore scale averaged on the REV sized sub volumes. For the first time, this provides direct validation of the inference of capillary pressure heterogeneities from mm-scale saturation distributions in heterogeneous cores and links pore-scale physics with the continuum scale.

\section{Procter and Gamble Student poster award:}

\section{References:}

1 S. J. Jackson, S. Agada, C. Reynolds and S. Krevor. Characterising Drainage Multiphase Flow in Heterogeneous Sandstones. Water Resources Research, 54 (4), 3139-3161, 2018.

2 R. Pini and S.M. Benson. Capillary pressure heterogeneity and hysteresis for the supercritical CO2/water system in a sandstone. Advances in Water Resources, 108:277-292, 2017.

3 M. Krause, S. Krevor \& S.M. Benson. A Procedure for the accurate determination of sub-core scale permeability distributions with error quantification. Transport in Porous Media, 98:3, 565 588, 2013. 
[4] R.T. Armstrong, M.L. Porter and D. Wildenschild, D. Linking pore-scale interfacial curvature to column-scale capillary pressure. Advances in Water Resources, 46, 55-62, 2012.

5 Q. Lin, B. Bijeljic, R. Pini, M.J. Blunt and S. Krevor. Imaging and Measurement of Pore-Scale Interfacial Curvature to Determine Capillary Pressure Simultaneously with Relative Permeability in Sandstones. Water Resources Research, 54 (4), 7046 - 7060, 2018. Acceptance of Terms and Conditions:

Click here to agree

\title{
Fostering microbial enhanced oil recovery? About general mech- anisms and technical tools to explore new avenues to prevent or stimulate bacterial adhesion
}

\author{
Author(s): Karin Jacobs ${ }^{1}$; Christian Spengler ${ }^{1}$ \\ Co-author(s): Thomas Faidt $^{1}$; Friederike Nolle ${ }^{1}$; Johannes Mischo ${ }^{1}$ \\ ${ }^{1}$ Saarland University
}

Corresponding Author(s): k.jacobs@physik.uni-saarland.de

\begin{abstract}
Understanding and controlling bacterial adhesion is highly relevant in many areas of life, especially since many processes in/of bacteria begin only if they are sessile. Since earlier studies approached these questions mostly with classical adsorption experiments, quantitative measurements of the interactions between bacterial cells and interfaces have so far only been carried out for a limited number of systems. Here, atomic force microscopy-based single cell force spectroscopy is used to determine adhesion forces of individual bacterial cells to different types of surfaces 1. As a basis, the adhesion of Staphylococcus aureus cells to very hydrophilic and strongly hydrophobic surfaces was characterized for a high number of individual cells and the influence of certain groups of cell wall molecules on adhesion was determined 2. With this knowledge, a method for measuring the contact area between bacterial cells and flat surfaces was developed and it was shown that the adhesive strength of an individual cell does depend its contact area 3. Propositions are developed to stimulate or prevent adhesion based on the resulted force analysis in each individual system of bacterium/medium/material surface. We now aim to move to more complex system such as those important for Microbial Enhanced Oil Recovery (MEOR) and are seeking collaborative partners providing interesting oil recovery systems.
\end{abstract}

Procter and Gamble Student poster award:

References:

1 N. Thewes et al., Soft Matter 11 (2015) 8913

2 J. Eisenbeis et al., Front. Cell. Infect. Microbiol. 8 (2018) 235

3 C. Spengler et al., Nanoscale 9 (2017) 10084 Acceptance of Terms and Conditions:

Click here to agree

\section{Application of numerical modelling and flow experiments to op- timize the design of microfluidic devices representing porous me- dia}

Author(s): Amir Jahanbakhsh ${ }^{1}$

Co-author(s): Omid Shahrokhi ${ }^{1}$; Rumbidzai A. E. Nhunduru ${ }^{2}$; Krystian L. Wlodarczyk ${ }^{3}$; Duncan P. Hand ${ }^{4}$; M. Mercedes Maroto-Valer ${ }^{1}$

\footnotetext{
${ }^{1}$ Research Centre for Carbon Solutions (RCCS), School of Engineering and Physical Sciences, Heriot-Watt University
} 


\footnotetext{
${ }^{2}$ Research Centre for Carbon Solutions (RCCS), School of Engineering and Physical Sciences, Heriot-Watt University

3 *Research Centre for Carbon Solutions (RCCS), School of Engineering and Physical Sciences, Heriot-Watt University

${ }^{4}$ School of Engineering and Physical Sciences, Heriot-Watt University
}

\section{Corresponding Author(s): a.jahanbakhsh@hw.ac.uk}

Understanding transport phenomena and governing mechanisms of different physical and chemical processes at the pore-scale is crucial for a wide range of applications including hydrocarbon recovery, $\mathrm{CO} 2$ sequestration and contaminant hydrology. Microfluidic devices, also known as micromodels, coupled with visualization techniques allow the investigation of these processes at pore-scale $[1,2]$. For the fabrication of micromodels, glass substrates are often preferred over silicon wafer and certain polymers due to their high transparency, thermal stability, hardness and chemical resistance. Recently, we have developed a laser-based process that can be used for the rapid prototyping of micromodels using glass substrates 3. Using this technique, we can fabricate porous structures containing fine elements, e.g. pores and channels with the width as small as $14 \mu \mathrm{m}$ (measured at FullWidth-Half-Maximum). Additionally, the process enables control of the depth of individual pores and channels.

It is desirable to have uniform migration of a front invading a pore pattern in micromodels. Control over the flow dynamics of a migrating front can be achieved by optimising the design of the inlet buffer. In our previous study, numerical simulations showed that the geometry of inlet buffers affects the distribution of fluids present in a micromodel and a triangular buffer can offer a more uniform distribution than a rectangular buffer [4]. In the present study, we integrate micromodel flow experiments and pore-scale numerical simulations to design and optimise micromodel fabrication. Simulations are particularly useful for guiding the prototyping of micromodels, since the geometry and dimensions of micro-channels and pores in the structure have a significant effect on fluid flow dynamics. In this work, we investigate the fluid displacement front, saturation distribution, and the influence of the micromodel imperfections on the bulk flow using a commercial computational fluid dynamics (CFD) code. Additionally, a set of dynamic fluid flow tests are performed on the fabricated micromodels with different inlet designs. Throughout these experiments, a 5.3 Megapixel colour camera is used to capture and visualize displacement events.

In this research, qualitative (flow images) and quantitative (flow rate) results from the dynamic flow tests of the fabricated micromodels are compared with predictions of the pore-scale numerical simulations. This promotes the validity of the numerical modelling assumptions and improves the predictions of the models. Moreover, integrating experimental results with numerical simulations pinpoints critically important features of the microfluidic pattern that have an impact on the fluid flow behaviour. This will help to identify optimal design of inlet buffers as well as the improvement of the fabrication of micromodels in order to avoid any unwanted artefacts in the experimental results.

\section{Acknowledgements}

This project has received funding from the European Research Council (ERC) under the European Union's Horizon 2020 research and innovation programme (MILEPOST, Grant agreement no.: 695070). This paper reflects only the authors' view and ERC is not responsible for any use that may be made of the information it contains.

\section{Procter and Gamble Student poster award:}

\section{References:}

1 Karadimitriou, N.K., Hassanizadeh, S.M., 2012. A Review of Micromodels and Their Use in Two-Phase Flow Studies. Vadose Zo. J. 11, vzj2011.0072. https://doi.org/10.2136/vzj2011.0072.

2 Anbari, A., Chien, H.-T., Datta, S.S., Deng, W., Weitz, D.A., Fan, J., 2018. Microfluidic Model Porous Media: Fabrication and Applications. Small 1703575, 1703575. https://doi.org/10.1002/smll.201703575 3 Wlodarczyk, K.L., Carter, R.M., Jahanbakhsh, A., Lopes, A.A., Mackenzie, M.D., J Maier, R.R., Hand, D.P., Mercedes Maroto-Valer, M., 2018. micromachines Rapid Laser Manufacturing of Microfluidic Devices from Glass Substrates 1-14. https://doi.org/10.3390/mi9080409

[4] Jahanbakhsh, A., Wlodarczyk, K.L., Maroto-valer, M.M., Hand, D.P., Maier, R.R.J., Macpherson, W.N., 2018. Coupling Numerical Modelling with Flow Experiments to Optimize Fabrication of Microfluidic Devices for Transport in Porous Media Applications. 10-11 Sep. 2018, Aberdeen, UK. Acceptance of Terms and Conditions:

Click here to agree 
873

\title{
Non-isothermal Reservoir Souring Simulations
}

\author{
moein Jahanbani veshareh $^{\text {None }} ;$ H. M. Nick ${ }^{1}$ \\ ${ }^{1}$ Danish Hydrocarbon Research and Technology Centre, Denmark
}

Corresponding Author(s): moein@dtu.dk

Primary derive mechanisms usually lead to a low oil recovery. Therefore, water injection is commonly applied to sustain the reservoir pressure and increase the ultimate oil recovery. Utilising seawater for waterflooding provides additional sulphate and lowers reservoir temperature. These provide a favourable condition for sulphate reducing bacteria (SRB) to reduce sulphate as an electron acceptor to H2S. This process is called reservoir souring, and is undesirable for oilfield operators since H2S is a toxic and corrosive gas [1,2]. Reservoir souring simulators have been developed to predict the associated risks and to design efficient mitigation strategies. Reservoir souring simulators utilising different numerical schemes for solving coupled reactive transport processes commonly assume a single reaction term for the activity of SRB. However contribution of more than one strain with different dependencies on environmental conditions such as temperature and $\mathrm{pH}$ lead to reservoir souring 3. In this work we introduce a method to upscale the impact of different strains under different reservoir conditions. We also show how the upscaled parameters can be used in both singleand two-phase flow for reservoir simulations for the field scale.

\section{Procter and Gamble Student poster award:}

I would like to compete in the Procter and Gamble Student award References:

1 Veshareh, M. Jahanbani, and H. M. Nick. "Learnings from Reservoir Souring Treatment by Nitrate Injection in the Halfdan Oil Field." 80th EAGE Conference and Exhibition 2018. 2018.

2 Veshareh, M. Jahanbani, and H. M. Nick. "Nitrate Treatment Strategy, Differences of the Reservoir Scale and the Lab Scale." First EAGE/IFPEN Conference on Sulfur Risk Management in Exploration and Production. 2018.

3 Vigneron, Adrien, et al. "Succession in the petroleum reservoir microbiome through an oil field production lifecycle." The ISME journal 11.9 (2017): 2141. Acceptance of Terms and Conditions:

Click here to agree

\section{Modeling of transport, accumulation and replication of bacteria in porous media}

Kartik Jain $^{1}$; Christian Holm

\footnotetext{
${ }^{1}$ University of Stuttgart
}

Corresponding Author(s): kartik.jain@icp.uni-stuttgart.de

Microorganisms like bacteria can propel, proliferate and accumulate in a number of media.

Not much is known about their dynamics although this is important for various applications ranging from engineering and biology, as well as from a fundamental point of view.

In addition to self-propulsion, bacteria can stick to each other and to surfaces where they can create fast growing colonies, called biofilm. Studies have shown that bacteria more commonly accumulate at surfaces and around obstacles.

Furthermore, the bacterial dynamics plays a pivotal role in microbial induced calcite precipitation (MICP) around surfaces in porous media on one hand, and on the other the trajectory of a single bacterium depends largely on the 
geometrical configuration of a given system.

The calcite precipitation only commences once the bacteria has formed a large enough biofilm to create sufficient concentrations of inorganic carbon in the form of carbonate ions by metabolising added urea.

In the present work we modeled dynamics of E.Coli bacteria in a water like underlying fluid.

In the model the bacteria are represented by molecular dynamics (MD) particles while the fluid in which they propel is represented by a lattice fluid via the lattice Boltzmann method (LBM).

The MD particles are coupled to the LB fluid using a frictional point coupling that ensures momentum conservation of the total system. We present results of transient dynamics of up to 600 bacteria in porous domain with various heterogeneous configuration of obstacles.

Our results show that the bacteria tend to a state of momentary stasis in regions where the underlying fluid recirculates, and thus result in a sticking behavior near the obstacles of the porous media.

This behavior can be fine-tuned based on the forces between particles, and between the particles and the obstacles.

Our findings indicate that such behavior is manifested mainly due to hydrodynamics interactions between a bacterium and the surfaces. We further developed a model of bacterial proliferation in relation to nutrient availability.

Preliminary results qualitatively demonstrate growth of bacteria during their transport through confined geometries.

This model is currently being investigated and enhanced with collaboration from experimentalists.

Procter and Gamble Student poster award:

\title{
References:
}

Acceptance of Terms and Conditions:

Click here to agree

\section{Fluid flow and heat transfer modeling through metal foams or lamella structures}

\author{
Author(s): Farshid Jamshidi ${ }^{1}$ \\ Co-author(s): Anastasia August ${ }^{2}$; Andreas Reiter ${ }^{1}$; Aron Kneer ${ }^{3}$; Michael Selzer $^{2}$; Britta Nestler $^{2}$ \\ ${ }^{1}$ Karlsruhe University of Applied Sciences \\ ${ }^{2}$ Karlsruhe Institute of Technology (KIT) \\ ${ }^{3}$ TinniT Technologies $\mathrm{GmbH}$
}

Corresponding Author(s): farshid.jamshidi@hs-karlsruhe.de

Modern condensing boilers fueled by oil or gas have been extensively in used to warm up water in apartments and residential buildings. A general problem associated with these boilers is relatively high $\mathrm{NO}_{\mathrm{x}}$ production during the initial stage of their performance; this happens due to low efficiency combustion. A more efficient and resource-saving method to do this is utilizing inductively heated structures inside the water pipes as heat exchangers. In designing these structures, an essential criterion, namely a larger wetted area (to enhance heat transfer) for a lower pressure drop should be considered. Recently, cellular materials are becoming popular in manufacturing these structures due to their lightweight constructions as well as noise reduction. Another advantage of using these materials is the easiness of their design using computers. Once a design is complete, it can be used in Computational Fluid Dynamics (CFD) simulations and manufactured using 3D printing. 
In this work, we selected water flow in stainless steel systems as a benchmark study and designed different digital models for the internal inductively heated structures. The reason to opt for the stainless steel, along with its high thermal conductivity, is that the selected material must neither be corrosive nor may support the development or spread of bacteria. We performed numerical simulations to test the flow and thermal properties of different structures and manufactured them using 3D printing. The samples were prepared for investment casting since open-pored metallic foams with a quite high porosity can be produced by the placeholder method (casting). By doing this, we can validate the simulation results of the synthetic design and select the most suitable model for the application. We generated specific open-pored metal foam structures with the help of a modified Voronoi tessellation to be inserted as components into water pipes. To conduct a comparative study, the porosity and specific surface area were selected as the fixed parameters to create other constructions such as lamellae and airfoil-shaped fins. For the simulations, the Navier-Stokes equations, as well as heat conduction/convection transport equation are numerically solved. The benefit of the performed simulations is that specific designs of cellular materials with appropriate heat transfer properties can be identified as candidates for production and experimental examinations. In the experiment, the structures are heated by a coil. The pressure and the temperature of the water are measured at different positions of the inserted component and pipe. The results obtained using numerical simulations agree reasonably with the experimental observations. We conclude that numerical simulation is a powerful tool to ascertain efficient foam designs used for heat exchange.

Procter and Gamble Student poster award:

I would like to compete in the Procter and Gamble Student award References:

Acceptance of Terms and Conditions:

Click here to agree

\section{Gas bubble migration and trapping in porous media - Pore-scale simulation}

Nariman Mahabadi ${ }^{1}$; Xianglei Zheng ${ }^{2}$; Tae Sup Yun ${ }^{3}$; Leon van Paassen ${ }^{1}$; Jaewon Jang ${ }^{4}$

${ }^{1}$ Arizona State University

${ }^{2}$ Municipal Testing Laboratory

${ }^{3}$ Yonsei University

${ }^{4}$ Hanyang University

Corresponding Author(s): jwj@hanyang.ac.kr

Gas bubbles can be naturally generated or intentionally introduced in sediments. Gas bubble migration and trapping affect the rate of gas emission into the atmosphere or modifies the sediment properties such as hydraulic and mechanical properties. In this study, the migration and trapping of gas bubbles are simulated using the pore-network model extracted from the 3D X-ray image of in-situ sediment. Two types of bubble size distribution (mono-sized and distributed-sized case) are used in the simulation. The spatial and statistical bubble size distribution, residual gas saturation, and hydraulic conductivity reduction due to the bubble trapping are investigated. The results show that the bubble size distribution becomes wider during the gas bubble migration due to bubble coalescence for both mono-sized and distributed-sized cases. And the trapped bubble fraction and the residual gas saturation increase as the bubble size increases. The hydraulic conductivity is reduced as a result of the gas bubble trapping. The reduction in hydraulic conductivity is apparently observed as bubble size and the number of nucleation points increase.

Procter and Gamble Student poster award:

References:

Acceptance of Terms and Conditions:

Click here to agree 


\title{
The significance of porous cell walls for water transport in plants
}

\author{
Steven Jansen ${ }^{1}$; Cora Carmesin ${ }^{1}$; Lucian Kaack $^{1}$; Zhang Ya ${ }^{1}$; Schenk H. Jochen ${ }^{2}$ \\ ${ }^{1}$ Ulm University \\ ${ }^{2}$ California State University Fullerton
}

Corresponding Author(s): steven.jansen@uni-ulm.de

Considering the global importance of water transport in plants for crop growth, primary productivity, uptake of atmospheric carbon dioxide, climate regulation of our planet, and a wide range of processes and factors that are essential to the human population, most people would be very surprised to hear that scientists do not fully understand how plants achieve long-distance water transport. The transport pathway, which generally runs from roots to leaves through the hollow conduits of a vascular plant, passes through thousands of conducting cells. Hydraulic transport between neighbouring conduits is enabled by nanoporous openings in cell walls with a fibrillar, non-woven matrix. These porous media or so-called "pit membranes" are composed of cellulose fibres, and create most of the resistance along the hydraulic pathway. It has long been assumed that redundancy of the hydraulic pathway provides hydraulic safety, since the porous cell walls are thought to prevent fast spreading of bubbles between adjacent water conducting cells. Bubble entry could indeed be problematic and potentially lead to hydraulic failure, especially because water transport is typically under negative pressure. However, the ultrastructure of porous cell walls remains poorly studied, and is typically viewed in a two-dimensional way only. Therefore, the central goal of our work is to investigate the three-dimensional ultrastructure of pit membranes, which would also allow us to better understand functional implications of water transport and bubble spreading between neighbouring conduits.

Based on transmission electron microscopy, we show that pit membranes show a 5 -fold variation in their thickness across flowering plant species, varying from ca. $200 \mu \mathrm{m}$ to $>1,000 \mu \mathrm{m}$, with species growing in wet environments having thinner pit membranes than species from dry environments (Li et al., 2016). This finding suggests that the thickness of the porous medium is associated with safety against hydraulic failure. Moreover, the pit membranes show a ca. $50 \%$ shrinkage upon dehydration, which is known to occur in plants in the field (Zhang et al., 2017). The shrinkage is largely irreversible, and driven by formation of hydrogen bonds between glucose chains of cellulose. Perfusion experiments with colloidal gold particles of known sizes $(5,10,20$, and $50 \mathrm{~nm})$ show that never-dried pit membranes show pore sizes that are up to $20 \mu \mathrm{m}$ in diameter, while shrunken membranes have a more compact cellulose network with pores below $20 \mathrm{~nm}$. A major challenge is the development of a realistic, three-dimensional pit membrane model at the nanoscale, which would also be required to obtain accurate estimations of the porosity, tortuosity, constrictivity, and the effective permeability. Because there is strong evidence for the presence surface active compounds on and/or within pit membranes (Schenk et al., 2017, 2018), such model will also be useful to conduct flow simulations in a multiphase environment (including cellulose, water, gas, and surface active compounds).

In conclusion, pit membrane structures are highly relevant to better understand how plants are able to transport water, and could also be useful to develop biomimetic transport systems such as evaporation-driven negative pressure devices.

\section{Procter and Gamble Student poster award:}

\section{References:}

Li S., Lens F., Espino S., Karimi Z., Klepsch M., Schenk H.J., Schmitt M., Schuldt B., Jansen S. (2016) Intervessel pit membrane thickness as a key determinant of embolism resistance in angiosperm xylem. IAWA Journal 37: 152-171.

Schenk H.J., Espino S., Romo D.M., Nima N., Do A.Y.T., Michaud J.M., Papahadjopoulos-Sternberg B., Yang J., Zuo Y.Y., Steppe K., Jansen S. (2017). Xylem surfactants introduce a new element to the cohesiontension theory. Plant Physiology 173: 1177-1196. 
Schenk H.J., Espino S., Rich-Cavazos S.M., Jansen S. (2018). From the sap's perspective: The nature of vessel surfaces in angiosperm xylem. American Journal of Botany 105: 174-187.

Zhang Y., Klepsch M., Jansen S. (2017) Bordered pits in xylem of vesselless angiosperms and their possible misinterpretation as perforation plates. Plant, Cell and Environment 40: 2133-2146. Acceptance of Terms and Conditions:

Click here to agree

\section{Molecular dynamics simulation of ink imbibition in porous me- dia}

Author(s): Ahmed Jarray ${ }^{1}$

Co-author(s): Wouter K. den Otter ${ }^{1}$; Stefan Luding ${ }^{1}$

${ }^{1}$ University of Twente

Corresponding Author(s): a.jarray@utwente.nl

Spontaneous imbibition of ink in paper is one of the key phenomena underlying the inkjet printing process. When a drop of water-based ink is deposited on a porous substrate, the imbibition of the droplet is strongly affected by the ink composition and the substrate physico-chemical properties. In this work, the influence of surfactants and pore properties on spontaneous water imbibition is investigated by Molecular Dynamics simulations.

To mimic the physicochemical structure of cellulose paper, a simple finite-length pore is modelled as an oxidized Graphene monolayer. A liquid reservoir is initially put in contact with the pore. Then, Molecular Dynamics simulations are performed and the imbibition of the fluid is investigated for different pore sizes, surface oxidation degrees and surfactant concentrations. Interestingly, we find that while the chemical properties of the porous structure highly affect the contact angle and the transport of fluid along the surface of the pore, they hardly affect the capillary imbibition rate. Furthermore, the simulations show that the addition of surfactants dramatically increases the displacement of the fluid into the capillary.

This study provides new insights on the fluid imbibition dynamics in the presence of surfactants, which is crucially important to ink-jet printing. Investigating the imbibition of liquids into pores with different oxidization degree at the nanoscale provides future guidelines for the design of more optimized paper coatings for water-based inks. 
a)

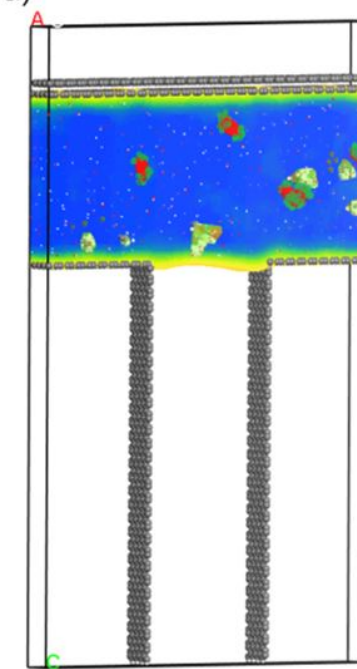

b)

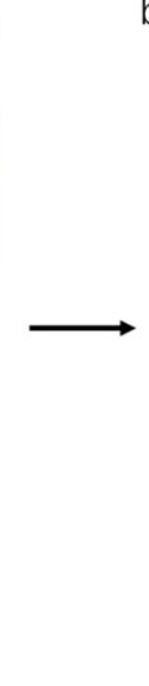

b)

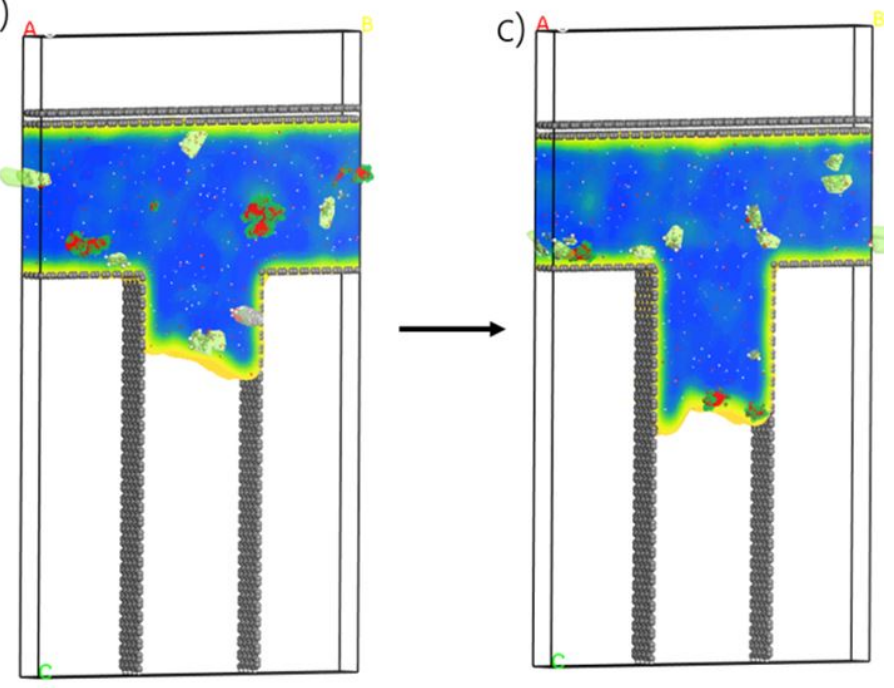

Figure 7: Molecular Dynamics simulation of water imbibition in a pore, with $\mathrm{N}=2556$ water molecules in the presence of few Surfinol 104 surfactants (in red), and a pressure controlled top-lid. a) $\mathrm{t}=0 \mathrm{ps}, \mathrm{b}) \mathrm{t}=50 \mathrm{ps}$, and c) $\mathrm{t}=200 \mathrm{ps}$.

\title{
Procter and Gamble Student poster award:
}

I would like to compete in the Procter and Gamble Student award References:

Acceptance of Terms and Conditions:

Click here to agree

\section{Effect of flow-path tortuosity and contact area fraction on perme- ability of a rough rock fracture}

\author{
Hoda Javanmard $^{1}$; Daniel Vogler ${ }^{\text {None }}$; Anozie Ebigbo ${ }^{2}$; Martin Saar ${ }^{1}$ \\ ${ }^{1}$ Geothermal Energy \& Geofluids, Institute of Geophysics, ETH Zurich, Zurich, Switzerland \\ ${ }^{2}$ ETH Zurich
}

Corresponding Author(s): jhoda@erdw.ethz.ch

Fluid flow in rock fractures is commonly described with the cubic law whose limitations are well documented in the literature $[1,2]$. The deviation of macroscopic hydraulic properties of rough fractures from the idealized parallel-plate model arises not only from the roughness properties of individual fracture surfaces, but also from the extent to which the asperities of the fractures are in touch. The contact area fraction of a fracture leads to a tortuous path of fluid flow which in turn results in a reduction of the effective permeability of the fracture 3. Very few available permeability models for rough fractures take the effect of the contact area or tortuosity into account [4]. These effects are particularly crucial to investigate in order to understand the dynamic behaviour of fractures under loading.We study the effect of the contact area on the permeability of a single, rough fracture, using a novel experimental apparatus, designed in-house, which enables visualization of the flow field in a transparent replica of rock fractures. The fractures are subjected to load, and the change in permeability versus dynamic evolution of the contact area is recorded. The applicability of the observed relationship between contact area and permeability is studied for a range of rough fractures with different morphological properties, employing hydraulic and mechanical numerical models, capable of capturing the contact problem in a rough rock under loading. 
I would like to compete in the Procter and Gamble Student award References:

1 Renshaw, C.E., (1995) On the relationship between mechanical and hydraulic apertures in roughwalled fractures,Journal of Geophysical Research,100 , pp. 24629-24636

2 Zimmerman, R.W. and Bodvarsson, G.S., (1996) Hydraulic conductivity of rock fractures,Transport in Porous Media,23, pp. 1-30

3 Zimmerman, R.W., Chen, D., and Cook, N.G.W., (1992), The effect of contact area on the permeability of fractures,Journal of Hydrology, 139, pp. 79-196

[4] Jin Y., Dong J., Zhang X. Li X. and Wu Y., (2017), Scale and size effects on fluid flow through selfaffine rough fractures,International Journal of Heat and Mass Transfer, 105, pp. 443-451 Acceptance of Terms and Conditions:

Click here to agree

\title{
Multiscale numerical simulation and analysis on reactive acid flow in carbonate rock.
}

${\text { Cunqi } \mathrm{Jia}^{1} \text {; Jun Yao }}^{1}$

${ }^{1}$ Research Centre of Multiphase Flow in Porous Media, China University of Petroleum (East China)

Corresponding Author(s): b17020093@s.upc.edu.cn

The study on transport of fluid system in porous media is always a fundamental and practical interest area. For acid fluid system the special is transport accompanying reaction process. Acidizing is one of reactive flow in porous media, which is widely employed in petroleum engineering to increasing conductivity of carbonate reservoir. Firstly in this paper a theoretical model is built to indicate the flowing mechanism of both solute and solvent on pore scale. Then the model is up-scaled through equivalent substitution on REV scale to gain macro control equation, which is utilized to quantitative characterize the mass, momentum and energy transport of acid fluid system in porous media. Finally based on above model the high-order simulation scheme is used to simulate the whole process and study the influence of independent variables. And the mathematic prove of numerical scheme is also given in this paper.

Procter and Gamble Student poster award:

References:

Acceptance of Terms and Conditions:

Click here to agree

8

\section{Gas-Water Flow of Tight Core Using Pore Scale Observation and Characterization}

\author{
Ying $\mathrm{Jia}^{1}$; Xiangyang $\mathrm{Hu}^{1}$; Lei Sun ${ }^{2}$ \\ ${ }^{1}$ E\&P Research Institute, SINOPEC \\ ${ }^{2}$ SouthWest Petroleum University
}

Corresponding Author(s): jiaying.syky@sinopec.com

The phenomena of tight gas reservoirs which exhibit the unique combination of very high initial water saturation combined with low to very low permeability (permeability of less than $0.1 \mathrm{mD}$ ) exist extensively in a number of regional sedimentary basins. However, due to the lack of sufficient insight vision, traditional method only reflects the macroscopic phenomena but fails to reveal some 
important microscopic behaviors inside the cores of different type of reservoir during multiphase flow process.

In this paper, we conducted gas-water flow experiments on 10 sandstones. Firstly, a series of core displacement experiments were carried out. Then, nuclear magnetic resonance and X-ray microcomputed tomography (micro-CT) experiments were introduced to effectively observe and characterize the water flow to various multiphase flow scenarios in different types of rocks, the pore size distribution of rock samples is converted by NMR T2 spectrum by T2 spectrum and micro-CT experiment. The distribution of the remaining water under different water saturation of different cores was analyzed and the water production mechanisms of tight high water saturation reservoir were revealed. Finally, we prove the relationship between gas \& water production and pore structure using effective flow formula.

This experiment and evaluation methods provided a valuable tool in the evaluation of whether given pay zones in an ultra-tight gas reservoir situation are worthy of completion and under what kind of pressure should be provided for production. It also provides information on how this new method can be used in the exploitation of this ever increasing area of tight gas reservoir production.

\section{References:}

Acceptance of Terms and Conditions:

Click here to agree

614

\section{Carbon aerogel composites with $\mathrm{SiCO}$ aerogels as inner coatings for ultra-high temperature thermal insulation}

Feng Jian $^{\text {None }} ;$ Li Xiafei $^{\text {None }} ;$ Feng Junzong ${ }^{\text {None }} ;$ Jiang Yonggang $^{\text {None }} ;$ Li Liangjun $^{\text {None }}$

Corresponding Author(s):

Carbon fiber-reinforced carbon aerogel composites $(\mathrm{C} / \mathrm{CA})$ are outstanding candidate materials for high-temperature thermal insulators, but the oxidation problem seriously limits their practical application. In order to improve the oxidative-resistant ability of $\mathrm{C} / \mathrm{CA}, \mathrm{SiCO}$ aerogels were used as in-situ ceramic coatings to cover the nanoskeleton structures of carbon aerogels and carbon fibers. Anti-oxidation carbon aerogel composites with SiCO aerogels as inner ceramic coating (C/CA-SiCO) were prepared through gelating, aging, solvent exchanging, ambient drying and pyrolysing stages. The density of the obtained C/CA-SiCO was $0.35 \mathrm{~g} / \mathrm{cm} 3$ with BET specific surface area $131.54 \mathrm{~m} 2 / \mathrm{g}$. The thermal insulation performance was characterized by transient plane source method with Fox 200 instrument for ambient temperature and laser flash method with LFA 427 instrument for high temperature. The solid thermal conductivity and ambient thermal conductivity of the $\mathrm{C} / \mathrm{CA}-\mathrm{SiCO}$ at room temperature was $0.05749 \mathrm{~W} \cdot \mathrm{m}-1 \cdot \mathrm{K}-1$ and $0.3194 \mathrm{~W} \cdot \mathrm{m}-1 \cdot \mathrm{K}-1$, respectively. The ambient thermal conductivity of $\mathrm{C} / \mathrm{CA}-\mathrm{SiCO}$ at $1000 \mathrm{oC}$ was $0.9192 \mathrm{~W} \cdot \mathrm{m}-1 \cdot \mathrm{K}-1$. In addition, the weight loss for the $\mathrm{C} / \mathrm{CA}-\mathrm{SiCO}$ after heating at $1600^{\circ} \mathrm{C}$ for $60 \mathrm{~min}$ in air was just $48.87 \%$ and the in-plane shrinkage ratio was less than $3 \%$. SiCO aerogels as nanoscale anti-oxidation coatings provides a new strategy for the protection of $\mathrm{C} / \mathrm{CA}$ for the high-end temperature thermal insulation application.

Procter and Gamble Student poster award:

I would like to compete in the Procter and Gamble Student award References:

1. Rocha R M, Cairo C A A, Grac M L A. Mat Sci Eng A, 2006, 437 (2): 268.

2. Feng J Z, Lv Y N, Jiang Y G, et al. Rare Metal Mat En, 2016, 45 (S1): 274.

Acceptance of Terms and Conditions:

Click here to agree 


\title{
Rock-typing of heterogeneous rock samples via Minkowski func- tionals and hydraulic attributes
}

\author{
Han Jiang ${ }^{1}$; Christoph Arns ${ }^{1}$ \\ ${ }^{1}$ The University of New South Wales \\ Corresponding Author(s): hanjiang_ss@163.com
}

The characterization of formations in the context of reservoir characterization typically relies on the choice of a hierarchical upscaling framework, assuming that homogeneous sections of each rocktype can be sampled and characterized. Recent developments in 3D tomography including micro-CT show that samples considered to be homogeneous frequently still have a high level of heterogeneity, challenging this assumption. This implies that measurements at the plug scale exhibit a high level of uncertainty, which may be caused e.g. by fine scale laminations. Characterizing these heterogeneities offers a better way of describing the formation characteristics and requires classification techniques sensitive to fine-scale structural details. Typically, when different rock samples are considered, rock-types may be defined on the basis of texture measures like grain sorting, angularity, size distributions of grains, and for example mercury intrusion capillary pressure (MICP) 1 . While this is done on sections of a sample, often using 2D information, 3D tomograms offer much more detail. In the past it has been shown that the Minkowski functionals are sensitive measures of microstructure 2. They were also applied to the characterization of thinly laminated sandstone, where however no saturation based attribute like MICP was utilized in the classification 3. This is significant, since a rock-type classification scheme including such attributes should better incorporate the scale of fluid connectivity: incorporating hydraulic attributes may reveal unique transporting ability through critical flow channels.

In this work we use regional measures based on the Minkowski functionals and local saturation information to partition heterogeneous rock samples into multiple distinct regions. Local hydraulic attributes are determined on the basis of capillary drainage transforms. We consider a range of artificial Boolean models to illustrate the effect of including hydraulic information on the resulting classifications scheme. This allows the determination of bias, since for these models local classes are known ab-initio. We test the classification framework by comparing upscaled petrophysical properties derived on the basis of the classification results with direct fine-scale computations.

Procter and Gamble Student poster award:

I would like to compete in the Procter and Gamble Student award References:

1 J. A. Rushing, K. E. Newsham, and T. A. Blasingame, "Rock Typing - keys to understanding productivity in tight gas sands," in 2008 SPE Unconventional Reservoirs Conference, Keystone, Colorado, 2008, p. SPE 114164.

2 Klaus Mecke and Christoph H. Arns; Fluids in porous media: a morphometric approach, J. Phys.: Condensed Matter, special issue (wetting), 17(9):S503-S534, (2005).

3 N. I. Ismail and C. H. Arns, "Rock-typing using the complete set of additive morphological descriptors," in SPE Reservoir Characterisation and Simulation Conference and Exhibition, Abu Dhabi, UAE, 2013, p. SPE 165989. Acceptance of Terms and Conditions:

Click here to agree

651

\section{Carbonate pore space segmentation and classification by a com- bination of multiscale imaging and deep learning}

Mingze Jiang $^{\text {None }}$; Jop Klaver ${ }^{\text {None }}$; Joyce Schmatz ${ }^{\text {None }}$; Janos Urai ${ }^{\text {None }}$; Moritz Suess ${ }^{\text {None }}$

Corresponding Author(s): mingze.jiang@rwth-aachen.de

Pore space in carbonate rocks can be heterogeneous at all scales; hence, their characterization is challenging, expensive and time-consuming. Petrophysical analyses cannot provide information on 
the full geometry and pore space connectivity. Moreover, these analyses can be time consuming and require evaluation by experts. Current imaging techniques may not cover a representative range of scales, or may not resolve microporosity, and are often not well integrated with the expert knowledge available. In this contribution we present a novel multi-scale workflow how we: 1) combine multimodal images by novel registration and alignment methods; 2 ) segment and classify pores, fractures, and grains automatically using deep learning.

Pore space from $\mathrm{cm}$ - to $\mathrm{nm}$-scale will be measured by integrating automated optical microscopy (Virtual Microscopy, ViP) with high resolution Broad Ion Beam Scanning Electron Microscopy (BIBSEM) image mapping. Pore connectivity will be assessed by applying liquid Metal Injection followed by BIB-SEM on twin samples. Validated pore space and pore type maps containing 100,000s of features will be the input for statistical analysis and used to train deep learning algorithms for pore segmentation and classification.

Procter and Gamble Student poster award:

I would like to compete in the Procter and Gamble Student award References:

Acceptance of Terms and Conditions:

Click here to agree

\title{
The influence of fracture on the gas reservoir development
}

\author{
Author(s): Chunyan Jiao None \\ Co-author(s): Yong $\mathrm{Hu}$; Xuan Xu \\ Corresponding Author(s): jiaochunyan69@petrochina.com.cn
}

There are two main roles for the fractures in the gas reservoir development. On the one hand, fractures will improve the permeability of the reservoir, and the gas recovery of the high permeability reservoir will be higher than that of the low permeability under the same abandonment gas production rate. On the other hand, the water will rush along the fractures and the gas-water two phases flow will occur in the gas reservoir, resulting in premature water production and low gas recovery. In this paper, the influence of fracture on the gas reservoir development is studied by the seepage experiment. Firstly, the influence of fracture on the permeability is studied by testing the physical properties of rock samples with fractures. And the fracture is characteristic with different fracture length, fracture width and fracture location. Secondly, influence of fracture on gas reservoir development is studied by gas and water flow in the fractured rock sample and mathematical simulation. The conclusions are that: (1) In the simple-phase flow, the penetrated fracture improved the rock sample permeability by over 10 times and the non-penetrated fracture improved the rock sample permeability by less than 2 times. (2) The two-phase flow physical simulation experiment shows that the water in the matrix rock is piston-type propulsion, and the propulsion speed is relatively slow. Water rushes in the fractures along the fractured core with a high speed. (3)The mathematical simulation shows that: when the permeability contrast which means the ratio of fracture permeability to matrix rock permeability reaches 20 , the effect of water invasion on the gas recovery is significantly increased, thus the gas recovery will be declined dramatically.

\section{Procter and Gamble Student poster award:}

\section{References:}

Acceptance of Terms and Conditions:

Click here to agree

\section{Graph theory to infer mixing capacity in complex systems}


Joaquin Jimenez-Martinez ${ }^{1}$; Christian Negre ${ }^{2}$; Jeffrey Hyman ${ }^{2}$

\author{
${ }^{1}$ EAWAG-ETHZ \\ ${ }^{2}$ Los Alamos National Laboratory
}

Corresponding Author(s): jjimenez@ethz.ch

Solving flow and transport through complex geometries such as porous and fractured media involves an extreme computational cost. Network theory approaches, where the complex network is conceptualized like a graph, can help to simplify and better understand fluid dynamics and transport in those media. We investigate the relative impact of topological, geometric, and hydraulic heterogeneity on transport processes in partially saturated porous media and in three-dimensional fracture networks. To address this issue, we propose a method based on eigenvector centrality and a measure of disorder in networks. The eigenvector centrality predictions show that this technique is computationally efficient and has potential for predicting preferential paths and stagnation zones for flow and transport. The measure of disorder, as a relative indicator by comparison between two networks, is a first approach to the dispersion potential and "mixing capacity" of a network.

Procter and Gamble Student poster award:

References:

Acceptance of Terms and Conditions:

Click here to agree

\title{
Fracture Modelling of Unconventional Reservoirs with X-ray micro- computed tomography
}

\author{
Author(s): Yu Jing ${ }^{1}$ \\ Co-author(s): Peyman Mostaghimi ${ }^{2}$ \\ ${ }^{1}$ UNSW \\ ${ }^{2}$ UNSW Sydney
}

Corresponding Author(s): yu.jing@unsw.edu.au

Unconventional reservoirs tend to have extremely low-permeability, thus fluid flow is mainly controlled by artificial or natural fractures. Therefore, the characterisation of fracture networks is of critical significance, which, however, is challenging due to its dramatic heterogeneity and complex porous structure. This paper aims to develop a novel framework of characterising fractured reservoir samples with micro-computed tomography (micro-CT) imaging technology.

A non-destructive imaging technology, X-ray micro-CT technology, is applied to acquire the 3D spatial representation of the fractured rock sample. As a result, rock compositions including fractures, matrix and minerals, are identified by respective grey values and segmented into unique phases for separate analysis. Based on the segmented micro-CT images, we develop a comprehensive micro-CT image analysis method to obtain the statistics of fracture geometrical properties, including orientation, length, spacing, aperture size. Apart from fracture network, the mineral phase is extracted and analysed with mineral size statistics and mineralisation degree. In order to represent the heterogonous fractured samples realistically, discrete fracture network models (DFN) are constructed, where geometrical properties are stochastically drawn from the measured statistics. The generated DFN model is discretised by a voxelisation process to provide the same format with original microCT images with the same resolution. Multiple DFN realisations are reconstructed stochastically with Monte Carlo method. Absolute permeability, porosity, connectivity, tortuosity and specific surface area of each realisation are computed and compared with original micro-CT images.

Our results show that the developed DFN models are sufficiently realistic representations of original micro-CT images in terms of fracture geometry, topology, mineralisation, and petrophysical properties. For example, according to the comparison between permeability of DFN realisations and original micro-CT scans, it is shown that our developed DFN models produce accurate permeability 
estimation with low error of approximately $6.5 \%$ on average. Since that the roughness and mineralisation information which are obtained from micro-CT images are additionally considered in our DFN modelling, thus the issue of transmissibility over-estimation associated with conventional DFN models is addressed. This framework of characterising fractured reservoir samples can be widely applied for any unconventional reservoirs, including coal seam gas and shale gas reservoirs. In addition, it is important for its application to digital core analysis because there are many difficulties with direct simulation of flow on micro-CT images because of limited image resolution. Therefore, the novelty of this work demonstrates its ability to be independent of the image resolution. Rather than struggling with the trade-off between the image resolution and scanned sample size, reconstructing DFN models to represent micro-CT images is no longer restricted with model dimension while also preserving resolution.

Procter and Gamble Student poster award:

References:

Acceptance of Terms and Conditions:

\title{
Porous Media Characterisation relavent to Enhanced Gas Recov- ery
}

Michael Johns $^{1}$; Eric May ${ }^{1}$; Vahab Honari ${ }^{2}$; Marco Zecca $^{1}$; Milan Patel $^{3}$

\author{
${ }^{1} U W A$ \\ ${ }^{2}$ University of Queensland \\ ${ }^{3}$ RMIT, Australia
}

Corresponding Author(s): michael.johns@uwa.edu.au

Enhanced Gas Recovery (EGR), in which injected (and hence sequestered) supercritical CO2 is used to increase natural gas production, has never been practiced industrially from the start of a gas field development despite presenting both significant operational (increased natural gas production) and environmental (secure CO2 sequestration) benefits 1. Primarily this lack of adoption is due to uncertainty regards the extent of mixing of the injected $\mathrm{CO} 2$ and the nascent natural gas, possibly resulting in unacceptable contamination of the natural gas product. Reservoir simulations that accurately capture the $\mathrm{CO} 2-$ natural gas mixing processes are essential if EGR is to be adopted 2. Here we report on the use of NMR measurements to determine critical inputs into EGR simulations; namely dispersivity and tortusoity relevant to $\mathrm{CO} 2-$ natural gas core flooding processes under reservoir/supercritical conditions.

Specifically we use in-situ time-resolved NMR 1D axial profiles to determine the dispersion coefficient for a CO2-CH4 core flood; via comparison with traditional effluent analysis using IR we are able to quantify and eliminate the erroneous contribution of entry/exit and edge effects in the core flood. We also measure tortusoity using traditional PFG techniques and use this measurement to validate the dispersion coefficient measurement as velocity approaches zero. This is performed for both a range of sandstone and carbonate rock cores 3 . We subsequently have proceeded to adapt our experimental apparatus and methodology to also incorporate residual or connate water $[4,5]$. Multidimensional MRI was used to confirm the even distribution of residual water in the rock cores; a variable direction centrifuge procedure was established to ensure this. NMR T2 relaxation measurements were used to confirm the pore size distribution occupied by the residual water phase. Residual water was found to distinctly occupy the smaller pores, which resulted in an increase in gas dispersion (of up to an order of magnitude) for all rock cores considered. We will also detail methodology to directly determine the tortuosity of these partially saturated rock cores, again using PFG techniques. Finally we present the inclusion of these experimental parameters into 3D reservoir simulations of an EGR process [6], as well as the adaptation of the apparatus to allow for the presence of clathrate hydrates during the $\mathrm{CO} 2-$ natural gas exchange process.

Procter and Gamble Student poster award:

References: 
1 Hughes T.J., Hanari, A., Graham, B.F., Chauhan, A.S., Johns, M.L. and May, E.F. (2012) CO2 Sequestration for Enhanced Gas Recovery: New Measurements of Supercritical CO2-CH4 Dispersion in Porous Media and a Review of Recent Research, Int. Jnl. Greenhouse Gas Control, 9, 457-468.

2 Patel, M.J., May E.F. and Johns, M.L. (2016), High-fidelity reservoir simulations of enhanced gas recovery with supercritical CO2, Energy, 111, 548-559.

3 Hanari, A., Bijeljic, B., Johns, M.L. and May, E.F. (2015) Enhanced Gas Recovery with CO2 Sequestration: the Effect of Medium Heterogeneity on the Dispersion of Supercritical CO2-CH4, Int. Jnl. Greenhouse Gas Control, 39, 39-50.।

[4] Zecca, M. Vogt, S.J., Honari, A., Xiao, G., Fridjonsson, E.O., May, E.F. and Johns M.L. (2017) Quantitative dependence of $\mathrm{CH} 4-\mathrm{CO} 2$ dispersion on immobile water fraction, AICHEJ, 63, 5159-5168.

5 Zecca, M., Vogt, S.J., Connolly, P.R.J., May, E.F. and Johns, M.L. (2018), NMR Measurements of Tortuosity in Partially Saturated Porous Media, Transport in Porous Media, 125 (2) 271-288.

[6] Patel, M.J., May E.F. and Johns, M.L. (2017), Inclusion of Connate Water in Enhanced Gas Recovery Reservoir Simulations, Energy, 141, 757-769. Acceptance of Terms and Conditions:

Click here to agree

119

\section{Simulation and Experimental Measurements of Internal Magnetic Field Gradients and NMR Transverse Relaxation Times (T2) in Sandstone Rocks}

Paul Connolly ${ }^{1}$; Weichao Yan $^{2}$; Mohammed Mahmoud ${ }^{3}$; Eric May ${ }^{4}$; Michael Johns ${ }^{4}$

${ }^{1} U W A$

${ }^{2}$ China University of Petroleum

${ }^{3}$ King Fahd University of Petroleum and Minerals

${ }^{4}$ University of Western Australia

Corresponding Author(s):

NMR T2 measurements are widely used to determine various petro-physical properties both in the laboratory and for in situ reservoir measurements using well logging tools. Internal magnetic field gradients, which occur in rock pores during NMR measurements due to magnetic susceptibility differences between the rock matrix and the pore fluids, can however distort these $\mathrm{T} 2$ measurements Hence, it is vital that a pore scale understanding of how these magnetic field gradients evolve with various rock types, and how they in turn affect T2 measurements, is realised.

Here we implement a FEM simulation of these internal magnetic field gradients on 3D digital $\triangle \mathrm{CT}$ images of five different sandstone rocks, coupled with a random walk simulation of the T2 NMR signal relaxation process. The FEM simulations required the magnetic susceptibility of each sandstone, this was directly measured using a superconducting quantum interference device (SQUID) over a range of magnetic field strengths. The resultant probability distributions of internal magnetic field gradients were then compared against equivalent experimental measurements for the first time. The results were generally in reasonable agreement, however the simulations failed to capture the larger magnetic field gradients that were observed experimentally.

Consideration of various potential reasons including; image resolution, model discretisation, the effect of heterogenous susceptibilities and the presence of trace ferromagnetic minerals are made. Based on scanning electron and magnetic force microscopy results coupled with simulations, we identify the assumption of a single mean magnetic susceptibility as being the primary source of the variation between simulated and measured results. Furthermore, we identified that small amounts of iron bearing clays situated at the pore surface can significantly increase local internal gradient fields, far exceeding what would be expected based on the bulk mineralogy. Simulations of the T2 relaxation process are shown however to be in good agreement with experimental measurements across the five sandstones studied for a standard $2 \mathrm{MHz}$ detection frequency.

Procter and Gamble Student poster award:

I would like to compete in the Procter and Gamble Student award References: 
Acceptance of Terms and Conditions:

Click here to agree

\title{
Enhanced convective dissolution flux due to an $A+B->C$ reaction
}

\author{
Mamta Jotkar ${ }^{1}$; Laurence Rongy ${ }^{2}$; Anne De $\mathrm{Wit}^{3}$ \\ ${ }^{1}$ Université Libre de Bruxelles \\ ${ }^{2}$ Universite libre de Bruxelles (ULB) \\ ${ }^{3} U L B$
}

Corresponding Author(s): mjotkar@ulb.ac.be

Chemical reactions are known to have a significant impact on convective dissolution in partially miscible density stratifications in a porous medium and to be able to enhance the steady-state flux with respect to the non-reactive case. We numerically study such reactive convective dissolution when species $A$ reacts with $B$ present in the host solution to produce $C$ via an $A+B \rightarrow C$ reaction. This work is divided into two parts. In the first one, we consider unstable non-reactive density stratifications where the dissolving species A increases the density of the host solution upon dissolution. We analyze the effect of reactions by classifying the density profiles and the nonlinear dynamics into distinct regimes in the parameter space. We show that upon varying the difference $\Delta R_{C B}=\mathrm{R}_{C}-$ $\mathrm{R}_{B}$ between the Rayleigh numbers of the product $\mathrm{C}$ and the reactant $\mathrm{B}$ and the ratio $\beta=B_{0} / A_{0}$ of the initial concentration of the reactant $B$ with respect to $A$, it is possible to increase the dissolution flux by an order of magnitude relative to the non-reactive case. In the second part, we consider nonreactive stable density stratifications where the dissolving species A decreases the density of the host solution. While the dominant transport mechanism in the absence of reactions is the diffusive one, we show that chemical reactions can induce convective dissolution and increasing $\Delta R_{C B}$ and $\beta$ can further enhance the steady-state dissolution flux. We obtain a critical value of $\Delta R_{C B}$ as a function of $\beta$ above which the reactions can initiate convective dissolution and we show that this value is in agreement with the theoretical predictions made using linear stability analysis. Additionally, the effects of hydrodynamic dispersion on the steady-state dissolution flux are studied. Our results are important in the context of $\mathrm{CO}_{2}$ geological sequestration where enhanced dissolution flux is favourable to improve the efficiency and safety of the process.

Procter and Gamble Student poster award:

References:

Acceptance of Terms and Conditions:

Click here to agree

\section{Imaging and modeling Martin Blunt at the pore scale}

Ruben Juanes ${ }^{1}$

${ }^{1}$ MIT

Corresponding Author(s): juanes@mit.edu

I reflect on some of the many contributions by Prof. Martin Blunt, and use them to highlight the profound influence he has had on my career and my life, from our early discussions on the esoteric elliptic regions in three-phase flow to the later ones on dynamic pore-scale imaging and modeling. 
Procter and Gamble Student poster award:

References:

Acceptance of Terms and Conditions:

Click here to agree

608

\title{
Study of contact angle variation considering geological CO2 se- questartion
}

\author{
Jongwon Jung ${ }^{1}$; Mohammad Jafari ${ }^{2}$ \\ ${ }^{1}$ Chungbuk National University \\ ${ }^{2}$ Louisiana State University
}

Corresponding Author(s): jjung@chungbuk.ac.kr

\begin{abstract}
Geological sequestration is one of the most effective methods of mitigating carbon dioxide emission. CO2 captured from the main sources is injected into deep underground siztes for long-term storage. There are many possible sites for $\mathrm{CO} 2$ seuqestration. Among them, saline aquifers is the most interested site due to its capacity. The capacity and safety of saline aquifers are governed by capillary force. Among the parameters influencing capillary pressure, the wettability of rock, which is quantified by the contact angle, has the highest uncertainty. The measurement of contact angles in real conditions inside the pores of a rock is ideal, but technical issues force researchers to measure the contact angle of a bubble of $\mathrm{CO} 2$ or a water/brine droplet on a flat surface, which cannot model the real fluid configuration inside the pores. In our experimental study, we used a transparent glass 2D micromodel with randomly patterned channels in order to inject $\mathrm{CO} 2$ and brine with different salinities and satureated conditions. And then, we measured the contact angle of the water-CO2 interface on glass channel wall surfaces. Results show that the effects of salinity and unsaturated condition on the dynamica contact angle including advancing- and receding-contact angles. Results implies that a higher contact angle increases the risk of the leakage through ground layers and the migration of $\mathrm{CO} 2$ back to the surface. Moreover, increased salinity results in an increased contact angle for a specific pressure, which must be considered for the safety of any storage site. Also, contact angle hysteresis, which is an important parameter for immobilizing CO2 bubbles inside of the rock pores, increases with pressure and decreases with salinity.
\end{abstract}

Procter and Gamble Student poster award:

References:

Acceptance of Terms and Conditions:

Click here to agree

644

\section{Monitoring Pressure Fluctuations in Artificial Porous Media.}

Author(s): Michael Jung ${ }^{1}$

Co-author(s): Martin Brinkmann ${ }^{2}$; Stephan Herminghaus ${ }^{3}$; Ralf Seemann ${ }^{1}$

\footnotetext{
1,2

${ }^{2} 1$

${ }^{3} 2$
} 
Corresponding Author(s): michael.jung@physik.uni-saarland.de

We aim to understand the microscopic mechanisms of individual capillary instabilities which govern slow displacement processes in permeable porous media. Therefore we investigate the influence of the pore space geometry and wettability on the "landscape" of pressure variations during slow displacement of fluid from an arrangement of glass beads by a second, immiscible fluid. For an experimental verification of the capillary pressure variations, we measure the pressure of a bursting meniscus in a single throat formed by three beads. Different wettability of the glass beads is realized by wetsilanization techniques as OTS (octadecyltrichlorosilane) or APTES (3-aminopropyltriethoxysilane)coating which result in a water-air contact angle on glass beads of $110^{\circ}$ and $60^{\circ}$, respectively. Heterogeneous wettability is achieved by throats assembled from beads with different coatings. The experiments shall be compared with numerical calculations of the capillary pressure in such a heterogeneous throat for various combination of contact angles. Understanding the characteristic pressure variations of single throats, we will extend our setup to small bead-packs consisting of a few layers of glass beads with heterogeneous wettability.

Procter and Gamble Student poster award:

References:

Acceptance of Terms and Conditions:

Click here to agree

612

\section{Carbon aerogels with high microporous specific surface area and low thermal conductivity prepared through self-sacrificial salt templating route with ambient drying}

Feng Junzong $^{\text {None }}$; Zhang Zhen $^{\text {None }}$; Jiang Yonggang ${ }^{\text {None }} ;$ Li Liangjun $^{\text {None }} ;$ Feng Jian $^{\text {None }}$

Corresponding Author(s):

The application of ultra-high temperature thermal insulation puts forward an urgent demand for carbon aerogels. The carbon aerogels with high microporous porosity were prepared through a simple and novel self-sacrificial salt templating routine to improve their thermal insulation performance. The salt templates could be removed during carbonization process and so carbon aerogel monoliths were directly obtained after ambient drying and pyrolyzing without washing removal of salts. The microporous specific surface area and BET specific surface area of the resultant carbon aerogels with density $0.23 \mathrm{~g} / \mathrm{cm} 3$ were dramatically increased up to $770 \mathrm{~m} 2 / \mathrm{g}$ and $1131 \mathrm{~m} 2 / \mathrm{g}$, respectively. The solid thermal conductivity of the carbon aerogels at room temperature was $0.0279 \mathrm{~W} \cdot \mathrm{m}-1 \cdot \mathrm{K}-1 \mathrm{with}$ gaseous thermal conductivity $0.0187 \mathrm{~W} \cdot \mathrm{m}-1 \cdot \mathrm{K}-1$ measured by laser flash method. At $1800 \mathrm{oC}$, the thermal conductivity at ambient pressure was $0.3721 \mathrm{~W} \cdot \mathrm{m}-1 \cdot \mathrm{K}-1$, in which gaseous thermal conductivity accounted for only about $11 \%$. The thermal insulation property of the obtained carbon aerogels was comparable to that of carbon aerogels synthesized through supercritical drying. Through improving the content of micropores to reduce thermal conductivity, the obtained carbon aerogels have great potential applications in the field of ultra-high thermal insulation.

Procter and Gamble Student poster award:

I would like to compete in the Procter and Gamble Student award References:

1. Junzong Feng, Cangrui Zhang, Jian Feng. Mater Lett, 2012; 67: 266.

2. Zhen Zhang, Junzong Feng, Yonggang Jiang, et al. Nanomaterials, 2018, 8: 255.

Acceptance of Terms and Conditions:

Click here to agree 


\title{
Multi-scale modeling in poroelasticity: Computational homoge- nization and Numerical Model Reduction
}

\author{
Ralf Jänicke $^{1}$; Fredrik Larsson ${ }^{1}$; Kenneth Runesson ${ }^{1}$ \\ ${ }^{1}$ Chalmers University of Technology
}

Corresponding Author(s): ralf.janicke@chalmers.se

In heterogeneous porous media, hydro-mechanical coupling between solid matrix and pore fluid under mechanical loading induces fluid pressure gradients and, accordingly, redistribution of fluid in the pore space in terms of a Darcy-type pore pressure diffusion.

In this contribution, we put particular emphasis on pore pressure diffusion in porous media on several length scales. To this end, we interpret the heterogeneous porous rock as a multi-scale material. We coin the name meso-scale for the length scale of heterogeneities (fractures, patchy saturation, ...) which are much larger than the microscopic pores and grains. The hydro-mechanical loading and the overall structural response are assumed to act and to be measured on the macro-scale (separation of scales between micro-, meso- and macro-scale).

In the case of a purely mechanical macroscopic loading, we observe a local redistribution of pore fluid without overall fluid transport. In other words, the diffusion length is, in this case, defined by the typical length of the mesoscopic heterogeneities. Thus, the macroscopic reaction is of viscoelastic nature. If, however, a hydro-mechanical loading is applied on the macro-scale, local fluid redistribution in the pore space is superimposed with a macroscopic fluid transport. This non-local interaction results in an overall poro-viscoelastic material behavior.

In this contribution, we investigate both, the local and the non-local scenario, by computational homogenization. Starting from [1,2] we develop a Numerical Model Reduction (NMR) technique similar to the Nonuniform Transformation Field Analysis (NTFA) to identify the macroscopic (poro)viscoelastic properties by a set of ("offline") training computations. Our machine learning algorithm allows us to establish a reduced $\mathrm{FE}^{2}$ scheme to solve macroscopic boundary value problems with full mesoscopic resolution in a numerically efficient and reliable way.

Procter and Gamble Student poster award:

I don't want to compete References:

1 Jänicke, R., F. Larsson, H. Steeb, \& K. Runesson [2016]. 'Numerical identification of a viscoelastic substitute model for heterogeneous poroelastic media by a reduced order homogenization approach.' Comp. Meth. Appl. Mech. Eng., 298, pp. 108-120.

2 Jänicke, R., F. Larsson, \& K. Runesson [2018]. 'Identification of viscoelastic properties from numerical model reduction of pressure diffusion in fluid-saturated porous rock with fractures.' Comp. Mech., DOI 10.1007/s00466-018-1584-7. Acceptance of Terms and Conditions:

Click here to agree

Impact of Heterogeneous Fracture Aperture on the Well Productivity of Deformable Fractured Porous Media

\author{
Author(s): T. Kadeethum ${ }^{1}$ \\ Co-author(s): H. M. Nick ${ }^{2}$; S. Salimzadeh ${ }^{3}$; S. Seyum ${ }^{2}$ \\ ${ }^{1}$ Danish Hydrocarbon Research and Technology Centre, Denmark \\ ${ }^{2}$ Danish Hydrocarbon Research and Technology Centre, Denmark \\ ${ }^{3}$ The Commonwealth Scientific and Industrial Research Organisation (CSIRO), Australia
}


Corresponding Author(s): teekad@dtu.dk

Several hydrocarbon production wells in the North Sea reservoirs suffer from productivity reduction during primary production. Since the affected reservoirs are highly fractured, closure of natu$\mathrm{ral} /$ induced fractures around wells, due to an increase in effective stress is expected to be one of the main reasons for this reduction. Traditionally, the fracture conductivity is determined by its aperture through a cubic law 1 . While the fracture aperture is commonly assumed either constant or uniformly distributed, it is well-known that the aperture distribution is heterogeneous and changes with varying contact stress at each point on the fracture surface. This heterogeneous aperture field can affect the flow performance, and fracture aperture evolution due to thermo-poroelastic stresses 2. Moreover, variance, prior distribution, and correlation length, which are used to populate the heterogeneous field, can further enhance this effect 3. Hence, this study aims to investigate and highlight the impacts of fracture aperture variation, including initial stage and deformed behaviour, on the well productivity through a conceptual steady-state single-fracture reservoir.

Coupled solid deformation and fluid flow in porous media is modelled utilising Complex Systems Modelling Platform (CSMP), an object-oriented application programme interface [4], 5. The investigation is separated into three main parts: (i) the effect of variance, (ii) the effect of prior distribution length, and (iii) the effect of correlation length/angle on well productivity index. Moreover, the comparison among the calculation of the well productivity using the homogeneous, heterogeneity aperture field, and its arithmetic average is also investigated. Taking into consideration the limitations and assumptions made in this study, the following findings are drawn: (i) well productivity tends to be higher when the heterogeneity aperture field is introduced than the homogeneous and arithmetic average ones, (ii) this effect is enhanced when the variance is increased, (iii) there is not much difference between the well productivity results when different prior distributions, i.e. uniform, normal, and log-normal distributions, are utilised, (iv) the increase in the correlation length that perpendicular to the well direction enhances the productivity of the system, and (v) the increase in the correlation length that parallel to the well direction hinders the productivity of the system.

Procter and Gamble Student poster award:

I would like to compete in the Procter and Gamble Student award References:

1 J. Jaeger, N. Cook, and R. Zimmerman, Fundamentals of Rock Mechanics, 4th ed. Wiley-Blackwell, 2010.

2 B. Guo, P. Fu, Y. Hao, C. A. Peters, and C. R. Carrigan, "Thermal drawdown-induced flow channeling in a single fracture in EGS," Geothermics, vol. 61, pp. 46-62, May 2016.

3 T. Kadeethum, S. Salimzadeh, and H. Nick, "Investigation on the Productivity Behaviour in Deformable Heterogeneous Fractured Reservoirs," in 2018 International Symposium on Energy Geotechnics, 2018.

[4] H. M. Nick and S. K. Matthäi, "A hybrid finite-element finite-volume method with embedded discontinuities for solute transport in heterogeneous media," Vadose Zo. J., vol. 10, no. 1, pp. 299-312, 2011.

5 S. Salimzadeh, A. Paluszny, H. M. Nick, and R. W. Zimmerman, "A three-dimensional coupled thermohydro-mechanical model for deformable fractured geothermal systems," Geothermics, vol. 71, pp. 212224, Jan. 2018. Acceptance of Terms and Conditions:

Click here to agree

\title{
A Mixed-dimensional Discontinuous Galerkin Method for Cou- pled Flow and Transport in Fractured Porous Media
}

\author{
Author(s): T. Kadeethum ${ }^{1}$ \\ Co-author(s): H. M. Nick ${ }^{1}$; S. Salimzadeh ${ }^{2}$; C. N. Richardson ${ }^{3}$; F. Ballarin ${ }^{4}$; S. Lee ${ }^{5}$ \\ ${ }^{1}$ Danish Hydrocarbon Research and Technology Centre, Denmark \\ ${ }^{2}$ The Commonwealth Scientific and Industrial Research Organisation (CSIRO), Australia \\ ${ }^{3}$ BP Institute, Cambridge University, United Kingdom \\ ${ }^{4}$ mathLab, Mathematics Area, SISSA, Italy \\ ${ }^{5}$ Department of Mathematics, Florida State University, United States of America
}


Corresponding Author(s): teekad@dtu.dk

Fluid flow and solute transport in fractured porous media are phenomena that control key processes in groundwater monitoring, generating thermal energy, earthquake prediction, and biomedical engineering, e.g. model activities of the heart or brain from noninvasive measurements on the chest or scalp. The mathematical representation of the fluid interaction between fracture and rock matrix domains is not straightforward because these two domains usually possess vastly different flow properties. For instance, fluid conductivity through a system of fractures can be much greater than that of the rock matrix. To tackle this issue, there are three main approaches, (i) equidimensional, (ii) mixed-dimensional representing fractures as split surfaces, and (iii) mixed-dimensional representing fractures as internal walls $[1,2]$. The first method demands excessively refinement of the fracture domain, more computationally burdens, while the second method requires a sophisticated contact model when mechanical deformation is included in the model 3. Hence, the third method is selected in this study because of its favourable computational cost and straightforward implementation when it is incorporated more phenomena.

Researchers have traditionally used the continuous Galerkin $(C G)$ method to solve flow and transport in fractured porous media problems for decades. However, it is not suitable for solving the transport equation because it may not satisfy mass conservation. Moreover, it cannot represent fractures that act as flow barriers [4]. This study aims to present the advantages of using the discontinuous Galerkin $(D G)$ method for solving coupled flow and transport in fractured porous media. A mixed $D G \times C G$ space is utilised to solve the pressure equation. Subsequently, a velocity field is established using $C G$ or Raviet - Thomas function space; then the transport equation is solved on $D G$ space. This procedure provides more accurate solutions in a convection-dominated regime, in which a sharp flood-front of the tracer is established, than those of $C G$ formulation. The result of this work is compared to relatively new numerical methods, including hybrid-finite-element-finitevolume, lowest order Raviart-Thomas mixed finite elements, and multi-Point flux approximation methods as part of "Verification benchmarks for single-phase flow in three-dimensional fractured porous media $[4,5,6]$."

Procter and Gamble Student poster award:

I would like to compete in the Procter and Gamble Student award References:

1 R. Juanes, J. Samper, J. Molinero, A general and efficient formulation of fractures and boundary conditions in the finite element method, International Journal for Numerical Methods in Engineering 54 (12) (2002) 1751-1774.

2 S. Salimzadeh, A. Paluszny, R. W. Zimmerman, Three-dimensional poroelastic effects during hydraulicfracturing in permeable rocks, International Journal of Solids and Structures 108 (2017) 153-163

3 M. Nejati, A. Paluszny, R. W. Zimmerman, A finite element framework for modeling internal frictional contact in three-dimensional fractured media using unstructured tetrahedral meshes. Computer Methods in Applied Mechanics and Engineering 306 (2016) 123-150.

[4] H. M. Nick, S. K. Matth“ai, Comparison of three FE-FV numerical schemes for single- and twophaseflow simulation of fractured porous media, Transport in Porous Media 90 (2) (2011) 421-444.

5 V. Martin, J. Jaffre, J. E. Roberts, Modeling Fractures and Barriers as Interfaces for Flow in Porous Media, SIAM J. Sci Comput. 26 (2005) 1667-1691.

[6] T. H. Sandve, I. Berre, J.M. Nordbotten, An efficient Multi-Point Flux Approximation based approach for Discrete Fracture Matrix simulation, Journal of Computational Physics 231 (9) (2012), 3784-3800. Acceptance of Terms and Conditions:

Click here to agree

\title{
Atomistic Simulation of the Adsorption and Transport of Natu- rally Occurring Radioactive Materials in Clay Nanopores in the Context of Shale Gas Exploration
}

\author{
Andrey G. Kalinichev ${ }^{1}$; Brice F. Ngouana-Wakou ${ }^{2}$; Iuliia Androniuk ${ }^{2}$

\footnotetext{
${ }^{1}$ Institut Mines-Telecom Atlantique, Nantes, France

${ }^{2}$ Laboratoire SUBATECH (UMR 6457 - Institut Mines-Telecom Atlantique, Université de Nantes, CNRS/IN2P3)
} 


\title{
Corresponding Author(s): kalinich@subatech.in2p3.fr
}

Nanoscale understanding and prediction of the structural, dynamic, and reactive properties of fluids confined in clay pores and the behavior of dissolved inorganic and organic aqueous species under these conditions is important for many geochemical, environmental, and technological applications, such as geological disposal of radioactive waste, shale oil and gas exploration, geological carbon sequestration, etc. Is extremely difficult to experimentally study such systems on the fundamental molecular scale, and the results of such studies are often subject to considerable interpretation usually based on certain atomistic models. At the same time, rapid development of atomistic computational modeling techniques in recent years 1 has already proven to be very efficient in providing significant new quantitative details of the specific effects of the clay mineral structure and composition on the structure, dynamics and reactivity of interfacial and nanoconfined fluids. However, in most computer simulations to date, clay particles are represented by their basal (001) surfaces only. Here we are using the recently developed modification 2 of the ClayFF force field 3 to realistically model the hydrated edges of finite size clay nanoparticles in large-scale classical molecular dynamics (MD) simulations. The structure of aqueous solutions and ion adsorption at the (010) and (110) edge surfaces of kaolinite, montmorillonite, and muscovite are investigated in detail for a number of mono- and di-valent cations relevant for the problems of shale gas exploration, where they are suspected to have negative environmental impact as potentially mobile naturally occurring radioactive materials (NORMs). Specific surface adsorption sites are initially identified, and then probed by the calculations of potentials of mean force [4], thus providing detailed site-specific quantitative information on the free energies of adsorption and the thermodynamics of interfacial cation exchange.

1 A.G.Kalinichev, X.Liu, R.T.Cygan (2016) Molecular simulations of clay-water interfaces: Recent progress, challenges, and opportunities. Clays and Clay Minerals, 64, 335-336.

2 M.Pouvreau, J.A.Greathouse, R.T.Cygan, A.G.Kalinichev (2017) Structure of hydrated gibbsite and brucite edge surfaces: DFT results and further development of the ClayFF classical force field with Metal-O-H bending terms. J. Phys. Chem. C., 121, 14757-14771.

3 R.T.Cygan, J.-J.Liang, A.G.Kalinichev (2004) Molecular models of hydroxide, oxy-hydroxide, and clay phases and the development of a general force field, J. Phys. Chem. B, 108, 1255-1266.

[4] N.Loganathan, A.G.Kalinichev (2017) Quantifying the mechanisms of site-specific ion exchange at an inhomogeneously charged surface: Case of $\mathrm{Cs}+/ \mathrm{K}+$ on hydrated muscovite mica, J. Phys. Chem. C, 121, 7829-7836.

Procter and Gamble Student poster award:

References:

Acceptance of Terms and Conditions:

Click here to agree

\section{Atomistic Computational Modeling of Fluid Transport in Cement Nanopores in the Context of Geological CO2 Sequestration}

\author{
Sylvia M. Mutisya ${ }^{1}$; Andrey G. Kalinichev ${ }^{2}$ \\ ${ }^{1}$ Laboratoire SUBATECH (UMR 6457 - Institut Mines-Telecom Atlantique, Université de Nantes, CNRS/IN2P3) \\ ${ }^{2}$ Institut Mines-Telecom Atlantique, Nantes, France
}

Corresponding Author(s): kalinich@subatech.in2p3.fr

The enormous potential of geological carbon sequestration technologies for reducing the carbon footprint of human activity is attracting an increasing attention in the recent years [1,2]. Cementitious materials are widely utilized in these technologies for zonal isolation of the CO2 storage wells. The degradation and deterioration of cement and concrete under the conditions of CO2 disposal and storage pose potential environmental risks such as fugitive gas leakages and groundwater contamination. To accurately understand, predict, and minimize these risks, a proper understanding of the 
fluid transport pathways in wellbore cement is particularly important.

Here we employ atomistic computational modeling techniques to study the molecular properties of $\mathrm{CO} 2 / \mathrm{H} 2 \mathrm{O}$ fluid mixtures nanoconfined in the porous space of cement's main hydration phase: calcium silicate hydrate (C-S-H). These molecular scale results contribute to a more accurate quantitative understanding of fluid migration through wellbore leakage pathways. The effects of the cement chemistry and pore size distribution are taken into account by varying the $\mathrm{Ca} / \mathrm{Si}$ ratio of the atomistic C-S-H models from 0.83 to 1.75 and the pore sizes from 1 to $5 \mathrm{~nm}$, respectively. At the first stage, the intercalation potential of $\mathrm{CO} 2 / \mathrm{H} 2 \mathrm{O}$ fluid mixtures is investigated using grand canonical Monte Carlo (GCMC) simulations for the $\mathrm{C}-\mathrm{S}-\mathrm{H}$ porous systems in equilibrium with bulk $\mathrm{CO} 2 / \mathrm{H} 2 \mathrm{O}$ mixtures at $(\mathrm{T}=323 \mathrm{~K}$ and $\mathrm{P}=90 \mathrm{bar})$ and $(\mathrm{T}=348 \mathrm{~K}$ and $\mathrm{P}=130 \mathrm{bar})$, mimicking the $\mathrm{T} / \mathrm{P}$ conditions of geological carbon sequestration. The increase of $\mathrm{Ca} / \mathrm{Si}$ ratio leads to lower $\mathrm{CO} 2$ adsorption in pores, as $\mathrm{H} 2 \mathrm{O}$ molecules competitively adsorb on the calcium cations, blocking access of $\mathrm{CO} 2$. The equilibrium compositions and structures obtained from the GCMC simulations are then used as an input for classical molecular dynamics (MD) simulations using the ClayFF force field 3 . The structural properties of fluids (i.e., atomic density profiles, preferential molecular orientations) and the diffusional mobility of $\mathrm{CO} 2$ and $\mathrm{H} 2 \mathrm{O}$ molecules in the pores are analyzed in detail.

References

1 Gíslason, S. R., Sigurdardóttir, H., Aradóttir, E. S., Oelkers, E. H. (2018). A brief history of CarbFix: Challenges and victories of the project's pilot phase. Energy Procedia, 146, 103-114.

2 Matter, J. M., Broecker, W. S., Gislason, S. R., Gunnlaugsson, E., Oelkers, E. H., Stute, M., Axelsson, G. (2011). The CarbFix Pilot Project-storing carbon dioxide in basalt. Energy Procedia, 4, 5579-5585. 3 Cygan, R. T., Liang, J. J., Kalinichev, A. G. (2004). Molecular models of hydroxide, oxyhydroxide, and clay phases and the development of a general force field. Journal of Physical Chemistry B, 108, 1255-1266.

Procter and Gamble Student poster award:

References:

Acceptance of Terms and Conditions:

Click here to agree

\title{
Adaptive Discontinuous Galerkin Methods for complex flow in porous media
}

\author{
Author(s): Birane Kane ${ }^{1}$; Birane Kane ${ }^{2}$ \\ Co-author(s): Robert Kloefkorn ${ }^{1}$ \\ ${ }^{1}$ NORCE Norwegian Research Centre AS \\ ${ }^{2} N O R C E$
}

Corresponding Author(s): birane.kane@norceresearch.no, birane.s.kane@gmail.com

\begin{abstract}
Modeling and simulation of multiphase flows arising in environmental problems such as infiltration and remediation requires careful numerical treatment due to the significant geologic complexity involved and the treatment of strongly heterogeneous soil properties.

The discretization of the PDE's arising from this class of problems requires locally conservative methods such as discontinuous Galerkin (DG) methods in order to be able to follow small concentration. We provide an adaptive interior penalty discontinuous Galerkin discretization for the solution of porous media flow problems extending our previous work on incompressible two-phase flow problems $[1,2]$ to compressible, reactive transport and multi-phase/multi-component models. We allow for refinement/coarsening in both the element size, the polynomial degree and the time step size. This adaptive strategy allows to refine the mesh when the solution is estimated to be rough and increase the local polynomial degree when the solution is estimated to be smooth. It also grants more flexibility with respect to the time step size.

To our knowledge, this is the first time the concept of local hp-adaptivity is incorporated in the study of such problems, thus, allowing to comprehend the advantages and disadvantages of using
\end{abstract}


hp-adaptive algorithms and higher order DG methods for the simulation of complex flows in porous media.

The implementation is based on the Open-Source PDE software framework 3.

\title{
Procter and Gamble Student poster award:
}

\section{References:}

1 B. Kane, R. Klöfkorn, and C. Gersbacher. hp-adaptive discontinuous galerkin methods for porous media flow. In International Conference on Finite Volumes for Complex Applications, 447-456. Springer, (2017).

2 A. Dedner, B. Kane, R. Klöfkorn and M. Nolte, Python framework for hp-adaptive discontinuous Galerkin methods for two-phase flow in porous media, Applied Mathematical Modelling 67: 179-200 (2018).

3 P. Bastian, M. Blatt, A. Dedner, C. Engwer, R. Kl“ofkorn, M. Ohlberger, and O. Sander. A generic interface for parallel and adaptive scientific computing. part i: Abstract framework. Computing, 82(23): 103-119, (2008). Acceptance of Terms and Conditions:

Click here to agree

\section{Stress-induced Anomalous Transport in Natural Fracture Networks}

\author{
Peter Kang ${ }^{1}$; Qinghua Lei ${ }^{2}$; Marco Dentz ${ }^{3}$; Ruben Juanes ${ }^{4}$ \\ ${ }^{1}$ University of Minnesota \\ ${ }^{2}$ Department of Earth Sciences, ETH Zurich \\ ${ }^{3}$ IDAEA-CSIC \\ ${ }^{4}$ MIT
}

Corresponding Author(s): pkkang@umn.edu

Fluid flow and transport in fractured rock controls many natural and engineered processes in the subsurface, including hydrocarbon production from fractured reservoir and the fate of radionuclides during geologic nuclear waste storage. However, characterizing flow and transport through fractured media is challenging due to the high uncertainty and large heterogeneity associated with fractured rock properties. In addition to these "static" challenges, geologic fractures are always under significant overburden stress, and changes in the stress state can lead to changes in the fracture's ability to conduct fluids. While confining stress has been shown to impact fluid flow through fractures in a fundamental way, the impact of confining stress on transport through fractured rock remains poorly understood. The link between anomalous (non-Fickian) transport and confining stress has been shown, only recently, at the level of a single rough fracture 1 .

Here, we investigate the impact of geologic stress on flow and tracer transport through natural fracture networks. We model geomechanical behavior of fractured rock using the finite-discrete element method (FDEM) 2, which can capture the deformation of intact rocks, displacement of pre-existing fractures and propagation of new cracks, upon changes in the stress field. We apply the FDEM to a fracture network extracted from the geological map of an actual rock outcrop to obtain aperture fields at different stress conditions. We then simulate fluid flow and particle transport through the stressed fracture networks. We observe that anomalous transport can emerge in response to geological stress on the fracture network, and show that the stress state is a powerful determinant of transport behavior: (1) The interplay between fracture network geometry and anisotropic stress states can induce preferential flow paths and anomalously early arrival of tracers through shear dilation; (2) An increase in geologic stress increases aperture heterogeneity due to the nonlinear relationship between fracture closure and stress magnitude which induces late-time tailing of particle breakthrough curves. Finally, we develop an effective transport model that captures anomalous transport through stressed fracture networks. Our results point to a heretofore unrecognized link between geomechanics and anomalous transport in natural fractured media. 
Procter and Gamble Student poster award:

I don't want to compete References:

1 P. K. Kang, S. Brown, and R. Juanes, Emergence of anomalous transport in stressed rough fractures. Earth and Planetary Science Letters, 454, 46-54 (2016).

2 Q. Lei, J. P. Latham, and C. F. Tsang, The use of discrete fracture networks for modelling coupled geomechanical and hydrological behaviour of fractured rocks. Computers and Geotechnics, 85, 151-176 (2017). Acceptance of Terms and Conditions:

Click here to agree

\title{
Understanding Mixing and Reaction at Rough Fracture Intersec- tions
}

\author{
Sanghyun Lee ${ }^{1}$; Peter Kang ${ }^{1}$ \\ ${ }^{1}$ University of Minnesota
}

Corresponding Author(s): pkkang@umn.edu

\begin{abstract}
Vigorous mixing and reaction of solutes can occur at fracture intersections (FI) since fluids with two very different properties can meet and react at FI. Such phenomena are of a great importance because reaction hotspots can emerge at FI which may control the biogeochemical cycles of elements and underground contaminant transport. Although the effects of fracture roughness and flow rate on solute transport through rough fractures have been widely studied, our understanding on mixing and reaction at FI is very limited. In this study, we investigate how the interplay between fracture roughness and flow rate controls mixing and reaction kinetics at FI by combining visual microfluidics experiments and direct numerical simulations.
\end{abstract}

Microfluidic devices with straight and rough fracture intersections are prepared and used as platforms to study hydrodynamics, mixing and reaction kinetics at varying flow rates. Since reactions should be fundamentally governed by transport and mixing of solutes, we first characterize flow fields by visualizing streamlines with fluorescent particles and quantify mixing with fluorescent tracers. The diffusive mixing of tracers was observed at low flow rates while chaotic mixing of tracers by eddies was observed at high flow rates. We then study reaction at FI with a fast bimolecular reaction based on a chemiluminescent reaction. Our results show that the interplay between roughness and flow rate yields complex flow patterns that initiates reaction hotspots which is not typically expected in straight FI. In addition, direct numerical simulations of fluid flow and tracer transport are performed to complement the experimental findings. We show that both roughness and flowrate can fundamentally change mixing and reaction kinetics. In summary, we combine microfluidics experiment and direct numerical simulations to elucidate how the interplay between roughness and flow rate controls mixing and reaction at FI.

Procter and Gamble Student poster award:

I don't want to compete References:

Acceptance of Terms and Conditions:

Click here to agree

615

\section{The effect of ionic strength on the development and evolution of two phase flow in an artificial porous medium}

Nikolaos Karadimitriou ${ }^{1}$; Vahid.J Niasar ${ }^{2}$; Hassan Mahani ${ }^{3}$; Holger Steeb ${ }^{1}$ 


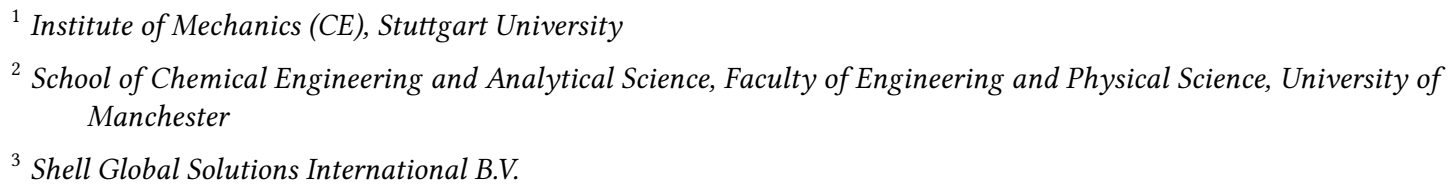

Corresponding Author(s): nkaradimitriou@gmail.com

Low Salinity Water Flooding (LSWF) is an enhanced oil recovery technique, where modified-salinity water, usually with lower salinity, is injected into the reservoir. This process can potentially lead to a higher oil production compared to conventional water flooding. There are many speculations regarding the mechanism(s) under which these effects take (or not) place. The most notable one is the one of wettability alteration which has been studied in several pore-scale and micromodel studies. Even though there is a substantial volume of works in the literature studying the LSWF effect, either experimentally or numerically, there has not been any experimental work which studies the time scale of oil recovery at different salinity concentrations, while offering direct observation of the effects taking place on the pore-scale.

In this work, we study the effect of the ionic strength on the time-scale of oil recovery in a closed network of capillaries forming the flow network of a micromodel. With the use of a custom-made microscope, we visualized the invasion of water at various ionic strengths in a hydrophobic micromodel initially filled with Fluorinert, which served as the wetting phase. Under different primary drainage conditions, we quantified the resulting change of saturation, in terms of recovery time and breakthrough values.

Our results clearly show that there is an optimal range of ionic strength where the recovery times are shorter, and for a value of ionic strength within this range, the breakthrough time and saturation are monotonically related to the flow rate. Finally, for a given injection rate, there is a clear non-monotonic trend between the breakthrough saturation, the breakthrough time and the ionic strength.

To our knowledge, this is the first reported REV-scale experiment with pore-scale, real-time observations in which the effect of LSWF is studied for a range of initial saturations and flow conditions in a micro-model, and the results can provide new insights into the non-linearities of oil recovery at different ionic strengths.

Procter and Gamble Student poster award:

\title{
References:
}

Acceptance of Terms and Conditions:

Click here to agree

\section{Impact of Time-dependent wettability alteration on Capillary pres- sure}

\author{
Author(s): Abay Kassa ${ }^{1}$ \\ Co-author(s): Sarah Gasda ${ }^{2}$; Kundan Kumar ${ }^{3}$; Florin Radu ${ }^{3}$ \\ ${ }^{1}$ NORCE \\ ${ }^{2}$ NORCE \\ ${ }^{3}$ University of Bergen
}

Corresponding Author(s): abka@norceresearch.no

Wettability plays an important role in porous media applications such as enhanced oil recovery and $\mathrm{CO} 2$ storage in terms of capillarity and residual trapping. In many applications, the wetting property of the rock surface is assumed to be static. However, the compositions of many fluids are capable of altering the wettability of rock surfaces continuously in time. In recent years, wettability has received attention in laboratory experiments and theoretical investigations. However, few studies have assessed the impact of wettability alteration (WA) on capillary pressure curves. Recently, core-scale experiments have shown a time-dependent WA that significantly alters the Darcy-scale 
two-phase flow constitutive relations. Due to the dynamics in WA, the standard capillary pressure model that assumes static wettability may be insufficient to describe the flow physics. We simulate capillary pressure curves using a bundle-of-tubes approach, where a Langmuir type model for timedependent WA is introduced at the pore-scale. The simulated curves include a pore-scale WA effect and used to understand how the equilibrium capillary pressure is altered by pore-scale mechanisms. Based on the information acquired from the simulated data, we proposed a new capillary pressure model that considers time-dependent WA at the pore-scale by adding a dynamic term to the standard capillary pressure model. Further, we showed that non-uniform WA can lead to significant hysteresis in capillary pressure-saturation relations which is not seen in the standard bundle-of-tubes model. This study shows the importance of time-dependent WA for determining capillary pressure over time scales of weeks and months. The impact of wettability has implications for experimental methodology as well as macro-scale simulation of wettability-altering fluids.

Procter and Gamble Student poster award:

I would like to compete in the Procter and Gamble Student award References:

Acceptance of Terms and Conditions:

Click here to agree

\title{
Robust Superhyrophobic/Superoleophilic Nanomaterial Coated Porous Medium for Selective Separation and Expulsion of Oil Pol- lutants from Water
}

\author{
Abdolreza Kazemi Abadshapoori ${ }^{1}$; Ali Maghsoudian ${ }^{2}$; Yousef Tamsilian $^{3}$; Zahra Ansariasl ${ }^{4}$ \\ ${ }^{1}$ Department of Petroleum Engineering, School of Chemical and Petroleum Engineering, Shiraz University, Shiraz, \\ Iran \\ ${ }^{2}$ Department of Petroleum Engineering, Faculty of Petroleum, Petroleum University of Technology, Ahvaz, Iran \\ ${ }^{3}$ Department of Oil, Gas \& Petrochemical Engineering (OGP), Faculty of Engineering, Shahid Chamran University of \\ Ahvaz (SCU), Ahvaz, Iran \\ ${ }^{4}$ Department of Chemistry, College of Science, Shahid Chamran University of Ahvaz (SCU), Ahvaz, Iran
}

Corresponding Author(s): reza.pixy@gmail.com, ali.maghsoudian@gmail.com

Oil spills have caused significant environmental and ecological problems. Oil-polluted water usually contains toxic chemicals, which may harm human's health or even have a disastrous impact on the ecosystem. Therefore, the development of methods for effective decontamination and cleanup is necessary for the protection of the environment and human health. Traditional cleanup techniques are useful for the separation of oil/water free mixtures, but suffer from the limits of low efficiency and high operating cost. Traditional techniques also absorb both water and oil during the separation process, thus displaying poor separation selectivity and efficiency, whereby separation efficiency is crucial to many applications.

Both modified and unmodified porous media such as fibers and cotton was previously subjected for separation of oil spills from water, but faced with some problems. In this work, a polyurethane sponge with homogenous distribution of porosity used as porous medium. Pore sizes are big enough that fluid droplets could flow throughout this porous medium. Unmodified porous medium can absorb both water and oil phases, but with modifying pore walls with nanomaterials, surface wettability alters, and as a result, just one phase could move through this porous media. Here, we modified our porous media with a synthesized functionalized nanoparticles, and altered surface wettability from neutral wettability, to highly oil-wet condition. Metal organic framework nanoparticles was first synthesized, and then was coated on wall surfaces of porous medium with PDMS polymer using dip-coating method. This sponge coated with functionalized nanoparticles (ZIF sponge) is used for expulsion of oil contaminant from water continuously.

In this study we used contact angle measurement for surface wettability determination. Contact angle results before and after coating with nanoparticles prove that wettability of porous medium has altered to highly oil-wet. Also SEM images before and after coating proves that pore walls have coated with nanoparticles, and this coating did not affect effective permeability of porous medium 
(because of small size of synthesized nanoparticles). In addition, EDAX results from different points of porous medium proves that our nanomaterials are well on the wall surfaces and altered its wettability. Its high crude oil sorption capacity and positive environmental footprint makes it ecologically friendly sorbent for oil spill cleanups. Furthermore, its high contact angle with water (about $160^{\circ}$ ), makes it an efficient filter to in-situ continuous separation other contaminants from water such as oil solvents. The results also demonstrate that in harsh conditions such as high temperature or acidic/basic environment and also in the vicinity of brine, it revealed a great performance. This implies that it can be used in marine mishaps or other pollutions.

\section{Procter and Gamble Student poster award}

I would like to compete in the Procter and Gamble Student award References:

Krupenkin T et al 2005 Electrically tunable superhydrophobic nanostructured surfaces Bell Labs Tech. J. 10 161-70 Young T 1805 Phil. Trans. 95 84; Works, edit by Peacock, 1,432

Jung Y C 2009 Natural and biomimetic artificial surfaces for superhydrophobicity, self-cleaning, low adhesion, and drag reduction $\mathrm{PhD}$ Dissertation Graduate Program in Mechanical Engineering The Ohio State University Simpson J T et al Method of making superhydrophobic/ superoleophilic paints, epoxies, and composites US Patent 20140090578 (Filed February 1, 2013, pub Apr 3, 2014 ORNL) Aytug T et al 2013 Optically transparent, mechanically durable, nanostructured superhydrophobic surfaces enabled by spinodally phase-separated glass thin films Nanotechnology Zhang X et al 2008 Superhydrophobic surfaces: from structural control to functional application J. Mater. Chem. Acceptance of Terms and Conditions:

Click here to agree

\section{Pore-Scale Flow of Water and Supercritical CO2 in 2D Porous Mi- cromodels: High-Speed Quantifications of Velocity Fields and In- terface Dynamics at Reservoir Conditions}

Yaofa $\mathrm{Li}^{1}$; Farzan Kazemifar ${ }^{2}$; Gianluca Blois ${ }^{1}$; Kenneth Christensen ${ }^{1}$; Yu Chen ${ }^{3}$; Amir Kohanpur ${ }^{4}$; Albert Valocchi $^{5}$

\footnotetext{
${ }^{1}$ University of Notre Dame

${ }^{2}$ California State University Sacramento

${ }^{3}$ Los Alamos National Lab

${ }^{4}$ University of Illinois at Urbana-Champaign

${ }^{5}$ Univ Illinois
}

Corresponding Author(s): farzan.kazemifar@csus.edu

Multiphase flow in porous media is relevant to a range of applications in the energy and environmental sectors. Recently, the interest in the topic has been greatly renewed by geological storage of $\mathrm{CO}_{2}$ within saline aquifers. While high-fidelity field-scale prediction of $\mathrm{CO}_{2}$ migration is central to a safe implementation of this technology, it is increasingly recognized that rigorous representation of porescale processes is critical to accurate modeling and prediction of such macroscopic flow behaviors. However, the complex pore-scale flow physics of such immiscible multiphase systems is yet to be fully understood. Moreover, recent evidence shows that transient flow events occur on the time scale of milliseconds, and the dynamics, such as rapid interfacial jumps and inertial effects, can greatly affect the accuracy of prediction if not accounted for properly in predictive models, necessitating spatially- and temporally-resolving those events using more advanced techniques 1. To this end, the pore-scale flow interactions of water and liquid/supercritical $\mathrm{CO}_{2}$ are experimentally quantified 
in $2 \mathrm{D}$ porous micromodels at reservoir-relevant conditions (i.e., $80 \mathrm{bar}, 20 \mathrm{degC}$ ), in an attempt to accurately mimics the process of $\mathrm{CO}_{2}$ injection into saline aquifers. High-speed microscopic particle image velocimetry (micro-PIV) and fluorescent microscopy are employed to measure the spatiallyand temporally-resolved instantaneous water velocity field and to quantify the interface dynamics, respectively 2. Furthermore, measurements are performed under a variety of wettability conditions, in order to quantify the impact of wettability on the physics and to evaluate the role of capillary effects. Initial results show that for capillary-dominated flow, the invasion of a single pore is dictated by its intrinsic dynamics which is independent of bulk flow, whereas within the viscous- or inertiadominated regime, pore drainage is overrun by bulk flow. It is also observed that changing wetting properties of the porous matrix significantly affects the pore-scale flow field and the interface dynamics both individually and statistically, therefore challenging their microscopic and macroscopic descriptions.

Procter and Gamble Student poster award:

I don't want to compete References:

1 Li, Y., Kazemifar, F., Blois, G., \& Christensen, K. T. (2017). Micro-PIV measurements of multiphase flow of water and liquid CO2 in 2-D heterogeneous porous micromodels. Water Resources Research, 53.

2 Kazemifar, F., Blois, G., Kyritsis, D. C., \& Christensen, K. T. (2015). A methodology for velocity field measurement in multiphase high-pressure flow of $\mathrm{CO} 2$ and water in micromodels. Water Resources Research, 51(4), 3017-3029. Acceptance of Terms and Conditions:

Click here to agree

\title{
Interfacial curvature, contact angle and capillary pressure mea- surements during drainage process in micromodels
}

\author{
Farzan Kazemifar ${ }^{1}$; Yaofa $\mathrm{Li}^{2}$; Gianluca Blois ${ }^{2}$; Kenneth Christensen ${ }^{2}$ \\ ${ }^{1}$ California State University Sacramento \\ ${ }^{2}$ University of Notre Dame
}

Corresponding Author(s): farzan.kazemifar@csus.edu

\begin{abstract}
Capillary pressure is one of the controlling parameters in immiscible displacement processes in porous media encountered in applications such as oil and gas production from hydrocarbon reservoirs and geological sequestration of $\mathrm{CO} 2$. At the micro-scale, capillary pressure is a function of interfacial tension and interfacial curvature for each meniscus, according to the Young-Laplace equation which is valid under static conditions. In this work, an immiscible displacement process is studied in a two-dimensional micromodel using high-speed imaging to resolve the motion and curvature of individual menisci. The correlation between the dynamic local capillary pressure measurements and pressure difference across the micromodel during the drainage process is examined in detail.
\end{abstract}

Procter and Gamble Student poster award:

References:

Acceptance of Terms and Conditions:

Click here to agree ity \& low permeability carbonate reservoir developed by WAGTake RS oil field in Abu Dhabi for example 
Wang $\mathrm{Ke}^{\text {None }}$; Zhang Junhan ${ }^{\text {None }}$

Corresponding Author(s): 837644664@qq.com

The Abu Dhabi's RS oil field is partially a middle porosity and low-permeate carbonate thick oil layer in the transition zone. At present, the horizontal well injection hydrocarbon gas WAG flooding is used for development. During the development process, there are rising problems of bottom water influx, poor displacement efficiency, and a large amount of remaining oil. In order to meet the requirements of high recovery, it is necessary to study the micro-structure and residual oil distribution. Through the numerical simulation software, the numerical simulation model of the oilfield is used to predict and simulate the oilfield's remaining oil. Combined with the dynamic and static data of the oilfield, it is found that on the plane, the remaining oil mainly exists between the wells and the wells lines. In the vertical direction, the remaining oil mainly affected by geological conditions, most of them exist in the lower part of the interlayer, the low point of the local structure, the vicinity of the fault, etc. In the three-dimensional space, due to the joint action of the WAG displacement method and the three-dimensional well pattern, the remaining oil is mainly a horn funnel shape exist between the production well row and the injection well row. The numerical simulation software is used to study the potential tapping of remaining oil. The different conditions of horizontal well productivity, horizontal well pattern deployment, and horizontal well production mode and formation pressure are studied. At last, we found the long horizontal well infilling is better, and pressure-keeping-development can achieve better results. Through Numerical simulation experiments, results have been concluded that when the horizontal well length is more than 1500 meters and the formation pressure is maintained at $30 \mathrm{MPa}$, a 3-5 years stable production time can be extended, and the Recovery factor can increase about $0.8 \%$.

Procter and Gamble Student poster award:

References:

Acceptance of Terms and Conditions:

Click here to agree

\section{One-particle layer drying kinetics to assess recycled concrete ag- gregates absorption}

Emmanuel Keita $^{1}$; Jennifer Naël-Redolfi ${ }^{2}$; Florian Théréné ${ }^{1}$; Nicolas Roussel ${ }^{1}$

${ }^{1}$ Laboratoire Navier

${ }^{2}$ Chryso

Corresponding Author(s): emmanuel.keita@ifsttar.fr

The environmental threat on non-renewable resources such as rounded natural aggregates strongly affects the materials available for the production of mortar or concrete. This leads to an increase in the relative contribution of alternative resources such as recycled or crushed aggregates, which are highly porous. To account for aggregates water content and correct the resulting water absorption, it is usual to correct the amount of mixing water added in the mixer. However, the traditional standard used to measure rounded aggregates water absorption is not suitable for crushed aggregates.

We develop a test based on drying for the assessment of water absorption and its kinetics in the case of crushed and recycled sands. We measure drying kinetics of one-particle layer composed of saturated porous aggregates. We show that this method is suitable to determine water absorption of crushed or recycled sands. Then, we aim at evaluating the robustness and the limit of such methods while understanding the general underlying physical processes. We therefore measure the drying kinetics of one-particle layer of non-porous and porous, real and model particles with various sizes and morphologies. We study specifically the effects of angularity and roughness of particles on drying rate changes over time. 


\title{
References:
}

J. Naël-Redolfi, E. Keita, N. Roussel, 'Water absorption measurement of fine porous aggregates using an evaporative method: Experimental results and physical analysis' Cement and Concrete Research, 2017 Acceptance of Terms and Conditions:

Click here to agree

\section{Influence of non-adsorbing polymers on drying of fresh mortars: measurement and physical origin}

\author{
Emmanuel Keita ${ }^{1}$; Yasser Rifaai ${ }^{1}$; Patrick Belin ${ }^{1}$; Nicolas Roussel $^{2}$ \\ ${ }^{1}$ Laboratoire Navier \\ ${ }^{2}$ laboratoire Navier
}

Corresponding Author(s): emmanuel.keita@ifsttar.fr

\begin{abstract}
We compare the drying of non-reactive materials (glass beads packing) with the one of fresh mortars. Through the sample drying process, the existing literature on the general underlying physical processes involved in drying of porous media allows for the identification of two distinct regimes. Capillary forces drive the drying kinetics. We then measure the drying kinetics of the same materials containing polymers, which do not absorb on the cement grains. We observe the formation of a crust at the free surface. Moreover, we measure the pore structure evolution with NMR spectroscopy; our results and analysis suggest that the increase in the viscosity of the interstitial water due to polymer addition is at the origin of a decrease in drying rate.

As drying of fresh cementitious materials strongly impacts the final properties in configuration with large exposed surfaces, we suggest that such polymer addition could slow down the overall drying of cementitious materials and improve their service life properties.
\end{abstract}

Procter and Gamble Student poster award:

References:

Keita, E., Rifaai, Y., Belin, P. \& Roussel, N. Influence of non-adsorbing polymers on drying of fresh mortars. Cem. Concr. Res. 116, 38-44 (2019). Acceptance of Terms and Conditions:

Click here to agree

159

\section{Understanding the Critical Role of Capillary Suction on Rock Me- chanical Responses during Post-fracturing Spontaneous Imbibi- tion in Tight Formations}

\author{
Reza Keshavarzi ${ }^{1}$; Rick Chalaturnyk ${ }^{1}$ \\ ${ }^{1}$ University of Alberta \\ Corresponding Author(s): rkeshava@ualberta.ca
}

Generally, most tight formations do not produce without fracturing. However, during hydraulic fracturing in this type of reservoirs, a large portion of the injected fracturing fluid cannot be recovered which would start interacting with the formation. Although spontaneous imbibition is mainly introduced as the main dominating mechanism but predicting the reservoir condition as a result of the formation and fracturing fluid interaction is a big concern. Therefore, understanding the rock 
responses as a result of post-fracturing spontaneous imbibition is a key to account for the behaviors observed in tight formations.

Since the main driving force for spontaneous imbibition is capillary suction and in another hand spontaneous imbibition can be considered as changing the saturation of the media, first through a novel integrated theoritical-experimental approach the values of capillary suction as a function of relative humidity and saturation have been determined for Montney tight gas samples (our case study) which shows very high values for capillary suction (more than 100MPa) and as the water saturation increases the capillary suction non-linearly decreases. Meantime, also based on the developed theoretical approach for the effective stress it is found that in partially saturated media decreasing the capillary suction would lead to local effective stress reduction followed by increasing the possibility of volumetric responses.

To tackle the above-addressed theoretical findings, a series of uniaxial compression experiments have been considered through the cycles of loading-unloading-reloading while the sample is being subjected to different degrees of relative humidity (or equivalently saturation) and it reaches to equilibrium in each cycle of loading-unloading. The volumetric behavior (axial and lateral strain) is monitored in each step where the capillary suction due to different relative humidity that the sample is being subjected to is changing. The results indicate a non-linear dependency of deformability on capillary suction (as expected from the theoretical analysis) and it highlights the critical role of reduction in capillary suction on rock mechanical properties (Young's modulus and Poisson's ratio) during spontaneous imbibition in tight formations. Based on the results it is clearly observed that Young's modulus and Poisson's ratio would decrease and increase respectively as the capillary suction is decreasing. Such response would suggest that the rock behavior is changing and it is getting more ductile.

One of the important points in this study is that the clays in Montney tight gas samples (which are mainly illite) are not expansive clays. It means that large volume changes (swelling) in clays as a result of increasing the water saturation would not occur which would be so beneficial to characterize the volumetric behaviors and quantify rock mechanical properties based on capillary suction.

\section{Procter and Gamble Student poster award:}

I would like to compete in the Procter and Gamble Student award References:

Acceptance of Terms and Conditions:

Click here to agree

19

\section{Geostatistical multi-scale estimating the effective elastic param- eters of sandstone from digital core images}

Tatiana Khachkova ${ }^{1}$; Yaroslav Bazaikin ${ }^{2}$; Boris Gurevich ${ }^{3}$; Dmitry Kolyukhin ${ }^{1}$; Vadim Lisitsa ${ }^{1}$; Maxim Lebedev ${ }^{3}$ ; Galina Reshetova ${ }^{4}$

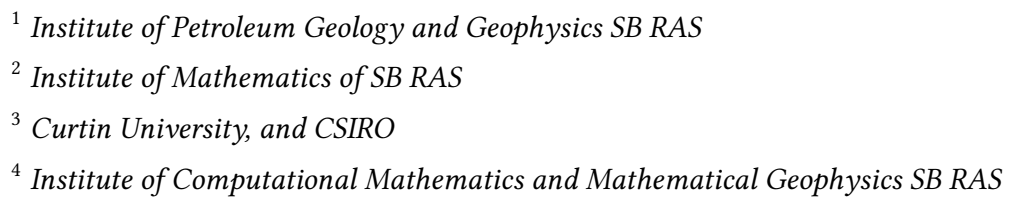

Corresponding Author(s): khachkovats@ipgg.sbras.ru

When evaluating the effective elastic parameters of a rock sample, it is essential to determine the appropriate image resolution. On the one hand, for a correct assessment, it is necessary to take into account the microstructure of the core - the surface roughness of the grains and the properties of the cementing material. On the other hand, images with such quality will be either unrepresentative or so large that numerical modeling of static loading tests will require substantial computational resources. 
We present a multi-scale numerical methodology for estimating the effective elastic parameters of a sandstone sample from digital core images that combines the numerical upscaling, computational topology and geostatistics.

Our methodology uses microscopic core images (SEM) to estimate the statistical parameters of rough cementing layers between grains and to calculate the effective elastic parameters of cement distribution for equivalent models with flat grain contacts. Then, a digital model (built on CT-images of medium resolution that preserves the representativeness of the sample) is divided into grains with an estimated thickness of the interlayer by computational topology (persistent homology method), and these layers are filled with effective cement. After that, the effective elastic parameters of the sample are estimated by the averaging method based on the principle of equivalence of elastic deformation energy, where the elastic moduli are determined by computation of potential energy of deformations arising in the model when homogeneous strains are applied to the boundary (simulating of the laboratory experiment). To solve the 3-dimensional static problem of the elasticity theory the iterative relaxation method is used.

Procter and Gamble Student poster award:

References:

Acceptance of Terms and Conditions:

Click here to agree

\title{
Effective Stress and Energetics of Unsaturated Soils
}

\author{
Nasser Khalili ${ }^{1}$ \\ ${ }^{1}$ School of Civil and Environmental Engineering, UNSW, Sydney 2052, Australia
}

Corresponding Author(s): n.khalili@unsw.edu.au

Tremendous contributions have been made to the mechanics of unsaturated soils in the past decade using the effective stress principle, including models for shear strength, volume change, elastoplasticity, coupled processes (thermo-hydraulic-mechanical), dynamic analysis, earth pressure, retaining wall, in situ tests interpretation, etc. The advantages of the effective stress is that i) the response of the soil to changes in total stress, pore water pressure and pore air pressure can be related to a single stress variable, ii) a single unified framework can be utilised for both saturated and unsaturated soil's behaviour, iii) the basic model parameters are reduced to exactly those used for saturated soils, except for two entities which can be determined in any soil physics laboratory, iv) complex and seemingly unrelated characteristics of unsaturated soils can be explained in a simple and unified manner. In this work, the effective stress expression for unsaturated soils is derived based on energetic equations of the system. The form of the effective stress is extracted based on the stress component that is conjugate to strain of the solid skeleton. It is shown that the deviation of the effective stress parameter from the degree of saturation is due to the rate of change on the specific area of air-water interface with degree of saturation. Validation of the expression is examined based on data from x-ray tomography of granular material taken at different degrees of saturation. It also shown that if effective stress parameter is correctly defined there may not be a need for separate consideration of the air-water interface as a separate variable.

\section{Procter and Gamble Student poster award:}

I don't want to compete References:

Acceptance of Terms and Conditions:

Click here to agree 


\title{
Performance modeling of Li-ion batteries using multiphysics-based pore network modeling
}

Jeff Gostick ${ }^{1}$; Zohaib Khan ${ }^{1}$; Ali Elkamel ${ }^{1}$; Pablo-Angel Garcia-Salaberri ${ }^{2}$; Rhodri Jervis ${ }^{3}$; Paul Shearing ${ }^{3}$; Dan Brett $^{3}$

\author{
${ }^{1}$ University of Waterloo \\ ${ }^{2}$ Universidad Carlos III de Madrid \\ ${ }^{3}$ University College London
}

Corresponding Author(s): zatiqkhan@uwaterloo.ca

Pore network modeling offers the potential to simulate the performance of electrochemical batteries, considering the entire device with pore-scale resolution at feasible computational cost. This work presents the application of pore network modeling to Li-ion battery electrodes, which present several interesting challenges. Cathode materials in lithium ion batteries consists of multiple phases including active material, carbonaceous binder, and electrolyte filled pores. Modern X-ray tomography and image processing is able to distinguish these phases in 3D tomograms. Applying pore-network modeling to these structures requires interpreting each of the phases as a unique pore network, and including the interactions between them all. For instance, the presence of carbon binder phase not only reduces the SEI layer between active material and electrolyte phase but also influences tortuosity and effective electronic conductivity in the pore and solid phase respectively. The present work extends the pore network modelling framework OpenPNM to handle 3 (or more) interpenetrating networks, then investigates the effect of carbon binder phase on Lithium Ion battery cathode. NMC811 cathode material is used as the test case, electrochemical performance with and without binder phase is simulated. Unlike previous models which account generally consider the carbon binder to have high electrical conductivity, we study the impact of binder conductivity on performance. The developed pore network model opens a new avenue for modelling complex electrochemical systems with less computational cost, enabling simulation of large electrode domains while considering structural heterogeneities and features of material in the computation. This approach is essential for designing electrode structures with optimized performance, capacity and durability.

Procter and Gamble Student poster award:

I don't want to compete References:

Acceptance of Terms and Conditions:

Click here to agree

\section{Fluid-solid interaction during particle aggregates drying}

\author{
Author(s): Abdolreza Kharaghani ${ }^{1}$; Son Thai Pham ${ }^{2}$ \\ Co-author(s): Bruno Chareyre ${ }^{3}$; Evangelos Tsotsas ${ }^{1}$ \\ ${ }^{1}$ Otto von Guericke University \\ ${ }^{2}$ Thermal Process Engineering, Otto von Guericke University Magdeburg \\ ${ }^{3}$ Univ Grenoble Alpes
}

Corresponding Author(s): abdolreza.kharaghani@ovgu.de, bchareyre@gmail.com

Spatial distributions of liquid and/or temperature during drying of particle aggregates may trigger undesired structural changes. In this work, a discrete pore-scale model is developed to study the influence of liquid phase distribution on deformation and damage of the aggregates during drying. The solid phase is represented by aggregates with different spatial and size distributions of primary particles that are bonded together at their contact points. The mechanical behavior of the aggregates is simulated by discrete element method. Liquid phase distributions are obtained from isothermal drying simulations with a complementary pore network. In a one-way coupling, capillary forces that are computed from the filling state of pores are applied as loads on a particle-particle basis. Bonds 
may break, resulting in micro cracks, if the load exceeds bond strength. Shrinkage can be modeled but is not yet accounted for in the complementary pore network. Simulations are conducted for aggregates with different mechanical properties. For stiff aggregates, formation of micro cracks is dominant. For soft aggregates, however, reversible shrinkage with no cracks is observed.

Procter and Gamble Student poster award:

I would like to compete in the Procter and Gamble Student award References:

Acceptance of Terms and Conditions:

Click here to agree

199

\title{
3D simulation of flow and active-passive tracer propagation in Voronoi tessellation-based fractured geometries
}

\author{
Siarhei Khirevich $^{1}$; Tadeusz Patzek ${ }^{1}$ \\ ${ }^{1}$ King Abdullah University of Science and Technology
}

Corresponding Author(s): khirevich@gmail.com

Tracer tests are a powerful tool for estimating various properties of geological formations. The idea behind these tests is i) to inject at least two chemical tracers into existing natural or artificial subsurface flow, and ii) to analyze exit times of tracers. These exit times can differ due to the differential thermal, biological, chemical and/or physical interactions of each tracer with the formation. Importantly, tracer tests are used by the oil industry to estimate oil saturation (Sor) remaining after waterflood. The reservoir rock may be saturated with immobile water and oil, and use of tracers with different solubility in water and oil phases provides information about remaining oil saturation.

In this work, we present a geometrical approach to fracturing a rock volume in three dimensions and simulating flow and tracer transport in the generated geometry. We vary geometrical properties of the generated fracture systems (e.g., porosity and heterogeneity) and address their impact on the oil saturation estimated from tracer injection tests. Due to high computational demand, the proposed simulation approach has been implemented for a highly scalable massively parallel execution.

Fracture generation includes i) distributing seed points in 3D, ii) calculating 3D polyhedra that surround each seed known as Voronoi tessellation, and iii) creating planar fractures after shrinking each polyhedron towards its center and releasing void space around it. The interior of each polyhedron is rock "matrix," while the space around it is "fractures". For each matrix-fracture geometry, we calculate a stationary flow field in the fractures with the lattice-Boltzmann method and then use the random-walk particle-tracking method (RWPT) to simulate the tracers. RWPT simulates the motion of a large number of tracer particles due to advection (in fractures) and diffusion (in fractures and matrix). Tracers can penetrate the complex matrix-fracture interface with some probability, which depends on the matrix porosity, oil saturation, and tracer-partitioning coefficient.

We have created a large a set of discrete fracture networks in 3D. By varying fracture porosity and heterogeneity (via spatial distribution of seed points), we addressed the impact of geometrical properties of fracture networks on the transport of active and passive tracers. This allowed us to estimate oil saturation in the generated geometries and compare it with the input known values of Sor. The efficient parallelized code scales to $10000+$ CPU cores on the KAUST Shaheen II supercomputer.

Procter and Gamble Student poster award:

References:

Acceptance of Terms and Conditions:

Click here to agree 


\title{
Analysis of numerical error in lattice-Boltzmann simulations of Stokes flow in porous media
}

\author{
Siarhei Khirevich ${ }^{1}$; Tadeusz Patzek ${ }^{1}$ \\ ${ }^{1}$ King Abdullah University of Science and Technology \\ Corresponding Author(s): khirevich@gmail.com
}

We perform Stokes flow simulations in random and regular (fcc, bcc, sc) sphere packings with the lattice Boltzmann method (LBM), considering packings of (non-)overlapping spheres over wide porosity range, $0.26-0.8$. We employ a two-relaxation-times (TRT) version of the lattice-Boltzmann method, as a most suitable choice for Stokes simulations. Use of a standard bounce-back boundary condition with some LBM implementations can result in viscosity-dependency of the simulation results (such as permeability). In TRT LBM this issue is resolved when a special combination of two relaxation parameters is fixed. This combination is known as "magic number" (L) and is a powerful tool in controlling simulation accuracy of TRT LBM simulations. In our study, we analyze the impact of $\mathrm{L}$ on numerical error convergence.

After defining a sphere packing via coordinates and radii of spheres, we map this packing on a discrete uniform cubic mesh. This approach seems to be simple, but its standard implementation is known to produce significant data scatter in resolution studies, especially in the case of regular (ordered) packings and low discretization resolutions. We use of an improved discretization approach (doi: $10.1016 /$ j.jcp.2014.10.038) which allowed us to significantly reduce this scatter and to observe smooth convergence behavior for practically any resolution.

Smooth convergence curves of numerical error demonstrate complex behavior, but still some similarity is found for all considered geometries - regular and irregular packings of (non-)overlapping spheres of various porosities. We provide a simple mathematical model, which captures key features of the error convergence curves and then suggest an extrapolation approach for error behavior as resolution goes to infinity. This approach and appropriate selection of the magic number resulted in significant reduction of numerical error, while using the most basic LBM scheme with bounce-back rule, demonstrating accuracies comparable with the higher-order boundary schemes (doi: 10.1063/1.5042229).

Procter and Gamble Student poster award:

References:

Acceptance of Terms and Conditions:

Click here to agree

\section{The effect of kinetics on the efficiency of biologically induced car- bonate precipitation via urea hydrolysis for soil improvement ap- plications}

Hamed Khodadadi Tirkolaei ${ }^{1}$; Leon van Paassen ${ }^{1}$; Edward Kavazanjian ${ }^{1}$

${ }^{1}$ Arizona State University

Corresponding Author(s): hkhodada@asu.edu

Microbially and enzyme induced carbonate precipitation (MICP and EICP) via urea hydrolysis are emerging biological soil improvement techniques. In these techniques, a treatment solution including calcium chloride $(\mathrm{CaCl} 2)$, urea, and either urease-producing bacteria or free urease enzyme are introduced into soil to precipitate calcium carbonate $(\mathrm{CaCO} 3)$ and modify the mechanical properties 
of the soil. Both chemical efficiency and mechanical efficiency affect the feasibility, cost, and environmental impact of these techniques. Chemical efficiency is defined as the percentage of substrates converted to the desired product. Mechanical efficiency describes the amount of improvement in the targeted mechanical property as a function of the amount (percent by dry weight) of precipitated $\mathrm{CaCO}$. To assess the efficiency of these techniques, the kinetics of urea hydrolysis and $\mathrm{CaCO} 3$ precipitation need to be considered. This study evaluates how the kinetics of these techniques affect both chemical and mechanical efficiency.

Chemical efficiency was evaluated through a systematic experimental design using different concentrations of substrate $(\mathrm{CaCl} 2$ and urea) and urease (bacterial cell or free enzyme). It was observed that chemical efficiency drastically drops when substrate concentration exceeds a specific value that is a function of the initial urease concentration. It was also demonstrated by these experiments and by experiments conducted by others that chemical efficiency of a microbial urease treatment solution is affected by the presence of seawater and by insufficient nutrient and air for microbial growth. Based upon these observations, a numerical model was developed to predict the effects of initial urease content, degradation and encapsulation of the cells or enzymes, $\mathrm{pH}$, temperature, urea, and calcium concentration on the rate of hydrolysis and precipitation. Model predictions are in good agreement with the experimental results.

Mechanical efficiency is typically characterized by empirical correlations relating strength and stiffness to $\mathrm{CaCO} 3$ content. It is difficult to distinguish the effect of the reaction rate on these empirical correlations as the mechanical properties of the improved soil also depend on many other factors, including density, grain size distribution, particle shape and saturation during treatment. However, experiments have been performed to evaluate how reaction rate influences treatment uniformity and crystal morphology, both of which have an influence upon mechanical properties. Uniform treatment requires uniform distribution of urease, substrate, and nuclei in the pores, which depends on the employed treatment method (e.g. injection, soaking, percolation, mix-and-compact) and the recipe for (i.e., the constituents concentrations of) the treatment solution. MICP at a low hydrolysis rate, which occurs at low initial cell concentration and low temperature, prolongs the induction time, delays the onset of precipitation, and reduces the number of crystals. For microbial solutions with high initial cell concentration and high $\mathrm{CaCl} 2$ concentration, the solubility product of $\mathrm{CaCO} 3$ is exceeded within a short period and the supersaturation of carbonate ions remains high for extended periods, resulting in prolonged nucleation time and consequently extended growth of non-stable $\mathrm{CaCO} 3$, which may adversely affect strength and durability. The effect of calcite seeds and the presence of seawater on morphology were also investigated. It was observed that presence of calcite seeds lowers the carbonate supersaturation level, facilitating calcite crystal formation. The presence of seawater was found to inhibit calcite crystal growth.

Procter and Gamble Student poster award:

References:

Acceptance of Terms and Conditions:

Click here to agree

\title{
Pulsed waterflood performance in oil reservoirs: a pore-scale vi- sualization experiment
}

\author{
Hossein Khorshidian $^{1}$; Hossein Hejazi ${ }^{1}$ \\ ${ }^{1}$ Department of Chemical and Petroleum Engineering
}

Corresponding Author(s): hossein.khorshidian@ucalgary.ca

Many oil reservoirs are waterflooded for maintaining an economic rate of production. The injected water may contain polymers and surface-active materials for mobility control and residual oil reduction. A waterflood operation can be affected by the presence of different scales of heterogeneities in a reservoir. At the pore-scale, water may bypass oil depending on the wettability state of the rock surface and the balance between viscous and capillary forces. Large-scale heterogeneities, such as high permeable zones and fractures, can create short paths for a rapid breakthrough of displacing fluids. Watered out reservoirs typically have an uneconomical rate of oil production due to a high water-oil ratio. Herein, the effect of pulsations, which are introduced to the injection rate, on the 
sweep performance of waterflood is investigated. A viscous oil is displaced by water in an oil-wet micromodel containing pore-level heterogeneities in the form of high permeability pathways embedded in a low perm matrix. In the drainage process, water tends to displace oil through larger paths that provide both less resistance to flow and lower capillary pressures. The advancing fingers of water, driven by the medium heterogeneity and/or viscosity contrast, bypass large zones of oil. Hence, in the waterflood process, a transition zone is formed where the water saturation gradually decreases from the trailing front to the leading end of advanced water fingers. In the pulsed waterflooding process, we reverse the direction of fluid flow and introduce variable injection rates, i.e. predefined functions for flowrates and switching frequency. Similar to the forward flow, most of the oil reversal flow occurs through larger pathways, especially when the backward flow rate is higher than that of a forward step. During the reversal flow period, the leading zones of the waterfront are discontinued by oil. The isolated water contributes to a local blockage of large pathways which consequently attenuates the growth of water fingers in the displacement front. In the reverse flow mode, most of the oil back-flow is in the leading zones of the water-front compared to the trailing zones which is at a farther distance to the outlet. In the forward flow, the oil displacement is more effective when the rate of waterflood is low due to the domination of capillary forces to viscous forces. Our experimental results reveal that the waterflood performance is significantly improved with a low forward and high backward flow rates. The period and amplitude of the flow rate swings are designed based on the feasibility of the backward flow to maintain an optimum oil production rate. Although increasing the frequency, i.e. reducing the interval time between forward and backward pulses, can improve the sweep performance by redirecting of waterfront to the bypassed zones, the time periods should be sufficiently long to sweep a certain number of pores by the flow of water and oil at each step of the pulsation.

Procter and Gamble Student poster award:

References:

Acceptance of Terms and Conditions:

Click here to agree

\title{
Experimental and theoretical study of formation of filtration struc- tures in swelling clays
}

\author{
Maxim Khramchenkov ${ }^{1}$; Eduard Khramchenkov ${ }^{1}$; Rustem Usmanov ${ }^{1}$ \\ ${ }^{1}$ Kazan Federal University
}

Corresponding Author(s): mkhramch@gmail.com

Modeling and experimental studies of flow in swelling porous media is a traditional research subject for various branches of such disciplines as soil physics, rock mechanics, oil and gas reservoir mechanics, and biomechanics. The reason of this interest is that flow in swelling porous media plays an important role in moisture transfer in the most fertile soil types and the formation of their water retaining properties. In this case, one has to deal with change of structure of pore space due to swelling, which enhances development of instability of a saturation front due to change of shapes and sizes of particles in the porous medium. Instability of a saturation front is also present during water displacement in low permeable reservoirs due to to partial swelling of a mineral phase. This process also plays important role in evolution of edema and hematoma in medical practice. Modeling of processes in swelling porous media requires adequate description language that reflects the complex multi-scale structure of the object of study. Development of such a language involves taking into account peculiarities of clay rocks evolution and issues of clay mechanics. It also requires taking into account interrelation between microscopic features of clay minerals and macroscopic description reflecting these features. Problems of modeling of flow in several interconnected porous systems should be considered as well. Heat and mass transfer in swelling clay is substantially non-linear which is reflected in corresponding differential equations. As a prototype model we use approach that leads to description of the process by Fisher's equation (also known as KolmogorovPetrovsky-Piskunov equation) 1 .

We propose mathematical model of flow in swelling porous media that uses equation of mass balance of fluid which differs from the equation used in classical models 2 . The necessity of usage of 
more complex equation is connected with formation of quasi-stable water films in clay microaggregates during the swelling. These films have, in particular, a density other than ordinary gravitational water, which must be taken into account when developing the mass balance equation. The model implement various variants of peaking flow regimes and describe the formation of long-existing zones with a modified flow structure. A series of experiments on flow in swelling porous media was performed using custom experimental setup. Experimental data was used to verify the validity of the developed mathematical model and the confirmation of formation of structures in filtration flows in accordance with the Fisher-KPP equation solution's properties.

Procter and Gamble Student poster award:

I don't want to compete References:

1. Krinari G.A., Khramchenkov M.G., Rakhmatulina Yu.Sh. Mechanisms of Inverse Transformations of Secondary Micas: Evidence from Changes in the Illite-Smectite Structure // Doklady Akademii Nauk, 2013, Vol. 452, No. 4, pp. 431-437. 2. Khramchenkov E., Khramchenkov M. Modeling of non-equilibrium mass-exchange processes in geo-systems // International Journal of Rock Mechanics and Mining Sciences, 2016 (86), pp. 1 -4.

Acceptance of Terms and Conditions:

Click here to agree

862

\title{
Stability and transport behavior of DNA-tagged silica particle trac- ers in undisturbed laboratory soil column experiments
}

\author{
Author(s): Bahareh Kianfar ${ }^{1}$ \\ Co-author(s): Jan Willem Foppen ${ }^{2}$; Bas Van der Zaan ${ }^{3}$; Joachim Rozemeijer ${ }^{3}$; Thom Bogaard ${ }^{1}$ \\ ${ }^{1}$ TU Delft, Civil Engineering and Geosciences, Water management \\ ${ }^{2}$ UNESCO-IHE, Institute for Water Education \\ ${ }^{3}$ Deltares
}

Corresponding Author(s):

Recently, DNA-tagged particles have been developed and applied as a tracer in environmental studies (1-3). This technique is a promising approach to investigate water and contaminant transport in subsurface and surface waters. The advantage of the DNA-tagged particle tracer technique over conventional hydrological tracers is in facilitating the fabrication of a large number of identical, but distinguishable tracers. These tracers can be tagged with different synthetic DNA sequences and do not have natural background concentrations. In addition, this approach enables us to conduct multipoint and multiplexing tracer experiments without convoluting the signal of the new test with previous ones $(1,2,4)$. As a result, a more enhanced insight about temporal and spatial variation in water and contaminant pathways in hydrological systems can be obtained (1).

In this study we tested the applicability of the DNA-tagged particles (5) in a coarse-sand and in a clayey fine sand column, each of $30 \mathrm{~cm}$ height under saturated conditions. The columns were excavated from two agricultural fields, one in the Pleistocene East and one in the Holocene West part of the Netherlands. The hypothesis was that the effect of grain size on the transport of the DNA-tagged tracer particles was negligible. The underlying assumption was the presence of dual porosity with interporosity flow or preferential flow in the clayey fine sand column. The other objective was to test the applicability of the DNA-tagged particles tracers in undisturbed columns. Various colloid transport models were fitted with measured breakthrough curves in order to identify typical transport parameters that described the data best.

Procter and Gamble Student poster award:

I would like to compete in the Procter and Gamble Student award References: 
1. Dahlke HE, Williamson AG, Georgakakos C, Leung S, Sharma AN, Lyon SW, et al. Using concurrent DNA tracer injections to infer glacial flow pathways. Hydrol Process. Wiley Online Library; 2015;29(25):5257-74.

2. Sharma AN, Luo D, Walter MT. Hydrological tracers using nanobiotechnology: proof of concept. Environ Sci Technol. ACS Publications; 2012;46(16):8928-36.

3. Mikutis G, Deuber CA, Schmid L, Kittilä A, Lobsiger N, Puddu M, et al. Silica-Encapsulated DNABased Tracers for Aquifer Characterization. Environ Sci Technol. ACS Publications; 2018;52(21):1214252.

4. Liao R, Yang P, Wu W, Luo D, Yang D. A DNA tracer system for hydrological environment investigations. Environ Sci Technol. ACS Publications; 2018;52(4):1695-703.

5. Paunescu D, Puddu M, Soellner JOB, Stoessel PR, Grass RN. Reversible DNA encapsulation in silica to produce ROS-resistant and heat-resistant synthetic DNA'fossils'. Nat Protoc. Nature Publishing Group; 2013;8(12):2440.

Acceptance of Terms and Conditions:

Click here to agree

\title{
Pore-scale Characterization of Biogenic Gas Formation in Porous Media: The Effect of Gas Production Rate
}

\author{
Author(s): Daehyun Kim ${ }^{1}$ \\ Co-author(s): Nariman Mahabadi Mahabad ${ }^{1}$; Jaewon Jang ${ }^{2}$; Leon van Paassen ${ }^{1}$ \\ 1 Arizona State University \\ ${ }^{2}$ Hanyang University
}

Corresponding Author(s): dkim147@asu.edu

Biogenic gas formation via microbially induced denitrification process has been investigated as a potential ground improvement technique towards liquefaction hazard mitigation. During denitrification, microorganisms reduce nitrate to dinitrogen gas and facilitate calcium carbonate precipitation as a by-product under adequate environmental conditions. The formation of dinitrogen gas desaturates soils and allows for potential pore pressures dampening during earthquake events. In addition, precipitation of calcium carbonate can improve the mechanical properties by filling the voids and cementing soil particles. Small changes in gas and mineral fractions within soil system can significantly affect the mechanical properties of soils. This study aims to explore the effect of gas production rate on the spatial distribution of gas in porous media and experimentally characterize the mechanisms. A microfluidic chip simulating a 2D homogeneous porous medium was used to visualize two sets of experiments. $\mathrm{CO} 2$ gas was produced by depressurizing a supersaturated solution at two different production rates. Biogenic gas was produced by denitrifying bacteria. The results show that very fast $\mathrm{CO} 2$ gas production led to nucleation of gas bubbles throughout the entire pore space, however, the lower rate of $\mathrm{CO} 2$ production promoted limited nucleation sites. Biogenic gas production had a single nucleation point and two percolation paths along the sides of the microfluidic chip. The experiment proved that gas production rate significantly affects the nucleation, migration and spatial distribution of produced gas through the porous media.

Procter and Gamble Student poster award:

References:

Acceptance of Terms and Conditions:

Click here to agree 


\title{
Deep learning-based fracture extraction from X-ray computed to- mographic images of fractured porous media
}

\author{
YEJIN KIM ${ }^{1}$; Seong Jun $\mathrm{Ha}^{1}$; Youngjong Sim ${ }^{\text {None }}$; Tae Sup Yun ${ }^{1}$ \\ 1 Yonsei University
}

Corresponding Author(s): yejin_kim@yonsei.ac.kr

\begin{abstract}
The recognition of fracture and its spatial development is vital to understand the fluid-driven fracturing mechanism in hydraulic stimulation. We present how the deep learning-based approach enables accurately extracting the fracture morphology from 3D x-ray computed tomographic images of fractured porous media and the quantitative measures of fracture aperture distribution and roughness. Although a number of fracture detection algorithm have been proposed in the field of computer vision for decades, it is still challenging to directly apply them to images when unavoidable image noises prevail and barely identifiable fractures are developed with low image contrasts. The convolutional neural network based deep learning has recently shown the outstanding performance in 'object detection' as the feature extractor by enhancing the intensity-contrast between the target object and background. We prepared three porous cementitious mortar specimens and each cylindrical specimen was hydraulically stimulated by three fluids of liquid carbon dioxide, water, and oil followed by X-ray computed tomographic imaging. It is virtually observed that the generated fracture from the internal borehole when carbon dioxide is used as a fracturing fluid is thinner and more tortuous than other cases because the less viscous fluid tends to be readily permeated into the porous matrix. We applied the encoder-decoder network for the automated fracture detection for all three image sets. The encoder uses the convolutional neural network $(\mathrm{CNN})$ of ResNet-152 as a feature extractor and the pixel-level detection of fracture is implemented using the concept of deconvolution and skip layer in the decoder. The convolutional neural network is further developed using weights of the ResNet pre-trained by ImageNet datasets (i.e., transfer-learning) because the deep CNN requires an enormous number of labeled data for training without overfitting. Then, we trained the proposed network by using 400 training images, in which visible fractures were manually and accurately detected, and evaluated the performance of proposed methods with representative test images. By the traditional image-processing algorithm, it is inevitable that fracture and void cannot be separated and they form the embossed plane of fracture when fractures cross the pre-existing void which has the similar intensity with fracture in X-ray CT images. Also, pre- or post-processing techniques should be applied in order to improve the image contrast between fracture and background and to eliminate noise. Compared to the typical detection algorithms, the proposed CNN learns the spatially invariant features regardless of location of target objects in the images so that this approach seems suitable for automating detection on the various types of image data with adjacent void or other noise, by accurately separating pre-existing void and induced fracture.
\end{abstract}

Procter and Gamble Student poster award:

References:

Acceptance of Terms and Conditions:

Click here to agree

486

\section{MICP in the Field: continuous injection to reduce permeability and enhance wellbore integrity}

Catherine Kirkland $^{1}$; Randy Hiebert ${ }^{2}$; Robert Hyatt ${ }^{3}$; Jay McCloskey ${ }^{2}$; Jim Kirksey ${ }^{4}$; Alfred B. Cunningham ${ }^{5}$; Robin Gerlach $^{1}$; Lee Spangler ${ }^{6}$; Adrienne Phillips ${ }^{1}$

\footnotetext{
${ }^{1}$ Montana State University

${ }^{2}$ Montana Emergent Technologies

${ }^{3}$ Montana Emergent Technologies
} 


\footnotetext{
${ }^{4}$ Loudon Technical Services, LLC.

${ }^{5}$ Center for Biofilm Engineering

${ }^{6}$ Energy Research Institute
}

Corresponding Author(s): catherine.kirkland@montana.edu

The first of two field demonstrations of microbially induced calcite precipitation (MICP) in a former water injection well in Indiana, USA, was performed in December 2017. Rather than entering the target oil-bearing sandstone, the injected water traveled through a leakage pathway in the wellbore cement to a higher permeability sandstone, or thief zone, located 30-50 feet $(9-15 \mathrm{~m})$ above the target formation. Our study team reduced flow in the leakage pathway using a 3.75 gal $(14.2 \mathrm{~L})$ slickline dump bailer to deliver the MICP-promoting fluids. The well was returned to service with an injection pressure of $700 \mathrm{psi}(4.8 \mathrm{MPa})$ at $4 \mathrm{gpm}(15 \mathrm{~L} / \mathrm{min})$. While this pressure-flow relationship represented a significant improvement, the first field demonstration was not able to restore the historical injection pressure of 1,400 psi $(9.6 \mathrm{MPa})$ at $1 \mathrm{gpm}(3.8 \mathrm{~L} / \mathrm{min})$. A second field demonstration was conducted in September 2018 to complete the sealing of the leakage pathway.

Since the volume of the wellbore cement channel and thief zone had proven to be larger than originally estimated, larger volumes of MICP-promoting fluids and a different delivery method were required for the second field demonstration. First, two strategies were employed to produce more ureolytic microbes. A total of $60 \mathrm{~L}$ of Sporosarcina pasteurii were grown in the lab at MSU, centrifuged, and frozen for resuspension in brine immediately prior to injection. In addition, the mobile laboratory was modified with an array of four 15 gal $(57 \mathrm{~L})$ bioreactors equipped with temperature control, mixing, aeration, and ventilation to cultivate up to 96 gal (363 L) of cultures per day. Second, the bailer delivery system was replaced with a direct injection strategy to convey the MICPpromoting fluids downhole. Multiple 'pulses' of microbes and urea-calcium containing medium were spotted into a string of 1-inch $(2.5 \mathrm{~cm})$ diameter tubing separated by brine 'spacers' and injected continuously at a flowrate of $3.2-1.4 \mathrm{gpm}(12-5.3 \mathrm{~L} / \mathrm{min})$.

Only moderate pressure increases were observed while the resuspended cells were injected, so on the second day freshly grown cells were used. An increase in pressure was observed and, during the third day of injection, the targeted pressure of close to $1400 \mathrm{psi}$ at $1.5 \mathrm{gpm}$ was achieved. Figure 1 shows the flow-pressure ratio recorded during injection in both field demonstrations as a function of the volume of fluids injected. A lower value means that it is more difficult to inject fluids into the formation and suggests that flow paths are restricted. A total of 280 gallons of frozen cell suspension, 156 gallons of fresh cell cultures, 955 gallons of urea - calcium medium, 647 gallons of brine, and 156 gallons of fresh water were injected over the 3 days of pumping.

This study demonstrates that MICP can be employed successfully in large-volume applications where the timeframe for the delivery of reactants is limited. This finding has significant relevance for commercialization of the MICP biotechnology in the oil and gas industry.

Figure 1. The September 2018 field demonstration began with injections of resuspended frozen cells and urea-calcium media. After injecting approximately 280 gallons of the frozen cell suspensions, only a moderate pressure increase and little change in flow - pressure ratio was observed. The flowpressure ratio decreased significantly during the injection of approximately 156 gallons of freshly cultured cells. A total of approximately 955 gallons of urea-calcium media was injected over the 3 days.

Procter and Gamble Student poster award:

\section{References:}

Acceptance of Terms and Conditions:

Click here to agree

\section{Imaging of porosity of concrete hydrates by (Cryo/LMI-) BIB-SEM}

Jop Klaver $^{1}$; Christiane Rößler ${ }^{2}$; Joyce Schmatz ${ }^{1}$; Christian Matthes ${ }^{2}$; Mingze Jiang ${ }^{1}$ 


\footnotetext{
${ }^{1} \mathrm{MaP}-$ Microstructure and Pores $\mathrm{GmbH}$

${ }^{2}$ F.A. Finger Institute for Building Materials Science, Bauhaus-University Weimar
}

Corresponding Author(s): jop@m-a-p.expert

Concrete strength and durability are manifested in the micro- to mesostructure of hydrate phases formed during reaction of Portland cement with water. The main hydrate phase origins from reaction of tricalcium silicate -the main constituent of Portland cement- with water. These hydrate phases are described as calcium-silicate-hydrate (C-S-H), indicating variable stoichiometry. The lack of understanding of the spatial arrangement (meso-structure) of these C-S-H phases hinders innovation in cementitious materials which is needed to reduce their enormous environmental impact. The understanding of spatial arrangement of the micro- to mesostructure, controlling the strength and durability, can be improved by investigating the pore space morphology using high resolution electron microscopy.

Scanning Electron Microscopy (SEM) imaging is used since decades to image the microstructure of cement and concrete. Cement hydrates are very sensitive to damage induced by mechanical polishing and electron bombardment during SEM investigations. This is one reason why the nano structure of cement hydrates is only qualitatively described.

The approach of the current study enables to visualize and quantify the porosity of hydrated tricalcium silicate at different stages of hardening by means of modern sample preparation, imaging and image analysis techniques established for clay materials (Desbois et al., 2009). First of all, by employing Broad Ion Beam (BIB) polishing a relatively large, representative and damage-free cross-section is prepared enabling high resolution image mapping of the microstructure using SEM. To reduce damage by electron bombardment the whole preparation and imaging protocol can be carried out at cryogenic conditions. For assessing the pore connectivity, a crucial parameter for durability evaluation, Liquid Metal Injected (LMI) samples are investigated in the SEM.

These combined methodologies lead to quantitative measures of porosity down to size of few nanometers enabling a better understanding of the changes in mechanical or transport properties at different stages of hardening. The first studies of standard Portland cement (CEM I Würfel 1016-881) indicate that: 1) the visible BIB-SEM porosity is on average $19.7 \%$ which is much lower than found with MIP (52\%); 2) BIB-SEM visible pore sizes follow power law behavior with small pores being most frequent but the largest pores account for most of the pore space; 3) the microstructure is affected by drying cracks and beam damage justifying the need for cryogenic investigations; 4) all BIB-SEM visible porosity is connected for the liquid metal; 5) pore geometry appear to change due to metal injection at high pressures (MIP and LMI).

Procter and Gamble Student poster award:

I don't want to compete References:

Desbois, G., Urai, J.L., Kukla, P.A., 2009. Morphology of the pore space in claystones - evidence from BIB/FIB ion beam sectioning and cryo-SEM observations. eEarth, 4, 15-22. Acceptance of Terms and Conditions:

Click here to agree

\section{On the scalability of MGSR- and CWY-based GMRES variants for the solver of two-phase flow in porous media using MHFEM dis- cretization}

Author(s): Jakub Klinkovský ${ }^{1}$

Co-author(s): Radek Fučík ${ }^{2}$; Tomáš Oberhuber ${ }^{1}$

${ }^{1}$ Department of Mathematics, Faculty of Nuclear Sciences and Physical Engineering, Czech Technical University in Prague

${ }^{2}$ Czech Technical University in Prague

Corresponding Author(s): klinkjak@fffi.cvut.cz 
We present a computational study comparing the parallel scalability of two GMRES variants when applied to the sparse linear systems arising from the MHFEM discretization of two-phase flow in porous media. For this particular problem, convergence of the traditional variant based on the modified Gramm-Schmidt procedure can be achieved only with reorthogonalization (MGSR), which imposes additional computational cost. Alternative approaches based on e.g. Householder transformations require computational cost comparable to the MGSR variant and provide more opportunities for efficient parallel implementation. In this talk, we focus on the variant using the compact WY representation (CWY) for the products of Householder transformations and evaluate the performance of the resulting GMRES variant on contemporary parallel hardware architectures.

\title{
Procter and Gamble Student poster award:
}

I don't want to compete References:

Acceptance of Terms and Conditions:

Click here to agree

\section{Beyond Kozeny Carman}

\author{
Peter Knabner ${ }^{1}$; Andreas Rupp ${ }^{2}$; Nadja Ray ${ }^{3}$; Raphael Schulz ${ }^{4}$ \\ ${ }^{1}$ University Erlangen-Nürnberg Department Mathematics \\ ${ }^{2}$ Friedrich-Alexander University Erlangen-Nürnberg \\ ${ }^{3}$ Friedrich-Alexander Universität Erlangen-Nürnberg \\ ${ }^{4}$ Universität Erlangen-Nürnberg
}

Corresponding Author(s): knabner@am.uni-erlangen.de

An accurate modeling of the permeability and diffusion coefficient is essential for predictive flow and transport modeling. Well-established relations are proposed by Kozeny-Carman for the permeability or by Buckingham, Penman, or Millington-Quirk for the diffusion, [1-3]. They relate the scalar permeability or diffusion coefficient to the porous medium's porosity. In order to capture the porous medium's structure in more detail, further models include fitting parameters, geometric, or shape factors. Some models additionally account for the tortuosity, e.g. via Archie's law.

Contrarily to these approaches, upscaling methods directly enable to calculate the full, potentially anisotropic, effective diffusion tensor without any fitting parameters. As input only the geometric information in terms of a representative elementary volume is needed. To compute the diffusionporosity relations, upplementary cell problems must be solved numerically on this volume and their (flux) solutions must be integrated. In [4] we applied this approach to provide easy to use quantitative diffusion-porosity relations that are based on representative single grain, platy, or blocky soil structures. As a discretization method we used discontinuous Galerkin method on structured grids. To make the relations explicit, interpolation of the obtained data was used. We furthermore compare the obtained diffusion-porosity relations with the well-established relations and also with the well-known Voigt-Reiss or Hashin-Shtrikman bounds. We discuss the ranges of validity and further provide the explicite relations between the diffusion and surface area and comment on role of a tortuosity - porosity relation.

\section{Procter and Gamble Student poster award:}

\section{References:}

1 J. Kozeny. “Über kapillare Leitung des Wassers im Boden.” Sitzungsber Akad. Wiss. Wien 136.2a (2004), pp. 271-306.

2 P. Carman. "Fluid flow through a granular bed." Trans. Inst. Chem. Eng. 15 (1937), pp. 150-167.

3 P. Moldrup, T. Olesen, S. Yoshikawa, T. Komatsu, and D. E. Rolston. "Three-porosity model for predicting the gas diffusion coefficient in undisturbed soil." Soil Science Society of America Journal 68.3 (2004), pp. 750-759.

[4] N. Ray, R. Schulz, A. Rupp, and P. Knabner: "Old and New Approaches Predicting the Diffusion in Porous Media”, Transport in Porous Media, 2018. (N. Ray, R. Schulz, A. Rupp, and P. Knabner: "Past 
and present approaches to calculate hydrodynamic parameters

in evolving porous media”, Preprint Series Angewandte Mathematik 395, ISSN: 2194-5127, 2016.) Acceptance of Terms and Conditions:

Click here to agree

\section{Hepatitis $C$ virus replication within the porous medium of the Endoplasmatic Reticulum}

Markus M. Knodel ${ }^{1}$; Paul Targett-Adams ${ }^{2}$; Alfio Grillo ${ }^{3}$; Eva Herrmann ${ }^{4}$; Gabriel Wittum ${ }^{5}$

${ }^{1}$ Department Mathematik, AM1, FAU Universität Erlangen

${ }^{2}$ Medivir AB, Huddinge, Sweden

${ }^{3}$ Politecnico di Torino

${ }^{4}$ Department Medizin, Universität Frankfurt, Germany

${ }^{5}$ GCSC Universität Frankfurt, Germany

Corresponding Author(s): markus.knodel@math.fau.de

Acute and chronic viral infections are a major challenge to human health and prosperity. The Hepatitis $\mathrm{C}$ virus (HCV) [Mo07] causes chronic liver diseases such as liver carcinoma and is the main reason for liver transplantations in the Western World. The HCV genome (viral RNA - vRNA) replication cycle is a dynamic process occurring in $3 \mathrm{D}$, which is difficult both to capture experimentally and to visualize conceptually. The vRNA replication cycle is strongly related to the Endoplasmatic Re1ticulum (ER), which is an intracellular porous medium. We created nonlinear fully 3D spatiotemporal resolved diffusion-reaction models of the major components of the HCV-vRNA replication cycle, namely the vRNA, the nonstructural viral proteins (NSPs), and a host factor. The processes are described by means of surface partial differential equations (sPDEs) evaluated upon realistically reconstructed ER surfaces [Ju11]. We present qualitative evaluations of the models [Kn17] and parameter estimations for the coefficients of the model, namely the diffusion coefficient estimation of the so-called NS5A protein [Kn18]. 


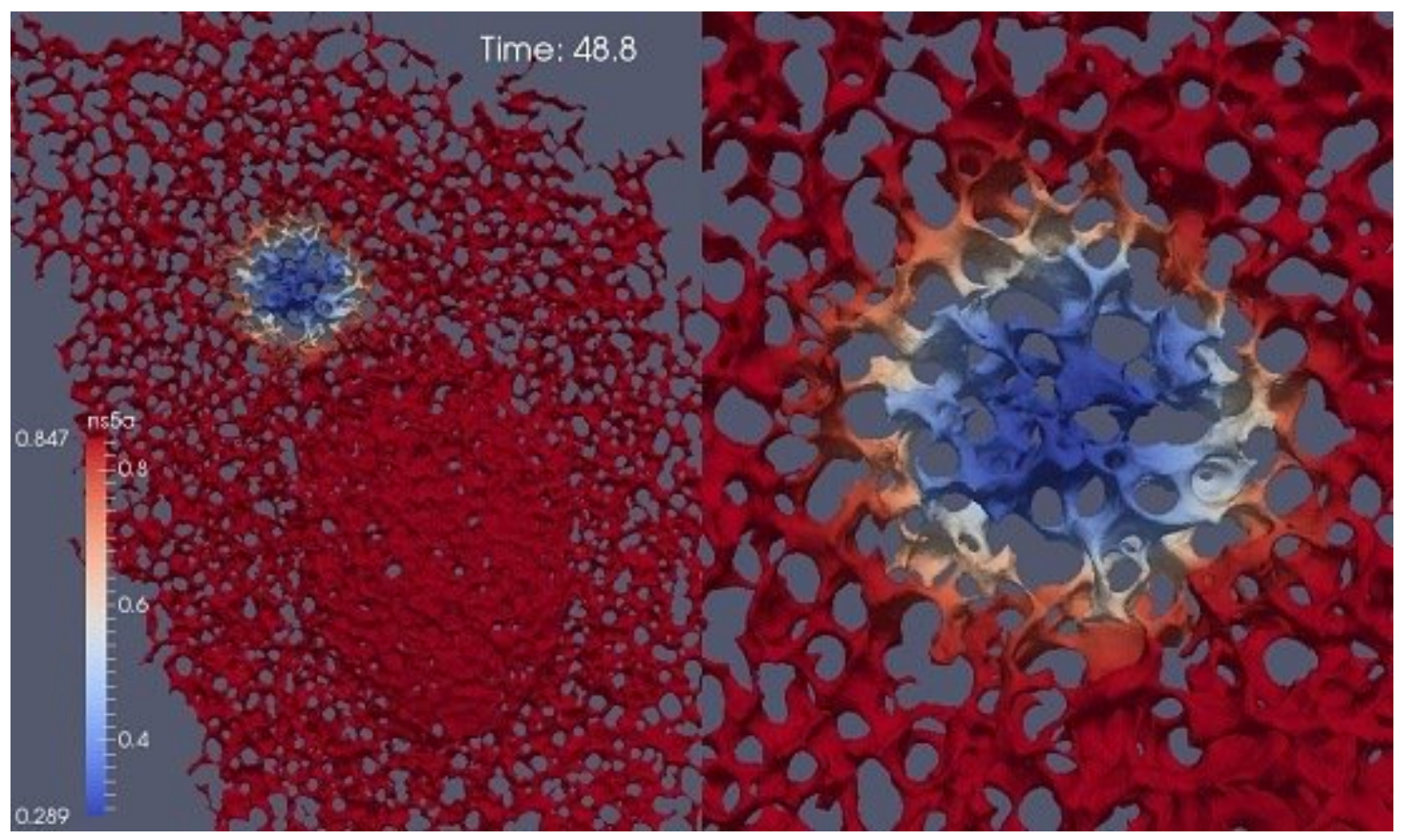

Figure 8: Simulation of a fluorescence experiment of the dynamics of the HCV NS5A viral protein.

The sPDEs are discretized with node-centered Finite Volumes. The linear equation systems arising from the application of the Newton method are solved with massively parallel geometric multigrid solvers. All computations are performed with the open source framework UG4 [Vo13].

Procter and Gamble Student poster award:

\section{References:}

[Mo07] D. Moradpour, F. Penin, and C. M. Rice. Replication of hepatitis c virus. Nat. Rev. Microbiol. 5, 453-463, 2007.

[Ju11] D Jungblut, G Queisser, and G Wittum. Inertia based filtering of high resolution images using a gpu cluster. Comp. Vis. Sci., 14;181-186., 2011.

[Kn17] M.M. Knodel, S. Reiter, P. Targett-Adams, A. Grillo, E. Herrmann, and G. Wittum. 3d spatially resolved models of the intracellular dynamics of the hepatitis c genome replication cycle. Viruses, 9(10):282, Sep 2017.

[Kn18] M. M. Knodel, A. Nägel, S. Reiter, M. Rupp, A. Vogel, P. Targett-Adams, J. McLauchlan, E. Herrmann, and G. Wittum. Quantitative analysis of hepatitis c ns5a viral protein dynamics on the er surface. Viruses 10(1):28, 2018.

[Vo13] A. Vogel, S. Reiter, M. Rupp, A. Nägel, and G. Wittum. Ug 4: A novel flexible software system for simulating pde based models on high performance computers. Comp. Vis. Sci. 16(4) 165-179, 2013. Acceptance of Terms and Conditions:

Click here to agree

\section{Multiphase multicomponent flow in porous media with general reactions}

Markus M. Knodel ${ }^{1}$; Serge Kräutle ${ }^{2}$; Peter Knabner ${ }^{3}$

\footnotetext{
${ }^{1}$ Department Mathematik, AM1, FAU Universität Erlangen
} 
${ }^{2}$ Universität Erlangen-Nürnberg, Department Mathematik

Corresponding Author(s): markus.knodel@math.fau.de

The prediction of $\mathrm{CO}_{2}$ storage behaviour in deep layers asks for the solution of highly nonlinear coupled diffusion-advection-reaction partial differential equations (PDEs). For a detailed description of the multiphase multicomponent flow, we model the interplay of various components not only in the fluid and solid phase, but moreover also in the gaseous phase, i.e., also the gaseous phase consists of various components. The Darcy velocity is a major characteristic of the gaseous as well as the fluid phase. Since the PDEs also include equilibrium and kinetic reactions, advanced solution techniques are required. We apply the reduction scheme of Kräutle and Knabner [Kr05, Kr07, Ho10, Br15] which performs specific linear combinations of equations in order to isolate each equilibrium reaction rate in one single transformed equation. Afterwards, these equations can be dropped. Finally, the equilibrium equations are used to eliminate certain variables by defining a resolution function, which further reduces the size of the system. The advantage of the method of Kräutle and Knabner compared to other transformation techniques is that in the transformed equations, all transport operators depend linearly on transformed concentration variables after solving and substituting the algebraic equations into the remaining equations. (This avoids the generation of additional nonzero entries in the Jacobian.) Since the final system splits into a global and a local system, we apply a nested semismooth Newton solver [Kr11] to the Finite Element discretized / Finite Volume stabilized PDE system and evaluate the arising linear equation systems with fast massively parallel solvers. In particular, we present the efficient computation of a recent benchmark [Si17] which is highly resolved in space and time.

\section{Indico rendering error}

Could not include image: Cannot read image data. Maybe not an image file?

Due to the similarities of the PDE structure, e.g., in case of petroleum industry applications, our algorithm implemented under the open source code $\mathrm{M}++[\mathrm{Wi10}]$ is highly flexible and applicable to various other problems within porous media.

\section{Procter and Gamble Student poster award:}

\section{References:}

[Kr05] Kräutle, S., Knabner, P.. A new numerical reduction scheme for fully coupled multicomponent transport-reaction problems in porous media, Water Resour. Res., 41, W09414, doi:10.1029/2004WR003624, 2005.

[Kr07] Kräutle, S., Knabner, P.: A reduction scheme for coupled multicomponent transport-reaction problems in porous media: Generalization to problems with heterogeneous equilibrium reactions, Water Resour. Res., 43, W03429, 2007. doi:10.1029/2005WR004465

[Ho10] Hoffmann, J, Kräutle, S, Knabner, P. A parallel global-implicit 2-D solver for reactive transport problems in porous media based on a reduction scheme and its application to the MoMaS benchmark problem. Comput Geosci (2010) 14:421-433 DOI 10.1007/s10596-009-9173-7

[Br15] Brunner F., PhD thesis, 2015, Universität Erlangen, Germany.

[Kr11] Kräutle, S.: The semismooth Newton method for multicomponent reactive transport with minerals, Advances Water Res., 34, 137-151, 2011. doi: 10.1016/j.advwatres.2010.10.004

[Si17] Sin, I, Lagneau, V, De Windt, L, Corvisier, J. 2D simulation of natural gas reservoir by two-phase multicomponent reactive flow and transport -description of a benchmarking exercise. Mathematics and Computers in Simulation Volume 137, July 2017, Pages 431-447.

[Wi10] Wieners, C. A geometric data structure for parallel finite elements and the application to multigrid methods with block smoothing. Computing and Visualization on Science, Vol.13, (2010) 161-175 Acceptance of Terms and Conditions: 


\title{
Pore-scale modeling of catalytic filters for automotive exhaust gas aftertreatment
}

Petr $\operatorname{Koci}^{1}$; Marie Placha ${ }^{1}$; Marek Vaclavik ${ }^{1}$; Martin Sourek ${ }^{1}$; Martin Isoz ${ }^{1}$; Milos Svoboda ${ }^{2}$; Emily Price ${ }^{3}$; David Thompsett ${ }^{3}$

\author{
${ }^{1}$ University of Chemistry and Technology, Prague \\ ${ }^{2}$ University of West Bohemia, Pilsen \\ ${ }^{3}$ Johnson Matthey Technology Centre
}

Corresponding Author(s): petr.koci@vscht.cz

Current automotive exhaust gas aftertreatment systems consist of catalytic converters for abatement of gaseous pollutants such as $\mathrm{CO}$, hydrocarbons (HC) and nitrogen oxides (NOx), and a filter trapping the particulate matter (PM). So far only cars with Diesel engines have been equipped with the filter (DPF) but the recently introduced EURO $6 \mathrm{c}$ particulate number limits enforce the use of particulate filters also with gasoline engines (GPF). Both catalyst and filter have the shape of a cylindrical monolith with a large number of parallel channels in a honeycomb arrangement. However, standard catalytic converters are flow-through while the filter channels are alternately plugged at the inlet or outlet so that the exhaust gas is forced to permeate through the porous wall from one channel to another, filtering out the soot. To make the system more compact, the catalytic material can be deposited directly into the filter as on-wall layer or inside the porous wall. The advantages of catalytic filters are space, weight and cost savings, reduction of overall heat-losses and easier soot combustion in the presence of catalyst. On the other hand, the distribution of catalytic material on or in the porous filter walls has to be carefully optimized to meet the opposing requirements of maximum filtration efficiency, high conversion, and minimum pressure drop.

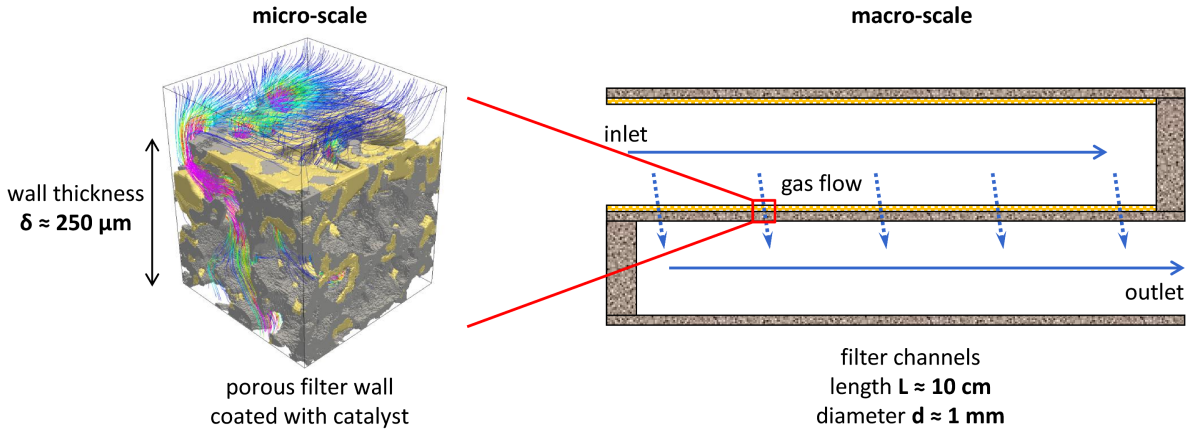

Figure 9: Schematics of catalytic filter for automotive exhaust gas aftertreatment.

In this contribution we introduce a newly developed methodology for the pore-scale simulation of gas flow, diffusion, reaction and soot filtration in the coated catalytic filter. 3D morphology of the porous filter wall including the actual distribution of catalytic material is reconstructed from X-ray tomography (XRT) images and further validated with the mercury intrusion porosimetry (MIP). The reconstructed medium is then transformed into simulation mesh for OpenFOAM. Flow through free pores in the substrate as well as through the coated zones is simulated by porousSimpleFoam solver, while an in-house developed solver is used for component diffusion and catalytic reactions. Transport and filtration of soot particles is calculated with Lagrangian model including Brownian motion. Three filter samples with different distribution of alumina-based coating ranging from in-wall to on-wall are examined. Velocity, pressure and component concentration profiles are calculated enabling the prediction of permeability, component conversion and filtration efficiency depending on the actual microstructure of the wall. The simulation results suggest that the gas predominantly flows through remaining free pores in the filter wall and cracks in the coated layer. The mass transport into the coated domains inside the filter wall is enabled mainly by diffusion. Large domains 
of compact catalytic coating covering complete channel wall result in a significant increase of pressure drop as the local permeability of the coating is two orders of magnitude smaller than that of bare filter wall. The spatially averaged results of pore-scale model are then employed in the entire device model. The predictions are in good agreement with the measured pressure drop, conversion and filtration efficiency. From the studied samples, the most promising structure is the one combining in-wall and partial on-wall coating. The developed models provide useful feedback for further optimization.

\title{
References:
}

Kočí P., Isoz M., Plachá M., Arvajová A., Václavík M., Svoboda M., Price E., Novák V., Thompsett D. 3D reconstruction and pore-scale modeling of coated catalytic filters for automotive exhaust gas aftertreatment. Catalysis Today 320 (2019), 165-174. DOI:10.1016/j.cattod.2017.12.025 ;

Václavík M., Plachá M., Kočí P., Svoboda M., Hotchkiss T., Novák V., Thompsett D. Structure characterisation of catalytic particulate filters for automotive exhaust gas aftertreatment. Materials Characterization 134 (2017), 311-318. DOI:10.1016/j.matchar.2017.11.011 Acceptance of Terms and Conditions:

Click here to agree

\section{Smart \& cost efficient solvers for poroelastic wave propagation}

\author{
Uwe Koecher $^{1}$; Markus Bause ${ }^{1}$ \\ ${ }^{1}$ Helmut-Schmidt-University Hamburg
}

Corresponding Author(s): koecher@hsu-hamburg.de

The accurate high-order approximation in space and time is of fundamental importance for the simulation of dynamic as well as quasi-static poroelastic models which include coupled fluid flow, deformation and wave propagation.

Dynamic poroelastic models appear for example Lithium-ion battery fast-charge simulations and include sharp concentration and pressure gradients, high mechanical stresses, elastic wave propagation, memory-effects on the permeability, multi-phase behaviour and electro-chemical reactions.

In this contribution our high-order space-time discretisations, including cost-efficient enriched Galerkin (eG) space-time finite elements for the variables, our monolithic solver technology, which includes a smart and automatically tuned preconditioning technology, as well as our novel implementation approach for goal-oriented space-time adaptive methods of the transport problem, are presented.

The performance properties and their potential for battery simulations and further applications of the subsurface are illustrated by challenging numerical experiments with physical relevance.

\section{Procter and Gamble Student poster award:}

\section{References:}

1 U. Koecher, M.P. Bruchhaeuser, M. Bause: Efficient and scalable data structures and algorithms for goal-oriented adaptivity of space-time FEM codes. Software X, submitted, p. 1-6, 2018.

2 U. Koecher, M. Bause: A mixed discontinuous-continuous Galerkin time discretisation for Biot's system, submitted, p. 1-19, 2018.

3 U. Koecher: Numerical investigation of the condition number of fully discrete systems from SIPG discretisations for elastic wave propagation. Numer. Math. Adv. Appl. ENUMATH 2017, in print, p. 1-8, 2017. 
[4] J. Both, U. Koecher: Numerical investigation on the fixed-stress splitting scheme for Biot's equations: Optimality of the tuning parameter, Numer. Math. Adv. Appl. ENUMATH 2017, in print, p. 1-8, 2017.

5 M. Bause, F. Radu, U. Koecher: Space-time finite element approximation of the Biot poroelasticity system with iterative coupling, Comput. Meth. Appl. Mech. Engrg. 320:745-768, 2017. Acceptance of Terms and Conditions:

Click here to agree

132

\title{
Hybrid models for simulating blood flow in microvascular net- works
}

Author(s): Tobias Koeppl ${ }^{1}$

Co-author(s): Barbara Wohlmuth ${ }^{2}$; Ettore Vidotto ${ }^{2}$; Rainer Helmig ${ }^{1}$; Timo Koch ${ }^{1}$

${ }^{1}$ University of Stuttgart

${ }^{2}$ Technical University of Munich

Corresponding Author(s): tobias.koeppl@iws.uni-stuttgart.de

In this talk, we present two modeling approaches for simulating blood flow in microvascular networks and the surrounding tissue. Reducing the computational complexity of this issue, the network structures are modeled by a one-dimensional graph, whose location in space is determined by the main axes of the three-dimensional vessels. The surrounding tissue is considered as a homogeneous porous medium and Darcy's equation is used to simulate flow within the tissue. The mass exchange with the blood vessels is accounted for by means of line source terms. On closer examination, this model reduction approach still causes high computational costs, in particular, when larger parts of an organ have to be simulated. This observation motivates the consideration of further model reduction steps. Thereby, we homogenize the fine scale structures of the microvascular networks resulting in a new hybrid approach modeling the fine scale structures as a heterogeneous porous medium and the flow in the larger vessels by one-dimensional flow equations. Both modeling approaches are compared with respect to mass fluxes and averaged pressures. The simulations have been performed on a microvascular network that has been extracted from a rat brain.

Procter and Gamble Student poster award:

References:

Acceptance of Terms and Conditions:

Click here to agree

409

\section{SoilJ - A software for the semi-automatic processing and analyses of X-ray images of soil samples}

\author{
John Koestel ${ }^{1}$ \\ ${ }^{1}$ Swedish University of Agricultural Sciences \\ Corresponding Author(s): john.koestel@slu.se
}

3-dimensional X-ray imaging is in the process of becoming a valuable new standard method in soil science. It allows establishing unequivocal links between soil structure and function. This will result in a better understanding of vadose zone processes and lead to improved simulation models 
and the establishment of more accurate pedotransfer functions. One prerequisite for a widespread and frequent use of X-ray imaging is that the required image processing and analyses time is reduced.

SoilJ aims at achieving this. SoilJ is a plugin for the free and open-source software ImageJ that is optimized for batch-more applications. It includes tools for automatic object recognition, artifact removal and gray-scale calibration, all tailor made for cylindrical soil samples. Moreover, SoilJ hosts routines for various image segmentation approaches, root extraction, mapping of the soil surface topography and morphological analyses. Recent additions have been functions for scale as well as network analyses. For the near future it is planned to integrate routines for MRI and neutron image processing and analyses as well as joint registration approaches for images obtained with different images techniques. In this presentation, the main functionalities of SoilJ are presented, using recent applications to soil pore structure evolution, percolation theory and representative elementary volume analyses.

Procter and Gamble Student poster award:

References:

Koestel, J. 2018. SoilJ: An ImageJ Plugin for the Semiautomatic Processing of Three-Dimensional X-ray Images of Soils. Vadose Zone J. 17. doi:10.2136/vzj2017.03.0062. Acceptance of Terms and Conditions:

Click here to agree

\title{
Quantifying and Analysing Transport Phenomena in Electrospun Electrodes by X-ray Computed Tomography
}

\author{
Author(s): Matthew Kok ${ }^{1}$ \\ Co-author(s): Rhodri Jervis ${ }^{1}$; Dan Brett ${ }^{1}$; Paul Shearing ${ }^{1}$; Jeff Gostick ${ }^{2}$ \\ ${ }^{1}$ University College London \\ ${ }^{2}$ University of Waterloo
}

Corresponding Author(s): m.kok@ucl.ac.uk

The redox flow battery (RFB) is attracting increasing interest as a grid scale energy storage device. The decoupled nature of their power and energy density allows a seemingly infinite amount of energy to be stored regardless of the power density of the cell. At the heart of the RFB is the electrode, responsible for electrolyte distribution in the cell as well as providing the conductive structure for the reaction to take place.

Electrodes made from carbonized electrospun materials offer a unique way of making highly customizable and tuneable electrodes with characteristics more suitable for flow batteries than would be expected from commercially available graphite felts and papers. Recently, X-ray computed tomography has been used to quantify a variety of morphological characteristics of electrospun electrodes, tracing material imperfections and heterogeneities.

Here we present simulations performed on X-ray computed tomographic volumes of electrospun electrodes. These simulations focus on quantifying the various transport properties in convective flow systems, such as the flow battery. Of key interest was analysing the different directional effects of fibre anisotropy on the permeability and mass transfer coefficient tensors. X-ray CT at very high resolution and magnification allows for incredibly detailed and thorough numerical analysis of processes with incredibly small scales, greatly increasing our understanding of these processes. The simulation results are contrasted and compared to experimental and well established data and good agreement was found in all cases. These studies demonstrate the considerable effect X-ray computed tomography coupled with numerical simulations can have on fully understanding the transport properties of complex porous flow systems.

Procter and Gamble Student poster award:

References: 
Kok, Matthew D. R., Rhodri Jervis, Tom G. Tranter, Mohammad A. Sadeghi, Dan J. L. Brett, Paul R. Shearing, and Jeff T. Gostick. "Mass Transfer in Fibrous Media with Varying Anisotropy for Flow Battery Electrodes: Direct Numerical Simulations with 3D X-Ray Computed Tomography." Chemical Engineering Science, October 29, 2018. https://doi.org/10.1016/j.ces.2018.10.049.

Kok Matt D. R., Jervis Rhodri, Brett Dan, Shearing Paul R., and Gostick Jeff T. "Insights into the Effect of Structural Heterogeneity in Carbonized Electrospun Fibrous Mats for Flow Battery Electrodes by X-Ray Tomography." Small 14, no. 9 (March 1, 2018): 1703616. https://doi.org/10.1002/smll.201703616.

Jervis, Rhodri, Matt D. R. Kok, Tobias P. Neville, Quentin Meyer, Leon D. Brown, Francesco Iacoviello, Jeff T. Gostick, Dan J. L. Brett, and Paul R. Shearing. "In Situ Compression and X-Ray Computed Tomography of Flow Battery Electrodes." Journal of Energy Chemistry, April 6, 2018. https://doi.org/10.1016/j.jechem.2018.03.022 Acceptance of Terms and Conditions:

Click here to agree

49

\section{Snap-Off Phenomenon in a Pore Network Model For Retrograde Gas Flow in Porous Media}

Paula Kozlowski Pitombeira Reis ${ }^{1}$; Marcio Carvalho ${ }^{1}$

${ }^{1}$ Pontifícia Universidade Católica do Rio de Janeiro

Corresponding Author(s): paulareis@lmmp.mec.puc-rio.br

Hydrocarbon recovery from retrograde gas reservoirs can be significantly reduced due to condensate blockage. As the dew point pressure of the reservoir fluid is reached, a liquid phase rich in heavy hydrocarbons condenses and builds up in the porous medium. The rise of this liquid phase reduces the availability of flowing paths for the gas phase, thus reducing its mobility. In order to assess the complex flow behavior of gas and condensate in a porous media, a fully-implicit isothermal compositional pore-scale network model for retrograde gas is presented and employed.

The porous medium comprised a 2D regular network of nodes and edges which represented, respectively, its pores and throats. The pore throats are described by capillaries with sinusoidally varying circular cross-sections. In the early stages of liquid phase formation, a thin liquid film is formed attached to the capillary walls. At critical flow conditions, e.g. liquid phase saturation and local capillary number, the converging-diverging feature of the throats leads to the formation and accommodation of stable liquid lenses across the capillary cross-section. The snap-off of the gas phase was implemented in the model by modifying both the gas and the condensate local conductance. This phenomenon can substantially hinder the gas flow, allowing for a better representation of condensate blockage.

After the snap-off occurrence, the condensate lens can be pushed away from the pore throats conceding that a critical pressure drop across the capillary is reached. This critical pressure drop was calculated as a function of the capillary geometry and the interfacial tension of the fluids contained in it. Hence, a multicomponent interfacial tension correlation was also included in the model.

With the model, relative permeability curves were generated and the impact of the snap-off inclusion was appraised. The influence of several parameters in the curves was investigated, such as the medium's pore size distribution, average pressure level throughout the network and pressure drop across the network. Additionally, fluids systems with different compositions were employed in the simulations in order to evaluate how the flow of different hydrocarbon mixtures responds to changes in the investigated parameters.

Procter and Gamble Student poster award:

I would like to compete in the Procter and Gamble Student award References:

Acceptance of Terms and Conditions:

Click here to agree 


\title{
Investigation of time dependent liquid property influence on pa- per wetting and penetration using pico-liter contact angle mea- surement
}

\author{
Author(s): Sarah Krainer ${ }^{1}$ \\ Co-author(s): Ulrich Hirn ${ }^{2}$ \\ ${ }^{1}$ Technical University of Graz \\ ${ }^{2}$ Graz University of Technology
}

Corresponding Author(s): sarah.krainer@tugraz.at

In this study, liquid penetration in the field of High Speed Inkjet (HSI) printing is investigated. In HSI printing, the characterisation of penetration speed and wetting of the ink is important, especially at short timescales $(<100 \mathrm{~ms})$. The investigation of parameters, which are responsible for the absorption behaviour and their impact, is necessary. Therefore, we study the influence of viscosity and surface tension on droplet behaviour during the first few milliseconds of contact with the paper. 1 Furthermore, the change of liquid property impact on drop behaviour over contact time is studied. The development of five model liquids was done using the Ohnesorge - Number and Reynolds Number to guarantee a jet-able fluid. 2, 3 The liquids have varying surface tension and viscosity to study the effect of these properties on penetration and wetting.

Not only the liquid property is affecting penetration behaviour, but also the paper grade significantly influences the penetration process. Therefore, we have tested the performance of model liquids on four different woodfree, uncoated, fine papers from an industrial supplier. These papers are an unsized und untreated paper, unsized HSI paper grade; an HSI unsized pigmented paper and an AKD sized paper.

A pico-liter contact angle measurement device is used for creating droplets in the lower $\mathrm{pl}$ - range. After the drop impinges on the surface of the paper the change of contact angle and change in volume of the drop is measured every $0.5 \mathrm{~ms}$ after contact. These results show that there is a change of main impact factor after longer contact times $(<20 \mathrm{~ms})$ with the paper.

Procter and Gamble Student poster award:

I would like to compete in the Procter and Gamble Student award References:

1 J. Blechschmidt, Taschenbuch der Papiertechnik. Carl Hanser Verlag München, 2013.

2 G. H. McKinley and M. Renardy, "Wolfgang von Ohnesorge," Phys. Fluids, vol. 23, no. 127101, 2011.

3 Y. Guo, H. S. Patanwala, B. Bognet, and A. W. K. Ma, "Inkjet and inkjet-based 3D printing: connecting fluid properties and printing performance," Rapid Prototyp. J., vol. 23, no. 3, pp. 562-576, 2017. Acceptance of Terms and Conditions:

Click here to agree

\section{Iterative coupling methods for multi-permeability poroelasticity models}

\author{
Author(s): Johannes Kraus ${ }^{1}$ \\ Co-author(s): Qingguo Hong $^{2}$; Maria Lymbery ${ }^{1}$; Mary Wheeler ${ }^{3}$ \\ ${ }^{1}$ University of Duisburg-Essen \\ ${ }^{2}$ The Pennsylvania State University \\ ${ }^{3}$ The University of Texas at Austin
}

Corresponding Author(s): johannes.kraus@uni-due.de 
Multiple-network poroelastic theory (MPET) has been introduced into geomechanics 2 to describe mechanical deformation and fluid flow in porous media as a generalization of Biot's theory [3,4]. The deformable elastic matrix is assumed to be permeated by multiple fluid networks of pores and fissures with differing porosity and permeability.

The biological MPET model captures flow across scales and networks in soft tissue and can be used as an embedding platform for more specific models, e.g. to describe water transport in the cerebral environment [7].

To solve the static problem occurring in each time step of a time-stepping algorithm, e.g., the implicit Euler method, one has to solve a coupled multi-field problem. In this talk we focus on flux-based formulations using strongly conservative and parameter-robust stable discretizations [5,6].

We analyze the convergence of iterative coupling methods, such as extensions of the fixed-stress splitting scheme 1 and variants of it and show that they often outperform fully implicit or simultaneous coupling methods while maintaining the stability of the latter ones. The theory is confirmed by numerical results.

This is joint work with Qingguo Hong (The Pennsylvania State University, USA), Maria Lymbery (University of Duisburg-Essen, Germany), Mary Wheeler (The University of Texas at Austin).

\title{
Procter and Gamble Student poster award:
}

\section{References:}

1 T. Almani, K. Kumar, M.F. Wheeler. Convergence and error analysis of fully discrete iterative coupling schemes for coupling flow with geomechanics. Comput. Geosci. 21 (2017), no. 5-6, 1157-1172.

2 G.I. Barenblatt, I.P. Zheltov, I.N. Kochina. Basic concepts in the theory of seepage of homogeneous liquids in fissured rocks [strata]. Journal of Applied Mathematics and Mechanics 24 (1960), no. 5, 12861303.

3 M.A. Biot. General theory of three-dimensional consolidation. J. Appl. Phys. 12 (1941), no. 2, 155-164. [4] M.A. Biot. Theory of elasticity and consolidation for a porous anisotropic solid. J. Appl. Phys. 26 (1955), no. 2, 182-185.

5 Q. Hong, J. Kraus. Parameter-robust stability of classical three-field formulation of Biot's consolidation model. Electronic Transactions on Numerical Analysis 48 (2018), 202-226.

[6] Q. Hong, J. Kraus, M. Lymbery, F. Philo. Conservative discretizations and parameter-robust preconditioners for Biot and multiple-network flux-based poroelastic models. Preprint arXiv:1806.00353, June 9, 2018.

[7] B. Tully, Y. Ventikos. Cerebral water transport using multiple-network poroelastic theory: application to normal pressure hydrocephalus. Journal of Fluid Mechanics 667 (2011), Cambridge University Press, 88-215. Acceptance of Terms and Conditions:

Click here to agree

\section{Influence of Small Scale Geological Heterogeneity on Capillary Trapping of $\mathrm{CO} 2$}

\author{
Prasanna Krishnamurthy ${ }^{1}$; Timothy Meckel $^{1}$; David Dicarlo ${ }^{1}$ \\ ${ }^{1}$ The University of Texas at Austin
}

Corresponding Author(s): prasannagk@utexas.edu

During geologic $\mathrm{CO} 2$ sequestration, most of the storage domain far from the injection sites is likely to be dominated by buoyancy and capillary forces. Under such flow regimes, small scale geological heterogeneities have been shown to dampen plume migration rates and cause trapping beneath capillary barriers. To understand the impact of such heterogeneities on $\mathrm{CO} 2$ trapping processes experimentally, many core-scale and lab scale flow studies have been conducted. Reservoir cores are limited by the scale of investigation possible and most lab experiments are conducted in macroheterogeneous media constructed by arranging homogeneous units to represent heterogeneity. However, most natural sedimentary facies display heterogeneity at a hierarchy of scales, and heterogeneity at 
the mesoscale ( $\mathrm{mm}$ to decimeters) goes unrepresented in laboratory experiments due to the difficulty in reproducibility.

This work presents results from buoyancy driven invasion experiments conducted at the meter scale using glass beads packed in a quasi 2D glass cell and complementary reduced physics simulations. We demonstrate a novel, automated technique to build beadpacks that mimic natural outcrop/rock like features in a reproducible manner. Fluid migration experiments are then conducted at ambient conditions using a surrogate fluid pair that mimics density and viscosity contrasts, and interfacial tension of in-situ reservoir brine and supercritical $\mathrm{CO} 2$. With the ability to generate different types of heterogeneous structures in a reproducible manner, we conduct a systematic investigation of the effect of grain size contrast and facies geometry on flow patterns and trapped saturation. We also comment on early and late time system behavior during invasion and the integrity of capillary trapping post imbibition.

Procter and Gamble Student poster award:

I would like to compete in the Procter and Gamble Student award References:

Acceptance of Terms and Conditions:

Click here to agree

84

\section{Low-Temperature Waste Heat Recovery by Reverse Electrodialy- sis Cells}

Author(s): Kim Roger Kristiansen ${ }^{1}$

Co-author(s): V. Maria Barragan ${ }^{2}$; Signe Kjelstrup ${ }^{3}$

${ }^{1}$ PoreLab, Department of Chemistry, Norwegian University of Science and Technology, NTNU

${ }^{2}$ Department of Structure of Matter, Thermal Physics and Electronics

${ }^{3}$ PoreLab, Department of Chemistry, Norwegian University of Science and Technology

Corresponding Author(s): kim.kristiansen@ntnu.no

Saline power plants exploit the Gibbs energy of mixing of sea- and brackish water. Two technologies are then relevant, reverse electrodialysis (RED) and pressure retarded osmosis (PRO). RED, which has stacked unit cells of alternating cation and anion exchange membranes, is considered to be somewhat better suited in the relevant concentration range 1. The energy efficiency of the concept is low, however. In this work, we investigated the possibility to use abundant low temperature waste heat, to enhance RED technology. We show for the first time that a raise in the temperature of the brackish water above the sea water temperature in the saline power plant, can substantially improve the cell performance of the RED cell. To show this, we measured the thermoelectric contributions to the RED cell potential using FUMASEP cation- and anion exchange membranes. Measurements with a test cell using water samples from the Nidelven river and the fjord near Trondheim, Norway, yielded a Seebeck coefficient of $1.8+-0.1 \mathrm{mV} / \mathrm{K}$ for the unit cell of a RED power plant. The isothermal concentration potential was approximately $150 \mathrm{mV}$ for the same water samples. This means that the thermal driving force gives more than $1 \%$ increase in cell emf per Kelvin of temperature difference. In addition there is a benefit by an increase in the conductivity of the solution with temperature 2, thereby reducing ohmic losses. By reversing the temperature difference, the energy required for water desalination by electrodialysis can be similarly lowered.

The authors are grateful to the Research Council of Norway through its Centres of Excellence funding scheme, project number 262644 PoreLab.

Procter and Gamble Student poster award:

\section{References:}

1 J. W. Post, J. Veerman, H. V. M. Hamelers, G. J. W.

Euverink, S. J. Metz, K. Nymeijer, and C. J. N. Buisman, 
J. Membrane Sci. 288, 218 (2007).

2 A. M. Benneker, T. Rijnaars, R. G. H. Lammertink, and

J. A. Wood, J. Membrane Sci. 548, 421 (2018). Acceptance of Terms and Conditions:

Click here to agree

691

\title{
Assessing pore network model performance for predicting flow and transport in three dimensional porous media
}

\author{
${\text { Quirine } \mathrm{Krol}^{1} \text {; Itzhak Fouxon }}^{1}$; Pascal Corso ${ }^{1}$; Markus Holzner \\ ${ }^{1}$ ETHZ
}

Corresponding Author(s): quirine.krol@protonmail.com

Resolving local velocities for laminar flow in porous media is essential to transport processes. Using high fidelity simulations is computationally costly for statistical representative volumes. Pore network models allow for much faster solutions and resolve much larger domains. However, the pore network architecture and constitutive model for the hydraulic conductivity heavily influence the permeability and the quality of the velocity predictions. Here, we investigate the suitability of network extraction methods and the validity of the constitutive law on the pore and the representative volume scale. We start by generating a range of three dimensional artificial geometries representing porous media. In this way we can control the geometrical parameters, such as the porosity, the averaged interface area and curvature measures. As a benchmark, we use high fidelity numerical simulations of the Navier-Stokes equations to resolve the pressure and velocity field for laminar flow in the pore-space of the artificial geometries. For the pore network model we use the same geometries from which we extract a network using a watershed segmentation algorithm. Subsequently, we solve for the local fluxes by using a Kirchoff model with Poiseuille-Hagen pipe flow as a constitutive law for the local hydraulic conductivity. This enables us to test the validity of the constitutive law by comparing it to the benchmark study. Whereas the Poiseuille-Hagen network model shows promising results for idealized two dimensional geometries, we show that it has limitations in more realistic three dimensional porous media. Lastly, we propose an novel network approach were we couple a pore-segmentation to iso-pressure surfaces obtained from the benchmark study. In this approach a local constitutive law based on energy dissipation is justified from a theoretical standpoint and therefore has the advantage of fewer assumptions over the Poiseuille-Hagen network model. The results of all models and their performance are discussed with respect to the geometrical parameters of the simulated porous media.

Procter and Gamble Student poster award:

I don't want to compete References:

Acceptance of Terms and Conditions:

Click here to agree

142

\section{Robust simulation of mineral precipitation-dissolution problems with non-Lipschitz mineral surface area}

\author{
Serge Kräutle ${ }^{1}$; Peter Knabner ${ }^{2}$ \\ ${ }^{1}$ Universität Erlangen-Nürnberg, Department Mathematik \\ ${ }^{2}$ University Erlangen-Nürnberg Department Mathematics
}

Corresponding Author(s): kraeutle@math.fau.de 
We are considering multi-species reactive transport problems in porous media with mobile (aqueous) and immobile (mineral) species. For the reaction rates we assume the law of mass action. Since reactions with minerals are involved, some specific challenges arise with respect to a) the mathematical model and b) its numerical solution. The main contribution of this talk is to propose a certain substitution which settles these difficulties.

First, for minerals, considered as constant activity species, it has to be distinguished between the two cases of a mineral being present, which allows for dissolution, and the mineral being completely dissolved, which prohibits any further dissolution. In a mathematical model these two cases can be formulated by a so-called complementarity condition. Complementarity formulations are useful both for a kinetic description and for an equilibrium formulation of the mineral reaction, and they guarantee nonnegative solutions. Numerical solution algorithms can be based on the Semismooth Newton method.

Second, often the assumption is made that the reaction rate depends on the size of the reactive surface, which again is related to the mineral volume. Measurements may lead to assumptions such as $A(m) \sim m^{\alpha}$ with $0<\alpha<1$, where $m$ is the mineral volume or mass and $A(m)$ is the surface area. Geometric arguments, e.g., assuming a hemispherical shape of (equi-sized) nuclei, particularly may lead to $A(m) \sim m^{2 / 3}$. However, since this term is not locally Lipschitz continuous, relations of this shape lead to models with non-unique solutions, i.e., to ill-posed problems. Starting from $m=0$ in an oversaturated fluid, the immediate precipitation, that is $m(t)>0$ for $t>0$, but also $m(t)=$ const $=0$ are mathematical solutions of the model. However, only the first one is 'reasonable'.

What happens when we base numerical simulations on such an ill-posed model?

Possible consequences are that numerical results are not reliable or that numerical difficulties (nonconvergence of Newton's method) for concentrations equal to zero or close to zero. To our knowledge, up to now the issue of non-uniqueness - and the numerical difficulties caused by this - are frequently handled for example by forcing $m \geq \epsilon$, for an $\epsilon>0$, or by a regularization of the nonLipschitz term. Both ways are modifications (approximations) of the original model. We propose a different way to deal with the issue, by proposing a simple substitution! This substitution lets the non-Lipschitz terms vanish. In order to also guarantee non-negativity of solutions, we introduce a complementarity formulation. The resulting model picks exactly the physically correct solution and is well-posed, and the solution is non-negative. We think that the substitution is an elegant way to deal with the issue:

- the complexity of the system is not increased at all,

- no model approximation error is introduced,

- we do not have to decide which value to take for $\epsilon$ or the regularization parameter.

We perform some numerical tests for the model without and with the transformation.

For a rigorous proof of existence and uniqueness of a global solution, also in the context of multispecies multi-reaction reactive transport problems (ODEs coupled to PDEs), a formulation using a Heaviside function is useful. (The Heaviside formulation is equivalent to the complementarity formulation.) For models with constant mineral surface area, such proofs are available. The substitution trick allows the extension of existence and uniqueness proofs also on reactive transport with minerals and variable (non-Lipschitz) mineral surface.

\section{Procter and Gamble Student poster award:}

\section{References:}

J. Hoffmann, S. Kräutle, P. Knabner: Existence and uniqueness of a global solution for reactive transport with mineral precipitation-dissolution and aquatic reactions in porous media, SIAM J. Math. Anal., 49, 4812-4837 (2017)

K. Kumar, M. Neuss-Radu, I. Pop: Homogenization of a pore scale model for precipitation and dissolution in porous media, IMA J. Appl. Math., 81, 877-897 (2016)

S. Kräutle, J. Hodai, P. Knabner: Robust simulation of mineral precipitation-dissolution problems with variable mineral surface area (currently in preparation)

M. Saaltink, J. Carrera: Reactive transport models without mathematical solution, Proceedings of the XVI internat. conf. on comput. methods in water resources (CMWR-XVI), Copenhagen, 2006 
S. Kräutle: The semismooth Newton method for multi-component reactive transport with minerals, Advances Water Res., 34, 137-151 (2011) Acceptance of Terms and Conditions:

Click here to agree

\title{
A numerical tool for design and explicit chemical interpretation of low salinity water flooding experiments
}

\author{
Author(s): Kata Kurgyis ${ }^{1}$; Holger Ott ${ }^{2}$ \\ Co-author(s): Bernd Flemisch ${ }^{3}$; Rainer Helmig ${ }^{3}$; Johannes Hommel ${ }^{3}$ \\ ${ }^{1}$ Depratment Petroleum Engineering, Montanuniversitat Leoben \\ ${ }^{2}$ Montanuniversität Leoben \\ ${ }^{3}$ University of Stuttgart
}

Corresponding Author(s): kata.kurgyis@unileoben.ac.at

In the past decades, many studies were conducted on the recovery of crude oil by water flooding both in secondary and tertiary recovery mode. Results implied that incremental oil could be achieved with such potentially low-cost and easy-to-implement techniques as low salinity water flood (LSWF) and alkaline flooding by modifying the ionic composition of the injected water. Low salinity effects are believed to be governed by the combination of several physiochemical processes with a common trait: the contribution to a shift in the wetting state of the reservoir because of reactions between constituents of the injected water and mineral-fluid interfaces.

The purpose of the present study is to provide a numerical capability for designing and interpreting LSW core flood experiments. This is achieved by linking measured relative permeability and capillary pressure saturation functions to salinity with a linear interpolation scheme.

As low saline water enters the pore space, polar hydrocarbon components bound to the rock surface desorb as ions dissolved in the brine adsorb to the mineral surface. The desorption of organic components, and hence, the concentration of adsorbed organic components, is proportional to the concentration of inorganic ions adsorbing from the aqueous phase. By coupling adsorption with the low salinity displacement, the interpolation coefficient is directly set as a function of total adsorbate concentration. The ad/desorption process is described by a set of equilibrium chemical reactions between the dissolved ions and the reactive solid surface resulting in adsorbed monovalent and divalent ions. These chemical reactions are linked to the wetting properties through the total adsorbate concentration.

In the presentation we describe the proposed mechanistic model (based on the work of Kuznetsov et al., 2015) and we discuss a numerical workflow to properly design an experimental program for investigating LSWF. Such a workflow would allow better screen capabilities for field scale LSW applications based on experimental results obtained from well-designed series of special core analyses (SCAL) including both pure aqueous flood experiments with varying salinity and two-phase SCAL displacements. Via single phase brine displacements, adsorption related parameters, such as the adsorption rate of salt ions to the reservoir rock and the concentration of the total reactive surface site in the pore system may be calibrated by monitoring the dissolved salts of the effluent. Steadystate SCAL measurements and Amott tests combined with CT scan provided saturation profiles can be used to assess the threshold conditions (including relative permeability and capillary pressure curves) of the in-situ high saline brine and the designed low saline water. Finally, the potential of the designed LSWF as tertiary recovery (oil bank formation, incremental recovery) could be evaluated through unsteady-state experimental setups.

\section{Procter and Gamble Student poster award:}

I don't want to compete References:

D. Kuznetsov, S. Cotterill, M. A. Giddins, M. J. Blunt: Low-Salinity Waterflood Simulation: Mechanistic and Phenomenological Models, Presented at SPE Asia Pacific Enhanced Oil Recovery Conference, 11-13 August 2015, Kuala Lumpur, Malaysia, SPE-174615-MS Acceptance of Terms and Conditions:

Click here to agree 


\title{
Experimental observations of the spatial structure of the concen- tration field during solute transport in reservoir rocks by Positron Emission Tomography
}

\author{
Author(s): Takeshi Kurotori ${ }^{1}$ \\ Co-author(s): Christopher Zahasky ${ }^{2}$; Sally M. Benson ${ }^{2}$; Ronny Pini ${ }^{1}$ \\ ${ }^{1}$ Imperial College London \\ 2 Stanford University
}

Corresponding Author(s): takeshi.kurotori13@imperial.ac.uk

Due to inherent heterogeneity of geologic porous media, it has been shown that the classical experimental approach for measuring solute dispersion can lead to significant overestimation of core-scale transport properties. The main reason for this is that traditional measuring techniques rely mostly on one-dimensional analysis of tracer flows (i.e. in terms of effluent breakthrough curves) that considers complete mixing of the solute plume in the radial direction. In this study, we deploy a unique combination of classic pulse-tracer tests in heterogeneous porous media with two imaging techniques, namely X-ray Computed Tomography (X-ray CT) and Positron Emission Tomography (PET). These provide in-situ characterisation of rock structure heterogeneity and real-time evolution of the tracer plume in three-dimensions. The validity of this experimental approach for quantitative analyses of solute transport in porous systems has been successfully demonstrated on both synthetic (i.e. beadpacks) and natural materials (i.e. Ketton Limestone, KL) 1.

Here, we extend our previous work to rock systems that present distinct physical features, including Bentheimer Sandstone (BS, uniform pore structure), Edwards Brown carbonate (EB, vuggy porosity) and Indiana Limestone (IL, significant degree of microporosity). Pulse-tracer tests using both brineand radio-tracers have been carried out over a range of Péclet numbers (20-600) and using brine as the carrier fluid. The extent of subcore-scale spreading and mixing has been evaluated directly from the PET images by using various quantitative measures, such as spatial moments, dilution index, scalar dissipation and their temporal variation. Significant differences are observed among the three rocks, with the degree of mixing increasing in the order $\mathrm{BS}<\mathrm{KL}<\mathrm{EB}<\mathrm{IL}$. Notably, when computed using experimental slice-averaged solute mass fractions, these properties become larger than the three-dimensional counterparts computed using voxel-scale values. We attribute this behaviour to the artificial ("numerical") mixing of the solute plume that has been significantly distorted by the presence of subcore-scale permeability heterogeneity. In this context, the difference between these two values are used in this study to assess the extent of solute spreading in heterogeneous porous media.

Procter and Gamble Student poster award:

References:

1 T. Kurotori, C. Zahasky, S. A. Hosseinzadeh Hejazi, S. M. Shah, S. M. Benson, R. Pini. (2018) Measuring, imaging and modelling solute transport in a microporous limestone, Chemical Engineering Science, https://doi.org/10.1016/j.ces.2018.11.001 Acceptance of Terms and Conditions:

Click here to agree

\section{A microfluidic study on biofilm and preferential flow path for- mation in porous media}

Author(s): Dorothee Luise Kurz ${ }^{1}$

Co-author(s): Eleonora Secchi ${ }^{2}$; Vicente Fernandez ${ }^{3}$; Roman Stocker ${ }^{2}$; Joaquin Jimenez-Martinez ${ }^{4}$ 
${ }^{1}$ ETH Zurich
${ }^{2}$ ETH Zürich
${ }^{3}$ ETHZ
${ }^{4}$ EAWAG-ETHZ

Corresponding Author(s): dkurz@ethz.ch

In environmental (bioremediation and ecology) and industrial (chemical engineering) applications, it is of growing importance to understand the interplay between hydrodynamics and biogeochemical processes. The mosaic of regions of high and low flow velocity in porous media permits soil microorganism a free swimming lifestyle and to form surface-attached communities known as biofilms. The growth of biofilms influences pore geometries by clogging them, and thus redirecting the flow, which in turn affects biofilm development and mass transport. Besides clogging of pore spaces, preferential flow paths can be formed which show a dynamic behavior. We study these phenomena with a soil-born microorganism, Bacillus subtilis, in porous media analogs created in microfluidic devices to obtain a mechanistic understanding of the interplay of the hydrodynamics and biofilm development at the microscale.

Experiments were performed in carefully designed porous geometries under different flow rates and pressure gradients. The devices were exposed to a flow of nutrient broth after being seeded with bacteria, and over a period of 48 hours. Biofilm growth was continuously imaged using phase-contrast microscopy.

The rate of biofilm growth is influenced by both hydraulic and geometric parameters of the porous medium. For the same porous geometry, the initiation of biofilm formation and the definition of preferential flow paths occurs earlier in time with increasing flow rate. Above a critical flow rate, this trend is inverted. The preferential paths for fluid flow through the biofilm show an intermittent opening and closing behavior, intermittent bioclogging. Preferential flow paths are characterized by the opening-closing frequency and the channel width on the pore scale. Besides the impact of flow rate and geometry of the porous medium, this intermittent behavior is also controlled by the biofilms' rheological properties, which enables it to accommodate flow and pressure differences. The rheological properties were measured with microrheology techniques to fully understand the observed phenomena. The results shed light on the mechanisms involved in biofilm formation, clogging and its impact on the hydraulic properties of porous media.

Procter and Gamble Student poster award:

References:

Acceptance of Terms and Conditions:

Click here to agree

871

\section{Influence of Porous Structure on Electrokinetic Properties of Poly- mer Membranes}

Christine Körner ${ }^{1}$; Thomas Luxbacher ${ }^{1}$

${ }^{1}$ Anton Paar GmbH

Corresponding Author(s): christine.koerner@anton-paar.com

Electrokinetic investigations such as zeta potential analysis provide a valuable tool for characterization of the interface of any surface in contact with a liquid. The zeta potential is a well-established parameter in the field of particle and solid surface characterization and gives information on the surface charge of a material. However, certain materials, among them electronically and ionically conductive samples, can make zeta potential analysis more complex and some care has to be taken when deducing conclusions based on such tests. In this work, we will elucidate the effect of the porous structure of polymer membranes on zeta potential analysis and will present a method to disentangle contributions from the outer surface and the inner pores of polymer membranes to the zeta potential result.

The zeta potential, as presented in this work, is determined from streaming potential and streaming 
current measurements using a SurPASS ${ }^{\mathrm{TM}} 3$ electrokinetic analyzer and its proprietary measuring cell for membrane analysis. For the analysis, the membrane sample is arranged to create a capillary flow channel between two measuring electrodes. An induced flow of electrolyte solution shears off the charge-compensating ions and gives rise to a streaming potential or streaming current signal, which is transferred into a zeta potential result.

The porous structure of polymer membranes contributes to the zeta potential by its electrical conductance 1. The conventional tangential measurement of the streaming potential thus leads to an apparent zeta potential only, which gets estimated too low. The measurement of streaming current takes into account the additional conductivity effects and improves the quality of zeta potential data. Determining the contributions of the streaming current inside the porous membrane structure and of the membrane body conductance requires the measurement of streaming current and electrical conductance at different gap heights of the flow channel. The proposed method allows for a differentiation of the "external" and "internal" streaming currents and the calculation of the correct zeta potential of the membrane surface 2 .

With this approach the measurement reproducibility is significantly improved thus increasing the reliability of the zeta potential analysis of porous membranes. It has the potential to provide significant insights into key issues in membrane science, including surface modification, ageing and fouling.

Procter and Gamble Student poster award:

I don't want to compete References:

1 Yaroshchuk A, Ribitsch V, Langmuir 2002, 18, 2036-2038.

2 Yaroshchuk A, Luxbacher T, Langmuir 2010, 26, 10882-10889. Acceptance of Terms and Conditions:

Click here to agree

\title{
A Domain Decomposition Projection Method for the Navier-Stokes Equations Based on the Multiscale Robin Coupled Method
}

\author{
Author(s): Camila Lages ${ }^{1}$ \\ Co-author(s): Fabricio Sousa ${ }^{1}$; Roberto Ausas ${ }^{1}$; Gustavo Buscaglia ${ }^{1}$; Felipe Pereira ${ }^{2}$ \\ ${ }^{1}$ University of Sao Paulo \\ ${ }^{2}$ Mathematical Sciences Department, The University of Texas at Dallas, Richardson, TX, USA \\ Corresponding Author(s): camilafalages@gmail.com
}

Different multiscale mixed methods for second order elliptic equations have been developed based on distinct approaches to address the continuity of pressure and velocity fields. We mention, for instance, the Multiscale Mortar Mixed Finite Element Method (MMMFEM) 1 and the Multiscale Hybrid-Mixed Method (MHM) 2, as some well known procedures of this type. In the MMMFEM, the pressure continuity is weakly satisfied in the fine-grid scale, while normal flux continuity is ensured on a much larger scale, usually associated with the size of the subdomains. Conversely, in the MHM, continuity of the normal fluxes is satisfied at the fine grid scale, while pressure continuity is only imposed at the larger scale. More recently the Multiscale Robin Coupled method (MRCM) has been introduced in the literature 3 where it was demonstrated that it can provide more accurate solutions than the existing methodologies.

Solving the Navier-Stokes equations for a large number of unknowns is a difficult task, usually done in parallel, but limited by convergence and speedup issues. We propose a new domain decomposition projection method based on the MRCM (to solve an elliptic equation that arises in this procedure), that allows for the independent computation of local problems, which are finally coupled by the solution of a much smaller interface problem, enforcing continuity in a scale larger than the fine grid scale. Results show that accurate solutions are obtained compared to the undecomposed case. As an application of the method that I am developing I am about to start using it to simulate two-phase flows in the pore scale where the domain for the PDEs is provided by images from CT scanners. 
Procter and Gamble Student poster award:

\title{
References:
}

1 T. Arbogast, G. Pencheva, M.F. Wheeler, and I. Yotov. A multiscale mortar mixed finite element method. SIAM Multiscale Model. Simul., 6(1):319-346, 2007.

2 R. Araya, C. Harder, D. Paredes, and F. Valentin. Multiscale hybrid- mixed method. SIAM J. Numer. Anal., 51(6):30505-3531, 2013.

3 R.T. Guiraldello, R.F. Ausas, F.S. Sousa, F. Pereira, and G.C. Buscaglia. The multiscale Robin coupled method for flows in porous media. J. Comput. Phys., 355:1-21, 2018. Acceptance of Terms and Conditions:

Click here to agree

\section{A relaxation projection analytical-numerical approach in hysteretic two-phase flows in porous media.}

\author{
Wanderson Lambert ${ }^{1}$; Eduardo Abreu $^{2}$; Abel Bustos $^{3}$; Paola Ferraz ${ }^{4}$ \\ ${ }^{1}$ UNIFAL \\ ${ }^{2}$ University of Campinas, Sao Paulo, Brazil \\ ${ }^{3}$ Pontificia Universidad Javeriana - Cali Colombia \\ ${ }^{4}$ University of Campinas (UNICAMP)
}

Corresponding Author(s): wanderson.lambert@gmail.com

Hysteresis phenomenon plays an important role in fluid flow through porous media and exhibits behavior that are often poorly understood and that is lacking of correct physical and mathematical modeling, rigorous mathematical/numerical analysis.

We present the key results obtained in 3, in which we study the hysteresis phenomenon for a twophase flow in 1D. In 3, we model this physical behavior by using a system of two equations with a forcing relaxation term in the equation describing the dynamics of hysteresis. The model is constructed in such way that solution tends to an equilibrium manifold in the phase space composed by the unknowns of the system: saturation of water or oil and the hysteresis parameter. This manifold is composed by three parts: two curves (drainage and imbibition) and an scanning relaxation region between them. On the drainage or imbibition curves, the system of two equations reduces to a single scalar equation for the two-phase flow. In the scanning region, the system of equations modeling the behavior is composed by two equations where the source term vanishes. A complication of this system is that on the equilibrium manifold the flux terms of equations are non-smooth functions. Our analysis encompasses all possible flow situations.

We propose a two-fold approach, by analysis and computing. We introduce a new analytical projection method for construction of the wave sequence in the Riemann problem for the system of equations. This analysis allows us to fully comprehend the interaction of the waves of the system between the relaxation terms and the equilibrium manifold. In 1 and 2 , we introduce a seminal technique that gave origin to the improved technique developed in 3 . We obtain the full Riemann solution and we prove the existence of traveling profiles to select the physical shocks satisfying an extended Oleinik's condition that we define. We stress that there is no classical entropy for this system. Next, we developed a new computational method to corroborate our analysis. This method calculates first the interaction of solution with the source terms, by solving the equation for hysteresis. We solve the saturation system using locally conservative discretizations by combining hybrid mixed finite method with finite volume within an effectively operator splitting formulation. We found that numerical method agrees nicely with the proposed projection technique. The approach can be extended for three-phase systems.

Procter and Gamble Student poster award:

\section{References:}

1 E. Abreu, A. Bustos and W. J. Lambert (2015), Non-monotonic traveling wave and computational solutions for gas dynamics Euler equations with stiff relaxation source terms. Computers \& Mathematics with Applications 70, 2155-2176. 
2 E. Abreu, A. Bustos and W. J. Lambert (2018), Asymptotic Behavior of a Solution of Relaxation system for Flow in Porous Media.

Klingenberg C., Westdickenberg M. (eds) Theory, Numerics and Applications of Hyperbolic Problems I. HYP 2016. Springer Proceedings in Mathematics \& Statistics, 236. Springer.

3 E. Abreu, A. Bustos, P. Ferraz, W. Lambert (2018), A relaxation projection analytical-numerical approach in hysteretic two-phase flows in porous media, under review. Acceptance of Terms and Conditions:

Click here to agree

\title{
Phase diagram of quasi-static immiscible displacement in disor- dered porous media
}

\author{
Tian Lan $^{1}$; Guanju Wei ${ }^{\text {None }}$; Ran $\mathrm{Hu}^{1}$; Yi-Feng $\mathrm{Chen}^{1}$ \\ ${ }^{1}$ Wuhan University
}

Corresponding Author(s): lantiannick@163.com

Immiscible displacement in porous media is an important phenomenon that impacts many natural and industrial processes including water infiltration into soil, the proton exchange membrane fuel cell, fluid mixing in microfluidics, enhanced oil recovery and geological $\mathrm{CO} 2$ sequestration. Under quasi-static conditions where the role of viscous force vanishes, the displacement pattern is controlled by the pore-scale disorder and the pore surface's wettability. Previous studies have gained insightful understanding on the impact of the wettability on the fluid displacement, but the impact of the interplay between wettability and disorder is not well understood. Here, we combine microfluidic experiments, pore-scale simulation and theoretical analysis to show how the pore-scale disorder together with wettability controls the fluid-fluid displacement patterns. Based on statistical analysis of pore-scale filling events, we propose a theoretical model that identifies the stable and unstable flow regimes and the crossover zone between them as functions of pore-scale disorder $\lambda$ and invading fluid contact angle $\theta$. The phase diagram predicted by the theoretical model shows a critical contact angle $\theta$ c below which increasing pore-scale disorder $\lambda$ destabilizes the displacement pattern whereas above which increasing $\lambda$ stabilizes the pattern. The diagram further demonstrates that crossover zone, in terms of the range of contact angle, significantly increases as porous media geometry shifts from uniform $(\lambda=0)$ to disordered $(\lambda>0)$. This diagram is evidenced by our microfluidic experiments and pore-scale simulations, including the quantitative analysis of invasion morphologies, specific fluid-fluid interface length and saturation. This work not only extends the classic phase diagram of multiphase flow in porous media under quasi-static conditions, but also has potential applications in predicting displacement pattern and manipulating porous media geometry in designing membranes, microfluidics etc. for desirable immiscible displacement behavior.

Procter and Gamble Student poster award:

References:

Acceptance of Terms and Conditions:

Click here to agree

\section{Biofilms in porous media: laboratory experiments, mathematical modelling, and upscaling.}

David Landa-Marbán ${ }^{1}$; Florin Adrian Radu ${ }^{2}$; Kundan Kumar $^{1}$; Iuliu Sorin Pop ${ }^{3}$; Liu Na ${ }^{4}$; Bødtker Gunhild ${ }^{4}$; Petterson Per $^{4}$; Vik Bartek Florczyk ${ }^{4}$ 


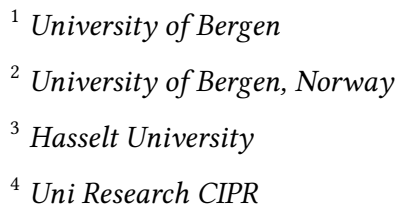

Corresponding Author(s): david.marban@uib.no

\begin{abstract}
A biofilm can be defined as an aggregation of bacteria, algae, fungi, and protozoa enclosed in a matrix consisting of a mixture of polymeric compounds, primarily polysaccharides, generally referred to as extracellular polymeric substance (EPS). Biofilms produce acids, biomass, gases, polymers, solvents, and biosurfactants. Biofilm formation is generally established through three steps: planktonic cell attachment to the surface, formation of a structured architecture with the assistance of EPS in the maturation stage, and cells leaving the biofilm in the dispersal stage. Different environmental factors affect the biofilm, such as temperature and $\mathrm{pH}$. Biofilms are complex systems, involving different physical, chemical, and biological processes such as bacterial decay, endogenous respiration, erosion, sloughing, abrasion, attachment, bacterial reproduction, and formation of metabolites. Biofilms are present in many systems, with beneficial applications in some areas, for example in medicine, food industry, and water quality.
\end{abstract}

In our research, we are interested in studying the biofilm to improve the oil extraction. Most of the biofilm models are based on simplifying assumptions, e.g. impermeability, a constant biofilm density, and accounting for diffusion but neglecting convection for transport of nutrients. In this work, we propose a pore-scale model for a permeable multi-component biofilm including a variable biofilm density, detachment, and transport of nutrients due to convection and diffusion. It is through laboratory experiments that we identify the key processes and variables that need to be considered. Accordingly, we compute some of the parameters (but not all due to the limited experimental observations) of the mathematical model through calibration. In addition, we study the sensitivity of the parameters in our model.

Pore-scale models are important because they aim to describe some of the physical phenomena in detail and one can derive core-scale models through upscaling. Two of the motivations to derive upscaled models are to determine constitutive relationships and to describe the average behaviour of the system in an accurate manner with relatively low computational effort compared to fully detailed calculations starting at the microscale. Then, we upscale this pore-scale model in two different geometries: a thin channel and a thin tube, in order to derive one-dimensional effective equations, by investigating the limit as the ratio of the height to the length approaches to zero.

In the macro-scale laboratory experiments, biofilm is growth in cylindrical cores. Permeability and porosity changes over time at different flow rates and nutrient concentrations are studied. Numerical simulations are performed to compare with the experimental results.

\title{
Procter and Gamble Student poster award:
}

I would like to compete in the Procter and Gamble Student award References:

A pore-scale model for permeable biofilm: numerical simulations and laboratory experiments (arXiv:1807.03400). An upscaled model for permeable biofilm in a thin channel and tube (arXiv:1810.06416). Acceptance of Terms and Conditions:

Click here to agree

\section{Tomographic PIV of low to high Re flow through quadratic and hexagonal arrangements of cylinders}

\author{
Sofia Larsson ${ }^{1}$; Staffan Lundström ${ }^{1}$; Henrik Lycksam ${ }^{1}$; Tobias Forslund ${ }^{1}$ \\ ${ }^{1}$ Fluid Mechanics, Luleå University of Technology, Sweden
}


Corresponding Author(s): sofia.larsson@ltu.se

Pressure-driven flow within the plane of a confined thin porous medium (TPM) takes place in a number of natural and industrial processes. This includes flow during manufacturing of fibre reinforced polymer composites with liquid moulding processes, passive mixing in microfluidic systems and paper making. During last year's Interpore conference in New Orleans we presented tomographic PIV measurements of low to high Reynolds number flows through well-ordered porous media 1. The work described here is a continuation of this project. The TPM considered is a simplified, wellstructured model of a porous media where the solid parts have the shape of vertical cylinders. The array of cylinders is confined between two parallel plates, meaning that the permeability is a function of the diameter and height of the cylinders, as well as their interspatial distance. The flow field is again investigated using tomographic particle image velocimetry. Refractive index matching is applied to enable measurements without optical distortion and a dummy cell is used for the calibration of the measurements. Two different cylinder arrangements are considered, quadratic and hexagonal, and the particle Reynolds number (based on the cylinder diameter and the interstitial velocity) is varied from 10 to 1700 . The pressure drop over the porous domain is measured simultaneously with the velocity. The results reveal that the averaged flow field changes substantially as $\operatorname{Re}$ increases, and under certain conditions the flow contains complex, three-dimensional vortex structures hard to visualize with only planar measurements. Tomographic PIV measurements of flow through models of porous materials, with different types of arrangements, provide a deeper understanding of the flow dynamics. Three-dimensional measurements also enable detailed studies where different general flow phenomena can be coupled to integrated quantities such as permeability and dispersion.

Procter and Gamble Student poster award:

\title{
References:
}

1 Larsson, I.A.S., Lundström, T.S. \& Lycksam, H. Tomographic PIV of flow through ordered thin porous media. Experiments in Fluids (2018) 59: 96. https://doi.org/10.1007/s00348-018-2548-6 Acceptance of Terms and Conditions:

Click here to agree

\section{Upscaling unsteady and inertial single-phase flow in homogeneous porous media}

\author{
Author(s): Didier Lasseux ${ }^{1}$ \\ Co-author(s): Francisco José Valdés-Parada ${ }^{2}$; Fabien Bellet ${ }^{3}$ \\ ${ }^{1}$ CNRS \\ ${ }^{2}$ UAM - Iztapalapa Mexico \\ ${ }^{3}$ CentraleSupélec
}

Corresponding Author(s): didier.lasseux@u-bordeaux.fr

A formal derivation of a macroscopic model for unsteady and inertial one-phase incompressible flow in rigid and periodic porous media is presented using an upscaling technique. The resulting model is non-local in time and involves two effective coefficients in the macroscopic filtration law, namely a dynamic apparent permeability tensor and a vector accounting for the time-decaying influence of the flow initial condition. This model generalizes previous non-local macroscale models restricted to creeping flow conditions. Ancillary closure problems are provided, which allow computing the effective coefficients. Symmetry and positiveness analyses of the apparent permeability are carried out, evidencing that this tensor is symmetric only in the creeping regime. The effective coefficients are functions of time, geometry, macroscopic forcings and the initial flow condition. Predictions of the effective coefficients are made on a simple periodic structure for a wide range of Reynolds numbers smaller than the critical value characterizing the first Hopf bifurcation. Finally, the performance of the macroscopic model for a variety of macroscopic forcing and initial conditions is examined in four case studies along with validation with direct numerical simulations. It is shown 
that the purely heuristic classical model, widely used for unsteady flow, consisting in a Darcy-like model complemented with an accumulation term on the filtration velocity, is inappropriate.

\title{
Procter and Gamble Student poster award:
}

I don't want to compete References:

Acceptance of Terms and Conditions:

Click here to agree

\section{Study of coupled finite volume schemes minimizing the grid ori- entation effect in reservoir simulation.}

\author{
Karine LAURENT ${ }^{1}$; Quang Huy TRAN ${ }^{1}$; Christophe BERTHON $^{2}$; Eric FLAURAUD ${ }^{1}$; Christophe PREUX ${ }^{1}$ \\ ${ }^{1}$ IFP Energies nouvelles \\ ${ }^{2}$ LMFL, Université de Nantes
}

Corresponding Author(s): karine.laurent@ifpen.fr

In this presentation, we propose an analysis method for the so-called grid orientation effect (GOE), which may occur when using coupled finite volumes schemes on structured grids for the simulation of two-phase flow in porous media. This effect is due to an instability on the modelling system and it is all the more visible as the mobility ratio is unfavorable, which is typically the case of the simulation of viscous oil recovery by injection of a more mobile fluid (water, steam,...). The GOE is characterized by a distortion of the solution that follows the axis of the mesh.

This problem is well known and is often studied by the engineers : historically, Yanosik and Mc Cracken [SPE Journal, vol. 19, pp. 253-262, 1979] has introduced a nine-point scheme for Cartesian meshes. This scheme is the result of a linear combination of two five-point schemes for parallel and diagonal grids. Several variants of this scheme were then proposed in the literature (Shah [SPE Reservoir Simulation Symposium, SPE-12251-MS, 1983] and Coats\&Modine [SPE Reservoir Simulation Symposium, SPE-12248-MS, 1983], ...) and more recently Eymard et al. [IMA J. Numer. Anal., vol. 33, pp.585-608, 2013] have developed a generalizable nine-point scheme for meshes that are not necessarily Cartesian. Finally, some other authors (Kozdon [Computational Geosciences, vol. 15, pp. 399-419, 2011],...) have explored multi-dimensional schemes to reduce the GOE. These schemes consist in upwinding the convective variables along the stream lines instead of the grid lines. Nevertheless, these schemes are not satisfactory enough and do not mitigate sufficiently the GOE.

Our contribution will be to study a new nine-point finite volume scheme and a multi-dimensional upwind scheme in order to reduce the GOE. In the nine-point scheme, the diagonal fluxes are constructed from a linear combination of vertical and horizontal fluxes and these fluxes can be coupled to an multi-dimensional upwind method. On those methods, we have worked out an approach to define a notion of angular error for all directions of the flow. It enables us to minimize the longitudinal error and tune an optimal parameter to single out the least anisotropic scheme.

Some numerical test problems testify to the improvement brought by the new construction. A radial test case is performed with an analytical solution and a five-well test case is carried out with two different meshes, where one is rotated from the other by an angle of $45^{\circ}$.

Procter and Gamble Student poster award:

\section{References:}

Acceptance of Terms and Conditions:

Click here to agree 


\title{
Non-linear biogeochemical reactions in chemical gradients
}

\author{
Author(s): Tanguy Le Borgne ${ }^{1}$ \\ Co-author(s): Charlotte Le Traon ${ }^{1}$; Antoine Hubert ${ }^{1}$; Hojjat Borhany ${ }^{2}$ \\ 1 University of Rennes \\ ${ }^{2}$ Université de Rennes 1, École nationale supérieure de chimie de Rennes
}

Corresponding Author(s): tanguy.le-borgne@univ-rennes1.fr

Porous media are natural reactors where fluid, solid and biological phases interact through redox reaction, precipitation, dissolution, adsorption and complexation. While reaction kinetics are often quantified in well mixed batch conditions, most porous media flows are characterized by chemical gradients, induced by broad velocity distributions and complex boundary conditions (Le Borgne et al. 2017, Bandopadhyay et al. 2018, Turuban et al. 2018). Yet, it is unclear how these chemical gradients affect the effective kinetics of reactions taking place between the fluid, solid and biological phases. Here we present new theoretical and numerical results linking the dynamics of mixing in porous media to biogeochemical reaction kinetics.

Considering non-linear reaction rates, including reversible and irreversible redox reactions, Monod kinetics and non-linear adsorption kinetics, we analyze the effect of different mixing dynamics on effective reaction kinetics. We use a stochastic framework in which the distributions of solute concentrations and reaction rates are quantified from Probability Density Functions (PDF). Depending on the form of the relationship between concentrations are reaction rates, mixing can either enhance or reduce significantly effective reaction rates compared to the well-mixed case. We quantify the effective kinetics laws in the different regimes that emerge from the coupling of mixing and nonlinear reactions. These results provide a general framework to understand and quantify a range of biogeochemical processes in chemical gradient environments.

References:

Le Borgne, T., Huck, P. D., Dentz, M., \& Villermaux, E. (2017). Scalar gradients in stirred mixtures and the deconstruction of random fields. Journal of Fluid Mechanics, 812, 578-610.

Turuban, R., Lester, D. R., Le Borgne, T., \& Méheust, Y. (2018). Space-Group Symmetries Generate Chaotic Fluid Advection in Crystalline Granular Media. Physical Review Letters, 120(2), 024501.

Bandopadhyay, A., Davy, P., \& Le Borgne, T (2018) Shear flows accelerate mixing dynamics in hyporheic zones and hillslopes. Geophysical Research Letters, in press

Procter and Gamble Student poster award:

References:

Acceptance of Terms and Conditions:

Click here to agree

\section{Viscoelastic multiphase flow simulations for tumor growth}

Vincent LE MAOUT ${ }^{1}$; Giuseppe Sciumè ${ }^{1}$; Henri Bertin ${ }^{1}$

${ }^{1} \mathrm{I} 2 M$

Corresponding Author(s): vincent.le-maout@u-bordeaux.fr

Recent years have seen a growing interest in the modelling of cancer from a mathematical and physical point of view. In this emerging field, a relevant analogy allows to model tumor cell agglomerates as viscoelastic fluids with interfacial tension 1 . These agglomerates coexist in tumor environment 
with other phases (death cells, living cells, healthy cells, interstitial fluids etc.) inside a biological scaffold, the extracellular matrix (ECM). The dynamic of the cancer is then governed by multiphase flow dynamics in porous media, and fluid-solid interaction can be described via extensions of Biot's theory. Governing equations are often formulated directly at the macroscale, or obtained by upscaling of microscale conservation equations. This second option is the most suitable because it allows to properly define the connection between micro- and macro- scales and to know all assumptions and simplifications needed to obtain the final system of macroscale conservation equations. Averaging theories provide well established frameworks to develop mathematical models for multiphase systems at any scale of interest, and assure a rigorous derivation of larger scale conservation laws based on microscale relations. However, once mathematical upscaling is achieved, a number of constitutive relationships are typically required to obtain a solvable system of equations; very often, identification of relevant closure relationships (e.g. pressure-saturation relationships, phase relative permeability, etc.) remains therefore an open issue.

With this overall aim, a microscale mathematical model for biphasic flow has been developed. The formulation consists in a set of partial differential equations coupling Cahn-Hilliard model with Navier-Stokes equations following the guidelines of 2 and Oldroyd-B constitutive law to account for viscoelasticity. The final model has been implemented on the FEniCS 3 platform and captures accurately the dynamic of tumor aggregates in vitro [4]. This approach permitted to provide original explanation on experimental data, showing the relevance of the multiphase flow framework for the tumor growth description and the predictive capacity of the model. Finally, the link between pore-scale simulation results and average phase properties in the porous media is studied. A set of numerical experiments is performed to identify macroscopic closure relationships for tumor growth modelling 5, in order to set up a model able to consider accurately the high spatial heterogeneity of the problem.

\title{
Procter and Gamble Student poster award:
}

\section{References:}

1 M. S. Steinberg, 'Differential adhesion in morphogenesis: a modern view', Curr. Opin. Genet. Dev., vol. 17, no. 4, pp. 281-286, Aug. 2007.

2 F. O. Alpak, B. Riviere, and F. Frank, 'A phase-field method for the direct simulation of two-phase flows in pore-scale media using a non-equilibrium wetting boundary condition', Comput. Geosci., vol. 20, no. 5, pp. 881-908, Oct. 2016.

3 A. Logg, K.-A. Mardal, and G. Wells, Eds., Automated Solution of Differential Equations by the Finite Element Method, vol. 84. Berlin, Heidelberg: Springer Berlin Heidelberg, 2012.

[4] K. Alessandri, 'The Cellular Capsules technology and its applications to investigate model tumor progression and to engineer tissues in vitro', Paris Descartes.

5 G. Sciumè et al., 'A multiphase model for three-dimensional tumor growth', New J. Phys., vol. 15, p. 015005, Jan. 2013. Acceptance of Terms and Conditions:

Click here to agree

\section{Optimal thickness of porous micro-electrodes operating a single reduction reaction}

\author{
Tien Dung Le ${ }^{\text {None }}$; Didier Lasseux ${ }^{1}$; Alexander $\mathrm{Kuhn}^{2}$; Nicolas Mano ${ }^{3}$; Gerard Vignoles ${ }^{4}$ \\ ${ }^{1}$ CNRS \\ ${ }^{2}$ Institute of Molecular Science \\ ${ }^{3}$ Centre de Recherche Paul \\ ${ }^{4}$ Université de Bordeaux - LCTS
}

Corresponding Author(s): letiendung.esgc@gmail.com

In the recent decades, porous electrodes with high specific surface, which are of major interest for the design of miniaturized electro-devices such as bio-batteries, have received a lot of attention both from modeling and experimental points of view. Such electrodes may provide much higher electrical current than classical flat electrodes of the same size 1 . Despite considerable progress in porous 
electrode manufacturing, effective tool for predicting the optimal thickness of such electrodes have not been developed in the literature. To fill this gap, this work aims at developing a thorough approach to estimate the optimal thickness of a porous electrode operating a single reduction reaction. This is achieved by making use of a macroscopic model formally derived from the microscopic coupled diffusion and electrochemical reaction model operating at the pore scale using an upscaling procedure 2 relying on the volume averaging method 3 . The solution of this macroscopic model was successfully compared to the solution of the microscale original model obtained from direct numerical simulations on the one hand and to experimental data on the other hand, validating our theoretical macroscale model.

The macroscopic model is subsequently used in the steady-state regime (a situation which is of wide practical interest) to derive an analytical solution for the concentration profile of the dilute species allowing to express the current intensity available at the electrode. On this basis, an optimization procedure is finally proposed to estimate the effective electrode thickness which is defined as the crossover value of two asymptotic regimes characterizing the volume current density dependence upon the electrode thickness. This yields an analytical expression of the optimal thickness that is general for a cylindrical porous electrode, regardless the type of microstructure of the electrode material. An illustration is provided for the type of electrode used in the experiments reported in this work.

Keywords: Porous electrode, Volume averaging, Optimal thickness

References

1 Walcarius A., Kuhn A., Ordered porous thin films in electrochemical analysis. TrAC Trends in Analytical Chemistry 27, 593-603 (2008).

2 Le T. D., Lasseux D. Nguyen X. P., Vignoles G. L., Mano N., Kuhn A., Multi-scale modeling of diffusion and electrochemical reactions in porous micro-electrode. Chemical Engineering Science 173, 153- 167 (2017).

3 Whitaker S., The method of volume averaging. Kluwer Academic Publishers, Dordrecht, the Netherlands (1999).

Procter and Gamble Student poster award:

I don't want to compete References:

Acceptance of Terms and Conditions:

Click here to agree

891

\section{Dynamic effect in capillary pressure for fractal porous media: a theoretical model}

gang $\operatorname{lei}^{1} ;$ liao qinzhuo ${ }^{1}$

${ }^{1} \mathrm{kfupm}$

Corresponding Author(s): gang.lei@kfupm.edu.sa

The dynamic capillary pressure effect in porous media has drawn tremendous attention in various scientific and engineering fields. However, though the dynamic capillary pressure has been intensively investigated in the last decades, the essential controls on dynamic effect in capillary pressure and the corresponding relative permeability are not determined. It is of practical significance to use analytic methods to study dynamic effect in capillary pressure and the corresponding relative permeability for fractal porous media. Unfortunately, because of the disordered and extremely complicated microstructures of porous media, the theoretical model for dynamic capillary pressure and the corresponding relative permeability is scarce.

The goal of this work is to establish a novel and reasonable quantitative model to determine the dynamic effect in capillary pressure and the corresponding relative permeability. The predictions of the theoretical model, derived from the Hassanizadeh's model and fractal geometry, agree well with the available experimental data. There is no empirical constant and every parameter in the model has specific physical significance. Compared with the previous models, our model takes into account more factors, including the influence of the microstructural parameters of the pore space. The proposed models can reveal more mechanisms that affect the dynamic effect in capillary pressure and 
the corresponding relative permeability for fractal porous media. This work provides a comprehensive theoretical study on dynamic effect in capillary pressure and relative permeability for fractal porous media, which is beneficial to accurate performance forecasts for the dynamic behavior in porous media.

Procter and Gamble Student poster award:

I don't want to compete References:

Acceptance of Terms and Conditions:

Click here to agree

\title{
Transport mechanism of emulsion micro-gel particles: Synergis- tic effect of emulsion and micro-gel particle
}

\author{
Wenhai Lei ${ }^{1}$; Chiyu Xie ${ }^{2}$; jinling Zhou ${ }^{1}$; Moran Wang ${ }^{1}$ \\ ${ }^{1}$ Tsinghua University \\ ${ }^{2}$ University of Texas Austin
}

Corresponding Author(s): leiwh16@mails.tsinghua.edu.cn

Non-uniform fluid displacements in porous media are commonly observed, while are unfavorable in oil/gas recovery processes. Recently, the micro-gel particle, such as preformed particle gel (PPG), soft micro-gel (SMG), elastic microsphere, etc., have been developed as a smart sweep improvement and profile modification agent under such bad conditions. The matching of particles and porous media will directly influence the displacement effect. Emulsions micro-gel particles (combining oil-inwater emulsion and micro-gel particle) will be formed automatically during the dilution procession of the micro-gel particle solution, which plays an important role in micro-gel particle transport in porous media. However, the synergistic effect of emulsion and micro-gel particle are ignored in the previous work, where only the micro-gel particle effect was considered.

In this study, the morphology, particle size analysis, and rheological property of emulsion micro-gel particles were characterized by Cryo-SEM, a Malvern Mastersizer 3000E Hydro, and Hakke MarsIII rheometer. Microscopic visualization models were used to study the oil-displacement effect and migration mechanism of emulsion micro-gel particles in the flow and displacement experiments. An Immersed Boundary-Lattice Boltzmann (IB-LBM) framework is established to capture the motion of the viscoelastic micro-gel particle in porous media in the pore scale.

Primarily, the experimental results of emulsion micro-gel particle characterization showed the different size of the micro-gel particle will influence the size of emulsion droplet in the micro-gel solution. The smaller size of the micro-gel particle, the stronger of the micro-gel particle aggregation phenomena (the larger size of emulsion micro-gel particle). Thence, the micromodel test indicated that emulsion micro-gel particles (small micro-gel particle will produce large emulsion micro-gel particle) can block the fluid channeling paths and diverting the displacing fluid from large channel to small channel. The pore-scale simulation showed that micro-gel particle can transport in small pores and cause pressure fluctuation due to its small size and good deformable performance. A synergistic "two-part" mechanism is important in the mixed emulsifier system, where both the micro-gel particle and emulsion components have specific functions in the relatively large and the small channel. These findings demonstrate that emulsion micro-gel particles can be adapted to different porous media stage classified by different pore sizes. Our results improve the understandings of the synergistic effect of emulsion and micro-gel particle in the micro-gel particle transport in the porous media, which is important to gel treatment for enhancing oil recovery.

\section{Procter and Gamble Student poster award:}

I would like to compete in the Procter and Gamble Student award References:

Acceptance of Terms and Conditions:

Click here to agree 


\title{
Hygromorphic response dynamics of oak: an NMR study
}

\author{
Pel Leo ${ }^{1}$; Arends Thomas ${ }^{1}$; Smeulders David ${ }^{1}$ \\ ${ }^{1}$ Eindhoven University of Technology
}

Corresponding Author(s): 1.pel@tue.nl

\begin{abstract}
Wooden boards, joined together by glue, dowels, and crosspieces were the most extensively used supports for portable paintings until the early 17th century. Typically, some layers of gesso and/or fabric were added to serve as a carrier for the paint layer. Well-known masterpieces have been constructed accordingly, e.g., by da Vinci, Rubens, or Rembrandt.

Since paint is much less permeable than wood, a panel painting can conceptually be approached as a board with an impermeable layer on one surface. Due to the hygroscopic nature of wood, a panel will exchange moisture with the ambient air upon changes in the relative humidity (RH). Consequently, the moisture content will be unevenly distributed throughout the thickness of the board which will result in differential expansion across the thickness, causing the wooden board to bend. This can give rise to permanent deformation or even failure, manifested, e.g., as cracks. Using a specialist Nuclear Magnetic Resonance (NMR) setup, which makes use of the magnet of a $1.5 \mathrm{~T}$ whole body MRI scanner, we have studied simultaneously both the moisture distribution in oak during changing relative humidity and the expansion. These results show that the asymmetry in the moisture distribution can be directly related to the expansion. In addition we have looked at the response of panel paintings to sinusoidal RH changes with different frequencies. Our goal is to come up with criteria for panels paintings for fluctuation frequency, and amplitude in RH which are safe or potentially harmful.
\end{abstract}

Procter and Gamble Student poster award:

References:

Acceptance of Terms and Conditions:

Click here to agree

803

\section{Multifunctional doubly-porous materials with controlled hydrophilic- ity}

Author(s): Thibault Lerouge ${ }^{1}$

Co-author(s): Benjamin Le Droumaguet ${ }^{2}$; Daniel Grande ${ }^{3}$; Olivier Pitois ${ }^{4}$; Philippe Coussot ${ }^{4}$

${ }^{1}$ Navier

${ }^{2}$ Institut de Chimie et des Matériaux Paris Est

${ }^{3}$ Institut de chimie et des Matériaux Paris-Est

${ }^{4}$ Univ. Paris-Est

Corresponding Author(s): thibault.lerouge@ifsttar.fr

Some multi-scale building materials, like wood and hemp concrete or daub, exhibit high potential properties in terms hygrometric diffusion (moisture expelling out of a house). However, the correlation between the imbibition and the multi porous structure is complex and still unclear. In order to get a better insight into the precise origin of such multifunctional properties, new model systems based on polymeric materials with double porosity have been envisioned.

Bi-porous polymer networks have been designed and elaborated with controlled pore morphology (interconnectivity, double pore size) and hydrophilicity (using different monomers, contact angle of water: $10^{\circ}<\theta<130^{\circ}$ ). To investigate the role of the doubly porous framework on the soaking/drying 
properties, the materials are compared to both monoporous counterparts: smallest pores (SP: $2 \mu \mathrm{m}$ ) and largest pores (LP: $200 \mu \mathrm{m})$ monoporous materials.

Absorption for SP material is well described by the classical Washburn theory. The LP monoporous material presents a high permeability due to the large connectivity of its porous morphology, obtained from high pressure sintering of cubic particles. However, it exhibits a drastic reduction in imbibition rate, which cannot be describe by Washburn model, with a succession of quick steps of imbibition and long pauses when the driving capillary pressure drops at constriction. This behavior is attributed to the slow breakthrough mechanism for the water interface at sharp edge connections between pores. Remarkably, this slow regime is suppressed for the biporous material, with identical large pore morphology, and imbibition rate is even higher than the sum of rates obtained for its monoporous counterparts. This highlights the synergy of mixed small and large pores actions that neutralize the sharp-edge constrictions effect described above, with the presence of two rising liquid fronts: one into the small pores skeleton, and one in the large pores network.

Procter and Gamble Student poster award:

I don't want to compete References:

Lerouge, Pitois, Grande, Le Droumaguet, Coussot Soft Matter 2018, 14, 8137-8146. Acceptance of Terms and Conditions:

Click here to agree

\title{
High Pressure Small Angle Scattering Investigation of Methane and $\mathrm{CO} 2$ Sorption in Shales and Nano-porous Shale Analogues
}

\author{
Leon Leu ${ }^{1}$; Gernot Rother ${ }^{2}$; Patrice Creux ${ }^{3}$; Michael F. Toney ${ }^{4}$ \\ ${ }^{1}$ Energy Resources Engineering, Satnford University \\ ${ }^{2}$ Oak Ridge National Laboratory \\ ${ }^{3}$ CNRS/TOTAL/Pau University \\ ${ }^{4}$ SIMES, Stanford Synchrotron Radiation Light Source
}

Corresponding Author(s): leuleon@slac.stanford.edu

Geologic shale formations are important sources for natural gas (containing a significant amount of methane) and play an important role for $\mathrm{CO} 2$ storage 1 . The nanoscopic pore network in shales, especially meso- and micropores $(50-2 \mathrm{~nm},<2 \mathrm{~nm}) 2$, plays a critical role for the total amount of fluid present, due to the high abundance and available surface area for sorption 3 . Efficient prediction of production and reservoir integrity prediction requires detailed understanding of the rock - fluid interactions under typical shale reservoir conditions of temperatures $20-90^{\circ} \mathrm{C}$ and pressures of 200 - 500 bar. In this study we investigate the high pressure sorption and fluid behaviour in organic rich shale samples using small angle neutron and X-ray scattering techniques (SANS/SAXS). To better understand the effects we observe for these heterogeneous materials (pore shapes, sizes, surface properties) we also use commercially available model materials such as carbon aerogel and controlled pore glass, allowing good control of surface properties and pore sizes/shapes. A series of (deuterated-)methane, and $\mathrm{CO} 2$ sorption experiments is performed at constant temperature of $50^{\circ} \mathrm{C}$ at different pressure steps ranging from $50-480$ bar (crossing the bulk critical point for both fluids). The experiments are complimented by laboratory gravimetric excess methane and $\mathrm{CO} 2$ sorption measurements at the same pressure and temperature conditions. We aim at providing experimental data for a fundamental understanding of the effects of the fluid rock interactions including, confinement, pore type and surface properties.

Procter and Gamble Student poster award:

\section{References:}

1 Gensterblum, Y. Ghanizadeh, A. Amann-Hildenbrand, A. Krooss, B.M. Clarkson, C. Harrington, J.F. Zoback, M. Gas transport and storage capacity in shale gas reservoirs - A review. Part A: Transport 
processes. Journal of Unconventional Oil and Gas Resources 12, 87-122. (2015)

2 Sing, K.. Reporting Pphysisorption Data for Gas/Solid Systems; with Special Reference to the Determination of Surface Area and Porosity. Pure \& Applied Chemistry 54, 2201-2218 (1982).

3 Clarkson, C.R., Jensen, J.L., Chipperfield, S. Unconventional Gas Reservoir Evaluation: What do We Have to Consider? Journal of Natural Gas Science and Engineering 8, 9-33. (2012.). Acceptance of Terms and Conditions:

Click here to agree

\title{
The influence of interfacial tension on spontaneous imbibition of low-permeability water-wet reservoir
}

\author{
Aifen $\mathrm{Li}^{1}$; Shuaishi $\mathrm{Fu}^{2}$; Bingqing $\mathrm{He}^{1}$; Qi Fang ${ }^{1}$ \\ ${ }^{1}$ China University of Petroleum \\ ${ }^{2}$ China University of Petroleum冈China University of Geosciences
}

Corresponding Author(s): aifenli@upc.edu.cn, fushuaishi@upc.edu.cn

To research the influence of interfacial tension of fracturing fluid on spontaneous imbibiton in lowpermeability water-wet reservoir, a spontaneous imbibition model was constructed upon a modified LW imbibition model includes hydrostatic pressure and flow resistance with fractal theory, and the relationship between spontaneous imbibition volume and imbibition time was described by the model. And the influence rules of spontaneous imbibition volume and interfacial tension was analysed. The imbibition model was verified by laboratory data. The experimental results and computing results reveal: the impact of interfacial tension to imbibition volume is not monotonous. Capillary force and flow resistance of oil phase increase with interfacial tension increasing, and meanwhile imbibition volume shows a trend from rise to decline. Each different kinds of fracturing fluid have optimal interfacial tension to reach the best imbibition effect, and imbibition volume is maximum.

Procter and Gamble Student poster award:

References:

Acceptance of Terms and Conditions:

Click here to agree

\section{Investigation on the multi-parameter of hydrate-bearing media using nano-focus $\mathrm{X}$-ray computed tomography}

\author{
Chengfeng $\mathrm{Li}^{\text {None }}$; Changling Liu ${ }^{\text {None }}$; Gaowei $\mathrm{Hu}^{\text {None }}$; Jianye Sun ${ }^{\text {None }}$; Lele Liu ${ }^{\text {None }}$
}

Corresponding Author(s): chengfenglee@163.com

A nano-focus X-ray computed tomography (X-CT) was used to observe methane hydrate formation in sands at pore-scale and to investigate the hydrate saturation, pore structure parameters, and permeability of hydrate-bearing sands. A new test technique was developed to improve the identification of water-hydrate boundary. It is observed that there are three hydrate accumulation habits in pore spaces. When there were no methane bubbles, hydrate distribution evolved from floating to contacting and then to cementing as hydrate saturation increasing. However, only contacting and cementing distribution patterns were observed in the pores with methane bubbles. We observed the gas/water/hydrate distributions and investigated the pore structure characteristics. The results show that the porosity, the maximal pore volume and diameter were decreasing as hydrates accumulating in the pores. However, the maximal pore surface, the total pore number, and the number of large 
pores ( $>100$ voxels) continued to increase until the hydrate saturation reached $35 \%$, and then began to decrease rapidly. The pore structure parameters show a detailed change of hydrate and water at porescale. The absolute permeability of hydrate-bearing porous media estimated based on X-CT digital images and Darcy's law agreed well with the permeability calculated from the Kozeny-Carman equation. The results provide a valuable reference for cognition of hydrate bearing reservoirs.

Procter and Gamble Student poster award:

References:

Acceptance of Terms and Conditions:

Click here to agree

\section{Genetic Mechanism of Diverse Pores in Bioclastic Limestone and Impacts on Reservoir Quality}

Fengfeng $\mathrm{Li}^{\text {None }}$; Rui Guo ${ }^{\text {None }}$; Wenju Sun ${ }^{\text {None }}$; Yichang $\mathrm{Yu}^{\text {None }}$

Corresponding Author(s): 1522188426@qq.com

The Mishrif Formation in M Oilfield was mainly characterized by porous carbonate reservoir with diverse biological skeletal and complex diagenesis. Reservoir in this area had complicated pore types such as interparticle pore, intraparticle pore, mouldic pore, vug, intercrystalline pore, fractures, matrix-hosted and grain-hosted micropore. Based on data from drilling core, cast thin-section,mercury injection experiment and scanning electron microscopy, the diagenetic mode of different microfacies had been established and the genetic mechanism of diverse pore had been analyzed. The microfacies controlled the biological skeletal types which had different ability in anti-resistance dissolution and compaction. Selective dissolution led to various moldic pore which had high porosity and low permeability. The dolomization could develop large amount of intercrystalline pore. If in a low energy environment, the sediments might had high matrix which result in matrix-hosted micropore and later nemorphism could result in grain-hosted micropore which both had little influence on reservor permeability. In meteoric environment, intense non-selective might develop interparticle pore and vug which not only had high porosity but also had good permeability. The bioturbation in marine environment mainly led to intraparticle pore which usually was poor-connected. The cementation was widely developed in diagenetic period, which was a double-edged sword. If cementation occurred in early stage, it could protect the rock structure and later dissolution could produce large amout of pore. If cementation occurred in late stage, it could infill the previous pore and pore throat, which result in low porosity and low permeability. In burial environment, compaction and pressolution were the main diagenesis, which could develop fractures in reservoir which had little influence on porosity but was favorable for permeability. It concluded that the environment determined the diagenetic types which had great influence on pore types. The complex pore system was the main reason of poor correlation of porosity and permeability. The diagenetic stage controlled the reservoir transform tendency. Reservoir pore types were the comprehensive superimposition of sedimentation and diagenesis. Sedimentation controlled the sediments types and diagenetic route. The types and period of diagenesis determined the reservoir quality. It was of great significance for the evaluation and the potential analysis of bioclastic limestone reservoir.

Procter and Gamble Student poster award:

I don't want to compete References:

Acceptance of Terms and Conditions:

Click here to agree

\section{Micro Gas Displacing potential of horizontal well in tight reser- voirs}


Author(s): Haibo Li ${ }^{\text {None }}$

Co-author(s): Hekun Guo ; zhengming yang ${ }^{1}$; TieNing Gao ; yapu zhang ; Yixin Dai

${ }^{1}$ PetroChina Research Institute of Petroleum Exploration \& Development

Corresponding Author(s): 76274491@qq.com

This study aim at tight oil reservoir cores of Erdos Basin, combining NMR technology and core displacement test, and analysis microscopic oil distribution characteristics both initial state and after gas flooding of reservoir. Research show that: Before gas flooding, the total oil percentage of target reservoirs is higher, but less oil(7.27\%) exist in micron pores (greater than $1 \mu \mathrm{m})$, oil mainly exist in the sub micron pores $(0.1 \sim 1 \mu \mathrm{m})$ and nanometer pores(less than $0.1 \mu \mathrm{m})$, which about $27.67 \%$ and $24.00 \%$ respectively. After $1 \mathrm{PV}$ gas flooding, reservoir recovery percent of reserves $\mathrm{R}$ of micro pores are higher(64.34\%), sub micron pores are second(37.27\%), nanometer pores are lower. In early stage of gas flooding, oil in the larger pores are removed first, and oil production in smaller pores are lower. After $50 \mathrm{PV}$ gas flooding, there are almost no residual oil in micro pores, $\mathrm{R}$ reaches over $90 \%$, remaining oil of submicron pores have a certain amount, $\mathrm{R}$ about $60 \%$, and $\mathrm{R}$ of nanometer pores are low, nanometer pores are the main storage space of residual oil. With the decrease of permeability, ratio of larger throat-pore in reservoir decreases, the microscopic heterogeneity decreases, lead to degree of gas flooding micro sweep increases, and the submicron oil recovery has an increasing trend. The gas injection capacity of tight reservoir is obviously better than that of water injection, and the lower the permeability reservoir have, the more obvious gas injection capacity get. The results provide theoretical basis for effective development and establishing rational development mode of tight oil reservoir.

Procter and Gamble Student poster award:

References:

Acceptance of Terms and Conditions:

Click here to agree

\title{
The analysis of the effect of ethane fraction on the carbon dioxide displacement in coal using molecular simulation
}

\author{
Author(s): JIAWEI LI ${ }^{\text {None }}$ \\ Co-author(s): Yuzhu Wang ; Zhixi Chen ; Sheik Rahman \\ Corresponding Author(s): jiawei.li4@student.unsw.edu.au
}

Coal seam gas reservoirs usually contain a divergent amount of ethane $\left(\mathrm{C}_{2} \mathrm{H}_{6}\right)$, propane, butane, etc. These gas components always change with the maturity and heterogeneous structure of coal. Carbon dioxide $\left(\mathrm{CO}_{2}\right)$ enhanced coal-bed methane $\left(\mathrm{CH}_{4}\right)$ recovery $(\mathrm{ECBM})$ is one of the most favorable and feasible techniques in coal seam gas development which also contributes to $\mathrm{CO}_{2}$ sequestration. However, the effect of the $\mathrm{C}_{2} \mathrm{H}_{6}$ mole fraction in coal seams on the $\mathrm{CO}_{2}$ displacement remains unclear currently. In this study, the mole fraction of $\mathrm{C}_{2} \mathrm{H}_{6}$ was varied to examine its effect on the adsorption behavior of $\mathrm{CH}_{4}$, and on the process of ECBM under given pressure and temperature using molecular simulation method. This procedure was carried out via two main steps. First, molecular dynamics was employed to obtain the geometry optimization of the unit cell of the carbon model. Then, Grand Canonical Monte Carlo (GCMC) simulation was undertaken to predict the gas adsorption behavior. Results showed that $\mathrm{C}_{2} \mathrm{H}_{6}$ had a similar adsorption behavior with that of $\mathrm{CO}_{2}$. It was, therefore, indicated that the adsorption selectivity of $\mathrm{CO}_{2} / \mathrm{CH}_{4}$ went down with a higher $\mathrm{CO}_{2}$ fraction concentration under the same temperature and pressure. The adsorption selectivity of $\mathrm{CO}_{2} / \mathrm{C}_{2} \mathrm{H}_{6}$ dispersed around 1.1, which suggested that $\mathrm{CO}_{2}$ could enhance the recovery of $\mathrm{C}_{2} \mathrm{H}_{6}$ to some extent during the ECBM process. In addition, the effect of pressure and temperature was analyzed. Results showed the selectivity of $\mathrm{CO}_{2} / \mathrm{CH}_{4}$, in our coal model, had a relatively high selectivity of $\mathrm{CO}_{2}$ at lower pressure, then it reached a plateau at an optimize pressure which was related to the 
favorable injection depth of the reservoir and it remained constant at higher pressure. However, the adsorption selectivity kept dropping under the same pressure with increasing temperature. This study revealed the mechanism and adsorption behavior of related gases and consequently provide basic data for $\mathrm{CO}_{2}$ enhanced coal seam gas development project.

Key words: $\mathrm{CH}_{4}, \mathrm{C}_{2} \mathrm{H}_{6}, \mathrm{CO}_{2}$, adsorption, Grand Canonical Monte Carlo simulation

Procter and Gamble Student poster award:

I would like to compete in the Procter and Gamble Student award References:

Acceptance of Terms and Conditions:

Click here to agree

\title{
Investigation of CO2 Huff-n-Puff EOR Mechanisms in Liquid-Rich Shale Reservoirs based on Microscopic Visualization Experiments
}

\author{
Author(s): Lei $\mathrm{Li}^{1}$ \\ Co-author(s): Yuliang Su ${ }^{1}$; Yongmao Hao ${ }^{1}$; Chengwei Wang ${ }^{1}$; Dongsheng $\mathrm{Li}^{1}$ \\ ${ }^{1}$ China University of Petroleum (East China)
}

Corresponding Author(s): lei.li@upc.edu.cn

$\mathrm{CO} 2$ huff-n-puff is an effective way to recover oil in liquid-rich shale reservoirs with multistage fractured horizontal wells. Numerous core-scale lab experiments have been conducted to prove its potential. There are many mechanisms during the CO2 huff-n-puff process such as pressure repressurization, miscibility, molecular diffusion, relative permeability hysteresis, gas dissolve and oil swelling, etc. It's difficult to analyze the mechanisms during the EOR process only through corescale lab experiments. New methods such as microscopic visualization experiment and numerical study should be designed and applied in this analysis. The purpose of this study is to investigate the functions of related mechanisms through microscopic visualization experimental and numerical methods.

In this experimental study, a new artificial visualization microscopic model was built to observe the $\mathrm{CO} 2$ diffusion and shale oil percolation in the nanoscale porous media during the CO2 huff-n-puff process. Different parameters such as oil composition, reservoir properties (permeability, porosity) and operation conditions (injection pressure, soaking time, depletion rate, gas injection cycles) were analyzed. After that, micro-nanofluidic chips were designed to mimic different cases (homogenous / heterogenous) during CO2 huff-n-puff. Numerical study was also applied to simulate the CO2 huff$\mathrm{n}$-puff process to investigate the functions of different mechanisms. Finally, the result is applied to fit some of CO2 field plots which were performed in Bakken formation of North Dakota. Various simulation cases were designed and established to investigate different mechanisms such as pressure repressurization, miscibility, relative permeability hysteresis, capillary pressure and oil swelling. This work clearly describes the $\mathrm{CO} 2$ diffusion and shale oil percolation process in the shale oil reservoir media through the microscopic experimental model. The micro-nanofluidic chips can simulate the nanopore to investigate the shale oil phase behavior in nanopores combined with hydraulic fracture and natural fractures. Visual observation of the $\mathrm{CO} 2 /$ shale oil displacements indicate that there is an interaction between phase behavior and microscopic heterogeneity. The experimental and numerical results show that the most important mechanism is pressure repressurization, followed by miscibility, diffusion, relative permeability hysteresis, and oil swelling. This study explains how CO2 diffusion in liquid-rich shale oil reservoirs is different in lab core scale level from microscale level, and how different mechanisms affects $\mathrm{CO} 2$ performance in improving oil recovery from unconventional resources.

Procter and Gamble Student poster award:

References:

Acceptance of Terms and Conditions: 


\title{
Advanced CO2 Injection Enhanced Oil Recovery in Shale Oil Reser- voirs: Experiments, Molecular Dynamic Simulations and Theo- retical Analysis
}

\author{
Author(s): Lei $\mathrm{Li}^{1}$ \\ Co-author(s): Yuliang Su ${ }^{1}$; Yongmao Hao ${ }^{1}$; Dongsheng $\mathrm{Li}^{1}$; Chengwei Wang ${ }^{1}$ \\ ${ }^{1}$ China University of Petroleum (East China)
}

Corresponding Author(s): lei.li@upc.edu.cn

Shale oil production has increased rapidly in last decades especially in United States and results in a revolution in the energy landscape. However, one main problem existing in the shale reservoir development is the sharp decline of liquids production in all the hydraulic wells. In recent years, CO2 injection has been experimentally and numerically studied to enhance the oil recovery. Some field pilots of $\mathrm{CO} 2$ injection were also conducted in Bakken unconventional plays and demonstrated great potential for CO2 enhanced oil recovery (EOR) in Bakken. However, the oil transport behavior and enhanced oil recovery mechanisms are still not well understood. The lack of understanding impedes the EOR strategy implement and production efficiency in the shale reservoirs.

This study includes experiment study and theoretical study. Firstly, experiments were conducted on twenty shale core samples. The microscopic pore characteristics, pore size distribution, mineral composition, totally organic carbon content and wettability were investigated. CO2 huff-n-puff experiments were implemented on shale core samples to investigate the EOR ability. Then dynamic molecular simulations were applied to analyze the interaction of $\mathrm{CO} 2$ with shale oil under reservoir pressure and temperature. The diffusion effect in organic clays and inorganic minerals were explored. Based upon the experiment and molecular simulation results, the oil flow behavior in organic matter, inorganic pores and oil dissolution were described in mathematic equations. After that, the $\mathrm{CO} 2$ injection process was investigated in both matrix and fracture systems based upon LBM method and N-S equation. From the lab experiments, the observed oil recovered in the latter huff-n-puff cycle was not significant, new development mode combined CO2 huff-n-puff with CO2 flooding was designed and evaluated.

The results show that considerable organic carbon presents $4-10 \mathrm{wt} \%$ in the shales. The pores in shale cores are mainly distributed in the junction region of inorganic minerals and organic clays. The pore sizes are among several nanometers to several micrometers and can be divided into three categories: inorganic pores, organic pores and micro-fractures. The oil produced in shale reservoirs was mainly come from inorganic pores in the early stage, and the oil production rate in inorganic pores is higher than that in organic materials. In the late stage, most of the produced oil is from organic pores. The molecular dynamics result shows that $\mathrm{CO} 2$ diffuse more rapidly with increases in temperature and pressure, but diffuse slowly with an increase in pore size. Based upon the studies of molecular dynamics simulations, liquid flow through carbon nanotubes, experiment results and theoretical analysis, a unified apparent permeability model of liquid hydrocarbon flow in shale is derived coupling different transport mechanisms in inorganic and organic nanopores. For the core scale CO2 injection experiments, the result shows that after four cycles of CO2 huff-n-puff, the application of $\mathrm{CO} 2$ flooding is more effective, which can increase oil recovery by another $5-10 \%$. This illustrates that after several CO2 huff-n-puff cycles, the injected CO2 only penetrate the relatively high permeability zones. Thus the oil in the small organic pores cannot be swept. The combination of huff-n-puff and CO2 flooding will increase both the sweep area and displacement efficiency, resulting in the increase of ultimate oil recovery.

This work includes a series of experiments, molecular dynamic simulations, theoretical analysis and development mode design. The oil transport behavior and enhance oil recovery mechanisms by $\mathrm{CO} 2$ injection were discussed in detail. This work is helpful to understand CO2 EOR mechanisms in shale 
reservoirs and fundamentally important for applying, evaluating and optimizing $\mathrm{CO} 2$ injection in field production.

\title{
Procter and Gamble Student poster award:
}

I would like to compete in the Procter and Gamble Student award References:

Acceptance of Terms and Conditions:

Click here to agree

\section{Simulation of Subsurface $\mathrm{CH} 4$ Storage if Using $\mathrm{CO} 2$ as Cushion Gas}

\author{
Qi $\mathrm{Li}^{1}$; Michael Kühn ${ }^{2}$; Jianli Ma ${ }^{2}$; Natalie Nakaten ${ }^{2}$ \\ ${ }^{1}$ Chinese Academy of Sciences \\ ${ }^{2}$ GFZ German Research Centre for Geosciences
}

Corresponding Author(s): kouzou_21st@hotmail.com

The climate change and energy shortage has attracted more and more attention. Power-to-Gas (PtG), a chemical energy storage technology, can convert the surplus electrical energy into a high energy density comestible gas. Subsurface energy storage is the critical process in a PtG system. Produced methane (CH4) can be storage in geological reservoir for further use. To avoid the waste of $\mathrm{CH} 4$ in the withdrawal season, carbon dioxide ( $\mathrm{CO} 2)$ is used as cushion gas to maintain the reservoir pressure due to its great compressibility near the critical pressure and temperature. However, the mixing problem between two kinds of gases in the same reservoir is inevitable. Therefore, the controlling of mixed region is of great necessary to improve the efficiency of a PtG system. In this paper, physical properties of $\mathrm{CH} 4, \mathrm{CO} 2$ and their mixtures are discussed in detail. Based on the interpolation calculations of the National Institute of Standards and Technology database and physical equations, a hydro-mechanical coupling model is established to study the mixing problem of two kinds of gases and mechanical stability of reservoir. Then the accuracy of this model is verified by comparing with the model of Curtis M. Oldenburg. In addition, the effects of reservoir thickness, geological structures and temperature of reservoir on the mixing degree of two kinds of gases and the distribution of the mixed region are studied and analyzed in detail. The results show that thick reservoir, anticline geological structure and relatively high temperature will be a good choice for subsurface energy storage by using $\mathrm{CO} 2$ as cushion gas. The mechanical stability and safety during the injection process is also important. The correlation between safe injection time and tensile strength is obtained based on von Mises failure criteria. Results of this paper may provide a theoretical and technical support for optimization of a PtG system.

Procter and Gamble Student poster award:

\section{References:}

Li, Q. et al., 2016. Positioning and Revision of CCUS Technology Development in China. International Journal of Greenhouse Gas Control, 46: 282-293.

Kühn, M., Li, Q., Christine Nakaten, N., Kempka, T., 2017. Integrated subsurface gas storage of CO2 and $\mathrm{CH} 4$ offers capacity and state-of-the-art technology for energy storage in China. Energy Procedia, 125: 14-18.

Ma, J., Li, Q., Kühn, M., Nakaten, N., 2018. Power-to-gas based subsurface energy storage: A review. Renewable and Sustainable Energy Reviews, 97: 478-496.

Ma, J., Li, Q., Kühn, M., Li, X., 2018. Mixed Region Simulation on Subsurface Gas Storage of CO2 and $\mathrm{CH} 4$ in a Power-to-Gas System. In: Zhan, L., Chen, Y., Bouazza, A. (Eds.), Proceedings of the 8th International Congress on Environmental Geotechnics Volume 3: Towards a Sustainable Geoenvironment. Environmental Engineering. Springer Singapore, Berlin, pp. 41-48. Acceptance of Terms and Conditions:

Click here to agree 


\section{Studying the generation of nanoparticle-stabilized foam at pore scale}

Qingjian $\mathrm{Li}^{1}$; Valentina Prigiobbe ${ }^{1}$

${ }^{1}$ Stevens Institute of Technology

Corresponding Author(s): qli39@stevens.edu

Foam injection into the subsurface is generally performed to improve gas mobility control in enhanced oil recovery (EOR) and contaminated aquifer remediation (Rossen,1996, Zhong, 2009). The additions of nanoparticles (NPs) in the presence of surfactant helps to enhance the foam stability (Binks, 2002, Worthen, 2013). However, the generation mechanism of a foam in the presence of nanoparticles and surfactant at the pore scale is has been well understood, yet.

Here, a study at the pore scale on the generation of nanoparticle-stabilized foam in the porous media is presented. A porous medium chip was used and drainage and co-injection tests were carried out. Sodium lauryl sulfate (SLS) and functionalized-silica nanoparticles were employed to stabilize a foam made of nitrogen $\left(\mathrm{N}_{2}\right)$ gas. The system was monitored with a pressure transducer and a high-resolution camera. The convolutional neural network method (Krizhevsky, 2012) was used to identify the bubble and, therefore, their density. In both types of tests, the same generation mechanism was observed with either SLS or SLS and NPs. In these tests, the results show overall that the generation rate, the total number of bubbles, and the uniformity of the bubble size distribution increase with the flow rate. However, in the drainage tests, the strong foam was generated only when stabilized with SLS due to a larger shear rate required to form bubbles stabilized with nanoparticles. These results suggest that when the system is not shear-rate limited the generation mechanisms is independent of the surface active materials used to stabilized the gas-bubbles and therefore the constitute equation currently used for surfactant-stabilized foams can be extended to the nanoparticle-stabilized foam.

Procter and Gamble Student poster award:

\section{References:}

Binks BP (2002) Particles as surfactants similarities and differences. Current Opinion in Colloid and Interface Science 7(1-2):21-41

Krizhevsky A, Sutskever I, Hinton GE (2012) Imagenet classification with deep convolutional neural networks. In: Advances in neural information processing systems, pp 1097-1105

Rossen WR (1996) Foams in enhanced oil recovery. Foams: Theory, Measurements and Applications 57:413-464

Worthen AJ, Bagaria HG, Chen Y, Bryant SL, Huh C, Johnston KP (2013) Nanoparticle-stabilized carbon dioxide-in-water foams with fine texture. Journal of Colloid and Interface Science 391:142-151

Zhong L, Qafoku NP, Szecsody JE, Dresel PE, Zhang ZF (2009) Foam delivery of calcium polysulfide to the vadose zone for chromium (vi) immobilization: A laboratory evaluation. Vadose Zone Journal 8(4):976-985 Acceptance of Terms and Conditions:

Click here to agree

\section{Modeling nanoparticle transport in porous media in the presence of a foam}

Qingjian $\mathrm{Li}^{1}$; Valentina Prigiobbe

\footnotetext{
${ }^{1}$ Stevens Institute of Technology
}

Corresponding Author(s): qli39@stevens.edu 
Nano-remediation is a promising in situ remediation technology. It consists in injecting reactive nanoparticles (NPs) into the subsurface for the displacement or the degradation of contaminants (Karn, 2009, O'Carroll, 2013, Tosco, 2014, Zhao, 2016). However, due to the poor mobility control of nanoparticle, the application of nano-remediation faces some major challenges, such as, loss of nanoparticle, override of the contamination, and particle aggregation, all of which can lead to a limited distance of influence. Previous experimental studies show the potential of combining nanoremediation with foam flooding to overcome these issues (Zhong, 2009, Zhong, 2010, Ding, 2013). In order to design and optimize the process, a model which couples nanoparticle and foam transport is necessary.

In this paper, a mechanistic model to describe the transport of NPs with and by a foam is presented. The model considers the delivery of nanoscale zero-valent iron (nZVI) and accounts for the processes of aggregation, attachment/detachment, and generation/destruction. Simulations show that when NPs are dispersed in the liquid phase, even in the presence of a foam, they may travel much slower than the NPs carried by the foam bubbles. This is because the nanoparticles in suspension are affected by the attachment onto the rock walls and straining at the pore-throats. When the nanoparticle surface is, instead, modified in order to favor their adsorption onto the gas bubbles, NPs are carried by the foam without retardation, except for the small fraction suspended in the liquid phase. Moreover, very stable high quality foam, i.e., 80-90 vol.\% of gas, can be attained using properly surface-modified nZVI (i.e., a nanoparticle-stabilized foam), allowing a significant reduction of water for the operation, while increasing the efficiency of nZVI delivery, even in a low permeability medium within the shallow subsurface.

\title{
Procter and Gamble Student poster award:
}

\section{References:}

Ding Y, Liu B, Shen X, Zhong L, Li X (2013) Foam-assisted delivery of nanoscale zero valent iron in porous media. Journal of Environmental Engineering 139(9):1206-1212

Karn B, Kuiken T, Otto M (2009) Nanotechnology and in situ remediation: a review of the benefits and potential risks. Environmental Health Perspectives 117(12):1813

O'Carroll D, Sleep B, Krol M, Boparai H, Kocur C (2013) Nanoscale zero valent iron and bimetallic particles for contaminated site remediation. Advances in Water Resources 51:104-122

Tosco T, Papini MP, Viggi CC, Sethi R (2014) Nanoscale zerovalent iron particles for groundwater remediation: a review. Journal of Cleaner Production4677:10-21

Zhao X, Liu W, Cai Z, Han B, Qian T, Zhao D (2016) An overview of preparation and applications of stabilized zero-valent iron nanoparticles for soil and groundwater remediation. Water Research 100:245-266 Zhong L, Qafoku NP, Szecsody JE, Dresel PE, Zhang ZF (2009) Foam delivery of calcium polysulfide to the vadose zone for chromium (vi) immobilization: A laboratory evaluation. Vadose Zone Journal 8(4):976-985

Zhong L, Szecsody JE, Zhang F, Mattigod SV (2010) Foam delivery of amendments for vadose zone remediation: Propagation performance in unsaturated sediments. Vadose Zone Journal 9(3):757-767 Acceptance of Terms and Conditions:

Click here to agree

\section{Role of the interaction of the calcite-water interface in the wetta- bility alteration during low salinity waterflooding}

\author{
Author(s): Shuai $\mathrm{Li}^{1}$ \\ Co-author(s): Matthew D. Jackson ${ }^{2}$ \\ ${ }^{1}$ China University of Geosciences \\ ${ }^{2}$ Imperial College London
}

Corresponding Author(s): lishuai@cug.edu.cn,

Wettability alteration has been widely accepted as the key process for the enhanced oil recovery during coreflooding test. There are several mechanisms proposed to affect the wetting behaviour 
of the calcite-water interface, such as $\mathrm{pH}$ variation, potential determined ions, fluid-fluid interaction. But what is the decisive mechanism during controlled salinity waterflooding, particularly for carbonate-crude oil-water system, is not fully understood yet. Zeta potential is a crucial electrochemical parameter for the mineral-water interface and sensitive to any physicochemical change at the interface (Vinogradov et al., 2010; Li et al., 2016; Al Mahrouqi et al., 2017; Li et al., 2018). In our study, we conducted two coreflooding tests associated with zeta potential measurements to characterize the surface wettability on the carbonate sample. Two types of carbonate (Carbonate-E and Carbonate-R) are employed in our experimental study, initially start with streaming potential test at fully saturated condition, then drain with crude oil to the irreducible water saturation, following the ageing procedure, spontaneous imbibition and the coreflooding test. Following the same coreflooding protocol with same crude oil and flooding brine, the two samples show a different behaviour in both zeta potential and coreflooding test.

The zeta potential of Carbonate-E and Carbonate-R with same mineralogy composition shows a different behaviour at high salinity brine, which is most likely caused by the different percentage of face and edge of calcite crystal exposed to brine. In the low permeability with nanoscale pores, the edges of calcite crystal are supposed to be the dominate surface exposed to brine within the Carbonate- $\mathrm{R}$ sample.

The results of low salinity waterflooding tests show that the incremental oil recovery can be positively observed in the oil-brine-Carbonate-R system while no response for Carbonate-E system. The electrokinetic behaviour of crude oil is similar to the positive oil as shown in previous study of Jackson et al (2016). However, the same crude oil shows a negative oil in Carbonate-R system cannot be explained well according to the polarity of oil-brine system yet.

Furthermore, the incremental oil recovery due to wettability alteration in the oil-brine- Carbonate- $R$ system can be confirmed by the change in the end-point water relative permeability. As the relative permeability is decreasing with increasing water saturation, it indicated that the wettability shifts towards to the less oil-wet which is consistent with the study of Nono et al (2014).

\title{
Procter and Gamble Student poster award:
}

\section{References:}

Al Mahrouqi, D., Vinogradov, J., \& Jackson, M. D. (2017). Zeta potential of artificial and natural calcite in aqueous solution. Advances in Colloid and Interface Science, 240, 60-76.

Jackson, M. D., Al-Mahrouqi, D., \& Vinogradov, J. (2016). Zeta potential in oil-water-carbonate systems and its impact on oil recovery during controlled salinity water-flooding. Scientific Reports, 6, 37363. doi:10.1038/srep37363

https://www.nature.com/articles/srep37363\#supplementary-information

Li, S., Collini, H., \& Jackson, M. D. (2018). Anomalous Zeta Potential Trends in Natural Sandstones. Geophysical Research Letters, 0(0). doi:doi:10.1029/2018GL079602

Li, S., Leroy, P., Heberling, F., Devau, N., Jougnot, D., \& Chiaberge, C. (2016). Influence of surface conductivity on the apparent zeta potential of calcite. Journal of Colloid and Interface Science, 468, 262-275. doi:10.1016/j.jcis.2016.01.075

Nono, F., Bertin, H., \& Hamon, G. (2014). An Experimental Investigation of the oil Recovery in the Transition Zone of Carbonate Reservoirs Taking Into Account Wettability Change. Paper presented at the International Petroleum Technology Conference, Doha, Qatar.

Vinogradov, J., Jaafar, M. Z., \& Jackson, M. D. (2010). Measurement of streaming potential coupling coefficient in sandstones saturated with natural and artificial brines at high salinity. Journal of Geophysical Research-Solid Earth, 115. Acceptance of Terms and Conditions:

Click here to agree

\section{Object-based modeling of braided river reservoir interbed con- strained by genetic density}

\author{
Author(s): Siyang Li ${ }^{\text {None }}$ \\ Co-author(s): Changbing Tian ; Xingjun Gao ; Zhuo Liu
}

Corresponding Author(s): 1sycugb@163.com

It mainly developed heavy oil reservoir in Fula North Oil Field in Sudan Muglad Basin. There were still considerable remaining oil in the braided river sandstone reservoir with high oil saturation due 
to the serious heterogeneity. This study aimed to establish a three-dimensional geological model of interbed in braided river sandstone reservoir, which included four steps. Firstly, the sequence, sedimentation, reservoir architectures, lithofacies and well patterns were analysed and different genesis of interbed with different geometric features, lithology and distirbution in braided river were identified based on the dynamic and static data including drlling core, cast thin sections, logging and production test. Secondly, the reservoir structure was built combinated with the lithofacies and the sequence which derived from the first step. Thirdly, density probability curve of different interbed were obtainted based on the statistics of geometric features and distirbution from well. Finally, under the constraints of density probability curve, based on the well data, the interbed model of the braided river reservoir was established using the object-based simulation method. The results showed there were three types of interbed that were the muddy interlayer developed in flood plains, argillaceous interbed developed between sand interval and argillaceous interbed developed in channel bar. the interbed with different genesis was different in their scale, continuty, physical property. Usually, the more continuous and thick the interbeds were, the better to the development. In the model, the interbed with different genesis were characterized in different sedimentary environment and showed different impacts on the development.

Procter and Gamble Student poster award:

I would like to compete in the Procter and Gamble Student award References:

Acceptance of Terms and Conditions:

Click here to agree

\title{
Calculating the exact volume of intersections between spheres and arbitrary polyhedra
}

\author{
Author(s): Weiwei $\mathrm{Li}^{1}$ \\ Co-author(s): Martin Brinkmann ${ }^{2}$; Michael Jung ${ }^{1}$; Ralf Seemann ${ }^{1}$; Stephan Herminghaus ${ }^{3}$ \\ ${ }^{1}$ Saarland University/MPIDS \\ ${ }^{2}$ Saarland University \\ ${ }^{3}$ MPIDS
}

Corresponding Author(s): weiwei.li@physik.uni-saarland.de, martin.brinkmann@physik.uni-saarland.de

Numerical models of immiscible fluid displacement in porous media often rely on an efficient and precise method to compute the intersection volume between spheres and the unit cells of a grid $[1,2]$. Here, we propose a robust and accurate approach to calculate the volume of intersections between spheres and general polyhedra of arbitrary topological genus. Applying the theorems of Gauss-Green and Gauss-Bonnet to a function whose divergence is unity inside the sphere but vanishes outside, we are able to express the total intersection volume by a sum of intersection volumes between the sphere and oriented elementary tetrahedra. Each tetrahedon is spanned by the three corners of a triangle and the center of the sphere. The results of extensive benchmark tests indicate that the numerical stability and accuracy of our method is superior to the decomposition of polyhedra into a number of shape primitives of known volume, as proposed in a recent publication 3 . Compared with previous approaches [2,3], our method is able to cover a wider range of size ratios between the dimension of a unit cell and the sphere diameter.

\section{Procter and Gamble Student poster award:}

I would like to compete in the Procter and Gamble Student award References:

1 H. Liu et al. "Multiphase lattice Boltzmann simulations for porous media applications-a review", Comput. Geosci. 20: 777-805 (2016).

2 B. D. Jones, J. R. Williams, "Fast computation of accurate sphere-cube intersection volume", Engineering Computations 34: 1204-1216 (2017). 
3 S. Strobl, A. Formella, T. Pöschel, "Exact calculation of the overlap volume of spheres and mesh elements", Journal of Computational Physics 311: 158 - 172 (2016). Acceptance of Terms and Conditions:

Click here to agree

\title{
Research on water and rock interaction of groundwater contam- ination in abandoned coal mine
}

\author{
Author(s): Xilin Li ${ }^{\text {None }}$ \\ Co-author(s): Laigui WANG ; Ling LIU ${ }^{1}$; Xiangfeng LIU \\ ${ }^{1}$ Liaoning Technical University
}

Corresponding Author(s): leexilin501@163.com

\begin{abstract}
Aiming at the existing problems of groundwater environment pollution in abandoned coal mines, taking Fuxin mining area - a representatively abandoned coal mining area in China as an object of study, the paper will study on the water and rock interaction mechanism of groundwater contamination in abandoned coal mines. The results of the study are as follows: (1) Tested by water immersion scanning electron microscopy, XRD analysis, white light speckle digital correlation method, soil column test and coupling model test, we studied the effect of mine water to the character of abandoned coal mine backfill, mudstone, sandstone and coal rock mechanics, and also analysed the rock stress variation, the characteristics of groundwater seepage and pollutant concentration changes, the water rock interaction characteristics and coupling characteristics, then the mechanism of water rock coupling of groundwater pollution is clarified. The results show that the mine water shows different mechanical damage to different rock mass ,and it also changes the physical, chemical and mechanical properties of rock mass; The process of infiltration of mine water from abandoned mine can cause pollution of groundwater in the aspects of total hardness, sulfate, chloride and iron.The heavy metal $\mathrm{Cr}(\mathrm{VI})$ was migrated by convection and diffusion in groundwater and the mechanism of pollution is mainly the migration, accumulation, adsorption and dissolution of soluble salts and ion exchange.(2)The governing equation of stress field of rock mass caused by mine drainage in abandoned coal mine,the groundwater seepage equation and the chemical field equation which is considered the convection dispersion, adsorption and desorption, precipitation and dissolution including supplies, chemical pollutants as source sink pollutants in the groundwater migration are established and coupled.A dynamic model of three - field coupling dynamics of groundwater pollution in abandoned coal mine is established .(3)Based on the coupled model,we simulated the groundwater pollution, pollution range, pollutant concentration distribution and development trend of Fuxin abandoned mine and combined with field monitoring data, the reliability of the model is verified. The findings provide scientific technical support for the protection of environment and ecological balance restoration in abandoned mining area.
\end{abstract}

Procter and Gamble Student poster award:

I don't want to compete References:

Acceptance of Terms and Conditions:

Click here to agree

927

\section{Heavy oil recovery and asphaltenes deposition mechanism in sol- vent based recovery technique}

Xuesong $\mathrm{Li}^{\text {None }}$; Steffen Berg ${ }^{\text {None }}$; Matthias Appel ${ }^{\text {None }}$

Corresponding Author(s): xuesong.x.li@shell.com, , 
In this study, micromodel experiments were used to assess the recovery factor and recovery rate of bitumen for different solvents. A solvent-vapor based technology for heavy oil and extra-heavy oil reservoirs gives promise to a substantially more energy efficient recovery. In this process, solvent vapor condenses on the immobile bitumen in the reservoir where a fraction of the bitumen is diluted by the solvent and recovered while the asphaltenes are intentionally precipitated and left behind. The produced oil quality is improved by the in-situ de-asphalting process, resulting in lower density, viscosity and heavy metal content. The recovery rate which - next to ultimate recovery - determines the overall economics and is controlled by processes in a very thin mixing zone where the condensed solvent diffuses into the bitumen, dissolves the less-heavy fraction and precipitates asphaltenes. The micromodel experimental results show that for propane the recovery factor is up to $70 \%$, and for pentane the factor is between $50 \%-65 \%$. The difference in recovery factor is related to the phase behavior and rheology of the precipitated asphaltenic phase which in the case of propane is a liquid and in the case of pentane is solid, which can cause plugging of the flow path. This study suggests that propane is the better candidate for the solvent-vapor recovery. The production from propane is comparably more stable, the recovery factor is higher, there is no evidence of severe asphaltene plugging to the production port.

Procter and Gamble Student poster award:

I don't want to compete References:

Acceptance of Terms and Conditions:

Click here to agree

\title{
Moisture and Heat Transfer in Layered soil: Implications for In- versely Estimating Layered Soil Hydraulic Property
}

\author{
Zhen $\mathrm{Li}^{\text {None }}$; Kathleen Smits ${ }^{1}$ \\ ${ }^{1}$ University of Texas Arlington \\ Corresponding Author(s): zhenli@mymail.mines.edu
}

Layered soil (i.e. vertical texture-contrast soil) is widely distributed in nature, as most soil systems are composed of soil layers, varying in types and compositions. Appropriate characterization of soil hydraulic properties is critical in the prediction of soil moisture, soil temperature, and soilatmosphere interactions, which further influences the hydrological cycle and energy cycle. Besides the normal soil sampling method, the inverse estimation strategy based on remotely sensed surface information (moisture, temperature, flux) is receiving more attention (Mohanty, 2013). While this method has been applied by various studies for vertically homogeneous soil, the question arises whether the inversely estimated effective soil properties can represent the characteristics of the layered soil (Dimitrov et al., 2015).

In this study, the bench-scale evaporation experiments were performed in the testing system consisting of a tank packed with varying layered sand configurations, a wind tunnel capable of simulating diurnal temperature environment and a sensor network which can measure variables in both the tank and in the free-flow. A model capable of simulating the coupled moisture-heat transport in layered soil was numerically solved and validated by comparing with the generated experimental data set. Then a series of field layered soil profiles under varying atmospheric forcing were simulated with the validated model, and were analyzed with the simulated surface information together.

Results show that, although it is difficult to use a single effective hydraulic parameter set to represent the layered soil system when a fine layer overlying a coarse layer $(\mathrm{F} / \mathrm{C})$, this property profile can be inversely estimated based on the evaporation flux or surface temperature of an entire drying cycle (fully saturated to dry condition). However, when a coarse layer overlies a fine layer, the soil property profile can hardly be inferred only based on surface information. Furthermore, if the inverse estimated property are used as the effective property, the evaporation flux can still be represented but may cause large deviation in terms of water storage prediction.

Procter and Gamble Student poster award:

References: 
1 Dimitrov, M., et al. "Soil hydraulic parameters of bare soil plots with different soil structure inversely derived from L-band brightness temperatures.” Vadose zone journal 14.8 (2015).

2 Mohanty, Binayak P. "Soil hydraulic property estimation using remote sensing: A review." Vadose Zone Journal 12.4 (2013). Acceptance of Terms and Conditions:

Click here to agree

\title{
Slip length of oil-water mixtures in organic nanopores
}

\author{
Author(s): Zheng Li ${ }^{1}$ \\ Co-author(s): Jun Yao ${ }^{2}$; Jianlong Kou ${ }^{3}$ \\ ${ }^{1}$ China University of Petroleum (East China) \\ ${ }^{2}$ China University of Petroleum \\ ${ }^{3}$ Zhejiang Normal University \\ Corresponding Author(s): 1zdyx361@163.com
}

Accurate characterization of oil flow through organic nanopores is significant to macroscale numerical simulation and enhance shale oil recovery. Since water can be present naturally and injected artificially underground, the previous studies investigating nanoscale flow of pure oil have much limitations. In this work, equilibrium and nonequilibrium molecular dynamic simulations have been used to study oil-water mixtures flow in organic nanopores and the slip length change with the mixture compositions is mainly focused on. With increasing the percentage of oil in a 6-nanometer pore, the slip length decreases initially. When the mass percent of oil is larger than $12 \%$, the slip length becomes a constant which is equal to that of pure oil. Based on the Green-Kubo relation, a mathematical relation between the slip length and the mixture composition has been proposed. Results of the mathematical model and molecular dynamics match well. The mathematical model is also applicable to nanopores with different sizes and is instructive to nanopores of different types.

Procter and Gamble Student poster award:

References:

Acceptance of Terms and Conditions:

Click here to agree

\section{Flow regulation analysis in rough fractures considering time vari- able conductivity}

Zongfa Li ${ }^{\text {None }}$

Corresponding Author(s): lizongfaupc@yeah.net

Under real reservoir condition, the fracture surface is not smooth and straight what's more, fracture conductivity is changing with time and spatial position because of stress change and fracture width change as a result of proppant sedimentation. These factors make it difficult to forecast production precisely. This paper estabilishes a fracture conductivity prediction model considering roughness of fracture surface, fracture tortuosity, fracture shape and conductivity loss caused by proppant change. Cone, fitting to fractal geometry characteristics, is used as roughness element to simulate roughness of fracture surface . Due to formation heterogeneity, fracture is not the straight one calculated by many fracture propagation models, Therefore, we extract an effective fluctuation angle from Barton's JRC fracture outline and derive an equation to describe the fracture outline's influence on conductivity .Considering shape variation of cross-section along the fracture ,We adjust the conductivity according to the variable fracture width along the fracture .we also build up an empirical 
equation according to the experiment during which fracture conductivity can drop to $10 \%$ of initial conductivity within 100 days, to embrace the conductivity loss caused by proppant change.

Based on the fracture conductivity prediction model and numerical simulation technique, we analyze the flow regulation difference under different well patterns, and indicate how the conductivity loss acts upon the flowing regulation. Compared with common fracture, It is found that flow flux in the model considering all the above factors is about at most $30 \%$ larger for the front $84 \%$ fracture yet it drops quickly for the following $16 \%$. Recovery rate near the fracture tip is lower, in the model considering all the above factors囚because lower conductivity at fracture tip due to narrower fracture cross-section shape at fracture tip weakens the fracture tip effect. What's more, recovery rate will be cut down by $7 \%$ percent because of heterogeneous and time-varying fracture conductivity .

In real reservoir condition, fracture conductivity is influenced by surface roughness, tortuosity, fracture shape and proppant change, which will change the flow flux distribution and reduce the recovery rate. Under real reservoir condition, Our research can help predict production rate for fractured wells accurately and contribute to adjust well distributions in order to maximize recovery rate.

\title{
Procter and Gamble Student poster award:
}

\section{References:}

1 Canals M., Meunier J D.. A model for porosity reduction in quartzite reservoirs by quartz cementation [J]. Geochemica et Cosmochimica Acta, 1995, 59(4): 699-709.

2 Cikes M.. Long-term hydraulic-fracture conductivities under extreme conditions [J]. Oil Production \& Facilities, 2000, 15(4): 255-261.

3Cobb S. L., Farrell J. J.. Evaluation of long-term proppant stability [A]. International Meeting on Petroleum Engineering [C]. Beijing, 1986.03.17-20.

[4] Garisto F.. Solid dissolution: Effect of mass transport-precipitation coupling [J]. Chemical Engineering Science, 1996, 41(12): 3219-3222.

5 McDaniel B. W.. Realistic fracture conductivities of proppants as a function of reservoir temperature [A]. Low Permeability Reservoirs Symposium [C]. Oakland, California, 1987.04.02-04. Acceptance of Terms and Conditions:

Click here to agree

\section{Data Assimilation Based on Ensemble Kalman Filters Using KL- Expansion and Stochastic Variable Transformation}

\author{
Author(s): Michael Liem ${ }^{1}$ \\ Co-author(s): Patrick Jenny ${ }^{1}$ \\ ${ }^{1}$ Institute of Fluid Dynamics, ETH Zurich \\ Corresponding Author(s): liemm@student.ethz.ch
}

Ensemble Kalman filters (EnKF) are widely used for history matching (respectively data assimilation) in the context of sub-surface flows. In general, the prime uncertainty lies in the permeability distribution, of which typically only sparse empirical data is available. Major difficulties are (i) proper regularization to avoid obtaining unphysical fields and (ii) dealing with non-Gaussian distributions of observed variables and model parameters (Vogt et al., 2012).

In this work, we consider cases where concentration measurements of an advected scalar are known at different times at selected locations. To integrate this empirical information, we calculate the Kalman update of the model parameters using all available measurements in time and space simultaneously. To deal with difficulty (i), we use KL-expansion to regularize the permeability fields as proposed by Chang and Zhang (2014). This has the additional and crucial advantage that the coefficients to be tuned by the EnKF are independent Gaussian variables. To deal with difficulty (ii), we apply a transformation of the concentration distribution in order obtain Gaussian observed variables. This is similar as presented in Schöniger et al. (2012), but here, instead of drawdown data for log-conductivity estimation, spatially and temporally correlated concentration values are used to estimate the KL coefficients. 
Comparative numerical studies are presented to show the effects of updating KL coefficients vs. permeability values directly, of the observation variable transformation and of considering temporal correlation of the observation variables in addition to their spatial correlation.

Procter and Gamble Student poster award:

I would like to compete in the Procter and Gamble Student award References:

CHANG, H. \& ZHANG, D. 2014. History matching of statistically anisotropic fields using the KarhunenLoeve expansion-based global parameterization technique. Computational Geosciences, 18, 265-282. doi:10.1007/s10596-014-9409-z

SCHÖNIGER, A., NOWAK, W. \& HENDRICKS FRANSSEN, H. J. 2012. Parameter estimation by ensemble Kalman filters with transformed data: Approach and application to hydraulic tomography. Water Resources Research, 48. doi:10.1029/2011WR010462

VOGT, C., MARQUART, G., KOSACK, C., WOLF, A. \& CLAUSER, C. 2012. Estimating the permeability distribution and its uncertainty at the EGS demonstration reservoir Soultz-sous-Forêts using the ensemble Kalman filter. Water Resources Research, 48. doi:10.1029/2011WR011673 Acceptance of Terms and Conditions:

Click here to agree

\section{Pore scale observations of the impact of wettability alteration on fluid interfacial curvature}

Qingyang $\operatorname{Lin}^{1}$; Branko Bijeljic ${ }^{2}$; Ronny Pini ${ }^{1}$; Martin Blunt ${ }^{1}$; Sam Krevor ${ }^{\text {None }}$

${ }^{1}$ Imperial College London

${ }^{2}$ Imperial College

Corresponding Author(s): q.lin11@imperial.ac.uk

The combination of pore-scale imaging with the measurement of Darcy-scale multiphase flow properties allows for a direct mapping of pore scale fluid morphology to macroscopic parameters like capillary pressure and relative permeability. In this study, we imaged the interfacial curvature of decalin-water interfaces within the pores of a Bentheimer sandstone. From the fluid curvature we inferred the local capillary pressure while simultaneous measurements of pressure drop allowed for a determination of relative permeability. To validate the workflow, a steady-state waterflood experiment was performed in a water-wet Bentheimer sandstone, where decalin and brine were simultaneously injected through the core at increasing brine fractional flows from 0 to 1 . The local saturation and the curvature of the oil-brine interface were imaged and determined from a wide field of view (10.4 $\mathrm{mm}$ in vertical length at a voxel size of $3.58 \mu \mathrm{m})$. The local capillary pressure was obtained using the Young-Laplace law and compared with independent core scale experimental measurements. The uncertainty of the curvature measurement has also been quantified, which is was up to approximately $9 \%$ with a minimum radius of 10 pixels.

Then the surface wettability of a Bentheimer sandstone was altered by sample ageing process with crude oil at $80{ }^{\circ} \mathrm{C}$ and $3 \mathrm{MPa}$. The same curvature measurement workflow was applied after the wettability alteration. The interfacial curvature and corresponding capillary pressures, as well as relative permeabilities, for this mixed-wet sample have been characterised and compared with the water-wet case. The fluid interface in a mixed-wet system was more complicated where both positive and negative principle curvatures resulting in a net zero curvature (and capillary pressure) have been measured (example interface in a mixed-wet system is shown in Figure 1). The overall measured capillary pressure of $0 \mathrm{kPa}$ from curvature measurement was also consistent with core-scale centrifuge measurement. The characterisation of the pore-scale fluid distribution and the fluid-fluid interface provide more insights for core analysis and enable a better understanding of the fluid displacement mechanisms in porous media.

Procter and Gamble Student poster award:

I would like to compete in the Procter and Gamble Student award References: 


\title{
Two-phase electrohydrodynamics in complex geometries - mod- elling and simulation
}

\author{
Gaute Linga $^{1}$ \\ ${ }^{1}$ SINTEF Digital \\ Corresponding Author(s): gaute.linga@gmail.com
}

Two-phase flow in porous media is associated with a number of important challenges, particularly since it constitutes a true multiscale problem where effects at the smallest scales are crucial to the overall macroscopic transport. At small scales, due to spatially inhomogeneous surface charge distributions and ions in solutions, electrokinetic effects come into play and influence the fluid flow.

In this talk, we discuss phenomenology and modelling strategies related to both single- and twophase electrohydrodynamic flow. To numerically resolve the governing equations, we introduce Bernaise - a numerical solver for electrohydrodynamic flow in complex geometries. In particular, we demonstrate for model systems how the combination of electric fields and ionic solutions can redirect flow patterns and alter the effective wetting properties.

The results show that the method can be applied to understand low salinity-enhanced oil recovery, but also that it can be used for systems outside geophysics, including modelling micro- and nanofluidic devices and electrocoalescence. We finally discuss improvements to the approach and suggest experiments where a direct comparison to theory and simulations is possible.

Procter and Gamble Student poster award:

References:

Acceptance of Terms and Conditions:

Click here to agree

\section{Coupling free flow and porous-medium flow: Comparison of so- lution strategies for solving saddle-point problems}

\author{
Author(s): Melanie Lipp ${ }^{1}$ \\ Co-author(s): Rainer Helmig ${ }^{1}$; Martin Schneider ${ }^{1}$; Bernd Flemisch ${ }^{1}$ \\ ${ }^{1}$ University of Stuttgart
}

Corresponding Author(s): melanie.lipp@iws.uni-stuttgart.de

Porous-medium flow is coupled to free-flow in a broad variety of medical, technical and environmental systems. Simulating the coupled flow and transport processes helps to understand e.g. evaporation dynamics from soils or some processes in fuel cells. So far, Fetzer et al. 1 have used a fully monolithic solution strategy for the coupled problem. The general goal is now to develop decoupling strategies in order to, among others, have smaller subsystems and be able to use different solvers suitable for the respective subsystems. This work presents a first step to decouple the system, which is to examine solvers suitable for the free-flow subsystem. 
One family of solution methods is algorithms of semi-implicit method for pressure-linked equations (SIMPLE) type, which are popular methods to solve the time-dependent, incompressible NavierStokes equations and which decouple pressure and velocity. Instead of solving the nonlinear, coupled system of the momentum and the mass balance simultaneously for the pressure and the velocity, every iteration step of the SIMPLE algorithm involves solving first one linear equation for a velocity correction and then another linear equation for a pressure correction. This way, velocity and pressure are corrected further in every iteration step, starting from an initial guess. The SIMPLE method, as well as some variants, have recently been implemented in the open source simulator DuMux 2 .

Different solvers and solution strategies for solving saddle-point problems, arising from the discretization of coupled problems, are presented and compared for various test cases, as for example for the lid-driven-cavity test case as well as for test cases based on Donea and Huberta 3 and Angeli et al. [4]

\title{
Procter and Gamble Student poster award:
}

\section{References:}

1 T Fetzer et al. FVCA, 347-356. Springer, 2017.

2 B Flemisch et al. Adv Water Resour, 34(9):1102-1112, 2011.

3 J Donea, A Huerta. Finite element methods for flow problems. John Wiley \& Sons, 2003.

[4] P E Angeli et al. FVCA, 181-202. Springer, 2017. Acceptance of Terms and Conditions:

Click here to agree

\section{Evaluation of pore space structure due to chemical dissolution of rock matrix}

\author{
Vadim Lisitsa $^{1}$; Dmitry Kolyukhin ${ }^{2}$; Kseniya Gadylshina ${ }^{2}$ \\ ${ }^{1}$ Novosibirsk State University and IPGG SB RAS \\ ${ }^{2}$ Institute of Petroleum Geology and Geophysics SB RAS
}

Corresponding Author(s): lisitsavv@ipgg.sbras.ru

\begin{abstract}
Standard approaches to the chemical transport at the Darcy scale resolve the porosity changes due to chemical fluid-matrix interaction, whereas permeability and diffusion are the model coefficients. However, they depend on the geometry of the pore space such as tortuosity, specific surface. Typically, empirical relations connecting porosity with all other parameters are used in modern reactive transport codes. Note that such relationships may not account for complex geometrical changes of pore space, for example, forming of wormholes leading to permeability anisotropy, which is not a subject of Cozeny-Carman relation (if the porosity-permeability connection is considered).

In our research, we present a numerical study of the pore space evolution for different flow and reaction rates. To simulate the reactive transport at the pore scale, we solve the Stokes equation (only low Reynolds numbers are subject of interest in underground transport). To account for the complex pore space geometry, when simulating fluid flow and reactant transport we use the immersed boundary conditions. Evolution of the pore space due to the chemical dissolution we apply the level-set method. Results of the simulation allow us to reconstruct the cross-correlation between porosity, permeability, specific surface area, and tortuosity for different flow regimes and reactions rates. Moreover, we manage to construct most probable "trajectories" in the phase space porositypermeability-specific surface area-tortuosity for different scenarios.
\end{abstract}

Procter and Gamble Student poster award:

References:

Acceptance of Terms and Conditions:

Click here to agree 


\title{
Coupling Continuum and Pore-Network Models to Study the Dis- persed Nanostructured Thin Film Electrodes of Polymer Electrolyte Fuel Cells
}

JIANGJIN LIU ${ }^{1}$; Ezequiel Médici ${ }^{2}$; Jeffrey S. Allen ${ }^{2}$; Andrew Haug ${ }^{3}$; Iryna Zenyuk ${ }^{4}$

\author{
${ }^{1}$ Tufts University \\ ${ }^{2}$ Michigan Technological University \\ ${ }^{3} 3 M$ \\ ${ }^{4}$ University of California, Irvine
}

Corresponding Author(s): jiangjin.liu@tufts.edu

Polymer electrolyte fuel cells (PEFCs) with high energy efficiency and zero tail-pipe emissions are promising replacement of the internal combustion engine. The dispersed nanostructured thin film (dNSTF) electrodes are developed by $3 \mathrm{M}$ and present a viable alternative to conventional electrodes with Pt nano-particles dispersed on carbon support. The nanostructured thin film (NSTF) electrodes are made of sputtered Pt film deposited on a perylene red support 1 . They are $0.5 \mu \mathrm{m}$ thick and showed to produce high power densities and mass activity. However, meeting the robustness targets remains a challenge, as these electrodes when implemented as cathode catalyst layer (cCL) suffer from water flooding at low temperatures when evaporative transport of water is low 2. To resolve the problem, $3 \mathrm{M}$ introduced dNSTF electrodes, where these Pt whiskers $(0.5 \mu \mathrm{m}$ in length $)$ are shaved off the liner and dispersed with ionomer and carbon support to make thicker, more hydrophobic cCLs. Understanding water management in cCL with these whiskers is critical as this specific cCL morphology has not been studied before.

We couple continuum and pore-network models to study the dNSTF electrodes' behavior during PEFC's operation to provide better understanding of the dNSTF's morphology and benefit of its design. The continuum model is a two-dimensional, two-phase, cross-sectional PEFC sandwich model3. It solves for gas, ion, electron, heat and water transport and also oxygen reduction reaction (ORR). The pore-network model (PNM) is used to better understand various physics processes in the dNSTF cCL on a pore-scale level. It is a two-dimensional network model that consists of a regular lattice of cylindrical tubes of constant length. The cylindrical tubes represent the PNM structure, which includes four components: ionomer, pore, support, catalyst. It solves for proton and water transport through the ionomer; water vapor, liquid water and oxygen transport through the pores; heat transport through the support. Its main advantage is the ability to show percolating water network for various current densities within the CL. In this coupled model, PNM focuses only on the cCL, whereas continuum model solves for all the other components.

The continuum model and PNM are coupled through boundary conditions. First, an initial guess of current density is put into the continuum model as a boundary condition. Then the continuum model is solved to get the CL-MEM and CL-MPL boundary conditions, e.g. the temperature, relative humidity and gas pressure. These boundary conditions serve as the input to PNM. A detailed simulation of the multi-physics processes is performed in PNM. The current density, heat flux, oxygen flux, vapor flux and water flux are evaluated and transferred into continuum model. Then the continuum model is calculated again with these inputs from PNM. The iteration loop is formed, and the convergence is studied.

The nano structure of the dNSTF electrodes is depicted by scanning electron microscopy (SEM). The PNM is constructed based on the morphological information evaluated from the SEM images. Then, the coupled model is applied to study the multi-physics processes. The study helps to better understand water management in dNSTF electrodes and its impact on fuel cell performance.

Procter and Gamble Student poster award:

I would like to compete in the Procter and Gamble Student award References:

1 M.K. Debe, Journal of The Electrochemical Society, 160 (2013) F522-F534. 
2 A.J. Steinbach, J.S. Allen, R.L. Borup, D.S. Hussey, D.L. Jacobson, A. Komlev, A. Kwong, J. MacDonald, R. Mukundan, M.J. Pejsa, M. Roos, A.D. Santamaria, J.M. Sieracki, D. Spernjak, I.V. Zenyuk, A.Z. Weber, Joule, 2 (2018) 1297-1312.

3 I.V. Zenyuk, P.K. Das, A.Z. Weber, Journal of The Electrochemical Society, 163 (2016) F691-F703. Acceptance of Terms and Conditions:

Click here to agree

\title{
A New Method for Analysis of Nano-Scale Dual-Pore-Shape Pore Size Distributions in shale and its application
}

\author{
Jianwu Liu ${ }^{1}$; Detang Lu ${ }^{1}$ \\ ${ }^{1}$ University of Science and Technology of China \\ Corresponding Author(s): ljw1005@mail.ustc.edu.cn
}

The conventional calculation methods of pore size distribution (PSD) are based on the simplistic assumption of single-pore-type. These methods may conclude inaccurate results when we deal with some heterogeneous porous materials like shale in nano-scale. We present a new dual-pore-shape method by utilizing both adsorption and desorption branches of nitrogen isotherms and deriving pore volume distributions of both cylindrical pores and slit pores. And this method will help us to better understand the micro-structure

Condensation occurs in capillary pores when the relative pressure reaches a critical value corresponding to the Kelvin radius. In fact, the adsorption branch and desorption branch of open cylindrical pores or open slit pores are not overlapped. They separate from each other forming a hysteresis loop. Meanwhile, there exist clear differences between the hysteresis loops of these two kinds of pores. Based on these facts, we develop the dual-pore-shape methods (DPS method) by utilizing both adsorption and desorption branches of the isotherms and deriving the volume distributions.

Obtaining the PSDs of both cylindrical pores and slit pores can be of great help to better understand shale reservoirs characteristics.

First, a more accurate mesopore volume distribution help us to better evaluate the gas storing and adsorption space at nano-scale. The comparison of pore volumes and specific surface areas obtained from $\mathrm{BJH}$ method and DPS method shows that it is easy to overestimate pore volume and specific surface area if only cylindrical pores are taken into account. Therefore, the gas content and ratio of free gas and adsorbed gas in shale reservoirs need to be re-estimated based on that.

Second, nano-scale pore structure and fractal characteristics are significant information to understand shale gas reservoirs. The correlations between them can now be meticulously classified into two categories, one with cylindrical pores, and the other with slit pores. The experiment results show that the connections in two shapes of pores can be very different, which can eventually reveal the unknown pore structure mystery.

Third, behaviors and properties of fluid flow in shale reservoirs cannot be simulated and predicted from traditional flow models because of the presence of nano-scale pore space. In this scale, pore geometry and pore size distribution can easily impact the flow in porous media. We provide a new approach to calculate apparent permeability of shale gas systems considering the factors of both two pore shapes and distribution of pore space simultaneously. The study processes show that considering dual-pore-shape pore size distributions have great impact on apparent permeability of shale in nano-scale.

Procter and Gamble Student poster award:

\section{References:}

Liu, J., Li, P., Sun, Z., Lu, Z., Du, Z., Liang, H. and Lu, D., 2017. A new method for analysis of dual pore size distributions in shale using nitrogen adsorption measurements. Fuel. 210: 446-454. Acceptance of Terms and Conditions:

Click here to agree 


\title{
Shale Oil Transport through Nano Slit of Kerogen by Molecular Dynamic Simulation
}

\author{
Author(s): JIe Liu ${ }^{1}$ \\ Co-author(s): Yongfei Yang ${ }^{2}$; Jun Yao ${ }^{3}$ \\ ${ }^{1}$ China University of Petroleum(East China) \\ ${ }^{2}$ China University of Petroleum (East China) \\ ${ }^{3}$ Research Centre of Multiphase Flow in Porous Media, China University of Petroleum (East China)
}

Corresponding Author(s): s18020122@s.upc.edu.cn, yangyongfei@upc.edu.cn

\begin{abstract}
In a context of further research for shale oil flow in unconventional reservoirs, the flowing machanism in organic matter (Kerogen) nano slit has to be studied. The nano slit is rough due to armorphous keorgen molecular structure, which impacts the stream law compared to the ideal model. In this paper, by means of molecular dynamic simulation, we unravel some patterns for transportation of shale oil that is multicomponent in realistic structure of kerogen. The kerogen matrix is freezed as the wall of slit, while the kerogen which close to slit is flexile to represent real condition of surface. Owing to tremendous adsorption effects of kerogen, the heavy components (n-octane,dodecane and asphalt) of oil are easy to get adsorbed on the surface of slit that hinder the transportation. The distribution of multicomponent has a impact on velocity profile, and potential of mean force (PMF) make it clear that light components (from methane to n-butane) are trend to assemble in the center of slit region that makes a parabolic profile for velocity. However, the unfixed kerogen layer affects the velocity of adsorbed layer, which enlarge gradient of velocity at the edge of slit. It is worthy to mention that analysis of energy can tell us the mechanism when macroscopic laws are invalid, which can explains the results of simulation precisely.
\end{abstract}

Procter and Gamble Student poster award:

References:

Acceptance of Terms and Conditions:

Click here to agree

\section{Hydrate growth in porous media - implications to the water per- meability of hydrate-bearing sediments}

\author{
${\text { Lele } \mathrm{Liu}^{1} \text {; Zhun Zhang }}^{\text {None }}$; Chengfeng $\mathrm{Li}^{\text {None }}$; Jianchao Cai ${ }^{\text {None }}$; Daigang Wang ${ }^{\text {None }}$; Changling Liu ${ }^{\text {None }}$ \\ ${ }^{1}$ The Key Laboratory of Gas Hydrate, Ministry of Natural Resources, Qingdao Institute of Marine Geology
}

Corresponding Author(s):

Natural gas hydrates have drawn enormous attention throughout the world as an alternative energy resource and as a potential trigger in submarine geohazards as well as global climate changes. Where and how natural gas hydrates form within pores play a critical role in pore-scale structures and corescale properties of hydrate-bearing sediments. In this study, natural gas hydrates are numerically simulated to grow within the pore space of marine sand slices obtained by an X-ray Computed Tomography (X-CT) scanner. Fractal parameters (i.e. area fractal dimension and maximum diameter) of the pore space in marine sands with coating and floating hydrates are characterized. These fractal parameters are furtherly extended to predict the water permeability of hydrate-bearing sediments. The result shows that: i) both the area fractal dimension and the maximum diameter of the pore space decrease with increasing hydrate saturation; ii) coating hydrates and floating hydrates have similar effects on the area fractal dimension evolution, but very different effects on the maximum diameter variation; iii) the maximum diameter is the key factor to the water permeability of hydrate-bearing sediments, while the area fractal dimension has minor effects on it. 
Procter and Gamble Student poster award:

References:

Acceptance of Terms and Conditions:

Click here to agree

\title{
Grain Boundary Effects on Mineral Dissolution in Porous Me- dia
}

Min Liu $^{1}$; Vitalii Starchenko ${ }^{1}$; Lawrence Anovitz ${ }^{2}$; Andrew Stack ${ }^{1}$

${ }^{1}$ Oak Ridge National Laboratory

${ }^{2}$ Oak Ridge National Laboratory

Corresponding Author(s): min6@outlook.com

Pore-scale reactive transport modelling has been attracting attention in mineral dissolution studies. However, the effect of anisotropic rock textures and chemical heterogeneity remains poorly understood. The goal of this research is to gain understanding of effects from nanoscale grain-boundary processes on macroscopic dissolution rates, wormhole formation and disaggregation in rocks. We employ reactive transport model based on the Darcy-Brinkman equation to simulate calcite dissolution in fractured media. Besides, the grain boundary dissolution-induced physical grain detachment with subsequent grain transport is included into simulation framework. Fractured media with different grain boundaries are generated. The effect of grain size distribution on dissolution are also investigated. Our preliminary results show that grain detachment can induce a significant decrease in permeability due to the pore clogging. We have also found that porous media with more uniform grain sizes have a higher average reaction rate than broad distributions of grain sizes

Procter and Gamble Student poster award:

References:

Acceptance of Terms and Conditions:

Click here to agree

\section{Pore-scale investigation of Bingham fluids flow through tight sand- stone on representative element volume}

\author{
Moran Wang ${ }^{\text {None }}$; Tong Liu ${ }^{\text {None }}$ \\ Corresponding Author(s): t-liu15@mails.tsinghua.edu.cn,
}

Deviation from Darcy's law for single phase flow can be caused by non-Newtonian viscosity of fluids. This non-Newtonian viscosity property can be from its origin bulk property or be a consequence of the confined pore space. Non-Newtonian property caused by confined pore space can lead to non-linear relationship between flow rate and pressure difference when Newtonian bulk fluid flows through tight rock samples. To investigate the non-Newtonian fluid flow through tight rock sample, a pore-scale investigation is conducted with applying Finite Volume Method (FVM) calculation on tight sandstone structures scanned by Computed Tomography (CT). For present study, the bulk non-Newtonian property is considered to investigate whether the sample is representative for this pore-scale modeling. The results show that when Bingham fluid flows through rock sample, the flow rate increases nonlinearly when pressure gradient is small and then it increases linearly with the pressure gradient, thus an effective permeability and start-up pressure gradient can be used to depict the flow. By conducting flow simulations at varying sample sizes, the representative element 
volume (REV) is obtained for effective permeability and start-up gradient. It is found that the REV size for effective permeability is the same as that of absolute permeability when Newtonian fluid is driven through the sample. For REV size of start-up pressure gradient, the size is much smaller than that of permeability. With the results, it is concluded that samples that are large enough to reach REV size for permeability can be used to investigate Bingham fluids flow through the sample as well. In the future work, non-Newtonian viscosity property related to pore size will be considered in this representative sample to investigate the flow rate and pressure gradient relation and the REV size in this case.

Procter and Gamble Student poster award:

I would like to compete in the Procter and Gamble Student award References:

Acceptance of Terms and Conditions:

Click here to agree

438

\title{
Poro-Elasto-Plastic Modeling of Hydraulic Fracture Propagation in Naturally Fractured Deep Shale Reservoir
}

\author{
Author(s): Wenzheng Liu $^{1}$ \\ Co-author(s): Jun Yao ${ }^{1}$ \\ ${ }^{1}$ China University of Petroleum (East China)冈Research Centre of Multiphase Flow in Porous Media
}

Corresponding Author(s): upc_lwz@163.com

With the increasing demand of oil and gas resource in the world, the development of deep shale reservoir has become an inevitable trend. For the development of shale reservoir, the hydraulic fracture pattern and geometry are the key factors to determine the stimulation efficiency. It is essential to accurately predict the behavior of hydraulic fractures based on the understanding of fundamental mechanisms governing the process. The prevailing approach for hydraulic fracturing simulation relies on linear-elastic fracture mechanics(LEFM), which can predict hydraulic fracture propagation in hard rock. In deep reservoir, however, shale deformation may change from brittleness to ductility under high stress, high pressure and high temperature. LEFM fails to give accurate predictions of fracture propagation in ductile shales, even in form of simple planar geometry. In order to investigate the coupled effects of rock elasto-plastic deformation and fluid flow in the process of hydraulic fracturing in deep shale reservoir, a fully coupled poro-elasto-plastic model for fracture propagation based on the theories of finite discrete element method (FDEM), cohesive zone model (CZM) and Drucker-Prager plasticity is presented, which could be able to capture hydraulic fracture geometry and rock plastic deformations. The model is verified against other analytical solutions of fracture propagation, stress concentration and classical consolidation problem. The effects of rock plasticity on the hydraulic fracture propagation are investigated. In addition, the interaction between natural fracture and hydraulic fracture in plastic formation is also studied. The results indicate that plastic deformations in ductile shale has a significant impact on final fracture geometry and fracturing pressure.

Procter and Gamble Student poster award:

References:

Acceptance of Terms and Conditions:

Click here to agree 


\title{
Study on modification experiment of pore structure of coal by response surface optimization method
}

\author{
Xiangfeng LIU ${ }^{\text {None }}$
}

Corresponding Author(s): 305066889@qq.com

\begin{abstract}
Coal has the complex pore structure and low permeability, it is often accompanied by accidents such as coal and gas outburst, gas explosion in the process of gas drainage. When the surfactant was immersed and penetrated into the coal, the penetration of the pores and cracks in the coal were increased, and the permeability of coal also be increased. Therefore, it is of great significance to study the effect of surfactant on the porosity of coal. In this paper, the long-flame coal was used as research object, and SDS solution was used as added surfactant. The effects of SDS solution mass concentration, modification time and modification temperature on the porosity of coal were determined by single factor experiment. However, The single factor could not analyze the continuous point, and there were limitations. Therefore, the Box-Behnken experiment design was used to optimize the modification conditions, and the porosity change of the modified coal was analyzed. The results showed that the single factor experiment initially determined the modification conditions were $0.5 \mathrm{wt} . \%$ of SDS solution mass concentration, $48 \mathrm{~h}$ of the modification time and 40 ${ }^{\circ} \mathrm{C}$ of the modification temperature. After the optimization of the response surface experiment, the modification conditions were $0.5 \mathrm{wt}$.\% of SDS solution mass concentration, $44 \mathrm{~h}$ of the modification time and $38{ }^{\circ} \mathrm{C}$ of the modification temperature. There were three parallel experiments were carried out to test the porosity of the coal under these conditions. It can be found that, the real porosity of the coal was $33.29 \%$, which was close to the predicted value $(33.37 \%)$. The results show that the fitting degree of the model was good and the modification conditions can be optimized well.
\end{abstract}

Procter and Gamble Student poster award:

References:

Acceptance of Terms and Conditions:

Click here to agree

\section{Characterization of pore interconnectivity and adsorption-induced deformation in mesoporous thin films by ellipsometry porosime- try : an experimental study}

\author{
Jérôme Loizillon ${ }^{1}$; David Grosso ${ }^{2}$ \\ ${ }^{1}$ IM2NP Aix-Marseille University \\ ${ }^{2} I M 2 N P$
}

Corresponding Author(s): jerome.loizillon@gmail.com

Thin films with ordered mesoporosity and controlled pore size and geometry can be elaborated on small and large surfaces through sol-gel process combined with evaporation-induced self-assembly (EISA) of templating agents. The assessment of this porosity is necessary to understand their interaction with their environment in many applications such as microelectronics, sensors or optics. While inert gas physisorption techniques are often used to characterize bulk nanoporous materials, they lack the sensitivity required to investigate thin films. SEM and TEM are also used to image nanoporous materials but the samples preparation require a considerable amount of time and often involve milling steps that will damage the porous structure.

Ellipsometry is a non-destructive spectroscopic characterization in which a polarized beam of light is reflected on the sample and recovered in a polarization sensitive analyser. The modification of the polarization allows the determination of the refractive index, extinction coefficient, thickness and porosity of thin layers by fitting with theoretical models. Ellipsometry can be combined with an environmental cell to observe in situ the adsorption of solvents, for instance water or alcohols. This technique, called ellipsometry porosimetry, can be used to determine the pore size distribution, give critical insights on surface chemistry, pore organization and interconnections which are not easily 
accessible by other techniques. Additionally; the adsorption-induced deformation of the nanoporous network due to capillary forces can be followed by ellipsometry using the variations of the thin film thickness.

In this oral communication, experimental work is presented where different types of self-assembled mesoporous silica thin layers are investigated by ellipsometry porosimetry. A new technique, desorption scanning, is employed to get additional information on the pore connectivities. Additionally, the adsorption-induced deformations of the thin films are exploited to determine their elastic properties and calculate their pore load modulus.

Procter and Gamble Student poster award:

I would like to compete in the Procter and Gamble Student award References:

Acceptance of Terms and Conditions:

Click here to agree

\title{
Adsorption-induced deformation in mesoporous materials: com- parison between pore-load modulus and Young's modulus
}

Jérôme Loizillon ${ }^{1}$; David Grosso ${ }^{2}$

\author{
${ }^{1}$ IM2NP Aix-Marseille University \\ ${ }^{2}$ IM2NP
}

Corresponding Author(s): jerome.loizillon@gmail.com

Metal oxides with ordered mesoporosity and controlled pore size and geometry can be elaborated through sol-gel process combined with evaporation-induced self-assembly (EISA) of templating agents.

Due to the confinement of the nanopores, capillary condensation of an adsorbate such as water occurs at a vapor partial pressure considerably lower than its saturated vapor pressure. This is accompanied with formation of menisci inside the pores and leads to adsorption-induced deformation of the whole porous network. These deformations are often purely elastic and can be exploited to determine the pore-load modulus of the porous material. In the case of a mesoporous thin film, the pore-load modulus can be obtained by the ellipsometry technique which determines the refractive index, porosity and thickness of the film. Additionally, since the Young's modulus of a porous thin film allows the assessment of its mechanical properties, obtaining an estimation of the latter from the pore-load modulus would be desirable.

In this poster, the deformation of mesoporous materials under adsorption stress will be explained and experimental work where the pore-load modulus of mesoporous silica thin films is determined by ellipsometry will be presented. Additionally, the pore load modulus will be compared with the Young's modulus obtained by nanoindentation measurements and their respective evolution with the porosity will be shown. The influence of the relative humidity on the determination of Young's modulus by nanoindentation will also be discussed.

will be shown. The influence of the relative humidity on the determination of Young's modulus by nanoindentation will also be discussed.

\section{Procter and Gamble Student poster award:}

I would like to compete in the Procter and Gamble Student award References:

\section{Acceptance of Terms and Conditions}

Click here to agree 


\title{
Pore-filling model for micro-structure of cement hydration using level-set
}

\author{
Nguyen-Tuan Long ${ }^{1}$; Christiane Roessler ${ }^{2}$; Merlin Etzold ${ }^{3}$; Horst-Michael Ludwig ${ }^{4}$ \\ ${ }^{1}$ Bauhaus-Universität Weimar \\ ${ }^{2}$ Bauhaus University Weimar \\ ${ }^{3}$ University of Cambridge, UK \\ ${ }^{4}$ Bauhaus-University Weimar
}

Corresponding Author(s): long.nguyen.tuan@uni-weimar.de

Tricalcium silicate (Ca3SiO5) is the most important constituent of Portland cement with a mass fraction between 50 and $70 \%$. Mixing tricalcium silicate with water results in fast dissolution of ions followed by massive precipitation of calcium silicate hydrates (C-S-H). The growing C-S-H phases subsequently fill the capillary pores between tricalcium silicate particles (Gartner et al, 2000). This is the origin of strength development of all calcium-silicate based cements.

Here we introduce a pore-filling model at micro-scale based on level-set propagation (Osher, S. \& Sethian, 1988), which can mimic the process of C-S-H precipitation and filling of pore space. Furthermore, the growth of C-S-H in parallel with the dissolution/reduction of tricalcium silicate particles is considered. In addition, as known from electron microscopic investigations two types of C-S-H are formed, i.e. one dense C-S-H layer that envelops unhydrated tricalcium silicate particles directly and a fibrous $\mathrm{C}-\mathrm{S}-\mathrm{H}$ that grows into the larger pores. Both types of C-S-H are numerically simulated in a representative elementary domain. The capillary pore-size distribution from numerical simulation is compared with those obtained experimentally (scanning electron microscopy imaging and mercury intrusion porosimetry). The densification process of C-S-H phases during the hydration process can also be compared with time-resolved X-ray diffraction and nuclear magnetic resonance observed.

Procter and Gamble Student poster award:

\section{References:}

Gartner, E.; Kurtis, K. \& Monteiro, P. Proposed mechanism of C-S-H growth tested by soft X-ray microscopy. Cement and Concrete Research, 2000, 30, 817 - 822.

Roessler, C.; Steiniger, F. \& Ludwig, H. Characterization of C-S-H and C-A-S-H phases by electron microscopy imaging, diffraction, and energy dispersive X-ray spectroscopy. J. Am. Ceram. Soc., 2017, 100, 1733-1742

Osher, S. \& Sethian, J. A. Fronts propagating with curvature-dependent speed: Algorithms based on Hamilton-Jacobi formulations. Journal of Computational Physics, 1988, 79, 12 - 49 . Acceptance of Terms and Conditions:

Click here to agree

\section{Experimental Investigations of Nanopore Adsorption Measure- ment by Low-field Nuclear Magnetic Resonance}

\author{
Peixue Jiang ${ }^{1}$; Ruina $\mathrm{Xu}^{1}$; Taojie $\mathrm{Lu}^{1}$ \\ ${ }^{1}$ Tsinghua University
}

Corresponding Author(s):

In recent ten years, shale gas has become more and more popular in the world because of its reach storage and cleanliness compared with traditional fossil fuels like coal. Gas shale is a porous rock composed primarily of compacted fine-grained clay particles which typically has porosoties less than $10 \%$, pore diameters of a few nanometers. Different from traditional natural gas, a large amount of methane is existed of adsorption state in the nanopores of shale. Thus, the measurement technique 
of adsorption methane in the shale nanopores is of great significance for future exploitation of shale gas.

However, there is currently limited understanding of the mechanism of absorption, especially in nanopores under $10 \mathrm{~nm}$. The traditional experiment investigation of adsorption process like volume method or weight method struggles in distinguishing the two phases of adsorption gas and free gas in nanopores.

Here, we try to find an effective quantitative measuring method for gas adsorption. NMR technology is a potential technique in the field of oil and gas exploration, especially Low-field NMR technology can quantitatively detect hydrogen-containing fluids in porous media without damage, and the transverse relaxation time of porous media is contributed by different atoms of different environments. In our study, we built an online NMR measurement system in order to measure the adsorption characteristics of nanopores in porous media. The NMR system we built mainly contains high pressure gas circuit up to $10 \mathrm{MPa}$, oil circuit of fluorine oil which provides a confining pressure and temperature environment, and a sample chamber of PEEK material. We use CPMG sequence to measure T2 map. The longest relaxation peak corresponds to the bulk methane in the free space of the sample cavity, and the adjacent middle peak corresponds to the methane in the pores of the particle packed bed, and may also include the methane signal of a large part of the sample. The shortest T2 peak is caused by methane in the shale pores. Compared with the solid background, the T2 time of the detected gas signal is about $0.35-1.35 \mathrm{~ms}$, and the corresponding area increases with the increase of pressure.

Combined with volumetric method and nuclear magnetic resonance technology, we can measure the shale isotherm adsorption. The advantage of this method is that the NMR experiment can distinguish the adsorption state and the free state to a certain extent. This work helps us to explore the mechanism of adsorption of methane in shale under different pressures or temperatures, which will be helpful to the shale gas exploitation in the future.

Procter and Gamble Student poster award:

I don't want to compete References:

Acceptance of Terms and Conditions:

Click here to agree

\title{
Insights into evaporation from the surface of capillary porous media gained by discrete pore network simulations
}

\author{
Author(s): Xiang $\mathrm{Lu}^{1}$ \\ Co-author(s): Abdolreza Kharaghani ${ }^{2}$; Evangelos Tsotsas ${ }^{2}$ \\ ${ }^{1}$ Otto von Guericke University Magdeburg \\ ${ }^{2}$ Otto von Guericke University Magdeburg
}

Corresponding Author(s): xiang.lu@ovgu.de

Understanding and prediction of the mass exchange between a heterogeneous capillary-porous medium surface and the adjacent gas-side boundary layer is still one of the remaining scientific challenges associated with evaporation of porous media. One rational way to tackle this issue is to opt for a meso-scale pore-network model. Such a model permits to explicitly represent the porous medium surface in a discrete way. Thus, the number of wet and dry patches on the surface and their statistical distribution during the evaporation process can be quantified. Our recent study conducted by three-dimensional pore network simulations indicates that both types of patches (wet and dry) contribute to the mass transfer from the surface 1 . This finding is in contrast with the Schlünder's classical model, which assumes that the contribution of dry pores to the mass exchange at the surface is negligible compared to the evaporation rate from the wetted area 2 . In this work, we push our previous study based on pore network simulations forward and thus derive a functional form of the mass transfer coefficient that depends on the surface saturation and on the pore structure. Moreover, a function is built between the vapor partial pressure at the surface and the surface saturation by pore network simulations. The results obtained in this study provide valuable insights into 
evaporation from the porous medium surface that can be used to improve the continuum modeling of the evaporation process.

\section{Procter and Gamble Student poster award:}

I would like to compete in the Procter and Gamble Student award References:

1. Attari Moghaddam A., Kharaghani A. Tsotsas E., Prat M. A pore network study of evaporation from the surface of a drying non-hygroscopic porous medium. AIChE Journal, 2018, 64(4):14351447.

2. Schlünder E.U. On the mechanism of the constant drying rate period and its relevance to diffusion controlled catalytic gas phase reactions. Chemical Engineering Science, 1988, 43(10):2685-2688.

Acceptance of Terms and Conditions:

Click here to agree

482

\section{Linearization and Domain Decomposition Methods for Two-Phase Flow in Porous Media}

Stephan Lunowa ${ }^{1}$

${ }^{1}$ Hasselt University, Computational Mathematics (CMAT)

Corresponding Author(s): stephan.lunowa@uhasselt.be

In this work, we discuss the mathematical model of two-phase flow in porous media including dynamic capillarity and static hysteresis, where the domain is composed of two regions with possibly different parameterizations leading to discontinuities in the coefficient values at the interface. These nonlinear differential equations within the subdomains are coupled by the continuity of the flux and the pressures at the interface. Based on this, we discretize the system in time by the implicit $\theta$-scheme and introduce two iterative schemes for finding the solutions to the semi-discrete equations, which combine a stabilized linearization iteration of fixedpoint type (L-scheme) and a non-overlapping domain decomposition method with Robin type transmission conditions. The proposed methods are independent of the concrete space discretization and avoid the use of derivatives as in Newton based iterations. In the subsequent analysis, we prove for both schemes the existence and uniqueness of solutions and rigorously show the convergence of the sequence of iterative solutions towards the solution of the semi-discrete equations under some mild constraints on the time step independently of the initial guesses. Finally, we present several numerical studies in two spatial dimensions to validate the stability, robustness and efficiency of the developed methods. The results confirm our theoretical analysis, and clearly demonstrate the practical advantages of the second scheme over the first.

Procter and Gamble Student poster award:

References:

Acceptance of Terms and Conditions:

Click here to agree

20

\section{An Ensemble-based Learning Framework For History Matching With Imperfect Forward Simulators}

Xiaodong Luo ${ }^{1}$ 
${ }^{1}$ Norwegian Research Centre (NORCE)

Corresponding Author(s): xluo@norceresearch.no

Objectives/Scope: Numerical models are widely deployed to tackle real-world challenges. For instance, in 4D seismic history matching problems, forward reservoir simulators and rock physics models play important roles. Model imperfection inevitably exists in such models, yet it remains to be a challenging problem that may substantially affect history matching results. Our objective is thus to develop an ensemble-based learning framework to handle model imperfection in history matching problems.

Methods, Procedures, Process: We divide our investigations into two steps. Firstly, we will study a class of supervised learning problems (SLP), and elaborate the similarities between SLP and the conventional variational data assimilation (VAR-DA) problems. This perspective naturally motivates us to develop an ensemble-based approach to solving SLP, as it inherits all the advantages of ensemblebased data assimilation approaches over VAR-DA methods. Secondly, we will show the link between SLP and the problem of identifying model imperfection, and thus apply the ensemble-based learning framework to handle model imperfection in history matching problems.

Results, Observations, Conclusions: We design two sets of experiments to demonstrate the performance of the ensemble-based learning framework. In the first experiment, we apply the proposed framework to a supervised learning problem, in which we aim to recover the underlying function that maps a set of inputs to another set of noisy outputs (labels). Experiment results indicate that the proposed framework not only correctly recovers the mapping function, but also provides good estimations of the associated uncertainties. In the second experiment, we adopt the proposed framework to handle model imperfection in a history matching problem. Our results show that, in all tested cases, the proposed framework helps improve the performance of history matching algorithms.

Novel/Additive Information: The novelties of the current work consist of the following two aspects. One is that, conventional machine learning algorithms typically do not deliver estimations of uncertainties associated with their final products. Here, we illustrate how uncertainty qualification can be conducted by solving SLP through an ensemble-based learning algorithm. The other is that, from the perspective of SLP, we develop simpler and more accurate approaches to handling model errors than existing methods.

Procter and Gamble Student poster award:

\title{
References:
}

Acceptance of Terms and Conditions:

Click here to agree

\section{Precisely Studies on Petrophysical Parameters and Interface Prop- erties of Cores from Tight Oil Reservoirs}

\author{
Author(s): Yutian Luo ${ }^{\text {None }}$ \\ Co-author(s): zhengming yang ${ }^{1}$; yapu zhang \\ ${ }^{1}$ PetroChina Research Institute of Petroleum Exploration \& Development
}

Corresponding Author(s): luoyutian@petrochina.com.cn

Tight oil reservoirs have got extremely low permeability and porosity, with complex microscopic pore structures and fine pore throats, relatively large force of fluids acting on the rock surface of the reservoir, and uncertain regularities of oil \& water distribution. Currently, tight oil reservoirs are mainly developed in the mode of quasi-natural energy exploitation using staged fracturing horizontal wells, where production declines rapidly, and the conventional water-flooding energy supplementation mode can be hardly effective. It is planned to carry out studies on microscopic pore 
structures of tight oil reservoirs using high and new technological approaches in the project to reveal fluid occurrence in full-scale pore space and percolation mechanism in micro-nano scale of tight oil reservoirs; proposed a new method for testing wettability of tight oil reservoirs, disclosing mixed wettability and a quantitative characterization of oil wet and water wet degree of tight oil reservoirs; build a relationship between NMR spectrum and fluid viscosity, establishing a mathematical model for viscosity and T2 spectra, drawing distribution of viscosity in typical tight oil reservoirs; with the adoption of surface work function and surface charge to evaluate interface properties of reservoirs, and proposed an optimized method for fracturing fluid formulation. The outcomes of this study will play an important fundamental role for the establishment of a full scale mathematical model for pores seepage flows, preferred selection of effective tight oil reservoir development modes and proposal of reasonable reservoir stimulation methods.

Procter and Gamble Student poster award:

References:

Acceptance of Terms and Conditions:

Click here to agree

514

\section{One-domain approach for immiscible two-phase dispersed flow in stratified porous media}

O. A. Luévano-Rivas ${ }^{1}$; Roel Hernandez-Rodriguez ${ }^{2}$; J. Alberto Ochoa-Tapia ${ }^{3}$

${ }^{1}$ Univerdad Autónoma Metropolitana

${ }^{2}$ Universidad Autónoma Metropolina

${ }^{3}$ Universidad Autónoma Metropolitana

Corresponding Author(s): iq.luevano@gmail.com

The importance of the immiscible two-phase dispersed flow (or emulsion flow) in porous media is present in several areas which include chemical engineering process, environmental sciences, petroleum recovery, among others. Recently, for the case of immiscible two-phase dispersed flow in homogeneous porous media, a multiphasic Darcy's law has been developed using the method of the volume averaging and under the assumption the local mechanical equilibrium is valid; the model is given in a closed scheme where the effective permeability can be computed by the solution of closure problems in representative unit cells 1 . However, this model is not necessarily valid near the porous media boundaries due to rapid changes of the effective properties (i.e. permeability, porosity or average velocity) occur in this zone, the so-called inter-region. In this work, a generalized transport equation is developed to analyze the immiscible two-phase dispersed flow between homogeneous regions, in particular two adjacent porous media. This model is developed upscaling the microscopic equations that describe this type of flow using the method of the volume averaging. In this formulation, the local mechanical equilibrium, which considers that only one average velocity is sufficient to describe the multiphasic flow, is assumed to be valid in the inter-region. The model obtained consists of a macroscopic equation for the equilibrium velocity, in terms of positiondepend coefficients, which resembles a Darcy-Brinkman equation and includes terms that reflect the interaction of the immiscible phases. Hence, only one equation is sufficient to describe the multiphasic flow in the inter-region and the homogenous regions under the one-domain approach 2 . The effective coefficients are computed on basis of the direct numerical solution of the microscopic equations using representative geometries of the inter-region. This solution domain requires to be large enough to represent the multiphase flow in this zone. The validation of the ODA model is carried out by the evaluation of the profile equilibrium velocity with those obtained via DNS. The results barely show any difference between the velocity profiles predictions from the ODA with respect the DNS. In addition, this work proposed the alternative methodology for the predictions of effective coefficients obtained by solving associated local closure problems in representative domains of the inter-region

Procter and Gamble Student poster award: 
I would like to compete in the Procter and Gamble Student award References:

1 O.A. Luevano Rivas and F.J. Valdés-Parada, Upscaling immiscible two-phase dispersed flow in homogeneous porous media: A mechanical equilibrium approach, Vol. 126, 116-131, 2015, Chemical Engineering Science.

2 Goyeau, B., Lhuillier, D., Gobin, D., \& Velarde, M. G. (2003). Momentum transport at a fluid-porous interface. International Journal of Heat and Mass Transfer, 46(21), 4071-4081. Acceptance of Terms and Conditions:

Click here to agree

\title{
Operator-based linearization method for modelling of multiphase flow with buoyancy and capillarity
}

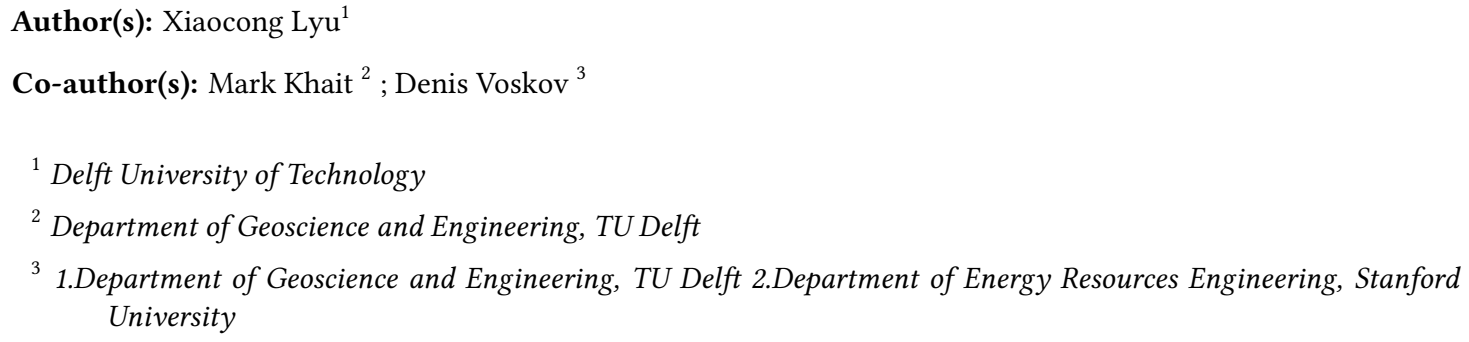

Corresponding Author(s): x.lyu@tudelft.nl

\begin{abstract}
Numerical simulation of coupling multiphase multi-component flow and transport in porous media is a crucial tool for understanding and forecasting of complex industrial applications related to the subsurface. The discretized governing equations become a highly nonlinear problem which usually needs to be solved with Newton's method and corresponds with high computational cost and complexity. With the presence of capillary and gravity forces, the nonlinearity of the problem amplifies even farther which corresponds with a larger number of nonlinear iterations. A recently proposed Operator Based Linearization (OBL) reduces the nonlinearity of complex physical problems. The OBL approach transforms the discretized mass conservation equations to space-dependent and state-dependent operators. While space-depended operators are treated conventionally, the statedepended operators are approximated by discrete representation on a uniform mesh in parameterspace. These state-dependent operators rely on current physical properties (e.g. density, viscosity, relative permeability) which represent the most nonlinear part of governing equations. The continuous representation of these operators is achieved through multilinear interpolation which provides a unique tool for approximate representation of exact physics of the problem. In this work, the OBL approach is then extended for multiphase multi-component isothermal systems with buoyancy and capillarity. Our numerical examples demonstrate that the extended OBL scheme significantly improves the computational efficiency with the accuracy of the conventional approach.
\end{abstract}

Procter and Gamble Student poster award:

I would like to compete in the Procter and Gamble Student award References:

(1) Voskov, D.V., 2017. Operator-based linearization approach for modeling of multiphase multi-component flow in porous media. Journal of Computational Physics, 337, pp.275-288.

(2) Khait, M. and Voskov, D., 2018. Adaptive Parameterization for Solving of Thermal/Compositional Nonlinear Flow and Transport With Buoyancy. SPE Journal. Acceptance of Terms and Conditions:

Click here to agree

\section{Numerical calculation of effective thermal conductivity for aero- gel porous materials at high temperature}




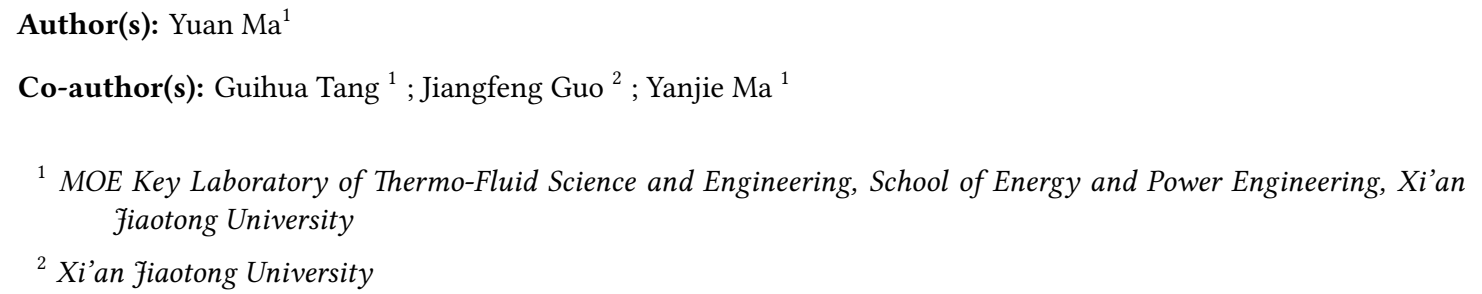

\section{Corresponding Author(s): 1298327127@qq.com}

Aerogels are porous materials with super insulation properties benefiting from the high porosity and the nanoscale pore size, making broad application in aeronautic and aerospace engineering and other fields. Fiber-loaded silica aerogels have a complex porous structure. The thermal conductivity of silica aerogel materials can be obtained by constructing silica aerogel materials and further calculating the effective thermal conductivity numerically.

In this work, an effective thermal conductivity of pure silica aerogels and fiber-loaded silica aerogels which combine heat conduction with radiation is investigated by numerical calculation. The pure silica aerogel structure model is established by DLA (Diffusion-limited Aggregation) method to simulate the real structure of pure silica aerogels. The structure model of fiber-loaded silica aerogels with different loading modes is also established. The spectral extinction coefficient of pure silica aerogels is calculated by GMM (Generalized Multiparticle Mie-solution) algorithm. The radiation heat flux of silica aerogel materials is calculated by two flux radiation model. Then, the effective thermal conductivity of silica aerogel materials is calculated by Fourier heat conduction equation coupled radiation heat flux. The numerical results provide an effective design for fiber-loaded silica aerogels. The results show that the fiber can effectively reduce the thermal conductivity of silica aerogels at high temperature. The fiber extinction coefficient decreases as the fiber incident ratio increases, and increases as the incident angle increases. The effective thermal conductivity of fiber-loaded silica aerogels can be reduced by moderately increasing the fiber content.

Procter and Gamble Student poster award:

References:

Acceptance of Terms and Conditions:

Click here to agree

\section{Research on influential characters of low- permeability coal rock under microwave radiation heating}

Yulin $\mathrm{Ma}^{\text {None }}$

Corresponding Author(s): hxwyaoyao@163.com

The application of microwave radiation technology to low- permeability coal-bed methane mining is a new research direction. In order to study the efficiency of low - permeability coal-bed methane by microwave heating, the temperature distribution range and influence law in low - permeability coal rock under microwave heating were analyzed by using different power microwave heating coal experiment and comparative numerical simulation method. The permeability and heating experiment under different microwave power and different radiation time conditions are carried out by a selfdesigned microwave radiation-infiltrating gas-permeable test system. The results show that under microwave radiation conditions, the microwave power determines the speed of heating, the higher the temperature, the power on the heating rate of the more obvious impact. And microwave heating efficiency is high, and the velocity of heat transfer is 1.5 times faster than that of electric heating. The relationship between microwave power and the coal temperature is linear. The microwave heating temperature distribution is chiefly characterized by regional and uneven. The highest temperature is nearby microwave port, and the temperature decreased rapidly with the increase of the distance. The maximum temperature of coal sample increases with the increase of frequency in the four frequency bands of $0.915 \mathrm{GHz} / 2.45 \mathrm{GHz} / 5.8 \mathrm{GHz} / 22.125 \mathrm{GHz}$, and the minimum value increases first and then decreases. The relationship between permeability and effective stress follows a negative exponential function, the fitting result R2 is greater than 0.98; The Permeability increases monotonously 
with the increase of microwave power and radiation time, and the linear fitting slope of data growth in the low effective stress region is greater than the high effective stress region; Under the radiation of microwave, the same energy input, the higher the microwave power, the greater the permeability of the coal sample. The results will provide a theoretical reference for the application of microwave radiation technology in the field of coal-bed methane extraction.

Procter and Gamble Student poster award:

I don't want to compete References:

Acceptance of Terms and Conditions:

Click here to agree

\title{
New Yield Stress fluid porosimetry Method (YSM)
}

\author{
Terence Emery MACKAYA ${ }^{1}$; Azita Ahmadi $^{2}$; Abdelaziz Omari $^{3}$ \\ ${ }^{1}$ PhD Candidate, I2M-TREFLE, ENSAM Bordeaux-Talence. \\ ${ }^{2}$ ENSAM - I2M \\ ${ }^{3} \mathrm{I} 2 \mathrm{M}$
}

Corresponding Author(s): terence.mackaya@u-bordeaux.fr

Imaging techniques like micro-tomography and Mercury Injection Porosimetry (MIP) are among the most utilized to characterize porous samples in terms of their pore-size distribution (PSD). While the former requires very expensive equipment, the latter uses a fluid with toxic vapors. Because of such reasons, research efforts have been dedicated to finding a non-toxic and cheaper alternative to these types of porosimetry techniques.

In this perspective, over the past decade, both theoretical and experimental improvements have been made in an innovative technique called the Yield Stress fluid porosimetry Method (YSM). Its main principle is that since Yield Stress Fluids (YSF) cannot flow below a certain stress threshold, by measuring the evolution of the flow rate versus the pressure gradient across a porous sample, its PSD can be retrieved by an inversion procedure 1 . In order to perform this inversion, the porous sample has been idealized as a bundle of rectilinear capillaries with circular cross-sections.

Different inversion methods have been developed in the literature (2, 3). The first objective of this talk is to present a new numerical inversion method, allowing to determine the PSD of a sample when characterized by the injection of a Herschel-Bulkley fluid. This method is based on GründwaldLetnikov partial derivatives, and has the advantage of being valid for a wider range of fluidity indexes than the one proposed by Oukhlef et al. 2 .

Furthermore, in an attempt to be more representative of real pore space when characterizing rock samples by YSM, more complexity has been introduced in our bundle of rectilinear capillaries by considering angular cross sections, thus allowing dead zones in the corners.

In order to do so, a set of flow rate/pressure gradient relationships have been derived for the flow of Herschel-Bulkley fluids in rectilinear capillaries of square and equilateral triangular cross sections. The approach adopted is based on two concepts: the critical Bingham number of a YSF, and the shape factor of a given rectilinear capillary (5, [6]). Numerical simulations performed with Openfoam have confirmed the reliability of those relationships, for a wide range of Bingham numbers.

Within the framework of YSM, those relationships offer the new possibility to model porous samples as bundles of capillaries with non-circular cross-sections. Therefore, the second objective of this presentation is to highlight the impact of the cross-sectional shape of capillaries on the PSD obtained by the inversion procedure.

Finally, it is well known that the characterization of rock samples by imaging techniques often reveals non-circular pore throats [4]. Therefore, the derived flow rate/pressure gradient relationships may bring new possibilities for the study of the flow of yield stress fluids in pore network models.

Procter and Gamble Student poster award:

References: 
1 Ambari, A., Benhamou, M., Roux, S., et Guyon, E., «Pore size distribution in a porous medium obtained by a non-Newtonian fluid flow characteristic,» C. R. Acad. Sci. Paris, t. 311, série II, pp. 1291-1295, (1990).

2 Oukhlef, A., Champmartin, S., et Ambari, A., Yield stress fluids method to determine the pore size distribution of a porous medium. Journal of Non-Newtonian Fluid Mechanics, vol. 204, p. 87-93, (2014).

3 Rodriguez de Castro, A., Omari, A., Ahmadi, A. et Bruneau, D, Towards a new method of porosimetry: principles and experiments. TiPM, 101, 349-364. (2014).

[4] Gundogar, A., Ross, CM ., Akin, C., Kovscek, AR., Multiscale pore structure characterization of middle east carbonates, Journal of Petroleum Science and Engineering, Vol 146 (2016).

5 Saramito, P. \& Roquet, N., An adaptive finite element method for viscoplastic fluid flows in pipes, Comp. Meth. Appl. Mech. Eng. 190 (40/41) 5391-5412 (2001).

[6] Huilgol R.R. A systematic procedure to determine the minimum pressure gradient required for the flow of viscoplastic fluids in pipes of symmetric cross-section. Journal of non-newtonian fluid mechanics, 136(2-3):140-146, (2006). Acceptance of Terms and Conditions:

Click here to agree

742

\title{
Viscous fingering in a soft porous medium
}

\author{
Christopher W. MacMinn ${ }^{1}$; Jian Hui Guan ${ }^{1}$ \\ ${ }^{1}$ University of Oxford
}

Corresponding Author(s): christopher.macminn@eng.ox.ac.uk

\begin{abstract}
Viscous fingering is a classical hydrodynamic instability that occurs when an invading fluid is injected into a porous medium or Hele-Shaw cell containing a more viscous defending fluid. Viscous fingering is an undesirable feature of many industrial processes, and various strategies have been proposed for suppressing or otherwise controlling it. In a Hele-Shaw cell, for example, viscous fingering can be suppressed by replacing one of the rigid walls with a flexible membrane. The result is a complex fluid-structure interaction problem, the dominant feature of which is inflation of the flow area near the inlet (high pressure) relative to the outlet (low pressure). The resulting negative permeability gradient is ultimately responsible for suppressing the instability. Here, we constrain this problem by working in a system that is deformable but not "inflatable" - A novel Hele-Shaw cell in which one of the rigid walls is coated with soft elastomer. Our system allows for local expansion of the flow area in response to the fluid pressure, but prohibits the generation of a global permeability gradient. We show that the two-way coupling between flow and deformation is nuanced in this constrained system, such that the instability is modified but not necessarily suppressed by softness. We then consider the implications of these results for two-phase flows through soft porous media.
\end{abstract}

Procter and Gamble Student poster award:

I don't want to compete References:

Acceptance of Terms and Conditions:

Click here to agree

41

\section{Direct Pore-scale modelling of $\mathrm{CO} 2$ solubility and mineral trap- ping}

Julien Maes ${ }^{1}$ 
${ }^{1}$ Heriot-Watt University

Corresponding Author(s): j.maes@hw.ac.uk

Solubility and mineral trapping are the two essential mechanisms for the long term security of geological CO2 capture and storage in saline aquifers. When modelling these processes, dissolution of $\mathrm{CO} 2$ in the surrounding brine is often assumed to be instantaneous with equilibrium phase partitioning. However, recent experiments in sandstone core samples have shown the importance of pore-scale concentration gradient. Therefore, resolving the underlying multiphysic equations at the micro-scale is critical to better constrain macro-scale models.

In this work, we present a fully coupled two-phase reactive multicomponent pore-scale model based on Direct Numerical Simulation of the Navier-Stokes equations using the Volume-Of-Fluid method. Mass transfer across fluid/fluid interfaces is accounted for using the Continuous Species Transfer method and the resulting phase change is computed and injected within the phase distribution equation. Chemical reactions in the water phase are modelled using the geochemical solver Phreeqc and mineral precipitation is accounted for by moving the solid interfaces using the Arbitrary Eulerian Lagrangian method. The model is validated by comparison with analytical solutions of simple set-ups. Then, the approach is used to study and upscale $\mathrm{CO} 2$ trapping in 2D micromodels.

Procter and Gamble Student poster award:

References:

Acceptance of Terms and Conditions:

Click here to agree

768

\section{Pore Network Simulation of Biogenic Gas Nucleation and Migra- tion in Porous Media}

Nariman Mahabadi ${ }^{1}$; Daehyun Kim ${ }^{\text {None }}$; Jaewon Jang ${ }^{2}$; Leon van Paassen ${ }^{1}$

\footnotetext{
${ }^{1}$ Arizona State University

${ }^{2}$ Hanyang University
}

Corresponding Author(s): nmahabad@asu.edu

Pore-scale simulations of various bio-geochemical processes such as mineral precipitation or dissolution and biomass growth or decay have been investigated in the literature. However, up to the author's knowledge, there is no available pore-scale model to simulate the formation and hydrodynamic behavior of biogenic gas bubbles in porous media. This is mainly due to the complex interactions governing the behavior of gas bubbles in porous media including nucleation, growth, migration, coalescence, and trapping. These processes are affected by the biogas generation rate, the distribution of reactive sites, and the pore-scale characteristics of the sediment. In this study, the behavior of gas bubbles in porous media is simulated using a pore-network model extracted from the 3D X-ray images of natural sediments. The classical nucleation theory is employed to simulate the nucleation and growth of biogenic gas bubbles. Several numerical algorithms and criteria are developed to model the expansion of gas bubbles by solute diffusion, size-dependent rising velocity of gas bubbles, bubble coalescence, slug formation, and movement, and bubble escaping and trapping in the pore space. The amount of produced gas bubbles, residual gas saturation, and hydraulic conductivity are also calculated during the simulations.

\section{Procter and Gamble Student poster award:}

I don't want to compete References:

Acceptance of Terms and Conditions:

Click here to agree 


\title{
An Integrated History-Matching of Enhanced Oil Recovery and Geochemical Processes for Carbonated Water Injection in Car- bonate Rocks
}

\author{
Author(s): Pedram Mahzari ${ }^{1}$; Thomas Mitchell ${ }^{1}$ \\ Co-author(s): Eric Oelkers ${ }^{1}$; Adrian Jones ${ }^{1}$ \\ ${ }^{1}$ University College London
}

Corresponding Author(s): p.mahzari@ucl.ac.uk

During carbonated water injection (CWI) and CO2-WAG scenarios, dynamic transfer of CO2 from aqueous phase into resident oil can cause complex $\mathrm{pH}$ variations, which can affect mineral dissolution around wellbore and precipitation in reservoir. Also, transfer of $\mathrm{CO} 2$ from carbonated water into live oil can liberate light components of the oil, which create gas phase in-situ. In this investigation, a novel methodology was developed to numerically simulate these complex processes in an integrated approach.

Taking account of CWI data of coreflood experiments performed on a carbonate rock under 3100 psi and $100 \mathrm{oC}$, profiles of ionic concentration of produced brine and rock weight during CWI into a dry core (in the absence of oil) were history-matched to tune reaction parameters. Also, two coreflood experiments performed to evaluate additional oil recovery by CWI were history-matched to obtain $\mathrm{CO} 2$ transfer parameters and relative permeabilities. Using the tuned parameters in a reservoir model, a series of simulations of CWI was performed to analyse wellbore stability and reservoir fluid flow due to dissolution and precipitation of calcite and anhydrite.

Calcite, dolomite, and anhydrite reaction parameters (surface area, volumetric content of mineral, and activation energy) were tuned to match $\mathrm{Ca}++, \mathrm{Mg}++$, and $\mathrm{SO} 4-2$ profiles. Despite XRD analysis and ESEM images, the effective surface area of anhydrite mineral was increased by $30 \%$ to match SO4-2 profile. On the other hand, for matching $\mathrm{Mg}++$, activation energy of dolomite reaction was increased by $23 \%$ to match dissolution at temperature of $100 \mathrm{oC}$. For additional oil recovery and $\mathrm{dP}$ profiles, secondary and tertiary CWI experiments were matched simultaneously to obtain relative permeabilities and $\mathrm{CO} 2$ mass transfer parameters.

The results of the sector model have demonstrated that dissolution of minerals would take place mostly in the vicinity of the injection wellbore. Local grid refinement was incorporated to capture the rock dissolution and in-situ gas formation around the wellbore. Taking into account the dissolution, wellbore stability analysis was performed, which indicated that extended injection of CWI (more than 5 years) may result in wellbore failure. However, the simulation results demonstrated that intermittent injection of plain water cycles would prevent wellbore failure, while significant additional oil recovery is achieved. For the precipitation throughout the reservoir, the simulation results showed that the permeability impairment would be insignificant.

Using the integrated approach to couple geochemistry and fluid flow, this study could present a step closer to realistic evaluations of dynamic $\mathrm{CO} 2$ transfer from carbonated water to resident oil and consequent interactions with the reactive minerals in a carbonate reservoir. The understanding produced in this work can be employed for practical implementation of CWI and CO2-WAG scenarios where low $\mathrm{pH}$ brine would interact with the rock while additional oil recovery is obtained.

Procter and Gamble Student poster award

References:

Acceptance of Terms and Conditions:

Click here to agree

\section{Two-Phase Flow Upscaling For Fractured Reservoirs}

Author(s): Christine Maier ${ }^{1}$

Co-author(s): Rafael March ${ }^{1}$; Florian Doster ${ }^{1}$; Sebastian Geiger ${ }^{1}$ 
${ }^{1}$ Heriot-Watt University

\title{
Corresponding Author(s): c.maier@hw.ac.uk
}

Simulation of multi-phase flow in fractured reservoirs is still challenging. For extensively fractured reservoirs, it is a common procedure to average the fractures into an effective medium. Accurate prediction of the flow in the upscaled medium relies on estimation of multi-phase flow properties such as upscaled relative permeability. Very little is known about the relative permeability of a fracture network and how it is related to the relative permeability of each individual fracture. Existing studies have shown that the relative permeability of a single fracture may be non-linear and is a function of its aperture. A fracture network will typically have a large range of aperture values. Moreover, it is well known that fracture apertures are sensitive to changes of in-situ stresses and fracture fluid pressures. In this work, we aim to understand the impact of heterogeneity in the fracture aperture distribution on the upscaled relative permeability of the fracture system. We use flow-based upscaling in synthetic fracture networks to compute the upscaled effective relative permeability. Here, the non-linear power law coefficient for the relative permeability of each fracture is related to its aperture via a theoretical correlation established in the literature. The findings of this study raise awareness of the intricate relationship between single fracture and fracture network multi-phase properties that may significantly affect the results of numerical simulation involving flow in fractured porous media.

Procter and Gamble Student poster award:

References:

Acceptance of Terms and Conditions:

Click here to agree

\section{Simulation of Flow and Transport in Microfractures using Dis- crete Fracture Network Approach}

\author{
Author(s): Nataliia Makedonska ${ }^{1}$ \\ Co-author(s): Satish Karra ${ }^{1}$; Hari S. Viswanathan ${ }^{1}$ \\ ${ }^{1}$ Los Alamos National Laboratory \\ Corresponding Author(s): nataliia@lanl.gov
}

Microfractures are small cracks and fractures, mostly visible under magnification, having lengths of millimeters or less and apertures generally less than $0.1 \mathrm{~mm}$. Microfractures are critical to a wide range of applications. For example, microfractures are a significant part of flow and transport pathways in an oil and natural gas reservoir, where flow mixing and fluid/gas blocking processes take place. Most numerical subsurface flow and transport simulations of fractured rock ignore implicit representation of microfractures in a model and the contributions from microfractures are often upscaled in the matrix parameters.

We present Discrete Fracture Network (DFN) simulations of microfractures at mm-scale performed using the dfnWorks simulation toolkit, where each microfracture is represented individually as $2 \mathrm{D}$ planar object in 3D simulation domain. DFN approach is known to be an accurate numerical approach for understanding the underground flow and transport in fractured rock, where fractures provide dominant flow and transport pathways. Recently developed at Los Alamos National Laboratory the computational suite based on DFN model, dfnWorks, has been successfully applied for studying contaminant transport in nuclear waste repository, $\mathrm{CO} 2$ sequestration and extraction of unconventional hydrocarbons at large scale.

In our simulations, the microfracture intensity in the DFN model varies representing different micro structure configurations. In each DFN configuration steady state pressure solution is obtained and particle tracking transport is used to model non-reactive tracer transport. Time Domain Random Walk (TDRW) technique is used to simulate particle movement through microfractures, where a particle's travel time is controlled by both processes, advection and diffusion. The simulated penetration profile of particles migration through micro structure is compared to those obtained in the 
Long-Term Diffusion Experiment (LTDE), conducted at the Äspö Hard Rock Laboratory, Sweden. The DFN simulation results show similar behavior with LTDE experimental penetration plot, where the advection term plays a significant role in the transport modeling, emphasizing the importance of microfractures in subsurface flow and transport simulations.

Procter and Gamble Student poster award:

I don't want to compete References:

Acceptance of Terms and Conditions:

Click here to agree

491

\title{
Particle tracking velocimetry for the multiphase fluid flow through a three-dimensional porous micromodel
}

\author{
Sojwal Manoorkar ${ }^{\text {None }}$; Simon Franchini ${ }^{1}$; Sam Krevor ${ }^{\text {None }}$ \\ ${ }^{1}$ Imperial College London \\ Corresponding Author(s): s.manoorkar@imperial.ac.uk
}

\begin{abstract}
Multiphase flow in permeable media is important in many industrial and natural processes such as water infiltration in soils, oil recovery from reservoir rocks and geological CO2 sequestration. As visualization is difficult in the actual rocks due to the opacity, we built an optically transparent micromodel. The microporous media is fabricated by sintering the glass beads inside the rectangular borosilicate glass capillary. The refractive index of the glass beads is matched with the wetting phase and non-wetting phase fluids to obtain the detailed pore-scale flow visualization. Polystyrene particles of diameter $2 \mu \mathrm{m}$ are used as the tracer for the particle tracking velocimetry. Experiments are carried out for a range of capillary number, viscosity ratio, and flow rate ratio. We use a novel technique based on generalized Gaussian feature extraction for particle tracking using only basic microscope components. We obtain the velocity magnitude and the velocity components both along and transverse to the imposed flow direction.
\end{abstract}

Procter and Gamble Student poster award:

References:

Acceptance of Terms and Conditions:

Click here to agree

\section{The impact of fracture slip on the fracture contact pressure}

\author{
Rafael March ${ }^{1}$; Florian Doster ${ }^{\text {None }}$ \\ ${ }^{1}$ Heriot-Watt University \\ Corresponding Author(s): rm208@hw.ac.uk
}

Simulation of fluid flow in fractured porous media under geomechanical forces has received considerable attention in the past few years due to its potential applications to the exploration of unconventional resources, enhanced geothermal systems and safety of $\mathrm{CO} 2$ storage operations. Accurate predictions of flow rates in fracture networks rely on the estimation of the aperture of each individual fracture. A common proxy for fracture aperture in mechanically-sensitive fracture networks is the normal contact pressure, which is the pressure experienced by the stressed fracture and is a common input for experimental models that relate the normal stress at the fracture to the hydraulic aperture. 
Accurate prediction of the contact pressure requires the implementation of complex non-linear contact mechanics algorithms, that prevent the penetration of the computational cells connected to the fractures. It is commonly assumed that the fracture contact pressure is significantly impacted by the normal and tangential displacements of neighboring fractures. In particular, the slip of a certain fracture may open or close neighboring fractures due to complex mechanical couplings. In this work we provide a quantitative assessment of the impact of fracture slip on the individual fracture apertures and on the upscaled fracture network permeability. We present a simple contact mechanics algorithm that was implemented in an open-source reservoir simulation package. We then evaluate the contact pressure distributions and the upscaled permeabilities for different fracture networks under different stress conditions. The understanding provided by this study will hopefully provide an insight about contact mechanics in fractured media that may motivate simplified implementations of contact algorithms.

Procter and Gamble Student poster award:

References:

Acceptance of Terms and Conditions:

Click here to agree

\title{
The Multiscale Restriction Smoothed Basis Method for Thermal Water-flooding in Porous Media
}

\author{
Author(s): Hassanali Marefat ${ }^{\text {None }}$ \\ Co-author(s): Olav Møyner ${ }^{1}$; Knut-Andreas Lie ${ }^{2}$ \\ ${ }^{1}$ SINTEF, NTNU \\ ${ }^{2}$ SINTEF
}

Corresponding Author(s): halimarefat@gmail.com, knut-andreas.lie@sintef.no

Thermal reservoir simulation plays a crucial role in hydrocarbon production, especially for heavy oil and deep-water reservoirs, but can be computationally time-consuming. So-called multiscale methods have been developed to accelerate the simulation of industry-standard black-oil and compositional simulation. Herein, we are presenting an extension of the state-of-the-art multiscale restrictedsmoothed basis (MsRSB) method to thermal water-flooding.

Our starting point is a fully implicit (FI) discretization of the two-phase thermal system, implemented using the object-oriented, automatic differentiation (AD-OO) framework of the open-source MATLAB Reservoir Simulation Toolbox (MRST). This constitutes a precise solution with a huge computer memory that will result in inefficiency. To accelerate simulation and reduce memory consumption, we formulate a sequential solution strategy that decouples the FI residual equations into separate equations for pressure, temperature, and transport of fluid phases and components, and then solve the three subsystems in sequence, possibly within an outer loop to ensure that the resulting solution converges to that of the full FI system.

The key idea of the MsRSB method is to numerically compute a set of basis functions that represent the effect of unresolved heterogeneities in a localized manner. Using these, we can restrict the pressure and thermal subsystems from the representative fine-scale grid to a coarse-scale grid with fewer unknowns and still obtain viscous and thermal fluxes that are mass conservative and have fine-scale resolution.

To validate the efficacy of the thermal MsRSB method, we present a series of thermal water-flooding test cases in which we compare the multiscale solutions and the sequential splitting solutions and demonstrate a very good match, even for complex and realistic reservoir models. The multiscale solutions are not only accurate, but also require noticeable less runtime.

The novelty of our work is the inclusion of a temperature equation in the MsRSB method for waterflooding simulation.

Procter and Gamble Student poster award:

I don't want to compete References: 


\title{
Acceptance of Terms and Conditions:
}

Click here to agree

62

\section{Analyzing tissue remodeling in avascular tumors}

Pietro Mascheroni ${ }^{1}$

${ }^{1}$ Helmholtz Center for Infection Research

Corresponding Author(s): pietro.mascheroni@helmholtz-hzi.de

Mechanical factors play a critical role in tumor development and response to treatment. This is evident in tumors grown in vivo, where cancer cells interact with the different components of the host tissue. Mathematical models are able to characterize the mechanical response of the tumor and provide a deeper understanding of these interactions. We present a model for tumor growth based on porous media mechanics. We consider a biphasic system, in which tumor cells and the extracellular matrix constitute a solid scaffold, filled with interstitial fluid. A nutrient is dispersed into the fluid phase, supporting the growth of the tumor. The internal reorganization of the tissue in response to external mechanical and chemical stimuli is described by enforcing the multiplicative decomposition of the deformation gradient tensor. In this way, we are able to distinguish the contributions of cell proliferation, rearrangement of cellular bonds, and elastic distortion occurring during tumor evolution. Results are shown for the growth of a tumor in a host tissue, focusing on the effects of different yield stresses on tumor progression.

Procter and Gamble Student poster award:

References:

Mascheroni, P., Carfagna, M., Grillo, A., Boso, D. P., Schrefler, B. A. (2018). An avascular tumor growth model based on porous media mechanics and evolving natural states. Mathematics and Mechanics of Solids, 23(4), 686-712. Acceptance of Terms and Conditions:

Click here to agree

471

\section{Pore-by-pore investigation of multiphase flow characteristics us- ing dynamic micro-computed tomography}

\author{
Arjen Mascini $^{1}$; Tom Bultreys ${ }^{1}$; Stefanie Van Offenwert ${ }^{1}$; Stephan Lunowa ${ }^{2}$; Iuliu Sorin Pop ${ }^{2}$; Veerle Cnudde \\ ${ }^{1}$ Ghent University, Centre for X-Ray Tomography (UGCT), Pore-scale Processes in Geomaterials Research Team \\ (PProGRess) \\ ${ }^{2}$ Hasselt University, Computational Mathematics (CMAT)
}

Corresponding Author(s): arjen.mascini@ugent.be

Multiphase flow has a wide range of applications in geoscience and engineering, including groundwater remediation and Carbon Capture and Storage (CSS). Recent developments in synchrotron (Berg et al., 2013) and lab based X-ray micro tomography (mCT) (Bultreys et al., 2016) have allowed to image multiphase flow in rock samples in near real time. While this has shed more light on the pore-scale dynamics, an all-encompassing theory which bridges the physics at this scale to the Darcy scale is still lacking. A crucial missing link is an empirical understanding of the evolution of the fluid distribution based on an appropriate measurement of the local pore geometry and wettability. Previous work which assessed the experimental repeatability of fluid distributions suggests that this should be approached in a stochastic framework (Bultreys et al., 2018). 
In this work, we address this problem by analyzing unsteady state displacements of brine by ndecane in porous samples (sintered glass). This process was continually imaged using an in-house developed mCT scanner (EMCT, (Bultreys et al., 2016)) with a high temporal resolution (between $12 \mathrm{~s}$ and $90 \mathrm{~s}$ acquisition time). The fluid distribution during the experiment was measured on a pore-by-pore basis. Contact angles and fluid-fluid interfacial curvatures of the non-wetting phase (AlRatrout et al., 2017) were tracked over time and linked to the geometrical descriptors and fluid invasion of the associated pores and throats. Finally, the non-wetting phase Euler characteristic was investigated. Special attention was given to the impact of the reduced signal-to-noise ratio which is commonly associated with fast imaging on the image analysis methodology proposed here. Followup experiments will be targeted at a similar analysis of imbibition and at the validation of pore-scale simulations of multi-phase flow.

\title{
Procter and Gamble Student poster award:
}

\section{References:}

AlRatrout, A., Raeini, A.Q., Bijeljic, B., Blunt, M.J., 2017. Automatic measurement of contact angle in pore-space images. Adv. Water Resour. 109, 158-169. https://doi.org/10.1016/j.advwatres.2017.07.018

Berg, S., Ott, H., Klapp, S.A., Schwing, A., Neiteler, R., Brussee, N., Makurat, A., Leu, L., Enzmann, F., Schwarz, J.-O., Kersten, M., Irvine, S., Stampanoni, M., 2013. Real-time 3D imaging of Haines jumps in porous media flow. Proc. Natl. Acad. Sci. 110, 3755-3759. https://doi.org/10.1073/pnas.1221373110

Bultreys, T., Boone, M.A., Boone, M.N., De Schryver, T., Masschaele, B., Van Hoorebeke, L., Cnudde, V., 2016. Fast laboratory-based micro-computed tomography for pore-scale research: Illustrative experiments and perspectives on the future. Adv. Water Resour. 95, 341-351. https://doi.org/10.1016/j.advwatres.2015.05.012

Bultreys, T., Lin, Q., Gao, Y., Raeini, A.Q., AlRatrout, A., Bijeljic, B., Blunt, M.J., 2018. Validation of model predictions of pore-scale fluid distributions during two-phase flow. Phys. Rev. E 97. https://doi.org/10.1103/PhysRevE.97.0 Acceptance of Terms and Conditions:

Click here to agree

\section{In-situ investigation of aging protocol effects on relative perme- ability measurements using high throughput experimentation meth- ods}

\author{
Matthieu Mascle ${ }^{1}$; Souhail Youssef ${ }^{1}$; Hervé Deschamps ${ }^{1}$; Olga Vizika ${ }^{1}$; Matthieu Mascle ${ }^{2}$ \\ ${ }^{1}$ IFP Energies Nouvelles \\ ${ }^{2}$ IFP Energies Nouvelles
}

Corresponding Author(s): matthieu.mascle@ifp.fr, mascle.matthieu@laposte.net

To be representative, relative permeabilities have to be measured at reservoir conditions. Among these conditions, the core wettability is a first order parameter that will strongly affect and control fluid distributions in the porous medium. A widely used method to restore wettability is aging core samples with crude oil. Yet, the result is dependent on many parameters such as crude oil composition, aging time and temperature as well as fluid distribution and aging protocols. In this study, we have investigate the effect of two aging protocols on oil/water relative permeabilities using small core samples (10 $\mathrm{mm}$ in diameter and $20 \mathrm{~mm}$ in length) and high throughput coreflood experimentation (HTCE).

Experiments were conducted on a set of initially strongly water-wet outcrop sandstone sample (Bentheimer and Berea). Small core samples were initially saturated with brine and then drained to Swi with crude oil using centrifugation. In the first protocol (static), samples were immerged in crude oil and aged at $80^{\circ} \mathrm{C}$ for three weeks. In the second protocol (dynamic) sample were mounted in a hassler type cell and continually flooded with crude oil at $80^{\circ} \mathrm{C}$ for three weeks. Steady-state relative permeabilities were then measured using a state of the art experimental setup (CAL-X). Using this setup, a typical coreflood experiment can be run in less than a day. The setup is equipped with an X-Ray radiography facility, enabling monitoring of 2D saturation profiles in real-time and thus giving access to fluid flow paths during flooding. 
Aged samples relative permeabilities curves show clear differences when compared to water-wet relative permeabilities, hence suggesting that the wettability has been effectively altered. However, the two aging protocols were unable to produce the same results. Dynamic aging produces symmetrical relative permeability curves, suggesting an intermediate-wet system, whereas the static aging protocol renders the curves more water-wet. The differences can be explained by analyzing a 2D saturation map. In the case of dynamic aging we observe a homogeneous distribution of fluids saturation during fractional flow. On the other hand, the static protocol results in heterogeneous flow paths, confirming that this protocol did not alter uniformly the wettability of the sample and generates a patchier system.

This study shows the potential of 2D in-situ observation of flow paths to detect wettability alteration irregularities and will help to improve our understanding of the mechanisms of laboratory wettability alteration. On top of that, HTCE will also enable optimization of time-consuming laboratory procedures and workflows.

Procter and Gamble Student poster award:

References:

Acceptance of Terms and Conditions:

Click here to agree

273

\title{
Pushing of a liquid drop through a non-wettable porous media
}

Maciej Matyka ${ }^{1}$

${ }^{1}$ Faculty of Physics and Astronomy

Corresponding Author(s): maciej.matyka@uwr.edu.pl

\begin{abstract}
Tortuosity is one of the key parameters in description of the flow through porous media at low Reynolds numbers. It is used in diffusion, fluid flow and electrical transport through complex media to account for non regular shape of the transport paths. Not much was done, however, in terms of multiphase flow and tortuosity, mostly because of tortuosity interpretation problems, but also because multiphase simulations are complex, require long simulation times and involves the problem of diffusive boundary at two phases. It is, however, interesting to know if tortuosity of another processes (different than fluid flow) scale with porosity in a similar way?
\end{abstract}
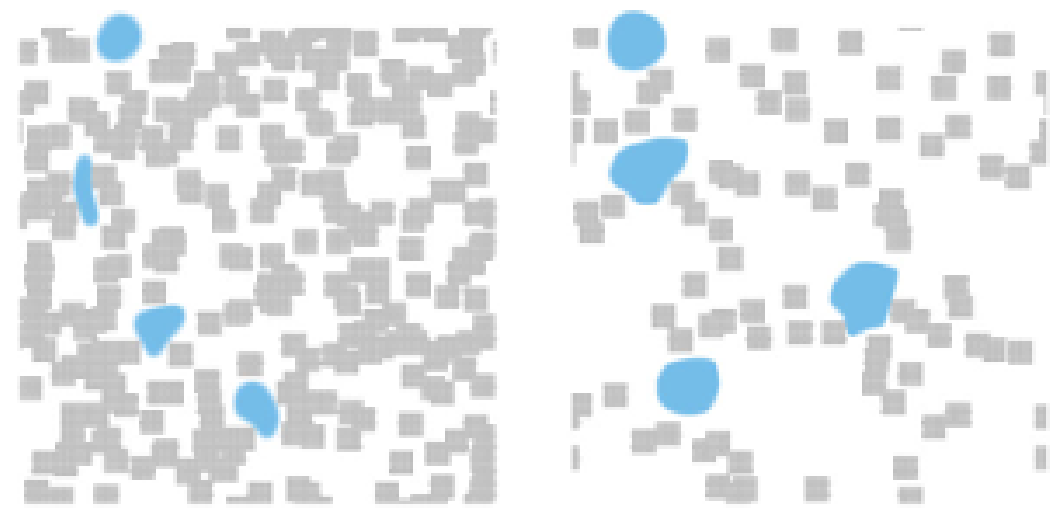

Figure 10: The concept of a pushing simulation based on the single droplet model put into random porous media. 
Here I will concentrate on tortuosity as a parameter that describes porous structure by itself (without linking to Carman-Kozeny equation). This will let me compute tortuosity directly from the transport of a single droplet that is pushed through the non-wettable media and provide numerical results at various conditions.

The concept is sketched in fig. 1. For modelling I will combine the mechanical model of pressure based soft body dynamics introduced in the context of video games and computer graphics before [Matyka03] with a random porous media [Matyka08].

As the main result I will provide snapshots of working simulations and preliminary results on tortuosity based on the simulated phenomena.

\title{
Literature
}

[Matyka03] Matyka, M. and Ollila, M., A pressure model for soft body simulation, Proc. of Sigrad (2003)

[Matyka08] Matyka, M., Khalili, A. and Koza, Z., Tortuosity-porosity relation in the porous media flow, Phys. Rev. E 78, 026306 (2008)

Procter and Gamble Student poster award:

References:

Acceptance of Terms and Conditions:

Click here to agree

884

\section{Irreversible salinization and sodification}

\author{
Author(s): Yair $\mathrm{Mau}^{1}$ \\ Co-author(s): Isaac Kramer ${ }^{1}$ \\ ${ }^{1}$ Hebrew University of Jerusalem \\ Corresponding Author(s): yair.mau@mail.huji.ac.il
}

Large swaths of agricultural land are abandoned every year because of irrigation-caused salinization and sodification. Among the main culprits of this kind of soil degradation is the deterioration of soil structure and the concomitant sharp decrease in hydraulic conductivity.

We examine the irreversible decline in saturated hydraulic conductivity (Ks) as a function of the soil's history regarding irrigation water usage. Through lab experiments, we characterized the hysteretic behavior of Ks for various soil types, as imposed by input water salinity and sodicity (electrical conductivity and sodium adsorption ratio, EC and SAR, respectively). There is no well-defined "point of no return" for Ks decline, but beyond a range in EC and SAR the hysteresis loops markedly increase in side. For clayey soils, the hysteretic paths of Ks may not close into loops, indicating the limits of chemical-based remediation.

We present a novel mathematical function for the decline in Ks and its hysteretic behavior. This function is then incorporated into a low-dimensional model of water and salt dynamics in the root zone, yielding predictions for soil deterioration and rehabilitation time scales, according to irrigation water quality and climatic conditions. Both the new function for Ks and the soil-water-salt dynamics model can be instrumental in devising irrigation policies that are both economically feasible and environmentally sustainable.

\section{Procter and Gamble Student poster award:}

I don't want to compete References:

Acceptance of Terms and Conditions: 


\title{
Efficient DG-based simulation of coupled surface subsurface flow
}

Dmitry Mazilkin ${ }^{1}$; Peter Bastian ${ }^{2}$; Dominic Kempf ${ }^{2}$; Olaf Ippisch ${ }^{1}$

\author{
${ }^{1}$ TU Clausthal, Institute for Mathematics \\ ${ }^{2}$ Heidelberg University, Interdisciplinary Center for Scientific
}

Corresponding Author(s): dmitry.mazilkin@tu-clausthal.de

\begin{abstract}
The simulation of coupled surface subsurface flow is a topic of high scientific and social relevance for flood protection, agriculture and weather prediction. Most existing numerical models use a kinematic wave approximation for the simulation of the surface runoff. We present an approach based on the diffusive wave approximation for surface and Richards' equation for subsurface flow. An operator splitting approach is used with a special boundary condition to achieve a mass conservative solution. The approach does not require tracking of the dry/wet boundary of the surface like other existing approaches. Spatial discretization of both flow equations is done with a Weighted Interior Penalty Discontinuous Galerkin scheme, while for the temporal discretization a semi-implicit scheme is used for the surface runoff and a diagonally implicit Runge-Kutta scheme for the subsurface flow. We present results obtained with the parallel numerical solver based on DUNE-PDELab and advanced in computational efficiency within the scope of EXA-DUNE project. The fully coupled model was tested in parallel simulations on structured and unstructured grids. A code generation framework developed by Dominic Kempf in the group at IWR Heidelberg was used to obtain an optimized version of the (expensive) subsurface solver. The replacement of the original material model including power functions by a cubic spline approximation allows a fast vectorized incorporation of flexible material functions. Sum-factorisation is used in the operator evaulations. A total speedup of 3 was measured in first test simulations. Even higher speedups are expected with higher-order base functions. On a 32 core Intel Haswell node, $20-35 \%$ of peak performance of the Dune-Codegen generated DG solver for subsurface flow is reached.
\end{abstract}

Procter and Gamble Student poster award:

References:

Acceptance of Terms and Conditions:

Click here to agree

\section{Lattice Boltzmann simulation of drop impact on textile materials placed on a substrate}

Ali Mazloomi Moqaddam ${ }^{1}$; Thijs De Goede ${ }^{2}$; Dominique Derome ${ }^{1}$; Noushine Shahidzadeh $^{3}$; Daniel Bonn ${ }^{3}$; Jan Carmeliet $^{4}$

\footnotetext{
${ }^{1}$ Laboratory for Multiscale Studies in Building Physics Empa, Swiss Federal Laboratories for Materials Science and Technology, 8600 Dübendorf, Switzerland

${ }^{2}$ an der Waals-Zeeman Institute, Institute of Physics, University of Amsterdam, Science Park 904, 1098 XH Amsterdam, Netherlands

${ }^{3}$ Van der Waals-Zeeman Institute, Institute of Physics, University of Amsterdam, Science Park 904, 1098 XH Amsterdam, Netherlands

${ }^{4}$ Chair of Building Physics, Department of Mechanical and Process Engineering, ETH Zurich, 8092 Zurich, Switzerland
} 
Corresponding Author(s): ali.mazloomi@empa.ch

Wetting and spreading of droplets impacting on solid flat surfaces have been extensively studied both numerically and experimentally for a long time. However, little efforts have been dedicated on studying such phenomena for droplets striking on textile materials placed on a substrate. Dynamics of impact on such surfaces is intrinsically complex. Compared to flat surfaces, on textile materials the liquid penetrates the holes in the textiles during spreading resulting in producing internal flows in the holes that may induce extra viscous dissipation and thus the energy of the droplet is modified compared to that on flat substrate which may influence on the maximum deformation of the droplet. Using our recent proposed lattice Boltzmann model1, we numerically investigate in detail the effect of different parameters such as the pore size, the thickness of yarns and the impact velocity on the dynamic behavior of the impacting droplets on textile materials. The simulation results are compared with the experimental observation of the same conditions and an excellent agreement is observed. We observe from our simulations that for textile substrates the drop spreads less in contrast with flat substrates. This suggests that the energy of the impacting droplets is modified for such surfaces. Using our numerical simulations, we compute the viscous dissipation of the droplet during spreading on both textile and flat substrates in a precise manner. The results obtained from simulations show that for droplets impacting on textile materials an extra viscous dissipation is observed compared with those on flat substrates. We attribute this extra dissipation to the internal flow produced inside the hole leading the impacting droplet to less deform on textile materials compared to those on flat substrates. Therefore, the maximum spreading scaling equation proposed in 2 for flat surfaces is corrected accordingly to take into account such extra dissipations and to properly predict the maximum deformation of droplets striking on textile material placed on a substrate.

Procter and Gamble Student poster award:

\title{
References:
}

Acceptance of Terms and Conditions:

Click here to agree

706

\section{Deep learning from multiphase digital rock simulations}

\author{
James McClure ${ }^{1}$; Ramstad Thomas ${ }^{2}$ \\ ${ }^{1}$ Virginia Tech \\ ${ }^{2}$ Equinor
}

Corresponding Author(s): mcclurej@vt.edu

Direct numerical simulations performed provide a basis to generate detailed pore-scale information that can be used to understand larger scale phenomena. The large amount of information that can be extracted from simulations provides an ideal basis to develop machine learning techniques that can used to automatically interpret simulation data. The application of such approaches to inform constitutive modeling is an important opportunity associated with pore-scale studies.

We review lattice Boltzmann simulation workflows available in the open source software package LBPM and discuss the steps needed to mimic traditional experimental protocols using a digital rockbased approach. We then demonstrate how data generated from simulations can be used as input for deep learning models as a way to gain additional insight.

While it is imperative that constitutive models be constructed in accordance with the laws of physics, many relationships of interest are phenomenological and cannot be derived a priori. We discuss how specific neural network structures can be designed with constitutive modeling and upscaling in mind, and present concrete examples and results. Deep learning techniques informed by simulation data are evaluated as a means to predict relative permeability and capillary pressure relationships that are essential input to large scale reservoir characterization. 
I don't want to compete References:

Acceptance of Terms and Conditions:

Click here to agree

509

\title{
The fluorescence microscopy toolkit in investigations of paper: High-speed, super-resolution and 3D imaging
}

\author{
Author(s): Tobias Meckel ${ }^{1}$ \\ Co-author(s): Marcel Krauße ${ }^{1}$; Andreas Geißler ${ }^{1}$; Markus Langhans $^{2}$; Samuel Schabel ${ }^{1}$; Markus Biesalski \\ ${ }^{1}$ Technische Universität Darmstadt \\ ${ }^{2}$ Technische Universität Darmstadt
}

Corresponding Author(s): meckel@cellulose.tu-darmstadt.de

Optical brightfield and electron microscopy have long been the predominant imaging methods to characterize the heterogeneous and complex structure of paper. Since the early 1990s, also fluorescence microscopy techniques and especially confocal laser scanning microscopy (CLSM), have seen an increasing application in investigations of paper (Bump et al. 2015). However, while impressive developments have advanced fluorescent microscopy over the last three decades into a method that has rightfully been honoured with the Nobel Prize in Chemistry in 2014, its full spectrum of capabilities has yet to be exploited in studies of paper.

With the aim to change that, this presentation will provide a brief overview of the most important advancements of the method in general and some of its applications to the microscopy of paper Among those will be (i) fast acquisitions of the imbibition and capillary transport of fluids inside the paper matrix, (ii) super-resolved images of fiber surfaces as acquired in-situ, i.e. in water, and (iii) $3 \mathrm{D}$ reconstructions of polymer functionalized paper.

Procter and Gamble Student poster award:

\section{References:}

Bump S, Böhm A, Babel L, Wendenburg S, Carstens F, Schabel S, Biesalski M, Meckel T: Spatial, spectral, radiometric, and temporal analysis of polymer-modified paper substrates using fluorescence microscopy. Cellulose. 2015;22: 73-88. Acceptance of Terms and Conditions:

Click here to agree

\section{Coreflood on a Chip: Scaling up of a Micromodel for EOR Exper- iments}

Lucas Mejia $^{\text {None }}$; Peixi Zhu ${ }^{1}$; Matthew Balhoff ${ }^{1}$; Kishore Mohanty ${ }^{2}$

${ }^{1}$ University of Texas at Austin

${ }^{2}$ The University of Texas at Austin

Corresponding Author(s): lmkerguelen@utexas.edu

Micromodels have been used for decades to study fundamental flow and transport behavior at the micrometer and nanometer scales of porous media. Due to their flexibility in fabrication methods, materials selection, and ease of visualization they are ideal tools for identifying transport mechanisms at the pore scale. The goal of this work is to create chips that are similar to cores in length and 
have internal pore structure and wettability similar to field cores. We designed and fabricated such micromodels, two-feet $(\sim 60 \mathrm{~cm})$ long porous medium (Figure 1$)$, with variable grain arrangements that emulated sandstones or carbonates. Additionally, our chip included 2.5D features where pore throats are much smaller than the pore bodies (Xu et al., 2017) and calcite coating for the limestone studies. We compare displacement experiments in our long chip to core floods to evaluate similarities and differences between the two platforms.

The two-foot long micromodel was used to evaluate the conditions for the formation of an oil bank during tertiary alkaline surfactant polymer (ASP) flooding. An initially brine-saturated micromodel was flooded with a light oil until residual brine saturation was reached. Then, the chip was waterflooded at $1 \mathrm{ft} /$ day until the oil saturation reached a residual value. Finally, a 0.3 pore volume (PV) ASP slug was injected and chased by polymer for $1 \mathrm{PV}$. Images of the floods were captured using a high resolution digital camera (Nikon D6500) and an optical microscope camera (Lumera Infinity 4). The results of the experiment were compared to corefloods for which CT saturation data was available.

Observations from the micromodel experiments such as the end effect saturations, breakthrough times, and displacement efficiency qualitatively matched coreflood results. However, the average residual saturation in the micromodel was higher than in the coreflood, and spatial distribution of residual oil was more erratic in the micromodel than in the coreflood. After comparing the micromodel results to the coreflood results, we investigated oil bank formation in our micromodel platform. By modifying the viscosity and the flow rate of the ASP slug, we determined the conditions at which an oil bank formed. Finally, we speculate on how the banking conditions achieved in our micromodel can be extrapolated to corefloods.

Procter and Gamble Student poster award:

I would like to compete in the Procter and Gamble Student award References:

Xu, K., Liang, T., Zhu, P., Qi, P., Lu, J., Huh, C., \& Balhoff, M. (2017). A 2.5-D glass micromodel for investigation of multi-phase flow in porous media. Lab on a Chip, 17(4), 640-646. https://doi.org/10.1039/C6LC01476C Acceptance of Terms and Conditions:

Click here to agree

\title{
DEPENDENCY OF STRESS REGIME AND ORIENTATION ON FRAC- TURE PERMEABILITY IN NATURALLY FRACTURED RESERVOIRS- A DISCRETE FRACTURE APPROACH
}

\author{
Alejandro Restrepo ${ }^{1}$; Lorena Diez $^{2}$; Juan D Vallejo ${ }^{3}$; Juan Mejía ${ }^{4}$ \\ ${ }^{1}$ Equion Energia, LTD \\ ${ }^{2}$ Facultad de Minas. Universidad Nacional de Colombia \\ ${ }^{3}$ Exergy Modeling and analytics \\ ${ }^{4}$ Universidad Nacional de Colombia
}

Corresponding Author(s): jmmejiaca@unal.edu.co

Fracture network systems provides the dominant permeability in naturally fractured reservoirs. One of the main reservoir variables impacting fracture permeability is the effective stress state in the near-wellbore region. The reservoir pressure declines as long as reservoir fluids are produced. Therefore, the stress distribution around the wellbore is increased, producing a fracture width reduction. As a consequence, the fracture conductivity is highly impacted on pressure depletion rate. In an anisotropic stress state, the permeability reduction is also dependent on the fracture orientation. The impact of permeability reduction will be more severe in fractures that are normally oriented to the maximum horizontal stress. An optimum reservoir management, based on the understanding of the underlying physics, is mandatory for increasing the final recovery factor of naturally fractured reservoirs.

We developed an analytical workflow that estimates the fracture permeability distribution of a set of discrete fracture network. The workflow is based on the evaluation of the stress state near the wellbore area, based on the modified Kirsch approach (Fjar et al., 2008). The effective stress is further calculated after solving the fluid pressure distribution around the wellbore. Then, the effective 
normal stress affecting each fracture is evaluated. The fracture width is further estimated based on the normal stress, using the Bandis model (Bandis et al., 1983), and the fracture permeability is calculated. The workflow was employed for analyzing a well producing from a formation having a strong tectonic activity in an oilfield of the Colombian foothills at the Llanos basin. The study shows that some of the fractures will restrict the permeability while others can improve permeability, depending on the fracture orientation and stress state. In addition, permeability variations along the fracture plane can be developed because of the effective stress distribution in the reservoir.

Sensitivity analysis are carried out on fracture properties and the main variables that affect the stress state around the wellbore. The difference between magnitude of principal stresses in-situ and azimuth and dip fracture, are the most relevant variables in fracture permeability variation. Other variables such as initial fracture width and Poisson's ratio, have a lesser effect in fracture permeability.

Procter and Gamble Student poster award:

I don't want to compete References:

Bandis, S.C., Lumsden, A. C., \& Barton, N. R. (1983). Fundamentals of rock joint deformation. International Journal of Rock Mechanics and Mining Sciences \& Geomechanics Abstracts (Vol. 20, No. 6, pp. 249-268). Pergamon.

Fjar, E., Holt, R. M., Raaen, A. M., Risnes, R., \& Horsrud, P. (2008). Petroelum related rock mechanics (vol. 53). Elsevier. Acceptance of Terms and Conditions:

Click here to agree

\title{
Numerical study of dispersed foamer injection in a gas stream for Enhanced Oil Recovery in Naturally Fractured Reservoirs
}

\author{
Hillmert Solano $^{1}$; Juan D Valencia ${ }^{2}$; Alonso Ocampo ${ }^{3}$; Juan M Mejía ${ }^{2}$ \\ ${ }^{1}$ Universidad Nacional de Colombia, sede Medellín \\ ${ }^{2}$ Universidad Nacional de Colombia \\ ${ }^{3}$ Equion Energia S.A.
}

Corresponding Author(s): jmmejiaca@unal.edu.co

\begin{abstract}
Naturally Fractured Reservoirs are highly heterogeneous porous media due to the permeability contrast between the matrix and the fractures. In Enhanced Oil Recovery processes, the fractures are preferential flow channels, letting high residual oil saturation in the matrix after a recovery process. For that reason, several methodologies are used for improving the swept in the Naturally Fractured Reservoir matrix. An example of those techniques is the in-situ foam generation which allows to block the fractures and sweep the matrix with the injection fluid. Foams are generated through some techniques, such as dispersed foamer injection in a gas stream. This novel technique has been evaluated through numerical simulation and core-flooding experiments, but its application in a Naturally Fractured Reservoir has a high level of uncertainty. For that reason, a mechanistic model, which was previously developed, is extended to evaluate the foam behavior in Naturally Fractured Reservoirs. This model involves constitutive and conservation equations that represent transport, transference and foaming mechanisms that describe the phenomenology of the process. The extension of the model is validated with data from core-flooding experiments and run for a proposed reservoir sector. As a conclusion of this work, foams generated through dispersed foamer injection in a gas stream leads to block the fractures temporally and improve the swept in the matrix.
\end{abstract}

Procter and Gamble Student poster award:

I don't want to compete References:

Acceptance of Terms and Conditions:

Click here to agree 


\title{
Reactive silicate mineral carbonation under diffusive transport and confining stress
}

\author{
Author(s): Anne Menefee ${ }^{1}$ \\ Co-author(s): Brian Ellis \\ ${ }^{1}$ University of Michigan, Ann Arbor, MI, USA \\ Corresponding Author(s): amenefee@umich.edu
}

Basalt and ultramafic reservoirs could provide a permanent sink for anthropogenic $\mathrm{CO} 2$ emissions through mineral trapping. In this study we designed a series of core flooding experiments targeting carbonation in diffusion-limited zones of reactive minerals where $\mathrm{CO} 2$ mineralization is expected to be most favorable. Whole flood basalt cores were saw-cut to create a single uniform fracture, and holes drilled parallel or perpendicular to the direction of flow were wet-packed with powders (30$425 \mu \mathrm{m}$ ) of olivine or wollastonite to simulate zones rich in reactive minerals adjacent to key flow paths. The reactive powders were separated from the injected fluid by a layer of non-reactive silica beads to prevent early washout from rapid dissolution and isolate $\mathrm{CO} 2$-driven carbonation reactions. Brines consisting of ultrapure water or $0.64 \mathrm{M} \mathrm{NaHCO} 3$ were pre-equilibrated with $\mathrm{CO} 2$ at $10 \mathrm{MPa}$ and injected through the cores, maintaining a pore pressure of $10 \mathrm{MPa}$ downstream. Pre- and postreaction $\mathrm{xCT}$ scans were processed to quantify porosity changes within the packed beds. Reaction products were further characterized with Raman spectroscopy, scanning electron microscopy, and thermogravimetric analysis (TGA). Complementary reactive transport models were developed using the geochemical code CrunchTope to isolate the roles of mineral reactivity, grain size (i.e., reactive surface area), and diffusion distance over longer time scales.

Consistent with prior work, the results indicated that carbonation of Ca-bearing silicates proceeds more rapidly than $\mathrm{Mg}$-bearing silicates, but may ultimately be limited by dissolution and CO2 supply. While carbonates formed at the ends of the dead-end packed beds in these experiments, indicating the reactions were not self-sealing, a general decrease in carbonation efficiency (determined through TGA) with diffusion distance from injection pathways suggests rapid precipitation at the front of $\mathrm{CO} 2$-charged brines may obstruct further $\mathrm{CO} 2$ trapping. Reactive transport models supported localized carbonation fronts, where carbonation peaked at 8-12 $\mathrm{mm}$ into the packed beds due to opposing geochemical gradients before decreasing with further distance as $\mathrm{CO} 2$ became more limited. In natural systems, such localization can form diffusion barriers to further $\mathrm{CO} 2$ penetration, which may limit long-term carbonation as pores fill and flow in diffusion-limited regions is insufficient to generate new transport pathways. Although in theory this limitation can be overcome if crystallizing carbonates generate sufficient stress to fracture the host rock, creating fresh reactive surface area and flow paths that can sustain reactions, no evidence of reaction-induced fracturing was observed in these experiments. Models also confirmed that the mineralogy of the host rock is more influential on carbonation efficiency than grain size and associated reactive surface area, highlighting the benefit of selecting reservoirs rich in $\mathrm{Ca}$-silicates to achieve rapid mineral carbonation relevant to the scale of $\mathrm{CO} 2$ emissions.

Procter and Gamble Student poster award:

I don't want to compete References:

Acceptance of Terms and Conditions:

Click here to agree

\section{Using nanoCT and high contrast imaging to inform microporos- ity permeability during Stokes-Brinkman single and two-phase flow simulations on microCT images}

Hannah Menke ${ }^{1}$; Ying Gao ${ }^{1}$; Sven Linden ${ }^{2}$; Matthew Andrew ${ }^{3}$ 


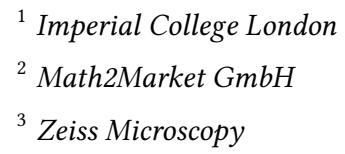

Corresponding Author(s): h.menke12@imperial.ac.uk

Understanding how micro porosity effects flow when upscaling to the pore-scale is imperative for accurate predictive modelling of flow in porous media. However, it is not currently possible to image at nanoscale resolutions on larger scales. Furthermore, models with trillions of voxels are computationally expensive, requiring high performance computing to solve their vast geometries. Carbonate rocks in particular often have complex and multiscale porosity structures that are poorly understood. In this study we use a combined experimental, modelling, and pore space generation methods to tackle the impact of micro porosity on the bulk flow properties of Estalliades limestone. First, a micro core of rock was scanned using x-ray microtomography $(\mu \mathrm{CT})$ and a representative microporous region was identified. A nano core of rock was then milled using a laser lathe and scanned using x-ray nano tomography (Nano CT). The Nano CT scan was then used as input into the pore space generator and the permeability field was simulated for a range of porosities to create a synthetic Kozeny-Carman porosity-permeability relationship for micro porosity. We found a good match between experimental and simulated Mercury Intrusion Capillary Pressure (MICP) range in the imaged geometry and a good match between the imaged and object generated permeabilities and MICP.

A micro-core of Estaillades was then scanned in the $\mu \mathrm{CT}$, the differential pressure was measured, and the rock was flooded with highly doped brine to elucidate where the micro porosity was connected and unconnected. The differential contrast between the dry and doped images was then used to assign a porosity to each voxel of connected micro porosity. The flow through the pore space was then solved using a Stokes-Brinkman solver while a second segmented image with no micro porosity was solved a Stokes solver. The differences between the measured permeability and the two computed permeabilities was evaluated. We found that there was good agreement between both the computed permeability of the Stokes and Stokes-Brinkman simulation with the measured permeability. However, there was considerable differences in the velocity fields with the Stokes-Brinkman simulation capturing stagnant regions of the pore space that were not present in the Stokes simulations.

Additionally we investigated the implications of including microporosity in estimations of relative permeability. Nitrogen was experimentally coinjected through the core with doped brine at a $50 \%$ fractional flow and imaged to find a single point on the two-phase relative permeability curve. This experimental measurement was then compared with the numerical permeability simulated using both Stokes and Stokes-Brinkman methods with several saturation points along the synthetic MICP injection curve. We found that the Stokes simulation was not able to predict relative permeability with this method due to the major flow paths being impeded by the injected non-wetting phase. However, the Stokes-Brinkman simulations allowed flow in the microporous regions around these blocked flow paths and was able to achieve a relative permeability curve that was a reasonable match to the experimental measurement. This method could be used to predict relative permeability in water wet pore-structures with high microporosity.

Procter and Gamble Student poster award:

References:

Acceptance of Terms and Conditions:

Click here to agree

\section{Regeneration of capillary state for water occluded in dual-porosity systems}

Lionel Mercury $^{\text {None }}$; Claudie HULIN ${ }^{1}$

\footnotetext{
${ }^{1}$ Universite d'Orléans
} 
Corresponding Author(s): lionel.mercury@univ-orleans.fr

Unsaturated zones are of growing interest due to global climate changes that modify the water balance in surficial areas (soils, exposed rocks, etc.), and to industrial drying processes targeting deep geological storage (CO2, nuclear wastes, gas temporary storages etc.) and/or extraction (tight gas reservoirs, water/oil uptakes, etc.) challenges. The consequent partitioning of the fluid phase into a biphasic air-water assemblage modifies the energy status of liquid water depending on the intensity of the capillary forces, itself linked to the pore size hosting the capillary bridges. With pore size heterogeneity in crustal or sedimentary rocks, it is possible to host significant volumes of capillary water in large pore bodies, the energy of which is controlled by capillary forces taking place at tiny pore throats. Getting strong capillary features on large volume of water can thus be obtained within a bimodal structure. However, pore bodies are too large with respect to establish equilibrium: the held-water is metastable and as such is time limited.

In the present work, experiments are developed using synthetic bimodal pore system to study the gas-water equilibria at capillary conditions. In particular, the pathway to generate and regenerate capillary state in body-throat porous assemblages is scrutinized by direct observations.

Two geochemical effects appear to cooperate for building the observables. First, capillary water has a higher solubility for gases: capillarity is a gas-in process. Second and most important, molar volume of water increases under tension, while the total volume of the gas-water assemblage remains constant. Consequently, 'capillarization' at the pore throats promote the homogenization of this closed system, as does an increasing temperature along the saturation curve.

This finding means that the pore heterogeneity in rocks submitted to drying processes (whichever the source of the drying wave), are conducive to long-term tensile state by repetition of the capillarization process for in-pores water. As a consequence, capillarity and its geochemical (gas and solid solubilities) and/or poromechanical (compaction, tensile stress, fracturing, etc.) properties is potentially one of the major player on the long term in any drying or wetting-drying pore structures.

Procter and Gamble Student poster award:

\title{
References:
}

Acceptance of Terms and Conditions:

Click here to agree

\section{Generalizing the Non-Local Darcy Formulation for Settings with Boundaries}

\author{
Author(s): Daniel Meyer ${ }^{1}$ \\ Co-author(s): Artur Gomolinski ${ }^{2}$ \\ ${ }^{1}$ Institute of Fluid Dynamics, ETH Zurich \\ ${ }^{2}$ Student at ETH Zurich
}

Corresponding Author(s): meyerda@ethz.ch,

Recently, we have proposed the non-local Darcy formulation, which bridges the gap between a discrete pore network description and a continuum Darcy-flow formulation 1 . Here, the conductivity distribution $T\left(x_{1}, x_{2}\right)$ is at the center of the formulation. It quantifies the flow in network tubes between two points $x_{1}$ and $x_{2}$. We showed that in the limit of vanishing characteristic throat lengths relative to the scale of interest, the non-local formulation reduces to the classical Darcy law with the permeability resulting from the conductivity distribution. Our past developments were focusing on space-stationary settings with $T\left(x_{1}, x_{2}\right)=T\left(\left|x_{2}-x_{1}\right|\right)$ and unbounded domains. In the present contribution, we inspect the impact of open network boundaries on the non-local formulation and show consistency with classical Darcy in the bounded setting. Moreover, we propose a Fourier-based technique to extract the conductivity distribution from pressure measurements. 


\title{
References:
}

1 Delgoshaie, A.H., D.W. Meyer, P. Jenny, and H.A. Tchelepi, Non-local formulation for multiscale flow in porous media. Journal of Hydrology, 2015. 531(3): p. 649-654. Acceptance of Terms and Conditions:

Click here to agree

\section{Development of a 3D dual pore-system leaching model with ap- plication on metal extraction from an oxide copper ore}

\author{
Author(s): Xiuxiu Miao ${ }^{1}$ \\ Co-author(s): Baohua Yang ${ }^{2}$; Guillermo Narsilio ${ }^{3}$; Aixiang Wu ${ }^{4}$ \\ ${ }^{1}$ State Key Laboratory for Geo-mechanics and Geo-technical Engineering, Institute of Rock and Soil Mechanics, Chi- \\ nese Academy of Sciences, Wuhan, Hubei Province 430071, China \\ ${ }^{2}$ Information Science and Engineering School, Hunan International Economics University, Changsha, Hunan Province \\ 410205, China \\ ${ }^{3}$ Department of Infrastructure Engineering, The University of Melbourne, Parkville, Melbourne, Victoria 3010, Aus- \\ tralia \\ ${ }^{4}$ Key Laboratory of High-efficient Mining and Safety of Metal Mines (Ministry of Education), University of Science \\ and Technology Beijing, Beijing 100083, China
}

Corresponding Author(s):

Ore heaps are typical multi-scale porosity structures, the heterogeneity of packed ore particle beds leads to complex flow, transport, reactions and heat transfer processes that are closely involved. A 3D dual pore-system leaching model based on heterogeneous ore pack geometry reconstructed from computed tomography (CT) images was specifically developed to account for such strongly coupled multi-physics across the scales of inter-particle macro-pores and intra-particle micro-pores. Free flow and porous media flow laws were adopted to depict fluid flow in macro-pore and micropore, respectively; convection, dispersion, diffusion transporting modes were selectively applied to macro- and micro-pore regions; shrinking-core reaction pattern with relaxation on spherical particle shape restriction and constant bulk solution concentration limitation was established and applied to lixiviant-identified mineral particle reaction; volumetric averaged reaction pattern was used to describe lixiviant-unidentified mineral particle reaction; convective and conductive heat transfer with heat source estimated according to the reaction thermodynamics was imposed on the micro and macro systems. Governing equations for each process were interlinked by the velocity field of lixiviant, concentrations of leaching agent and target metal, and temperature profile of the ore pack The model was deployed to investigate the leaching kinetics of an oxide copper ore by sulphuric acid. CT scans of the ore column and ore particles were implemented to capture the structures and analyse the physical properties of the macro- and micro-pores. Mineralogical analysis was carried out to identify reactive minerals from CT slices, and then incorporated in the dual pore-system to solve the dissolution of copper and gangue minerals. Ultimately, the velocity field, mineral dissolving rate, metal ion $(\mathrm{Cu} 2+$ and $\mathrm{Ca} / \mathrm{Mg} 2+)$ concentration, $\mathrm{pH}$ and temperature profile were solved numerically by finite element method, and the results were physically reasonable. The authors believe the dual pore-system model is able to provide insights into the details of the complicate leaching behaviours that otherwise cannot be revealed by models built on basis of uniform geometries. A miniature leaching device with strict control on leaching conditions was drafted using a core holder (as an insulated reactor) and a peristaltic pump for in-situ CT imaging, which can be deployed to improve and validate the model in the future.

Keywords: Computed tomography $\bullet$ Dual pore-system model $\bullet$ Pore scale modelling $\bullet$ Coupled flotransport-reaction-heat transfer processes $\bullet$ Leaching kinetics

Procter and Gamble Student poster award:

References: 
1. Ackermann, S., Scheffe, J., Duss, J., \& Steinfeld, A. (2014). Morphological characterization and effective thermal conductivity of dual-scale reticulated porous structures. Materials, 7(11), 71737195.

2. Anderson, M. P. (1984). Movement of contaminants in groundwater: groundwater transportadvection and dispersion. Groundwater Contamination, 37-45.

3. Bennett, C. R., Mcbride, D., Cross, M., \& Gebhardt, J. E. (2012). A comprehensive model for copper sulphide heap leaching : Part 1 Basic formulation and validation through column test simulation. Hydrometallurgy, s 127-128(18), 150-161.

4. Burnett, R., \& Frind, E. (1987). Simulation of contaminant transport in three dimensions: 1 . The alternating direction Galerkin technique. Water Resources Research, 23(4), 683-694.

5. Cariaga, E., Concha, F., \& Sepúlveda, M. (2005). Flow through porous media with applications to heap leaching of copper ores. Chemical Engineering Journal, 111(2-3), 151-165.

6. Cariaga, E., Concha, F., \& Sepúlveda, M. (2007). Convergence of a MFE-FV method for two phase flow with applications to heap leaching of copper ores. Computer Methods in Applied Mechanics \& Engineering, 196(25), 2541-2554.

7. Casas, J. M., Vargas, T., Martinez, J., \& Moreno, L. (1998). Bioleaching model of a copper-sulfide ore bed in heap and dump configurations. Metallurgical and Materials Transactions B, 29(4), 899-909.

8. Chae, D., \& Wadsworth, M. (1979). Modeling of the leaching of Oxide Copper Ores.

9. Cross, M., Bennett, C. R., Croft, T. N., Mcbride, D., \& Gebhardt, J. E. (2006). Computational modeling of reactive multi-phase flows in porous media: Applications to metals extraction and environmental recovery processes. Minerals Engineering, 19(10), 1098-1108.

10. Dixon, D. G., \& Hendrix, J. L. (1993). A general model for leaching of one or more solid reactants from porous ore particles. Metallurgical and Materials Transactions B, 24(1), 157-169.

11. Genty, A., \& Pot, V. (2014). Numerical calculation of effective diffusion in unsaturated porous media by the TRT lattice boltzmann method. Transport in Porous Media, 105(2), 391-410.

12. Han, K. N., \& ebrary Inc. (2002). Fundamentals of aqueous metallurgy.

13. HsiaoChingKuo. (1970). Molecular volumes and the Stokes-Einstein equation. Journal of Chemical Education, 47(4), 5857-5860.

14. Leahy, M. J., Davidson, M. R., \& Schwarz, M. P. (2004). A column bioleaching model for chalcocite - An investigation of oxygen limitation and bacterial inoculation on leaching. Paper presented at the Bac-min 2004, Victoria, Australia.

15. Leahy, M. J., Davidson, M. R., \& Schwarz, M. P. (2005). A model for heap bioleaching of chalcocite with heat balance: Bacterial temperature dependence. Minerals Engineering, 18(13), 1239-1252.

16. Leahy, M. J., Davidson, M. R., \& Schwarz, M. P. (2007). A model for heap bioleaching of chalcocite with heat balance: Mesophiles and moderate thermophiles. Hydrometallurgy, 85(1), 24-41.

17. Leahy, M. J., \& Schwarz, M. P. (2009). Modelling jarosite precipitation in isothermal chalcopyrite bioleaching columns. Hydrometallurgy, 98(1), 181-191.

18. Leahy, M. J., Schwarz, M. P., \& Davidson, M. R. (2003). An air sparging CFD model for heap bioleaching of copper-sulphide. Applied Mathematical Modelling, 30(11), 1428-1444.

19. Leahy, M. J., Schwarz, M. P., \& Davidson, M. R. (2006). An air sparging CFD model for heap bioleaching of chalcocite. Applied Mathematical Modelling, 30(11), 1428-1444.

20. Levenspiel, O. (1972). Chemical reaction engineering (2d ed.). New York: Wiley.

21. Liddell, K. C. (2005). Shrinking core models in hydrometallurgy: What students are not being told about the pseudo-steady approximation. Hydrometallurgy, 79(1-2), 62-68.

22. Lin, C. L., Miller, J. D., \& Garcia, C. (2005). Saturated flow characteristics in column leaching as described by LB simulation. Minerals Engineering, 18(10), 1045-1051.

23. Lin, C. L., Videla, A. R., \& Miller, J. D. (2010). Advanced three-dimensional multiphase flow simulation in porous media reconstructed from X-ray microtomography using the He-ChenZhang lattice boltzmann model. Flow Measurement \& Instrumentation, 21(3), 255-261. 
24. Lin, Q., Barker, D. J., Dobson, K. J., Lee, P. D., \& Neethling, S. J. (2016). Modelling particle scale leach kinetics based on X-ray computed micro-tomography images. Hydrometallurgy, 162, 25-36.

25. Madsen, B. W., \& Wadsworth, M. E. (1980). A mixed kinetics dump leaching model for ores containing a variety of copper sulfide minerals. U. S. Dept. of Interior, 44.

26. Miao, X. X., Narsilio, G. A., Wu, A. X., \& Yang, B. H. (2017). A 3D dual pore-system leaching model. Part 1: Study on fluid flow. Hydrometallurgy, 167, 173-182.

27. Miao, X. X., Wu, A. X., \& Yang, B. H. (2014). Unsaturated flow and solute transport in a porous column using spherical ore particles. International Journal of Minerals, Metallurgy, and Materials, 21(2), 113-121.

28. Miller, G. M. (2003). Ore geotechnical effects on copper heap leach kinetics. Paper presented at the proceedings of the 5th International Symposium on Hydrometallurgy, Vancouver, Canada.

29. Millington, R., \& Quirk, J. P. (1961). Permeability of Porous Solids. Transactions of the Faraday Society, 57(8), 1200-1207.

30. Pantelis, G., Ritchie, A. I. M., \& Stepanyants, Y. A. (2002). A conceptual model for the description of oxidation and transport processes in sulphidic waste rock dumps. Applied Mathematical Modelling, 26(7), 751-770.

31. Paul, B. C., Sohn, H., \& Mccarter, M. K. (1992). Model for ferric sulfate leaching of copper ores containing a variety of sulfide minerals: Part I. Modeling uniform size ore fragments. Metallurgical Transactions B, 23(5), 537-548.

32. Raeini, A. Q., Blunt, M. J., \& Bijeljic, B. (2014). Direct simulations of two-phase flow on micro-CT images of porous media and upscaling of pore-scale forces. Advances in Water Resources, 74, 116-126.

33. Safari, V., Arzpeyma, G., Rashchi, F., \& Mostoufi, N. (2009). A shrinking particle - shrinking core model for leaching of a zinc ore containing silica. International Journal of Mineral Processing, 93(1), 79-83.

34. Sohn, H., \& Szekely, J. (1972). A general dimensionless representation of the irreversible reaction between a porous solid and a reactant gas. Chemical Engneering Science, 27, 763-778.

35. Szekely, J., Evans, J. W., \& Hong, Y. S. (1976). Gas-solid reactions. New York: Academic Press.

36. Wadsworth, M. E., \& Miller, J. D. (1979). Hydrometallurgical processes Rate Processes of Extractive Metallurgy (pp. 133-244): Springer.

37. Wagman, D. D., Evans, W. H., Parker, V. B., Schumm, R. H., Halow, I., Bailey, S. M., Nuttall, R. L. (1982). The Nbs tables of chemical thermodynamic properties - Selected values for inorganic and C-1 and C-2 organic-substances in SI units. Journal of Physical and Chemical Reference Data.

38. Yang, B. H., Wu, A. X., Wang, C. L., Niu, W. X., \& Liu, J. Z. (2012). Three-dimensional simulation of pore scale fluid flow in granular ore media with realistic geometry. Transactions of Nonferrous Metals Society of China, 22(12), 3081-3086.

39. Zaretskiy, Y., Geiger, S., Sorbie, K., \& Förster, M. (2010). Efficient flow and transport simulations in reconstructed 3D pore geometries. Advances in Water Resources, 33(12), 1508-1516.

Acceptance of Terms and Conditions:

Click here to agree

\section{Modelling of the liquid water transport in the porous layers of a PEM fuel cell using pore-network modelling techniques}

Author(s): Cynthia Michalkowski ${ }^{1}$

Co-author(s): Veronika Schleper ; Rainer Helmig ${ }^{2}$ 


\footnotetext{
${ }^{1}$ Robert Bosch $\mathrm{GmbH}$

${ }^{2}$ University of Stuttgart
}

\title{
Corresponding Author(s): cynthia.michalkowski@de.bosch.com
}

For optimal operating conditions of a polymer electrolyte membrane fuel cell (PEM FC), a sophisticated water management is crucial. Therefore, it is necessary to understand the transport mechanisms of water throughout the cell constituents especially on the cathode side where the excess water has to be removed. Microscale modeling of diffusion layers and flow field has been established as a favorable technique to investigate the ongoing processes.

So far, only the gas diffusion layer is considered as a porous medium while the flow in the flow field is considered using a free flow approach. This concept reduces the computation domain to a very small section (usually including only one channel and one land) and does not allow the inclusion of spatial heterogeneities and dependencies inside the cell. Considering the gas diffusion layer as well as the flow field as porous media allows a more flexible and more efficient handling of the flow regimes and occurring phenomena, using homogenized models. This allows the coherent simulation of large cells with a active area of $200-400 \mathrm{~cm}^{2}$, as used in the automotive context. To extract such homogenized models with sufficient accuracy, a correct description of all transport phenomena at the interface of GDL and flow field is mandatory. Additionally, concerning the development of a full cell model, upscaling techniques are necessary to gain efficiency in the simulation.

Investigating the interface between the porous layers, a particular challenge is the combination and interaction of the different material structures and wetting properties at the interface and its influence on the flow.

In this work, a watershed segmentation algorithm 1 is used to convert real structures to a pore network on which a multi-phase and multi-component flow simulation [2,3] is performed for drainage and imbibition scenarios. The different wetting and structure properties of the considered materials are included in the model and first results for a combination of the two layers are presented.

\section{Procter and Gamble Student poster award:}

I would like to compete in the Procter and Gamble Student award References:

1 Gostick, J., Versatile and efficient pore network extraction method using marker-based watershed segmentation, Physical Review E 96, 023307 (2017)

2 Weishaupt, K., Helmig, R., Using a pore-network model to couple mass, momentum and energy at the interface between free flow and porous media flow, Proceedings of the 7th GACM Colloquium on Computational Mechanics for Young Scientists from Academia and Industry (2017)

3 Joekar-Niasar, V., Hassanizadeh, S. M., Dahle, H. K., Non-equilibrium effects in capillarity and interfacial area in two-phase flow: Dynamic pore-network modelling, Journal of Fluid Mechanics 655, 38-71 (2010) Acceptance of Terms and Conditions:

Click here to agree

\section{Comparative study of voids in minerals aggregates of virtual ag- gregate assembly using a game physics' engine}

\author{
Sonia Michot Roberto ${ }^{\text {None }}$; Alvaro Garcia Hernandez ${ }^{1}$; Andrew Dawson ${ }^{2}$ \\ ${ }^{1}$ University of nottingham \\ ${ }^{2}$ University of Nottingham
}

Corresponding Author(s): sonieli_85@hotmail.com

Aggregate assemblies have a great importance in civil engineering materials, such as ballast or asphalt mixture, since they influence how stresses are distributed depending on the stone-stone contact and the porosity of the material 1 , which affect strength and permeability. The design and optimisation of aggregate structures is complex, since it depends on the gradation and shape of aggregates and the compaction's methods to which they are subjected 2. To simplify the evaluation of ballast and asphalt mixture, the present work has the objective to generate an algorithm that re-creates virtually the mixing and compaction process of ballast and aggregates and evaluates the influence 
of aggregates' shape on the porosity of ballast and asphalt after compaction.

This problem has been previously analysed using the Discrete Element Modelling (DEM) method 4 , which is very computationally intensive. To accelerate the simulation of asphalt's virtual aggregate packings, the computers game engine NVIDIA's physX has been used. This method can simulate the discrete nature of the granular mixture, so as use particles with complex shapes and concavities without the high computational costs involved in DEM [6][7]. In addition, the game engine performs calculations based on physical laws and takes into account the dynamics of the rigid aggregates [8]. The algorithm developed in the game physics' engine allows to include aggregates of a range of sizes and shapes, to evaluate their influence on the final porosity and, has been used to produce virtual ballast and asphalt aggregate packings with realistic Void in Mineral Aggregates (VMA), which is the space between the aggregates that is occupied by bitumen and air voids [9]. While ballast has been simulated by simply subjecting the virtual aggregates to vibration, the effect of binder in asphalt mixture had to be simulated by changing the gravity values during compaction.

To evaluate the precision of the algorithm, ballast and asphalt granular materials have been produced experimentally, X-rayed, and their VMA structures evaluated and compared to those of virtual materials. The VMA parameters that have been analysed are the total volume of pores, their specific surface area, pore size average, global connectivity and tortuosity and, the results obtained indicated that the game physics' engine is able to simulate realistically the VMA of asphalt.

\section{Procter and Gamble Student poster award:}

I would like to compete in the Procter and Gamble Student award References:

1 N. Shashidhar and K. Gopalakrishnan, "computer modeling and particle packing simulations Evaluating the aggregate structure in hot-mix asphalt using three-dimensional computer modeling and particle packing simulations," no. March 2014, 2011.

2 J. M. Rodriguez, Importance of the Particle Shape on Mechanical Properties of Soil Materials. .

3 S. Analysis, "Influence of Size and Shape Properties of Railroad Ballast on Aggregate Packing."

[4] P. Stroeven, "Packing of non-spherical aggregate particles by DEM," pp. 809-816.

5 J. E. Lane, P. T. Metzger, and R. A. Wilkinson, A Review of Discrete Element Method (DEM) Particle Shapes and Size Distributions for Lunar Soil, no. December. 2010.

[6] A. Seugling and M. Rolin, "Evaluation of physics engines and implementation of a physics module in a 3d-authoring tool," Umea Univ., p. 89, 2006.

[7] S. M. Longshaw, M. J. Turner, E. Finch, and R. Gawthorpe, "Analysing the use of Real-time Physics Engines for Scientific Simulation: Exploring the Theoretical and Practical Benefits for Discrete Element Modelling," Acme2010, 18Th ..., no. March, pp. 18-21, 2010.

[8] A. H. Maciel, Z. Lu, L. P. Nedel, and S. De, "Using the PhysX engine for physics-based virtual surgery with force feedback," Int. J. Med. Robot. Comput. Assist. Surg., vol. Volume 5, 2009.

[9] L. Miranda, B. Rubio, R. Jiménez, L. Enríquez, and J. Loma, "Método De Dosificación Bailey. Aplica Acceptance of Terms and Conditions:

Click here to agree

183

\section{Filtration by porous media: the role of flow disorder}

Author(s): Filippo Miele ${ }^{1}$

Co-author(s): Pietro de Anna ${ }^{1}$; Marco Dentz ${ }^{2}$; Hervé Tabuteau ${ }^{3}$

${ }^{1}$ UNIL University

${ }^{2}$ IDAEA-CSIC

${ }^{3}$ Université de Rennes

Corresponding Author(s): miele.filippo@gmail.com

The understanding of colloid filtration, transport by fluid flow and their attachment to solid surfaces, is a long standing problem that affect the development of efficient strategies for bio-remediation, production and storage of fresh water. Classical approaches, like the Colloid Filtration Theory (CFT), do not capture the complex behaviors that has been often observed in colloid transport experiments through porous media. In particular, column experiments exhibits anomalous - long tailing - phenomena in Breakthrough curves (BTC) and power law decay in spatial deposition profile. However, 
on the one side: stochastic approaches are often invoked to recover consistency with experimental data, but often without a direct link with the pore-scale processes, where filtration takes place. On the other side: a proper characterization of such anomalous transport and retention is hard to obtain since most of the available data were collected over short temporal and spatial scales. We propose a novel theoretical model and a new experimental set-up for filtration by porous media. The derived model and our experimental data enable us to investigate how the microscopic processes of transport and attachment impact the macroscopic phenomenon of filtration.

Procter and Gamble Student poster award:

I would like to compete in the Procter and Gamble Student award References:

Acceptance of Terms and Conditions:

Click here to agree

616

\title{
Modeling of asphaltene precipitation and deposition in porous medium under a natural gas injection process.
}

\author{
Jesús Millán ${ }^{1} ;$ Liang $\mathrm{Yu}^{2}$; Wendong Wang ${ }^{1} ; \operatorname{Lei~Li}^{1}$ \\ ${ }^{1}$ China University of Petroleum (East) \\ ${ }^{2}$ CHina University of Petroleum (East)
}

Corresponding Author(s): jemc447@gmail.com

The Carito-Mulata is a Venezuelan field with a complex compositional system which changes from gas condensate at the top to an under saturated black oil in the flank. Asphaltene precipitation has becomes a serious problem in this field, plugging the porous medium, wellbore and production facilities which strongly affect the productivity and final recovery of the area. Recently, the implementation of some EOR strategies such as $\mathrm{CO} 2$ flooding, nitrogen flooding and WAG (natural gas) has been evaluated as alternatives to increase the recovery factor of the reservoir. However, it has been observed that most of those miscible solvents potentially aggravate asphaltene precipitation. Therefore, it has been considered necessary to define a representative numerical model to predict the phase behavior of asphaltene precipitation and deposition.

In this study, the effect of natural gas injection (at different methane concentrations) on the asphaltene precipitation process was investigated. PVT analysis was used to match a Peng-Robinson EOS in order to reproduce the phase behavior of the system. Asphaltene precipitation as function of pressure was modeled by a pure solid model which was matched by measurements of precipitation onset pressures. Then, predictions of the effect of natural gas injection on asphaltene precipitation behavior were performed. A solid deposition model was matched based on core analysis to reproduce the porosity and permeability reduction by asphaltene deposition.

The provided model was able to describe the phase behavior of reservoir oil encountered with a compositional disturbance caused by injecting light fluids into the reservoir. The changes in the onset of asphaltene precipitation for different injections showed that asphaltene precipitation effect is aggravated as the concentration of methane decrease, which in turns aggravated the porosity and permeability reduction.

Procter and Gamble Student poster award:

References:

Acceptance of Terms and Conditions:

Click here to agree 


\title{
Experimental observations of Sporosarcina Pasteurii attachment in sands and soils for a range of MICP treatment strategies
}

\author{
James Minto ${ }^{1}$; Rebecca Lunn ${ }^{1}$; Grainne El Mountassir ${ }^{1}$ \\ ${ }^{1}$ University of Strathclyde
}

Corresponding Author(s): james.minto@strath.ac.uk

\begin{abstract}
Microbially induced $\mathrm{CaCO} 3$ precipitation by urea hydrolysis is a proposed ground improvement technique that relies on either growing a community of ureolytic bacteria in-situ (bio-stimulation) or injecting a pure strain of lab-grown ureolytic bacteria (bio-augmentation), then using these bacteria to precipitate $\mathrm{CaCO} 3$ in the soil. For the bio-augmentation approach to be effective and efficient, it is crucial that we can predict the bacterial attachment properties for a range of soil types and environmental conditions as the location of attachment will largely determine where $\mathrm{CaCO} 3$ precipitation occurs.
\end{abstract}

To answer this question, we used a modified chromatography system consisting of an HPLC pump, HPLC columns of $4 \mathrm{~mm}$ internal diameter and $250 \mathrm{~mm}$ length, and a high sensitivity scanning fluorescence detector. The system was used to inject Sporosarcina pasteurii into the HPLC columns packed with sand or soil, monitor effluent breakthrough curves, and compare transport characteristics with a conservative tracer. Measurements of cell size distribution in the bacterial suspension before and after injection were made with dynamic light scattering, as was the suspensions zeta potential.

Parameters investigated were: soil type, particle size distribution, and degree of compaction; bacterial concentration, suspending media composition, $\mathrm{pH}$, and ionic strength; the effect of a 'fixation fluid' (Harkes et al., 2010) before and after bacterial injection; and the effect of flow velocity (Tobler et al., 2014) as well as length of no-flow attachment periods.

Breakthrough curves were modelled with the two-region non-equilibrium convection-dispersion equation (Tang et al., 2010). The difference between bacterial transport and conservative tracer transport was used to derive bacterial attachment parameters. The validity of these parameters was tested by using them as input for a field-scale model of MICP (Minto et al., under review) and comparing with field-scale experiment results from the literature.

Results indicate that bacterial attachment is a complex process and highly dependent on soil properties and environmental conditions, however those intending to use MICP for ground improvement will have some control on where Sporosarcina pasteurii attachment occurs by controlling ionic strength, injection flow rate, and injection point spacing.

Procter and Gamble Student poster award:

References:

Harkes, van Paassen, Booster, Whiffin \& van Loosdrecht (2010). DOI: /10.1016/j.ecoleng.2009.01.004 Minto, El Mountassir \& Lunn (under review). Field-scale simulation of ground improvement by microbially induced calcite precipitation. Submitted to Water Resources Research.

Tang, Mayes, Parker \& Jardine (2010). DOI: /10.1016/j.cageo.2010.01.013

Tobler, Cuthbert \& Phoenix (2014). DOI: /10.1016/j.apgeochem.2014.01.004 Acceptance of Terms and Conditions:

Click here to agree

\section{Pore network modeling in unsaturated soil mechanics: current state, challenges, and future perspectives}

Ehsan Nikooee ${ }^{1}$; Ghassem Habibagahi $^{1}$; Rasoul Mirghafari ${ }^{1}$; S. Sajjad Sajjadian ${ }^{1}$; Thomas Sweijen $^{2}$

\footnotetext{
${ }^{1}$ Unsaturated Soil Mechanical Laboratory, Dept. of Civil and Environmental Engineering, Shiraz University
} 
${ }^{2}$ Department of Earth Sciences, Utrecht University

Corresponding Author(s): rasmir90@gmail.com

Having vastly utilized in other disciplines such as hydrogeology, and petroleum engineering, pore networks have faced less attention in geotechnical engineering and particularly unsaturated soil mechanics. Unsaturated soil mechanics deals with soils in their two-phase state where two or more than two fluid phases coexist and flow inside soil porous medium. In order to obtain various fundamental characteristics of unsaturated soils such as soil water retention curve, unsaturated hydraulic conductivity as well as effective stress parameter, one would need detailed information about fluid phases and their interfaces [1-3]. Such information is not easily available. Sophisticated experimental set up such as triaxial testing equipped with simultaneous micro-tomography imaging may be one possibility for such purposes to look at hydromechanical behavior of unsaturated soil while detailed information from the fluid fabric, phases and interfaces inside the porous skeleton is obtained. However, such advanced techniques are not yet available due to complexity of having enough resolution as well as imaging and loading at the same time. Pore network models (PNMs) which target capturing structural features of soil porous skeleton can be utilized to model multiphase flow therein at the same time the porous skeleton can be updated and used as a mean to model coupled processes such as hydro-mechanical or chemo-hydromechanical processes. However, the pore network models which consist of pores and throats as idealization of the soil porous skeleton still suffer from mathematical and geometrical simplifications which can influence their simulations. Furthermore, extraction of the required information to construct a pore network is another challenge. In this presentation, a review of the state of art in applications of pore networks in unsaturated soil mechanics is briefly offered [4-7]. Next, major challenges in application of pore networks for modeling hydraulic processes as well as hydro-mechanical coupling of unsaturated soils are presented. Possible solutions to alleviate these shortcomings are discussed. The presentation ends with future perspectives and possibilities for the use of PNMs in modeling various processes in unsaturated soils.

Keywords: Pore network models, unsaturated soils, soil water retention curve, hydro-mechanical coupling, soil porous skeleton

\section{Procter and Gamble Student poster award:}

I don't want to compete References:

1 Likos, W. J. (2014). Effective stress in unsaturated soil: Accounting for surface tension and interfacial area. Vadose Zone Journal, 13(5).

2 Manahiloh, K. N., \& Muhunthan, B. (2012). Characterizing liquid phase fabric of unsaturated specimens from X-ray computed tomography images. In Unsaturated soils: Research and applications (pp. 71-80). Springer, Berlin, Heidelberg.

3 Nikooee, E., Habibagahi, G., Hassanizadeh, S. M., \& Ghahramani, A. (2013). Effective stress in unsaturated soils: A thermodynamic approach based on the interfacial energy and hydromechanical coupling. Transport in porous media, 96(2), 369-396.

[4] Khaksar, H., Habibagahi, G., \& Nikooee, E. (2013). SWRC modeling in unsaturated soils: A pore network approach. In Poromechanics V: Proceedings of the Fifth Biot Conference on Poromechanics (pp. 1570-1579).

5 Nikooee, E., Sweijen, T., \& Hassanizadeh, S. M. (2016). Determination of the relationship among capillary pressure, saturation and interfacial area: a pore unit assembly approach. In E3S Web of Conferences (Vol. 9, p. 02002). EDP Sciences.

[6] Rostami, A., Habibagahi, G., Ajdari, M., \& Nikooee, E. (2013). Pore network investigation on hysteresis phenomena and influence of stress state on the SWRC. International Journal of Geomechanics, 15(5), 04014072.

[7] Sweijen, T., Nikooee, E., Hassanizadeh, S. M., \& Chareyre, B. (2016). The effects of swelling and porosity change on capillarity: DEM coupled with a pore-unit assembly method. Transport in porous media, 113(1), 207-226. Acceptance of Terms and Conditions:

Click here to agree

\section{The impact of intraformational baffles on carbon mineralization in $\mathrm{CO} 2$ storage reservoirs}


Author(s): Achyut Mishra ${ }^{1}$

Co-author(s): Ralf Haese ${ }^{1}$; Abhijit Chaudhuri ${ }^{2}$

${ }^{1}$ Peter Cook Centre for CCS Research and The University of Melbourne

${ }^{2}$ Indian Institute of Technology Madras

Corresponding Author(s): achyutm@student.unimelb.edu.au

Lithological heterogeneity plays an important role in geological carbon storage $[1,2]$. The impact of heterogeneity at reservoir scale upon flow and $\mathrm{CO} 2$ trapping capacity has been well recognised 1 . But there exists a knowledge gap regarding the quantitative impact of sub-meter scale lithological heterogeneity on the mechanisms and the volume of CO2 stored in the sub-surface. This mainly results from the lack of relevant field data documenting heterogeneity at sub-metre scales as well as computationally intensive nature of dynamic simulations implementing small scale lithological heterogeneity in reservoir models. As a consequence, lithological heterogeneity in the form of intraformational baffles often gets neglected $\mathrm{CO} 2$ trapping studies at reservoir scale. Carbon mineralisation is one trapping mechanism which appears to be enhanced at the lower boundary of intraformational baffles. In this case, divalent cations are mobilised from the higher amount of clay minerals and (partly) reprecipitate as carbonate cements [3,4,5,6]. Therefore, neglecting baffles in reservoir scale models might lead to an underestimation of the volume of $\mathrm{CO} 2$ permanently trapped in the sub-surface. This study addresses the need to better understand the role of small-scale lithological heterogeneity on $\mathrm{CO} 2$ trapping, specifically carbon mineralisation. Geostatic models with common sedimentary structures and rock types are developed and used in multiphase reactive transport simulations to assess the nature of geochemical reactions and the rate of mineralisation in baffles.

Initial core analysis was used to deduce the nature of $\mathrm{cm}$-scale heterogeneity, which occurs in the form of sedimentary structures like cross bedding, planar bedding and massive bedding. The observed sedimentary structures are replicated in $\mathrm{cm}$-scale static models developed within the software SBED v4.3. Rock types are defined and characterised based on the analysis of physical and chemical properties like porosity, permeability, capillary entry pressure and mineral composition. The models are then populated with different rock type classes and their respective properties. A total of 80 model realizations were built to assess the complete impact of heterogeneity in sedimentary structure, porosity, permeability, mineral composition and specific surface area of clay minerals upon carbon mineralization. Static model realizations were then exported to TOUGHREACT v1.2 where multiphase multicomponent reactive transport simulations were performed. $\mathrm{CO} 2$ was injected as a supercritical phase, dissolved to the point of saturation and the $\mathrm{CO} 2$ enriched water reacted with the mineral assemblages of the different rock types implemented in various model realizations. The changes in volume fractions of different minerals as well as the rates of porosity and permeability evolution were studied over a simulation period of 1000 years.

Results from large number of simulations were utilised to derive an upscaled law relating the change in volume fraction of carbonate minerals as a function of heterogeneity in porosity, permeability and mineral composition. The latter was quantified in terms of dimensionless parameters referred to as heterogeneity factors. The models honour facies, sedimentary structure and rock type distribution based on field data and hence give confidence in realistically quantifying the volume of carbon trapped via mineral trapping after incorporating the effect of lithological heterogeneity at sub-meter scales.

\section{Procter and Gamble Student poster award:}

I would like to compete in the Procter and Gamble Student award References:

1 Frykman, P., Bech, N., Soresen, A.T., Nielsen, L.H., Nielsen, C.M., Kristensen, L., Bidstrup, T., 2009. Geological modelling and dynamic flow analysis as initial site investigation for large-scale CO2 injection at the Vedted structure, NW Denmark. Energy Procedia 1, 2975-2982.

2 Metz, B., Davidson, O., Coninck, H.d., Loos, M., Meyer, L., 2005. IPCC Special Report on Carbon Dioxide Capture and Storage. Intergovernmental Panel on Climate Change.

3 Higgs K.E et al., 2012. Changes in reservoir heterogeneity and quality as a response to high partial pressure of CO2 in a gas reservoir, New Zealand. J. Mar. Petr. Geol. 32: 110-137.

[4] Higgs K.E. et al., 2015. The Pretty Hill Formation as a natural analogue for CO2 storage: An investigation of mineralogical and isotopic changes associated with sandstones exposed to low, intermediate and high CO2 concentrations over geological time. Chem. Geol. 399: 36-64.

$5 \mathrm{Lu}$ J. et al., 2012, Complex fluid flow revealed by monitoring CO2injection in a fluvial formation. J. Geophys. Res. 117, B03208, doi: 10.1029/2011JB008939 
[6] Watson, M.N., 2012. Natural CO2 accumulations as analogues for $\mathrm{CO} 2$ geological storage and $\mathrm{CO} 2$ induced diagenesis in the Otway Basin, Australia. PhD Thesis. Acceptance of Terms and Conditions:

Click here to agree

\title{
Laboratory Investigation of Infiltration of Non-Newtonian Flu- ids through Cold Porous Media for Effective Remediation of Ad- sorbed Contaminants
}

\author{
Fawad Naseer $^{1}$; Debasmita Misra ${ }^{1}$; Majdi Abou Najm ${ }^{2}$; Obadare Awoleke $^{1}$ \\ ${ }^{1}$ University of Alaska Fairbanks \\ ${ }^{2}$ University of California Davis
}

Corresponding Author(s): dmisra@alaska.edu

\begin{abstract}
Non-Newtonian fluids such as biopolymers (e.g., Polyacrylamide solution) have been used for soil and groundwater remediation and enhanced oil recovery. While laboratory and field scale studies including modeling of non-Newtonian fluid flow in porous media as a remediation agent has been studied, but those have been limited to temperatures at or above $30^{\circ} \mathrm{C}$. Little is known about the flow characteristics of biopolymer solutions through a porous medium, especially at the range of low temperatures that are experienced in cold regions. Hence, it is critical to understand the impact of temperature on the flow or rheology of non-Newtonian fluids in soils, because the adsorption kinetic of each contaminant is different. Once the flow characteristic is understood, the effectiveness of non-Newtonian fluids in remediation of adsorbed contaminants can be assessed. Our hypothesis is that the difference in rheological characteristics between Newtonian and non-Newtonian fluids make the latter a better candidate for remediation of adsorbed contaminants from soils at different thermal regimes. We have investigated the rheological characteristics of non-Newtonian fluids in comparison to Newtonian fluids at $0.6^{\circ} \mathrm{C}, 5^{\circ} \mathrm{C}, 15^{\circ} \mathrm{C}, 19^{\circ} \mathrm{C}$ and $30.6^{\circ} \mathrm{C}$ to understand their strain, contact angle and viscosity changes with at different stress and concentration levels. We used both Guar gum and Xanthan gum solutions as non-Newtonian fluids. OFITE model 900 viscometer and Tantec contact angle meter were used to record the changes in viscosity and contact angle for concentrations of $0.5 \mathrm{~g} / \mathrm{l}, 1 \mathrm{~g} / \mathrm{l}, 3 \mathrm{~g} / \mathrm{l}, 6 \mathrm{~g} / \mathrm{l}$ and $7 \mathrm{~g} / \mathrm{l}$ of the polymer solution. The range of shear rate applied varied from $17.02 \mathrm{~s}-1$ to $1021.38 \mathrm{~s}-1$. Effect of salt on rheological characteristics were studied by separately adding $\mathrm{NaCl}(10 \mathrm{~g} / \mathrm{l})$ and $\mathrm{KCl}(10 \mathrm{~g} / \mathrm{l})$ to each polymer solution. It was observed that the sample solutions with high concentrations $(3 \mathrm{~g} / \mathrm{l}, 6 \mathrm{~g} / \mathrm{l}$, and $7 \mathrm{~g} / \mathrm{l})$ behaved as non-Newtonian shear-thinning fluids. Shear thinning behavior decreased with decrease in concentration. Currently, flow characteristic of Newtonian fluid and non-Newtonian fluids in a synthetic (glass-tube-bundle setup) porous media is being studied at temperatures of $5^{\circ} \mathrm{C}$ and $19^{\circ} \mathrm{C}$. Through these experiments, the mobility and behavior of non-Newtonian fluids under various thermal regimes will be characterized and its effectiveness in removing contaminants from porous media will be understood. Results of laboratory studies of rheological and flow characteristics of non-Newtonian fluids in synthetic porous media will be presented.
\end{abstract}

Procter and Gamble Student poster award:

References:

Acceptance of Terms and Conditions:

Click here to agree

\section{Fronts in two-phase flow in porous media: hysteresis and dy- namic capillarity}




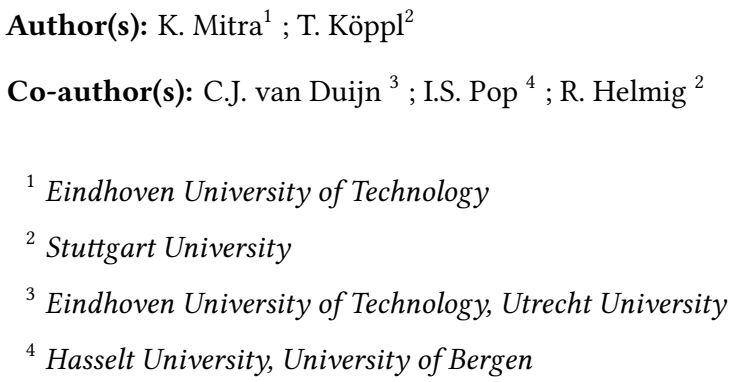

Corresponding Author(s): k.mitra@tue.nl

In two-phase porous media flow, different type of fronts can be observed in injection or drainage experiments in long columns and inside viscous fingers. For a homogeneous one-dimensional nondeformable media with a constant total influx, the dimensionless governing differential equations can be reduced to

begin\{equation\}

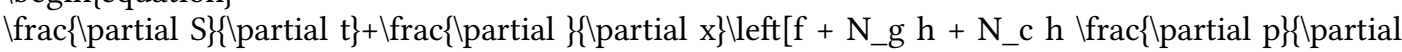
$\mathrm{x}\} \backslash$ right $]=0 . \backslash$ phantom $\{$ abcdefgh $\} \backslash \operatorname{text}\{(1)\}$

lend\{equation\}

Here $N_{c}$ is the capillary number, $N_{g}$ is the gravity number and $f, h$ are fractional flow functions and $x$ points along gravity. Two unknowns are involved: $S$, the saturation of the wetting phase and $p$, the difference between pressures of the two phases. Commonly it is assumed that fractional flow functions, as well as the phase pressure difference, are functions of the saturation of the wetting phase, i.e.

lbegin $\{$ equation

$\mathrm{f}=\mathrm{f}(\mathrm{S}), \backslash ; \mathrm{h}=\mathrm{h}(\mathrm{S}), \backslash ; \mathrm{p}=\mathrm{P} \_\mathrm{c}(\mathrm{S}) . \backslash$ phantom $\{$ abcdefgh $\} \backslash \operatorname{text}\{(2)\}$

lend $\{$ equation $\}$

However, models based on this assumption cannot explain phenomena like saturation overshoot or finger formation, which are observed experimentally but ruled out as solutions to (1)-(2) 1. Moreover, history dependent effects are disregarded both in $p$ and in $f, h 2$.

In this work, we consider non-standard models, where dynamic and hysteretic effects are included in (2). As an example, the following expression is used to expand $p$ :

begin $\{$ equation $\}$

p $\backslash$ in $\mathrm{P}^{\wedge}+$ _c(S)-P^__c(S) $\backslash$ cdot $\backslash$ mathrm $\{\operatorname{sign}\}(\backslash$ partial_t S)-N_c $\backslash$ tau $\backslash$ partial_t S. $\backslash$ phantom $\{$ abcdefgh $\} \backslash$ text $\{(3)\}$ lend\{equation\}

To understand how the behaviour of fronts changes upon adding these effects, we consider travelling wave (TW) formulation [3,4,5]. First, the situation when $N_{g}=0$ and hysteresis is not present in $f$, is analyzed. Next, $N_{g}$ large case is investigated where the flux function becomes non-monotone. Finally, the effects of adding hysteresis in $f$ and $h$ are discussed. The entropy solution of (1) in the hyperbolic limit $N_{c} \searrow 0$ are derived using the TW analysis. These solutions have much wider variety compared to the ones derived using Oleinik entropy conditions, e.g. they include non-monotone profiles and up to three shocks. In the end, numerical results are presented that support the analytical findings.

\section{Procter and Gamble Student poster award:}

I would like to compete in the Procter and Gamble Student award References:

1 S.M. Hassanizadeh and W.G. Gray. Thermodynamic basis of capillary pressure in porous media. Water Resources Research, 29(10):3389-3405, 1993.

2 J.M. Nordbotten, and M.A. Celia, Geological Storage of CO2: Modeling Approaches for Large-Scale Simulation, Wiley, 2011.

3 C.J. Van Duijn, L.A. Peletier, and I.S. Pop. A new class of entropy solutions of the Buckley-Leverett equation. SIAM Journal on Mathematical Analysis, 39(2): 507-536,

2007.

[4] C.J. van Duijn, Y. Fan, L.A. Peletier, and I.S. Pop. Travelling wave solutions for degenerate pseudo-parabolic equations modelling two-phase ow in porous media. Nonlinear Analysis: Real World Applications, 14(3):1361-1383, 2013.

5 C.J. van Duijn, K. Mitra, and I.S. Pop. Travelling wave solutions for the Richards equation incorporating non-equilibrium effects in the capillarity pressure. Nonlinear Analysis: Real World Applications, 41: 232-268, 2018. Acceptance of Terms and Conditions:

Click here to agree 


\title{
Microbial ecology of seawater flooded petroleum reservoirs: a modelling and field study
}

\author{
Jahanbani Veshareh Moein ${ }^{1}$; Hamid M. Nick ${ }^{1}$ \\ ${ }^{1}$ Danish Hydrocarbon Research and Technology Center \\ Corresponding Author(s): moein@dtu.dk
}

For decades, water injection has been utilized by different petroleum operators to increase ultimate oil recovery. Considering the limitation of available fresh water resources, especially for offshore water injection platforms, seawater is often injected. Seawater is full of oxidants such as sulphate and it cools down reservoir temperature; therefore, it changes microbial ecology of petroleum reservoirs. Nitrate, as a stronger oxidant has been injected in some of seawater injection projects to reduce the environmental footprint of seawater injection by dominating nitrate reducers over sulphate reducers $[1,2]$. Literature works through gene analysis of injection water and production water samples have revealed that nitrate injection significantly shift the microbial community composition toward nitrate reducing microorganisms [3,4]. This change in microbial community is suggested to be due to different mechanisms. For example, heterotrophic nitrate reducing microorganisms can cut down in the electron donor available for sulphate reducing microorganisms. They also produce nitrite that has an inhibitory effect on sulphate reducing microorganisms. On the other hand, reports from oil operators show an insignificant effect of nitrate injection on the activity of sulphate reducing microorganisms 5. Therefore, it seems that genomic-based experiments cannot represent the active microbial community that is developed through seawater flooding.

In this work we employ a coupled thermal-hydrodynamic-microbial model to reveal the distribution of different active microorganisms in a model reservoir (corresponding to an industrial case). We show that in current nitrate injection concentrations, nitrate effect on microbial ecology is insignificant. We will also address how biologic processes induced by seawater injection and nitrate treatment affect porous media characteristics. Utilizing a reactive transport model help decision makers in petroleum companies to design more effective reservoir souring mitigation strategies. On top of that, it contribute in less production of toxic H2S gas, and less consumption of toxic H2S scavengers.

Procter and Gamble Student poster award:

\section{References:}

1 Veshareh, M. Jahanbani, and H. M. Nick. "Learnings from Reservoir Souring Treatment by Nitrate Injection in the Halfdan Oil Field." 80th EAGE Conference and Exhibition 2018. 2018.

2 Veshareh, M. Jahanbani, and H. M. Nick. "Nitrate Treatment Strategy, Differences of the Reservoir Scale and the Lab Scale." First EAGE/IFPEN Conference on Sulfur Risk Management in Exploration and Production. 2018.

3 Vigneron, Adrien, et al. "Succession in the petroleum reservoir microbiome through an oil field production lifecycle.” The ISME journal 11.9 (2017): 2141.

[4] Gittel, Antje, et al. "Prokaryotic community structure and sulfate reducer activity in water from high-temperature oil reservoirs with and without nitrate treatment." Applied and Environmental Microbiology 75.22 (2009): 7086-7096.

5 Mitchell, Anthony F., et al. “A review of reservoir souring for three North Sea fields." CORROSION 2010. NACE International, 2010. Acceptance of Terms and Conditions:

Click here to agree

\section{Modified Low Salinity Water Efficiency by Advanced Core-Flooding under X-Ray Tomographic Monitoring}

Author(s): Leili Moghadasi ${ }^{1}$ 
Co-author(s): Dario Renna ${ }^{1}$; Sara Scagliotti ${ }^{1}$; Patrizia Pisicchio ${ }^{1}$; Martin Bartosek ${ }^{1}$; Franco Masserano ${ }^{1}$; Michela De Simoni ${ }^{1}$

${ }^{1}$ ENI SpA

Corresponding Author(s): leili.moghadasi@eni.com

Low Salinity Modified Water (LSMW) injection is considered as promising advanced water based Enhanced Oil Recovery (EOR) technique. Understanding the mechanism of modified water injection and its impact is complex due to several chemical liquid-solid interactions. In this work, we focus on the experimental study to investigate the effects of Low Salinity Modified Water injection on oil recovery by in situ monitoring of fluid displacement relying on X-Ray CT imaging.

We present sets of core-flooding experiments carried out on outcrop sandstone plugs to investigate the effect of formation water, Low Salinity water and modifying its composition on oil recovery. All the core-flooding tests were conducted in tertiary mode by reducing the salinity and modifying the ionic composition of injected water. The dynamic interaction between different injected water and rock minerals in absence and in presence of crude oil were also tested.

The results of core-flooding experiments document the differential effects of Low Salinity Modified Water on increasing the $\mathrm{pH}$ and wettability alteration toward water wet, reducing residual oil saturation therefore resulting oil recovery improvement. We also emphasized the crucial influences of different injected waters and crude oil interaction with pore surface minerals to better assess the LSMW efficiency on oil recovery. With the aid of advanced core-flooding setup integrated with industrial X-Ray tomography, we were able to deeper understand and access the complex dynamics displacements according to diverse resolutions, leading to advanced characterization of the effects of local sample inhomogeneity on oil recovery.

By optimizing the water salinity and composition, LSMW injection as advanced water based EOR technique, can be implemented to enhance oil recovery in favorable conditions.

Procter and Gamble Student poster award:

References:

Acceptance of Terms and Conditions:

Click here to agree

184

\section{Bayesian Validation of a Coupled Porous Medium/Free-flow Model using Adaptive arbitrary Polynomial Chaos Expansion}

Farid Mohammadi ${ }^{1}$; Sergey Oladyshkin ${ }^{2}$; Bernd Flemisch ${ }^{1}$

${ }^{1}$ Institute for Modeling Hydraulic and Environmental Systems (IWS), University of Stuttgart

${ }^{2}$ Institute for Modeling Hydraulic and Environmental Systems Dept. of Stochastic Simulation and Safety Research for Hydrosystems, University of Stuttgart

Corresponding Author(s): farid.mohammadi@iws.uni-stuttgart.de

Assessment of the computational model performance against experimental data can be extremely informative, but cumbersome since the experiment in question has probably not been designed to serve as a basis for the validation of the computational model. On the other hand, pure code inter-comparison studies cannot ensure that a successfully participating model indeed maps the reality.

Investigating the correctness of the software representing such a model constitutes an important part of the model validation process. The main challenge in the validation of computational models arises from the possibly large uncertainties that are present in the experimental data as well as in the simulation results.

To formulate and conduct benchmarks which assist in the uncertainty-aware validation of computational models, we propose a Bayesian validation framework that incorporates parameter and conceptual uncertainty. Incorporation of a fully Bayesian approach will yield an optimal bias-variance 
trade-off against the experimental data and will provide an integrative quantity of the model validation.

Additionally, in order to guarantee the feasibility of the proposed framework for computational demanding models, we incorporate model reduction techniques using an Adaptive arbitrary Polynomial Chaos Expansion (AaPCE). An adaptive algorithm is formulated for automatic identification of the significant coefficients of the aPC expansion by employing Bayesian model selection in a Bayesian updating of reduced model. Hence, a rather few aPC terms are eventually preserved, which obtained at a reduced computational cost compared to the full aPC expansion.

The method is illustrated on the validation of a model developed by Fetzer et al. (2016) to investigate the effect of turbulence and roughness on coupled porous-medium/free-flow exchange processes. The experimental results are taken from Davarzani et al. (2014).

Procter and Gamble Student poster award:

\title{
References:
}

1 Fetzer, T., Smits, K. M., \& Helmig, R. (2016). Effect of turbulence and roughness on coupled porous medium/free-flow exchange processes. Transport in Porous Media, 114(2), 395-424.

2 Davarzani, H., Smits, K., Tolene, R. M., \& Illangasekare, T. (2014). Study of the effect of wind speed on evaporation from soil through integrated modeling of the atmospheric boundary layer and shallow subsurface. Water resources research, 50(1), 661-680. Acceptance of Terms and Conditions:

Click here to agree

\section{A General, Data-Driven Dispersion Model for Stochastic Trans- port in Heterogeneous Media}

\author{
Author(s): Ranit Monga ${ }^{1}$ \\ Co-author(s): Oliver Brenner ${ }^{1}$; Daniel Meyer ${ }^{2}$; Patrick Jenny ${ }^{2}$ \\ ${ }^{1}$ Institute of Fluid Dynamics, ETH Zürich \\ ${ }^{2}$ Institute of Fluid Dynamics, ETH Zurich
}

Corresponding Author(s): rmonga@student.ethz.ch

Dispersion associated with fluid flows in porous media is of broad interest owing to its implications in, e.g. contaminant transport, nuclear waste disposal, exploration of fossil fuels and geothermal energy extraction etc. For systematic studies of such systems, a good understanding and predictive numerical models of dispersion are essential.

Subsurface formations exhibit, typically, a high degree of spatial variability in their properties over by a broad range of length scales. To deal with the associated inherent uncertainties and with their average effects on the contaminant transport, various stochastic dispersion models have been developed. A major difficulty, thereby, is that the relevant properties, e.g. the permeability, are spatially correlated.

Continuous Time Random Walk (CTRW) [Berkowitz et al. (1995), (1998)] models and its variants, thereof, have enjoyed considerable success in predicting the non-Fickian stochastic dispersion in flow networks. Le Borgne et al. (2008) have introduced a correlated CTRW method for continuous heterogeneous porous media which, in some cases, proved to be predictive for longitudinal stochastic dispersion. No convincing CTRW results have been published for transverse dispersion in continua, however.

The Markovian Velocity Process (MVP) model [Meyer and Tchelepi (2010)] and the Polar MVP (PMVP) model [Meyer et al. (2013)] are based on Langevin equations for the velocity, which are used for the tracer particle evolution. The coefficients, in its formulations, can be parametrized based on the permeability and the velocity statistics obtained from a limited number of Monte-Carlo 
realizations. Numerical studies with exponentially correlated log-normally distributed permeability fields show that these methods provide very accurate longitudinal and transverse dispersion profiles at a small fraction of the cost required for the MC simulation. However, for more complicated permeability distributions, e.g. with Gaussian correlation structure or with channels, the use of Langevin equations becomes inadequate.

Recently, a data driven Discrete Temporal Markov Velocity Process (DTMVP) model was presented [Delgoshaie et al. (2018)], which proved to accurately predict stochastic dispersion in porous media with log-normal permeability distribution having Gaussian- or exponential correlation structures.

Our current work integrates the ideas from CTRW, PMVP and DTMVP, and aims at porous media with multi-modal permeability distributions and more complex multi-point statistics. Unlike in CTRW for continua, variable step lengths are used in the discrete particle trajectories and the effect of the transverse particle coordinates is taken into account, as was established in the PMVP model formulation. Numerical studies with the proposed model and comparisons with reference MC data demonstrate the range of applicability.

\section{Procter and Gamble Student poster award:}

I would like to compete in the Procter and Gamble Student award References:

1. Berkowitz, B., \& Scher, H. (1995). On characterization of anomalous dispersion in porous and fractured media. Water Resources Research, 31(6), 1461-1466.

2. Berkowitz, B., \& Scher, H. (1998). Theory of anomalous chemical transport in random fracture networks. Physical Review E, 57(5), 5858.

3. Le Borgne, T., Dentz, M., \& Carrera, J. (2008). Spatial Markov processes for modeling Lagrangian particle dynamics in heterogeneous porous media. Physical Review E, 78(2), 026308.

4. Meyer, D. W., \& Tchelepi, H. A. (2010). Particle-based transport model with Markovian velocity processes for tracer dispersion in highly heterogeneous porous media. Water resources research, 46(11).

5. Meyer, D. W., Tchelepi, H. A., \& Jenny, P. (2013). A fast simulation method for uncertainty quantification of subsurface flow and transport. Water Resources Research, 49(5), 2359-2379.

6. Delgoshaie, A. H., Glynn, P. W., Jenny, P., \& Tchelepi, H. A. (2018). A flexible temporal velocity model for fast contaminant transport simulations in porous media. Water Resources Research, 54(10), 8500-8513.

Acceptance of Terms and Conditions:

Click here to agree

901

\section{Coupled Thermally-Enhanced Bioremediation and Renewable En- ergy Storage System: Conceptual Framework and Modeling In- vestigation}

Kathleen Smits ${ }^{1}$; Ali Moradi ${ }^{2}$

${ }^{1}$ The University of Texas at Arlington

${ }^{2}$ Humboldt State University

Corresponding Author(s): am651@hsu.edu

A Novel method to couple an environmental bioremediation system with a subsurface renewable energy storage system is presented. This method involves first treating unsaturated contaminated soil using in-situ thermally enhanced bioremediation; the thermal system is powered by renewable energy and coupled with a long-term energy storage system for follow on energy requirements 
after remediation is achieved. After remediation goals are achieved, the thermal system can then be used to store renewable energy in the form of heat in the subsurface for later use. This method can be used for enhanced treatment of environmental pollutants for which temperature is considered a limiting factor. For instance, this system can be used at a wide variety of petroleum-related sites that are likely contaminated with hydrocarbons. A case-study example was analyzed using a previously developed numerical model of heat transfer in unsaturated soil. Results demonstrate that coupling energy storage and thermally-enhanced bioremediation systems offer an efficient and sustainable way to achieve desired temperature-moisture distribution in soil that will ultimately enhance the microbial activity.

Procter and Gamble Student poster award:

I don't want to compete References:

Acceptance of Terms and Conditions:

Click here to agree

\title{
Correlations between cross-over and critical flow conditions for steady-state two-phase flows in porous media
}

\author{
Alexandros Morfopoulos ${ }^{1}$; Marios Valavanides ${ }^{1}$ \\ ${ }^{1}$ University of West Attica \\ Corresponding Author(s): alekosmorf@gmail.com
}

During routine /special core analysis (R/SCAL) plots of relative permeabilities against saturation always intersect at the so-called cross-over point, indicating there is a certain flow set-up whereby relative permeability of the NWP becomes equal to that of the WP 1 . A virtual saturation value can then be identified. In addition, so long as the mobility ratio equals flow rate ratio (a direct result of flow analysis for steady-state conditions), the corresponding, cross-over value of the flow rate ratio, can be indentified to be equal to the inverse of the NWP/WP viscosity ratio 2. In addition, analysis of steady-state two-phase flow, from an energy efficiency point of view, has revealed a universal flow characteristic, the existence of a unique locus of the so-called critical flow conditions, whereby the energy efficiency of the process (NWP flow rate per unit power spent) attains locally maximum values. Both of these universal theoretical results are verified against ample experimental evidence 1. The existence of a unique locus of critical flow conditions per N/W/PM system has opened new perspectives in effectively describing the sought process in terms of the actual independent variables, i.e. the NWP and WP flow rate intensities or -equivalently- the capillary number and the flow rate ratio 3. Corresponding, critical values of the saturation and the flow rate ratio can also be identified for different N/W/PM systems and flow conditions.

In an effort to improve our understanding of the behavior of the sought process and its inherent, universal flow characteristics, we have plotted cross-over against critical values of saturation and flow rate ratio. The values have been retrieved from a review of 180 published relative permeability diagrams, pertaining to different N/W/PM systems and flow conditions 1 . Preliminary results indicate a latent, weak correlation between the cross-over and critical values of the saturation. The correlation becomes linear when the viscosity ratio approaches unity, i.e. when the viscosity of the NWP approaches that of the WP. Similar, but more weak correlations have been observed for the cross-over /critical values between saturation and flow rate ratio.

Procter and Gamble Student poster award:

I don't want to compete References:

1. Valavanides, M.S., Totaj, E., Tsokopoulos, M. Energy efficiency characteristics in steady-state relative permeability diagrams of two-phase flow in porous media. J. Petrol. Sci. Eng. 147, 181201 (2016). https://doi.org/10.1016/j.petrol.2016.04.039

2. Valavanides, M.S. Review of steady-state two-phase flow in porous media: independent variables, universal energy efficiency map, critical flow conditions, effective characterization of flow and 
pore network. Transp. in Porous Media 123 (1), 42-99 (2018), https://doi.org/10.1007/S11242-0181026-1

3. Valavanides, M.S. Universal, true to mechanism, flow dependent relative permeability scaling functional form for steady-state 2-phase flows in p.m.”, Intern. Symposium of the Society of Core Analysts, Trondheim, Norway, 27-30/8/2018, http://users.uniwa.gr/marval/publ/Valavanides_SCA2018_066.pdf

Acceptance of Terms and Conditions:

Click here to agree

138

\title{
Binary solvents in nanoporous confinement: how different are they?
}

\author{
Author(s): Denis Morineau ${ }^{1}$ \\ Patrick Huber ${ }^{3}$; Michael Fröba ${ }^{4}$; Bernhard Frick ${ }^{5}$; Laurence Noirez ${ }^{6}$ \\ ${ }^{1}$ CNRS - Institute of Physics of Rennes \\ ${ }^{2}$ Institute of Physics of Rennes, CNRS-University of Rennes 1, France \\ ${ }^{3}$ Hamburg University of Technology \\ ${ }^{4}$ University of Hamburg \\ ${ }^{5}$ Institute Laue-Langevin, Grenoble, France \\ ${ }^{6}$ Laboratoire Léon Brillouin, CEA-CNRS, Gif-Sur-Yvette, France
}

Co-author(s): Aicha Jani ${ }^{2}$; Ramona Mhanna ${ }^{2}$; Sujeet Dutta ${ }^{2}$; Ronan Lefort ${ }^{2}$; Ilham Essafri $^{2}$; Aziz Ghoufi ${ }^{2}$;

Corresponding Author(s): denis.morineau@univ-rennes1.fr

Fluids confined in nanometer-size porous geometry exhibit unique properties that have no equivalent in the corresponding bulk systems. As such, they deserve an extensive interest for their high potential of technological innovation.

Fundamentally, it turns out that the exact nature of the surface-liquid interaction is one key-parameter, which affects the phase behavior, structure, dynamics and fluid flow.

In this presentation, we report how the precise control of the liquid/liquid and liquid/surface interactions can be used to direct the structural and dynamical properties of nanoconfined fluids. Our strategy is based on the use of carefully designed mesostructured porous materials along with binary solvents with amphiphilic interactions. It relies on the combination of an extensive number of complementary methods, both experimental and numerical, encompassing temporal and spatial windows that range from the molecular to the macroscopic scales.

\section{Procter and Gamble Student poster award:}

I would like to compete in the Procter and Gamble Student award References:

1 Press release of the American Institute of Physics, January 10th (2017): https://publishing.aip.org/publishing/journalhighlights/zeroing-true-nature-fluids-within-nanocapillaries

2 Micro-Phase Separation of Binary Liquids Confined in Cylindrical Pores A. R. Abdel Hamid, R. Mhanna, R. Lefort, A. Ghoufi, C. Alba-Simionesco, B. Frick and D. Morineau, J. Phys. Chem. C, 120, 9245 (2016). 3 More room for microphase separation: An extended study on binary liquids confined in SBA-15 cylindrical pores, R. Mhanna, A. R. Abdel Hamid, S. Dutta, R. Lefort, L. Noirez, B. Frick and D. Morineau, J. Chem. Phys., 146024501 (2017).

[4] Multiple Glass Transitions of Microphase Separed Binary Liquids Confined in MCM-41, A. R. Abdel Hamid, R. Mhanna, P. Catrou, Y. Bulteau, R. Lefort and D. Morineau, J. Phys. Chem. C, 120, 11049 (2016).

5 Thermodynamics of binary gas adsorption in nanopores, S. Dutta, R. Lefort, D. Morineau, R. Mhanna, O. Merdrignac-Conanec, Arnaud Saint-Jalmes and T. Leclercq, Phys. Chem. Chem. Phys., 18, 24361

(2016). Acceptance of Terms and Conditions:

Click here to agree 


\title{
Multiphysics gas transport in coal seams
}

\author{
Peyman Mostaghimi ${ }^{1}$; Ryan Armstrong ${ }^{\text {None }}$; Xiao Lu ${ }^{\text {None }}$; Dylan Shaw ${ }^{\text {None }}$ \\ ${ }^{1}$ UNSW Sydney
}

Corresponding Author(s): peyman@unsw.edu.au

\begin{abstract}
A significant unconventional resource for energy is the methane gas stored in shallow coalbeds, known as coal seam gas. An integrated experimental and numerical framework is developed for evaluation of unique transport phenomena in coal and quantify its pertophysical properties. Two scales of gas flow exist in coal cores: flow in fractures and diffusion in matrix. The diffusion process is quantified by the gas diffusion coefficient while flow in fractures determines gas permeability. We explore dynamic permeability of coals as subsurface reservoir ages and apply special core analysis techniques to identify several trends apparent in coal relative permeability data as coal transitions from a low to high effective stress state. These observed trends are related to the cleat width distribution, high irreducible water saturation, gas slippage and matrix deformation linked to the poroelasticity of coal. We use Krypton saturated X-ray images to evaluate the diffusion process in coal matrix, estimate diffusion coefficient and employ them to interpret unique dynamic petrophysics of coal. Overall, our results provide novel physics-based evidences for coal properties and we suggest practical dynamics trends of permeability and relative permeability curves of coal to be used for reservoir engineering and simulation.
\end{abstract}

Procter and Gamble Student poster award:

References:

Acceptance of Terms and Conditions:

Click here to agree

\section{D model-based interpretation of tracer tests in large undisturbed columns from fractured clayey till}

\author{
Klaus Mosthaf $^{1}$; Peter Jørgensen ${ }^{2}$; Chloe Lanters ${ }^{3}$; Nora Badawi ${ }^{4}$; Jens Aamand ${ }^{4}$; Massimo Rolle ${ }^{1}$ \\ ${ }^{1}$ Technical University of Denmark \\ ${ }^{2}$ Pf Bluetech \\ ${ }^{3}$ DTU Environment \\ ${ }^{4}$ Geological Survey of Denmark and Greenland
}

Corresponding Author(s): klmos@env.dtu.dk

Large undisturbed columns (LUC) provide the possibility of well-controlled laboratory experiments of macroporous and fractured media to investigate transport pathways and the risk of pesticide leaching to the groundwater. We have conducted several flow and solute transport tests in large undisturbed columns (diameter $0.5 \mathrm{~m}$, height $0.5 \mathrm{~m}$ ), which were excavated from an agricultural field site in Denmark with a fractured clayey till geology. Hydraulic tests were performed on the columns to determine the main hydraulic properties. A series of solute transport tests was performed under in-situ pressure and temperature conditions. First a mixture of different pesticides (bentazone, MCPA, tebuconacole) was injected, followed by an injection of a conservative bromide tracer and finally by an injection of the color tracer brilliant blue, all under constant flow conditions. After the brilliant-blue tracer injection, the column was opened and segmented, which allowed to map the location of conductive fractures and to inspect the column interior. 
Distinct macropores and fractures could be identified as major transport pathways, with a strong hydraulic conductivity contrast between fractures and matrix. Several fractures contained iron precipitate that partly blocked the fractures and channeled the flow in the open parts. Based on the mapping and on the characterization of the clayey till matrix, a detailed 3D discrete fracture model was setup to simulate the flow and solute transport in the column considering both the preferential flow through the fractures and the interaction with the clayey-till matrix. The pesticides used in the flow-through experiments have different sorption and degradation characteristics, which were analyzed in the laboratory and included in the model simulations. The model allowed interpreting the resulting breakthrough curves of the pesticides and of the bromide tracer in the LUC setups taking into account the physical and biogeochemical processes controlling the transport and breakthrough of the different compounds. Furthermore, the influence of various parameters on solute transport through macroporous fractured clayey till could be analyzed with a sensitivity study using the calibrated model.

Procter and Gamble Student poster award:

References:

Acceptance of Terms and Conditions:

Click here to agree

\title{
A Novel Method to Evaluate Reservoir Net pay by Using Cut-offs and Hydro Flow Units
}

\author{
Mohadeseh Motie ${ }^{1}$; Ramin Soltanmohammadi ${ }^{2}$ \\ ${ }^{1}$ M.Sc student of reservoir engineering, Iran University of Science and Engineering \\ ${ }^{2}$ M.Sc student of Petroleum Engineering, Politecnico di Torino
}

Corresponding Author(s): mohadeseh.motie@gmail.com, raminsoltanmohammadi@gmail.com

Net pay (NP) is a reservoir interval containing the most recoverable hydrocarbons. Determining this thickness would be a key parameter of the volumetric calculations and predicting reservoir behavior. It is also really practical in controlling and optimizing drilling processes and well perforation by providing more accurate quantities. Hence, it is essential to precisely measure the location of the NP and estimating the correct hydrocarbons volume. To do that, cut-offs identification is considered as a suitable way to eliminate unwanted reservoir segments which do not contribute to oil and gas production.

In current work, a synthetic reservoir is first modeled and classified into an optimum number of hydro flow units (HFUs), using flow zone indicator (FZI) method. Regarding to any HFU, various cut-off values are defined to form the different cases. The effects of the different amounts of the cut-offs on cumulative oil production are then evaluated by employing dynamic simulation of the reservoir. This is done using Eclipse coupled with MATLAB. Meanwhile, Genetic Algorithm (GA) is applied to find the optimum value of cut-offs. The discrepancy with the base case value of the cumulative oil production is assumed as an objective function to be the minimum.

Despite the lack of general procedure for cut-offs identification, the results showed that great improvement in the extreme reservoir heterogeneity. This is achieved by dividing reservoir into different flow units. More important, via implementing cut-off limitations, it can be seen that not only results will not face to a significant changes, but a better perception of net pay interval can be provided.

This study was aimed to introduce a new method for estimating reservoir net pay beyond the conventional methods and rule of thumbs. In case of requiring reliable approximation of the volume of oil in place, it is necessary to prohibit non-reservoir segments or subcritical character rocks contributions. To meet this, it is essential to use a dynamic method which is introduced in current study.

\section{Procter and Gamble Student poster award:}

I would like to compete in the Procter and Gamble Student award References:

Acceptance of Terms and Conditions: 


\title{
Unconventional transport mechanisms in porous media: connec- tivity enhancement due to thin film flow
}

\author{
Marcel Moura ${ }^{1}$; Knut Jørgen Måløy ${ }^{1}$; Eirik Flekkøy ${ }^{1}$; Gerhard Schäfer $^{2}$; Renaud Toussaint ${ }^{3}$ \\ ${ }^{1}$ PoreLab, The Njord Center, Department of Physics, University Oslo \\ ${ }^{2}$ LHyGeS, CNRS, University of Strasbourg, France \\ ${ }^{3}$ IPGS, CNRS, Univ. Strasbourg \& PoreLab, Univ. Oslo
}

Corresponding Author(s): marcelmoura@yahoo.com.br

\begin{abstract}
The standard liquid transport processes in porous media happen through the usual network of interconnected pore bodies and pore throats (here called the primary network). When a non-wetting phase displaces a wetting phase from a porous sample (drainage), thin films of the wetting phase are bound to be left on the surface of the constituting grains (for example when air displaces water from a porous rock, thin films of water are left behind, covering the rock grains). Under certain conditions, isolated liquid films can eventually merge, forming a secondary network of interconnected films and capillary bridges that can effectively enhance the overall connectivity of the medium and act as a new pathway for fluid transport. We have performed experiments using transparent networks with the objective of studying these unconventional transport processes. After the usual drainage process, a set of trapped liquid clusters remains in the sample, but we observe that a small portion of them can surprisingly still be drained. This kind of unconventional drainage event can only be possible due to the enhanced connectivity introduced by the secondary network of thin films and capillary bridges. We have observed the existence of an active zone behind the main drainage front where the probability of these unconventional drainage events is maximized.
\end{abstract}

Procter and Gamble Student poster award:

References:

M. Moura, K. J. Måløy, E. G. Flekkøy, Gerhard Schäfer and R. Toussaint, "Connectivity enhancement due to film flow processes in porous media," in preparation. Acceptance of Terms and Conditions:

Click here to agree

197

\section{Examining the limitations and advantages of multiphase flow meters}

\author{
Author(s): S.Amin Mousavi ${ }^{\text {None }}$ \\ Co-author(s): Hamidreza Asaadian ${ }^{1}$ \\ ${ }^{1}$ Petroleum University of Technology
}

Corresponding Author(s): aminmoosavi30@gmail.com, hamidreza.asaadian@hotmail.com

In order to maintain production capacity, it is essential to obtain sufficient knowledge about in situ fluids and the production of liquids from the reservoir. In addition to the importance of measuring the multiphase flow, there are also major problems. As the complexity of the multiphase flow has created difficulties for modeling this flow, measuring these flows in the topics related to their recognition, has created a new chapter. Multiphase flow meters technology has created new capabilities using other technologies; this technology has solved many problems of conventional methods and in addition have been able to respond well to the industry's needs in this field. In this paper, these technologies are studied and the limitations and advantages of multiphase flow meters are examined. 
Procter and Gamble Student poster award:

I would like to compete in the Procter and Gamble Student award References:

Acceptance of Terms and Conditions:

Click here to agree

501

\title{
Mathematical model of microbiological oil recovery with wetting inversion by bio-surfactants
}

\author{
Akerke Mukhamediarova $^{1}$; Mikhail Panfilov ${ }^{2}$ \\ ${ }^{1}$ Institut Elie Cartan, Université de Lorraine \\ ${ }^{2}$ Institut Elie Cartan - Université de Lorraine; Institut fean le Rond d'Alembert, Sorbonne Universités
}

Corresponding Author(s): akerke.mukhamediyarova@univ-lorraine.fr

Oil displacement by water which contains microorganisms able to produce bio-surfactants is one of the most promising methods of oil recovery. The bio-surfactant significantly reduces the surface tension and weakens the negative role of capillary oil trapping. The second effect caused by surfactants is inversion of wetting, which is even more important for oil recovery, since it allows separating oil from pore walls, making it non-wetting (in carbonate reservoirs). We develop the mathematical model of this process, which takes into account both mentioned effects. The model of wetting alternation is its key point. On the macroscale this effect leads to the modification of the relative permeability curves, which may be modeled by special kinetic relationships. The closure relationships for the characteristic time of wetting inversion has been obtained by modelling this process at the pore-scale. The numerical method of diffuse interface was applied to system water-surfactant-oil separated by a meniscus on a solid surface.

For the kinetics of bacterial population grow and decay, we suggest new nonlinear relationships, which enables to model various physiological stages, including the lag stage.

The results of modeling have shown the appearance of specific regimes of self-organization proved in the form of auto-oscillatory waves in time and in space.

Procter and Gamble Student poster award:

I would like to compete in the Procter and Gamble Student award References:

Panfilov M. Underground storage of hydrogen: in situ self-organization and methane generation. Transp. Porous Media, v. 85, n 3, (2010): $841-865$

Panfilov M. Physicochemical Fluid Dynamics in porous Media (apprication in geosciences and Petroleum Engineering). Wiley VCH, 2018.

Nielsen S.M., Shapiro A., Stenby E.H., and Michelsen M.L. Microbial Enhanced Oil Recovery - Advanced Reservoir Simulation. PhD thesis, Technical University of Denmark (DTU), 2010. Acceptance of Terms and Conditions:

Click here to agree

806

\section{Convective Flow in Fuel Cell Gas Diffusion Layers and its Impact on Evaporation}

Author(s): Adrian Mularczyk ${ }^{1}$

Co-author(s): Felix N. Büchi ${ }^{2}$; Jens Eller ${ }^{2}$; Thomas J. Schmidt ${ }^{2}$

\footnotetext{
${ }^{1}$ Paul Scherrer Institut

${ }^{2}$ Electrochemistry Laboratory, Paul Scherrer Institut, Villigen, Switzerland
} 
Corresponding Author(s): adrian.mularczyk@psi.ch

The large potential that fuel cell technology offers, not only in mobility, but also in terms of storage of renewable and excess energy has resulted in a high demand for research in this field, to increase the performance of fuel cells and extend their areas of applicability. Amongst these research efforts is the study of the gas diffusion layer (GDL), a porous domain between the flow field and the catalyst layer of a fuel cell. Alongside mechanical stabilization of the membrane electrode assembly and the reduction of electrical and thermal resistances, its main role is the optimization of reactant gas distribution as well as improved removal of product water from the cathode catalyst. This results in a non-trivial mass transfer occurring inside the GDL which is further complicated by the accumulation of liquid water through condensation, which has been shown to block pores and thus reduce the overall mass transport. The exact mechanisms with which the different species are traversing this porous medium is still not fully understood and holds strong potential for optimization. The characterization of the evaporative transport and removal of water from the GDL in particular could help to increase fuel cell performance. The benefit would stem on the one hand from a reduction of liquid water in the GDL domain, leaving more open pore space for the transport of fresh gas to the catalyst layer, and on the other hand, the large latent heat consumed by an optimized evaporation process could be used as primary cooling mechanism for fuel cells, reducing their overall size and ancillary equipment needed. Recent numeric investigations by Safi et al. 1 suggest the contribution of a convective transport region in the GDL to the overall mass transfer. This convection reduces the overall diffusive path length the gases have to overcome in the GDL and has the potential to influence future GDL design to take full advantage of this effect. Experimental as well as computational investigations of different GDL types, operational conditions and evaporation geometries are carried out to understand their influence on this convective region and its effect on evaporation. An $\mathrm{X}$-ray tomographic microscopy compatible ex-situ cell setup is used to emulate the conditions found on the cathode side of a fuel cell. This enables monitoring of the water front in the GDL. Water is supplied to the cell at a controlled pressure using an injection pathway allowing the evaluation of the evaporative flux.

References:

1 M. A. Safi, J. Mantzaras, N. Prasinakis, A. Lamibrac, F. N. Büchi, International Journal of Heat and Mass Transfer, February 2019, Volume 129, 1250-1262

2 S. Lal, A. Lamibrac, J. Eller, F. N. Büchi, Journal of Electrochemical Society, 2018, Volume 165(9), F652-F661

Procter and Gamble Student poster award:

I don't want to compete References:

Acceptance of Terms and Conditions:

Click here to agree

65

\title{
Multiphase multi-rate mass transfer model for anomalous trans- port in fractured and heterogeneous media
}

\author{
Matteo Icardi ${ }^{1}$; Federico Municchi ${ }^{1}$ \\ ${ }^{1}$ University of Nottingham
}

Corresponding Author(s):

Anomalous transport phenomena occur in a wide range of industrial and environmental applications. For example, such phenomena are encountered in the study of heterogeneous porous media, which are of pivotal importance in modern oil\&gas and aquifer remediation research. Generally, standard Fickian diffusion models fail to accurately model solute transport in complex porous media like natural soil or fracture matrices, due to the wide range of mass transfer rates between zones of mobile contaminant (advection dominated) and immobile contaminant (diffusion dominated). In 
fact, these transfer rates depends on the complexity and variety of the pore geometry (for example polydisperse and clustered particles or randomly shaped fractures) which is not accounted for in Fickian diffusion models.

A model that account for porous systems with multiple transfer rates between one mobile zone and a set of immobile zones is the Multi-Rate Mass Transfer (MRMT) model (Haggerty \& Gorelick 1995). The MRMT is based on the multi-continuum concept, where different zones are represented as different continua communicating through first order transfer functions or linear mechanisms such as the Fick's first law (Carrera et al. 1998). In the latter case, an integral formulation of the coupling term is often employed, which takes the form of the convolution of the solute concentration with a memory function which reflects the geometry and the heterogeneity of the immobile region. While this method was initially developed for groundwater applications as a natural extension of the dual-porosity model, it has also been applied to fracture-matrix transfer (Geiger et al. 2011).

In this work, we present an implementation of the MRMT model based on the opensource finite volume library OpenFOAM that is suitable for simulating scalar transport in subsurface multiphase flows. This implementation comes as an extension of the open-source toolbox porousMultiphaseFoam (Horgue et al. 2015) that provides a series of novel features. First, we extend the Implicit Pressure Explicit Saturation (IMPES) method employed in the toolbox to non-orthogonal grids and strongly coupled systems by mean of a set of fixed point Picard iterations. Second, we implement a MRMT model for the solute concentration, which gives rise to a set of coupled equations, one for each immobile region. These equations are discretized implicitly in time, resulting in a semi-implicit method consisting in a pressure coupling loop enclosing a concentration coupling loop.

We discuss the numerical aspects of the MRMT model, comparing accuracy and performance of a monolithic solution of the MRMT equations (which are linearly coupled) against a segregated algorithm. Furthermore, results from the MRMT are compared against a range of test cases to assess its accuracy and efficiency. Our code is made available to the public through GitHub.

Procter and Gamble Student poster award:

\section{References:}

Carrera, J. et al., 1998. On matrix diffusion: formulations, solution methods and qualitative effects. Hydrogeology journal, 6(1), pp.178-190.

Geiger, S., Dentz, M. \& Neuweiler, I., 2011. A Novel Multi-rate Dual-porosity Model for Improved Simulation of Fractured and Multi-porosity Reservoirs. In SPE Reservoir Characterisation and Simulation Conference and Exhibition. SPE Reservoir Characterisation and Simulation Conference and Exhibition. Society of Petroleum Engineers. Available at: http://www.onepetro.org/doi/10.2118/148130-MS.

Haggerty, R. \& Gorelick, S.M., 1995. Multiple-Rate Mass Transfer for Modeling Diffusion and Surface Reactions in Media with Pore-Scale Heterogeneity. Water resources research, 31(10), pp.2383-2400.

Horgue, P. et al., 2015. An open-source toolbox for multiphase flow in porous media. Computer physics communications, 187, pp.217-226. Acceptance of Terms and Conditions:

Click here to agree

\section{Water sorption into and evaporation from thin porous media- a thermographic study}

Vignesh Murali $^{1}$; J. C. H. Zeegers ${ }^{1}$; Anton. A. Darhuber ${ }^{1}$

${ }^{1}$ TU/e

Corresponding Author(s): v.murali@tue.nl

Abstract for interpore Minisymposium MS16 Fluid interactions with thin porous media 
Water sorption into and evaporation from thin porous media- a thermographic study

\author{
Vignesh Murali, Jos. C. H. Zeegers and Anton. A. Darhuber \\ Department of Applied Physics, Eindhoven University of Technology, Eindhoven, The Nether- \\ lands
}

In the context of ink-jet printing, the interaction of water based inks with paper remains a subject where detailed understanding of the interplay of all the dynamic processes involved is lacking. In general, the study of the transient wetting or uptake of water by a porous medium is complicated due to the complex interplay between the movement of the wetting front and the interfacial effects arising from vapor transport, evaporation and the subsequent re-condensation or sorption. To this end, we have studied the evaporative cooling and sorptive heating of thin porous media as a function of the substrate speed (representing the printing speed in ink-jet printing) with the aim of obtaining the specific thermal signature of water-vapor-paper interaction(s).

The experimental set-up consists of a sheet of paper fastened onto a motorized translation stage. A stationary injector is connected to a motorized syringe pump that supplies water onto the suspended sheet of paper. The constant speed of the motion $U_{\text {sub }}$ of the substrate is varied in the range 0.2 to 3 $\mathrm{mm} / \mathrm{s}$. An infrared (IR) camera is used to measure the temperature field $\mathrm{T}(\mathrm{x}, \mathrm{t})$ of the substrate upon injection of water. The volume of water on the paper is kept constant in time by the syringe pump to compensate for the loss due to absorption. Different types of printing paper were used in the study. The salient feature of the experiment is the maintenance of a steady liquid front (wet zone) into which the dry substrate is moved at pre-defined and controlled speeds $\left(U_{\text {sub }}\right)$. This implies that the transient effects will die out and the system reaches a steady state. Moreover, the rigid body like motion of the substrate aids in maintaining stationary boundary conditions. The speed of the substrate, amongst other parameters, controls how far ahead of the nozzle the wetting front can propagate. Furthermore, we developed a 1D continuum model for water imbibition into unsaturated thin porous media including evaporation/condensation and heat transfer, which is coupled to a $2 \mathrm{D}$ model accounting for buoyant gas phase advection, vapor transport and heat transfer in the atmosphere above the porous media. We have obtained an excellent agreement between the results of the experiments and the results of the numerical model.

\title{
Procter and Gamble Student poster award:
}

I would like to compete in the Procter and Gamble Student award References:

Acceptance of Terms and Conditions:

Click here to agree

\section{Analysis of species mass transport in fibrous electrodes of redox flow batteries}

Miguel Muñoz-Lorente ${ }^{1}$; José M. Rubio-Hammo ${ }^{1}$; Fikile R. Brushett ${ }^{2}$; Antoni Forner-Cuenca ${ }^{2}$; Pablo A. GarcíaSalaberri ${ }^{1}$

\footnotetext{
${ }^{1}$ Departamento de Ingeniería Térmica y de Fluidos, Universidad Carlos III de Madrid

${ }^{2}$ Department of Chemical Engineering, Massachusetts Institute of Technology
}

Corresponding Author(s): 100346819@alumnos.uc3m.es

Redox flow batteries (RFBs) are regarded as one of the most promising energy storage technologies for stationary applications due to the possibility of decoupling power (dependent on the performance of the electrochemical cell) and energy (dependent on the size of the storage tanks). The majority of existing and emerging RFBs use porous carbonaceous electrodes where redox reactions take place at the fiber surface, which is both electrochemically active and electronically conductive. However, these porous electrodes (e.g., carbon-fiber papers and felts) are re-purposed gas diffusion layers (GDLs) traditionally used in fuel cells and have not been designed to meet all RFB requirements. This fundamentally limits the performance and cost of these devices, making it necessary the synthesis and design of novel porous materials for RFBs. 
In this work, mass transport of active species to the reactive surface area of thin fibrous electrodes (thickness $102 \mu \mathrm{m}$ ) used in RFBs is examined under convection-diffusion-reaction conditions by means of direct numerical simulation (finite-volume method). Pressure-driven laminar flow is modeled together with the convection-diffusion equation for species transport, considering a heterogeneous first-order reaction rate at the fiber surface. A comprehensive parametric analysis is carried out to assess the dependence of the drag coefficient, Sherwood number and electrode utilization on Reynolds, Schmidt and Damköhler numbers. Furthermore, the impact of key geometrical parameters is analyzed, including the porosity and the roughness of the electrode (defined as the ratio of the electrochemically active area, i.e., the micrometer-scale surface area of the fibers, to the macroscopic planform area of the electrode).

The proposed "computer-to-battery" approach is aimed to provide fundamental knowledge for the construction of optimized electrodes in the lab with improved power output and reduced pumping losses. The study will also provide important information for porous media manufacturers and vendors to establish a best-practice guideline, which can link a desired battery chemistry to an optimized electrode design.

Procter and Gamble Student poster award:

\title{
References:
}

Acceptance of Terms and Conditions:

Click here to agree

161

\section{Effect of inhomogeneous compression on pressure drop and species transport in electrodes of redox flow batteries}

\author{
José M. Rubio-Hammo ${ }^{1}$; Miguel Muñoz-Lorente ${ }^{2} ;$ Paul R. Shearing $^{3} ;$ Rhodri Jervis $^{3} ;$ Pablo Ángel García-Salaberri $^{2}$
}

\author{
${ }^{1}$ Departamento de Ingeniería Térmica y de Fluidos, Universidad Carlos III de Madrid, Leganés \\ ${ }^{2}$ Departamento de Ingeniería Térmica y de Fluidos, Universidad Carlos III de Madrid \\ ${ }^{3}$ Electrochemical Innovation Lab, University College London
}

Corresponding Author(s): 100346819@alumnos.uc3m.es

Assembly compression induces large deformations in fibrous electrodes of redox flow batteries (RFBs), leading to significant changes in its mass and electrical transport properties, which impact cell performance and lifetime. Moreover, the rib-channel pattern of the bipolar plate results in a highly inhomogeneous compressive load on the electrode, so that while large strains are produced in the region under the rib, the virtually uncompressed region under the channel partially blocks the flow field. This creates important spatial variations in the mass and charge transport rates through the electrode and increases the pressure drop in the cell. Therefore, a thorough understanding of the effects of assembly compression is essential for an appropriate description of the physicochemical processes and pumping losses that take place in RFBs.

In this work, micro X-ray computed tomography images of inhomogeneously compressed carbonfelt electrodes $(0.89 \mu \mathrm{m} / \mathrm{voxel})$, acquired at the Zeiss Centre for Correlative Microscopy in the Electrochemical Innovation Lab, are examined. Seven compression ratios are considered, ranging from $0 \%$ to $90 \%$. The pressure drop and mass transport of active species are modeled using direct numerical simulation of the mass, momentum and species conservation equations on the 3D segmented data sets. Specifically, the velocity field is first determined by simulating a laminar flow through the electrode. Then, the species convection-diffusion equation with a heterogeneous reaction term on the fiber surface is solved. A detailed analysis of the effect of Reynolds, Schmidt and Damköhler numbers on the Sherwood number and the utilization factor (defined as the ratio of the actual to the maximum reaction rate) is performed in randomly selected sub-domains extracted from the regions under the channel and the land, as well as the transition region between them. 
The results extracted from this analysis will provide key information about the behavior of carbonfelt electrodes in real-life applications, where the compression ratio and the flow-field width are important parameters to be optimized.

Procter and Gamble Student poster award:

I would like to compete in the Procter and Gamble Student award References:

Acceptance of Terms and Conditions:

Click here to agree

831

\section{Pattern formation of frictional fingers in a gravitational poten- tial}

Knut Jørgen Måløy ${ }^{1}$; Eirik Grude Flekkøy ${ }^{2}$; Jon Alm Eriksen ${ }^{3}$; Bjørnar Sandnes ${ }^{4}$; Toussaint Renaud ${ }^{5}$; Olivier Galland $^{6}$

${ }^{1}$ PoreLab, The Njord Center, Department, Department of Physics, University of Oslo
${ }^{2}$ PoreLab, The Njord Center, Department of Physics, University of Oslo
${ }^{3}$ PoreLab, The Njord Center, Department of Physics, University of Oslo
${ }^{4}$ College of Engineering, Swansea University
${ }^{5}$ Institute de Physique du Globe de Strasbourg, University of Strasbourg
${ }^{6}$ Physics of Geological Processes, The Njord Center, Department of Geosciences, University of Oslo

Corresponding Author(s): maloy@fys.uio.no

Aligned finger structures, with a characteristic width, emerge during the slow drainage of a liquidgranular mixture in a tilted Hele-Shaw cell. A transition from vertical to horizontal alignment of the finger structures is observed as the tilting angle and the granular density are varied. An analytical model is presented, demonstrating that the alignment properties are the result of the competition between fluctuating granular stresses and the hydrostatic pressure. The dynamics is reproduced in simulations. We also show how the system may explain patterns observed in nature, created during the early stages of a dike formation.

Procter and Gamble Student poster award:

\section{References:}

Eriksen J.A., Toussaint R., Måløy K.J., Flekkøy E., Galland O., Sandnes B., Pattern formation of frictional fingers in a gravitational potential. Phys. Rev. Fluids 3, 013801, (2018)

B. Sandnes, E.G. Flekkøy, H.A. Knudsen, K.J. Måløy, and H. See, Pattern and flow in frictional fluid dynamics. Nat. Commun. 2:288 doi:10.1038/ncomms1289 (2011)

B. Sandnes, H.A. Knudsen, K.J. Måløy, and E.G. Flekkøy, Labyrinth patterns in confined granular-fluid systems. Phys. Rev. Lett. 99, 038001, (2007). Acceptance of Terms and Conditions:

Click here to agree

\section{Environmental Constraints of Non-Mechanical Excavation of Wa- ter from Gypsum on the Martian Surface}

Shahab Bayani Ahangar ${ }^{1}$; Ezequiel F. Médici ${ }^{1}$; Paul J. van Susante ${ }^{1}$; Timothy C. Eisele $^{1}$; Jeffrey S. Allen ${ }^{1}$

${ }^{1}$ Michigan Technological University 
Corresponding Author(s): efmedici@mtu.edu

Access to water on Mars plays a critical role in making a manned mission to this planet more affordable, sustainable, and feasible. On Mars, water exists in the form of buried glacial ice, subsurface ice, as part of minerals in common regolith, and poly-hydrated sulphate minerals and clays such as gypsum and smectite. Gypsum is identified as one of the most efficient resources of water due to low water release temperature at $150^{\circ} \mathrm{C}$ and contains $20 \%$ water by mass. This work was supported by an Early Stage Innovations grant from NASA's Space Technology Research Grants Program (Award: 80NSSC18K0252) and explores the possibility of using non-mechanical excavation of water by disaggregating the Martian gypsum. In this paper, a transient pore network model is developed to determine the optimal excavation size, and to predict the heat and mass leaks occurring during the disaggregating process. Water transport in the gypsum is modeled through a regular grid of pores where each pore is regarded as a cylindrical tube with constant length and randomized cross-sectional areas following Gaussian distribution. The pore-network structure is developed by connecting four pores at each node. The solid matrix network was built by considering a network where the cross-sectional area distribution follows the same pore size Gaussian distribution. The solid network and the pore network are coupled to allow interaction between thermal and mass transport in the modeled gypsum rock. The heat transfer between the two networks is modeled with the assumption that heat transfer only occurs through conduction. Boundary conditions on the gypsum deposit near the excavation site are obtained by solving the Laplace equation in the far field. The domain of the model was confined to the volume of $1 \mathrm{~m} 3$. The temperature under the enclosure at the disaggregation site interface is set at $25^{\circ} \mathrm{C}$ and held constant, and the ambient temperature is extracted from available data of Mars atmosphere. The first analysis performed with this model was the identification of the frost isotherm $\left(0^{\circ} \mathrm{C}\right)$ line. The frost line at the extreme ambient temperature of $-55^{\circ} \mathrm{C}$ is close (less than $0.2 \mathrm{~m}$ ) to the gypsum disaggregation interface which restricts the percolation of water through the gypsum rock, i.e., water loss is confined through the gypsum. The frost line moves farther down from the excavation site when the Martian temperature reaches $-10^{\circ} \mathrm{C}$ but it is still within reach of the expected excavation depth of one meter.

Procter and Gamble Student poster award:

References:

Acceptance of Terms and Conditions:

Click here to agree

\section{Water Vapor Diffusive Transport in a Smectite Clay: Cationic Control of Normal vs. Anomalous Diffusion\}}

Leander Michels ${ }^{1}$; Yves Méheust ${ }^{2}$; Mario A. S. Altoé ${ }^{3}$; Everton C. dos Santos ${ }^{1}$; Henrik Hemmen ${ }^{1}$; Roosevelt Droppa $^{4}$; Jon O. Fossum ${ }^{1}$; Geraldo J. da Silva ${ }^{5}$

\footnotetext{
${ }^{1}$ Norwegian University of Science and Technology (NTNU), Trondheim, Norway

${ }^{2}$ Géosciences Rennes, Univ. of Rennes

${ }^{3}$ Universidade de Brasilia, Instituto de Fisica, Brasilia, Brazil

${ }^{4}$ Universidade Federal do ABC, Santo André, Brazil

${ }^{5}$ Universidade de Brazilia, Instituto de Fisica, Brasilia, Brazil
}

Corresponding Author(s): yves.meheust@univ-rennes1.fr

The transport of chemical species in porous media is ubiquitous in subsurface processes, including contaminant transport, soil drying and soil remediation. We study vapor transport in a multiscale porosity material, a smectite clay, in which water molecules travel in mesopores/macropores between the clay grains but can also intercalate inside the nanoporous grains, making them swell. The intercalation dynamics is known to be controlled by the type of cation that is present in the nanopores; in this case exchanging the cations from $\mathrm{Na}+$ to $\mathrm{Li}+$ accelerates the dynamics. By inferring spatial profiles of mesoporous humidity from a space-resolved measurement of grain swelling, and analyzing them with a fractional diffusion equation, we show that exchanging the cations 
changes mesoporous transport from Fickian to markedly subdiffusive. This results both from modifying the exchange dynamics between the mesoporous and nanoporous phases, and from the feedback of transport on the medium's permeability due to grain swelling. An important practical implication is a large difference in the time needed for vapor to permeate a given length of the clay depending on the type of intercalated cation.

Reference:

L. Michels, Y. Méheust, M. A. S. Altoé, E. C. dos Santos, H. Hemmen=, R. Droppa Jr., J. O. Fossum, G. J. da Silva (2018), Water vapor diffusive transport in a smectite clay: cationic control of normal vs. anomalous Diffusion, accepted in Phys. Rev. E.

Procter and Gamble Student poster award:

References:

Acceptance of Terms and Conditions:

Click here to agree

924

\title{
Specific Surface Area Determination on Chalk Drill Cuttings
}

\author{
Author(s): Dirk Müter ${ }^{1}$ \\ Co-author(s): Stefan Bruns ${ }^{1}$; Henning O. Sørensen ${ }^{1}$ \\ ${ }^{1}$ Technical University of Denmark
}

Corresponding Author(s): mueter@fysik.dtu.dk

Chalk is a biogenic limestone comprising a large part of the subsurface in the North Sea Basin. It hosts both groundwater and hydrocarbon reservoirs and is of importance to a number of industrial applications. Thus, knowledge of basic petrophysical parameters such as porosity and surface area is crucial to decide e.g. whether exploitation of a particular field is economically viable. Traditionally, these parameters would be determined by extracting core plug samples from larger rock cores and performing macroscale laboratory experiments on these. Due to the extensive costs of coring an exploration well, the core log of many fields is sparse and presumably marginally viable fields have not been logged but drilled through. This rotary drilling process produces small chips of the rock that are unusable for classical core plug analysis but large enough for imaging by X-ray nanotomography. In this presentation, we show data for the specific surface area of chalk produced by image analysis of nanotomography data and compare them to values determined by nitrogen physisorption (BET method). The tomography data were recorded at BL47XU at SPring-8 (Japan) at a voxel size of $\sim 40 \mathrm{~nm}$ and subsequently denoised, segmented and meshed. Surface area was calculated from the triangular surface meshes. In BET, the surface area is determined by adsorbing monolayers of nitrogen on to the pore surface of a previously degassed sample and measuring the amount of adsorbed nitrogen. Given that the probe and sample sizes in the two methods (image resolution for tomography and nitrogen molecules for BET) are orders of magnitude different we still find good agreement for about half of the samples, i.e. samples with specific surface area of $\sim 1 \mathrm{~m} 2 / \mathrm{g}$. For the remaining samples, BET delivers values that are a factor of 2-3 larger than estimated by nanotomography. Both sets of samples span about the same range of porosity, so we can conclude that the higher surface area samples contain a large amount of pores below the resolution limit of tomography.

Procter and Gamble Student poster award:

I don't want to compete References:

Acceptance of Terms and Conditions:

Click here to agree 


\title{
Upscaling transport of nanoparticles in porous media
}

Seetha $\mathrm{N}^{1}$

${ }^{1}$ Assistant Professor, Department of Civil Engineering, Indian Institute of Technology Hyderabad

Corresponding Author(s): seetha@iith.ac.in

\begin{abstract}
Nanoparticle transport in porous media is a multi-scale problem, with length scales spanning from the nano scale, where the nanoparticle-soil interaction forces control the deposition, up to the Darcy scale, where the macroscopic equations governing nanoparticle transport are formulated. Hence, it is important to study nanoparticle transport at different length scales in order to understand the dominant mechanisms controlling particle retention at each of these length scales, and the relationship between these mechanisms. In this study, a comprehensive mechanistic approach is employed by integrating pore-scale processes into Darcy-scale models through pore-network modeling to upscale nanoparticle transport in porous media to the Darcy scale. The first step in upscaling using pore-network modeling is to obtain relationships between attached mass and aqueous mass for a single pore. Therefore a mathematical model is developed to simulate nanoparticle transport in a cylindrical pore by considering various processes such as advection, diffusion, hydrodynamic wall effects, and nanoparticle-collector surface interactions. Pore-scale results are then used to develop correlation equations for the averaged deposition rate coefficients of nanoparticles in a cylindrical pore under unfavorable conditions as a function of various pore-scale parameters. At the next step, nanoparticle transport is upscaled from pore scale to the Darcy scale by incorporating the correlation equations for the pore-averaged deposition rate coefficients into a multi-directional pore-network model. Pore-network modeling provides correlation equations for the Darcy-scale deposition rate coefficients under unfavorable conditions as a function of various measurable Darcy-scale parameters, including: porosity, mean pore throat radius, mean pore water velocity, nanoparticle radius, ionic strength, dielectric constant, viscosity, temperature, and surface potentials on the nanoparticle and grain surface. The developed correlation equations are found to be consistent with the available experimental results, and in qualitative agreement with colloid filtration theory for all parameters except for the mean pore water velocity and nanoparticle radius. Such correlation equations provide fundamental insights for predicting the movement of pathogenic viruses and engineered nanoparticles in the subsurface, and thereby to protect drinking water wells from contamination.
\end{abstract}

Procter and Gamble Student poster award:

References:

Seetha, N., Raoof, A., Mohan Kumar, M.S., Hassanizadeh, S.M., 2017. Upscaling of nanoparticle transport in porous media under unfavourable conditions: Pore scale to Darcy scale. Journal of Contaminant Hydrology, 200, 1-14.

Seetha, N., Hassanizadeh, S.M., Mohan Kumar, M.S., Raoof, A., 2015. Correlation equations for average deposition rate coefficients of nanoparticles in a cylindrical pore. Water Resources Research, 51, 80348059. Acceptance of Terms and Conditions:

Click here to agree

\section{Mathematical Models for Skin Penetration}

\author{
Arne Naegel ${ }^{1}$ \\ ${ }^{1}$ Goethe-University Frankfurt \\ Corresponding Author(s): naegel@gcsc.uni-frankfurt.de
}

The skin is an organ with an ordered, highly differentiated structure, providing vitally important barrier properties for the organism. In particular the stratified epithelial layer of the epidermis includes various sources of heterogeneities both with respect to morphology as well as to function. 
Complementing the experiment, mathematical modelling and corresponding simulations are attractive tools for improving our understanding the skin and its barrier properties, e.g., by identifying rate-limiting steps, providing predictions and guiding the experimental design.

In this spirit, the focus of this presentation will be on a mechanistic bottom-up description. The corresponding model is based on first principles from physics, thermodynamic considerations, and comprises microscopic sub-structures. This yields a multi-scale model in which structural effects spanning a variety length and time scales need to be included. We present examples of on-going research in the field, provide application-oriented results and also comment on the underlying mathematical tools and numerical algorithms.

Procter and Gamble Student poster award:

References:

NAEGEL, A. et al.: ADDR 2013, 65:191-207 Acceptance of Terms and Conditions:

Click here to agree

\title{
Uncertainty Quantification in Multiphysical Process Simulation of Porous and Granular Media for Geotechnical Applications
}

\author{
Nandor Tamaskovics ${ }^{1}$; Thomas Nagel ${ }^{1}$ \\ ${ }^{1}$ Technische Universität Bergakademie Freiberg \\ Corresponding Author(s): thomas.nagel@ifgt.tu-freiberg.de
}

In many scientific fields, certain input quantities in the analysis of physical processes have a stochastic ("unsharp") nature and cannot be adequately represented by sharp values. With appropriate techniques, such as the Monte Carlo method, the influence from the stochastic nature of input values can be taken into account, at the expense of a high number of computations. In order to limit the required computational effort depending on the characteristics of the individual physical problem, the number of input values with stochastic nature can be limited a priori with a sensitivity analysis. The Random Set Theory combines interval analysis and a simple probabilistic formulation of physical quantities, leading to an attractive method for computations with "unsharp" input values at significantly lower cost than Monte Carlo simulations. Of particular importance for the simulation of multiphysical processes in porous media is the fact that the method does not pose any restrictions on the nature of the modelled process in terms of boundary conditions, process non-linearity and constitutive models in contrast to some alternative approaches such as the Stochastic (SFEM) or the Random Finite Element Methods (RFEM). Depending on the available input data, the interval-based approach can be practically more feasible than methods pased on probability density functions. Here, we demonstrate the capabilities of the Random Set Method by applying it to analytical solutions of classical geotechnical problems and by linking it to the Finite Element Method (RSFEM) for the simulation of multiphysical problems in geotechnics and geoscience using Cast3M and OpenGeoSys. The results will be discussed in the context of alternative approaches.

Procter and Gamble Student poster award:

References:

Acceptance of Terms and Conditions:

Click here to agree

\section{Modelling deformation and flow during fault-reactivation in argilla- cieous rocks following fluid injection}


Thomas Nagel ${ }^{1}$; Dmitri Naumov ${ }^{2}$; Keita Yoshioka ${ }^{2}$; Olaf Kolditz ${ }^{2}$; Jobst Maßmann ${ }^{3}$

${ }^{1}$ Technische Universität Bergakademie Freiberg

${ }^{2}$ Helmholtz Centre for Environmental Research - UFZ

${ }^{3}$ Federal Institute for Geosciences and Natural Ressources (BGR)

Corresponding Author(s): thomas.nagel@ifgt.tu-freiberg.de

The creation and evolution of flow-paths are one of the dominant factors to consider when evaluating the safety of deep geological repositories. These flow paths consist of the pore space in the geotechnical barriers as well as in the host rocks on the one hand, and of discrete networks of existing fault zones and newly developed fractures on the other. The degree to which major and minor faults contribute to the overall flow and transport behaviour can change abruptly when faults become activated by mechanical, thermal or hydraulic perturbations. In the international project Decovalex, a task is dedicated to studying the topic of fault reactivation in low-permeable argillaceous rocks using field data from a fault slip experiment in the Mont Terri underground research laboratory in Switzerland (Gulielmi, 2016). Here, we present the results of the BGR/UFZ team modelling different aspects of the in-situ experiments using an enriched finite element formulation to represent the geological discontinuities (faults and fractures), which has been implemented into the open-source finite element framework OpenGeoSys. The fault reactivation process is modelled using cohesive zone formulations. We discuss the ability of different constitutive formulations for the activated/non-activated fault zones in capturing the data obtained from the field tests. We further highlight numerical challenges associated with the transition to stimulated properties at the edge of the activated fault domain present in the current implementation and suggest alternative numerical schemes. Finally, we put the results in the context of a novel phase-field implementation available in OpenGeoSys in which discontinuities are represented using a phase-field variable.

Procter and Gamble Student poster award:

References:

Acceptance of Terms and Conditions:

Click here to agree

\title{
Flow through a regular arrays of complex shapes
}

\author{
Prapanch Nair ${ }^{1}$; Sebastian Mühlbauer ${ }^{2}$; Thorsten Pöschel ${ }^{2}$ \\ ${ }^{1}$ Institute for Multiscale Simulation, Erlangen \\ ${ }^{2}$ Institute for Multiscale Simulation
}

Corresponding Author(s): prapanch.nair@gmail.com,

Permeability of a porous media is known to be a function of some aspect of the geometry of the pores. However, since geometry is difficult to quantify, permeability is often related to empirical and non-unique features of the porous structure such as tortuosity, void fraction and Euler characteristics. Minkowski functionals can be used to quantify the interface between two phases (solid-fluid). Using the data from flow simulations about thousands of complex shaped bodies, we present a statistical relationship between the Minkowski functionals and permeability, keeping the porosity and other flow features constant. We make some remarks about the Darcy and Darcy-Forchheimer laws and show how boundary layer separation plays a role in such a system. We then train a Neural Network to predict permeabilities in order to assess the relevance of Minkowski functionals to truly homogeneous porous media.

\section{Procter and Gamble Student poster award:}

I would like to compete in the Procter and Gamble Student award References:

Acceptance of Terms and Conditions: 
Click here to agree

639

\title{
Is it possible to introduce $3 \mathrm{D}$ complex heterogeneities in a real transient groundwater model used to manage a sedimentary aquifer?
}

\author{
Nuria Naranjo-Fernández ${ }^{1}$; Carolina Guardiola-Albert ${ }^{2}$; Esperanza Montero ${ }^{1}$ \\ ${ }^{1}$ University Complutense of Madrid \\ ${ }^{2}$ Spanish Geological Survey
}

Corresponding Author(s): nuria.naranjo.phd@gmail.com, c.guardiola@igme.es

\begin{abstract}
Almonte-Marismas aquifer system maintains the biodiversity richness of one of the most sensible wetlands in Spain, which depends on groundwater quantity and quality. Spanish Geological Survey has been studied the aquifer for more than 40 years and plenty of different hydrogeological numerical models have been built. Now, the new challenge is to change the concept of previous homogeneous models and take into account the heterogeneous hydrogeology in order to make an accurate water resources management. The consideration of $3 \mathrm{D}$ geostatistical hydrofacies simulation has been the latest improvement of the management mathematical model. The restructuring of the sedimentary system of the Almonte-Marismas aquifer into seven hydrofacies contributed to a more realistic characterization of the hydrogeological properties of each geological unit in the model, leading to a more natural flow pattern. This previous hydrological model, with a detailed description of the units in a 7 layer vertical discretization, was tested in a stationary state framework. The present work wants to go further in the use of this model, integrating this new spatial variable discretization into the transient state. NWT MODFLOW package and UCODE software will be used to automatically interpolate hydrogeological parameters and perform sensitivity analysis. ModelMuse and ModelMate software (USGS) will allow running the mentioned packages to solve the transient groundwater flux related to the complex sedimentary Almonte-Marismas aquifer system.
\end{abstract}

Procter and Gamble Student poster award:

I would like to compete in the Procter and Gamble Student award References:

Acceptance of Terms and Conditions:

Click here to agree

\section{Pore size distribution effects on the mechanical behaviour of nanoporous silicon structures}

\author{
Mohammad Nasr Esfahani ${ }^{\text {None }}$; Masoud Jabbari ${ }^{\text {None }}$ \\ Corresponding Author(s): mohammad.nasr-esfahani@warwick.ac.uk
}

The full potential of nanostructures is yet to be realized in the multi-disciplinary world of nanotechnology for introducing new functionalities. Porous media is one such domain emerging as truly nanoscale building blocks through nanoporous structures. A remarkable surface-to-volume ratio in nanoporous materials offers many applications in the field of batteries and fuel cell technology. Nanoporous silicon (np-Si) is among those nanostructures enabling on-chip integration with electronic systems. Furthermore, bio-compatibility in np-Si facilitates high resolution detections of chemicals, biomolecules and cells for new-generation diagnostic devices, where the mechanical interactions play a critical role on bio-detections. Therefore, the determination of the np-Si mechanical behaviour has become challenging for improving the performance in sensing purposes. Although a few studies have been carried out regarding pore geometry effects on the np-Si mechanical response, the pore distribution effect on mechanical properties remains to be addressed for further 
insight into nanomechanical characterisations. In this study, we perform molecular dynamics simulations to study pore size distribution effects on the mechanical behaviour of np-Si crystal structures. Elastic modulus and ultimate strength are determined through uniaxial tensile tests. The influence of pore volume fraction on mechanical response is investigated by stress analysis along the ligament thickness. Results show a decrease on the ultimate strength through increasing the pore size. A non-uniform stress distribution along the ligament thickness reduces the Young's modulus and fracture strength. Results in this work can provide a guideline for future design and fabrication of $\mathrm{np}$-Si structures for ultra-sensitive detections.

Procter and Gamble Student poster award:

References:

Acceptance of Terms and Conditions:

Click here to agree

\section{test}

Sourena Nasrollahi ${ }^{\text {None }}$; Mani Kamalzadeh ${ }^{1}$

${ }^{1}$ Sharif university

Corresponding Author(s): sourena@itecsco.com

this is a test

Procter and Gamble Student poster award:

References:

Acceptance of Terms and Conditions:

Click here to agree

952

\section{Phase-field method vs. volume of fluid (VOF) for simulating im- miscible two-phase flow in porous media}

Taofik Nassan ${ }^{1}$; Mohammad Amro ${ }^{1}$

\footnotetext{
${ }^{1}$ Freiberg University of Technology
}

Corresponding Author(s): taofik.nassan@student.tu-freiberg.de

Understanding fluid flow phenomena in porous media is a crucial issue in petroleum, chemical, and material engineering. In oil and gas reservoirs, fluid flow simulation is one of the most powerful tools to predict reservoir behavior in the future and the response of the reservoir to different production scenarios and enhanced oil recovery (EOR) schemes that may be planned after primary recovery of the oilfield.

Volume averaging method is always used to simulate different transport phenomena in porous media including oil and gas reservoirs. However, in the last decade, pore scale modelling started to gain more attention because of the advances in pore scale-imaging, developed mathematical algorithms, and the exponential growth of computational power.

In this short communication, we solve phase-field model in a single pore channel and compare the results to volume of fluid method (VOF) on the same domain from a selected publication from the lietrature. The immiscible two phases are water and oil with different contact angles and viscosities The results show some difference in residual oil/water distribution within the pore channel, which 
urges more research in this direction to understand the flow behavior at pore scale and predict it numerically more precisely.

Procter and Gamble Student poster award:

I would like to compete in the Procter and Gamble Student award References:

Acceptance of Terms and Conditions:

Click here to agree

\title{
Finite element analysis of LTE and LTNE processes during steam injection process in porous media
}

\author{
Taofik Nassan ${ }^{1}$; Mohammad Amro \\ ${ }^{1}$ Freiberg University of Technology \\ Corresponding Author(s): taofik.nassan@student.tu-freiberg.de
}

Hot fluid injection in porous media has a wide range of applications in chemical and petroleum industries. Whether to consider the medium undergoes local thermal equilibrium (LTE) or local thermal nonequilibrium (LTNE) depends on different parameters like velocity of fluid, thermal properties of fluid and solid materials, just to name a few. LTE and LTNE are intensively discussed in the literature but until now, there is no consensus where to use both mathematical models. In this paper a comparison between both cases will be illustrated with an example related to thermal enhanced oil recovery in 1D where the steam is injected in a permeable formation (saturated with heavy oil) and condenses into hot water where it will displace the heavy oil to the production well after decreasing its viscosity to a reasonable value. The results show that at the first stage of steam injection where the velocity of steam is very high the LTNE model is the best to describe the process while later on the velocity will drop down and LTE model is accurate enough to describe the process.

Procter and Gamble Student poster award:

I would like to compete in the Procter and Gamble Student award References:

Acceptance of Terms and Conditions:

Click here to agree

649

\section{A versatile model for mass transport abnormalities applicable to molecular displacements complemented by tracer test}

Marie-Christine Neel ${ }^{1}$; Marc Fleury ${ }^{2}$; Daniela Bauer ${ }^{3}$

\footnotetext{
${ }^{1}$ Univerité Avignon

${ }^{2}$ IFP Energies Nouvelles

${ }^{3}$ IFP Energies nouvelles
}

Corresponding Author(s): marie-christine.neel@inra.fr, marc.fleury@ifpen.fr

Mass transport in porous media often reveals deviations from Advection-Dispersion Equation : strong assymetry of propagators and break-through curves suggest small scale motions described by stochastic process equivalent to stable motion subjected to random time change. Pulsed Field Gradient Nuclear Magnetic Resonance determines the Fourier transform of molecular displacements, and tracer 
tests (possibly deduced from other NMR measurements) document tracer density : we describe numerical approaches for Fourier transform and density of such process in order to interpret these two observables attached to molecular motion.

Stable motion is the simplest generalization of Brownian motion. As the latter, it is a Markov process, has zero average, and represents random fluctuations around some mean flow. Yet, it allows very large displacements in some privileged direction compensated by many small opposite motions. The density of particles performing stable motion satisfies a Partial Differential Equation incorporating spatial derivatives of non-integer order beside the first order time derivative. The particular case of Brownian motion corresponds to the ADE.

A random time change modifies the time schedule of the sample paths of any stable motion (possibly Brownian) by inserting immobile steps of random duration. This modifies the above PDE by adding a memory operator equal to the convolution of the fist order time derivative with some kernel determined by the distribution of the immobile steps. The new process is not Markovian. A well-known particular case is the mobile-immobile model used since decades in porous media: it corresponds to Brownian motion and to exponentially distributed pausing times (of finite mean). Other stable motion combined with the same time change was observed in some saturated porous media. Many other time changes are possible: for immobile steps of stable duration (of infinite mean) the memory operator is a time derivative of fractional order, also observed in porous media.

Stable motion subjected to random time change form a huge family of models : they are characterized by the 4 parameters of stable motion (including the average velocity), and by the pausing time distribution which can belong to infinitely many families indexed by their own parameters. Two simple examples of such families are cited above. Each of them depends on two parameters. We discuss a method that finds which of these two families is the best adapted to mass transport in a medium in which data document molecular displacements Fourier transform and break-through curve. It is based on a very general expression of displacement Fourier transform, not obvious for non-Markovian process. This observable is measured at very small time-scale, and data analysis finds the optimal parameters in each family of time changes, a preliminary stage before comparing the merits of the families. However, some indeterminacy (with one degree of freedom) remains because some parameters are better detected at larger time scale : comparing tracer tests removes the indeterminacy.

Procter and Gamble Student poster award:

\title{
References:
}

1 H.A. Deans, Soc. Pet. Eng. J. 3, p. 49 (1963).

2 D. A. Benson, M. M. Meerschaert, Adv. Water Res. 32 pp 532-539 (2009).

3 B. Maryshev, A. Cartalade, C. Latrille, M.C.Néel, TIPM 116(1), (2017) pp 53-71

[4] M.C.Néel, D . Bauer, M. Fleury, PRE 89, 062121 (2014)

5 S.H. Rakotonasy, M.C.Néel, M. Joelson, Commun Nonlinear Sci Numer Simulat 19(2014) 2284-2293

Acceptance of Terms and Conditions:

Click here to agree

311

\section{Accuracy of Hydraulic Rock Properties derived from X-ray-Tomographic Images - Insights from Laboratory Experiments on Porous and Fractured Rocks}

\author{
Author(s): Mathias Nehler ${ }^{\text {None }}$ \\ Co-author(s): Ferdinand Stöckhert ${ }^{1}$; Mandy Duda ${ }^{2}$; Erik Saenger ${ }^{2}$; Jörg Renner ${ }^{1}$; Rolf Bracke ${ }^{2}$ \\ ${ }^{1}$ Ruhr-Universität Bochum \\ ${ }^{2}$ International Geothermal Centre
}

Corresponding Author(s): mathias.nehler@hs-bochum.de

Hydraulic properties of rocks are controlled by the geometry of open pores and fractures. The geometry of individual pores and fractures, but also the tortuosity of the conduit network affect the flow 
properties and may change in response to the state of stress or chemical exchange between solid and fluid and thus may evolve with time. Nondestructive imaging methods, such as X-ray tomography, provide insight into the geometrical characteristics of pore networks on the laboratory scale. Today these methods can be applied to samples subjected to reservoir conditions. Combining imaging and flow-through experiments will help to predict the development of liquid-resources reservoirs and thus to increase the efficiency of reservoir exploitation.

Porosity and permeability of different sandstone varieties were investigated in standardized laboratory experiments and compared to their counterparts calculated from X-ray tomography images. The total porosity was determined from the ratio between bulk and matrix density. Effective porosity was independently deduced from water imbibition. We investigated the sensitivity of segmentation of images gained from X-ray tomography with respect to (a) resolution of the intensity images, (b) denoising filters (median and non-local means), and (c) different global thresholding methods.

Porosity characterization was complemented by flow-through experiments to derive permeability of low-porosity samples with a single natural or artificial fracture subjected to confining pressures between $2.5 \mathrm{MPa}$ and $30 \mathrm{MPa}$. Fractures were oriented parallel to the flow direction. At each pressure level, effective permeability was determined from steady-state flow based on Darcy's law. In addition, a full 3D image was recorded by a $\mu \mathrm{CT}$ scanner with a $225 \mathrm{kV}$ multifocal X-ray tube to gain information on the fracture aperture. The flow-through cell was placed inside the $\mu \mathrm{CT}$ scanner. Samples measured $10 \mathrm{~mm}$ in diameter resulting in a voxel resolution of approximately $10 \mu \mathrm{m}$. The mean fracture aperture was derived from the intensity images and used to estimate fracture permeability using the cubic law.

The porosity of the sandstone varieties ranged between $3 \%$ and $25 \%$. The reconstructed intensity images of the highly porous sandstones exhibited distinctly bimodal intensity distributions enabling fairly reliable porosity estimates. Low-porosity sandstones exhibited unimodal or skewed intensity distributions. The applied global thresholding algorithms yield highly variable porosity estimates.

A resolution exceeding the characteristic length scale of pores is mandatory for accurate porosity estimates. Filtering of datasets does not improve segmentation results and thresholding algorithms are biased by the amount of unresolved pore space. Resolution is equally significant for the geometrical characterization offractures, characterized by two adjacent surfaces of large lateral extension at a small distance. The limited resolution of X-ray micro-tomography leads to non-resolved apertures that in turn lead to erroneous permeability estimates when using the cubic law.

Procter and Gamble Student poster award:

References:

Acceptance of Terms and Conditions:

Click here to agree

\title{
Quantifying the influence of microstructure on effective conduc- tivity and permeability: virtual materials testing
}

\author{
Matthias Neumann $^{1}$; Ole Stenzel ${ }^{2}$; François Willot ${ }^{3}$; Lorenz Holzer ${ }^{2}$; Volker Schmidt ${ }^{1}$ \\ ${ }^{1}$ Ulm University \\ ${ }^{2}$ ZHAW Winterthur \\ ${ }^{3}$ MINES Paris Tech Fontainbleau
}

Corresponding Author(s): matthias.neumann@uni-ulm.de, volker.schmidt@uni-ulm.de

Effective conductivity and permeability of a versatile, graph-based model of random structures are investigated numerically. This model, originally introduced in Gaiselmann et al. (2014) allows one to simulate a wide class of realistic materials. In the present work, an extensive dataset of twophase microstructures with wide-ranging morphological features is used to assess the relationship 
between microstructure and effective transport properties, which are computed using Fourier-based methods on digital images. Our main morphological descriptors are phase volume fractions, mean geodesic tortuosity, two "hydraulic radii" for characterizing the length scales of heterogeneities, and a "constrictivity" parameter that describes bottleneck effects. This additional parameter, usually not considered in homogenization theories, is an essential ingredient for predicting transport properties, as observed in Gaiselmann et al. (2014). We modify the formula originally developed in Stenzel et al. (2016) for predicting the effective conductivity and propose a formula for permeability. For the latter one, different geometrical definitions of the hydraulic radius are compared. Our predictions are validated using tomographic image data of fuel cells.

\title{
Procter and Gamble Student poster award:
}

\section{References:}

G. Gaiselmann, M. Neumann, O. M. Pecho, T. Hocker, V. Schmidt, and L. Holzer. Quantitative relationships between microstructure and effective transport properties based on virtual materials testing. AIChE Journal, 60:1983-1999, 2014.

O. Stenzel, O. M. Pecho, L. Holzer, M. Neumann, and V. Schmidt. Predicting effective conductivities based on geometric microstructure characteristics. AIChE Journal, 62:1834-1843, 2016.

M. Neumann, O. Stenzel, F. Willot, L. Holzer and V. Schmidt. Quantifying the influence of microstructure on effective conductivity and permeability: virtual materials testing. Preprint 2018 (submitted) Acceptance of Terms and Conditions:

Click here to agree

\section{Permanent Deformation of Permeable Base Based on Repeated Load Triaxial Test}

\author{
Author(s): Tan Hung Nguyen ${ }^{1}$ \\ Co-author(s): Jaehun Ahn ${ }^{2}$ \\ ${ }^{1}$ Pusan National University, South Korea \\ ${ }^{2}$ Pusan National University
}

Corresponding Author(s): nthung010189@gmail.com, jahn@pusan.ac.kr

\begin{abstract}
In permeable pavement, open-graded aggregate materials contribute largely to the mechanical behavior of the system as base/subbase layers. Under the traffic loading, the accumulation of permanent deformation is one of the main forms of distress of pavement structure. This study investigated the permanent deformation in open-graded aggregate materials based on multistage repeated load triaxial tests. Two materials with different particle size distribution were tested and then the accumulation of permanent deformation were modelled using the time-hardening approach. The materials parameters of the predictive model were optimized using the repeated loading triaxial test data. The results showed that the multistage repeated loading triaxial test procedure has the potentials to be used for characterizing the permanent deformation of open-graded aggregate materials.
\end{abstract}

Keywords: permanent deformation; open-graded aggregate, triaxial test.

\section{ACKNOWLEDGEMENTS}

This research was supported by a grant from Basic Science Research Program (grant no. 2017R1D1A3B03034563) through the National Research Foundation of Korea (NRF) funded by the Ministry of Education.

Procter and Gamble Student poster award:

References: 
Ba, M., Tinjum, J. M., \& Fall, M. (2015). Prediction of permanent deformation model parameters of unbound base course aggregates under repeated loading. Road Materials and Pavement Design, 16(4), pp. 854-869.

EN, B. 13286-7 (2004) Unbound and Hydraulically Bound Mixtures-Cyclic Load Triaxial Test for Unbound Mixtures. British Standard Institute, UK

Erlingsson, S., \& Rahman, M. S. (2013). Evaluation of permanent deformation characteristics of unbound granular materials by means of multistage repeated-load triaxial tests. Transportation Research Record, 2369(1), pp. 11-19.

Hornych, P., Chazallon, C., Allou, F., \& El Abd, A. (2007). Prediction of permanent deformations of unbound granular materials in low traffic pavements. Road Materials and Pavement Design, 8(4), pp. 643-666.

Rahman, M. S., \& Erlingsson, S. (2014). Permanent deformation models of unbound granular materials: a comparative study based on multi-stage repeated-load triaxial tests. Transportation Research Board 93rd Annual Meeting, Washington DC, USA, Report.

Rahman, M. S., \& Erlingsson, S. (2015a). A model for predicting permanent deformation of unbound granular materials. Road Materials and Pavement Design, 16(3), pp. 653-673.

Rahman, M. S., \& Erlingsson, S. (2015b). Predicting permanent deformation behaviour of unbound granular materials. International Journal of Pavement Engineering, 16(7), pp. 587-601.

Rahman, M. S., \& Erlingsson, S. (2016). Modelling the moisture dependent permanent deformation behavior of unbound granular materials. Procedia engineering, 143, pp. 921-928.

Salour, F., \& Erlingsson, S. (2017). Permanent deformation characteristics of silty sand subgrades from multistage RLT tests. International Journal of Pavement Engineering, 18(3), pp. 236-246. Acceptance of Terms and Conditions:

Click here to agree

\section{The Effect of Injection Rate on Fluid Invasion Patterns: Immis- cible Two-Phase Fluid Displacement in 2D Laser-Manufactured Micromodels}

Rumbidzai A. E. Nhunduru ${ }^{1}$; Amir Jahanbakhsh ${ }^{2}$; Omid Shahrokhi ${ }^{3}$; Krystian L. Wlodarczyk ${ }^{4}$; Duncan P. Hand ${ }^{5}$ ; William N. MacPherson ${ }^{2}$; Susana Garcia ${ }^{2}$; M. Mercedes Maroto-Valer ${ }^{3}$

\footnotetext{
${ }^{1}$ Research Centre for Carbon Solutions (RCCS), School of Engineering and Physical Sciences, Heriot-Watt University

${ }^{2}$ Heriot-Watt University

${ }^{3}$ Research Centre for Carbon Solutions (RCCS), School of Engineering and Physical Sciences, Heriot-Watt University

4 *Research Centre for Carbon Solutions (RCCS), School of Engineering and Physical Sciences, Heriot-Watt University

${ }^{5}$ School of Engineering and Physical Sciences, Heriot-Watt University
}

Corresponding Author(s): ran4@hw.ac.uk

Keywords: CO2 Storage, Micromodel, Capillary Number, Invasion Pattern, Modelling

The physical process whereby an immiscible fluid phase replaces a second resident fluid in a porous medium is characteristic of many subsurface processes that include remediation of non-aqueous phase liquids, enhanced oil recovery, and $\mathrm{CO} 2$ storage(1). Therefore, understanding of fluid displacement mechanisms at the pore level is essential to improve existing technologies in the petroleum and hydrology industries. The aim of this study is to enhance our understanding of invasion processes of immiscible fluids at the pore scale. To do so, we use laser-manufactured micromodels that are made of transparent, borosilicate glass substrates(2). Micromodels are simplified, two-dimensional (2D) representations of natural porous media that enable direct visualization of processes within patterned microstructures(3). An experimental visualization setup is used to vary injection rates and observe fluid invasion patterns in immiscible two-phase fluid displacement experiments in lasermachined micromodels. The components of the set-up include a syringe pump for fluid injection, a uniform light source, and a camera mounted on a translation stage for image acquisition. To imitate reservoir conditions, fluid displacement experiments are conducted at capillary numbers ranging from $9.5 \times 10-6$ to $1.9 \times 10-5$. Direct numerical simulations are a useful tool to improve our ability to predict the dynamics of immiscible two-phase flow in porous media. Accordingly, in this work the 
micromodel visualization studies are complimented with simulations and results from the experiments are used to validate the numerical models. The Cahn-Hilliard phase-field method is applied for direct numerical simulation of the two-phase flow experiments.

\title{
Procter and Gamble Student poster award:
}

I would like to compete in the Procter and Gamble Student award References:

1. Edery Y, Weitz D, Berg S. Surfactant Variations in Porous Media Localize Capillary Instabilities during Haines Jumps. Phys Rev Lett [Internet]. American Physical Society; 2018;120(2):28005.https://doi.org/10.1103/PhysRe

2. Wlodarczyk KL, Carter RM, Jahanbakhsh A, Lopes AA, Mackenzie MD, Maier RRJ, et al. Rapid laser manufacturing of microfluidic devices from glass substrates. Micromachines. 2018;9(8). 409. https://doi.org/10.3390/mi9080409

3. Grate JW, Zhang C, Wietsma TW, Warner MG, Anheier NC, Bernacki BE, et al. A note on the visualization of wetting film structures and a nonwetting immiscible fluid in a pore network micromodel using a solvatochromic dye. Water Resour Res. 2010;46(11):1-6. https://doi.org/10.1029/2010WR009419

Acceptance of Terms and Conditions:

Click here to agree

414

\section{Novel insights into two-phase flow dynamics due to wettability alteration during low salinity waterflooding}

\author{
Rimsha Aziz ${ }^{1}$; Vahid.J Niasar ${ }^{\text {None }}$; Pedro Martinez ${ }^{2}$; Omar Emmanuel Godinez Brizuela ${ }^{3}$; Hassan Mahani ${ }^{4}$ \\ ${ }^{1}$ University of Manchester \\ ${ }^{2}$ Barcelona Supercomputing Center \\ ${ }^{3}$ The University of Manchester \\ ${ }^{4}$ Shell Global Solutions International B.V.
}

Corresponding Author(s): vahid.niasar@manchester.ac.uk

\begin{abstract}
Low salinity waterflooding has proven to accelerate oil production at core and field scales. Wettability alteration from a more oil-wetting to a more water-wetting condition has been established as one of the most notable effects of low salinity waterflooding. While wettability alteration at the interface between fluids and rock has been critically studied from chemical point-of-view, there is no clear understanding of (i) signature of wettability alteration on dynamics of two-phase flow, (ii) role of hydrodynamic transport and mixing between the low salinity water and the formation brine (high salinity water) in wettability alteration, (iii) role of pore heterogeneity in two-phase dynamics due to wettability alteration.

To address these objectives, computational fluid dynamic simulations of coupled dynamic two-phase flow, hydrodynamic transport and wettability alteration in a 2D domain were carried out using the volume of fluid method. The numerical simulations imply that the presence of stagnant regions in the tertiary oil recovery impedes the potential of wettability alteration for additional oil recovery. Hence, it would be favourable to inject low salinity water from the beginning of waterflooding to avoid stagnant saturation. Also, the role of pore-scale heterogeneity in additional oil recovery due to low salinity waterflooding has been discussed.
\end{abstract}

Procter and Gamble Student poster award:

References:

Acceptance of Terms and Conditions:

Click here to agree 


\title{
Using direct numerical simulations to uncover the mechanisms of two-phase flow between GDL interfaces in fuel cells
}

Daniel Niblett $^{\text {None }}$; Vahid.J Niasar ${ }^{\text {None }}$; Stuart Holmes ${ }^{1}$

${ }^{1}$ University of Manchester

Corresponding Author(s): daniel.niblett@manchester.ac.uk

Understanding the mechanisms of two phase flow in fuel cell porous transport layers is necessary if the challenges related to water management in fuel cells are to be addressed. In this talk, the mechanisms of two-phase flow are evaluated with connections to the micro-porous layer and the gas channels.

Using the Finite Volume Method of OpenFOAM and the volume of fluid method solver with IsoAdvector interface reconstruction, air-water two-phase flow within a reconstructed fuel cell gas diffusion layer is performed to understand the coupling between the microporous layer and gas channel. Although the method is not efficient to simulate operation (low $\mathrm{Ca}$ ), it can give insight into the development of mechanistic models through understanding of the local scale coupling between layers. Often the GDL is modelled isolated from the adjacent layers resulting in mechanisms not being fully resolved. Coupling of the pore scale resolved porous layer and gas channel allows for the effect of droplet growth and detachment to be seen internally on the porous media. Using the sharper interface method, numerical diffusion and mass conservation are improved, allowing for faster and more accurate simulations to be performed.

The concept of applying an artificial MPL is evaluated by addition, allowing the quantification and visualisation of the water management properties it adds, along with the importance of MPL cracks in the two-phase flow dynamics. This work provides insights in to the complex interplay between the free flow and porous media flow under two-phase flow conditions, which has not been addressed in detail.

Procter and Gamble Student poster award:

I would like to compete in the Procter and Gamble Student award References:

Acceptance of Terms and Conditions:

Click here to agree

\section{How seismic faults and well test data analysis may help to bet- ter model the sub-seismic faults: a study of impact of a fractal dimension choice on flow.}

\author{
André Fourno ${ }^{1}$; Alexandre De Lima ${ }^{2}$; Denis José Schiozer ${ }^{2}$; Benoit Noetinger \\ ${ }^{1}$ IFPEN \\ ${ }^{2}$ State University of Campinas
}

Corresponding Author(s): benoit.noetinger@ifpen.fr

Carbonate reservoirs presents a large number of faults that cannot be fully identified due the lack of seismic resolution. So one of the key points for modeling fractured reservoirs is the characterization of sub-seismic faults, which are almost invisible and that may impact significantly fluid flow behavior. An approach (Verscheure et al. 2010, Verscheure et al. 2012) was proposed to characterize the fault network and to model the sub-seismic faults keeping a geological consistency of seismic faults. This approach captures the spatial fault organization thank to a fractal dimension. A stochastic algorithm based on multiplicative cascades (Darcel et al. 2003) is then used to generate the sub-seismic faults. 
The poster presents a synthetic carbonate reservoir with its associated fault network. A fault analysis is performed. The results show a variability of fractal dimension according to different reservoir areas. After, each fault network is characterized by fractal dimensions taking into account the different portions of the reservoir and also the full fault network. A key aspect of modeling the sub-seismic faults is to make a good choice of the fractal dimension. Consequently, it raises questions about if we should use a single fractal dimension for the whole reservoir or one fractal dimension by area. In order to illustrate the impact of this choice several sub-seismic fault models are built. Subsequently for each associated sub-seismic fault networks an upscaling step is done. The upscaling step is performed using an analytical upscaling (Oda 1985). The results of this upscaling step is then studied and commented from a flow point of view. Thus, the $\mathrm{KH}$ variability and the equivalent block sizes have been studied. In a second time, well data is also analyzed for identifying the hydraulic conductivity $(\mathrm{KH})$, the presence or not of seal faults, the presence of major conductive area and the distance of these objects from the well. Both well test analysis and fault network analysis are used for better choosing the sub-seismic model, whose better fits the conceptual model of flow behavior.

The current study found that for this reservoir the best option is to use a single fractal dimension for representing the whole reservoir area. The poster shows the importance of using multi-disciplinary competences (geostatistical and well test analysis) in the process of understanding the reservoir behavior.

\title{
Procter and Gamble Student poster award:
}

I don't want to compete References:

M. Verscheure, A. Fourno and J.-P. Chilès. Joint Inversion of Fracture Model Properties for CO2 Storage Monitoring or Oil Recovery History Matching. Oil Gas Sci. Technol. Rev. IFP Energies nouvelles 67 (2) 221-235 (2012). DOI: http://dx.doi.org/10.2516/ogst/2011176

M. Verscheure, A. Fourno ; J.-P. Chilès. History matching of a realistic stochastic fault model using fractal geometry and the gradual deformation method. SPE-129759. SPE EUROPEC/EAGE Annual Conference and Exhibition held in Barcelona, Spain, 14-17 June 2010.

Darcel C., Bour O., Davy P., de Dreuzy J.R. (2003) Connectivity properties of two-dimensional fracture networks with stochastic fractal correlation, Water Resour. Res. 39, 10, 1272

Oda M. (1985) Permeability tensor for discontinuous rock masses, Geotechnique 35, 4, 483-495 Acceptance of Terms and Conditions:

Click here to agree

\section{Experimental gas permeability studies on synthetic microporous materials}

\author{
Author(s): Steffen Nolte ${ }^{1}$ \\ Co-author(s): Yue Wang ${ }^{2}$; Reinhard Fink ${ }^{1}$; Bernhard M. Krooss ; Alexandra Amann-Hildenbrand ; Moran Wang \\ ${ }^{2}$; Joyce Schmatz ${ }^{3}$; Jop Klaver ${ }^{3}$ \\ ${ }^{1}$ RWTH Aachen University \\ ${ }^{2}$ Tsinghua University \\ ${ }^{3} \mathrm{MaP}-$ Microstructure and Pores $\mathrm{GmbH}$
}

Corresponding Author(s): steffen.nolte@emr.rwth-aachen.de

Gas transport in tight rocks is of relevance in various geotechnical contexts such as carbon dioxide sequestration, nuclear waste disposal and the exploitation of shale gas and coalbed methane. These transport processes are typically controlled by fluid-dynamic (e.g. slip flow) and rock mechanic (pore compressibility) effects.

Numerous modeling approaches have addressed various aspects of gas transport in gas shales, but there is a notorious shortage of reliable experimental data to validate or verify the modeling results. Some major issues are uncertainties regarding the experimental starting and boundary conditions and material properties (pore size distributions, mechanical properties etc.). Recent studies (Fink 
et al., 2017) have shown that interpretations of transport mechanisms in low-permeable rocks are still controversial. In order to reduce the complexity encountered in natural rocks one approach is to reduce the amount of undefined parameters by conducting fluid flow experiments on synthetic, homogeneous and well-defined porous media. The "NanGasPor" project has been initiated to link the modeling side and the experimental side, improve communication and create feedback.

A first set of flow experiments with different gases (Helium, Nitrogen, Argon, Hydrogen) was carried out using fused silica capillaries with diameters in the $\mu \mathrm{m}$-range to assess reproducibility of measurements and consistency with established flow models. Using the Hagen-Poiseuille law for cylindrical capillaries and compressible fluids the viscosity values were calculated for the different gases and compared to literature data (NIST database). For a capillary with a nominal diameter of 10 $\mu \mathrm{m}$ the results were found to be in good agreement with the literature data. The same measurements with a capillary $2 \mu \mathrm{m}$, nominal ID, significant offsets were observed. These could be compensated consistently by adjusting the diameter value to $1.62 \mu \mathrm{m}$. BIB-SEM investigations of the material yielded an average effective capillary diameter of $1.4 \mu \mathrm{m}$. The experimental work is now focusing on the identification of the causes and consequences of these inconsistencies for interpretation and modeling of fluid transport in narrow capillaries.

Another series of flow tests is being conducted on sintered ceramic plugs that provide a homogeneous pore network with a narrow pore-throat distribution. Helium pycnometry, low pressure nitrogen adsorption (BET), mercury intrusion porosimetry (MIP) and BIB-SEM are used for a comprehensive characterization of the specimens. Porosity values range around $29 \%$ with the most prominent pore diameter, determined by MIP, of $70 \mathrm{~nm}$ as compared to a nominal diameter of $50 \mathrm{~nm}$. Determination of effective transport pore size and permeability by means of fluid flow experiments yielded values between 34 and $65 \mathrm{~nm}$, respectively, depending on the pore shape model. BIB-SEM images show irregular and angular pore shapes. Permeability coefficients measured with different gases range around $30 \mu \mathrm{D}\left(3.1017 \mathrm{~m}^{2}\right)$ and are not affected by induced stress changes. Due to the rigidity of the ceramic material fluid-dynamic effects (1st or 2nd order slip flow, transitional flow) can thus be investigated without interference of poro-elastic stress effects.

\title{
Procter and Gamble Student poster award:
}

\section{References:}

Fink, R., Krooss, B.M., Gensterblum, Y., Amann-Hildenbrand, A., 2017. Apparent permeability of gas shales-superposition of fluid-dynamic and poro-elastic effects, Fuel 199, 532-550. Acceptance of Terms and Conditions:

Click here to agree

\section{Apparent Permeability of Gas Shales - Caution when comparing simulation results with experimental data}

\author{
Author(s): Steffen Nolte ${ }^{1}$; Reinhard Fink ${ }^{1}$ \\ Co-author(s): Bernhard M. Krooss ; Alexandra Amann-Hildenbrand \\ ${ }^{1}$ RWTH Aachen University
}

Corresponding Author(s): steffen.nolte@emr.rwth-aachen.de, reinhard.fink@emr.rwth-aachen.de

For decades experimentalists found that apparent gas permeability coefficients can be successfully approximated by the linear Klinkenberg equation under laboratory conditions. In recent years, however, an increasing number of simulation (e.g. lattice Boltzmann) and experimental studies found deviations from the 1st order slip model claiming that these deviations can be attributed to 2nd order fluid dynamic effects in the transitional flow regime.

Here we show based on careful examination of available literature data that deviations from the 1st order slip model result from a misinterpretation of the effective stress conditions. Apparent permeability coefficients measured with gases on low-permeable rocks always reflect a combination of fluid-dynamic behavior and the stress state of the sample. Therefore, fluid dynamic effects are evaluated over a range of pore pressures at constant effective stress conditions. This is done by assuming Terzaghi's principle to be valid, which is highly inaccurate for gas shales, as stress dependent permeability rather follows a more complicated modified effective stress relationship [1,2]: 
$\sigma^{\prime}=\operatorname{Pc}-\chi \operatorname{Pp}(1)$

The permeability effective stress coefficients $\chi$ is usually $\leq 1$, indicating that pore pressure has a lesser influence on effective stress than confining pressure. Inaccurate $\chi$ values (e.g. assuming Terzaghi's principle) lead to "apparent" deviations from the 1st order slip model. This is confirmed by careful examination of the available literature data.

To our understanding, the sensitivity of apparent permeability coefficients to changing $\chi$ values should be examined before simulating experimental 2nd order slippage models. The error that is introduced strongly differs between samples and stress levels and can be reduced by measuring on stress-insensitive samples at high stresses.

Procter and Gamble Student poster award:

References:

1 Heller, R., Vermylen, J., Zoback, M., 2014. Experimental investigation of matrix permeability of gas shales. AAPG Bulletin 98, 975-995.

2 Fink, R., Krooss, B.M., Gensterblum, Y., Amann-Hildenbrand, A., 2017. Apparent permeability of gas shales-superposition of fluid-dynamic and poro-elastic effects, Fuel 199, 532-550. Acceptance of Terms and Conditions:

Click here to agree

\title{
Formation dry-out and salt precipitation in porous and fractured media: Laboratory insights on physics and dynamics of $\mathrm{CO} 2$-induced halite accumulations
}

\author{
Mohammad Nooraiepour ${ }^{1}$; Hossein Fazeli ${ }^{2}$; Rohaldin Miri $^{1}$; Helge Hellevang ${ }^{1}$ \\ ${ }^{1}$ University of Oslo (UiO) \\ ${ }^{2}$ University of Oslo
}

Corresponding Author(s): nooraiepour@gmail.com

We have conducted a series of microfluidic experiments on the glass and geomaterial micromodels at ambient and HPHT conditions to investigate physics and dynamics of salt precipitation, governing mechanisms, and influencing factors. We have shown that the trapped water films in porous or fractured media have enough continuity and conductivity to transport residual brine to an evaporating front, and cause an increase in the rate and amount of precipitated halite crystals. The pressure gradient imposed by capillary-back flow and imbibition processes can produce significant conductivity and stability of the water films. Laboratory observations suggest that the salt precipitation during $\mathrm{CO}_{2}$ injection is a time-evolving and self-enhancing phenomenon which has the following characteristics: (a) in addition to the aqueous phase, salt crystals can precipitate and grow on the interface of rock and $\mathrm{CO}_{2}$ flow pathway. (b) salt crystals are covered with a thin water film of brine that is attracted by surface energy effects and hydrophilic nature of salt crystals. (c) micrometer-sized salts have a porous structure of densely precipitated aggregates with narrow pore throats between the crystals, which provides a potentially large capillarity to the salt aggregates to imbibe water over long distances. (d) micrometer-sized crystals that precipitate on the interface of solid and $\mathrm{CO}_{2}$ stream enhance the distribution of brine, increase the surface area for evaporation and growth, and hence, accelerate the evaporation rate. (e) evaporation, precipitation, and growth of salt bodies induce further nucleation and precipitation, which in turn contributes to an increase in capillary transport and suction. The results also indicate that $\mathrm{CO}_{2}$ phase states and pressure-temperature conditions govern the magnitude, distribution and precipitation patterns of salt precipitates. Injection of gaseous $\mathrm{CO}_{2}$ resulted in higher salt precipitation compared to liquid and supercritical $\mathrm{CO}_{2}$. The thermodynamic conditions influence salt precipitation via water solubility in $\mathrm{CO}_{2}$, maximum water flux into $\mathrm{CO}_{2}$ stream, and balance between the imposed viscous forces and capillary-driven backflow. The $\mathrm{CO}_{2}$ phase states also affect the relationship between the injection rate and extent of precipitated salts It is shown the higher the injection flow rate, the lower the salt coverage. A conceptual framework was introduced that suggests salt precipitation may be not only a near-well phenomenon but also a sealing mechanism that can impede $\mathrm{CO}_{2}$ leakage from fracture networks. The research outcome highlights the mechanisms and processes that are crucial to consider during the investigation of salt 
precipitation induced by $\mathrm{CO}_{2}$ injection because it has implications for both injectivity and containment assessments. For a better reservoir-scale numerical modeling, such mechanisms need to be incorporated and scaled-up in the reservoir simulator. The present approach for modeling salt precipitation using the volumetric approach in the reservoir-scale numerical simulator may not reflect the required physics for investigation of salt precipitation induced by $\mathrm{CO}_{2}$ injection.

\section{Procter and Gamble Student poster award:}

I would like to compete in the Procter and Gamble Student award References:

Nooraiepour, M., Fazeli, H., Miri, R., Hellevang, H., 2018. Effect of CO2 Phase States and Flow Rate on Salt Precipitation in Shale Caprocks-A Microfluidic Study. Environmental Science \& Technology 52, 6050-6060. DOI: 10.1016/j.ijggc.2015.10.004

Nooraiepour, M., Fazeli, H., Miri, R., Hellevang, H., 2018. Salt Precipitation during Injection of CO2 into Saline Aquifers: Lab-on-chip Experiments on Glass and Geomaterial Microfluidic Specimens. 14th International Conference on Greenhouse Gas Control Technologies (GHGT-14), 21-25 October 2018, Melbourne, Australia.

Miri, R., van Noort, R., Aagaard, P., Hellevang, H., 2015. New insights on the physics of salt precipitation during injection of CO2 into saline aquifers. International Journal of Greenhouse Gas Control 43, 10-21. DOI: $10.1021 /$ acs.est.8b00251

Nooraiepour, M., 2018. Rock Properties and Sealing Efficiency in Fine-grained Siliciclastic Caprocks Implications for CCS and Petroleum Industry, Faculty of Mathematics and Natural Sciences, Department of Geosciences. University of Oslo, Oslo, Norway, p. 231. Acceptance of Terms and Conditions:

Click here to agree

\section{Numerical methods for detection of kidney disease using tissue deformation fields}

Jan Martin Nordbotten ${ }^{1}$; Erlend Hodneland ${ }^{2}$; Erik Hanson ${ }^{1}$; Eirik Keilegavlen ${ }^{1}$; Arvid Lundervold ${ }^{1}$; Antonella Zanna Munthe-Kaas ${ }^{1}$

\footnotetext{
${ }^{1}$ University of Bergen

${ }^{2}$ Norce Research
}

Corresponding Author(s): jan.nordbotten@math.uib.no

Medical imaging is of key relevance in both the understanding of organ function as well as defection of disease. Several challenges arise in the use of time-series data, in particular related to the deformation of the observed organ as a consequence of the person breathing during the acquisition.

In a novel perspective, we see the deformation field not as an artifact to be removed, but rather as a potential source of data. Recognizing that chronic kidney disease may manifest itself in alterations in the mechanical properties of the kidney, we propose to use inverse modeling of time-sequences of MRI images as a non-invasive diagnostic tool.

In this talk, we review the development of an inverse modeling framework and efficient numerical methods applicable to large data sets of the form typically encountered in medical imaging. Furthermore, we show the applicability of our approach both in terms of phantom data, as well as by analyzing newly acquired MRI sequences of healthy volunteers and patients.

\section{Procter and Gamble Student poster award:}

\section{References:}

Nordbotten, J. M. (2016), Stable cell-centered finite volume discretization for Biot's equations, SIAM Journal of Numerical Analysis, 54(2), 942-968 
Hodneland, E., E. A. Hanson, A. Z. Munthe-Kaas, A. Lundervold, J. M. Nordbotten (2016), Physical models and their effect on human tissue deformation fields - Theory and MRI simulations of the moving kidneys, IEEE Transactions on Biomedical Engineering, 63(10), 2200-2210

Hodneland, E. E. Keilegavlen, E. A. Hanson, E. Andersen, J. A. Monssen, J. Rørvik, S. Leh, H.-P. Marti, A. Lundervold, E. Svarstad, and J. M. Nordbotten, In vivo detection of chronic kidney disease using tissue deformation fields from dynamic MR imaging, IEEE Transactions on Biomedical Engineering Acceptance of Terms and Conditions:

Click here to agree

18

\section{Numerical modeling of wave propagation in fractured porous fluid- saturated media - fracture connectivity effect on seismic wave attenuation}

Mikhail Novikov $^{1}$; Vadim Lisitsa ${ }^{1}$

${ }^{1}$ Novosibirsk State University, and Institute of Petroleum Geology \& Geophysics SB RAS

Corresponding Author(s): novikovma@ipgg.sbras.ru

Wave-induced fluid flow study is actual nowadays because seismic attenuation may indicate fractured highly-permeable reservoirs. We propose a numerical algorithm for wave propagation in porous fluid-saturated media with complex systems of connected fractures to study the connectivity effect on the wave attenuation.

First, we generate a discrete fracture network using simulated annealing approach, with fracture connectivity varying from almost nonintersecting fractures to the models with high percolation length. After that, we perform statistical analysis for generated models with different percolation length to study the geometrical and topological features of the obtained structures. Resulting models contain mesoscopic fracture clusters. Generated models are used for further numerical modeling of seismic wave propagation to observe the influence of fracture connectivity on the resulting wavefields and wave attenuation.

Proposed numerical technique for modeling the plane wave propagation is based on the finitedifference approximation of Biot's dynamic equations. We model the fast $\mathrm{P}$-wave propagation within a broad frequency range from 1 to $10 \mathrm{kHz}$. Set of numerical experiments is performed for the case of relatively high background permeability and higher permeability of the fracture-filling material. Results show the presence of intensive fracture-to-background flow between fracture clusters and the background. Resulting wavefields are used to estimate the frequency-dependent inverse quality factor demonstrating the attenuation. Obtained estimations show a significant increase of the wave attenuation due to intensifying FB-WIFF. For the other set of experiments, we use models with no FB-WIFF due to very low permeability and are performed to investigate the impact of fractureto-fracture fluid flow appearing within the connected fractures. Fracture-filling material models present here the cases of the rock before and after $\mathrm{CO} 2$ sequestration, which affects the stiffness and permeability of the material. Analogous estimations of the attenuation are obtained demonstrating the relative increase of FF-WIFF due to partial dissolution of the fractured material, but almost no attenuation dependence on the fracture system geometry and percolation length. Besides, in both cases, the value of the attenuation is negligibly small, and the propagating wave has no significant change in amplitude as well and velocity.

This research was supported by Russian Foundation for Basic Research grants no. 18-05-00031, 1605-00800, 18-01-00579, 18-35-00253.

Procter and Gamble Student poster award:

References:

Acceptance of Terms and Conditions:

Click here to agree 


\title{
Numerical assessment of organic source rocks' flexibility at the atomistic scale an its implications on transport at the nanoscale
}

\author{
Amael Obliger $^{\text {None }}$; Jérémie Berthonneau ${ }^{1}$; Pierre-Louis Valdenaire ${ }^{1}$; Roland Pellenq ${ }^{2}$; Jean-Marc Leyssale ${ }^{3}$; Franz \\ Ulm $^{1}$ \\ ${ }^{1}$ MIT \\ ${ }^{2}$ MIT / CNRS \\ ${ }^{3} \mathrm{MIT} / \mathrm{CNRS}$
}

Corresponding Author(s): amael.obliger@gmail.com

Shale-gas recovery from unconventional reservoirs is a very slow process that exhibits anomalous behaviour at large scales such as non-Fickian productivity decline. Recent studies, focusing on the subnanoporosity of the organic matter called kerogen (where the hydrocarbons are produced and trapped during maturation), has been carried out to elucidate these features $[1,2,3,4]$. Unfortunately, the molecular force field used in these studies is not adapted to capture flexibility effects such as swelling upon fluid adsorption. Those findings are thus restricted to the case of very mature matrices wher flexibility effects are negligible. In this work 5 we present a re-parameterization of the non-bonded part of the AIREBO $\mathrm{C} / \mathrm{H}$ potential, achieved to reproduce experimental equations of state for alkane chains, and use it to study the mechanical properties of two - raw and fluid-filled porous amorphous carbon matrices obtained from quench molecular dynamics simulations, serving as proxys for mature and immature kerogens. While, as expected, the mature matrix is somehow insensitive to pressure and tempreature effects, we observe a huge dependence of the density (and thus porosity) of the immature matrix on applied pressure, temperature and fluid loading. Especially, while some open porosity might be observed at atmospheric pressure, the raw immature matrix significantly shrinks in volume at geological pressures (25-100 MPa) where only traces of closed porosity remains. However, the presence of trapped/adsorbed hydrocarbons can significantly open the porosity.

As a consequence, the adsorption isotherms for the immature matrix does not plateau at large fluid pressure and simply increases linearly and the diffusion coefficient increases with the quantity of adsorbed fluid instead of decreasing as in the rigid case. We upscale these results with a Continuous Time Random Walk method on lattices built on 3d tomograms obtained by Transmission Electron Microscopy [6] with a subnanometric resolution to account for the impact of the mesopores ( $2 \mathrm{~nm}<$ $\mathrm{d}<50 \mathrm{~nm}$ ) which are scarcely distributed among the microporous carbon matrix. We show that the transport properties are not qualitatively impacted by the presence of the mesopores. An analytical model is derived for the upscaled diffusion coefficient that accounts for sorption between the two porous phases.

1 K. Falk et al., Nature Communications, 6:6949, 2015.

2 J. Collell et al., Journal of Physical Chemistry C, 119(39):22587-95, 2015.

3 A. Obliger et al., Journal of Physical Chemistry Letters, pp. 3712-3717, 2016.

[4] A. Obliger et al., Nano Letters, 18(2):832-7, 2018.

5 A. Obliger et al., Langmuir, 34(45):13766-80, 2018.

[6] J. Berthonneau et al., Procceedings of the National Academy of Sciences of the USA, 2018.

Procter and Gamble Student poster award:

References:

Acceptance of Terms and Conditions:

Click here to agree 


\title{
Impact of Physical Heterogeneity on Multispecies Reactive Trans- port in a Microporous Carbonate
}

\author{
Thomas David Serafini de Oliveira ${ }^{1}$; Martin J. Blunt ${ }^{1}$; Branko Bijeljic $^{1}$ \\ ${ }^{1}$ Imperial College London
}

Corresponding Author(s): thomas.oliveira@centraliens.net

A key determinant impacting mixing and reaction in subsurface media is heterogeneity of pore space. We use X-ray imaging and direct numerical simulation to examine the impact of physical heterogeneity on mixing-driven fluid-fluid multispecies chemical reaction in porous media. Exemplars of a homogeneous medium (bead pack) and a highly complex heterogeneous medium (microporous Portland limestone) are used to have a variety of pore-scale complexity which is reflected in different physical heterogeneity.

In bead pack we solve the Navier-Stokes equations and the advection-diffusion equation for concentration fields using the finite volume method and we consider each grid cell as a completely mixed batch reactor (Oliveira et al., 2018). We compute homogeneous reversible reactions in the liquid phase using a geochemical model Reaktoro (Leal et al., 2016). We simulate flow through the Portland image using a methodology which consists of direct numerical simulation in the complex macro- and micropore space resolved by differential X-ray imaging, and anchoring porosity measurements to the throat (and pore) sizes obtained from mercury injection porosimetry (Bijeljic et al., 2018). Flow in dual porosity media is described using Stokes equation in macropore space combined with Darcy term representing flow in microporosity. Diffusion coefficients in microporous regions are a function of porosity.

We quantify the effect of heterogeneity on mixing by analysing spatially resolved maps of concentration of species and their reactor ratio. In contrast to the bead pack, the heterogeneous flow field in the carbonate led to much more transverse mixing, with reactants provided throughout the simulation domain.

The impact of heterogeneity on reaction dynamics in the carbonate is observed from the comparison of the spatially resolved maps of the rates of formation and consumption of species after different numbers of pore volumes injected. We observe a highly non-uniform spatial distribution of reaction rates. The evolution of the species reaction rates reveals new insights on the reaction dynamics: while some of the species reach an asymptotic rate, others still show transient non-monotonic behaviour. This implies that in multispecies reversible reactive transport in natural porous media reaction rates exhibit much more complex behaviour in comparison to bimolecular irreversible reaction, which needs to be considered in analysis.

We demonstrate that PDFs of velocity in voxels in which reactions occur reveal different impacts of transport in macroscopic pore space and microporosity on both formation and consumption rates of species. For both bead pack and carbonate, we observe different relative yield values for each species, implying that the difference from the fully mixed system is species dependent. However, due to different nature of mixing in the carbonate, we observe a much more fluctuating behaviour in comparison to the bead pack.

Overall, we demonstrate that multispecies reactive transport at the pore scale can have highly interesting features of coupled transport and reversible reaction dynamics that can be used in describing larger scale behaviour.

\section{Procter and Gamble Student poster award:}

\section{References:}

Bijeljic, B., Raeini, A.Q., Lin, Q. \& Blunt, M.J. (2018) Multimodal Functions as Flow Signatures in Complex Porous Media. Available from: http://arxiv.org/abs/1807.07611.

Leal, A.M.M., Kulik, D.A. \& Kosakowski, G. (2016) Computational methods for reactive transport modeling: A Gibbs energy minimization approach for multiphase equilibrium calculations. Advances in Water Resources, 88, 231-240. Available from: doi:10.1016/j.advwatres.2015.11.021 
Oliveira, T. D. S., Blunt, M. J., and Bijeljic, B. Modelling of multispecies reactive transport on images of pore space. Advances in Water Resources. (In review) Acceptance of Terms and Conditions:

Click here to agree

\title{
Non-Newtonian behaviour of foam flow in porous media: exper- iments and upscaling
}

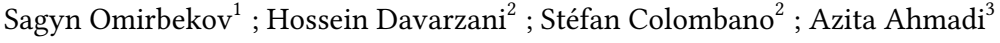 \\ ${ }^{1}$ BRGM (French geological survey); Institut de Mécanique et Ingénierie de Bordeaux (I2M, TREFLE), Arts et Métiers \\ ParisTech \\ ${ }^{2}$ BRGM (French geological survey) \\ ${ }^{3}$ Institut de Mécanique et Ingénierie de Bordeaux (I2M, TREFLE), Arts et Métiers ParisTech
}

Corresponding Author(s): s.omirbekov@brgm.fr

Foam injection in porous media has been investigated for a variety of applications in the oil industry, especially, for Enhanced Oil Recovery (EOR). Foam injection technics developed for EOR can also be applied to soil remediation processes to remove Non-Aqueous Phase Liquids (NAPL) from aquifers. The primary purpose of foam injection in soil remediation operations is to control the permeability of porous media to divert the flow from high to low permeable zones in aquifers. There are some major differences between oil reservoirs and aquifers. For instance, porous media in oil reservoirs are mainly low permeable and consolidated, and under high pressure and temperature conditions. While, polluted aquifers are mainly unconsolidated and highly permeable. As a consequence of these differences, in-situ foam generation in aquifers is questionable, and also foam flow in aquifers calls upon further investigation.

Generally, understanding of foam flow in porous media is hampered due to complex behaviour of foam and apparent contradictions in foam studies. For example, some authors indicated Newtonian 1 behaviour of foam flowing in porous media, others shear-thinning 2 , shear-thickening 3 , a mixture of Newtonian and shear-thinning [4] or dependent on foam quality (foam gas volume fraction) 5. In addition, it must be noted that most studies presented in the literature concern low permeable media for EOR applications.

The objective of this study is to determine, experimentally, the rheological behaviour of bulk foam and to investigate foam flow in high permeable unconsolidated porous media by performing numerous laboratory experiments and finally to scale-up, theoretically and/or numerically, the foam flow from pore to Darcy scales.

At first, the rheological parameters of the bulk foam, pre-generated using a fine sand-packed column, were measured by a rheometer. The bulk foam for $85 \%$ foam quality was found to have a non-Newtonian yield stress fluid behaviour, which could be correctly represented by the HerschelBulkley model. Then, the pre-generated foam has been injected into fine sand and also calibrated glass beads packed columns ( $4 \mathrm{~cm}$ internal diameter, $40 \mathrm{~cm}$ height). In each experiment, a fixed quality foam is injected for different total flow rates, where gas and surfactant solution rates are changed simultaneously. Pressure drops are measured over the 1D column for each flow rate, thus allowing to investigate the macroscopic flow rate $(\mathrm{Q})$ - pressure gradient $(\nabla \mathrm{P})$ relationship. The macroscopic flow of $85 \%$ and below foam quality for various unconsolidated porous media clearly leads to $Q(\nabla P)$ featuring a yield stress behaviour.

The experimental results are the basis to validate foam upscaling in porous media, where foam was considered as a single-phase Herschel-Bulkley fluid. This assumption is valid when the bubble size is much smaller than the pore size. The closure problem obtained by homogenization from the general case of non-Newtonian fluids flow in porous media [6] is solved numerically for yield stress fluids for different 2D and 3D unit cell geometries.

In this talk, experimental and numerical results will be presented, and the experimental data will be compared to the upscaled results. 
Procter and Gamble Student poster award:

I would like to compete in the Procter and Gamble Student award References:

1 P. Persoff, C. J. Radke, K. Pruess, S. M. Benson, and P. A. Witherspoon, “A Laboratory Investigation of Foam Flow in Sandstone at Elevated Pressure.," Society of Petroleum Engineers., Aug. 1991.

2 G. J. Hirasaki and J. B. Lawson, "Mechanisms of Foam Flow in Porous Media: Apparent Viscosity in Smooth Capillaries,” Society of Petroleum Engineers, 1985.

3 T. W. Patzek and M. T. Koinis, "Kern River Steam-Foam Pilots," Society of Petroleum Engineers, 1990. [4] F. Vassenden and T. Holt, "Experimental Foundation for Relative Permeability Modeling of Foam," Society of Petroleum Engineers, 2000.

5 J. M. Alvarez, H. J. Rivas, and W. R. Rossen, "Unified Model for Steady-State Foam Behavior at High and Low Foam Qualities," Society of Petroleum Engineers, 2001.

[6] L. Orgéas, C. Geindreau, J.-L. Auriault, and J.-F. Bloch, "Upscaling the flow of generalised Newtonian fluids through anisotropic porous media," Journal of Non-Newtonian Fluid Mechanics, vol. 145, no. 1, pp. 15-29, 2007. Acceptance of Terms and Conditions:

Click here to agree

596

\title{
Soil Structure - the elusive and fragile scaffolding for soil ecolog- ical functioning
}

Dani $\mathrm{Or}^{1}$; Thomas Keller ${ }^{2}$

\author{
${ }^{1}$ ETH Zurich \\ ${ }^{2}$ Department of Soil and Environment, Swedish University of Agricultural Sciences, Sweden
}

Corresponding Author(s): dani.or@env.ethz.ch

Soil biological activity often imparts structural signatures such as biopore networks and aggregation of soil particles with a nonrandom spatial arrangement of soil constituents that reflect numerous ecological and hydrological feedbacks. A simple and intuitive definition of soil structure is the binding and spatial arrangement of soil constituents that support physical and biological traits not found in a disturbed soil with the same constituents (i.e., texture, organic matter). As a fragile product of soil biological activity, it encompasses traits invisible to the eye (mechanical and ecological) that contribute to the difficulty of defining soil structure rigorously. The picture is further complicated by the existence of "managed" soil structure by tillage and land management that only marginally relies on biological activity. We will examine the similarities and differences between natural and managed soil structure in terms of goals and impacts on eco-hydrological processes. Of particular interest for the mini symposium is to examine how maximizing yields for increased food production would affect soil structure management (tillage) decisions in the presence of a glaring gap in the scientific basis and quantification of soil structure resulting from the largest geo-engineering operation on Earth - the annual tillage of arable lands.

Procter and Gamble Student poster award:

References:

Amilcare Porporato Acceptance of Terms and Conditions:

Click here to agree

\section{Observation and modelling of capillary instabilities, front pin- ning and drainage/imbibition hysteresis in two-phase displace- ments across a single constriction}

Author(s): Jordi Ortín ${ }^{1}$ 
Co-author(s): Ramon Planet ${ }^{1}$; Lautaro Díaz-Piola ${ }^{2}$

${ }^{1}$ Universitat de Barcelona

${ }^{2}$ Universitat de Barcelona

Corresponding Author(s): jordi.ortin@ub.edu

Two-phase fluid displacements in porous and fractured solid materials are essential to environmentally relevant processes such as secondary oil recovery, soil irrigation, filtering and $\mathrm{CO}_{2}$ sequestration. Displacements proceed by fluid invasion of individual pores or gap openings, most often through sudden interfacial motions or Haines jumps induced by capillary instabilities. Localised jumps give rise to fluid rearrangements that may themselves trigger new instabilities, leading cooperatively to spatially-extended avalanches of fluid invasions (Haines jumps). The relevance of local capillary instabilities and Haines jumps in the fluid invasion of porous media has led to an increasing number of in-depth investigations using sophisticated experimental techniques with improved spatial and temporal resolution. At large scales these irreversible events give rise to hysteresis of imbibition-drainage cycles, of capital importance to engineering applications and hydrology.

In this work we focus on the physics of the capillary phenomena involved in the fluid invasion or withdrawal of a single constriction in a laboratory model of an open fracture. This simple setting features elementary Haines jumps in the form of irreversible pinning-depinning events, piston-like steady displacements, and reversible front pinning. The sequence of events depends on the displacement direction, so that two opposite depinning events in an imbibition-drainage cycle give rise to an elementary hysteresis cycle, which we identify with the physical building block of macroscopic pressure-saturation hysteresis cycles.

The simplicity of the experiment makes it suitable to theoretical modelling. We write down a quasistatic local pressure balance at the two-phase fluid interface that reproduces all the features observed experimentally. It provides also quantitative predictions that are satisfied experimentally with remarkable accuracy, using only material and geometrical properties of the experiment with no fitting parameters. We believe that the modelling approach proposed in this work may represent the microscopic building block of physically grounded (as opposed to phenomenological) macroscopic models of hysteresis of multiphase flows in disordered media.

Procter and Gamble Student poster award:

References:

Acceptance of Terms and Conditions:

Click here to agree

93

\title{
Controlling the drying Induced Peeling of Colloidal Films
}

\author{
Author(s): Abdulkadir Osman ${ }^{1}$ \\ Co-author(s): Nima Shokri ${ }^{2}$; Hugh Stitt ${ }^{3}$; Lucas Goehring ${ }^{4}$ \\ ${ }^{1}$ The University of Manchester \\ ${ }^{2}$ School of Chemical Engineering and Analytical Science, The University of Manchester, Manchester, UK \\ ${ }^{3}$ Johnson Matthey Technology Centre \\ ${ }^{4}$ Nottingham Trent University
}

Corresponding Author(s): abdulkadir.osman@postgrad.manchester.ac.uk

Understanding the drying of colloidal dispersions is of great importance to a variety of processes such as the production of particulate matters and in the drying of coated layers which has several factors to consider that impacts the product quality 1 . The solidification of these complex systems during drying can lead to the formation of various mechanical instabilities, such as cracking and 
buckling of films, as a result of the build-up of stress [2-4]. In the present study, the effect of the suspension properties on the competition between the binding and peeling of colloidal films on solid surface was investigated. To do so, we conducted a comprehensive series of experiments with aqueous mixtures of silica nano-dispersions and polyethylene oxide (PEO) polymers. Time-lapse digital microscope images of the droplets show how an increase in polymer concentration and molecular weight reduced the curvature of the peeled films until the critical point is reached at which debonding is arrested. This is the result of the polymers changing the suspension properties which in turn influences the stress distribution acting across the surface. Thus, we show that the peeling of films can be controlled by modifying the properties of the suspension.

Procter and Gamble Student poster award:

I would like to compete in the Procter and Gamble Student award References:

Acceptance of Terms and Conditions:

Click here to agree

\title{
Experimental study of the influence of flow rate, temperature and pressure on the carbonation of natural serpentinites induced by $\mathrm{CO} 2$ geological storage
}

\author{
Author(s): Florian Osselin ${ }^{1}$ \\ Co-author(s): Michel Pichavant ${ }^{2}$; Mohamed Azaroual ${ }^{3}$ \\ ${ }^{1}$ Université d'Orléans, ISTO \\ ${ }^{2}$ ISTO, Institut des Sciences de la Terre d'Orléans \\ ${ }^{3} B R G M$
}

Corresponding Author(s): florian.osselin@cnrs-orleans.fr

With the recent GIEC 1 report about global warming urging humanity to increase its efforts on the reduction of greenhouse gas emissions in the atmosphere, in order to limit the temperature increase to $2^{\circ} \mathrm{C}$ maximum, research on the geological storage of carbon dioxide appears more sustained than ever. Geological storage of $\mathrm{CO} 2$ consists in injecting pure $\mathrm{CO} 2$ or $\mathrm{CO} 2$-saturated water in deep geological formations where it is expected to be trapped over several hundred or thousand years and can thus no longer participate in global warming 2. This storage can be performed in a variety of geological settings such as depleted gas and oil fields, deep saline aquifers or very reactive mafic and ultramafic geological formations 3 .

However, since the injection in the deep saline aquifers stores the CO2 in the porosity of the rock under gas or supercritical state, legitimate concern is raised about the safety and long-term behavior of such dynamic multiphase hydrosystems. Indeed, in case of failure of the caprock, $\mathrm{CO} 2$ would be able to leak to the surface with potentially dramatic effects [4]. The storage of CO2 in mafic and ultramafic formations appears then as a very appealing technology since it involves the mineralization of the carbon by precipitation with the major alkaline earth metals (i.e., $\mathrm{Mg}$, Ca...) leached from the formation itself. Carbonation of mafic rocks can be done either ex-situ (carbonation of mine tailing) or in-situ (carbonation of the peridotite in natural ophiolite fields or carbonation of basalt formations). Large ophiolite formations (Oman, Papua New Guinea, east coast of Adriatic Sea...) are expected to have a storage capacity of several billion tons of $\mathrm{CO} 2$, which combined with other geological storage technologies may help to limit global warming to acceptable levels.

However, experimental results show that the feasibility of such a process is limited by an inherent negative feedback due to the passivation of the reactive surface and the clogging of the porosity by magnesite and amorphous silica precipitation [5,6]. Indeed, it appears that magnesite and silica precipitate directly on the surface of the reactive minerals and isolate it from the incipient flow of enriched carbon dioxide brine. Additionally, the precipitation of magnesite was observed to clog the natural fractures and decrease the overall permeability of the sample. As a result, only a small portion of the geological formation will react with $\mathrm{CO} 2$ bearing brines, limiting strongly the potential storage capacity of such geochemically reactive reservoirs.

In this study, we present experimental results on the carbonation of natural cores of serpentinites and 
peridotites, by the continuous injection of carbon-saturated water. We show, similarly to previous numerical studies [7], that the Péclet (relative importance of diffusion and convection processes) and Damköhler (relative importance of convection and chemical processes) numbers control the localization of the magnesite precipitation as well as the initial geometry of the porosity and permeability (in particular the grain size and the potential presence of natural or engineered factures). A careful control of the key parameters such as the temperature, the confining pressure and the flow rate of the $\mathrm{CO} 2$-saturated water permits then an optimization of the reaction, by limiting the clogging and the potential passivation of the serpentine reactant. Such results are particularly interesting for the design and the optimization of pilot sites and the development of this technology at industrial scale. Furthermore, the experimental results are useful for numerical modelling, risk analysis and the management of the carbon dioxide storage reservoirs.

\title{
Procter and Gamble Student poster award:
}

\section{References:}

1 IPCC, 2018: Summary for Policymakers. In: Global warming of $1.5^{\circ} \mathrm{C}$. An IPCC Special Report on the impacts of global warming of $1.5^{\circ} \mathrm{C}$ above pre-industrial levels and related global greenhouse gas emission pathways, in the context of strengthening the global response to the threat of climate change, sustainable development, and efforts to eradicate poverty

[V. Masson-Delmotte, P. Zhai, H. O. Pörtner, D. Roberts, J. Skea, P. R. Shukla, A. Pirani, W. MoufoumaOkia, C. Péan, R. Pidcock, S. Connors, J. B. R. Matthews, Y. Chen, X. Zhou, M. I. Gomis, E. Lonnoy, T. Maycock, M. Tignor, T. Waterfield (eds.)].

World Meteorological Organization, Geneva, Switzerland, $32 \mathrm{pp}$

$2 \mathrm{~S}$. Bachu and J.J. Adams. Sequestration of CO2 in geological media in response to climate change: Capacity of deep saline aquifers to sequester CO2 in solution. Energy Conversion and Management, 44:3151-3175, 2003.

3 Kelemen, P.B. et al. In situ carbon mineralization in ultramafic rocks: Natural processes and possible engineered methods, Energy Procedia, 146 :92-102, 2018

[4] C.R. Nelson et al. Factors affecting the potential for CO2 leakage from geologic sinks. Plains Reduction Partnership, 2005.

5 Andreani et al. Experimental Study of Carbon Sequestration Reactions Controlled by the Percolation of CO 2 -Rich Brine through Peridotites Environmental Science \& Technology, 43(4):1226-1231, 2009

[6] Hövelmann et al. Microstructure and porosity evolution during experimental carbonation of a natural peridotite Chemical Geology, 334:254-265, 2012

[7] Kondratiuk, $\mathrm{P}$ et al. Instabilities and finger formation in replacement fronts driven by an oversaturated solution, J. Geophys. Res. Solid Earth, 122, 5972-5991, 2017 Acceptance of Terms and Conditions:

Click here to agree

\section{Screening of EOR Potential on the Pore Scale - Application of Mi- crofluidics to Alkaline Flooding}

\author{
Holger Ott ${ }^{1}$; Ahmad Kharrat ${ }^{1}$; Mostafa Borji ${ }^{1}$; Torsten Clemens ${ }^{2}$; Pit Arnold ${ }^{1}$ \\ ${ }^{1}$ Montanuniversität Leoben \\ ${ }^{2}$ OMV AG
}

Corresponding Author(s): holger.ott@unileoben.ac.at

Complex chemical EOR processes, such as in alkaline or surfactant flooding, are typically optimized on their phase behavior and by core flood experiments. However, the information from classical experiments are rather limited, because they do not directly give insight the details of oil mobilization 
and displacements - in core floods, typically oil production and differential pressure are measured, which are both $1 \mathrm{D}$ data sets. The phase behavior is typically measured in test tubes and not under realistic flow (mixing) conditions in porous media flow.

Chemical EOR is changing interactions between fluids and the porous medium and is therefore manifested on the pore scale, where fluids are actually displaced. However, pore scale observations are typically suffering from a limited field of view especially for multiphase flow effects, which may not be representative for the overall system or the displacement.

In the frame of this study, we investigate displacements of crude oil by water and alkali solutions in order to optimize injection-water compositions for tertiary recovery. The study takes advantage of the high spatial and temporal resolution of microfluidics in order to observe fluid phases in the pore space, their distribution and displacements. Changes of the wetting state, breaking of oil clusters and the formation of emulsion phases as characteristic for the displacements have been observed. In order to overcome the limitation of the relatively small field of view, oil clusters have been analyzed by statistical and topological means showing a systematic change form water flooding to EOR.

The study shows that (a) cluster analysis can be used for EOR screening and - in the present case is more indicative with respect to EOR performance than production data from the same experiment. The study might be a first step towards statistical fingerprinting for optimizing EOR processes. (b) classical phase behavior experiments do not reflect (or just partly) the phase behavior in the porous medium under flow conditions. (c) the formation of (micro) emulsions in the pore space leads to pinning effects and is therefore of disadvantage for the displacement.

Procter and Gamble Student poster award:

References:

Acceptance of Terms and Conditions:

Click here to agree

\title{
Cross-property relations between elastic properties and thermal conductivity of partially sintered ceramics and fractured rocks
}

\author{
Willi Pabst $^{1}$; Eva Gregorová ${ }^{2}$; Tereza Uhlířová ${ }^{2} ;$ Vojtěch Nečina $^{2}$ \\ ${ }^{1}$ University of Chemistry and Technology, Prague \\ ${ }^{2}$ UCT Prague
}

Corresponding Author(s): pabstw@vscht.cz

Cross-property relations are relations between two different relative properties, the latter being defined as non-dimensional ratios of the effective properties of porous or fractured materials and their dense and compact (i.e. pore-free and non-fractured) counterparts. In this contribution we recall that, as long as the microstructure as a whole is statistically isotropic, our cross-property relation, which has originally been derived for correlating the Young modulus and thermal conductivity of porous ceramics with convex isometric pores 1 and has later been generalized to other elastic moduli (shear and bulk modulus) 2, can be used also for estimating the effective properties of partially sintered ceramics (where the pores are essentially concave) [3-5]. Since partially sintered ceramics are metrically and topologically similar to porous sandstone and other (potentially oil-bearing) sedimentary rocks, the cross-property relations can be used in these cases as well. Moreover, we show that our generalized cross-property relation is able to capture extreme deviations from spherical and isometric pore shape 2, so that it can be used even for estimating the relative properties of fractured ceramics and rocks (where the pores degenerate to extremely oblate shapes with negligible volume fraction). This is possible, because the exact single-inclusion solutions for spheroidal pores can be implemented in effective medium approximations for elastic properties and thermal conductivity [6,7]. The present contribution is based on extensive numerical calculations with computer-generated digital microstructures of virtual materials, using the commercial software package GeoDict ${ }^{\circledR}$ both for microstructure generation and effective property calculation, and takes into account the whole range of possible solid Poisson ratios from extremely auxetic $(-0.99)$ to extremely incompressible $(+0.49)$, so that the results are essentially of general validity, irrespective of the special material in question. 
Procter and Gamble Student poster award:

I don't want to compete References:

1. Pabst W., Gregorová E.: A cross-property relation between the tensile modulus and the thermal conductivity of porous materials, Ceram. Intern. 33 (1), 9凶12 (2007).

2. Pabst W., Gregorová E.: A generalized cross-property relation between the elastic moduli and conductivity of isotropic porous materials with spheroidal pores, Ceram. Silik. 61 (1), 74-80 (2017).

3. Pabst W., Uhlířová T., Gregorová E., Wiegmann A.: Young's modulus and thermal conductivity of model materials with convex or concave pores - from analytical predictions to numerical results, J. Eur. Ceram. Soc. 38 (7), 2694-2707 (2018).

4. Uhlířová T., Nečina V., Pabst W.: Modeling of Young's modulus and thermal conductivity evolution of partially sintered alumina ceramics with pore changes from concave to convex, J. Eur. Ceram. Soc. 38 (8), 3004-3011 (2018).

5. Pabst W., Uhlírová T., Gregorová E.: Shear and bulk moduli of isotropic porous and cellular alumina ceramics predicted from thermal conductivity via cross-property relations, Ceram. Int. 44 (7), 8100-8108 (2018).

6. Pabst W., Gregorová E.: Conductivity of porous materials with spheroidal pores, J. Eur. Ceram. Soc. 34, 2757-2766 (2014).

7. Pabst W., Gregorová E.: Young's modulus of isotropic porous materials with spheroidal pores, J. Eur. Ceram. Soc. 34, 3195-3207 (2014).

Acceptance of Terms and Conditions:

Click here to agree

\title{
Pore shape influence on the thermal conductivity of porous ce- ramics and sedimentary rocks with empty, water-saturated or high-conductivity pore space
}

\author{
Willi Pabst ${ }^{1}$; Eva Gregorová ${ }^{2}$; Tereza Uhlířová ${ }^{2}$; Vojtěch Nečina ${ }^{2}$ \\ ${ }^{1}$ University of Chemistry and Technology, Prague \\ ${ }^{2}$ UCT Prague
}

Corresponding Author(s): pabstw@vscht.cz

Porous ceramics and sedimentary rocks can have many common microstructural features. In particular, partially sintered ceramics exhibit an interconnected pore space with essentially concave pores, resulting in very low thermal conductivity (compared to porous materials with convex pores), when the pores are empty (vacuum voids) [1,2]. Diagenetically solidified sedimentary rocks exhibit very similar microstructures and properties. By contrast, materials with convex pores exhibit much higher thermal conductivity as long as the pores are empty and isometric [1,2]. In this contribution we investigate the influence of the pore shape on the porosity dependence of thermal conductivity of porous ceramics and sedimentary rocks with empty, water-saturated or high-conductivity pore space. As long as the pores are empty (vacuum voids) and spheroidal, the whole analytical apparatus of micromechanical porous media theory can be applied, i.e. single-inclusion solutions (which are in this case identical with the self-consistent model), Maxwell-type models, Coble-Kingery relations, differential models, and exponential relations 3. Also for the opposite, high-contrast, case (i.e. highly conductive pores in a low-conductivity solid) simple asymptotic relations are available [4-6], whereas for the low-contrast case the two-point (Hashin-Shtrikman) and three-point (Beran) bounds, and the self-consistent model (Bruggeman-Landauer relation), can be invoked, i.e. the analytical apparatus of composite theory is available (not necessarily restricted to spheroidal pores) [4-6]. In this contribution the analytical predictions based on the single-inclusion solution for spheroidal 
pores are confronted with results obtained from numerical calculations on virtual materials with a wide range of different computer-generated model microstructures (isotropic, random): overlapping convex pores or concave pores between overlapping solid particles of spherical or spheroidal (prolate or oblate) shape. The commercial software package GeoDict ${ }^{\circledR}$ is used both for microstructure generation and effective property calculation.

Procter and Gamble Student poster award:

I don't want to compete References:

1. Pabst W., Uhlírová T., Gregorová E., Wiegmann A.: Young's modulus and thermal conductivity of model materials with convex or concave pores - from analytical predictions to numerical results, J. Eur. Ceram. Soc. 38 (7), 2694-2707 (2018).

2. Uhlírová T., Nečina V., Pabst W.: Modeling of Young's modulus and thermal conductivity evolution of partially sintered alumina ceramics with pore changes from concave to convex, J. Eur. Ceram. Soc. 38 (8), 3004-3011 (2018).

3. Pabst W., Gregorová E.: Elastic and thermal properties of porous materials - rigorous bounds and cross-property relations (Critical assessment 18), Mater. Sci. Technol. 31 (15), 1801-1808 (2015).

4. Torquato S.: Random Heterogeneous Materials - Microstructure and Macroscopic Properties. Springer, New York 2002.

5. Milton G. W.: The Theory of Composites. Cambridge University Press, Cambridge 2002.

6. Sahimi M.: Heterogeneous Materials I - Linear Transport and Optical Properties. Springer, New York 2003.

Acceptance of Terms and Conditions:

Click here to agree

\title{
Groundwater Remediation using Nanotechnology
}

\author{
Tannaz $\mathrm{Pak}^{1}$; Luiz Fernando de Lima Luz $\mathrm{Jr}^{2}$; Tiziana Tosco ${ }^{3}$; Gabriel Schubert Ruiz Costa ${ }^{4}$; Paola Rosa ${ }^{4}$; Nathaly \\ Lopes Archilha ${ }^{4}$ \\ ${ }^{1}$ Teesside University \\ ${ }^{2}$ Federal University of Parana \\ ${ }^{3}$ Politecnico di Torino \\ ${ }^{4}$ Brazilian Center for Research in Energy and Materials
}

Corresponding Author(s): t.pak@tees.ac.uk, nathaly.archilha@lnls.br

Chlorinated solvent contaminants are among the most recalcitrant aquifer contaminants which can cause serious health problems (e.g. kidney and liver damage) and some are considered as carcinogenic. They are classified as DNAPLs, i.e. dense non-aqueous phase liquids. The scale of the problem posed by these contaminants is globally significant due to their wide industrial use since the beginning of 20th century e.g. in metal processing plants. Removal of chlorinated contaminants from the host aquifers can be done by water injection (soil flushing). In an aquifer water is the wetting phase and the chlorinated solvent acts as the non-wetting phase. It is known that such a displacement is always less than $100 \%$ efficient due to capillary action, therefore, a portion of these contaminants will remain trapped in the host aquifer1. Previous studies have shown that a non-wetting oil phase becomes disconnected into small oil droplets trapped in pores of the host rock as water is injected into the rock. Aquifer remediation technologies are designed to target this trapped contaminant to either (i) pump it out for ex-situ treatment, or (ii) degrade it, in-situ, into less harmful substances. The latter has received more attention recently as it offers a non-invasive means for contamination elimination. 
Nanoremediation is an emerging technology with great potential for in-situ degradation of chlorinated contaminants and many other metal contaminants (e.g. Cr, As, etc.). The technology injects $\mathrm{Fe} 0$ nanoparticle in form of aqueous suspensions into contaminant bearing sediments 2 . These nanoparticles are highly reactive and excellent electron donors $\left(\mathrm{Fe} 0 \otimes \mathrm{Fe} 2++2 \mathrm{e}^{-}\right)$. Chlorinated solvents can readily accept those electrons and release their chlorine atoms in form of ions. Example reaction: $\left(\mathrm{C} 2 \mathrm{H} 2 \mathrm{Cl} 2+\mathrm{Fe} 0+2 \mathrm{H}+\bigotimes \mathrm{C} 2 \mathrm{H} 4+2 \mathrm{Cl}^{-}+\mathrm{Fe} 2+\right)$. Specifically, nanoremediation has benefited from recent developments in industrial scale manufacturing of engineered nanoparticles at low cost. While nanoremediation concept is proven to be successful at laboratory, pilot, and field scales, the existing practice is far from optimised. This study contributes to the design of an optimum nanoremediation process, targeting remediation of the contamination source that contains the chlorinated solvent in form of a disconnected residual phase. We have studied the mechanisms that occur within the pore space of the contaminant-bearing sediment as the chemical reactions explained above occur.

We present the outcomes of our 4D (time-resolved, 3D) experiments comprising flow injections and simultaneous 3D imaging using X-ray computed micro-tomography (micro-CT) technique. The study has been conducted at the Brazilian synchrotron. The specific chlorinated contaminant under study is Trichloroethane (known as TCE). For the first time, we have captured the evolution of TCE phase structure/distribution, in 3D, during the nanoremediation process. Our data show that the gas phase released during the chemical reaction remobilises the trapped TCE phase, facilitating its complete removal in subsequent soil flushing processes. Our findings provide new insights into the pore-scale physics of the nanoremediation process and contribute to optimisation of this process.

Procter and Gamble Student poster award:

\title{
References:
}

References

1. Pak, T., Butler, I. B., Geiger, S., van Dijke, M. I. J. \& Sorbie, K. S. Droplet fragmentation: 3D imaging of a previously unidentified pore-scale process during multiphase flow in porous media. Proc. Natl. Acad. Sci. 112, 1947-1952 (2015).

2. Tosco, T., Petrangeli Papini, M., Cruz Viggi, C. \& Sethi, R. Nanoscale zerovalent iron particles for groundwater remediation: a review. J. Clean. Prod. 77, 10-21 (2014). Acceptance of Terms and Conditions:

Click here to agree

\section{Mobility of zero-valent iron nanoparticles in porous media - A study using $X$-ray computed micro-tomography}

\author{
Tannaz $\mathrm{Pak}^{1}$; Luiz Fernando de Lima Luz $\mathrm{Jr}^{2}$; Nathaly Lopes Archilha ${ }^{3}$; Gabriel Schubert ${ }^{4}$; Tiziana Tosco $^{5}$
}

\author{
${ }^{1}$ Teesside University \\ ${ }^{2}$ Federal University of Parana \\ ${ }^{3}$ Brazilian Center for Research in Energy and Materials \\ ${ }^{4}$ Brazilian Centre for Research in Energy and Materials (CNPEM) \\ ${ }^{5}$ Politecnico di Torino
}

Corresponding Author(s): t.pak@tees.ac.uk, nathaly.archilha@lnls.br

In this contribution we present the results of our study on the progressive porous media clogging induced by deposition of zero-valent iron nanoparticles (nZVI). We study this specific particle since it has proven to deliver effective degradation of chlorinated hydrocarbons when injected in sub-surface layers contaminated by these organic-based toxic substances. The technology is known as nanoremediation and is an emerging technology with great potential for in-situ remediation of contaminated aquifers. For the degradation to occur the nZVI particles need to be injected (in form of aqueous suspensions) into the contaminant bearing sediments1. nZVI nanoparticles are highly reactive and excellent degraders of the chlorinated hydrocarbon contaminants by reduction reactions. 
While the nanoremediation concept is proven to be successful at laboratory, pilot, and field scales, there are a number of limitations to the technique, including the mobility of nZVI suspensions in subsurface sediment layers. Particle agglomeration and retention in porous media restricts efficient delivery of nZVI nanoparticles to the contaminated zones. The two primary challenges of working with nanoparticles in porous media: (i) particle aggregation and (ii) particle retention causing clogging1. These challenges are, mainly, dealt with by controlling the size and surface charge of nanoparticles, the chemistry of fluids (ionic strength and $\mathrm{pH}$ ), and the sediment surface charge. Given their small sizes $(<100 \mathrm{~nm})$ compared to typical pore-throat sizes of naturally occurring sediment layer $(\geq 10 \mathrm{~s}$ $\mu \mathrm{m})$, nanoparticles should cause no clogging issues, if suspended properly. However, nanoparticles form aggregates ( $\sim \mu \mathrm{m}$ size) which reduces mobility, surface area, and reactivity.

Darcy-scale modelling approaches commonly used to describe nanoparticle transport in porous media are based on a modified advection-dispersion equation that incorporates exchanges with the porous matrix, namely deposition and release (DR-ADE). The DR-ADE formulations (e.g. clean bed deposition, straining, ripening, blocking) are derived assuming a range of pore-scale processes that, in common laboratory tests (column tests), cannot be investigated and verified in details, but only inferred from large-scale observations. Here we used the experimental results obtained at pore-scale (using X-ray computed micro-tomography2) to obtain darcy-scale data, averaged on our sample, which were then fitted with a DR-ADE, similar to column test results, using the software MNMs. MNMs are the particle transport models developed at the Polytechnic University of Turin (Politecnico di Torino). This software (freely available) is the only coupled flow and particle transport model currently available. It models porous media clogging through a range of mechanisms as outlined above. By combining the fitting results and the detailed information on real pore-scale processes occurring in our sample, we verified the validity of the attachment/detachment kinetic models typically implemented in the DR-ADE. Our data is a sequence of 3D images which were captured before, during, and after injection of nZVI suspensions in a sand column. Analysing these images shows how accurate and inclusive the previously identified clogging mechanisms included in the MNMs are.

Procter and Gamble Student poster award:

\title{
References:
}

References

1. Pak, T., Butler, I. B., Geiger, S., van Dijke, M. I. J. \& Sorbie, K. S. Droplet fragmentation: 3D imaging of a previously unidentified pore-scale process during multiphase flow in porous media. Proc. Natl. Acad. Sci. 112, 1947-1952 (2015).

2. Tosco, T., Petrangeli Papini, M., Cruz Viggi, C. \& Sethi, R. Nanoscale zerovalent iron particles for groundwater remediation: a review. J. Clean. Prod. 77, 10-21 (2014). Acceptance of Terms and Conditions:

Click here to agree

\section{Experimental and Numerical Studies of Chemical Flooding Un- der Impacts of Solutal Marangoni Effects}

\author{
Sepideh Palizdan ${ }^{1} ;$ Jassem Abbasi $^{2} ;$ Masoud Riazi $^{3} ;$ Mohammad Reza Malayeri $^{4}$ \\ ${ }^{1}$ Student \\ ${ }^{2}$ Research Assistant \\ ${ }^{3}$ Associate Professor \\ ${ }^{4}$ Professor
}

Corresponding Author(s): palizdansepideh@gmail.com

Chemical flooding is gaining increased attention for the purpose of enhanced oil recovery. In the experimental part of this study, the impact of solutal Marangoni effects on improving the oil production in a micromodel is investigated by conducting several alkaline chemical floodings. To do so, and to make a surface tension gradient, two alkaline flooding experiments under identical initial and injection conditions but two different injection fluids were carried out. In the first experiment, a 
solution of magnesium sulfate [MgSO4], and in the second experiment, sodium carbonate ( $\mathrm{Na} 2 \mathrm{CO} 3)$ solution were used. Moreover, a simulation study is performed using computational fluid dynamics (CFD) to verify the results obtained in the laboratory. Observations after stopping the injection of fluids showed that in the first experiment, the distribution of fluids were completely stationary, while after stopping the injection of sodium carbonate, a spontaneous and twirling movement of fluids in the porous medium was detected. Tracking of changes in distribution of fluids showed that the circular movements of fluid due to Marangoni effects can be effective in draining of the unswept regions, that can be a powerful mechanism for improving the oil recovery factor. Moreover, it accelerates the mass diffusion of the chemicals at locations with less available concentrations in the fluid. This would have implications for wettability alteration process that is another important mechanism in improving the oil recovery factor. The CFD results also approved that the Marangoni effects exist in chemical flooding processes for which the wettability alteration would profoundly be influenced.

Procter and Gamble Student poster award:

I would like to compete in the Procter and Gamble Student award References:

Acceptance of Terms and Conditions:

Click here to agree

\title{
On the impact of asphaltene and resin on stability of water in oil emulsions
}

Sepideh Palizdan ${ }^{1}$; Mohamad Reza Malayeri ${ }^{2}$; Masoud Riazi ${ }^{3}$

\author{
${ }^{1}$ Student \\ ${ }^{2}$ Head of Department of Petroleum Engineering, School of Chemical and Petroleum Engineering, Shiraz University, \\ Shiraz, I.R. Iran, \\ ${ }^{3}$ Associate Professor
}

Corresponding Author(s): palizdansepideh@gmail.com

Oil production from wells suffers from formation of water in oil (water-oil) emulsion which would result in increased oil viscosity and consequently higher pressure drops along the well. These are frequently reported to link closely to chemistry of oil in terms of asphaltene and resin contents of crude oil. Asphaltenes are the polar molecules of the crude oil which may serve as stabilizers for water-oil emulsions. In this study, the stability of water-oil emulsions is investigated by means of varying the concentration of asphaltene and resin. Thereafter the pertinent mechanisms of emulsion stability are proposed. The water-oil emulsions are prepared from water and crude oil naturally without the use of any surfactant. The colloidal instability index (CII) is used to investigate the effects of oil compounds on stability of emulsions. The utilized emulsion has exhibited higher stability for the $\mathrm{CII}$ index value of 0.87 as asphaltene is about to become unstable and precipitate around this point. The formed emulsion has its highest number of dispersed droplets in the CII index of 1.08. However, this emulsion becomes severely unstable as time elapses due to the accelerated precipitation of asphaltene.

Procter and Gamble Student poster award:

\section{References:}

Acceptance of Terms and Conditions:

Click here to agree 


\section{Supershear rupture promoted by poroelastic coupling in induced earthquakes}

Pedro Pampillon Alonso de Velasco ${ }^{1}$; Luis Cueto-Felgueroso Landeira ${ }^{1}$; David Santillán Sánchez ${ }^{1}$; Juan Carlos Mosquera Feijóo ${ }^{1}$

${ }^{1}$ Universidad Politécnica de Madrid

Corresponding Author(s): pedropampillonalonso@gmail.com

The complexity of earthquake ruptures in poroelastic media arises from the interaction between stick-slip frictional instabilities and poromechanical couplings. One of the most fascinating features of natural and triggered earthquakes is the possibility of supershear ruptures, where rupture fronts propagate faster than the shear wave speed. Supershear earthquakes typically lead to largemagnitude events, with potentially more damaging impact on ground infrastructure.

Understanding elastodynamic processes during the rupture of induced earthquakes requires numerical simulations where a realistic fault constitutive behavior and rock poroelasticity including inertia are coupled. We simulate earthquake sequences induced by fluid injection near a strike-slip fault, assuming a rate-and-state fault and the Kelvin-Voigt viscoelastic model for the porous rock. We are able to resolve the full earthquake cycle, interseismic, aseismic/nucleation and dynamic-rupture phases, by using a monolithically-coupled, adaptive time-stepping scheme.

We observe that poroelastic coupling acts as an enhanced coseismic weakening mechanism during earthquake rupture, due to the undrained pressure response leading to a significant pore pressure increase localized near the compressional rupture front. This undrained pressure increase may promote further failure ahead of the advancing front, leading to supershear rupture propagation. By simulating earthquakes with different confinements and degree of poroelastic coupling, we characterize the hydromechanical factors controlling the sub-Rayleigh to supershear transition.

Our results show that there is an abrupt transition from sub- to super-shear rupture regimes as the effect of the undrained coseismic response decreases. In particular, the compressibility of injected and resident fluids around the fault emerges as a key parameter to understand the occurrence of supershear earthquakes in natural and induced earthquakes.

Procter and Gamble Student poster award:

I would like to compete in the Procter and Gamble Student award References:

Acceptance of Terms and Conditions:

Click here to agree

\section{A novel nano-based filter to combat oil spills}

Author(s): Aliasghar Parsaie ${ }^{1}$

Co-author(s): Abdolreza Kazemi Abadshapoori ${ }^{2}$; Mohammad Mohammadi-Khanaposhtani ${ }^{1}$; Masoud Riazi $^{3}$; Yousef Tamsilian ${ }^{4}$; Mohamad Reza Malayeri $^{5}$

${ }^{1}$ Faculty of Fouman, College of Engineering, University of Tehran, PO Box 43515-1155, Fouman, Iran

${ }^{2}$ Department of Petroleum Engineering, School of Chemical and Petroleum Engineering, Shiraz University, Shiraz, Iran

${ }^{3}$ Vice-dean of Research, Director at EOR research center, School of Chemical and Petroleum Engineering, Shiraz University, Shiraz, I. R. Iran

${ }^{4}$ Department of Oil, Gas \& Petrochemical Engineering (OGP), Faculty of Engineering, Shahid Chamran University of Ahvaz (SCU), Ahvaz, Iran

${ }^{5}$ Head of Department of Petroleum Engineering, School of Chemical and Petroleum Engineering, Shiraz University, Shiraz, I.R. Iran,

Corresponding Author(s): aliasghar.parsaie@ut.ac.ir, reza.pixy@gmail.com 
Oil production, storage, and transportation are always prone to pollution such as oil spills with detrimental and long-lasting impacts on the environment. Oil pollution in marine operations can seriously endanger the livelihood of aquatics. This requires stringent legislation and tangible implementation of approaches to counteract such hazardous circumstances. Present approaches including the use of oil adsorbents are usually costly and also are overshadowed by the lack of continuous oil separation. Accordingly, new methods for oil spill removal from water phase are required to remove oil from water phase continuously and also be cost effective. In this study, a new method is proposed which uses a porous medium as a robust filter for continuous separation of oil from water.

A nanoparticle-modified porous medium is developed as a filter to remove oil from water phase. For achieving this purpose, a polyurethane sponge is used as a porous medium which, in turn, can simultaneously absorb both oil and water phases. By the use of magnesium stearate nanoparticles as surface modifier agent, and phenol-formaldehyde resin as the sticker agent, the surface wettability of porous medium was then altered from neutral wettability to highly oil wet. Once this is accomplished the wettability and surface roughness of porous medium were measured using drop shape analysis (DSA) apparatus. After that, produced nanoparticles were dispersed in the base fluid (ethanol) using ultrasonic apparatus. Then, phenol-formaldehyde resin was used to stick nanoparticles onto pore walls. The next stage was to coat the pore walls with produced solution containing nanoparticles and the sticker, and then drain the porous medium. After coating porous medium, contact angle measurements and absorption efficiency were evaluated using DSA apparatus. The results demonstrated that the absorption time for oil phase was decreased from 5 seconds to less than half a second. Water contact angle measurements also showed an increase in contact angle from $105^{\circ}$ to $167^{\circ}$. The resultant porous medium was able to absorb oil phase spontaneously and can be used as a filter for oil removal from water phase.

The coating method poses some challenges. Here, the cost effective immersion method was used to coat pore walls with nano-particles. SEM images prove that this method was able to coat pore walls throughout porous medium. At the final stage, the modified sponge was used as a filter, and a pump was used for continuous oil removal from water phase. The resultant findings demonstrated that 500 cc of oil was removed from water phase without producing any water, and also continue the suction using pump, resulted no water production. This approach can be employed in many situations such as marine tanker accidents to separate oil from water phase and also to minimize costs of respective pollution.

Procter and Gamble Student poster award:

I would like to compete in the Procter and Gamble Student award References:

Acceptance of Terms and Conditions:

Click here to agree

\title{
Evaluating Storage Capacity of a Depleted Oil Reservoir during $\mathrm{CO} 2$ sequestration process using advanced numerical simulation technique
}

\author{
Author(s): Saeed Parvin ${ }^{1}$ \\ Co-author(s): Shahin $\operatorname{Kord}^{1}$; Rohaldin Miri ${ }^{2}$ \\ ${ }^{1}$ Petroleum University of Technology \\ ${ }^{2}$ University of Oslo
}

Corresponding Author(s): saeed.parvin.20@gmail.com

$\mathrm{CO} 2$ concentration in the atmosphere has increased drastically due to human activities, especially the worldwide use of fossil fuels. It is now rising at a rate of $1 \mathrm{ppm}$ per year, leading ahead to notable global warming (Yang Fang, 2010) which in turn causes several phenomena such as increasing sea level, loss of fragile ecosystems, increased floodings and droughts (Pachauri et al., 2014). One of the best midterm techniques to resolve the issues related to emission of $\mathrm{CO} 2$ gas, is Carbon Capture and Storage (CCS) in geological reservoirs (Bruant et al., 2002). The long-term storage of CO2 in the terrestrial biosphere, underground or in the oceans to decrease the increasing rate of $\mathrm{CO} 2$ concentration in the atmosphere, is known as CO2 sequestration (Reichle, 1999). Recent studies by 
the International Energy Agency have indicated that CCS can contribute to $14 \%$ of the reduction in global greenhouse gas emissions required by 2050, in order to limit global warming to $2{ }^{\circ} \mathrm{C}$ (Miri, 2015).

Different parameters of a candidate reservoir, such as injectivity, storage capacity and containment, should be assessed to conduct a successful $\mathrm{CO} 2$ sequestration project. Among these parameters, storage capacity is evaluated by numerical reservoir simulation. In this work, flow of $\mathrm{CO} 2$ through one of the Middle East depleted oil reservoirs was simulated and resulted storage capacity and storage efficiency of this reservoir were investigated. Matlab reservoir simulation toolbox (MRST), an open source framework with finite volume compositional capability, was applied to examine storage capacity, storage efficiency of the reservoir and flow path of $\mathrm{CO} 2$ in the porous media. In the simulated model of the reservoir, $\mathrm{CO} 2$ enters from one open boundary of the reservoir and rises continuously up-structure. The simulated model was run for a two-year period and results revealed that $11 \%$ of the total reservoir pore volume can be occupied by $\mathrm{CO} 2$. Furthermore, it was found that $80 \%$ of all trapped $\mathrm{CO} 2$ in pore volume is mobile that resides in up-structure of the reservoir.

\section{Procter and Gamble Student poster award:}

I would like to compete in the Procter and Gamble Student award References:

1. Yang F, Bai B, Tang D, Shari DN, David W. Characteristics of CO 2 sequestration in saline aquifers. Petroleum Science. 2010 Mar 1;7(1):83-92.

2. Pachauri RK, Allen MR, Barros VR, Broome J, Cramer W, Christ R, Church JA, Clarke L, Dahe Q, Dasgupta P, Dubash NK. Climate change 2014: synthesis report. Contribution of Working Groups I, II and III to the fifth assessment report of the Intergovernmental Panel on Climate Change. IPCC; 2014.

3. Bruant R, Guswa A, Celia M, Peters C. Safe Storage of CO2 in Deep Saline Aquifers. ENVIRONMENTAL SCIENCE AND TECHNOLOGY-WASHINGTON DC-. 2002 Jun 1;36(11):240A-5A.

4. Reichle D. Carbon sequestration research and development. US Department of Energy Report DOE/SC/FE-1, Washington DC, USA. 1999

5. Miri, R., 2015. Effects of CO2-Brine-Rock Interactions on CO2 Injectivity-Implications for CCS.

\section{Acceptance of Terms and Conditions:}

Click here to agree

879

\section{Beyond failure of geomaterials}

Author(s): Manuel Pastor ${ }^{1}$

Co-author(s): Diego Guillermo Manzanal ${ }^{1}$; Miguel Martin Stickle ; P. Navas ${ }^{1}$; Agustin Yagüe ${ }^{2}$; Saeed S.M. Tayyebi ${ }^{1}$; M. Molinos ${ }^{2}$; J.A.Fernández Merodo ${ }^{3}$; Pablo Mira Mc Williams ${ }^{4}$

${ }^{1}$ Universidad Politécnica de Madrid

${ }^{2}$ Universidad Politécnica de Madrid

${ }^{3}$ Universidad Politécnica de Madrid and IGME

${ }^{4}$ CEDEX

Corresponding Author(s): manuel.pastor@upm.es

Geotechnical engineers have been concerned for many years for determining the conditions under which a geostructure would fail. In order to determine the failure load and the mechanism type, mathematical, constitutive and numerical models have been used. As an example, we can consider the case of a slope subjected to seismic loading. Here, the mathematical model has to describe the coupling between the solid skeleton and the pore fluids. The contributions of Olek Zienkiewicz and Bernardo Schrefler have been of paramount importance for both saturated and unsaturated soils. To describe soil behaviour, constitutive relations are used. We will consider here Generalized Plasticity 
models for both saturated and unsaturated soils where we have included a state parameter. Regarding numerical models, most of practical cases have been modelled using coupled finite elements. Special techniques have been proposed in the past years to improve the accuracy of the models.

Once failure has been triggered, large deformations and displacements can occur. New mathematical models enlarging the domain of application of the classical pre-failure models have been derived, taking into account large relative displacements between phases. Regarding the rheological behaviour of fluidized soil, the progress has been much slower, and much work lies ahead of the concerned researchers. During last years, we have explored the similarity between rheological models and viscoplastic constitutive equations of Perzyna's type, which seem to provide a suitable bridge between solid and fluidized geomaterials behaviour.

Regarding numerical modelling, lagrangian meshless techniques such as SPH provide a suitable framework. In cases of landslides propagating distances much larger than their initial length, thin layer approximations provide suitable compromises between accuracy and cost of computation.

Procter and Gamble Student poster award:

I don't want to compete References:

Acceptance of Terms and Conditions:

Click here to agree

\title{
About the Use of 3D Printed Micromodels to Investigate single- and two-phase Flow Processes
}

\author{
Author(s): Alexandros Patsoukis Dimou ${ }^{1}$ \\ Co-author(s): Julien Maes ${ }^{2}$; Mohamed Arab ${ }^{3}$; Sebastian Geiger ; Thomas McGravie ${ }^{3}$ \\ ${ }^{1}$ Heriot Watt Univesrity \\ ${ }^{2}$ Heriot-Watt University \\ ${ }^{3}$ Heriot Watt University
}

Corresponding Author(s): ap92@hw.ac.uk

In order to understand subsurface processes in porous media at the continuum scale, it is firstly vital to understand how these processes behave at the pore scale. Microfluidic devices are an essential tool to conduct flow experiments that can be visualised on a pore-by-pore level. These experiments, underpinned by numerical simulations, have the potential to bring new insights into the processes and help to better constraints macro-scale models. Pore-scale numerical models have greatly improved in recent years and are now capable of reproducing micromodel flow experiments qualitatively. However, pore-by-pore quantitative comparison cannot be achieved due to the absence of a fully controlled repeatable benchmark for pore-scale multiphase flow modelling. Three dimensional (3D) printing allows for cheap and fast manufacturing of complex porosity models, enabling investigation of specific flow processes. However, in order for 3D printed micromodels to be used for creating a fully controlled repeatable benchmark, there are two major challenges to overcome: resolution and wettability control. Although 3D printer has recently been used to manufacture porosity models at a resolution up to $200 \mu \mathrm{m}$, it is still unknown if sufficient repeatability can be achieved to benchmark numerical models. Moreover, the ability of current 3D printer to print micromodels with different wettability has not yet been investigated. In this work, we assess the capability of a FormLab Form2 stereolithography printer to generate homogeneous and heterogeneous pore structures in a repeatable fashion at resolutions form 500 to 200 microns. Control of wettability may be achieved by printing using different available materials such as standard or ceramic resins. A workflow to analyse the variability of experiments is presented and a map of "fully repeatable" features is generated, than can then be used to compare quantitatively with numerical modelling.

Procter and Gamble Student poster award:

I would like to compete in the Procter and Gamble Student award References: 


\title{
A study on the microstructure of colloidal silica hydrogel when used as soil grouting material for ground barriers
}

\author{
Matteo Pedrotti ${ }^{1}$; Christopher Wong ${ }^{\text {None }}$; Eleonora Cali ${ }^{2}$; Bots Pieter $^{1}$; El Mountassir Grainne ${ }^{1}$; Ryan Mary ${ }^{2}$; \\ Lunn Rebecca J. ${ }^{1}$ \\ ${ }^{1}$ University of Strathclyde \\ ${ }^{2}$ Imperial College
}

Corresponding Author(s): matteo.pedrotti@strath.ac.uk

Colloidal silica hydrogel based grouts are being investigated for formation of ground barriers, especially for nuclear decommissioning purposes. Potential applications include pre-treatment of the ground beneath, and surrounding, legacy structures prior to the retrieval of hazardous wastes and the formation of horizontal and vertical barriers surrounding unlined waste disposal trenches. Over the last thirty years, colloidal silica hydrogel has been investigated, and more recently deployed, as a low viscosity grout for permeation grouting in soils and for grouting fractured rock. Colloidal silica hydrogel has a number of properties that make it attractive. It has an initially low viscosity (close to water) which means that very low injection pressures are required. The gel time of colloidal silica hydrogel can be controlled from minutes to several days and, once gelled, it has a hydraulic conductivity lower than $10^{\wedge}-10 \mathrm{~m} / \mathrm{s}$ In addition, it is considered to be environmentally inert and with particle sizes $<100 \mathrm{~nm}$, it has high penetrability.

Colloidal silica is an aqueous dispersion of silica particles, which are generally uniform in size and can range from several, to hundreds, of nanometres. A colloidal silica dispersion can be destabilized via the controlled application of an electrolyte. Once destabilized, silica particles form siloxane bonds ( $\mathrm{Si}-\mathrm{O}-\mathrm{Si}$ ), resulting in an increase in the viscosity and eventually a connected matrix of nanoparticles, i.e. a gel.

Given the near-surface conditions the grout is meant to be deployed, the effects on hydro-mechanical properties of colloidal silica hydrogel upon the interaction with the environmental conditions are of particular interest, especially given the severe cracking conditions colloidal silica might show.

This research aims to put forward a conceptual model able to interpret the cracking (upon drying) and swelling (upon leaching) mechanisms of colloidal silica, when coupled with different soils types (e.g. sand, silt and clay). A microstructural analysis has been carried out in order to investigate the different stages of a colloidal silica grouting in soils: from colloidal silica gelling (gel formation) to the behaviour of colloidal silica during repeated cycles of drying and wetting. A gelling mechanism is proposed based on the comparison between a time-lapse sequence of TEM images on colloidal silica during gelling and the respective mesoscopic and macroscopic measurements, such as particle aggregate size and dynamic viscosity. Once gelled, the colloidal silica particle configuration and their interaction with different soil particles is investigated by means of SEM images and $\mathrm{X}$-CT tomography. This microscopic conceptual model is then used to interpret X-CT tomography and synchrotron X-ray tomography that shows cracking and swelling patterns in grouted soil samples during cycles of drying and wetting. These results are related to the measured changes in bulk hydraulic conductivity.

Procter and Gamble Student poster award:

I don't want to compete References:

Acceptance of Terms and Conditions:

Click here to agree 


\title{
Fluid-Particle Interactions: Image-based Discrete Element Method (iDEM)
}

\author{
Xiaoming Zhang ${ }^{1}$; Tahmasebi Pejman ${ }^{1}$ \\ ${ }^{1}$ University of Wyoming \\ Corresponding Author(s): ptahmase@uwyo.edu
}

The solid system in deformable porous media undergoes deformation with the flow of fluid. Most of the current methods for modeling of granular geomaterials try to infer some statistical properties to describe the complexities and then regenerate the medium. These techniques, however, cannot extract very complex information and, thus, they produce unrealistic packing, which are physically unlikely. Eventually, the final models for simulations are usually not similar to the real materials and they make a significant underestimation for risk assessments and other related evaluations. In this presentation, for the first time, the available granular materials are used directly, without any parameters' extraction amongst, which results in much more accurate models of such materials for any specific scale. Consequently, our new method based on Discrete Element Method and actual particles for dealing with the models with realistic shapes and morphologies will be presented, which will incorporate various physics such as flow, and irregular particles. The developed computational model will be discussed using a few 3D examples.

Procter and Gamble Student poster award:

References:

Acceptance of Terms and Conditions:

Click here to agree

204

\section{Direct observation of the moisture distribution in castables dur- ing first dry out at high temperatures: an NMR study}

\author{
Leo $\mathrm{Pel}^{1}$; Barakat Ahmed ${ }^{1}$; Adan Olaf ${ }^{1}$ \\ ${ }^{1}$ Eindhoven University of Technology \\ Corresponding Author(s): 1.pel@tue.nl
}

In this study, the drying behaviour for a variety of calcium alumina- and hydratable alumina-bonded refractory castables was investigated in the temperature regime of first-drying, i.e., the initial drying up to 300 oC. Using a specialized high-temperature Nuclear Magnetic Resonance (NMR) setup, we were able to directly and non-destructively measure the spatially and temporally resolved moisture distribution, while simultaneously measuring the temperature distribution as well. These measurements give for the first time a direct insight into the drying behaviour of castables and show that the drying front speed and temperature are strongly correlated with control of key material parameters such a as binder content and water demand. Based on the measurements and a simplified model one can directly determine the permeability at high temperatures. These measurements provide a direct insight into the steam pressures which are generated within the samples and hence in the risk of explosion.

Procter and Gamble Student poster award:

References:

Acceptance of Terms and Conditions:

Click here to agree 


\title{
Coupled evolution of ecohydrologic processes and pore-size dis- tributions
}

\author{
Norman Pelak ${ }^{\text {None }}$; Amilcare Porporato ${ }^{1}$ \\ ${ }^{1}$ Princeton University \\ Corresponding Author(s): npelak@princeton.edu
}

\begin{abstract}
Soil functions are determined by a complex arrangement of pores, particles, and aggregates, which may change in time, as a result of both ecohydrological dynamics and human activity. The soil pore size distribution (PSD) is a key determinant of soil functions, and its accurate representation has the potential to improve hydrological and crop models. A modeling framework is proposed for the time evolution of the PSD which takes into account both natural and artificial processes, including tillage, consolidation, and changes in organic matter. The power-law PSD is shown to be the solution of a master equation for the pore radii, and the equation is parameterized to capture the changes in the PSD as a result of the soil processes considered. The benefit of this method for determining soil functions over the widely used pedo-transfer functions (PTFs) is that it allows for the history of the soil, rather than only its present state, to be taken into account. The PSD model is coupled to a minimal ecohydrologic model which is used to explore the impact of contrasting management strategies (e.g. irrigation, fertilization, organic matter inputs) on the soil structure and function.
\end{abstract}

Procter and Gamble Student poster award:

I would like to compete in the Procter and Gamble Student award References:

Acceptance of Terms and Conditions:

Click here to agree

310

\section{Analyzing ultrasonic data of nanoporous materials without using effective medium theories: pore sizes and pressure-dependence of elastic moduli.}

\author{
Author(s): Rolf Pelster ${ }^{1}$ \\ Co-author(s): Klaus Schappert ${ }^{1}$ \\ ${ }^{1}$ Saarland University, Department of Physics \\ Corresponding Author(s): rolf.pelster@mx.uni-saarland.de
}

\begin{abstract}
We discuss a method to evaluate pores sizes in nanoporous material and compare it to volumetric procedures. In particular, we focus on differences regarding the respective contributions of surface layers and capillary condensate as well as the assumptions used in the analysis. Next, we show that the pressure dependence of the adiabatic modulus of argon confined in Vycor glass, $\beta^{\text {ad }}$, is enhanced compared to bulk liquid. We discuss whether the pore pressure also affects the isothermal modulus, $\beta^{\text {iso }}$, or the heat capacity ratio, $c_{p} / c_{V}=\beta^{a d} / \beta^{i s o} 1$.
\end{abstract}

1 K. Schappert and R. Pelster, J. Phys. Chem. C, DOI: 10.1021/acs.jpcc.8b08136

Procter and Gamble Student poster award:

\section{References:}

Acceptance of Terms and Conditions:

Click here to agree 


\title{
Influence of sub-micrometer porosity on permeabilty of Rotliegend reservoir sandstones
}

\author{
Author(s): Markus Peltz ${ }^{1}$ \\ Heinrich Grathoff ${ }^{1}$ \\ ${ }^{1}$ University of Greifswald \\ 2 fohannes Gutenberg-University Mainz \\ ${ }^{3}$ Karlsruhe Institute of Technology \\ ${ }^{4}$ fohannes Gutenberg University Mainz
}

Co-author(s): Arne Jacob ${ }^{2}$; Sina Hale ${ }^{3}$; Frieder Enzmann ${ }^{4}$; Philipp Blum ${ }^{3}$; Laurence Noel Warr ${ }^{1}$; Georg

Corresponding Author(s): markus.peltz@uni-greifswald.de

\begin{abstract}
Heterogeneity of geological materials poses various problems when evaluating reservoir quality and storage potential. We analysed samples of different sedimentary facies of a Rotliegend sandstone from the Flechtingen High (Northern Germany) to determine the influence of depositional environment and diagenetic history on mineralogical composition and its impact on porosity and permeability.

We employed high resolution computer tomography (CT) (voxel size: $2.4 \mu \mathrm{m}$ ) and focussed ion beam - scanning electron microscopy (FIB-SEM) (voxel size: $0.01-0.03 \mu \mathrm{m}$ ) for direct pore space and mineral distribution analyses with focus on sub-micrometer zones like feldspar cement boundaries and diagenetically grown illite meshwork pore fillings. As shown by 1 , sub-resolution porosity in CT models can drastically influence flow properties. We found that about $20-30 \%$ of the segmented initial pore space is rather porous $(\varphi<100 \%)$ than pure void $(\varphi=100 \%)$. Thus, we utilized a NavierStokes-Brinkmann approach that allows to refine flow properties of observed CT models using void and porous domains. Porous domain properties were derived from Navier-Stokes simulations on FIB-SEM models. For comparison low temperature $\mathrm{N}_{2}$ adsorption and He-porosimetry data were used to characterize bulk permeability $\left(\mathrm{K}_{\text {bulk }}\right)$, total porosity $\left(\varphi_{\text {total }}\right)$, BET surface area and pore size distributions (PSD). X-ray diffraction (XRD) Rietveld analyses gave quantitative mineralogical information about the different facies and the clay mineral pore fillings.

Cross bedded layers show slightly higher Kbulk values (3.8 - 5.1 mD) compared to laminated layers $(1.5-2.3 \mathrm{mD})$ which correlates with feldspar cement content. However, calcite and clay cement phases show variance between the samples but no correlation with permeability and porosity. This can be attributed to the pore structure of these cement/grain interfaces with pore radii below $0.05 \mu \mathrm{m}$ as observed by FIB-SEM. Interfaces connected to feldspar cements show open pore networks with pore radii up to $0.5 \mu \mathrm{m}$. Such areas are particularly relevant for quantifying fluid flow as treating these spaces as pure voids, when in fact they represent semi-porous rock, will lead to an overestimation of simulated permeability values (compared to measured $K_{\text {bulk }}$ ) by more than one order of magnitude $(40-50 \mathrm{mD})$.
\end{abstract}

\section{Acknowledgements:}

This work was supported by the Bundesministerium für Bildung und Forschung (BMBF, Grant number: 03G0871B).

\section{Procter and Gamble Student poster award:}

I would like to compete in the Procter and Gamble Student award References:

1 Soulaine C., Gjetvaj, F., Garing C., Roman S., Russian A., Gouze P., Tchelepi H. A.: The Impact of Sub-Resolution Porosity of X-ray Microtomography Images on the Permeability (2016). Transp Porous Med (2016) 113:227-243 DOI 10.1007/s11242-016-0690-2 Acceptance of Terms and Conditions:

Click here to agree 


\title{
Homogenized modeling for vascularized poroelastic materials
}

\author{
Raimondo Penta ${ }^{1}$; José Merodio ${ }^{2}$ \\ ${ }^{1}$ University of Glasgow \\ ${ }^{2}$ Universidad Politécnica de Madrid
}

Corresponding Author(s): raimondo.penta@glasgow.ac.uk

A new mathematical model for the macroscopic behavior of a material composed of a poroelastic solid embedding a Newtonian fluid network phase (also referred to as vascularized poroelastic material), with fluid transport between them, is derived via asymptotic homogenization. The typical distance between the vessels/channels (microscale) is much smaller than the average size of a whole domain (macroscale). The homogeneous and isotropic Biot's equation (in the quasi-static case and in absence of volume forces) for the poroelastic phase and the Stokes' problem for the fluid network are coupled through a fluid-structure interaction problem which accounts for fluid transport between the two phases; the latter is driven by the pressure difference between the two compartments. The averaging process results in a new system of partial differential equations that formally reads as a double poroelastic, globally mass conserving, model, together with a new constitutive relationship for the whole material which encodes the role of both pore and fluid network pressures. The mathematical model describes the mutual interplay among fluid filling the pores, flow in the network, transport between compartments, and linear elastic deformation of the (potentially compressible) elastic matrix comprising the poroelastic phase. Assuming periodicity at the microscale level, the model is computationally feasible, as it holds on the macroscale only (where the microstructure is smoothed out), and encodes geometrical information on the microvessels in its coefficients, which are to be computed solving classical periodic cell problems. Recently developed double porosity models are recovered when deformations of the elastic matrix are neglected. The new model is relevant to a wide range of applications, such as fluid in porous, fractured rocks, blood transport in vascularized, deformable tumors, and interactions across different hierarchical levels of porosity in the bone.

Procter and Gamble Student poster award:

\section{References:}

Penta, Raimondo, and José Merodio. "Homogenized modeling for vascularized poroelastic materials." Meccanica 52.14 (2017): 3321-3343. Acceptance of Terms and Conditions:

Click here to agree

\section{A comparative study of elastic, single- and two-phase REV of porous rocks}

Joao Paulo Pereira Nunes ${ }^{1}$; Rodrigo Surmas ${ }^{1}$; Thomas Oliveira ${ }^{1}$

${ }^{1}$ Petrobras

Corresponding Author(s): jppn@petrobras.com.br

Sampling the reservoir formation is performed at an early stage of reservoir characterization. The choice of sample size (sidewall, plugs or whole-cores) has important impacts for the operation of a hydrocarbon reservoir. Increasing the sample size requires dedicated and complex bottomhole operations to extract whole-cores, which significantly impact the operational costs and poses operational risks. When designing a data acquisition campaign, reservoir engineers must weigh the need for larger samples size against the costs, typically using methods such as value of information analysis. In this work, we discuss such constraints in the light of the REV concept. Some typical questions faced by geoscientists are: 1) to what extent larger samples are necessary to describe the reservoir? 2) are the laboratory results meaningful, i.e, do they represent real physical properties? 
We performed a systematic comparison of representative elementary volumes (REV) for single- and two-phase flow and elastic properties on a variety of sandstone and carbonate reservoir rocks. All the relevant petrophysical properties (flow fields, absolute, stress fields and capillary pressure curves) were calculated through direct numerical simulation on tomographic images, at the pore and the core scale. Velocity fields are obtained by solving of Stokes equation in the pores. Stress fields are the result of finite element solutions which consider the voxel images as finite elements. Finally, capillary pressure curves are obtained through a geometric method (maximum spheres).

This work presents a thorough comparison between the flow and elastic REVs. We will discuss how the presence of multiscale heterogeneity (present in some carbonate rocks) affects the REV. To conclude, we will also examine how the choice of the sample size must be made based on the kind of information desired (single or multi-phase, flow or elastic) and the complexity of the reservoir rock.

Procter and Gamble Student poster award:

\title{
References:
}

Acceptance of Terms and Conditions:

Click here to agree

\section{Effective elastic parameters of porous heterogeneous materials computed from tomographic image large datasets using personal computers}

\author{
Andre Pereira $^{1}$; Ricardo Leiderman ${ }^{1}$; Rodrigo Azeredo ${ }^{1}$ \\ ${ }^{1}$ Universidade Federal Fluminense
}

Corresponding Author(s): andremaues@gmail.com

We present a computational implementation we developed to estimate the effective elastic parameters from segmented microstructural images, with focus on the use of ordinary personal computers. The effective elastic parameters are computed using computational homogenization framework with the aid of the finite element method (FEM). Micro-scale X-ray computed tomography (micro$\mathrm{CT}$ ) were used to image porous heterogeneous materials, e.g. composite materials and rock samples. One of the main challenges in the present context is the generation of a finite element (FE) mesh for a representative volume element (RVE) of the actual material. Here, we convert the micro-CT images into input data through a direct voxel to FE transcription. Thus, each voxel is adopted as an eight-node hexahedral element. To use the FEM, the element size needs to be significantly smaller than the pores and/or grains sizes, typically a few microns. With this discretization, millions of degrees of freedoms (DOFs) are required in the FEM model, to analyze a small material block. The large number of FEs restricts the application of a standard FEM, due to limitations in computer-processing time and memory storage. Therefore, the analyses performed here are carried out by means of an inhouse written software, using $\mathrm{C}++$ programming language and OpenMP extension for parallelism. To handle large linear systems, a special technique known as element-by-element (EBE) implementation is adopted. The implementation takes advantage of the fact that all elements in the mesh are of the same size. In addition, as is common for the EBE implementation, the resulting linear system (that is not assembled) is solved with the aid of a preconditioned conjugate gradient (PCG) algorithm. Furthermore, the computations for each element are performed in parallel. However, as the tasks may not be distributed arbitrarily between the processors, the elements are divided into different "groups" (or "colors") such that elements of the same group do not share nodes, avoiding attempt to update the same vector entry simultaneously, causing memory contention or dirty read/write entries. Finally, to consider the asymptotic multiscale homogenization theory, we applied the so-called periodic boundary conditions (PBC). The basic assumption behind the use of PBC is that the numerical model is a RVE of an unbounded (statistically homogeneous) medium, i.e. a periodic microstructure is assumed throughout the medium. In the context of the FEM, PBC can be applied by assigning the same equation number to corresponding nodes at opposite sides, forcing the displacements at these corresponding nodes to be equal and satisfying equilibrium. Our implementation is able to handle 
comfortably images of $400 \times 400 \times 400$ voxels $(\sim 2 \mathrm{E} 8 \mathrm{DOF})$. Each analysis (one simulation for a unidirectional uniform-strain field with PBC) takes approximately 2 min to run for a $100 \times 100 \times 100$ voxels input image ( 3 million DOFs), 20 min for a $200 \times 200 \times 200$ voxel input image ( 24 million DOFs), and 2 $\mathrm{h}$ for a $400 \times 400 \times 400$ voxel input image (193 million DOFs). In all the analyses, the PCG convergence criterion used was $1.0 \mathrm{E}-8$ for the relative norm of the residue vector. We also validated the results obtained numerically by means of experimental tests.

Procter and Gamble Student poster award:

I don't want to compete References:

Acceptance of Terms and Conditions:

Click here to agree

\title{
A Two-Stage Markov Chain Monte Carlo Method for Full Wave- form Inversion
}

\author{
Author(s): Felipe Pereira ${ }^{1}$ \\ Co-author(s): Susan Minkoff ${ }^{2}$; Georgia Stuart ${ }^{2}$ \\ ${ }^{1}$ Mathematical Sciences Department, The University of Texas at Dallas, Richardson, TX, USA \\ ${ }^{2}$ The University of Texas at Dallas
}

Corresponding Author(s): luisfelipe.pereira@utdallas.edu

\begin{abstract}
Bayesian methods for full waveform inversion facilitate uncertainty quantification, including allowing us to construct interval estimates and characterize the posterior distributions of the model unknowns. Markov chain Monte Carlo (MCMC) methods produce posterior distributions subject to fewer assumptions, such as normality, than deterministic Bayesian methods. However, MCMC is computationally a very expensive process that requires repeated solution of the wave equation. Ultimately, a large proportion of these models (often 90\%) are rejected. To reduce computational expense, we apply a two-stage MCMC algorithm, which uses a coarse-grid filter to quickly reject unacceptable proposals generated in the process, to the seismic velocity inversion problem. Our filter stage uses operator upscaling applied to the acoustic wave equation which provides near-perfect speedup in parallel with essentially no communication between processors. The coarse grid wave equation solution produces data that is highly correlated with the data obtained from the full fine-grid solution, thereby ensuring that the upscaled solution is a good filter. Three numerical experiments demonstrate the efficiency and accuracy of the method: one assuming velocities are known while inverting for unknown laterally varying interfaces, one assuming interfaces are known while inverting for velocities in a model with an angular unconformity, and one where both velocities and interface positions are unknown in a flat-layered model. We find that the two-stage MCMC produces the same results (i.e., posterior distributions, constructed by kernel density estimation, and uncertainty information, namely medians and highest posterior density intervals) as the traditional MetropolisHastings MCMC. Furthermore, the two stage substantially reduces the time-per-trial by $40 \%$ while increasing the acceptance rate from $9.1 \%$ to $89.4 \%$ for the flat-layered experiment. Therefore, the two-stage MCMC method for seismic full waveform inversion is an appropriate, less computationally expensive replacement for Metropolis-Hastings MCMC.
\end{abstract}

Procter and Gamble Student poster award:

I would like to compete in the Procter and Gamble Student award References:

Acceptance of Terms and Conditions:

Click here to agree 


\title{
Mixing-limited bimolecular chemical reactions at pore-scale
}

\author{
Author(s): Lazaro Perez ${ }^{1}$ \\ Co-author(s): Alexandre Puyguiraud ${ }^{1}$; Juan J. Hidalgo ${ }^{1}$; Joaquin Jimenez-Martinez ${ }^{2}$; Marco Dentz ${ }^{1}$ \\ ${ }^{1}$ IDAEA-CSIC \\ ${ }^{2}$ EAWAG-ETHZ
}

Corresponding Author(s): lazarojorgeperez@gmail.com

Mixing processes control chemical transformations such as precipitation/dissolution or degradation reactions that are fast compared to mass transfer processes. Chemical reactions are intrinsically local phenomena, while many applications require predictions at large scales. Physical and chemical heterogeneities are found at all scales and are at the root of complex spatial concentration distributions, segregation of reactants and phenomena related to the notion of incomplete mixing.

In order to assess the impact of medium and flow heterogeneity at pore-scale on mixingcontrolled reactions, we study the bimolecular irreversible chemical reaction $\mathrm{A}+\mathrm{B} \rightarrow$ $\mathrm{C}$. We consider the reactive displacement of $\mathrm{B}$ by a continuous injection of $\mathrm{A}$ in a 2-dimensional porous medium characterized by a random distribution of grain size and position. We use a reactive random walk particle tracking (RWPT) method to simulate the reactive transport problem. This approach is fully equivalent to the advection-diffusionreaction-equation. We observe three different regimes for the evolution of the product mass $\mathrm{mC}(\mathrm{t})$. In the first regime the reaction is controlled by diffusion, in the intermediate regime it is dominated by advective heterogeneity and characterized by incomplete mixing, in the third, asymptotic regime, mass production is controlled by hydrodynamic dispersion. We quantify the full evolution of the product mass through the dispersive lamella model (Perez et al., 2018), based on an effective dispersion coefficient, which captures the features of stretching, compression and coalescence of the mixing front. The effective model predicts accurately the total mass of $\mathrm{C}$. The developed methodology is applied to the pore-scale experiments reported by Jimenez-Martinez et al. (2015).

Procter and Gamble Student poster award:

References:

Acceptance of Terms and Conditions:

Click here to agree

\section{Network models for physical and biological processes in porous media}

Francisco Perez-Reche Perez-Reche ${ }^{\text {None }}$

Corresponding Author(s): fperez-reche@abdn.ac.uk

The structure of porous media can be remarkably complex and understanding how such complexity affects processes occurring in the porous space can be extremely challenging. In this talk, I will illustrate how modelling the pore space as a complex network can help understanding the effect of intricate structure on physical and biological processes in porous media. In particular, I will summarise our methods to investigate biological invasions in soil and capillary condensation in technologically important porous media. Or modelling of soil biological invasions is based on network representations of the pore space derived from 3D digital images of soil samples scanned with X-ray micro-tomography. Networks are then used as a substrate to describe the spread of generic microorganisms in terms of epidemic-like mathematical models which we studied through numerical simulations. We found that the remarkable structural heterogeneity of soil typically favours microbial invasions. The mechanisms behind this general observation are closely related to the topology of the pore space network. Our description of capillary condensation is also based on network models of porous media but condensation is described in terms of lattice gas models that can be mapped 
to spin models with heterogeneity. Such mapping allowed us to obtain exact solutions for relatively simple network topologies that are sufficient to explain important features of sorption-desorption curves at the macroscopic level.

Procter and Gamble Student poster award:

References:

Acceptance of Terms and Conditions:

\title{
Adaptive Stratified Sampling in Geomechanics and Fractured Porous Media
}

\author{
Author(s): Per Pettersson ${ }^{1}$ \\ Co-author(s): Eirik Keilegavlen ${ }^{2}$ \\ ${ }^{1}$ NORCE Norwegian Research Centre \\ ${ }^{2}$ University of Bergen
}

Corresponding Author(s): pepe@norceresearch.no, eirik.keilegavlen@uib.no

When large volumes of fluid are injected into subsurface reservoirs the enhanced fluid pressure can interact with existing mechanical stresses to cause shear movement of faults. This shear movement is associated both with seismic activity and enhanced permeability, thus leakage potential, of the fault, and the process is therefore a major safety concern for engineering operations such as carbon storage and wastewater injection. Numerical simulations of injection scenarios can be used to better understand, and ideally quantify, uncertainty related to the injection. However, the computational cost of the simulations is high even in the deterministic case. The problem has multiple classes of uncertain parameters, including the position and orientation of subseismic faults, the flow properties of the reservoir, existing stresses, and the frictional resistance to sliding of the faults. This calls for efficient approaches to uncertainty quantification in a high-dimensional parameter space.

Monte Carlo based sampling methods converge with rate $c N^{-1 / 2}$, where $c$ is typically well approximated by a constant, and $N$ is the number of samples. Increasing the number of samples with two orders of magnitude to get a single order of magnitude error reduction is often numerically intractable. Variance reduction methods aim at reducing the computational cost by decreasing the constant $c$, rather than increasing $N$. These methods include Multi-Level Monte Carlo methods that are suitable when a hierarchy of correlated models of different degree of fidelity can be established, e.g., multiple physical grids of different resolution. For problems in fractured porous media, it is cumbersome to introduce conforming grids of different degrees of refinement.

An alternative means to obtain variance reduction that does not rely on a hierarchy of different models (for instance when only a single unstructured grid is available) is offered by stratified sampling methods. The idea is straightforward: the stochastic domain is partitioned into disjoint subsets, socalled strata, and a suitable number of samples are drawn from each stratum. The number of samples can be chosen differently from standard Monte Carlo sampling to achieve an estimator with significantly reduced standard error. Thus, the number of times an expensive numerical simulator needs to be called, can be reduced with significant computational speedup as a result.

We introduce two novel methods for variance reduction via stratified sampling. The first method uses ideas from adaptive mesh refinement, applied to the stochastic instead of the physical model. The stochastic domain is adaptively stratified, and the samples are sequentially allocated to the strata for optimal distribution.

Theoretically optimal stratification is obtained by defining strata based on the level curves of the function to be approximated. However, this function is in general unknown since it is exactly what we want to estimate. The second method we present relies on this idea of adaptive stratification based on successively more accurate contour level functions. 
The proposed methodology is demonstrated on geomechanics problems of fractured reservoirs. Computational speedup with respect to brute force Monte Carlo sampling is obtained, and explained in terms of the underlying function we wish to compute.

Procter and Gamble Student poster award:

\title{
References:
}

Acceptance of Terms and Conditions:

Click here to agree

\section{In Situ Bioremediation of Selenium and Nitrate for Mine Waste Management in the Elk Valley, British Columbia}

\author{
Brent Peyton ${ }^{1}$; Lisa Kirk ${ }^{2}$; Seth D’Imperio ${ }^{2}$; Chiachi Hwang ${ }^{1}$ \\ ${ }^{1}$ Montana State University \\ ${ }^{2}$ Enviromin, Inc.
}

Corresponding Author(s): bpeyton@montana.edu

\begin{abstract}
Microbial reduction of selenate to less mobile forms (selenite and elemental selenium) can stabilize selenium (Se) in mined waste rock, which can also provide reactor substrate for in situ treatment of mine-affected water. These biogeochemical processes require careful balancing of oxidants (oxygen and nitrate, NO3) and reductants (carbon). Molecular biology and biological engineering methods have been used to characterize the microbial ecology and metabolic capacity of waste rock and mineaffected water for mining operations in the Elk Valley, located in southern British Columbia, Canada. Batch and column studies of native microbial capacity to reduce Se in saturated waste rock showed that oxygen and NO3 inhibition of Se reduction is overcome via carbon addition as methanol, glycerol or coal-derived native carbon in no-carbon controls. Biofilm grown on waste rock in saturated aerobic column tests was capable of 50 to $99 \%$ NO3 reduction followed by 40 to $95 \%$ Se removal; Se was sequestered in the biofilm as selenite. Microaerobic NO3 reduction and selenate removal increased to 95 and $75 \%$, respectively, with 25\% sulfate removal. Denitrification and Se reduction to elemental Se was most rapid and efficient under suboxic conditions, as high as $99 \%$. The extent of denitrification and Se reduction also varied in response to the form and concentration of carbon added to the column tests.

Microbial community structure in nitrate and Se-reducing biofilm from saturated waste rock backfill was described using 16S rRNA sequencing. Microaerophilic biofilm contained sequences belonging to NO3-reducing, iron-oxidizing Thiobacillus and iron-reducing Albidiferax, with most sequences similar to the hydrocarbon-degrading genus Polaromonas. Less abundant sulfate-reducing Desulfosporosinus were observed with the sulfur-oxidizing genera Sulfuritalea, especially in suboxic columns. Methylotenera were common in methanol-fed columns. Abundance of Se-reducing bacteria such as Dechloromonas, Anaeromyxobacter, and Acidovorax increased as oxygen decreased. The Se-reducing genera were less abundant than nitrogen, iron, and sulfur-cycling genera, which represent more of the energy and mass cycled in mined environments.

Applying the tools of biotechnology and principles of microbial ecology to the understanding of biomineralization is effective not only for management of NO3 and Se in mining, but also for management of sulfur, iron and other metals in a wide variety of industrial settings.
\end{abstract}

Procter and Gamble Student poster award:

I don't want to compete References:

Acceptance of Terms and Conditions:

Click here to agree 


\title{
Two-fluid model for supercritically adsorbed films of hydrogen and methane in nanoporous materials
}

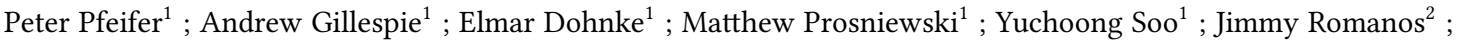
Lucyna Firlej ${ }^{3}$; Bogdan Kuchta ${ }^{4}$

${ }^{1}$ Dept. of Physics, University of Missouri

${ }^{2}$ Natural Sciences Dept., Lebanese American University

${ }^{3}$ Laboratoire Charles Coulomb, Université de Montpellier

${ }^{4}$ MADIREL, Aix-Marseille Université

Corresponding Author(s): pfeiferp@missouri.edu

\begin{abstract}
Sorption-based hydrogen storage for hydrogen fuel cell vehicles and methane storage for low-carbonfootprint, near-zero-emission internal combustion engines are at the forefront of energy storage for advanced, clean, sustainable transportation. Storage by adsorption is fully reversible, achieves fast fill/discharge by simple pressurization/depressurization, and operates at much lower pressure than compressed hydrogen or methane. We have investigated the density, thickness, and volume of adsorbed hydrogen films at $77 \mathrm{~K}$ and methane films at $298 \mathrm{~K}$ in a variety of porous carbons synthesized at the University of Missouri [1,2]. Samples include monoliths, powders, activated carbons, and pyrolytic carbons. The films, adsorbed at supercritical conditions, are monomolecular. The analysis decomposes stored hydrogen and methane into a high-density adsorbed film and low-density nonadsorbed gas (two-fluid model), coexisting in the pore space, and determines the fraction of pore volume occupied by the two phases. Saturated film densities, film thicknesses, and film volumes are determined from high-pressure excess adsorption isotherms.
\end{abstract}

Saturated hydrogen film densities at $77 \mathrm{~K}$ are $100-120 \mathrm{~g} / \mathrm{L}$ across all samples at pressures as low as 35-70 bar. This is 1.4-1.7 times the density of liquid hydrogen at its normal boiling point, $71 \mathrm{~g} / \mathrm{L}$ (20 $\mathrm{K})$. Experimental film thicknesses are $0.30-0.32 \mathrm{~nm}$, and fractions of total pore volume filled with high-density film are $0.25-0.53$. Thus high storage capacities, in excess of liquid hydrogen, can be achieved at $77 \mathrm{~K}$ in appropriately engineered nanoporous carbons.

Saturated methane film densities at $298 \mathrm{~K}$ are 390-420 g/L, comparable to that of liquid methane at its normal boiling point, $420 \mathrm{~g} / \mathrm{L}(112 \mathrm{~K})$. Experimental film thicknesses are $0.40-0.41 \mathrm{~nm}$. We paraphrase adsorbed methane films as "liquid methane at room temperature."

The dense films occur at a temperature $\mathrm{T} / \mathrm{Tc}=2.3$ and 1.6 , for hydrogen and methane, where $\mathrm{Tc}=33$ $\mathrm{K}$ and $190 \mathrm{~K}$ is the liquid-gas critical temperature, above which no bulk liquid exists at any pressure. The high-density films above Tc do not contradict the non-existence of bulk liquid: the film is not a bulk, 3D phase, but a monomolecular 2D phase. Monte Carlo simulations confirm the observed high density and small film thickness. The hydrogen film thickness, $\sim 0.30 \mathrm{~nm}$, equals the $\mathrm{H} 2-\mathrm{H} 2$ closest approach distance. The resulting picture is that the adsorption potential pins hydrogen molecules to the surface as densely packed layer short of lateral repulsion setting in.

Applications include:

- Parametrization of the Ono-Kondo isotherm for excess adsorption in terms of experimental film volume, saturated film density, and binding energy;

- Binding energy (heat of adsorption) from excess adsorption at a single temperature;

- Transition from "storage by high film density" to "storage by high gas density" in arbitrary pore geometries;

- Film volume divided by film thickness ("two-fluid-weighted" surface area) is smaller than the BET surface area because it counts area in narrow/wide pores with a large/small weight, while BET counts area in narrow and wide pores equally. For $\mathrm{H} 2$ at $77 \mathrm{~K}$, the difference is $\sim 20 \%$.

- When is high surface area per unit volume a reliable predictor of high volumetric storage capacity of an adsorbent?

Procter and Gamble Student poster award:

I don't want to compete References:

1 P. Pfeifer, A. Gillespie, D. Stalla, E. Dohnke, "Multiply Surface-Functionalized Nanoporous Carbon for Vehicular Hydrogen Storage-Final Technical Report.” U.S. Department of Energy, Contract DE-FG3608GO18142 (Washington, DC, 2017). https://www.osti.gov/scitech/biblio/1344383 
2 P. Pfeifer, R. Little, T. Rash, J. Romanos, and B. Maland, “Advanced Natural Gas Fuel Tank Project." California Energy Commission, Energy Research and Development Division, Publication No. CEC-5002016-038 (Sacramento, CA, 2016). http://www.energy.ca.gov/2016publications/CEC-500-2016-038/CEC500-2016-038.pdf Acceptance of Terms and Conditions:

Click here to agree

\title{
Numerical and experimental immiscible two-phase flow under non-isothermal conditions: application to thermally enhanced DNAPLs recovery
}

Hossein Davarzani $^{1}$; Malorie Dierick ${ }^{2}$; Manuel Marcoux ${ }^{3}$; Nicolas Philippe ${ }^{4}$; Pierre-Yves Klein ${ }^{2}$; Stéfan Colombano ${ }^{1}$

\author{
${ }^{1}$ BRGM \\ ${ }^{2}$ REMEA \\ ${ }^{3} I M F T$ \\ ${ }^{4}$ BRGM, REMEA
}

Corresponding Author(s): n.philippe@brgm.fr

In soil remediation applications, the cleaning of highly viscous DNAPLs is still a pending problem due to low recovery rates and high operational costs 1 . It is shown that decreasing the dynamic viscosity of DNAPLs (Dense Non-Aqueous Phase Liquids), using hot water flooding technique, increases its recovery rate 2 . The technique investigated in the current work consists in a moderate pre-heating up to $50{ }^{\circ} \mathrm{C}$ of the subsurface using electrical resistances followed by DNAPL pumping. The main objective of this work is to study the temperature effect on a viscous DNAPL recovery at both Darcy and field scales. The highly viscous DNAPL, sampled from a real polluted site, has a dynamic viscosity ranging from $0.01 \mathrm{~Pa} \cdot \mathrm{s}$ at $50^{\circ} \mathrm{C}$ and $0.1 \mathrm{~Pa} \cdot \mathrm{s}$ at $10{ }^{\circ} \mathrm{C}$.

A one-dimensional Tempe cell $(5.8 \mathrm{~cm}$ diameter, $5.6 \mathrm{~cm}$ height) has been used to measure capillary pressure versus saturation curves at $20{ }^{\circ} \mathrm{C}$ and $50{ }^{\circ} \mathrm{C}$. The experimental setup consists of a twodimensional transparent tank (50 cm length, $30 \mathrm{~cm}$ height, $7.8 \mathrm{~cm}$ width) filled with glass beads. The bottom $15 \mathrm{~cm}$ of the tank height is saturated with DNAPL while the top part is filled with water to model DNAPL pumping tests. An electrical resistance heats the tank from its left side until a steady state is achieved while the tank is kept at room temperature. Saturation fields are obtained with imaging technique, geophysical sensors, and frequency domain reflectometry methods and the temperature fields are measured with thermocouples.

Given that the DNAPL from the polluted site is a mixture of different chemical compounds where some of them are not identified, another couple of fluids, ethanol and canola oil, with similar thermophysical behavior have been chosen for preliminary experiments. These experiments are used to validate a numerical model developed via COMSOL Multiphysics ${ }^{\circledR}$. Muskat's extension of Darcy's law for two-phase flow has been used, coupled with transient heat transfer via convection and conduction. Thermal dispersion and local thermal non-equilibrium effects have been taken into account in this numerical model. Non-isothermal pumping experiments with DNAPL and water fluids have been performed.

The preliminary results show an asymmetric pumping cone with lower DNAPL saturation values in higher temperature zones (left side of the tank). In addition, it shows that heating the tank from 20 ${ }^{\circ} \mathrm{C}$ to $50^{\circ} \mathrm{C}$ increases the DNAPL recovery rate by about $20 \%$. However, the simulated temperature fields are not completely matching the experimental ones, in particular, near the water and DNAPL interface. This may be due to the use of the empirical interfacial heat transfer and dispersion coefficients. The simulated recovery rate is also underestimated compared to experimental recovery results.

Therefore, a one dimensional column experimental study is under investigation in order to measure the effective thermal properties of above-mentioned couple of liquids in porous media. Moreover 
field scale experiments on a $10 \mathrm{~m}$ per $10 \mathrm{~m}$ contaminated site are under study to quantify the effect of temperature on DNAPLs recovery rates in industrial conditions on a formerly contaminated soil.

Procter and Gamble Student poster award:

References:

1 Mackay, D. M., \& Cherry, J. A. (1989). Groundwater contamination: Pump-and-treat remediation. Environmental Science and Technology, 23(6), 630-636.

2 O'Carroll, D. M., \& Sleep, B. E. (2009). Role of NAPL thermal properties in the effectiveness of hot water flooding. Transport in porous media, 79(3), 393-405. Acceptance of Terms and Conditions:

Click here to agree

\title{
Uncertainty Quantification in Discrete Fracture Networks flow simulations
}

\author{
Stefano Berrone ${ }^{1}$; Sandra Pieraccini ${ }^{\text {None }}$; Stefano Scialò ${ }^{2}$ \\ ${ }^{1}$ Politecnico di Torino, Italy \\ ${ }^{2}$ Politecnico di Torino
}

Corresponding Author(s): sandra.pieraccini@polito.it

In the framework of underground flow in low permeability rocks, fractures act as preferential pathways for the flow. Within this framework, the discrete fracture network (DFN) models explicitly represent all the fractures in the network, endowing each fracture with its own geometrical features (position, size, orientation...) and hydro-geological properties (fracture trasmissivity). Geometrical and hydro-geological properties of the fractures are typically not (fully) known, and the fractures forming the network for underground flow simulations in DFNs are sampled from given probabilistic distributions. Due to the large amount of uncertainty affecting the description of the fractures, uncertainty quantification (UQ) is a crucial issue.

The complex geometry of the computational domain makes UQ a challenging issue on several respects: firstly, the high computational cost required by a single simulation can make the overall UQ analysis an unfeasible task, if a naive method is adopted; second, the stochastic generation of the fractures is likely to generate critical geometrical configurations which can result very challenging for the meshing process, which is known to be a major issue in flow simulations in DFNs; finally, since the network connectivity largely depends on geometrical parameters, a stochastic description of the geometry of the network can result in abrupt changes of the connectivity, yielding a nonsmooth behavior of the quantity of interest in the stochastic parameter space, which prevents the successful application of, e.g., stochastic collocation strategies.

We propose a viable approach for performing UQ analyses on the output of DFN models; the approach is based on Multi Level Monte Carlo (MLMC) method, applied in conjunction with a well assessed underlying solver for performing DFN flow simulations, which is extremely robust with respect to geometrical complexities in the network. The ability of the solver to handle complex geometries with no mesh constraints, also allowing the use of coarse meshes, makes its combination with MLMC a quite effective tool for UQ analyses in fracture networks.

Procter and Gamble Student poster award:

References:

Acceptance of Terms and Conditions:

Click here to agree 


\section{Phase Separation of Hydrogen}

André Pingel ${ }^{\text {None }} ;$ Michael E. Dreyer ${ }^{\text {None }}$

Corresponding Author(s): andre.pingel@zarm.uni-bremen.de

\section{Introduction}

The phase separation of hydrogen is a current research theme in the field of space technology which is of special interest concerning two different applications in future cryogenic propulsion systems: on one side for the gas- or vapor-free delivery of the liquid propellant to the combustion chamber and on the other side for the liquid-free venting to condition the propellant. Phase separation can be realized using the retention capability of a screen or double screen against liquid as shown by Behruzi et al. 1.

Both applications are conceivable with autogenic pressurization in a one species two-phase system consisting of liquid hydrogen and hydrogen vapor as well as pressurization with a non-condensable gas in a two species two-phase system consisting of liquid hydrogen and gaseous helium.

In this project a cryogenic test facility has been developed which allows to analyze the physical effects which are combined with the retention capability of a double screen against a sloshing of liquid hydrogen in hydrogen vapor environment during ground and microgravity tests.

\section{Numerical test predictions}

The development of the test facility required the provision of test predictions of the expected physical effects.

Using the commercial computational fluid dynamics program Flow-3D, a first two dimensional, numerical, model could be produced. The model depicts the physical effects radial wicking, capillary rise with overlaid pressurization, bubble point pressure and screen cross flow pressure loss in combination under isothermal, incompressible conditions.

\section{Cryogenic test facility}

With the aid of the test predictions, the development and building of the cryogenic test facility could be accomplished.

The double screen consists of two cylindrical dutch twilled weave metal screens with 200 warp and 1400 weft wires per square inch. The screens are fixed inside an inner glass tube, which is immersed into an amount of liquid hydrogen provided inside an outer glass cylinder.

The cryogenic thermal environment has been provided by a helium bath cryostat which contains the described test section and which can be housed inside a drop capsule.

\section{Experiments}

An experimental campaign consisting of 14 tests in earth gravity and three drop tests in microgravity using the drop tower at the University of Bremen has been conducted.

With the aid of an external pressurization system different differential pressures acting at the vapor phase of the outer glass cylinder have been applied to accelerate the liquid hydrogen towards the double screen.

During all tests wall and screens temperatures have been measured. In addition to that the differential pressure of the hydrogen vapor phases between the inner and outer glass cylinder has been recorded as well as the absolute pressure of the hydrogen vapor phase at the inner glass cylinder. Using a laser for illumination and an endoscope with connection to a CCD camera, videos could be recorded to track the liquid movement.

The experimental results confirm the predicted governing physical effects. In addition to that, influences due to evaporation and condensation have been observed. Both, numerical and experimental results will be presented. 


\title{
References
}

1 P. Behruzi, J. Klatte, G. Netter, Passive Phase Separation in Cryogenic Upper Stage Tanks, AIAA 2013-3905, (2013).

Procter and Gamble Student poster award:

I would like to compete in the Procter and Gamble Student award References:

Acceptance of Terms and Conditions:

Click here to agree

302

\section{Dynamic measurements of drainage capillary pressure curves in carbonate rocks}

\author{
Author(s): Sayed Alireza Hosseinzadeh Hejazi ${ }^{1}$ \\ Co-author(s): Saurabh Shah ${ }^{1}$; Ronny Pini ${ }^{1}$ \\ ${ }^{1}$ Imperial College London \\ Corresponding Author(s): r.pini@imperial.ac.uk
}

The heterogeneity of rocks represents a challenge for interpreting and using outcomes from multiphase flow experiments carried out on laboratory samples. While the capillary pressure-saturation function, $\mathrm{pc}(\mathrm{S})$, is known to vary spatially and cause local saturation development during immiscible displacements, its variation remains difficult to measure. This is particularly challenging for rocks with complex fabrics, such as carbonates. In this study, we present a workflow for the dynamic measurement of core- and subcore-scale drainage $\mathrm{pc}(\mathrm{S})$ curves in heterogeneous porous media. Multi-rate, two-phase core-flooding tests are conducted on three carbonate rocks with direct observations of local saturation data using X-ray computed tomography. The interpretation of the experiments is done by fitting the parameters of the $\mathrm{pc}(\mathrm{S})$ curve, while describing both steady-state saturation and pressure profiles with a detailed one-dimensional model that accounts for the variation of subcore-scale properties in the direction of displacement. Workflow validation is achieved by means of synthetic data, thereby demonstrating the uniqueness of the solution of the resulting multi-objective optimisation problem. The model accurately reproduces experimental data on the three rocks and enables computing the effective core-scale $\mathrm{pc}(\mathrm{S})$ curve in the limit of zero velocity, as it would be expected during a porous plate experiment. The output of the proposed technique is however much richer and includes the relative $\mathrm{pc}(\mathrm{S})$ curve that is universal and independent of the specific pattern of heterogeneity, in addition to a set of scaling factors. The latter describe the distribution of the $\mathrm{pc}(\mathrm{S})$ curves at the subcore-scale due to heterogeneity and form the statistical basis needed for upscaling studies.

Procter and Gamble Student poster award:

References:

Acceptance of Terms and Conditions:

Click here to agree 
Agnese Piovesan ${ }^{1}$; Tim Van De Looverbosch ${ }^{2}$; Pieter Verboven ${ }^{3}$; Clement Achille $^{4}$; Elodie Boller ${ }^{5}$; Yin Cheng ${ }^{5}$; Rob Ameloot ${ }^{4}$; Bart Nicolai ${ }^{2}$

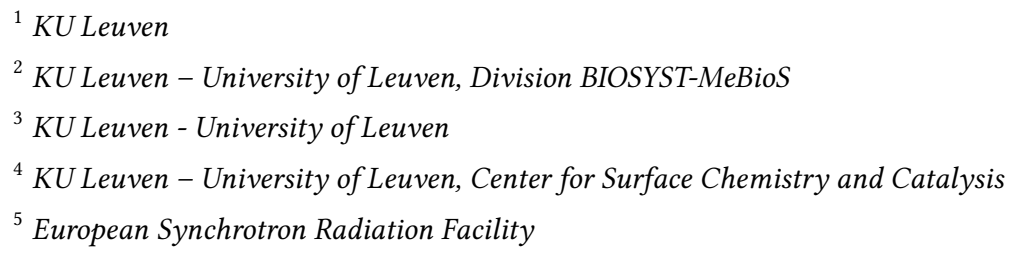

Corresponding Author(s): agnese.piovesan@kuleuven.be

Capillarity driven lab-on-chips (LOC) are small cheap devices in which fluids autonomously flow in functionalized porous channels. LOC performances are strongly dependent on the microscale fluid dynamics, which is in turn influenced by factors such as pore geometry and surface chemistry. Deep understanding and characterization of pore scale fluid flow are therefore important to optimize capillarity driven LOCs. In this framework an experimental study was carried out at the European Synchrotron Radiation Facility (ESRF) to visualize the dynamics of water imbibition in the LOC porous channels through fast X-Ray micro computed tomography $(\mu \mathrm{CT})$. A total of 10 poly-methyl methacrylate (PMMA) samples with different hydrophilic properties (i.e. different contact angels) were continuously imaged during water imbibition at the beamline ID19 of the ESRF, with space resolution of $1 \mu \mathrm{m}$ and maximum time resolution of $0.5 \mathrm{~s}$ for a total of $12 \mathrm{~s}$. To ensure sufficient contrast, a solution of water and potassium iodide was used. The reconstructed images at different time frames were imported in Avizo 9.5 (Visual Sciences Group, www.vsg3d.com) where phases (air, water and solid phase) could be segmented. In the first place, this allowed us to visualize in situ and with sufficient time resolution the flow of water in the porous matrix. From this the presence of a preferential wicking directions in the LOC, probably due to the fabrication technique, was observed. Secondly, it was possible to investigate the pore filling mechanisms and to study the evolution of water saturation both of the pore space and of single pores which were identified through a watershed algorithm. These results, moreover, provided a powerful tool to validate multiphase pore-network (PN) models. PN models are relatively cheap computational model useful to study fluid transport properties at the pore scale but direct validation of multiphase PN is quite difficult. A PN was extracted from the imaged samples and used to develop a multiphase dynamic imbibition model, whose results were compared to those obtained from dynamic $\mu \mathrm{CT}$ images.

Procter and Gamble Student poster award:

References:

Acceptance of Terms and Conditions:

Click here to agree

344

\title{
Experimental modelling of the oscillating multiphase flow in porous medium with chemically active skeleton
}

\author{
Ivan Zavialov ${ }^{1}$; Plyashkov Plavnik ${ }^{1}$; Rinat Plavnik ${ }^{1}$ \\ ${ }^{1}$ Moscow Institute of Physics and Technology \\ Corresponding Author(s): egor.plyashkov@mail.ru, plavnik.ra@phystech.edu
}

Chemical methods to increase permeability of a skeleton are used to intensify hydrocarbons extraction. One of these technologies is in-situ combustion via the injection of an oxidizer, capable to dissolve a part of the skeleton and make high-permeability channels. However, the process of multiphase flow in porous medium with the formation of a gaseous phase is related with the development of different unstable phenomena. The influence of these phenomena on parameters of the flow determines a high interest for studying such processes both experimentally and theoretically [1-5]. During the laboratory experiment a self-oscillating mode of reaction was observed when the rate of the flow in the porous medium and the pore pressure began to fluctuate quasi-periodically. The 
forming of gas phase occurs in waves as called secondary oxidation waves [6-8].

The purpose of the work was to find a correlation between amplitude, period of these waves and the pressure gradient experimentally. Also, the ratio of concentrations of acid and oxidizer at which a certain flow regime occurred was experimentally studied.

The experiment was carried out in a flat cell formed by two transparent polycarbonate plates. The external size is $350 \times 200 \times 45 \mathrm{~mm}$, the gap size between two plates $-350 \times 160 \times 15 \mathrm{~mm}$. There are two outlets for tubes at the top of the cell. One of the tubes is used for the mineral oil and oxidant solution injection and another one - for keeping pressure constant by removing excess oxidant solution and oil. Injection is performed with a peristaltic pump LOIPLS-301 with consumption $70 \mathrm{ml} / \mathrm{min}$ The bottom of the cell is covered with a grid. There are 15 pressure sensors Honeywell 24PC15SMT in the center of the one of the plates with the interval $20 \mathrm{~mm}$.

As a model of chemically active skeleton technical glass beads (diameter - 300-400 $\mu \mathrm{m}$ ) uniformly mixed with sodium bicarbonate are used. Mineral oil models a highly viscous fracture. For chemical reaction with the gas phase discharge a citric acid solution is used.

First, a mixture of glass beads and sodium bicarbonate is poured into the cell. Then mineral oil is pumped in the cell through the inlet. After the whole mixture gets saturated with oil, pumping oil stops and the acid solution is pumped. As a result of the flow of the oxidizer secondary oxidation waves arising with a constant period were observed.

\section{Indico rendering error}

Could not include image: Cannot read image data. Maybe not an image file?

shows the readings from pressure sensors that confirm the presence of a periodic process. As a result of experimental work, it was found that the period of pressure fluctuation in the secondary oxidation waves weekly depends on the pressure gradient and the amplitude of these waves increases with decreasing pressure gradient in the cell

Indico rendering error

Could not include image: Cannot read image data. Maybe not an image file?

Indico rendering error

Could not include image: Cannot read image data. Maybe not an image file?

The curve separating the two flow regimes: with pressure oscillations and the regime of stable flow, was found experimentally

Indico rendering error

Could not include image: Cannot read image data. Maybe not an image file?

. For the existence of secondary waves some critical concentrations of oxidizer and reducing agent are necessary.

\section{Procter and Gamble Student poster award:}

I would like to compete in the Procter and Gamble Student award References:

1Akhatov I.S., Kovaleva L.A., Valiullin R.A. [et al.].\} Experimental and Mathematical Workflow in Modeling In-situ Combustion Processes for Unconventional Resources Recovery // IOR 2013-17th European Symposium on Improved Oil Recovery. - 2013. 
2Chen B., Castanier L.M., Kovscek A.R.\} Consistency measures for isoconversional interpretation of insitu combustion reaction kinetics // Energy $\backslash \&$ Fuels. - 2014. - T. 28, №. 2. - C. 868-876.

3Ijioma E.R., Muntean A., Ogawa T.\} Effect of material anisotropy on the fingering instability in reverse smoldering combustion //International Journal of Heat and Mass Transfer. - 2015. - T. 81. - C. 924-938. 4 Kovscek A., Castanier L.M., Gerritsen M.\} Improved predictability of in-situ-combustion enhanced oil recovery // SPE Reservoir Evaluation \\& Engineering. - 2013. - T. 16, № 02. - C. 172-182.

5Quah F.W., Qi Y., Nagatsu Y.\} Experimental study on effects of effective interfacial tension on miscible viscous fingering // APS Meeting Abstracts. - 2014.

[6]Haudin F., Riolfo L.A., Knaepen B., Homsy G.M., De Wit A.\} Experimental study of a buoyancy-driven instability of a miscible horizontal displacement in a Hele-Shaw cell // Physics of fluids. - 2014. - T. 26, №. 4. - C. 044102.

[7]Konyukhov A.V., Zavialov I.N. Numerical investigation of oscillatory multiphase flow in porous medium with chemically active skeleton // Journal of Physics: Conference Series. - IOP Publishing, 2016. - T. 774, №. 1. - C. 012059.

[8]Zavialov I. et al. LABORATORY MODELING OF FLOW WITH GAS-PRODUCING REACTION IN POROUS MEDIA //Journal of Porous Media. - 2018. - T. 21. - №. 10. Acceptance of Terms and Conditions:

Click here to agree

\title{
Magnetic resonance imaging to assess changes in transport prop- erties of porous media due to dissolution and precipitation pro- cesses
}

\author{
Jenna Poonoosamy ${ }^{1}$; Andreas Pohlmeier ${ }^{2}$; Sabine Haber Pohlmeier ${ }^{2}$; Martina Klinkenberg ${ }^{3}$; Dirk Bosbach ${ }^{1}$; \\ Guido Deissmann ${ }^{1}$ \\ ${ }^{1}$ Institute of Energy and Climate Research (IEK-6): Nuclear Waste Management and Reactor Safety, Forschungszen- \\ trum fülich $\mathrm{GmbH}$ \\ ${ }^{2}$ Institute of Bio- and Geosciences (IBG-3): Agrosphere, Forschungszentrum fülich GmbH \\ ${ }^{3}$ Institute of Energy and Climate Research (IEK-6): Nuclear Waste Management and Reactor Safety
}

Corresponding Author(s): j.poonoosamy@fz-juelich.de

The understanding of the porosity evolution in porous media due to mineral reactions and its impact on the transport of fluids and solutes is important, since this is a key factor, for example, regarding the long-term behaviour of engineered systems in the underground. The implementation of such coupled processes into numerical codes requires a mechanistic understanding of the relevant precipitation/dissolution processes in porous media and model validation with quantitative experiments. In this context, we conducted two sets of flow through column experiments - following the experimental concept of Poonoosamy et al. (2015) and Singurindy \& Berkowitz (2003) - to investigate the effect of barite and gypsum precipitation following dissolution of celestine and calcite, respectively, and consequential permeability changes. These experiments were modelled using the reactive transport code OpenGeosys-GEM. Although the Kozeny-Carman equation used in this context is widely applied in numerical models to describe porosity and permeability changes due to mineral dissolution/precipitation, it distinctively underestimates the permeability changes observed in our experiments. Instead, a porosity-permeability relationship involving a critical porosity at which the porous medium becomes impermeable had to be considered in the model to reproduce the experimental findings. Currently, we are developing experimental approaches combining in-situ imaging methods such as magnetic resonance imaging (MRI) to gain a mechanistic understanding of the physical meaning of the so called critical porosity. For this purpose, the experiments were repeated in a Bruker super wide bore MRI scanner with a field strength of 4.7T. By conducting multi-slice multi echo measurements (Pohlmeier et al. 2010) for the determination of translational relaxation time maps we monitored the evolution of the pore size distribution during the precipitation progress. In addition, the influence on the flow patterns was monitored using deuterium as tracer. This approach will enable to investigate process specific porosity/permeability relations, in this case due to mineral precipitation, which can be introduced into reactive transport simulations. 


\section{References:}

Poonoosamy J., Kosakowski G., Van Loon L. R. and Mäder U. (2015) Dissolution-precipitation processes in tank experiments for testing numerical models for reactive transport calculations: Experiments and modelling. J. Contam. Hydrol. 177-178, 1-17.

Pohlmeier, A., F. Vergeldt, E. Gerkema, H. Van As, D. Van Dusschoten and H. Vereecken (2010). MRI in Soils: Determination of Water Content Changes Due to Root Water Uptake by Means of a Multi-SliceMulti-Echo Sequence (MSME). The Open Magn. Res. J. 3, 96-74.

Singurindy O. and Berkowitz B. (2003) Evolution of hydraulic conductivity by precipitation and dissolution in carbonate rocks. Water Resour. Res. 39, WE001055. Acceptance of Terms and Conditions:

Click here to agree

981

\section{Ecohydrology: interactions of life and water around a porous medium}

Amilcare Porporato ${ }^{1}$

${ }^{1}$ Princeton University, USA

Corresponding Author(s): aporpora@princeton.edu

As a quintessential example of porous media, soils act as complex filters of hydroclimatic forcing, giving rise to moisture variability at different spatial and temporal scales. These dynamics exert strong controls on vegetation and soil microbial life and in turn feed back on meteorological and hydroclimatic conditions, thus propagating to larger scales with profound impacts on ecosystems and society.

We discuss upscaling of such processes in a way that preserves the essential nonlinearities, originating from pore-scale processes in the soil and plant tissues, while surrogating high-dimensional hydroclimatic variability by means of probabilistic components. The resulting description offers useful simplifications for ecohydrological, biogeochemical, geomorphological and climatic analysis and allows us to integrate from daily to decadal timescales and beyond to address problems related to land-use and water resources planning. We discuss examples related to plant water stress and plant productivity as well as soil carbon and nutrient dynamics. The challenges for rigorous upscaling and coupling of ecohydrological processes in soils offer a unique opportunity for the scientific community to contribute to the pressing need of using soil and water resources in sustainable way.

Procter and Gamble Student poster award:

References:

Acceptance of Terms and Conditions:

Click here to agree

5

\section{Impact of wind action and medium physical properties on hori- zontal pore gas flow in near-surface porous media.}

Tjalfe Poulsen ${ }^{1}$

${ }^{1}$ Guangdong Technion Israel Institute of Technology

Corresponding Author(s): tjalfe.poulsen@gtiit.edu.cn 
Wind action at the soil surface potentially controls the movement of gases (including greenhouse gases and water vapor) in the near-surface soil as well as exchange of these gases with the atmosphere. Part of the mechanism behind this gas movement has recently been shown to be windinduced horizontal pore gas movement within the near-surface soil.

The objective of this study was to investigate how these wind-induced horizontal pore gas velocities depend on near-surface wind speed, wind gustiness, soil gas permeability, and distance to the soil surface.

Velocity profiles for wind-induced, horizontal pore gas flow as a function of soil depth in the top $15 \mathrm{~cm}$ of the soil, were determined using a recently presented tracer tracking method for measuring wind-induced pore gas velocity profiles in porous media. Measurements were carried out using two dry, granular porous media for different combinations of wind speed, wind gust frequency and medium particle size, in a wind tunnel to assure controlled conditions. N2 was used as tracer gas. Media particle sizes were $1.18-2.36 \mathrm{~mm}$ and $4.75-10 \mathrm{~mm}$, exposed to wind speeds of up to $6 \mathrm{~ms}-1$ and gust frequencies up to $1 \mathrm{~Hz}$. Experiments were based on the $2 \mathrm{k}$ factorial design.

Results indicate, that average wind speed, wind gustiness and soil medium physical properties, all have a significant influence on both absolute horizontal pore gas velocities, as well as on the overall shape of the pore gas velocity profiles as a function of soil depth.

Procter and Gamble Student poster award:

References:

Acceptance of Terms and Conditions:

Click here to agree

\section{Swelling behavior of shale/clay: Discrete element modeling, based on Monte-Carlo technique}

Srutarshi Pradhan ${ }^{1}$

${ }^{1}$ Researcher, PoreLab, NTNU

Corresponding Author(s): srutarshi.pradhan@ntnu.no

Swelling of Shale-rocks create several problems 1 during underground drilling operations, such as stuck-pipe/drill-bit. However, swelling of shale-rocks can close the gaps between rock (wellbore) and casing -therefore no cementing is needed - which can save a lot of time and money and such a "natural" closing ensures "no-leakage" during further drilling and production phases (i.e., gas producing wells). The field experience reveals that some shale-rocks are good candidate for swelling and some are not. Understanding clay swelling and clay consolidation are important for clay deposit management works -especially in Port areas. There are several parameters influence the swelling and consolidation of clay/shale, such as- porosity, clay-quartz contents, stress difference between field and drilling zone etc. Therefore, to plan a safe and efficient drilling operation through shalerocks and to manage clay deposits, we should understand the swelling mechanism of shale and the consolidation mechanism of clay. In this work, we have introduced a discrete element model, based on Monte-Carlo technique. We define a probability of swelling for all the clay grains in the clay/shale sample that includes the effect of stress-difference, porosity, temperature etc. The time evolution of grain swelling results in bulk swelling behavior of the sample and the simulation result qualitatively matches with the observations of shale/clay swelling experiments [2,3].

\section{Procter and Gamble Student poster award:}

\section{References:}

1. E. Fjær, R. M. Holt, P. Horsrud, A. M. Raaen and R. Risnes, Petrolum Related Rock Mechanics (Elsevier, 2008).

2. M. Deriszadeh and R.C.K. Wong, Transp Porous Med (2014) 101:35-52 DOI 10.1007/s11242-0130229-8. 
3. E. Rybacki, J. Herrmann, R. Wirth and G. Dresen, Rock Mech Rock Eng (2017) 50:3121-3140.

Acceptance of Terms and Conditions:

Click here to agree

207

\section{Dissolution - precipitation driven migration of a salt crust}

Marc Prat ${ }^{\text {None }}$; Glad Licsandru ${ }^{\text {None }}$

Corresponding Author(s): mprat@imft.fr

Evaporation of saline solutions in porous media leads very often to the formation of typically thin and porous salt crusts at the surface 1 . The crust formation has a great impact on the evaporation rate, which can be reduced by more than one order of magnitude in comparison to the evaporation period preceding the crust development 2, 3. The mechanisms leading to this strong reduction are not yet fully understood. One particular point of interest is the formation of an air layer below the crust. This layer offers a much greater resistance to water transport which has to occur in vapour phase, consistent with the observed very strong decrease in the evaporation rate. To elucidate the mechanisms leading to the air layer formation, we present a new specific experiment where a suspended crust is first generated in a Hele-Shaw cell. After which the suspended crust is exposed to humid air on its bottom side and to dry air on its top side. This configuration leads to a dissolutionprecipitation process characterized by dissolution (deliquescence) at the crust bottom as well as precipitation on top due to ion transport through the crust and surface evaporation.

The experiment shows that the crust must be considered as a two layered thin porous medium with possible in-between separation as a result of upward migration of its top layer. This dissolution precipitation driven migration of the top layer leads to the formation of an air gap between the two layers of the crust. Thus, the experiment very clearly shows how an air barrier can form below the crust.

The crust dynamics resulting from the dissolution - precipitation process will be discussed in detail with the aid of optical image processing techniques, SEM images of the bottom and top layers and a model helping delineate the crust dynamics various regimes depending on the competition between the dissolution and precipitation processes.

A major conclusion is that the mechanical effects are of second importance in the dynamics of salt crusts, which essentially are driven by solute transport within the crust and dissolution-precipitation phenomena. This study of the salt crust opens up the route towards a much better understanding of evaporation from a porous medium with salt crust formation as well as a variety of particular crust patterns, such as the polygonal patterns at the surface of salars 4 .

Procter and Gamble Student poster award:

\section{References:}

1 U.Nachshon, N. Weisbrod, Roee Katzir, A.Nasser, $\mathrm{NaCl}$ crust architecture and its impact on evaporation - three dimensional insights, Geophysical Research letters, 45, 6100-6108, (2018).

2 H. Eloukabi, N.Sghaier, S. Ben Nasrallah, M. Prat, Experimental study of the effect of sodium chloride on drying of porous media: the crusty-patchy efflorescence transition, Int. J. of Heat and Mass Tr., 56, 80-93, (2013)

3 S.Veran-Tissoires and M. Prat, Evaporation of a sodium chloride solution from a saturated porous medium with efflorescence formation, J. of Fluid Mechanics, 749, 701-749, (2014).

4 J.B.Svensen, Parabolic halite dunes on the Salar de Uyuni, Bolivia, Sedimentary Geology, 155, 147-156, (2003) Acceptance of Terms and Conditions:

Click here to agree

\section{Modelling cell migration in confined environemnts}


${ }^{1}$ Politecnico di $>$ Torino

Corresponding Author(s): luigi.preziosi@polito.it

Cell-extracellular matrix interaction and the mechanical properties of cell nucleus have been demonstrated to play a fundamental role in cell movement across fibre networks and micro-channels. From the point of view of application understanding this process is important to describe on one hand the spread of cancer metastases and on the other hand to optimize medical scaffold that can be use to cure chronic wounds. From the point of view of mathematics, the problem need be addressed using different methods, starting from modelling cell adhesion mechanics to the inclusion of influence of nucleus stiffness in the motion of cells.

Procter and Gamble Student poster award:

I don't want to compete References:

Acceptance of Terms and Conditions:

Click here to agree

\title{
On the Upscaling of Reactive Flow in Plug-Flow Experiments in the Project ResKin
}

\author{
Torben Prill $^{1}$; Oleg Iliev ${ }^{\text {None }}$; Pavel Gavrilenko ${ }^{1}$ \\ ${ }^{1}$ Fraunhofer ITWM \\ Corresponding Author(s): prill@itwm.fraunhofer.de
}

In this talk, we will discuss the upscaling of reactive transport in evolving pore-spaces within the project ResKin. The overall goal of the project is, to predict the change of permeability and porosity due to calcite dissolution in a Plug-Flow experiment, using numerical upscaling on $\mu C T$-Image Data. We will give an overview over the project and discuss the newly developed methods used in the project and show early results.

\section{Pore-Scale Simulation}

As the upscaling process relies on pore-scale information, direct numerical simulations of the dissolution process are needed. All pore-scale simulations needed for the project are done with PoreChem 1, a software package developed at Fraunhofer ITWM, dedicated to the simulation of reactive flow on the pore-scale. The software can compute the flow of a fluid in the pore-space, as well as the diffusive and advective transport of solute species. PoreChem solves the Navier-Stokes-Brinkman equations in the pore-space, then transport and reactions are simulated by solving the reactiondiffusion-advection equation with the resulting velocity solution. All equations are discretized with the finite volume method on a regular grid, where a fast voxel based solver enables calculations directly on $\mu$ CT-Images. For the project, the software has been amended to compute reactive transport by coupling it to the geochemistry solver Reaktoro. Also, a numerical scheme has been implemented to simulate the evolution of the pore-space due to the dissolution of the calcite. In the first part of the talk, we will introduce the numerical schemes used for the pore scale simulation and present results on the direct numerical simulation of the dissolution process.

\section{Numerical Upscaling of Reactive Transport}

The second part of the talk concerns the upscaling of the reactive transport. The problem of upscaling of reactive transport has been studied for a long time and is a well-established field. In the talk, we will shortly review the application of the homogenization method to transport problems, and show how different scaling regimes lead to different cell-problems, which have to be solved to compute effective diffusion and dispersion tensors 2. Then we show how, using the software PoreChem, the relevant cell problems can be solved numerically on the pore scale for large 3D data sets and macroscale diffusion and dispersion tensors can be computed. 


\section{Upscaling Reactive Transport With Evolving Geometries}

In the final part of the talk, he will give an outlook on the extension of the numerical upscaling to evolving pore-spaces resulting from the dissolution of the calcite phase, as this is a major objective of the project. To this end, we plan to implement a coupled micro-macro simulation algorithm 3 , where the effective transport parameters are recomputed after each time step and in each grid cell. However, as solving the full model at every instance is computationally infeasible, we are planning to implement reduced order modeling methods to solve the required cell problems. In this talk we will discuss the numerical methods and the challenges presented by this approach, such as extending existing methods to problems with evolving computational domains.

\section{Procter and Gamble Student poster award:}

I don't want to compete References:

1 Iliev, O., Lakdawala, Z., Neßler, K. H., Prill, T., Vutov, Y., Yang, Y., \& Yao, J. (2017). On the PoreScale Modeling and Simulation of Reactive Transport in 3D Geometries. Mathematical Modelling and Analysis, 22(5), 671-694

2 Homogenization Approach to the Dispersion Theory for Reactive Transport through Porous Media Grégoire Allaire, Andro Mikelić, and Andrey Piatnitski

SIAM Journal on Mathematical Analysis 2010 42:1, 125-144

3 On a multiscale approach for filter efficiency simulations

O Iliev, Z Lakdawala, G Printsypar - Computers \& Mathematics with Applications, 2014 Acceptance of Terms and Conditions:

Click here to agree

\section{Experimental Study of Gas Mass Transfer between Gas Bubbles and Water in Otherwise Saturated Sand}

Tomas Princ $^{1}$; Michal Snehota ${ }^{1}$

${ }^{1}$ Czech Technical University in Prague, Civil Engineering

Corresponding Author(s): tomas.princ@fsv.cvut.cz

Aim of this study is obtaining time development of the mass transfer coefficient and relationship between the actual hydraulic conductivity and the saturation by the entrapped gas bubbles during the course of entrapped air dissolution in otherwise water-saturated sand. The changes in gas saturation occur only because of the gas mass transfer between bubbles and partly degassed water flowing through the sand column. In the experimental set-up water driven by a peristaltic pump first passes through vacuum membrane degasser into total dissolved gas probe. Then, it enters vessel with submerged sand column. Given the water level in the vessel is kept at a constant level, mass of water and air in the sample can be determined gravimetrically. The samples were prepared from the coarse sand. Initial full saturation of the samples was achieved by packing the samples under degassed deionized water. Samples were subsequently drained at suction pressure $5 \mathrm{kPa}$ and then submerged in air-saturated water for re-saturation that induced bubbles trapping. Then series of inflow outflow experiments was conducted. Firstly, the ponded infiltration experiment with water in equilibrium with atmosphere removed the mobile bubbles from the porous space. Then the degasser was turned on and air bubbles dissolution phase of the experiment started. Total dissolved gas probes recorded the concentration of dissolved air at the inflow and at outflow. The hydraulic conductivity was determined using a Darcy law from known hydraulic gradient and measured water flux. The experiment was maintained until the sample mass (and therefore the water and gas saturation) remained constant. The outcome of the experiment is a replicated record of time development of the mass transfer between air bubbles and flowing water in a coarse sand. Additionally, the relationship between hydraulic conductivity and gas saturation was developed during the same experiment.

Procter and Gamble Student poster award:

References: 


\title{
Pathways of heat and mass transfer in adsorption of methane in nanoporous monolithic carbon
}

\author{
Matthew Prosniewski ${ }^{1}$; Jimmy Romanos ${ }^{2}$; Peter Pfeifer $^{1}$ \\ ${ }^{1}$ Dept. of Physics, University of Missouri \\ ${ }^{2}$ Dept. of Natural Sciences, Lebanese American University
}

Corresponding Author(s): mjpbtd@mail.missouri.edu

The search for clean and sustainable automotive fuels has driven the study of carbon-based adsorbed natural gas (ANG) tanks, for next-generation clean vehicles [1-8]. Storage of methane in such a tank is fully reversible under the standard fill/discharge protocol: pressurization to $35 \mathrm{bar}$ at ambient temperature to fill the tank (adsorption); depressurization to 2 bar at ambient temperature to empty the tank (desorption) 5. The low pressure of 35 bar, compared to storage as compressed natural gas $(\mathrm{CNG})$ at 250 bar, allows for much thinner tank walls, which reduces tank weight, volume, and compression costs and enables a conformable tank geometry. An Al-based ANG tank filled with high-performance carbon monoliths [5-7] weighs 50\% less and occupies a volume $20 \%$ less than an Al-based CNG tank with the same fuel capacity 5.

The filling process of the tank is accompanied by a large thermal response (heat release) from loading and compression of gas into the pore space (pump $4.2 \mathrm{~kg} \mathrm{CH} 4$ into 40-liter vessel holding $21 \mathrm{~kg}$ of carbon monoliths) and exothermic adsorption of gas from the pore space onto the carbon (adsorb 3.9 $\mathrm{kg} \mathrm{CH} 4$ onto $47 \mathrm{~km} 2$ of surface area). During a fast fill ( $80 \%$ full in $2 \mathrm{~min}$ ), the temperature rises from $23^{\circ} \mathrm{C}$ to over $90^{\circ} \mathrm{C}$ in $2 \mathrm{~min}$ [7], and one seeks to reduce this high temperature by fast heat transport from the inside of the tank to the outside. In this paper we analyze data for the temperature vs. pressure evolution during the first $2 \mathrm{~min}$, which shows a quasi-linear increase $\mathrm{T}(\mathrm{p})$ up to $\mathrm{p}=30 \mathrm{bar}$ $(t=0.5 \mathrm{~min})$ and then a sharp rise in $\mathrm{T}$, in terms of three different models of mass and heat transfer for $\mathrm{t}=0-0.5$ min: (I) Adiabatic compression of gas in the pore space without heat sink (fast gas transport into pores, slow heat transfer in carbon matrix); (II) Adiabatic compression of gas with carbon as heat sink (fast gas transport into pores, fast heat transfer in carbon matrix); (III) Adiabatic compression of gas with carbon as heat sink, but compression only in volume outside of the monoliths (slow gas transport into pores, fast heat transfer in carbon matrix). We find that experimental $\mathrm{T}(\mathrm{p})$ data follows Model II with high precision (also for filling with natural gas instead of pure methane) and that Models I, III give vastly different T(p) profiles. Heat release due to adsorption sets in only at $\mathrm{t}>$ $0.5 \mathrm{~min}$. The overall conclusion for $\mathrm{t}=0-0.5 \mathrm{~min}$ is: gas transport into pores is fast; heat transport in carbon matrix is fast; adsorption of gas on pore walls is slow.

\section{Procter and Gamble Student poster award:}

I don't want to compete References:

1 P. Pfeifer et al., "High-surface-area biocarbons for reversible on-board storage of natural gas and hydrogen.” Mater. Res. Soc. Symp. Proc. 1041, 1041-R02-02 (2008).

2 J. Romanos, S. Sweany, T. Rash, L. Firlej, B. Kuchta, J.C. Idrobo, P. Pfeifer, "Engineered porous carbon for high volumetric methane storage.” Adsorpt. Sci. \& Technol. 32, 981-991 (2014).

[3a] P. Pfeifer, G.J. Suppes, P. Shah, J.W. Burress, "High surface area carbon and process for its production.” U.S. Patent No. 8,691,177 (2014) and No. 8,926,932 (2015).

[3b] J. Romanos, J. Burress, P. Pfeifer, T. Rash, P. Shah, G. Suppes, "High surface area carbon and process for its production.” U.S. Patent No. 9,517,445 (2016).

4 J.A. Mason, M. Veenstra, J.R. Long, "Evaluating metal-organic frameworks for natural gas storage." Chem. Sci. 5, 32-51(2014).

5 P. Pfeifer, R. Little, T. Rash, J. Romanos, B. Maland, “Advanced Natural Gas Fuel Tank Project.” California Energy Commission, Energy Research and Development Division, Publication No. CEC-500-2016038 (Sacramento, CA, 2016).

http://www.energy.ca.gov/publications/displayOneReport.php?pubNum=CEC-500-2016-038 
[6] T.A. Rash, A. Gillespie, B.P. Holbrook, L.H. Hiltzik, J. Romanos, Y.C. Soo, S. Sweany, and P. Pfeifer, "Microporous carbon monolith synthesis and production for methane storage." Fuel 200, 371-379 (2017). [7] M.J. Prosniewski, T.A. Rash, E.W. Knight, A.K. Gillespie, D. Stalla, C.J. Schulz, and P. Pfeifer, "Controlled charge and discharge of a 40-L monolithic adsorbed natural gas tank." Adsorption 24, 541-550 (2018).

[8] J. Romanos, T. Rash, S. Abou Dargham, M. Prosniewski, F. Barakat, and P. Pfeifer, "Cycling and regeneration of adsorbed natural gas in microporous materials.” Energy Fuels 31, 14332-14337 (2017). Acceptance of Terms and Conditions:

Click here to agree

\section{Experimental determination of fluid and solute transport param- eters in textiles}

Sabine Przybilla ${ }^{1}$; Tobias Wybranietz ${ }^{1}$; Joachim Villwock ${ }^{1}$

${ }^{1}$ Beuth University of Applied Sciences Berlin

Corresponding Author(s): sabine.przybilla@beuth-hochschule.de

To model and simulate transport processes in porous media the flow characteristics such as the permeability and the capillary pressure as well as their hysteresis effects need to be quantified. Also, the solute transport is restrained by the tortuosity of the pore structure. All those physical parameters and effects must be considered saturation dependent. The characterisation of the transport parameters for textiles is very challenging. Especially natural fibres like cotton are complex, due to their natural growth and varying pore scales. For many materials the hydrophily and hygroscopy need to be considered. Another difficulty is the characterization of the planar textile structures. The aim of this contribution is to show experimental methods to characterize porous transport parameters for textiles using the example of cotton.

To characterize the saturation dependent permeability and capillary pressure for a textile stripe the experimental setup of 1 is applied. It consists of an upward, a horizontal and a downward section which use different physical phenomena to characterize the fabric. The horizontal and the downward section show a characteristic mass flux, which is recorded and utilized to determine the capillary pressure and permeability. A high humidity is adjusted to reduce evaporation effects. Consequential, the hygroscopy of the cotton and the initial saturation need to be considered.

Additionally, the hysteresis effect of the capillary pressure is investigated in capillary height experiments, which are elucidated in 2. The saturation is determined for dry and pre-wetted textiles for different height segments. The results for imbibition are compared to the capillary pressure saturation relation of the previously described setup in 1 .

Furthermore, capabilities to expand the setup of 1 to quantify the saturation dependency of the effective diffusion are demonstrated.

Prospectively, further physical aspects will be investigated and quantified experimentally, including deformation, dispersion and adsorption processes. As a next step, textile continua will be examined and a link to planar textile structures will be modelled depending on the compression ratio. The described experimental methods offer the opportunity to model, simulate and validate transport processes in textiles. 3 shows the utilization of the particle-based simulation method smoothed particle hydrodynamics (SPH) to simulate the moisturization of a porous medium, which will be extended with additional transport processes.

Procter and Gamble Student poster award:

\section{References:}

1 C. B. Simile and H. W. Beckham, "Permeability-saturation-capillary pressure relations in textile fabrics from an integrated upward-horizontal-downward wicking test”, The Journal of The Textile Institute, vol. 103, pp. 945-951, November 2011

2 K. Ghali, B. Jones and J. Tracy, "Experimental Techniques for Measuring Parameters Describing Wetting and Wicking in Fabrics", Textile Research Journal, vol. 64, pp. 106-111, February 1994

3 T. B. Wybranietz, P. Sabrowski, S. Przybilla, J. Villwock, "Numerical SPH Simulation of porous media flow considering permeability, capillarity and saturation relations", Proceedings of the 13th International SPHERIC Workshop, pp. 133-140, June 2018 Acceptance of Terms and Conditions: 
Click here to agree

\title{
Pore-network - lattice Boltzmann method hybrid model for mul- tiphase flow
}

\author{
Eduard Puig Montella ${ }^{1}$; Bruno Chareyre ${ }^{2}$; Antonio Gens ${ }^{3}$ \\ ${ }^{1}$ Université Grenoble-Alpes (UGA) \\ ${ }^{2}$ Univ Grenoble Alpes \\ ${ }^{3}$ Universitat Politecnica de Catalunya
}

Corresponding Author(s): eduard.puigmontella@3sr-grenoble.fr

Two-phase flow through porous media can be simulated by means of the multicomponent ShanChen lattice Boltzmann method [1-2] (see figure 1). However, such simulations require a significant computation time. Thus, in order to optimize the computation resources, we present a hybrid model that combines the efficiency of the pore-network approach and the accuracy of the lattice Boltzmann method at the pore scale 3 .

In this work, the evolution of capillary forces, fluid morphology and water content are evaluated during the drainage of a granular assembly made up of 40 spheres. The sphere-pack system is designed with a porous membrane located at the bottom of the sample to ensure a complete drainage. A pressure gradient is imposed to mimic the displacement of the interface. This method enhances a continuous description of the complex liquid morphologies that are formed during the drainage. The results are compared with a new model based on the decomposition of the granular assembly into small subsets (see figure 2), in which lattice Boltzmann simulations are performed to determine the entry pressure $P e$, the primary drainage curve and the liquid morphology for each pore throat. In each elementary problem that is solved with the LBM, both phases (typically water and air) are initially in equilibrium. Then, the fluid-fluid interface is displaced as the capillary pressure increases. When the capillary pressure reaches the entry pressure $P e$, the non-wetting phase (air) invades the pore body. $P e$ is determined for all the subsets and the global problem is assembled and solved at the network scale 3 .

This technique leads to a complete analysis with a smaller computation domain. Besides, some areas of the granular assembly can be excluded from the simulation (empty pores and isolated cluster with no flux). Thus, the multiscale coupling takes advantage of both Pore-Network and LBM reducing the computational cost. The work is completed with results obtained with approximations (such as the Incircle and the MS-P method) that predict hydrostatic properties within very reduced computing time computation time, therefore, suitable to replace LBM simulations when the computation resources are limited.

\section{Indico rendering error}

Could not include image: Cannot read image data. Maybe not an image file?

\section{Indico rendering error}

Could not include image: Cannot read image data. Maybe not an image file?

\section{Procter and Gamble Student poster award:}

I would like to compete in the Procter and Gamble Student award References:

1 Shan, X., \& Chen, H. (1994). Simulation of nonideal gases and liquid-gas phase transitions by the lattice Boltzmann equation. Physical Review E, 49(4), 2941.

2 Shan, X., \& Chen, H. (1993). Lattice Boltzmann model for simulating flows with multiple phases and components. Physical Review E, 47(3), 1815. 
3 Chareyre, B., Yuan, C., Montella, E. P., \& Salager, S. (2017). Toward multiscale modelings of grain-fluid systems. In EPJ Web of Conferences (Vol. 140, p. 09027). EDP Sciences. Acceptance of Terms and Conditions:

Click here to agree

\title{
Pore-network - lattice Boltzmann method hybrid model for mul- tiphase flow
}

\author{
Eduard Puig Montella ${ }^{1}$; Bruno Chareyre ${ }^{2}$; Antonio Gens $^{3}$ \\ ${ }^{1}$ Université Grenoble-Alpes (UGA) \\ ${ }^{2}$ Univ Grenoble Alpes \\ ${ }^{3}$ Universitat Politecnica de Catalunya
}

Corresponding Author(s): eduard.puigmontella@3sr-grenoble.fr

Two-phase flow through porous media can be simulated by means of the multicomponent ShanChen lattice Boltzmann method [1-2] (see figure 1). However, such simulations require a significant computation time. In order to optimize the computation resources, we present a hybrid model that combines the efficiency of the pore-network approach and the accuracy of the lattice Boltzmann method at the pore scale 3 .

In this work, the evolution of capillary forces, fluid morphology and water content are evaluated during the drainage of a granular assembly made up of 40 spheres. The sphere-pack system is designed with a porous membrane located at the bottom of the sample to ensure a complete drainage. A pressure gradient is imposed to mimic the displacement of the interface. This method enhances a continuous description of the complex liquid morphologies that are formed during the drainage. The results are compared with a new model based on the decomposition of the granular assembly into small subsets (see figure 2), in which lattice Boltzmann simulations are performed to determine the entry pressure $P_{e}$, the primary drainage curve and the liquid morphology for each pore throat. In each elementary problem that is solved with the LBM, both phases (typically water and air) are initially in equilibrium. Then, the fluid-fluid interface is displaced as the capillary pressure increases. When the capillary pressure reaches the entry pressure $P_{e}$, the non-wetting phase (air) invades the pore body. $P_{e}$ is determined for all the subsets and the global problem is assembled and solved at the network scale 3 .

This technique leads to a complete analysis with a smaller computation domain. Besides, some areas of the granular assembly can be excluded from the simulation (empty pores and isolated cluster with no flux). Thus, the multiscale coupling takes advantage of both Pore-Network and LBM reducing the computational cost. The work is completed with results obtained with approximations (such as the Incircle and the MS-P method) that predict hydrostatic properties within very reduced computing time computation time, therefore, suitable to replace LBM simulations when the computation resources are limited.

\section{Figures:}

Indico rendering error

Could not include image: Cannot read image data. Maybe not an image file?

Indico rendering error

Could not include image: Cannot read image data. Maybe not an image file?

Procter and Gamble Student poster award:

I would like to compete in the Procter and Gamble Student award References: 
1 Shan, X., \& Chen, H. (1994). Simulation of nonideal gases and liquid-gas phase transitions by the lattice Boltzmann equation. Physical Review E, 49(4), 2941.

2 Shan, X., \& Chen, H. (1993). Lattice Boltzmann model for simulating flows with multiple phases and components. Physical Review E, 47(3), 1815.

3 Chareyre, B., Yuan, C., Montella, E. P., \& Salager, S. (2017). Toward multiscale modelings of grain-fluid systems. In EPJ Web of Conferences (Vol. 140, p. 09027). EDP Sciences. Acceptance of Terms and Conditions:

Click here to agree

902

\section{Comparative Analysis of Image Segmentation Techniques Applied for Measuring Pore-Scale Parameters in Saturated Media}

Prakash Purswani $^{1}$; Khaled Enab ${ }^{1}$; Zuleima Karpyn ${ }^{1}$

${ }^{1}$ The Pennsylvania State University

Corresponding Author(s): prakash.purswani506@gmail.com

With the growing access to sophisticated X-ray imaging capabilities, there has been an increase in pore-scale research to more accurately capture fluid flow behavior in permeable media. Image processing has, therefore, become an integral part of porous media research. In particular, segmentation of the various phases (oil/brine/rock) is critical for the estimation of fluid saturation, fluid and rock topology, and pore connectivity, among other pore-scale parameters. Standard thresholding techniques that operate by marking a shift in the grayscale intensity among the involved phases, fail to give superior performance when segmenting these phases. However, thresholding techniques continue to be heavily used due to their ease of use. Together with this, newer machine learning techniques, being more involved, are less commonly utilized. A comparison showing the tradeoffs and benefits of these techniques is lacking. Therefore, the purpose of this research is to compare the effectiveness of different image segmentation techniques in view of applications to porous media research. Three techniques, namely, thresholding, supervised machine learning, and unsupervised machine learning, were compared. Performance comparisons were made on bulk estimates, such as porosity, saturation, total phase surface area, as well as pore-scale estimates, such as interfacial areas, and Euler characteristic. For this, two-phase (air/brine) X-ray micro-computed tomography images were acquired at rest in a sintered glass bead pack. The images were captured at two different voxel resolutions, namely, $6 \mu \mathrm{m}$ and $18 \mu \mathrm{m}$. The high-resolution images were used as a target case for comparison purposes. The results showed that average thresholding and supervised machine learning techniques result in porosity, saturation, and phase fraction estimates that are closer to the target case. With the exception of total surface area measurements, segmentation results from unsupervised machine learning were found to be mostly away from the target case. Furthermore, supervised machine learning provided good measures of air-brine interfacial areas by accurately capturing three-phase contact regions. Lastly, all segmentation techniques gave close measures of air phase Euler characteristic, but the results were unreliable for the measure of the solid phase, likely due to the sensitive nature of the measure. Overall, we find that although computationally intensive, supervised machine learning approach for image segmentation appears to be the most reliable, whereas the unsupervised machine learning approach appears to be the least reliable. This research provides the reader an awareness of some of the newer image segmentation techniques, their implementation, their success, and their limitation with regards to application in porous media research.

Procter and Gamble Student poster award:

References:

Acceptance of Terms and Conditions:

Click here to agree 


\title{
Upscaling hydrodynamic transport in heterogeneous porous me- dia from fully advective to small Péclet number regimes
}

Author(s): Alexandre Puyguiraud ${ }^{1}$

Co-author(s): Marco Dentz ${ }^{1}$; Philippe Gouze ${ }^{2}$

${ }^{1}$ IDAEA - CSIC

${ }^{2}$ Géosciences Montpellier, CNRS - Université de Montpellier

Corresponding Author(s): alexandre.puyguiraud@gmail.com

\begin{abstract}
Upscaling transport processes in porous media from the pore- to the Darcy scale is challenging. It is well known that transport often displays non-Fickian behavior at laboratory and field scales. This can be traced back to the geometric heterogeneity at pore-scale that triggers highly heterogeneous spatial distribution of the velocity and thus a broad range of solute particle transition times and arrival times. An upscaling model must be able to reproduce the behavior of advection and diffusion processes that control the velocity that solute particles experience during their transport into the porous media, using measurable macroscopic properties. To study these mechanisms, we run particle tracking simulations on a 3D real rock digitized sample and investigate the transport properties through a wide range of Péclet regimes. For infinite Péclet cases, the velocities remain correlated on spatial scale imprinted in the pore structure. The transport in these conditions is anomalous and the breakthrough curves for instance exhibit an early peak and a pronounced tailing. We show that a one-dimensional Continuous Time Random Walk (CTRW) based on a Markov process for the prediction of the particle velocity captures and predicts transport properties such as breakthrough curves, propagators and moments. This velocity process is based on a relaxation scheme that enforces the velocity correlation on a spatial scale related to the average pore length of our sample. Conversely, for finite Péclet numbers, diffusion affects noticeably the transport behavior. Breakthrough curves exhibit new features such as earlier arrivals, a delayed peak, and a large time cutoff. The features are linked to the diffusive motion that occurs on a temporal scale and enforces particles to jump from a streamline to another, and thus modify the spatial and temporal velocity series. For upscaling, we propose a one-dimensional CTRW model coupling the advective velocity change (occurring with a give spatial frequency) and the diffusive velocity change (occurring with a given temporal frequency). The model is able to reproduce the observables, such as the breakthrough curves, modeled by the $3 \mathrm{D}$ pore scale simulation for a wide range of Péclet regimes.
\end{abstract}

Procter and Gamble Student poster award:

I would like to compete in the Procter and Gamble Student award References:

Acceptance of Terms and Conditions:

Click here to agree

838

\section{Generalized network modelling: validation using micro-CT im- ages of two-phase flow}

Ali Qaseminejad Raeini ${ }^{1}$; Jianhui Yang ${ }^{2}$; Igor Bondino ${ }^{2}$; Ying Gao ${ }^{3}$; Qingyang Lin $^{3}$; Kamaljit Singh ${ }^{\text {None }}$; Branko Bijeljic $^{4}$; Martin Blunt ${ }^{3}$

\footnotetext{
${ }^{1}$ Research Associate

${ }^{2}$ TOTAL

${ }^{3}$ Imperial College London

${ }^{4}$ Imperial College
} 
Corresponding Author(s): a.qaseminejad-raeini09@imperial.ac.uk

The generalized network model is an efficient yet flexible tool for prediction of two-phase flow through porous media at the pore scale, as well as a platform to characterize and analyze of micro-CT images of multiphase flow. In this work we present a validation of this model against micro-CT images of two-phase flow experiments. We first quantify the uncertainty in experimental data, mapped onto the pore-network as an analysis tool. Then we compare the network model predictions with the experimental measurements on a pore-by-pore basis as well as in terms of average/upscaled flow properties. These comparisons are used to evaluate the accuracy of the network model and identify potentials for further improvement. We conclude with a discussion on how this validation work moves the pore-network model toward a more predictive tool by utilizing the data obtained from micro-CT imaging and the core-scale measurements such as capillary pressure and relative permeability.

Procter and Gamble Student poster award:

I don't want to compete References:

Acceptance of Terms and Conditions:

Click here to agree

\title{
Quantitative Predictions of Two-Phase Flow Dynamics in Porous Media by the Pore-Network Modeling
}

\author{
Chao-Zhong Qin ${ }^{1}$; Mahmoud Hefny ${ }^{2}$; E. Harald van Brummelen ${ }^{3}$ \\ 1 TU/e \\ ${ }^{2}$ ETH Zurich \\ ${ }^{3}$ Technology University of Eindhoven
}

Corresponding Author(s): c.qin@tue.nl

Two-phase flow dynamics in porous media plays a pivotal role in many industrial applications, such as enhanced oil recovery, geological carbon storage, and inkjet printing. There have been many pore-scale studies of two-phase flow dynamics in porous media by direct numerical simulation (DNS) models like VOF, LBM, and SPH. Their practical importance, however, is usually compromised by a small computational domain due to the heavy computational demand, and discrepancy of flow dynamics due to the oversimplification of contact line dynamics.

Alternatively, the pore-network modeling, as an efficient meso-scale model, has also been widely used in porous media study. Most of previous pore-network models are percolation-based models, which were mainly used to obtain materials properties like relative permeability and capillary pressure relationship (Patzek, 2001). Though a few dynamic pore-network models have been developed, they are limited by the oversimplification of pore-network elements (i.e., regular polyhedrons) (Qin, 2015). Therefore, they are not suitable to the study of a realistic porous medium.

In this work, we aim to develop a pore-network model which can be used to quantitatively study twophase flow dynamics in porous media. The first and crucial step is to properly represent complex pore spaces with well-defined pore-network elements. We start with the pore-network extraction from a Synchrotronic X-ray scanning. We will discuss how complex pore structures are approximated by tubes of various cross sections. Then, we will introduce our state-of-the-art dynamic pore-network model for both drainage and imbibition. Finally, we will report our numerical results in terms of temporal phase distributions, drainage and imbibition rates, dynamic capillary and phase pressures, and interfacial areas.

\section{Procter and Gamble Student poster award:}

I don't want to compete References:

Patzek, T.W., 2001. Verification of a Complete Pore Network Simulator of Drainage and Imbibition. SPE J. 6, 144-156. https://doi.org/10.2118/71310-PA

Qin, C., 2015. Water Transport in the Gas Diffusion Layer of a Polymer Electrolyte Fuel Cell: Dynamic 
Click here to agree

\title{
Hybrid LBM simulation of non-isothermal drying of colloid sus- pension in a micro-pore structure
}

Feifei Qin $^{1}$; Luca Del Carro ${ }^{2}$; Ali Mazloomi Moqaddam ${ }^{\text {None }}$; Qinjun Kang ${ }^{3}$; Dominique Derome ${ }^{4}$; Jan Carmeliet ${ }^{5}$

\author{
${ }^{1}$ ETH/Empa \\ ${ }^{2}$ IBM Zurich \\ ${ }^{3}$ Los Alamos National Laboratory \\ ${ }^{4}$ Empa \\ ${ }^{5}$ ETHZ
}

Corresponding Author(s): feifei.qin@empa.ch

To study the physics of colloidal liquid evaporation and colloidal particle deposition during the nonisothermal drying of colloidal suspension in micro-pore structures, a hybrid lattice Boltzmann model (LBM) is developed here. An entropic multiple relaxation-time multirange pseudopotential twophase LBM (EMRT-MP LBM) 1 for isothermal interfacial flow is firstly coupled to an extended temperature equation (ETE) 2 for simulating non-isothermal liquid drying. Then the coupling model is further coupled with a modified convection diffusion equation (MCDE) to consider non-isothermal drying of colloid suspension 3. The ETE and MCDE are solved by the finite difference scheme (FDM) with the same lattices as in LBM. With all the sub-models, this coupled model is capable of considering the main physical phenomena at play: two-phase fluid flow, heat/mass transport and solidification.

We consider two drying events. First, drying of colloid suspension in a two-pillar micro-pore structure is simulated, and the final configuration of colloidal particles is compared with experimental one. A good agreement is observed between each other. Second, under the heating temperature of 70 Celsius degree, drying of colloid suspension in a complex spiral-shaped micro-pore structure containing 220 pillars is simulated. The drying pattern follows the spiral shape due to the capillary pumping, which indicates the liquid is transported from large pore to small ones driven by capillary pressure difference. Since the colloidal particles are passively carried with liquid, they accumulate at the small menisci during the drying process. When the local liquid is dried out at the small menisci, the colloidal particles are deposited as solid structures between the pillars (mainly), or around the surfaces of pillars (slightly). As a result, the particle deposition exhibits the spiral route. Qualitatively, our simulated liquid and particle configurations agree well with experimental ones during the entire drying process. Quantitatively, our model captures that the evaporation rate and the particle accumulation rate decreasing slowly during drying similar to ones in experiment, which is due to the deduction of liquid-vapor interface area.

In conclusion, our nested model has shown the capability and accuracy by simulating non-isothermal drying of colloid suspension in a complex micro-pore structure both qualitatively and quantitatively, as it includes all the required physics and captures all the complex features observed experimentally. Such a tri-coupled LBM has a high potential to become an efficient numerical tool for further investigation of the real and complex engineering problems with drying of colloids suspension in porous media.

Procter and Gamble Student poster award:

\section{References:}

1 F. Qin, A. Mazloomi Moqaddam, Q. Kang, D. Derome and J. Carmeliet, Phys. Fluids 30, (2018).

2 Q. Li, P. Zhou, and H. J. Yan, Langmuir 32, 9389 (2016).

3 F. Qin, A. Mazloomi Moqaddam, Q. Kang, D. Derome and J. Carmeliet, Transp. Porous Media, (2018). Acceptance of Terms and Conditions:

Click here to agree 


\title{
Impact of subcore-scale permeability heterogeneity on solute mix- ing in a microporous limestone: experiments and numerical study
}

\author{
Author(s): Swapna S. Rabha ${ }^{1}$ \\ Co-author(s): Takeshi Kurotori ${ }^{2}$; Ronny Pini ${ }^{3}$ \\ ${ }^{1}$ Research Associate \\ ${ }^{2}$ Research Postgraduate \\ ${ }^{3}$ Senior Lecturer
}

Corresponding Author(s): s.rabha@imperial.ac.uk

In the recent years, because of the requirement to transition towards a low carbon economy for climate change mitigation, saline aquifer storage of $\mathrm{CO} 2$ has emerged as an important strategy for $\mathrm{CO} 2$ sequestration. Apart from the large storage capacity, the potential storage reservoirs for $\mathrm{CO} 2$ must have sufficiently high porosity and permeability to provide sufficient volume for $\mathrm{CO} 2$ and to allow injection of the $\mathrm{CO} 21$. Therefore, it is vital to characterise these reservoir storage sites and conduct detailed experiments to analyse how heterogeneities in rock properties at sub-seismic resolution can influence the spatial extent of the plume migration and secondary trapping processes, such as dissolution. Core-flooding experiments have been routinely used in the last decades [2,3] for analysing these rock formations. The ability to validate observations from core-flooding experiments with suitable numerical simulations can provide key insight on the underlying physics beyond the experimental measurements, including sub-metre scale distributions of velocity and other rock properties, such as longitudinal and transverse dispersivity.

In this work, we explore a novel approach where the imagery generated during core-flooding experiments is analysed with detailed numerical simulations of the transport process within the rock core. To this aim, two and three-dimensional numerical simulations are conducted using the reactive transport code CrunchFlow 4 . For both experiments and simulations, a Ketton Limestone rock core of diameter $5 \mathrm{~cm}$ and length of $10 \mathrm{~cm}$ is used. The geometry and operation conditions are exactly same as the experiments used in 5 where the transport of a solute was imaged dynamically by Position Emission Tomography (PET).

In the simulator, each grid cell in the solution domain was assigned a permeability value, which was calculated using different methods, namely by using (i) the Kozeny-Carman based permeability model [6], (ii) Fractal-Geometry permeability model [7] and a (iii) thresholding method that uses the corresponding gas saturation maps acquired during a drainage test to identify low and high permeability regions. The predicted results of the simulations are compared with 3D images of the solute plume obtained by PET and show that the use of a porosity-based permeability distribution gives a good agreement with the experimental results in terms of the predicted residence time distribution and averaged internal concentration profiles within the core, However, these model-based simulations fail at capturing the spreading of the solute caused by the subcore scale heterogeneity in the rocks. On the other hand, simulations where the gas saturation-based permeability map was used show a better agreement with the experiments in terms of both averaged values and in the spatial distribution of the predicted solute plume migration. These are further analysed in terms of spatial measures of mixing, such as the scalar dissipation and the dilution index. Various realisations of the calibrated permeability map are built by varying the spatial distribution of heterogeneity in the numerical model, thereby providing a statistical basis to the experimental observations on one rock sample.

Procter and Gamble Student poster award:

\section{References:}

1] Bentham, M., \& Kirby, G. (2005). Oil \& Gas science and technology. Oil \& Gas science and technology -Rev. IFP, 60(3),559 - 567.

2] Akin, S., \& Kovscek, A. (2003). Computer tomography in petroleum engineering research. Geological Society Special Publication, 215, 23-38.

3] Shafer, J. (2013). Recent advances in core analysis. Petrophysics, 54(6), 554-579. 
4] Steefel, C., Appelo, C. A., Arora, B., D, J., Kalbacher, T., O, K., . . Yeh, G. T. (2014). Reactive transport codes for subsurface environmental simulation. Computational Geosciences, 19(3), 445-478.

5] Kurotori, T., Zahasky, C., Hejazia, S. A., Shah, S. M., Benson, S. M., \& Pini, R. (2018). Measuring, imaging and modelling solute transport in a microporous limestone. Chemical Engineering Science. doi:10.1016/j.ces.2018.11.001.

6] Mavko, G., \& Nur, A. (1997). The effect of a percolation threshold in the Kozeny-Carman Relation. Geophysics, 62(5), 1480-1482.

7] Pape, H., Clauser, C., \& Iffland, J. (1999). Permeability prediction based on fractal pore space geometry. Geophysics, 64(5), 1447-1460. Acceptance of Terms and Conditions:

Click here to agree

456

\title{
The effect of carbonate cementation on the pore space structure
}

\author{
Andrzej Radlinski ${ }^{1}$; Tomas Blach ${ }^{2}$; Matej Lipar ${ }^{3}$; Silvana Magni ${ }^{1}$; Schweins Ralf ${ }^{4}$; Hartmut Lemmel $^{5}$; Piotr \\ Szymczak ${ }^{6}$ \\ ${ }^{1}$ University of Warsaw, Faculty of Physics, Warsaw, Poland \\ ${ }^{2}$ University of New South Wales, Minerals and Energy Resources Engineering, Sydney, Australia \\ ${ }^{3}$ Anton Melik Geographical Institute, Ljubljana, Slovenia \\ ${ }^{4}$ Institut Laue-Langevin \\ ${ }^{5}$ TU Wien \\ ${ }^{6}$ University of Warsaw, Faculty of Physics
}

Corresponding Author(s): andrtzej.radlinski@gmail.com

One of the possible solutions for the $\mathrm{CO} 2$ sequestration is its mineralization. During this process, secondary calcite precipitates in the pore space. This may eventually lead to clogging of the flow pathways, which is undesirable, as it prevents $\mathrm{CO} 2$ from spreading further into the underground reservoir. To understand better the impact of carbonate cementation on the pore-space geometry, we have analyzed the natural cementation on the rims of solution pipes formed in the Middle Upper Pliocene coastal calcarenite in Apulia (Italy) and compared the pore-space characteristics with those of uncemented host rock.

We used small angle neutron scattering techniques combined with the measurements of composition (XRD), porosity (He pycnometry) and permeability (MICP, mercury intrusion) to quantify the impact of cementation on pore space characteristics. We find a significant drop in porosity in the cemented rocks ( $17 \%$ vs $49 \%$ in the host rock) and an associated 20 -fold decrease in permeability. Interestingly, however, the specific surface area (SSA) in the cemented samples is almost 10 times larger than that in the host rocks, which indicates that roughness of the rock-pore interface markedly increases during the cementation. The combination of MICP and neutron scattering techniques have shown that this increase in the roughness of pore interfaces takes place on all scales - down to the nanometer scale, thus the reactions leading to cementation are not limited to the regions of the fastest flow, largest porosity and high permeability.

Finally, the analysis of neutron scattering data allowed us to calculate the fractal dimension of the pore interface, D. For each sample, this parameter is different for the population of accessible pores and inaccessible pores. In the host rock $\mathrm{D}=2.30$ (2.65) for accessible (inaccessible) pores. In the cemented sample, D is markedly increased and reaches respectively 2.7(3.00). Large values of D indicate rough and convoluted interface. A particularly striking result here is that $\mathrm{D}=3$ for inaccessible pores in the cemented sample, meaning that the surface of the internal pore network fills almost all the internal space. We hypothesize that such a highly convoluted pore structure results from the negative coupling between flow and permeability during the calcite precipitation processes. The deposition of reaction products forces the rock penetrating fluid to find new pathways of increasing tortuosity, which eventually forms a structure akin to a D=3 interface devoid of bulk.

Procter and Gamble Student poster award:

References: 
Some recent publications on small angle scattering of neutrons in rocks:

Radlinski, A.P. (2006). Rev. Mineral. Geochem 63, 363-397;

Clarkson, C.R. et al. (2013). Fuel 103, 606-616;

Blach, T. et al. (2018). I.J. Coal Geol. 186, 135-144; Acceptance of Terms and Conditions:

Click here to agree

\title{
A Convergence Analysis of MCMC Methods for Porous Media Flows
}

\author{
Abdullah Mamun $^{1}$; Felipe Pereira ${ }^{1}$; Arunasalam Rahunanthan ${ }^{2}$ \\ ${ }^{1}$ University of Texas at Dallas \\ ${ }^{2}$ Central State University
}

Corresponding Author(s): arahunanthan@centralstate.edu

In subsurface characterization, its properties are reconstructed with a set of limited data using a history-matching algorithm. In our study we focus on the characterization of the permeability field in an aquifer using Markov Chain Monte Carlo (MCMC) algorithms, which are reliable procedures for such reconstruction. The MCMC algorithm is serial in nature due to its Markovian property. Moreover, the calculation of the likelihood information in the MCMC is computationally expensive for flow problems in the aquifer. Running a long MCMC chain for a very long period makes the method less attractive for the characterization of the aquifer. In contrast, several shorter MCMC chains can substantially reduce computation time and can make the method more suitable for the characterization. However, the convergence of such MCMC chains should be carefully studied. In this talk, we consider multiple MCMC chains for a single-phase flow problem in the aquifer and analyze the convergence of the chains.

Procter and Gamble Student poster award:

References:

Acceptance of Terms and Conditions:

Click here to agree

\section{Injection Strategies and Benefits of Using Flue Gas as Working fluid for Enhanced Geothermal Systems}

\author{
Hariharan Ramachandran ${ }^{1}$ \\ ${ }^{1}$ The University of Texas at Austin \\ Corresponding Author(s): hariharan@utexas.edu
}

Cost efficient carbon negative technologies will play an important role in GHG mitigation. Injection of flue gases from industrial sources and power stations into geothermal reservoirs achieves heat recovery and gas storage. Numerical reservoir modeling of this option is important to improve existing understanding of subsurface interactions and forecast energy recovery. The main objective of this study is to estimate optimal injection strategies, overall energy balance and cost benefits for a representative flue gas EGS project. Non-isothermal numerical reservoir simulator was developed for this study. Complete equations and solution algorithms are presented. The conservation equations describe the overall mass and energy balance coupled with component transport. Appropriate models have been used to estimate the effect of this thermal gradient on rock and fluid properties. A highly accurate multi-parameter equation of state (EOS) based on the span-wagner approach is used 
to describe the multicomponent $(\mathrm{H} 2 \mathrm{O}, \mathrm{CO} 2, \mathrm{~N} 2, \mathrm{CH} 4$, other flue gas components) phase behavior. This EOS predicts accurate fluid property and phase conditions for wide pressures and temperatures. Literature values are used. The coupled differential equations are implemented in Python and solved in a sequentially implicit method. The results from this study will focus on well placement, well type (vertical, inclined, horizontal), pressure control, when and how much and how long can be injected and how soon will the breakthrough occur at production wells. One strategy of note is to use horizontal wells to inject gases at the bottom of the reservoir and produce at the top of the reservoir to control pressure. One possibility to dispose of the produced brine is to dissolve flue gases at the surface and reinject into the reservoir. This will immediately reduce the pressure buildup associated with injection. All of these strategies depend on the geology, geomechanics, petrophysics, fluid properties and other conditions unique to the reservoir.

Procter and Gamble Student poster award:

References:

Acceptance of Terms and Conditions:

Click here to agree

\title{
Effect of fractures geometrical and hydrodynamic characteristics on natural convection in fractured porous enclosure
}

\author{
Fanilo Ramasomanana ${ }^{1}$; Marwan Fahs ${ }^{2}$; Husam Baalousha ${ }^{3}$; Vincent Fontaine ${ }^{4}$ \\ ${ }^{1}$ Qatar Environment \& Energy Research Institute, Hamad Bin Khalifa University, Qatar Foundation \\ ${ }^{2}$ ENGEES-LHYGES \\ ${ }^{3}$ Qatar Environment \& Energy Research Institute, Hamad Bin Khalifa University, Qatar Foundation, Doha, Qatar \\ ${ }^{4}$ PIMENT - Physique et Ingénierie Mathématique pour l'Énergie, l'environnemeNt et le bâtimenT, Université de La \\ Réunion, France
}

Corresponding Author(s): framasomanana@hbku.edu.qa

\begin{abstract}
Natural convection in fractured porous media has become an important focus of scientific research in the last decades. Important engineering applications can be found in several industrial and environmental applications such as in oil and gas recovery, fuel cells, geothermal energy, groundwater contamination, saltwater intrusion, nuclear waste disposal, flow through salt formations, among others. Thus, there is a need to understand the physical processes of natural convection in fractured porous media.

The objective of this work is to provide better understanding of the effect of fractures on natural convection in porous enclosure. Particularly, we aim to understand the effect of fractures locations, density, aperture and hydrodynamic properties on the heat transfer and convective flow processes. We consider the problem of square porous cavity with differentially heated vertical walls. This problem is widely used in the literature to understand the physical processes of natural convection in heterogeneous porous media. However, to the best of our knowledge, fractured configuration, which is one of the most challenging form of heterogeneity has been never studied in this context. Thus, we consider several fractured configurations and we develop numerical simulations to investigate the effect of fractures on velocity field, temperature distribution and heat flux as measured by the Nusselt number.

To correctly represent fracture embedded in the porous matrix, we use the Discrete Facture Model (DFM). This model is highly accurate as it can represent fractures explicitly without any simplification. However, numerical solution of this model with standard numerical methods can lead to numerical artifacts that affect the solution to various degrees and in consequence the physical behavior of the system. To avoid this problem, we implement a high accurate solution based on the Eulerian-Lagrangian Localized Adjoint Method (ELLAM). For verification issue, we tested our numerical solution against a standard finite element model based on Discrete Facture Network model developed in the frame of COMSOL Multiphysics ${ }^{\circledR}$. We use the ELLAM model to establish relation between heat transfer and fluid flow characteristics and the fracture properties.
\end{abstract}

Procter and Gamble Student poster award: 


\title{
References:
}

Acceptance of Terms and Conditions:

Click here to agree

43

\section{A multi-scale asymptotic homogenization technique to the mate- rial remodeling of heterogeneous media}

Ariel Ramírez-Torres ${ }^{1}$; Salvatore Di Stefano ${ }^{1}$; Alfio Grillo ${ }^{1}$; Reinaldo Rodríguez-Ramos ${ }^{2}$; José Merodio ${ }^{3}$; Raimondo Penta $^{4}$

\footnotetext{
${ }^{1}$ Politecnico di Torino

${ }^{2}$ Universidad de La Habana

${ }^{3}$ Universidad Politécnica de Madrid

${ }^{4}$ University of Glasgow
}

\section{Corresponding Author(s): ariel.ramirez@polito.it}

We apply the asymptotic homogenization technique to the equations describing the dynamics of a heterogeneous material with evolution of its internal structure. We refer to this phenomenon as to material remodeling and we interpret it with the production of plastic-like distortions. The latter ones being accounted for by means of the Bilby-Kröner-Lee decomposition. The asymptotic homogenization approach is applied to a heterogeneous body comprising two hyperelastic materials. The whole study is framed within the limit of small elastic distortions, and provides a robust framework that can be readily generalized to growth and remodeling of nonlinear composites. We complete our theoretical model by performing numerical simulations.

Procter and Gamble Student poster award:

\section{References:}

Acceptance of Terms and Conditions:

Click here to agree

\section{Viscous fingering instability in oscillating systems}

\author{
Author(s): CHINAR RANA ${ }^{1}$ \\ Co-author(s): Anne De Wit ${ }^{2}$ \\ ${ }^{1}$ Université libre de Bruxelles \\ ${ }^{2} U L B$ \\ Corresponding Author(s): ranachinar@gmail.com
}

In a porous medium, understanding the evolution of the interface between two miscible fluids, when one fluid displaces the other one is of fundamental importance. Localized spatiotemporal patterns can develop with the interplay of reaction and diffusion processes, when two reactants A and B of an oscillating reaction are placed in contact. Using the classical Brusselator model, we show numerically the existence of localized oscillations in the reaction zone depending on the initial concentrations of the reactants. We further explore the influence of hydrodynamic destabilization by introducing a viscosity gradient on the initially oscillating patterns. The interface between the fluids deforms into finger-like patterns leading to a viscous fingering instability, when a less viscous fluid displaces a more viscous one. Reactive systems are able to trigger this instability as soon as 
the chemical reaction changes the viscosity of the interplaying fluids. Nonlinear simulations of the related reaction-diffusion-convection equations with the fluid viscosity varying with the inhibitor concentration show an active coupling between the oscillatory kinetics and the viscously-driven instability. The periodic oscillations in the concentration of the intermediate species induce localized changes in the viscosity, which in turn affects the fingering pattern. Guided by the properties of the underlying reaction-diffusion dynamics, we show that localized changes in the viscosity profiles can induce oscillations in an initially non-oscillating reactive system while, as a corollar, nonlinear kinetics can trigger viscous fingering in an initially viscously stable stratification.

Procter and Gamble Student poster award:

I would like to compete in the Procter and Gamble Student award References:

Acceptance of Terms and Conditions:

Click here to agree

890

\title{
Some improvements to projection-based embedded discrete frac- ture model (pEDFM)
}

\author{
Author(s): Xiang Rao ${ }^{1}$ \\ Co-author(s): Linsong Cheng $^{2}$; Renyi Cao ${ }^{2}$ \\ ${ }^{1}$ China University of Petroleum, Beijing, China \\ ${ }^{2}$ China University of Petroleum, Beijing
}

Corresponding Author(s): raoxiang0103@gmail.com, lscheng@cup.edu.cn, caorenyi@cup.edu.cn

\begin{abstract}
pEDFM is firstly proposed by Tene et al. to extend EDFM to be adaptive to a wide range of fracture permeabilities (i.e. from flow barriers to highly conductive fractures). Jiang and Younis made several improvements upon the original pEDFM and applied the improved pEDFM to resolve the limitation that EDFM could induce large errors for multiphase displacement processes. Their works indicate that original EDFM is only adaptive to the production simulation of multistage fractured well, but pEDFM may be superior because it can be adaptive to more general cases.

This paper firstly points out that there are still some problems in current pEDFM, lying on the algorithm in pre-process for three-dimensional (3D) cases, the formula of transmissibilities of additional fracture-matrix ( $\mathrm{f}-\mathrm{m})$ connections and lack of additional fracture-fracture (f-f) connections. Then, corresponding improvements are presented to resolve these problems, and these improvements includes practical algorithm to select projected faces of fracture cells for 3D cases, practical algorithm to calculate the area of the union of projections for 3D cases, the modified physical transmissibilities of additional $\mathrm{f}-\mathrm{m}$ connections and the extended pEDFM considering additional $\mathrm{f}-\mathrm{f}$ connections. Some numerical examples are implemented to illustrate the necessity and validness of these improvements.
\end{abstract}

Procter and Gamble Student poster award:

I would like to compete in the Procter and Gamble Student award References:

Acceptance of Terms and Conditions:

Click here to agree

117

\section{Numerical simulation of coupling flow and geomechanics for frac- tured reservoirs}

Xiang Rao ${ }^{1}$; Linsong Cheng ${ }^{1}{\text {; Renyi } \mathrm{Cao}^{2} \text {; Shijun Huang }}^{1}$; Yongchao Xue $^{1}$ 


\footnotetext{
${ }^{1}$ China University of Petroleum-Beijing

${ }^{2}$ China University of Petroleum
}

Corresponding Author(s): raoxiang0103@gmail.com, lscheng@cup.edu.cn, caorenyi@126.com, hshj@cup.edu.cn, xyc75@cup.edu.cn

Natural fractured reservoirs (NFRs) now contribute a great deal of world's proven hydrocarbon reserves. Compared with conventional reservoirs, NFRs exhibit a high degree of heterogeneity and complexity created by fractures, and geomechanical effects play an important role in production, which is observed from field practice. Therefore, accurate modeling and simulation of the flow in fractured reservoirs are quite challenging, and an accurate and efficient numerical simulator of coupling flow and geomechanics for fractured reservoirs is necessary.

This paper presented a hybrid numerical model of extended finite element method (XFEM) and embedded discrete fracture model (EDFM), the novelty of which lies on coupling flow and geomechanics for fractured reservoirs, especially considering the effect of the dynamic propagation of waterflooding induced fracture. In the proposed hybrid model, XFEM is applied to numerically solve the displacement, stress and strain fields in fractured media, and corresponding geomechanics models for different flow media including matrix, natural fractures, hydraulic fractures and water-injection induced fractures, especially the water-injection induced fracture propagation model, are presented to link the stress-field parameters with flow parameters and determine the length and direction of propagating water-flooding induced fracture. Based on the flow parameters, the pressure and saturation fields in the reservoir can be computed by EDFM. For the next time step, the boundary conditions of the stress field are updated by calculated fluid pressure in this time step, and above calculation steps are iterated to compute the stress and flow fields of each time step. Some numerical examples are implemented to illustrate the validness of this hybrid numerical model.

\section{Procter and Gamble Student poster award:}

I would like to compete in the Procter and Gamble Student award References:

1 Bai M. On equivalence of dual-porosity poroelastic parameters[J]. Journal of Geophysical Research Solid Earth, 1999, 104(B5):10461-10466.

2 Belytschko T, Black T. Elastic crack growth in finite elements with minimal remeshing[J]. International Journal for Numerical Method in Engineering, 1999, 45(5)凶601-620.

3 Berryman J G. Extension of poroelastic analysis to double-porosity materials: New technique in microgeomechanics[J]. Journal of Engineering Mechanics, 2002, 128(8):840-847.

4 Beskos D E, Aifantis E C. On the theory of consolidation with double porosity-III A finite element formulation[J]. International Journal of Engineering Science, 1986, 24(11):1697-1716.

5 Biot M A. General Theory of Three-Dimensional Consolidation[J]. Journal of Applied Physics, 1941, 12(2):155-164.

[6] Daux C, Moës N, Dolbow J, et al. Arbitrary branched and intersecting cracks with the extended finite element method[J]. International Journal for Numerical Methods in Engineering, 2000, 48囚1741-1760.

[7] Garipov T T, Karimi-Fard M, Tchelepi H A. Fully coupled flow and geomechanics model for fractured porous media[J]. 2014.

[8] Khalili N. Coupling effects in double porosity media with deformable matrix[J]. Geophysical Research Letters, 2003, 30(22).

[9] Khoei A R. Extended Finite Element Method: Theory and Applications[J]. John Wiley \& Sons Inc, 2015(1):1-565.

[10] Khoei, A. R., Hosseini, N., and Mohammadnejad, T. Numerical modeling of two-phase fluid flow in deformable fractured porous media using the extended finite element method and an equivalent continuum model. Advance in water resource. 94 (August):510-528.

[11] Kim J, Moridis G J. Numerical studies on coupled flow and geomechanics with the multiple porosity model for naturally fractured tight and shale gas reservoirs. Presented at the 46th US Rock Mechanics/Geomechanics Symposium, Chicago, 24-27 June.

[12] Kim J, Moridis G J. Gas Flow Tightly Coupled to Elastoplastic Geomechanics for Tight and Shale Gas Reservoirs: Material Failure and Enhanced Permeability[J]. Spe Journal, 2014, 19.

[13] Kim J, Sonnenthal E L, Rutqvist J. Formulation and sequential numerical algorithms of coupled fluid/heat flow and geomechanics for multiple porosity materials[J]. International Journal for Numerical Methods in Engineering, 2012, 92(5):425-456.

[14] Melenk J M, Babuska I. The Partition of Unity Finite Element Method冈Basic Theory and Applications[C].Seminar fur Angewandte Mathematik, Eidgenossische Technische Hochschule, Switzerland, 1996.

[15] Moës N, Dolbow J, Belytschko T. A finite element method for crack growth without remeshing[J]. International Journal for Numerical Methods in Engineering, 1999, 46囚131-150.

[16] Moghaddam R N, Aghabozorgi S, Foroozesh J. Numerical Simulation of Gas Production From Tight, Ultratight and Shale Gas Reservoirs: Flow Regimes and Geomechanical Effects[C]// Society of Petroleum 
Engineers, EUROPEC 2015, 01-04 June, Madrid, Spain. 2015.

[17] Omidi O, Abedi R, Enayatpour S. An Adaptive Meshing Approach to Capture Hydraulic Fracturing[C]// U.s. Rock Mechanics/geomechanics Symposium. 2015.

[18] Ren G, Jiang J, Younis R M. Fully Coupled Geomechanics and Reservoir Simulation for Naturally and Hydraulically Fractured Reservoirs[C]// U.s. Rock Mechanics/geomechanics Symposium. 2016.

[19] Ren G, Jiang J, Younis R. A Fully Coupled XFEM-EDFM Model for Multiphase Flow and Geomechanics in Fractured Tight Gas Reservoirs $\otimes[J]$. Procedia Computer Science, 2016, 80:1404-1415.

[20] Ren G, Jiang J, Younis R M. Fully-Coupled XFEM-EDFM Hybrid Model for Geomechanics and Flow in Fractured Reservoirs[C]// SPE Reservoir Simulation Conference. 2017.

[21] Savitski A A, Lin M, Riahi A, et al. Explicit Modeling of Hydraulic Fracture Propagation in Fractured Shales[C]// International Petroleum Technology Conference. 2013.

[22] Wu K. Simultaneous Multi-Frac Treatments:Fully Coupled Fluid Flow and Fracture Mechanics for Horizontal Wells[J]. Spe Journal, 2015, 20(2):337-346.

[23] Yan Xia, et al. An Efficient Numerical Hybrid Model for Multiphase Flow in Deformable Fracturedshale Reservoirs. Society of Petroleum Engineers, 2018.

[24] Zhang X, Jeffrey R G. The role of friction and secondary flaws on deflection and re-initiation of hydraulic fractures at orthogonal pre-existing fractures $[\mathrm{J}]$. Geophysical Journal of the Royal Astronomical Society, 2010, 167(1):1454-1465.

[25] Zhang X, Jeffrey R G. Reinitiation or termination of fluid-driven fractures at frictional bedding interfaces[J]. Journal of Geophysical Research Solid Earth, 2008, 113(B8):-.

[26] Zhang X, Jeffrey R G, Thiercelin M. Mechanics of fluid-driven fracture growth in naturally fractured reservoirs with simple network geometries[J]. Journal of Geophysical Research Solid Earth, 2009, 114(B12):-

[27] Zhang X, Jeffrey R G. Role of overpressurized fluid and fluid-driven fractures in forming fracture networks[J]. Journal of Geochemical Exploration, 2014, 144:194-207. Acceptance of Terms and Conditions:

Click here to agree

975

\section{Thermal Driving Forces for Transport in Porous Media}

Author(s): Michael Rauter ${ }^{1}$

Co-author(s): Olav Galteland ${ }^{1}$; Bjørn Hafskjold ${ }^{1}$; Sondre K. Schnell ${ }^{2}$; Signe Kjelstrup ${ }^{1}$

${ }^{1}$ PoreLab (NTNU)

${ }^{2} N T N U$

Corresponding Author(s): michael.t.rauter@ntnu.no

Thermodynamic driving forces, as chemical, osmotic and thermal forces can cause transport of fluids in porous media. So far, most of the two-phase flows in porous media have been investigated at isothermal conditions, with the pressure gradient as the only driving force. The fluid transport in porous media due to a temperature gradient is therefore still posing unsolved practical as well as theoretical problems. In fact, it was shown in the literature that a temperature difference of only $40^{\circ} \mathrm{C}$ can overcome a hydraulic pressure difference of more than 10 bar. Internal simulations suggest that an even higher hydraulic pressure might be achievable at a smaller temperature difference. The understanding of this phenomenon may open up a possibility for further generalizations of the description of the two-phase flow and could also give valuable information for practical applications like sea water desalination, fuel cells or oil and natural gas recovery. The aim of the project is to understand the role of thermal driving forces for fluid transport in porous media, when it is acting alone or in combination with mechanical forces. The problem will be investigated with nonequilibrium molecular dynamics simulations and non-equilibrium thermodynamic modeling of one or more immiscible fluids in a porous membrane. By coupling the two simulation approaches, an improved description of the two-phase flow in porous media is expected which can be used to optimize properties of porous media in a more realistic manner and may even lead to the development of new applications.

Procter and Gamble Student poster award:

I don't want to compete References: 
Anthony P. Straub, Ngai Yin Yip, Shihong Lin, Jongho Lee \& Menachem Elimelech - Harvesting lowgrade heat energy using thermo-osmotic vapour transport through nanoporous membranes Acceptance of Terms and Conditions:

Click here to agree

\title{
Upscaling reactive transport in an evolving porous medium
}

\author{
Nadja Ray $^{1}$; Peter Frolkovic ${ }^{2}$; Jens Oberlander ${ }^{1}$ \\ ${ }^{1}$ Friedrich-Alexander Universität Erlangen-Nürnberg \\ ${ }^{2}$ Slovak University of Technology
}

Corresponding Author(s): ray@math.fau.de

We investigate porous media applications that contain evolving solid phases due to heterogeneous reaction. At the pore scale, we consider a coupled system of partial differential equations. It consists of transport equations for the species' concentrations while taking the processes of convection and diffusion into account. The interface between an attached layer of immobile chemical species and the fluid is characterized by means of a level-set. Applying two-scale asymptotic expansion in a level set framework, a micro-macro model emerges that is comprised of several levels of couplings: Macroscopic equations describing transport at the scale of the porous medium (macro scale) include averaged time- and space-dependent coefficient functions. These functions may explicitly be computed by means of auxiliary cell problems (micro scale). Finally, the pore space in which the cell problems are defined is time- and space dependent. The pore space's geometry is determined by means of the level-set equation and information from the transport equation's solutions is used to determine the explicit geometric structure (micro-macro scale). We complement our theoretical results with numerical computations. For the level set equation an upwind scheme described by Rouy and Tourin is applied. (Extended) Finite Element Methods are used for the evaluation of the cell problems and the transport equation. Ultimately, we investigate the dissolution of an array of calcite grains in the micro-macro context.

Procter and Gamble Student poster award:

References:

Acceptance of Terms and Conditions:

Click here to agree

80

\section{Discrete-continuum multiscale model for evolving porous media}

\author{
Nadja Ray ${ }^{1}$; Andreas Rupp ${ }^{2}$; Alexander Prechtel ${ }^{3}$ \\ ${ }^{1}$ Friedrich-Alexander Universität Erlangen-Nürnberg \\ ${ }^{2}$ Friedrich-Alexander University Erlangen-Nürnberg \\ ${ }^{3}$ Universität Erlangen-Nürnberg
}

Corresponding Author(s): ray@math.fau.de

Microaggregates are the fundamental building blocks of soils and thus important for its structure, properties and functions. Although there has been much research on the links, dynamics, stability, and structure of soil microaggregates, there is still a substantial lack of quantifying the relationships between the key factors of their dynamics. Those key factors are soil fauna, microorganisms, roots, 
inorganics, and physical processes [Totsche et al. 2017]. We assess the complex coupling of biological, chemical and physical processes at different scales with the help of a mechanistic modeling approach in order to gain a model-based mechanistic understanding of the formation, build-up, composition, properties and stability of microaggregates.

Our dynamic framework combines biomass development and structural changes in the solid originating from stabilizing sticky agents or electric effects in a comprehensive micro-macro model [Ray et al. 2017]. It uses a versatile discrete cellular automaton method (CAM) on the microscale with a continuous PDE formulation on the micro and the macroscale. This means that the underlying time-dependent computational domain for the pore scale, i.e. the distribution of a solid, biomass, a wetting, and a non-wetting fluid is determined discretely by means of a CAM. The diffusion of mobile bacteria, possibly transforming into immobile biomass, nutrients, and ions are prescribed by means of PDEs, likewise, the surface concentration of a sticky agent tightening together solid or bio cells (in the cellular automaton context) is considered. The idea of using a CAM setting with biofilm growth at the pore scale goes back to work in [Tang / Valocchi, Tang et al].

To omit the explicit tracking of interfaces as it is necessary in level-set approaches we use this combined discrete-continuum approach. As the soil is evolving in this setting we have to use a discretization for the PDE systems in the fluid that is robust and handles discrete discontinuities. The local discontinuous Galerkin (LDG) method is suitable for this task.

One main objective of this research is to examine the strong interplay between functional properties and geometric structure. To that end standard homogenization results are used to compute the soil's characteristic properties such as porosity or effective diffusion tensors for the resulting complex and time-dependent geometries.

Procter and Gamble Student poster award:

\section{References:}

References:

John W. Crawford et al, "Microbial diversity affects self-organization of the soil-microbe system with consequences for function”, J. R. Soc. Interface 9, (2012)

Nadja Ray, Andreas Rupp and Alexander Prechtel, "Discrete-continuum multiscale model for transport, biomass development and solid restructuring in porous media”, Advances in Water Resources 107, (2017) Youneng Tang and Albert J. Valocchi, "An improved cellular automaton method to model multispecies biofilms", Water Research 47, (2013)

Youneng Tang et al, "An improved pore-scale biofilm model and comparison with a microfluidic flow cell experiment”, Water Resources Research 49, (2013)

Kai U. Totsche et al, "Microaggregates in Soils (Review article)", J. Plant Nutr. Soil Sci. (2017) Acceptance of Terms and Conditions:

Click here to agree

\section{A pore-scale model of microbially induced calcium carbonate pre- cipitation}

Mohammad Amin Razbani ${ }^{1}$; Jennifer Zehner ${ }^{2}$; Espen Jettestuen ${ }^{3}$; Anja Røyne ${ }^{1}$; Pawel Sikorski ${ }^{2}$; Anders MaltheSørenssen ${ }^{1}$

\footnotetext{
${ }^{1}$ Physics of Geological Processes (PGP), The NFORD Centre, Department of Physics, University of Oslo, Oslo, Norway

${ }^{2}$ Department of Physics, Norwegian University of Science and Technology, NTNU, Trondheim, Norway

${ }^{3}$ Norwegian Research Centre AS (NORCE), Bergen, Norway
}

Corresponding Author(s): m.a.razbani@fys.uio.no

Microbially induced calcium carbonate precipitation (MICP) is a process in which bacterial processes are used for cementation of granular media for applications in geotechnical engineering, soil consolidation, and environmentally friendly alternatives to concrete 1 . In our work, we apply lactic acid producing bacteria to dissolve calcium carbonate, then in the next step calcium carbonate is reprecipitated due to hydrolysis of urea which induces consolidation of the material. The final product is called bio-concrete. In this process, urea is hydrolyzed and as a result the carbonate content and 
$\mathrm{pH}$ increases, which consequently induces crystallization of calcium carbonate. The complex interplay of different factors related to living organisms and geochemical processes has made it difficult to develop an accurate model for MICP 2. A multi-component reactive transport model need to be supplemented by kinetic expressions for ureolysis and precipitation, as well as explicit modeling of microbial attachment and detachment 3 .

We will present a lattice Boltzmann (LB) approach to model the MICP process described above on the pore-scale. We will describe how to couple the LB model to a full geomechanical solver, so that the chemical and mineralogical reactions can be accurately modeled. Bacteria are added to the model as point sources for lactic acid production and hydrolysis of urea. The rate of reactions is given by the local chemical environment of the bacteria and amount of nutrients.

Nucleation criteria, based on thermodynamic surface properties, will be incorporated to simulate mineral growth and gas production. A semi-empirical cementation criterion based on tracking of new solid-solid contact area will be used as a proxy for the strength of the material. The temporal development of porosity, permeability, and strength will be studied for a range of parameters. This work is integrated with a coordinated laboratory project, which studies MICP on grain and micro-scale 4. The model developed is benchmarked against experimental data. The main aim of the modelling work is to numerically investigate the consolidation process, which is important for the strength of the final product, as a function of bacteria concentration, bacteria distribution and activity, required amount of nutrition and other process parameters. Applicability of our modelling approach to study these processes will be demonstrated.

\title{
Procter and Gamble Student poster award:
}

I would like to compete in the Procter and Gamble Student award References:

1De Muynck, W., N. De Belie, and W. Verstraete, Microbial carbonate precipitation in construction materials: A review. Ecological Engineering, 2010. 36(2): p. 118-136.

2 Hommel, J., Ebigbo, A., Gerlach, R., Cunningham, A. B., Helmig, R., \& Class, H. (2016). Finding a balance between accuracy and effort for modeling biomineralization. European Geosciences Union General Assembly 2016, 97, 379-386.

3 Barkouki, T. H., Martinez, B. C., Mortensen, B. M., Weathers, T. S., De Jong, J. D., Ginn, T. R., . . . Fujita, Y. (2011). Forward and Inverse Bio-Geochemical Modeling of Microbially Induced Calcite Precipitation in Half-Meter Column Experiments. Transport in Porous Media, 90(1), 23-39.

4 Jennifer Zehner, Mohammad Amin Razbani, Simone Balzer Le, Alexander Wentzel, Espen Jettestuen, Anja Røyne, Pawel Sikorski (2019). Experimental investigation of microbial induced calcium carbonate precipitation induced by Sporosarcina pasteurii on the microscale. InterPore2019. Valencia. Acceptance of Terms and Conditions:

Click here to agree

\section{Flexible Framework for Two-Phase-Flow in Extremely Heteroge- neous Media}

Tatiana Reiche $e^{\text {None }}$

\author{
Corresponding Author(s): tatiana.reiche@grs.de
}

The assessment of the long-term safety of a deep underground repository for radioactive waste requires a comprehensive understanding of the system and appropriate numerical tools. RepoTREND is a numerical tool being developed by GRS for simulating

- the release of contaminants and

- their transport through the near-field and far-field to the biosphere, including

- the estimation of the radiological consequences for man and environment.

It is applicable to different concepts of final repositories in different host formations.

For a typical repository model the model area is extremely heterogeneous. Neighbouring grid elements can essentially differ with respect to their properties and the relevance of the effects that have to be considered additionally to the underlying basic processes, such as the two-phase flow in porous media. Such heterogeneities can lead to the necessity to vary the basic equations over the model regions. For instance, the rock convergence dynamically changes the pore volume in some region. The convergence process is controlled by a number of additional factors, and the change of 
the pore volume is described by a nonlinear differential equation that has to be integrated into the global equation system for the relevant region. The relevance of effects depends on the environment parameters and may change during the simulation time. For example, rock convergence gets irrelevant as soon as a final value of porosity is reached.

During a simulation of the processes in a repository for radioactive waste, numerous different effects have to be considered additionally to the basic process. Various physical models that use different equations and variables have to be implemented. Specific challenges in developing the structure of a simulator program are to enable a flexible choice of models for different regions of the modeled area, their combination during a simulation and an easy way to extend the program by new models and effects.

The program code of RepoTREND is designed as a framework for solving a general nonlinear equation set. Different physics are realized as models in a library form. A model is defined as a base model using a certain set of variables with the possibility to build other models from this base model by variable switching (according to the current system state) and by modifications due to the additional effects (additional terms, modified equation parameters etc.).

Therefore, a model is defined by certain equation(s) of state and certain routines for taking account of the relevant effects, organized as a library of equations and a library of effects. This code structure makes it easy to incorporate new equations and effects.

Different models can be assigned to different grid blocks. Any grid block is defined by its own set of equations. The full set can be reduced:

- by Schur-complement method, which reduces the full set to a primary set relevant for the appropriate block row in the common matrix system,

- by reduction of the implicit level of the primary set.

These operations are performed on individual grid blocks.

The coupling of physical models is described in implicit manner: directly solve the linear couplings between variables by including all equations (block rows prepared for any grid block) in the same matrix system.

Procter and Gamble Student poster award:

\title{
References:
}

Reiche, T. RepoTREND - Das Programmpaket zur integrierten Langzeitsicherheitsanalyse von Endlagersystemen, Report GRS-413, GRS Braunschweig, 2016 (https://www.grs.de/publikation/grs-413)

Homepage RepoTREND: https:/www.grs.de/en/research-development/waste-management-repotrend Acceptance of Terms and Conditions:

Click here to agree

\section{Modeling of wavefields in saturated elastic porous medium based on thermodynamically compatible system theory for multiphase mixture}

\author{
Author(s): Galina Reshetova ${ }^{1}$ \\ Co-author(s): Evgeniy Romenski ${ }^{2}$ \\ ${ }^{1}$ Institute of Computational Mathematics and Mathematical Geophysics SB RAS \\ ${ }^{2}$ Sobolev Institute of Mathematics
}

Corresponding Author(s): kgv@nmsf.sscc.ru

A multiphase model and its application to the wavefields numerical simulation are discussed for the description of compressible fluid flow in elastic porous medium. The proposed model is an extension of the unified model of continuum mechanics proposed in [1,2]. The derivation is based on the theory of thermodynamically compatible systems and on the model of nonlinear elastoplasticity combined with the multiphase compressible flow model 3. The governing equations of the model include the phase mass conservation laws, total momentum conservation law, equation for relative velocities of phases, equations for deformation gradient of the medium and balance equation for porosity. They 
form a hyperbolic system of conservation form equations and satisfy fundamental laws of thermodynamics. Two types of phase interaction are introduced in the model: phase pressure relaxation to the common value and interfacial friction. The inelastic deformation also can be accounted by the source terms in the equation for the deformation gradient. The formulated model can be used for study a compressible fluid flow in a deformable elastoplastic porous medium in general, and for modeling wave propagation in a saturated porous medium in particular.

The governing equations for small amplitude wave propagation in the uniform porous medium saturated with a single fluid medium are derived. They form the first-order hyperbolic PDE system written in terms of stress and velocities and, like in Biot's model, predict three type of waves: fast and slow longitudinal waves and shear wave existing in the fluid-saturated porous medium. For the numerical solution of these equations an efficient numerical method based on staggered-grid finite difference scheme is used. The solution of some numerical test problems is presented and discussed.

1. Dumbser, M., Peshkov, I., Romenski, E., Zanotti, O. High order ADER schemes for a unified first order hyperbolic formulation of continuum mechanics: Viscous heat-conducting fluids and elastic solids, Journal of Computational Physics, Vol. 314, P. 824-862, 2016.

2. Dumbser, M., Peshkov, I., Romenski, E., Zanotti, O. High order ADER schemes for a unified first order hyperbolic formulation of Newtonian continuum mechanics coupled with electrodynamics, Journal of Computational Physics, Vol. 348, P. 298-342, 2017.

3. Romenski, E., Belozerov, A.A., Peshkov, I.M. Conservative formulation for compressible multiphase flows, Quarterly of Applied Mathematics, Vol. 74(1), P. 113-136, 2016.

Procter and Gamble Student poster award

References:

Acceptance of Terms and Conditions:

Click here to agree

\title{
Machine Learning for Data Assimilation, Uncertainty Quantifica- tion of System Performance and Optimization of Controls
}

\author{
Albert Reynolds ${ }^{1}$; Zhenyu Guo ${ }^{1}$; Emilio Sousa ${ }^{1}$ \\ ${ }^{1}$ University of Tulsa
}

Corresponding Author(s): reynolds@utulsa.edu

We consider a system whose measurable outputs (observed data) depend on parameters (system variables) and controllable inputs (controls), where the system is represented by a system of discrete equations obtained by applying the finite volume method to an initial-boundary-value problem for a set of coupled evolutionary nonlinear partial differential equations. This discrete system represents the forward model for predicting the behavior of the system given the input controls and the coefficients (dependent parameters) in the discrete system. These coefficients (parameters) are the components of the model, or vector of model parameters, $\mathrm{m}$, where $\mathrm{m}$ is treated as a random vector. It is assumed that information independent of the evolutionary behavior of the system is available in the form of a prior model for $m$ where the prior model is represented by a prior probability density function (pdf). The posterior pdf, conditional to the observed data, can then be formally constructed by Bayes theorem.

In problems of interest, there are an infinite number of plausible models, i.e., models that are consistent with both the prior information and the observed dynamic data. Generation of such models using data assimilation techniques is relatively easy, e.g., by gradient-based randomized maximum 
likelihood, however, the goal is not simply to find such models but to find an ensemble of models that characterizes the posterior uncertainty in $\mathrm{m}$, i.e., in the Bayesian framework, the ensemble members should be samples from the posterior pdf. Given a correct sampling of the posterior pdf, the uncertainty in future performance predictions of the system can be characterized by solving the forward model for each model in the ensemble.

We provide a framework for data assimilation and sampling the posterior pdf that uses machine learning throughout. Our focus is on support vector regression (SVR) although, potentially, online deep learning neural networks also can be used. Two basic ideas are that (i) one can develop a reliable SVR proxy for the forward model of the system during the data assimilation process by following the iterative procedure developed in which one updates the proxy model as the data assimilation proceeds; (ii) by using the final SVR proxy developed to replace the computationally expensive forward model, one can afford to perform Metropolis-Hasting Markov chain Monte Carlo (MH-MCMC) to construct parallel Markov chains of sufficiently long length to ensure that the chains converge to the posterior pdf. Using the SVR proxy, the cost of computing the value of the posterior pdf at any proposed state $\mathrm{m}$ is very inexpensive and correct uncertainty characterization incurs a computational cost which is equivalent to at most a few thousand runs of the forward model. Using an SVR training algorithm with a different iterative updating designed for the specific application, we develop a computationally efficient procedure to optimize controls. One lesson learned is that insight into the mathematics/physics/objectives of the problem considered informs the proper application of SVR. Examples presented pertain to reservoir history matching, prediction and uncertainty quantification.

\section{Procter and Gamble Student poster award:}

\section{References:}

Acceptance of Terms and Conditions:

Click here to agree

\section{Test}

Corresponding Author(s): sabastian.riedel@interpore.org

test

Procter and Gamble Student poster award:

References:

Acceptance of Terms and Conditions:

993

\section{test contribution}

Corresponding Author(s): sabastian.riedel@interpore.org

Procter and Gamble Student poster award:

References:

Acceptance of Terms and Conditions: 


\title{
Experimental and numerical study of the compression dependency of the permeability of nonwoven
}

\author{
Sarah Staub $^{1}$; Ralf Kirsch ${ }^{1}$; Stefan Rief ${ }^{1}$ \\ ${ }^{1}$ Fraunhofer ITWM \\ Corresponding Author(s): rief@itwm.fraunhofer.de
}

Nonwoven media play a crucial role in several technical applications, e.g. filtration processes. Due to large pressure drops, compression may occur leading to the decrease of the flow permeability of the medium. The permeability dependency is quite important when it comes to predicting the behavior of a filter element by means of simulation.

In this talk, we study the compression dependency of the permeability of a nonwoven. Therefore, we use the DMA measuring device to plastically deform the nonwoven. In a second step, the permeability is measured by the TEXTEST FX 3300.

The numerical study is based on $\mu \mathrm{CT}$ images of the uncompressed nonwoven and of the plastically deformed structures. The permeability is computed by the software GeoDict. The second simulation approach uses the image of the uncompressed nonwoven and virtually compresses it by the Fraunhofer structural mechanics code FeelMath and, finally, computes the permeability.

In the presentation, we will illustrate the three approaches to determine the compression dependency of the permeability of a nonwoven and give a comparative study of the results.

Procter and Gamble Student poster award:

I don't want to compete References:

Acceptance of Terms and Conditions:

Click here to agree

\section{The Multiscale Robin Coupled Method for two-phase flows in porous media}

\author{
Author(s): Franciane Rocha ${ }^{1}$ \\ Co-author(s): Fabricio Sousa ${ }^{1}$; Roberto Ausas ${ }^{1}$; Gustavo Buscaglia ${ }^{1}$; Felipe Pereira ${ }^{2}$ \\ ${ }^{1}$ University of Sao Paulo \\ ${ }^{2}$ Mathematical Sciences Department, The University of Texas at Dallas, Richardson, TX, USA \\ Corresponding Author(s): fr.franciane@gmail.com
}

The Multiscale Robin Coupled Method (MRCM) is a domain decomposition procedure that has been developed to efficiently approximate velocity and pressure fields for single-phase flows in highly heterogeneous porous media (see 1). It generalizes other well-established multiscale mixed methods $(2,3)$ that are based on domain decomposition and it adds great flexibility for the choice of interface spaces.

We investigate the approximation of two-phase flows in porous media using the MRCM to compute velocity fields. We consider an operator splitting strategy, where a scalar conservation law for the saturation of one of the phases and the velocity field are updated sequentially. The choice of parameters for the MRCM is thoroughly investigated, demonstrating its accuracy compared to other popular methods. We vary both the order of polynomials for interface spaces and the Robin parameter (that allows us to produce MMMFEM-like 2 and MHM-like 3 approximations for the velocity fields). The improved accuracy that the MRCM produces for single phase flow problems in the approximation of velocity and pressure fields is also observed for the saturation field. Thus, the original findings for the MRCM are fully preserved in the approximation of two-phase flows. Moreover, we produce a more accurate solution for problems with high contrast permeability coefficients when compared to other existing multiscale methods. The efficiency and accuracy of the MRCM make it a competitive scheme for uncertainty quantification in subsurface problems. 
Procter and Gamble Student poster award:

\title{
References:
}

1 R.T. Guiraldello, R.F. Ausas, F.S. Sousa, F. Pereira, and G.C. Buscaglia. The multiscale Robin coupled method for flows in porous media. J. Comput. Phys., 355:1-21, 2018.

2 T. Arbogast, G. Pencheva, M.F. Wheeler, and I. Yotov. A multiscale mortar mixed finite element method. SIAM Multiscale Model. Simul., 6(1):319-346, 2007.

3 R. Araya, C. Harder, D. Paredes, and F. Valentin. Multiscale hybrid- mixed method. SIAM J. Numer. Anal., 51(6):30505-3531, 2013. Acceptance of Terms and Conditions:

Click here to agree

980

\section{Robust discretizations for fluid-flow problems in deformable porous media}

\author{
Carmen Rodrigo $^{1}$; Francisco Gaspar ${ }^{2}$; Xiaozhe $\mathrm{Hu}^{3}$; James Adler ${ }^{3}$; Peter Ohm ${ }^{3}$; Ludmil Zikatanov ${ }^{4}$ \\ ${ }^{1}$ Universidad de Zaragoza \\ ${ }^{2}$ University of Zaragoza \\ ${ }^{3}$ Tufts University \\ ${ }^{4}$ The Pennsylvania State University
}

Corresponding Author(s): carmenr@unizar.es

The numerical simulation of the coupling between mechanical deformation and fluid flow in porous media has become of increasing importance due to the wide application of these models in different fields ranging from geomechanics and petroleum engineering, to biomechanics.

The governing systems of partial differential equations of poroelastic models involve parameters which typically vary over several orders of magnitude from one application to another, making its stable discretization and efficient solution a challenging task.

Robust discretizations with respect to all the physical parameters are needed for this type of problems to obtain reliable numerical solutions. This is a very important task, and some efforts are being carried out in this address by the scientific community.

In this talk, we discuss about stable discretizations for poroelastic problems, for which stability estimates uniformly independent on the physical and discretization parameters are derived.

Procter and Gamble Student poster award:

References:

Acceptance of Terms and Conditions:

Click here to agree

598

\section{Understanding the behavior of BTEX on the vadose zone using simple 1-D finite differences modeling}

\author{
JAVIER RODRIGO-ILARRI $^{1}$; María-Elena Rodrigo-Clavero ${ }^{2}$; Luis Romero-Ballesteros ${ }^{\text {None }}$ \\ ${ }^{1}$ UNIVERSITAT POLITECNICA VALENCIA \\ ${ }^{2}$ Universitat Politècnica de València
}


Corresponding Author(s): jrodrigoilarri@gmail.com

This work shows the results obtained when applying VLEACH, a simple one-dimensional finitedifference model, to analyze the contamination caused by a BTEX spill coming from a set of underground storage tanks located on a new fuel station in Valencia.

Specific studies of the impact of fuel spills on the vadose zone are currently required when trying to obtain the environmental permits for new fuel stations. The development of One-Dimensional mathematical models of fate and transport of BTEX on the vadose zone can therefore be used to understand the behavior of the pollutants and identify the main environmental consequences that should be addressed and controlled during the operational phase of the facilities.

The VLEACH model is used. VLEACH - a simple One-Dimensional Finite Different Vadose Zone Leaching Model - uses an numerical approximation of the Millington Equation, a theoretical based model for gaseous diffusion in porous media. This equation has been widely used in the fields of soil physics and hydrology to calculate the gaseous or vapor diffusion in porous media.

The model describes the movement of organic contaminants within and between three different phases: (1) as a solute dissolved in water, (2) as a gas in the vapor phase, and (3) as an absorbed compound in the soil phase. Initially, the equilibrium distribution of contaminant mass between liquid, gas and sorbed phases is calculated. Transport processes are then simulated. Liquid advective transport is calculated based on values defined by the user for infiltration and soil water content. The contaminant in the vapor phase migrates into or out of adjacent cells based on the calculated concentration gradients that exist between adjacent cells. After the mass is exchanged between the cells, the total mass in each cell is recalculated and re-equilibrated between the different phases. At the end of the simulation, (1) an overall area-weighted groundwater impact for the entire modeled area and (2) the concentration profile of BTEX on the vadose zone are calculated.

Procter and Gamble Student poster award:

I would like to compete in the Procter and Gamble Student award References:

Acceptance of Terms and Conditions:

Click here to agree

\section{Application of Yield Stress fluids porosimetry Method and pore- network modelling to characterize the pore size distribution of packs of spherical beads}

Antonio RODRÍGUEZ DE CASTRO ${ }^{1}$; Mehrez AGNAOU $^{2}$; Azita AHMADI-SÉNICHAULT ${ }^{1}$; Abdelaziz OMARI $^{3}$

\footnotetext{
${ }^{1}$ Arts et Métiers ParisTech

${ }^{2}$ University of Waterloo

${ }^{3}$ Bordeaux-INP
}

Corresponding Author(s): antonio.rodriguezdecastro@ensam.eu

The Pore Size Distribution (PSD) is known to profoundly influence the movement and distribution of fluids inside a porous medium as well as the transport and reaction of chemicals. In this respect, some properties that are intimately linked to the PSD, e.g. capillary pressure curves, are important inputs to reservoir simulation software and provide valuable information for management and decision making. Despite the rapid progress in non-destructive imaging techniques such as X-ray Computed Tomography, Mercury Intrusion Porosimetry (MIP) is still the most commonly used method to determine PSDs although its toxicity is well established. Recently, Yield Stress fluids porosimetry Method (YSM) has been identified as a promising clean alternative to MIP 1. This technique is based on the particular percolation patterns followed by yield stress fluids, which only flow through certain pores when injected at a given pressure gradient. Indeed, as the pressure gradient is increased, smaller and smaller pores are open to the flow of these fluids. In previous works, YSM was used to characterize 
natural and synthetic porous media [2,3], and the results were compared with MIP showing reasonable agreement. However, considerable uncertainty still remains regarding the pore dimension characterized by each method. This arises from the highly complex geometry of the interstices in real porous media, which contain tortuous pathways of extremely variable cross-sections and irregular shapes, and the different physical phenomena at stake in each method. Therefore, a critical stage for the validation of YSM consists in achieving successful characterisation of model porous media with well-known pore morphology and topology. With this objective in mind, a set of four packs of glass beads were characterized in the present work using different porosimetry methods. First, pore and throat size distributions were computed from a 3D digital image by using a pore-network modelling approach. Then, the generated pore networks were used to numerically simulate a MIP test. Finally, a set of YSM laboratory tests were performed in which a yield stress fluid was injected through the four packs of spherical beads at different flow rates and the stationary pressure drop was measured. The PSD determined from YSM tests was then compared to the results of MIP and network modelling, allowing the identification of the pore dimensions being characterized by each technique. The results of this research elucidate the causes of the discrepancies between the considered porosimetry methods and demonstrate the usefulness of the PSD provided by YSM when predicting transport in porous media.

Procter and Gamble Student poster award:

\section{References:}

1 Rodríguez de Castro, A., Omari, A., Ahmadi-Sénichault, A., Bruneau, D., Toward a New method of Porosimetry: Principles and Experiments, Transport in Porous Media, 101, 349-364 (2014). http://dx.doi.org/10.1007/s11242013-0248-5

2 Rodríguez de Castro, A., Ahmadi-Sénichault, A., Omari, A., Savin, S., Madariaga, L.-F., Characterizing porous media with the Yield Stress Fluids porosimetry Method, Transport in Porous Media 114, 213-233 (2016).http://dx.doi.org/10.1007/s11242-016-0734-7

3 Rodríguez de Castro, A., Ahmadi-Sénichault, A., Omari, A., Using Xanthan Gum Solutions to Characterize Porous Media with the Yield Stress Fluid Porosimetry Method: Robustness of the Method and Effects of Polymer Concentration, Transport in Porous Media 122(2), 357 - 374 (2018).http://doi.org/10.1007/s11242018-1011-8 Acceptance of Terms and Conditions:

Click here to agree

\section{Dynamics and reversibility of tracer dispersion in time depen- dent flows inside rough fractures}

Yanina Lucrecia Roht ${ }^{1}$; Ricardo Chertcoff ${ }^{2}$; Jean-Pierre Hulin $^{3}$; Harold Auradou ${ }^{\text {None }}$; Irene Ippolito ${ }^{2}$

${ }^{1}$ Grupo de Medios Porosos, Facultad de Ingeniería, Universidad de Buenos Aires

${ }^{2}$ Universidad de Buenos Aires, Facultad de Ingeniería, Grupo de Medios Porosos

${ }^{3}$ FAST Laboratory CNRS

Corresponding Author(s): lucrecia.roht@gmail.com

We compare the mixing of a dyed and a clear fluid by constant and time-dependent flows inside a transparent Hele-Shaw cell (length: $400 \mathrm{~mm}$, width $50 \mathrm{~mm}$, aperture $\mathrm{H}=0.4 \mathrm{~mm}$ ) with randomly distributed circular obstacles (diameter $\mathrm{d}=1.4 \mathrm{~mm}$, height $\mathrm{H}$ ) covering $20 \%$ of the area of the cell walls $^{\wedge}\{5\}$. A dyed and a transparent solution of same viscosity $(1.8 \mathrm{mPa} . \mathrm{s})$ and density $\left(1.95 \mathrm{~g} / \mathrm{cm}^{\wedge}\{3\}\right)$ with a dye diffusion coefficient D_ $\{\mathrm{m}\}=4.0610-4 \mathrm{~mm}^{\wedge}\{2\} / \mathrm{s}$ are initially separated by a linear front perpendicular to the mean flow parallel to the length. Three types of experiments are performed: transmission in which the mean flow velocity $\mathrm{U}$ is constant, $e c h o^{\wedge}\{2\}$ in which flow is reversed after a preset time Tinv (penetration distance T_\{inv $\}$ U) and oscillating flow $w^{\wedge}\{4\}$ with a sinusoidal flow rate variation of period $\mathrm{T}$, amplitude $\mathrm{A}$ and zero mean value. The instantaneous concentration map is obtained by measuring light absorption through the cell with a suitable calibration. One determines at any given time the mean location and width of the front: their variation with time over a chosen time lapse provides a global dispersivity ${ }_{-}\{\mathrm{d}\}=\mathrm{D} / \mathrm{U}$ and mean velocity $\mathrm{U}$. 
Reference transmission experiments performed at different Péclet numbers $\left(\mathrm{Pe}=\mathrm{UH} / \mathrm{D} \_\{\mathrm{m}\}\right)$ show, for 1 lt Pe \lt 30 a geometrical dispersion regime (ld leqsim cst) ${ }^{\wedge}\{1,3\}$ associated to the flow disorder introduced by the obstacles and, for Pe \gt 30, Taylor dispersion with ld \propto Pe (for Pe lt 1 molecular diffusion would be dominant with ld \propto $1 / \mathrm{Pe}$ ).

Echo dispersion experiments with only one injection-suction cycle provide a dispersivity significantly larger at the end of the injection ( $\left.\mathrm{t}=\mathrm{T}_{-}\{\mathrm{inv}\}\right)$ than of the cycle $(\mathrm{t}=2 \mathrm{~T}\{$ inv $\})$, implying a partial reversibility of dispersion with respect to the flow reversal. Both $\operatorname{ld}\left(\mathrm{T}_{-}\{\mathrm{inv}\}\right)$ and $\mathrm{ld}\left(2 \mathrm{~T} \_\right.$inv $\left.\}\right)$ increase with Tinv and reach different limits $1 \_\{d\}^{\wedge}\{\operatorname{trans}\}>1 \_\{d\}^{\wedge}\{e c o\}$ at long times This is illustrated by comparing the the displacement fronts contours at $\mathrm{t}=\mathrm{T}_{-}\{$inv $\}$and $2 \mathrm{~T}\{$ inv $\}$

For oscillating flows, geometrical dispersion only occurs for large oscillation amplitudes $(\mathrm{A}=8$ and $40 \mathrm{~mm}$ ) and up to Péclet numbers $\mathrm{Pec}=80$ and 300 increasing with A: the corresponding dispersivity is smaller than for transmission and increases with $\mathrm{A}$. Above $\mathrm{Pe} \_\{\mathrm{c}\}(\mathrm{A})$, the results are similar to those obtained for flat cell walls ${ }^{\wedge}\{4\}$ and depend of the ratio $\backslash$ tau_ $\{\mathrm{m}\} / \mathrm{T}$ of the transverse diffusion time $\mathrm{H}^{\wedge}\{2\} / \mathrm{D}_{-}\{\mathrm{m}\}$ and the period T. For $\backslash \operatorname{tau} \_\{\mathrm{m}\} / \mathrm{T} \backslash \mathrm{ll} 1$ one has quasi-stationary Taylor dispersion modulated with the period T and, for $\backslash$ tau_\{m\}/T $\backslash$ gg 1, partly reversible Taylor dispersion in which the periodic distortions of the front follow those of the Poiseuille profile. Compared to echo experiments, oscillating flows provide additional information when the number of oscillations increases: $1 \_\{d\}$ oscillates and reaches a limit intermediate between $1 \_\{d\}^{\wedge}\{\operatorname{trans}\}$ and $1 \_\{d\}^{\wedge}\{e c o\}$.

\title{
Procter and Gamble Student poster award:
}

\section{References:}

1) Boschan, A., Ippolito, I., Chertcoff, R., Auradou, H., Hulin, J.P., Water Resour. Res. 44, W06420 (2008)

2) Hulin, J.P., Plona, T.J., Phys. Fluids A 1, 1341-1347 (1989)

3) Ippolito, I., Daccord, G., Hinch, E.J., Hulin, J.P., J. Contam. Hydrol. 16, 87-108 (1994)

4) Roht, Y.L., Auradou, H., Hulin, J.-P., Salin, D., Chertcoff, R., Ippolito, I. Phys. Fluids 27, 103602 (2015)

5) Roht, Y.L., Chertcoff, R., Hulin, J-P., Auradou, H., I. Ippolito, I., Transp. Porous Med. 122(2), 421-436 (2018). Acceptance of Terms and Conditions:

Click here to agree

\section{Emptying of mesoporous materials: direct evidence for cavita- tion}

Etienne Rolley ${ }^{1}$; Victor Doebele ${ }^{2}$; Fabien Souris ${ }^{2}$; Laurent Cagnon ${ }^{2}$; Panayotis Spathis ${ }^{2}$; Pierre-Eienne Wolf ${ }^{2}$; Annie Grosman ${ }^{3}$; Isabelle Trimaille ${ }^{3}$

\author{
${ }^{1}$ LPENS, Paris, France \\ ${ }^{2}$ Institut Néel, Grenoble, France \\ ${ }^{3}$ INSP, Paris, France
}

Corresponding Author(s): rolley@lps.ens.fr

Cavitation, the formation of a vapor bubble in a metastable liquid, is relevant in many fields, ranging from engineering to natural sciences. In most cases, the occurrence of cavitation in porous materials is disregarded. Standard models assume that the evaporation of a fluid confined in a porous material requires the creation of a vapor path from the surface to the core of the material, via the propagation of the liquid-vapor interface. However, recent experiments have shown that cavitation does occur in some mesoporous silica materials. On the other hand, In model materials such as porous silicon ( $\mathrm{p}-\mathrm{Si}$ ) or porous alumina ( $\mathrm{p}-\mathrm{Al})$, cavitation has never been observed except for a single experiment on $\mathrm{p}-\mathrm{Si}$.

In order to clarify this fundamental issue, we have undertaken systematic experiments, using porous alumina ( $\mathrm{p}-\mathrm{Al}$ ) and porous silicon ( $\mathrm{p}-\mathrm{Si})$ as model materials. $\mathrm{p}-\mathrm{Al}$ and $\mathrm{p}$-Si present unconnected pores, which can be tailored in an ink-bottle geometry, that is cavities in contact with the gas reservoir through small constrictions. We use a large variety of fluids (helium, nitrogen up to the critical point, alkanes). Classical volumetric adsorption isotherms are measured together with optical interferometry and light scattering to obtain a direct signature of cavitation events. We present here the first set of results from these systematic studies.

First, we have studied hexane condensation in p-Al pores with diameter in the range 10-40 $\mathrm{nm}$. 
When closed with very small apertures, all samples exhibit a brutal emptying at the vapor pressure $\mathrm{P}=0.33$ Psat, a signature of cavitation. This value is close to the one expected for homogeneous cavitation in classical nucleation theory, suggesting that confinement effects are small down to 10 nm pore size.

Second, we have studied hexane and nitrogen condensation in $\mathrm{p}-\mathrm{Si}$ « duplex » samples made of a layer of large pores (mean cavity diameter 25 or $50 \mathrm{~nm}$ ) in contact with the vapor phase through an upper layer of small pores (mean neck diameter $12 \mathrm{~nm}$ ). In that case, white light interferometry allows to track independently the liquid fraction in both layers. We find that cavities exhibit a brutal emptying while the necks remain filled. This is again a signature of cavitation. It occurs at a vapor pressure in the range $0.5-0.7$ Psat, which rather points to heterogeneous cavitation. Surprisingly, we find that the cavitation thresholds strongly depend on the cavity geometry. Optical imaging of monolithic p-Si samples reveals that cavitation events are spatially correlated. This might be an evidence for pore-pore coupling effects in $\mathrm{p}$-Si.

Procter and Gamble Student poster award:

I don't want to compete References:

Acceptance of Terms and Conditions:

Click here to agree

\title{
Dissipative processes during two-phase flows: microfluidic ex- periments
}

\author{
Sophie Roman ${ }^{1}$; Cyprien Soulaine ${ }^{2}$; Anthony Kovscek ${ }^{3}$ \\ ${ }^{1}$ University of Orleans \\ ${ }^{2}$ BRGM, the French Geological Survey \\ ${ }^{3}$ Stanford University
}

Corresponding Author(s): sophie.roman@univ-orleans.fr

Multiphase flow in porous media is important in a number of environmental and industrial applications such as $\mathrm{CO} 2$ sequestration in geological formations, enhanced oil recovery, soil remediation, and energy storage technologies. Capillary, viscous and gravitational forces determine the dynamics of two-phase immiscible flow and the competition between these forces can lead to highly unstable flow. Previous studies of unstable two-phase flow at the core-scale have demonstrated that these processes are not described adequately by the standard two-phase extension of Darcy's law 1 . This is not surprising given that this Darcy-based approach relies on very strong assumptions, including scale separation between the local events and the large-scale phenomena as well as that the fluidfluid interface is locally stable at the pore-scale. More importantly, it is assumed that the shear stress exerted at the non-wetting/wetting fluid interface has no impact on large-scale behavior. Indeed, in the extension of Darcy's law the interactions between the fluids are neglected and the relative permeabilities represent the drag force due to the flow of each fluid over the solid surface. This approach neglects the possibility that momentum transfer between the two flowing phases may act also as a driving force. The interactions between both fluids, however, can be significant and the drag due to the momentum transfer across the fluid-fluid interfaces might be important. For more than 60 years, authors have questioned whether this viscous coupling effect is important or not. Recently, we observed that during drainage experiments, when the displaced fluid is being trapped in a porous medium, this phase is not immobile but shows a driven cavity flow due to the shear stress resulting from the displacement of the other phase that is still flowing, thus showing interfacial viscous coupling effects 2 .

In this work, we used microfluidic experiments to provide a detailed understanding of the magnitude of viscous coupling effects during two-phase flows and to understand the underlying flow mechanisms in order to develop a satisfactory quantitative model. For drainage experiments in micromodels, using the micro-PIV (Particle Image Velocimetry) technique, we measured velocity profiles and the profiles of local rate of dissipation of mechanical energy within trapped globules of water. We identified different regimes of viscous coupling that depend on the pore topology, the interfacial area, and the viscosity ratio between the phases. We found that the localization of the rate of viscous dissipation strongly depends on the topology of the pore space. 
By performing a detailed study of these viscous dissipative events, we explored the origins of dissipative processes at pore scale and their consequences on the upscaling of rock and fluid properties, i.e. relative permeabilities.

\title{
Procter and Gamble Student poster award:
}

\section{References:}

1 Aryana, S. A., and Kovscek A. R. (2012), Experiments and analysis of drainage displacement processes relevant to carbon dioxide injection, Physical Review E 86.

2 Roman S., Soulaine C., Abu AlSaud M. A., Kovscek A. R., and Tchelepi H. (2016), Particle velocimetry analysis

of immiscible two-phase flow in micromodels, Advances in Water Resources, 95, 199-211. Acceptance of Terms and Conditions:

Click here to agree

\section{Do deformation bands influence CO2 storage volume?}

\author{
Author(s): Carla Romano ${ }^{1}$; Carla Romano ${ }^{1}$ \\ Co-author(s): James Minto ${ }^{1}$; Charlotte Garing ${ }^{2}$; Christopher Zahasky ${ }^{2}$; Sally M. Benson ${ }^{2}$; Zoe K. Shipton ${ }^{1}$; \\ Rebecca Lunn ${ }^{1}$ \\ ${ }^{1}$ University of Strathclyde \\ ${ }^{2}$ Stanford University
}

Corresponding Author(s): carla.romano@strath.ac.uk, carla.romano17@gmail.com

Understanding the role of geologic heterogeneities in porous media is a key factor for providing accurate estimation of $\mathrm{CO} 2$ storage capacity and integrity. Multiple studies have been conducted to understand the effect of heterogeneities on $\mathrm{CO} 2$ distribution and migration. None have yet focused on investigating the influence of small-scale structural heterogeneities within the reservoir. In highly porous sandstones (porosity $>12 \%$ ) strain localization results in the development of deformation bands, rather than fractures. Since it is common that porous sandstones in aquifers are affected by deformation, we should expect that deformation bands may be present in potential storage sites. Individual deformation bands form due to fracturing and sliding of the grains, generally decreasing porosity and permeability of the rock. As strain increases, clusters of deformation bands may coalesce, often accompanied by dissolution and cementation, further reducing the porosity and permeability. These structures are well below the resolution of seismic data, and are clustered so may not be detected in borehole surveys, yet if the capillary entry pressure of the deformation bands is not exceeded, the potential reservoir storage volume is reduced.

To understand the role of deformation bands on fluid flow, core flooding experiments were conducted on cores of Navajo sandstone taken from the damage zone surrounding a small fault. The first core sample is characterized by a single diagonal deformation band; the second has a dense network of deformation band clusters which divide the rock into several compartments. We conducted both single-phase radiotracer pulse experiments using micro-PET imaging and two-phase core flooding experiments using medical X-ray CT scanner. For the multiphase experiment at reservoir pressure conditions, $100 \% \mathrm{CO} 2$ or $\mathrm{N} 2$ were injected into water saturated core samples, imposing progressively higher flow rates and measuring the capillary pressure curves. For better understanding the complicated geometry of the deformation bands in $3 \mathrm{D}$, we also acquired scan images with higher resolution micro-CT scanner.

Experiments show that in both samples, the bands hinder the fluid flow. However, the effect on flow depends on the deformation band network geometry and the properties of the bands. In the single deformation band sample, different steady state $\mathrm{CO} 2$ saturation levels occur in the host rock due to the presence of the band. However, in the core containing clusters of bands with pressure solution, the fluid is compartmentalized despite a high differential pressure across the core. At the end of the drainage experiment, several compartments have zero N2 saturation. These results have implications for $\mathrm{CO} 2$ storage projects: unanticipated compartmentalization in the storage reservoir could strongly effects the injectivity and migration of the $\mathrm{CO} 2$ plume by reducing potential $\mathrm{CO} 2$ storage volume. 
Procter and Gamble Student poster award:

References:

Acceptance of Terms and Conditions:

Click here to agree

592

\title{
Chemically-driven convective dissolution in a porous medium
}

\author{
Author(s): Laurence Rongy ${ }^{1}$ \\ Co-author(s): Mamta Jotkar ${ }^{2}$; Anne De Wit ${ }^{3}$ \\ ${ }^{1}$ Universite libre de Bruxelles (ULB) \\ ${ }^{2}$ Université Libre de Bruxelles \\ ${ }^{3} U L B$
}

Corresponding Author(s): lrongy@ulb.ac.be

\begin{abstract}
Chemical reactions are known to induce convective dissolution in partially miscible stable density stratifications in a porous medium. We conduct a linear stability analysis using frozen time approximation to identify the critical conditions for instability and numerically study the convective dynamics of a system where the dissolving species A decreases the density of the host phase upon dissolution, reacts with a solute $\mathrm{B}$ to produce $\mathrm{C}$ via an $\mathrm{A}+\mathrm{B} \rightarrow \mathrm{C}$ type of reaction. While the equivalent non-reactive case is dominated by diffusion alone, it is possible to achieve enhanced convective dissolution and increase the flux of dissolution of species A by increasing the difference between the Rayleigh numbers of the product $\mathrm{C}$ and reactant $\mathrm{B}$ above a certain critical value. This critical value obtained from the linear stability analysis is consistent with nonlinear simulations. We show that the dissolution flux can be further enhanced by increasing the ratio of the initial concentration of reactant B with respect to A. Results presented here show that chemical reactions can initiate and strongly influence convective mixing, which has to be accounted for in various geological situations such as the dissolution of porous rocks or the dissolution of carbon dioxide at the bottom of oceans.
\end{abstract}

Procter and Gamble Student poster award:

I would like to compete in the Procter and Gamble Student award References:

Acceptance of Terms and Conditions:

Click here to agree

939

\section{Multiscale Image Based Modelling of Plant-Soil Interaction}

Tiina Roose ${ }^{1}$

${ }^{1}$ University of Southampton

Corresponding Author(s): t.roose@soton.ac.uk

In this talk I will describe a state of the art image based model of the soil-root interactions, i.e., a quantitative, model of the rhizosphere based on fundamental scientific laws. This will be realised by a combination of data rich fusion of structural and chemical imaging methods, integration of experimental efforts to both support and challenge modelling capabilities at the scale of underpinning biophysical processes, and application of mathematically sound homogenisation/scale-up techniques 
to translate knowledge from rhizosphere to field scale. The specific science question I will address with these techniques is how to translate this knowledge from the single root scale to root system, field and ecosystem scale in order to predict how the climate change, different soil management strategies and plant breeding will influence the soil fertility.

Procter and Gamble Student poster award:

I don't want to compete References:

Acceptance of Terms and Conditions:

Click here to agree

\title{
Flow velocity distribution of a shear-thinning fluid in porous me- dia
}

\author{
Clément Roques $^{1}$; Noud Kuilder ${ }^{1}$; Filippo Miele ${ }^{2}$; Pietro De Anna ${ }^{\text {None }}$ \\ ${ }^{1}$ Department of Earth Sciences, Institute of Geology, ETH Zürich, Zürich, Switzerland \\ ${ }^{2}$ UNIL University
}

Corresponding Author(s): clement.roques@erdw.ethz.ch

The complexity of flow topology in natural environments, chemical transport and resulting reactivity, arises from the random spatial organization of the host medium, the interaction at the fluid-solid interfaces and the rheological fluid properties. The rheology of non-Newtonian fluids (that show a shear rate dependence of their viscosity) combined with the complex pore structure present in most man-made and natural porous media makes challenging to predict the flow distribution and the associated solutes spreading. We present a microfluidics set-up that is used to quantify the porescale velocity distribution of Newtonian and a shear-thinning fluid. The method is based on tracking suspended fluorescent particles, via time-lapse video-microscopy, flowing through a microfluidics replica of confined media. The objective is to investigate how the fluid rheology impacts its velocity distribution and macroscopic dispersion and how these behaviors diverge from what is known for Newtonian fluids.

Procter and Gamble Student poster award:

References:

Acceptance of Terms and Conditions:

Click here to agree

\section{A numerical tool for computing the thermal radiative properties of digitally generated cellular materials}

\author{
Benoit Rousseau $^{1}$; Jerome Vicente ${ }^{2}$ \\ ${ }^{1}$ LTeN UMR CNRS 6607 \\ ${ }^{2}$ IUSTI UMR CNRS 7343 \\ Corresponding Author(s): benoit.rousseau@univ-nantes.fr
}

Refractory ceramic-based open-cell foams know a growing interest for the conception of high-temperatures energy conversion systems in reason of their remarkable characteristics such as high thermal-shock resistance and good corrosion resistance. These porous materials (porosity spans from 70 up to $95 \%$ ) 
can be described by an interconnected network of struts or ligaments delimiting cells, the association of cells in space ensuring the propagation of gaseous fluids. The foams are used in the design of volumetric solar receivers, heat exchangers, burner stabilizers, catalyst support among others. In this context, it appears crucial to propose an accurate determination of the thermo-physical properties of the foams at high temperatures (up to $1800 \mathrm{~K}$ ), in order to propose appropriate prediction of heat transfer behaviours for dimensioning afterwards suitable systems. From all the thermo-physical physical properties, the thermal radiative properties are less investigated because of the huge efforts required to directly measure them with experimental set-up. This framework makes that today numerical modelling is the best way today to provide the missing data.

On the last decade, the emergence of X-ray $\mu$-tomography in the field of thermal sciences, has resulted in the handling of 3D digitalized images, from which thermal properties have been quantitatively calculated. The same images are also able to provide a comprehensive description of the 3D statistical organization of the solid matter, the latter information being useful to generate virtual porous media. In this presentation, a home-made software named genMat, $(\mathrm{C}++, \mathrm{Qt})$ aiming at computing the thermal radiative properties of any cellular ceramics will be detailed. The foam generation is initialized, here, by seeding the centers of the cells according to a regular distribution following a tetrahedral layout. The positions of each center are perturbed according to a criteria that respects the distributions of distances between neighbouring centers, measured beforehand in real samples. A fast-marching technique coupled to a watershed algorithm governs then the growth and the segmentation of the pores. The strut geometry is finally controlled by a thickness growth method 1 . At the end of the numerical process, the final porosity can be adjusted with a fast-marching algorithm and the surface mesh can be provided through a marching cube method for subsequent computations.

genMat allows therefore the calculation, from the latter 3D reconstructed images of the macroscopic thermal radiative properties and in particular of the quantities (absorption and scattering coefficient, scattering phase function) useful for solving the Radiative Transfer Equation. A Monte Carlo algorithm allows the propagation of photons at the mesoscopic scale by taking account the spectral dependence of the complex refractive index of each solid phases composing the solid foam. The versatility of genMat is for example useful for connecting the extinctions coefficients computed on a set of 96 samples 2 with their respective textural parameters (nominal pore diameter and porosity among others). The resulting law and its accuracy will be will be compared to the other relationships available in the literature since the pioneering work of Hendricks and Howell in 19963.

\title{
Procter and Gamble Student poster award:
}

\section{I don't want to compete References:}

1 Guevelou, Simon, et al. "Evolution of the homogenized volumetric radiative properties of a family of $\alpha$-SiC foams with growing nominal pore diameter.” Journal of Porous Media 18.10 (2015).

2 Guévelou, Simon, et al. "A simple expression for the normal spectral emittance of open-cell foams composed of optically thick and smooth struts." Journal of Quantitative Spectroscopy and Radiative Transfer 189 (2017): 329-338.

3 Hendricks, T. J., and J. R. Howell. "Absorption/scattering coefficients and scattering phase functions in reticulated porous ceramics." Journal of Heat Transfer 118.1 (1996): 79-87. Acceptance of Terms and Conditions:

Click here to agree

\section{Study of the coupled liquid and oxygen transfer in wood: Appli- cation to oak for cooperage}

\author{
Author(s): Claire Roussey ${ }^{1}$ \\ Co-author(s): Julien Colin ${ }^{1}$; Rémi Teissier du $\operatorname{Cros}^{2}$; Joel Casalinho ${ }^{1}$; Patrick Perré ${ }^{1}$ \\ ${ }^{1}$ Laboratoire de Génie des Procédés et Matériaux \\ ${ }^{2}$ Chêne\&Cie
}

Corresponding Author(s): claire.roussey@centralesupelec.fr 
Wine or spirit aging in oak barrel modifies organoleptic quality of the beverage due to two main phenomena: firstly, the wood releases aromatic compounds which enrich the liquid and, secondly, transfer properties of wood allow a mild oxygenation all along maturation. This oxygenation may be affected by the width of the wood rings, the oak species and intensity of toasting in cooperage. In this paper, a set of experiments is presented for a better understanding of $\mathrm{O} 2$ transfer in wood, in relation with its intrinsic parameters.

The oxygen diffusion through wood is analysed using a custom experimental device. Wood samples are placed in an airtight holder to obtain 1-D gaseous transfer. To that purpose, a plug is placed at the back face of the sample to avoid mass transfer. The measurement principle consists in submitting the front face to a sudden change of $\mathrm{O} 2$ concentration and measuring the evolution of $\mathrm{O} 2$ concentration at the back face using optical oxygen sensors (First Sensors, XYO). This experiment reveals that the growth ring width has a strong impact on oxygen diffusion (ten times faster for fine grain than for coarse grain).

Oxygen transfer is not only related to the intrinsic properties of the wood. It also depends on the physical state of this porous media, mainly due to its hygroscopic properties. The oxygen diffusion through the barrel is reduced by the presence of liquid in wood. Thus, it is necessary to investigate the depth of impregnation front during maturation in barrels.

The moisture content field in wood is studied using a 2-D X-ray imaging system composed of an X-ray microfocus source (Hamamatsu L8601-01) and a digital X-ray detector (Fibre Optic Imagestar, Photonic Science). One face of the sample is in contact with either distilled water or a $40 \%$ diluted hydro-alcoholic solution. Images are recorded at different impregnation times for several months. An image processing has been developed to automatically analyse the raw data. In particular, image correlation is used to follow the deformation field. So, we can monitor the migration of the hygroscopic water - the so-called bound water causing the swelling of wood cell walls - and of the liquid flow in the cell lumens. Depending on the wood anatomy and the nature of liquid, the depth of liquid impregnation varies from $3 \mathrm{~mm}$ to $12 \mathrm{~mm}$.

Finally, the coupled liquid and oxygen transfer is intended to be studied by combining the two experimental devices, which is a great challenge due to additive experimental constraints. During the impregnation of liquid, followed by the X-ray imaging system, the oxygen content is measured on the front - inside liquid phase - and on the back side of the sample - inside gaseous phase - through non-invasive optical oxygen sensor spots (PreSens, PSt6). Several changes of O2 rate are submitted to the front face. This schedule will enable to assess the evolution of the average coefficient of O2 diffusion during wine and spirit maturation.

Procter and Gamble Student poster award:

References:

Acceptance of Terms and Conditions:

Click here to agree

\title{
Modeling moisture sorption and desorption in porous materials: A finite volume approach
}

\author{
Author(s): Pratanu Roy ${ }^{1}$; Yunwei Sun ${ }^{1}$; Stephen Castonguay ${ }^{1}$ \\ Co-author(s): Hom Sharma ${ }^{1}$; Elizabeth Glascoe ${ }^{1}$; Jennifer Knipe ${ }^{1}$ \\ ${ }^{1}$ Lawrence Livermore National Laboraotry
}

Corresponding Author(s): roy23@llnl.gov

Understanding the process of moisture sorption and desorption in porous materials is important for a variety of applications, such as electronic packaging, food packaging, hygrothermal building performance, and material aging and compatibility. In this work we model the sorption-desorption process in porous materials by coupling diffusion with equilibrium Henry's absorption and kinetic Langmuir adsorption. A finite volume method with implicit Euler time-stepping scheme was applied to solve the coupled unsteady diffusion-sorption equations. A level set framework with smoothed Heaviside function was used to implicitly treat the moisture concentration and flux at the material interface. The code was validated against existing experimental data for moisture uptake and 
outgassing in polymeric materials. Simulations were run to predict the vapor outgassing from an initially saturated polymeric material inside a closed container. The simulation results were compared with the experimental results and good agreement was observed. The proposed numerical method is shown to be well adapted for predicting interfacial mass transfer during sorption-desorption in multi-material system.

This work was performed under the auspices of the U.S. Department of Energy by Lawrence Livermore National Laboratory under Contract DE-AC52-07NA27344.

Procter and Gamble Student poster award:

I don't want to compete References:

Acceptance of Terms and Conditions:

Click here to agree

\section{A Study in Earthquake and Porous Medium: A Numerical Ap- proach in Disordered Systems}

Subhadeep Roy ${ }^{1}$

${ }^{1}$ Post Doctor , NTNU (Norwegian University of Science and Technology)

Corresponding Author(s): subhadeep.roy@ntnu.no

Disorder plays a crucial role while understanding the dynamics of statistical mechanical models, guided through threshold activated process. Such systems are observed to be useful to explain many phenomena that includes avalanches with time or external parameters like stress, pressure etc. One on such model, namely the fiber bundle model is a simple yet useful disordered system that can explain many aspects of failure processes, starting from laboratory scale (acoustic emissions) to a very large scale like earthquake. One part of the poster concentrates on how the model can explain the dynamics of fore-shocks and after shocks, observed in seismic events. Also the poster concentrates on the analog of this model in fluid mechanics, called the capillary tube model. This model takes account of the capillary pressure during flow of fluid within the tube. The capillary tube model is operated with a constant flow rate and the jump in pressure difference is observed across a number of such tubes interlinked with each other. A capillary tube model can be explored extensively in order understand the flow fluid (both single phase and two phase flow) through a porous medium. Such models can be observed in mean field limit, one dimension and higher dimensions as well as for complicated networks.

Procter and Gamble Student poster award:

References:

Disordered systems

Systems out of equilibrium

Threshold activated dynamics Acceptance of Terms and Conditions:

Click here to agree

\section{The effect of pore-scale heterogeneities on capillary trapping for geological storage of $\mathrm{CO} 2$}

Leonardo Ruspini ${ }^{1}$; Pål-Eric Øren ${ }^{1}$; Robert Sok $^{2}$; M. Saadatfar ${ }^{2}$; Anna Herring ${ }^{2}$; Mark Knackstedt ${ }^{2}$

\footnotetext{
${ }^{1}$ Petricore Norway
} 
${ }^{2} A N U$

Corresponding Author(s): leonardo.ruspini@petricore.com

Improving our understanding of the impacts of small-scale heterogeneities on residual or capillary trapping is crucial for assessing the potential for long term secure geological storage of $\mathrm{CO} 2$ in saline aquifers. In this work we describe an imaging and pore scale modelling study of the effect of pore scale heterogeneities (lamination) on capillary trapping of $\mathrm{CO} 2$ in laminated sandstone samples from the Precipice formation in the Surat basin. 3D X-ray computed tomography (micro-CT) was used to characterize the pore structure of the reservoir cores. We obtain in-situ pore-scale images of the distribution of super critical $\mathrm{CO} 2$ and brine within the samples during low capillary number drainage and imbibition flooding experiments under reservoir conditions. The 3D images are used directly as input to a quasi-static pore-scale simulation model. We simulate $\mathrm{CO} 2$ injection and subsequent brine imbibition and investigate the predictive capabilities of the simulation model by comparing on a pore by pore basis the simulated and CT-imaged fluid distributions. The simulated and experimental pore filling states are in good agreement for both the drainage and imbibition displacements. The comparison shows that the quasi-static model, that accounts for piston-type displacement, capillary dominated pore filling and throat snap-off, provides a good representation of both drainage and imbibition fluid distributions in laminated samples at low capillary numbers. Both the in-situ CT-images and the pore-scale simulations show that the presence of laminations result in heterogenous capillary pressure and non-uniform saturation distributions. At any given global capillary pressure, the $\mathrm{CO} 2$ saturation is higher in the coarse lamina than in the fine lamina. This delays $\mathrm{CO} 2$ breakthrough and strongly reduces drainage brine relative permeability. For imbibition, the non-uniform initial $\mathrm{CO} 2$ saturation distribution in the different laminas leads to increased capillary trapping of $\mathrm{CO} 2$. Our results show that laminations strongly affect both post-drainage and post-imbibition saturation distribution.

Procter and Gamble Student poster award:

\section{References:}

Acceptance of Terms and Conditions:

Click here to agree

\section{Reduced Darcy-Stokes model for flow in fractured porous me- dia}

Iryna Rybak $^{1}$; Stefan Metzger ${ }^{2}$

\footnotetext{
${ }^{1}$ University of Stuttgart

${ }^{2}$ Illinois Institute of Technology
}

Corresponding Author(s): rybak@ians.uni-stuttgart.de

Fractures are heterogeneities in geological formations whose transversal extension is significantly smaller than the lateral one. This fact motivates us to model fractures as lower-dimensional inclusions in the surrounding rock matrix. Usually, the flow in the fracture and in the matrix is described by the same model, which is the single- or two-phase Darcy's law. When the flow rate in the fracture is large enough, the Forchheimer extension of Darcy's law is applied in the fracture, while Darcy's law is used in the matrix. For modelling highly permeable fractures and open channels, a reduced model is proposed in 1, where the Brinkman equation is applied to describe the fluid flow in the fracture, and Darcy's law is used in the rock matrix. This model includes the conservation of mass, balance of normal forces and a simplified Beavers-Joseph-Saffman condition with zero tangential velocity at the fracture-matrix interface. However, assuming tangential velocity equal to zero at the interface is often non-physical. Therefore, we consider a Darcy-Stokes model with the Beavers-Joseph and Beavers-Joseph-Saffman interface conditions instead, and develop the corresponding reduced model considering the fracture as a lower-dimensional domain. 
In this talk, we will present a reduced model to describe fluid flow in fractured porous media. The fractures are modelled as lower-dimensional inclusions, which can store and transport fluid. The flow system of interest is single-phase, non-compositional and isothermal. The reduced model is obtained by averaging the Stokes equations across the fracture and coupling them to the full-dimensional Darcy's equations in the surrounding rock matrix. We prove the well-posedness of the proposed coupled model and validate the model via numerical simulations.

Procter and Gamble Student poster award:

References:

1 M. Lesinigo, C. D’Angelo, and A. Quarteroni. A multiscale

Darcy-Brinkman model for fluid flow in fractured porous media. Numer. Math., 117:717-752, 2011. Acceptance of Terms and Conditions:

Click here to agree

\title{
Wettability - Capillary Pressure Relationships in Ketton Rock: From Nano-Scale Fluid Films to Core-Scale
}

\author{
Maja Rücker ${ }^{1}$; Willem-Bart Bartels ${ }^{2}$; Tom Bultreys ${ }^{3}$; Marijn Boone ${ }^{4}$; Martin Blunt ${ }^{3}$; Ove Wilson ${ }^{5}$; Veerle Cnudde $^{6}$ \\ ; Steffen Berg ${ }^{7}$; Apostolos Georgiadis ${ }^{8}$; Paul Luckham ${ }^{9}$ \\ ${ }^{1}$ Imperial College London \\ ${ }^{2}$ Earth Sciences Department, Utrecht University \\ ${ }^{3}$ Department of Earth Science and Engineering, Imperial College London \\ ${ }^{4}$ Tescan XRE \\ ${ }^{5}$ Shell Global Solutions B.V. \\ ${ }^{6}$ UGCT-PProGRess, Ghent University \\ ${ }^{7}$ Shell Global Solutions International B.V, \\ ${ }^{8}$ Shell Global Solutions International B.V. \\ ${ }^{9}$ Department of Chemical Engineering, Imperial College London
}

Corresponding Author(s): m.rucker15@imperial.ac.uk

Commonly, capillary pressure - and relative permeability - saturation functions are determined to characterize the flow behaviour of two fluids in porous media. Close to the saturation end points the fluid films dominate the flow dynamics [1,2]. The properties of these fluid films depend on the chemical characteristics of the two fluids and the porous media, the rock structure and initial fluid distribution, as well as the corresponding wettability and wettability alteration processes of the system $[3,4,5]$. For example, in most oil-reservoirs, the oil-phase behaves as non-wetting phase when it penetrates a brine saturated rock. The thin brine-films formed during this process impact the wettability-alteration of the rock by the oil and consequently the wettability dependent flow dynamics occurring during flooding $[4,5,6]$. In this work we investigated the formation of fluid films during sample initialization by modelling the coverage of the rock surface by thin water films for capillary pressures close to connate water saturation. Furthermore, the relationship of surface coverage and wettability was assessed.

The nano-scale topography was obtained by AFM, and the coverage was computed by a sphere fitting algorithm from GeoDict. The results were compared to Amott spontaneous imbibition tests on samples prepared with a similar range of capillary pressures [6]. The tests were monitored with microCT [7] to assess the impact of wettability. Dynamic processes were captured with the Environmental MicroCT (EMCT) [8,9] while full core-scale images were acquired with HECTOR [10]micro-CT scanner at the Center for X-ray Tomography of Ghent University (UGCT, www.ugct.ugent.be).

The simulations on the nano-sale revealed that the surface coverage of water varies from $1 \%$ to $50 \%$ depending on the capillary pressure applied. Based on the pore-size distribution of the studied rock, the applied range of capillary pressure would be associated with a minor change in saturation. However, as the wettability alteration process depends on the contact between oil and the rock surface, the difference in coverage could cause significant differences in the core-scale wettability state [11]. 
Following this line of reasoning, the lower the capillary pressure at oil injection in a brine saturated sample, the higher the surface coverage of the rock by water films, and consequently less wettability alteration towards oil-wet can be expected. This hypothesis was assessed using production vs. time curves and droplet shapes from Amott-tests with samples prepared with a similar range of capillary pressures. The scan results confirmed the results based on the AFM image: The sample initialized at lower capillary pressure responded more water-wet while the sample initialized with higher capillary pressure appeared more oil-wet.

The results obtained at core- and sub-pore-scale show that the wettability of the system strongly depends on the capillary pressure applied during initialization and the surface structure of the rock. Such small-scale surface features cannot be resolved with microCT but only with techniques targeting smaller length-scales such as AFM. To correctly measure and model the core-scale wettability response, these features cannot be disregarded.

\title{
Procter and Gamble Student poster award:
}

\section{References:}

1 Lenormand, R., Zarcone, C., \& Sarr, A. (1983). Mechanisms of the displacement of one fluid by another in a network of capillary ducts. Journal of Fluid Mechanics, 135, 337-353.

2 Rücker, M., S. Berg, R. T. Armstrong, A. Georgiadis, H. Ott, A. Schwing, R. Neiteler, N. Brussee, A. Makurat, L. Leu . M. Wolf, F. Khan, F. Enzmann, M. Kersten (2015). From connected pathway flow to ganglion dynamics. Geophysical Research Letters, 42(10), 3888-3894.

3 Herminghaus, S. (2000). Roughness-induced non-wetting. EPL (Europhysics Letters), 52(2), 165.

4 Buckley, J. S. (2001). Effective wettability of minerals exposed to crude oil. Current opinion in colloid \& interface science, 6(3), 191-196.

5 AlRatrout, A., Raeini, A. Q., Bijeljic, B., \& Blunt, M. J. (2017). Automatic measurement of contact angle in pore-space images. Advances in Water Resources, 109, 158-169.

[6] Bartels, W. B., Rücker, M., Berg, S., Mahani, H., Georgiadis, A., Fadili, A., Brussee, N., Coorn, A., van der Linde, H., Hinz, C., Jacob, A., Wagner, C., Henkel, S., Enzmann, F., Bonnin, A., Stampanoni, M., Ott, H., Blunt, M., \& Hassanizadeh, S. M. (2017). Fast X-ray micro-CT study of the impact of brine salinity on the pore-scale fluid distribution during waterflooding. Petrophysics, 58(01), 36-47.

[7] Cnudde, V., \& Boone, M. N. (2013). High-resolution X-ray computed tomography in geosciences: A review of the current technology and applications. Earth-Science Reviews, 123, 1-17.

[8] Dierick, M., Van Loo, D., Masschaele, B., Van den Bulcke, J., Van Acker, J., Cnudde, V. \& Van Hoorebeke, L. (2014). Recent micro-CT scanner developments at UGCT. Nuclear Instruments and Methods in Physics Research B, 324, 35-40.

[9] Bultreys, T., Boone, M. A., Boone, M. N., De Schryver, T., Masschaele, B., Van Hoorebeke, L., \& Cnudde, V. (2016). Fast laboratory-based micro-computed tomography for pore-scale research: illustrative experiments and perspectives on the future. Advances in water resources, 95, 341-351.

[10] Masschaele, B., Dierick, M., Van Loo, D., Boone, M. N., Brabant, L., Pauwels, E., Cnudde, V. \& Van Hoorebeke, L. (2013). HECTOR: A 240kV micro-CT setup optimized for research. Journal of Physics: Conference Series, 463, 1, 012012.

[11] Bartels, W., Rücker, M., Boone, M., Bultreys, T., Mahani, H., Berg, S., Hassanizadeh, S., \& Cnudde, V. (2017). Pore-scale displacement during fast imaging of spontaneous imbibition. Paper presented at the International Symposium of the Society of Core Analysts, Vienna, Austria. Acceptance of Terms and Conditions:

Click here to agree

\section{Dynamics of Capillarity-Driven Water Invasion into Mixed-Wet Angular Pores}

\author{
Author(s): Ahmed M. Saad \\ Co-author(s): Maxim Yutkin ${ }^{2}$; TADEUSZ PATZEK ${ }^{3}$ \\ ${ }^{1} \mathrm{Mr}$ \\ ${ }^{2}$ King Abdullah University of Science and Technology \\ ${ }^{3}$ KAUST
}

Corresponding Author(s): ahmed.mohamedsaad@kaust.edu.sa 
Piston-type (PT) imbibition drives water invasion into porous media and incremental hydrocarbon recovery. Due to its importance, the process was well studied experimentally and theoretically down to pore level, e.g., [Oren and Bakke (1998), Patzek (2001)]. It was shown that other mechanisms, for example snap-off, are unfavorable for prolonged hydrocarbon recovery, because they trap and disconnect oil phase. Many researchers have tried to address the effects of wettability alteration on multiphase flow in porous media. The problem has been studied either by changing the wettability uniformly throughout micromodels [Zhao et al. (2016)] or by altering the wettability in different regions to produce a heterogeneous media [Lee et al. (2015)]. Yet, in these studies the wettability within individual pores is uniform.

We, in contrast, introduce heterogeneous wettability inside pores in a highly controlled and repeatable fashion. Here, we present a technique of fabrication of mixed-wet square capillaries with a selective silane coating. Capillary corners are left uncoated and preserve their water wetness, while capillary walls are coated and become oil-wet. Such a wettability distribution in pores mimics the microscopic state of wettability of asphaltenic oil reservoirs.

We have characterized the coating using various high-resolution tools: Atomic Force Microscopy (AFM), X-ray Photoelectron Spectroscopy (XPS) and Environmental Scanning Electron Microscopy (ESEM). All measurements confirm the success of the coating procedure, high quality of the coating and sharpness of its boundaries. AFM in force modulation mode shows a strong and sharp contrast in phase and amplitude values across the boundary between the coated and non-coated silica surfaces. XPS indicates an increased carbon concentration and a decrease of silicon concentration on the coated surface, compared with the non-coated side. Controlled condensation of water droplets with ESEM generates tiny droplets ranging in size from few to 50 microns. Again, a sharp boundary and an increase in the contact angle on the coated side are observed.

We study water invasion into square capillaries and observed differences between mixed wet capillaries, with sides having contact angle around $100^{\circ}$ and water-wet corners with contact angle around $40^{\circ}$, and fully oil-wet capillaries. First, we notice that upon drainage the fabricated mixed wet capillaries retain water corner filaments, which is a characteristic feature of water-wet capillaries. Fully oil-wet capillaries do not demonstrate such behavior. In addition, we study capillary rise dynamics of water in square capillaries and quantify the differences between mixed wet and fully water-wet capillaries. We also investigate the impact of corner water films on the imbibition dynamics.

In this study, for the first time, we present the 3D high-speed imaging of water/oil interface during forced-water-PT imbibition into the square cross-section tubes. We employ fluorescence laser sheet imaging in a plane orthogonal to the direction of flow. The spatial resolution in the direction parallel to flow is controlled by the frame rate of the camera and the thickness of the laser sheet. This technique eliminates the speed limit imposed by conventional laser 3D imaging techniques, which involve slow movement of the laser source/detector.

Indico rendering error

Could not include image: Cannot read image data. Maybe not an image file?

\section{Procter and Gamble Student poster award:}

\section{References:}

Øren, P. E., S. Bakke, and O. J. Arntzen, Extending predictive capabilities to network models, Soc. Pet. Eng. J., 3(4), 324-336, 1998.

Patzek, T. W., Verification of a complete pore network model of drainage and imbibition, Soc. Pet. Eng. J., 6(2), 144-156, 2001.

Lee, Hyundo, Seung Goo Lee, and Patrick S. Doyle. "Photopatterned oil-reservoir micromodels with tailored wetting properties." Lab on a Chip 15.14, 3047-3055, 2015.

Zhao, Benzhong, Christopher W. MacMinn, and Ruben Juanes. "Wettability control on multiphase flow in patterned microfluidics." Proceedings of the National Academy of Sciences of the United States of America 113.37, 2016. Acceptance of Terms and Conditions:

Click here to agree 


\title{
Mathematical Modeling and Simulation of Asphaltene Precipita- tion and Deposition during Miscible Displacement of Fluids in Porous Media
}

\author{
Nasser Sabet ${ }^{1}$; Hassan Hassanzadeh ${ }^{2}$; Jalal Abedi ${ }^{2}$ \\ ${ }^{1}$ Research Assistant \\ ${ }^{2}$ University of Calgary
}

Corresponding Author(s): nsabet@ucalgary.ca

\begin{abstract}
Asphaltenes are a solubility class of petroleum fluids, soluble in benzene or toluene and insoluble in excess amounts of low-molecular-weight alkanes such as n-pentane and n-heptane. The formation of solid or liquid asphaltene phase, known as asphaltene precipitation, occurs when the thermodynamic condition of oil sample changes. The precipitated asphaltene particles may attach to the walls of porous media, and therefore asphaltene deposition takes place. Light hydrocarbons such as light paraffins are widely used as diluents in the production and upgrading of heavy crudes. The addition of a diluent to heavy oil or bitumen alters the chemical forces within the mixture leading to the aggregation of asphaltenes. Precipitation of asphaltene from oil changes the viscosity behavior of the mixture and therefore influences the dynamics of viscous fingering. Also, asphaltene deposition alters the porosity and permeability of the porous media and modifies the flow paths leading to possible formation damage.

In this study, we model viscous fingering in porous media accounting for asphaltene precipitation and deposition. To do so, the mass balance equations for solvent and asphaltene are defined, and the highly nonlinear system of equations is solved numerically. Using the results of numerical simulations, we explain how asphaltene precipitation and the resulting formation damage caused by asphaltene deposition influence the growth of viscous fingers and subsequently the breakthrough time. This study provides a deeper insight into the influence of in situ generated particles (asphaltenes) on the dynamics of viscous fingering.
\end{abstract}

Procter and Gamble Student poster award:

References:

Acceptance of Terms and Conditions:

Click here to agree

\section{Mixing by dispersion in heterogeneous porous medium}

\author{
Chunendra K. Sahu ${ }^{1}$; Mike J. Bickle ${ }^{1}$; Jerome A. Neufeld ${ }^{1}$ \\ ${ }^{1}$ University of Cambridge
}

Corresponding Author(s): cks34@cam.ac.uk

Dispersion of chemicals in the sub-surface flows is common, for example, in the leakages of contaminants into ground water or during carbon sequestration where dispersion plays an important role in effective mixing between injected $\mathrm{CO}_{2}$ and brine. For large Peclet number flows, dispersion is quantified by the dispersion coefficient $D=\alpha U$, where $\alpha$ is the dispersivity and $U$ is the velocity. This problem has been studied previously in cases where the medium is homogeneous (constant $\alpha$ ) and the velocity is uniform (constant $U$ ). Therefore, advection-dispersion equation, $\frac{\partial C}{\partial t}+U \frac{\partial C}{\partial x}=\frac{\partial}{\partial x}\left(D \frac{\partial C}{\partial x}\right)$, is generally solved using a method of moving frame of reference, considering that the solute disperses equally in the forward and backward directions from the mean advective length, where $x=x_{m}=U t$ (Ogata \& Banks 1961). In contrast, numerous field data shows that $\alpha$ increases due to heterogeneity with the distance, $x$, away from the source and generally follows a 
power law (Neuman 1990). As a result, the rate of dispersion at field scale is significantly enhanced in comparison to classical predictions.

Here, we investigate models of flow-path dependent dispersivity using analytical and theoretical approaches to enable parameterisation of field-scale dispersion by suitably simple expressions. We assume that the dispersivity increases with a power of the length of the flow path such that $\alpha=$ $\alpha_{0} x^{\gamma}$, where $\alpha_{0}$ is the reference dispersivity at $x=0$ and $\gamma$ is the power law constant.

When $\gamma=0$, which means $\alpha$ does not depend on the flow path, the problem reduces to the classical case of mixing through molecular diffusion or constant dispersion. In this case, and as reported by previous researchers like Ogata \& Banks (1961), Gelhar \& Collins (1971) and others, we get a symmetrical error function solution for the concentration profile around the mean advective length $x_{m}$, where the dimensionless concentration, $C\left(x_{m}\right)$, is 0.5 (see the enclosed figure). When $0<$ $\gamma<1$, which means $\alpha$ increases with $x$, the concentration profile still obeys error function forms, however, with an asymmetric dispersion across $x_{m}$, resulting in $C\left(x_{m}\right)<0.5$. On the other hand, when $\gamma \geq 1$ such that $\alpha$ increases with $x$ linearly or with an even higher rate, the concentration decreases exponentially away from the source and does not exhibit error function behaviour. More precisely, for $\gamma=1, C=e^{-x / U t}$. In general, we find that $C\left(x_{m}\right)<0.5$ at $x_{m}$ for $\gamma>0$. As a result, dimensionless concentration is equal to unity only at the source and smaller elsewhere in the flow field, as shown in the figure.

In conclusion, as $\gamma$ increases, $C\left(x_{m}\right)$ decreases: This signifies that, with increasing heterogeneity of the medium, field scale values diverge further from the predictions of classical models. Therefore, variation of $\alpha$ with $x$ must be accounted in the calculations of concentration.

Procter and Gamble Student poster award:

I don't want to compete References:

Ogata, A. and Banks, R. B. 1961. A solution of the differential equation of longitudinal dispersion in porous media. U.S. Geol. Surv. Prof. Pap. 411-A, pp. A1-A7.

Neuman, S. P. 1990. Universal scaling of hydraulic conductivities and dispersivities in Geologic Media. Water Resour. Res. 26-8, pp. 1749-1758.

Gelhar, L. W. and Collins, M. A. 1971. General analysis of longitudinal dispersion in nonuniform flow. Water Resour. Res. 7-6, pp. 1511-1521. Acceptance of Terms and Conditions:

Click here to agree

\section{Scale-up of Laboratory Data for Surfactant-Alternating-Gas Foam EOR}

Rodrigo Orlando Salazar Castillo ${ }^{1}$; William R. Rossen ${ }^{2}$

${ }^{1}$ TU Delft

${ }^{2}$ Dept. of Geoscience and Engineering, TU Delft

Corresponding Author(s): r.o.salazar@tudelft.nl, w.r.rossen@tudelft.nl

Foam increases sweep efficiency during gas injection in enhanced oil recovery (EOR). Surfactant Alternating Gas, or SAG, is the preferred method to inject foam for both operational and injectivity reasons. Dynamic SAG corefloods are unreliable for direct scale-up to the field because of core-scale artefacts. In this study we report, fit and scale-up steady-state data taken in a Bentheimer core for different surfactant concentrations and total superficial velocities.

We measured steady-state data and fit them to a Local-Equilibrium foam model for scale-up to a dynamic foam process on the field scale using fractional-flow theory. We apply different parameterfitting methods (least-squares fit to entire range of foam qualities (gas fractional flow) and the method of Boeije and Rossen (2015)) and compare their fits to data and predictions for scale-up. We also test the implications for injectivity of complete foam collapse at irreducible water saturation.

Each of these sets of data predicts a shock front with sufficient mobility control at the leading edge 
of the foam bank. Mobility control improves with increasing surfactant concentration. In every case injectivity is better than with co-injection of gas and liquid. The results illustrate how a foam model without foam collapse at irreducible water saturation (Namdar-Zanganeh and Rossen, 2013) can greatly underestimate injectivity.

For the first time we examine how the method of fitting the parameters to coreflood data affects scaleup to field behaviour. The method of Boeije and Rossen (2015) does not give a unique parameter fit, but the predicted mobility at the foam front is nearly the same among the fits. Predicted injectivity does vary, however. The least-squares fit to all foam qualities is sensitive to the weighting applied to data at different foam quality. Gas injection in a SAG process depends especially on data at low injected water fraction and whether foam collapses at irreducible water saturation, which may not be apparent from a conventional scan of foam mobility as a function of gas fraction in the injected foam.

About half the previously published data used for this kind of study find a non-monotonic fractionalflow function, which would predict a failure of mobility control at the foam front (Rossen and Bruining, 2007). Many coreflood studies, starting with Apaydin and Kovscek (2001), find initial propagation of foam to the core outlet, and subsequent eruption of a much stronger foam there, followed by upstream propagation of that foam to fill the core. The initial eruption of stronger foam evidently reflects the capillary end effect. We also observed this behaviour in some cases. In fact, in our study, all cases of a non-monotonic fractional-flow function at steady state were preceded by this event earlier in the coreflood. When the event was did not occur, or steady-state data could be taken before it occurred, the fractional-flow function was monotonic and a successful SAG process was predicted. If it is found more generally that the non-monotonic behaviour reflects behaviour dependent on the capillary end effect, its relevance to field application would be moot.

\title{
Procter and Gamble Student poster award:
}

\section{References:}

Apaydin, O.G. and Kovscek, A.R. "Surfactant Concentration and End Effects on Foam Flow in Porous Media” Transport in Porous Media (2001). https://doi.org/10.1023/A:1010740811277

Namdar-Zanganeh, M. N., Kam, S. I., LaForce, T. C. and Rossen W.R., “The Method of Characteristics Applied to Oil Displacement by Foam”. SPE Journal (2011). SPE-121580-PA. https://doi.org/10.2118/121580PA

Rossen, W. R., \& Bruining, J. "Foam Displacements with Multiple Steady States”. Society of Petroleum Engineers (2007). SPE-89397-PA. https:/doi.org/10.2118/89397-PA

Rossen, W. R. and Boeije, C. S. Fitting Foam-Simulation-Model Parameters to Data: II. SurfactantAlternating-Gas Foam Applications. SPE Reservoir Evaluation \& Engineering (2015). SPE 165282. https://doi.org/10.2118/ PA Acceptance of Terms and Conditions:

Click here to agree

\section{Potential application of fungal biogeotechnology as irrigation canal lining material for agricultural water conservation}

\author{
Author(s): Emmanuel Salifu ${ }^{1}$ \\ Co-author(s): Gráinne El Mountassir ${ }^{2}$ \\ ${ }^{1}$ University of Strathclyde Glasgow and Università di Napoli Federico II, Napoli \\ ${ }^{2}$ University of Strathclyde, Glasgow UK
}

Corresponding Author(s): emmanuel.salifu@strath.ac.uk

Irrigated agriculture accounts for the largest consumptive water use globally. However, the irrigation water requirement is far less than the water withdrawal for irrigation purposes; this is because significant amount of water losses occur during distribution and application of irrigation water to crops. With increasing pressure on water resources globally there is a need to conserve available water, especially in arid and semi-arid irrigated lands. On farms, irrigation water from different sources 
is transported to the crops through canals or channels. Depending on the prevailing climatic conditions and the characteristics of the canal, irrigation water is often lost during conveyance due to evaporation and/or seepage, with significant losses due to seepage compared to evaporation. While it might be difficult to control climatic conditions responsible for evaporation losses, research efforts have focussed on reduction of seepage via canal beds and side slopes, considerably improving conveyance efficiencies. Seepage is affected by: soil porosity and structure, moisture content, soil chemistry, microbial activity, water temperature, flow rate and the evolution of soil structure due to erosion and siltation. In response to these factors, canal beds and side slopes are typically lined using impermeable materials including bricks, concrete and geosynthetics, among others.

Besides minimising seepage of water from the channel, a suitable lining material should in general: i) maintain the structural integrity of the channel, ii) prevent growth of vegetation iii) prevent soil erosion and iv) be durable. Additional considerations include financial, environmental and operational implications. For instance, concrete linings are susceptible to cracking over time, therefore requiring maintenance. Also, concrete is not considered to be an environmentally friendly option given that its production involves the release of $\mathrm{CO} 2$ in significant quantities contributing to global carbon emissions. On the other hand, geosynthetic materials are less durable in adverse weather conditions and are susceptible to damage from debris and animals. There is a need for low carbon, environmentally sustainable technologies that can be easily and cheaply deployed for irrigation channel lining in a range of climatic conditions.

At the University of Strathclyde, we are developing a technology which engineers the growth of fungal hyphae in soils. Hyphae are thin, tubular-structured, thread-like filamentous branches, which are capable of forming massive fungal hyphal networks (mycelia) over extensive areas. We are investigating the influence of fungal mycelia (Pleurotus ostreatus) on the hydraulic, mechanical and erosion characteristics of soils. Findings from our laboratory studies show that the presence of fungal mycelia can: 1) induce water repellency in soils, delaying the water breakthrough time for infiltration, 2) reduce soil infiltration due to a reduction in permeability as pores have become filled with fungi and their associated products, 3) reduce soil erosion by enmeshment and aggregation of soil grains into larger aggregates, therefore increasing the critical shear stress required to initiate the motion of soil aggregates and 4) reduce the rate of water loss from soils via evaporation.

These results suggest that fungal hyphal networks have the potential to be deployed to create impermeable linings for irrigation channels. The advantages of this novel technology are that it is environmentally friendly and does not involve the release of toxic materials to the environment, it is cheap, and could be deployed in an easy manner. Fungi have been found to survive (and even thrive) in extreme environments and hence they may be suitable for use even in arid regions. With the provision of minimal growth requirements, fungi can grow over large areas of land, implying that canals conveying water across long distances could be lined by engineering the growth of fungal mycelia

Procter and Gamble Student poster award:

References:

Acceptance of Terms and Conditions:

Click here to agree

\section{Constitutive relations for primary, secondary drainage and imbi- bition from percolation theory}

Hamidreza Salimi $^{1}$; Karl-Heinz Wolf ${ }^{2}$; Hans Bruining ${ }^{3}$

\footnotetext{
${ }^{1}$ Panterra

${ }^{2}$ TU-Delft

${ }^{3}$ TUdelft
}

Corresponding Author(s): hamidreza_salimi@yahoo.com

Constitutive relations like relative-permeability and capillary-pressure functions play an important role in multiphase flow in porous media. They are used in a variety of applications such as oil recovery, geothermal energy recovery and carbon dioxide storage. The relations depend on the detailed 
pore geometry, topology, and the physical properties of porous media (Bedrikovetskii and J. Bruining (2)). Primary (secondary) drainage Brooks-Corey and modified Brooks-Corey for imbibition can be used as empirical relations.

Recent P,T multi-phase flow experiments in a dual beam CT-scanner using a heterogeneous porous sandstone and image analysis (4), has revealed better insight in the pore structure and the effect of the distribution. Pore-network modeling based on the pore structure has shown that derived relative permeabilities are in line with experimentally determined rel-perms. Our interest, however, is to emphasize the difference of the relative permeabilities for primary, secondary drainage and imbibition. Barenblatt, Patzek and Silin1,(3) have emphasized that the imbibition water the viscous forces favor the large pores, whereas capillary forces favor the small pores. Consequently the pore filling sequence for imbibition is less straightforward than for drainage where both viscous and capillary forces favor the large pores. For imbibition the viscous forces start filling the large pores, but capillary forces lead to a delayed filling of the small pores. For drainage the non-wetting phase resides in the large pores, whereas for imbibition some non-wetting phase may end up in the small pores. Consequently, the drainage permeability exceeds the imbibition wetting phase permeability. For the same reason some wetting phase may end up in the large pores, and consequently the imbibition wetting phase permeability exceeds the drainage permeability. This inspired Barenblatt, Patzek and Silin to define an effective wetting phase saturation that exceeds the actual saturation, leading to a similar description as the dynamic capillary pressure defined by Hassanizadeh et al.

Percolation theory (2) gives a straightforward procedure to model non-wetting phase drainage permeabilities, for various shapes of the ducts connecting the pore bodies. Percolation theory shows results for large REV's. It confirms the finite permeabilities for very low oil saturations. Moreover its shows that the connate water saturation is extremely small, leading to finite water permeabilities at very low water saturations; in fact it shows that the connate water saturation approaches zero. Striking features of percolation theory for the obtained constitutive relations are that the water relative permeabilities exhibit a marked reduction below a critical water saturation Swc, but continue to be finite even at very low water saturations. The capillary pressure remains finite even at near zero water saturations. Primary-drainage oil relative permeabilities are non-zero at low oil saturations. Imbibition relative permeabilities are derived from some empirical models of pore filling sequence. Once the pore filling sequence is established we can apply percolation theory to determine the constitutive (rel-perms and capillary pressure). Percolation theory confirms that imbibtion rel-perms exceed the drainage wetting phase rel-perms, whereas the drainage non-wetting rel-perms exceed the imbibition non-wetting phase rel-perms as proposed in the paper of Barenblatt, Patzek and Silin. Our approach differs from previous work in that we use various shapes of non-circular pores and also combinations of different shapes. Indeed, the porous medium is approximated by a network of ducts (prisms). We use a finite-element approach to compute the conductivity of arbitrarily shaped ducts, which are partly filled with oil and water. We use the effective-medium approximation to obtain the conductivity of oil and water of the whole network. Comparison to a simple network model shows that qualitatively the same behavior is obtained as with percolation theory except that for primary drainage non-zero non-wetting phase primary permeabilities occur at oil saturations much larger than zero.

\section{Procter and Gamble Student poster award:}

\section{References:}

1. Barenblatt, G.I. and Patzek, T. W. and Silin, D. B., The mathematical model of nonequilibrium effects in water-oil displacement, Spe Journal, 8 (04), 409-416, (2003)

2. Bedrikovetsky, P., Bruining, J.: "A percolation based upscaling technique for viscous force dominated waterflooding in uncorrelated heterogeneous reservoirs", Proceedings, Vol. 2 of the 8th European Symposium on Improved Oil Recovery; European Association of Petroleum Geoscientist \& Engineers (EAPG),Vienna, Austria, p. 316-326 (15-17 May1995).

3. W. G. Gray, S.M. Hassanizadeh , Paradoxes and realities in unsaturated flow theory - Water resources research, 1991 - Wiley Online Library, Water Resources Research, Vol. 27, No. 8, Pages 1847-1854

4. F. A. L. Saadi, M. Hurkmans, C. van Kruijsdijk, K-H.Wolf. "Fontainebleau Porous Media: Visualization of Oil Bank in MicroModels.", Abstract Interpore, May 2017, Rotterdam - Netherlands.

\section{Acceptance of Terms and Conditions:}

Click here to agree 


\title{
Hydraulic Fracturing In Layered Media: Comparison of Different Scenarios
}

Saeed Salimzadeh ${ }^{1}$

${ }^{1}$ CSIRO

Corresponding Author(s): saeed.salimzadeh@csiro.au

Hydraulic fracturing has been proven as an efficient method to improve recovery from unconventional reservoirs. The real reservoirs are made of multiple, soft and stiff layers, which makes the hydraulic fracturing process more complex. Stress gradient and stress jumps due to the layering affect the hydraulic fracture geometry, and its efficiency to improve reservoir productivity (Figure 1). Furthermore, the local stresses can be engineered by injection and production from the reservoir in order to align the minimum stress in the desired direction and towards desired layers. Hence, accurate modelling of hydraulic fracture growth in the presence of stress gradients and sudden stress jumps is a fundamental requirement for treatment design. In this study, the problem of hydraulic fracturing in a layered reservoir under stress gradient and stress jumps is investigated using a robust finite element model (Salimzadeh et al., 2017). Results show under some circumstances the vertical growth of the fracture may be blocked due to stress gradient and jump. Results also show that the altered stress distribution can be favourable for forcing the hydraulic fracture growth in the desired direction and towards desired layers.

Procter and Gamble Student poster award:

\section{References:}

Salimzadeh S., Paluszny A., Zimmerman R.W., 2017. Finite Element Simulations of Interactions between Multiple Hydraulic Fractures in a Poroelastic Rock, Int. J. Rock Mech. Min. Sci. 99, 9-20. Acceptance of Terms and Conditions:

Click here to agree

145

\section{Numerical Modelling of Coupled Flow and Fluid-Driven Fractur- ing in Fractured Porous Media using the Immersed Body Method}

\author{
Asiri Obeysekara $^{1}$; Pablo Salinas ${ }^{\text {None }}$; Jiansheng Xiang ${ }^{2}$; J.P. Latham ${ }^{2}$; Christopher C. Pain ${ }^{\text {None }}$ \\ ${ }^{1}$ Imperial college London \\ ${ }^{2}$ Imperial College London
}

Corresponding Author(s): pablo.salinas@imperial.ac.uk

Fracture discontinuities are ubiquitous in reservoirs as a result of geological processes such as jointing and faulting. Due to the intrinsic nature of the fractures and its possible interconnections it is very common that these geological features play a key role in how the fluids move through the reservoirs and affects a variety of engineering applications such as geothermal production, $\mathrm{CO} 2 \mathrm{se}$ questration, mining operations and oil and gas production. Therefore, it is very important to model and understand how fractures are generated and propagate through the porous medium under different geomechanical effects such as in situ stresses or tectonic deformation.

Here, a novel approach for hydro-geomechanical modelling of fractured rocks is presented. This framework is developed by linking a solid mechanics model with a multi-phase fluid flow model using the immersed-body approach for fluid-solid interactions. The method uses non-conforming mesh-to-mesh projection to pass variables between an adaptive mesh refining couple multi-phase fluid-flow solver, and a deformation and fracturing solid geomechanics solver. The adaptive mesh refining and coarsening of the fluid model further permits the flow within and near to fractures (e.g. 
localised flow, leak-off) to be represented using dynamically adapting mesh. The mass conservation between the coupled fluid and solid fields is ensured through a globally conservative Galerkin projection-based mesh interpolation.

To simulate the nonlinear deformation of natural fractures in rock, the geomechanics model is integrated with a 'joint-constitutive model' that can capture the aperture variation of rough fractures under normal and/or shear loading. The solid mechanics is modelled using a Lagrangian specification in a Finite-Discrete Element Method (FEMDEM) framework called Solidity Project. Multi-phase (incompressible and compressible) fluid flow in porous media is considered through a Control-Volume Finite-Element Method (CV-FEM) based Darcy Flow solver and is called the Imperial College FiniteElement Reservoir Simulator (IC-FERST).

The coupling framework work in 3D and 2D. The solid domain is meshed independently but fully immersed in an 'extended' coupled fluid domain with the information of the two fields transferred via the shell-mesh surrounding the solid. To inform the fluid solver with the stress-dependent permeability of pre-existing or propagating fractures, a layer of shell-mesh with the thickness defined by the local hydraulic aperture is generated automatically at the fracture walls. Finally, numerical results are in both $2 \mathrm{D}$ and $3 \mathrm{D}$ against experimental results and in more complex scenarios, showing that the presented framework can model fracture propagation with a very good accuracy.

\title{
Procter and Gamble Student poster award:
}

\section{References:}

Obeysekara, A. et al., 2018. Modelling stress-dependent single and multi-phase flows in fractured porous media based on an immersed-body method with mesh adaptivity. Computers and Geotechnics, 103, pp.229-241.

Obeysekara, A, 2018. Numerical Modelling of Hydraulic Fracturing in Naturally Fractured Rock. PhD Thesis. Imperial College London Acceptance of Terms and Conditions:

Click here to agree

\section{An efficient control volume finite element method for multi-phase flow in fractured porous media}

\author{
Hossam Osman $^{1}$; Pablo Salinas ${ }^{\text {None }}$; Christopher C. Pain ${ }^{\text {None }}$; Matthew D. Jackson ${ }^{\text {None }}$ \\ ${ }^{1}$ Imperial College London
}

Corresponding Author(s): pablo.salinas@imperial.ac.uk

Modelling multiphase porous media flows is important in many engineering areas such as unconfined aquifers, geothermal energy extraction, magma reservoirs, CO2 storage, and hydrocarbon reservoirs. These models are typically composed of several different geological materials, and among them fractures normally play a key role due to their potential to act as conduits of the fluids. Nonetheless, due to the difference in the length scales to be considered when modelling a reservoir, fractures tend to be upscaled and not represented. Moreover, when modelled, it can be challenging to generate good quality unstructured meshes for fractured porous media models since domains typically have very large aspect ratios, complex geometries and many length scales need to be considered. Another important problem to represent highly fractured system using unstructured grids is the fact that the classical control volume finite element method (CVFEM) has continuous pressure representation, and it requires control volumes that span different elements, which creates an artificial "leakage" for highly heterogeneous domains. These two last problems have been addressed separately for unstructured meshes in Salinas et al. 2017, and Salinas et al. 2018. In the former a novel formulation based on CVFEM for simulating multi-phase flow in heterogeneous porous media for challenging meshes was presented, named double control volume finite element method (DCVFEM). In the latter, a CVFEM for multi-phase porous media flow with discontinuous pressure representation was presented. The method does not require control volumes (CVs) that span the boundaries between elements with potentially differing material properties, enabling an efficient simulation of flow in highly fractured models. 
Here, we introduce a new efficient CVFEM that combines both approaches, DCVFEM and CVs that do not span different elements. The method uses discontinuous piecewise linear functions enriched with bubble functions for velocity and discontinuous piecewise linear functions for pressure evaluated on control volumes (CVs). LBB stability is maintained with a very efficient velocity:pressure degrees of freedom ratio of 1.25 on tetrahedral meshes. The method, as in Salinas et al. 2018, does not require CVs to span element boundaries and as a result it is able to accurately preserve saturation discontinuities across material boundaries. Moreover, the use of control volume representation for pressure, likewise Salinas et al. 2017, yields significant improvements in stability of the method on challenging meshes. Our approach ensures mass conservation and the use of implicit time integration allows the method to efficiently converge using highly anisotropic meshes without reducing the time-step. Results are presented showing the robustness of the presented method under a set of highly fractured and complex geometries, requiring very high aspect ratio elements, and using the black-box iterative solvers provided in the PETSc library.

Procter and Gamble Student poster award:

References:

Salinas, P., Pavlidis, D., Xie, Z., Osman, H., Pain, C., Jackson, M.:A discontinuous control volume finite element method for multi-phase flow in heterogeneous porous media. J. Comput. Phys. 352, 602-614 (2018). https://doi.org/10.1016/j.jcp.2017.09.058

Salinas, P., Pavlidis, D., Xie, Z., Jacquemyn, C., Melnikova, Y.,Pain, C.C., Jackson, M.D.: Improving the robustness of the control volume finite element method with application to multiphase porous media flow. Int. J. Numer. Methods Fluids 0, 1-12 (2017). https://doi.org/10.1002/fld.4381 Acceptance of Terms and Conditions:

Click here to agree

140

\section{The Effects of Coating Morphology on Complex Liquid - Porous Substrates Interaction}

Helder Salvador $^{1}$; Hamid Mansouri ${ }^{1}$; Herman Wijshoff ${ }^{2}$; Nicolae Tomozeiu ${ }^{1}$

${ }^{1}$ Oce

${ }^{2}$ Oce, TU/e

Corresponding Author(s): helder.marquessalvador@oce.com

Nowadays printing water based inks is becoming the main printing technology due to its print quality / cost and environment care attributes. Understanding the physical mechanism of ink-paper interaction is crucial in order to optimize the printing process. The coated paper - frequently used in graphic arts printing - consists in two different porous layers: a coating and a fibrous. Moreover, studying in more detail the interaction between ink droplets and the coating layer, allows understanding the dynamics of liquid penetration into coated media. This work proposes that, by coupling droplet spreading and absorption rate measurements of complex fluids allows a better understanding of the dynamics of liquid absorption in the porous coated media.

The coating of the coated paper is $\mathrm{CaCO} 3$ based, but via the fabrication processes two different structures can emerge: grounding will results in a "spherical" granular structure while precipitation will create a needle like structure. Furthermore additives are used in the fabrication process to enhance absorption of liquid. If the additives enhance the absorption of water based mixtures, the paper is called inkjet treated; if the absorption enhancement is for oil based mixture then the paper is offset treated.

As complex fluids water-glycerol-surfactant mixtures are used in this work. The droplets have been generated with a dispenser in the range of $0.1 \mathrm{uL}$ to $10 \mathrm{uL}$. The investigation methods employed for this work are Scanning Electron Microscopy, Automatic Scanner Absorptometer, Droplet Shape Analyser and Optical Microscopy. 
The results of this work are summarized as:

I. For a granular structure, decreasing the surface tension of the mixture increases the absorption rate for offset coated and decreases the absorption rate of inkjet coated media. This is expected due to the surface energy of the coatings;

II. The structure of the coating strongly affects the way how the liquid is distributed inside the porous media. A correlation between spreading and absorption measurements was performed:

- for a granular structure a positive correlation was obtained;

- for the needle-like shape (heterogeneous structure), a negative correlation was observed. As we propose in this work, the heterogeneity of the coating determines a larger absorption in the direction of the needles (horizontal), rather than on the depth of the paper (vertical).

Procter and Gamble Student poster award:

References:

Acceptance of Terms and Conditions:

Click here to agree

\section{A Conservative Streamline Method for In-Situ Combustion Pro- cesses}

Luiz Sampaio $^{1}$; Margot Gerritsen ${ }^{1}$; Anthony Kovscek ${ }^{1}$

${ }^{1}$ ERE-Stanford University

Corresponding Author(s): luizebs@stanford.edu

The end goal of this project is to have an opensource framework based on the Matlab Reservoir Simulator Toolkit (MRST, from Sintef) that implements the streamline method for the solution of highly complex multiphysics flow in porous media. We are particularly interested in the In Situ Combustion (ISC) process as an Enhanced Oil Recovery (EOR) technique, where oxygen or air is injected and heat triggers the initial combustion of the oil in place. A small percentage of the oil in place is burned providing enough energy to maintain and propagate the combustion front that pushes an oil bank ahead, of improved quality and mobility. This EOR technique is very challenging for numerical simulations, as it involves physical subprocesses with significant variations in spatial and temporal scales, which are coupled in a highly non-linear fashion. The simulation code must be able to deal with the transport of energy and mass of multiple components. These components may react and undergo phase behavior, which can lead to severe volume changes.

The system of equations presents a mix of parabolic/elliptic and hyperbolic behavior. The latter is responsible for the presence of sharp gradients in temperature and compositions. Capturing this is critical for the solver overall accuracy as reactions are very important and sensitive to local levels of temperature and concentrations.

For this reason, we pursue an implementation that combines the streamline method for advective transport with a finite volume method for the non-advective terms in the governing equations. In the first step, pressure and energy equations are solved on an Eulerian grid, followed by a streamline tracing step that utilizes pressure gradients and Darcy's law. Then, energy and mass are transported along the streamlines, ignoring cross-streamline processes. This system of decoupled 1D problems can be effectively solved in parallel.

Apart from parallelization, we also like this approach as it allows for tailored solution approaches on each of the grids, which can be designed, for example, to reduce grid orientation effects that represent a typical obstacle for ISC and similar processes. A challenge with this approach is that solutions must be regularly mapped between grids. If not done carefully, these mappings can lead to excessive smearing as well as convergence problems in the pressure and energy solvers on the Eulerian grid. It also typically leads to loss of conservation.

In this talk, we will focus on two aspects of the code. The first is a new intrinsically conservative formulation, that guarantees mass and energy conservation. The second is a specialized 1D solver 
that can accurately capture self-sharpening fronts. The results show that this is achievable without implementing grid refinement or higher order numerical schemes.

Procter and Gamble Student poster award:

I don't want to compete References:

Acceptance of Terms and Conditions:

Click here to agree

\title{
Phase-field modelling of desiccation cracks in multiphase porous media
}

\author{
Tuanny Cajuhi ${ }^{1}$; Claudio Gavagnin ${ }^{2}$; Laura De Lorenzis ${ }^{1}$; Lorenzo Sanavia $^{2}$ \\ ${ }^{1}$ Technische Universität Braunschweig \\ ${ }^{2}$ University of Padova
}

Corresponding Author(s): lorenzo.sanavia@unipd.it

Porous media such as soil, rocks and concrete are of great importance in the context of civil engineering and environmental geomechanics. They consist of a solid skeleton and pores filled with fluids, e.g. air and water. Complex mechanisms of flow and transport take place within the pore network and can lead to deformation of the solid skeleton and eventually to fracture phenomena 1 .

Phase-field modeling of fracture has recently emerged as an alternative to conventional approaches such as remeshing, extended finite element methods or cohesive zone modeling. The phase-field framework can be considered a special type of gradient damage modeling approach, where a diffusive approximation of the crack is taken into account and the continuous phase-field parameter is used to describe the material integrity. The essential advantages are the possibility to describe arbitrarily complicated fracture patterns such as nucleation, branching and merging, without ad-hoc criteria on a fixed mesh, through the solution of partial differential equations derived from variational principles [2-5].

Phase-field modeling of fracture in porous media has been addressed in some recent publications [6-7], which however have only focused on the fully saturated case. Objective of this contribution is to describe fracture in partially saturated porous media using a phase-field approach [8]. In this study the air phase is assumed at constant atmospheric pressure with negligible density (passive air phase assumption) and the solid skeleton is described by its linear-elastic properties. Quasi-statics processes are studied. The equilibrium equations of the porous media, the mass balance equation of the liquid water and the phase-field evolution equation constitute a nonlinear coupled and timedependent system of equations, which needs to be discretized and linearized. We formulate the coupled non-linear system of partial differential equations governing the problem with displacements, capillary pressure and crack phase-field as unknowns. The spatial discretization is carried out with finite elements of appropriate order for the different unknowns. We discuss its solution and present some relevant examples on desiccation tests [8].

The previous model has recently been extended taking into account the contribution of the air phase and the dynamics (u-p approach). The first preliminary numerical results will be shown and discussed.

Procter and Gamble Student poster award:

\section{References:}

1 L. Simoni, B.A. Schrefler, Multi field simulation of fracture. Advances in Applied Mechanics, 2014.

2] B. Bourdin, G.A. Francfort, J-J. Marigo, Numerical experiments in revisited brittle fracture. Journal of the Mechanics and Physics of Solids, 48, 797-826, 2000.

3 C. Miehe , F. Welschinger, M. Hofacker, Thermodynamically consistent phase-field models of fracture: variational principles and multi-field FE implementations. Int. J. Num. Meth in Eng, 83, 1273-1311, 2010. 4 C. Kuhn, R. Muller, A continuum phase field model for fracture. Eng. Fracture Mech. 3625-3634, 2010. 5 M. Ambati, T. Gerasimov, L. De Lorenzis, A review on phase-field models of brittle fracture and a new fast hybrid formulation. Computational Mechanics 55, 383-405, 2014. 
[6] A. Mikelić, M.F. Wheeler, T. Wick, A phase-field method for propagating fluid-filled fractures coupled to a surrounding porous medium. SIAM Multiscale Modeling and Simulation 13(1), 367-398, 2014. [7] A. Mikelić, M.F. Wheeler, T. Wick, Phase-field modeling of a fluid-driven fracture in a poroelastic medium. Computational Geosciences.1-25, 2015.

[8] Cajuhi T., Sanavia L., De Lorenzis L. (2018) Phase-field modeling of fracture in variably saturated porous media, Computational Mechanics. https://doi.org/10.1007/s00466-017-1459-3 Acceptance of Terms and Conditions:

Click here to agree

\title{
Multiscale, pore-scale modeling of fluid mechanics using digital images and direct numerical solutions on unstructured meshes
}

\author{
Author(s): Karsten Thompson ${ }^{\text {None }}$ \\ Co-author(s): Paula C. Sanematsu ${ }^{1}$ \\ ${ }^{1}$ Louisiana State University
}

Corresponding Author(s): psanem1@lsu.edu

Multiscale modeling is important in porous media applications because of the enormous range of length scales that affects many processes. The term "multiscale modeling" is broad, encompassing a variety of theories and computational methods. In this work, we focus specifically on modeling disparate length scales that occur in pore-scale modeling of fluid flow and solute transport, starting from the nm scale (e.g,. surface and wetting films) and spanning up to mm-to-cm length scales (e.g., multiple pore dimensions necessary to capture representative volumes and predict spatially averaged parameters). A few examples of processes where multiscale processes are coupled over these length scales include solute transport in dual porosity systems, nanoparticle transport and retention in heterogeneous media, in-situ condensation, drainage at low wetting-phase saturations, and slow-release solute delivery from nanoporous structures.

While many multiscale techniques rely on integrating different methods for different scales, a promising technique for some applications of pore-scale modeling is direct modeling using unstructured meshes with various levels of refinement. This approach involves selective application of orders-ofmagnitude mesh refinement to resolve the different scales, followed by computational methods that remain accurate over the entire range. This meshing methodology is ideally suited for multiscale digital images and, if implemented with a technique such as the finite element method (FEM), it is well suited for direct methods for flow and particle transport as well as multiphysics models.

In this work, we use a multiscale meshing technique and a high-order FEM for Stokes equations to efficiently model $\mathrm{nm}$ - and mm-scale fluid mechanics in a single direct numerical simulation. The meshing is performed using a new point-insertion technique that can operate on multiscale digital images (e.g., spatially registered CT images) or on geometric images with multiple scales (e.g., computer-generated porous media or CAD files for microfluidics). The point-insertion algorithm is coupled to a black-box signed distance function for the material surface, which in turn allows high levels of refinement for complex geometries, while controlling mesh quality - an important factor for contrasting length scales. Low-Reynolds number flow is simulated using FEM with P2P1 elements, which provides high accuracy and is not overly sensitive to mesh quality. In order to verify accuracy of velocity fields at orders-of-magnitude smaller scales, we use sphere packings with known velocity profiles to quantify mm-scale flow patterns and evaluate flow within artificial nm-scale features with known geometry such as cylindrical "pinholes". Results indicate that this approach is an efficient and effective method for image-based modeling using multiscale digital images, and for capturing fluid mechanics over dramatically different length scales within a system.

\section{Procter and Gamble Student poster award:}

I don't want to compete References:

Acceptance of Terms and Conditions: 


\title{
Structural characterization of nanoporous materials by small-angle X-ray scattering
}

Heiner Santner ${ }^{1}$

${ }^{1}$ Anton Paar GmbH

Corresponding Author(s): heiner.santner@anton-paar.com

Small-angle X-ray scattering (SAXS) is a well-established method used for determining structural information on inhomogeneities in nanoscaled materials. The typical measured size range is between $1 \mathrm{~nm}$ and $200 \mathrm{~nm}$ making SAXS perfectly suited for analyzing nanoporous and nanoparticulate systems which include mesoporous (pore width $<50 \mathrm{~nm}$ ) and macroporous (pore width $>50 \mathrm{~nm}$ ) materials. In the scattering process SAXS is sensitive to sample domains or areas of different electron density, therefore it can probe various parameters within a nanoporous material, provided that the size range is within the resolution limit of the SAXS method: pore size/diameter, inter-pore distance, radial electron density profile and also the specific surface area. After all SAXS is an important complementary technique with respect to other characterization methods of porous materials such as microscopy and gas sorption methods. A particular advantage of SAXS is that it can measure specific surface of inaccessible, closed pores or inclusions. Furthermore SAXS provides averaged results which are representative over a large sample area or volume.

In this contribution we will present different applications of SAXS for characterizing porous materials. A high internal surface makes such materials very attractive for their application in e.g., energy storage, catalysis, molecular sieving. Their ability to selectively adsorb atoms or molecules of certain size and shape is closely related to their internal pore size and geometry. In addition to bulk materials, mesoporous system are also applied and studied as thin films on solid substrates. The porous nature of these films results in high interfacial surface areas which also make them applicable for various applications. Such thin film surfaces are analyzed using the grazing-incidence (GI) SAXS method in which the incident X-ray beam is directed onto the sample at a very shallow angle and therefore probes the surface of the material.

Procter and Gamble Student poster award:

I don't want to compete References:

Acceptance of Terms and Conditions:

Click here to agree

631

\section{Finite element/finite volume framework for phase-field fracture in saturated porous media}

\author{
Author(s): Juan Michael Sargado ${ }^{1}$ \\ Co-author(s): Eirik Keilegavlen ${ }^{2}$; Inga Berre ${ }^{2}$; Jan Martin Nordbotten ${ }^{2}$; Robert Kloefkorn ${ }^{3}$ \\ ${ }^{1}$ NORCE Norwegian Research Centre AS \\ ${ }^{2}$ University of Bergen \\ ${ }^{3}$ NORCE Norwegian Resarch Centre AS
}

Corresponding Author(s): jusa@norceresearch.no

Understanding the fluid-driven evolution of crack networks in a permeable medium is of prime importance for many scientific and engineering problems, among them unconventional oil and gas 
recovery, enhanced geothermal systems, water flooding processes and magma effusion. In this talk, we present a combined finite element/finite volume approach for solving the Biot poroelasticity equations together with a phase-field model for crack propagation. The proposed approach monolithically couples a finite element discretization of stress equilibrium to a control volume form of the fluid mass balance, which is further coupled iteratively to the phase-field equation in finite volume form via alternate minimization. The main goal of the formulation is to allow for discretization length scales that are much larger than the fracture apertures. To achieve this, we make use of alternative degradation functions that minimize loss of stiffness in the mechanical response prior to fracture for coarser meshes, and upscale the effect of flow in open fractures to the surrounding medium. The proposed model is applied to several numerical examples, and in addition we investigate the performance of different linear solvers for the resulting systems of equations.

Procter and Gamble Student poster award:

\title{
References:
}

Acceptance of Terms and Conditions:

Click here to agree

34

\section{Seeking minimum entropy production for flow-field patterns and their geometries in fuel cells}

\author{
Author(s): Marco Sauermoser ${ }^{1}$ \\ Co-author(s): Natalya Kizilova ${ }^{2}$; Eirik Flekkøy ${ }^{3}$; Bruno G. Pollet ${ }^{4}$; Signe Kjelstrup ${ }^{1}$ \\ ${ }^{1}$ PoreLab, Department of Chemistry, Norwegian University of Science and Technology, Trondheim, Norway \\ ${ }^{2}$ Department of Aeronautics and Applied Mechanics, Warsaw University of Technology, Poland \\ ${ }^{3}$ PoreLab, Dep. Physic University of Oslo, Norway \\ ${ }^{4}$ Department of Energy and Process Engineering, Norwegian University of Science and Technology, Trondheim, Nor- \\ way
}

Corresponding Author(s): marco.sauermoser@ntnu.no

The flow field plates in a fuel cell still have potential for optimization which can lead to an increase in fuel cell performance, one of the targets set by the U.S. Department of Energy. One way to optimize this flow field plate is, to develop a flow pattern, which diminishes the drawbacks from the current industry standards like serpentine and parallel pattern, like non-uniform reactant distribution and water management problems.

For this reason, a tree-shaped flow pattern, inspired by biological transportation networks, was picked, enabling the authors to achieve a uniform distribution of reactants in the fuel cell, increasing its performance 1 . The diameters of the channels in the bifurcations were related by the Murray's law providing minimal entropy production 2. In this work we varied the width and depth of the flow channels, and the number of generations was determined by the Peclet number providing the diffusion transport in the gas diffusion layer. The energy loss due to the T-shape bifurcations and non-laminar entrance flow in each channel were accounted for. Both 1D and 3D computations of the pressures and velocities in the channels has been used for detailed calculations of the energy dissipation due to viscous and hydraulic losses in the flow field plate. The optimal design was determined from the observation that certain units with optimal efficiency have a uniform distribution of entropy production [2,3]. The best designs with constant and gradually diminishing depth of the consequent channels were compared to the classical serpentine design and proposed for improvement of the fuel cell efficiency.

Acknowledgement:

The authors are grateful to the Research Council of Norway through its Centres of Excellence funding scheme, project number 262644, PoreLab. 


\title{
Procter and Gamble Student poster award:
}

I would like to compete in the Procter and Gamble Student award References:

1 P. Trogadas, J. I. S. Cho, T. P. Neville, J. Marquis, B. Wu, D. J. L. Brett and M.-O. Coppens. A lunginspired approach to scalable and robust fuel cell design. Energy Environ. Sci., 11(1), 2018, pp. 136-143 2 S. Gheorghiu, S. Kjelstrup, P. Pfeifer and M.-O. Coppens. Is the lung an optimal gas exchanger? In: Fractals in Biology and Medicine, ed. G. A. Losa, D. Merlini, T. F. Nonnenmacher and E. R. Weibel, Birkhauser Basel, Basel, 2005, pp. 31-42.

3 S. Kjelstrup, D. Bedeaux, E. Johannessen and J. Gross. Non-Equlibrium Thermodynamics for Engineers. World Scientific Publishing, 2. edition, 2017. Acceptance of Terms and Conditions:

Click here to agree

\section{Three-Phase Flow Visualization and Characterization for Water- and Mixed-Wet Carbonate Rocks}

\author{
Author(s): Alessio Scanziani ${ }^{1}$ \\ Co-author(s): Kamaljit Singh ; Amer Alhammadi ${ }^{2}$; Hannah Menke $^{1}$; Branko Bijeljic $^{3}$; Martin Blunt ${ }^{1}$ \\ ${ }^{1}$ Imperial College London \\ ${ }^{2}$ Earth Science and Engineering, Imperial College London \\ ${ }^{3}$ Imperial College
}

Corresponding Author(s): alessio.scanziani16@imperial.ac.uk

Three-phase flow is ubiquitous in many energy-related physical processes such as oil recovery, carbon dioxide geo-sequestration, fuel cells optimisation and contaminant removal (Blunt, 2017). Modern world keeps requiring more energy due to the increase of the population, to be balanced with a decrease in the emissions of greenhouse gases due to the efforts in mitigating climate change. For these reasons, the combination of gas driven enhanced oil recovery (EOR) with CO2 storage (CCS) is one of the challenges the world has to tackle for its sustainable development (IEA, 2017).

Recently, X-ray micro-CT tomography has allowed for major improvements in the understanding of two-phase flow (AlRatrout et al., 2018; Blunt et al., 2013; Bultreys et al., 2016). In our studies we not only apply the techniques developed for two phases to systems where three phases - water, oil and gas, involved in EOR and CCS - are present, but also develop new methods required by the additional complexities in three-phase flow. We were able to image the flow of the three phases during and after their injection in porous rocks, for different rock geometries and wettability (Scanziani et al., 2018).

The first experiment was the injection of oil in a water-wet Ketton sample fully saturated with brine, followed by first waterflooding, gas injection, and second waterflooding. The sample was imaged after each injection step using a laboratory micro-CT scanner. The second experiment was similar to the first one, but was performed at Diamond synchrotron facility, allowing dynamic imaging with time resolution of 50 seconds. The third experiment was designed to be at the reservoir conditions, where the reservoir carbonate sample, the brine and the crude oil were used from a producing oil field in the Middle East. This sample was aged, causing a change in wettability towards oil-wet conditions and the experiment was performed in laboratory based micro-CT scanner.

The results of the three experiments allowed us to have a deeper understanding of respectively (i) the fundamentals of fluid arrangement, recovery and trapping in three-phase flow for a water-wet sample (Figure 1); (ii) dynamics, namely evolution of the oil layer thickness, connectivity and interfacial area, and (iii) how the change in wettability - measured by an in situ contact angle method (Scanziani et al., 2017) - affects the wettability order, and hence all the other quantities previously mentioned (as an example, the pore occupancy changes are shown in Figure 2).

Indico rendering error

Could not include image: Cannot read image data. Maybe not an image file? 
Could not include image: Cannot read image data. Maybe not an image file?

\title{
Procter and Gamble Student poster award:
}

I don't want to compete References:

AlRatrout, A., Blunt, M.J., Bijeljic, B., 2018. Wettability in complex porous materials, the mixed-wet state, and its relationship to surface roughness. Proc. Natl. Acad. Sci. U. S. A. 115, 8901-8906.

Blunt, M.J., 2017. Multiphase Flow in Permeable Media. Cambridge University Press.

Blunt, M.J., Bijeljic, B., Dong, H., Gharbi, O., Iglauer, S., Mostaghimi, P., Paluszny, A., Pentland, C., 2013. Pore-scale imaging and modelling. Adv. Water Resour. 51, 197-216.

Bultreys, T., De Boever, W., Cnudde, V., 2016. Imaging and image-based fluid transport modeling at the pore scale in geological materials: A practical introduction to the current state-of-the-art. Earth-Science Rev.

IEA, 2017. Energy Technology Perspectives 2017 - Executive Summary, Iea.

Scanziani, A., Singh, K., Blunt, M.J., Guadagnini, A., 2017. Automatic method for estimation of in situ effective contact angle from X-ray micro tomography images of two-phase flow in porous media. J. Colloid Interface Sci. 496, 51-59.

Scanziani, A., Singh, K., Bultreys, T., Bijeljic, B., Blunt, M.J., 2018. In situ characterization of immiscible three-phase flow at the pore scale for a water-wet carbonate rock. Adv. Water Resour. 121, 446-455. Acceptance of Terms and Conditions:

Click here to agree

\section{Biofilm architectural differentiation in porous systems}

\author{
david scheidweiler ${ }^{1}$; Hannes Peter $^{1}$; Pietro De Anna ${ }^{\text {None }}$; Tom Battin ${ }^{\text {None }}$ \\ ${ }^{1}$ EPFL
}

Corresponding Author(s): david.scheidweiler@epfl.ch

Hydrated porous systems with their high surface availability are attractive substrates for biofilm growth. A prime example of such a system is the hyporheic zone of streams hosting diverse microbial communities concealed within the sediment interstices. Within this sedimentary environment, fluid flow constrains microorganism dispersal and biofilm growth. However, the interplay between hydrodynamics, biofilm architecture and dispersal in porous systems remain poorly understood. To better appreciate these links, we designed porous-like fluidic devices, exposed to streamwater flow containing bacterial cells. Biofilm formation and local hydrodynamics were investigated using time-lapse microscopy and micro-particle image velocimetry. We additionally studied the transport dynamics of motile and non-motile cells through the porous landscape in presence and absence of mature stream biofilm. We found two different architectures: a biofilm coating the grains and streamers extending into the pore space. We show that the architectural differentiation was the result of biofilm growth on the grains and of cell retention by the streamers. Our work advances previous studies on biofilm architectural formation in porous systems and discusses the ecological relevance of different dispersal strategies.

\section{Procter and Gamble Student poster award:}

I would like to compete in the Procter and Gamble Student award References:

\section{Acceptance of Terms and Conditions:}

Click here to agree 


\title{
Coupling of Adsorption and Transport in Hierachical Porous Ma- terials
}

\author{
Alexander Schlaich $^{1}$; Benoit Coasne ${ }^{2}$ \\ ${ }^{1}$ Laboratoire Interdisciplinaire de Physique, CNRS and University Grenoble Alpes \\ ${ }^{2}$ CNRS/University Grenoble Alpes
}

Corresponding Author(s): alexander.schlaich@univ-grenoble-alpes.fr

\begin{abstract}
Hierarchical porous materials are widely used for adsorption, separation, catalysis etc. as they combine several porosity scales to overcome slow diffusion in their nanoporosity while maintaining a large specific surface area. Fluids in such multiscale confinement exhibit rich thermodynamic and dynamical behaviors that are significantly different from their bulk counterpart: surface forces and reduced dimensions affect phase transitions due to capillary condensation, freezing, etc. and transport in such nanoporous media exhibits a broad range of novel phenomena. As a result, while adsorption and transport in a nanopore is reasonably well understood in the limiting cases of liquid filled pores, the complex interplay between thermodynamics and dynamics still lacks satisfactory description for multiscale porous media such as hierarchical porous solids.

In this work, atom-scale simulations were coupled to a multiscale lattice model to obtain explicit descriptions for the coupling between macroscopic transport and the thermodynamics of the confined fluid. By investigating the role of pore size, pressure, temperature and surface chemistry, we are able to sketch a 'transport phase diagram'. More in detail, the microscopic behaviors obtained at the molecular scale allow us to construct a hierarchical lattice model with transport coefficients explicitly derived from Statistical Mechanics. We address how macroscopic transport arises from contributions where gas and liquid phases contribute. Experimental adsorption and dynamical measurements at different scales (from the $\mathrm{nm}$ to the micron scales) can readily be included in our model. Successive upscaling allows us to predict the macroscopic transport in hierachical porous materials from simple experimental topological data as obtained from tomography, electron microscopy, etc. We demonstrate that our simple approach can be employed to optimize the adsorption and transport of fluids confined in multiscale porous solids.
\end{abstract}

Procter and Gamble Student poster award:

References:

Acceptance of Terms and Conditions:

Click here to agree

\section{The role of porous media in PEM fuel cells}

\author{
Veronika Schleper ${ }^{\text {None }}$
}

Corresponding Author(s): veronika.schleper@de.bosch.com

Polymer electrolyte membrane (PEM) fuel cells are one of the major technologies for electrified mobility. From a fluid dynamic perspective, fuel cells are a rather complicated multi-component multi-phase flow problem in a variety of thin porous layers, in general in thermal non-equilibrium. This leads to a variety of complex processes that interact and have to be described correctly in order to predict the behaviour of the fuel cell under operation.

In my talk, I will explain the role of the different layers of a PEM fuel cell and point our the major physical effects, that have to be taken into account. The intention is to give an overview of the technology for those participants that are not so familiar with fuel cells, but would like to know more and maybe start their own research in the field of fuel cells.

Procter and Gamble Student poster award: 
I don't want to compete References:

Acceptance of Terms and Conditions:

Click here to agree

\section{Imaging and quantifying the microstructure of polymers and com- posites using Cryo-BIB-SEM}

Joyce Schmatz ${ }^{1}$

${ }^{1} \mathrm{MaP}$ - Microstructure and Pores $\mathrm{GmbH}$

Corresponding Author(s): joyce.schmatz@m-a-p.expert

Polymers are used in a wide field of industrial applications such as fuel cells, building materials, and fibers. High-resolution characterization and quantification of polymer microstructure is essential to improve our understanding in modification of naturally occurring polymers and in the manufacturing processes of synthetic polymers. The microstructure controls the chemical reactivity, the mechanical properties, and the transport properties of polymers, and thus governs properties such as the durability of fuel cells, the resistivity against weathering and heat of plastics and building materials.

In the recent years a range of imaging methods were established to study the properties of polymers, such as wide range X-ray scattering, small angle X-ray scattering, small angle neutron scattering to study the crystalline structure and FTIR and Raman spectroscopy to study the composition. Generally, Cryo-SEM is ideal to study microstructure and porosity of polymer composites and liquid polymers. However, the typically rough specimen surfaces - resulting from the freeze fracturing process - often do not allow a reliable investigation and quantification of the nanostructures, such as nanoparticles, phase contacts and nanoporosity from the SEM micrographs. The identification of these structures is important since they affect physical processes such as absorption and diffusion, and their accurate assessment is required for process simulations.

Cryo-BIB-SEM (Broad Ion Beam milling and Scanning Electron Microscopy) is a newly established imaging method (Schmatz et al., 2015, 2017, Jaiser at al., 2017) that allows studying the microstructure of cryogenically stabilized samples on large (up to $4 \mathrm{~mm}^{2}$ ) planar, and damage-free sample crosssections. The technology allows, among others, the ex-situ investigation of drying processes in polymer thin films and studying the formation of bio films on fiber surfaces.

In this contribution a range of application examples of the Cryo-BIB-SEM technology will be presented comprising paint, fuel cells, plants, biofilms, bentonite/polymer composites, and liquid crystals.

\section{Procter and Gamble Student poster award:}

\section{References:}

Schmatz, J., J. L. Urai, S. Berg, and H. Ott (2015), Nanoscale imaging of pore-scale fluid-fluid-solid contacts in sandstone. Geophys. Res. Lett., 42, 2189-2195. doi: 10.1002/2015GL063354.

Schmatz, J., Klaver, J., Jiang, M., \& Urai, J. L. (2017). Nanoscale Morphology of Brine/Oil/Mineral Contacts in Connected Pores of Carbonate Reservoirs: Insights on Wettability From Cryo-BIB-SEM. Society of Petroleum Engineers. doi:10.2118/180049-PA.

Jaiser, S., Kumberg, J., Klaver,J., Urai,J.L., Schabel,W. Schmatz,J., Scharfer,P. (2017) Microstructure formation of lithium-ion battery electrodes during drying - An ex-situ study using cryogenic broad ion beam slope-cutting and scanning electron microscopy (Cryo-BIB-SEM), Journal of Power Sources, Volume 345, 2017, Pages 97-107. Acceptance of Terms and Conditions:

Click here to agree 


\title{
Depth-dependent Flow and Pressure Characteristics in Brain Mi- crovascular Networks
}

\author{
Author(s): Franca Schmid ${ }^{1}$ \\ Co-author(s): Bruno Weber ${ }^{1}$; Patrick Jenny ${ }^{2}$ \\ ${ }^{1}$ University of Zurich \\ ${ }^{2}$ ETH Zurich
}

Corresponding Author(s): frschmid@ethz.ch

\begin{abstract}
A detailed knowledge of flow in brain microvascular networks (MVNs) is crucial for various fundamental aspects related to nutrient supply in the brain, for which the cortical vasculature is crucial. However, until today, a profound understanding of topology and perfusion of the cortical vasculature is lacking 1 .

We simulate flow in three realistic MVNs from the mouse somatosensory cortex, which have been obtained by two-photon laser scanning microscopy 2 . The realistic microvascular networks are embedded in a tissue volume of $\sim 1 \mathrm{~mm} 3$ and consist of up to 16,000 nodes connected by tortuous vessels. The bi-phasic nature of blood originates from the presence of red blood cells (RBCs). Our numerical model has the unique feature of tracking individual RBCs 3, which is in contrast to other commonly used blood flow models [4,5]. This approach enables us to minimize the number of empirical functions required and to resolve fluctuations resulting from varying $\mathrm{RBC}$ concentrations. To assign suitable pressure boundary conditions over the entire depth we embedded the realistic MVN into a large, but representative artificial microvascular network [6].

Our study focuses on: (1) The pressure drop in different vessel types of the MVN, (2) the trajectories of individual RBCs through the MVN and (3) on general flow properties, such as velocity and capillary transit time. All aspects are analyzed with respect to cortical depth.

The trajectories of individual RBCs reveal that RBCs tend to exit the capillary bed at a similar cortical depth at which entering it. Hence, in the capillary bed the preferential RBC flow direction is horizontal. It remains to be shown if the capillary bed is built to enforce this preferential flow direction or if it solely results from the applied pressure field.

Furthermore, we show that close to the cortical surface the pressure drop is largest in the capillary bed, while deeper in the cortex it becomes dominant in arterioles. We postulate that these differences are relevant for the up-regulation of blood flow in response to neuronal activation (neurovascular coupling). Indeed, we suggest that different regulation mechanisms might be in place depending on cortical depth.

Interestingly, we observe a significant decrease in $\mathrm{RBC}$ velocity with depth. As oxygen discharge from $\mathrm{RBCs}$ to the surrounding tissue is a diffusion driven process, the observed velocity differences will also affect oxygen diffusion from RBCs to tissue. It seems likely that RBCs travelling through deeper cortical layers will be less saturated with oxygen than close to the cortical surface. Consequently, deeper cortical layers might be more sensitive to disturbances in perfusion and thus more prone to oxygen depletion.
\end{abstract}

Procter and Gamble Student poster award:

I don't want to compete References:

1 Schmid et al., Neuroimage, 2017, doi: 10.1016/j.neuroimage.2017.06.046

2 Blinder et al., Nature Neuroscience, 2013, doi: 10.1038/nn.3426

3 Schmid et al., AJP-Heart and Circulatory Physiology, 2015, doi: 10.1152/ajpheart.00335.2014

4 Lorthois et al., Neuroimage, 2011, doi: 10.1016/j.neuroimage.2010.09.032

5 Guibert et al., Journal of Cerebral Blood Flow and Metabolism, 2010, doi: 10.1038/jcbfm.2010.105

[6] Schmid et al., PLOS Computational Biology, 2017, doi: 10.1371/journal.pcbi.1005392 Acceptance of Terms and Conditions:

Click here to agree

\section{Modeling Mass Transport in Porous Transport Layers of PEM Wa- ter Electrolysis Cells}


Author(s): Gergely Schmidt ${ }^{1}$

Co-author(s): Michel Suermann ${ }^{2}$; Boris Bensmann ${ }^{2}$; Richard Hanke-Rauschenbach ${ }^{2} ;$ Insa Neuweiler $^{1}$

${ }^{1}$ Institute of Fluid Dynamics and Environmental Physics in Civil Engineering, Leibniz University Hannover

${ }^{2}$ Institute of Electric Power Systems, Leibniz University Hannover

Corresponding Author(s): schmidt@hydromech.uni-hannover.de

A combination of power-to-gas technology and proton exchange membrane (PEM) water electrolysis is an energy storage option which is versatile regarding fluctuating electrical input, storage durations and distributed energy demands 1. PEM water electrolysis' efficiency is however diminished by overpotentials resulting from mass transport through porous transport layers (PTL).

Feed water is transported through the PTL to the anode catalyst where it is split into protons, electrons and oxygen. Oxygen forms a gas phase and needs to be removed through the PTL in countercurrent direction as remaining oxygen interferes with further electrolysis. Consequently, the optimization of PTL properties requires identification of dominant transport mechanisms which are to date unknown due to the lack of empirical or measured data.

We therefore examine several Titanium, sintered powder PTLs which have been characterized by means of 3D tomography and in situ, electrochemical measurements [2,3]. From the latter, overpotentials accounting for mass transport through PTL are extracted based on experimental observations at varying operating pressures 2 .

We show that novel results of a 1D, multiphase, multicomponent, porous medium continuum model describing fluid fluxes and component transport are consistent with experimental data for different operating conditions and varying PTL properties. Model results also show that diffusive transport is negligible over a wide range of operating conditions. Calibrated capillary pressure and relative permeability functions differ significantly from standard model predictions based on contact angle and pores size distribution (PSD) [4,5], yet they agree well with capillary flow porometry measurements for similar PTL materials [6]. Model parameters could, however, only be transferred between materials to predict mass transport overpotentials, when materials had different PSD but identical particle size distributions and assumingly equal wettability. This fact leaves the model's and overpotential extraction's overall validity undecided, yet it might also be a result of highly diverse wettabilities amongst considered materials.

References:

1. M. Carmo, D. L. Fritz, J. Mergel, D. Stolten. Int. J. Hydrog. Energy 38 (2013), 12, 4901

2. M. Suermann, K. Takanohashi, A. Lamibrac, T. J. Schmidt, F. N. Büchi. J. Electrochem. Soc. 164 (2017), 9, F973

3. U. Babic, T. J. Schmidt, L. Gubler. J. Electrochem. Soc. 165 (2018), 15, J3016

4. M. Aubertin, M. Mbonimpa, B. Bussière, R. P. Chapuis, Can. Geotech. J. 40 (2003), 6, 1104

5. N. T. Burdine, J. Petrol. Tech. 5 (1953), 71

6. K. Bromberger, J. Ghinaiya, T. Lickert, A. Fallisch, T. Smolinka, Int. J. Hydrog. Energy 43 (2018), 5,2556

Procter and Gamble Student poster award:

I would like to compete in the Procter and Gamble Student award References:

Acceptance of Terms and Conditions:

Click here to agree

618

\title{
Non-linear hydro-mechanics of fluid-filled fractures
}

\author{
Patrick Schmidt ${ }^{1} ;$ Holger Steeb ${ }^{1}$ \\ ${ }^{1}$ University of Stuttgart \\ Corresponding Author(s): patrick.schmidt@mechbau.uni-stuttgart.de
}


Numerical investigations of flow processes in fractured porous media are of interest to determine properties of underground matter and heat transport and underground fluid storage. The determination process of hydraulic properties includes generation of transient field data by hydraulic stimulations such as pumping operations and inverse numerical simulations. Numerical calculations are commonly based on diffusion models; hence hydro-mechanical coupling is not taken into account and even extended diffusion-based formulations often fail, when the numerical results are compared to experimental observation. Immediate non-local volumetric, pressure-induced changes caused by the (elastic) deformation of the fractures act faster than pressure diffusion in the fluid. The different propagation dimensions in time result in inverse water level fluctuations also known as Noordbergum effect on the reservoir scale. Besides volumetrically-triggered phenomena, mechanical fracture deformations might lead to local permeability changes. In case of large fracture deformations, the effective fracture permeability changes have an immediate impact on the diffusion process, i.e. the characteristic diffusion time. In order to capture both, non-local and local phenomena, a highly non-linear fracture flow model for deformable high aspect ratio fractures (length vs. aperture, i.e., $\delta>1000)$ is introduced. The hybrid-dimensional model is motivated by low Reynold numbers flow and assumes pressure driven Poiseuille-type flow within fluid-filled fractures. This leads to a numerical efficient dimension-reduction of the fracture domain. Coupling of the surrounding porous matrix and the hybrid-dimensional flow model results in a numerically stiff system. Interface element formulations are used to solve the resulting system monolithically in order to guarantee numerical stability and efficiency, respectively. The work closes with a discussion of numerical results obtained for various characteristic hydro-mechanical scenarios. We will show, that large aperture changes of fluid-filled fractures lead to non-linear effects which qualitatively affect pressure-fluxes relationships.

Procter and Gamble Student poster award:

References:

Acceptance of Terms and Conditions:

Click here to agree

\section{Measurement of bare soil evaporation using outdoor lysimeter data}

Author(s): Jana Schneider ${ }^{1}$

Co-author(s): Jan Vanderborght ; Youri Rothfuss

${ }^{1}$ Forschungszentrum

Corresponding Author(s): jan.schneider@fz-juelich.de

Long-term lysimeter data of 12 weighed lysimeters are collected, processed and analysed to study the effect of soil properties on evaporation rates. Therefore, data of bare soil periods occurring during fallows of cropland lysimeters are studied. Data of five years are selected (2014-2018). The soil columns in the lysimeters are undisturbed, originating from different sites in Germany. In general four different soil types can be distinguished. The soil texture varies between sandy silt and silty sand.

The lysimeters' weighing systems have an accuracy of $\pm 0.01 \mathrm{~mm}$ and a resolution of $\pm 0.001 \mathrm{~mm}$ for the seepage water reservoir mass. Data are recorded in one minute intervals. Hence, actual evaporation can be determined with a high accuracy. Within the depths of 10, 30, 50 and $140 \mathrm{~cm}$ soil water content, heat flux, water potential and temperature are recorded. Moreover, a climate station is collecting information about wind speed, precipitation, air temperature and radiation in 10 minute intervals.

The diverse measurement methods and the collected data about soil parameters and climate give us further information about the soil water fluxes at different temporal scales. Because of the long time periods which are evaluated a comparison of evaporation in wet and dry seasons can be made.

This outdoor experiment makes it possible to observe the soil state variables and evaporation under natural conditions. Thus, model simulations and phenomena observed in laboratory can be compared with the outdoor experiments to determine how well they display the natural conditions. 
The evaporation data is analysed statistically to compare the diurnal evaporation rates between the different soil types. Moreover, a model developed with HYDRUS-1D including water vapour transport is used to reproduce the evaporation rate of the observed soils. Model results and observations are compared.

Preliminary evaluation of the data is showing, surprisingly, that there is no statistical significant difference of evaporation rates between the soils with different texture.

Procter and Gamble Student poster award:

I don't want to compete References:

Acceptance of Terms and Conditions:

Click here to agree

\title{
Modelling microbially enhanced coal-bed methane production: From a validated batch model to column scale
}

\author{
Simon Scholz ${ }^{1}$; Katherine Davis ${ }^{2}$; Johannes Hommel $^{3}$; Robin Gerlach ${ }^{2}$; Al Cunningham ${ }^{4}$; Holger Class $^{3}$; Rainer \\ Helmig $^{3}$ \\ ${ }^{1}$ LH2, University of Stuttgart \\ ${ }^{2}$ Montana State University \\ ${ }^{3}$ University of Stuttgart \\ ${ }^{4}$ Center for Biofilm Engineering, Montana State University
}

Corresponding Author(s): simon.scholz@iws.uni-stuttgart.de

Microbially enhanced coal-bed methane (MECBM) production is an innovative idea to stimulate biogenic coal-bed methane production by providing nutrients to the native microbial community. Through additional substrate in the subsurface, a stimulation of microbes occurs, which leads to an increase in methane production well beyond the amount that could be expected from the addition of substrate alone. Experimental studies, performed at Montana State University, provide the basis for modelling MECBM production with two-phase multi-component transport processes using the numerical simulator DuMuX 1 .

We will present the calibrated and validated numerical batch model that was obtained using experimental results from 2. The model is able to capture the interaction between microbial activity, coal bioavailability, biofilm growth and decay as well as $\mathrm{CH}_{4}$ production.

The numerical batch model is extended to simulate column studies. The batch and column models are used to test various hypotheses on different processes e.g. coal bioavailability, biofilm conditions and retardation effects when injecting substrate. The numerical model aims to provide further understanding of the relevant processes involved in MECBM as well as a general understanding of biochemical reactions coupled with possibly changing flow and transport conditions in the subsurface.

\section{Procter and Gamble Student poster award:}

\section{References:}

1 Flemisch, Bernd, et al. "DuMux: DUNE for multi-\{phase, component, scale, physics,...\} flow and transport in porous media." Advances in Water Resources 34.9 (2011): 1102-1112.

2 Davis, Katherine J., et al. "Biogenic coal-to-methane conversion efficiency decreases after repeated organic amendment.” Energy \& Fuels 32.3 (2018): 2916-2925. Acceptance of Terms and Conditions:

Click here to agree 


\section{Microscale Analysis of deforming saturated porous media: Terza- ghi stress principle and the Significance of Shear and Plasticity in Hydrostatic Compression}

Howard Schreyer ${ }^{1}$; Brandon Lampe ${ }^{2}$; Lynn Schreyer ${ }^{3}$; John Stormont ${ }^{\text {None }}$

${ }^{1}$ University of New Mexico

${ }^{2}$ Lead Cavern Engineer, WSP USA

${ }^{3}$ Washington State University

Corresponding Author(s): lynn.schreyer@wsu.edu

At the macroscale, the governing equations often include the Terzaghi effective stress principle or a generalization of the principle containing a Biot coefficient. These equations are often used in geotechnical engineering and bioengineering, and can be derived using concepts from multivariable calculus (total differential), but in many applications the model does not predict well what is observed. Experimental data from geophysics literature exhibit nonlinear pressure-volumetric strain relations and a strong dependence on just the Terzaghi effective pressure, defined as confining pressure minus pore pressure. A microscale analysis demonstrates a plausible reason for why the Terzaghi effective pressure (without the Biot coefficient) plays such a dominant role. The results show that the stress field within a relatively small region around a pore contains shear stress proportional to the effective stress that produces a cascade of effects including the increased likelihood of the material entering into a plastic regime and pore sizes changing significantly.

Procter and Gamble Student poster award:

References:

Acceptance of Terms and Conditions:

Click here to agree

\section{Comparing Upscaled Governing Equations for Transport and Flow Derived via Hybrid Mixture Theory and Homogenization}

Lynn Schreyer ${ }^{1}$

${ }^{1}$ Washington State University

Corresponding Author(s): lynn.schreyer@wsu.edu

The macroscale governing equations for charged porous media are not yet standardized - different upscaling approaches yield different results, although not as different as one might expect upon first examination. Here we compare two approaches: hybrid mixture theory [1,2] and homogenization 3, to show that at its core, the two approaches give remarkably similar results for the generalized Darcy's equation and the generalized convection diffusion equation.

Procter and Gamble Student poster award:

I don't want to compete References:

1. Bennethum, L.S., Cushman, J.H.: Multicomponent, multiphase thermodynamics of swelling porous media with electroquasistatics: I. macroscale field equations. Transport in Porous Media 47(3), 309-336 (2002)

2. Bennethum, L.S., Cushman, J.H.: Multicomponent, multiphase thermodynamics of swelling porous media with electroquasistatics: II. constitutive theory. Transport in Porous Media 47(3), 337-362 (2002) 
3. Moyne, C., Murad, M.A.: A two-scale model for coupled electro-chemo-mechanical phenomena and Onsager's reciprocity relations in expansive clays: I Homogenization analysis. Transport in Porous Media 62(3), 333-380 (2006)

Acceptance of Terms and Conditions:

Click here to agree

286

\section{Comparing simple and complex continuum-mechanical models for lung-cancer proliferation}

Patrick Schröder $^{1}$; Arndt Wagner ${ }^{1}$; Wolfgang Ehlers ${ }^{1}$

${ }^{1}$ University of Stuttgart

Corresponding Author(s): patrick.schroeder@mechbau.uni-stuttgart.de, arndt.wagner@mechbau.uni-stuttgart.de, wolfgang.ehlers@mechbau.uni-stuttgart.de

Like other cells, lung-cancer cells need a variety of substrates to keep up their metabolism and even start the proliferation process. In particular, a sustainable energy supply is necessary for proliferating cells. As a result, the increasing nutrient consumption leads to a depletion in the infested tissue, and a blood-vessel growth is triggered. Thereby, vascular growth factors are expressed from the cancer cells stimulating the proliferation of endothelial cells.

These processes can be described using a macroscopic continuum-mechanical model on the basis of the Theory of Porous Media. Therein, the solid constituents, namely the tissue cells as well as metastases, compose the porous skeleton which is saturated by immiscible pore liquids, the blood and the interstitial fluid. Furthermore, the latter is considered as a real mixture consisting of the energy supplying nutrients, migrating cancer cells and the vascular growth factors (VEGF) as well as the solvent. The process of main interest is the proliferation and its link to vascular growth. During the proliferation, VEGF initiates the blood-vessel growth increasing the nutrient concentration and speeding up the proliferation. Furtheron, this general and rather complex continuum-mechanical model is reduced. Since the additional nutrient demand is counteracted by modifying the massproduction terms of the nutrients and the cancer cells, the blood vessel growth is omitted leading to a much simpler and computationally cheaper model. Both models are governed by their mass balances and the overall momentum balance and solved within the finite element tool PANDAS. Numerically, the system of equations is spatially discretised by applying Taylor-Hood elements. The temporal discretisation is realised with an Euler time-integration scheme. In the numerical examples, the results of both models are adjusted to the same proliferation data set enabling the comparison between the different approaches. Therefore, the maximum likelihood method is utilised to optimise the parameters of the corresponding mass-production terms.

Procter and Gamble Student poster award:

I would like to compete in the Procter and Gamble Student award References:

Acceptance of Terms and Conditions:

Click here to agree

96

\section{Effective Model for Crystal Precipitation and Dissolution in a Porous Medium with Perforated Solid Matrix}

Raphael Schulz ${ }^{1}$

${ }^{1}$ Friedrich-Alexander-Universität Erlangen-Nürnberg 
Corresponding Author(s): raphael.schulz@math.fau.de

This talk presents an upscaled model for crystal precipitation and dissolution in a saturated porous medium with a perforated solid matrix. In case of high flow rates and a not strictly impermeable solid matrix (e.g. rocks) the importance of flow within the grains increases such that it needs to be included. Therefore, we model the solid matrix itself at the pore-scale as a porous medium. Hence, at the pore-scale we consider a Darcy-Stokes system, where the Beavers-Joseph boundary condition at the corresponding interface is proposed. The model contains a free boundary allowing changes of the microstructure due to crystal precipitation and dissolution, which is captured by a level-set approach. Asymptotic expansions leads to an upscaled model describing the process via Darcy's law, a transport equation and corresponding effective coefficients given by the evolution of the microstructure. The macroscopic Darcy permeability as well as the diffusivity are computable from solutions of underlying cell problems. Moreover, well-posedness of the upscaled model is investigated.

Procter and Gamble Student poster award:

References:

Acceptance of Terms and Conditions:

Click here to agree

\section{Digital Rock Physics for Carbonate Reservoirs - Simulation of Acidizing Treatments}

Jens-Oliver Schwarz ${ }^{1}$; Liping Cheng ${ }^{\text {None }}$; Tom Cvjetkovic ${ }^{1}$; Andreas Wiegmann ${ }^{1}$

${ }^{1}$ Math2Market $\mathrm{GmbH}$

Corresponding Author(s): jens-oliver.schwarz@math2market.de

Digital rock physics (DRP), like other analytical techniques, relies on the assumption that the examined samples are representative for the property of interest. With the contrasting requirements of large sample volume and high image resolution for DRP, representative elementary volumes (REV) often result in computational domains that exceed 10243 voxels.

Here, we present and evaluate a new reactive transport model for the simulation of acidizing treatments. With this model we can handle large REV up to $3072^{3}$ voxels.

The injection of hydrochloric acid is used to stimulate a carbonate reservoir by enlarging the pore space with the goal of increasing the permeability. Different dissolution patterns develop in the rock, depending on the injection rate and the concentration of the acid.

Indico rendering error

Could not include image: [404] Error fetching image

These patterns can be predicted with reactive flow simulations and the injection parameters leading to the most favorable dissolution of the reservoir rock and the correlated permeability increase can be determined. In our approach, the mineral dissolution process is modeled as a combined continuum/particle method, using the numerical code(s) in the DRP software GeoDict ${ }^{\circledR}$ and the scientific software MATLAB®.

In this ongoing project, numerical experiments qualitatively verify the numerical model. The model reproduces the dissolution patterns from the literature 1 . We simulate on a carbonate sample from the Grosmont formation (Alberta, Canada) published in a DRP benchmark study 2.

Four numerical experiments replicate the patterns described in the literature 1 . They correspond to the four distinct dissolution patterns: face dissolution, conical wormhole, wormhole, and uniform dissolution. The formation of the patterns depends on the fluid velocity and acid concentration. 
Although with the currently existing numerical code, the accessible computational domains are already larger than the ones reported in the literature 3, we are presently further optimizing the code for performance. Our aim is to compare our results with time sequences of $\mu \mathrm{CT}$-scans of real dissolution processes.

Procter and Gamble Student poster award:

\title{
References:
}

1 Maheshwari, P., Ratnakar, R.R., Kalia, N., Balakotaiah, V. 3-D simulation and analysis of reactive dissolution and wormhole formation in carbonate rocks. Chemical Engineering Science 90, 258-274, (2013). 2 Andrä, H., Combaret, N., Dvorkin, J., Glatt, E., Junehee, H., Kabel, M., Keehm, Y., Krzikalla, F., Lee, M., Madonna, C., Marsh, M., Mukerji, T., Saenger, E., Sain, R., Saxena, N., Ricker, S., Wiegmann, A., Zhan, A. Digital rock physics benchmarks Part I: Imaging and segmentation. Computers \& Geosciences 43, 25-32, (2013).

3 Pereira Nunes, J.P., Blunt, M.J., Bijeljic, B. Pore-scale simulation of carbonate dissolution in micro-CT images. Journal of Geophysical Research: Solid Earth 121, 558-576, (2016). Acceptance of Terms and Conditions:

Click here to agree

\section{A Lagrange multiplier method for single-phase flow in 3D dis- crete fractured porous media}

\author{
Philipp Schädle ${ }^{1}$; Patrick Zulian ${ }^{2}$; Daniel Vogler ${ }^{\text {None }}$; Maria G.C. Nestola ${ }^{2}$; Anozie Ebigbo ${ }^{3}$; Rolf Krause $^{2}$; Martin \\ O. Saar ${ }^{4}$ \\ ${ }^{1}$ ETH Zürich \\ ${ }^{2}$ Institute of Computational Science, Università della Svizzera italiana \\ ${ }^{3}$ ETH Zurich \\ ${ }^{4}$ Geothermal Energy and Geofluids Group, Institute of Geophysics, ETH Zurich, Zurich, Switzerland
}

Corresponding Author(s): philipp.schaedle@erdw.ethz.ch

Fractured rock formations are of crucial importance in a variety of reservoir applications, such as geothermal energy, $\mathrm{CO}_{2}$ sequestration, nuclear waste storage or unconventional oil and gas recovery. The fracture configuration and hydraulic properties of individual fractures dominate preferential fluid flow paths in fractured media, since fractures of particularly high or low conductivity act as flow conduits and barriers, respectively. Fracture configurations in fracture networks are often highly complex, which makes mesh generation for discrete fracture networks and the background matrix difficult.

In recent years, this has lead to an increasing focus on the development of numerical methods which allow generation of separate non-conforming meshes for fractures and matrix. For example, Köppel et al. 2 proposed a Lagrange multiplier method for a non-conforming finite element formulation, which they successfully applied to a benchmark suite of 2D cases. However, flow through fracture networks is governed by $3 \mathrm{D}$ effects.

This work therefore expands on their previous studies by presenting a distributed Lagrange multiplier/fictitious domain method for single-phase flow in 3D discrete fractured porous media on a parallel computing framework. Next to Köppel et al. 2, we build on work by Krause and Zulian 3 , who developed a $\mathrm{L}^{2}$-projection variational transfer method for $3 \mathrm{D}$, scalable, parallel information transfer. We combine the Lagrange multiplier method with the $\mathrm{L}^{2}$-projection to model single-phase fluid flow through 3D fracture networks. As the fractures are represented by lower-dimensional manifolds, the $\mathrm{L}^{2}$-projection algorithm has been extended for surface-volume interaction, to be able to transfer information between surface elements and volume elements for fractures and matrix, respectively. To this end, the method has been implemented successfully and a comparison of 2D results to state-of-the-art methods 1 in the field shows good agreement. Furthermore, we validate the method for 3D fracture networks by comparing to results from conforming mesh simulations which we used as a reference. Moreover, different discretization schemes and mesh convergence will be discussed. Finally, we apply the method to realistic fracture networks with hundreds of fractures. 
Our results demonstrate that the Lagrange multiplier method is capable of modeling single-phase flow through realistic 3D fracture networks.

Procter and Gamble Student poster award:

I don't want to compete References:

1 Flemisch, Bernd; Berre, Inga; Boon, Wietse; Fumagalli, Alessio; Schwenck, Nicolas; Scotti, Anna; Stefansson, Ivarand Tatomir, Alexandru. Benchmarks for single-phase flow in fractured porous media.Advances in Water Resources,239-258, (2018).

2 Köppel, Markus; Martin, Vincent; Jaffré, Jérôme and Roberts, Jean E. A Lagrange multiplier method for a discretefracture model for flow in porous media.Computational Geosciences, 1573-1499, (2018).

3 Krause, Rolf and Zulian, Patrick. A Parallel Approach to the Variational Transfer of Discrete Fields between ArbitrarilyDistributed Unstructured Finite Element Meshes.SIAM Journal on Scientific Computing, 38(3), C307-C333, (2016). Acceptance of Terms and Conditions:

Click here to agree

\title{
Simulation of fluid flow in porous materials with embedded frac- tures via an optimization approach
}

\author{
Stefano Scialò ${ }^{1}$; Stefano Berrone ${ }^{2}$; Sandra Pieraccini ${ }^{\text {None }}$ \\ ${ }^{1}$ Politecnico di Torino \\ ${ }^{2}$ Politecnico di Torino, Italy
}

Corresponding Author(s): stefano.scialo@polito.it

Flow simulations in fractured porous materials are extremely challenging for the geometrical complexity and multi-scale nature of typical domains of interest. Fractures are regions in a porous material characterized by a dramatic change of material properties, with one spatial dimension, the thickness, which is orders of magnitude smaller than the other two dimensions and than the whole domain. On the other hand, fractures might have a significant impact on relevant flow characteristics, such as flow directionality, and for this reason, in many circumstances, their explicit representation is to be preferred to the use of upscaling techniques defining homogenized material properties. According to Discrete Fracture and Matrix (DFM) models, fractures in a porous material can be represented as planar interfaces of co-dimension one, thus overcoming the difficulty related to the simultaneous representation of the scale of fracture thickness and of domain size. Nevertheless, fractures can form an intricate network of intersections, thus generating extremely challenging computational domains. Fluid flow in DFMs is governed by the 3D Darcy's law in the bulk domain and by a 2D reduced Darcy's law on a tangential reference system to each fracture, coupled by fracture/matrix and fracture/fracture interface laws. Standard numerical approaches for the simulation of flow in DFMs are based on finite element discretizations on meshes conforming to the various interfaces, in order to enforce the required matching conditions. But, as fractures can arbitrarily intersect each other, a variety of geometrical entities might be generated, such as fractures intersecting with extremely narrow angles, small fractures intersecting much larger fractures, simultaneous presence of very small and very large fracture intersections, and so on. This geometrical complexity and multi-scale nature of the domain can severely limit the applicability of numerical methods based on mesh conformity, for the impossibility of generating good-quality conforming mesh of such complex domains.

A new approach for the simulation of the flow in fractured porous materials is here presented and analyzed. The method is based on the numerical optimization in order to enforce matching conditions at fracture/matrix and fracture/fracture interfaces in such a way that no mesh conformity is needed. The solution is obtained as the minimum of a cost functional expressing the error in the fulfillment of interface conditions, constrained by multi-dimensional Dacy's law in the bulk domain and on the fractures. The resulting method is extremely flexible and robust, thus providing a viable option for simulations on large scales.

Procter and Gamble Student poster award:

References: 
S. Berrone, S. Pieraccini, S. Scialò, "Flow simulations in porous media with immersed intersecting fractures”, J. Comput. Phys. 345 (2017), pp 768-791. Acceptance of Terms and Conditions:

Click here to agree

\section{A bi-compartment porous medium approach for modeling of vas- cularized solid tumors}

Giuseppe Sciumè

${ }^{1}$ University of Bordeaux

Corresponding Author(s): giuseppe.sciume@u-bordeaux.fr

Nowadays it is well accepted by the scientific community that physical forces have a primary role on cancer evolution. Tumor growth is impeded by the anatomical structure of afflicted organs and the properties of the tissue specific extracellular matrix. The growing tumor mass presses the stroma and reduces the matrix permeability for drugs and nutrients while matrix stiffening and mechanical forces impact proliferation rates and drug performance [1,2]. Analogies with inert soft condensed matter (e.g. colloids, polymers, viscoelastic fluids, etc.) have been considered to understand mechano-biological responses of tumor cells to physical forces; these studies during the last fifteen/twenty years have successfully explained several complex behaviors of cells and tissue dynamics, such as reorganization of cell populations and cancer invasion 3 demonstrating the effectiveness of mechanistic approaches.

A mechanistic model for numerical simulation of tumor growth has been recently proposed by Sciumè et al. 4. This model is founded in the rigorous theoretical framework provided by Thermodynamically Constrained Averaging Theory (Gray and Miller 5). Tumor mass is considered as an open thermodynamic system exchanging mass and energy with the host tissue and its vascular and lymphatic systems. The mathematical formulation originally developed for tumor growth during the avascular stage has been recently developed including angiogenesis and blood vessels as an additional phase. These new features allow modeling also the vascular stage and evolution of blood flow due to the presence of the tumor and angiogenesis.

Tumor and its microenvironment are modeled as a multiphase continuum including five phases: the extracellular matrix, ECM, tumor cells, TC (mixture of living and necrotic tumor cells), healthy tissue cells, HC, interstitial fluid, IF and blood B. The ECM is the solid phase of the system and is modeled as a deformable porous continuum, while TC, HC, IF and B are modeled as immiscible fluid phases. Two porous compartments are considered: the vascular porosity saturated by the blood phase and the extravascular porosity saturated by cells and interstitial fluid. Despite the two porosities are not connected, they can exchange mass (e.g oxygen) through vessels' walls, which constitute the interface between the two porous compartments and sustain pressure difference between blood and fluids in the extravascular space. An internal variable describes formation of new capillary vessels and the consequent increase of vascular porosity and permeability. Release of tumor angiogenic factors by hypoxic cells induces production of a migratory phenotype of endothelial cells that sustains the angiogenesis process.

Numerical studies of glioma growth in different culture conditions are shown to highlight potentialities of the presented model. As a perspective, thanks to the introduction of microvasculature, the model can be further improved for study of drugs delivery within tumor and its surroundings.

Procter and Gamble Student poster award:

\section{References:}

1 Ferrari M, (2010). Frontiers in cancer nanomedicine: directing mass transport through biological barriers. Trends in Biotechnology.

2 Michor F., Liphardt J., Ferrari M. \& Widom J.(2011). What does physics have to do with cancer? Nature Reviews Cancer.

3 Gonzalez-Rodriguez, D., K. Guevorkian, S. Douezan, and Brochard-Wyart F.(2012) Soft matter models of developing tissues and tumors. Science 338:910-917

4 Sciumè G, Shelton S., Gray W. G., Miller C. T., Hussain F., Ferrari M., Decuzzi P., Schrefler B. A. (2013).

A multiphase model for three-dimensional tumor growth. New Journal of Physics, 15, 015005. 
5 Gray WG \& Miller CT (2005). Thermodynamically constrained averaging theory approach for modelling flow and transport phenomena in porous medium systems: 1. Motivation and overview Advances in Water Resources. Acceptance of Terms and Conditions:

Click here to agree

\title{
The influence of flow on bacterial transport and surface colonisa- tion in a model porous medium
}

\author{
Author(s): Eleonora Secchi ${ }^{1}$ \\ Co-author(s): Rusconi Roberto ${ }^{2}$; Roman Stocker ${ }^{1}$ \\ ${ }^{1}$ ETH Zürich \\ ${ }^{2}$ Department of Biomedical Sciences, Humanitas University
}

Corresponding Author(s): esecchi@ethz.ch

The vast majority of microorganisms are exposed to fluid flow, whether in natural environments, the human body, or artificial systems. Flow plays an important role in a broad variety of microbial processes, including nutrient uptake and fertilisation, as well as in many industrial applications, ranging from wastewater treatment to the production of biofuels. However, despite the pervasive occurrence and implications of a fluid dynamic environment, its influence on the transport and attachment of bacteria to surfaces remains poorly investigated and understood, especially in complex geometries that best describe real systems. The aim of this work is studying the influence of flow on bacterial transport and surface attachment in porous structures.

To examine surface colonisation in model porous media, we investigated the effect of laminar flow on motile bacterial suspension around a single pillar in a microfluidics channel, mimicking a single pore of a porous medium. We developed a microfluidics platform where we could study both the effect of pillar diameter and of the local flow velocity on the transport and surface attachment of the opportunistic pathogen Pseudomonas aeruginosa. In order to broaden the generality of this study, we considered also corrugated surfaces.

First, we present a phenomenon by which the combination of bacterial motility and shear results in a higher cell concentration near the walls of a channel and consequently in a strong enhancement of bacterial attachment to surfaces compared to quiescent conditions 1 . Thanks to the same mechanism, the topological features of the flow in porous structures promote the attachment of bacteria to specific regions of the surface and shape their distribution.

With a systematic experimental and numerical study, we show that the combined effect of flow past pillars of different dimensions and bacterial motility can increase the capture efficiency at imposed flow velocity compared to bacterial swimming speed. These results underscore the importance of fluid flow in triggering bacterial attachment and biofilm formation under common environmental conditions, with significant consequences in a broad range of ecological, industrial, and medical problems.

Procter and Gamble Student poster award:

\section{References:}

1Rusconi, R., Guasto, J.S. \& Stocker, R.; 2014; Nature Communications; 10: 212-217. Acceptance of Terms and Conditions:

Click here to agree

\section{Effects of adsorption-induced deformation of microstructure on fluid flow in smectite}

Majid Sedighi $^{1}$; Tian $_{\text {Chen }}{ }^{1}$; Andrey Jivkov ${ }^{1}$; Suresh Seetharam ${ }^{2}$ 
${ }^{1}$ The University of Manchester
${ }^{2}$ Belgian Nuclear Research Centre

Corresponding Author(s): majid.sedighi@manchester.ac.uk

Developing a sound theoretical description for the flow processes in smectite-rich soils and rocks have remained a challenge due to the complex microstructure effects of smectite clay minerals and adsorption-induced swelling phenomena. The pore system of compacted smectite can be simplified by two scales of porosity: i) the interlayer porosity which includes the spaces between the unit layers of smectite (microstructure) and ii) the macro porosity which represents the pore spaces between the particles and aggregates of particles. Water adsorption/desorption in the interlayer spaces between the clay platelets (interlayer hydration/dehydration) can induce microstructure deformation, affecting the fluid flow in macro pores. Understanding the evolution of fluid flow in partially saturated system of smectite is crucial for developing accurate predictive models for water flow and chemical transport in this material.

We present a model to describe the evolution of hydraulic conductivity of compacted smectite by including the theoretical explanations of adsorption induced microstructure deformation. The model is based on describing the evaluation of pore system variations of smectite with relative humidity using a geochemical approach for describing the underlying mechanisms of hydration/dehydration. We will present a hydraulic conductivity model for compacted smectite by revisiting the Kozeny-Carman $(\mathrm{KC})$ relationship with regards to the microstructure properties and the effects of adsorption-induced deformation/fabric evolution. The hydraulic conductivity model presented provides strong correlations with the experimental data reported on the evolution of unsaturated hydraulic conductivity with suction for compacted smectite-rich clays (bentonite).

We present a series of numerical analysis of the water flow in partially compacted bentonite under confined hydration. The accuracy of the new model for hydraulic conductivity will be examined through the numerical analysis presented against experimental data reported in the literature. The importance of adsorption-induced deformation of microstructure on the overall rate of macroscopic saturation process will be demonstrated. The developments in the research presented, provide further theoretical explanations and new insights on the mechanisms controlling the flow behaviour in smectite-rich geo-materials.

Procter and Gamble Student poster award:

References:

Acceptance of Terms and Conditions:

Click here to agree

\title{
Model coupling in thermochemical heat storage
}

\author{
Author(s): Gabriele Seitz ${ }^{1}$ \\ Co-author(s): Holger Class ${ }^{1}$; Rainer Helmig ${ }^{1}$ \\ ${ }^{1}$ University of Stuttgart
}

Corresponding Author(s): gabriele.seitz@iws.uni-stuttgart.de

Energy storage is regarded as one key solution for handling the fluctuating renewable sources of energy. Whereas a lot of research in energy storage focuses on electricity, there is also a huge potential in the storage of heat, e.g. as seasonal storage in house heating or as short-time storage for enhancing the efficiency in industrial processes.

Within the different technologies for heat storage, thermochemical heat storage stands out due to its large storage densities and the theoretical possibility of a nearly loss-free storage.

Thermochemical heat storage uses the difference in chemical potential of a reversible reaction. Different materials are suitable for thermochemical heat storage and span a large temperature range thus allowing a wide range of applications. The storage material usually is handled in form of powders or particles to ensure a large surface area for a fast and controllable chemical reaction. 
Modeling this systems is a tool to investigate and promote this promising technology. However it is challenging as a lot of processes interact: flow in a porous medium, transport of gaseous components, chemical reaction of solid and gaseous components under enormous release of heat which affects the fluid properties and changes the properties of the solid. Furthermore, the thermochemical reactor itself has to be represented sufficiently accurately in the model as it affects crucially the different mentioned processes.

In order to handle this complexity different approaches of model coupling are envisaged:

- Iterative coupling of transport and reaction is compared to a monolithic approach in terms of accuracy and efficiency.

- If the heat is released at the reactor surface, this boundary is best represented by coupling the porous medium flow heat-conductively to a free flow domain. This coupling is challenging, as it either necessitates an expensive spatial resolution of the free flow or a sophisticated averaged or analytical representation, e. g. by so called wall-functions.

Procter and Gamble Student poster award:

\section{References:}

Acceptance of Terms and Conditions:

Click here to agree

713

\section{Microscale modeling of high-temperature heat transfer in anisotropic porous materials}

Federico Semeraro ${ }^{1}$; Joseph Ferguson ${ }^{2}$; Sadaf Sobhani ${ }^{3}$; Arnaud Borner ${ }^{2}$; Francesco Panerai $^{4}$; Nagi Mansour ${ }^{5}$

${ }^{1}$ UIUC at NASA Ames Research Center

${ }^{2}$ Science and Technology Corp at NASA Ames Research Center

${ }^{3}$ Department of Mechanical Engineering, Stanford University

${ }^{4}$ Department of Aerospace Engineering, University of Illinois Urbana-Champaign

${ }^{5}$ Advanced Supercomputing Division, NASA Ames Research Center

Corresponding Author(s): federico.semeraro@nasa.gov

$\backslash$ documentclass[final,3p,times]\{elsarticle\}

$\% \% \% \% \% \% \% \% \% \% \% \% \% \% \% \% \% \% \% \% \% \% \% \% \% \% \% \% \% \% \% \% \% \% \% \% \% \% \% \%$

lusepackage[usenames,dvipsnames]\{xcolor\}

Inewcommand $\{\backslash \operatorname{rev}\} 1\{\{\backslash$ color $\{$ red $\} \# 1\}\}$

DeclareFontFamily\{U\}\{euc\}\{\}\%

DeclareFontShape $\{U\}\{$ euc $\}\{\mathrm{m}\}\{\mathrm{n}\}\{<-6>$ eurm 5<6-8>eurm7<8->eurm10\}\{\}\%

DeclareSymbolFont $\{$ AMSc $\}$ U $\}\{$ euc $\}\{\mathrm{m}\}\{\mathrm{n}\}$

DeclareMathSymbol\{|umu\}\{|mathord\}\{AMSc\}\}"16\}

\%\%\%\%\%\%\%\%\%\%\%\%\%\%\%\%\%\%\%\%\%\%\%\%\%\%\%\%\%\%\%\%\%\%\%\%\%\%\%\%

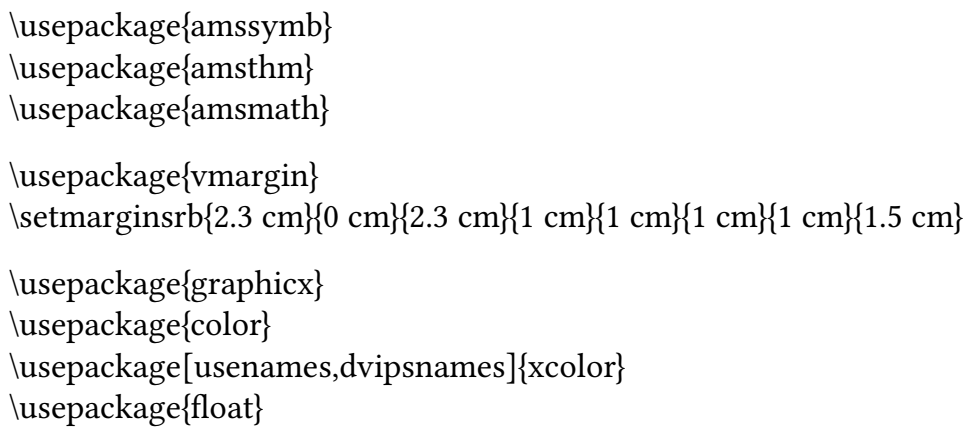


lusepackage $\{\mathrm{bm}\}$

lusepackage\{caption\}

lusepackage\{subfigure\}

lusepackage\{booktabs\}

lusepackage\{setspace\}

lusepackage[intoc] \{nomencl\}

lusepackage $\{$ makeidx\}

Imakenomenclature

lusepackage\{multicol\} \% Multiple columns environment

lusepackage $\{$ framed $\} \%$ Framing content

lusepackage $\{$ soul $\}$

ljournal\{InterPore 2019\}

\begin\{document\} }

।begin\{frontmatter\}

\title $\{\backslash$ flushleft Microscale modeling of high-temperature heat transfer in anisotropic porous materials\}

lauthor[first] $\{$ Federico Semerarolcorref $\{\operatorname{cor} 1\}\}$

lead\{federico.semeraro@nasa.gov\}

lauthor[second] \{Joseph C. Ferguson\}

lauthor[third] \{Sadaf Sobhani\}

lauthor[second]\{Arnaud Borner\}

lauthor[fourth]\{Francesco Panerai\}

lauthor[fifth]\{Nagi N. Mansour\}

laddress[first]\{UIUC, NASA Ames Research Center, Moffett Field, CA 94035, USA\}

laddress[second] \{Science and Technology Corp, NASA Ames Research Center, Moffett Field, CA 94035, USA

laddress[third]\{Department of Mechanical Engineering, Stanford University, Palo Alto CA 94305, USA

laddress[fourth]\{Department of Aerospace Engineering, University of Illinois Urbana-Champaign, Champaign, IL\}

laddress[fifth]\{Advanced Supercomputing Division, NASA Ames Research Center, Moffett Field, CA 94035, USA

lcortext[cor1]\{Corresponding author.\}

lbegin $\{$ abstract $\}$

Modeling heat transfer in porous media at high-temperatures, as in thermal protection systems during atmospheric entry, requires the understanding of both solid and radiative conduction. At lower temperatures, the primary mode of heat transfer is solid conduction through both material and gas phases. At high temperatures, however, the radiative component becomes dominant. To model these phenomena at the micro-scale, a high-fidelity representation of the materials microstructure is obtained through the use of X-ray micro-tomography. The micro-tomographic scans provide high resolution 3D reconstructions, upon which simulations are conducted on Cartesian grids.

A common assumption for solid conduction is that the thermal conductivity of the various phases is isotropic. Although this assumption is often valid, there are many materials that have anisotropic properties at the micro-scale, including fiber-based and woven materials. To simulate the isotropic or anisotropic solid conduction, a fully-conservative finite volume scheme based on the Multipoint flux approximation (MPFA) technique $\sim \operatorname{cite}\{\mathrm{mpfa}\}$ has been formulated and implemented into the Porous Microstructure Analysis (PuMA) software $\backslash$ cite\{puma\}. One challenge in modeling solid conductivity of porous materials is determining the constituent thermal conductivities, which often change with temperature. Experimental and numerical techniques to determine these values will be discussed.

For the computation of the radiative component of the thermal conductivity, the tomographic scans are processed using a Marching Cubes algorithm and the relevant view-factors are then obtained using a collision based Monte-Carlo method. The final steady-state heat fluxes into and out of each face are solutions to an algebraic system of coupled equations, with the view-factors as coefficients, 
solved using an under-relaxation iterative method. The radiative conductivity computed is combined with the solid conduction component calculated using PuMA to determine the total effective conductivity.

The simulation tools were verified against several analytical steady-state solutions and compared to previous computational studies. The conduction and radiative transfer models were then applied to fibrous and woven materials used by NASA as thermal protection systems and the results were compared to experimental data when available. IIII

lend $\{$ abstract $\}$

lbegin $\{$ keyword $\}$

Conduction $\backslash$ sep $\backslash$, Radiation $\backslash$ sep $\backslash$, Micro-tomography $\backslash$ sep $\backslash$, Finite Volume $\backslash$ sep $\backslash$, Effective Thermal Conductivity

lend $\{$ keyword $\}$

lend\{frontmatter\}

Isection*\{References\}

।bibliographystyle\{elsarticle-num\}

bibliography\{AWS_template\}

lend\{document\}

\section{Procter and Gamble Student poster award:}

I don't want to compete References:

@inproceedings\{mpfa,

title $=\{$ Multipoint flux approximation methods for quadrilateral grids $\}$,

author $=\{$ Aavatsmark, Ivar $\}$,

booktitle $=\{9$ th International forum on reservoir simulation, Abu Dhabi $\}$,

pages $=\{9-13\}$,

year $=\{2007\}$

@article\{puma,

title $=$ "\{PuMA: the Porous Microstructure Analysis software $\} "$,

journal = "SoftwareX",

volume $=$ " 7 ",

pages $=$ " $81-87$ ",

year $=$ “2018”,

issn $=$ "2352-7110",

author = "Joseph C. Ferguson and Francesco Panerai and Arnaud Borner and Nagi N. Mansour",

keywords = "Microtomography, Oxidation, Conductivity, Tortuosity factor, Porous media",

abstract $=$ "The Porous Microstructure Analysis (PuMA) software has been developed in order to compute effective material properties and perform material response simulations on digitized microstructures of porous media. PuMA is able to import digital three-dimensional images obtained from X-ray microtomography or to generate artificial microstructures. PuMA also provides a module for interactive 3D visualizations. Version 2.1 includes modules to compute porosity, volume fractions, and surface area. Two finite difference Laplace solvers have been implemented to compute the continuum tortuosity factor, effective thermal conductivity, and effective electrical conductivity. A random method has been developed to compute tortuosity factors from the continuum to rarefied regimes. Representative elementary volume analysis can be performed on each property. The software also includes a timedependent, particle-based model for the oxidation of fibrous materials. PuMA was developed for Linux operating systems and is available as a NASA software under a US \& Foreign release."

@article\{anisotropictestcase,

title $=\{$ Stationary temperature and stress fields in an anisotropic elastic slab $\}$,

author $=\{$ Tauchert, TR and Akoz, AY $\}$,

journal $=\{$ Journal of Applied Mechanics $\}$,

volume $=\{42\}$,

number $=\{3\}$,

pages $=\{647-650\}$, 
year $=\{1975\}$, publisher $=\{$ American Society of Mechanical Engineers $\}$

\} Acceptance of Terms and Conditions:

Click here to agree

\title{
Opposite effects of water repellency phenomenon on transport and release of Escherichia coli and PTCC1767 Rhodococcus ery- thropolis through dry natural soils
}

\author{
Author(s): Nasrollah Sepehrnia ${ }^{\text {None }}$ \\ Co-author(s): Jörg Bachmann ; Mohammad Ali Hajabbasi ; Fereidoun Rezanezhad ; Lubomir Lichner ; Paul Hallett \\ ; Marc Coyne \\ Corresponding Author(s): sepehrnia@ifbk.uni-hannover.de
}

Transport of bacteria is still an ongoing problem. This study explored the effect of initial air-dried condition of natural soil on the transport and removal of microorganisms that has not been studied by other researchers. Transport and release of bacteria were investigated before and after soil water repellency (SWR) breakdown under a consecutive but separate leaching process for the saturated (at suction of $0 \mathrm{~cm}$ ) and unsaturated flows (at suction of $-5 \mathrm{~cm}$ ). Hydrophilic Escherichia coli and hydrophobic Rhodococcus erythropolis (PTCC 1767) were separately infiltrated into the air-dried (at suction of $-15000 \mathrm{~cm}$ ) wettable and water repellent natural soil columns. Cell-free leaching was performed by tap water using a disk infiltrometer for a four-pore volumes $(0-4 \mathrm{PVs})$ when an influent concentration of bacteria $(3 \times 108 \mathrm{CFU} \mathrm{ml}-1)$ was poured on the column surface. That was immediately continued by further six-PVs (4.10-10 PVs) as the second influent injection performed. Bromide (Br-) was simultaneously infiltrated into the columns at the rate of $10 \mathrm{mmol} \mathrm{L}-1$ for the both pulses. $\mathrm{Br}$ - transport was influenced by the initial air-dried and respective wet conditions of both media for different hydraulic conditions. The wettable soil had a significant capacity to retain both due to higher free surface energy as the result of clay content $(p<0.001)$. The water repellent soil, however, released considerable amount of bacteria after breakdown of water repellency $(\mathrm{p}<0.001)$. Hydrophobic R. erythropolis transport was greater than hydrophilic E. coli in both soils with different manner, but, E. coli was more released. The retention patterns of bacteria were uniform and exponential; depending on soil, strain, and water flow type. The data illustrated bioaugmentation programs using R. erythropolis can be promising for subsurface media under low water content conditions. Further research using micro-tomography and scanning electron microscope (SEM) is warranted to illustrate the fate of such high retention rate of bacteria in the air-dried wettable soil in the short and long spells.

Procter and Gamble Student poster award:

References:

Acceptance of Terms and Conditions:

Click here to agree

453

\section{Numerical simulation of Santa Olalla lagoon through MODFLOW and Local Grid Refinement package.}

\author{
Author(s): Carmen Serrano Hidalgo ${ }^{1}$ \\ Co-author(s): Carolina Guardiola Albert ${ }^{2}$; Javier Heredia díaz ${ }^{2}$; Francisco Javier Elorza Tenreiro ${ }^{1}$

\footnotetext{
${ }^{1}$ Universidad Politécnica de Madrid

${ }^{2}$ Instituto Geológico y Minero de España
} 
Corresponding Author(s): carmen.serrano.hidalgo@alumnos.upm.es

Santa Olalla lagoon, situated in Doñana National Park (Spain), is an important reservoir of biodiversity. The lagoon is a hypogenic wetland which receives the discharge of the aquifer of the aeolic sands on a regional scale. This fact allows the maintenance of a permanent water regime without suffering a reduction in the volume of the lagoon's water. This situation contrasts with the general condition of other small wetlands in the Park whose hydrological degradation is, in some cases, extreme due to the intense pumping.

The evolution of the current state of the aquifer could impact into the Santa Olalla lagoon, degrading its water reservoirs and causing the loss of ecosystem services. In the framework of global change scenarios and exploitation conditions, numerical modeling offers a powerful tool to study how the lagoon would be affected.

Presently, the current model of the regional aquifer does not represent the lagoon and its relations with the aquifer. The proposal of this study is to identify the adequate numerical representation of Santa Olalla lagoon in both, a steady-state and a transient model. The present works proposes a finer spatial discretization and the implementation of different boundary conditions in the model (i.e. LAKE and DRAIN packages). The identification of the appropriate model structure to represent the lagoon was based on contrasting between the calculated and observed levels in piezometers of the lagoon's environment and the water balance of the lagoon, which have been obtained in the different variants of the model. The MODFLOW code was applied in its implementation with the ModelMuse interface and the Local Grid Refinament-2 (LGR-2) package, to refine the mesh in the lagoon's environment.

Procter and Gamble Student poster award:

References:

Acceptance of Terms and Conditions:

Click here to agree

\title{
An LDD method for coupled two-phase flow in porous media
}

\author{
David Seus $^{\text {None }}$; Florin Adrian Radu ${ }^{1}$; Christian Rohde ${ }^{2}$ \\ ${ }^{1}$ University of Bergen, Norway \\ ${ }^{2}$ University of Stuttgart
}

Corresponding Author(s): david.seus@mathematik.uni-stuttgart.de

Keywords: domain decomposition, fix point iteration, nonlinear model coupling, L-scheme, robust solvers, heterogeneous soil

Soil remediation, enhanced oil recovery, $\mathrm{CO} 2$ storage and geothermal energy are among the most important applications of porous media research and are notable examples of multiphase flow processes through porous media. In these situations mathematical modelling and simulation are among the most important tools available to predict subsurface processes and to asses feasibility and risk, since measurements below surface are very difficult or not possible at all. The mathematical and computational problems then appearing are most challenging. The modelling leads to coupled nonlinear partial differential equations, which change type and involve largely varying physical properties of the soil, such as porosity, permeability as well as soil composition. Moreover, the sheer size and scale of the domains pose computational challenges in their own right and make the design of robust discretisation methods a non-trivial task.

In the recent years, coupled problem where different models are coupled, have become one of the main focuses of porous media research. Therefore, further exploring the techniques and ideas laid out in [2,3], we present advances in the development of robust solvers for such problems on the basis of model based domain decomposition. We combine a globally convergent L-scheme with a nonoverlapping domain decomposition of the two-phase flow system as well as the Richards equation, making use of a linearised Robin type interface condition to decouple both problems and obtain a globally convergent scheme which is robust and allows to account for very heterogeneous soil properties such as vastly different and even discontinuous permeabilities. 
On this poster We present a convergence result and discuss a range of numerical experiments for the coupled full two-phase flow and Richards case. Due to its robustness the presented scheme, called the LDD scheme, is well suited to handle couplings of different physics such as porous media flow with poromechanics, either as a solver in its own right as presented here or a as a preconditioner in conjunction with a Newton based solver, as is explored in 1 .

Thus, the LDD scheme constitutes a notable contribution to handling these types of problems.

\title{
Procter and Gamble Student poster award:
}

I would like to compete in the Procter and Gamble Student award References:

1 J. Both, M. Borregales, J. M. Nordbotten, K. Kumar, F. Radu Robust fixed stress splitting for Biots equations in

heterogeneous media, Applied Mathematics Letters 68, (2017), 101 - 108.

2 D. Seus, K. Mitra, S. Pop, F. Radu, C. Rohde A linear domain decomposition method for partially saturated flow in

porous media, Comput. Methods in Appl. Mech. Eng. 333, (2018), 331-355.

3 D. Seus, F. Radu, C. Rohde A linear domain decomposition method for two-phase flow in porous media, Numerical

Mathematics and Advanced Applications ENUMATH 2017, (accepted).

4 F. A. Radu, J. M. Nordbotten, I. S. Pop, K. Kumar, A robust linearization scheme for finite volume based discretiza-

tions for simulation of two-phase flow in porous media, J. Comput. Appl. Math. 289 (2015) 134-141.

5 H. Berninger, O. Sander, Substructuring of a Signorini-type problem and Robin's method for the Richards equation

in heterogeneous soil , Computing and Visualization in Science 13 (5) (2010) 187-205.

[6] F. A. Radu, K. Kumar, J. M. Nordbotten, I. S. Pop, A robust, mass conservative scheme for two-phase flow in porous

media including $\mathrm{H}^{*}$

older continuous nonlinearities, IMA Journal of Numerical Analysis (2017) 1-37. Acceptance of Terms and Conditions:

Click here to agree

\section{Creating synthetic porous media for multiphase flow experiments under controlled conditions}

\author{
Swej Shah ${ }^{1}$; Alexander van Ballaer ${ }^{1}$; Amin Askarinejad ${ }^{1}$; William Rossen $^{1}$; Karl-Heinz Wolf ${ }^{1}$ \\ ${ }^{1}$ Delft University of Technology
}

Corresponding Author(s): s.y.shah@tudelft.nl

Heterogeneity in sedimentary rock makes prediction of subsurface flow behaviour difficult. Sharp changes in permeability can be found as unconformities such as layer boundaries or laminations and cross-laminations within individual subsurface formations. They contribute to effects such as capillary entrapment, channelling or early breakthrough and foam generation. They can have a significant impact on the successful application of a displacement process, be it for oil recovery or for aquifer remediation. It is desirable to conduct flow experiments under controlled conditions with pre-defined model porous media with the desired features of heterogeneity represented. Laboratory experiments studying subsurface processes are often conducted with tubular sand-packs or rock tubes called cores. Naturally found rocks often have a variety of structural features that affect experimental results and make the modelling of the effects of a single feature difficult. Sand-packs, on the other hand, are unconsolidated and extremely difficult to compact. Therefore, in this study, we explore several methods of preparing controlled synthetic pore frameworks of pre-defined dimensions in tube-shaped samples. The degrees of freedom in parameters are limited by the degree of resolution expected for certain type of experiments. In this work, our attempts are directed towards creating layered cores with homogeneous individual layers and sharp, monotonic changes in permeability in between, for experiments concerning foam generation and gas trapping. We tested synthetic glasses of mono material, heterogeneous materials and both, with high/low permeability interfaces. We determined the quality of the interfaces, heterogeneity and pore surface qualities. 
Next to this, high temperature preparations of clay rich grain aggregates were prepared and their pore structure analysed by using micro-computed tomography. The grains are fused at elevated temperatures and contact points are transformed to contact planes. Lastly, well-characterized, compacted and consolidated sand-packs were created. These packs initially hold point contacts between the grains, creating a maximum porosity. Compaction is achieved using centrifugal force and consolidation is achieved by drainage of a wetting fluid under vacuum, during compaction. Advantages and disadvantages of these techniques, together with the suitability of the end-result with respect to our experimental requirements, is discussed. Every sample was analysed through permeability measurements and X-ray computed tomography. The layered sintered glass cores were also used to conduct core-flooding experiments with foam generation across abrupt increments in permeability. The sintering process is sensitive to the physical dimensions of the core such as length and diameter. Repeatability is limited and imperfect sintering near the edges of a core can significantly affect the relative permeability of individual phases. The structural integrity of cores made from sintered clay rich grain aggregates is sensitive to the heating and cooling cycles. Inhomogeneous temperature profiles often lead to fractures within the core and repeatability can not be ensured. The compacted sand-packs, using a centrifuge, produced the most promising results in terms of homogeneity within the samples. On average, a 15-35\% reduction in permeability is achieved as a result of compaction.

Procter and Gamble Student poster award:

I would like to compete in the Procter and Gamble Student award References:

Acceptance of Terms and Conditions:

Click here to agree

664

\section{Biological activity affects rheological properties in surface water sediments}

Florian Zander $^{1}$; Ahmad Shakeel $^{1}$; Alex Kirichek $^{2}$; Claire Chassagne $^{1}$; Julia Gebert ${ }^{1}$

${ }^{1}$ TU Delft

${ }^{2}$ alex.kirichek@deltares.nl

Corresponding Author(s): a.shakeel@tudelft.nl, j.gebert@tudelft.nl

The microbial turnover of organic matter in surface waters is suspected to affect the processes of flocculation, sedimentation and consolidation, leading to the formation of layers of different rheological properties in the transition zone between the water phase and the genuine river bed. Under anaerobic conditions, degradation of organic matter leads to gas formation, obstructing sonic depth finding, impeding sedimentation and delaying consolidation. Gas generation reduces sediment density, viscosity and shear strength, thereby impacting detection and maintenance of the navigable depth and decreasing the stability of subaquatic constructions 1 . The gas production in sediments due to the degradation of organic matter can have a significant effect on the sediment rheological properties, such as yield stress and thixotropy. The extent of organic matter degradation depends on the amount (source term) and the degradability of the organic matter. Degradability is, amongst others, controlled by the extent of immobilization of organic matter in complexes with the mineral phase (silt, clay) and the subsequent shift of available organic matter to the less accessible, higherdensity pool 2 .

In a vertical cut, four layers can be distinguished between the upper (water) and the lower (riverbed) boundary: suspended particulate matter (SPM), fluid mud (FM), pre-consolidated sediment (PS) and consolidated sediment (CS). The role of the physicochemical properties of the organic and mineral phases in these layers for organic matter degradability and its influence on the rheological properties are investigated in the framework of the projects BIOMUD and RHEOMUD (www.mudnet.eu). Over four years, the spatial and temporal variability of biogeochemical and rheological properties of sediments from nine locations in the area of the Port of Hamburg are investigated. First results show a clear decreasing gradient in organic matter degradability from upstream to downstream of 
the port 3. In the vertical cut, a decrease in organic matter degradability from upper layers (fluid mud) to deeper layers (consolidated sediment) is associated with an increase in the shear stress at given shear rates. The direct effect of gas production on the rheological properties is analyzed by performing rheological analyses on fresh and differently degraded samples using two different geometries (Couette and Vane), which were proven to be the most suited for this type of experiments 4. Stress sweep tests are carried out to determine the yield stress of the samples whereas shear rate increasing and decreasing ramps are performed to analyze the thixotropic behavior of the samples. This study enables to directly link the degradation of organic matter to the rheological properties of the sediments.

References

1 A. Kirichek, C. Chassagne, H. Winterwerp, A. Noordijk, R. Rutgers, C. Schot, K. Nipius, T. Vellinga (2018): How navigable are fluid mud layers? TERRA ET AQUA.

2 J. Gebert, C. Knoblauch, A. Gröngröft (2018): Gas production from dredged sediment. Waste Management, under review.

3 F. Zander, J. Gebert (2018): Turnover of organic matter in ports and waterways: Project BIOMUD. Poster presented at Rostock Dredging seminar, September 2018.

4 A. Shakeel, A. Kirichek, C. Chassagne, Yield stress measurements of natural mud suspensions using different rheological methods and geometries: A comparison (to be published).

Procter and Gamble Student poster award:

I don't want to compete References:

Acceptance of Terms and Conditions:

Click here to agree

\title{
Microscopic investigation of polymer flow in porous media
}

\author{
Author(s): Pegah Shakeri ${ }^{1}$ \\ Co-author(s): Michael Jung ${ }^{1}$; Martin Brinkmann ${ }^{2}$; Stephan Herminghaus ${ }^{3}$; Ralf Seemann ${ }^{1}$ \\ ${ }^{1} 1,2$ \\ ${ }^{2} 1$ \\ ${ }^{3} 2$
}

Corresponding Author(s): pegah.shakeri@physik.uni-saarland.de

Polymer flooding is established as a promising, low risk and efficient enhanced oil recovery technique. The general consensus in the petroleum industry suggests that volumetric sweep efficiency improvement due to the favorable mobility ratio of polymer flooding on the macroscale is the main mechanism of increasing oil recovery and microscopic sweep efficiency is not affected by polymers 1. This means that a polymer solution would not reduce the residual oil saturation on the scale of individual pores beyond the residual saturation after water flooding. However, recent experimental and field results indicate that the microscopic sweep efficiency of polymer flooding could be substantially higher than that of water flooding [1-3]. Thus a microscopic investigation of the polymer flow and of the characteristic properties of a polymer solution are needed to gain clear insight into the pore scale mechanism of oil displacement by a polymer solution in porous media.

In our experiments, we have studied the flow of aqueous polymer solutions in a quasi-two-dimensional microfluidic device. The microfluidic cell was fabricated by soft lithography techniques and consist of oil wet UV-curable glue. Initially the cell was completely saturated with oil, which is subsequently displaced by flushing a polymer solution. Primary observations indicated unstable and fluctuating (wobbling) oil-polymer solution interfaces and lower residual saturations, in comparison to a flood of water or aqueous glycerol solution having the same zero shear viscosity as the polymer solutions. Qualitative observation of the flow path lines occurring during a polymer flush in the microfluidic device was performed by adding fluorescent particle to the aqueous phase. The results indicated the occurrence of fluctuations in the flow path lines deviating from laminar flow even in the creeping flow domain (low Reynolds number), in contrast to Newtonian fluids in the same flow condition. 
Further focus on the oil-polymer solution interface shows that the observed instabilities in the bulk flow destabilize the oil-polymer interface and act in favor of displacement of entrapped oil ganglia by exerting an extra force to the interface and consequently are capable of improving the microscopic displacement. These fluctuations and unsteady pattern of flow path lines seem to originate from viscoelastic properties and elasticity of the polymer solution and depend on flow rate, the design of the polymer solution (polymer concentration, salinity, etc.) and geometry of the porous media.

Procter and Gamble Student poster award:

I would like to compete in the Procter and Gamble Student award References:

1. Wei, Bing, et al. "Oil displacement mechanisms of viscoelastic polymers in enhanced oil recovery (EOR): a review.” Journal of Petroleum Exploration and Production Technology 4.2 (2014): 113121.1.

2. Qi, Pengpeng, et al. "Reduction of residual oil saturation in sandstone cores by use of viscoelastic polymers." SPE Journal 22.02 (2017): 447-458.

3. Wever, D., Picchioni, F. \& Broekhuis, A., 2011. Polymers for enhanced oil recovery: a paradigm for structure-property relationship in aqueous solution. Progress in Polymer Science, 36(11), pp.1558-1628.

Acceptance of Terms and Conditions:

Click here to agree

153

\title{
Direct numerical simulation of two-phase flow at the micro-scale using a volume-of-fluid method
}

\author{
Mosayeb Shams $^{\text {None }}$; Branko Bijeljic ${ }^{\text {None }} ;$ Martin Blunt $^{\text {None }}$
}

Corresponding Author(s): m.shams14@imperial.ac.uk

We present a numerical scheme to model two-phase flow in porous media where capillary forces dominate over viscous effects. The volume-of-fluid method is employed to capture the fluid-fluid interface whose dynamics are described based on a finite volume discretization of the Navier-Stokes equations. Interfacial forces are calculated directly on reconstructed interface elements such that the total curvature is preserved. The computed interfacial forces are explicitly added to the NavierStokes equations using a sharp formulation which effectively eliminates spurious currents. The numerical model is validated in terms of physics, robustness, and mesh convergence, using an extensive hierarchy of static and dynamic test cases including wetting effects at the solid interface in two and three space dimensions.

Procter and Gamble Student poster award:

References:

Acceptance of Terms and Conditions:

Click here to agree

970

\section{Reservoir Physical Analysis of EIlj Gas Field in West Amu Darya}

Yunpeng Shan ${ }^{\text {None }}$

Corresponding Author(s): 704410267@qq.com 
Amu Darya is a Mesozoic-Cenozoic large depression basin spanning Eastern Turkmenistan and southwestern Uzbekistan. EIlj Gas Field is located in the north of Amhe Basin. The reservoirs are carbonate rocks deposited by Jurassic Carlovian gentle slope platform and Oxford marginal shelf platform. The main sedimentary facies are overlapping shoal and platform margin levee reef. The reservoir space is mainly primary pore due to the undeveloped tectonic movement in this area. In this paper, the cores drilled from exploration wells in EIlj Gas Field are selected for reservoir physical property testing. The measurement of porosity includes microscopic observation of the cast slices of cores, displacement testing of cores and crushing measurement. For permeability measurements, gas permeability is measured by nitrogen. Based on the experimental results, intersection diagrams of porosity and permeability and relative permeability-oil saturation curves are plotted. Four types of cementation of pore were identified by microscopic slice observation, namely, base cementation, pore cementation, contact cementation and mosaic cementation. Textural maturity is at a high level. The physical property evaluation criteria of primary pore reservoirs in carbonate reservoirs can be established by the experimental results in this paper. And the relationship between porosity and permeability of carbonate reservoir is positively correlated. This conclusion can guide oil and gas exploration and give priority to the exploration of primary porous sedimentary facies zones with high porosity, which is conducive not only to the discovery of oil and gas fields with large reserves, but also to the oil and gas exploitation after putting into production.

\title{
Procter and Gamble Student poster award:
}

I would like to compete in the Procter and Gamble Student award References:

Acceptance of Terms and Conditions:

Click here to agree

\section{Field application of micellar flooding for LNAPL recovery}

\author{
Pushpesh Sharma ${ }^{1}$; Konstantinos Kostarelos ${ }^{1}$; Anders Christensen ${ }^{2}$; Søren Lenschow ${ }^{2}$; Phillip de Blanc ${ }^{3}$ \\ ${ }^{1}$ University of Houston \\ ${ }^{2}$ Niras $A / S$ \\ ${ }^{3}$ GSI Environmental Inc.
}

Corresponding Author(s): psharma19@uh.edu, kkostare@central.uh.edu

NAPL remediation techniques using surfactants, such as enhanced pump and treat (“SEAR”) and micellar flooding, provide a faster and more efficient way to recover NAPL from subsurface. Micellar flooding is a recovery technique that relies on the ability of surfactants to mobilize the NAPL phase by reducing the interfacial tension between the aqueous and NAPL phases. The application of micellar flooding for NAPL recovery has been limited to laboratory studies and some pilot scale/full field applications. This paper presents results from a full-scale field application of the micellar flood process designed to recover a LNAPL (Jet fuel) from a surficial sandy aquifer located at a tank facility in western Jutland, Denmark.

Phase behavior and flow experiments were conducted with field samples to identify suitable surfactant formulations. A field-scale simulation model was developed that indicated that a line-drive pattern with hydraulic control wells would be optimal for NAPL recovery. Based on the experimental and simulation studies, the micellar flood was implemented in 8 stages on the full field. In addition to monitoring during the field implementation, monitoring was conducted immediately after and for a period of more than 1 year. Post surfactant flood site monitoring consisted of sampling water from multi-levels and from recovery wells. Groundwater samples were analyzed for Total Petroleum Hydrocarbon (TPH) and benzene, toluene, ethylbenzene and xylene (BTEX).

The field implementation resulted in more than $90 \%$ recovery (approximately $32,700 \mathrm{~kg}$ of LNAPL) based on the mass balance using Laser Induced Fluorescence (LIF) and chemical soil analysis (Total Petroleum Hydrocarbon, TPH and BTEX) data. Both pre-flood and post-flood LIF data are presented and discussed. The pre-treatment and post-treatment mass discharges were also monitored, which led to a relationship between mass discharge with the mass reduction in the source zone. This paper discusses the results of the field application and presents the monitoring methods and their implementation in the field, as well as the lessons learned from the field operation. Also, mass discharge 
$\Gamma$-model commonly used for DNAPL modelling, was successfully implemented for LNAPL remediation. Studies of field applications of surfactant remediation processes are not readily available; it is especially rare to present a study of micellar flooding implementation for remediation processes. This paper provides insights about the micellar flooding process that will be useful in designing future applications.

Procter and Gamble Student poster award:

I would like to compete in the Procter and Gamble Student award References:

Acceptance of Terms and Conditions:

Click here to agree

\title{
Upscaling of two-phase flow models in porous media: from the pore scale to the Darcy scale
}

\author{
Author(s): Sohely Sharmin ${ }^{1}$ \\ Co-author(s): Iuliu Sorin Pop ${ }^{1}$; Carina Bringedal \\ ${ }^{1}$ Hasselt University
}

Corresponding Author(s): sohely.sharmin@uhasselt.be

We consider the flow of two immiscible phases in a porous medium. At the scale of pores, these fluids are separating by an interface, of which properties are influencing the flow. Since on its turn this interface moves with the flow, its location is not known apriori. In mathematical terms, this translates into a free boundary problem.

For many practical applications, the main interest is in the behaviour of the system, as averaged at the Darcy scale. This is usually modelled through the mass balance equations for the two fluids, and through constitutive relationships relating the phase pressures and velocities to the phase saturations, respectively the phase pressure difference and their phase saturations. Such relationships are often postulated beforehand, and then validated experimentally under suitable conditions. Often, the connection to the processes at the scale of pores is diluted.

This presentation is focusing on the scale transition when deriving the Darcy scale models. Specifically, we start with the model at the scale of pores, where the flow of each fluid phase is described by the Navier-Stokes equations. At the interface separating the fluids we impose the continuity of the fluid velocities and of the tangential components of the normal stresses, whereas the jump in their normal components depend on the surface tension effects. In particular, Marangoni effects are considered.

In a first attempt, a simple pore geometry is considered: a long, thin pore. Assuming that the ratio between the pore width and its length is small, we use asymptotic expansions to derive effective equations valid at the Darcy scale. These equations are derived for different capillary numbers and viscosity ratios. In particular, we show that the surface tension effects are important for small capillary numbers whereas Marangoni effects are visible for moderate capillary numbers. Finally, the Darcy scale models are validated by numerical experiments comparing the outcome of the upscaled models with the averaged results of the direct pore scale simulations.

Procter and Gamble Student poster award:

I would like to compete in the Procter and Gamble Student award References:

Acceptance of Terms and Conditions:

Click here to agree 


\title{
In-Situ High Resolution Dynamic X-ray Microtomographic Imag- ing of Olive Oil Removal in Kitchen Sponges by Squeezing and Rinsing
}

\author{
Author(s): Abhishek Shastry ${ }^{1}$ \\ Assche ${ }^{1}$; Eric Robles ${ }^{5}$; Veerle Cnudde ${ }^{4}$; Luc Van Hoorebeke ${ }^{4}$; Matthieu N Boone ${ }^{1}$ \\ ${ }^{1}$ Ghent university \\ ${ }^{2}$ Procter and Gamble Corporate Functions, Surface Imaging and Microscopy Department, \\ ${ }^{3}$ The Procter and Gamble Company, Brussels Innovation Center \\ ${ }^{4}$ Ghent University \\ ${ }^{5}$ The Procter and Gamble Company, Newcastle Innovation Center
}

Co-author(s): Paolo E Palacio-Mancheno ${ }^{2}$; Karl Braeckman ${ }^{3}$; Ivan Josipovic ${ }^{4}$; Sander Vanheule ${ }^{1}$; Frederic Van

Corresponding Author(s): abhishek.shastry@ugent.be

High resolution X-ray Computed Tomography $(\mu \mathrm{CT})$ is an imaging technique to visualize 3D structures, non-destructively and non-invasively. Recently, the possibilities to visualize dynamic processes in-situ by adding a time dimension has gained interest. This study illustrates the method to visualize the removal of olive oil from kitchen sponges made of polyurethane under dynamic conditions (rinsing, squeezing and cleaning by adding detergents) and the results can be used as modelling inputs for the evaluation of sponge behaviour during absorption and removal of food soil. However, the possibilities of imaging fatty liquids (olive oil) in water simultaneously are limited due to the low difference in X-ray attenuating coefficients at the X-ray energies commonly used in $\mu \mathrm{CT} 1$. For the first time, time-dependent imaging of this type of system was established with sufficiently large contrast gradient between water (with/without detergent) and olive oil (model fatty liquid) by the application of suitable fat-sensitive X-ray contrast agents. Two different contrast agents (magnetite powder (Fe3O4) and brominated vegetable oil) were evaluated. The contrast agent and olive oil were mixed and subsequently added on to the sponge. The contrasted olive oil filled sponges were then rinsed and squeezed in a unique laboratory loading device with a fluid flow channel designed to fit inside a rotating gantry-based X-ray $\mu \mathrm{CT}$ system. Results suggest the use of brominated vegetable oil as a preferred contrast agent over magnetite powder for enhancing the attenuation coefficient of olive oil in a multi fluid filled kitchen sponge. The contrasted olive oil solution does not phase separate and does not adsorb strongly to the sponge inner surfaces during the cleaning process by detergents. The application of contrast agents also helps in accurately tracking the movement and volume changes of soils in compressed open cell structures. With the in house-built cleaning device, it was quantified that almost $99 \%$ of cleaning was possible for contrasted olive oil (brominated vegetable oil with olive oil) dispersed in the sponge. This $4 \mathrm{D}-\mu \mathrm{CT}$ (time dependent imaging) approach allowed for realistic mimicking of the cleaning process and provided closer evaluation of the effectiveness of cleaning by detergents to minimize bacterial growth.

References:

1 Brown, K.; Schlüter, S.; Sheppard, A.; Wildenschild, D. On the challenges of measuring interfacial characteristics of three-phase fluid flow with X-ray microtomography. J. Microsc. 2014, 253, 171182.

Procter and Gamble Student poster award:

I would like to compete in the Procter and Gamble Student award References:

Acceptance of Terms and Conditions:

Click here to agree

\section{Cellular 3D printed concrete with disconnected pores}

Shashank Shekhar ${ }^{1}$ 
${ }^{1}$ IIT Gandhinagar

Corresponding Author(s): shashank.shekhar@iitgn.ac.in

Pore size and permeability of the concrete are governing factors that control properties such as corrosion resistance, chemical attack resistance, fire resistance, thermal resistance and acoustic resistance. A disconnected pore network is the primary requirement for having the above-mentioned properties in abundance. Researchers have developed 3D printed cellular structures using cement paste, which is the main binding agent in concrete. However, the pores are connected and do not offer the properties as mentioned in the beginning. The present work is focused on the development of 3D printed concrete structure with disconnected pores or cells. The pore structure of the autoclaved aerated concrete is taken as the reference to 3D print the structure. The size of the pores is dependent on the rheology of the concrete mix. It is demonstrated that this $3 \mathrm{D}$ printed concrete structure with disconnected pores has better strength to mass ratio, thermal insulation, accoustic resistance, and flow resistance for ingressing liquid.

Procter and Gamble Student poster award:

I would like to compete in the Procter and Gamble Student award References:

Acceptance of Terms and Conditions:

Click here to agree

\section{Gas flows in nano channels: coupling molecular dynamics to a kinetic method}

Qiang Sheng ${ }^{1}$; Minh Tuan $\mathrm{Ho}^{2}$; Jun $\mathrm{Li}^{3}$; Matthew Borg ${ }^{4}$; Yonghao Zhang ${ }^{2}$; Jason Reese ${ }^{1}$

${ }^{1}$ The University of Edinburgh

${ }^{2}$ University of Strathclyde

${ }^{3}$ King Fahd University of Petroleum and Minerals

${ }^{4}$ The university of Edinburgh

Corresponding Author(s): qiang.sheng@ed.ac.uk

The physics at fluid-solid interfaces 1 dominates the fluid dynamics and heat transfer in micro and nano flow systems. However, we still do not understand fully how to characterize the fluid-solid interactions and evaluate their effects on the flow (for example, gas adsorption and surface diffusion). Molecular Dynamics (MD) is arguably the best tool to explore nanoscale interfaces, provided accuratelycalibrated intermolecular potential functions are available. Unfortunately, it is very computationally intense, so various solvers that couple MD to continuum-fluid computational fluid dynamics (CFD) have been proposed. These aim to balance accuracy against computational cost by only using MD in interfacial regions while the bulk flow is solved using CFD.

Continuum-fluid CFD is inappropriate for microscale gas flows, due to nonequilibrium (or rarefaction) arising, when the mean free path of molecules is comparable to the height of channels. For example, velocity slip and temperature jump occur at bounding surfaces, which are widely modelled using

diffusive boundary conditions 2 . However, a detailed role of solid walls on thelocal gas behavior cannot be characterized accurately by such a general boundary model 3 . The gas transport in the bulk in micro-confined geometries can be described using the Boltzmann equation. Simplified models, e.g. the BGK equation, are often adopted in such cases, with the Discrete Velocity Method (DVM) employed for a numerical solution deterministically. The DVM has been shown to produce reliable data for rarefied gas flows from low to high speed 4 .

This work addresses some of the challenges in describing, at the same time, both the non-equilibrium behavior in the gas and the complex gas-solid interactions. We propose a hybrid model that couples MD to DVM: accurate MD simulations calculate the velocity distribution function (VDF) for gas molecules in the vicinity of molecularly-resolved solid walls; then, this VDF is introduced as a numerical boundary condition to a DVM solver for the BGK equation for the bulk flows. We show results of validations of this $\mathrm{MD} / \mathrm{DVM}$ 
hybrid method for different benchmark flows: Fourier, Couette, and Poiseuille. Comparisons with the solutions produced purely by DVM or MD with diffusive boundary conditions demonstrate the advantages and limitations of this hybrid approach.

Procter and Gamble Student poster award:

\title{
References:
}

References

1 E. Bormashenkoa. Physics of solid-liquid interfaces: from the Young equation to the super-hydrophobicity. Low Temperature Physics, 42, 622, 2016.

2 X. J. Gu and D. R. Emerson. A high-order moment approach for capturing non-equilibrium phenomena in the transition regime. Journal of Fluid Mechanics, 636, 177-216, 2006.

$3 \mathrm{~S}$. Wang et al. Fast mass transport of oil and supercritical carbon dioxide through organic nanopores in shale. Fuel, 181, 741-758, 2016.

4 M. T. Ho et al. A comparative study of the DSBGK and DVM methods for low-speed rarefied gas flows. Computers \& Fluids, 181, 143-159, 2019. Acceptance of Terms and Conditions:

Click here to agree

\section{A Dual Domain Stochastic Lagrangian Model for Predicting Trans- port in Open Channels with Hyporheic Exchange}

\author{
Author(s): Thomas Sherman ${ }^{\text {None }}$ \\ Co-author(s): Aaron Packman ${ }^{1}$; David Richter ${ }^{2}$; Diogo Bolster ${ }^{3}$; Kevin Roche ${ }^{4}$ \\ ${ }^{1}$ Northwestern University \\ ${ }^{2}$ University of Notre Dame \\ ${ }^{3}$ Notre Dame \\ ${ }^{4}$ University of Notre Dame
}

\section{Corresponding Author(s): tsherma3@nd.edu}

The exchange of surface and subsurface waters is important in understanding and predicting large scale transport processes in streams and rivers. Accurately capturing the influence of small-scale features associated with turbulent dispersion on exchange in an upscaled framework is necessary for developing reliable predictive models at the reach scale. Using direct numerical simulations (DNS), we fully resolve turbulent flow and hyporheic exchange in an open channel. The spatio-temporally averaged DNS velocity and scalar diffusivity profiles are used to parameterized a 2D particle tracking model. Breakthrough curves and rate of surface mass loss to the subsurface in the 2D model agree with the DNS after a sufficient distance downstream from particle injection. Additionally, we parameterize a 1D dual domain coupled Continuous Time Random Walk (ddc-CTRW) with surface and subsurface travel time/distance joint pdfs and successfully reproduce the behavior of both the DNS and the 2D particle tracking model. Once fully parameterized, the ddc-CTRW enables accurate predictions of breakthrough curves, while reducing cpu time by orders of magnitude when compared with DNS.

\section{Procter and Gamble Student poster award:}

I would like to compete in the Procter and Gamble Student award References:

Acceptance of Terms and Conditions:

Click here to agree 


\title{
Production Forecasting of Horizontal Wells with Acid Etching Hole Completion in Unconventional Reservoirs
}

\author{
Juntai Shi ${ }^{1}$; Xuechen You ${ }^{1}$; Jinhui Fang ${ }^{2}$; Ming Lv ${ }^{1}$; Yanran Jia $^{3}$; Zheng Sun ${ }^{\text {None }}$ \\ ${ }^{1}$ China University of Petroleum Beijing \\ ${ }^{2}$ China University of Petroleum (Beijing) \\ ${ }^{3}$ China Unversity of Petroleum Beijing
}

Corresponding Author(s): juntai.shi@gmail.com,615807453@qq.com, szcup611@163.com

The development of unconventional resources has been boosted by the advancement in horizontal multi-stage fracturing technology. However, horizontal multi-stage fracturing need a lot of water and low water flow back after fracturing decreases oil and gas productivity greatly. Horizontal wells with acid etching hole completion may be as a good choice to handle this issue. A detailed study for production forecasting of horizontal wells with acid etching hole completion in unconventional reservoirs is significantly necessary.

This paper presents a new approach for predicting production of horizontal wells with acid etching hole completion in unconventional reservoirs. The transient pressure model for horizontal wells with acid etching hole completion is derived by utilizing the point source function, Newman Green's function and Laplace transformation. Well bottom hole pressure is calculated based on the numerical inversion algorithm and a template curve is made. Production decline template curve is also made based on relationship between the transient pressure and production. Production rate by horizontal wells with acid etching hole completion and that by multi-fractured horizontal well is compared. Results demonstrate that, in the log-log plots of dimensionless pressure derivative versus dimensionless time and the log-log plot of dimensionless production versus dimensionless time, wellbore storage flow, radial flow to acid etching hole, linear flow to acid etching hole assemble, bilinear flow from the stimulated reservoir volume (SRV) to acid etching hole assembles and from outer reservoir to the SRV, and pseudo radial flow of the whole reservoir including the outer region. Comparison of production rate by horizontal wells with acid etching hole completion and that by multi-fractured horizontal well indicates that horizontal wells with acid etching hole completion gives a higher production rate at the middle and late stage. If multiple assemble of acid etching hole is designed, the production rate at early stage may become also higher than that by multi-fractured horizontal well. This work can provide another choice for improving production and a new approach for production forecasting of horizontal wells with acid etching hole completion in unconventional reservoirs Also, it can provide guidance on optimization of acid etching hole completion design in horizontal wells.

Procter and Gamble Student poster award:

References:

Acceptance of Terms and Conditions:

Click here to agree

\section{A New Relative Permeability Correlation for Gas Condensate Reser- voirs Based on Microscale Force Analysis}

\author{
Juntai Shi ${ }^{1}$; Chengyuan Liu $^{2}$; Zheng Sun ${ }^{\text {None }}$; Tao Zhang ${ }^{2}$ \\ ${ }^{1}$ China University of Petroleum Beijing \\ ${ }^{2}$ China University of Petroleum (Beijing)
}

Corresponding Author(s): juntai.shi@gmail.com,1663272226@qq.com, szcup611@163.com, tobiascheuing@163.com

The relative permeability is an important parameter in oil and gas production forecasting. People often acquire the relative permeability from experiments or correlations. For gas condensate reservoir, the current experiment costs a lot if considering gradual condensation. The correlations of 
relative permeability are often used these years, such as X-model, Corey or BrookseCorey model, viscous-coupling model and Chima's model. However, for gas and condensate reservoirs, if using these correlations, the condensate blocking and gas and condensate oil deliverability reduction will be overestimated. Hence, it is extremely important to propose a new gas-condensate relative permeability correlation for purpose of accurately forecasting gas and condensate oil production.

In this paper, we firstly analyze the gas and condensate occurrence and their filtration mechanism in the porous media. Secondly, we develop microscopic filtration models and relative permeability models for the condensate oil droplets, oil film and oil plug based on force analysis. Thirdly, we establish the final relative permeability correlation for condensate oil by combining these three models together and derive the gas relative permeability correlation. Finally, we validate the proposed model by experimental data in literatures and reservoir simulation in Yakela gas condensate reservoir.

Microscale force analyses show that forces acting on the condensate oil droplets, the condensate film and the condensate plug include gravity, interface force, gas flow carrying force, pressure driving force, supporting force of the water film adhering to the pore wall and viscous force. Condensate oils flow pattern (including the condensate oil droplets, the condensate films and the condensate plugs) in porous media is microscopic non-linear and the corresponding model consists of three parts: threshold pressure gradient term, Darcy flow term and inertial flow term. Both experimental data and results evaluated by the proposed model show that the condensate relative permeability is concave-convex-concave in shape with increasing condensate oil saturation, especially in low permeability condensate gas reservoirs. Good agreements between experimental relative permeabilities and those evaluated by the proposed model are obtained, and the gas and oil productions forecasted by reservoir simulation incorporating the proposed relative permeability also agree the real gas well production performance. Results also indicate that the condensate relative permeability curve does not always monotonically increase with condensate saturation for low permeability condensate gas reservoirs, the highest condensate relative permeability is between $30 \%$ and $70 \%$. Thus, for a gas condensate reservoir, large gas and condensate production rates in middle stage of development process are recommended.

The relative permeability model proposed in this paper is theoretical derived based on microscale force analysis and has been validated by experimental data and reservoir simulation. It is an essential parameter in well performance forecasting and reservoir simulation. Also, it can be as a reference to determination of the rational working system for condensate gas reservoirs.

Procter and Gamble Student poster award:

References:

Acceptance of Terms and Conditions:

Click here to agree

\section{Porous materials for nutrient recirculation from industry to agri- culture}

Mirian Chieko Shinzato $^{1}$; Juliana Gardenalli de Freitas ${ }^{1}$; Tereza da Silva Martins ${ }^{1}$; Luis Fernando $\mathrm{Wu}^{1}$; Thais Oliveira Mariano ${ }^{1}$

${ }^{1}$ Universidade Federal de São Paulo

Corresponding Author(s): mirianshinzato@gmail.com

Brazil is one of the largest fertilizer consumers in the world, since the agriculture is an important economic activity. However most of fertilizers are imported and, in the case of N-NH4+, more than $85 \%$ were imported in 2017. On the other hand, the discharge of wastewater containing ammoniacal nitrogen $(\mathrm{N}-\mathrm{NH} 3)$ from various anthropogenic activities is contaminating and increasingly degrading the water quality. Because most of them present high ammonia concentration, alkaline $\mathrm{pH}$ and high 
salinity, the efficiency of the traditional biological process in removing nitrogen is affected. Therefore, the use of natural porous material with high cation exchange capacity as filters may solve this problem. This study tested the use of a natural zeolitic material (clinoptilolite and mordenite) and a clay mineral (vermiculite) for NH4+ removal from solution and, after this treatment, its application as a slow release fertilizer in soil. This kind of fertilizer is more proper to the tropical conditions, since the traditional fertilizers are very soluble, and the plants cannot absorb all available nutrients. Zeolites and vermiculites are aluminosilicate with high cationic exchange capacity. This property is due to the large replacement of $\mathrm{Si} 4+$ by $\mathrm{Al} 3+$ in their tetrahedral sheet, which is compensated by the presence of cations inside the cavities of zeolite and in the interlamellar space of vermiculite - which can be exchanged for other ones from the external environment. The NH4+ removal efficiency by both materials was investigated in batch tests using a synthetic solution with $100 \mathrm{mg} \mathrm{L}-1 \mathrm{of}$ NH4+. The best efficiency in ammonium removal by zeolite and vermiculite were, respectively, 85\% (4.25 $\mathrm{mg}$ g-1) reached in $30 \mathrm{~min}$, and $67.26 \%$ (3.8 $\mathrm{mg} \mathrm{g}-1)$ in $24 \mathrm{~h}$; and the optimum $\mathrm{pH}$ was 4 to 8 for zeolites and 7 for vermiculite. The best efficiency of zeolite than vermiculite is because the porous structure did not change during the exchange process, while the interlamellar space of vermiculites changes according to the ion radii involved in the process. Therefore, in addition to removing NH4+ from wastewater, the used zeolite and vermiculite filters media were also be tested as slow release fertilizer. The capacity of these materials in releasing NH4+ was tested by leaching methods using distilled water and acid solution (acetic solution with $\mathrm{pH} 4.9$ ) according to the Brazilian regulations. This test revealed that the acid solutions leached more NH4+ $(10 \mathrm{mg} \mathrm{L}-1$ for zeolite and $50 \mathrm{mg} \mathrm{L}-1$ for vermiculite) than distilled water ( $2 \mathrm{mg} \mathrm{L}-1$ for both zeolite and vermiculite). Although the acid solution released more $\mathrm{NH} 4+$, this loss represented, respectively, only $12 \%$ and $29 \%$ of the total NH4+ retained in the zeolite and vermiculite structures. Therefore, the results of this work revealed that the porous minerals as zeolite and vermiculite can transform an industrial contaminant into a valuable nutrient for plants.

Procter and Gamble Student poster award:

References:

Acceptance of Terms and Conditions:

Click here to agree

\section{Foam: A potential vehicle for fertilizer delivery in soil}

Mohammad Javad Shojaei ${ }^{1}$; Nima Shokri ${ }^{2}$

${ }^{1}$ The University of Manchester

${ }^{2}$ School of Chemical Engineering and Analytical Science, The University of Manchester, Manchester, UK

Corresponding Author(s): mohammadjavad.shojaei@postgrad.manchester.ac.uk

Fertilizers are commonly used in agriculture to increase crop yield. Excessive fertilizers application may result in unnecessary soil enrichment, their release into environment, groundwater pollution and a higher cost for crop production. When fertilizers are applied to soil, a part of it may drain through the soil profile via gravity. Additionally, preferential flow paths developed in soil may hinder the uniform distribution of fertilizer. Root zones occupy a relatively small volume in soil profile so it would be of great importance to target the root zone for fertilizers application and not the entire soil profile. Therefore, developing novel methods for minimizing fertilizers usage and effective application will impact positively human health, environment and economy.

Foam (defined as a dispersion of gas in a continuous liquid phase) may offer an excellent opportunity for targeted fertilizer delivery to the root zone due to its unique mobility characteristics. Foam exhibits apparent viscosity of a few orders of magnitude higher than its constituent gas and liquid in porous media leading to low mobility. This results in formation of a more uniform displacement front moving into soil profile and thus more uniform distribution of fertilizers in soil. Foam bubbles have a low density and are less susceptible to gravity and consequently leaching. Moreover, the higher surface area of foam bubble could also improve the adsorption of fertilizer around plant roots. The aim of this research is to evaluate the potentials of foam to serve as a targeted fertilizer delivery 
vehicle in soil. To do so, leaching experiments were conducted using cylindrical soil columns $(30 \mathrm{~cm}$ in height, $4 \mathrm{~cm}$ in diameter). Two sets of experiments were performed. In the first and second set, fertilizers were applied to the soil profiles mixed with liquid surfactant and foam, respectively. Foam was generated by injecting gas into the mixture of fertilizers and surfactant. In both sets, the leachate was collected from the bottom of the columns every 24 hours. Our preliminary results suggest less leaching when fertilizers were applied into the soil with foam. Extra analysis and experiments using foam with different properties are required to investigate the potential of foams for targeted fertilizers delivery to improve the retention and absorption of fertilizers in the region of interest as well as maintaining high local concentration, retarding deep drainage and increasing residence time in the root zone.

Grassia, P., E. Mas-Hernandez, N. Shokri, S.J. Cox, G. Mishuris W.R. Rossen (2014), Analysis of a Model for Foam Improved Oil Recovery, J. Fluid Mech., 751, 346-405.

Osei-Bonsu, K., P. Grassia, N. Shokri (2017), Relationship between bulk foam stability, surfactant formulation and oil displacement efficiency in porous media, Fuel, 203, 403-410.

\title{
Procter and Gamble Student poster award:
}

I would like to compete in the Procter and Gamble Student award References:

Acceptance of Terms and Conditions:

Click here to agree

\section{Water table depth and soil salinity: Pore-scale physics defining large-scale responses}

Salome M.S. Shokri-Kuehni ${ }^{1}$; Bernadette Raaijmakers ${ }^{2}$; ${\text { Theresa } \mathrm{Kurz}^{3} \text {; Rainer Helmig }}^{3}$; Nima Shokri ${ }^{1}$

\author{
${ }^{1}$ The University of Manchester \\ 2 (2) Department of Earth Sciences, Utrecht University, Utrecht, The Netherlands \\ 3 (3) Department of Hydromechanics and Modelling of Hydrosystems, University of Stuttgart, Stuttgart, Germany
}

Corresponding Author(s): salome.shokri@gmail.com

The excess accumulation of salt in soil is a global problem and is one of the most widespread soil degradation processes in the world. Accumulation of salt in soil adversely influences the plant growth, vegetation and crop production. As water evaporates, salt concentration in the pore space increases continually until it precipitates. Recent studies confirmed the porous nature of the precipitated salt and the complex dynamics of its evolution during evaporation (Shokri-Kuehni et al., 2017, 2018). The presence of porous salt at the surface causes top-supplied creeping of the solution feeding the growth of subsequent precipitation. Such a phenomenon leads to additional water evaporation from soil and will impact the interaction between water table, soil surface and the evaporative fluxes.

In the present study, we have investigated saline water evaporation from porous media in the presence of water tables fixed at various depths below the surface. Our results illustrate the significant impact of the presence of hydraulically connected precipitated salt at the surface with the water table on the total cumulative water losses. High-resolution thermal imaging enabled us to investigate the rapid temperature changes of the surface precipitated salt in the presence of a water table, providing further confirmation of precipitated salt contribution to the evaporation. Our results showed that as long as the water table was hydraulically connected to the surface, more salt precipitation occurred at the surface when the water table was deeper. This was consistent with our results obtained by continuum-scale numerical simulation (Jambhekar et al., 2015) where higher solute concentration close to the surface was obtained when the water table was deeper. Moreover, we made use of two global-scale databases containing topsoil salinity, soil texture and water table depth data to reveal the relevance of our findings to the large-scale responses and the relationships between the water table depth and soil salinity at the global-scale. Our multiscale analyses illustrate how the pore-scale physics constrain the responses at the global scale. 
Procter and Gamble Student poster award:

\section{References:}

Jambhekar, V.A., R. Helmig, Natalie Schroder, N. Shokri (2015), Free-flow-porous-media coupling for evaporation-driven transport and precipitation of salt, Trans. Porous. Med., 110(2), 251-280.

Shokri-Kuehni, S.M.S., T. Vetter, C. Webb, N. Shokri (2017), New insights into saline water evaporation from porous media: Complex interaction between evaporation rates, precipitation and surface temperature, Geophys. Res. Lett., 44, 5504-5510.

Shokri-Kuehni, S.M.S., M. Bergstad, M. Sahimi, C. Webb, N. Shokri (2018), Iodine k-edge dual energy imaging reveals the influence of particle size distribution on solute transport in drying porous media, Sci. Rep., 10, 10731, London: Nature Publishing Group. Acceptance of Terms and Conditions:

Click here to agree

917

\section{Quasi-2D Phase Transition of Methane Adsorbed in Cylindrical Silica Mesopores}

Daniel Siderius $^{1}$; William Krekelberg ${ }^{1}$; Wei-Shan Chiang ${ }^{1}$; Vincent Shen ${ }^{1}$; Yun Liu ${ }^{1}$

${ }^{1}$ National Institute of Standards and Technology

Corresponding Author(s): daniel.siderius@nist.gov

Recently, experimental small-angle neutron scattering (SANS) experiments identified a possible phase transition of methane adsorbed in MCM-41 and SBA-15, two cylindrical silica mesoporous adsorbents, indicated by a unexpected increased in the adsorbed fluid density at liquid-like conditions 1. To better understand this observed phenomena, we used Monte Carlo and molecular dynamics simulations to examine the adsorption of methane in cylindrical silica mesopores of varying size and at varying temperatures and pressures. Our initial simulation results identified a roughly 10 $\%$ increase in the density of the liquidlike adsorbed phase for either an isotherm with increasing pressure or an isobar with decreasing temperature and that this densification is associated with a local maximum in the isosteric enthalpy of adsorption. Subsequent analysis of the simulated fluid, via computation of bond-orientational order parameters of specific annular layers of the adsorbed fluid, showed that the layers undergo an ordering transition from a disordered, amorphous state to one with two-dimensional hexagonal structure. Furthermore, this two-dimensional restructuring of the fluid occurs at the same thermodynamic state points as the aforementioned densification and local maximum in the isosteric enthalpy of adsorption. We thus conclude that the densification of the fluid is the result of structural reorganization, which is signaled by the maximum in the isosteric enthalpy. Owing to the qualitative similarity of the structural transitions in the simulated and experimental methane fluids, we propose this hexagonal reorganization as a plausible explanation of the densification observed in SANS measurements 2. Lastly, we discuss why this structural transition has never been previously observed and how it may impact the transport properties of the adsorbed fluid.

1 W-S. Chiang et al., Langmuir, 32:8849 (2016).

2 D.W. Siderius, W.P. Krekelberg, W-S. Chiang, V.K. Shen, and Y. Liu, Langmuir, 33:14252 (2017).

Procter and Gamble Student poster award:

References:

Acceptance of Terms and Conditions:

Click here to agree 


\title{
Natural designs of efficient heat and mass exchangers
}

\author{
Author(s): Solberg Simon B. b. ${ }^{1}$; Simon Solberg ${ }^{\text {None }}$ \\ Co-author(s): Elisa Magnanelli ${ }^{2}$; Natalya Kizilova $^{3}$; Signe Kjelstrup $^{1}$; Mario Acquarone ${ }^{4}$; Lars Folkow ${ }^{4}$ \\ ${ }^{1}$ 2. PoreLab, Department of Chemistry, Norwegian University of Science and Technology, NTNU \\ 2 SINTEF Energy AS \\ ${ }^{3}$ 3. Department of Aeronautics and Applied Mechanics, Warsaw University of Technology \\ ${ }^{4}$ Department of Arctic and Marine Biology,
}

Corresponding Author(s): sbsolber@stud.ntnu.no, simon.birger@gmail.com

Fluid flow systems for delivery of oxygen and nutrition, as well as removal of waste, can be found in all natural living systems. Respiratory and blood circulation systems in animals, trophic fluid systems in sponges, and veins in plants are examples of optimal transportation systems with minimum energy losses 1. The nasal cavity in reindeers gives an example of efficient heat and mass exchange, able to quickly heat up the ambient air from $-30^{\circ} \mathrm{C}$ to $38^{\circ} \mathrm{C}$ and to moisturize it from $\sim 20 \%$ to $100 \%$ 2. An equivalent $1 \mathrm{D}$ model based on measured data of perimeters, cross-section areas and distribution of blood vessels along the nasal airway of the reindeer showed better performance during respiration, than a simple cylindrical shaped reference nose [2, 3]. Moreover, while the fully developed reindeer's nasal geometry is more efficient at lower temperatures, the reindeer calf's nasal geometry showed an opposite trend. In both cases, performance was quantified in terms of entropy production.

In this work, we use the equivalent 1D model to study the influence that nasal geometries of polar and non-polar animals have on heat and mass transfer. In addition, we estimate their efficiency as air heaters and moisturizers. We show that the use of the equivalent tube hydraulic diameter Dh does not properly describe the influence of the complex non-convex cross sections on heat and mass transport. Thus, a new parameter accounting for the tortuosity $\mathrm{Td}$ of the different cross sections is proposed. The nasal airways of the juvenile reindeer, reindeer calf, and a white-tailed deer are classified in terms of Td, and the entropy production during respiration is computed taking this new parameter into account. The geometry of these efficient natural systems can be used as inspiration for the design of energy-efficient mass- and heat-exchange processes.

The authors are grateful to the Research Council of Norway (RCN) through its Centres of Excellence funding scheme, project number 262644, PoreLab. NK is grateful to the HighEFF Centre for Environmental-friendly Energy Research, RCN project no 257632/E20

\section{Procter and Gamble Student poster award:}

I would like to compete in the Procter and Gamble Student award References:

1 S. Gheorghiu, S. Kjelstrup, P. Pfeifer, and M.-O. Coppens. Is the lung an optimal gas exchanger? In: Fractals in Biology and Medicine, ed. G. A. Losa, D. Merlini, T. F. Nonnenmacher and E. R. Weibel, Birkhauser Basel, Basel, 2005; 31-42.

2 E. Magnanelli. Ø. Wilhelmsen, M. Acquarone, L.P. Folkow, S. Kjelstrup The Nasal Geometry of the Reindeer Gives Energy-Efficient Respiration. J. Non-Equilibr. Thermodyn. 2017; 42, 59-78.

3 S. Kjelstrup, D. Bedeaux, E. Johannessen, J. Gross, Non-Equilibrium Thermodynamics for Engineers. World Scientific, 2. edition, 2017. Acceptance of Terms and Conditions:

Click here to agree

\section{Representative Elementary Volume using Second-order Grayscale Entropy: A Sandstone and Carbonate case study}

Ankita Singh $^{1}$; Klaus Regenauer-Lieb ${ }^{2}$; Ryan Armstrong ${ }^{\text {None }}$; Peyman Mostaghimi ${ }^{\text {None }}$ 
${ }^{1}$ UNSW Sydney
${ }^{2}$ School of Minerals and Energy Resources Engineering, UNSW Sydney, Australia;

Corresponding Author(s): ankita.unsw@gmail.com

Reservoir engineers use field-scale properties for simulations and production predictions. Inaccuracies in prediction could lead to considerable uncertainty in reserve estimation. Field-scale properties of a reservoir are calculated by upscaling macroscopic properties such as permeability, porosity and surface area. These properties are calculated from numerical simulations on a representative elementary volume (REV). Unless the REV has captured essential information about the rock microstructure, computing macroscopic properties and upscaling to field-scale would be futile.

The current technique for identifying a REV of porous media is based on binary images and needs computationally heavy numerical simulations. Using binary images discards essential information about the rock micro-structure and there exists no ground-truth for validation; one must rely on laboratory measurements. In fact, without defining a REV, the laboratory analyses that are reported for petroleum reservoirs are not representative of the large-scale subsurface.

In this work, an alternative direct interpretation method of grayscale images obtained after micro-CT reconstruction is presented. The method allows capturing inherent heterogeneities present in the reservoir rocks in its original form and provides a more realistic estimation of macroscopic properties for robust upscaling to field-scale. The novel method uses second-order entropy to define a REV for the case of Berea sandstone. This entropy is calculated from the Grey-Level Co-occurrence Matrix, a visual descriptor of the micro-CT images based on the grey-level intensities and their occurrences. This method allows automation of REV selection process and incorporates information which is otherwise lost during binary segmentation of micro-CT images.

Procter and Gamble Student poster award:

I would like to compete in the Procter and Gamble Student award References:

Acceptance of Terms and Conditions:

Click here to agree

\title{
Dynamics of multiphase flow in porous media: pore-filling I-n mechanisms, snap-off and trapping
}

\author{
Author(s): Kamaljit Singh ${ }^{\text {None }}$ \\ Co-author(s): Tom Bultreys ${ }^{1}$; Ali Q. Raeini ${ }^{2}$; Branko Bijeljic ${ }^{3}$; Martin Blunt ${ }^{2}$ \\ ${ }^{1}$ Ghent University \\ ${ }^{2}$ Imperial College London \\ ${ }^{3}$ Imperial College
}

Corresponding Author(s): kamaljit.singh@imperial.ac.uk

Understanding the pore-scale dynamics of multiphase flow in porous media is important in many processes such as water infiltration in soils, oil recovery and geological CO2 storage. These dynamics have been studied in two dimensions for decades; however, the pore-scale processes leading to snap-off and trapping have not been investigated in a realistic three-dimensional geometry in such details. The time-dependent information is important to validate models of pore-scale displacement and to quantify how the balance of viscous and capillary forces control the exact nature of trapping. Using fast synchrotron X-ray micro-tomography, we visualize and quantify pore-to-pore displacement during secondary imbibition in a Ketton limestone sample. By superimposing the pore-throat network extracted from the segmented pore space on time-resolved tomographic images, we have obtained information about various pore-filling mechanisms, called $I n$, (where $n$ is the number of 
throats filled with non-wetting fluid), snap-off and trapping events. Our findings suggest that porefilling and snap-off events strongly correlate with the aspect ratio (the ratio of pore radius to throat radius). A high value of the aspect ratio ( $>2.1)$ is found to be favourable for snap-off over pore-filling for $I 1$ events. On the other hand, throat shape factor shows a weaker correlation. This analysis is further being conducted on the complete time-series datasets to obtain statistics on $I 2$ to In events. In addition, we have found a few unexpected pore-filling events leading to the formation of sub-singlet ganglion, which will be explored further by performing direct numerical simulations.

Indico rendering error

Could not include image: Cannot read image data. Maybe not an image file?

Procter and Gamble Student poster award:

I don't want to compete References:

Acceptance of Terms and Conditions:

Click here to agree

\title{
Nonisothermal modeling of $\mathrm{CO} 2$ dissolution trapping in deep saline aquifers
}

\author{
Mrityunjay Singh $^{1}$; Abhijit Chaudhuri ${ }^{1}$; Philip Stauffer ${ }^{2}$; Rajesh Pawar ${ }^{2}$ \\ ${ }^{1}$ Department of Applied Mechanics, Indian Institute of Technology Madras \\ ${ }^{2}$ Earth \& Environmental Sciences Division, Los Alamos National Laboratory
}

Corresponding Author(s): 56mems@gmail.com

To control the catastrophic increase of anthropogenic $\mathrm{CO}_{2}$ in the atmosphere, $\mathrm{CO}_{2}$ can be sequestered in deep saline aquifers as one of the safest and economical mechanism. Due to very deep injection, the injected $\mathrm{CO}_{2}$ is in supercritical or liquid phase and injection temperature is much lower than reservoir temperature. Supercritical $\mathrm{CO}_{2}$ is lighter than brine. A saline aquifer with overlaying caprock is the most suitable formation for $\mathrm{CO}_{2}$ sequestration. Injected $\mathrm{CO}_{2}$ moves upward and spreads below a low permeability hard caprock, which behaves as a barrier. The supercritical $\mathrm{CO}_{2}$ dissolves in the brine by molecular diffusion. In the deep saline aquifer, gravity assisted plume migration and gravitational fingering process assist the dissolution trapping. For a very large range of temperature, the density of brine with dissolved $\mathrm{CO}_{2}$ is higher than fresh brine. Existing literature discussed the effect of capillary pressure at reservoir scale for the isothermal condition. We numerically simulated $\mathrm{CO}_{2}$ dissolution process in brine by continuous injection of supercritical/liquid $\mathrm{CO}_{2}$ at the lower temperature than reservoir temperature. The reservoir was considered fully saturated with brine and no $\mathrm{CO}_{2}$ was present initially. It is known that the density of brine and solubility of $\mathrm{CO}_{2}$ in brine are the function of temperature. Hence the density variation within the reservoir depends on both temperature and dissolved $\mathrm{CO}_{2}$ concentration fields. This forms a density imbalance along with the evolution of the capillary transition zone. We coupled the buoyancy-driven convection with heat transport stimulated by cold injected $\mathrm{CO}_{2}$. We found that the temperature distribution was largely controlled by the injection rate and reservoir permeability. The vertical density gradient which is responsible for gravitational instability and buoyant convection are affected by the reservoir temperature, injection temperature, reservoir permeability, and injection mass flow rate. Thermal effects are negligible at lower reservoir temperatures and gravitational fingering and dissolved $\mathrm{CO}_{2}$ plume migration is very similar to those in isothermal case. When the reservoir temperature is very high compared to injection temperature, overall $\mathrm{CO}_{2}$ dissolution process is significantly different from the isothermal condition. In such cases, unsteady convection and bulging of the plume are observed depending on the formation permeability.

Procter and Gamble Student poster award:

I would like to compete in the Procter and Gamble Student award References: 


\title{
Effective Viscosity of Immiscible Two-phase Flow in Porous Me- dia at High Capillary Number
}

\author{
Santanu Sinha ${ }^{1}$; Magnus Aa. Gjennestad ${ }^{2}$; Morten Vassvik ${ }^{\text {None }}$; Mathias Mathias ${ }^{\text {None }}$; Alex Hansen ${ }^{3}$; eirik \\ flekkøy $^{4}$ \\ ${ }^{1}$ Beijing Computational Science Research Center \\ ${ }^{2}$ Norwegian University of Science and Technology \\ ${ }^{3} N T N U$ \\ ${ }^{4}$ PoreLab, Dep. Physic University of Oslo, Norway
}

Corresponding Author(s): santanu.sinha@ntnu.no

\begin{abstract}
The interplay between capillary and viscous forces in immiscible two-phase flow in porous media makes the flow rate non-linear with change in the pressure drop and the volumetric saturation of the two fluids $[1,2]$. Change in the fluid saturation alters the viscous forces due to the difference in the viscosities of the individual fluids, as well as the capillary forces due to the surface tension at the interfaces. However, in the regime of high capillary number, one can neglect the capillary forces and the flow should only depend on the viscosities of the two individual fluids while changing their saturations. We demonstrate that, at high capillary numbers, the two-phase flow in porous media may be characterized by an effective viscosity that is well described by the LichteneckerRother equation that connects the effective viscosity with the individual fluid viscosities and their saturations by an exponent $\alpha$. Moreover, the exponent $\alpha$ characterizes different regimes of the flow depending on the pore geometry, wettability and viscosity of the fluids. We find $\alpha=1$ when fluids are well mixed with small bubbles, $\alpha=0.6$ in two- and $\alpha=0.5$ in three-dimensional systems when there is less mixing with the appearance of big bubbles, and $\alpha=-0.5$ when there are lubrication layers exist along the pore walls 3. Our arguments are based on analytical and numerical methods, namely the pore-network modelling and lattice Boltzmann simulations.
\end{abstract}

Procter and Gamble Student poster award:

References:

1 A. Hansen, S. Sinha, D. Bedeaux, S. Kjelstrup, M. Aa. Gjennestad and M. Vassvik, Transp. Porous Med. 125, 565 (2018).

2 S. Sinha, A. T. Bender, M. Danczyk, K. Keepseagle, C. A. Prather, J. M. Bray, L. W. Thrane, J. D. Seymour, S. L. Codd and A. Hansen, Transp. Porous Med. 119, 77 (2017).

3 S. Sinha, M. Aa. Gjennestad, M. Vassvik, M. Winkler, A. Hansen and E. G. Flekkøy, arXiv:1712.06826. Acceptance of Terms and Conditions:

Click here to agree

\section{An alternative approach for solving the VTN-stability testing prob- lem of a multicomponent mixture}

\author{
Tomáš Smejkal ${ }^{\text {None }}$ \\ Corresponding Author(s): smejkto5@fjfi.cvut.cz
}

In this contribution we present various methods for solving the phase stability testing problem at constant volume, temperature, and moles (VTN-specification) of a multicomponent mixture. This problem can be formulated as a nonlinear unconstrained optimization problem. Different method 
including the Cuckoo search or the Differential evolution will be shown. Comparison with the standard Newton-Raphson method with line-search will be given. The performance of the algorithms will be shown on examples from literature with different levels of difficulty.

\title{
Procter and Gamble Student poster award:
}

I would like to compete in the Procter and Gamble Student award References:

\section{Acceptance of Terms and Conditions:}

Click here to agree

\section{Dissolution Dynamics of Air Bubbles Entrained in Porous Me- dia}

\author{
Author(s): Scott Smith ${ }^{1}$ \\ Co-author(s): Matthieu Vandamme ${ }^{2}$; Kimberly Kurtis ${ }^{1}$ \\ ${ }^{1}$ Georgia Institute of Technology \\ ${ }^{2}$ Ecole des Ponts ParisTech
}

Corresponding Author(s): ssmith314@gatech.edu

The long-term saturation of porous media, such as concrete or limestone, immersed in water has been theorized to be governed by dissolution and diffusion of compressed air in spherically entrained voids 1. Modeling such a process is of practical interest as higher water saturation results in an increased likelihood of failure if the medium is exposed to freezing [2,3]. Prior to predicting the long-term saturation rate for a macroscopic and polydisperse material system 4 , it is first necessary to clearly detail how compressed air bubbles dissolve and then diffuse through a porous shell. A novel set of coupled equations is derived to predict the size reduction of compressed gases in an individual air void in a porous medium. The derived model is general and can be compared to the Epstein-Plesset equation 5 and Duncan and Needham's experimental results [6] when the porous shell is sufficiently large and treated as water. The results clearly highlight the difference in mechanisms of bubble dissolution dynamics in fluids and porous media. The derived model is then used to approximate bubble dissolution dynamics expected for porous materials with spherically entrained air void systems and a parametric study is carried out to investigate the influence of air void size, diffusivity of the porous media, and initial dissolved gas concentration in the porous shell.

Procter and Gamble Student poster award:

I would like to compete in the Procter and Gamble Student award References:

1 G. Fagerlund, The long time water absorption in the air-pore structure of concrete, Division of Building technology, Lund Institute of Technology (1993).

2 W. Li, M. Pour-Ghaz, J. Castro, J. Weiss, Water absorption and critical degree of saturation relating to freeze-thaw damage in concrete pavement joints, J. Mater. Civ. Eng. 24 (2012) 299-307. doi:10.1061/(ASCE)MT.19435533.0000383.

3 T.C. Powers, R.A. Helmuth, Theory of volume changes in hardened portland-cement paste during freezing, Highway Research Board Proceedings Vol. 32 (1953).

4 D. Eriksson, T. Gasch, A. Ansell, A Hygro-Thermo-Mechanical Multiphase Model for Long-Term Water Absorption into Air-Entrained Concrete, Transp Porous Med (2018) https://doi.org/10.1007/s11242-018$1182-3$

5 P.S. Epstein, M.S. Plesset, On the Stability of Gas Bubbles in Liquid-Gas Solutions, J. Chem Phys., (1950) 18:1505-1509.

[6] P.B. Duncan, D. Needham, Test of the Epstein-Plesset Model for Gas Microparticle Dissolution in Aqueous Media: Effect of Surface Tension and Gas Undersaturation in Solution, Langmuir, (2004) 20:2567-2578. Acceptance of Terms and Conditions:

Click here to agree 


\title{
Effects of Land-Atmosphere Interactions on Methane Emissions from Natural Gas Pipelines and Implications for Detection Sys- tems
}

\author{
Kathleen Smits ${ }^{1}$; Bridget Ulrich ${ }^{2}$ \\ ${ }^{1}$ The University of Texas at Arlington \\ ${ }^{2}$ Colorado School of Mines
}

Corresponding Author(s):

\begin{abstract}
Natural gas (NG) is often regarded as a "transition fuel" as we move to replace high-carbon fossil fuels with renewable energy sources due to its lower cost and cleaner burning. Yet, fugitive emissions of methane (the main component of NG and a very potent greenhouse gas) from NG infrastructure could compromise these potential environmental benefits, as well as pose significant health and safety concerns. Improved methane leak detection technologies will be essential to ensure a prompt response, allowing timely mitigation of environmental impacts. Atmospheric methane emissions from underground NG distribution pipelines are particularly difficult to evaluate due to the complex nature of subsurface methane transport in natural soils, variable atmospheric conditions, and interactions at the land-atmosphere interface. Yet, accurate and timely detection of such emissions are vital in identifying underground pipeline leak locations, which in turn can have regulatory, environmental, and health implications.

To better inform leak detection technology development, a field-scale testbed was developed to simulate NG emissions from underground low-pressure pipelines. The testbed is configured such that methane concentrations can be measured both in the subsurface (using methane sensors as well as underground sampling ports) and in the free flow at and above the soil surface (using a Picarro G2203 methane analyzer) in tandem with other relevant subsurface and atmospheric properties (e.g., soil moisture, near-surface wind, and temperature). A series of experiments are underway to assess the effects of subsurface (i.e. soil moisture, leak rate, pipeline burial depth), surface (i.e. vegetation, roadways), and atmospheric (i.e. wind speed and temperature) conditions on the transport of methane through the subsurface and the development of the concentration profiles in the free flow close to the soil surface. The findings from these field studies, in combination with numerical modeling efforts, will provide useful insight to increase the accuracy in detecting, locating, and quantifying leaks from NG distribution lines.
\end{abstract}

Procter and Gamble Student poster award:

I don't want to compete References:

Acceptance of Terms and Conditions:

Click here to agree

\section{Magnetic resonance imaging of freezing and thawing of water in two contrasting porous media}

Michal Snehota $^{1}$; Andreas Pohlmeier ${ }^{2}$; John Koestel ${ }^{\text {None }}$; Tomas Princ ${ }^{3}$; Martina Sobotkova ${ }^{\text {None }}$; Milena Cislerova ${ }^{4}$

\footnotetext{
${ }^{1}$ Czech Technical University in Prague, Civil Engineering

${ }^{2}$ Research Center Jülich

${ }^{3}$ Czech Technical Univerzity in Prague

${ }^{4}$ Czech Technical University in Prague
}

Corresponding Author(s): michal.snehota@fsv.cvut.cz 
Magnetic resonance imaging (MRI) of freezing and thawing process was performed on two samples of different porous materials, each one in two replicates. The soil freeze sample assembly consisted of two Plexiglas cylinders, the outer dimensions of the set-up was $6 \mathrm{~cm}$ in diameter and $12.5 \mathrm{~cm}$ high. The inner cylindrical container $(3 \mathrm{~cm}$ in diameter, $6 \mathrm{~cm}$ high) was filled with the sample material. The inner cylinder was insulated by vacuum. On the top of the porous media, a glass disk $(2.8 \mathrm{~cm}$ in diameter, $1 \mathrm{~cm}$ thickness) was placed to define the upper boundary of a material during freezing and thawing. One inflow tube and two outflow tubes at the top of the inner cylinder were used for circulation of cold nitrogen gas as a freezing medium to the top of the sample. The temperatures of the freezing medium were continuously recorded by the temperature sensors located outside of the MR coil. The first sample packing consisted of 72 glass beads, $0.8 \mathrm{~cm}$ in diameter immersed in the 1 $\mathrm{mM} / \mathrm{L}$ GdDTP2--2Na+. In the second sample, the coarse sand was packed in $0.5 \mathrm{~cm}$ thick layers in the same sample solute. Total eight freezing-thawing cycles were performed and recorded on the samples. As a result, time-lapse series of 3D MR images were obtained. The geometric distortion of these 4D MR images due to magnetic field inhomogeneity had to be elaborated prior to the process analysis. The analyses of the freezing-thawing process on glass beads revealed interesting effects while thawing, where the thin layers on the glass beads surface exhibited faster melting in otherwise homogeneous ice. The impact of MRI heat transfers has to be evaluated. The freezing-thawing fronts recorded of sand samples were relatively uniform. Small changes in sand structure as a consequence of volumetric ice-water changes are studied. The spatiotemporal analysis of the frozen water volume is done. The data are available for a two-phase ice-water simulation models evaluation.

Procter and Gamble Student poster award:

References:

Acceptance of Terms and Conditions:

Click here to agree

\title{
Direct numerical simulation and characterization of flame prop- agation regimes in porous inert media
}

\author{
Sadaf Sobhani ${ }^{1}$; Joseph Ferguson ${ }^{1}$; Matthias Ihme ${ }^{1}$ \\ ${ }^{1}$ Stanford University \\ Corresponding Author(s): ssobhani@stanford.edu
}

Cleaner and more efficient combustion strategies are integral in the transition to a global low-carbon energy system. Matrix-stabilized combustion in porous media has been identified as an opportunity with the potential for overcoming technological barriers associated with conventional combustion systems. Matrix-stabilized combustion is facilitated in porous-media burners (PMBs), consisting of a solid porous structure (typically made of alumina, zirconia, silicon carbide, or other heat-conducting materials) to enhance the internal heat-recirculation, thereby achieving excess-enthalpy combustion. Matrix-stabilized combustion is an advanced combustion strategy, offering the following advantages (i) Stable and robust operation of flexible and low-calorific fuels; (ii) Extended power modulation and turn-down ratios; (iii) Improved thermal efficiency and extension of lean flammability limits; (iv) Increased volumetric heat release; and (v) Significant reductions of emissions of CO and NOx. These attractive properties are achieved through the enhanced heat exchange between gas phase and porous structure as a result of the large solid-gas interfacial surface area of the porous matrix. Furthermore, the porous matrix alters the hydrodynamics of the flow by enhancing the mixing and perturbing the flame structure and surface area. To investigate this latter phenomenon, pore-scale direct numerical simulations of premixed combustion in a two-dimensional array of adiabatic disks are conducted. Numerical calculations are obtained by solving the reactive Navier-Stokes equations with finite-rate chemistry. The pore-space of the random and ordered configurations are characterized by means of the Delaunay triangulation using the disks center points, where the edges approximate the pore channel lengths between neighboring obstacles. The flame consumption speed is computed for a range of inlet velocities, array configurations, and disk diameters, varying from half to three times the flame thickness. From these results, a combustion-regime diagram is developed that characterizes the combustion mode in terms of the porous media microstructure, flame kinematics, and global combustion properties. Since adiabatic boundaries between the solid and gas are imposed in these 
simulations, the incoming flow is not preheated from the solid. Therefore, the results isolate the hydrodynamic interaction of the porous media and the flame and quantify the lower limits of the consumption speed in porous media burners.

Procter and Gamble Student poster award:

I would like to compete in the Procter and Gamble Student award References:

Acceptance of Terms and Conditions:

Click here to agree

\title{
Freezing and thawing of water in fully saturated packed sand sample
}

Author(s): Martina Sobotkova ${ }^{1}$

Co-author(s): Alexandr Zak ; Michal Snehota ; Michal Benes

${ }^{1}$ CTU in Prague

Corresponding Author(s): martina.sobotkova@fsv.cvut.cz

\begin{abstract}
Horizontal freezing and thawing of water were carried out in the laboratory on fully saturated packed sand sample (15 $\mathrm{cm}$ in diameter and $20 \mathrm{~cm}$ in height). The experimental setup consisted of plastic tube covered on its sides and bottom by insulation layers. The sample assembly was placed into the precisely controlled freezer chamber. The top of the sample was covered by a stainless steel plate. Initially the sample was equilibrated at +5 and $+10^{\circ} \mathrm{C}$ then the temperature inside the chamber was changed to -5 and $-10{ }^{\circ} \mathrm{C}$. The inner temperature of sand sample was monitored in three depths by thin temperature sensors (109 SS, Campbell Scientific) horizontally inserted into the sample. Temperature development in three temperature sensors was obtained during freezing and thawing cycles. The experiment aims to provide information on freezing dynamics and thermo-mechanical changes during the freezing and thawing cycles. The data were compared with simulations obtained by a numerical model. The model is based on the heat balance within the sample assembly and a modified heat equation for the porous medium temperature allowing for the phase transition below the freezing point depression. The comparisons of the thermal behavior show good agreements both in quantitative and qualitative sense and are presented.
\end{abstract}

Procter and Gamble Student poster award:

References:

Acceptance of Terms and Conditions:

Click here to agree

340

\section{A Lagrangian Model of Mixing-Limited Reactive Transport}

\author{
Author(s): Guillem Sole-Mari ${ }^{1}$ \\ Co-author(s): Diogo Bolster ${ }^{2}$; Daniel Fernàndez-Garcia ${ }^{1}$; Xavier Sanchez-Vila ${ }^{1}$ \\ ${ }^{1}$ Universitat Politècnica de Catalunya \\ ${ }^{2}$ Notre Dame
}




\title{
Corresponding Author(s): guillem.sole.mari@upc.edu
}

Experiments such as those of Gramling et al. (2002) are a good example of how the upscaled Advection Dispersion Reaction Equation (ADRE) can fail to predict reactive transport of solutes in porous media - even in seemingly homogeneous settings. This is mainly due to the inability of the upscaled dispersion term in the ADRE to treat spreading and mixing separately, leading to over-predictions of mixing and hence reaction. Recently, several models have been proposed to account for imperfectly mixed conditions at the subscale. However, these typically artificially impose a time-dependency on the mixing state of the system, either through an "unmixed" initial condition (e.g., Ginn, 2018) or in the form of a time-evolving reaction rate (Sanchez-Vila et al., 2010). This limitation might prevent these models from being readily generalizable to other scenarios, where a single "time origin" of the subscale mixing process may not be possible to define. That is, in a real system it is not just incomplete mixing associated with the initial condition that matters; complex flow through the porous medium may trigger events that continuously induce incomplete mixing states. Here, to account for this, we propose a Random Walk model that naturally accounts for the generation and destruction of subscale concentration variability. We first show how the occurrence of subscale mixing events mathematically leads to a memory function describing the time evolution of a concentration perturbation experienced by a particle. Then, motivated by parsimony, we introduce a simplification to render our model memoryless (Markovian). To demonstrate the model's veracity, we show its ability to effectively reproduce real data from simple experiments. Finally, we apply it to more complex reactive transport problems and use it to analyze the implications of mixing limitation in more realistic settings.

\section{Procter and Gamble Student poster award:}

I would like to compete in the Procter and Gamble Student award References:

Ginn, T. R. (2018). Modeling bimolecular reactive transport with mixing-limitation: Theory and application to column experiments. Water Resources Research, 54, 256-270. https://doi.org/10.1002/2017WR022120

Gramling, C. M., Harvey, C. F., \& Meigs, L. C. (2002). Reactive Transport in Porous Media: A Comparison of Model Prediction with Laboratory Visualization. Environmental Science \& Technology, 36(11), 25082514. https://doi.org/10.1021/es0157144

Sanchez-Vila, X., D. Fernàndez-Garcia, and A. Guadagnini (2010), Interpretation of column experiments of transport of solutes undergoing an irreversible bimolecular reaction using a continuum approximation, Water Resour. Res., 46, W12510, doi: 10.1029/2010WR009539. Acceptance of Terms and Conditions:

Click here to agree

\section{Lagrangian Modeling of Solute Transport with Equilibrium Reac- tions}

\author{
Author(s): Guillem Sole-Mari ${ }^{1}$ \\ Co-author(s): Diogo Bolster ${ }^{2}$; Daniel Fernàndez-Garcia ${ }^{1}$; Xavier Sanchez-Vila ${ }^{3}$ \\ ${ }^{1}$ Universitat Politècnica de Catalunya \\ ${ }^{2}$ Notre Dame \\ ${ }^{3}$ University Politecnica de Catalunya
}

Corresponding Author(s): guillem_sm@hotmail.com

Lagrangian methods such as Random Walk Particle Tracking (RWPT) can be an attractive alternative to Eulerian methods for simulating solute transport in porous media. These methods are virtually free of numerical dispersion and instabilities, which makes them particularly well-suited to advection-dominated problems. Recent advances have allowed us to incorporate increasingly complex non-linear reactions in RWPT models, which were traditionally limited to linear reactions. To date, however, standard random walks could only simulate chemical equilibrium by explicitly modeling the pertinent forward and backward kinetic reactions involved in the equilibrium. To 
avoid unphysical temporal fluctuations this can require overly restrictive small time steps, as, by definition, the reaction rates of equilibrium reactions are very fast compared to the time-scales of interest. Here, we present a novel random walk method that overcomes these limitations and is capable of simulating transport while incorporating equilibrium reactions. In our approach, numerical particles represent conservative components, whose transport parameters at any given time reflect chemical speciation. The proposed methodology can also simulate the interaction between kinetic and equilibrium reactions. To demonstrate its applicability, we present some simple examples, as well as a more complex case study of heavy metal mobilization caused by the leakage of dissolved $\mathrm{CO} 2$.

Procter and Gamble Student poster award:

I would like to compete in the Procter and Gamble Student award References:

Acceptance of Terms and Conditions:

Click here to agree

811

\title{
MHFEM with BDDC for two-phase flow in porous media in 2D and 3D
}

\author{
Author(s): Jakub Solovský ${ }^{1}$ \\ Co-author(s): Radek Fučík ${ }^{2}$ \\ ${ }^{1}$ FNSPE, CTU in Prague \\ ${ }^{2}$ Czech Technical University in Prague
}

Corresponding Author(s): jakubsolovsky@gmail.com

This work deals with the application of the BDDC method for two-phase flow problems in porous media.

We briefly describe the spatial discretization of the problem which is based on the mixed-hybrid finite element method (MHFEM) and semi-implicit time discretization. Then in detail, we describe the BDDC method, discuss the differences between the 2D and 3D cases, and present necessary modifications of the algorithm to improve its efficiency for a more complicated 3D case. We describe the parallel implementation of the method and highlight the critical steps of the algorithm that affects the performance and scalability.

The parallel implementation is then tested on benchmark problems in $2 \mathrm{D}$ and $3 \mathrm{D}$ and its efficiency is investigated on various meshes.

The numerical results on the computational cluster Galileo at CINECA indicate that the method preserves high computational efficiency for increasing numbers of processes and, therefore, allows solving problems on very fine meshes.

Procter and Gamble Student poster award:

I would like to compete in the Procter and Gamble Student award References:

Acceptance of Terms and Conditions:

Click here to agree

111

\section{Concentration fluctuations and dilution of non-reactive solutes in hierarchical and multiscale geological media}

Reza Soltanian $^{1}$; Faranak Behzadi $^{1}$; Felipe de Barros $^{2}$ 
${ }^{1}$ University of Cincinnati
${ }^{2}$ University of Southern California, Los Angeles

Corresponding Author(s): soltanma@uc.edu

Hydrogeological heterogeneity exists over multiple spatial scales. Such heterogeneity leads to irregular spreading of solute clouds resulting in larger surface area which in turn augment the dilution rate. In this work, we quantify the dilution index and concentration fluctuations of solute bodies in geological formations displaying hierarchical sedimentary architecture through the use of the Lagrangian concentration framework. This allows for a fundamental understanding of how dilution arises from the hierarchical architecture of sedimentary facies, and allows for a quantitative decomposition of dilution into facies-related contributions at different scales within the hierarchy. The proposed Lagrangian model is based on hierarchical expressions of the spatial covariance of the log-permeability $(Y)$. The spatial correlation structure in the covariance expression is the probability of transitioning across facies types of different scales, and they are parameterized by independent and quantifiable physical attributes of sediments including univariate statistics for $Y$, and the proportions and lengths of facies. The model is illustrated in the well-documented tracer test at the Borden research site. We show how different scale of sedimentary architecture contributes to the dilution enhancement of the solute cloud at the relatively homogeneous Borden site. By quantitatively decomposing the dilution and concentration fluctuations into facies-related contributions, we gain fundamental insights on the key factors controlling the time-dependent rate of dilution.

Procter and Gamble Student poster award:

\section{References:}

Acceptance of Terms and Conditions:

Click here to agree

\section{STUDY ON THE FLOW CHARACTERISTICS OF OIL IN NANO- CHANNELS}

Fuqan Song ${ }^{1}$; Kai $\mathrm{Ji}^{1}$; Haoze $\mathrm{Gao}^{1}$

${ }^{1}$ School of Petrochemical and Energy Engineering, Zhejiang Ocean University

Corresponding Author(s): songfuquan@zjou.edu.cn

The flow characteristics of oil in nanometer pores play an important role in porous membrane separation and purification, biological membrane ion channels, microfluidic chip, mems research manufacture and development of unconventional oil and gas fields, especially in the development of low permeability and ultra low permeability reservoirs. In this paper, anodic alumina membranes with pore sizes of $26.1 \mathrm{~nm}, 67.0 \mathrm{~nm}, 89.2 \mathrm{~nm}$, and $124.0 \mathrm{~nm}$ are used as flow channels to conduct singlephase oil flow experiments, and the characteristics of oil flow at nanoscale are analyzed. The results show that the flow of single-phase oil in the nanochannel is in line with the linear characteristics described by the Hagen-Poiseuille equation, but the experimental flow is smaller than the theoretical flow. When the shear rate is close to 0 , the experimental results are consistent with the theory: when the proportion of the boundary layer is 1, and the proportion of the boundary layer decreases rapidly with the increase of shear rate when the shear rate is small, and the proportion of the boundary layer decreases slowly with the increase of shear rate and gradually becomes unchanged when the shear rate is large; The ratio of resistance coefficient decreases nonlinearly with the increase of shear rate, while at the high shear rate, the ratio of resistance coefficient decreases slowly with the increase of shear rate and approximates the ratio of resistance coefficient under ideal conditions; The flow of a single-phase oil in the nanochannel has a quasi-threshold pressure gradient; There is a quasi-start-up pressure gradient in the flow of single-phase oil in nanotubes, and the quasi-start-up pressure gradient decreases with the increase of nanometer pore diameter. 
Corresponding author: Fuquan Song

Email:songfuquan@zjou.edu.cn

Procter and Gamble Student poster award:

References:

Acceptance of Terms and Conditions:

Click here to agree

\title{
Understanding fluid transport through hardened cement paste from 3D image observation
}

\author{
Yang SONG ${ }^{\text {None }}$
}

Corresponding Author(s): 121948267@qq.com

\begin{abstract}
Hardened cement paste contains the most important pore structure of concrete, which plays a vital role in durability and permeability of concrete. To reveal the pore characteristic of hardened cement paste and analyze its permeability, we scan and reconstruct the pore structure of hardened cement paste by Focusing Ion Beam/Scanning Electron Microscope (FIB/SEM) and 3D reconstruction software Avizo, respectively. The resolution of the two FIB/SEM samples is $10 \times 10 \times 10 \mathrm{~nm} 3$ and the sample size is $6 \times 6 \times 8 \mu \mathrm{m} 3$. Then, equal area method and continuous pore size distribution (PSD) method are applied to analyze the pore size distribution of hardened cement paste. And the pore structure is studied by the simulated mercury porosimetry and compared with results of mercury porosimetry test. Finally, permeability simulation is applied to the 3D pore structure based on Lattice Boltzmann Method (LBM) and the simulated results are compared with the measured values of hardened cement paste. Results show that the observed size of two specimens can only partially represent the pore structure characteristics of the hardened cement paste and thus more FIB/SEM observation should be focused for the representativeness The PSD of the hardened cement paste obtained by the equal area method is larger and mainly concentrated in the $110 \bigotimes 120 \mathrm{~nm}$, while the continuous PSD method can better simulate the complexity of the pore structure and "invade" from the inner pore structure. Its diameter is mainly concentrated in $50 \sim 90 \mathrm{~nm}$. Based on the pore structure image, the simulated mercury porosimetry is to simulate the physical mercury intrusion process. The peak pore diameter is $50 \mathrm{~nm}$ and the result is similar to that of mercury porosimetry test. Based on the 3D pore structures of two samples, the simulated permeability results are $2.17 \times 10-18 \mathrm{~m} 2$ and $3.12 \times 10-18 \mathrm{~m} 2$, which is lower than the measured one of $2.57-8.51 \times 10-17 \mathrm{~m} 2$. This is mainly because the samples for permeability test generate some microcracks during the sample drying and thus enhance the connectivity of pore network.
\end{abstract}

Procter and Gamble Student poster award:

References:

Acceptance of Terms and Conditions:

Click here to agree

\section{Towards a continuum-mechanical model of fluid-driven fracture for fully and partially saturated porous materials}

\author{
Alixa Sonntag $^{1}$; Arndt Wagner ${ }^{1}$; Chenyi Luo $^{1}$; Wolfgang Ehlers ${ }^{1}$ \\ ${ }^{1}$ University of Stuttgart
}

Corresponding Author(s): alixa.sonntag@mechbau.uni-stuttgart.de 
Fluid-driven fractures are of specific interest for various fields of the energy sector. In particular, for enhanced geothermal energy plants, naturally occurring cracks of the rock are widened by the injection of highly pressurised cold water to augment the rock's permeability. Thus, the flow rate of the heated water can be increased leading to a higher energy output. Furthermore, in hydrocarbon exploitation, additional artificial fissures and cracks are created by fluid injections to enhance the availability of oil and gas in subterranean natural reservoirs. The control of these exploitation techniques is still widely based on empirical methods. To enlarge the knowledge of the ongoing processes and prevent severe geological consequences, a systematic and theoretical understanding of fluid-driven fractures is necessary.

In this regard, a continuum-mechanical model for fluid-driven fracturing is presented on the basis of the Theory of Porous Media and the phase-field approach to fracture. Hereby, the material is described on the macroscopic scale as an immiscible mixture of a solid phase, representing the porous skeleton, and fluid constituents. By use of this model, either a single pore fluid saturates the solid, or a liquid and a gaseous pore fluid inhibit the solid skeleton for the additional consideration of partially saturated porous material. Moreover, a scalar phase-field variable is introduced to describe the fracturing process, characterising a diffuse crack pattern. Hereby, the crack propagation in the solid skeleton is induced by the pressure field of the injected fluid. The presented continuum-mechanical model is governed by the momentum balances together with the mass and volume balances of the constituents, and an evolution equation for the phase-field variable.

The resulting set of coupled partial differential equations is solved using the numerical finite-element solver PANDAS. Hereby, the spatial discretisation is realised by Taylor-Hood elements, while the time is discretised using a backward Euler scheme. Finally, two- and three-dimensional numerical examples of crack propagation are presented to show the capabilities of the model.

Procter and Gamble Student poster award:

References:

Acceptance of Terms and Conditions:

Click here to agree

\title{
Pore-scale modeling of coupled hydro-geochemical processes
}

\author{
Cyprien Soulaine $^{1}$; Sophie Roman ${ }^{2}$; Christophe Tournassat ${ }^{3}$ \\ ${ }^{1}$ BRGM, the French Geological Survey \\ ${ }^{2}$ Institut des Sciences de la Terre d'Orléans \\ ${ }^{3} B R G M$
}

Corresponding Author(s): c.soulaine@brgm.fr

The development of predictive capabilities for reactive multiphase flow in fractured formations is crucial to assess the long-term integrity of storage systems in the subsurface as well as the safe exploitation of subsurface energy resources. As of today, such capabilities remain limited for fractured porous rock in the sense that important phenomena such as the multiphase flow in the fracture, the evolution of the fracture aperture, and the effects of the rock mineral composition are poorly represented or simply neglected. In this presentation, we discuss the recent progress we made in the understanding and the modeling of the coupled hydro-geochemical processes including dissolution and precipitation in heterogeneous rock starting at the pore-scale.

\section{Procter and Gamble Student poster award:}

\section{References:}

Acceptance of Terms and Conditions:

Click here to agree 


\title{
The multiscale perturbation method for the solution of time-dependent porous media flows
}

\author{
Fabricio Sousa $^{1}$; Het Mankad ${ }^{2}$; Ali Alsadig ${ }^{2}$; Felipe Pereira ${ }^{2}$ \\ ${ }^{1}$ University of São Paulo, Brazil \\ ${ }^{2}$ The University of Texas at Dallas
}

Corresponding Author(s): fsimeoni@icmc.usp.br

In the numerical solution of two-phase flows in porous media, multiscale domain decomposition methods based on mixed formulation (see MMMFEM 1, MHM 2, MuMM 3 and MRCM 4) offer the possibility of improving the speedup in parallel computations. This is achieved by decomposing the PDE domain in non-overlapping subdomains, where a local set of multiscale basis functions is usually pre-computed. The final solution is obtained as a linear combination of these multiscale basis functions by solving a reduced problem to impose continuity at the interfaces between subdomains, which is much cheaper than solving the whole problem. When simulating fully saturated incompressible two-phase flows, due to the coupling between the elliptic equation modeling the Darcy law and the hyperbolic conservation law modeling the transport of the saturation of one of the phases, this set of multiscale basis functions has to be recomputed every time step of a simulation. The Multiscale Perturbation Method (MPM) is then employed to avoid the recalculation of all multiscale basis functions. The method consists of applying regular perturbation theory to compute an approximation in a give time step, in terms of multiscale basis functions computed at earlier time steps, resulting in a much faster procedure as compared to the recalculation of all multiscale basis functions.

Procter and Gamble Student poster award:

I don't want to compete References:

1 T. Arbogast, G. Pencheva, M.F. Wheeler, I. Yotov, A multiscale mortar mixed finite element method, SIAM Multiscale Model. Simul. 6 (1) (2007) 319-346.

2 R. Araya, C. Harder, D. Paredes, and F. Valentin. Multiscale hybrid- mixed method. SIAM J. Numer. Anal., 51(6) (2013) 3505-3531.

3 A. Francisco, V. Ginting, F. Pereira and J. Rigelo, Design and implementation of a multiscale mixed method based on a nonoverlapping domain decomposition procedure, Math. Comput. Simul., 99, (2014) 125-138.

4 R.T. Guiraldello, R.F. Ausas, F.S. Sousa, F. Pereira, G.C. Buscaglia, The Multiscale Robin Coupled Method for flows in porous media, In J. Comput. Phys. 355 (2018) 1-21. Acceptance of Terms and Conditions

Click here to agree

\section{Dynamic interface rearrangement during steady-state multi-phase flow in porous media}

Author(s): Catherine Spurin

Co-author(s): Sam Krevor ; Martin Blunt ${ }^{1}$; Branko Bijeljic ${ }^{2}$; Tom Bultreys ${ }^{1}$

\footnotetext{
${ }^{1}$ Imperial College London

${ }^{2}$ Imperial College
}

Corresponding Author(s): cls13@ic.ac.uk 
Fluid flows in the subsurface of the Earth's crust are central to several pressing environmental and societal challenges including the geological storage of carbon dioxide, hydrocarbon recovery and aquifer contamination containment. Current macroscopic flow behaviour is described using a continuum mechanical description. During multiphase flow, constitutive properties, capillary pressure and relative permeability, are described using an extended form of Darcy's law. The longstanding justification for this is that the fluids flow in connected pathways, and the interface between phases is an invariant partition 1 .

However, it has been observed that this partition is not always stable, and that the fluid phase interfaces can dynamically rearrange, even during steady-state flow. The dynamic rearrangement of flow pathways leads to variations in the connectivity of the fluid phases which governs the key characteristics of subsurface multiphase flow - energy dissipation during flow and trapping. By understanding the controls on the displacement dynamics within pores, we will be able to link their role to larger scale manifestations of flow such as relative permeability. In this work we observed drainage using X-ray micro-CT in a water wet bioclastic Estaillades carbonate rock. We explored the role of capillary, viscous and inertial forces on intermittent pathways through variation in the capillary number, and groups of observations with either nitrogen or decane as the non-wetting phase. We have developed a technique to identify intermittency in time-averaged images and have assessed the role of intermittency for a range of fractional flows for two different total flow rates for both fluid pairings.

We found that a range of parameters are controlling the location and frequency of dynamic interface fluctuations. These intermittent connections are important in controlling the connectivity of the non-wetting phase. The inclusion of intermittent areas with the non-wetting phase, reduces the number of disconnected non-wetting phase ganglia significantly (see Figure 1). It also is responsible for providing a connected pathway across the subvolume analysed for the lower fractional flow of the non-wetting phase. Capillary forces play an important role in intermittency, with the nonwetting phase always occupying the largest pores, brine always occupying the smallest pores and intermittency occurring in the intermediate sized pores. As the capillary number of the non-wetting phase increases it can invade smaller pores and intermittency then occurs in smaller pores (Figure 2).

The length of the non-wetting phase ganglia being connected by intermittent pores was used as a proxy for the inertial forces for that ganglia. This was compared to the pore radii for the intermittent pores causing the connection (Figure 3). Figure 3 shows that although there is no distinct relationship between the length of ganglia and the intermittent pore size, for a single ganglia, the range of pore sizes being occupied is narrowly distributed. This suggests the force in a ganglia is controlling the intermittency, but between ganglia, there are other factors, such as pore geometry, that are also influencing intermittency.

Indico rendering error

Could not include image: Cannot read image data. Maybe not an image file?

Indico rendering error

Could not include image: Cannot read image data. Maybe not an image file?

Indico rendering error

Could not include image: Cannot read image data. Maybe not an image file?

\section{Procter and Gamble Student poster award:}

I would like to compete in the Procter and Gamble Student award References:

1 Armstrong, R.T., McClure, J.E., Berrill, M.A., Rücker, M., Schlüter, S. and Berg, S., 2016. Beyond Darcy’s law: The role of phase topology and ganglion dynamics for two-fluid flow. Physical Review E, 94(4), p.043113. Acceptance of Terms and Conditions:

Click here to agree 


\title{
INVESTIGATING THE ENHANCED MASS FLOW RATES IN PRESSURE- DRIVEN WATER FLOW THROUGH NANOPORES
}

\author{
Author(s): Alexandros Stamatiou ${ }^{\text {None }}$ \\ Co-author(s): Kokou Dadzie ${ }^{1}$ \\ ${ }^{1}$ Heriot-Watt University
}

Corresponding Author(s): alexandros.stamatiou@hw.ac.uk

Over the past two decades, several researchers have presented experimental data from pressuredriven water flow through nanotubes. They quote flow velocities which are four to five orders of magnitude higher than those predicted by the Navier-Stokes equations. Thus far, attempts to explain these enhanced mass flow rates at the nanoscale have focused mainly on the introduction of wall-slip boundary conditions for the velocity field. In this paper, we present a different theory. A change of variable on the velocity field within the classical Navier-Stokes is adopted to transform the equations into mathematically and physically different equations. The resulting equations termed recasted Navier-Stokes equations contain additional diffusion terms whose expressions depend upon the driving mechanism. The new equations are then solved for the pressure driven flow configuration in the case of water flow in a long nanochannel. Analogous to previous studies of gas flow in microand nano-channels, a perturbation expansion in the aspect ratio allows for the construction of a $2 \mathrm{D}$ analytical solution for the streamwise component of the new velocity. In contrast to the slip-flow models, this solution is specified by a no-slip boundary condition at the channel walls. The mass flow rate can be calculated explicitly and compared to available data.

Procter and Gamble Student poster award:

I don't want to compete References:

Acceptance of Terms and Conditions:

Click here to agree

191

\section{CONSISTENT DISCRETIZATION OF FLOW FOR INHOMOGENEOUS GRAVITATIONAL FIELDS}

Michele Starnoni $^{1}$; Inga Berre ${ }^{1}$; Eirik Keilegavlen ${ }^{1} ;$ Jan Martin Nordbotten $^{\text {None }}$

${ }^{1}$ University of Bergen

Corresponding Author(s): michele.starnoni@uib.no

We present a consistent discretization of flow that includes a, generally heterogeneous, gravity field. So far, the traditional approach has been to represent the pressure as a potential, and let the discretization consider only deviations from the potential, by ignoring gravity effects in the discretizations. This approach is however inconsistent for inhomogeneous gravitational fields, as caused e.g. by twophase effects, density variations and certain variants of vertically averaged models for $\mathrm{CO} 2$ storage. Our discretization, termed gravitationally consistent multipoint flux approximation (GCMFA), is based on the idea that jumps in gravitational forces over the faces act as flux imbalance, and thus induce an additional pressure gradient in the sub-cells. Here, the traditional formulation is extended to the case including gravity by introducing an additional set of right hand side to the local linear system solved in the MPFA construction, thus obtaining an expression of the fluxes in terms of jumps in cell-centers gravity. It is shown that when the material properties are piecewise constant, the discrete flux operator is exact for piecewise linear functions. Applications to two-phase flow are also presented and discussed. 
References:

Acceptance of Terms and Conditions:

Click here to agree

\title{
Two-scale approach for the simulation of fluid-structure interac- tion in thin fibrous filters
}

\author{
Author(s): Sarah Staub ${ }^{1}$ \\ Co-author(s): Heiko Andrä ${ }^{1}$; Julia Orlik ${ }^{1}$; Ralf Kirsch ${ }^{1}$; Stefan Rief ${ }^{1}$ \\ ${ }^{1}$ Fraunhofer ITWM \\ Corresponding Author(s): sarah.staub@itwm.fraunhofer.de
}

Filter media can be produced based on several materials, e.g. non-woven, woven and spacer fabrics. All variants have in common that they are built-up by fiber networks, whose effective behavior like permeability, membrane and bending stiffness strongly depend on the microscopic morphology. These morphologic parameters are given e.g. by porosity, fiber anisotropy and length of unsupported fiber paths related to their thickness. Furthermore, the structural aspects can change during the deformation of the filter medium due to the fluid-structure interaction induced by the fluid pressure. Therefore, a coupled problem accounting for the filter deformation and the perfusion properties is solved. Implicitly, the effective filter permeability and stiffness depend on the applied pressure difference. Thus, different length scales are taken into account. On the one hand the microstructure is regarded with highly resolved fibers and on the other hand the complete filter is captured in terms of effective permeabilities. The scale bridging between the two-scales is elucidated.

We propose an asymptotic analysis for the coupled linearized problems with evolving back-coupling of the filter bending. At the microscale a Fast-Fourier transform (FFT) based approach for the computation of the effective bending stiffness of filters is applied. To this end, an extension of existing FFT solvers for tensile and shear loading to bending is presented. Finally, a coupled two-scale algorithm for the iterative computation of the effective filter properties depending on the actual pressure difference is presented.

The talk is illustrated by several numerical examples, which are validated by analytical formulas.

Procter and Gamble Student poster award:

References:

Acceptance of Terms and Conditions:

Click here to agree

719

\section{Calcium carbonate crystal/biofilm attachment in MICP grouted sands under different wetting conditions}

Author(s): Fabian Steinacher ${ }^{1}$

Co-author(s): Mary Mcinnes Anderson ${ }^{1}$; Gráinne El Mountassir ${ }^{1}$; James M. Minto ${ }^{1}$; Vernon R. Phoenix ${ }^{1}$; Rebecca J. Lunn ${ }^{1}$

\footnotetext{
${ }^{1}$ University of Strathclyde
}

Corresponding Author(s): fabian.steinacher@strath.ac.uk 
KEYWORDS: Microbial induced carbonate precipitation, S. pasteurii, biotechnology, bacteria/calcite attachment, different wetting conditions

\begin{abstract}
The interest in developing microbial induced carbonate precipitation as a cement repair alternative in oil and gas wells stems from the fact that this biotechnology can penetrate pore networks and fractures that conventional cement grouts cannot, due to their low viscosity. Currently, researchers are investigating the potential of MICP as a wellbore barrier technology to repair and mitigate hydrocarbon leakage through a) micro-channels in the cement matrix b) micro-annuli as communication pathway between the casing and the wellbore cement sheath. Research to-date assumes that the treated volume is fully water saturated. However, in subsurface applications for hydrocarbon leakage and repair, multiple fluids may be present, and the properties of the wetting fluid may effect bacterial and calcite crystal attachment. The impact of hydrocarbons and other wellbore treatment fluids on biomineralization processes at the microstructural scale, including bacterial attachment and subsequent carbonate precipitation are largely unknown.
\end{abstract}

During an MICP treatment process, the spatial distribution and strength of calcite attachment is key to the hydraulic and mechanical performance of the treated material. Carbonates that precipitate in pore throats can form bridges in between the individual sand gains which result in increased strength of the porous media and some reduction in permeability. Carbonate biominerals that precipitate on grain boundaries within the pore space provide additional grain roughness, which leads to an increase in nucleation sites for further precipitation of calcium carbonate crystal polymorphs. Continued biomineral treatment results in a decrease in rock porosity. But how do changes in the wetting phase downhole affect the precipitation processes?

In this experimental study, we explore the effect of biomineralization on the microstructural and physical properties of a series of batch cemented samples in the presence of typical downhole fluids (hydrocarbons and wellbore treatment fluids) on MICP and determine how these affect the formation of a "biomineral-seal" in the subsurface. We use Scanning Electron Microscopy to evaluate the spatial location and extent of the carbonate crystal/bacteria attachment.

We use UCS tests to compare the mechanical behavior of the bio-grouted sands in terms of compressive strength due to different wetting agents.

To investigate the attachment of precipitated carbonates and bacterial cells (entombed in calcium carbonate) to grain boundaries or calcium carbonate (free-floating) precipitated within pores, cryoFocused Ion Beam Scanning Microscopy (cryo-FIB/SEM) is deployed. These results will open a new way in the investigation of the 3D structures of micro and nano-structural features of a MICP cemented sand. Moreover, the suggested application of FIB/SEM technology will reveal new insights on biofilm/carbonate crystal attachment and is going to represent a new way of imaging microscopy of biological and geological material.

Procter and Gamble Student poster award:

I don't want to compete References:

Acceptance of Terms and Conditions:

Click here to agree

Multiphysics modeling of porous media acid dissolution: the effects of acid fluid properties and geometry on wormhole formation and directionality

\author{
Aslak Stubsgaard $^{1}$; H. M. Nick ${ }^{2}$ \\ ${ }^{1} D T U$ \\ ${ }^{2}$ Danish Hydrocarbon Research and Technology Centre, Denmark
}

Corresponding Author(s): aslakstubsgaard@gmail.com 
An OpenFOAM solver has been developed, capable of modeling buoyancy, advection, diffusion, and reaction of acid in geometries consisting of both fully fluid and heterogeneous porous media regions. Furthermore, the dissolution of porous media and with it the transition from Darcy-Forchheimer to fully fluid flow is accurately captured. With this solver, along with meshing schemes capable of constructing geometries consisting of fully fluid, porous media, and unaffected solid regions from three-dimensional CAD drawings, the impact on wormhole formation and directionality is shown to depend heavily on: Fluid properties, flow characteristics, and domain geometry. The importance of modeling three-dimensional domains, as opposed to two-dimensional domains is also observed. Full well completion models, including liner, perforations, and heterogeneous porous media, were analyzed to assess the feasibility of affecting wormhole directionality with heavy acids.

Procter and Gamble Student poster award:

I don't want to compete References:

Acceptance of Terms and Conditions:

Click here to agree

\title{
Relative permeability model of two-phase flow in nanopores con- sidering liquid-solid molecular interactions
}

\author{
Han Wang $^{\text {None }}$; Yuliang Su ${ }^{1}$ \\ ${ }^{1}$ China University of Petroleum (East China)
}

Corresponding Author(s): suyuliang@upc.edu.cn

Understanding and accurately calculating relative permeability is of critical importance to clarify the multiphase flow behaviors in porous media, and relative permeability is predominantly affected by the liquid-solid molecular interactions (contact angle of wet-phase). In this paper, a relative permeability model is proposed to investigate the oil-water flow behaviors in water-wet nanopores based on modified no-slip Hagen-Poisenille equation. The proposed model takes into account the water-wall interactions, viscosity of water and oil, pore wall roughness and pore size distribution (PSD). The velocity distribution and relative permeability of proposed model are validated with the results of molecular simulations and experiments. The results show that the relative permeability strongly depends on the contact angle: the increasing contact angle reduces the adsorbed water viscosity and increases the slip length that result in the different increasing values of permeability of single water phase and relative permeability of oil phase, thus, the oil phase relative permeability increases first and then decreases with an increasing contact angle, and with contact angle of 40$50 \mathrm{o}$, the relative permeability of oil phase reaches the maximum and that of water phase reaches the minimum; the increasing oil viscosity increases the oil phase permeability, due to the "lubricating" of the water phase, the oil phase relative permeability is greater than the permeability of single phase of water at low water saturation and with great ratio of oil viscosity to water viscosity; the pore wall roughness predominantly affected the contact angle, therefore, the relative permeability strongly depends on the roughness which reduce the relative permeability of oil phase and increase that of water phase; according the PSD analysis, the smaller the average pore radii, the smaller the relative permeability of oil phase and the greater the relative permeability of water phase. This work focuses on the relative permeability of two-phase flow in porous media and can be used in shale or tight oil recovery and ground water flow.

\section{Procter and Gamble Student poster award:}

I would like to compete in the Procter and Gamble Student award References:

Zhang T, Li X, Sun Z, et al. An analytical model for relative permeability in water-wet nanoporous media[J]. Chemical Engineering Science, 2017, 174: 1-12.

Zhang Q, Su Y, Wang W, et al. Apparent permeability for liquid transport in nanopores of shale reservoirs: coupling flow enhancement and near wall flow[J]. International Journal of Heat and Mass Transfer, 2017, 115: 224-234.

Xu P, Yu B. Developing a new form of permeability and Kozeny-Carman constant for homogeneous 
porous media by means of fractal geometry[J]. Advances in water resources, 2008, 31(1): 74-81.

Zhao J, Kang Q, Yao J, et al. The Effect of Wettability Heterogeneity on Relative Permeability of Two-Phase Flow in Porous Media: A Lattice Boltzmann Study[J]. Water Resources Research, 2018, 54(2): 1295-1311. Mattia D, Calabrò F. Explaining high flow rate of water in carbon nanotubes via solid-liquid molecular interactions[J]. Microfluidics and Nanofluidics, 2012, 13(1): 125-130.

Yiotis A G, Psihogios J, Kainourgiakis M E, et al. A lattice Boltzmann study of viscous coupling effects in immiscible two-phase flow in porous media[J]. Colloids and Surfaces A: Physicochemical and Engineering Aspects, 2007, 300(1-2): 35-49.

Wang S, Javadpour F, Feng Q. Molecular dynamics simulations of oil transport through inorganic nanopores in shale[J]. Fuel, 2016, 171: 74-86.

Huang D M, Sendner C, Horinek D, et al. Water slippage versus contact angle: A quasiuniversal relationship[J]. Physical review letters, 2008, 101(22): 226101.

$\mathrm{Wu} \mathrm{K}$, Chen Z, Li J, et al. Wettability effect on nanoconfined water flow[J]. Proceedings of the National Academy of Sciences, 2017: 201612608.

Li T, Song H, Wang J, et al. An analytical method for modeling and analysis gas-water relative permeability in nanoscale pores with interfacial effects[J]. International Journal of Coal Geology, 2016, 159: 71-81.

Wang S, Shi J, Wang K, et al. Apparent permeability model for gas transport in shale reservoirs with nano-scale porous media[J]. Journal of Natural Gas Science and Engineering, 2018, 55: 508-519.

Feng D, Li X, Wang X, et al. Capillary filling of confined water in nanopores: Coupling the increased viscosity and slippage[J]. Chemical Engineering Science, 2018, 186: 228-239.

Jin L, Pu H, Wang Y, et al. The consideration of pore size distribution in organic-rich unconventional formations may increase oil production and reserve by $25 \%$, eagle ford case study[C]//Unconventional Resources Technology Conference, San Antonio, Texas, 20-22 July 2015. Society of Exploration Geophysicists, American Association of Petroleum Geologists, Society of Petroleum Engineers, 2015: 17701782 .

Arshadi M, Zolfaghari A, Piri M, et al. The effect of deformation on two-phase flow through proppantpacked fractured shale samples: A micro-scale experimental investigation[J]. Advances in water resources, 2017, 105: 108-131. Acceptance of Terms and Conditions:

Click here to agree

525

\title{
NMR Experimental Study of CO2 Huff-Puff Characteristics on Matrix and Fractured Tight Sand Based on Four-Pore Model
}

\author{
Yuli Sun ${ }^{1} ;$ Xu Dong ${ }^{1}$; Dongxing Du ${ }^{1}$ \\ ${ }^{1}$ Qingdao University of Science and Technology
}

Corresponding Author(s): 15762181816@163.com

Tight sandstone reservoir is an important part of unconventional resources. The flooding is difficult to be carried out on the rock due to its low-permeability. CO2 huff-puff is a good solution to improve the recovery of such reservoir. Manual fracturing is often used to provide high-speed seepage channels for underground fluids to furtherly enhance the oil recovery. In this study, the tight sand aliquots are fractured with the Brazilian splitting method, and saturated with the simulation oil (10.5 $\mathrm{cp}, 0.821 \mathrm{~g} / \mathrm{cm} 3)$. CO2 huff-puff experiments under constant injection pressure (15 MPa) are made on the matrix prior to the splitting and the fractured plugs. The CO2 huff-n-puff characteristics in these two aliquots are investigated using the NMR (TE=60 us, TW=10 s). A four-pore model based on the T2 spectrum is constructed to illustrate the distribution of oil in the full-scale pores.

Results showed that the T2 range at about $0.05-200 \mathrm{~ms}$, and the T2 cutoff (T2c) values are about $10-30$ $\mathrm{ms}$ for the unfractured aliquots. Pores in this abstract are divided into four parts according to the relationships between T2 and pore diameter: fracture $(200 \mathrm{~ms}<\mathrm{T} 2<400 \mathrm{~ms})$, macropore (T2c $<\mathrm{T} 2<$ $200 \mathrm{~ms})$, mesopore $(1 \mathrm{~ms}<\mathrm{T} 2<\mathrm{T} 2 \mathrm{c})$, and micropore $(\mathrm{T} 2<1 \mathrm{~ms})$. Contrast to the pore distributions in the matrix, splitting introduces new fractures and decreases some components in the macropores, but changes the meso and micro pores unobviously. It suggests that some of the macropores in the original matrix evolved into fractures after splitting. 3 rounds of $\mathrm{CO} 2$ huff-puff can basically stable the residual oil saturations in both kinds of samples. The fracturing does not change the oil displacement law in pores significantly. The oil is recovered first from the fractures and then from the macropores and mesopores in turn. But not all oil in the macropores can be recovered. After 3 rounds of huff-puff, the right edge of all T2 spectrums do not move close to the T2c simply, but shrink 
gradually and remain stable at about $110-140 \mathrm{~ms}$. The right boundaries of residual oil in moveable pores cannot be obviously changed by using the matrix or fractured aliquots. The peak represents the residual oil adhered to the surface of the particles in the form of film, which is resulted by the wettability differences between the gas and oil to the particles. The enhanced recovery in the last rounds of huff-puff is mainly contributed by the oil recovered from the mesopores. The unchangeable of $\mathrm{T} 2$ of the micropores means the $\mathrm{CO} 2$ huff-puff could not recover the oil in clay particle pores no matter in the matrix or the fractured aliquots. Compared to the matrix, a higher final residual oil saturation (about 18\%) is observed in the fractured plug. The splitting mode used in the study plays a critical role for the decreasing of oil recovery. The four-pore mode based on NMR described in this study provides new insights into the understanding of the dynamic distribution of oil in matrix and splitting fractures on the $\mathrm{CO} 2$ huff-puff processes in the full pore scale of tight oil reservoirs.

\title{
Procter and Gamble Student poster award:
}

I would like to compete in the Procter and Gamble Student award References:

Acceptance of Terms and Conditions:

Click here to agree

\section{A hybrid numerical model (CFD-DEM) for proppant transport in dynamically propagating hydraulic fractures in shale gas reser- voirs}

\author{
Author(s): Yatin Suri ${ }^{1}$ \\ Co-author(s): Sheikh Islam ${ }^{1}$; Mamdud Hossain ${ }^{1}$ \\ ${ }^{1}$ Robert Gordon University
}

Corresponding Author(s): y.suri@rgu.ac.uk

\begin{abstract}
For hydraulic fracturing design in unconventional reservoirs, it is of significant importance to accurately predict proppant distribution in a fracture, as the distribution of proppant affects fracture conductivity and fractured well productivity. In this paper, a three-dimensional multiphase modelling approach has been applied, and the equations defining the fluid-proppant and inter-proppant interaction have been solved using the finite volume technique that uses hybrid Computational Fluid Dynamics model (Euler model coupled with Discrete Element Method) to simulate proppant distribution in hydraulic fractures in the unconventional reservoir. The predicted simulation results were validated against the experimental study by Tong and Mohanty 1. Based on the previous literature [2-4], the existing studies used planar and fixed fracture geometry to study proppant transport neglecting the fluid leak off from the fracture wall or fracture-matrix interface and fracture tip screen out. In this work, a realistic fracture geometry is developed with fluid leak off rate defined along the fracture length to mimic the fluid leak-off from the fracture into the surrounding porous reservoir. Additionally, the effect of fracture roughness and dynamic fracture propagation is included in the model.
\end{abstract}

Next, a case study is designed to use foam (Non-Newtonian fluid) as fracturing fluid and mitigate the challenge faced by petroleum industry of quick deposition of the proppant transport and suspension in thin fracturing fluid due to the very low viscosity of thin fracturing fluid. A parametric study is then performed to investigate the effect of variation in proppant properties (proppant addition schedule, proppant size, fractional flow of proppant), fracturing fluid properties (fluid viscosity, fluid leak-off rate, injection rate) and geomechanical properties (fracture width, fracture tip screen out). In addition, the placement of multi-size proppant distribution in horizontal and vertical well configuration was investigated.

The proppant transport in hydraulic fractures has been studied comprehensively. Lighter proppants can be injected early followed by heavier proppants to maintain high propping efficiency. Based on the simulation results, it is recommended that a composite process of over-flushing and tail-in with large proppant particles at a low injection rate can improve proppant distribution in the fracture, 
which will greatly increase the productivity of fractured wells. Only when the equilibrium height of the proppant dune is reached can the later injected proppant be transported farther into the fracture. This study has solved the challenge of early deposition of the proppant transport in thin fracturing fluid used in shale gas reservoirs and enhanced the understanding of the interaction phenomenon between proppant and fracturing fluid. Depending on the nature and properties of the proppant and hydraulic fracture, this study can provide a basis for understanding the ways to characterize the fluid flow, proppant transport, and distribution through the hydraulic fracture in the unconventional reservoir and accordingly optimize the hydraulic fracturing design.

\section{Procter and Gamble Student poster award:}

I would like to compete in the Procter and Gamble Student award References:

1 Tong, S. and Mohanty, K.K., 2016. Proppant transport study in fractures with intersections. Fuel, 181, pp.463-477.

2 Zhang, G., Gutierrez, M., and Li, M., 2017. A coupled CFD-DEM approach to model particle-fluid mixture transport between two parallel plates to improve understanding of proppant micromechanics in hydraulic fractures. Powder Technology, 308, pp.235-248.

3 Blyton, C.A., Gala, D.P. and Sharma, M.M., 2015, September. A comprehensive study of proppant transport in a hydraulic fracture. In SPE Annual Technical Conference and Exhibition. Society of Petroleum Engineers.

4 Zeng, J., Li, H. and Zhang, D., 2016. Numerical simulation of proppant transport in hydraulic fracture with the upscaling CFD-DEM method. Journal of Natural Gas Science and Engineering, 33, pp.264-277. Acceptance of Terms and Conditions:

Click here to agree

\section{Topological characterization of 3D printing fracture networks}

Author(s): Anna Suzuki ${ }^{1}$

Co-author(s): Miyuki Miyazawa ${ }^{1}$; Megumi Konno ${ }^{1}$; Takatoshi Ito ${ }^{1}$

${ }^{1}$ Tohoku University

Corresponding Author(s): anna.suzuki@tohoku.ac.jp

Detailed characterization of flow and transport processes within complex fracture networks remains many challenges. The fluid flow is mainly controlled by the fracture structures. Fractures have complex shapes, and their intersections and connectivities with other fractures also lead to complex flow. We aim at quantifying the complex fracture structures by topological data analysis and finding interaction between the structure and flow with 3D fracture network models. We use persistent homology, which is a tool for evaluating complex and multi-scale features in the geometry and is usually used to analyze connected components, holes/tunnels, and voids in data of any dimension. We also conduct flow experiment using synthetic fracture network models created by a 3D printer. 3D printer technologies allow us to control any shapes of structures. Our study clarifies relationships between the geometries that can be detected by persistent homology and hydraulic properties obtained from the fracture network models.

Procter and Gamble Student poster award:

References:

Acceptance of Terms and Conditions:

Click here to agree 


\title{
Karst-on-a-chip: microfluidic studies of dissolution of an ana- logue fracture
}

\author{
Piotr Szymczak $^{1}$; Florian Osselin ${ }^{2}$; Filip Dutka ${ }^{1}$; Silvana Magni ${ }^{1}$; Max Cooper $^{1}$ \\ ${ }^{1}$ University of Warsaw \\ ${ }^{2}$ Institut des Sciences de la Terre d'Orleans
}

Corresponding Author(s): pszymczak@gmail.com

Dissolution of fractured and porous media introduces a positive feedback between fluid transport and chemical reactions at mineral surfaces leading to self-focusing of the flow in pronounced wormholelike channels $[1,2]$. We study the flow-induced dissolution in a simple microfluidic setup, with a gypsum block inserted in between two polycarbonate plates, which is the simplest model of a fracture 3 . This gives us a unique opportunity to observe the evolution of the dissolution patterns in-situ and in real-time. By changing the flow rate and the aperture of the fracture we can scan a relatively wide range of Peclet and Damkohler numbers, characterizing the relative magnitude of advection, diffusion and reaction in the system. Additionally, as the aperture is increased, a transition is observed between the fractal and regular dissolution patterns. For small gaps, the patterns are ramified fractals. For larger gaps, the dissolution fingers are found to have regular forms of two different kinds: either linear (for high flow rates) or parabolic (for lower flow rates). The experiments are supplemented with numerical simulations and analytical modeling which allow for a better understanding of evolving flow patterns. In particular, we find the shapes and propagation velocities of dominant fingers for different widths of the system, flow rates and reaction rates. Finally, we comment on the link between the experimentally observed patterns and the natural karst systems - both cave conduits and epikarst solution pipes.

1 Hoefner, M. L. and Fogler, H. S. Pore evolution and channel formation during flow and reaction in porous media. AIChE J. 34, 45-54, 1988

2 P. Szymczak, A. J. C. Ladd, Wormhole formation in dissolving fractures, J. Geophys. Res., 114, B06203, 2009

3 F. Osselin, P. Kondratiuk, A Budek, O. Cybulski, P. Garstecki, P. Szymczak Microfluidic observation of the onset of reactive infiltration instability in an analog fracture, Geophys. Res. Lett., 43, 69076915,2016

Procter and Gamble Student poster award:

References:

Acceptance of Terms and Conditions:

Click here to agree

714

\section{Natural Time Scales of the Monod type kinetics: experimental support by photocatalysis and oxygen consumption in saturated porous media}

Elena Sánchez-Badorrey ${ }^{1}$; Ronald E. Vargas Balda ${ }^{2}$; Lucía L. Sánchez Sánchez ${ }^{3}$; María Ojeda ${ }^{4}$

\author{
${ }^{1}$ Dpto. M.E. e Ing. Hidráulica - Instituto del Agua. Universidad de Granada \\ ${ }^{2}$ Dpto. Química, Universidad Simón Bolivar. \\ ${ }^{3}$ Instituto del Agua, Universidad de Granada. \\ ${ }^{4}$ Dpto. Química, Universidad Simón Bolivar
}

Corresponding Author(s): elenasb@ugr.es

An accurate characterization of kinetic parameters related to degradation processes in saturated porous media is key for a proper modeling of reactive solute transport and the coupled mixing and 
dispersion processes. Degradation processes, as well as microbial growth, are often described by non-linear kinetics with consumption rate laws, $r= \pm d C / d t$, of the form $r=k K C /(1+K C)$. This is the case, for example, of: (1) the Monod model, describing degradation processes related to organic matter preservation, denitrifcation or trace metal speciation and mobility linked to the iron cycle in shallow-water sediment, (2) the Langmuir-Hinselwook $(\mathrm{L}-\mathrm{H})$ model, describing heterogeneous catalysis processes and widely used in water treatment or, (3) the Michaellis-Menten (M-M) model describing enzymatic catalysis. The kinetic parameters $k([\mathrm{M}][\mathrm{T}]-1)$ and $K([\mathrm{M}]-1)$ are commonly estimated from measured time $(t)$ series of the reactant concentration $(C)$ using non-linear regression analysis. However, data heterogeneity in time, low data density and the intrinsic non-linearity of the method can result in a substantial parameter uncertainty.

By identifying the Natural Time Scales (NTS) of the general reactive Monod type cycles, this work presents a new procedure, the NTS method, to extract robust kinetic information from arbitrary distributed experimental reactant concentration-time data. Two natural scales are identified: (i) $t f=k K$, describing the fastest time to perform one run of the catalytic cycle, and (ii) $t s=C 0 / k$, the slowest time it would take to chemically transform an initial amount of matter, being $\mathrm{C} 0$ the initial concentration. Thus, the reactive transport equation results in a linear combination of functions weighted by the tf and ts time scales, from which the kinetic parameters can be easily computed using a linear regression. The NTS method has been first tested using experimental data from the heterogeneous photocatalysis of naphthalene (as reactant) and $\mathrm{TiO} 2$ (as photocatalyst) in a tubular bench reactor under different physical, chemical and flow conditions. Then, the method was tested using field measurements of the dissolved oxygen (DO) consumption in heterogeneous sediments of a shallow lagoon under different climate conditions. In both cases, the NTS method shows an excellent behavior, with smaller regression errors than those of the standard non-linear analysis. Moreover, the density, distribution or extension of the data has no significant influence on the estimation of the kinetic parameters.

Finally, the Kinetic Family (KF) concept is presented to analyze the roll of the inhibition factor, KC, on the natural time scales ( $t f$ to $t s$ ) calculated for the two study cases. The KF concept is probed to be useful to analyze the influence of physicochemical variables, such as flow, radiance and temperature, on the NTS and the kinetic behaviour of complex biogeochemical processes.

Acknowledgements: MICINN (project-CTM2011-28984) and Coimbra Group.

Keywords: natural time scales, kinetic family, reaction rates, heterogeneous photocatalysis, shallow lagoon.

Procter and Gamble Student poster award:

References:

Acceptance of Terms and Conditions:

Click here to agree

520

\title{
Mathematical modelling of modified salinity water flooding in carbonate rocks
}

\author{
Author(s): Mirhossein Taheri ${ }^{1}$ \\ Co-author(s): Maria Bonto ${ }^{1}$; Karen Louise Feilberg ${ }^{2}$; Ali Akbar Eftekhari ${ }^{3}$; Hamid Nick ${ }^{2}$ \\ ${ }^{1}$ PhD student at DTU university \\ 2 senior researcher at center for oil and gas \\ ${ }^{3}$ Researcher at center for oil and gas
}

Corresponding Author(s): mirtah@dtu.dk

We develop a 1D-two phase analytical model that considers the impact of transport and potential determining ions (PDI) interactions with carbonate rock to identify the underlying mechanisms of the modified salinity water flooding; to that end, we utilize a large set of core flooding tests including several in-house experiments on the reservoir chalk (Taheri et al., 2019). Based on the experimental 
conditions of the tests, e.g., the ionic composition of the injecting brine and rock surface composition, we quantify the adsorption of PDI's on the rock surface using an in-house surface complexation model (Eftekhari et al., 2017). Further, we tune different models for oil and water relative permeability with and without the adsorption effect using a modern search-based optimization algorithm to match the oil recovery and pressure drop history. We found that an acceptable match during the tertiary and post tertiary mode can only be obtained by including the wettability modification as a consequence of PDI adsorption. The analyses of the core flooding tests show that the oil breakthrough is not always commenced immediately when the brine composition is altered in tertiary mode. In most cases, a delay in the oil breakthrough was observed which may be due to the wettability modification processes. This wettability change can be due to the adsorption of PDI or reduction of total salinity of the injecting brine. This observation, combined with our ability to accurately model the adsorption of PDIs on the carbonate surface enables us to identify the important PDIs that may cause the wettability change in carbonates.

Procter and Gamble Student poster award:

I would like to compete in the Procter and Gamble Student award References:

Eftekhari, A.A., Thomsen, K., Stenby, E.H., Nick, H.M., 2017. Thermodynamic Analysis of Chalk-BrineOil Interactions. Energy and Fuels 31, 11773-11782. doi:10.1021/acs.energyfuels.7b02019

Taheri, M. , Bonto, M. „Eftekhari, A.A., Nick, H., 2019. Towards Identifying the Mechanisms of the Modified-Salinity Water Flooding by a Novel Combination of Core-Flooding and Mathematical Modeling. MEOR 2019, Society of petroleum engineering Acceptance of Terms and Conditions:

Click here to agree

\title{
Modeling the effect of time-dependent wettability modification in modified salinity water flooding
}

\author{
Author(s): Mirhossein Taheriotaghsara ${ }^{1}$ \\ Co-author(s): Hamid Nick ${ }^{2}$; Ali Akbar Eftekhari ${ }^{3}$; Maria Bonto ${ }^{4}$ \\ ${ }^{1}$ PhD student at DTU university \\ ${ }^{2}$ Senior researcher-DHRTC \\ ${ }^{3}$ researcher-DHRTC \\ ${ }^{4}$ PhD student at DTU university
}

Corresponding Author(s): mirtah@dtu.dk

Most of the current models treat wettability change through adsorption as an equilibrium process, i.e., an instantaneous adsorption of ions at the rock-brine and oil-brine interface that alters the surface properties towards a more water-wet or oil-wet behaviour. However, there is plenty of experimental evidence that points to a much slower wettability change than a process that can be described by a simple chemical equilibrium. Here, a mathematical model is presented to analyze the influence of salinity reduction and brine composition modification on the wettability alteration of carbonate rocks. A one dimension-two phase flow model is developed that couples an in-house Surface Complexation Model (SCM)(Eftekhari, 2015) with analytical and numerical solutions of oil-water flow and ionic species transport equations. The adsorption of potential determining ions is described by either the predicted adsorption isotherms by the optimized SCM or by a rate-dependent equilibrium reaction. The model is empowered by a modern search based algorithm to fit the oil recovery and pressure drop curves in core flooding tests by adjusting the Corey's relative permeability parameters and the reaction rate constants. The outcome of our analysis from a large set of reported core flooding experiments and some in-house tests shows that carbonate rock wettability modification during modified salinity water flooding is a time-dependent phenomenon. We precisely modelled the observed delay in the occurrence of oil breakthrough in the tertiary mode in many tests by including the interactions of potential determining ions (PDIs) with the carbonate rock surface. Our mathematical analysis of the modified salinity water flooding combined with our precise estimation of the equilibrium interactions of PDI's with the rock surface shows that the observed delay in the mobilization of oil in the core flooding experiments can be explained by an equilibrium or rate-dependent adsorption curve for the PDI's, depending on the experimental conditions. 
Procter and Gamble Student poster award:

I would like to compete in the Procter and Gamble Student award References:

Eftekhari, A.A., Thomsen, K., Stenby, E.H., Nick, H.M., 2017. Thermodynamic Analysis of Chalk-BrineOil Interactions. Energy and Fuels 31, 11773-11782. doi:10.1021/acs.energyfuels.7b02019 Acceptance of Terms and Conditions:

Click here to agree

\section{Effective Rheology of Bi-Viscous Non-Newtonian Fluids in Porous Media}

Laurent Talon ${ }^{1} ;$ Alex Hansen $^{2}$

${ }^{1}$ Lab. FAST, Univ. Paris-Sud, CNRS

${ }^{2}$ PoreLab, NTNU

Corresponding Author(s): talon@fast.u-psud.fr

The behavior of complex fluids inside porous media may be very different from when they are not. This is a problem encountered in many biological or industrial applications ranging from impregnation of fibrous materials to immiscible multi-phase flow in porous media.

Of all the different types of non-Newtonian fluids, many undergo behavioral changes depending on the stress or strain applied. One may cite the Carreau rheology which is Newtonian at low shear rate but behaves as a power law fluid above a certain shear rate. Another example is the yield stress fluid that responds as a solid below a critical stress and as a power law fluid above.

At the mesocopic level, this rheological approach can also be extended to immiscible displacements in porous media. In this case, the fluids may each be Newtonian but the interfacial tension between them makes them effectively behave in a non-Newtonian way. For instance, a minimum level of stress is required for a non-wetting phase to invade small pore throats. The local flow rate will then be different below and above this threshold.

The purpose of this presentation is to study the coupling between the heterogeneity of a porous medium and a rheology with a change in behavior. We study a very simple model called bi-viscous, where the fluid is Newtonian but with a change in viscosity at a particular shear rate (or shear stress). The coupling between the disorder and such a simple rheological model leads to a very rich problem. In particular, two limit cases of this problem present universal behaviors sharing the universality class of percolation or the directed polymer problem.

Procter and Gamble Student poster award:

References:

Acceptance of Terms and Conditions:

Click here to agree

\section{New Capillary Number Definition for Micromodels: the Impact of Pore Microstructure}

Author(s): Jinyu Tang ${ }^{1}$

Co-author(s): Michiel Smit ${ }^{1}$; Sebastien Vincent-Bonnieu ${ }^{2}$; Willaim R. Rossen ${ }^{1}$ 
${ }^{1}$ Dept. of Geoscience and Engineering, TU Delft
${ }^{2}$ Shell Global Solutions International, the Netherlands

Corresponding Author(s): j.tang-4@tudelft.nl

The capillary desaturation curve (CDC), which indicates nonwetting-phase saturation as a function of capillary number (Nca), is one of the most fundamental concepts in describing fluid transport and flow in porous media. The conventional definitions of Nca were initially proposed for 3D porous media, yet many experimental studies use these definitions for 2D pore networks. Experimental observations and theoretical analysis show that flow in a 2D pore network, e.g. a microfluidic device, is very different from that in $3 \mathrm{D}$ porous rock. Direct application of the conventional Nca may lead to incorrect conclusions for fluid transport in geological formations.

We propose a new capillary number (Nca) definition for 2D etched micromodels. The new definition is derived from a force balance on a nonwetting ganglion trapped by capillarity. It incorporates the impact of pore microstructure on mobilization. The geometrical factors introduced can be estimated directly from image analysis of the pore network etched in the micromodel, without conducting flow experiments. The improved fit of the new Nca to published data supports its validity. The new definition yields a consistent trend in the CDC. The conventional Nca definitions proposed for porous rock give a large scatter in the CDC for data in micromodels. This is due to the different type of flow in micromodels, as 2D networks, relative to 3D geological porous media. In particular, permeability is dominated by channel depth in micromodels with shallow depth of etching, and generally there is no simultaneous multiphase flow under capillary-dominated conditions. We compare the conventional and new Nca, showing that the conventional CDC overestimates the velocity at the mobilization by a factor of tens to thousands for a variety of micromodels in published studies.

The new definition could be applied as a mobilization condition for nonwetting phase in microfluidic evaluations across a variety of processes: removal of DNAPL (Dense Non-Aqueous Phase Liquid) contaminants in aquifers and soils; enhanced recovery of oil in reservoirs; or trapping of CO2 in CCUS (Carbon Capture Utilization and Storage).

\title{
Procter and Gamble Student poster award:
}

I would like to compete in the Procter and Gamble Student award References:

AlQuaimi, B., \& Rossen, W. (2017). New Capillary Number Definition for Displacement of Residual Nonwetting Phase in Natural Fractures. Geophysical Research Letters. Acceptance of Terms and Conditions:

Click here to agree

\section{Three-Phase Fractional-Flow Theory of Oil Displacement by Foam with Multiple Steady States}

\author{
Author(s): Jinyu Tang ${ }^{1}$ \\ Co-author(s): Pablo Castañeda ${ }^{2}$; Dan Marchesin ${ }^{3}$; William R. Rossen ${ }^{4}$ \\ ${ }^{1}$ Dept. of Geoscience and Engineering, TU Delft \\ ${ }^{2}$ Dept. of Mathematics, ITAM, Mexico \\ ${ }^{3}$ Instituto Nacional de Matemática Pura e Aplicada, Brazil \\ ${ }^{4}$ Dept. of Geoscience and Engieering, TU Delft, The Netherlands
}

Corresponding Author(s): j.tang-4@tudelft.nl

Foam flow with oil in porous media is involved in numerous processes across a variety of fields, i.e. aquifer and soil remediation, oil displacement, and carbon storage. Understanding the interplay of foam and oil or other non-aqueous phases in porous media is key to improving prediction and control of these processes. A widely used implicit-texture foam model predicts a surface describing foam 
apparent viscosity as a function of fractional flows. The surface folds on itself, that is reminiscent of catastrophe theory. This means, in the folded region, that there are multiple foam states fitting same injected fractional flows. A displacement therefore corresponds to more than one possible solution. Numerical simulations suggest the displacing state among the multiple possible injection states but do not explain the reason.

We address the issue of multiple steady states from the perspective of wave propagation, using three-phase fractional-flow theory. The wave-curve method is applied to solve the two conservation equations for composition paths and wave speeds in 1D foam flow with oil. There is a composition path satisfying the conservation equations from each possible injection state $\mathrm{J}$ to the initial state I. In all cases examined with multiple steady states, two of the paths feature negative wave velocity at $\mathrm{J}$; such a solution does not correspond to the physical injection conditions, i.e. forward propagation of the given state. The stable displacement is the one with wave speeds (characteristic velocities) all positive along the path from $\mathrm{J}$ to I. We observe that either a state with strong foam or a state with nearly collapsed foam gives a stable displacement, but never the intermediate, unstable state. Which state makes the displacement depends on the initial state of a reservoir. A boundary curve in ternary saturation space defined here captures the dependence of the choice of the displacing foam state (strong or collapsed) on initial condition.

In applications, foam is not co-injected with oil, but it mostly likely flows concurrently with oil at some distance away from the injection well, resulting in multiple possible foam states for the same local fractional flows. In particular, in oil recovery, complexities in fluids and rock leave part of the oil in place after initial efforts of flooding. In environmental application, injected foam is in direct contact with non-aqueous contaminants in soils and aquifers. The findings here could identify the displacing state among the multiple foam states given the initial conditions. This could help to designers to optimize and maximize the benefit of foam processes.

Procter and Gamble Student poster award:

I would like to compete in the Procter and Gamble Student award References:

Tang, J., Ansari, M. N., \& Rossen, W. R. (2016). Modelling the Effect of Oil on Foam for EOR. EAGE, XV ECMOR, Amsterdam. https://doi.org/10.3997/2214-4609.201601877

Castañeda, P., Abreu, E., Furtado, F., \& Marchesin, D. (2016). On a universal structure for immiscible three-phase flow in virgin reservoirs. Computational Geosciences, 20(1), 171-185.

Gauglitz, P.A., Friedmann, F., Kam, S. I, and Rossen, W.R. (2002). Foam generation in homogeneous porous media. Chem. Eng. Sci. 57(19), 4037-4052. https://doi.org/10.1016/S0009-2509(02)00340-8 Acceptance of Terms and Conditions:

Click here to agree

\section{Experimental study on effect of effective stress on coal and gas outburst in crosscutting}

Jupeng Tang $^{1}$; Liping $\mathrm{Li}^{1}$

${ }^{1}$ Liaoning Technical University, P.R.China

Corresponding Author(s): jupengt@126.com

Coal is typical porous media. The coal and gas outburst in crosscutting is the most serious dynamic disaster in coal mine in China. The relationship formula between the critical effective stress and the exposed area was deduced according to the fold catastrophe model. The maximum exposed areas of Fuxin Sunjiawan coal mine were presented under the different mining depth. The relationship between the exposed area and the critical effective stress was analyzed. The $-1100 \mathrm{~m}$ outburst coal seam in Sunjiawan mine of Fuxin was regarded as the research object. The influence experiment of the exposed area on the coal and gas outburst was performed using triaxial outburst instrument designed and developed by ourselves $\$ the coal briquette was put in the outburst instrument. The triaxial pressures were applied to the coal samples to simulate the stress environment of coal seam. 
The different exposed area was simulated by changing the weak surface area(30.19凶34.21 and 38.48 $\mathrm{cm} 2$ ). The results show that the critical effective stress increases with the exposed area increase based on the experiment and theory. The formula validity of the critical effective stress is verified. The critical effective stress and exposed area are main factors of the intensity of coal and gas outburst in crosscutting. The distribution of coal powder after outburst has the characteristics of fluctuation. The outburst energy and intensity near the exposed face both increase with the increment of exposed area and critical effective stress. The conclusion has important reference significance to prevent the outburst in crosscutting.

Procter and Gamble Student poster award:

References:

Acceptance of Terms and Conditions:

Click here to agree

\title{
Accurate thermodynamic analysis of supercritical fluids adsorp- tion on shale and coal
}

\author{
Xu Tang ${ }^{1} ;$ Robert Mokaya ${ }^{1}$ \\ ${ }^{1}$ University of Nottingham
}

Corresponding Author(s): xutang2050@outlook.com

Understanding supercritical fluids $(\mathrm{scCH} 4 / \mathrm{scCO} 2)$ adsorption mechanism on shale and coal lays the foundation for unconventional gas resource estimation and enhanced gas recovery via carbon dioxide injection. However, accurate thermodynamic potentials of scCH4/scCO2 adsorption on shale and coal were and are rarely reported because of the lack of a reliable approach for obtaining the true adsorption uptake from measured adsorption isotherms and the routinely used but oversimplified Clausius-Clapeyron (C-C) approximation. This work develops a rigorous framework for direct description of measured scCH4/scCO2 adsorption isotherms (up to $27 \mathrm{MPa}$ and $355 \mathrm{~K}$ ) on shale and coal and for straightforward calculation of the intrinsic thermodynamic potentials by considering non-ideal gas behaviour and the contribution of the adsorbed phase. By this method, the temperature dependence as well as the uptake dependence of the thermodynamic potentials can be readily investigated, where the former cannot be revealed using the $\mathrm{C}-\mathrm{C}$ approximation. The influence of the adsorbed phase and the gas behaviour (real gas or ideal gas) on the thermodynamic potentials are also investigated, which shows that neglecting either the real gas behaviour or the adsorbed phase volume always results in an overestimation of the thermodynamic potentials. This work therefore justifies the method to obtain the true thermodynamic potentials for $\mathrm{scCH} 4 / \mathrm{scCO} 2$ adsorption on shale and coal. The developed framework also lays the foundation for future investigations of the thermodynamics and heat transfer characteristics of the interaction between $\mathrm{scCH} 4 / \mathrm{scCO} 2$ and shale.

Procter and Gamble Student poster award:

References:

Acceptance of Terms and Conditions:

Click here to agree

988

\section{Learning Parameters and Constitutive Relationships with Physics- Informed Deep Neural Networks}

Alexandre M Tartakovsky ${ }^{\text {None }}$ 
Corresponding Author(s): alexandre.tartakovsky@pnnl.gov

I will present a physics-informed deep neural network (DNN) method for estimating parameters and unknown physics (constitutive relationships) in partial differential equation (PDE) models. We use PDEs in addition to measurements to train DNNs to approximate unknown parameters and constitutive relationships as well as states. The proposed approach increases the accuracy of DNN approximations of partially known functions when a limited number of measurements is available and allows for training DNNs when no direct measurements of the functions of interest are available. Physics-informed DNNs are used to estimate the unknown space-dependent diffusion coefficient in a linear diffusion equation and an unknown constitutive relationship in a non-linear diffusion equation. For the parameter estimation problem, we assume that partial measurements of the coefficient and states are available and demonstrate that under these conditions, the proposed method is more accurate than state-of-the-art methods. For the non-linear diffusion PDE model with a fully unknown constitutive relationship (i.e., no measurements of constitutive relationship are available), the physics informed DNN method can accurately estimate the non-linear constitutive relationship based on state measurements only. Finally, I will demonstrate that the proposed method remains accurate in the presence of measurement noise.

Procter and Gamble Student poster award:

References:

Acceptance of Terms and Conditions:

\title{
Introducing a New Conceptual-Physical Model for Simulation of Groundwater Flow in a Porous Medium Using a Network of Pipes
}

\author{
Parjang Monajemi ${ }^{1} ;$ Hamed Tavakolipour $^{1}$ \\ ${ }^{1}$ Fasa University
}

Corresponding Author(s): hamedtp@gmail.com

In this paper, a new analog model is introduced in order to simulate groundwater flow under steady state condition. The model is a 2D network of horizontal and vertical pipes connected by junctions. Pipes and junctions in this model are simplifications of the throats and pores of a real porous medium. By assuming that the model follows Hagen-Poiseuille law, it is shown that the governing equation of the model is the Laplace equation which governs steady-state flow in a saturated homogenous porous medium. Further, the physical apparatus of the pipe network is constructed in a laboratory scale by connecting real pipes and junctions.

Similar scenarios are studied by conceptual analysis and the constructed lab pipe network model. In this regard, groundwater flow under a dam and groundwater flow in confined aquifers are modeled. The results are then compared to numerical solutions to illustrate the accuracy of the proposed model which shows an excellent agreement, making the pipe network model a novel tool for simulating groundwater flow.

Finally, it is notable that unlike other analog groundwater models, pipe network model is a simplification of a real aquifer as it clearly shows how groundwater flows in confined aquifers. Besides the visual aspect of the model, another great advantage of pipe network model over contemporary models is the simplicity in simulating impermeable barriers and heterogeneity through a porous medium which makes it highly applicable for observing numerous observations in a great variety for groundwater modeling and educational purposes.

Procter and Gamble Student poster award:

I would like to compete in the Procter and Gamble Student award References:

Acceptance of Terms and Conditions: 


\title{
Permeability change during the growth of dense 3D geomechan- ical fracture networks
}

\author{
Author(s): Robin N Thomas ${ }^{1}$ \\ Co-author(s): Adriana Paluszny ${ }^{1}$; Robert W Zimmerman ${ }^{1}$ \\ ${ }^{1}$ Imperial College London \\ Corresponding Author(s): robin.thomas11@imperial.ac.uk
}

Fractured rock permeability is known to vary significantly due to the change in connectivity and morphology of individual fractures, which emerge from the mechanical process of fracture propagation. We demonstrate the use of three-dimensional finite element simulations of fracture growth to generate geomechanical discrete fracture networks (GDFNs), and then quantify the flow properties and effective permeability of these networks. By directly simulating the physics of rock deformation and fracturing, mechanical features that are routinely observed in fractured rocks emerge naturally from the simulations, including anisotropy, non-planarity, and fracture intersections. Following advances in methods for fast and accurate calculation of fracture tip stress intensity factors using the virtual disk integration technique, handling fracture intersections, and accurate simulation of fracture surface contact, geomechanical networks can now be generated at unprecedented fracture densities. GDFNs are generated through forty to fifty individual growth steps, and contain over 150 individual fractures. To achieve this, a cubic volume is first initialised with two sets of disc-shaped flaws. In set one, fractures have small initial radii and are oriented perpendicular to the direction of tension. Fractures in set two are larger and are oriented almost parallel to the direction of tension, reflecting an earlier phase of deformation. A geometric representation of the domain is stored separately from the discretising mesh, and updated as growth progresses. The rock is assumed to be granite, and its deformation in tension is simulated using the finite element method, treating the matrix domain as homogeneous, isotropic and linear-elastic. Fracture propagation is a function of the local changes in stress intensity factors along the fracture tips. Fractures perturb their local stress field, leading to activation of otherwise inactive (set two) fractures due to stress amplification, and suppression of other fractures in shadow zones. The final shape of geomechanical fractures is determined primarily by the local fracture neighbourhood, rather than by their initial shape. In set one, final fracture surface areas of the different fractures vary over an order of magnitude. Over the course of many growth steps, the fracture intensity (P32) of the networks increases by an order of magnitude, to reach ranges comparable to the densities observed in deep crystalline rocks. The flow properties and effective permeabilities of several dense GDFNs are examined at different growth steps and fracture intensities, assuming Darcy flow in the matrix and laminar flow in the fractures. The steeply inclined fractures (set two) provide important vertical connectivity for flow in the network. At later growth stages, the dense network becomes sufficiently interconnected, and percolates. The permeability tensor of a GDFN is shown to be a function of both the stress state during network formation, and present stress state.

Procter and Gamble Student poster award:

I don't want to compete References:

Acceptance of Terms and Conditions

Click here to agree

\section{Modeling dynamic particle filtration processes using coupled pore- to-continuum multiscale modeling}

John Blears ${ }^{1}$; Karsten Thompson ${ }^{\text {None }}$ 
${ }^{1}$ Louisiana State University

Corresponding Author(s): karsten@lsu.edu

Mechanical filtration of particles by a porous media is important in applications such as emissions control, pharmaceutical sterilization, and sand control during oil production. Modeling this process using first principles is difficult because of the strong interplay between particle- and pore-scale phenomena and behavior at the much larger length scales relevant to many of these problems. For example, retention efficiency generally decreases over time, which in turn alters the distribution of particle sizes removed from the flow. These factors, along with corresponding changes in permeability and dispersion behavior, impact the macroscopic distribution of flow and particle sizes. Despite the range of scales involved, all of these effects tie back to interactions between specific particle sizes and pore geometries at the microscopic scale.

We have studied the use of a concurrently coupled pore-to-continuum computational model for this problem. Particle retention, particle dispersion, permeability and porosity changes are modeled from first principles using an image-based physically representative network model. Macroscopic behavior is modeled using a traditional numerical implementation of the advection-dispersion-reaction (ADR) equation. Concurrent coupling occurs between these two models by embedding an independent network model within each grid block of the numerical ADR model and allowing the network to advance in time alongside the ADR. The ADR model passes boundary conditions to the embedded network models (such as the incoming particle concentration and size distribution); the network models pass back the aforementioned continuum parameters. In this way, each embedded network model represents the current state of its host grid block (describing a pore structure that has been altered by whatever history, sequence, and size distribution of particles may have been filtered up until that time at that particular location). Additionally, because the pore-scale models operate on first principles, they are able to accept whatever sequence of particles may still come from their upstream neighbors - something almost impossible to envision with an empirical approach.

In this work, we present network modeling algorithms and the concurrent coupling scheme that allows efficient communication between the pore and continuum scales.

A 1:1 concurrently coupled model (i.e., identical spatial dimensions as well as timesteps for the pore-scale domain and the continuum grid block) was used for validation and testing of parameter communication. The methodology was then applied to situations where the pore-scale modeling occurs on much smaller and shorter scales than in the continuum model, and the challenges and errors introduced by the process are discussed. Finally, the potential of this multiscale modeling process is demonstrated by applying it to situations in which a traditional empirical approach is not viable.

Procter and Gamble Student poster award:

References:

Acceptance of Terms and Conditions:

Click here to agree

\section{Advances in conformal mixed finite element methods for gener- alized poroelasticity}

Kent-Andre Mardal $^{1}$; Marie E. Rognes ${ }^{2}$; Travis Thompson ${ }^{2}$

\footnotetext{
${ }^{1}$ Simula Research Laboratory, University of Oslo

${ }^{2}$ Simula Research Laboratory
}

Corresponding Author(s): tthompson@simula.no

The equations of generalized poroelasticity, or MPET, describe the flow through multiple fluid networks in a porous media. In the quasi-static case, these equations read as: for a set of $N$ fluid 
networks, find the displacement $u$ and the network pressures $p_{n}$ for $n=1, \ldots, N$ such that begin equation\}

।begin $\{$ array $\}\{1 \mathrm{lr}\}$

- $\backslash \operatorname{text}\{\operatorname{div}\} \backslash$ left $(2 \backslash \mathrm{mu} \backslash$ varepsilon$(\mathrm{u})+\backslash \operatorname{lambda} \backslash \operatorname{text}\{\operatorname{div}\} \mathrm{u} \backslash$ right $)+\backslash$ sum_ $\{\mathrm{n}\} \backslash$ alpha_ $\{\mathrm{n}\} \backslash$ nabla $\mathrm{p} \_\{\mathrm{n}\}$ $=\mathrm{f} \&(1) \backslash$

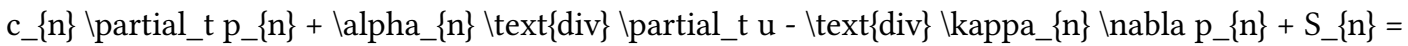
g_\{n\} \& (2)

lend\{array\}

lend\{equation\}

for $n=1, \ldots, N$. The characterizing parameters for each fluid network are the Biot-Willis coefficient $\alpha_{n}$, the storage coefficient $c_{n} \geq 0$, and the hydraulic conductivity tensor $\kappa_{n}$, while $S_{n}$ represent transfer terms in/out of network $n$. When $\mathrm{N}=1$, the system (1)-(2) reduces to the classical Biot equations.

The MPET equations, long utilized in geomechanics, are seeing increased use in biomechanics. Indeed, the multiple network poroelasticity theory aptly models the multiple fluid networks encountered in e.g. the brain: such as extracellular spaces, vasculature and paravasculature 1. In biomechanics application the storage coefficients, permeability tensors and Lam $\{\backslash$ 'e $\}$ coefficient $\lambda$ can pose challenges for numerical discretizations. In the brain, for instance, storage coefficients and permeability tensors may typically be very small and with possible jumps. Solving (1)-(2) using standard finite element discretizations can present numerical issues such as the loss of discrete stability. This fact has spurred recent interest in developing numerical methods, and preconditioners 2, which are robust in the intrinsic limits of elastic, and poroelastic models; including $\lambda \rightarrow \infty, \kappa_{n} \rightarrow 0$, and $c_{n} \rightarrow 0$.

In this talk we overview recent advances in conformal mixed methods for the MPET equations. Topics to be overviewed include a-posteriori error estimates for (1)-(2), uniform stability for the Poisson-type subproblem arising from introducing network fluxes of the form:

$\backslash$ begin\{equation\} \begin\{array\}\{lr\} }

$\mathrm{z} \_\mathrm{n}=$ - \kappa_n $\backslash$ nabla p_n, \& (3)

lend\{array\}

lend\{equation\}

and the role of this result in the context in the discrete stability of (1)-(3) for $0<\kappa \ll 1$.

\section{Procter and Gamble Student poster award:}

\section{References:}

1 Tully, B. and Ventikos, Y. Cerebral water transport using multiple-network poroelastic theory: application to normal pressure hydrocelphalus. J. Fluid Mech, 2011: 667. pp. 543-561

2 Hong, Q. and Kraus, J. and Lymbery, M. and Philo, F. Conservative discretizations and parameterrobust preconditioners for Biot and multiple-network flux-based poroelastic models. arXiv:1706.00724, 2018. Acceptance of Terms and Conditions:

Click here to agree

\section{Plug formation of a ferromagnetic and glass beads mix}

Louison Thorens ${ }^{1}$; Knut Jørgen Måløy ${ }^{2}$; Mickaël Bourgoin ${ }^{3}$; Stéphane Santucci ${ }^{3}$

\footnotetext{
${ }^{1}$ PoreLab - University of Oslo

${ }^{2}$ University of Oslo

${ }^{3}$ ENS de Lyon
}

Corresponding Author(s): louison.thorens@ens-lyon.fr

We study experimentally the flow of a granular mixture, composed of glass beads and ferromagnetic particles immersed in a glycerol solution, slowly dragged inside a narrow tube. The invading airliquid interface loads a portion of granular material that clogs the tube due to friction with the 
confining walls. When the pressure is too high, the packing is destabilized and creates a "plug" of granular material. The process repeats, such that the advancing interface leaves a trail of plugs along the tube.

We investigate the formation of the first plug necessarily forced by the use of an external magnetic field, leading to the start-up of the process all along the tube. Finally, we show the impact of the different concentrations of particles on the different lengths visible in the obtained patterns.

Procter and Gamble Student poster award:

I would like to compete in the Procter and Gamble Student award References:

Acceptance of Terms and Conditions:

Click here to agree

403

\title{
Numerical estimation of compacted illite multiphase transport properties from pore-scale simulations using FIB-SEM images
}

\author{
Aliaksei Pazdniakou ${ }^{1}$; Anne-Julie Tinet $^{2}$; Magdalena Dymitrowska ${ }^{1}$; Fabrice GOLFIER ${ }^{3}$; Jerome Vicente ${ }^{4}$; Marc \\ Prat $^{\text {None }}$ \\ ${ }^{1}$ IRSN, PRP-DGE/SRTG/LETIS \\ ${ }^{2}$ GeoRessources - Université de Lorraine \\ ${ }^{3}$ Université de Lorraine - GeoRessources Laboratory \\ ${ }^{4}$ IUSTI - Aix-Marseille University
}

Corresponding Author(s): anne-julie.tinet@univ-lorraine.fr

\begin{abstract}
The low permeability of sedimentary clays as well as their chemical characteristics make them particularly suitable to serve as a natural barrier for radioactive waste geological storage (Andra, 2005). Due to the low permeability, the gas, mainly the hydrogen, originating from the bacterial corrosion of the metallic parts of the facility can be accumulated through time causing significant pressure rise at the interface between sedimentary clay and the facility components. If the pressure is high enough, the gas can enter the water saturated pores by triggering various transport mechanisms (Cuss et al. 2014). For low gas pressures, the gas transport is dominated by advection-dispersion (Bardelli et al. 2014), while for higher pressures the gas is able to penetrate the water saturated pores leading to the continuous visco-capillary flow. If the pressure is too high, gas may cause a modification of the pore space geometry (Cuss et al. 2014). In this work, we will focus on the intermediate multiphase flow without structure evolution. Developments in digital rock physics allow us to study this phenomenon numerically. In this study the multiphase transport properties (relative permeabilities and retention curve) were numerically calculated using pore-scale simulation method inside a 3D sample reconstructed from the Focused Ion Beam - Scanning Electron Microscopy (FIBSEM) images of a compacted illite. For this purpose, the Lattice Boltzmann Model (LBM) (Pazdniakou et al. 2018), the Smooth Particle Hydrodynamics (SPH) (Pazdniakou and Dymitrowska 2018) and a morphological model based on Pore Network Model (PNM) were applied. The results obtained by these numerical methods are compared and analysed. We demonstrate that the LBM and SPH provide similar predictions of the relative permeabilities and capillary pressures for carefully chosen parametrization which results in similar dimensionless numbers. The curves obtained by PNM are also rather similar to those obtained through direct modeling. We also demonstrate the importance of decent geometrical discretization, especially needed to calculate precisely the capillary pressure for low water saturations.
\end{abstract}

Procter and Gamble Student poster award:

\section{References:}

ANDRA Dossier 2005 Argile. Synthesis. Evaluation of the feasibility of a geological repository in an argillaceous formation. ANDRA Report Series, 782 p., ISBM 2-916162-00-3, (2005).

R. Cuss, J. Harrington, R. Giot, C. Auvray. Experimental observations of mechanical dilatation at the onset of gas flow in Callovo-Oxfordian claystone. Clays in Natural and Engineered Barriers for Radioactive Waste Confinement, 400, 507-519, (2014). 
F. Bardelli, C. Mondelli, M. Didier, J.G. Vitillo, D.R. Cavicchia, J.C. Robinet, L. Leone, L. Charlet. Hydrogen uptake and diffusion in Callovo-Oxfordian clay rock nuclear waste disposal technology. Applied Geochemistry, 49, 168-177, (2014).

A. Pazdniakou, A-J. Tinet, F. Golfier, K. Kalo, S. Gaboreau, P. Gaire. Numerical efficiency assessment of the Lattice Boltzmann model for digital nano-porous rock applications. Advances in Water Resources, 121, 44-56, (2018).

A. Pazdniakou, M. Dymitrowska. Migration of gas in water-saturated clays by coupled hydraulicmechanical model. Geofluids, 2018, ID 6873298, 25 p., (2018). Acceptance of Terms and Conditions:

Click here to agree

\title{
Insights into the physical interactions of water based ink with porous paper
}

\author{
Nicolae TOMOZEIU ${ }^{1}$
}

${ }^{1}$ Océ-Technologies / A Canon Group Company, AM department

Corresponding Author(s): nicolae.tomozeiu@oce.com

Nowadays the world of printing is rapidly evolving, especially due to the inkjet technologies, which offer the flexibility of digital printing at a breakthrough cost price, productivity, print quality and versatility. Water based inks add to all these characteristics a very important feature: environmental care. The R\&D department of Océ Technologies, a Canon company, is a major player in the development of inkjet technologies for many different applications.

The main purpose of the research described in this work is to gain a deeper un-derstanding of the physical processes that characterize the ink - porous paper interaction. Understanding processes as ink spreading, evaporation and imbibition into porous substrate is vital to have prints of high quality. Using both experimental and computational modeling, the overall objective is to determine the printing parameters that control quality of the print considering the ink physical properties and the paper characteristics. The paper is characterized in terms of porosity, surface roughness and surface energy. Droplets and thin layers of complex liquids (mixtures of aqueous liquids with polymericcolloidal particles) deposited on coated paper have been investigated during drying. Experimental methods as optical spectroscopy, light microscopy, Scanning Electron Microscopy (SEM), Automatic Scanning Absorptometer (ASA) and Electrical Impedance Spectroscopy (EIS) are employed for this study. For each method of investigation the main models, including their strengths and limitations, are presented.

It is well known that fast absorption of the ink carrier liquid into the paper and fast fixation of the ink pigment on the surface are crucial in high speed and high quality inkjet printing. This study reveals which parameters affect the absorption process to be able to control the mechanisms and to optimize the print quality.

The results can be considered as a base for future studies of ink absorption into porous coated paper, as well as of aggregation of colloidal particles on surfaces.

Keywords: inkjet printing, porous paper, droplet spreading, evaporation, ab-sorption in porous paper

Procter and Gamble Student poster award:

References:

Acceptance of Terms and Conditions:

Click here to agree 


\title{
Microemulsion-Based Conformance Treatment: Fluid Optimiza- tion and Microfluidics Implementation
}

\author{
Victor Torrealba ${ }^{1}$; Hussein Hoteit ${ }^{2}$ \\ ${ }^{1}$ King Abdullah University of Science and Technology \\ ${ }^{2}$ King Abdullah University of Science \& Tech (KAUST) \\ Corresponding Author(s): victor.torrealba@kaust.edu.sa
}

Waterflooding from reservoirs with high permeability layers or thief zones is challenging owing to preferential water channeling that leads to poor sweep efficiency and high water cut. Recently, a novel microemulsion-based conformance technique was proposed showing promising results from numerical simulations. In this paper, we show experimentally for the first time the effectiveness of the proposed treatment using a microfluidics approach.

We use hard lithography nanofabrication workflow to make a micromodel on a silicon wafer. The micromodel consists of a low permeability model with a high permeability thief layer running through the middle. We use brines ranging from 10,000-100,000ppm of $\mathrm{NaCl}$, pure decane oil, and different ratios of sodium dodecylbenzene sulfonate surfactant and isobutanol. Phase behavior scans are conducted for four surfactant-alcohol ratios, while maintaining a constant mass of amphiphilic components.

The hard lithography workflow we present allows for the fabrication of high-fidelity micromodels for conformance treatment studies. Scanning electron microscopy characterization shows that the thickness of the fabricated micromodel is greater than $80 \mu \mathrm{m}$, with pore throat sizes of 7 and $14 \mu \mathrm{m}$ for the low and high permeability regions, respectively. For the fluid characterization, we observe that the resulting microemulsions are more viscous for the lower alcohol content case and as the micellar morphologies approach cylindrical structures. The microfluidics experiment showed that injection of small volumes of the target formulation was successful in generating a high viscosity microemulsion in the high permeability channel away from the injection site, which helped both in diverting flow to the previously unswept regions and keeping injection pressures low.

In this paper, we show experimentally for the first time the impact of a newly proposed microemulsionbased conformance treatment using a microfluidics approach. Our results indicate that the injection of small volumes of chemicals is sufficient to generate a high viscosity microemulsion in the thief layer, which diverts flow to the unswept areas to improve oil recovery.

Procter and Gamble Student poster award:

I would like to compete in the Procter and Gamble Student award References:

Acceptance of Terms and Conditions:

Click here to agree

960

\section{Direct Visualization of the Inaccessible Pore Volume Effect of Polymer Chains in a Dual Porosity Medium Using a Microfluidic Technique}

\author{
Victor Torrealba ${ }^{1}$; Hussein Hoteit ${ }^{1}$ \\ ${ }^{1}$ King Abdullah University of Science and Technology \\ Corresponding Author(s): victor.torrealba@kaust.edu.sa
}

Polymer flooding improves oil recovery by decreasing the displacement mobility ratio. However, the interaction of polymer chains with porous media is not fully understood. One example is inaccessible pore volume (IPV), causing polymer components to accelerate compared to a tracer component (e.g., 
salt). This effect can be harnessed to improve the effectiveness of polymer flooding. In this paper we shed light into the relationship between polymer chain size and inaccessible pore using a microfluidic technique.

We employ a hard lithography approach to fabricate a micromodel on a silicon wafer. The micromodel consists of a representative elementary volume that has large and small square features describing both the high and low porosity media. The corresponding pore throat sizes for both high and low porosity media are 10 and $1 \mu \mathrm{m}$, respectively. The size distribution of polymer chains is characterized using dynamic light scattering. The inaccessible pore volume effect is directly visualized using a particle velocimetry technique.

The presented hard lithography workflow allows for the fabrication of high-fidelity micromodels for enhanced oil recovery studies. Scanning electron microscopy characterization shows that the thickness of the fabricated micromodel is greater than $20 \mu \mathrm{m}$. The particle size distribution study shows the dependence of polymer aggregate size with both salinity and concentration for two HPAM polymer of different molecular weights. As expected from scaling arguments, the increase in aggregate size coincides with higher polymer concentration, lower salinity, and higher molecular weight. From the microfluidics experiments, we observed a pronounced inaccessible pore volume effect for solutions with aggregate size comparable to the pore throat size of the low porosity medium.

The insights from this research allow for an improved understanding of polymer-rock interactions, with emphasis in the inaccessible pore volume effect. We highlight how polymer selection can be harnessed to improve the effectiveness of a recently proposed slug-based polymer flooding method.

Procter and Gamble Student poster award:

I would like to compete in the Procter and Gamble Student award References:

Acceptance of Terms and Conditions:

Click here to agree

\title{
Experimental investigation of the impacts of microtopographic and vegetative roughness elements on near-surface airflow and soil moisture dynamics using a coupled wind tunnel-porous me- dia test-system
}

\author{
Author(s): Andrew Trautz ${ }^{1}$

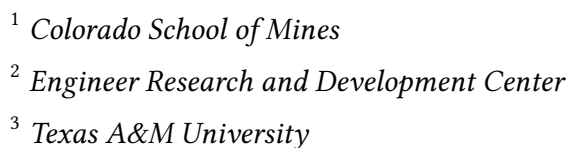

Co-author(s): Tissa Illangasekare ; Stacy Howington ${ }^{2}$; Matthew Reimann ${ }^{1}$; Ignacio Rodriguez-Iturbe ${ }^{3}$

Corresponding Author(s): atrautz@mines.edu

Vegetation, topography, or other natural/anthropogenic structures act as roughness elements which alter near-surface airflow patterns and velocity profiles by extracting kinetic energy from the flow. As air flows around, over, or through an obstructing roughness element, regions of accelerated flow develop above and at its sides. A sheltering "quiet" zone, or a wake zone of asymptotic recovery, simultaneously develops downstream of the roughness element that has been consistently shown to extend to a distance approximately equal to 7-10 times the height of the roughness element. Within this wake zone, the velocity decreases significantly, and the turbulence intensity increases compared to the undisturbed upstream profile - a recirculating eddy may even form. Understanding such flow phenomena is important to many near-surface problems involving coupled heat, was, and momentum exchange processes including: sedimentation/erosion, land-atmospheric interactions (evapotranspiration), ecohydrology, and agricultural water management. The impact of localized changes to the fluid dynamics and flow profile has been extensively studied in the context of aeolian transport 
in both the field and wind tunnel setting. Very little work in contrast has focused on the effects of the feedbacks associated with flow variability on local evaporation dynamics affecting water availability at the scale of a single or limited number of roughness elements. This study explores how microtopographic and vegetative elements individually, and collectively, impact local aboveground airflow and the associated near-surface soil moisture dynamics (i.e., availability and spatiotemporal distribution). Data generated from a series of published and unpublished experiments conducted over the last two years at the Center for Experimental Study of Subsurface Environmental Processes low wind speed micrometeorological wind tunnel-porous media test-facility are used in this investigation. This facility allowed high spatiotemporal resolution datasets to be generated in both the atmosphere and subsurface at a 7-meter length scale (of which a 3-meter subset was used) under carefully prescribed and controlled conditions. Key flow variables (i.e., velocity, turbulence intensity, shear stress) measured in the atmosphere with laser Doppler velocimetry and autonomously monitored soil moisture distributions are analyzed herein upstream and downstream of the roughness elements. Results demonstrate that the roughness elements create different flow regions and patterns that affect the local soil moisture dynamics to varying degree. Comparison of the results further shows that the shape and size of the roughness elements are important factors affecting the atmospheric air flow field and the subsequent distribution and availability of near-surface soil moisture. It is the belief of the authors that collection of experimental datasets can help lead to the development of better conceptual and numerical coupled heat, mass, and momentum porous media-free fluid models and improve the observation strategies and predictive tools currently used by researchers involved in the study of soil-plant-atmosphere continuum processes and agricultural water management.

Procter and Gamble Student poster award:

I don't want to compete References:

Acceptance of Terms and Conditions:

Click here to agree

\section{Downscaling Strategies for Multiscale Mixed Methods}

Rafael Trevisanuto Guiraldello ${ }^{1}$; Roberto Ausas $^{2}$; Fabricio Sousa ${ }^{3}$; Felipe Pereira ${ }^{4}$; Gustavo Buscaglia ${ }^{5}$

${ }^{1}$ University of São Paulo - São Carlos

${ }^{2}$ Universiade de São Paulo

${ }^{3}$ University of Sao Paulo

${ }^{4}$ Mathematical Sciences Department, The University of Texas at Dallas, Richardson, TX, USA

${ }^{5}$ Universidade de São Paulo

Corresponding Author(s): trevi@usp.br

Multiscale domain decomposition methods for the simulation of multiphase flows in porous media, aiming at high performance and scalability, has been the focus of intensive research motivated by the practical needs of the energy and environmental sectors. The main idea is to decompose the computational domain into non-overlapping subdomains in which local small problems are solved. In order to recover a global solution, continuity of flux and pressure need to be fulfilled at the interface between subdomains. These compatibility conditions are then relaxed and enforced through low-dimensional spaces, reducing the number of unknowns to be solved, turning it to a relatively inexpensive task. Examples of such type of methods are the MMMFEM 1 (Multiscale Mixed Mortar Finite Element Method) and the MRCM (Multiscale Robin Coupled Method) 2. The drawback associated with such procedures is a discrepancy in continuity (of pressure or normal fluxes, or both) in fine scales. Downscaling procedures are then needed to recover local conservation of the velocity field at the fine grid level between subdomains interfaces.

In this presentation, we propose two new downscaling procedures based on a minimum overlapping for the velocity field. We investigate the applicability of such methods when tested on singlephase flow problems using the MRCM, and compare them in terms of accuracy to a standard technique. 
Procter and Gamble Student poster award:

I don't want to compete References:

1 T. Arbogast, G. Pencheva, M.F. Wheeler, I. Yotov, A multiscale mortar mixed finite element method, SIAM Multiscale Model. Simul. 6 (1) (2007) 319-346.

2 R.T. Guiraldello, R.F. Ausas, F.S. Sousa, F. Pereira, G.C. Buscaglia, The Multiscale Robin Coupled Method for flows in porous media, In J. Comput. Phys. 355 (2018) 1-21. Acceptance of Terms and Conditions:

Click here to agree

\title{
Modelling of bone-cement-injection processes in cancellous ver- tebral bones
}

\author{
Author(s): Zubin Trivedi ${ }^{1}$ \\ Co-author(s): Christian Bleiler ${ }^{1}$; Arndt Wagner ${ }^{1}$; Oliver Röhrle ${ }^{1}$ \\ ${ }^{1}$ University of Stuttgart
}

Corresponding Author(s): zubin.trivedi@mechbau.uni-stuttgart.de

Percutaneous vertebroplasty is a commonly used practice for restoring the structural strength of vertebral bones affected by osteoporosis via the injection of polymethylmethacrylate (PMMA) into the cancellous structure of the bone. However, in some cases, the bone cement can leak outside the bone leading to complications like pulmonary embolism. A model that can simulate the cement flow inside the cancellous structure of the bone is beneficial to predict the cement filling pattern and prevent such cases. A three-dimensional multiphasic model of a vertebral bone using the Theory of Porous Media has been developed that can simulate the injection of the bone cement. However, there is always the question of underlying permeability values and material properties to be used in the model. In particular, the properties of the bone and its permeability can vary for individual patients.

Towards the solution of this problem, a parametric investigation is investigated to characterise the permeability with respect to basic geometric parameters. This study is carried out using a porous medium with a regular microstructure since its permeability is easy to measure and the geometric parameters can be analysed. Firstly, a simple method is established to determine the permeability of a regular microstructure using two models in tandem: a pore-scale model and a REV-scale model, giving the resultant permeability of the microstructure. Subsequently, a parametric investigation is carried out, using closed-packed structures of uniform-sized spheres, wherein the packing structures and the radii of spheres were varied to tune the porosity and the specific surface area respectively in a controlled manner. The permeability was computed and plotted against a range of values of the parameters for analysis. The tortuosity values of the packing structures were computed using the Kozeny-Carman equation. The values were found to be constant for the most part, implying agreement of the permeability computation with the Kozeny-Carman equation. The values were also found to be close to the measured values of tortuosity found in the literature for packed beds of random spheres.

\section{Procter and Gamble Student poster award:}

I would like to compete in the Procter and Gamble Student award References:

\section{Acceptance of Terms and Conditions:}

Click here to agree 


\title{
Numerical investigation of fracture propagation in porous medium under shear stimulation of fluid flow
}

\author{
Hau Trung Dang ${ }^{1}$; Inga Berre ${ }^{1}$; Eirik Keilegavlen ${ }^{1}$ \\ ${ }^{1}$ University of Bergen
}

Corresponding Author(s): hau.dang@uib.no

\begin{abstract}
We are interested in low-pressure stimulation of naturally fractured porous media, as applied e.g. in enhanced geothermal systems. Naturally occurring fractures are considered joint surfaces that can withstand tectonic stresses due to friction by asperities in the fracture walls. Hydraulic stimulation by the injection of fluids in the fracture network can overcome the frictional resistance, leading to shear deformation along the fracture surfaces. The shearing is associated with a dilation of the fracture aperture and increased permeability. Here we study how the shear deformation will alter the local stress fields, and can potentially trigger fracture propagation. The porous medium and fluid flow are modeled by the discrete fracture-matrices model that the fractures are treated as interfaces and allows the fluid transport from high-permeable conductive fractures to the rock matrix and vice versa. We treat the rock matrix as a linearly elastic medium. The governing equations are discretized by a combination of finite element and finite volume methods. We discuss the numerical modelling of fracture propagation in this setting, with an emphasis on representation of the new fracture in the computational grid by a novel adaptive re-meshing technique. Numerical examples shows the interaction between fluid pressure and mechanical forces.
\end{abstract}

Procter and Gamble Student poster award:

References:

Acceptance of Terms and Conditions:

Click here to agree

\section{Green synthesis of nZVI suspensions from plant extracts for in situ remediation of polluted groundwater}

Christos Tsakiroglou ${ }^{1}$; Mihalis Karavasilis ${ }^{1}$

${ }^{1}$ Foundation for Reserach and Technology Hellas - Institute of Chemical Engineering Sciences

Corresponding Author(s): ctsakir@iceht.forth.gr, mkaravas@iceht.forth.gr

Fruits, vegetables, herbs and grains (e.g. tea, grapes, sorghum bran) contain polyphenols at high concentration, and the plant extracts may act both as reducing agents of iron salt and as capping agent for iron nanoparticles. Beyond the high solubility, low toxicity and biodegradability of polyphenols, during the nano-iron green synthesis, neither hazardous materials are used for the reductant or capping agent, nor any hazardous wastes are generated. Compared to other nZVI manufacturing processes, the resulting suspension is not undergone oxidative corrosion during its storage, shipping or use. This new synthetic method is an extremely simple green approach that generates bulk quantities of relatively stable nanocrystals of iron (Fe) using plant extract at room temperature [1-3]. In the present work, plant extracts were produced from Camelia synesis (Green Tea) and Punica Granatum (Pomegranate), and the total concentration of polyphenols was measured in terms of equivalent concentration of Gallic acid by using the Folin-Ciocalteu method. nZVI suspensions were synthesized in semi-batch reactor by injecting, at constant influx rate of $4 \mathrm{~mL} / \mathrm{min}$, a pre-specified volume of plant extract in an aqueous solution of FeSO4 7H20. To monitor the kinetics of nZVI synthesis reaction, the $\mathrm{pH}$ and redox potential (Eh) of the solution were recorded as functions of time with the aid of a data acquisition system. The transient responses of $\mathrm{pH}$ and Eh could be regarded as "signatures" of the overall nZVI synthesis process and used in a "pattern recognition" procedure to forecast the properties of synthesized nano-materials. The nanoparticles were char-

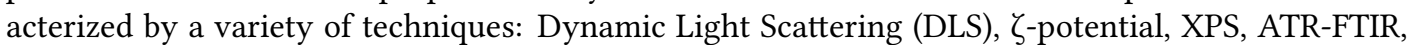


TEM. The reactivity of nZVIs assessed through their ability to reduce hexavalent chromium Cr (VI). In aqueous solution of $\mathrm{Cr}(\mathrm{VI})$ prepared by using $\mathrm{K} \neg 2 \mathrm{Cr} 2 \mathrm{O} 7$ salt and $\mathrm{Cr}$ (VI) reduction tests were performed in batch reactor through their mixing with the synthesized nZVI suspension nano-iron effused in it. The concentration of dissolved hexavalent chromium was determined by using 7196A method.

\section{ACKNOWLEDGMENTS}

We acknowledge support of this work by the project "Innovative Actions in Environmental Research and Development (PErAn)" (MIS 5002358) which is implemented under the "Action for the Strategic Development on the Research and Technological Sector", funded by the Operational Program "Competitiveness, Entrepreneurship and Innovation" (NSRF 2014-2020) and co-financed by Greece and the European Union (European Regional Development Fund).

\section{Procter and Gamble Student poster award:}

I would like to compete in the Procter and Gamble Student award References:

1. Hoag, G.E., J. B. Collins, J.L. Holcomb, J.R. Hoag, M.N. Nadagouda, and R.S. Varma, Degradation of bromothymol blue by 'greener' nano-scale zero-valent iron synthesized using tea polyphenols. J. Mater. Chem. (2009) 19 (45), 8671-8677.

2. Machado, S., S.L. Pinto, J.P. Grosso, H.P.A. Nouws, J.T. Albergaria, C. Delerue-Matos, Green production of zero-valent iron nanoparticles using tree leaf extracts, Sci. Total Environ. (2013) 445$446,1-8$.

3. Lin, J., X. Weng, R. Dharmarajan, and Z. Chen, Characterization and reactivity of iron based nanoparticles synthesized by tea extracts under various atmospheres, Chemosphere (2017) 169, 413-417.

Acceptance of Terms and Conditions:

Click here to agree

896

\section{Two-dimensional flows in fluid coupled with porous medium}

Kirill Tsiberkin $^{1}$; Irina Tyulkina ${ }^{1}$

${ }^{1}$ Perm State University

Corresponding Author(s): kbtsiberkin@psu.ru

We consider the stationary 2D flows in a channel with rigid boundaries is partially filled with a porous medium. The pure fluid is bounded by solid or free rigid boundary, while the channel bottom is solid. The method uses Berman transformation to build the 2D solution. Free flow is described by the Navier-Stokes equations, the filtration flow is described by Darcy-Brinkman equations, and the interface boundary conditions by Ochoa-Tapia and Whitaker model. Numerical modeling shows the transversal flow into the porous medium exists in the wide parameter range. The transversal velocity magnitude is of order $1 /$ Re. The dependence of velocity on porous medium permeability and thickness of the porous medium is examined. Stability of 2D flow is analysed numerically; the flow is more stable than pure plane-parallel. The transversal flow becomes significant at low Reynolds numbers; it also should be taken into account in long channels.

The study is financially supported by the Russian Science Foundation (project 18-71-00057).

Procter and Gamble Student poster award:

I don't want to compete References:

Acceptance of Terms and Conditions:

Click here to agree 


\section{Boundary layers at "fluid - porous medium" interface}

Kirill Tsiberkin $^{1}$; Irina Tyulkina ${ }^{2}$

${ }^{1}$ Perm State University

${ }^{2}$ Perm State Universty

Corresponding Author(s): kbtsiberkin@psu.ru

We present an analysis of 2D convective flows near the interface between free fluid and porous medium. There are series of problems considered:

- forced flow over the porous medium;

- free convection near heated boundary;

- free convective plume over point heat source at the boundary.

All the problems permit self-similar transform when the permeability changes along boundary by the power dependence. The profiles of flow velocity and temperature (in convective problems) are investigated under the different system parameters. It is found the non-linear convective terms are the important for boundary-layer formation.

The study is financially supported by the Russian Science Foundation (project 18-71-00057).

Procter and Gamble Student poster award:

I don't want to compete References:

Acceptance of Terms and Conditions:

Click here to agree

33

\section{Bone Dry: Hydroxyapatite as a Water-Resistant Cement Coating Material}

Author(s): Ronald Turner ${ }^{1}$

Co-author(s): Joanna Renshaw ${ }^{1}$; Alan Richardson ${ }^{2}$; Andrea Hamilton ${ }^{1}$

${ }^{1}$ University of Strathclyde

${ }^{2}$ University of Northumbria

Corresponding Author(s): ronald.turner@strath.ac.uk

Cements and concretes are porous materials used in many varied applications, ranging from the built environment to within the human body. The permeability of cements and concretes to aqueous solutions is of significant relevance to many of these practical applications. For example, reduced permeability will reduce ingress salt water in coastal or maritime infrastructure; minimising chloride attack and reducing the degradation of the concrete.(1,2) Consequently, the generation of hydrophobic surface coatings for concretes has been of significant recent research interest.(2-5)

Biomineralisation provides a method to coat cements with various materials, such as calcite [CaCO3] or hydroxyapatite [Ca5(PO4)3(OH)2]. In this work we demonstrate that biological deposition of hydroxyapatite directly onto a cement substrate generates a hydrophobic mineral layer which completely coats the surface, minimising water ingress into the cement substrate. We also describe the biochemical reactions which underpin this deposition process, which involves synergistic interactions between the cement, growth medium, and bacterial culture. We establish that the roughness of this coating can be altered by varying between biological and non-biological synthesis methods. 
Procter and Gamble Student poster award:

\title{
References:
}

\section{References}

1. Ahmad, S. Reinforcement corrosion in concrete structures, its monitoring and service life prediction - A review. Cem. Concr. Compos. 25, 459-471 (2003).

2. Barnat-Hunek, D. \& Smarzewski, P. Influence of hydrophobisation on surface free energy of hybrid fiber reinforced ultra-high performance concrete. Constr. Build. Mater. 102, 367-377 (2016).

3. Wong, H. S., Barakat, R., Alhilali, A., Saleh, M. \& Cheeseman, C. R. Hydrophobic concrete using waste paper sludge ash. Cem. Concr. Res. 70, 9-20 (2015).

4. Muzenski, S., Flores-Vivian, I. \& Sobolev, K. Durability of superhydrophobic engineered cementitious composites. Constr. Build. Mater. 81, 291-297 (2015).

5. Grumbein, S. et al. Hydrophobic Properties of Biofilm-Enriched Hybrid Mortar. Adv. Mater. 81388143 (2016). doi:10.1002/adma.201602123 Acceptance of Terms and Conditions:

Click here to agree

\section{Numerical characterization of intrinsic permeability based on $2 \mathrm{D}$ and 3D data}

\author{
Samaneh Vahid Dastjerdi ${ }^{1}$; Maria Osorno ${ }^{1}$; Nikolaos Karadimitriou ${ }^{1}$; Holger Steeb $^{1}$ \\ ${ }^{1}$ Institute of Mechanics (CE), Stuttgart University, Pfaffenwaldring 7, 70569, Stuttgart, Germany
}

Corresponding Author(s): samaneh.vahiddastjerdi@mechbau.uni-stuttgart.de

It has been a matter of debate in the literature, whether two-dimensional (2D) approaches, either experimental or numerical, can sufficiently describe three-dimensional (3D) phenomena in porous media, such as flow and transport, precipitation, biofilm growth and its effect on the intrinsic properties of the porous medium, etc. 1 .

When it comes to physical experiments where imaging is of essence, there are some limitations in the quantitative characterization of the third dimension. In the case of optical microscopy, there is practically no information on the depth of the domain under observation. However, techniques like fluorescent and confocal microscopy, as well as X-Ray Computed micro-Tomography

$(\mu \mathrm{XRCT})$, can effectively give physical and morphological information on the (otherwise) invisible third dimension. Still, even in these techniques, there are some physical limitations regarding the field of view, refractive index matching, the acquisition time in comparison to the time scale of the effect under observation, etc. These issues make the use of such techniques not always a practical choice.

In order to investigate the effect of the visualization method on the estimation of three- dimensional concepts, such as intrinsic permeability and flow patterns, the limitations of each imaging technique need to be addressed and accounted for. Under this scope, a (2D) micro-model made of glass (using the wet-etching technique) replicating the flow network of a natural porous medium, with dimensions of $20 \times 10 \times 0.02 \mathrm{~mm} 3$, was used in micro-fluidic investigations.

First, the intrinsic permeability of the flow network was experimentally measured. Then, the pore morphology of the micro-model was visualized with an optical microscope (2D), and with $\mu$ XRCT (3D). Given the limitations of each approach, such as the small field of view in $\mu$ XRCT or the lack of information in depth in optical microscopy, combinations of imaging methods and domain dimensions derived from the same microfluidic flow cell were taken into account.

Based on the morphological data acquired with both techniques, creeping flow (Stokes) simulations have been performed in $2 \mathrm{D}$ and 3D. The output of both types of simulation is e.g. the effective (coarse-grained) intrinsic permeability. The 2D/3D numerical scheme is a Cartesian-grid-based MPIparallelised Finite Differences Stokes solver which allows for large-scale simulations and slip boundary conditions. 2

To conclude, the results from the numerical simulations were compared to the experimental data for intrinsic permeability, in order to develop a new efficient dimensionally-reduced numerical model which will potentially be able to incorporate the morphological and geometrical features acquired from both imaging approaches to a model which can effectively approximate the experimentally measured values. 


\title{
Procter and Gamble Student poster award:
}

I would like to compete in the Procter and Gamble Student award References:

1 N. K. Karadimitriou and S. M. Hassanizadeh. A review of micromodels and their use in two-phase flow studies. Vadose Zone Journal, 11(3):0, 2012. doi: 10.2136/vzj2011.0072.

2 Maria Osorno, David Uribe, Oscar E. Ruiz, and Holger Steeb. Finite difference calculations of permeability in large domains in a wide porosity range. Archive of Applied Mechanics, 85(8):1043-1054, jul 2015. doi: 10.1007/s00419-015-1025-4. Acceptance of Terms and Conditions:

Click here to agree

\section{Recent advances in the DeProF theoretical framework for two- phase flows in porous media - Where we stand and where we could go.}

\author{
Marios Valavanides ${ }^{1}$ \\ ${ }^{1}$ Universit of West Attika \\ Corresponding Author(s): marval@uniiwa.gr
}

The DeProF tentative theory for two-phase flow in porous media is built around a hybrid mechanistic/stochastic model 1 that is based on the concept of decomposition in prototype constituent flows, namely, connected pathways, ganglion dynamics and emulsion-type /drop traffic flows, each showing different levels of disconnectedness of the non-wetting phase. The model accounts all essential pore-scale phenomena on a mechanistic basis and up-scales these into corresponding network-wide cooperative effects using a stochastic approach. The momentum balance in the entire flow is regulated by the relative intensities of the NWP and WP flows (inducing viscous flow resistances within the bulk of the NWP and WP) and the degree of disconnection of the NWP (inducing capillary resistance across the N/W interfaces). Steady-state flow conditions settle-in when each dissipative term is counterbalanced against the other two. The balance point is regulated by the flow conditions. This is so because viscous and capillary resistances do not depend on the flow conditions in the same way. The DeProF model takes into account the contribution of all system properties correctly in appropriate reduced numbers; in particular, viscosity disparity vis-à-vis capillarity, wettability and pore network structure, leading to the derivation of an improved, system-reduced, capillary number. In that context, modeling redundancies associated to the system properties are reduced to a minimum. Being very efficient in correctly and consistently predicting, not only the relative permeabilities on the Darcy scale, but also, the interstitial structure of the flow, the DeProF model evolved in a research tool. With extensive simulations across orders of magnitudes in terms of flow conditions and over different fluid systems it was possible to reveal the underlying, inherent systematic structure of biphasic flows in porous media across different flow regimes 2. Just recently, it was possible to derive universal scaling functions for flow-dependent relative permeabilities 3 ; the corresponding closed form expressions can now be easily integrated into true-to-mechanism, FEM solvers showing improved performance in terms of specificity.

In parallel, energy efficiency analysis of the sought process revealed a universal flow characteristic, the existence of a unique locus of critical flow conditions whereby the energy efficiency of the process (NWP flow rate per kW spent) attains locally -in terms of flow conditions- maximum values. This characteristic property was remaining in latency until revealed in an extensive retrospective examination of relative permeability diagrams 4 . The existence of a unique locus of critical flow conditions per N/W/PM system has opened new perspectives: (a) in describing the process in terms of the actual independent variables (the two flow rate intensities); (b) in deriving universal energy efficiency and relative permeability maps; (c) in developing methodologies for the normative characterization of biphasic flows as to the predominance of capillary or viscosity effects, the taxonomic classification of relative permeability diagrams and the effective characterization of pore network structures, using just a few, carefully selected, characteristic numbers 5; and (d), in terms of practical applications, by providing a powerful tool in designing more energy efficient interventions in process design (e.g. in EOR displacements or filter applications).

To provide a concrete theoretical justification of the existence of critical flow conditions, the DeProF framework implements the maximum entropy production principle and accounts the total entropy as the sum of two terms: the thermal entropy production at the molecular level scale and the configurational entropy production at the scale of the ensemble of physically admissible interstitial flows 
[6]. The former is estimated directly by viscous and capillary hysteresis dissipation; the latter has been estimated by deriving an appropriate Boltzmann-Gibbs type expression accounting the process microstates.

The presentation will conclude with a list of open problems and research directions to explore.

\section{Procter and Gamble Student poster award:}

I don't want to compete References:

1. Valavanides, M.S., Payatakes, A.C. True-to-mechanism model of steady-state two-phase flow in porous media, using decomposition into prototype flows. Adv. Water Resour. 24(3-4), 385-407 (2001), https://doi.org/10.1016/S0309-1708(00)00063-4

2. Valavanides, M.S. Flow structure maps for two-phase flowinmodel pore networks. Predictions based on extensive, DeProF model simulations. Oil Gas Sci. Technol. Rev. IFP Energies nouvelles 73 (6), 1-36 (2018), https://doi.org/10.2516/ogst/2017033

3. Valavanides, M.S. Universal, true to mechanism, flow dependent relative permeability scaling functional form for steady-state 2-phase flows in p.m.”, Intern. Symposium of the Society of Core Analysts, Trondheim, Norway, 27-30 Aug. (2018), http://users.uniwa.gr/marval/publ/Valavanides_SCA2018_066.pd

4. Valavanides, M.S., Totaj, E., Tsokopoulos, M. Energy efficiency characteristics in steady-state relative permeability diagrams of two-phase flow in porous media. J. Petrol. Sci. Eng. 147, 181201 (2016). https://doi.org/10.1016/j.petrol.2016.04.039

5. Valavanides, M.S. Review of steady-state two-phase flow in porous media: independent variables, universal energy efficiency map, critical flow conditions, effective characterization of flow and pore network. Transp. in Porous Media 123 (1), 42-99 (2018), https://doi.org/10.1007/S11242-0181026-1

6. Valavanides, M.S., Daras, T. Definition and counting of configurational microstates in steadystate two-phase flows in pore networks. Entropy 18 (054), 1-28 (2016). https://doi.org/10.3390/e18020054

Acceptance of Terms and Conditions:

Click here to agree

809

\section{Energy efficiency optimization in n-spot recovery processes based on critical flow conditions and integration of flow-dependent rel- ative permeability scaling functions.}

Marios Valavanides ${ }^{1}$; Eugene Skouras ${ }^{2}$

\footnotetext{
${ }^{1}$ University of West Attica

${ }^{2}$ FORTH/ICE-HT
}

Corresponding Author(s): marval@uniwa.gr

We show the potential applications of the recently furnished, universal, energy efficiency map for two-phase flows in porous media and the associated locus of critical flow conditions 1, as an analysis and design tool for processes of immiscible two-phase flow in porous media. In particular, we have chosen a typical case in n-spot remediation campaigns as a paradigm, and selected the best flow set-up aiming to achieve maximum energy efficiency in terms of the flow rate of the non-wetting phase (NWP) per unit power spent in the pumps. In particular, we have considered the case of recovering the NWP from an initially uniformly polluted (saturated) formation implementing typical n-spot arrangements 2 .

At first, the best set-up for the central well, i.e. to operate as a source (injector) or as a sink (producer), is deduced. That result came straightforward from an energy efficiency analysis, considering the form of the two-dimensional locus of critical flow conditions over the process domain of the 
inherently independent variables of the process, namely the conventional capillary number of the $\mathrm{WP}, \mathrm{Ca}$, and the NWP/WP flow rate ratio, $\mathrm{r}$.

Then, by incorporating the universal, flow dependent relative permeability scaling functions, recently revealed 3 from systematic, extended simulations 4 with the DeProF hybrid mechanisticstochastic model for two-phase flow in pore networks 5, into FEM flow integrators, we were able to predict the flow field within the formation (streamlines and flow intensities for the NWP and WP) for a variety of characteristic flow scenarios (operating the central well as a source or sink, different ratios of flow intensities, etc.). The incorporation of closed-form analytical expressions for the relative permeability scaling functions (true-to-mechanism across the entire domain of the two-phase flow regime) has substantially improved the performance of the FEM integrator when compared to previous adaptations that implemented look-up tables for the relative permeability values.

The two-phase flow problem was treated as an equivalent one-phase (saturated) flow problem of a virtual fluid having a local effective mobility equal to the sum of the local mobilities of NWP and WP. The FEM algorithm solves the equivalent 1-ph (saturated) flow problem (potential) combining Darcy's law with the continuity equation and momentum equation, while considering dependence of the local effective hydraulic conductivity on the local flow conditions. The local flow conditions are described by the total flow rate intensity, i.e. the effective (equivalent one-phase) superficial velocity, equal to the sum of the NWP and WP velocities. Now, for a local set of WNP and WP velocities, the local values of $\mathrm{Ca} \& \mathrm{r}$ are readily computed and the local value of the effective mobility is estimated as the sum of the local individual mobilities of NWP \& WP. The mobilities (or, equivalently, the relative permeabilities), and the reduced pressure gradient, are now provided analytically by flow-dependent relative permeability scaling functions. Implementation of standard one-phase Darcy velocity vs pressure gradient relation for the equivalent phase (a virtual mixture of NWP \& WP) delivers the new effective superficial velocity (of the equivalent one phase flow). This latter velocity is decomposed into a set of local NWP \& WP superficial velocities according to the value of the local flow rate ratio. The procedure is integrated along the effective flow streamlines which coincide with the actual 2-ph flow streamlines.

The integration scheme has been applied to a variety of injection/production patterns, solitary wells, direct and staggered line drives, 5-, 7- and 9-spot well arrangements 2 and for different orientations, including gravity effects. We present results for the predicted flow fields and corresponding global energy efficiency values attained.

Procter and Gamble Student poster award:

I don't want to compete References:

Valavanides, M.S. Review of steady-state two-phase flow in porous media: independent variables, universal energy efficiency map, critical flow conditions, effective characterization of flow and pore network. Transp. in Porous Media 123 (1), 42-99 (2018), https://doi.org/10.1007/S11242-018-1026-1

2 Langnes, G.L., Robertson, J.O., Mehdizadeh, A., Torabzadeh, J. "Waterflooding" in "Enhanced Oil Recovery I, Fundamentals and Analyses (Developments in Petroleum Science; 17A)" Donaldson, E.C. , Chilingarian, G.V., Yen, T.F. (Editors), ISBN 0444-42206-4 Elsevier Science Publishers B.V. (1985) pp. 260262

3 Valavanides, M.S. Universal, true to mechanism, flow dependent relative permeability scaling functional form for steady-state 2-phase flows in p.m.”, Intern. Symposium of the Society of Core Analysts, Trondheim, Norway, 27-30/8/2018, http://users.uniwa.gr/marval/publ/Valavanides_SCA2018_066.pdf 4 Valavanides, M.S. Flow structure maps for two-phase flowinmodel pore networks. Predictions based on extensive, DeProF model simulations. Oil Gas Sci. Technol. Rev. IFP Energies nouvelles 73 (6), 1-36 (2018), https://doi.org/10.2516/ogst/2017033

5 Valavanides, M.S., Payatakes, A.C. True-to-mechanism model of steady-state two-phase flow in porous media, using decomposition into prototype flows. Adv. Water Resour. 24(3-4), 385-407 (2001), https://doi.org/10.1016/S03091708(00)00063-4 Acceptance of Terms and Conditions:

Click here to agree

\section{Pore-scale visualization and quantification of saturated solute trans- port using fast micro-computed tomography}

Stefanie Van Offenwert ${ }^{1}$; Tom Bultreys ${ }^{1}$; Arjen Mascini ${ }^{1}$; Marijn Boone ${ }^{2}$; Veerle Cnudde $^{1}$

\footnotetext{
${ }^{1}$ Ghent University
} 
${ }^{2}$ Tescan XRE

\title{
Corresponding Author(s): stefanie.vanoffenwert@ugent.be
}

Understanding solute transport in porous media is key for various applications in engineered and natural porous media, such as groundwater management and building stone performance. Pore scale heterogeneity has a significant impact on spreading and mixing during solute transport, which complicates upscaling (Dentz et al., 2011). Boon et al. (2017) indicate that three-dimensional experimental CT-data at the core scale can characterize the impact of rock heterogeneity on solute spreading and mixing. Direct visualization and quantification of solute concentration fields at the micron-scale has however remained difficult. In 2018, Van Offenwert et al. presented first results on quantitative correctness of dynamic micro-CT experiments of solute transport in sintered glass. A basic methodology to determine transport properties such as the dispersion coefficient was also provided.

In this study, we extend this research by quantifying solute spreading patterns separately in the pores of a sintered glass sample using dynamic laboratory based X-ray micro-CT (Dierick et al., 2014). First, the sample was fully saturated with demineralized water and a high quality micro-CT image was acquired prior to the experiment. Then the sample was injected with a tracer solution of $10 \mathrm{wt} \% \mathrm{CsCl}$ at a flowrate of either $0.25 \mu \mathrm{l} / \mathrm{s}$ or $0.5 \mu \mathrm{l} / \mathrm{s}$. The high quality pre-scan was segmented and used to identify pores and pore throats in the sample, by applying a pore network extraction algorithm (Raeini et al., 2017). The average grey value in each pore throughout the solute injection experiment was then used to determine the local tracer concentration in each individual pore over time. For every pore a breakthrough curve thus defines the evolution of the tracer concentration versus time. Parameters describing these curves can be used to characterize the internal velocity and dispersion of the solute in the pore space.

Sintered glass samples were used in our first experiments to minimize image artefacts. Similar experiments were then also performed on more challenging rock samples with higher levels of heterogeneity. The results can be used to evaluate the influence of pore-scale heterogeneity on solute transport and to validate pore-scale simulations.

Procter and Gamble Student poster award:

\section{References:}

Boon, M., Bijeljic, B. \& Krevor, S., 2017. Observations of the impact of rock heterogeneity on solute spreading and mixing. Water Resources Research, 53: $4624-4642$.

Dentz, M., Le Borgne, T., Englert, A. \& Bijeljic, B., 2011. Mixing, spreading and reaction in heterogeneous media: A brief review. Journal of Contaminant Hydrology, 120-121:1-17.

Dierick, M., Van Loo, D., Masschaele, B., Van den Bulcke, J., Van Acker, J., Cnudde, V. \& Van Hoorebeke, L., 2014. Recent micro-CT scanner developments at UGCT. Nuclear Instruments and Methods in Physics Research B, 324: 35-40.

Raeini, A.Q., Bijeljic, B. \& Blunt, M.J., 2017. Generalized network modelling: Network extraction as a coarse-scale discretization of the void space of porous media. Physical review E, 96: 013312.

Van Offenwert, S., Bultreys, T., Van Stappen, J., Boone, M. \& Cnudde, V., 2018. Visualisation of solute transport and determination of its transport properties in porous sintered glass. InterPore2018, New Orleans, Louisiana, USA, 14-18 May 2018. Acceptance of Terms and Conditions:

Click here to agree

\section{Liquefaction mitigation of Fraser River sand with Microbial In- duced Desaturation and Precipitation (MIDP)}

\author{
Liya Wang $^{1}$; Leon van Paassen ${ }^{2}$ \\ ${ }^{1}$ Hohai University, Arizona State University \\ ${ }^{2}$ Arizona State University
}


Corresponding Author(s): leon.vanpaassen@asu.edu

A new alternative bio-mediated ground improvement known as microbial-induced desaturation and precipitation (MIDP) can be used to stimulate the formation of nitrogen gas and precipitation of calcium carbonate minerals in the soil. A framework on how to assess the potential of MIDP for field applications is presented. Cone penetration tests were performed and samples were collected near the embankment along the south arm of the Fraser River in Richmond, BC Canada. Results from the cone penetration tests were used to assess the cyclic shear resistance. Consolidated undrained triaxial tests were conducted reconstituted Fraser river sand to investigate the effect of MIDP on liquefaction triggering. Results showed that biogenic gas desaturates the soil, dampens excess pore pressures and influences the phase transformation stress. Stiffness can be improved by both the gas production and calcium carbonate precipitation. Cyclic tests show that MIDP can significantly improve the dynamic response of sand. The definition for the onset of liquefaction changes from pores pressure ratio criteria to shear strain criteria at higher CSR values.

Procter and Gamble Student poster award:

References:

Acceptance of Terms and Conditions:

Click here to agree

\title{
Long-term deformation rate of sealed concrete samples as a vis- cous response of the material to capillary forces
}

\author{
Abudushalamu Aili ${ }^{1}$; Matthieu Vandamme ${ }^{2}$; Jean-Michel Torrenti ${ }^{1} ;$ Benoit Masson $^{3}$ \\ ${ }^{1}$ IFSTTAR \\ ${ }^{2}$ Ecole des Ponts ParisTech \\ ${ }^{3}$ EDF-DIN-SEPTEN
}

Corresponding Author(s): matthieu.vandamme@enpc.fr

Concrete and cement paste samples that are kept free from any mechanical loading and in sealed conditions (i.e., exchange no moisture with their surroundings) deform over time. This deformation is called autogenous shrinkage. Experiments show that, in the long term, autogenous shrinkage evolves linearly with the logarithm of time. In this study, we aim at shedding some light on the physical origin of this long-term logarithmic kinetics. More specifically, we aim at verifying, through an exhaustive analysis of experiments from the literature, whether this kinetics can be explained by a creep (i.e., a viscous deformation) of the solid skeleton under the action of capillary forces due to self-desiccation -an idea first proposed by Hua et al. (Cem. Conc. Res., 1995)-.

Indeed, when a cement-based sample is kept in autogenous conditions, hydration consumes the pore liquid, which leads to the partial desaturation of the pore space, to the creation of curved interfaces (i.e., menisci) between the pore liquid and its vapor, and hence to a decrease of internal relative humidity. Consequently, the pressure of the pore liquid becomes more and more negative (w.r.t. the atmospheric pressure), which translates into a compressive stress applied to the solid skeleton. Since the solid skeleton of cement-based materials is viscous, under the action of this stress induced by capillary effects, one expects the sample to shrink over time. Here we aim at verifying whether the long-term rate of autogenous shrinkage one would predict based on this physical explanation is consistent with the long-term rate of autogenous shrinkage measured experimentally. To do so, we perform an exhaustive analysis of autogenous shrinkage data from the literature.

In a first step, based on an estimation of the viscous properties of the various concrete and cement paste samples whose autogenous shrinkage was measured, we calculate to what stress their solid skeleton had to be submitted, in the long term, to explain the measured long-term rate of autogenous shrinkage. Then, we calculate an estimate of the stress induced by capillary effects, as $b^{*} S_{-} L^{*}{ }^{*} \_C$, where $b$ is the Biot coefficient, S_L the liquid saturation, and $p_{-} C$ the capillary pressure. The various 
terms are calculated through a combined used of measured evolutions of internal relative humidity, Power's model, Kelvin's law, and Eshelby-based homogenization tools. We estimate that this stress can reach up to about $10 \mathrm{MPa}$ in the materials with the lowest water-to-cement mass ratios. We eventually find out that this stress induced by capillary effects compares well with the stress to which the solid skeleton must be submitted in the long term to explain the measured long-term rate of autogenous shrinkage. We conclude that considering that the long-term rate of autogenous shrinkage is a viscous response of the sample to capillary forces induced by self-desiccation seems to be a reasonable assumption.

Related reference:

Aili, A., Vandamme, M., Torrenti, J.-M., \& Masson, B. (2018). Is long-term autogenous shrinkage a creep phenomenon induced by capillary effects due to self-desiccation? Cement and Concrete Research, 108, 186-200. https://doi.org/10.1016/j.cemconres.2018.02.023

Procter and Gamble Student poster award:

\section{References:}

Acceptance of Terms and Conditions:

Click here to agree

\section{Validation of functional-structural root system models using MRI- monitored tracer experiments.}

Jan Vanderborght ${ }^{1}$; Axelle Koch $^{2}$; Felicien Meunier ${ }^{3}$; Sarah Garré ${ }^{4}$; Andreas Pohlmeier ${ }^{1}$; Mathieu Javaux ${ }^{2}$

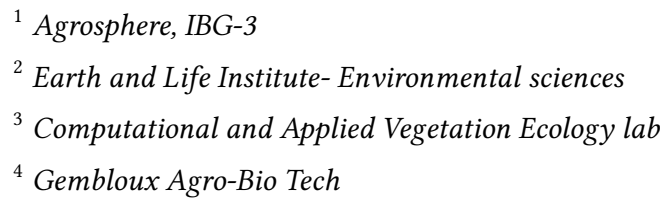

Corresponding Author(s): j.vanderborght@fz-juelich.de

The root system is the plant's organ that has the important function of taking up water and plant nutrients from the soil. Root system functioning depends on its multiscale structure: the size and structure of cells in the conductive tissues, the development and spatial organization of tissues, and the network of root segments that make up the root system. Breeders select for these structural properties of roots and root systems to develop crop varieties with high water and nutrient efficiencies that give a high or stable yield under non-controllable adverse environmental conditions (droughts, nutrient poor soils, soil salinity, ...). But, a direct relation between the functioning of a root system and its structure is not at hand. Functional-structural root system models that simulate the relations between root architectural and hydraulic properties, and the spatio-temporal patterns of water and solute fluxes in the root zone can therefore play an important role in this selection process. However, it must be first demonstrated that these models accurately reproduce the processes they intend to describe but this is a challenging task for two reasons. First, the root system is the so-called 'hidden halve' of the plant so that its structure and the flow and transport processes in the soil towards individual roots are hard to observe. Secondly, the properties that control water flow in root segments and that are required as input parameters in structural-functional root models vary with age and root order and quantitative information about these relations is scarce. In this paper, we demonstrate how this deadlock could be broken by combining co-registered root structure and tracer distributions obtained from magnetic resonance imaging, a functional-structural root system model, and inverse modeling. The main features in the tracer patterns were well reproduced by the model using root hydraulic parameters that were obtained using inverse modeling and that were found to correspond with available information about these parameters in the literature. The simulation results further demonstrated that water uptake location and intensity cannot be directly derived from neither observations of tracer accumulation nor water depletion. This proves that functionalstructural root system model simulations, combined with observations, are required to translate the observed variables (tracer accumulation and water depletion) into information about local processes and root system properties. 
Procter and Gamble Student poster award:

I don't want to compete References:

Acceptance of Terms and Conditions:

Click here to agree

775

\title{
Pore-scale modelling of carbonation reaction in fractured hard- ened cement paste
}

\author{
Anna Varzina \\ ${ }^{1}$ KU Leuven \\ Corresponding Author(s): anna.varzina@kuleuven.be
}

\begin{abstract}
Carbonation is one of the degradation processes in concrete that destroys passivation layer around reinforcement bars, which then leads to corrosion of reinforced steel and to the end-of-life of concrete structures. Moreover, cementitious materials always tend to cracking, which accelerates carbonation, because fractures form a free flow path for gaseous water and $\mathrm{CO} 2$. However the link between fracture properties and carbonation reaction remains undetermined yet. Carbonation reaction in concrete involves dissolution of calciferous compounds, such as portlandite and CSH phases and precipitation of calcium carbonate. Such dissolution-precipitation reactions alter pore and fracture geometry at pore scale, and thus affect the reactive transport inside the porous medium. Change of material geometry alters effective transport properties at higher scales. In order to correctly estimate the change of transport properties during carbonation reaction, pore-scale modelling was used. In this research work carbonation on rough fracture surface has been modelled at pore-scale. The numerical model is based on lattice Boltzmann method and combines diffusive transport of reactive species, mineral dissolution-precipitation reactions of cement phases and geometry update. Fracture surface and capillary pores build the material geometry, while small pores are not resolved explicitly and only mineral mass and volume are tracked. Reactive transport was coupled with the geochemical solver PHREEQC and pore size controlled solubility was incorporated, which allowed transport through a calcite layer and precipitation in larger pores instead. The results intend to serve as input for further continuum-scale model and provide better understanding of carbonation reaction in fractured cement pastes.
\end{abstract}

Procter and Gamble Student poster award:

I would like to compete in the Procter and Gamble Student award References:

Acceptance of Terms and Conditions:

Click here to agree

977

\section{A Multi-instrument Approach to the Characterisation of Irradi- ated and Virgin Nuclear Graphite}

Ben Veater $^{1}$; Giuliano Maurizio Laudone ${ }^{1}$; Katie Louise Jones ${ }^{1}$; Natasha Stephen ${ }^{1}$

${ }^{1}$ University of Plymouth

Corresponding Author(s): ben.veater@plymouth.ac.uk

Graphite is used in a number of reactor designs as an essential component. It serves two purposes, as a moderator and as a structural component of the reactor core. Under temperature and irradiation, graphite is subjected to a number of physical and chemical processes which compromise the 
integrity of the material over time. This is a cause for concern in the industry, especially as the fleet of reactors in the UK reaches and surpasses its predicted operating life-time.

Radiolytic oxidation is a process in which graphite subjected to irradiation in the presence of carbon dioxide coolant, is oxidised. This results in weight-loss and reduction in density. The mechanism for this process is unknown, but its effects are well documented. High energy collisions dislocate carbon atoms, relocating them to neighbouring, energetically favourable positions. The net result of this process is an expansion in the material along the $C$ axis (perpendicular to the graphite lattice) and shrinkage along the $\mathrm{A}$ axis (parallel to the graphite lattice). This irradiation-induced dimensional change causes a challenge due to graphites structural role in the nuclear reactors core.

As a result, research has been commissioned to investigate this material in a variety of novel approaches. Historically, studies focused on individual techniques, which concentrated on limited scales (micro / meso / macro). This research is unique in that it attempts to span all scales using a multi-instrument method. Previous research by the EFMG group using pycnometry, mercury porosimetry and gas sorption, has shown this is a viable approach. This project continues to build upon the work done prior, adding to it multi-scale image analysis using state of the art FIB-SEM and high resolution X-ray CT scanning instrumentation. Instrumental data was used in conjunction with in house proprietary software PoreXpert to model a variety of phenomena including permeability, tortuosity and diffusion. This data will be compared to models using the tomography meshes and a physics modelling suit such as OpenFoam.

Procter and Gamble Student poster award:

I would like to compete in the Procter and Gamble Student award References:

Acceptance of Terms and Conditions:

Click here to agree

\title{
Effects of spatial heterogeneity on flow and transport in variably saturated porous media
}

\author{
Author(s): Andres Velasquez-Parra ${ }^{1}$ \\ Co-author(s): Matthias Willmann ${ }^{2}$; Tanguy Le Borgne ${ }^{3}$; Yves Méheust $^{3}$; Joaquin Jimenez-Martinez ${ }^{4}$ \\ ${ }^{1}$ EAWAG - ETH Zurich \\ ${ }^{2}$ ETH Zurich - Simultec AG \\ ${ }^{3}$ Géosciences Rennes \\ ${ }^{4}$ EAWAG-ETHZ
}

Corresponding Author(s): veandres@student.ethz.ch

The description of the flow field in unsaturated porous media is essential to understand subsurface solute transport and reactive phenomena. Heterogeneity and connectivity of the medium lead to a distinct spatial distribution of the fluid flow velocities. This distribution dictates the development of preferential pathways and, hence, controls both the spatial distribution of chemicals and their residence time. 2D images of phase distributions (water and air) from steady-state millifluidic experiments, in combination with computational fluid dynamics, were used to study the impact of phase saturation on the velocity field and the particle transport through the porous medium. We found that the velocity distribution falls off exponentially at high velocities independent of phase saturation, showing an increasing characteristic velocity as saturation decreases. However, low velocities in the distribution drop-off algebraically following a similar power-law behavior independent of saturation. The observed longitudinal spreading of particles, which turns non-Fickian as soon as the medium is slightly desaturated, is theoretically explained from the velocity distribution in the stagnation zones.

\section{Procter and Gamble Student poster award:}

I would like to compete in the Procter and Gamble Student award References:

Acceptance of Terms and Conditions: 


\section{Effect of surfactants on film formation in inkjet printing on dense and porous media}

Gianmarco Venditti ${ }^{1}$; Myroslava Hanyak ${ }^{\text {None }}$; Anton Darhuber ${ }^{1}$

${ }^{1} \mathrm{TU} / \mathrm{e}$

Corresponding Author(s): g.venditti@tue.nl

Inkjet printing is one of the most flexible techniques for printing and for contactless deposition of fluids on various substrates in general. Patterns are obtained by depositing a multitude of closely spaced droplets that coalescence and form a continuous film. Ripples and undulations are present initially, which will disappear if the ink has enough time to 'settle' before solvent evaporation or solidification. We show that in the case of water-based inks, the presence of surfactants tends to slow down this levelling process significantly. We studied the impact of surface-active solutes on the levelling of ink ripples on porous and non-porous substrates. We present a theoretical model as well as numerical simulations that yield an estimate of the retardation of up to a factor of 8 .

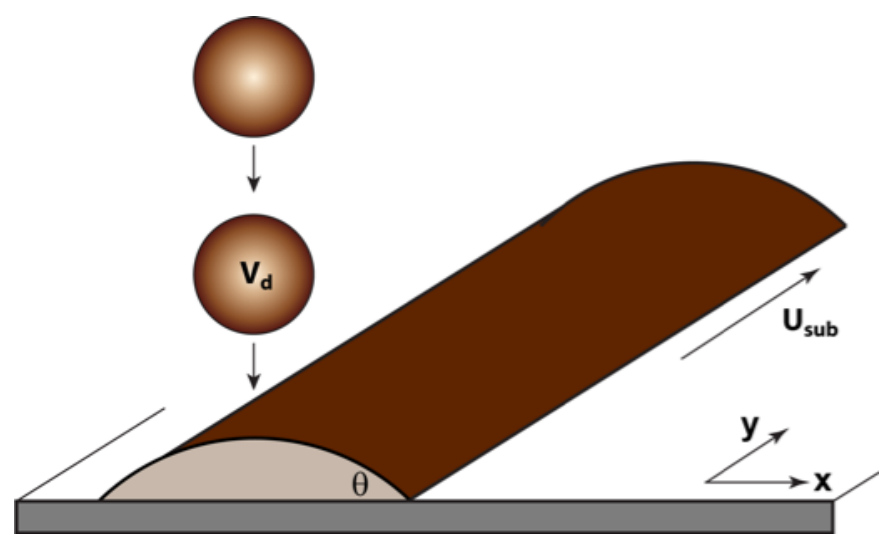

Figure 11: enter image description here

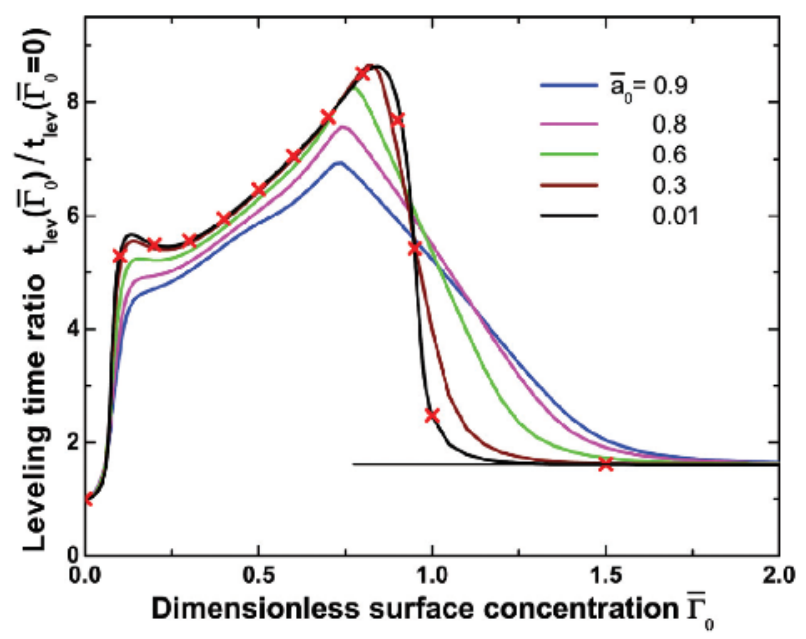

Figure 12: Non-dimensional levelling time as a function of non-dimensional surfactant concentration. 


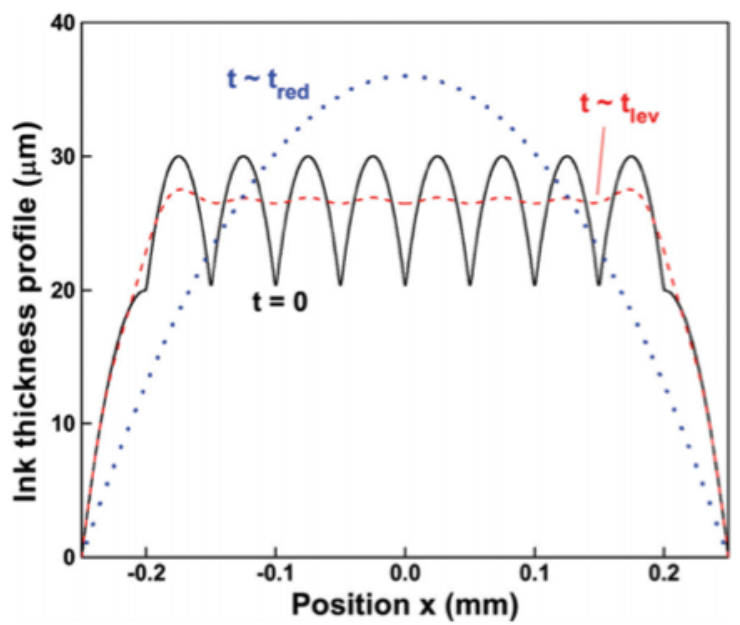

Figure 13: Typical ink thickness profile of $\mathrm{N}=9$ overlapping inkjet-deposited lines. The black solid line represents the initial distribution immediately after deposition. The dashed and dotted lines represent the height profile after leveling ( $t$ tlev) and after capillary redistribution ( $t$ tred), respectively.

Procter and Gamble Student poster award:

I would like to compete in the Procter and Gamble Student award References:

Acceptance of Terms and Conditions:

Click here to agree

\title{
Multiscale dentinal porous medium modeling using image restora- tion techniques
}

\author{
Elsa Vennat ${ }^{1}$; Denis Aubry ${ }^{2}$ \\ ${ }^{1}$ Centrale-Supelec \\ ${ }^{2}$ Ecole CentraleSupelec
}

Corresponding Author(s): elsa.vennat@centralesupelec.fr

Restoring teeth is part of dental practitioner daily practice. The longevity of the restorations is impacted by the mechanical and physical environment of the tooth. However, how the loads are macroscopically distributed in the tooth is not well known and restorations are still not durable enough. Our final aim is to investigate the stress distribution taking account the microscopical scale knowledge (microstructure and local mechanical properties).

In this study, we focus on the main tissue of the tooth, the dentin which is a hierarchical porous media (Vennat et al., 2009). At the tissue scale, it is made of three main structures: the tubules (micrometric cylindrical holes filled with physiological fluids), the peritubular collar (a micrometric highly mineralized cuff surrounding the tubules) and intertubular dentin. At a lower scale, intertubular dentine is itself a composite made of collagen fibers and hydroxyapatite crystals with characteristics length of tenth of nanometers order of magnitude.

The mechanical properties of the dentinal tissue depend on the structure of its porosity and heterogeneity surrounding the tubules (Vennat et al., 2017). But image acquisition only provides a partial/localized view of the microstructure.

Here, it is proposed to build an extension to these known areas using an image restoration technique such as inpainting with texture (Schönlieb, 2015). Then it will possible to affect local mechanical properties to the microstructure features and study the tissue global mechanical behavior. 
Procter and Gamble Student poster award:

\title{
References:
}

Vennat, E., Bogicevic, C., Fleureau, J. M., \& Degrange, M. (2009). Demineralized dentin 3D porosity and pore size distribution using mercury porosimetry. Dental Materials, 25(6), 729-735.

Vennat, E., Wang, W., Genthial, R., David, B., Dursun, E., \& Gourrier, A. (2017). Mesoscale porosity at the dentin-enamel junction could affect the biomechanical properties of teeth. Acta biomaterialia, 51, 418-432.

Schönlieb, C. B. (2015). Partial Differential Equation Methods for Image Inpainting (Vol. 29). Cambridge University Press. Acceptance of Terms and Conditions:

Click here to agree

\section{Application of Micellar Flooding for Soil Remediation at a DNAPL contaminated site}

Enjelia Veony ${ }^{1} ;$ Konstantinos Kostarelos $^{1}$; Pushpesh Sharma ${ }^{1}$

${ }^{1}$ University of Houston

Corresponding Author(s): eveony@uh.edu, kkostare@central.uh.edu

\begin{abstract}
Chlorinated hydrocarbons are the most common contaminants in the USA. Perchloroethylene (PCE), a dense non-aqueous phase liquid (DNAPL), is used in abundance as a solvent in dry cleaning industry and is also used as a degreaser. Soil contamination by PCE can take place due to leak in PCE storage facilities and by improper disposal. Recovery of leaked DNAPLs is problematic due to capillary trapping phenomena resulting from high interfacial tension (IFT) between the DNAPL and aqueous phases rendering the DNAPL immobile; thus, the only effective means of recovery relies on dissolution of the DNAPL into the groundwater that is pumped and treated above ground (P\&T). To date, the only surfactant based method used full-scale is known as Surfactant-Enhanced Aquifer Remediation (SEAR) focuses solely on increasing the apparent solubility of DNAPL into the aqueous phase and shorten the time needed to recover the DNAPL. However, surfactants can also be used to decrease the IFT between the aqueous and non-aqueous phases. Micellar flooding relies on mobilizing trapped DNAPL by reducing the IFT. This approach can shorten the DNAPL time further by eliminating capillary trapping thereby mobilizing the DNAPL. The work presented in this paper will describe the screening and phase behavior studies conducted to identify suitable surfactant formulation. The selected formulation achieved a solubilization parameter (SP) of 7.8 at a salinity of 4,650 $\mathrm{mg} / \mathrm{L} \mathrm{NaCl}$. A 1-D flow experiment was performed to evaluate the performance of the selected surfactant formulation in dynamic conditions. The column experiment resulted in mobilizing trapped PCE in sand, recovering 79\% of the residual PCE as mobilized DNAPL bank. Additional 20.5\% PCE was recovered as solubilized in the microemulsion. In total $99.5 \%$ of the residual PCE was recovered by micellar flooding process. Furthermore, no gel was observed.
\end{abstract}

Procter and Gamble Student poster award:

I don't want to compete References:

Acceptance of Terms and Conditions:

Click here to agree

99

\section{Elucidating the effects of tapered flow channels on the perfor- mance of vanadium redox flow batteries}

Carlos Moreno-Carrero ${ }^{1}$; Tugba Ceren Gokoglan ${ }^{2}$; Marcos Vera $^{1}$; Ertan $_{\text {Agar }}{ }^{2}$; Pablo A. García-Salaberri ${ }^{1}$ 


\footnotetext{
${ }^{1}$ Departamento de Ingeniería Térmica y de Fluidos, Universidad Carlos III de Madrid

${ }^{2}$ Department of Mechanical Engineering, University of Massachusetts Lowell
}

Corresponding Author(s): mvcoello@ing.uc3m.es

The recent growth of intermittent renewable energy sources, such as wind and solar, requires a simultaneous growth of grid-scale energy storage facilities to provide energy resilience and reliability. Redox flow batteries (RFBs) are electrochemical devices that stand out as potential candidates for the storage of large amounts of energy in an economical and flexible way due to their ability to decouple power and energy capacity. However, one of the main drawbacks that hinders the widespread commercialization of RFBs is their relatively low power density, which results in large stack size and high system cost. In recent years, the optimization of engineering aspects, such as electrode materials and cell architectures, has become a key element on the roadmap towards high-performance and costeffective RFBs. In particular, flow fields are responsible for the transport of active species to/from the electrochemically active area of the electrode (i.e., the surface area of the fibers) and, therefore, their design significantly affects the overall power output and efficiency of the system.

In this work, the effects of interdigitated channel tapering on the performance of sulfuric acid-based vanadium RFBs are examined experimentally, numerically and theoretically. Critical performance metrics, such as peak power density, limiting current density, round-trip efficiencies and pressure drop are obtained experimentally using flow cells with the tapered flow fields and compared to the ones obtained with the conventional (i.e., non-tapered) flow fields. The results are further examined by means of a macroscopic multiphysics model. According to the dilute solution theory, the transport of charged species in the electrodes (V2+, V3+, VO2+, VO2+, H+, HSO4-, SO42-) is described by the Nernst-Planck equation, where the convection term is determined through the Navier-Stokes equations (including Darcy's viscous resistance). Besides, the volumetric consumption/production rate of species due to the electrochemical reactions is modeled by Butler-Volmer equations, and the dissociation of sulfuric acid (H2SO4) into bisulfate (HSO4-) and sulfate (SO42-) ions and protons (H+) is modeled by a dissociation source term. The electronic (solid) and ionic (liquid) potentials are obtained from the conservation of charge, where the effective ionic conductivity of the electrolyte is a function of species concentration. A simplified model is adopted for the membrane and its interfaces, considering that the fully-saturated membrane is impermeable to all species except protons, and that the proton concentration and ionic potential are continuous across the membrane-electrolyte interface. Under these hypotheses, the ionic potential in the membrane is determined from the charge conservation equation (i.e., the ionic current due to proton transport) with an effective ionic conductivity that depends on the proton concentration. The latter is assumed equal to the concentration of fixed sulfonic acid groups in the membrane, as given by the electro-neutrality condition.

Preliminary results show that the use of novel flow-field designs can be an effective way to develop RFBs with superior performance, while maintaining pumping losses in acceptable levels.

Procter and Gamble Student poster award:

References:

Acceptance of Terms and Conditions:

Click here to agree

\section{Permeation of gas through single-layer nanoporous graphene mem- branes: theory and molecular simulations.}

Romain Vermorel $^{1}$; Fouad Oulebsir ${ }^{1}$; Juncheng GUO ${ }^{\text {None }}$; Guillaume Galliero ${ }^{1}$

${ }^{1}$ LFCR, E2S-UPPA, University of Pau and Pays de l'Adour

Corresponding Author(s): romain.vermorel@univ-pau.fr 
With the advent of graphene material, membranes based on single-layer nanoporous solids appear as promising devices for fluid separation, be it liquid or gaseous mixtures. The design of such architectured porous materials would greatly benefit from accurate models that can predict their transport and separation properties. More specifically, there is no universal understanding of how parameters such as temperature, fluid loading conditions or the ratio of the pore size to the fluid molecular diameter influence the permeation process. In this study, we address the problem of supercritical fluids diffusing through both simplified models of single-layer porous materials 1 and realistic porous graphene molecular structures.

We performed extensive Equilibrium (EMD) [1,2] and Non Equilibrium (NEMD) 3 simulations to document the physical mechanisms involved at the molecular scale. We propose a general constitutive equation for the diffusional transport coefficient derived from classical statistical mechanics and kinetic theory, which can be further simplified in the ideal gas limit. This transport coefficient relates the molecular flux to the fluid density jump across the single-layer membrane. It is found to be proportional to the accessible surface porosity of the single-layer porous solid and to a thermodynamic factor accounting for the inhomogeneity of the fluid close to the pore entrance. Both quantities directly depend on the Potential of Mean Force that results from molecular interactions between solid and fluid atoms.

Comparisons with EMD simulations performed on simplified models show that the kinetic model captures how narrowing the pore size below the fluid molecular diameter lowers dramatically the value of the transport coefficient 1 . We also demonstrate that our general constitutive equation allows for a consistent interpretation of the intricate effects of temperature and fluid loading conditions on the permeation process. Furthermore, we performed NEMD simulations to investigate the range of applicability of our theoretical model in the case of pressure gradient driven flows 3 . We show that discrepancies should be expected as the pore size increases, as purely non-equilibrium effects come into play.

Recently, we have extended this work to realistic models of single-layer nanoporous graphene membranes 4 . We will report our latest results and show how our theoretical approach pertains to the case of gas separation (methane, carbon dioxide, nitrogen, etc) by porous graphenes.

Procter and Gamble Student poster award:

\section{References:}

1 F. Oulebsir, R. Vermorel, G. Galliero, Diffusion of supercritical fluids through single-layer nanoporous solids: theory and molecular simulations, Langmuir, 34 (2), 561-571 (2017)

2 R. Vermorel, F. Oulebsir, G. Galliero, Communication: A method to compute the transport coefficient of pure fluids diffusing through planar interfaces from equilibrium molecular dynamics simulations, J. Chem. Phys., 147, 101102 (2017).

3 R. Vermorel, to be published (2019).

4 J. Guo, R. Vermorel, G. Galliero, to be published (2019) Acceptance of Terms and Conditions:

Click here to agree

\section{Novel Unsupervised Machine Learning Methods for Characteri- zation Information Content of Data and Model Results}

Velimir Vesselinov ${ }^{1}$

${ }^{1}$ Los Alamos National Laboratory

Corresponding Author(s): vvv@lanl.gov

Currently, the integration of large datasets and powerful computational capabilities has resulted in widespread use of machine learning (ML) in science, technology, and industry. Most of the recent 
ML developments focus on supervised methods which require huge training tests. However, these supervised ML methods are not highly applicable to science-driven applications where typically available datasets for ML training are very limited. The supervised ML methods are also impacted by adversarial problems which can cause inaccurate ML predictions when a random noise is present in the training data which also a major limitation of science applications. In contrast, unsupervised ML methods allow for unbiased extraction of hidden (latent) features that characterize key spatial and temporal processes present in the analyzed datasets. In this way, the unsupervised ML methods are relevant problems in the general area of data exploratorily, data analytics, and model diagnostics. The unsupervised ML methods can be also applied for detection of disruptions and anomalies, and discovery of unknown dependencies and phenomena represented in the datasets. The unsupervised ML methods are also powerful tools for development of physics and reduced-order models representing data and model outputs.

Recently, we have developed a series of novel unsupervised machine learning (ML) methods based on matrix and tensor factorizations coupled with custom k-means clustering and sparcity constraints, called NMFk and NTFk. Our novel unsupervised ML techniques are powerful tools for objective, unbiased, data analyses to extract essential hidden (latent) features in data and model outputs. Our methodology is capable of identifying the unknown number of features charactering the analyzed datasets, as well as the spatial footprints and temporal signatures of the features in the explored data domain.

Here, we present (1) detailed discussion of the developed NMFk/NTFk methodology, (2) extensive testing and verification of the novel methods and developed computational tools, (3) a series of real-world applications of the NMFk and NTFk methods. The applications include diverse sets of problems including multi-phase flow, fluid and geothermal extraction, material characterization, polymer phase transitions, groundwater contamination transport, and fast irreversible bimolecular reactions. NMFk and NTFk are capable to process large datasets (GB/TB's) utilizing advanced computational frameworks such as TensorFlow, PyTorch, and MXNet to efficiently utilize GPU and TPU hardware.

\section{Procter and Gamble Student poster award:}

I don't want to compete References:

Stanev, Vesselinov, Kusne, Antoszewski, Takeuchi, Alexandrov, Unsupervised Phase Mapping of X-ray Diffraction Data by Nonnegative Matrix Factorization Integrated with Custom Clustering, Itextm\{Nature Computational Materials\}, 2018.

Vesselinov, Munuduru, Karra, O’Maley, Alexandrov, Unsupervised Machine Learning Based on NonNegative Tensor Factorization for Analyzing Reactive-Mixing, \textm\{Journal of Computational Physics\}, (in review), 2018.

Vesselinov, O’Malley, Alexandrov, Nonnegative Tensor Factorization for Contaminant Source Identification, \textm\{Journal of Contaminant Hydrology\}, (accepted), 2018.

O’Malley, Vesselinov, Alexandrov, Alexandrov, Nonnegative/binary matrix factorization with a D-Wave quantum annealer, Itextm\{PLOS ONE\}, (accepted), 2018.

Vesselinov, O’Malley, Alexandrov, Contaminant source identification using semi-supervised machine learning, \textm\{Journal of Contaminant Hydrology\}, 10.1016/j.jconhyd.2017.11.002, 2017.

Alexandrov, Vesselinov, Blind source separation for groundwater level analysis based on nonnegative matrix factorization, $\backslash$ textm $\{W R R\}, 10.1002 / 2013 W R 015037,2014$. Acceptance of Terms and Conditions:

Click here to agree

\section{Imbibition, pervaporation and drying in $3 \mathrm{~nm}$ diameter pores}

Olivier Vincent ${ }^{1}$; Bastien Marguet ${ }^{2}$; Alexandre Szenicer ${ }^{2}$; Théo Tassin ${ }^{2}$; Abraham Stroock $^{2}$ 
${ }^{1}$ CNRS
${ }^{2}$ Cornell University

Corresponding Author(s): olivier.vincent@univ-lyon1.fr

We studied water transport in mesoporous silicon layers (pore size $\sim 3 \mathrm{~nm}$ in diameter) in three different situations: spontaneous imbibition triggered by capillary condensation 1, steady-state pervaporation where liquid is fed on one side of the medium and evaporated at the other end 2, and drying in air and vacuum [unpublished].

Both imbibition and pervaporation display a coherent set of results, with different regimes as a function of vapor pressure (relative humidity) allowing an independent measure of both the permeability and the intinsic capillary pressure of these nanoporous layers. Both values are consistent with $3 \mathrm{~nm}$ pores with values in the $10-20 \mathrm{~m}^{2}$ and $-100 \mathrm{MPa}$ range, respectively.

Drying, on the other hand, is much faster than expected from a receding liquid front limited by Knudsen diffusion in the nanopores, which suggests the potential importance of surface flows or other mechanisms. Even more surprisingly, the drying front progresses linearly in time even in vacuum, which is difficult to account for with simple drying models that I will discuss.

Procter and Gamble Student poster award:

References:

1 Vincent, O., Marguet, B., Stroock, A. D., Imbibition triggered by capillary condensation in nanopores, Langmuir 33(7), 2017.

2 Vincent, O., Szenicer, A., Stroock, A. D., Capillarity-driven flows at the continuum limit, Soft Matter 12, 2016. Acceptance of Terms and Conditions:

Click here to agree

\section{Microfluidics Experiments and Lattice Boltzmann Simulations to Characterize Multi-phase Flow and Particle Transport in Frac- ture Networks}

Hari Viswanathan ${ }^{1}$; Phong Nguyen ${ }^{2}$; Bill Carey ${ }^{1}$; Duncan Ryan ${ }^{1}$; Qinjun Kang ${ }^{1}$; Yu Chen ${ }^{2}$; Jim Werner ${ }^{\text {None }}$

\footnotetext{
${ }^{1}$ Los Alamos National Laboratory
}

${ }^{2}$ Los Alamos National Lab

Corresponding Author(s): viswana@lanl.gov

Multi-phase flow and particle transport in fractured systems are critical processes to characterize and optimize for efficient extraction of subsurface resources. Multi-phase flow in fracture networks is critical in evaluating sweep efficiency for hydrocarbon recovery and is a current topic of interest since alternate fracturing fluids to water are being considered such as nitrogen and carbon dioxide. For many subsurface energy applications, particles called proppants are introduced into the flow to prop open fractures and play a critical role in optimizing hydrocarbon or heat extraction in hydraulic fracturing and geothermal operations. Proppant effectiveness and fate is not well known since operations typically take place kilometers underground making in situ proppant transport measurements infeasible. We use microfluidic experiments and Lattice Boltzmann simulations to 1) explore sweep efficiency of oil using nitrogen and water at high T,P in 2D fracture networks and 2) characterize proppant transport in 3D fracture networks.

We conduct direct visualization experiments with a microfluidic system to reveal the mechanisms and to quantify the recovery rates of oil from fracture networks. We compare the effectiveness of water, nitrogen and supercritical carbon dioxide at reservoir conditions in a process mimicking the huff-and-puff method in both dead-end and connected fracture systems. Injection of supercritical 
supercritical carbon dioxide results in the highest recovery rate with an average end-point recovery of about $90 \%$ in the connected fracture network and $60 \%$ in the dead-end fracture network. N2 has lower solubility in oil and hence showed a lower recovery rate of $40 \%$ in the connected fracture network and $25 \%$ in the dead-end fracture network. Injection of water had no effect on oil mobilization since water is insoluble, immiscible and incompressible. The main mechanism of enhanced recovery is gas exsolution from the liquid phase as pressure was decreased below the bubble point pressure. Because the gas is distributed throughout the oil phase, bubble nucleation, growth, coalescence, and elongation occurred throughout the fracture network.

In addition to $2 \mathrm{D}$ microfluidics experiments at high $\mathrm{P}, \mathrm{T}$, we conduct $3 \mathrm{D}$ microfluidics experiments to characterize proppant transport under ambient conditions. Fluid flow and particle transport through porous media is inherently a three-dimensional problem but experiments have been hindered by the challenge of tracking particles in three dimensions. High fidelity simulations have been hindered both by the lack of experimental validation and the high computational cost of three-dimensional simulations with the proper flow physics. We are integrating 3D fluid flow simulation with threedimensional experimental particle tracking methods to characterize complex fluid and particle transport in synthetic fractured media with the aim to optimize proppant dispersal within a fracture network. High resolution, three-dimensional particle tracking experiments integrated with threedimensional particle flow simulations aim to move beyond the two-dimensional microfluidic and lattice Boltzmann studies. We present our first attempts at tracking particles through synthetic fracture networks and Lattice Boltzmann simulations of these experiments to illuminate 3D processes that were not captured in our previous 2D simulations and models.

Procter and Gamble Student poster award:

\title{
References:
}

Acceptance of Terms and Conditions:

Click here to agree

\section{Computing Fluid Flow through Discrete Fracture Networks by Using Combinatorial Optimization on Graph Networks}

\author{
Alex Hobé ${ }^{1}$; Daniel Vogler ${ }^{2}$; Martin P. Seybold ${ }^{3}$; Anozie Ebigbo ${ }^{4}$; Randolph R. Settgast ${ }^{5}$; Martin O. Saar ${ }^{6}$ \\ ${ }^{1}$ Uppsala University, Department of Earth Science, Uppsala, Sweden \\ ${ }^{2}$ ETH Zurich, Zurich, Switzerland \\ ${ }^{3}$ University of Stuttgart, Institute of Formal Methods in Computer Science, Stuttgart, Germany \\ ${ }^{4}$ ETH Zurich \\ ${ }^{5}$ Lawrence Livermore National Laboratory, Livermore, CA, USA \\ ${ }^{6}$ Geothermal Energy and Geofluids Group, Institute of Geophysics, ETH Zurich, Zurich, Switzerland
}

Corresponding Author(s): davogler@ethz.ch

Fracture networks often dominate fluid flow through subsurface reservoirs (e.g., geothermal energy applications, unconventional oil and gas recovery, radioactive waste disposal, and $\mathrm{CO}_{2}$ sequestration). This is due to the significantly higher permeabilities of fractures, vs the surrounding rock matrix. To understand and quantify flow structures in the subsurface, it is therefore important to asses fracture network geometry and the influence of its hydraulic properties. Gaining specific knowledge of fracture network properties is difficult, however, since monitoring hydraulic properties at great depth is both difficult and associated with uncertainties.

We therefore present two methods to rapidly compute fluid flow rates in discrete fracture networks. Both methods rely on algorithms for combinatorial optimization in graph networks and are called the Hanan Shortest Path Maxflow (HSPM) and the Intersection Shortest Path Maxflow (ISPM).

Both methods translate discrete fracture network geometries and properties to an abstract graph network. Next, an adapted max flow algorithm is used to estimate the overall fluid flow rate through 
the fracture network and individual fractures. This is achieved with a shortest path decomposition of the max flow algorithm's flow assignment.

We compare HSPM and ISPM flow calculations to benchmarks of explicit numerical results for individual fracture network test cases and stochastic realizations. Results of the computed flow rates by the HSPM and ISPM show high accuracy, while not requiring long run times or meshgeneration.

Procter and Gamble Student poster award:

References:

Acceptance of Terms and Conditions:

Click here to agree

408

\title{
Immersed boundary methods for HM-simulations of fractures
}

\author{
Author(s): Cyrill von Planta ${ }^{1}$ \\ Co-author(s): Daniel Vogler ; Maria Giuseppe Chiara Nestola ${ }^{1}$; Martin O. Saar ${ }^{2}$; Rolf Krause ${ }^{1}$ \\ ${ }^{1}$ Institute of Computational Science \\ ${ }^{2}$ Geothermal Energy and Geofluids Group, Institute of Geophysics, ETH Zurich, Zurich, Switzerland
}

Corresponding Author(s): cyrill.planta@gmail.com, davogler@ethz.ch

Immersed boundary methods provide an efficient way to simulate fluid structure interaction. No remeshing of the fluid domain is required in case of solid displacements and no a-priori knowledge of the solid fluid boundary is necessary, thus immensely simplifying the set up of HM simulations with complex surface geometries. Fluid and solid processes are simulated on different domains. The domains are superimposed in such a way, that the solid is immersed into the fluid. They have non-matching meshes and are coupled using $L^{2}$-projections, which transfer the physical properties between the meshes.

We show with geometries sampled from granitic rock specimens how immersed boundary methods can replicate the change of fluid flow patterns of a fracture under increasing normal pressure and how increased fluid pressure leads to solid displacements in the rock.

Procter and Gamble Student poster award:

References:

Planta et al. 2018, Variational Parallel Information Transfer between Unstructured Grids in GeophysicsApplications and Solutions Methods, PROCEEDINGS, 43rd Workshop on Geothermal Reservoir Engineering

Nestola et al. 2018, An immersed boundary method for fluid-structure interaction based on overlapping domain decomposition, ArXiv e-prints, 1810.13046 Acceptance of Terms and Conditions:

Click here to agree

\section{Pore-Scale Phase Field Models for Multi-Phase Flow with Precip- itation}

Lars von Wolff ${ }^{1}$ 
${ }^{1}$ University of Stuttgart

Corresponding Author(s): lars.von-wolff@mathematik.uni-stuttgart.de

Multi-phase flow and reactive transport processes in porous media are of importance in many engineering applications. We are interested in the situation when evolving interfaces are encountered on the pore scale. This is the case for interfaces separating two immiscible fluids, or when the pore geometry is changing in time due to processes like dissolution or deposition.

Instead of a standard sharp-interface approach we focus on phase field models to describe these interfaces: Additional phase field variables are introduced as a smooth approximation to the indicator for each of the phases. The Cahn-Hilliard equation determining the evolution of these phase field variables is then coupled with the incompressible Navier-Stokes equations.

We discuss the construction of a phase field model for three phases (fluid-fluid-solid). An important criterion is thermodynamical consistency, i.e. obeying the second law of thermodynamics. For justification of the model the sharp interface limit is identified through asymptotic analysis.

An advantage of the phase field ansatz is that homogenization techniques are easier to apply. We conclude with the upscaling of a fluid-solid phase field model as well as some remarks on the numerical implementation.

Parts of this work have been done in cooperation with Carina Bringedal, Sorin Pop, and Christian Rohde.

Procter and Gamble Student poster award:

References:

Acceptance of Terms and Conditions:

Click here to agree

56

\section{Lyphilization front structures in thin layers of frozen solutions}

Nicole Vorhauer ${ }^{1}$; Petra Först $^{2}$; Harald Schuchmann ${ }^{3}$; Evangelos Tsotsas ${ }^{4}$

${ }^{1}$ Otto-von-Guericke University

${ }^{2}$ Chair of Process Systems Engineering, TU Munich

${ }^{3}$ Wilhelm Büchner Hochschule

${ }^{4}$ Otto von Guericke University

Corresponding Author(s): nicole.vorhauer@ovgu.de

Vacuum freeze drying is a process that is applied if products with high sensitivity to water activity and temperature shall be dried very gently. Examples are pharmaceutical or food products. As this process is carried out at temperatures and pressures below the triple point of water, capillary forces that would lead to the shrinkage of product or degradation of the biological components are excluded. However, to decrease drying time and to increase the throughput of dryers, drying temperature is usually set close to its maximum. This procedure indeed demands a careful control of process conditions if product collapse and degradation shall be avoided. For this reason, current research focusses on the development of predictive models that help to estimate optimum drying conditions at maximum process efficiency.

The lyomicroscope is an experimental tool that is often applied to study drying of frozen solutions. It provides a vacuum chamber cooled by liquid nitrogen and de-pressurized by a rotary vane pump. The frozen solutions are confined in the chamber between the transparent glass surface plate (in front of the microscopy lenses) and the heating shelf at the bottom [1,2]. The investigated area typically has the form of a cylindrical disk with diameter $d=3 \mathrm{~mm}$ and a height of only several 100 microns. The frozen disks are essentially porous structures formed by the solid skeleton of the amorphous solute and pores saturated with frozen water 3. So far, drying of these disks is principally described with macroscopic approaches, such as $v(\mathrm{~m} / \mathrm{s})=k\left(P^{\prime}-P\right) / s$ (with drying front velocity $v$, thickness of the dry solid $s$, saturation vapor pressure $P^{\prime}$ at the sublimation front and lyomicroscope chamber pressure $P$ ), with the aim to identify the transport coefficient $k$ of the porous dry zone and to study 
collapse phenomena. The macroscopic approaches, however, strongly simplify the situation because size of the ice crystals is basically distributed with a standard deviation depending on the freezing conditions (in 4 the pore sizes evolving from the sublimation of ice crystals varied roughly between $6 \mu \mathrm{m}$ and $28 \mu \mathrm{m}$ to $48 \mu \mathrm{m}$ ). The pore size distribution of the dry solid though strongly affects the vapor transport through this zone as it essentially occurs in the transient regime between Knudsen diffusion and viscous flow 5 . If additionally temperature varies only slightly with depth, the vapor transport and thus drying might be accelerated at the bottom side of the frozen disk, i.e. where the latent heat for sublimation is provided, due to the higher vapor transfer rates. This implies that the velocity of the sublimation front $v$ varies with depth. Obviously, the continuum approach appears to be not appropriate to study lyophilization, even in such a simple situation as the thin frozen disk in the lyomicroscope. We therefore propose a pore network model of lyophilization. In a first step, we will show, based on the assumption of quasi-steady heat transfer and vapor flow in the transient regime, that the local sublimation front velocity depends on the pore size distribution and temperature.

\title{
Procter and Gamble Student poster award:
}

\section{References:}

1 Zhai, S., Taylor, R., Sanches, R., Slater, N.K.H.: Measurement of lyophilisation primary drying rates by freeze-drying microscopy. Chem Eng Sci 58 (2003) 2313-2323

2 Raman, P., Rielly, C.D., Stapley, A.G.F.: Freeze drying microscopy as a tool to study the sublimation kinetics of a freeze drying process. In Proceedings of the 19th International Drying Symposium (IDS 2014), Lyon, France, August 24-27, 2014.

3 Raman, P., Rielly, C.D., Stapley, A.G.F.: Freeze drying microscopy to study the kinetics of sublimation in a model lactose system. In Proceedings of the 19th International Drying Symposium (IDS 2014), Lyon, France, August 24-27, 2014.

4 Lechner, M.: Relationship between pore size distribution and drying rate of freeze-drying process. Master Thesis, TU Munich, Germany.

5 Vorhauer, N., Först, P., Schuchmann, H., Tsotsas, E.: Pore network model of primary freeze drying. In: Proceedings of the 21st International Drying Symposium (IDS2018), Valencia, Spain, September 11-14, 2018. DOI: 10.4995/ids2018.2018 Acceptance of Terms and Conditions:

Click here to agree

40

\section{Experiment based pore network study on secondary capillary trans- port through liquid films of different morphology}

\author{
Nicole Vorhauer ${ }^{1}$; Yi Ding ${ }^{2}$; Helmut Geistlinger ${ }^{3}$; Evangelos Tsotsas ${ }^{4}$; Marc Prat $^{\text {None }}$ \\ ${ }^{1}$ Otto-von-Guericke University \\ ${ }^{2}$ Helmholtz-Centre for Environmental Research - UFZ \\ ${ }^{3}$ elmholtz-Centre for Environmental Research - UFZ \\ ${ }^{4}$ Otto von Guericke University
}

Corresponding Author(s): nicole.vorhauer@ovgu.de

In drying of porous media (such as soils, building walls, clay, wood, foods, pharmaceuticals etc.) a partially saturated zone temporally evolves, in which the liquid phase (to be removed from the medium) is fragmented by gas capillary fingers penetrating this zone.

The mass transfer through the partially saturated zone can be separated into three different transport mechanisms: i) the transport of liquid through liquid clusters spanning the partially saturated zone, ii) the transport of liquid through liquid films spanning this zone and iii) vapor diffusion, the latter being important only in the presence of thermal gradients 1. In previous analyses, e.g. [2-3], the liquid films were essentially modelled as a system of continuous corner films, also referred to as thick or capillary films, wetting the crevices and corners of the void space. These films can be distinguished from the very thin films driven by the disjoining pressure. The latter have no impact on the drying rate and are neglected. However, contrary to model systems such as straight channels, e.g. [4-5], it is not obvious that corner films can really form a system of continuous films in a porous medium. Actually, another type of secondary capillary structures, referred to as discrete secondary capillary 
structures (DSCS), can form. Liquid bridges at contact points between particles, [6], or liquid rings around pillars in model systems, [7], are two examples. Although liquid bridges can form long chains in certain geometries, e.g. [8], the liquid rings and bridges are most often considered as discrete objects in the analysis of the drying process. A key question is the hydraulic connectivity of the DSCS. As shown in [7], rings are only present in the partially saturated zone, given hydraulic connectivity by liquid bridges. In the dry zone, however, where hydraulic connectivity is absent, the DSCS dry out. This is in contrast with the continuous corner film model, e.g. [2-3], which predicts that the continuous film region can also develop into a predominantly gas occupied zone upstream of the invasion front. In brief, the continuous corner film model leads to consistent results with the experiments as regards the drying kinetics but does not truly correspond to the DSCS generally expected in the pore space.

To overcome this contradiction, we study drying of pore networks with liquid films of different morphologies under quasi-isothermal conditions in 2 and 3 dimensions using the different concepts from the literature as well as the novel concept of virtual pore network models representing the films as a sub-network. The simulation results are compared to the results of microfluidic experiments with $2 \mathrm{D}$ pore networks. We demonstrate that the liquid permeability through the partially saturated zone is significantly increased in presence of the liquid films if they can form an interconnected liquid region spanning the two-phase zone between the invasion and the evaporation fronts.

\title{
Procter and Gamble Student poster award:
}

\section{References:}

1 Vorhauer, N., Tsotsas, E., Prat, M.: Temperature gradient induced double stabilization of the evaporation front within a drying porous medium. Phys Rev Fluids 3 (2018), 114201.

2 Yiotis, A. G., Boudouvis, A. G., Stubos, A. K., Tsimpanogiannis, I. N., Yortsos, Y. C.: Effect of liquid films on the drying of porous media. AIChE J 50 (2004), 2721-2731.

3 Prat, M.: On the influence of pore shape, contact angle and film flows on drying of capillary porous media. Int. J. Heat Mass Trans. 50 (2007), 1455-1468.

4 Eijkel, J. C. T., Dan, B., Reemeijer, H.W., Hermes, D.C., Bomer, J.G., van den Berg, A.: Strongly accelerated and humidity-independent drying of nanochannels induced by sharp corners. Phys Rev Lett 95 (2005), 256107.

5 Chauvet, F., Duru, P., Geoffroy, S., Prat, M.: Three periods of drying of a single square capillary tube. Phys. Rev. Lett. 103 (2009), 124502.

[6] Soulié, F., Cherblanc, M.S., Youssoufi, El, Saix, C.: Influence of liquid bridges on the mechanical behaviour of polydisperse granular materials. Int J Numer Anal Methods Geomech 30 (2006), 213-228.

[7] Vorhauer, N., Wang, Y., Kharaghani, R., Tsotsas E., Prat, M.: Drying with formation of capillary rings in a model porous medium. Trans Porous Media 110 (2015), 197-223.

[8] Chen, C., Duru, P., Joseph, P., Geoffroy, S., Prat, M.: Control of evaporation by geometry in capillary structures. From confined pillar arrays in a gap radial gradient to phyllotaxy-inspired geometry. Scientific Reports 7 (1) (2017), 15110. Acceptance of Terms and Conditions:

Click here to agree

\section{Pore network analysis of the steady state water saturation of hy- drophilic GDLs at high current densities}

\author{
Nicole Vorhauer ${ }^{1} ;$ Haashir Altaf $^{2}$; Tanja Vidakovic-Koch ${ }^{3}$; Evangelos Tsotsas ${ }^{2}$ \\ ${ }^{1}$ Otto-von-Guericke University \\ ${ }^{2}$ Otto von Guericke University \\ ${ }^{3}$ Max-Planck-Institute Magdeburg
}

Corresponding Author(s): nicole.vorhauer@ovgu.de

The performance of an electrolyser drastically decreases at high current densities mainly because of kinetic losses associated with the mass transfer resistances through the GDL 1 . To overcome these limitations the GDL should conceptually be designed so as to allow maximum supply of the electrode with water (educt) and simultaneous removal of oxygen (product), flowing countercurrently through the GDL. This requires a deep understanding of the pore scale distribution of gas and liquid phase at 
certain operating conditions. To achieve this, pore scale study of mass transfer through the porous medium based on pore network models and microfluidic experiments can be helpful 2. From previous studies it is known that the gas-liquid distribution can be controlled by the pore size distribution and the geometrical arrangement of larger and smaller pores, temperature gradients and wettability, e.g. in drying 3, drainage $[4,5]$ or imbibition $[5,6]$. The aim is thus to show the impact of these parameters on the steady state water saturation of the GDL for different operation conditions. For this purpose, we apply pore network modeling with drainage and imbibition concepts. As the GDL is a thin porous medium, with only a few pore rows over the thickness of the GDL, the size of the pore network can be adjusted to the size of the GDL. This allows us to simulate the through plane mass transfer and to identify the steady state liquid saturation for different properties of the GDL.

Procter and Gamble Student poster award:

References:

1 Lee, C.H., Banerjee, R., Arbabi, F., Hinebaugh J., Bazylak, A.: Porous transport layer related mass transport losses in polymer electrolyte membrane electrolysis: A review. In: Proceedings of the ASME 2016 14th International Conference on Nanochannels, Microchannels, and Minichannels, Washington, DC, USA, July 10-14, 2016, DOI:10.1115/ICNMM2016-7974.

2 Arbabi, F., Kalantarian A., Abouatallah, R., Wang, R. Wallace, J.S., Bazylak A.: Feasibility study of using microfluidic platforms for visualizing bubble flows in electrolyzer gas diffusion layers. J Power Sources 258 (2014) 142-149.

3 Prat, M.: On the influence of pore shape, contact angle and film flows on drying of capillary porous media. Int J Heat Mass Trans 50 (2017) 1455-1468.

4 Laurindo, J.B., Prat, M.: Numerical and experimental network study of evaporation in capillary porous media: Phase distributions. Chem Eng Sci 51 (1996) 5171-5185.

5 Fenwick, D.H., Blunt, M.: Three-dimensional modeling of three phase imbibition and drainage. Adv Water Res 21 (1998), 121-143.

[6] Sun, Y., Kharagani, A., Tsotsas, E.: Micro-model experiments and pore network simulations of liquid imbibition in porous media. Chem Eng Sci 150 (2016) 41-53. Acceptance of Terms and Conditions:

Click here to agree

\section{Improved performance of geothermal systems through modelling with uncertainties}

Denis Voskov ${ }^{1}$; Daniilidis Alex ${ }^{2}$

\footnotetext{
${ }^{1}$ TU Delft

${ }^{2}$ Tu Delft
}

Corresponding Author(s): d.v.voskov@tudelft.nl

The production of geothermal energy is a renewable low-carbon energy source for heating and electricity production widely available around the world. In low-enthalpy conduction-dominated settings, geothermal operations typically use doublets where the water extracted from the production well is re-injected back into the reservoir at an injection well to maintain reservoir pressure. With time, the cooled water reaches the production well, and due to the resulting temperature drop, the doublet will eventually be abandoned. The uncertainty in the timing of the cold-water breakthrough complicates the estimation of the system lifetime and thereby its profitability.

The reduction in the economic risk of geothermal projects directly depends on computer-assisted simulations. Studies performed in the hydrocarbon industry indicate a large potential for an enhanced economic output of projects by application of uncertainty reduction and dynamic optimization methods. These improvements are strongly required for geothermal energy to become more affordable and feasible for industrial developments.

In this presentation, I will demonstrate how uncertainty quantification and risk analysis can be addressed in practical simulation studies for geothermal operations. These studies generally can help in further improving the economic output of projects and the wider deployment of geothermal energy. A sensitivity analysis with ranking effects influencing the thermal breakthrough in low-enthalpy 
geothermal projects will be presented. In addition, the application of in-depth water diversion technologies will be suggested for an extension of geothermal doublet lifetime.

Procter and Gamble Student poster award:

I don't want to compete References:

Acceptance of Terms and Conditions:

Click here to agree

673

\title{
Exploring the Impact of Electrode Microstructure on the Perfor- mance of Non-Aqueous Redox Flow Batteries
}

\author{
Antoni Forner-Cuenca ${ }^{1}$; Charles Tai-Chieh Wan ${ }^{1}$; Fikile Brushett ${ }^{1}$ \\ ${ }^{1}$ Massachusetts Institute of Technology
}

Corresponding Author(s): ctwan@mit.edu

Redox flow batteries (RFBs) are promising rechargeable electrochemical devices for grid-scale energy storage, but further cost reductions are needed for ubiquitous adoption of this technology. Research efforts have primarily focused on molecular discovery, there has been significantly less attention paid to the development of other critical system components. Of particular importance are the porous electrodes used in the electrochemical stack. Today's electrodes largely draw from the fuel cell material set, but, within a RFB, the porous electrode must perform a number of different roles including providing active surfaces for electrochemical reactions, facilitating uniform liquid electrolyte distribution, and supporting low pressure drops. Thus, before advanced electrodes can be developed for RFB applications, performance-limiting factors for the present materials set must be quantified. However, unambiguous analysis is challenging in an operating RFB due to the complex coupling of transport and reactions which varies as a function of state of charge during cell cycling. Deconvoluting the role of electrode properties on flow battery performance requires the development of diagnostic techniques that enable electrode characterization under well-controlled but application-relevant conditions.

To this end, we systematically compare the operando performance of RFBs containing selected carbon paper, felt, and cloth electrodes using the single-electrolyte cell configuration 1 and a model organic redox couple (TEMPO/TEMPO+) 2. Using polarization and electrochemical impedance spectroscopy, we quantify the impact of electrode microstructure on battery performance. We find that, depending on the electrode choice and flow conditions, current densities as high as $450 \mathrm{~mA} \mathrm{~cm}-2$ can be achieved at an overpotential of $0.3 \mathrm{~V}$ with a cell area specific resistance as low as $0.7 \Omega \mathrm{cm} 2$. This result suggests that, through appropriate cell engineering and materials selection, high power performance may be realized in nonaqueous flow batteries. Finally, building on this acquired knowledge, I will discuss our recent efforts in the bottom-up fabrication of hierarchically-organized porous electrodes.

Acknowledgements

We gratefully acknowledge the financial support of the Swiss National Science Foundation (P2EZP2_172183) and the Joint Center for Energy Storage Research (JCESR), an Energy Innovation Hub funded by the United States Department of Energy.

References

1 R. Darling et al., Energy Environ. Sci., 7, 3459 (2014).

2 J. A. Kowalski et al., Curr. Op. Chem. En., 13, 45 (2016).

Procter and Gamble Student poster award:

\section{References:}

Acceptance of Terms and Conditions: 


\title{
A new method of quantifying pore structure and extracting pore networks from 2D images
}

\author{
Author(s): Chenhui Wang ${ }^{1}$ \\ Co-author(s): Kejian $\mathrm{Wu}^{1}$; Gilbert Scott ${ }^{1}$ \\ ${ }^{1}$ University of Aberdeen
}

Corresponding Author(s): r02cw17@abdn.ac.uk

Pore structures are complex but fundamental to fluid flow and developing a better understanding of single and multi-phase flow largely relies on quantifying the pore structure. We introduce a new pore structure quantification method which employs 2D scanning electron microscopy (SEM) images with higher resolutions. SEM provides a larger scales of pore sizes ranging from nano size to centimetre and the complex pore structures can be captured as well. The method discretizes the pore space into a set of connected pore elements which capture the pore geometry and topology.

This new quantification method combines the advantages of the maximal ball and medial axis methods and overcomes their problems. By employing the maximal ball concept, pore bodies are defined sufficiently to coincide with the geometrical pores. The method also uses the medial axis transformation to preserve the topology of the pore structure. The method discretizes the pore space into a set of connected pore elements which capture the pore geometry and topology. The distinction between pore bodies and pore throats is discussed and an alternative methodology proposed where we discard the difference and treat them altogether as pore elements. These pore elements can also be used to construct a bond and node network, however, we suggest that the distinction between pore bodies (network nodes) and pore throats (network bonds) should be discarded in favour of connected pore elements.

To demonstrate the value and functionality of the pore structure quantification method, it is applied to a set of 2D SEM images of a heterogeneous sandstone. The relationship between pore structures quantified by the new method and fluid flow properties is discussed. The results show fluid transport properties are highly related to the geometrical and topological properties of pore structures. And the pore networks constructed by the new quantification method can predict reasonable agreement of absolute permeability with the Lattice Boltzmann method. Overall, complex pore structures can be quantified completely by implementing the new quantification method.

In future work, we plan to extend the method to 3D and apply it to X-ray micro-CT volumes. We also propose to investigate the relationship between $2 \mathrm{D}$ and 3D topological properties and perform simulations to validate the treatment of the pore space as a single set of pore elements rather than introducing a distinction between pore bodies and pore throats.

Procter and Gamble Student poster award:

I would like to compete in the Procter and Gamble Student award References:

1 Blunt, M. J., Bijeljic, B., Dong, H., Gharbi, O., Iglauer, S., Mostaghimi, P., ... Pentland, C. (2013). Porescale imaging and modelling. Advances in Water Resources, 51, 197-216. https://doi.org/10.1016/J.ADVWATRES.2012.03.003 2 Dong, H., \& Blunt, M. J. (2009). Pore-network extraction from micro-computerized-tomography images. Physical Review E - Statistical, Nonlinear, and Soft Matter Physics, 80(3), 1-11. https://doi.org/10.1103/PhysRevE.80.036307 3 Jiang, Z., Wu, K., Couples, G., Van Dijke, M. I. J., Sorbie, K. S., \& Ma, J. (2007). Efficient extraction of networks from three-dimensional porous media. Water Resources Research, 43(12), 1-17. https://doi.org/10.1029/2006WR005780 4 Lindquist, W. B., \& Venkatarangan, A. (1999). Investigating 3D geometry of porous media from high resolution images. Physics and Chemistry of the Earth, Part A: Solid Earth and Geodesy, 24(7), 593-599. https://doi.org/10.1016/S1464-1895(99)00085-X Acceptance of Terms and Conditions:

Click here to agree 


\section{Effect of gas hydrate formation and decomposition on flow prop- erties of fine-grained sediments using X-CT based pore network modeling}

Daigang Wang ${ }^{\text {None }}$; Chenchen Wang ${ }^{\text {None }}$; Chengfeng Li $^{\text {None }}$; Changling Liu ${ }^{\text {None }}$; Hailong Lu ${ }^{\text {None }}$

Corresponding Author(s):

Experiments are performed to study the closed-loop effect of gas hydrate formation and decomposition on the flow properties of a fine-grained quartz sand specimen. The high resolution X-ray CT images of the test specimen at different experimental stages are acquired. In order to elucidate the changes in pore structure of the test specimen, topologically representative pore networks are established. The evolution of the flow properties during gas hydrate formation and decomposition is further evaluated. The results show that, gas hydrates occupancy in pore space exhibit different modes; they grow mainly as the grain-cementing mode except some intermediate stages, where pore-filling or load-bearing hydrates are observed. It is also found that the formation and decomposition of gas hydrates can cause the pore structure and flow properties changed. Increase of gas hydrate saturation results in a sharp decline in water relative permeability, larger irreducible water saturation and smaller gas-water percolation zone, while gas relative permeability does not exhibit obvious changing law. The decomposition of gas hydrates will exert a greater influence on the flow properties described above than gas hydrate formation does. The increase in frequency percentage of $10 \sim 20 \mu \mathrm{m}$ pores within the fine-grained test specimen after experiments might be caused by gas hydrate decomposition induced damage of pore structure.

Procter and Gamble Student poster award:

I would like to compete in the Procter and Gamble Student award References:

Acceptance of Terms and Conditions:

Click here to agree

\section{A New Approach to Enhance Oil Recovery in the Tight Oil Reser- voir: Advance Gas Injection}

Deqiang Wang $^{\text {None }}$; Linsong Cheng ${ }^{\text {None }}$; Renyi Cao ${ }^{\text {None }}$; Shijun Huang ${ }^{\text {None }}$; Yongchao Xue $^{\text {None }}$

Corresponding Author(s):

The application of multi-fractured horizontal well has made great progress in the development of tight oil reservoirs. However, the oil production will decrease rapidly and the estimated ultimate recovery is still low in the tight reservoir with low pressure in china when the well is on production using depletion. To improve this situation, the advance gas injection (AGI), which is a process of gas injection and soak before production, is evaluated to analysis the effect and mechanism.

The simulation model is built to describe exactly the flow behavior of oil swelling and viscosity reduction after crude oil dissolved $\mathrm{CO} 2$ in the tight matrix. Oil viscosity can be decreased after advance gas injection and soak, oil relative permeability increased by calculating in the simulation model. The soak time and injection rate are optimized by a series of comparison and sensitive analysis. AGI can enhance the pressure of wellbore and fracture connecting with wellbore by gas injection, which can decrease the effectiveness of stress sensibility and extent the stable production period. Oil recovery can be enhanced $10 \%$ by the comparison between depletion and depletion after AGI. And oil recovery can be enhanced $5 \%$ by the comparison between huff-n-puff and huff-n-puff after AGI. The approach of AGI can be regard as an effective EOR measure because it can enhance pressure near the wellbore, and decrease the oil viscosity and replace the oil in the matrix.

Procter and Gamble Student poster award:

References: 


\title{
Analysis of Dynamic Structure Characteristics of Foam Fluid Trans- port in Porous Media Based on Fractal Theory
}

\author{
Author(s): Fei Wang ${ }^{1}$ \\ Co-author(s): Dongxing Du ${ }^{1}$; Xu Dong ${ }^{1}$ \\ ${ }^{1}$ Qingdao University of Science and Technology \\ Corresponding Author(s): wangfeiupc@163.com
}

\begin{abstract}
The evaluation and simulation of foam fluid are still mattering of significant debate despite the large number of available studies due to the excellent properties of foam and its successful applications, especially in oil and gas field development which include enhanced oil recovery, matrix acidizing, gas breakthrough control, plugging removal, etc. The control of gas mobility and the liquid production profile show the great potential of foam as an intelligent fluid. The properties of foam fluid are substantially determined by its dynamic structure in porous media; however only a few studies that investigate and perform measurements related to such structure have been reported. In this research, a new method based on fractal theory is proposed for evaluation of aqueous foam in porous media. As a first step, we attempt to carry out a quantitative investigation of foam structure in porous media based on fractal theory directly without bothering to consider the changing of gas and liquid or lamella and the fractal characteristics of foam in porous media are confirmed by image processing and calculations. Accordingly, the foam dynamic structure is quantitatively studied by defining and calculating the foam fractal dimension. Secondly, a concise relation is established between the foam fractal dimension and time. Finally, a sensitivity analysis is carried out by discussing three major factors affecting foam structure in porous media and bubble size was also analyzed. The results show that:(1) Foam fluids have fractal characteristics in porous media, exhibiting a box-counting fractal dimension between 1 and 2 based on 2-dimensional images.(2) The fractal dimension of aqueous foam is nearly time-independent during displacement.(3) Three factors affecting the foam structure, namely temperature, concentration and pore structure were separately analyzed. The trend in fractal dimension was still constant with the changing of time, but the value was different at different conditions.(4) Analyses of bubble size illustrated that a smaller foam fractal dimension indicates a more uniform distribution of bubbles throughout the foam.
\end{abstract}

Procter and Gamble Student poster award:

References:

Acceptance of Terms and Conditions:

Click here to agree

72

\section{Experimental Study on the Variation of Micro structure of Coal under surfactant}

Author(s): Laigui WANG ${ }^{\text {None }}$

Co-author(s): Wenbo An

Corresponding Author(s): 1522468690@qq.com

Abstract: Coal is a low permeability solid medium with pores and fracture structures. In this paper, the surfactants were used to modify the coal to improve the coal permeability, porosity and gas extraction efficiency. The change of physical and mechanical properties of coal after modified 
by surfactants were analyzed via the combination of macroscopic methods (contact angle, mercury injection, permeability and uniaxial compression) and microscopic methods (X-ray diffraction, scanning electron microscope, fourier transform infrared spectrum). The micro modification mechanism of coal was revealed via the calculating method of quantum chemistry. The results showed the following facts, (1). The best modification condition were $0.5 \mathrm{wt}$.\% of SDS solution mass concentration, 48 hours of modification time, and $40{ }^{\circ} \mathrm{C}$ of modification temperature by contact angle test, and the effect of SDS solution on coal modification was better than that of CTAB solution and NP-40 solution. (2). The permeability and porosity of coal were increased ans the uniaxial compression strength and elastic modulus of coal were decreased after modified by surfactants. (3). The micro experiments showed that the pore distribution of coal changed from micro pore or transition pore to medium pore or large pore, which could explain why the porosity increased. The surface functional groups of coal were changed and the the hydrophilicity was increased, which was the reason why the contact angle of coal was decreased. (4). The mineral content of coal was changed, the carbonate mineral was decreased and the silicate mineral was increased, which was also the essential reason of the macroscopic physical and mechanical change of coal.

Procter and Gamble Student poster award:

References:

Acceptance of Terms and Conditions:

Click here to agree

\title{
Research on Non-linear Porous Seepage Model of Multi-Fractured Horizontal Well in Shale Gas Reservoir
}

\author{
QIANG WANG ${ }^{1}$
}

${ }^{1}$ reservoir engineer

Corresponding Author(s): dream2008@petrochina.com.cn

The technology of multi-fractured horizontal well is the one of the most effective and efficient methods to develop shale gas reservoir. Referring to absorption/diffusion effect, stress sensitivity effect in shale formation and slippage effect and, a compound flow model of multi-fractured horizontal well is built and its analytic solution in Laplace space is also obtained. Using Stefest numerical reversion technology and Duhamel principle, taking wellbore storage and skin effect into consideration, a non-dimensional productivity model in real space is built and its solution is resolved, thus drawing standard template of non-dimensional productivity model and carrying out sensitive analysis of influencing factors. According to the consequences of realistic application, this model can predict real productivity of a multi-fractured horizontal well and the forecast accuracy is high enough to solve real problem. The result shows that production of multi-fractured horizontal well in shale gas can be divided into three seepage zones and five stages. In the first stage when linear flow in artificial fractures is dominant, production relatively high whereas the decline rate is also high; in the middle stage when linear flow in nature fractures or micro-fractures is dominant, production and decline rate are both decreasing; in the final stage when linear flow in matrix is predominant, production period long with low production rate. This paper builds foundation for learning complicated seepage law of multi-fractured horizontal well, forecasting productivity of multi-fractured horizontal well in shale gas reservoir, revaluating result of artificial fracturing and optimizing fracturing parameters of horizontal well.

Procter and Gamble Student poster award:

I would like to compete in the Procter and Gamble Student award References:

Acceptance of Terms and Conditions:

Click here to agree 


\title{
Hysteresis in Adsorption and Desorption Isotherm for Shale Gas in Realistic Kerogen models
}

\author{
Runxi Wang ${ }^{1}$; Qiang Sheng ${ }^{2}$; Jun $\mathrm{Li}^{3}$; Matthew Borg ${ }^{4}$; Yonghao Zhang ${ }^{5}$; Jason Reese ${ }^{2}$ \\ ${ }^{1}$ University of Edinburgh \\ 2 The University of Edinburgh \\ ${ }^{3}$ King Fahd University of Petroleum and Minerals \\ 4 The university of Edinburgh \\ ${ }^{5}$ Department of Mechanical and Aerospace Engineering, University of Strathclyde
}

Corresponding Author(s): runxi.wang@ed.ac.uk

Shale gas production from the unconventional reservoir has received significant attention. The complex mechanisms of gas transport and multiscale hierarchy of porous structures make the understanding and prediction of gas recovery a challenging question. In organic-rich shales, methane molecules can accumulate in nanoscale cracks or slits, which is called free gas, while they can be adsorbed on the solid surface of nanoscale cracks/slits or in molecular nanopores of kerogens, called absorbed gas. Kerogens, where petroleum and natural gas form, are a mixture of organic chemical compounds that make up a portion of the organic matter in sedimentary rocks. 1 The absorbed molecules can supplement the free gas molecules after desorption while the free gas molecules can be absorbed into nanopores during their transport process. However, we have not obtained complete understanding about the dynamic properties of gas adsorption or desorption in kerogens. Thus, it is essential to explore the adsorption and desorption of methane molecules in kerogens to understand the shale gas behaviours.

In this work, molecular dynamics (MD) simulation is employed to investigate the adsorption and desorption dynamics of methane molecules between kerogen structures 2 and a chamber under a certain pressure. As shown in Fig. 1, on the right of this chamber, a solid plate, with external force applied, is used to maintain controllable pressure during adsorption/desorption. The effects of maturity level and kerogen densities on adsorption/desorption will be investigated. 3 We expect to observe a hysteresis between the adsorption isotherm and desorption isotherms, 4 based on which, the adsorption and desorption mechanisms will be analysed. We intend to identify the type of adsorption isotherm of methane in kerogens and examine the effect of the pore size distribution on the hysteresis phenomenon. Finally, we plan to theoretically analyse dynamics evolutions during adsorption or desorption, to help understand the interaction between adsorbed gas and free gas in shales.

\section{Procter and Gamble Student poster award:}

I would like to compete in the Procter and Gamble Student award References:

1. Vandenbroucke, M.; Largeau, C., Kerogen origin, evolution and structure. Org. Geochem. 2007, 38 (5), 719-833.

2. Bousige, C.; Ghimbeu, C. M.; Vix-Guterl, C.; Pomerantz, A. E.; Suleimenova, A.; Vaughan, G.; Garbarino, G.; Feygenson, M.; Wildgruber, C.; Ulm, F.-J., Realistic molecular model of kerogen's nanostructure. Nat. Mater. 2016, 15 (5), 576.

3. Zhang, T.; Ellis, G. S.; Ruppel, S. C.; Milliken, K.; Yang, R., Effect of organic-matter type and thermal maturity on methane adsorption in shale-gas systems. Org. Geochem. 2012, 47, 120-131.

4. Burgess, C. G.; Everett, D. H.; Nuttall, S., Adsorption hysteresis in porous materials. Pure Appl. Chem. 1989, 61 (11), 1845-1852.

\section{Acceptance of Terms and Conditions:}

Click here to agree 


\title{
Hydraulic fracture optimization in tight oil reservoirs using arti- ficial intelligence
}

\author{
Author(s): Sen Wang ${ }^{1}$ \\ Co-author(s): Qihong Feng ${ }^{1}$; Farzam Javadpour ${ }^{2}$; Shiqian Xu \\ ${ }^{1}$ China University of Petroleum (East China) \\ ${ }^{2}$ The University of Texas at Austin
}

Corresponding Author(s): wangsena1@126.com

Optimizing the parameters of multistage fractured horizontal wells is essential for the economic development of tight oil reservoirs. In general, this process is performed by integrating reservoir numerical simulator and optimization algorithm. However, this task is time-consuming and expensive because hundreds of simulations cases, or even more, are required to forecast the production performance during each simulation. Given that the powerful capacity of artificial intelligence (AI), developing fast proxy model on the basis of advanced AI techniques may provide an alternative avenue to alleviate the computational cost and improve the optimization performance. In this study, we build a fast proxy model using Long Short-term Memory (LSTM) network to predict hydrocarbon production, and then this model is coupled with the Covariance Matrix Adaptation Evolution Strategy (CMA-ES) to optimize hydraulic fractures in tight oil reservoirs.

We first generated the production data set from a generic hydraulic fractured model using an industrystandard simulator. The ranges of reservoir parameters resemble real tight oil plays in China. A total of 168 cases were simulated to obtain the production performance. Then we conducted a statistical correlation analyses to determine the controlling factor in tight oil production. We found that reservoir permeability, initial pressure, hydraulic fracture length, fracture conductivity, horizontal well length, dissolved gas-oil ratio, and bottomhole pressure are the most influential factors. Therefore, in our proxy model, we considered these seven parameters as the input factors and the production data in the 15 years as the output factor. The LSTM network model is trained using $75 \%$ of the data and the other 25\% was used for testing. Meanwhile, to examine the performance of our model, two commonly-used AI methods, i.e., the Support Vector Machine (SVM) and the Artificial Neural Networks (ANN), were also employed to train these data. We evaluated these models through the average absolute relative deviation (AARD), coefficient of determination (R2), and the normalized root mean square error (NRMSE). Our results indicate that in comparison to SVM and ANN, whose AARDs are $13.6 \%$ and $18.4 \%$, respectively, the prediction performance of LSTM is much superior $(7.1 \%)$.

We then coupled LSTM network and optimization algorithm to tune fracture parameters to maximize net present value of tight oil. CMA-ES was utilized to solve this optimization problem because this choice has been demonstrated in our previous work. We showed that the combination of LSTM model and CMA-ES is very efficient, which decreases the computational cost of traditional numerical simulation method by at least $60 \%$, and the optimum value is also better. This method shed light on the production optimization of unconventional reservoirs, but more generally for porous media-related prediction.

Procter and Gamble Student poster award:

I don't want to compete References:

Acceptance of Terms and Conditions:

Click here to agree

697

\section{Pressure-driven two-phase flow of Gas and water in shale nanopores}

\author{
Author(s): Sen Wang ${ }^{1}$ \\ Co-author(s): Qihong Feng ${ }^{1}$; Farzam Javadpour ${ }^{2}$; Wei Zhang \\ ${ }^{1}$ China University of Petroleum (East China)
}


${ }^{2}$ The University of Texas at Austin

Corresponding Author(s): wangsena1@126.com

Gas production from shale systems has emerged as a pivotal energy resource. Owing to the widespread nanopore and complex mineralogical composition, mass transfer through shale matrix is extremely complex. Up to now, our knowledge on the physics of fluid transport in nanoporous shale mainly focuses on single gas phase, whereas the influences of water introduced during hydraulic fracturing on the gas migration has seldom been explored. Here, on the basis of grand canonical Monte Carlo (GCMC) simulations and molecular dynamics, we studied methane and water two-phase flow in both organic and inorganic nanopores of shale.

Although amorphous kerogen model has been proposed for shale, we used carbon nanotube to mimic organic nanopores in shale matrix because the sizes of pores located in shale kerogen $(<2 \mathrm{~nm})$ are much smaller than those commonly observed in shale $(2-100 \mathrm{~nm})$. For the inorganic nanopores, we explored montmorillonite (clay) substrate. We first conducted GCMC simulations to study gas adsorption in shale nanopores containing various amounts of preadsorbed water molecules, from which the static configuration without driving force were obtained. Although the introduction of water molecules reduces gas adsorption, different phenomena are observed in shale organic and inorganic nanopores. These water molecules tend to form a cluster within carbon nanotube, which acts as a piston and tremendously impede gas diffusion. However, an adsorbed water membrane is present at the clay surface, causing less impact on gas transport. We attributed the reason to the weak water-carbon affinities and strong hydrogen bonds.

Then this model was used in subsequent flow simulations. We applied a constant gravitylike force, in the direction parallel to the basal planes, to all the gas and water molecules to study the pressuredriven flow behavior in shale nanopores. Similar to the single phase flow, we observed interfacial slip between gas/water and solid substrate. However, two-phase flow pattern changes as the pressure gradient increases above a size-dependent threshold value, leading to the deviation of linear flow. In the inorganic nanopore, as the pressure gradient is smaller, the pattern is similar to the slug flow observed for two-phase flow at the macroscale, whereas as the gradient increases, the gas phase breaks through the water and the pattern resembles an annular flow. Because the behavior of two-phase flow depends on the structure of each phase, the linear flow fails when the flow pattern changes. However, when confined in carbon nanotube, the flow pattern remains almost unaltered because of its ultrasmooth surface and perfect molecular structure. We computed the velocity profiles of both phase and estimated the slip length, from which we build a mathematical model to characterize the two-phase flow behavior in shale nanopore. This work, which explores gas-water two phase flow in shale organic and inorganic nanopores, has implications for the development of shale gas but more generally for mass transfer through nanoporous media.

Procter and Gamble Student poster award:

I don't want to compete References:

Acceptance of Terms and Conditions:

Click here to agree

\title{
Data Driven Models Using Machine Learning Methods to Predict Well Productivity in Montney Formations
}

\author{
SHUHUA WANG ${ }^{\text {None }}$; SHENGNAN CHEN ${ }^{\text {None }}$ \\ Corresponding Author(s): shuhwang@ucalgary.ca
}

With the commercial development of the unconventional tight and shale reservoirs, data on geology, well completion, stimulation, and production are also accumulating rapidly. In this talk, data-driven models are built for Montney formation to predict the first year cumulative production and optimize the fracturing parameters, such as the proppant tonnage and the fracturing fluid volume. Data of a total of 4790 horizontal wells in the Montney formation were firstly collected. General data visualization and statistical data analysis are conducted to qualitatively and quantitatively interpret the relationships between the stimulation design strategy and the 12 months well production. Then, 6 features are identified as the most important variables for fractured well performance by using the recursive feature elimination with cross validation (RFECV) method. Based on these features, 
four supervised learning approaches including random forest (RF), adaptive boosting (AdaBoost), support vector machine (SVM), and neural network (NN) are evaluated to predict the first-year oil production in the Montney Formation. Results show that $88.6 \%$ of observations fall into the $90 \%$ prediction intervals, indicating that the developed production forecasting model is reasonable and robust for further fracture stimulation design. Furthermore, the newly developed prediction models are successfully used to identify the optimal range for the mass of proppant and volume of fracturing fluid.

The AI-based fracture design system is of practical useful for reservoir engineers to design the hydraulic fracturing process when drilling a new horizontal well in Montney Formation. Furthermore, such AI-based system can also be used to design fracturing parameters for other Formations as long as corresponding data sets are fed into the system and re-trained the models.

\title{
Procter and Gamble Student poster award:
}

I would like to compete in the Procter and Gamble Student award References:

1 LaFollette, R.R., Holcomb, W.D., Aragon, J., 2012. Impact of completion system, staging, and hydraulic fracturing trends in the Bakken formation of the eastern Willison Basin. In: SPE Hydraulic Fracturing Technology Conference, Woodlands, Texas.

2 Lolon, E., Hamidieh, K., Weijers, L., Mayerhofer, M., Melcher, H., Oduba, O., 2016. Evaluating the relationship between well parameters and production using multivariate statistical models: a middle Bakken and Three Forks case history. In: SPE Hydraulic Fracturing Technology Conference, Woodlands, Texas.

3 Ma, Z., Leung, J.Y., Zanon, S., Dzurman, P., 2015. Practical implementation of knowledge-based approaches for steam-assisted gravity drainage production analysis. Expert Systems with Applications 42, 7326-7343.

4 Schuetter, J., Mishra, S., Zhong, M., LaFollette, R., 2015. Data analytics for production optimization in unconventional reservoirs. In: Unconventional Resources Technology Conference, San Antonio, Texas. 5 Shaheen, M., Shahbaz, M., ur Rehman, Z., Guergachi, A., 2011. Data mining applications in hydrocarbon exploration. Artificial Intelligence Review 35, 1-18. Acceptance of Terms and Conditions:

Click here to agree

\section{Effect of cyclic injection on migration and trapping mechanisms of immiscible fluids in porous media}

\author{
Sookyun Wang ${ }^{1}$; Minhee Lee ${ }^{2}$; Jung Gi Um ${ }^{1}$; Seon-ok Kim² \\ ${ }^{1}$ Pukyong National University \\ ${ }^{2}$ Pukyong National university
}

Corresponding Author(s): sookyun@pknu.ac.kr

In geological $\mathrm{CO} 2$ sequestration, the behavior of $\mathrm{CO} 2$ within a reservoir can be characterized as twophase flow in a porous media. For two phase flow, these processes include drainage, when a wetting fluid is displaced by a non-wetting fluid and imbibition, when a non-wetting fluid is displaced by a wetting fluid. In CO2 sequestration, an understanding of drainage and imbibition processes and the resulting NW phase residual trapping are of critical importance to evaluate the impacts and efficiencies of these displacement process. This study aimed to observe migration and residual trapping of immiscible fluids in porous media via cyclic injection of drainage-imbibition. For this purpose, cyclic injection experiments by applying $\mathrm{n}$-hexane and deionized water used as proxy fluid of scCO2 and pore water were conducted in the two dimensional micromodel. The images from experiment were used to estimate the saturation and observed distribution of n-hexane and deionized water over the course drainage-imbibition cycles. Experimental results showed that n-hexane and deionized water are trapped by wettability, capillarity, dead end zone, entrapment and bypassing during 1st drainageimbibition cycle. Also, as cyclic injection proceeds, the flow path is simplified around the main flow path in the micromodel, and the saturation of injection fluid converges to remain constant. Experimental observation results can be used to predict the migration and distribution of $\mathrm{CO} 2$ and pore water by reservoir environmental conditions and drainage-imbibition cycles. 
Procter and Gamble Student poster award:

References:

Acceptance of Terms and Conditions:

Click here to agree

575

\title{
Application of Machine Learning Ideas to Shale Oil Reservoir Per- meability Estimation
}

\author{
Author(s): Jilong $\mathrm{Xu}^{1}$ \\ Co-author(s): Wendong Wang ${ }^{2}$; Yuliang Su ${ }^{2}$; Han Wang ${ }^{2}$; Shiyuan Zhan ${ }^{2}$ \\ ${ }^{1}$ Chinese University of Petroleum (East China) \\ ${ }^{2}$ China University of Petroleum (East China)
}

Corresponding Author(s): wwdong@upc.edu.cn

\begin{abstract}
It is important to correctly determine the shale oil apparent permeability in the development of shale oil reservoirs. However, due to the extremely low pore size and complex pore network (organic and inorganic), it is difficult to accurately determine the apparent permeability of shale oil by experimental methods. Moreover, because of the limited number of coring, at present, we can only obtain the permeability at the core location. How to theoretically calculate and predict all permeability of the whole reservoir scale is still an unsolved problem. In this study, a stochastic apparent permeability (ALP) model of shale oil was established based on SEM images and nitrogen adsorption test data. The model considers structural parameters including roughness, tortuosity, total organic carbon (TOC), and pore distribution in organic matrix and inorganic matrix. Additionally, in organic pore, the model considers velocity slip and near wall flow, while in inorganic pore, the model incorporates physical adsorption. Then an artificial neural network has been designed that is able to predict the apparent permeability of the formations using the data provided by stochastic model. The results show that the oil molecules in adsorbed layers can imped flow for organic matrix especially the matrix is strong lipophilic, while, if inorganic matrix is strong oleophobic, the large slip length in inorganic pore can significantly increase the ALP. With the increase of TOC content, tortuosity and roughness, the ALP all decrease. The input data used in the training include SEM images, nitrogen adsorption test data, structural parameters, and oil properties such as viscosity and density. Trained models were developed using the data and used to predict ALP was not part of the training. The results also indicate that when enough valid data is obtained, machine learning techniques offer promise for formation permeability estimation.
\end{abstract}

Procter and Gamble Student poster award:

I don't want to compete References:

Acceptance of Terms and Conditions:

Click here to agree

174

\section{An research and application of yield variation law of fractured wells in tight oil straight well $\searrow$ A case of the Daqing Oilfield, China}

Author(s): zhengming yang ${ }^{1}$; Xinli Zhao ${ }^{\text {None }}$; Ting Chen $^{2}$; Yutian Luo ${ }^{\text {None }}$

Co-author(s): Wenming Wang ; Lei Ren 


\footnotetext{
${ }^{1}$ PetroChina Research Institute of Petroleum Exploration \& Development

${ }^{2}$ University of Chinese Academy of Sciences
}

Corresponding Author(s): ,

Using the reservoir engineering method, we established a mathematical model for the production capacity of straight well seam fracturing wells in tight reservoirs considering the sorption effect. The flow patterns of straight wells under the conditions of joints were analyzed, and the seepage and crack complexity were analyzed for the impact of capacity. Studies have suggested that the osmotic action accelerates the recovery of crude oil in the matrix of the pressure zone. The stronger the osmotic effect, the more complicated the fracture network, the larger the fracturing scale and the higher the yield. On this basis, combined with the production status of the fractured wells of 10 vertical wells in Daqing Oilfield, the production curve of cumulative production and dimensionless production was established. The contribution of cracks and matrix to productivity was analyzed, and different factors were analyzed. We also analyzed the effect of fracturing scale, matrix permeability, water content, initial productivity and fracturing well location on the fracturing effect of straight seams. The research suggested that in the tight oil block, the production decline of the fracturing wells in the seam network is in line with the exponential decline. After 12 months, the output is reduced to $50 \%$ of the initial output, and the substrate yield accounts for $20 \%$ of the maximum output. Fracturing scale and water content are the decisive factors affecting the fracture fracturing capacity. The initial capacity is the stable production time of the fracturing wells and the matrix permeability is the later production capacity of the fracturing wells. In tight oil areas, only seam-controlled reserves can be effectively developed.

Procter and Gamble Student poster award:

References:

Acceptance of Terms and Conditions:

Click here to agree

13

\title{
Pore Scale Investigating of Multi-phase Flow in Tight Reservoirs
}

\author{
Xiukun Wang ${ }^{1}$; James Sheng ${ }^{2}$ \\ ${ }^{1}$ China University of Petroleum(Beijing) \\ ${ }^{2}$ Texas Tech University \\ Corresponding Author(s): xiukunwang@cup.edu.cn
}

Multiphase flow is commonly encountered within tight reservoirs, however, the previous studies are more focused on single phase flow. Due to the extremely low permeability of tight formations, the experimental measurements are not capable to be performed. Attempts are made by applying the techniques of digital rock analysis and pore network modeling to investigate the multiphase flow mechanisms in tight reservoirs. This work follows this way. Firstly, a stochastic pore network generation algorithm and a two-phase flow simulation method are presented. This algorithm is applied and validated in Berea sandstone. By applying the introduced algorithm, the micro and macro pore networks are generated for tight formations, then the multi-scaled pore network is established after upscaling the micro pores to a micro-patch. Finally, the oil-water flow is simulated within this established multi-scaled pore network and some analysis and discussions are conducted. The results imply that the characteristics of pore systems in tight formations pose an extreme impact on the multi-phase flow mechanisms, especially the wettability of micro-pores and the pore-throat aspect ratios.

Procter and Gamble Student poster award:

\section{References:}

Acceptance of Terms and Conditions: 


\title{
CO2-Induced Structure Alteration of Oil Well Cement: a Micro CT Study
}

\author{
Yan $\mathrm{WANG}^{1}$; Liwei Zhang ${ }^{2}$; Xiuxiu Miao ${ }^{3}$; Xiaochun $\mathrm{LI}^{1}$ \\ ${ }^{1}$ State Key Laboratory of Geomechanics and Geotechnical Engineering, Institute of Rock and Soil Mechanics, Chinese \\ Academy of Sciences \\ ${ }^{2}$ Institute of Rock and Soil Mechanics, Chinese Academy of Sciences \\ ${ }^{3}$ Wuhan Institute of Soil and Rock Mechanics, Chinese Academy of Sciences
}

Corresponding Author(s): ywang@whrsm.ac.cn

Wellbore cement integrity under $\mathrm{CO} 2$ geologic storage (CGS) conditions is a key factor to assure safe and permanent storage of $\mathrm{CO} 2$. Wellbore cement integrity may be impaired and the structure of cement may be altered as a result of $\mathrm{CO} 2$ attack. To understand how $\mathrm{CO} 2$-induced structure alteration in oil well cement under CGS conditions affects well integrity in CGS projects, this paper reports an experiment of reaction between $\mathrm{CO} 2$ and oil well cement under CGS conditions. Samples were scanned by Micro CT before and after reaction. The Micro CT is capable of operating at $140 \mathrm{KV}$ and $10 \mathrm{~W}$, has a maximum resolution of $10 \mu \mathrm{m}$. To simulate the reaction between $\mathrm{CO} 2$ rich brine and oil well cement at CGS conditions, our team has developed a testing system which provides the storage temperature and pressure.

The samples were made by standard class G oil well cement used for CGS pilot projects. The cement was cured at $\mathrm{CO} 2$ storage formation conditions: $62^{\circ} \mathrm{C}, 17 \mathrm{MPa}$, and $1 \mathrm{wt} \% \mathrm{NaCl}$ solution. The curing was maintained for 14 days. The diameter of the samples was $10 \mathrm{~mm}$. Every sample contained a small borehole at center (around $1 \mathrm{~mm}$ diameter) that made the samples suitable for examining seepage through small leakage pathways within cement. During the reaction experiment, the samples were placed in the high-pressure, high-temperaure testing system for 14 days, given a temperature of $62^{\circ} \mathrm{C}$ and a CO2 partial pressure of $17 \mathrm{MPa}$. The goal of this experiment is to evaluate how the geochemical reactions between dissolved $\mathrm{CO} 2$ and cement affect structure of the cement. Change of borehole geometry was not observed in the Micro CT images. However, a region with decreased porosity around the borehole due to $\mathrm{CaCO} 3$ precipitation and a region with increased porosity around the borehole due to $\mathrm{Ca}(\mathrm{OH}) 2$ and $\mathrm{C}-\mathrm{S}-\mathrm{H}$ dissolution were observable. Initial distribution of cementitious materials and solution buffering governed the width of the high-porosity region and $\mathrm{CaCO} 3$ precipitation region. This study demonstrates a 3-D sample characterization technique that can be used to investigate $\mathrm{CO} 2$-induced structure alteration of oil well cement.

Procter and Gamble Student poster award:

References:

Acceptance of Terms and Conditions:

Click here to agree

\section{High-enthalpy Geothermal Simulation with Multi-level Physics Parameterization}

Author(s): Yang Wang ${ }^{1}$

Co-author(s): Denis Voskov ${ }^{2}$; David Bruhn ${ }^{3}$

${ }^{1}$ Department of Geoscience Engineering, TU Delft 


\footnotetext{
${ }^{2}$ Department of Geoscience Engineering, TU Delft; Department of Energy Resources Engineering, Stanford University

${ }^{3}$ Department of Geoscience Engineering, TU Delft; Helmholtz Centre Potsdam, GFZ German Research Centre for Geosciences
}

Corresponding Author(s): y.wang-18@tudelft.nl

When modeling the process of cold water injection into a steam-saturated high-enthalpy geothermal reservoir, numerical simulation often experiences convergence issues when target time step needs to be reduced. The key point behind this behavior is the volume reduction due to the condensation process which can temporarily decrease grid-block pressure in a particular range of physical and numerical parameters. This phenomenon is commonly known as 'negative compressibility'. As a consequence, this effect creates a region in the solution space where the gradient of the residual governing equations does not point to the direction of the solution. As the consequence, if the initial guess (which is often taken from the solution at previous time-step) is located in this region, the nonlinear iterations based on Newton-Raphson approach will guide the solution towards the wrong direction which prevents the convergence at the targeted timestep. Therefore, the time-step should be severely restricted in the simulation process. To tackle this problem, we propose the continuation method with multi-level physics using Operator-Based Linearization (OBL) technique. In OBL approach, the continuous operators in the governing equations, related to different physical mechanisms (e.g. convection or conduction), are translated into multidimensional tables. In the course of the simulation, only the supporting points are evaluated based on the reference physics while points between them are interpolated. This provides a unique mechanism where nonlinear physics can be represented at different levels of accuracy. The coarser the representation is, the more linear the operators become. In our continuation approach, we start with the coarse approximation of the governing physics in pressure-enthalpy solution space. Due to a more linear form of operators, the negative compressibility effect is absent and only a few nonlinear iterations reduce residual below the predefined tolerance. Next, the OBL approximation is modified towards the reference nonlinear physics by increasing resolution in OBL tables. With the refinement in physics, the solution will gradually approach the final results where residual will satisfy the convergence criteria of the reference physics. As the result, the total number of timesteps and nonlinear iterations is significantly reduced in comparison to conventional simulation with fine resolution physics.

Procter and Gamble Student poster award:

I would like to compete in the Procter and Gamble Student award References:

Acceptance of Terms and Conditions:

Click here to agree

899

\title{
Dynamic Effects of Capillary Number on Relative Permeability of Porous Sandstone and Carbonate Rocks
}

\author{
Ying Da Wang ${ }^{1}$; Traiwit Chung ${ }^{\text {None }}$; James McClure ${ }^{2}$; Ryan Armstrong ${ }^{\text {None }}$; Peyman Mostaghimi ${ }^{\text {None }}$ \\ ${ }^{1}$ UNSW \\ ${ }^{2}$ Virginia Tech
}

Corresponding Author(s): yingdawang56@gmail.com

The comparative ability for phases to flow in the presence of each other in multiphase porous media is best described by the relative permeability. It is used as an upscaled parameter from microscopic pore scale flow to the macroscale Darcy flow of practical porous systems. Traditionally measured at low capillary numbers that result in a static, flow independent relative permeability, the impact of the dynamic nature of relative permeability at intermediate capillary numbers is underexplored and underused. This is becoming increasingly important to characterise as natural gas reservoirs and EOR techniques such as polymer flooding become more prolific. We apply Lattice Boltzmann Methods for Porous Media (LBPM) to numerically determine the dynamic effect that capillary number has on the relative permeability of sandstone and carbonate rocks imaged by micro-computed tomography (micro-CT). By applying a morphologically adaptive simulation algorithm, relative permeability curves are reliably obtained numerically within 24 hours for 600-cubed domains, run with 
$1000 \mathrm{CPU}$ cores. We show results that match with laboratory experiments, and the dynamic relative permeability curves are used to generate a type curve extension to Corey parameters for reservoir scale simulations. We run case studies with a modified IMPES routine, showing differences in production profiles and project NPV and expect the application of dynamic relative permeability models in reservoir simulation to continue gaining relevance.

Procter and Gamble Student poster award:

I don't want to compete References:

Acceptance of Terms and Conditions:

Click here to agree

973

\section{Derivation of new water drive characteristic curve formula for ultra high water-cut oilfield}

Yingsheng Wang ${ }^{1}$

${ }^{1} 17611452667$

Corresponding Author(s): 511970934@qq.com

Currently part of the domestic water drive reservoir has entered the stage of extra high water cut ,for which, the ratio of relative permeability and water saturation relationship curves upward happens, this also lead to water drive characteristic curve upward. So using the conventional water flooding characteristic curve to forecast the actual oil field production will produce large deviation. So this article is based on the actual data of oil field, analyzing a number of the back part of the ratio of relative permeability and water saturation curve upward from different oil block, gives a new expression of the ratio of relative permeability and water saturation, at the same time, the author deduces the new water drive characteristic curve with the new expression of the ratio of relative permeability and water saturation, and applied it in the actual oil field production, the results show that the new water drive characteristic curve can predict the oil production well for the back part of conventional water flooding characteristic curve, it has good applicability for the extra high water cut stage forecasting.

Procter and Gamble Student poster award:

References:

Acceptance of Terms and Conditions:

Click here to agree

190

\section{A semi-analytical interpretation model of pulse decay measure- ments on ultra-tight rocks}

Author(s): Yue Wang ${ }^{1}$

Co-author(s): Steffen Nolte ; Garri Gaus ; Alexandra Amann ; Bernhard Krooss ; Moran Wang ${ }^{1}$

${ }^{1}$ Tsinghua University

Corresponding Author(s): wangyue14thu@163.com

Unconventional natural gas has become an increasingly important energy source in recent years and attracted active research and development accordingly. One key problem in unconventional natural 
gas reservoir exploitation is the determination of viability for commercial production, where permeability is a critical parameter. The pulse decay method is one of the most widely-used experimental methods for permeability measurements and various interpretive models have been established to process the recorded pressure data. However, most models only evaluate the late stage of a pressure pulse decay test. This makes the measurements very time-consuming and nearly impracticable for ultra-tight rocks like shales.

In this contribution we present a new semi-analytical model aimed at the determination of apparent gas permeability coefficients of ultra-tight rocks. This method is derived from the unidirectional unsteady porous flow equation, incorporating the consideration of gas compressibility and slippage effects. Unlike previous models, this analytical interpretation accounts for the early stage data of pulse decay test, namely the time before significant pressure increase is observed on the downstream pressure side.

In order to validate the proposed method, measurements were performed on a core sample of the Cretaceous Eagle Ford shale, Texas, USA, under different pore and confining pressures. Helium was used as the test fluid to minimize the effects of gas adsorption on the pore walls. Permeability coefficients obtained from this new approach agree well with those from the classical pulse decay evaluation while the duration of the tests was reduced from hours to minutes. The model presented is a good supplement for the pulse decay method and suitable for the measurement of ultra-low permeability coefficients, down to the nanodarcy range.

Procter and Gamble Student poster award:

I would like to compete in the Procter and Gamble Student award References:

Acceptance of Terms and Conditions:

Click here to agree

387

\title{
Disorder characterization of porous media and its effect on fluid displacement
}

\author{
Zhongzheng Wang ${ }^{1}$; Yixiang Gan ${ }^{1}$; Kapil Chauhan ${ }^{1}$ \\ ${ }^{1}$ The University of Sydney \\ Corresponding Author(s): zwan4662@uni.sydney.edu.au
}

Most porous media have intrinsic random pore structure. A modified disorder index I_v is proposed here to characterize the disorder of the media. Different displacement patterns (stable displacement and fingering) under the same flow condition and fluid property are obtained by simulation using Lattice Boltzmann method. We analytically demonstrate how increase in disorder promotes fingering due to the uneven distribution of local capillary pressure. It is shown that the displacement efficiency under different wetting conditions and disorder well correlates with the distribution of capillary pressure. A power law relation between the fluid-fluid interfacial length and saturation of invading fluid is proposed by taking geometry into account. The parameters in the power law relation can be predicted by a single index, the capillary index I_c, which captures the combined effects of the topology disorder and wettability.

Procter and Gamble Student poster award:

\section{References:}

\section{Acceptance of Terms and Conditions:}

Click here to agree 


\title{
Reduced Complexity Modelling of $\mathrm{CO}_{2}$ Leakage Through Faults
}

Francesca Watson $^{1}$; Mary Kang ${ }^{2}$; Florian Doster $^{\text {None }}$; Mike Celia ${ }^{\text {None }}$; Jan Martin Nordbotten ${ }^{3}$

\author{
${ }^{1}$ SINTEF Digital \\ ${ }^{2}$ McGill University \\ ${ }^{3}$ University of Bergen
}

Corresponding Author(s): francesca.watson@sintef.no

Quick and efficient numerical simulations are required for risk analysis and characterisation of $\mathrm{CO}_{2}$ storage sites. Vertical equilibrium (VE) modelling is an efficient form of reduced complexity modelling. It exploits the fact that in typical $\mathrm{CO}_{2}$ storage scenarios $\mathrm{CO}_{2}$ floats on resident brine due to buoyancy. This allows us to reduce the explicitly represented dimensions from 3 to 2 by modelling horizontal flow in the reservoir numerically and then reconstructing the vertical configuration of fluids analytically. However it is not possible to model vertical flow in VE models and this might be important if injected $\mathrm{CO}_{2}$ encounters a leaking vertical fault. We have developed an analytical solution for fault zone leakage which can be included in VE models. Fine scale simulations have been carried out using the Matlab Reservoir Simulation Toolbox (MRST) to model two-phase leakage of $\mathrm{CO}_{2}$ and brine through a fault zone. Results have then been used to develop an explicit model relating fault geometry, total fault leakage rate and $\mathrm{CO}_{2}$ layer thickness with $\mathrm{CO}_{2}$ leakage rate through the fault. This leakage rate can be included as a sub-grid scale source term in the VE modelling framework, thus allowing us to quickly model many realisations of $\mathrm{CO}_{2}$ injection into faulted reservoirs.

Procter and Gamble Student poster award:

References:

Acceptance of Terms and Conditions:

Click here to agree

\section{Fast artificial $\mu \mathrm{CT}$-scans from 3D-structure models using Gaus- sian Random Fields}

Andreas Weber $^{\text {None }}$; Andreas Wiegmann ${ }^{1}$; Nicolas Harttig ${ }^{2}$; Christian Wagner ${ }^{2}$

\footnotetext{
${ }^{1}$ Math2Market $\mathrm{GmbH}$

${ }^{2}$ math2market
}

Corresponding Author(s): andreas.weber@math2market.de

The measurement of segmentation quality is a challenge in 3D-image processing of e.g. micro computed tomography $(\mu \mathrm{CT})$ scans or FIB-SEM images. A precise evaluation of the results of segmentation (or postprocessing) is often not possible because level of noise, signal strength, and position of image artifacts are unknown in real 3D $\mu$ CT-scans. A common method to overcome this challenge is to examine a ground truth data set, where artificial noise and artifacts are added. The source of the ground truth data set can either be a generated 3D-model of a structure or segmented 3D-images from a real $\mu \mathrm{CT}$-scan.

Recently, different ways of simulating 3D-images from real $\mu \mathrm{CT}$-scans have been introduced, often using a tomographic forward projection to obtain the x-ray projection images. Noise and other artifacts are added on the projection space images to reproduce those found in real images obtained from $\mu \mathrm{CT}$ scanners. Then, these images are reconstructed, resulting in typically defective $3 \mathrm{D} \mu \mathrm{CT}$ scans with well-known ground truth.

Here, we present a different technique to generate these artificial $3 \mathrm{D} \mu \mathrm{CT}$-scans starting from a 3D structure model using GeoDict ${ }^{\circledR}$. Instead of back-transformation to the projection space, this technique uses Gaussian Random Fields (GRF) and Euclidean Distance Transform (EDT). Common $\mu \mathrm{CT}$-scan artifacts, such as beam hardening, are added by modifying gray values based on the EDT 
of solid and pore space voxels. Typical image noise in 3D $\mu$ CT-scans is achieved by adding a GRF, which can be parametrized to generate fine, coarse, and multi-level noise. Other artifacts, such as stripes and shadows, are simulated by applying certain FFT filters.

If a real $\mu \mathrm{CT}$-scan is available for comparison, the generated artificial $\mu \mathrm{CT}$-scan can be modified further to resemble the original as exact as possible. This is accomplished by histogram matching of the generated $\mu \mathrm{CT}$-scan, such that the same gray value distribution is achieved.

The use of structure generation like in GeoDict to obtain the artificial $\mu \mathrm{CT}$-scans yields the possibility to alter one parameter while keeping others unchanged. For example, in a generated granular structure model, the positions of the grains can be randomly modified while keeping the overall solid volume fraction and/or tortuosity of the structure unchanged. In this way, a large set of similar artificial 3D $\mu \mathrm{CT}$-scans can be generated to optimize image segmentation workflows or to train neuronal net segmentation algorithms.

Further, our approach is not limited to generating $\mu \mathrm{CT}$ scans and could be used for other types of image acquisition methods, such as FIB-SEM or Confocal Microscopy.

Procter and Gamble Student poster award:

References:

Acceptance of Terms and Conditions:

Click here to agree

179

\title{
The critical factor for relationship between permeability and for- mation factor of porous media: Body-to-throat ratio, tortuosity and connectivity
}

\author{
Author(s): Wei Wei ${ }^{\text {None }}$ \\ Co-author(s): Jianchao Cai ; Yuxuan Xia \\ Corresponding Author(s): weiw2015@gmail.com
}

Characterizing transport characteristics of porous media becomes a significant role in a wide range of geophysical fields. It is significant and valuable for the exploitation of petroleum resources, water resources and geothermal energy. An analytical permeability model of pore-throat ratio is established with capillary bundle fractal model in this work. The model combines with permeability and electrical conductivity to explore the function of pore size and pore-throat size. Furthermore, a novel method with tortuosity and connectivity to characterize permeability of porous media. The pore structural factors information is extracted and exactly express the influence for permeability. The results are agreement with permeability-formation factor model. Considering these deficiencies, the final part provides some advices for the further study on the relationship between electrical conductivity and hydraulic properties.

Procter and Gamble Student poster award:

References:

Acceptance of Terms and Conditions:

Click here to agree

920

\section{Numerical simulations of turbulent flows and heat transfer in regular porous structures}

Bernhard Weigand ${ }^{1} ;$ Yang $^{\text {None }} ; \mathrm{Chu}^{\text {None }} ;$ Helimg $^{\text {None }}$ 
${ }^{1}$ University of Stuttgart

Corresponding Author(s): bernhard.weigand@itlr.uni-stuttgart.de

Porous media can be found in nature with a broad range of geometrical characteristics and are also involved in many industrial and scientific applications with simplified regular structures. Such regular porous structures receive increasing importance because of manufacturing techniques based on selective laser melting. In several industrial applications, the pore-scale Reynolds number exceed the critical Reynolds number (which is about 300) and the flow in the porous structure is found to be turbulent. For such applications, the pore-scale flow and heat and mass transfer behavior is still not well understood. Here Direct Numerical Simulations (DNS) help to understand the complex flow features. For example, for a regular porous medium, consisting of square cylinders, DNS can be used for a very detailed flow analysis. In such investigations, it was found e.g. by anisotropy analysis using a barycentric map that the turbulence in porous media is highly isotropic in the macro-scale, but not in the micro-scale. After looking into the microscopic behavior, it is also very important to investigate the interaction between a porous structure and a free-flow. For example, the flow in a T-junction with a flow vertically discharged through a structured porous medium can be analyzed numerically. For such larger domains of course DNS would not be appropriate for case studies. Thus, Reynolds averaged Navier-Stokes (RANS) methods can be used. For such investigations, it is of particular interest to study the interface between porous structure and free channel flow. Numerical investigations can help in such configurations to understand e.g. the limitations of the Beaver-Joseph interface conditions and can help to find ways to improve it. This will then help improving models based on REVs to better capture the link between porous structures and free flows.

Procter and Gamble Student poster award:

I don't want to compete References:

Acceptance of Terms and Conditions:

Click here to agree

\title{
A pore-scale approach to couple mass, momentum and energy at the interface between free flow and porous-medium flow
}

\author{
Author(s): Kilian Weishaupt ${ }^{1}$ \\ Co-author(s): Timo Koch ; Vahid.J Niasar ; S. Majid Hassanizadeh ${ }^{2}$; Rainer Helmig ${ }^{1}$ \\ ${ }^{1}$ University of Stuttgart \\ ${ }^{2}$ Utrecht University
}

Corresponding Author(s): kilian.weishaupt@iws.uni-stuttgart.de

Systems of coupled free flow and porous-medium flow appear ubiquitously in nature and in technical applications. Examples for interface-driven transport and exchange processes include soil evaporation, fuel cell water management or food drying.

A pore-scale description of these systems (e.g. by means of DNS) is very often unfeasible due to the highly complex geometry of the porous material.

Averaged REV-scale approaches often fail to describe the region

close to the interface to the free flow in sufficient detail which might be required to account for porescale effects pore-scale effects like local saturation distribution patterns that can strongly affect the global behavior of the coupled system.

Here, we propose a novel hybrid model that is able to capture pore-scale effects at the interface in ample detail while maintaining a comparatively low computational effort which allows the simulation of rather realistic scenarios. The key feature of this approach is a pore-network model which represents the transition region between the porous matrix and the free flow. The model comprises three computational domains: the free-flow region where the (Navier-) Stokes equations are solved, the transition zone described by the pore-network model and a bulk porous domain accounted for 
by Darcy's or Forchheimer's law. Appropriate coupling conditions ensure the continuity of mass, momentum and energy fluxes as well as thermodynamic consistency between the respective subdomains.

Procter and Gamble Student poster award:

I would like to compete in the Procter and Gamble Student award References:

Acceptance of Terms and Conditions:

Click here to agree

\title{
Controlling the mechanics of nanoporous metals by adsorption and superficial electric charging
}

Jörg Weissmüller ${ }^{1}$

${ }^{1}$ Hamburg University of Technology and Helmhotz-Centre Geesthacht

Corresponding Author(s): weissmueller@tuhh.de

Nanoporous metals may rightly be considered wallflowers in a symposium focusing on natural materials, concrete and the like. Yet, these materials exhibit a rich bunch of phenomena in the field of adsorption-induced deformation phenomena. This is partly the result of the electric conductivity of metals. When the pore spaces filled with aqueous electrolyte, the resulting metal-water hybrid nanomaterials contain two separate electric conduction channels - electrons in the metal, ions in the electrolyte - that are capacitively coupled along the entire pore surface and that afford a precise and reversible control of the state (adsorption, electric charging) of the pore surfaces. This enables the operando control of many of the macroscopic materials properties 1 . With respect to mechanics, it is possible to control the external dimensions, the stiffness, and the mechanical strength of samples. Furthermore, the sorption-strain coupling can be inverted - external loads then create electric signals. These signals are unusually robust, suggesting that metals can be effectively turned into efficient piezoelectrics by incorporating them into a hybrid nanomaterials design strategy. Appealing scientific issues also live in the nanoscale self organization processes behind making nanoporous metal by dealloying, and in the coarsening phenomena that underlie the opportunities for precisely tuning the structure size between $5 \mathrm{~nm}$ and $500 \mathrm{~nm}$. The talk will provide an overview over this active field.

Procter and Gamble Student poster award:

\section{References:}

1 J. Weissmüller, K. Sieradzki, Dealloyed nanoporous materials with interface-controlled behavior. MRS Bulletin 43 (2018) 14. Acceptance of Terms and Conditions:

Click here to agree

\section{Microbial processes that allow pollutant biodegradation in toxic and inaccessible micro-environments within porous media}

\author{
Author(s): Charles J Werth ${ }^{\text {None }}$ \\ Co-author(s): Kyle Michelson ; Reinaldo E. Alcalde ; Lang Zhou ; Robert A. Sanford ; Albert Valocchi ${ }^{1}$ \\ ${ }^{1}$ Univ Illinois
}


Corresponding Author(s): werth@utexas.edu

Pollutants and metal oxides sequestered deep within narrow pores too small for cell access, and in toxic microenvironments deemed inhabitable, are often thought to be inaccessible to microbial degradation. However, dissimilatory metal reducing bacteria have evolved the capacity for extracellular electron transfer. In this presentation, I show results demonstrating diffusion based electron shuttling between the bacterium Shewanella oneidensis MR-1 and a metal oxide across a physical separation created by a nanoporous barrier in a microfluidic reactor. Real-time quantification of electron flux across this barrier by MR-1 strains with different electron transfer capabilities revealed that this bacterium exports flavins to its surroundings when faced with no direct physical access to an electron acceptor, allowing it to reduce metals via diffusion at distances exceeding 60 microns. An energy balance indicates flavins must be recycled for S. oneidensis MR-1 to yield energy from lactate oxidation coupled to flavin reduction. Both experimental and modeling results indicate that flavins are recycled at least 24 times, depending on flow conditions. This energy saving strategy, which until now had not been systematically tested or captured in environmentally relevant systems, suggests that electron shuttling microorganisms have the capacity promote bioremediation in physically distant or potentially toxic microenvironments.

Procter and Gamble Student poster award:

References:

Acceptance of Terms and Conditions:

982

\section{Scalable Solvers for Multiphase Poromechanics}

Joshua White $^{1}$; Nicola Castelletto $^{\text {None }}$; Hamdi Tchelepi $^{\text {None }}$; Sergey Klevtsov ${ }^{\text {None }}$; Quan M. Bui ${ }^{\text {None }}$; Daniel OseiKuffuor ${ }^{\text {None }}$

${ }^{1}$ Lawrence Livermore National Lab., USA

Corresponding Author(s): white230@llnl.gov

Many applications involving porous media-notably reservoir engineering and geologic applicationsinvolve tight coupling between multiphase fluid flow, transport, and poromechanical deformation. While numerical models for these processes have become commonplace in research and industry, the poor scalability of existing solution algorithms has limited the size and resolution of models that may be practically solved. In this work, we propose a multistage Newton-Krylov solution algorithm to address this shortfall. The proposed solver exhibits rapid convergence, good parallel scalability, and is robust in the presence of highly heterogeneous material properties. The key to success of the solver is a block-preconditioning strategy that breaks the fully-coupled system of mass and momentum balance equations into simpler sub-problems that may be readily addressed using targeted algebraic methods. Numerical results are presented to illustrate the performance of the solver on challenging benchmark problems.

Procter and Gamble Student poster award:

References:

Acceptance of Terms and Conditions:

Click here to agree

\section{AI-based identification of binder and fibers in 3D images of non- woven}

Author(s): Andreas Wiegmann ${ }^{1}$ 
Co-author(s): Andreas Grießer ${ }^{2}$; Hans Hagen ${ }^{3}$; Christian Wagner ${ }^{1}$; Rolf Westerteiger ${ }^{1}$

\author{
${ }^{1}$ Math2Market GmbH \\ ${ }^{2}$ Math2Market \\ ${ }^{3}$ TU Kaiserslautern
}

Corresponding Author(s): andreas.wiegmann@math2market.de

Fibrous structures are present in many materials, including nonwoven media used for filtration, nonwoven in diapers, or gas-diffusion layers used in fuel cells. An important characteristic of nonwoven media is how binder is distributed in them. Resolving this question can be tackled by using 3D imaging techniques such as $\mu \mathrm{CT}$-scans or synchrotron radiation scans. One crucial issue is that the binder often has the same gray values in images as the fibers and thus, cannot be identified using global thresholding.

Here, we present an alternative technique using machine learning to identify the binder through the shape of the solids in the 3D image. The method works on segmented images but is currently being extended to work directly on gray value images.

The idea of the method is to start by using the built-in structure generation capability of the GeoDict ${ }^{\circledR}$ software to create realistic 3D structure models of gas diffusion layers. In these models, every individual fiber and the location of the binder are known exactly through the entered generation parameters. In this manner, pairs of images with individual fibers and binder in separate colors vs. images with only solid and empty information can be used to train neural networks to identify the detailed information in the black and white images.

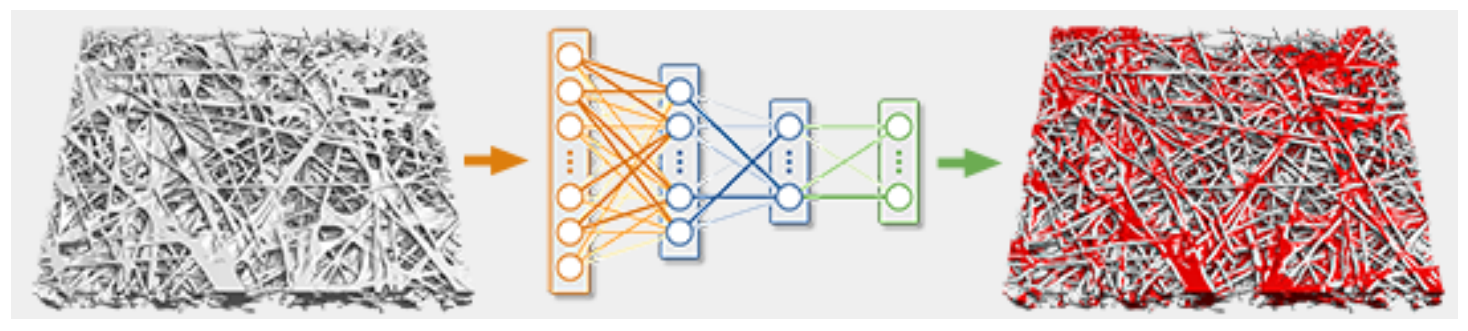

Figure 14: Figure: Setup for training and using neural networks. Left: binder and fibers have the same gray value. Middle: neural network. Right: binder and fibers have different labels.

The figure presents how, in the training phase, the neural network learns from the realisticallylooking generated or artificial images. In the training phase, both the image on the left and the image on the right are generated with GeoDict ${ }^{\circledR}$. The same figure also illustrates how during the usage phase the neural network predicts the image on the right from the input of the segmented image on the left. For the usage phase, the segmented image is obtained from a $\mu \mathrm{CT}$ scan or synchrotron radiation scan.

The talk describes the models for the fibers and anisotropic binder distribution, as well as the choice of topology of the neural networks, the validation of the quality of the determination of the binder for models. Finally, the talk proceeds to show the use of machine learning to identify the individual fibers in the scans.

Procter and Gamble Student poster award:

References:

Acceptance of Terms and Conditions:

Click here to agree

\title{
Time-Correlation Functions of Immiscible Two-Phase Flow in Porous Media
}


Mathias Winkler ${ }^{1}$; Magnus Aashammer Gjennestad ${ }^{1}$; Dick D. Bedeaux ${ }^{2}$; Raffaela Cabriolu ${ }^{3}$; Signe Kjelstrup ${ }^{2}$; Alex Hansen ${ }^{1}$

${ }^{1}$ Porelab, Department of Physics, Norwegian University of Science and Technology - NTNU, Trondheim, Norway

${ }^{2}$ Porelab, Department of Chemistry, Norwegian University of Science and Technology - NTNU, Trondheim, Norway

${ }^{3}$ Department of Chemistry, Norwegian University of Science and Technology - NTNU, Trondheim, Norway

Corresponding Author(s): mathias.winkler@ntnu.no

In a steady state of immiscible two-phase flow in porous media, the instantaneous flow rate undergoes fluctuations around a steady mean. These fluctuations are due to the constantly changing saturation in the individual pores of the porous media and the random distribution of pore sizes. Such a situation has much in common with equilibrium and steady states in atomistic statistical mechanics where fluctuations around the steady mean arise from random thermal motion.

Linear response theory implies that the decay of such microscopic fluctuations is governed by the same physics as the relaxation of a macroscopic system driven out of equilibrium. Thus, one can calculate transport coefficients from time-correlation functions of the equilibrium system via the Green-Kubo method. The latter finds application in not only equilibrium and steady state situation but can be extended to stationary systems with slowly decaying power law correlations and even to aging system 1. It appears that time-correlation functions of multiphase flow in porous media have not been studied in this context, which may open a novel route to determine transport properties of such flows.

In this study, we use a periodic network model 2 to investigate the behavior of time-correlations function of the flow rates and velocity in steady state. We show that such time-correlation functions do indeed converge and that the transport matrix is symmetric, thus obeying the Onsager relations. These findings imply that the assumption of local equilibrium is valid and that the framework of non-equilibrium thermodynamics applies to steady state immiscible two-phase flow. The findings therefore support a recent effort to extend non-equilibrium thermodynamics to flow in porous media 3.

\title{
Procter and Gamble Student poster award:
}

\section{References:}

1 A. Dechant, E. Lutz, D. A. Kessler and E. Barkai, "Scaling Green-Kubo Relation and Application to Three Aging Systems”, Phys. Rev. X 4 (2014) 011022.

2 M. Aa. Gjennestad, M. Vassvik, S. Kjelstrup, A. Hansen, "Stable and efficient time integration of a dynamic pore network model for two-phase flow in porous media", Frontiers in Physics 6 (2018), Doi:10.3389/fphy.2018.00056.

3 S. Kjelstrup, D. Bedeaux, A. Hansen, B. Hafskjold, O. Galteland, "Non-isothermal Transport of Multiphase Fluids in Porous Media. The Entropy Production”, Frontiers in Physics 6 (2018), Doi:10.3389/fphy.2018.00126

Acceptance of Terms and Conditions:

10

\section{Taylor Dispersion: Evolution from the Initial Condition}

\author{
Brian Wood $^{1}$; Ehsan Taghizadeh ${ }^{1}$; Fransisco Valdés-Parada ${ }^{2}$ \\ ${ }^{1}$ Oregon State University \\ ${ }^{2}$ Departamento de Ingeniería de Procesos e Hidráulica. Divisíon de ciencias básicas e ingeniería. Universidad Autónoma \\ Metropolitana-Iztapalapa
}

Corresponding Author(s): brian.wood@oregonstate.edu

After nearly 65 years of research on Taylor dispersion and literally thousands of papers on the subject, it is reasonable to ask if there is anything interesting left to be said about it. However, there still remains one outstanding problem in Taylor dispersion theory that has resisted a very satisfactory theoretical description. This is the problem of the relaxation of the initial condition at so-called 
"early" times in the Taylor dispersion process. Although a number of methods have been proposed, the constraints on the results are usually quite severe (e.g., asymptotic expansions that are only valid for exceptionally small times, or formulations that apply only to particularly simple configurations).

In this talk, we will discuss some of the history of this problem, and recent results that have been obtained by our group for describing the full range of the transport process from early to asymptoticallylong times. Our approach is based in conventional PDE theory, and a somewhat unconventional approach to averaging. In particular, we derive an effective mass balance evolution equation not scale invariant upon averaging. The averaged equation contains an exponentially decaying (in time) source term that does not appear in the original microscale balance. The role of this source term is to account for the relaxation of the initial condition by redistributing mass within the domain (hence, the integral of the source term over the entire domain is zero). This leads to a final result that is able to represent spatially non-symmetric distributions of the average concentration at early times, and approaches the conventional Taylor-Aris result at asymptotic times. We compare the results of our upscaled representation with those computed from direct numerical simulation at the microscale; good fidelity between the two methods is observed. The approach has interesting applications to other kinds of systems where upscaling in the proximity to specific initial configurations (e.g., reactive transport; ecological dynamics) is important.

Procter and Gamble Student poster award:

References:

Acceptance of Terms and Conditions:

Click here to agree

\title{
Hydrogel-Enhanced EICP Application to Fugitive Dust Mitigation: Impacts on Surface Permeability and Erosion Resistance
}

\author{
Author(s): Miriam Woolley ${ }^{1}$ \\ Co-author(s): Edward Kavazanjian ${ }^{1}$; Leon van Paassen ${ }^{1}$ \\ ${ }^{1}$ Arizona State University
}

Corresponding Author(s): mawoolle@asu.edu, leon.vanpaassen@asu.edu

Enzyme Induced Carbonate Precipitation (EICP) has been shown to be an effective means of suppressing fugitive dust through wind tunnel testing. In EICP, agriculturally derived urease enzyme catalyzes the hydrolysis of urea to induce the precipitation of calcium carbonate in the presence of calcium ions in an aqueous solution. The EICP solution is sprayed on the soil surface to form a crust that is resistant to wind erosion. In coarse-grained granular soils, the EICP solution penetrates the surface too quickly to develop a well-defined crust. Bench scale testing showed that addition of a hydrogel (a hydrophilic polymer) to the EICP solution slowed the penetration rate and enhanced formation of the crust on the surface of a granular soil. Additional benefits include reducing water loss (and increasing reaction time) and localization of the reaction products around inter-particle contacts during crust formation. The resulting crust has a greater resistance to wind erosion than the EICP solution without addition of a hydrogel. Besides enhancing the erosion resistance, creation of a surficial crust via urea hydrolysis may also affect the soil permeability. Previous work has demonstrated that crust formation can establish a water-impermeable surface (Stabnikov et al. 2011), however, a resistant crust can also be obtained with negligible changes to the hydraulic conductivity (Montoya et al. 2018). Preliminary testing with small-scale EICP treated samples show nonuniform dispersion of the solution through nearly the entire sample depth. Addition of the polymer to the EICP solution produces a more uniform crust and a consistently defined treatment zone, but further limits permeability by inducing pore clogging. We present the results of experiments which were performed to characterize the crust in terms of thickness, composition, penetration resistance,and permeability. Methods for characterizing the crust include SEM imaging to determine crystal morphology, acid washing to determine calcium carbonate content, and thickness will be measured via a penetrometration analysis and supplemented with calipers. This analysis compares current research 
of EICP and hydrogel-enhanced EICP solutions with previous work evaluating an effective crust that limits the effects on soil permeability while increasing the surficial strength for erosion control and dust suppression.

Procter and Gamble Student poster award:

I would like to compete in the Procter and Gamble Student award References:

Stabnikov V, Naeimi M, Ivanov V, Chu J (2011) Formation of waterimpermeable crust on sand surface using biocement. Cem Concr

Res 41:1143-1149

Montoya, B. M., Do, J. \& Gabr, M. M., 2018. Erodibility of Microbial Induced Carbonate PrecipitationStabilized Sand under Submerged Impinging Jet. s.l., IFCEE. Acceptance of Terms and Conditions:

Click here to agree

110

\section{Pore network modeling of cathode catalyst layer of proton ex- change membrane fuel cell}

Rui $\mathrm{Wu}^{1}$

${ }^{1}$ Shanghai fiao Tong University

Corresponding Author(s): ruiwu@sjtu.edu.cn

In this paper, a pore network model is developed to investigate the coupled oxygen and proton transport and reaction process in the cathode catalyst layer (CCL) of proton exchange membrane fuel cell (PEMFC). The developed model is validated by comparing the predicted polarization curve with the experimental data, and the parametric studies are carried out to elucidate the effects of CCL design parameters. The results show that with decreasing the CCL thickness and the Nafion content, the cell voltage reduces at the low the current density but becomes increased when the current density is higher. The cell performance also can be improved by increasing the proton conductivity of Nafion in the CCL. Furthermore, it is revealed that the CCL with the Nafion volume decreasing along the thickness direction exhibits better performance as compared to the one with uniformly distributed Nafion at the high current density.

Procter and Gamble Student poster award:

References:

Acceptance of Terms and Conditions:

Click here to agree

113

\section{Evaporation from thin porous media with mixed intermediately- wet and hydrophobic networks}

Rui $\mathrm{Wu}^{1}$

\footnotetext{
${ }^{1}$ Shanghai fiao Tong University
}

Corresponding Author(s): ruiwu@sjtu.edu.cn

Evaporation from porous media with mixed wettability is still unclear, and different results have been reported experimentally. To address this issue, a pore network (PN) model with the capillary 
valve effect is developed to shed light on evaporation from thin porous media with intermediatelywet and hydrophobic networks. The PN is composed cubic pore bodies connected by cylindrical pore throats. Gas invasion in the PN is dominated by capillary forces and shows the same pattern in the purely hydrophilic and hydrophobic PNs. Effects of the hydrophilic fraction on evaporation are trivial if hydrophilic and hydrophobic pore bodies have the similar threshold pressure. When hydrophobic pores have a lower threshold pressure than hydrophilic pore bodies, gas invasion during evaporation can be divided into two stages; and the evaporation rate at the constant rate period increases first and then reduces with the increase of the hydrophilic fraction. Furthermore, we also discuss effects of pore wettability and structure on evaporation in mixed wet PNs with superhydrophilic and hydrophobic pores, and reveal three limiting cases for gas invasion. We find that effects of the hydrophilic fraction on evaporation in porous media depend on not only the pore wettability but also the pore structure.

Procter and Gamble Student poster award:

References:

Acceptance of Terms and Conditions:

Click here to agree

497

\title{
Numerical Simulation of fluid flow in unsaturated porous media using the SPH method
}

\author{
Tobias bernd Wybranietz ${ }^{1}$; Sabine Przybilla ${ }^{\text {None }}$; Pierre Sabrowski ${ }^{\text {None }}$; Joachim Villwock ${ }^{\text {None }}$ \\ ${ }^{1}$ Technische Universität Berlin
}

Corresponding Author(s): twybranietz@beuth-hochschule.de

The aim of the presented work is to apply Smoothed Particle Hydrodynamics (SPH) to the moisture penetration of porous solids. SPH is a meshfree Lagrangian Computational Fluid Dynamics (CFD) method. It is based on the solution of the Navier-Stokes equations by calculating particle interaction based on smoothed physical fields. The resulting model reproduces precisely the physical behaviour and allows numerical scalability and efficiency.

Suitable SPH formulations were used to discretize the macroscopic description of porous fluid transport processes, including the saturation dependency of capillary pressure and permeability. To realize the interaction between adjacent fluid and porous medium, different physical and numerical approaches were applied. Similar to 5 , porous properties were assigned to the water field, allowing mass exchange between the two phases. Additionally, a pore pressure based on hydrological approaches was introduced to include external fluid dynamics [7]. Based on the mass transition between fluid and porous media, particles with different masses arise near the interface. The interaction between those particles is handled by a merging algorithm [9] and correction methods to increase mathematical consistency and avoid numerical instabilities [6].

Furthermore, the concept of a new boundary condition is presented, which could allow the interaction between SPH fluid particles and the Standard Triangulation Language (STL) format and shows many advantages with respect to the interface modelling between fluid and porous media 3 . This interfacial treatment not only increases numerical accuracy compared to ghost particle models 1 , but also has the potential to couple SPH with other methods such as Finite Element Method (FEM). Thus, a performant numerical modelling for the combination of free surface flows, deformations of porous media and transport processes in the connected porosity would be possible prospectively.

As a foundation, the dive.sph software is extended by the presented models. It provides a state of the art SPH framework including the pressure extrapolation wall boundary 1 , pressure or volume flow defined open boundaries [8], the delta-SPH correction method 2 and an efficient CPU-parallelized neighbour search algorithm 4 . 
The model is validated by comparing simulation results to experimental data. Finally, the verifiability and applicability of porous media simulations for water absorption in soils und textiles were presented. Future work regards the modelling of hysteresis effects, the inclusion of the correlation between wetting and mechanical behaviour, solute transport and adsorption mechanisms.

Procter and Gamble Student poster award:

References:

1 Adami, S., Hu, X. Y., Adams, N. A., 2012, “ A generalized wall boundary condition for smoothed particle hydrodynamics", Journal of Computational Physics, Vol. 231, pp 7057-7075.

2 Antuono, M., Colagrossi, A., Marrone, S., Molteni, D., 2010, "Free-surface flows solved by means of SPH schemes with numerical diffusive terms", Computer Physics Communications, Vol. 181, pp. 532549.

3 Chiron, L., de Leffe, M., Oger, G., Le Touzé, D., Fast and accurate SPH modelling of 3D complex wall boundaries in viscous and non viscous flows, Computer Physics Communications (2018), https://doi.org/10.1016/j.cpc.2018.08.001 4 Ihmsen, M., Akinci, N., Becker, M., and Teschner, M., 2010, "A parallel SPH implementation on Multicore CPUs”, Computer Graphics Forum, Vol. 30, No. 1, pp. 99-112.

5 Lenaerts, T., "Unified Particle Simulations and Interactions in Computer Animation," PhD thesis. Katholieke Universiteit Leuven, 2009.

[6] Oger, G., Marrone, S., Le Touzé, D., de Leffe, M., SPH accuracy improvement through the combination of a quasi-Lagrangian shifting transport velocity and consistent ALE formalisms, Journal of Computational Physics 313, pp. 76-98.

[7] Stauffer, F. 1998, "Strömungsprozesse im Grundwasser Konzepte und Modelle“, vdf Hochschulverlag AG an der ETH Zürich, pp. 18-21.

[8] Tafuni, A., Domínguez, J. M., 1998, “Accurate and efficient SPH open boundary conditions for real 3-D engineering problems ", 12th International SPHERIC Workshop, pp. 346-354.

[9] Vacondio, R., Rogers, B. D., Stansby, P. K., and Mignosa, P., 2016, "Variable resolution for SPH in three dimensions: Towards optimal splitting and coalescing for dynamic adaptivity", Computer methods in applied mechanics and engineering, Vol. 300, pp. 442-460. Acceptance of Terms and Conditions:

Click here to agree

\title{
Simultaneous determination of pore space and tortuosity fractal dimensions based on 3D CT images
}

Author(s): Yuxuan Xia None

Co-author(s): Jianchao Cai ${ }^{1}$; Wei Wei

${ }^{1}$ China University of Geosciences (Wuhan)

Corresponding Author(s): xiayx@cug.edu.cn

\begin{abstract}
The petrophysical properties of rocks, such as thermal conductivity, electrical conductivity, and fluid transport, have been studied based on fractal geometry theory in many areas over several decades and determination of fractal dimensions is always the focus of researches and applications by means of fractal-based methods. In this work, a new method for calculating pore space fractal dimension and tortuosity fractal dimension of porous media is derived based on fractal tortuous capillary model assumption. The presented work establishes relationship between fractal dimensions and pore size distribution, which can be directly used to calculate the fractal dimensions. The published pore size distribution data for 8 sandstone samples are used to calculate the fractal dimensions and simultaneously compared with prediction results from analytical expression. In addition, the proposed fractal dimension method is also tested through Micro-CT images of three sandstone cores, and are compared with fractal dimensions by box-counting algorithm. The good consistency of two comparisons proved our new method and also proved that there is a self-similar fractal range in sandstone when excluding smaller pores and the fractal dimensions are intimate related to the microstructures of porous media.
\end{abstract}

Procter and Gamble Student poster award: 


\title{
References:
}

1. Yu, B. and J. Li, Some fractal characters of porous media. Fractals, 2001. 9(3): p. 365-372.

2. Cai, J. and B. Yu, A Discussion of the Effect of Tortuosity on the Capillary Imbibition in Porous Media. Transport in Porous Media, 2011. 89(2): p. 251-263.

3. Pia, G. and U. Sanna, An intermingled fractal units model and method to predict permeability in porous rock. International Journal of Engineering Science, 2014. 75: p. 31-39.

4. Cai, J., et al., Electrical conductivity models in saturated porous media: A review. Earth-Science Reviews, 2017. 171: p. 419-433.

Acceptance of Terms and Conditions:

Click here to agree

\section{A Machine-learning Based Subdomain POD-TPWL for Reservoir History Matching}

\author{
Author(s): Cong Xiao ${ }^{1}$ \\ Co-author(s): Arnold Heemink ${ }^{1}$; Hai Xiang Lin ${ }^{1}$; Olwijn Leeuwenburgh ${ }^{2}$ \\ ${ }^{1}$ Delft University of Technology \\ ${ }^{2}$ TNO, and Delft University of Technology
}

Corresponding Author(s): c.xiao@tudelft.nl

History matching is a major challenge for a large number of uncertain parameters. In general, if the adjoint model can be implemented, the adjoint-based minimization algorithm is one of the most efficient approaches nowadays to handle large-scale inversion problems. However, the implementation of the adjoint model requires an overwhelming programming effort, and the legacy code of forward simulation model is not always available. We have recently developed a machine-learning based subdomain POD-TPWL to assist an adjoint-based history matching without the need of model intrusion. Here a machine-learning based RBF interpolation approach is integrated with domain decomposition to efficiently construct subdomain reduced-order model. The adjoint of this reducedorder model can be implemented very easy.

From a computational point of view, a local parameterization where the parameters are defined in each subdomain separately is very attractive. In this study, we presents a local parameterization through integrating principle component analysis and domain decomposition to independently represent the spatial parameter field within low-order parameter subspaces in each subdomain. This local parameterization allows us to perturb parameters in each subdomain simultaneously and the effects of all these perturbations can be computed with very few full-order model simulations.

The performance of this SubDomain POD-TPWL with Local Parameterization, referred to LP-SD POD-TPWL, has been assessed through the benchmark SAIGUP model. Comparisons with the classic finite-difference based history matching show that this proposed subdomain history matching approach obtains comparable history matching results much faster. This local parameterization has high scalability, since the number of sampling points depends primarily on the number of the local parameters in each subdomain and not on the dimension of the underlying full-order model. Activating more subdomains results in much less local parameter patterns and enables us to run less model simulations. Given the need for training input and output in machine learning as well as model order reduction approaches, our proposed subdomain reduced-order model reduces the required number of training cases relative to previous approaches by use of subdomain instead of global representation of dynamics, and of a radial basis function model with coefficients that are learned from a limited number of training runs. For the cases studied in this work, to optimize 78 global PCA patterns, LP-SD POD-TPWL needs $56=22+(2 \times 12+1)+9$ simulations, among them, 22 simulations are used to collect the snapshots for POD, 25 simulations are used to construct the initial subdomain 
reduced-order model, and the remaining 9 simulations are used to update the reduced-order model in additional 9 outer-loops.

Procter and Gamble Student poster award:

I would like to compete in the Procter and Gamble Student award References:

1. Cong Xiao, Olwijn Leeuwenburgh, Hai Xiang Lin, and Arnold Heemink. Non-intrusive subdomain POD-TPWL algorithm for reservoir history matching. arXiv preprint arXiv:1806.04135, 2018.

Acceptance of Terms and Conditions:

Click here to agree

86

\title{
Particle Transport and Deposition Characteristics in 3D Horizon- tal Fracture with Roughness
}

\author{
Author(s): Wang Xiaoyu ${ }^{1}$ \\ Co-author(s): Jun Yao ${ }^{1}$; Hai Sun ${ }^{1}$; Liang Gong ${ }^{1}$; Lei Zhang ${ }^{1}$; Wen chao Liu ${ }^{2}$; Yang Li $^{3}$ \\ ${ }^{1}$ Research Centre of Multiphase Flow in Porous Media, China University of Petroleum (East China) \\ ${ }^{2}$ School of Civil and Environmental Engineering, University of Science and Technology Beijing \\ ${ }^{3}$ Department of Oilfield Exploration \& Development, Sinopec, Beijing, China
}

Corresponding Author(s): b17020095@s.upc.edu.cn, sunhaiupc@sina.com, lgong@upc.edu.cn, zhlei84@163.com,

\begin{abstract}
The problem about the deposition and transport of particles in horizontal fracture or mini-channel is widely exists in both environmental and industrial applications, such as the particle migration and deposition progress existing in the fracturing development of unconventional oil and gas and related pollution treatment issues in groundwater reservoir with fractures. The roughness of fracture roughness is a significant feature which have effect on the flow in channels. Thus, in this paper, the particle flow characteristics in rough fractures is investigated. The Gauss Model is used to construct the rough surfaces of fracture, based on it, the movement of dispersed particles is simulated by CFD-DEM (Computational Fluid Dynamics- Discrete Element Method) coupling method. Particles experience up and down motion, swaying around in the three-dimensional rough channel and their overall movement is a spiral trajectory. In addition, one comprehensive analysis of the sedimentary particles indicated that the deposition distance of particles is inversely proportional to the particle size and density ratio. Moreover, the impacts of both the rough bottom and the sides are considered. The uneven distribution of granular sediment is captured by simulation and the mechanism that particles tend to settle in the valley of rough bottom and the corners of rough sides is analyzed from the perspective of the particles collisions and kinetic energy dissipation. The trajectory morphogenesis of flowing sparse particles and distribution of deposited particles in fracture are impacted with wall roughness while the law of particle separation is less affected which is more related to the particle parameters. Overall, this work can give a clear insight in to the sparse particles flow in horizontal fracture with rough surfaces with the aid of CFD-DEM simulation strategy.

Keywords: Fracture; Roughness; Particles; CFD-DEM coupling; unconventional reservoirs.
\end{abstract}

Procter and Gamble Student poster award:

References:

Acceptance of Terms and Conditions:

Click here to agree 


\title{
Multi-scale Extended Finite Element Method For Fractured Geo- logical Formations
}

\author{
Fanxiang $\mathrm{Xu}^{1}$; Hadi Hajibeygi ${ }^{1}$; Bert Sluys ${ }^{1}$ \\ ${ }^{1}$ TU Delft \\ Corresponding Author(s): f.xu-4@tudelft.nl
}

Underground reservoir fluid pressure change, resulting from either resource extraction or fluid injection, leads to stress field change within the subsurface and its surrounding formations. The stressfield change can cause unfavorable surface displacements (i.e., subsidence or uplift) and activate faults and fractures (i.e., induced seismicity). These consequences can lead to growing opposition in the society towards the use of underground resources for supply of affordable energy (e.g., as in hydrocarbon) and renewable energy (e.g. geothermal production and energy storage). The geological formations span large length scales and are typically highly heterogeneous and massively fractured at different scales. As such, accurate and efficient simulation of mechanical deformation in heterogeneous fractured geological formations is a key factor in maintaining the safety of operations. For mechanics analysis the finite element methods (FEM) have been developed extensively in the literature. To account for fractures accurately, extended finite element method (XFEM) provides an advantage of avoiding complex meshes. For geoscientific applications, there exists several fractures over large $(\mathrm{km})$ length scales. For such application, having a scalable and accurate XFEM method is extremely important. To achieve this, we present the first multiscale extended finite element (MSXFEM) method. MS-XFEM develops local multiscale basis functions which allow for constructing accurate coarse-scale systems. The coarse-scale system is then solved, and its solution is interpolated (prolonged) back to the original fine-scale resolution by the means of the same multiscale basis functions. Both enrichment functions along the fracture nodes and tips are considered. In the MSXFEM independent coarse grids for matrix and fractures are used, which results in a convenient strategy for large-scale applications with complex fracture networks. For several test cases, the MSXFEM performance is investigated compared with the classical XFEM method. MS-XFEM casts a promising approach for real-field analyses of mechanical deformation influenced by the reservoir pore-pressure change.

Procter and Gamble Student poster award:

I would like to compete in the Procter and Gamble Student award References:

Acceptance of Terms and Conditions:

Click here to agree

\section{Flow and heat transfer in a microchannel partially filled with a microporous foam involving effects of flow inertia, flow/thermal slips, thermal non-equilibrium and thermal asymmetry}

\author{
Huijin $\mathrm{Xu}^{1}$; Changying $\mathrm{Zhao}^{1}$; Kambiz Vafai ${ }^{2}$ \\ ${ }^{1}$ Shanghai fiao Tong University \\ ${ }^{2}$ University of California, Riverside
}

Corresponding Author(s): xuhuijin@sjtu.edu.cn

A theoretical study of forced convection in a parallel-plate microchannel partially filled with a porous medium is performed based on the local thermal non-equilibrium (LTNE) model. The two walls sandwiching the channel are exposed to thermal asymmetry boundary conditions, and the flexible porous medium is not connected to the walls. Effects of flow inertia in porous medium, velocity jump at the porous/fluid interface, and flow slip and thermal slip at the solid/fluid interface are involved. Exact solutions are obtained for velocity and temperature in both porous medium region and fluid 
region. The flow heterogeneity coefficient defined for describing the nonuniform distribution of fluid flow is especially considered, and the effect of flow heterogeneity on heat transfer is revealed. The entropy generation analysis is performed for heat transfer and fluid friction irreversibilities in the channel partially filled with a porous medium. The benchmark solution provided in this work can be used for improving numerical scheme accuracy and validating similar research.

Procter and Gamble Student poster award:

References:

Acceptance of Terms and Conditions:

Click here to agree

\title{
The laplace transform embedded discrete fracture model method for the simulation of complex fracture system
}

\author{
Author(s): Jianchun $\mathrm{Xu}^{1}$ \\ Co-author(s): Baojiang Sun ${ }^{2}$; Wei Zhang ${ }^{3}$ \\ ${ }^{1}$ China University of Petroleum (East China)

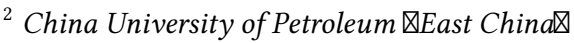 \\ ${ }^{3}$ The Pennsylvania State University
}

Corresponding Author(s): 20170048@upc.edu.cn

A new numerical method, the Laplace Transform Embedded Discrete Fracture Model (LTEDFM) method, is presented for the simulation of fluid through porous media with complex fracture system. The flow is governed by Darcy's flow in both matrix and fracture. The cell centered structured grid is employed to discretize the spatial domain for matrix. The fracture is embedded to the matrix grids. The fractures are modelled by lower-dimension line in 2D transport problem and interface in $3 \mathrm{D}$ problem in spatial discretization. In the computational process, the fractures keep the equivalent dimension. By taking Laplace Transform to time, the backward Euler difference for time discretization is avoided and is replaced by a semi-analytical method. This guarantees the accuracy and stability for time domain. The projection-based embedded-discrete-fracture method (pEDFM) is used to efficiently model the fractures and barriers. The method is tested by the existing semi-analytical methods, discrete fracrure models and standard finite difference simulators. Some numerical experiments are showed and the results are presented in detail. It is demonstrated that the proposed LTEDFM method is accurate for single flow in complex geology situations. The computational efficiency and robustness is also assessed.

Procter and Gamble Student poster award:

References:

Acceptance of Terms and Conditions:

Click here to agree

\section{A machine learning method for joint optimization of well loca- tion and hydraulic fracture parameter design}

Author(s): Jianchun $\mathrm{Xu}^{1}$

Co-author(s): Qian Ding ${ }^{1}$ 
${ }^{1}$ China University of Petroleum (East China)

Corresponding Author(s): 20170048@upc.edu.cn

Unconventional tight oil/gas have been developed during recent years, and a large number of hydraulic fractured wells have been drilled to improve the reservoir recovery. However, poorly designed well location and hydraulic fracture parameter in a low permeability oil/gas reservoir development can result in suboptimal production performance.

Focusing on well location and hydraulic fracture parameter (e.g., size, conductivity, location) optimization, we developed a machine learning approach to maximize the life-cycle net-present-value (NPV). Firstly, the reduced order models (ROM) is developed. The Latin Hypercube Sampling method is used to sample the parameters that need to be optimized. The Improved Projection-based Embedded Discrete Fracture Model (pEDFM) (Jiang J, Younis R M. An improved projection-based embedded discrete fracture model (pEDFM) for multiphase flow in fractured reservoirs[J]. Advances in Water Resources, 2017, 109: 267-289.) based simulator is employed for the simulation run of each training samples. After all the training simulations are completed, the input parameters and the calculated NPV are collected. The Multivariate Adaptive Regression Splines (MARS) algorithm is applied to generate the ROMs.

Then, we validate the predictions by ROMs with the results computed from improved pEDFM base simulator. The StoSAG (Fonseca, R. R. M., Chen, B., Jansen, J. D., \& Reynolds, A. (2017). A stochastic simplex approximate gradient (StoSAG) for optimization under uncertainty. International Journal for Numerical Methods in Engineering, 109(13), 1756-1776.) is chosen to estimate the optimal variables (i.e., well locations and hydraulic fracture parameters). Different synthetic reservoir examples generated with geostatistical modelling methods are tested to validate the accuracy of the constructed ROMs. The accuracy and computational efficiency are both analyzed. Finally, we apply the methodology to the optimization of a real low permeability gas reservoir. With the aid of ROMs, the performances for production are optimized.

The proposed machine learning approach associated with StoSAG optimization algorithm is confirmed to be a good optimization tool to achieve optimal well location and hydraulic fracture parameters for low permeability oil/gas reservoir development. To the best of our knowledge, this work represents the first step to the optimization of well location optimization and hydraulic fracture parameter in a low permeability oil/gas reservoir by combing the advanced numerical simulator and machine learning method.

Procter and Gamble Student poster award:

\title{
References:
}

Acceptance of Terms and Conditions:

Click here to agree

\section{Experimental Study on Gas Accumulation Mechanism of Sand- stone Gas Reservoirs in Sulige Gas Field, Ordos Basin, China}

\author{
$\mathrm{Xuan} \mathrm{Xu}^{\text {None }}$; Yong $\mathrm{Hu}^{\text {None }}$; Weijun Shen ${ }^{\text {None }}$; Chunyan Jiao ${ }^{\text {None }}$; Jiping Wang ${ }^{\text {None }}$; Yingli Chen ${ }^{\text {None }}$ \\ Corresponding Author(s): cugxuxuan@163.com
}

The understanding of gas accumulation in sandstone gas reservoirs is crucial for developing and optimizing the gas productivity. The gas accumulation in sandstone gas reservoirs is influenced by reservoir properties such as reservoir permeability, filling power and temperature. In this study, the experimental system of gas accumulation in gas reservoirs was established, and twelve full diameter core samples from Sulige gas field were designed to simulate the process of gas accumulation. The effects of the reservoir permeability, filling power and temperature, were analyzed. The results show that there exists a threshold pressure of the hydrocarbon accumulation in the low-permeability tight sandstone reservoirs. The reservoir with the permeability of $0.1 \mathrm{mD}$ is about $2.0 \mathrm{MPa}$ while the one with $0.01 \mathrm{mD}$ is $10.0 \mathrm{MPa}$. The lower the permeability is, the higher the threshold pressure is. The 
slower balance process will lead to a higher balanced pressure. The filling process is divided into three different stages, including critical fill stage, rapid increase stage and gentle increase stage. The rising velocity and range of gas saturation as well as water yielding pores, are quite different in the different stages. The gas-bearing property has a positive correlation with reservoir quality and filling power, which shows a very good logarithmic function relationship. These results can provide a better understanding of gas accumulation for improving the gas recovery and development benefit in sandstone gas reservoirs.

Procter and Gamble Student poster award:

References:

Acceptance of Terms and Conditions:

Click here to agree

83

\title{
Enhanced Thermal Recovery Mechanism of CO2 - Assisted Steam
}

\author{
Zhengxiao $\mathrm{Xu}^{1}$; Zhaomin $\mathrm{Li}^{1}$; Teng $\mathrm{Lu}^{1}$; Mingxuan $\mathrm{Wu}^{1}$ \\ ${ }^{1}$ China University of Petroleum(East China)
}

Corresponding Author(s): b18020040@s.upc.edu.cn

\begin{abstract}
Steam injection is an effective method of thermal oil recovery and plays an important role in the development of heavy oil reservoirs. Steam condensation experiment with $\mathrm{CO} 2$ is carried out to investigate the enhanced thermal recovery mechanism of $\mathrm{CO} 2$ - assisted steam. The heat transfer process between pure steam, mixed vapor and solid wall was studied by condensation heat transfer experiments. The effects of coagulation method, $\mathrm{CO} 2$ content in mixed vapor and mixed vapor flow rate on condensation heat transfer were analyzed. The results show that the condensation between pure steam and the wall is dropwise condensation, while the condensation between the mixed vapor and the wall is film condensation. Compared with dropwise condensation, the heat transfer enhancement mechanism of film condensation includes increasing the heat transfer resistance during condensation, reducing the condensation heat transfer coefficient of steam, and slowing the heat exchange rate between steam and wall. As the $\mathrm{CO} 2$ content in the mixed vapor increases, the condensation heat transfer between the steam and wall is deteriorated. As the flow rate of the mixed vapor increases, the condensation heat transfer between the steam and the wall is enhanced. This study is conducive to optimizing steam development methods and achieving geological storage of $\mathrm{CO} 2$.
\end{abstract}

Procter and Gamble Student poster award:

I would like to compete in the Procter and Gamble Student award References:

Acceptance of Terms and Conditions:

Click here to agree

6

\section{The Effect of Rock Deformation during CO2 Flooding and Storage Using Pore Scale Characterization}

\author{
Jin Yan ${ }^{1}$; Ying Jia ${ }^{1}$; Yunqing Shi ${ }^{1}$; Shenglai Yang ${ }^{2}$; Qian Wang ${ }^{2}$ \\ ${ }^{1}$ E\&P Research Institute, SINOPEC \\ ${ }^{2}$ China University of Petroleum
}


Corresponding Author(s): yanjin.syky@sinopec.com

Understanding the influence of $\mathrm{CO} 2$ injection on rock deformation is one of the key elements to analyze $\mathrm{CO} 2$ Enhanced gas recovery and long term CO2-storage in tight sand gas reservoirs. Rock deformation is a pore scale phenomenon, yet previous studies did not conduct comprehensive pore scale characterization during CO2 Flooding and Storage. This study utilizes integrated pore scale characterization techniques to study the phenomenon.

We present $\mathrm{CO} 2$ injection experiments performed on 14 sandstone samples, which include sand stones with natural fissures, shear fissures and artificial sanding crack fissures. Firstly, the core samples are characterized using nitrogen permeability and X-ray micro-computed tomography (micro$\mathrm{CT}$ ). Then, the core samples were flooded with freshwater, then natural gas, and finally supercritical $\mathrm{CO} 2(\mathrm{scCO} 2)$. To calculate permeability, the pressure difference across the core samples was monitored during these fluid injections. After the flooding experiment, nitrogen permeability and micro-CT was repeated to characterize pore scale deformation. After the experiments, the numerical simulations coupled with nonlinear rock deformation model with fluid flow were performed. The simulation results give a detailed understanding of the experimental mechanic system in pore scale. The results show that the damage rate of permeability decreases with the increase of initial permeability and increases linearly with the increase of irreducible water saturation. The recovery rate of permeability increases with the increase of initial permeability during $\mathrm{CO} 2$ flooding. Matrix and fissure cores showed respectively $29-47 \%$ and $40-86 \%$ decrease in permeability in production process. While after CO2 flooding, Matrix and fissure cores showed respectively $74-87 \%$ and $32-73 \%$ recovery in permeability. The permeability damage of the sample can be explained by the stress path followed by the reservoir controls the evolution of the effective stress state, and with it the changes in deviatoric stresses.

The evolution of the stress state, captured as a stress path, significantly affects the gas production profile and $\mathrm{CO} 2$ storage capability. This research of spatial and temporal changes in stress state laid the basis for studying $\mathrm{CO} 2$ storage and enhanced gas recovery.

\section{References:}

Acceptance of Terms and Conditions:

Click here to agree

\section{Experimental investigation on the stress sensitivity of permeabil- ity in naturally fractured shale}

Diansen Yang ${ }^{1}$

1 1. State Key Laboratory of Geomechanics and Geotechnical Engineering, Institute of Rock and Soil Mechanics, Chinese Academy of Sciences, Wuhan, Hubei 430071, China

Corresponding Author(s): dsyang@whrsm.ac.cn

In this paper, we present an experimental investigation regarding the stress sensitivity of permeability in naturally fractured shale. Gas permeability tests were performed on the fractured cylindrical shale samples under loading and unloading conditions. Different hydrostatic stress and gas pressure levels were chosen to investigate the dependence of permeability on stress. The permeability of the fractured shale decreases with increasing hydrostatic stress, re-increases during unloading and is irreversible during loading and unloading processes. The gas pressure exhibits a significant effect on the permeability in comparison with the hydrostatic stress. Small gas pressure changes (e.g., $2 \mathrm{MPa}$ ) induce a comparable change in permeability with a large hydrostatic stress change (e.g., $40 \mathrm{MPa}$ ). The gas pressure gradient on the permeability will be discussed. The fracture aperture was estimated by recording the volume change during loading and shows that the aperture change is consistent with the permeability evolution during loading, which is more complicated at a higher hydrostatic stress value. The roughness of the fractured surface was also analyzed and will be discussed in combination with the permeability evolution. 
Procter and Gamble Student poster award:

I don't want to compete References:

Acceptance of Terms and Conditions:

Click here to agree

107

\title{
Experimental investigation on anisotropic permeability and its relationship with anisotropic thermal cracking of oil shale under high temperature and triaxial stress
}

\author{
Author(s): Dong Yang ${ }^{1}$ \\ Co-author(s): Guoying Wang ${ }^{2}$; Zhiqin Kang ${ }^{2}$ \\ ${ }^{1}$ Taiyuan Univresity of Technology \\ ${ }^{2}$ Taiyuan University of Technology \\ Corresponding Author(s): ydscience@hotmail.com
}

\begin{abstract}
A high-temperature triaxial permeability testing machine was used to study anisotropic permeability of oil shale at stresses that emulate in-situ conditions. The threshold temperature of permeability in the direction perpendicular to bedding is $450^{\circ} \mathrm{C}$ and in the direction parallel to bedding is $400^{\circ} \mathrm{C}$. The magnitude of permeability of perpendicular bedding is low than $10-20 \mathrm{~m} 2$ below $450^{\circ} \mathrm{C}$, and increase from $10-19$ to $10-17 \mathrm{~m} 2$ at $450^{\circ} \mathrm{C}-600^{\circ} \mathrm{C}$. The magnitude of permeability of parallel bedding is $10-19$ to $10-18 \mathrm{~m} 2$ below $400^{\circ} \mathrm{C}$, and increase form $10-16$ to $10-15 \mathrm{~m} 2$ at 400 to $600^{\circ} \mathrm{C}$. The ratio of permeability of parallel bedding to permeability of perpendicular bedding was maximal at $450^{\circ} \mathrm{C}$. Simultaneously, sample microstructure was characterized by X-ray computed tomography. Thermal cracks mainly occur in the bedding direction, and cracks perpendicular to the bedding are rare. Thermal cracks caused by increased temperature are the main reason for the change of permeability in the direction parallel to bedding, and the connection of macropores is the main reason for the change of permeability in the direction perpendicular to bedding.
\end{abstract}

Procter and Gamble Student poster award:

References:

Acceptance of Terms and Conditions:

Click here to agree

411

\section{Integrated simulation of Darcy and Stokes flow for porous rock by lattice Boltzmann method}

\author{
Eomzi Yang $^{1}$; Dong Hun $\mathrm{Kang}^{2}$; Tae Sup Yun ${ }^{3}$ \\ ${ }^{1}$ Graduate student, Department of Civil and Environmental Engineering, Yonsei University, Yonsei-ro 50, Seodaemun- \\ gu, Seoul, 03722, Republic of Korea \\ ${ }^{2}$ Yonsei university \\ ${ }^{3}$ Yonsei University
}

Corresponding Author(s): did8810@yonsei.ac.kr

It is common to directly import 3D pore structure taken from high-resolution X-ray computed tomographic images and to evaluate the pore morphology and flow characteristics in porous rocks. The 
16-bit gray-level X-ray images are subjected to the series of image processing to segment the binary pore structure which serves as the analysis domain and this workflow has been widely adopted for the micro-scale studies on hydraulic phenomena in porous media. This appears intuitive and straightforward while the segmented pore domain may change depending on the image resolution and segmentation methods. As the range of pore size varies, the sufficiently large pore can be captured as it is while the small pore could belong to either pore or solid phase with respect to bimodal-shaped CT number histogram. Also, how the virtually apparent pores are captured highly depends upon the segmentation approaches. Therefore, it leads the uncertainty in the connectivity, smoothness, and volume of pore and can cause the misinterpretation of transport properties. We herein a new methodology to reasonably estimate the permeability of porous rock by providing the scientifically sound decision-making process. Based on the pore size distribution curve obtained from the experimental measure, we set the separating diameter (ds) which puts the boundary between 'apparent pore' and 'gray pore'. Herein, the apparent pore voxel should have a porosity of 1 while gray pore voxel has a porosity from 0 to 1 so that the summation of pore volume from both apparent and pore voxels should be equivalent to the experimentally measured porosity. Then, any other voxels become 'solid'. The determination of apparent and gray pores therefore depends upon the image resolution under analysis and the separating diameter. Once ternary components (i.e., apparent pore, gray pore, and solid) are assigned to each voxel, Brinkmann force lattice Boltzmann method (BF-LBM) is implemented for the flow simulation. In this study, the X-ray CT images of Navajo sandstone from Utah, U.S.A. are taken with the resolution of $4.17 \mu \mathrm{m}$. Results highlight that the separating diameter explicitly determines which pores correspond to 'gray pore' so that the regime of Darcy flow largely changes. However, the variation of computed permeability resides within acceptable ranges compared with values computed by other conventional methods. Also, the computed permeability seems most analogous to the experimentally measured one when the separating diameter is close to the mean pore diameter. This observation suggests that either under or over-estimated permeability by conventional approaches can be correctly calibrated regardless of image resolution by solving both Darcy and Stokes flow regime using BF-LBM.

Procter and Gamble Student poster award:

References:

Acceptance of Terms and Conditions:

Click here to agree

\title{
A new method for simultaneous determination of in situ porosity and permeability under reservoir conditions: insights from gas diffusion on gas shales with nanopores
}

\author{
Feng Yang ${ }^{1}$ \\ ${ }^{1}$ China University of Geosciences, Wuhan \\ Corresponding Author(s): fengyang@cug.edu.cn
}

Accurate estimation of in situ porosity and permeability is crucial for successful evaluation and development of unconventional reservoirs. However, gas porosity measured on cores using a helium pycnometer (Boyle's Law) or rock fragments using the buoyancy method (Archimedes principle) is commonly performed at ambient pressure. Many studies have shown that the porosity and permeability can be strongly dependent on the effective stress, and thus the porosity measured on core/crushed samples at ambient conditions will be larger than in situ porosity. There are now multiple methods for laboratory determination of permeability for unconventional tight oil/gas reservoirs. The conventional (steady-state flow) method for permeability determination is not adequate for low-permeability shale cores. The low flow rates across the core plug are difficult to measure, and the tests are quite time consuming. The crushed-rock permeability (GRI) determined from helium expansion is performed on crushed samples under unconfined conditions. Pulse-decay technique is commonly preferred to determine the permeability of tight rocks.

The ideal method of evaluating the petrophysical properties of rocks under stress would be to determine permeability and porosity simultaneously. In this paper, we derive an analytical model for non-steady-state gas flow through a core based on gas diffusion theory. Diffusion equation that 
describes gas diffusion as a function of porosity and permeability are presented. Shale samples from Lower Silurian strata of Sichuan basin, China are characterized using TOC analysis, helium pycnometer (grain density, and porosity), low-pressure gas adsorption (BET specific surface, pore size distribution, and pore volume). A laboratory apparatus was constructed to measure the pressure decay as a function of time as gas diffuses to the core samples. The pressure decay can be used to determine the in situ porosity and diffusion coefficients. Diffusion coefficients are sensitive to both pore volume and permeability and are used to determine the gas transport property. Simultaneously, pulse-decay measurements and crushed-rock permeability experiments are also performed to compare the results of different methods.

Results show that the porosity derived from diffusion experiments at ambient conditions agree with the porosity determined on helium pycnometer. However, there are significant difference between unconfined pycnometry porosity and in situ porosity. The specific pore volume of shale plugs decrease more than $10 \%$ from unconfined state to in situ condition. The diffusion coefficients of helium on these shale plugs are controlled by pore structure and TOC content, and are at the magnitude of $10-11-10-8 \mathrm{~m} 2 / \mathrm{s}$ at room temperature. The permeability values derived from gas diffusion on core plugs under different effective pressure agree with pulse-decay permeability values, and are higher than crush-rock permeability values.

The developed method only mount sample once and can be used to measure the porosity and permeability simultaneously under in situ stress conditions, which reduce test time and samples required. Furthermore, the porosity and permeability are evaluated at the same stress cycle, which eliminates any concern over permanent stress-hysteresis effects on the core and provide an intrinsically consistent porosity-permeability data.

Procter and Gamble Student poster award:

References:

Acceptance of Terms and Conditions:

Click here to agree

930

\title{
A Digital Image Correlation Analysis on the Rapid Reduction Mech- anism of Permeability in Low-Permeability Sandstone Reservoirs
}

\author{
Jing Yang ${ }^{1}$; Jianwen $\mathrm{Ye}^{1} ; \mathrm{Yao} \mathrm{Li}^{1}$ \\ ${ }^{1}$ Chengdu University of Technology
}

Corresponding Author(s): jingyang1125@gmail.com

Low-permeability sandstone reservoirs play a great role in the exploration and production of oil and gas resources, but most of them show a rapid reduction on permeability at the beginning of production process and present a depleted production rate along with the time. To figure out the rapid reduction mechanism of permeability, this research performed an experimental study using low-permeability sandstone specimens. Using a digital image correlation method, a binary image process was used to capture a fluid flow passage through the microscopic pore structures. Combining with a laser engraving technique, a simplified displacement experiment was conducted and found that the rapid reduction on permeability was caused by the loose grain particles, which can be transferred with the fluid and thus block the pore throats. These preliminary findings can provide guidance for improving the prediction accuracy on the well productivity and the understanding on a rapid depleted production rate at the beginning of the production history.

Procter and Gamble Student poster award:

References:

Acceptance of Terms and Conditions:

Click here to agree 


\title{
Multiphase SPH simulation based on an improved boundary model
}

\author{
Author(s): Qianhong Yang ${ }^{\text {None }}$ \\ Co-author(s): Jun Yao \\ Corresponding Author(s): s17020254@s.upc.edu.cn
}

\begin{abstract}
Smoothed particle hydrodynamics is a Lagrangian meshless method,which has unique advantages in modelling various interface phenomena such as interface fragmentation and large deformation. In this paper, under the background of multiphase flow in oil production, a numerical model is established using SPH multiphase method. Various interface phenomena are explored by solving the continuous surface tension model (CSF). Firstly, the governing equation of multiphase SPH model are given, as well as the SPH discrete format of pressure gradient, viscous force, surface tension, and various numerical processing techniques. Meanwhile, the viscous force proposed by $\mathrm{Hu}$ and Adams1 is modified in this paper. Base on the work of Qiang hongfu2 and Krimi3, a new boundary condition is proposed. In order to verify the correctness of this model, the contraction and concussion of square droplet at different density ratios are tested. The results are consistent with Laplace solution. On this basis, the simulation of rising bubble at a high density ratio( $\mathrm{DR}=1000)$ is performed. Finally, the three-dimensional cases are tested. Taking highly computational features of this method into consideration, techniques such as parallel computing are applied to accelerate the simulation. The research in this paper provides an effective numerical method for multiphase flow in petroleum engineering and expands the application of SPH method in engineering field.

Keywords: smoothed particle hydrodynamics; multiphase flow; continuous surface tension model; large density ratio; parallel computing
\end{abstract}

Procter and Gamble Student poster award:

\section{References:}

$1 \mathrm{Hu}, \mathrm{Y}$ X., A N. A multi-phase SPH method for macroscopic and mesoscopic flows[J]. J.comput.phys, 2006, 213(2): 844-861.

2Han Ya-Wei, Qiang Hongfu, et al. A new repulsive model for solid boundary condition in smoothed particle hydrodynamics[J]. Acta Physica Sinica, 2013, 62(4):326-336.

3Abdelkader Krimi, Mehdi Rezoug, ofiane Khelladi, et al. Smoothed Particle Hydrodynamics: A consistent model for interfacial multiphase fluid flow simulations[J]. Journal of Computational Physics, 2018, 358: 53-87. Acceptance of Terms and Conditions:

Click here to agree

\section{The Research of Microscopic Remaining Oil on the Basis of Dis- placement Experiment and Digital Core}

\author{
SHAN YANG ${ }^{\text {None }}$; YI ZHAO ${ }^{\text {None }}$ \\ Corresponding Author(s): 15153242100@163.com
}

The research of microscopic remaining oil on the basis of displacement experiment and digital core is based on micro-model manufacturing and microscopic remaining oil displacement experiment. Combined with three-dimensional digital cores obtained by X-ray computed tomography and analysis of pore network extraction, microscopic displacement mechanism and remaining oil entrapment mechanism are utilized to predict the distribution of microscopic remaining oil.

On the basis of pore network, micro-model is made of glass and sculpted by laser, which is convenient to be observed and recorded and is capable of conducting experiment under the circumstance of high temperature and high pressure to explore the microscopic displacement and remaining oil entrapment mechanism. Samples are carefully selected and through a series of steps including two dimension to three dimension reconstruction, sub-volume extraction, filtering, segmentation, opening, skeletonization冈three dimensional digital core pore-network is constructed. In order to extract 
pore-network model precisely, the improved NLM filtering algorithm is applied based on the structural comparison, and multi-constraint binary segmentation and micro-pore throat identification are utilized to enhance the boundary recognition.Experiment simulation and numerical modeling are combined to promote the accuracy and reliability.

Tazhong 402 reservoir is in the extra-high water-cut stage, whose characteristics are summarized as "five high and one low", namely, high water-cut, high recovery level, high temperature, high degree of mineralization and low heterogeneity. After a long period of recovery, the pore network, grains and minerals are experiencing transformation, which leads to the complicated distribution patterns of microscopic remaining oil. Therefore, microscopic displacement experiments are conducted and $\mathrm{X}$-ray computed tomography is utilized to reveal the microscopic displacement and remaining oil entrapment mechanism, thus to predict the distribution of remaining oil, providing evidence and reliability of further tertiary recovery.

Procter and Gamble Student poster award:

References:

1. Cense A, Berg S, Bakker K, et al. Direct visualization of designer water flooding in model experiments [C]//SPE Enhanced Oil Recovery Conference. Society of Petroleum Engineers, 2011.

2 N.Rezaei, O.Mohamadzadeh, RParsaei, I.Chatzis. The Effect of Reservoir Wettability on the Production Characteristics of the VAPEX Process [J]. SOCIETY OF PETROLEUM ENGINEERS OF AIME,2010,SPE130096: $1-12$.

3 Rebert F Li, George J. Hirasaki, Clarence A Miller, et al. Wettability alteration and foam mobility control in a layered 2-D heterogeneous system [J]. SOCIETY OF PETROLEUM ENGINEERS OF AIME, 2011, spe141462: 1-17.

4 Talal Al-Aulaqi, Carlos Grattoni, Quentin Fisher, et al. Effect of temperature,oil asphaltene content, and water salinity on wettability alteration [J]. SOCIETY OF PETROLEUM ENGINEERS OF AIME, 2011, spe149071: 1-19.

5 M. Sohrabi, D.H.Tehrani, A. Danesh , G.D.Henderson. Visualisation of Oil Recovery by Water Alternating Gas (WAG) Injection Using HighPressure Micromodels - Oil-Wet \& Mixed-Wet Systems [J]. SOCIETY OF PETROLEUM ENGINEERS OF AIME, 2001 SPE71494: 1-7.

[6] N.B.jomdalen, J.M.Alvarez, W.E.Jossy. An Experimental Study of the Pore Blocking Mechanisms of Aphron Drilling Fluids using Micromodels [J]. SOCIETY OF PETROLEUM ENGINEERS OF AIME, 2009 SPE121417: 1-16.

[7] Alireza Mollaei, M. Haghighi, B. Maini. Experimental investigation and nerwork modeling simulation of free fall gravity drainage in single-matrix and fractured-blocks models [J]. SOCIETY OF PETROLEUM ENGINEERS OF AIME, 2006, SPE100400: 1-17.

[8] A.A.Dehghan, M.H.Ghazanfari, S.A.Farzaneh. Studying the Effects of Pore Geometry Wettability and Co-Solvent Types on the Efficiency of Sovent Flooding to Heavy Oil in Five-Spot Models [J]. SOCIETY OF PETROLEUM ENGINEERS OF AIME, 2009 SPE123315: 1-11.

[9] S.F.Shariatpanahi, A.Dastyari, B.Bashukooh, M.Haghighi, M.Sahimi. Visualization Expetiments on Immiscible Gas and Water Injection by Using 2D-Fractured Glass Micromodels [J]. SOCIETY OF PETROLEUM ENGINEERS OF AIME, 2005 SPE93537: 2-9.

[10] Laura Rometo, Apostolos Kantzas. The effect of wettability and pore geometry on foamed gel blockage performance in gas and water producing zones [J]. SOCIETY OF PETROLEUM ENGINEERS OF AIME, 2004, SPE89388: 1-17. Acceptance of Terms and Conditions:

Click here to agree

\section{Fluid displacement and trapping during two-phase steady-state flow in complex carbonate imaged by synchrotron $x$-ray micro- tomography}

YILI YANG $^{1}$; Ian Butler ${ }^{1}$; Florian Fusseis ${ }^{1}$; Rink van Dijke ${ }^{2}$; Sebastian Geiger ${ }^{\text {None }}$; Xianghui Xiao ${ }^{3}$

\footnotetext{
${ }^{1}$ University of Edinburgh

${ }^{2}$ Heriot-Watt University

${ }^{3}$ Argonne National Laboratory
}

Corresponding Author(s): yili.yang@ed.ac.uk 
Multiphase fluid flow has been intensively studied within relatively homogeneous pore structures such as bead packs and sandstones. It has been shown that pore network characteristics such as the network topology, the pore size distribution and surface morphology can strongly affect the displacement and trapping of fluids by varying local capillary pressure and thereby changing the preferable path of fluids. In more complex media such as carbonates, fluid displacement and trapping in highly tortuous pore networks comprised of multiscale porosity are less-well understood. To find out how these factors impact the process, we conducted in-situ two phase core-flooding experiments in an oolitic Indiana Limestone with multiscale pore size and two different pore surface morphologies at the microtomography beam lime 2-BM at the Advanced Photon Source. The $3 \mathrm{~mm}$ diameter, $10 \mathrm{~mm}$ long core was saturated with oil, then flooded with brine to an irreducible oil saturation, followed by oil re-injection and steady-state (oil and water simultaneously) injection, using potassium iodide brine and dodecane as aqueous and oil phase, respectively. The entire injection process was imaged in $4 \mathrm{D}$ using 1-second 3D data acquisition in 20 second intervals. The excellent microtomographic data with a spatial resolution of 2.2 micron per pixel allowed visualisation of fluid flow in the sample and quantitative analysis of pore-scale fluid displacement processes. Here we show 4-dimensional data of 1) fluid trapping and displacement in a complex pore structure during drainage and imbibition. 2) The effect of two different surface morphologies, smooth, drusy calcite surfaces and rougher oolite surfaces, on the contact angle and fluid saturation. We found that in this specific pore structure, steady state injection of two fluids does not lead to a monotonic saturation change that occurs in nonsteady-state injection or reaching a steady saturation level, but a sinusoid saturation - desaturation - resaturation behaviour in the recorded time-lapse. At this stage of our ongoing interpretation we believe that this is caused by a dynamic competition of two immiscible fluids that both have the access to the same complex pore space. The pore surface morphology may influence this competition by partially altering local contact angles within pores, therefore causing a complex local capillary pressure. This study provides insights on fluid behaviour in complex pore structures and varied pore surface topology during steady-state flow, which are beneficial for improving fluid simulation predictions.

\title{
References:
}

Acceptance of Terms and Conditions:

Click here to agree

\section{Pore-scale simulation of shale oil flow based on pore network model}

\author{
Author(s): Ke Wang ${ }^{1}$ \\ Co-author(s): Yongfei Yang ${ }^{2}$; Jun Yao ${ }^{3}$; Yaohao Guo ; Zhihui Liu ${ }^{4}$ \\ ${ }^{1}$ Research Center of Multiphase Flow in Porous Media, China University of Petroleum (East China) \\ ${ }^{2}$ China University of Petroleum (East China) \\ ${ }^{3}$ Research Centre of Multiphase Flow in Porous Media, China University of Petroleum (East China) \\ ${ }^{4}$ China University of petroleum (East China)
}

Corresponding Author(s): yangyongfei@upc.edu.cn

The characteristics of shale reservoirs are complex: nano-scale pore throats, multi-scale, rich in organic matter. Shale oil flow in nanoporous media is affected by micro-scale effect, which is described by slippage and adsorption phenomenon. In addition, micro-fractures make shale oil flow more complicated. In this study, a pore network model with different organic matter contents was established, and corrected shale oil flow equation was introduced. The effects of slip length, viscosity ratio of adsorbed phase to bulk phase, thickness of adsorption layer on network permeability were analyzed under different organic matter contents. Shale permeability has positive correlation with slip length and negative correlation with viscosity ratio and thickness of adsorption layer. The flow capacity of inorganic and organic pore throats was different, which lead to distinct effect of organic matter content on permeability. With organic matter content increases, connectivity of organic pore throats increases, permeability was further improved. A method of integrating micro-fractures in 
pore network model was proposed to build pore network model with micro-fractures. Effects of angle, aperture and amount of fracture on shale oil flow combined with slippage and adsorption were analysed. The result show that fracture significantly improved network permeability, the mechanism of slippage and adsorption further enhanced permeability.

Procter and Gamble Student poster award:

References:

Acceptance of Terms and Conditions:

Click here to agree

\title{
Impact of Temperature on Sandstone based on CT images
}

\author{
Author(s): Haiyuan Yang ${ }^{1}$ \\ Co-author(s): Yongfei Yang ${ }^{2}$; Jun Yao ${ }^{3}$ \\ ${ }^{1}$ China University of Petroleum $\bowtie$ East China》 \\ ${ }^{2}$ China University of Petroleum (East China) \\ ${ }^{3}$ China University of Petroleum
}

Corresponding Author(s): yangyongfei@upc.edu.cn

\begin{abstract}
Rock thermal damage induces the physical and mechanical changes, which are crucial to thermal recovery. To investigate the changes due to heat, three sandstone samples were heated to $100^{\circ} \mathrm{C}$, $200^{\circ} \mathrm{C}, 300^{\circ} \mathrm{C}, 400^{\circ} \mathrm{C}, 500^{\circ} \mathrm{C}$ and $600^{\circ} \mathrm{C}$, respectively. The porosity and permeability measurements and CT scanning were conducted after samples natural cooling at various temperature points. The results show that as the temperature increases, the porosity and permeability of sandstone show the fluctuating increase which can be categorized in three stages. The recrystallization of minerals occur at $200^{\circ} \mathrm{C}$ from CT images. With the temperature increase, the direction of rearrangement change. For thermal damage mechanism, thermal expansion is the initial reason of porosity and permeability increase. The interaction between thermal expansion and recrystallization result in the decrease of porosity and permeability. The rearrangement of minerals is the main reason of porosity and permeability increase at high temperature. The change of fracture aperture can be explained by the mechanism.
\end{abstract}

Procter and Gamble Student poster award:

References:

Acceptance of Terms and Conditions:

Click here to agree

889

\section{Characterizing the Plugging Capability of New Bilayer-Coating Microspheres for In-Depth Conformance Control: Mathematical Modeling and Laboratory Study}

\author{
Yulong Yang ${ }^{1}$; Tingting Cheng ${ }^{1}$; Jirui Hou ${ }^{1}$; Zhenjiang You ${ }^{2}$; Yang Liu ${ }^{1}$; Fenglan Zhao ${ }^{1}$; Jun Li $^{1}$ \\ ${ }^{1}$ China University of Petroleum, Beijing \\ ${ }^{2}$ University of Queensland
}


Corresponding Author(s): yulong.yang2016@outlook.com

Microspheres are commonly applied for conformance control within heterogeneous reservoirs. However, due to the premature aggregation in wellbore neighborhood, the injected particles cannot transport deeply into the reservoir. To solve this problem, microparticles covered with two layers of functional coatings are developed for in-depth conformance control. The proposed bilayer-coating microspheres (BCMS) are expected to transport deeply into porous media, given that the rigid outer layer is designed to protect the adhesive inner coating. The objective of the present work is to characterize the adhesion property and plugging behavior of BCMS at reservoir temperature and fluid salinity. The Derjaguin-Landau-Verwey-Overbeek (DLVO) theory is used to calculate interactions between particles, which reveals a significant repulsion between the external coatings of different particles, indicating the elimination of premature particle aggregation. The static tests show that BCMS exhibits resistance to high temperature and salinity. Laboratory coreflood tests and mathematical modeling on BCMS injection are performed. We present the governing equation system for the deep-bed filtration of BCMS in porous media, accounting for the limited retention concentration and the velocity difference between the suspended particles and the carrier fluid. The exact solution for one-dimensional flow is derived. The measured pressure drops across different core sections agree with the modeling results with high accuracy, which validates the analytical model. BCMS shows promising potential application for in-depth conformance control, owing to its ability of deep transport into porous media.

Procter and Gamble Student poster award:

I don't want to compete References:

Acceptance of Terms and Conditions:

Click here to agree

\title{
Research and Application of Numerical Method of Evaluation of Fracturing Effects in Large Scale Volume Reform of Vertical Wells
}

\author{
Author(s): Zhengming Yang ${ }^{1}$ \\ Co-author(s): Debin Xia ; Anshun Zhang ; Yapu Zhang ; Yutian Luo \\ ${ }^{1}$ PetroChina Research Institute of Petroleum Exploration \& Development
}

Corresponding Author(s): yzhm69@petrochina.com.cn

At present, the evaluation methods of fracturing effects under large-scale volumetric reforming of vertical wells are commonly used for direct fracturing effects evaluation methods such as microearthquake technology and inclinometers, but they can only evaluate the fracturing effect at a certain point in time, can't evaluate the whole process of fracturing effect. This paper is based on the characteristics of "Fracture network" flow in the large scale volume reform of vertical wells, through studied the intelligent PEBI mesh division, the relationship between the permeability of different zones and the pressure and pressure derivative curves, and the influence of different parameters on the pressure curve. established numerical method for evaluation of fracturing effects of large scale frature network of vertical wells. Based on the data of fracturing fluid returning, well testing and production, the numerical evaluation of fracturing effect of four repetitive volume reforming wells in different periods of ChangQing was carried out, and the scale and permeability transformation features of volume fracturing in different periods were given , these well guided oilfield production.

\section{Procter and Gamble Student poster award:}

\section{References:}

Acceptance of Terms and Conditions:

Click here to agree 


\title{
Unsaturated flow through fracture intersections: competition be- tween gravity and capillary force controls fluid splitting of droplets
}

\author{
Zhibing Yang $^{1}$; Song Xue ${ }^{1}$; Yi-Feng Chen ${ }^{1}$ \\ ${ }^{1}$ Wuhan University \\ Corresponding Author(s): zbyang@whu.edu.cn
}

\begin{abstract}
We present an experimental study on the flow splitting behavior at a fracture intersection under unsaturated condition. The flow cell is composed of a vertical fracture intersected by a horizontal fracture, forming an overturned T-shape. Deionized water is injected at the top of the vertical fracture. Different combinations of apertures of the vertical $\left(b_{v}\right)$ and the horizontal $\left(b_{h}\right)$ fractures are considered. Experimental results confirm that the gravity-driven flow in the vertical fracture transitions from droplet to rivulet mode as the flow rate increases. By image analysis we quantify the flow dynamics through the intersection, and especially focus on the partitioning efficiency () defined as the percentage of flow partitioned into the horizontal fracture. When $b_{v}<b_{h}$, we find that the droplets completely imbibe into the horizontal fracture (almost equals to 1 ) for injection rate smaller than a threshold value. For higher flow rate, the droplets split in such a way that the partitioning efficiency is proportional to the injection rate. This behavior can be well explained by a force balance analysis at the fracture intersection. The findings are further supported by numerical simulations of droplet flow through intersections.
\end{abstract}

Procter and Gamble Student poster award:

References:

Acceptance of Terms and Conditions:

Click here to agree

85

\section{Atomic Force Microscopy (AFM) Study of Wettability Modifica- tion and Mineralogy of Bandera Brown.}

\author{
Author(s): Sherifat Yesufu ${ }^{1}$ \\ Co-author(s): Maja Ruecker ${ }^{1}$; Fons Marcelis ${ }^{2}$; Paul Luckham ${ }^{1}$ \\ ${ }^{1}$ Imperial College London \\ ${ }^{2}$ Shell Global Solutions International B.V.
}

Corresponding Author(s): s.yesufu17@imperial.ac.uk

One characteristic of porous media is the large surface-area to volume ratio, which enhances surfacefluid interactions of any kind, e.g. in catalysts, adsorption studies, Enhanced Oil Recovery (EOR) in oil reservoirs, etc. Atomic Force Microscopy (AFM) is a technique to characterise such surfaces at length scales ranging from $\mu \mathrm{m}$ to the $\mathrm{nm}$. The use of AFM to characterise rock surfaces though has been very limited. It has the potential to carry out 3D imaging of the surface topography and give information about interaction forces at the pore surfaces. In this study, the capabilities to investigate porous media with AFM will be presented based on an example of mineral modification in Bandera Brown rock.

In AFM, the forces between the sample surface and a microscopically sharp tip can be determined from the deflection of a flexible cantilever which holds the tip and, in essence, acts as a spring of known force constant. Thus, by utilizing an AFM tip functionalised with oil components, the wettability of a mineral surface can be ascertained from the adhesion forces between its pore surfaces 
and these oil components. Basically, simulating the interactions between crude oil and the mineral surface. This process is quite useful in EOR for ascertaining conditions in the pores of reservoir rocks. However, the difficulty lies within the fact that reservoir cores (particularly sandstones that consist of iron-bearing clay minerals), once brought to the surface, become oxidized, thus deviating from anaerobic, reducing conditions within the rock formation. Expectedly, experimental results obtained using these oxidised cores differ substantially from field tests, as wettability is observed to vary depending on the redox state.

In this work, the capability of the AFM for characterisation of the mineralogy of an outcrop sandstone, Bandera Brown, at the pore scale from 3D imaging, as a direct corroboration of Scanning Electron Microscopy (SEM) analysis will be explored. Furthermore, this technique will be used to probe the possibility for modifying the wettability of the pore surfaces of Bandera Brown, by measuring the interactions with oil components such as the hydrophobic methyl end groups (-CH3) and hydrophilic carboxyl end groups (-COOH).

Keywords: 3D imaging. AFM. SEM. Wettability. Adhesion. Sandstones.

Procter and Gamble Student poster award:

I would like to compete in the Procter and Gamble Student award References:

Acceptance of Terms and Conditions:

Click here to agree

\section{Asymptotic mathematical model of gas filtration through thin porous wall}

Dmytro Yevdokymov ${ }^{1}$

${ }^{1}$ Oles Honchar Dnipro National University

Corresponding Author(s): devd@ukr.net

Classical filtration theory considers a lot of porous media in a great variety of possible configurations, including thin porous bodies. The mentioned thin bodies, in particular plates, membranes and shells, are often occurred in different applications in modern sciences and technologies. As a result, processes of filtration across such bodies are interesting from the practical point of view and well investigated at the moment by experimental and theoretical methods. However filtration in nonasymptotically thin bodies did not attract so attention of investigators and, as a rule, such bodies were investigated on the base of general model of porous body filtration. At the same time, it is evident that small thickness of the considered bodies in comparison of their other reference sizes gives an opportunity to build an asymptotic mathematical model, using the relation of correspondent reference sizes as a small parameter. The present work represents such attempt concerning gas filtration through non-asymptotic thin porous body (porous layer). It is necessary to note, that the authors of the present work had preliminary experience in the heat conduction across thin solid body problem, which is similar to the considered problem, and correspondent analysis tools were developed in previous works by the authors. Classical gas filtration theory mainly deals with isothermal filtration process. However in the case of thin body there is possibility a sufficient temperature difference between opposite sides of the wall. In dependence of parameters of the rigid frame and gas filtration flow, there are three mathematical models of the investigated phenomena: The first one is the mentioned isothermal case, what means that the temperature gap is absent and mass of gas is negligibly less than the mass of the rigid frame. The second one is one-temperature model, what means that the temperature distribution in the wall is completely determined only by heat conduction of rigid frame, since the mass of gas in negligibly small similar to the first case. And the third one is two-temperature, which takes into account a heat transfer between the gas and the rigid frame. However, the last model is very seldom occurred in applications. Beside of that, it is extremely complicated, therefore it is not considered here.

According to asymptotic approach, the solution is founded as a small parameter expansion. The 
obtained zero approximation is described by boundary-value problem for ordinary differential equation, which can always be solved analytically. The following approximations can be treated analytically too, but this procedure can occur too complex and it is better to obtain numerical solution in such case. The proposed approach is formulated in local coordinate system connected with the body surface and, as a result, it is effective for bodies of complex geometrical shapes. Another advantage of the approach is opportunity of analytical treatment of relevant nonlinear terms.

The described above approach is illustrated by several examples of calculations.

Procter and Gamble Student poster award:

I don't want to compete References:

Acceptance of Terms and Conditions:

Click here to agree

611

\title{
On volume averaging in porous electrode theory
}

\author{
Author(s): Xiaoguang Yin ${ }^{\text {None }}$ \\ Co-author(s): Baohua $\mathrm{Li}^{1}$; S.Majid Hassanizadeh ${ }^{2}$ \\ ${ }^{1}$ Tsinghua University \\ ${ }^{2}$ Utrecht University
}

Corresponding Author(s): xiaoguang.yin@sz.tsinghua.edu.cn

Porous electrode is widely used in commercial electrochemical devices. The porous configuration allows high interfacial area for electrochemical reactions. Considering the complex geometry and topology together with the coupled kinetic, transport and thermodynamic processes, detailed treatment of the electrochemical cell at particle scale is formidable, if not impossible. Volume averaging technique developed for describing flow and transport in porous media has been employed for modelling of batteries and fuel cells. Averaging the particle-scale equations over a representative elementary volume (REV) results in the so-called porous electrode theory. This theory has seen successful applications in simulation of electrochemical systems. Over the years, efforts have been made on extending the theory to non-ideal active materials, on comparing Newman's porous electrode model with upscaled equations from volume averaging of generalized Poisson-Nernst-Plank (PNP) equations, and on thermodynamics based derivation of Butler-Volmer equations for lithium intercalation kinetics, to name a few.

Volume averaging adopted in porous electrode theory will be re-examined theoretically. Issues with current porous electrode theory will be discussed and demonstrated through numerical examples. Relevance to upscaling of electrochemical systems, like identifying macroscopic parameters from particle-scale simulations, will also be covered.

\section{Procter and Gamble Student poster award:}

\section{References:}

1J. C. Slattery. Flow of viscoelastic fluids through porous media. AIChE Journal, 13(6):1066-1071, 1967. $2 \mathrm{~S}$. Whitaker. Diffusion and dispersion in porous media. AIChE Journal, 13(3):420-427, 1969.

3 J. Newman and W. Tiedeman. Porouse-Electrode Theory with Battery Applications. AIChE Journal, 21(1):25-41, 1975.

4 P. De Vidts and R. E. White. Governing Equations for Transport in

Porous Electrodes. Journal of The Electrochemical Society, 144(4):1343, 1997.

5 C. Y. Wang, W. B. Gu, and B. Y. Liaw. Micro-Macroscopic Coupled Modeling of Batteries and Fuel Cells: I. Model Development. Journal of The Electrochemical Society, 145(10):3407, 1998.

[6] S.M. Hassanizadeh and W.G. Gray. General conservation equations 
for multi-phase systems: 1. averaging procedure. Advances in Water Resources, 2(1):131-144, 1979.

[7] Marc Doyle. Modeling of Galvanostatic Charge and Discharge of the Lithium/Polymer/Insertion Cell. Journal of The Electrochemical Society, 140(6):1526, 1993.

[8] Marc Doyle, John Newman, Antoni S. Gozdz, Caroline N. Schmutz, and Jean-Marie Tarascon. Comparison of Modeling Predictions with Experimental Data from Plastic Lithium Ion Cells. Journal of The Electrochemical Society, 143(6):1890, 1996.

[9] C. Y. Wang. Fundamental models for fuel cell engineering. Chemical Reviews, 104(10):4727-4765, 2004.

[10] W. B. Gu, C. Y. Wang, and B. Y. Liaw. Micro-Macroscopic Coupled Modeling of Batteries and Fuel Cells: II. Application to Nickel-Cadmium and Nickel-Metal Hydride Cells. Journal of The Electrochemical Society, 145(10):3418-3427, 1998.

[11] Pauline De Vidts, Javier Delgado, and Ralph E. White. Mathematical modeling for the discharge of a metal hydride electrode. Journal of The Electrochemical Society, 142(12):40064013, 1995.

[12] R. E. Garc' 1a and Y M Chiang. Spatially resolved modeling of microstructurally complex battery architectures. Journal of the Electrochemical Society, 154(9):A856-A864, 2007.

[13] T. R. Ferguson and M. Z. Bazant. Nonequilibrium Thermodynamics of Porous Electrodes. 159(12), 2012.

[14] Graham M. Goldin, Andrew M. Colclasure, Andreas H. Wiedemann, 202 and Robert J. Kee. Three-dimensional particle-resolved models of liion batteries to assist the evaluation of empirical parameters in onedimensional models. Electrochimica Acta, 64:118 - 129, 2012. 205

[15] Andrew M. Colclasure and Robert J. Kee. Thermodynamically consistent modeling of elementary electrochemistry in lithium-ion batteries. Electrochimica Acta, 55(28):8960 - 8973, 2010.

[16] Wei Lai and Francesco Ciucci. Mathematical modeling of porous 187

battery electrodes-Revisit of Newman's model. Electrochimica Acta,

56(11):4369-4377, 2011.

[17] A. Latz and J. Zausch. Electrochimica Acta Thermodynamic deriva-

tion of a Butler - Volmer model for intercalation in Li-ion batteries.

Electrochimica Acta, 110:358-362, 2013. Acceptance of Terms and Conditions:

Click here to agree

\title{
A numerical and experimental study of drainage processes in pre- dominantly 2D microfluidic porous domains
}

\author{
Andreas Yiotis $^{\text {None }}$; Nikolaos Karadimitriou ${ }^{1}$; Ioannis Zarikos ${ }^{2}$; Holger Steeb ${ }^{3}$ \\ ${ }^{1}$ Stuttgart University \\ ${ }^{2}$ Department of Mechanical Engineering, Eindhoven University of Technology \\ ${ }^{3}$ University of Stuttgart
}

Corresponding Author(s): yiotis@ipta.demokritos.gr, nkaradimitriou@gmail.com

Immiscible two-phase flow in porous materials is a process of significant scientific and applied interest, encountered in numerous technological applications related to energy and the environment (e.g. subsurface water flow, oil recovery, soil remediation and geologic carbon sequestration). Unlike processes that include only single-phase flow, which are typically approximated at the REV-scale using Darcy's law (thus exhibiting a linear dependence between flow rates and the applied pressure difference), in two-phase flow the combined effects of viscous, capillary and gravity forces within the heterogeneous/tortuous pore space render a highly non-linear flow problem which strongly depends on pore-scale properties. As such, immiscible flow processes within porous materials can be efficiently treated using novel pore-scale-resolved Direct Numerical Simulations (DNS), as well as pore-scale microfluidic experiments. Such approaches provide significant potential for upscaling the results for use in the context of field-scale simulators.

In this contribution, we study the dynamics of immiscible two-phase flow in PDMS microfluidic porous structures that consist of randomly distributed circular or polygonal pillars to "mimic" the 
tortuous nature of a natural porous material. A non-wetting fluid (i.e. water) is injected from one side of the microfluidic structure to displace the initially resident wetting phase (i.e. fluorinert) at different flow rates in a drainage-like process. During the displacement process we record the dynamics of the phase distributions and the corresponding inlet pressure. We also calculate the average capillary pressure by using a novel methodology that relies on the recorded dynamics of the radii of curvature across interfaces. Our experimental results are then compared against a series of 2D numerical simulations performed at similar flow conditions with a commercial finite element simulator. The latter solves directly for flow and interfacial flow dynamics using a coupled implementation of the level set method and the laminar Navier-Stokes equations. The numerical simulations are found in very good agreement with the experimental results in terms of saturation topology, pressure distribution, and overall time scales over a wide range of capillary numbers, while they also offer significant physical insight on the effects of the domain depth (which is not explicitly accounted for in the simulations) on the dynamics of the drainage process. Furthermore, we recover a rich diagram of phase distribution patterns with respect to the Capillary number and viscosity ratio despite the small/finite dimensions of the computational/experimental microfluidic domain.

Procter and Gamble Student poster award:

References:

Acceptance of Terms and Conditions:

Click here to agree

904

\section{Study on occurrence state of fluid in nanopores by combining nanofluidic and simplified local density model}

Liu Yishan ${ }^{1}$

${ }^{1}$ China University of Petroleum-Beijing

Corresponding Author(s): lys2010021519@163.com

Study on occurrence state of tight oil is important to reservoir evaluation and development and many methods are proposed to study it. However, intuitive means and multi-factor analysis are extremely rare. In this study, a nanofluidic technology with a capability of describing pore structure of tight oil was applied to characterize the fluid occurrence state directly, and a SLD (simplified local density) model coupled with various of influencing factors was revised to describe how fluid exists in nanopores.

Nanofluidic experiments and SLD model were combined to investigate the occurrence state of nanopore fluid. Nanofluidic chips fabricated with photolithography and reactive-ion etching were made with silicon and silica, respectively. The adsorbed film saturated fluid in nanofluidic chips was imaged by ESEM under different materials, temperatures, pressures, pore sizes and fluid components (C5, C8, C10). Then the thickness of adsorbed film was measured from SEM images, which can directly illustrate the influences of wettability, formation conditions and fluid properties on occurrence state. Based on the experiments, the SLD model was revised by coupling with wettability changes under different conditions.

The nanofluidic experiment results indicated that the density profile was greatly influenced by wettability, temperature, pressure, fluid component and pore size. First, wettability determined the fluid density proflie at pore wall (adsorption phase) and pore center (bulk phase): under hydrophilic silica chip, the adsorbed phase density was smaller than the bulk phase density. Second, the density of adsorbed phase decreased as the temperature increased, and it increased with an increase in pressure. Third, an increase in heavy components led to an increase in the density of the adsorbed phase. Last, a larger pore size resulted in a thicker adsorbed film. The revised SLD model demonstrated the density of adsorbed phase and wettability were affected by temperature and pressure. Specifically , the wettability of the pore wall tended to be hydrophilic, and the density of adsorbed phase decreases with an increase in temperature. Additionally, the density of adsorbed phase increased, the wettability tends to be hydrophilic with an increase in pressure, but the influence of wettability is rather small, thus the density still increased.

Procter and Gamble Student poster award: 


\title{
References:
}

Acceptance of Terms and Conditions:

Click here to agree

692

\section{D printing application for flow and mechanical deformation in a single fracture network}

\author{
Author(s): Hongkyu Yoon ${ }^{1}$ \\ Co-author(s): Mario J. Martinez ${ }^{1}$; J. Eric Bower ${ }^{1}$; Alec Kucala ${ }^{1}$ \\ ${ }^{1}$ Sandia National Laboratories
}

Corresponding Author(s): hyoon@sandia.gov

\begin{abstract}
3D printing of geomaterial analog has the potential to enhance hydrogeological and mechanical interpretations by generating engineered samples in testable configurations with reproducible structures and tunable surface and mechanical properties. The use of 3D printed samples allows us to overcome sample-to-sample heterogeneity that plague rock physics testing and to test material response independent from material variability. In this work, we used two different printing techniques (stereolithography (SLA) and inkjet printing) to create single fracture network systems. For single fracture system, a set of X-ray computed microtomography (micro-CT) images of a shale core sample with single fracture network was segmented to create a digital fracture system for 3D printing. Aperture sizes in a single fracture network range from $\sim 0.2$ to $\sim 1 \mathrm{~mm}$. In particular, transparent fractured specimens based on 3D reconstruction of micro-fractured rock (i.e., 3D digital rock) were printed to study fluid flow characterization and manipulation. Here we describe the design of single fracture network and the progress of printing practices. CAD features have been integrated into the printing, including sample end pieces with a luer lock. With printed fracture samples, water inflow was tested to evaluate the impact of fracture aperture distribution on the 3D meniscus with the micro-CT system. Contact angle of water phase distribution at different locations was analyzed for different printed fractures with varying aperture sizes. Various finite element and lattice Boltzmann numerical simulations including (non-)reactive transport and multiphase flow cases are performed to study fluid flow characterization in a single fracture network. For multiphase flow, the Conformal Decomposition Finite Element Method (CDFEM) has been employed to capture water flow in the fracture network with a dynamic contact angle. We will discuss these integrated approaches using 3D printing and numerical simulations of fractured porous media to establish a science-based, rather than empirical, workflow for understanding and predicting fluid-rock interactions in fractured media.
\end{abstract}

Sandia National Laboratories is a multimission laboratory managed and operated by National Technology and Engineering Solutions of Sandia, LLC, a wholly owned subsidiary of Honeywell International, Inc., for the U.S. Department of Energy's National Nuclear Security Administration under contract DE-NA-0003525.

\section{Procter and Gamble Student poster award:}

\section{References:}

Acceptance of Terms and Conditions:

Click here to agree 


\section{Interplay among Roughness, Flow, and Transport in Rough Sin- gle Fractures: The Critical Role of Velocity Correlation on Anoma- lous Transport}

Seonkyoo Yoon $^{\text {None }}$; Peter Kang ${ }^{\text {None }}$

Corresponding Author(s): yoonx213@umn.edu

Fractures in rocks often act as major fluid pathways thereby exerting dominant control over many important subsurface processes. The complex interplay among fracture roughness, fluid flow physics and mass transport is known to cause anomalous transport that cannot be described with the Fickian framework. However, the current understanding on how the interplay controls anomalous transport through single fractures is still fragmental. We systematically investigate the complex interplay among roughness, flow, and transport in rough single fractures, and demonstrate that velocity correlation is the key mechanism that controls anomalous transport. We quantify fracture roughness with Hurst exponent $(H)$, fluid flow conditions with Reynolds number $(R e)$, and transport conditions with Péclet number $(\mathrm{Pe})$. To gain a complete picture of the complex interplay, we analyze all combinations of the three parameters in the range of $H: 0.7-0.9, \operatorname{Re}: 1-100$, and Pe: 100-100000. In the rough-walled condition $(H=0.7)$, the size of eddies grow significantly as $R e$ increases, whereas in the smoother conditions there is no significant emergence of eddies. We use a particle tracking approach for solute transport that provides Lagrangian information. We also implemented an algorithm that identifies eddy zones and distinguishes residence times in the eddy zone and in the main flow channel. When $P e$ is smaller than 10,000 , the eddies caused by roughness induce heavy tailing in residence time distribution (RTD) since the eddies trap solute particles. This confirms the previous studies which reported eddies as the origin of the heavy tailing. However, when $P e$ is larger than 10,000, we find the opposite result: the tail of RTD in the low-roughness conditions are heavier than the high-roughness conditions. When $P e$ is high enough, the eddies no longer act as trapping zones since particles hardly enter the eddy zones. The eddies rather act as a slip boundary that decreases the Lagrangian velocity correlation thereby reducing tailing. This implies that the eddies caused by fracture roughness can reduce late time tailing via velocity correlation breaking, which is contrary to the previous studies that identifies eddies as the origin of late time tailing. We further show the critical importance of velocity correlation across Re and validate our findings with a correlated CTRW that honors the velocity correlation.

Procter and Gamble Student poster award:

References:

Acceptance of Terms and Conditions:

Click here to agree

97

\section{Flow and transport in fractured poroelastic media}

${\text { Ivan } \text { Yotov }^{1} \text {; Ilona Ambartsumyan }}^{2}$; Eldar Khattatov ${ }^{2}$; Truong Nguyen ${ }^{1}$

${ }^{1}$ University of Pittsburgh

${ }^{2}$ The University of Texas at Austin

Corresponding Author(s): yotov@math.pitt.edu

We study flow and transport in fractured poroelastic media using Stokes flow in the fractures and the Biot model in the porous media. The Stokes-Biot model is coupled with an advection-diffusion equation for modeling transport of chemical species within the fluid. The continuity of flux on the fracture-matrix interfaces is imposed via a Lagrange multiplier. The coupled system is discretized by a finite element method using Stokes elements, mixed Darcy elements, conforming displacement elements, and discontinuous Galerkin for transport. The stability and convergence of the coupled scheme are analyzed. Computational results verifying the theory as well as 
simulations of flow and transport in fractured poroelastic media are presented.

Procter and Gamble Student poster award:

References:

Acceptance of Terms and Conditions:

Click here to agree

\section{Foam Propagation at Low Superficial Velocity: Implications for Long-Distance Foam Propagation}

Guanqun $\mathrm{Yu}^{1}$; William Rossen ${ }^{1}$; Sebastien Vincent-Bonnieu ${ }^{\text {None }}$

${ }^{1}$ Delft University of Technology

Corresponding Author(s): g.yu@tudelft.nl

Since the 1980s experimental and field studies have found anomalously slow propagation of foam that cannot be explained by surfactant adsorption. Friedmann et al. (1994) conducted foam propagation experiments in a cone-shaped sandpack and concluded that foam, once formed in the narrow inlet, was unable to propagate at all at lower superficial velocities towards the wider outlet. They hence concluded that long-distance foam propagation in radial flow from an injection well is in doubt (or questionable).

Ashoori et al. (2012) provides a theoretical explanation for slower or non-propagation of foam at decreasing superficial velocity. Their explanation connects foam propagation to the minimum velocity utmin or pressure gradient $\bigotimes$ Pmin required for foam generation in homogeneous porous media (Gauglitz et al., 2002). The conditions for propagation of foam are less demanding than those for creation of new foam. However, there still can be a minimum superficial velocity necessary for propagation of foam, except that it could be significantly smaller than the minimum velocity for foam generation from an initial state of no-foam.

In this study, we extend the experimental approach of Friedmann et al. in the context of the theory of Ashoori et al. We use a cylindrical core with stepwise increasing diameters such that the superficial velocity in the outlet section is $1 / 16$ of that in the inlet. N2 foam is created and stabilized by the widely used surfactant formulation C14-16 alpha olefin sulfonate. Previously, we map the conditions for foam generation, in a Bentheimer sandstone core, as a function of total superficial velocity (foam trigger), surfactant concentration and injected gas fraction (foam quality). In this study, we enrich the content of the map with the conditions for downstream propagation of foam, after its creation in the narrow inlet section at greater superficial velocity. Meanwhile, we are also interested in interpreting our results for both foam generation and propagation in terms of local pressure gradient, which plays a dominant role in the mobilization and creation of foam.

Our results suggest that the minimum superficial velocities for both foam generation and propagation increase with increasing foam quality and decreasing surfactant concentration, in agreement with theory. The minimum velocity for propagation of foam is much less than that for foam generation. In the end, the implications drawn from our lab results for field application of foam are discussed, by translation between linear core-floods and radial propagation of foam around injection well.

Procter and Gamble Student poster award:

\section{References:}

Friedmann, F., Smith, M.E., Guice, W.R., Gump, J.M., Nelson, D.G. 1994. Steam-foam mechanistic field trial in the midway-sunset field. SPERE 9(4), 297-304.

Ashoori, E., Marchesin, D., \& Rossen, W. (2012, December 1). Multiple Foam States and Long-Distance Foam Propagation in Porous Media. Society of Petroleum Engineers. doi:10.2118/154024-PA Acceptance of Terms and Conditions:

Click here to agree 


\title{
Reactive Flow in Unconsolidated Sandstone: Implications for Car- bon Geosequestration
}

\author{
Hongyan $\mathrm{Yu}^{1}$; Lebedev Maxim ${ }^{2}$; Iglauer Stefan ${ }^{3}$ \\ ${ }^{1}$ Northwest University \\ ${ }^{2}$ Curtin University \\ ${ }^{3}$ Edith Cowan University Western Australia
}

Corresponding Author(s): amelia-yu@hotmail.com

Carbon dioxide (CO2) injection into deep saline aquifers is considered a viable solution for largescale $\mathrm{CO} 2$ storage. However, $\mathrm{CO} 2$ mixed with water (live brine) creates a medium strength acid at high pressure and temperature. Thus, injecting CO2 into a deep reservoir leads to reactive flow. Consequently, it is thus of great importance that these pore-scale mechanisms are understood in detail. We thus imaged an unconsolidated sandstone samples at reservoir conditions before and after $\mathrm{CO} 2$ saturated (live) brine injection in situ with X-ray micro computed tomography (micro-CT) in 3D at high resolution, and also conducted X-ray diffraction (XRD) and Scanning electron microscope (SEM) to explore the effects on the pore structure, associated with that the permeability, mineral morphology and composition, and $\mathrm{CO} 2$ injectivity. All these properties may change due to fines migration, rock dissolution and/or solid re-precipitation. We observed that the size of the large pores increased and the size of small pores decreased, thus enhancing inter-pore connections, drastically increasing permeability. The main reason can be explained the alternation in pore structure and permeability is the mechanically fines migration. Some fines particle flush out the core due to the critical flow velocity beyond the release of fines is triggered and some clay minerals dissolution during the live brine injection, yielding the porosity and permeability increased. Furthermore, many fractures were generated in the quartz grain after live brine flooding. We conclude that the pore structure of unconsolidated highly porous rock can significantly change after live brine injection, although porosity is only slightly affected. Injecting $\mathrm{CO} 2$ into unconsolidated sandstone can dramatically change the pore structure and increase the permeability of the rock, which will significantly enhance CO2 injectivity for economic performance.

Procter and Gamble Student poster award:

I don't want to compete References:

Acceptance of Terms and Conditions:

Click here to agree

\section{A dynamic simulator for gas diffusion and surface adsorption- desorption in fractured coal}

\author{
$\mathrm{Xu} \mathrm{YU}{ }^{\text {None }}$; Klaus Regenauer-Lieb ${ }^{1}$ \\ ${ }^{1}$ School of Minerals and Energy Resources Engineering, UNSW Sydney, Australia;
}

Corresponding Author(s): xu.yu@student.unsw.edu.au

\begin{abstract}
Coalbed methane is a cause of concern for safety in mines, as well as an unconventional resource. It is mainly stored in micropores of the matrix, as well as adsorbed on the interface of cleats. The recovery process of coalbed methane is the flow and diffusion of desorbed gas. To date, no dynamic simulation environment exists that is capable of simulating the multi-scale thermo-hydro-chemical feedbacks of gas diffusion and adsorption in a fractured coal. In this work, a pore-scale model of gas flow and diffusion in fractured coal is proposed. This model is capable of solving the gas diffusion problem, considering the effects of cross-scale surface adsorption and desorption between methane
\end{abstract}


molecules, the coal pores, the matrix during gas flow through the cleat network.

The gas flow and diffusion are governed by the advection-diffusion-reaction equation. The gas velocity and concentration in cleats are controlled by the incompressible Navier-Stokes equations and advection-diffusion equation which are solved by the lattice Boltzmann method (LBM). The natural cleats are assumed are to be the primary pathways for the gas and water flow through the reservoirs. The immersed boundary method allows high flexibility as it efficiently deals with curved boundaries. It is used to solve the momentum and mass exchange on the cleat-matrix interface. The surface sorption of methane is considered as a chemical reaction only on existing boundary nodes. The reaction rate for adsorption is given by the linear driven force equation which has been validated and is equal to the kinetic form of Langmuir isotherm.

As gas adsorption and desorption are significant for the coal bed methane recovery, about $98 \%$ of methane is in the adsorbed state in the micropores. With the numerical model, the effects of surface area, surface roughness, aperture of cleat and adsorption coefficient on the adsorption amount are studied and show good agreements with the experimental data. Gas diffusivity is the key factor for the rate of methane production in petroleum engineering. The relationship between adsorption/desorption and gas diffusivity is also investigated based on this model. The numerical model is a robust tool to study the kinetic process of gas diffusion and adsorption. Results of the research can help people understand the effects of gas adsorption/ desorption on the gas diffusion at small scale and industrial production at reservoir scale.

Procter and Gamble Student poster award:

I would like to compete in the Procter and Gamble Student award References:

Acceptance of Terms and Conditions:

Click here to agree

\title{
High resolution sequence stratigraphic characteristics of ahe for- mation,Tarim basin
}

\author{
Chun Yuan ${ }^{1}$ \\ ${ }^{1}$ Research Instuite of Petroleum Exploration and Development \\ Corresponding Author(s): yuanchun1996@163.com
}

High resolution sequence stratigraphy is based on the principle of base-level cycle and the principle of accommodating spatial variation to reveal the relationship between base-level cycle sequence and sedimentary dynamics and stratigraphic response process, as well as the corresponding sedimentary microfacies evolution sequence, preservation status and the development location and output characteristics of favorable reservoir sand bodies, source and interval layers.Use drilling core, logging (YiNan 4, 2 well and deep YiNan 4 Wells) and outcrop (g kyzyl nur and vomit, Ming) etc, through changes in vertical lithologic facies, vertical phase sequence and the change of the combined cycle of the overlay style and geometric relations of strata cycle identification, lower Jurassic in kuche depression formed can be identified six long-term base level cycle (equivalent to Vail III sequence) and 20 to 23 mid-term base-level cycle (equivalent to Vail IV sequence);Among them, well Yinan 2 has 20 medium-term base level cycles, well Yinan 4 has 23 medium-term base level cycles, and well Yinan 4 has 22 medium-term base level cycles .Long-term base level cycle is the foundation and key to establish high-resolution sequence stratigraphic correlation framework in this area. The variation of phase combanation,thickness and symmetry is small,thus the whole area can be tracked and compared.Medium-term base level cycle is the basic unit of reservoir heterogeneity research, reservoir prediction and combination analysis of source, reservoir and cap.

The determination of the medium-term datum level cycle in the drilling profile is marked by: the scour phenomenon in the stratigraphic profile and the overlying sediment, the transformation of lithofacies type or facies combination on the vertical profile, and the change of the thickness cycle of sand and mudstone;Characteristics of natural gamma curve and apparent resistivity curve.The sequence interface of the medium-term base level cycle is the small scour surface or intermittent exposed surface formed when the medium-term base level drops to the lowest point.It can also be 
the transition surface of the medium-term base level decline and rise.According to the characteristics of the sequence interface and the phase sequence, the medium-term base level cycle can be roughly divided into three types with obviously different structures:An asymmetric cycle sequence with upward "deepening",An asymmetric upward "shallower" cyclic sequence.Symmetric cyclic sequence

Procter and Gamble Student poster award:

I don't want to compete References:

Acceptance of Terms and Conditions:

Click here to agree

947

\title{
High resolution sequence stratigraphic characteristics of ahe for- mation, Tarim basin
}

Chun Yuan ${ }^{1}$

${ }^{1}$ Research Instuite of Petroleum Exploration and Development

Corresponding Author(s): yuanchun1996@163.com

High resolution sequence stratigraphy is based on the principle of base-level cycle and the principle of accommodating spatial variation to reveal the relationship between base-level cycle sequence and sedimentary dynamics and stratigraphic response process, as well as the corresponding sedimentary microfacies evolution sequence, preservation status and the development location and output characteristics of favorable reservoir sand bodies, source and interval layers. Use drilling core, logging (Yinan 4, 2 well and Yishen 4 Wells) and outcrop (Kezlenur group and Tugerming group) etc, through analyzing changes in vertical lithologic facies, vertical phase sequence and the change of the combined cycle of the overlay style and geometric relations of strata cycle identification, lower Jurassic in kuche depression formed can be identified as six long-term base level cycle (equivalent to Vail III sequence) and 20 to 23 mid-term base-level cycle (equivalent to Vail IV sequence);Among them, well Yinan 2 has 20 medium-term base level cycles, well Yinan 4 has 23 medium-term base level cycles, and well Yinan 4 has 22 medium-term base level cycles .Long-term base level cycle is the foundation and key to establish high-resolution sequence stratigraphic correlation framework in this area. The variation of phase combination, thickness and symmetry is small, thus the whole area can be tracked and compared. Medium-term base level cycle is the basic unit of reservoir heterogeneity research, reservoir prediction and combination analysis of source, reservoir and cap.

Procter and Gamble Student poster award:

I don't want to compete References:

Acceptance of Terms and Conditions:

Click here to agree

599

\section{An innovative workflow for designing ion-tuned water flooding of carbonate reservoirs}

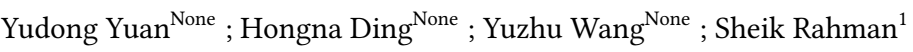 \\ ${ }^{1}$ School of Minerals and Energy Resources Engineering, UNSW Sydney
}

Corresponding Author(s): yydcug@163.com 
1. Objectives/Scope

Numerous authors have contributed to improve our understanding of wettability alternation by low salinity or smart and ion-tuned water flooding in carbonate reservoirs. The underlying concept of these approaches is to alter the surface wettability by electro-chemical interactions between oil, specific ions in brine and carbonate minerals. This can be done by manipulating the composition of injection water. To date, however, there a general lack of quantitative analysis of how different ions contribute to wettability alternation. In this study, we propose an innovative approach which combines experimental and numerical methods to capture the mechanisms of wettability alteration by different individual or combination of ions and quantitatively assess their capacity to enhance oil recovery from an oil-wet carbonate rock.

2. Methods, Procedures, Process

Atomic force microscopy (AFM) with "soft tip" technique, zeta potential and contact angle measurements are carried out to estimate intermolecular forces between oil tip and cleaved calcite at different brine solutions ( $\mathrm{NaCl}, \mathrm{MgCl} 2, \mathrm{CaCl} 2$, and $\mathrm{Na} 2 \mathrm{SO} 4$ at a constant ionic strength). These data are used to guide us to manipulate the individual ion. Surface complexes that contributed to surface charge change are then numerically simulated to optimise the composition of ion-tuned water. Water flooding is carried out with $6 \mathrm{~mm}$ carbonate samples and oil recovery is monitored and the relative permeability is estimated. At different stages of water flooding, micro-CT images are taken at resolution of $2 \mu \mathrm{m}$ to visualise pore scale distribution of contact angles using an in-housed image processing algorithm. We have also used our in-house reactive transport simulator to reproduce the relative permeability and the production profile of the optimised ion-tuned water flooding experiment.

3. Results, Observations, Conclusions

AFM force measurements have demonstrated that Mg2+ ions generate less adhesion and stronger repulsion. In contrast, oil is more likely to adhere onto calcite surface in $\mathrm{Ca} 2+$ ions. Therefore we manipulate seawater containing more $\mathrm{SO} 42$ - and less $\mathrm{Mg} 2+/ \mathrm{Ca} 2+$. Zeta potential results and contact angle data show that only SW4SO and SW1/4Ca can modify the water-wet to a larger magnitude. These two waters are selected for the IPhreeqc reactive-transport modelling and porescale flow simulation. The simulation results correlate well with the water-flooding experiments, which show an additional recovery of up to $15 \%$.

4. Novel/Additive Information

(1) AFM and pore scale micro-CT analysis have allowed us to quantitatively determine the intermolecular forces contribute to the changes of wettability and even to visualise these changes. (2) The current work focuses on carbonate rocks on which very limited amount of studies have been carried out to date. (3) We suggest a work flow of optimising ions in water (ion-tuned water) for enhancing oil recovery. (4) The combination of experimental and numerical work flow ensures target oil recovery, such as the one achieved as part of this study.

Procter and Gamble Student poster award:

References:

Acceptance of Terms and Conditions:

Click here to agree

\title{
Dust suppression by enzyme induced carbonate precipitation (EICP) in soils
}

\author{
Author(s): Jun Young Song ${ }^{\text {None }}$; Youngjong Sim ${ }^{\text {None }}$; Jaewon Jang ${ }^{1}$ \\ Co-author(s): Tae Sup Yun ${ }^{2}$ \\ ${ }^{1}$ Hanyang University \\ ${ }^{2}$ Yonsei University
}


Corresponding Author(s): taesup@yonsei.ac.kr

The fine dust scattering originated from the open-air storage yard and transportation of heavy trucks brings about the serious civil complaints at the early stage of construction site. The most common methods to mitigate the fine dust are watering and chemical treatment which are often temporary, expensive and contaminating groundwater and soil environment. To overcome these issues, we apply the enzyme-induced carbonate precipitation method to possibly suppress the fine dust. Bacteria and enzyme can activate the hydrolysis whereas the in-situ bacteria may be disadvantageous because of uncertainties in bacterial activity, sensitivity to the soil environment such as bacterial competition and nutritional status, and bacterial trapping. Therefore, the direct usage of enzyme can reduce the reaction process of bacterial usage, and allows appropriately controlling the degree of carbonation. When spraying the EICP solution on the soil surface, one expects that soil particles are cemented by the precipitated calcium carbonate and fine dust are suppressed during precipitation. We collect the in-situ soil from the construction site which is categorized as SP. The sample tray has $20305 \mathrm{~cm} 3$ and SP soil is compacted to achieve $50 \%$ relative density. The EICP solution is composed of $0.5 \mathrm{M} \mathrm{CaCl} 2,0.75 \mathrm{M}$ urea, and $0.5 \mathrm{~g} / \mathrm{L}$ urease is sprayed to the soil surface with varying amount from $1 \mathrm{~L} / \mathrm{m} 2$ to $9 \mathrm{~L} / \mathrm{m} 2$. The reaction is allowed for 30 hours and the sample is dried in a $60{ }^{\circ} \mathrm{C}$ for 24 hours. The tray is then placed $180 \mathrm{~cm}$ away from the inlet within the wind tunnel which as 40 $40250 \mathrm{~cm} 3$ in dimension. The wind velocity of $5 \mathrm{~m} / \mathrm{s}$ is continuously applied to both untreated and treated samples. The tray and instrument measuring dust concentration are placed at the location of $180 \mathrm{~cm}$ and $250 \mathrm{~cm}$ away from the wind fan. Also, we measure the cone-resistance of treated sample by customized cone penetration test to examine the sustainability of surface-treated soil and the effectiveness along the depth. The samples made with the same sequence are prepared in the cylindrical chamber of $20 \mathrm{~cm}$ in diameter and $30 \mathrm{~cm}$ in height. The fine dust of treated sample is significantly decreased compared with untreated sample and the specimens treated with more than $3 \mathrm{~L} / \mathrm{m} 2$ showed similar values shown in the sample treated with water or salt. The peak value of the cone resistance near surface sharply increases followed by gradual reduction for EICP treated sample, exhibiting the high resistance to surface load. From these results, EICP treatment is effective in find dust suppression with improved resistance and it can further prevent any potential corrosion of construction machinery due to salt-treated.

Procter and Gamble Student poster award:

References:

Acceptance of Terms and Conditions:

Click here to agree

506

\title{
Equilibrium Constraints in Low Salinity Waterflooding of Car- bonate Reservoirs
}

\author{
Maxim Yutkin ${ }^{1}$; Clayton Radke ${ }^{2}$; Tadeusz Patzek \\ ${ }^{1}$ King Abdullah University of Science and Technology \\ ${ }^{2}$ Professor - University of California, Berkeley
}

Corresponding Author(s): maxim.yutkin@gmail.com

Success of low salinity waterflooding (LSW) is attributed to increasing water wetness of the reservoir rock. Consequently, surface chemistry of the crude oil/rock/brine interfaces is a critical component of the process. Many studies, therefore, focus on wettability measurements including, for example, contact angles, surface charge densities, ion exchange, and zeta potentials, in addition to oil-recovery flooding. In carbonate rocks, however, injected chemistry is drastically altered by reaction with reservoir minerals, making injection chemistry irrelevant to in-situ conditions. This presentation argues that in all laboratory experiments involving carbonate rock, the aqueous flooding solution must be pre-equilibrated with the reservoir minerals. Otherwise, process chemistry is not that actually occurring in the reservoir; all diagnostic wettability assessments become misleading. 
To reveal the critical importance of reactive-mineral dissolution/precipitation in LSW, we consider brine injection into a one-dimensional porous calcite rock containing movable oil. Conservation of calcium ion of concentration $C(t, z)$ in the aqueous flowing phase reads

$\varphi S_{w} \frac{\partial C}{\partial t}+u f_{w} \frac{\partial C}{\partial z}=(1-\varphi) a_{V} r_{A}$

where $t$ is time, $z$ is axial distance, $\varphi$ is porosity, $S_{w}$ is water saturation, $f_{w}$ is fractional water flow, $u$ is superficial velocity (i.e., frontal advance rate), $a_{V}$ is rock surface area per solids volume, and $r_{A}$ is the net dissolution rate of calcite (i.e., the difference between dissolution and precipitation rates) per unit solids area $\left(\mathrm{mol} / \mathrm{m}^{2} / \mathrm{s}\right)$.

The key to understanding reactive-mineral equilibration is through the reaction rate, $r_{A}$. We establish that for small deviations from equilibrium the governing rate expression is

$\mathrm{r}=\mathrm{k}_{m}\left[\left(K_{S P} / C_{C O_{3}^{2-}}\right)-C_{C a^{2+}}\right] /\left[1+k_{m} \sqrt{K_{S P}} /\left(k_{r x n} C_{C O_{3}^{2-}}\right)\right] ; \quad \mathrm{pH}<10$

where $k_{m}(\mathrm{~m} / \mathrm{s})$ is the convective-diffusion mass transfer coefficient appropriate to flow through porous media, $k_{r x n}$ is the intrinsic dissolution reaction-rate constant $(\mathrm{m} / \mathrm{s}), C_{i}$ is the equilibrium concentration, and $K_{S P}$ is the solubility product of calcium carbonate.

The reactive transport model is solved at constant (residual) oil saturation and water fractional flow equal to 1 . For a step lowering of injected brine hardness, our predictions are shown as lines in fig. 1 on a semi-logarithmic scale. Effluent calcium concentration from the carbonate core is seen as a step change from that initially present in the medium $(0.1 \mathrm{M})$. For $D a_{r x n}$ numbers larger than 10 , equilibrium is achieved in the pore-fluid composition. Since experimental $k_{r x n}$ is approximately

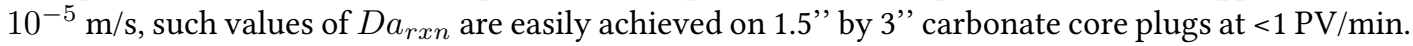
The calcium concentration front moves as an equilibrium shock wave through the core (with neglect of dispersion). There is no rock dissolution/precipitation except immediately at the very beginning of the core (or reservoir). We conclude that aqueous equilibration with carbonate rock is fast.

This conclusion has major implications for understanding LSW. First, injected flooding concentration is not that appearing in the reservoir pore fluids. Regardless of the injected $\mathrm{pH}$, the in-situ $\mathrm{pH}$ is reset by the equilibrium to a value that depends on the $\mathrm{CO}_{2}$ and $\mathrm{Ca}^{+2}$ content. Such value is usually lower than 9, as presence of any of these components lowers the $\mathrm{pH}$, and therefore saponification is unlikely (Yutkin et al. 2018). Second, the proposed dissolution or surface reconstruction (Chen et al. 2017) recovery mechanisms are not possible. We assert that nowhere in the reservoir, except directly at the wellbore, there is rock dissolution/precipitation. Any recovery mechanism that relies on rock dissolution/precipitation cannot occur on reservoir scale. The suggested dissolution mechanisms can only take place in laboratory experiments. Next, we emphasize that all characterization laboratory experiments, such as zeta potentials, contact angles, surface charges, etc., must be performed with rock pre-equilibrated aqueous solutions so that reservoir conditions are correctly mimicked. Otherwise, generated results are unrealistic. Care must be taken to ensure that pore-fluid equilibrium is achieved in laboratory studies. 

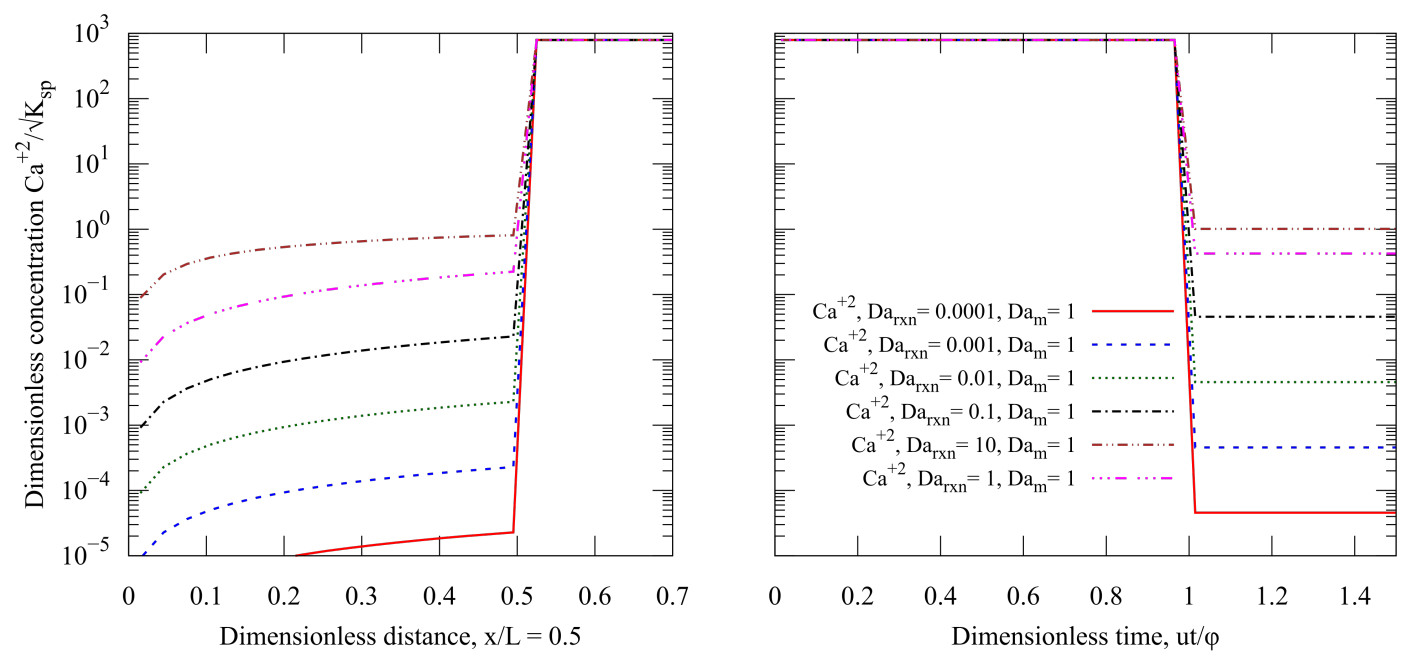

Figure 15: Calcium concentration profile and history predicted from reactive transport model in Eqns. 1, 2. Effluent calcium concentration from a carbonate core is a step change from that initially in the reactive porous medium to that at reaction steady state. Breakthrough occurs at 1 pore volume

\section{Procter and Gamble Student poster award:}

\section{References:}

Chen, Szu-Ying, Yair Kaufman, Kai Kristiansen, Dongjin Seo, Alex M Schrader, Mohammed B. Alotaibi, Howard A Dobbs, et al. 2017. "Effects of Salinity on Oil Recovery (the 'Dilution Effect'): Experimental and Theoretical Studies of Crude Oil/Brine/Carbonate Surface Restructuring and Associated Physicochemical Interactions." Energy \& Fuels 31 (9): 8925-41. https://doi.org/10.1021/acs.energyfuels.7b00869.

Yutkin, Maxim P., Himanshu Mishra, Tadeusz W. Patzek, John Lee, and Clayton J. Radke. 2018. "Bulk and Surface Aqueous Speciation of Calcite: Implications for Low-Salinity Waterflooding of Carbonate Reservoirs." SPE Journal 23 (01): 084-101. https://doi.org/10.2118/182829-PA. Acceptance of Terms and Conditions:

Click here to agree

\section{Thermodynamic modeling of adsorption kinetics}

Zaineb Zaafouri ${ }^{1}$; Daniela Bauer ${ }^{2}$; Guillaume Batôt ${ }^{1}$; Carlos Nietos Draghi ${ }^{1}$; Benoit Coasne ${ }^{3}$

${ }^{1}$ IFP Energies Nouvelles

${ }^{2}$ IFP Energies nouvelles

${ }^{3}$ CNRS/University Grenoble Alpes

Corresponding Author(s): zaineb.zaafouri@ifpen.fr

Adsorption is a surface phenomenon playing a key role in industrial applications such as heterogeneous catalysis, separation, purification or enhanced oil recovery. It consists in the accumulation on a solid surface of atoms, ions or molecular species from a gas or a liquid phase. Pore sizes in porous media range from nanometers to millimeters, thus the specific surface can be extremely high leading to an important quantity of adsorbed molecules.

The adsorption phenomenon is usually studied through the determination of experimental adsorption isotherms which relate the amount of adsorbed molecules to their constant bulk concentration 
and temperature. The nature of the molecular interactions with the surfaces at play varies from one system to another, so that there are many adsorption isotherm shapes that can be analyzed by different models. Each model consists of a number of parameters, which define the specific adsorption isotherm of the different chemical components. If the adsorption isotherms can be well reproduced by an appropriate model, different information can be obtained about the type of adsorption (physical or chemical), and the nature of the fluid-solid interactions (exothermic or endothermic).

In this work, we will first report experimental data for static adsorption of AOT (ionic surfactant: bis (diéthyle-2, hexyl) sodium sulfosuccinate) in amorphous silica. The experimental results reveal a S-shaped adsorption isotherm which is different from the standard Langmuir isotherm. The aim of this work is to propose a new isotherm model to adjust the data.

The problem of fitting adsorption isotherms that are not of the Langmuir type is not new. Previous works already address the problem of modelling non-Langmuir isotherms to match the $\mathrm{H}, \mathrm{L}, \mathrm{S}$ and $\mathrm{C}$ types 1. However, these equations still do not fit adsorption isotherms for surfactants in porous medium. In order to address this issue, we modify the Langmuir Kinetic model defined by:

$\frac{*}{t}=k_{a}\left(Q_{a}^{*}-{ }^{*}\right)-k_{d}^{*}$

where ${ }^{*}$ is the amount of adsorbed surfactant, $k=k_{a} / k_{d}$ the adsorption-desorption rate, $k_{a}, k_{d}$ adsorption/desorption constants, and $Q_{a}^{*}$ the maximum adsorption capacity. We show that the experimental data can be accounted for if cooperative effects are included in the Langmuir model. To this goal, we replace the adsorption/desorption rate constant $k$ in the adsorption kinetics by a concentration-dependent one (in practice, a power law dependence is used). We show that this can lead under certain hypothesis to a Sips adsorption isotherm in static conditions. The power exponent is linked to the cooperative interactions where adsorbed molecules enhance the adsorption of new additional molecules.

This model opens perspectives to present a large variety of analytical isotherms which lead to fit a wider range of experimental data. Furthermore, the proposed isotherms have been used in a Lattice Boltzmann model in order to study the transport properties of adsorbent tracer in porous media.

\title{
Procter and Gamble Student poster award:
}

I would like to compete in the Procter and Gamble Student award References:

1: GRANT, Patrick G., LEMKE, Shawna L., DWYER, Maxene R., et al. Modified Langmuir equation for S-shaped and multisite isotherm plots. Langmuir, 1998, vol. 14, no 15, p. 4292-4299 Acceptance of Terms and Conditions:

Click here to agree

\section{Lattice Boltzmann Modelling of surfactant adsorption and trans- port in porous media}

\author{
Zaineb Zaafouri ${ }^{1}$; Benoit Coasne ${ }^{2}$; Guillaume Batôt ${ }^{1}$; Carlos Nietos Draghi ${ }^{1}$; Daniela Bauer ${ }^{3}$ \\ ${ }^{1}$ IFP Energies Nouvelles \\ ${ }^{2}$ CNRS/University Grenoble Alpes \\ ${ }^{3}$ IFP Energies nouvelles
}

Corresponding Author(s): zaineb.zaafouri@ifpen.fr

Adsorption under flow conditions plays an important role in many industrial processes. An example is the enhanced oil recovery where surfactants are injected in the reservoir to lower the surface tension between the oil and the water. However, they also tend to be adsorbed at the solid surface. Furthermore, surfactants are subjected to a varying velocity field due to the heterogeneity of porous rocks. In fact, the complex rock structure strongly affects the velocity and therefore the transport characteristics.

The present work aims to model and understand the influence of the interplay between transport properties and specific adsorption kinetics on the adsorbed quantity of surfactants in porous media. We use a Lattice-Boltzmann code based on the two relaxations time (TRT) variant 1 . The main advantage of this scheme is that the physical parameters (viscosity or molecular diffusion) do not depend on the numerical ones, thus limiting numerical uncertainties. 
We study transport of an adsorbing tracer (e.g. surfactant) in 2D geometries (between two parallel plates and in porous media). To this goal, we solve the Advection-Diffusion Equation (ADE) coupled with adsorption kinetics:

$\frac{c}{t}+u c-\left(D_{m} c\right)+\frac{*}{t}=0$,

where ${ }^{*}$ is the quantity of adsorbed surfactant, $c$ is the surfactant concentration in the liquid phase, $u$ is the velocity and $D_{m}$ is the molecular diffusion coefficient. We consider Langmuir adsorption kinetics given by:

$\frac{{ }^{*}}{t}=k_{a}\left(Q_{a}^{*}-{ }^{*}\right)-k_{d}^{*}$,

where the corresponding isotherm is defined as ${ }^{*}=\frac{Q_{a}^{*} k c_{e}}{1+k c_{e}}$ with $c_{e}$ being the concentration at equilibrium, $k=k_{a} / k_{d}$ the adsorption-desorption rate, $k_{a}, k_{d}$ the adsorption/desorption constants and $Q_{a}^{*}$ the maximum adsorption capacity.

At first, in order to compare the transport of passive tracer with the transport of adsorbing tracer, a pulse is injected between the two parallel plates. Following the spreading of the pulse in the fluid, we compare the evolution in time and space of the adsorbed and non-adsorbed quantities for different velocities and $k$ values. Results show that the adsorption slows down part of the molecules causing tailing in the concentration profile.

We also compute the evolution of the displacement variance as a function of time ${ }_{r}^{2}(t)$ and determine its time derivative as $D(t)=\frac{1}{2} \frac{d_{r}^{2}(t)}{d t}$. As expected, for the non-adsorbing tracer, we obtain $D(t)=D_{\text {eff }}$ for $t \rightarrow \infty$ where $D_{\text {eff }}$ is the effective Taylor dispersion coefficient. For the adsorbing tracer, the results of the simulation show that for large time, $D(t)$ also reaches a plateau with $D(t)=D_{e f f}^{a d s}$. This result stands for the fact that even in the case of transport of an adsorbing tracer an effective dispersion regime exists. It is finally observed that the effective dispersion coefficient $D_{\text {eff }}^{a d s}$ depends on the ratio $k=k_{a} / k_{d}$, it increases when the adsorption dominates.

We then simulate transport of the adsorbing tracer in porous media to study the influence of $\mathrm{k}$ on the spatial concentration distribution. The simulations show that the transport is highly dependent on adsorption/desorption ratio due to heterogeneity of the velocity field.

Thus, in conclusion, to correctly simulate the transport in porous media, introducing adequate adsorption kinetics is crucial.

\section{Procter and Gamble Student poster award:}

I would like to compete in the Procter and Gamble Student award References:

1 Ginzburg, I., Verhaeghe, F., \& d'Humières, D. (2008). Two-relaxation-time lattice boltzmann scheme: About parametrization, velocity, pressure and mixed boundary conditions. Communications in Computational Physics, 3(2), 427-478. Acceptance of Terms and Conditions:

Click here to agree

\section{Advanced X-ray Computed Tomography in Additive Manufactur- ing}

Gerhard Zacher ${ }^{1}$

${ }^{1}$ GE Sensing \& Inspection

Corresponding Author(s): gerhard.zacher@bhge.com

Additive manufacturing is increasingly used for producing high performance parts, such as turbine blades, medical implants and tools, and for these reasons quality control is an essential part of it. Hereby X-ray computed tomography (CT) adds value at different stages of the whole process chain as it offers non-destructive testing at high precision. For instance, prior to manufacturing, powder for metal printing is analyzed in terms of size distribution, sphericity, foreign particles and porosity inside the grains. Furthermore, manufactured samples and components are controlled by threedimensional (3D) failure analysis and dimensional measuring. An iterative process of X-ray CT control and printing parameter changes help optimizing the final product. Later in the manufacturing process, powder residues in the interior structures is easily detected as well. The X-ray CT is also used to regularly verify the system performance of 3D printing machines.

In summary, there is an increasing tendency for Additive Manufacturing for larger series as well as for critical products which requires new quality control methods in the whole productive chain. 
Therefore, high precision and non-destructive techniques like X-ray CT are in growing demand. This contribution shows several case studies highlighting the power of various X-ray CT analytics.

Procter and Gamble Student poster award:

References:

Acceptance of Terms and Conditions:

Click here to agree

104

\title{
Positron Emission Tomography in Water Resources and Subsur- face Energy Resources Engineering Research
}

\author{
Author(s): Christopher Zahasky ${ }^{1}$ \\ Co-author(s): Takeshi Kurotori ${ }^{2}$; Ronny Pini ${ }^{2}$; Sally M. Benson ${ }^{1}$ \\ ${ }^{1}$ Stanford University \\ ${ }^{2}$ Imperial College London \\ Corresponding Author(s): zahas002@stanford.edu
}

Recent studies have demonstrated that positron emission tomography (PET) is a valuable tool for insitu characterization of fluid transport in porous and fractured geologic media at the laboratory scale. While PET imaging is routinely used for clinical cancer diagnosis and preclinical medical researchand therefore imaging facilities are available at most research institutes-widespread adoption for applications in water resources and subsurface energy resources engineering have been limited by real and perceived challenges of working with this imaging modality. In this study we discuss and address these challenges, and provide detailed analysis highlighting how positron emission tomography can complement and improve laboratory characterization of different subsurface fluid transport problems. Specifically, unique experimental design aspects of PET studies will be discussed such as radiotracer selection and radiotracer handling safety, and a new protocol for radioactivity dosing optimization for imaging in geologic materials. Signal-to-noise and sensitivity analysis comparisons between PET and clinical computed tomography are performed to highlight how PET data can complement more traditional characterization methods. PET imaging is shown to be especially well suited for solute mixing and spreading quantification due to the high signal-to-noise ratio, excellent temporal resolution, and negligible influence of radiotracer on fluid transport properties. Analysis of solute pulse injection experiments indicate that with optimal radioactivity dosing PET has a signalto-noise ratio over 50 times greater than that of clinical CT. Examples and analysis will provide a better understanding of the strengths and weakness of PET and how to best utilize PET-derived data for future studies.

Procter and Gamble Student poster award:

References:

Acceptance of Terms and Conditions:

Click here to agree

\section{Lithium Ion Battery Separators: understanding the importance of surface interactions and pore space topology for cell operation and manufacturing}

Raphael Zahn ${ }^{1}$; Christina Sauter ${ }^{1}$; Marie Francine Lagadec ${ }^{1}$; Vanessa Wood ${ }^{1}$ 
${ }^{1}$ ETH Zurich

Corresponding Author(s): rzahn@ethz.ch

Li-ion battery (LIB) separators are porous membranes that electronically isolate the battery's electrodes yet allow ionic transport between them. As a result, the optimal separator design is a tradeoff between high cycling performance and battery safety 1 .

We developed a method to obtain quantitative 3D datasets of LIB separators using focus-ion-beam scanning electron microscopic (FIB-SEM) tomography 2. We have applied this to wet-stretched polyethylene (PE) and dry-stretched polypropylene (PP) separators, which exhibit distinct morphologies that stem from the different processes used to manufacture them. This allows us, for example, to quantify the isotopic nature of the PE separator and the anisotropic structure of PP, to run diffusion and mechanical simulations on realistic structures, and to simulate imbibition of the separators with electrolyte.

Traditionally, when discussing separator performance, diffusion of lithium ions in the electrolytefilled pore space is given by the diffusion of lithium ions in the electrolyte scaled by the effective transport coefficient of the microstructure, which is the ratio of porosity and tortuosity. Surprisingly, the effective transport parameters of most separators as measured by experimental methods are significant lower compared to values determined by steady-state diffusion simulations or geometrical approaches $[3,4]$.

Here, we show that interactions between the separator surface and the electrolyte are responsible for the differences in effective transport. We modify commercial polyethylene separators with ultrathin layers $(\sim 1 \mathrm{~nm})$ of selected polyelectrolytes and characterize their performance by EIS, and by steady-state diffusion simulations $[2,4,5]$. We show that these coatings do not alter the separators' geometry, but considerably change their wetting behavior and their ion transport properties. In imbibition/drainage simulations we demonstrate, that differences in the physico-chemical properties of the separator-electrolyte interface cause different amounts of gas to be entrapped in the separator pores during electrolyte infilling. These gas inclusions cause the observed differences in performance.

We also show that characterizing separators solely by their porosity and tortuosity is insufficient, and that pore space connectivity should be considered as an additional parameter for separator performance. Our analysis of tomographic data of commercial separators reveals that different polyolefin separators have similar porosity and tortuosity, which, in the homogenized picture of lithium ion cell operation, would imply that these different separators exhibit similar performance. However, we see in numerical diffusion simulations that these separators strongly differ in their ability to smoothen local ion gradients. A topological and network analysis of the separators' pore space shows that high pore space connectivity more effectively equalizes differences in ion concentration that can be caused, e.g., by locally blocked pores. We propose that pore space connectivity should be considered along with porosity and tortuosity in separators, to understand and optimize separator performance, and that topological and network theory should be applied regularly to characterize the porous structures in energy applications.

\section{Procter and Gamble Student poster award:}

I don't want to compete References:

1. Zhang, S.S., A review on the separators of liquid electrolyte Li-ion batteries. Journal of Power Sources, 2007. 164(1): p. 351-364.

2. Lagadec, M.F., et al., Communication-Technique for Visualization and Quantification of LithiumIon Battery Separator Microstructure. Journal of The Electrochemical Society, 2016. 163(6): p. A992-A994.

3. Landesfeind, J., et al., Tortuosity Determination of Battery Electrodes and Separators by Impedance Spectroscopy. Journal of the Electrochemical Society, 2016. 163(7): p. A1373-A1387.

4. Zahn, R., et al., Improving Ionic Conductivity and Lithium-Ion Transference Number in LithiumIon Battery Separators. ACS Appl Mater Interfaces, 2016. 8(48): p. 32637-32642.

5. Zahn, R., M.F. Lagadec, and V. Wood, Transport in Lithium Ion Batteries: Reconciling Impedance and Structural Analysis. ACS Energy Letters, 2017. 2(10): p. 2452-2453. 
Acceptance of Terms and Conditions:

Click here to agree

\title{
Micro-Scale Mathematical Model of Freezing in a Porous Medium
}

\author{
Alexandr $\mathrm{Zak}^{1}$; Michal Benes ${ }^{2}$; Martina Sobotkova ${ }^{3}$; Tissa H. Illangasekare ${ }^{4}$ \\ ${ }^{1}$ Czech Technical University in Prague, Faculty of Nuclear Sciences and Physical Engineering \\ ${ }^{2}$ Czech Technical University in Prague \\ ${ }^{3}$ CTU in Prague \\ ${ }^{4}$ Colorado School of Mines, Center for Experimental Study of Subsurface Environmental Processes
}

Corresponding Author(s): alexandr.zak@fjfi.cvut.cz

\begin{abstract}
In the contribution, we present a model of freezing and thawing in a fully saturated porous medium. The phase transition occurs in pores of the porous medium with grains intact but participating in the heat transfer, and is accompanied by mechanical effects. The research is motivated by the climate changes inducing thawing of permanently frozen land with further environmental impact as well as by the development of advanced materials. Freezing and thawing inside the porous medium is accompanied by complex processes affected by the material composition, micro-scale interfaces between phases within the porous medium, bulk properties of the present phases, and ambient physical conditions. Volumetric changes of the liquid presented in pores subjected to phase change conditions is one of crucial phenomena.

Due to the generic inhomogeneity of the volume occupied by the freezing porous medium, we focus on treating the phase transition at microscale. We have developed a micro-scale model describing mechanical, thermal, and phase change processes within a small sample of a porous medium. The phase

change is described in the Lagrangian framework by means of the energy, Navier, and phase-field equations. A coupling of multi-physics and multiple phases is introduced. The model provides spatio-temporal dependencies of primary variables, the resulting forces exerted on grain surfaces by the change in specific volume due to phase transition, and possibly, the mean values of the key quantities useful for upscaling. The model is discussed in the context of small-scale experiments, and its functionality is demonstrated on several computational studies which follow the recently published results.
\end{abstract}

\section{Procter and Gamble Student poster award:}

\section{References:}

Žák A., Beneš M. and Illangasekare T.H. Analysis of Model of Soil Freezing and Thawing, IAENG International Journal of Applied Mathematics, Volume 43, Issue 3, pp. 127-134, September 2013.

A. Žák, M. Beneš, T. H. Illangasekare, and A. C. Trautz. Mathematical Model of Freezing in a Porous Medium at Micro-Scale, Communications in Computational Physics, 24/2:557-575, 2018.

A. Žák, and M. Beneš. Micro-Scale Model of Thermomechanics in Solidifying Saturated Porous Media. Acta Physica Polonica A - to appear, 2018. Acceptance of Terms and Conditions:

Click here to agree

\section{Improved understanding of microbe-mineral interactions using droplet-based microfluidics}


Author(s): Neerja Zambare ${ }^{\text {None }}$

Co-author(s): Robin Gerlach ${ }^{1}$; Nada Naser ${ }^{1}$; Connie Chang

${ }^{1}$ Montana State University

Corresponding Author(s): neerja.zambare@montana.edu

Subsurface porous media present an ideal application environment for the precipitation-based technology called Microbially Induced Calcium carbonate Precipitation (MICP). During MICP, precipitation is facilitated by an increase in alkalinity which can result from the ureolytic activity of certain microbes. Such ureolytic biomineralization has been of interest for several subsurface applications such as soil strengthening and stabilization, concrete and limestone remediation, subsurface barrier creation as well as groundwater remediation.

Meso-scale flow reactors allow for an understanding of the overall process and how it can be controlled on relevant scales. This is undeniably important from an application standpoint, but mesoscale studies do not offer insight into microbe-mineral interactions at the micro-scale. Phenomena at the micro-scale indeed will determine the success of MICP at the larger scale. For instance, it is hypothesized that precipitation starts at the bacterial cell itself because the negatively charged cell attracts calcium ions. However, this has not been shown definitively at the single cell level, other than with post-mortem scanning electron microscopy images of dried precipitates with cell-sized indentations, which might be prone to artifacts. The main goal of this project was to monitor cell growth and MICP at the single cell level and visualize the precipitation process, including, crystal nucleation, aggregate growth and microbe-mineral associations. To investigate such microbe-mineral interactions, we developed microfluidic chips to generate miniature droplets containing single cells of the ureolytic bacterium Escherichia coli MJK2, a strain modified to express a green fluorescent protein as well as to carry out ureolysis. The droplets contained dissolved urea, calcium and other nutrients to promote MICP. Bacterial growth as well as calcium carbonate nucleation and growth in stable droplets were recorded via real-time microscopy using Confocal Scanning Laser Microscopy. Calcium carbonate was detected by its ability to auto-fluoresce under UV light, and the precipitate polymorph was identified using Raman Spectromicroscopy.

Droplets provide a means to study real-time cell attachment and growth as well as crystal nucleation. These processes are impossible to visualize or quantify in the subsurface, which is where a majority of MICP applications are proposed to occur. Droplet based studies can thus be used to manipulate cell attachment, thus promoting biofilm formation in porous media. Also, it is important to control which calcium carbonate polymorph forms during MICP because it affects the stability of the precipitate. With the limited potential for sampling during field-scale applications of MICP, it is difficult to control, or even identify calcium carbonate polymorphism. Droplet microfluidics is a novel approach to monitor real-time changes in calcium carbonate crystal phases. The potential to control biofilm formation or polymorphism can be explored readily using droplets by changing the chemical make-up or environmental conditions. The ability to control these factors could aid in maximizing the efficacy of MICP treatments in field applications.

Procter and Gamble Student poster award:

I would like to compete in the Procter and Gamble Student award References:

Acceptance of Terms and Conditions:

Click here to agree

804

\title{
Multipoint flux mixed finite element methods for a double-porosity model of single-phase flow in naturally fractured reservoirs
}

\author{
Andrés Arrarás $^{1}$; Laura Portero ${ }^{1}$; Javier Zaratiegui ${ }^{1}$ \\ ${ }^{1}$ Universidad Pública de Navarra
}

Corresponding Author(s): javizaraurda@outlook.com 
In this work, we consider mixed finite element discretizations for a double-porosity model of singlephase Darcy flow in naturally fractured reservoirs. The model is suitable for regularly distributed micro-fractures showing interconnections with the surrounding porous matrix. This interconnectivity is represented by a mass transfer function between the fractures and the porous matrix. The spatial approximation is based on the so-called multipoint flux mixed finite element method. Such a method, which has been successfully applied for solving single-porosity flow models (cf. [1, 2]), is extended here to the double-porosity case. In a two-dimensional setting, it considers the lowest order Brezzi-Douglas-Marini spaces in combination with special quadrature rules. This strategy allows for local flux elimination and reduction to a cell-centered pressure scheme, in the spirit of multipoint flux approximation schemes. Depending on the symmetry of the quadrature rule under consideration, two variants of the method are derived: a symmetric scheme, suitable on spatial meshes consisting of quadrilateral elements obtained as $\mathcal{O}\left(h^{2}\right)$-perturbations of parallelograms, and a non-symmetric scheme, designed to preserve the accuracy on general distorted meshes. Numerical experiments show first-order convergence for both the fracture and porous matrix pressures and velocities, and second-order superconvergence for the pressures at the cell centers. In addition, firstorder convergence is observed for the normal components of the velocities on the element edges. Further applications of practical interest, such as quarter five-spot configuration problems, are also discussed.

\section{Procter and Gamble Student poster award:}

\section{References:}

1 M. Wheeler, G. Xue, I. Yotov, A multipoint flux mixed finite element method on distorted quadrilaterals and hexahedra, Numer. Math. 121 (2012), 165-204.

2 M.F. Wheeler, I. Yotov, A multipoint flux mixed finite element method, SIAM J. Numer. Anal. 44 (2006), 2082-2106. Acceptance of Terms and Conditions:

Click here to agree

\section{Modeling of lithium ion batteries with emphasis on spatial fluc- tuations and mechanical effects on the electrode pore scale}

Jan Lammel $^{1}$; Tobias Hofmann ${ }^{1}$; Jochen Zausch ${ }^{1}$

${ }^{1}$ Fraunhofer ITWM

Corresponding Author(s): jochen.zausch@itwm.fraunhofer.de

Although lithium ion batteries are state-of-the-art technology there are several aspects that are still not well understood e.g. degradation and aging mechanisms. However, not only these effects are a matter of current research, engineering applications require also an understanding of how the cell architecture and production as well as the system design influence the cells properties. In all these respects physical modeling and computer simulation can help to gain more understanding and to improved batteries.

Based on our theory on ion, charge and heat transport 1 in active electrode materials and electrolyte we have developed the "Battery and Electrochemistry Simulation Tool" (BEST), which allows for three-dimensional simulation of lithium ion batteries with explicit consideration of virtual or measured electrode microstructures. In this model the species transport is consistently coupled to thermal effects. Based on the microscopic model we have derived a homogenized electrochemicalthermal 3d-model for cell scale simulation 2, usable for instance in cell design studies.

In this contribution we will briefly review the model and discuss thermal effects like the different sources of heat and how they vary with space and time (i.e. soc). Due to the implications for degradation phenomena, we will pay attention to spatial fluctuations of several quantities and study their dependence on microstructural properties. Furthermore, the results from the microscopic simulations will be compared to the cell scale computations, where fluctuations are much less pronounced. Besides an increase in temperature there are also mechanical phenomena that accelerate degradation and reduce cell life time. Therefore, we present our approach to couple electrochemistry to effects 
resulting from volume change in the active material 3. By simulations we show how mechanical stresses increase during charging in the granular microstructure. Stress invariants are calculated and the effect on the cell performance is discussed.

1 A. Latz and J. Zausch, Journal of Power Sources 196 (2011) 3296.

2 A. Latz and J. Zausch, Beilstein Journal of Nanotechnology 6, (2015) 987.

3 T. Hofmann, H. Andrä, R. Müller and J. Zausch., 7th Proceedings of GACM (2017)

Procter and Gamble Student poster award:

References:

1 A. Latz and J. Zausch, Journal of Power Sources 196 (2011) 3296.

2 A. Latz and J. Zausch, Beilstein Journal of Nanotechnology 6, (2015) 987.

3 T. Hofmann, H. Andrä, R. Müller and J. Zausch., 7th Proceedings of GACM (2017) Acceptance of Terms and Conditions:

Click here to agree

\title{
Experimental investigation of microbial induced calcium carbon- ate precipitation induced by Sporosarcina pasteurii on the mi- croscale
}

\author{
Author(s): Jennifer Zehner ${ }^{1}$ \\ Co-author(s): Mohammed Amin Razbani ${ }^{2}$; Simone Balzer Le ${ }^{3}$; Alexander Wentzel ${ }^{4}$; Espen Jettestuen ${ }^{5}$; Anja \\ Røyne ${ }^{2}$; Pawel Sikorski ${ }^{6}$ \\ ${ }^{1}$ Department of Physics, Norwegian University of Science and Technology, NTNU, Trondheim, Norway \\ ${ }^{2}$ Physics of Geological Processes (PGP), The NFORD Centre, Department of Physics, University of Oslo, Oslo, Norway \\ ${ }^{3}$ Department of Biotechnology and Nanomedicine, SINTEF Industry, Trondheim, Norway \\ ${ }^{4}$ Department of Biotechnology and Nanomedicine, SINTEF Industry, Trondheim, Norway \\ ${ }^{5}$ Norwegian Research Centre AS (NORCE), Bergen, Norway \\ ${ }^{6}$ Department of Physics, Norwegian University of Science and Technology, NTNU, Trondheim, Norway
}

Corresponding Author(s): jennifer.s.zehner@ntnu.no

A promising alternative to conventional concrete production methods uses microbial induced carbonate precipitation (MICP) via urea hydrolysis to form a concrete-like consolidated material, often referred to as bio-concrete 1 . This type of material is especially interesting in high volume, low strength applications, such as soil consolidation and geotechnical engineering 2 . The urea hydrolysis reaction is catalyzed by the enzyme urease and results in a $\mathrm{pH}$ increase and the production of carbonate ions. In the presence of calcium ions, this can result in calcium carbonate precipitation. If the crystallization occurs in a granular medium a solid material can be produced. We aim to combine urea hydrolysis catalyzed by urease-producing bacteria with calcium ions provided by the dissolution of calcium carbonate (chalk), induced by acid-producing bacteria, to obtain bio-concrete that does not require the use of an external calcium source. Well known ureolytic bacteria are Sporosarcina pasteurii. Key factors for controlling the urea hydrolysis reaction and $\mathrm{CaCO} 3$ precipitation are enzyme concentration and activity, urea concentration, the amount of dissolved inorganic carbon, $\mathrm{pH}$ value, calcium concentration, and the availability of nucleation sites 3. To get a better understanding of how those factors influence the properties of the consolidation of granular material like sand or soil, it is important to understand the formation of the consolidated material on the microscale. Factors like crystal morphology, crystal size and distribution, porosity and adhesion to the granular media will determine the mechanical properties of the consolidated material.

Here we describe an experimental methodology to study microscale consolidation processes in realtime and in situ. Sporosarcina pasteurii are used as the source of urease, and calcium carbonate (chalk) is dissolved in lactic acid as a calcium source, mimicking a calcium source obtainable by growing lactic acid-producing bacteria in the presence of chalk. Real-time $\mathrm{pH}$ monitoring of the crystallization process is performed with the help of a $\mathrm{pH}$ sensitive dye in small volumes to get a better understanding of the kinetics of the underlying urea hydrolysis reaction. Crystal growth is monitored by light 
microscopy to study crystal growth kinetics. The morphology, shape and size of the formed crystals are characterized by micro-Raman spectroscopy and scanning electron microscopy. In addition, experiments are performed in a sample cell that is compatible with optical microscopy. This allows studying crystallization in the presence of a granular medium with spatial and temporal resolution, and to understand how dissolution and crystallization processes depend on the concentration and the distribution of bacteria. The experimental data obtained are used to develop pore-scale numerical models of the dissolution and precipitation processes 4 for a more targeted optimization of the consolidation process.

\title{
Procter and Gamble Student poster award:
}

I would like to compete in the Procter and Gamble Student award References:

1 De Muynck, Willem, Nele De Belie, and Willy Verstraete. "Microbial carbonate precipitation in construction materials: a review.” Ecological Engineering 36.2 (2010): 118-136.

2 Adrienne J. Phillips, Robin Gerlach, Ellen Lauchnor, Andrew C. Mitchell, Alfred B. Cunningham \& Lee Spangler (2013) Engineered applications of ureolytic biomineralization: a review, Biofouling, 29:6,715733

3 Hammes, Frederik, and Willy Verstraete. "Key roles of $\mathrm{pH}$ and calcium metabolism in microbial carbonate precipitation.” Reviews in environmental science and biotechnology 1.1 (2002): 3-7.

4 Mohammad Amin Razbani, Jennifer Zehner, Pawel Sikorski, Espen Jettestuen and Anja Røyne (2019). A pore-scale model of microbially induced calcium carbonate precipitation. InterPore2019. Valencia. Acceptance of Terms and Conditions:

Click here to agree

\section{Numerical modelling of microbially induced calcite precipitation in field trials}

\author{
Leon van Paassen ${ }^{1}$; Chen Zeng ${ }^{2}$; Jun-jie Zheng ${ }^{3}$ \\ ${ }^{1}$ Arizona State University \\ ${ }^{2}$ Huazhong University of Science and Technology \\ ${ }^{3}$ Huezhong University of Science and Technology
}

Corresponding Author(s): zengchen@hust.edu.cn

Microbially induced calcite precipitation (MICP) by urea hydrolysis has potential as ground improvement method in geotechnical and environmental applications. Field trials of MICP were performed in Toronto. The suspension of locally enriched ureolytic bacteria was injected into three plots and each plot had six injection wells surrounding a central extraction well. The injection of bacteria was followed by injection of chemical solutions containing urea and calcium chloride. The extracted groundwater was used to dilute the added solutions. Each plot had a different target amount of precipitation. A numerical model was developed using COMSOL Multiphysics to interpret the obtained results after MICP treatment and evaluated the effect of different process variables. The applied flow rates, injection strategy and concentrations were used as variable boundary conditions. The urease activity and cumulative height of permeable layers were used to fit the simulated results to the measured concentrations and electrical conductivity. The simulation results provide insight on the treatment efficiency, distribution of substrates and products throughout the treated area.

Procter and Gamble Student poster award:

I would like to compete in the Procter and Gamble Student award References:

Acceptance of Terms and Conditions:

Click here to agree 


\title{
Apparent Permeability Model of Complex Fracture Network in Shale Reservoir
}

\author{
Fanhui Zeng ${ }^{1}$; Fan Peng ${ }^{2}$; Jianchun Guo ${ }^{1}$ \\ ${ }^{1}$ State Key Laboratory of Oil and Gas Reservoir Geology and Exploitation, Southwest Petroleum University, Chengdu, \\ China \\ ${ }^{2}$ State Key Laboratory of Oil and Gas Reservoir Geology and Exploitation, Southwest Petroleum UniversitySouthwest \\ Petroleum University, Chengdu, China
}

Corresponding Author(s): zengfanhui023024@126.com, swpupengfan@163.com,guojianchun@163.com

\begin{abstract}
In shale reservoir, complex fracture network stimulated from horizontal well multiple fracturing is a major gas flow channel in the stimulated reservoir volume region. The direction of fracture distribution is arbitrary and the extending trajectory is complex, meanwhile, the gas flow in shale complex fracture network has the characteristic of multi-scale flow including continuous flow, Knudsen diffusion and surface diffusion. Previous researchers did not fully consider the two characteristics in calculating apparent permeability of complex fracture network. Currently, accurate apparent permeability calculation of complex fracture network is of great significance for productivity evaluation and formulation of reasonable shale gas development plan. In this paper, first, an apparent permeability calculation model for single fracture is established, which considers different transport mechanisms. Then, by simplifying fractures of the complex fracture network to be parallel fracture clusters, based on the fractal theory and generalized Darcy law, a comprehensive apparent permeability calculation model for different scale fractures is established, and this model can be used to calculate the apparent permeability of complex fracture network, which comprehensively fuses the dynamic fracture width variation and gas effective viscosity. The reliability of this model is verified via molecular simulations. Finally, the influence of different factors on complex fracture network apparent permeability is discussed in detail. The results demonstrate the following findings. (1) The comprehensive apparent permeability calculation model in this paper can well calculate the apparent permeability of complex fracture network in shale stimulated region. (2) Under low formation pressure (less than $5 \mathrm{MPa}$ ), the effective gas viscosity rapidly decreases with the decrease of formation pressure, and it has an obvious reducing effect on apparent permeability of complex fracture network. (3) The apparent permeability of complex fracture network is positively correlation with fracture density, shale Young's modulus and Poisson's ratio. (4) As the important parameters used in fractal theory, the values of maximum fracture width and minimum fracture width in complex fracture network have the vital effect on comprehensive apparent permeability.
\end{abstract}

Procter and Gamble Student poster award:

References:

Acceptance of Terms and Conditions:

Click here to agree

\section{Fractal Forced Spontaneous Imbibition Model of Water Phase in Shale}

Fanhui Zeng ${ }^{1}$; Chao Wen ${ }^{1}$; Jianchun Guo ${ }^{1}$

${ }^{1}$ State Key Laboratory of Oil and Gas Reservoir Geology and Exploitation, Southwest Petroleum University

Corresponding Author(s): zengfanhui023024@126.com

Massive hydraulic fracturing is the core technology for effective development of shale reservoir. The water-based fracturing fluid used in the fracturing process will be absorbed by nanoporous media under the effect of capillary force and osmotic pressure. In addition, the spontaneous imbibition process is also influenced by the external force caused by fracturing operation and viscosity changes of water phase. Previous researchers did not fully consider the two factors in the model of spontaneous imbibition. This paper systematically carries out a research on spontaneous imbibition mode 
of shale gas and analysis of engineering influential factors.

The work in this paper can be summarized as follows: Firstly, the shale pores are divided into nonclay pores (including organic pores, brittle mineral pores) and clay pores according to the force of water phase spontaneous imbibition. Secondly, based on the Hagen-Poiseuille equation and the Navier-Stoke equation, a non-clay porous elliptical capillary bundle spontaneous imbibition model, considering the non-circular characteristics of the capillary, viscosity changes and the boundary slip effect, is established. What's more, taking the fractal characteristics of the pore size and tortuosity factor into consideration, we have built up a fractal forced spontaneous imbibition model of nonclay pore, in which the capillary force and the external force plays an important role. Thirdly, based on the Fritz semi-permeable membrane efficiency model, van't Hoff osmotic pressure equation and equivalent salinity model, the calculation model of pore osmotic pressure of shale clay is established. Meanwhile, on the basis of the N-S equation, we have constructed a model of water phase flow of the flat capillary bundle, in which the high aspect ratio of clay pores, viscosity changes and the boundary slip effect are considered. Furthermore, a fractal forced spontaneous imbibition model of clay pore is also established with the capillary force, osmotic pressure and the external force. Finally, we build a fractal forced spontaneous imbibition model of nanoporous media of shale by combining the two spontaneous imbibition models above.

The reasonableness of this model has been verified by theoretical analysis and experiments. The characteristics of spontaneous imbibition and backflow in fracturing wells can be also explained. Factors affecting spontaneous imbibition of water phase are analyzed. The result shows that the volume of spontaneous imbibition is positively correlated with time and area of spontaneous imbibition, porosity, external force and slip length, and inversely related to tortuosity, fractal dimension, viscosity, ratio of pore length and width. At the same time, we carries out an engineering scale prediction of backflow rate, as well as optimization of engineering parameter.

This paper effectively reveals the mechanism of fractal forced spontaneous imbibition in shale, which can provide guidance for optimization of the backflow process and hydraulic fracturing parameters. Keywords: nanoporous media; spontaneous imbibition; fractal feature; external force; viscosity changes

Procter and Gamble Student poster award:

I would like to compete in the Procter and Gamble Student award References:

Acceptance of Terms and Conditions:

Click here to agree

\title{
Molecular Simulation of Water Adsorption in Shale Organic Nano- Pores
}

\author{
Kecheng Zeng $^{1}$; Ruina $\mathrm{Xu}^{1}$; Peixue Jiang ${ }^{1}$ \\ ${ }^{1}$ Department of Energy and Power Engineering, Tsinghua University
}

Corresponding Author(s): zkc16@mails.tsinghua.edu.cn

Hydraulic fracturing is an important technique in the exploration of shale gas and used widely, water could displace the methane stored in the shale nanopores and might influence the physical properties of shale. Complex shale pore surface with different material composition, different geometry, it's necessary to consider how the water adsorbs on the surface of shale nanopores. All the shale matrix pores are in nanoscale, in which the organic kerogen pore radii are mostly less than 10 $\mathrm{nm}$. The nanoscale kerogen pores provide huge specific surface area for shale reservoir. Comparing with the methane fluid stored in shale kerogen organic pores, water shows stronger adsorption affinity because of its intermolecular interaction with the pore surfaces. Based on a novel molecular simulation workflow, the kerogen pores are constructed using Molecular Dynamics under typical reservoir condition (up to $20 \mathrm{MPa}$ ). In the confined space of shale nanopores, the pore surface curvature has important implications for fluid phase change, the theory for phase change in macro scale is no longer suitable for the water phase change in shale nanopore. The surface of confined space has strong effect on fluid density fluctuations, and in nanoscale the fluctuation of fluid surface tension weakens the stability of the condensate liquid, we use Molecular Dynamics and Monte Carlo methods to model the nanoscale water/kerogen system and research the water adsorption and phase 
change in shale nanopores.

Shale gas has become an increasingly important energy source as a result of the depletion of the conventional oil and gas resources and an urgent call for cleaner fuels. With the successful application of hydraulic fracturing and horizontal drilling, shale gas has attracted more and more attention in the last decade. As a kind of unconventional fossil energy resource, shale gas account for a great proportion of the total natural gas amount and production, such as in the U.S., the production of shale gas has reached to 420 billion cubic meter in 2016 and made up a significant share, which is up to $56 \%$, of total natural gas production. As a result of the fast development of shale gas industry, energy structure of the U.S. has changed from importing LNG to exporting it. However, due to the ultra-low permeability, ultra-low pore connectivity, ultra-fine grain size sand ultra-complex pore structures in the shale's rock, assessing and exploring shale's reservoirs encounters tremendous obstacles.

It was well recognized in the literature that sufficient hydraulic fractured shale's reservoir is typically consisted of four porosity systems: organic pores in the kerogen, inorganic pores, natural fracture and induced fracture. Fluid confined in the nanopores, which includes methane, $\mathrm{CO} 2$ and water vapor, exist as adsorbed state on the surface of organic content and as free-state in the shale's pores and fractures systems. With the depletion of the shale's reservoir, adsorbed fluid desorbs from the organic kerogen into the matrix's pores as free fluid, then the free fluid transports to the wellbore through diffusion and advection flow driven by the difference of concentration and pressure respectively. However, hydraulic fracture technique helps enlarge the production rate and amount of shale gas, it's shown from field experience that not all of the injected water flows back to the ground and the recovery of the flowback water only account for $10-30 \%$ of the injected volume in the first few months, which means that a great portion of the injected water is left behind the fractured shale porous system, then the irreducible water would have a large impact on the transport and storage of shale gas. For the purpose of assessing the amount of irreducible water in shale fracture system and revealing the influence of water on the transport of shale gas, in this paper, we conduct MD simulation about water adsorption in shale organic pores.

Procter and Gamble Student poster award:

I don't want to compete References:

Acceptance of Terms and Conditions:

Click here to agree

\section{Coupling virus and salinity transport through porous media.}

Dong Zhang ${ }^{1}$; Michael Zabarankin ${ }^{1}$; Valentina Prigiobbe ${ }^{1}$

${ }^{1}$ Stevens Institute of Technology

Corresponding Author(s): dzhang18@stevens.edu

The reuse of wastewater in agricultural is a common practice worldwide, in particular in arid and semiarid regions. However, untreated or partially treated wastewater may contain significant concentration of fecal microorganism and, therefore, cause microbial contamination with serious public health concern 1. Therefore, it is important to understand the fate of fecal microorganisms in soil. The fate and the transport of viruses in soil is governed by attachment and detachment processes at the solid-liquid interface, which are controlled by the soil properties and the chemical composition of the solution. The salinity (or ionic strength) is one of the most important factor as it controls the electrostatic interactions between the microorganisms and the porous medium surface and, therefore, the speed of their migration. Salinity can be significant in untreated or partially treated wastewater. At high salinity, the attachment of viruses onto a porous medium surface is the dominant process. However, few studies $[2,3,4]$ quantitatively analyze the effect of the salinity on the transport behavior of virus in porous media. Moreover, these studies do not account for the coupling of the viruses and salinity, which is evident from experimental observations 2. Missing therefore the possibility to describe the effect of the variations of salinity with their models. 
Here, a study that explores the effect of salinity on the instability of the virus front during transport in saturated porous media is presented. One dimensional transport model was developed to couple microorganism and salinity transport through porous media. The model consists of two mass conservation equations for viruses and a salt coupled through the constitutive equations of attachment/detachment mechanism. Numeric and analytic solutions of the coupled model were derived and compared with the numeric solution of the classical uncoupled system. The first two solutions agree very well, but the latter misses important features of the concentration fronts. In particular, under salinity reduction, a contaminated soil with virus exhibits a significant concentration peak due to the detachment of viruses from the solid surface, which is instead not predicted by the uncoupled system. This work helps to gain an insight into the understanding of the effect of salinity gradients on virus transport which may occur during wastewater reuse in agriculture.

\title{
Procter and Gamble Student poster award:
}

I would like to compete in the Procter and Gamble Student award References:

1 Gotkowitz, Madeline B., et al. (2016) Effects of climate and sewer condition on virus transport to groundwater. Environmental science \& technology 50.16, 8497-8504.

2 Cao, H., et al. (2010) Salinity and soluble organic matter on virus sorption in sand and soil columns. Groundwater 48, 42-52.

3 Schijven, Jack F., et al. (2002) Two-site kinetic modeling of bacteriophages transport through columns ofsaturated dune sand. Journal of Contaminant Hydrology 57, 259-279.

4 Bradford, Scott A., et al. (2014) Modeling microorganism transport and survival in the subsurface." Journal of environmental quality 43.2, 421-440. Acceptance of Terms and Conditions:

Click here to agree

\section{Numerical study on the mechanism of fluid flow through thin rough fractures}

\author{
Ge Zhang ${ }^{1}$ \\ ${ }^{1}$ Beijing Institute of Applied Physics and Computational Mathematics
}

\section{Corresponding Author(s): kdzhangge@163.com}

The fluid flow through fractured rock mass has a wide range of engineering applications. In this case, fracture flow has been concerned from different points. As the fracture network is complex, researches mostly concentrate on the single fracture which is the base of the fracture network system 1. For a single fracture, the roughness on its surface exerts significant effects on the flow behavior and consequently influences the permeability. When the aperture of a rough fracture reduces to a relatively small size compared with the mean free path of the fluid molecular, the flow shows microscale characters. This paper intends to shed a light on the flow through thin fractures, of which both the micro effect and the surface roughness effect should be considered. Previous empirical roughness models for the hydraulic aperture are modified with consideration of the micro effect, and they show a preliminary pattern of the relationship between two effects. Direct numerical simulation using the lattice Boltzmann method is conducted on artificially created fractures with standard roughness following the Gaussian distribution to reveal the competitive relationship of the two effects on the flow. The result confirms the relationship: for very rough fractures with the standard deviation of the aperture $(\sigma)$ greater than about $60 \mu \mathrm{m}, e / E$ decreases with the reduction of $E$. However, if the fracture is relatively smooth and $\sigma$ is smaller than $60 \mu \mathrm{m}, e / E$ shows a positive relationship. Fractures with $\sigma$ around $60 \mu \mathrm{m}$ is an equilibrium state of $e / E$ which is not apparently affected by the change of $E 2$. The result reveals the mechanism of the fracture flow both affected by the roughness effect and the micro effect, and it also provides a reference for engineering problems like the transport of natural gas through microfractures.

\section{Procter and Gamble Student poster award:}

I would like to compete in the Procter and Gamble Student award References: 
1 Tsang Y. W., Tsang C. Channel model of flow through fractured media. Water Resources Research, 1987, 23(3): 467-479.

2 Zhang G., Liu B., Xu A., et al. Morphology Effect of Surface Structures on Microchannel Flow Using Lattice Boltzmann Method, Geofluids, 2019. Acceptance of Terms and Conditions:

Click here to agree

\title{
A discrete Boltzmann method investigation for the micro-compressible- thermal flow in porous media with nonequilibrium effect
}

\author{
Ge Zhang ${ }^{1}$; Aiguo $\mathrm{Xu}^{1}$; Xiaomian $\mathrm{Hu}^{1}$; Yingjun $\mathrm{Li}^{2}$ \\ ${ }^{1}$ Institute of Applied Physics and Computational Mathematics, Beijing \\ ${ }^{2}$ China University of Mining and Technology, Beijing \\ Corresponding Author(s): kdzhangge@163.com
}

\begin{abstract}
The multiscale intrinsic of the flow in porous media lead to a great challenge on the simulation method. Especially for the cases when the flow endures different flow regimes and encounters complex solid matrix, the drastic change on the density and temperature would happen. In this case, a credible method is in great need. Originated from a two-step coarse-grained modeling from Boltzmann equation, discrete Boltzmann method (DBM) was proposed and applied to several complex flow cases in previous works 1. According to the degree of the nonequilibrium of the flow, various DB model can be set up and target on specific problems. This method easily couples thermal effect and adjusts itself with Knudsen effect 2. However, it has not been used for the flow in porous media. In this paper, a first attempt of the DBM simulation for the flow in porous is shown both with the modeling processes and benchmarks.
\end{abstract}

1 Gan, Yanbiao, et al. "Discrete Boltzmann modeling of multiphase flows: hydrodynamic and thermodynamic non-equilibrium effects.” Soft Matter 11.26 (2015): 5336-5345.

2 Zhang, Yu-Dong, et al. "Discrete Boltzmann Method with Maxwell-Type Boundary Condition for Slip Flow." Communications in Theoretical Physics 69.1 (2018): 77.

Procter and Gamble Student poster award:

I would like to compete in the Procter and Gamble Student award References:

Acceptance of Terms and Conditions:

Click here to agree

574

\section{A coupled flow-DEM approach to investigate the effect of embed- ded gravel on the propagation of hydraulic fractures in glutenite reservoirs}

Guodong Zhang ${ }^{1}$; Ruobing $\mathrm{Niu}^{1}$; Bingjie Liu ${ }^{1}$; Kun Chao ${ }^{1}$

${ }^{1}$ Qingdao University of Science and Technology

Corresponding Author(s): zhang_gd1987@hotmail.com

Glutenite is widely found in tight gas reservoirs, but because the permeability and porosity of glutenite reservoirs are extremely low, hydraulic fracturing treatments are necessary to commercially develop the gas in glutenite reservoirs. Due to the presence of embedded gravel, stress concentration and reorientation usually occur, and the stress heterogeneity can significantly affect the 
mechanical response of an intact rock. Because the nucleation, propagation and coalescence of a hydraulic fracture in glutenite reservoirs involve complicated interactions of particles-gravel and inter-particles with different sizes, the fracturing mechanisms in glutenite reservoirs are still unclear. A coupled flow-DEM approach was used to simulate the hydraulic fracturing process to improve the understanding of fracturing mechanisms in glutenite reservoirs. The microscale geometry heterogeneity induced by embedded gravel and multi-sized particles was accurately characterized in DEM, while a flow algorithm was used to model the fluid flow in glutenite specimens. Gravel with different sizes and shapes were randomly embedded in the specimens, the interaction mechanisms between propagating fracture and encountering gravel were investigated, and the effects of geo-stress difference, gravel strength and grain size distribution were studied.

As can be expected, the macroscale fracture morphology is dominated by geo-stress state, but the microscale mechanical responses of the glutenite specimens under hydraulic loading are significantly affected by stress and strength heterogeneities induced by the geometry heterogeneity. At earlier stage of the simulations, the hydraulic pressure causes sufficient energy in the vicinity of the wellbore, so cracks can be easily initiated. The stress distribution near the wellbore is governed by geostress state and geometry heterogeneities, for large geo-stress difference, two cracks initiate at the wellbore wall and finally propagate to form a bi-wing fracture along the direction of the maximum principle stress, while multiple cracks positions occur at small geo-stress difference, forming complicated fracture morphology with branched fracture. However, when hydraulic fracture propagates to the far-field region of the specimen, geo-stress difference dominates the global propagation, while the embedded gravel can cause stress concentration at the interfaces between gravel and matrix, and the propagating fracture is intended to be attracted into the interface following three interactions mechanisms (penetration, deflection and termination) with encountering gravel. Although the probability of a propagating fracture being arrested by encountering gravel increases with the increase in gravel strength, the hydraulic fracture always can find a least resistance path to propagate, so the terminal fracture morphologies are similar for specimens with different gravel strengths. In addition, compared with heterogeneous specimens, more initiation positions form in homogeneous specimens due to small stress and strength heterogeneities, and stress reorientation frequently occurs.

Procter and Gamble Student poster award:

References:

Acceptance of Terms and Conditions:

Click here to agree

\title{
Dynamic simulation of water invasion in multi-well collabora- tive mining of gas reservoirs with edge and bottom water
}

\author{
Jie ZHANG ${ }^{1}$; Qi $\mathrm{LI}^{1}$; Shusheng $\mathrm{GAO}^{2}$; Liyou $\mathrm{YE}^{3}$; Huaxun $\mathrm{LIU}^{2}$ \\ ${ }^{1}$ University of Chinese Academy of Sciences, Beijing 100190, China; \\ ${ }^{2}$ Research institute of petroleum exploration \& development-Langfang, CNPC, Langfang 065007, China) \\ ${ }^{3}$ Research institute of petroleum exploration \& development-Langfang, CNPC, Langfang 065007, China)
}

Corresponding Author(s): 437314297@qq.com

Gas reservoirs with edge and bottom water need to drill wells simultaneously in different areas or to perforate in different physical reservoirs at the same time. Due to the differences in reservoir properties and connectivity, the overall water invasion characteristics of the gas reservoirs will be manifested by the law of water invasion under different types of reservoir combinations. In this respect, use multiple full-diameter cores in parallel with permeability difference, and connect to a simulation device with a volume of $20000 \mathrm{ml}$ water, which is to simulate multi-well collaborative development process for different physical reservoirs in water flooding, study the mechanism and dynamics of water invasion. According to the gas reservoir engineering method, the continuous measurement of gas water yield is realized through the pressure data conversion of the intermediate container at the outlet end of the experiment. The results showed that: (1) In the process of multiwell collaborative mining, the water first invaded along the high permeability reservoir and water first appears in the high-permeability reservoir, followed by the medium-permeability reservoir, and 
the low-permeability reservoir did not produce water. (2) After the gas well produced water, the gas production rate gradually decreases, the water production rate drops rapidly first and becomes flat gradually, the water vapor ratio tends to stabilize after decreasing. (3) In the early stage of water invasion, the bottom pressure drops slowly, the water supplies energy to the gas reservoir, and the pressure drops rapidly in the later stage of water invasion. (4) The gas wells continue to be mined after the production of water, and the ultimate recovery rate can reach $59.25 \%$. The wells are shut down immediately after the water production, and the ultimate recovery can reach $43.5 \%$. The research results have enriched the understanding of water invasion dynamics and laws in the multi-well cooperative mining process of edge and bottom water gas reservoirs, which has certain guiding significance for the exploitation of this kind of gas reservoirs.

Procter and Gamble Student poster award:

I would like to compete in the Procter and Gamble Student award References:

Acceptance of Terms and Conditions:

Click here to agree

974

\title{
Study on the Factors Affecting the Productivity of Coal-bed Gas Well -A Case Study of Block S of D Gas Field in Australia
}

\author{
JUNHAN ZHANG ${ }^{1}$ \\ ${ }^{1}$ Research Institute of Petroleum Exploration and Development
}

Corresponding Author(s): 15689131211@163.com

\begin{abstract}
Australian S block belongs to the low coal rank coal seam group, with large block area, many longitudinal coal seams, thin single coal seam and complex plane distribution. For the many physical parameters of coal reservoirs, it is difficult to select the main controlling factors, including porosity, permeability and pore structure etc, affecting the productivity of coalbed methane wells. Firstly, taking the $\mathrm{S}$ block of the D gas field in Australia as an example, based on the oilfield production data, using the geological modeling and simulation modeling software-Petrel RE, the porosity, permeability and pore structure, coal seam thickness, etc. are correlated with the peak gas production of a single well. Next, quantitative research and comparison of the factors affecting the above production capacity, using the gray correlation analysis method to determine the primary and secondary relationship of various factors, to find out the main controlling factors affecting the production capacity of coal-bed methane wells.
\end{abstract}

Procter and Gamble Student poster award:

References:

Acceptance of Terms and Conditions:

Click here to agree

118

\section{Pore Scale Simulation of Coupled multiple physicochemical pro- cesses for Methane Hydrate Dissociation using Lattice Boltzmann Method}

Lei Zhang ${ }^{1}$; Chuangde Zhang $^{2}$; Kai Zhang ${ }^{2}$; Jun Yao ${ }^{1}$

\footnotetext{
${ }^{1}$ China University of Petroleum

${ }^{2}$ China University of Petroleum $\bowtie$ East China囚
} 


\title{
Corresponding Author(s): zhlei84@163.com
}

Methane hydrate is a new energy source with great potential and abundant reserves. In this paper, a pore scale model based on the Lattice Boltzmann Method (LBM) is established for methane hydrate dissociation process. The model combines the single phase flow LB model, the mass transport LB model, the heat transfer LB model and gas hydrate dissociation kinetics model. The pore scale model can describe the methane hydrate dissociation behavior coupled nonlinear non-isothermal multiple physical chemical processes, including intrinsic dissociation dynamics, gas flow, mass transport, phase change heat transfer, conjugate heat transfer at fluid solid interface and dynamic evolution of the pore geometries. The various submodels that make up the pore scale model is validated using the Poiseuille flow and several convection diffusion problems. The results show that the numerical solution agrees well with the analytical solution which indicates that the model can be used to simulate the methane gas hydrate coupled multiple physics. The pore scale studies provide deep understanding of $\mathrm{MH}$ dissociation process in porous media, and also allow to quantitatively predict some important empirical relationships, such as permeability-saturation variation relation, for continuum models.

Procter and Gamble Student poster award:

References:

Acceptance of Terms and Conditions:

Click here to agree

\section{The Adaptability of Foam Profile Control in Low Permeable Het- erogeneous Reservoir}

\author{
Lijuan Zhang $^{1}$; Xiangan Yue ${ }^{1}$ \\ ${ }^{1}$ China University of Petroleum, Beijing \\ Corresponding Author(s): zhangljbj2001@sina.com
}

Foam has dual properties of oil displacement and profile control, which has shown potential advantages and wide application prospect in medium and high permeability reservoirs. However, the profile control capacity of foam in low permeability reservoirs is different from that in medium and high permeability reservoirs. This paper aims to simulate the adaptability of foam profile control in low permeable heterogeneous reservoir. For a particular low permeability reservoir, foam flow experiments were performed to evaluate its effectiveness of diversion and determine suitable heterogeneity range. Heterogeneous model composed of two parallel homogeneous layers: matrix layer and water channeling layer. The permeability of matrix layer was $1.34 \mathrm{mD}$, and that of water channeling layer was $5.6 \mathrm{mD}, 49.8 \mathrm{mD}$ and $464 \mathrm{mD}$ with permeability contrasts of 4.2:1, 37.1:1 and 346:1. Additionally, we examined the effects of injection ways on foam profile control ability. One way was injecting foam (simulating foam generation on the ground); the other way was injecting in SAG (surfactant-alternating-gas, simulating foam generation in the formation). Moreover, Oil displacement experiments were performed to confirm foam diversion effectiveness in communicating layers using man-made three-layer cores with each layer permeability of $5 \mathrm{mD}, 80 \mathrm{mD}$ and $200 \mathrm{mD}$. Research results showed that from the perspective of improving water entering into the matrix, foam flooding favors plugging water channeling passage. There is optimum heterogeneity range: more heterogeneous and weaker foam plugging strength is in water flowing channeling layer; less heterogeneous, more foam into matrix layer and mobility is reduced. In low permeability reservoir of matrix permeability of $1-5 \mathrm{mD}$, foam profile control is suitable for water channeling and the matrix permeability contrasts of 30:1-40:1. The fractional flow ratio into matrix increases more than $8 \%$ during subsequent water flooding. Injecting in SAG have better profile control effectiveness than foam injection. Relatively less foam enters into the matrix layer and there is less blocking effect on the subsequent water flooding into matrix layer. Foam flooding in SAG leads to an oil recovery by $13 \%$ incremental oil, while an oil recovery by $9 \%$ incremental oil is due to foam injection. 
I would like to compete in the Procter and Gamble Student award References:

1 Singh, Robin, and K. K. Mohanty. Foam flow in a layered, heterogeneous porous medium: A visualization study. Fuel, 197(2017):58-69.

2 A. H. Al Ayesh, Saudi Aramco, R. Salazar, R. Farajzadeh, S. Vincent-Bonnieu, W. R. Rossen. Foam diversion in heterogeneous reservoirs: Effect of permeability and injection method. SPE-179650-MS. The SPE improved oil recovery conference held in Tulsa, Oklahoma, USA, 11-13 April 2016 Acceptance of Terms and Conditions:

Click here to agree

166

\title{
Uranium release and migration in porous rock under geologic CO2 storage conditions: a numerical investigation
}

\author{
Liwei Zhang $^{1}$; Xiuxiu Miao ${ }^{\text {None }}$; Yan Wang ${ }^{\text {None }}$; Manguang Gan ${ }^{\text {None }}$; Hejuan Liu ${ }^{\text {None }}$; Xiaochun LI $^{2}$ \\ ${ }^{1}$ Institute of Rock and Soil Mechanics, Chinese Academy of Sciences \\ ${ }^{2}$ State Key Laboratory of Geomechanics and Geotechnical Engineering, Institute of Rock and Soil Mechanics, Chinese \\ Academy of Sciences
}

Corresponding Author(s): zlw_85@foxmail.com

\begin{abstract}
Geologic CO2 storage (GCS) is widely recognized as a promising strategy to reduce emissions of greenhouse gas and mitigate global temperature increase. Potential $\mathrm{CO} 2$ sinks, such as saline aquifers, depleted coalbeds and unminable coalbeds, may contain trace amount of U-bearing minerals, and $\mathrm{CO} 2$ injection may trigger mobilization of uranium from U-bearing minerals. Therefore, it is necessary to investigate the potential for uranium mobilization from U-bearing minerals in deep subsurface due to $\mathrm{CO} 2$ injection. In this study, A TOUGHREACT model was developed to investigate the potential of U-bearing mineral (UO2) dissolution in a hypothetical deep $\mathrm{CO} 2$ storage reservoir. The reservoir had an average reservoir temperature of $67.5^{\circ} \mathrm{C}$ and an average reservoir pressure of 18.7 $\mathrm{MPa}$. Two uranium release mechanisms, i.e., $\mathrm{H}+$-induced $\mathrm{UO} 2$ dissolution and $\mathrm{HCO} 3$-induced $\mathrm{UO} 2$ dissolution, were investigated in this study. Numerical simulation results show that $\mathrm{HCO}$-induced UO2 dissolution is the dominant mechanism to cause uranium release. Given a low content of UO2 $(3 \times 10-4 \mathrm{vol} \%)$ in reservoir rock and a low $\mathrm{CO} 2$ injection rate (0.1 million tonnes $\mathrm{CO} 2$ per year), the maximum released uranium concentration in $\mathrm{CO} 2$ storage reservoir increased to $6.10 \times 10-5 \mathrm{~mol} / \mathrm{L}$ at $\mathrm{t}=1000$ years. In the worst-case scenario (UO2 content was $2 \mathrm{vol} \%$ and the $\mathrm{CO} 2$ injection rate was 1 million tonnes $\mathrm{CO} 2$ per year), the maximum released uranium concentration reached $0.262 \mathrm{~mol} / \mathrm{L}$ at $\mathrm{t}=1000$ years. However, released uranium did not migrate toward the shallow aquifer through leakage pathways specified in the model, while $\mathrm{CO} 2$ could migrate upward through the leakage pathways. In summary, uranium contamination is restrained in deep subsurface and notable migration of uranium to shallow aquifer caused by $\mathrm{CO} 2$ injection does not occur.
\end{abstract}

Procter and Gamble Student poster award:

References:

Prof. David Dzombak dzombak@cmu.edu

Prof. Martin Blunt m.blunt@imperial.ac.uk Acceptance of Terms and Conditions:

Click here to agree

4

Development and Calibration of an Analytical Model for Uncon-
ventional Wells Using Machine Learning Techniques

Qi Zhang ${ }^{1}$; Yu Zhao ${ }^{2}$; Yuliang $\mathrm{Su}^{3}$ 


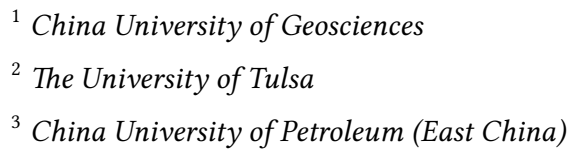

Corresponding Author(s): qizhang@cug.edu.cn

Given reliable parameters, a newly developed analytical model could offer an efficient option to predict the performance of the multi-fractured horizontal wells (MFHWs) in unconventional reservoirs. However, one major challenge comes from the significant uncertainty in the fracture network parameters, e.g., permeability, porosity, stimulated volume size, and fluid characteristics. The objective of this work is to develop and calibrate an analytical model using data integration methods for the uncertainty reduction in the description and forecasting of MFHWs.

Considering single MFHW with multi-scale flow and heterogeneous hydraulic fractures in the unconventional reservoirs, an analytical model is developed and verified by a synthetic case. Then, different flow regimes are diagnosed by analyzing the production data of a real field case. What follows is employing machine learning predictive data analytics techniques to history match in order to calibrate the parameters of the analytical model and reduce the uncertainty in the forecasting of unconventional reservoir performance. Lastly, the effects of heterogeneity of hydraulic fractures, gas adsorption/diffusion on the data integration results are explored and discussed.

Based on the linear flow assumptions, the presented analytical model is shown to simulate and predict the performance of MFHWs more reasonably by considering the stimulated reservoir volume (SRV) and fluid characteristics. Through analyzing the results of synthetic case, we are able to identify the approximate solution and the mainly influencing factors of the individual flow regime. From the results of comparison, the presented analytical model is shown to well simulate the critical linear flow regimes which dominate the production of MFHWs in the unconventional formation, and other flow regimes (e.g., the linear flow in the fractures) that are harder to be observed in the numerical model. In addition, the least square approach presents desirable capability of reducing the uncertainty in the modeling parameters. Moreover, using the analytical model with the data integration method, the modeling parameters are adjusted by matching the actual field dynamic data in different flow regimes, especially the uncertain parameters in stimulated volume and shales.

This work considers the effective region of stimulated volume and develops a linear flow model to better simulate different flow regimes in the shale reservoirs where MFHWs are applied. An effective and simple data integration workflow is proposed for uncertainty reduction and calibration of the formation parameters in the fractured unconventional reservoirs. A real field case is investigated to assess the performance of the proposed method in practical applications.

Procter and Gamble Student poster award:

References:

Acceptance of Terms and Conditions:

Click here to agree

\title{
Oil adsorption and diffusion in shale nanopores: Insight of molec- ular dynamics simulation
}

\author{
WEI ZHANG
}

${ }^{1}$ China University of Petroleum

Corresponding Author(s): weizhang0919@hotmail.com

Shale contains various minerals. The occurrence behavior and flow mechanism of gas and oil in organic matters, inorganic material and clay minerals have significant differences. In this study, the diffusivity of oil in shale pores composed by various mineral types was investigated. The effects of pore size, temperature, pressure, alkane types, mineral types and moisture content on shale oil diffusion were discussed. The results revealed that the diffusion coefficients of octane in shale nanopores were on the order of 10-9 m/s2. The adsorption amount of oil in organic matters was almost two folds larger than that in inorganic composition and clay minerals. However, the diffusivity of oil 
decreases in the order of organic matter, inorganic material and clay mineral due to the ultrasmooth structure of organic surface. The oil diffusivity increased with the increase of pore size and temperature. Long chain alkane presented a weaker diffusivity due to the larger molecular weight and intermolecular forces. The temperature effect on long chain alkane diffusion was less evident comparing with shorter chain alkane. The exist of water inhibited the adsorption and diffusion of oil in the pores composed by all kinds of materials. The water inhibiting effect of oil diffusivity was much more significant in organic pores since the water accumulated as cluster in organic slit, providing a piston-like process, while a water membrane was presented at the surface of inorganic materials. This study provided a better understanding of oil diffusion behavior in shale nanopores, which is helpful for shale oil exploration and development.

Procter and Gamble Student poster award:

I would like to compete in the Procter and Gamble Student award References:

Acceptance of Terms and Conditions:

Click here to agree

95

\title{
Numerical simulation of fluid flow and heat extraction in En- hanced Geothermal System based on discrete fractures model
}

\author{
Xu Zhang ${ }^{1}$; Jun Yao ${ }^{1}$; Zhaoqin Huang ${ }^{2}$; Zhixue Sun ${ }^{2}$ \\ ${ }^{1}$ Research Centre of Multiphase Flow in Porous Media, China University of Petroleum (East China) \\ ${ }^{2}$ Research Centre of Multiphase Flow in Porous Media, China University of Petroleum (East China), China
}

Corresponding Author(s): upczhangxu@163.com

The enhanced geothermal system (EGS) creates an artificial heat reservoir by hydraulic fracturing and allows heat transmission fluids to be circulated in the reservoir to extract heat from hot dry rock (HDR), which involves complex thermal-hydraulic-mechanical (THM) coupling process in fractured rock mass. A THM coupling model based on local thermal non-equilibrium theory is presented with treating the fractured rock as discrete fractures network and matrix rock. In addition, Wellbores in enhanced geothermal system provide large heat exchange areas between circulating fluids and enclosing formation, therefore successful management of subsurface geothermal resources involves a system comprising wellbores, a hot artificial reservoir and a surrounding formation. A coupled wellbore-reservoir 3D model considering discrete fractures network is demonstrated to investigate the heat extraction performance of EGS. The proposed models are validated by comparing it with several analytical solutions. The characteristics of fluid flow, heat transfer, mechanical response and heat extraction performance in geothermal reservoir are investigated. The main parameters controlling the extraction temperature of EGS are also studied by sensitivity analysis. The results show the significance of taking into account the THM coupling effects and wellbore heat transfer when investigating the efficiency and performance of EGS.

Procter and Gamble Student poster award:

References:

Acceptance of Terms and Conditions:

Click here to agree

Why permeability reduction during carbon geosequestration in coal seam: an experimental study via direct in-situ microCT scanning 


\author{
Author(s): Yihuai Zhang ${ }^{1}$ \\ Co-author(s): Stefan IGLAUER ; Maxim Lebedev \\ ${ }^{1}$ Curtin University
}

Corresponding Author(s): yihuai.zhang@postgrad.curtin.edu.au

$\mathrm{CO} 2$ enhanced coalbed methane recovery (ECBM) and $\mathrm{CO} 2$ geo-sequestration in deep coal seams has received considerable interest in recent years. However, the permeability decreases during $\mathrm{CO} 2$ injection limits technical implementation in many cases. Recently, via direct in-situ microCT scanning at reservoir conditions, we demonstrated that matrix swelling (due to $\mathrm{CO} 2$ adsorption in the coal matrix) leads to coal cleat closure and associated permeability loss. Moreover, we observed that the internal swelling stress (due to matrix swelling) initiated mineral fracturing (note that minerals are embedded in the coal). Here we also present Discrete Element Method (DEM) simulations with which we show how the swelling stress was generated, and how the resulting stress field led to compressive failure of the mineral phase. These new fundamental insights enable the development of advanced ECBM models, which again will further improve methane production and CO2 geo-storage in deep unmineable coal seams.

\title{
Procter and Gamble Student poster award:
}

I would like to compete in the Procter and Gamble Student award References:

Acceptance of Terms and Conditions:

Click here to agree

\section{Porous beds to mitigate fluctuated fluid across channel flow}

Yingyi Zhang $^{1}$; Mohammad Aminpour ${ }^{2}$; Alexander Scheuermann ${ }^{3}$

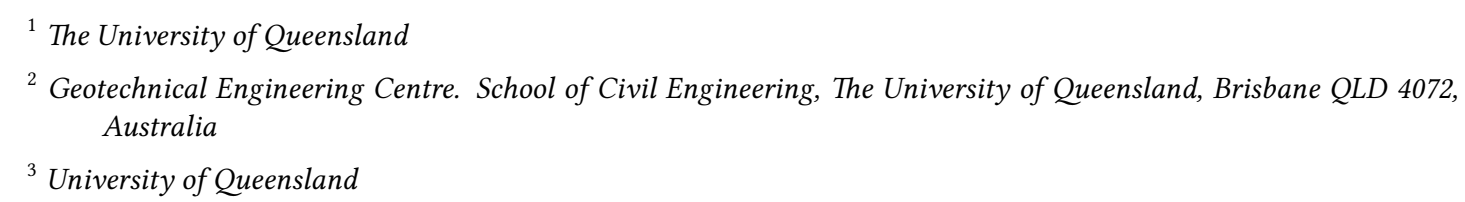

Corresponding Author(s): yingyi.zhang@uq.net.au

When the boundary conditions are fluctuating, streamlines towards the opposite direction can be observed across a channel flow. A non-steady boundary condition can be common in flow through natural porous systems. Moreover, this phenomenon is important when a channelized flow is required for experimental purposes. The study focuses on how porous medium beds inside the channel influences dispersion of the opposite velocities caused by fluctuation. Velocity distribution is studied experimentally using Particle Image Velocimetry for fluid flows through different bedding permeabilities under different flow rates. Results show that the porous medium retains the countercurrents caused by fluctuation depending on the permeability and flow rates, while the opposite velocities are also mitigated after traveling through a hollow channel. The results could provide insights into the behavior of naturally fluctuated flow in porous media, with effects extended to transport regimes.

\section{Procter and Gamble Student poster award:}

I would like to compete in the Procter and Gamble Student award References:

\section{Acceptance of Terms and Conditions:}

Click here to agree 


\title{
Computational exploration of the performances of mechanically constrained MOFs for gas separation
}

\author{
Author(s): Hengli Zhao ${ }^{1}$ \\ Co-author(s): Aziz GHOUFI ${ }^{2}$; MAURIN Guillaume ${ }^{3}$; Bin Zheng ${ }^{3}$ \\ ${ }^{1}$ University of Rennes 1 \\ ${ }^{2}$ University of Rennes 1. \\ ${ }^{3}$ University of Montpellier II
}

Corresponding Author(s): hengli.zhao@univ-rennes1.fr

Since the first study in the 1990s 1, the hybrid materials, namely the MOFs (Metal organic frameworks) have attracted a great attention owing to their unprecedented chemical and topological richness. These MOFs show many potential applications in various domains, such as purification, separation process, gas storage, sensor application, catalyst etc.

Here, we aim to understand at the molecular level theadsorption/separation of propane/propylene confined into the ZIF-8 (Zeolitic Imidazolate Framework) MOFs material (see Figure). To do so, Molecular Dynamics and Monte Carlo Simulations in osmotic ensemble were carried out. Adsorption isotherms of pure components and binary mixtures were predicted whereas dynamics and structure were investigated from MD simulations. We related the kinetic and thermodynamics aspects ruling the propane/propylene adsorption and separation.

Procter and Gamble Student poster award:

I would like to compete in the Procter and Gamble Student award References:

1 Hoskins, B. F, Robson, R. J. Am. Chem. Soc. 1989, 111, 5962

2 A. Ghoufi, A. Subercaze, Q. Ma, P. G. Yot, Y. Ke, I. Puente-Orench, T. Devic, V. Guillerm, C. Zhong, C. Serre, G. Férey, G. Maurin. J. Phys. Chem. C 2012, 116, 24, 13289-13295

3 Ross J. Verploegh, Sankar Nair, David S. Sholl. J. Am. Chem. Soc. 2015, 137, 50, 15760-15771 Acceptance of Terms and Conditions:

Click here to agree

508

\section{Pore-scale Two-phase Flow Simulation in Porous Media Coupling Pore Network Model and Lattice Boltzmann Method}

Jianlin Zhao $^{1}$; Ali Mazloomi Moqaddam ${ }^{\text {None }}$; Dominique Derome ${ }^{2}$; Jan Carmeliet $^{3}$

${ }^{1}$ ETH Zürich (Swiss Federal Institute of Technology in Zürich)

${ }^{2}$ Empa

${ }^{3} \mathrm{ETHZ}$

Corresponding Author(s): zhaojia@ethz.ch

Pore network model (PNM) and lattice Boltzmann method (LBM) are two types of pore-scale simulation methods for two-phase flow in porous media. PNM simulates fluid flow based on simplified pore bodies and throat bonds extracted from real pore structures. It is very computationally efficient but not accurate enough to describe the flow details. On the contrary, LBM simulates fluid flow directly based on the real pore structures. Therefore, it is more accurate but much less computationally efficient. To combine the advantages of both PNM and LBM, we propose to couple PNM and LBM to simulate two-phase flow in porous media. First, the real porous media is segmented into single pore-throat elements without simplification of the pore geometry. Then LBM is used to simulate two-phase flow in each pore-throat element to explore the pore filling mechanisms in each single pore. Both the critical capillary entry pressure and capillary pressure - saturation relationship can 
be obtained. Finally, these relationships are incorporated into the PNM to simulate two-phase flow in the whole pore network composed of all pore-throat elements. To validate the proposed coupling method, we simulate two-phase displacement in both regular pore networks and real porous media with the three different methods: LBM, original PNM and the newly proposed method. Both the drainage and imbibition processes are simulated. Because of the pore geometry simplification and inaccurate pore filling mechanisms, the displacement patterns obtained by the original PNM shows significant difference with the LBM simulation results, especially for the imbibition process. As the newly proposed method incorporates the pore filling mechanisms obtained by LBM simulations, it can produce similar displacement patterns with the LBM simulation results. In addition, the computational cost of the new model is much less than only using the LBM. In sum, we propose a pore-scale simulation method for two-phase flow in porous media coupling PNM and LBM without simplification of the porous media, which is more accurate than the original PNM and more computationally efficient than the LBM.

Procter and Gamble Student poster award:

\title{
References:
}

Acceptance of Terms and Conditions:

Click here to agree

\section{Study on the Change of Seepage Field in the Condition of Ex- treme Water Consumption}

\author{
Author(s): Liang Zhao ${ }^{1}$ \\ Co-author(s): Jianwei $\mathrm{Gu}^{1}$ \\ ${ }^{1}$ China University of Petroleum(East China)
}

Corresponding Author(s): 243099884@qq.com

For oilfields where the reservoir type is mainly terrestrial clastic rocks, in the process of water flooding, due to factors such as reservoir heterogeneity, water-oil mobility ratio, rhythm, and injectionproduction imbalance, with more and more water injection, the reservoir permeability will become larger and larger, and an extreme water consumption zone will be formed at the bottom of the oil layer. And then, an advantageous flow channel will appear, and the injected water will preferentially flow along the dominant flow channel to generate turbulence. Finally, a weak seepage zone is formed at the top of the oil layer, causing the remaining oil to be enriched in the weak seepage zone. In this paper, a typical loose reservoirs' five-point well model is established, and the seepage field parameters are calculated to describe the water flooding development effect and seepage field characteristics. The numerical simulation method is used to calculate the dynamics of water wells and oil wells after plugging, and the characteristics of pressure field, saturation field change and flow field change after plugging are analyzed, and the variation law of displacement pressure gradient field in stratum is described. The mechanism of the flow field adjustment under the threshold of plugging regulation is summarized.

Procter and Gamble Student poster award:

References:

Acceptance of Terms and Conditions:

Click here to agree 


\title{
Seepage characteristics and fracture numerical experiments on the action of stress-seepage coupling of the rock with micro ran- dom fractures
}

\author{
Na ZHAO $^{1}$; Lai-gui WANG ${ }^{1}$ \\ ${ }^{1}$ College of Mechanics and Engineering, Liaoning Technical University
}

Corresponding Author(s): zhaona24@163.com

\begin{abstract}
Rock is a naturally formed porous medium material. There are a large number of irregular and multi-scale micro-pores in the rock. These micro-pores and fractures are closely related to the physical and chemical properties, pore structure and the seepage characteristics of fluid in rock. Firstly, through theoretical analysis, the influence on seepage characteristics of the size and structure characteristics of cracks and pore is given. Blockdyna, a block numerical simulation software based on Lagrangian mechanics, is used to generate a random fracture network with Weibull distribution to simulate fractured rock mass. The fractured rock is regarded as an equivalent continuum, and the permeability tensor and permeability representative elementary volume(REV) are studied. Based on the geometric characteristics of fractures, the effects of fracture density, trace length and dip angle on permeability coefficient of the rock mass are studied, and the permeability tensor and the representative elementary volume (REV) are determined. The results show that the the representative elementary volume decreases with the increase of fracture density, decreases first and then increases with the increase of fracture angle, and decreases gradually and tends to be stable with the increase of trace length. At the same time, numerical simulation of stress-seepage fracture of the rock mass with different fracture distribution is carried out. The coupling mechanism of heterogeneous fracture network and pore water pressure is analyzed from micro-scale. The influence of pore water pressure on rock fracture process and permeability characteristics is analyzed, and the fracture evolution law and crack propagation mechanism are given. The results show that the increase of stress-strain corresponding to linear elasticity and non-linear stage is relatively slow after considering pore water pressure. After the peak strength, the rock ruptures instantaneously, the stress drops sharply, and the permeability coefficient increases sharply. The peak strength and residual strength of the rock both decrease and tend to brittle failure. The calculation of seepage and stress-seepage fracture in fractured rock mass can provide theoretical guidance for the exploitation and utilization of fractured rock mass resources, such as oil and gas.

Keywords: random fracture; seepage characteristic; stress-seepage coupling; numerical experiment Acknowledge:

The research presented in this paper has been supported by National key R \& D plan \2017YFC1503102 \ХNational Natural Science Fund (51474121), General research projects of Liaoning Provincial Department of Education (LJYL035), Youth Fund of National Natural Science Fund (51304108).

Contact information:

Tel:+86-13591999886 E-mail: zhaona24@163.com
\end{abstract}

Procter and Gamble Student poster award:

References:

Acceptance of Terms and Conditions:

Click here to agree

163

\section{Fractal analysis of gas relative diffusion coefficient in porous nanofibers with rough surfaces}

Qian Zheng None

Corresponding Author(s): tbftbfzhengqian@163.com

Fractal model of gas diffusion through porous nanofibers with rough surfaces is derived, in which the porous structure is simplified to be composed of a bundle of tortuous capillaries whose pore size distribution and surface roughness follow the fractal scaling laws. The analytical expression for gas relative diffusion coefficient is a function of the relative roughness, fiber radius and the other 
microstructural parameters (porosity, the fractal dimension for pore size distribution and tortuosity, the maximum and minimum pore diameter and the characteristic length). The proposed fractal model is validated by comparison with available experimental data and correlations. At the same time, the effect of microstructural parameters of porous fibrous materials on gas diffusion has been studied in detail. It is believed that the current model may be extended to porous materials other than fibrous materials.

Procter and Gamble Student poster award:

References:

Acceptance of Terms and Conditions:

Click here to agree

270

\title{
Microscopic Residual Oil Recovery Effect by Different Displace- ment Methods
}

Taiyi Zheng ${ }^{\text {None }}$

Corresponding Author(s): 247672813@qq.com

The content and distribution of remaining oil is the important basis for designing and adjusting the development plan, and also the main basis for evaluating the economic benefit of oilfield. Through comparison of parallel experimental, research the state of microscopic remaining oil distribution, the morphological types and the oil displacement efficiency, after polymer(P) flooding, surfactant polymer compound system(SP) flooding and alkaline surfactant polymer compound system(ASP) flooding, further elaborate the different compound system flooding displacement mechanism clearly. The microscopic residual oil contains five kinds of microscopic remaining oil types, namely, columnarshaped residual oil, oil droplet residual oil, film-shaped residual oil, corner-shaped residual oil and cluster-shaped residual oil. The experimental results show that the remaining oil content after water flooding, from large to small, is cluster-shaped, columnar-shaped, film-shaped, corner-shaped and oil droplet residual oil. Further use of aqueous chemical flooding on the basis of water flooding, polymer flooding mainly displace cluster-shaped residual oil, SP flooding system can effective displace cluster-shaped, columnar-shaped, oil droplet remaining oil, and ASP flooding system have a greatly displacement effect for all types of remaining oil

Procter and Gamble Student poster award:

References:

Acceptance of Terms and Conditions:

Click here to agree

\section{Direct Visualization of Fluid in Nanopores - Fundamental and Ap- plication in Shale with Nanofluidics}

\author{
Junjie Zhong ${ }^{1}$; David Sinton ${ }^{1}$ \\ ${ }^{1}$ University of Toronto, MIE \\ Corresponding Author(s): junjie.zhong@mail.utoronto.ca
}

Phase change and fluid transport at nanoscale play important roles in various natural processes and engineering practices (e.g., shale gas/tight oil production). Fundamentally, they are different from that at the bulk scale. For the phase change, when the system size decreases to nanoscale, the 
saturation condition will change because of the rapid increase of capillarity and surface adsorption selectivity. For fluid transport, when the characteristic length of the system is close to the main free path of the fluid molecule, the description of transport behaviors can fundamentally deviate from NS equations.

Historically, most of the investigations on nanoscale phase change and fluid transport were carried out through gravimetric or volumetric measurement of adsorption, in fixed bed geometries, by interferometry or through surface force apparatus. Recent advances in nanotechnology allow for the direct observation of fluid behavior in deterministic structures such as nanochannel. In this presentation, we show our experimental works on studying the fundamental hydrocarbon thermodynamics and fluid mechanics in nanoconfinement, as well as many potential applications in helping industry understand/optimize primary or enhanced oil/gas recovery in tight formations.

Procter and Gamble Student poster award:

I don't want to compete References:

Acceptance of Terms and Conditions:

Click here to agree

100

\title{
Capillary imbibition governed by water adsorption in hygroscopic plant-like structure
}

\author{
Philippe Coussot $^{1}$; Meng Zhou ${ }^{2}$; Sabine Caré ${ }^{1}$ \\ ${ }^{1}$ Univ. Paris-Est \\ ${ }^{2}$ Saint-Gobain
}

Corresponding Author(s): meng.zhou720@gmail.com

Plant matter is being used increasingly in construction and in various other applications thanks to its remarkable porous and mechanical properties, but water transfers play a critical role on these properties and their possible alteration. Water in plants may be either in a "free" liquid state in capillaries, or in a "bound" state after adsorption in cell-walls, associated with significant deformation of the structure. Here we show that the coexistence of these two effects strongly affect the transport properties.

We demonstrate this from Synchrotron and MRI observation in hardwoods, which exhibit a relatively simple hydraulic structure. Capillary imbibition dynamics appears to be dramatically damped (velocity decreased by several orders of magnitude), but the liquid can still climb over significant heights (in contradiction with its dynamics) as soon as sufficient bound water has been adsorbed This contradiction is confirmed by 3D Synchrotron images of the internal structure obtained during imbibition, which show that the liquid-air interfaces in the capillary vessels remain planar, which implies negligible Laplace pressure, but significantly advance along the vessels, again unexpectedly. From MRI measurements allowing to distinguish bound and free water, but also direct measurements of the induced macroscopic deformation distribution in time, we then show that this contradiction is explained by the adsorption of a slight amount of bound water in the capillary walls. This adsorption governs the process: it momentarily damps wetting and then allows further advance later when the walls are saturated with bound water. The generality of the process for hygroscopic systems is demonstrated with a model material, i.e. hydrogel, from which both the position and shape evolution of liquid-air interface and the adsorption and propagation of bound water may be directly observed (see below). This suggests the development of bio-inspired porous materials able to absorb liquid with a tunable timing, for pharmaceutical or chemical engineering applications.

Procter and Gamble Student poster award:

\section{References:}

Acceptance of Terms and Conditions:

Click here to agree 


\title{
Similarity between Modeling of Solute and Heat Transport in Frac- tured Reservoirs with a Generalized Multirate Memory Function
}

\author{
Quanlin $\mathrm{ZHOU}^{1}$; Curtis Oldenburg ${ }^{1}$ \\ ${ }^{1}$ Lawrence Berkeley National Laboratory \\ Corresponding Author(s): qzhou@lbl.gov
}

Modeling solute and heat transport in fractured reservoirs has been conducted separately by two different communities. However, there are several similarities between solute and heat transport modeling: (1) solute diffusion and heat conduction in the rock matrix, (2) solute advection-dispersion and heat convection-dispersion in fractures, and (3) diffusive exchange of solute and heat between fractures and the rock matrix. The diffusive exchange can be handled by a generalized multirate memory function that takes into account the dimensionality, shape, size, and properties of individual matrix blocks in a representative elementary volume of fractured reservoirs (Zhou et al., 2017a, b). Rectangular matrix blocks can be formed by 1,2, and 3 orthogonal sets of parallel fractures with different spacing. The global transfer functions in the Laplace domain account for the solute or heat transport in the fracture network. A suite of semi-analytical solutions is developed by plugging the multirate memory function into the global transfer functions with various (1-D linear, 1-D radial, 2-D dipole, and single-well injection-withdrawal) flow fields, thereby greatly simplifying solution development (Zhou et al., 2018).

The huge difference between solute and heat transport modeling is in their matrix diffusivity and retardation factor, leading to a significant difference in the time scale of fracture-matrix equilibration. Such a significant difference can improve our understanding of the solute/heat transport in fractured reservoirs and the sensitivities of geometric and rock properties of matrix blocks in different transport regimes. The geometric sensitivities include (1) dimensionless surface area-to-volume ratio in the early-time regime of diffusion, (2) aspect ratios in the middle-time regime, and (3) shape factor in the late-time regime. The early-time regime is often involved in tracer tests because of small matrix diffusivity on the order of $1.0 \mathrm{E}-10 \mathrm{~m} 2 / \mathrm{s}$, while the late-time regime is often involved in thermal tests because of higher thermal matrix diffusivity on the order of $1.0 \mathrm{E}-6 \mathrm{~m} 2 / \mathrm{s}$. The tracer and thermal tests can be combined to better characterize the properties of the matrix blocks.

This presentation will focus on various sensitivities of solute and heat transport to the geometric and rock properties of matrix blocks. Both uniform flow and channelized flow in the fracture network are considered. These sensitivities will help show the values of monitoring in different diffusion regimes.

\section{Procter and Gamble Student poster award:}

I would like to compete in the Procter and Gamble Student award References:

Zhou, Q., C.M. Oldenburg, and J. Rutqvist, 2018. Revisiting the analytical solutions of heat transport in fractured reservoirs using a generalized multirate memory function, Water Resources Research (submitted on 9/24/2018).

Zhou, Q., C.M. Oldenburg, J. Rutqvist, J.T. Birkholzer, 2017. Revisiting the fundamental analytical solutions of heat and mass transfer: The kernel of multirate and multidimensional diffusion, Water Resources Research 53, 9960-9979, doi:10.1002/2017WR021040.

Zhou, Q., C.M. Oldenburg, L.H. Spangler, and J.T. Birkholzer, 2017. Approximate solutions for diffusive fracture-matrix transfer: Application to storage of dissolved CO2 in fractured rocks, Water Resources Research, 53(2), 1746-1762, doi:10.1002/2016WR019868 Acceptance of Terms and Conditions:

Click here to agree 


\section{A phase-field moving contact line model with soluble surfactants}

Guangpu Zhu ${ }^{1}$; Jun $\mathrm{Yao}^{2}$

${ }^{1}$ China University of Petroleum (East China)

${ }^{2}$ China University of Petroleum

Corresponding Author(s): b16020069@s.upc.edu.cn

A phase-field moving contact line model is proposed for a multiphase system with soluble surfactants. With the introduction of some scalar auxiliary variables, the original free energy functional is transformed into an equivalent form, and then a new governing system is obtained. The resulting model consists of two Cahn-Hilliard-type equations and incompressible Navier-Stokes equation with variable densities, together with the general Navier boundary condition for the moving contact line. We prove that the proposed model satisfies the total energy dissipation with time. To numerically solve such a complex system, we develop a nonlinearly coupled scheme with unconditional energy stability. A splitting method based on pressure stabilization is used to solve the Navier-Stokes equation. Some subtle implicit-explicit treatments are adopted to discretize convention and stress terms. We rigorously prove that the proposed scheme can preserve the discrete energy dissipation. An efficient finite difference method on staggered grids is used for the spatial discretization. Numerical results in both two and three dimensions demonstrate the accuracy and energy stability of the proposed scheme. Using our model and numerical scheme, we investigate the wetting behavior of droplets on the solid wall. Numerical results indicate that surfactants can affect the wetting properties of droplet by altering the value of contact angles.

Procter and Gamble Student poster award:

I would like to compete in the Procter and Gamble Student award References:

1 J.-J. Xu, W. Ren, A level-set method for two-phase flows with moving contact line and insoluble surfactant, J. Comput. Phys. , 263 (2014) 71-90.

2 S. Engblom, M. Do-Quang, G. Amberg, A.-K. Tornberg, On diffuse interface modeling and simulation of surfactants in two-phase fluid flow, Communications in Computational Physics, 14 (2013) 879-915. Acceptance of Terms and Conditions:

Click here to agree

\section{Pore-scale simulation of imbibition process in a 3D digital rock}

Guangpu Zhu' ${ }^{1}$; Jun Yao ${ }^{1}$

${ }^{1}$ China University of Petroleum (East China)

Corresponding Author(s): b16020069@s.upc.edu.cn

Carbon dioxide (CO2) enhanced oil recovery is a green and promising way to produce oil and reduce the rapid growth of carbon dioxide released to atmosphere. A pore-scale understanding of CO2 displacement phenomena is important to enhance oil recovery in porous media. In this work, a direct numerical simulation method is used to investigate the imbibition process of $\mathrm{CO} 2$ in a three dimensional digital rock. The interface between the oil and $\mathrm{CO} 2$ is tracked by the volume of fluid (VOF) method. The capacity and accuracy of the model are validated using a classic benchmark. A series of numerical experiments were performed over a large range of values of capillary number, viscosity ratio and contact angle to investigate the imbibition process of $\mathrm{CO} 2$ in a digital rock.

Procter and Gamble Student poster award:

References:

Acceptance of Terms and Conditions: 
Click here to agree

150

\title{
Pore-scale direct numerical simulations of non-continuum flows by solving kinetic model equations in OpenFOAM
}

\author{
Author(s): Lianhua Zhu ${ }^{1}$ \\ Co-author(s): Minh-Tuan $\mathrm{Ho}^{1}$; Peng Wang ${ }^{1}$; Yonghao Zhang ${ }^{1}$ \\ ${ }^{1}$ Department of Mechanical and Aerospace Engineering, University of Strathclyde \\ Corresponding Author(s): 1.zhu@strath.ac.uk
}

\begin{abstract}
Due to the ultra-small pores in tight porous media such as shale, the gas transport in those porous space falls into non-continuum flow regimes, and those porous media's permeability depends both on the geometry and on the gas media. Accurate pore-scale simulations based on the gas-kinetic equations can predicate apparent permeability directly. The direct simulation Monte Carlo (DSMC) method for rarefied gas dynamics has been applied to study pore-scale non-continuum flows, but the ultra-low speed flow in micro-scale porous media make the method extremely expensive. Until recently, direct Boltzmann kinetic model equation solver has been proposed as an effective alternative method [1,2]. In this talk, we will present another pore-scale kinetic equation solver developed in the OpenFOAM framework 3. The advantage of using arbitrary unstructured mesh in OpenFOAM allows us to resolve the gas-solid boundary with much higher accuracy than the stair-case approximation used in previous finite difference kinetic schemes. The factors that affect the solver's performance including the pore-space mesh resolution the discrete velocity set will be examined in detail. In addition, as a simple and direct application of the solver, we investigated the proper definition of the effective pore size (or characteristic Knudsen number) that should be used in many apparent permeability models, which are usually derived from simple geometry, to achieve consistent and better fitting.
\end{abstract}

Procter and Gamble Student poster award:

\section{References:}

$1 \mathrm{Wu}$, Lei, Minh Tuan Ho, Lefki Germanou, Xiao-Jun Gu, Chang Liu, Kun Xu, and Yonghao Zhang. "On the Apparent Permeability of Porous Media in Rarefied Gas Flows.” Journal of Fluid Mechanics 822 (2017): 398-417. https://doi.org/10.1017/jfm.2017.300.

2 Ho, Minh Tuan, Lianhua Zhu, Lei Wu, Peng Wang, Zhaoli Guo, Zhi-Hui Li, and Yonghao Zhang. "A Multi-Level Parallel Solver for Rarefied Gas Flows in Porous Media." Computer Physics Communications 234 (January 1, 2019): 14-25. https://doi.org/10.1016/j.cpc.2018.08.009.

3 Zhu, Lianhua, Songze Chen, and Zhaoli Guo. "DugksFoam: An Open Source OpenFOAM Solver for the Boltzmann Model Equation.” Computer Physics Communications 213 (April 2017): 155-64. https://doi.org/10.1016/j.cpc.2016.11.010. Acceptance of Terms and Conditions:

Click here to agree

\section{Experimental Studies of the Hydraulic Properties of Thin Porous Media}

\author{
Luwen Zhuang ${ }^{1}$; Majid Hassanizadeh ${ }^{2}$ \\ ${ }^{1}$ Utrecht University \\ ${ }^{2}$ Environmental Hydrogeology, Faculty of Geosciences, Utrecht University
}


Corresponding Author(s): 1.zhuang@uu.nl

Nowadays, thin porous media have attracted much attention because of their importance to many various industries. Hygiene products, paper, filters, fuel cells, membranes, textiles, muscular tissues, and other biological or manufacturing thin compositions widely exist in daily life. The typical characteristic of a thin porous layer is that its thickness is much smaller than its in-plane dimensions. A stack of thin porous layers creates contact interfaces, whose properties are quite different with thin layers. Furthermore, the hydraulic properties of a thin porous layer may vary along through-plane or in-plane directions. Determining the hydraulic properties is essential to understand and model fluid flow in thin porous media. Regarding the thickness and deformation, it is very difficult to measure the absolute permeability of a thin fibrous layer. In this work, we constructed a relatively simple experimental setup for measuring the absolute permeability of a thin fibrous layer. We performed a series of experiments using water or gas phase. The experimental results were compared with empirical formulas.

Procter and Gamble Student poster award:

References:

Acceptance of Terms and Conditions:

Click here to agree

320

\title{
An Auxiliary Space Method for Mixed Finite Element Discretiza- tions
}

\author{
Batista Juan $^{1}$; Francisco Gaspar ${ }^{2}$; Xiaozhe $\mathrm{Hu}^{3}$; Carmen Rodrigo $^{4}$; Ludmil Zikatanov ${ }^{1}$ \\ ${ }^{1}$ The Pennsylvania State University \\ ${ }^{2}$ University of Zaragoza \\ ${ }^{3}$ Tufts University \\ ${ }^{4}$ Universidad de Zaragoza
}

Corresponding Author(s): ludmil@psu.edu

We propose a an auxiliary space method for the solution of the indefinite problem arising from mixed method finite element discretizations of scalar elliptic problems. The proposed technique uses conforming elements as auxiliary space and utilizes special interpolation operators for the transfer of residuals and errors between the spaces. We show that the corresponding method provides optimal solver for the indefinite problem by only solving symmetric and positive definite auxiliary problems.

Procter and Gamble Student poster award:

I would like to compete in the Procter and Gamble Student award References:

Acceptance of Terms and Conditions:

Click here to agree

147

\section{Wicking simulations of cryogenic liquid into superheated porous structures}

Dawid Zimnik None

Corresponding Author(s): dawid.zimnik@zarm.uni-bremen.de 
Phase separation in spacecrafts is a challenge in microgravity, because spacecraft engines require a gas-free supply of propellant over a wide range of thermal environments. A method to separate phases is to use propellant management devices. They use porous materials to separate gas from liquid and to transport the liquid to the tank outlet. Cryogenic liquids are common as propellants for spacecraft engines, but they are often subjected to thermal gradients, which leads to evaporation in a porous structure. Evaporation is an undesirable effect, because it can dry out the porous structure and therefore reduce the performance of such a structure. Therefore, the wicking process subjected to evaporation has to be understood.

Numerical simulations of the wicking process in a porous material, subjected to evaporation, are performed to predict the liquid behavior inside the porous material. A superheated porous structure is dipped into a cryogenic liquid at saturation temperature. Due to the heat transfer between the superheated structure and the liquid, the liquid start to evaporate, which affect the wicking process. Wicking experiments with liquid nitrogen were performed to validate the numerical model.

Procter and Gamble Student poster award:

References:

Acceptance of Terms and Conditions:

Click here to agree

\title{
Three-Dimensional Simulation of Density-Driven Natural Con- vection in Porous media for CO2 Geological Storage
}

\author{
Author(s): Pacelli Zitha ${ }^{1}$ \\ Co-author(s): Panneerselvam Ranganathan ${ }^{2}$ \\ ${ }^{1}$ TU Delft \\ ${ }^{2}$ National Institute of Technology Calicut \\ Corresponding Author(s): pacelli.zitha@bingagroup.com
}

The dissolution of $\mathrm{CO} 2$ in the aqueous phase is one of the most significant physical processes during geological storage of $\mathrm{CO} 2$. It causes density-driven convective mixing, which enables a permanent sequestration of $\mathrm{CO} 2$, reduces the risk of leakage of the stored $\mathrm{CO} 2$ and ensures a long-term $\mathrm{CO} 2$. This paper reports a three-dimensional numerical simulation of density-driven natural convection during geological $\mathrm{CO} 2$ storage in homogeneous and heterogeneous formations. The heterogeneity of the formations is represented by spatial variations of the permeability, generated using sequential gaussian simulation (SGSIM) method. The convective motion of dissolved CO2-brine mixture is investigated in terms of $\mathrm{CO} 2$ concentration, isosurface profiles and the average $\mathrm{CO} 2$ mass flux at top boundary of the flow domain. At early times, the $\mathrm{CO} 2$ concentration profiles show the formation of many small fingers. The $\mathrm{CO} 2$ isosurface profiles, they indicate the primarily transverse mixing process at the early stages where the formation of transverse roll cell is dominant and at later stage the vertical mixing where the formation of large-scale fingering is enhanced. At later times the number of fingers diminishes as fingers merge together. The average $\mathrm{CO} 2$ mass flux at the top boundary decreases quickly at earlier time, then it increases slowly towards stabilised value at the later times. The effect of Rayleigh number on convective motions is further investigated in this study.

Procter and Gamble Student poster award:

I don't want to compete References:

Acceptance of Terms and Conditions:

Click here to agree 


\section{Capillary-driven transport of dissolved salt to the drying zone during $\mathrm{CO} 2$ injection in homogeneous and layered porous me- dia.}

Pacelli Zitha ${ }^{1}$; Saskia Roels ${ }^{2}$

${ }^{1}$ TU Delft

${ }^{2}$ SRK Consulting

Corresponding Author(s): pacelli.zitha@bingagroup.com

$\mathrm{CO} 2$ injection into saline aquifers poses a serious risk of formation clogging due to ensuing salt precipitation. Capillary-driven flow of brine can provide a continuous transport of dissolved salt toward the dry zone around the injection well where it ultimately precipitates due to evaporation. In this study, core flooding experiments were performed in homogeneous coarse-textured cores and in layered cores consisting of a coarse-textured layer overlying a fine-textured layer. CO2 was injected through a well in the upper part of the cores, and the bottom parts functioned as brine sources. Impairment in injectivity was found due to accumulation of precipitated salt caused by capillarydriven flow from the brine sources to the upper dryer region. Compared to flow domains without a brine source, we found that capillary-driven upward flow at first prevents complete clogging because the porous medium remains wet, but eventually leads to a more severe clogging of the entire domain. The results show that after sufficient dry-out, a coarse-textured injection layer can draw brine from an underlying fine-textured layer by capillary forces. A connected fine-textured layer can therefore contribute to salt precipitation and clogging of the injection layer.

Procter and Gamble Student poster award:

\section{References:}

H. Ott, S. M. Roels, K. de Kloe., (2015). Salt precipitation due to supercritical gas injection: capillarydriven flow in unimodal sandstone. International Journal of Greenhouse Gas Control, 2015.

Roels, S.M., Ott, H., Zitha, P.L.J., (2014). $\mu$-CT analysis and numerical simulation of CO2 injection into brine-saturated sandstone. Int. Journal of Greenhouse Gas Control 27, 146-154. Acceptance of Terms and Conditions:

Click here to agree

\section{Multiscale and multiphysics models for geomechanics}

Author(s): Paolo Zunino ${ }^{1}$

Co-author(s): Daniele Cerroni ${ }^{1}$; Luca Formaggia $^{1}$; Anna Scotti ${ }^{1}$

${ }^{1}$ MOX, Department of Mathematics, Politecnico di Milano

Corresponding Author(s): paolo.zunino@polimi.it

The coupled multiphase flow-geomechanics behavior in the presence of a highly heterogeneous media involving different spatial and temporal scales presents difficulties for reliable numerical simulations. In a reservoir, the fluid flow behavior and the deformation of solid skeleton are strongly coupled, thereby requiring the mathematical models and numerical methods to take this into account. Furthermore, both the naturally occurring and induced fractures have strong influences on both flow and mechanical behavior of the reservoir. On the other hand, the flow in a reservoir may cause a change in situ stress conditions and pore pressures leading to formation of new fractures and other geomechanical effects. The coupling of processes, however, complicates the development of efficient and high fidelity numerical simulation tools. Present capabilities in commercial simulators are limited to ad hoc coupling of solid mechanics and flow modules. We introduce and discuss new approaches and algorithms for multiscale simulations of coupled multiphase porous media flow and geomechanics in complex domains. 
Procter and Gamble Student poster award:

\section{References:}

(1) Ambartsumyan, I., Khattatov, E., Yotov, I., Zunino, P.

A Lagrange multiplier method for a Stokes-Biot fluid-poroelastic structure interaction model (2018) Numerische Mathematik, 140 (2), pp. 513-553.

(2) Bukač, M., Yotov, I., Zunino, P. 55521654500;6701409727;18839699700;

Dimensional model reduction for flow through fractures in poroelastic media (2017) ESAIM: Mathematical Modelling and Numerical Analysis, 51 (4), pp. 1429-1471.

(3) Giovanardi, B., Formaggia, L., Scotti, A., Zunino, P.

Unfitted FEM for modelling the interaction of multiple fractures in a poroelastic medium (2017) Lecture Notes in Computational Science and Engineering, 121, pp. 331-352.

(4) Guadagnini, A., Guadagnini, L., Porta, G., Cerroni, D., Formaggia, L., Scotti, A., Zunino, P., Ruffo, P. Modeling the feedback between glaciation, geochemical and mechanical compaction on sedimentary basin evolution

(2017) Offshore Mediterranean Conference and Exhibition 2017, OMC 2017.

(5) Flemisch, B., Berre, I., Boon, W., Fumagalli, A., Schwenck, N., Scotti, A., Stefansson, I., Tatomir, A. Benchmarks for single-phase flow in fractured porous media (2018) Advances in Water Resources, 111, pp. 239-258. Acceptance of Terms and Conditions:

Click here to agree 